Combined Loadings in

the Theory of Plasticity 


\section{Combined Loadings in the Theory of Plasticity}

Michał Życzkowski

Cracow Technical University 
The first English edition based on

Obciażenia zlożone $w$ teorii plastyczności

published in 1973 by Państwowe Wydawnictwo Naukowe, Warszawa

Translation: Michal Życzkowski

Graphic design: Zygmunt Ziemka

Copyright (C) 1981 by PWN-Polish Scientific Publishers, Warszawa

All rights reserved. No part of this publication may be reproduced, stored in a retrieval system, or transmitted, in any form or by any means, electronic, mechanical, photocopying, recording or otherwise, without the prior permission of the copyright owner.

Distributor:

ARS POLONA-RUCH

Krakowskie Przedmieście 7, 00-068 Warszawa, Poland

ISBN 83-01-01818-6

Printed in Poland by D.R.P. 


\section{To my Family}

for their patience and help 


\section{Preface}

The analysis of combined loadings in the plastic range, very important both from the theoretical and from the engineering viewpoint, is one of the more difficult branches of the theory of plasticity. Recently the number of publications in this field has increased so rapidly that the lack of a monographic treatment is evident.

The purpose of the present book is to fill this gap. It aims at a systematization of the basic concepts, a description of methods of solution of typical problems and a presentation of the more important solutions; above all, however, it furnishes research workers and engineers with a guide to a very extensive bibliography. A classification of combined loadings has been introduced-successively at the levels of a point of the body $\mathscr{P}$, of a cross-section $\mathscr{S}$, and of the body as a whole $\mathscr{B}$-and has been used consistently throughout the book. As a rule the generally accepted concepts and ideas have been used, but in certain cases the author has considered it appropriate to introduce some new definitions, e.g. that of a spontaneous process, of a statically partially admissible stress field, of a stress-quasi-cycle, of a strainquasi-cycle, of the decohesive carrying capacity, etc.

Particular attention has been paid to the provision of a comprehensive list of references. The enclosed bibliography, given with full details, is allocated to individual chapters, but gathered at the end of the book. It has more than 3000 entries and is estimated by the author to constitute about 90 per cent of world literature in the field of combined loadings in plasticity. Unfortunately, for technical reasons the list of references had to be closed with the years 1975-1977 (to the successive chapters), and more recent entries could not be included. The references to each chapter are ordered alphabetically, but in the' text they are discussed in chronological order. The titles of the papers published 'in Latin alphabet are given in the original language; in the case of other alphabets they were translated into English (mainly papers published in Russian, Ukrainian, Bulgarian, Chinese and Japanese). The titles of journals, proceedings, etc. are abbreviated; index of abbreviations introduced is enclosed at the end of Bibliography.

Formally, the book is a translation of the Polish original, published by the Polish Scientific Publishers, PWN, in 1973. In fact, it is a new book: the introduction of over 1800 new references and of numerous new topics resulted in an increased volume.

The book, in spite of its monographic character, pays particular attention to 
the didactic aspects of the subject and may also be used as auxiliary material in teaching a course in Plasticity. The reader is expected to have taken the usual course in the Strength of Materials, as given by the engineering faculties of the universities, and also to have been given an introduction to the theory of elasticity and plasticity and to the tensorial calculus.

Finally, the author would like to express his deep gratitude to the reviewers and scientific editors of the book, Professors W. Szczepiński, A. Sawczuk and J. A. König from the Institute of Fundamental Technological Research of the Polish Academy of Sciences in Warsaw, and to numerous colleagues from the Technical University of Cracow-above all, to Professors M. Chrzanowski, Z. Kordas, W. Krzyś, J. Orkisz, S. Piechnik, J. Ryś, J. Skrzypek, G. Szefer and Z. Waszczyszyn, to Doctors J. Białkiewicz, K. Kowalczyk, T. Liszka, K. Szuwalski, R. Wojdanowska and A. Zieliński, as well as to Mr. Mr. E. Cegielski, A. Dudek, J. Kraus, J. Krużelecki, M. Kur, T. Kurtyka, S. Laczek, M. Rysz, H. Sanecki, M. Stodulski and A. Trojnacki-for reading the individual chapters of the book and offering many valuable remarks, and for their help in drawing the figures. Grants 05.12 and J-23 are also gratefully acknowledged.

Michał Życzkowski Cracow, June 1979 


\section{Contents}

Preface

Index of more important symbols

\section{CHAPTER I Basic concepts of the theory of combined loadings}

1 Levels of analysis of plastic deformations

1.1 Introductory remarks . . . . . . . . . . . . . . . . . . . . . . . . . 1

$\begin{array}{ll}\text { 1.2 Definitions of the levels of analysis and the classification symbolism adopted } 2 & 2\end{array}$

1.3 Exertion factors at the level of a point of the body $(\mathscr{P})$. . . . . . . . . . . 3

1.4 Exertion factors at the level of a cross-section $(\mathscr{P})$. . . . . . . . . . . 4

1.5 Exertion factors at the level of the body as a whole $(\mathscr{B})$. . . . . . . . . . 8

1.6 Basic exertion factors . . . . . . . . . . . . . . . . . . 9

2 Active and passive processes. Loading and unloading

2.1 Definition of plastically active and plastically passive processes . . . . . . 9

2.2 Loading and unloading in the case of one exertion factor . . . . . . . . . 10

2.3 Loading and unloading in the case of several exertion factors . . . . . . . 13

2.4 Interrelation between loading and unloading, active and passive processes at various levels ................. 15

3 Concept of the interaction surface (limit surface)

3.1 Space of the exertion factors . . . . . . . . . . . . . . . . . . . . 16

3.2 Typical interaction surfaces . . . . . . . . . . . . . . . . . . . 17

3.3 Distance function. Exertion of the material and of the structure . . . . . . 18

4 Classification of combined loadings

4.1 Symbolism of the classification . . . . . . . . . . . . . . . . . . . . 21

4.2 Examples of the classification . . . . . . . . . . . . . . . 22

4.3 The problem of independence of the exertion factors . . . . . . . . 25

\section{CHAPTER II Basic equations of the theory of plasticity}

5 Systems of invariants of second-order tensors

5.1 Number of independent invariants . . . . . . . . . . . . . . . . . . . 26

5.2 Basic invariants of the tensor . . . . . . . . . . . . . . . . . . . . 27

5.3 Principal invariants of the tensor . . . . . . . . . . . . . . . . . . . 28

5.4 Cylindrical invariants of the tensor . . . . . . . . . . . . . . . . . . 29

5.5 Invariants of the axiator and of the deviator . . . . . . . . . . . . . 31

5.6 The Lode parameter . . . . . . . . . . . . . . . . . . . . . . 32 
5.7 Tensor of directions and similarity . . . . . . . . . . . . . . . . . 33

5.8 Calculation of the principal values of the tensor . . . . . . . . . . . 33

5.9 Common invariants of several tensors . . . . . . . . . . . . . . 34

6 Stress and stress rate

6.1 Engineering and indicial notation ... . . . . . . . . . . 35

6.2 Transformation of stresses, stress invariants . . . . . . . . . . . . . . . 36

6.3 Equations of internal equilibrium . . . . . . . . . . . . . 37

6.4 Stress rates . . . . . . . . . . . . . . . . . . . . 39

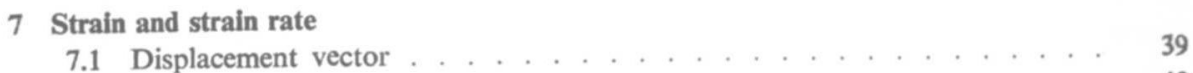

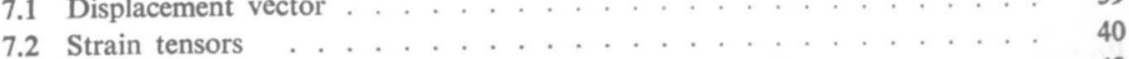

7.3 Small strains in cylindrical and spherical coordinates . . . . . . . . . . . 43

7.4 Decomposition of the strain tensor. Dilatation . . . . . . . . . . . . . . 44

7.5 Invariants of the small-strain tensor . . . . . . . . . . . . . . . . . . 44

7.6 Strain rates . . . . . . . . . . . . . . . . 46

7.7 Logarithmic or natural strain . . . . . . . . . . . . . . . . . . . . . 47

7.8 Equations of compatibility . . . . . . . . . . . . . . . . . . . 49

8 Uniaxial plasticity

8.1 True stress-strain diagram . . . . . . . . . . . . . . . . . . . 51

8.2 Perfectly plastic solids . . . . . . . . . . . . . . . . . . . . . . 53

8.3 Schematizations of plastic hardening . . . . . . . . . . . . . . . 55

8.4 Special schematizations . . . . . . . . . . . . . . . . . . . 57

8.5 The Bauschinger effect . . . . . . . . . . . . . . . . . . . . 58

8.6 Schematizations of cyclic plasticity . . . . . . . . . . . . . . . 60

8.7 Models of plastic bodies . . . . . . . . . . . . . . . . . . . . . . 62

8.8 Plastic strain. Plastic work . . . . . . . . . . . . . . . . . . 64

9 Classical theories of perfect plasticity

9.1 Elastic range Hooke's law _. . . . . . . . . . . . . . . . . . . . . . . . . $\quad 67$

9.2 Limitation of the elastic range . . . . . . . . . . . . . . . $\quad 69$

9.3 Conditions of perfect plasticity (yield conditions) . . . . . . . . . . $\quad 70$

9.4 The law of volume change. Coefficient of transversal deformation . . . . . $\quad 72$
9.5 The

$\begin{array}{lll}\text { 9.5 The Hencky-Ilyushin theory of small elastic-plastic deformations . . . . . . } \quad 73 & 78\end{array}$

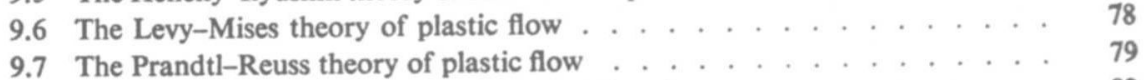

9.8 Coincidence of the deformation and flow theories . . . . . . . . . . . 82

9.9 Example of a comparison of the classical theories . . . . . . . . . . . 85

\section{CHAPTER III Combined loadings at the level 9 of a point of the body}

10 Auxiliary spaces at the level $\mathscr{P}$

10.1 Nine-dimensional spaces

10.2 Six-dimensional spaces $\quad 88$

10.3 Five-dimensional spaces . . . . . . . . . . . . . . . . . . . . . 89

10.4 Three-dimensional spaces . . . . . . . . . . . . . . . . . . 90

10.5 Two-dimensional spaces, planes . . . . . . . . . . . . . . . . . . . 91

10.6 Isotropy and isosensitivity . . . . . . . . . . . . . . . . . . 93

11 Conditions of perfect plasticity for isotropic bodies

11.1 General remarks ... . . . . . . . . . . . . . . . . . 94

11.2 Cylindrical limit surfaces . . . . . . . . . . . . . . . . . 95 
11.3 Rotationally symmetric limit surfaces . . . . . . . . . . . . . . . . . 100

11.4 Other limit surfaces in the space of principal stresses . . . . . . . . . . . 103

11.5 General form of the "ad hoc" elliptic yield condition . . . . . . . . . . 104

11.6 The influence of accessory exertion factors . . . . . . . . . . . . . . . 105

11.7 The range of applicability of the yield conditions . . . . . . . . . . . 107

12 Conditions of perfect plasticity for anisotropic bodies

12.1 Generalizations of the Huber-Mises-Hencky yield condition . . . . . . . 109

12.2 Generalizations of the Tresca-Guest yield condition . . . . . . . . . . 112

12.3 Generalizations of the Burzyński yield condition . . . . . . . . . . . . 113

12.4 Yield conditions in the case of structural anisotropy . . . . . . . . . . 113

13 Drucker's definition of a stable plastic material. Constitutive equations of perfect plasticity

13.1 Plastic potential . . . . . . . . . . . . . . . . . . . 115

13.2 The notions of cycles in the theory of plasticity . . . . . . . . . . . 119

13.3 Drucker's definition of a stable plastic material (Drucker's postulate) . . . . 120

13.4 Implications of the Drucker postulate . . . . . . . . . . . . . . . . . 122

13.5 Deviations from the Drucker postulate . . . . . . . . . . . . . . . . . 124

13.6 Material stability of a perfectly elastic-plastic solid . . . . . . . . . . . 126

13.7 The Drucker postulate in the case of approximate yield conditions . . . . . 129

13.8 General constitutive equations of perfect plasticity . . . . . . . . 135

14 Subsequent neutral surfaces during plastic hardening

14.1 Hypothesis of a uniform curve of plastic hardening . . . . . . . . . . . 137

14.2 Hypotheses of isotropic hardening . . . . . . . . . . . . . . . . . . . 141

14.3 Generalized isotropic hardening . . . . . . . . . . . . . . . 144

14.4 Hypotheses of kinematic hardening . . . . . . . . . . . . . . . . . 145

14.5 A triple analogy for kinematic hardening . . . . . . . . . . . . . . . 149

14.6 Hypotheses of mixed hardening . . . . . . . . . . . . . . . . . . 154

14.7 Problem of the formation of plastic corners . . . . . . . . . . . . . . . 155

14.8 Hypotheses of general anisotropic hardening . . . . . . . . . . . . 157

14.9 Concept of double neutral surfaces . . . . . . . . . . . . . . . 163

14.10 Neutral surfaces in the strain space . . . . . . . . . . . . . . . . 165

14.11 Experimental investigations of neutral surfaces and difficulties in their interpretation

15 Constitutive equations of plastic hardening

15.1 General remarks . . . . . . . . . . . . . . . . . . . . 172

15.2 Total (deformation, holonomic) theories of plasticity . . . . . . . . . 172

15.3 Incremental (flow, anholonomic) theories of plasticity . . . . . . . . . . 175

15.4 Generalizations of incremental theories . . . . . . . . . . . . . . . 181

15.5 The Ilyushin geometrical theory of plasticity . . . . . . . . . . . . . 185

15.6 Theories based on the concept of slip .. . . . . . . . . . . . . . . . . . . 189

15.7 Plastic deformations at variable temperature . . . . . . . . . . . . . 193

15.8 Theories of plastic softening . . . . . . . . . . . . . . . . . 195

15.9 Theories of finite plastic strains . . . . . . . . . . . . . . . . . . . 196

16 Constitutive equations of the theory of plastically anisotropic materials

16.1 Flow rules for perfectly plastic anisotropic bodies . . . . . . . . . . . . 199

16.2 Hypothesis of a uniform curve of plastic hardening in the case of anisotropy 201

16.3 Subsequent neutral surfaces for anisotropic bodies . . . . . . . . . . 202

16.4 Total (deformation) theories of anisotropic plasticity . . . . . . . . . . . 203

16.5 Incremental theories of anisotropic plasticity . . . . . . . . . . . . . . 204 


\section{CHAPTER IV Transition from the local level $\mathscr{P}$ to the integral levels $\mathscr{S}$ and $\mathscr{B}$}

17 Limit carrying capacity

17.1 Concept of the limit carrying capacity . . . . . . . . . . . . . . . . . 206

17.2 Influence of elastic strains . . . . . . . . . . . . . . . . . 209

17.3 Influence of geometry changes (abandonment of the principle of rigidification) . . . . . . . . . . . . . . . . . . 210

17.4 Influence of plastic hardening . . . . . . . . . . . . . . . . . . 214

17.5 Maximal carrying capacity resulting in necking or bulging . . . . . . . 216

17.6 Limit carrying capacity and full plastification of the body . . . . . . . 217

18 Decohesive carrying capacity

18.1 Cases of non-existence of solutions in perfect plasticity and the concept of decohesive carrying capacity . . . . . . . . . . . . . . . . 221

18.2 Bars and bar systems . . . . . . . . . . . . . . . . . . . . . . . . 223

18.3 The Stüssi-Kollbrunner paradox . . . . . . . . . . . . . . . . . . . 229

18.4 Decohesive carrying capacity of disks and infinite circularly-symmetric sheets 233

18.5 Decohesive carrying capacity of disks and sheets in the light of finite-strain theory ................... 240

18.6 Stages of typical deformation processes at the levels $\mathscr{S}$ and $\mathscr{O}$. . . . . 243

18.7 Physical criteria of decohesion under static (monotonic) loadings . . . . . 244

18.8 Decohesion as a result of variable repeated loadings . . . . . . . . . 246

19 Methods of reduction of the number of plasticity equations

19.1 List of the equations of the classical theory of perfect plasticity . . . . . . . . 251

19.2 Displacement equations of the theory of plasticity . . . . . . . . . . . . 253

19.3 Stress functions . . . . . . . . . . . . . . . . . . 255

19.4 Parametrization of the yield condition . . . . . . . . . . . . 260

20 Analytical methods of solution

20.1 General remarks. Geometrical hypotheses . . . . . . . . . . . . . . 262

20.2 Statically admissible solutions . . . . . . . . . . . . . . . . . . . 263

20.3 Kinematically admissible solutions . . . . . . . . . . . . . . . . . 266

20.4 Statically partially admissible solutions . . . . . . . . . . . . . 269

20.5 Variational methods . . . . . . . . . . . . . . . . . . . 270

20.6 Perturbation (small parameter) method . . . . . . . . . . . . . . . . 273

20.7 Approximation of the Hermitian type . . . . . . . . . . . . . . . 276

20.8 Onefold optimal approximation . . . . . . . . . . . . . . . . . . 277

21 Numerical methods in plasticity

21.1 General remarks . . . . . . . . . . . . . . . . . . . . . . . . 277

21.2 Direct integration method. Integration along characteristics . . . . . . . 278

21.3 Finite difference method . . . . . . . . . . . . . . . . . . . . . . 281

21.4 Finite element method . . . . . . . . . . . . . . . . . . . . . . . . 282

21.5 Iteration and incremental (step-by-step) procedures . . . . . . . . . . 286

21.6 Mathematical programming methods . . . . . . . . . . . . . . . . 289

21.7 Other methods . . . . . . . . . . . . . . . . . 292

CHAPTER $V$ Combined loadings at the level $\mathscr{S}$ of a cross-section of a bar; type $\mathscr{P}_{1} / \mathscr{S}_{n}$

22 Elastic-plastic bending with normal force

22.1 General remarks

22.2 Constitutive equations of perfectly elastic-plastic bending with tension or compression of bars with an arbitrary cross-section 
22.3 Approximate assumption of unstretchability of the axis . . . . . . . . . . 299

22.4 Constitutive equations of perfect plasticity for a rectangular cross-section 300

22.5 Limit curves . . . . . . . . . . . . . . . . . . . . 302

22.6 Subsequent neutral curves. Shakedown problems . . . . . . . . . . 306

22.7 Finite deflections of beams with a rectangular cross-section . . . . . . . . 311

22.8 Multipoint substitutive cross-sections . . . . . . . . . . . . . . . 316

22.9 Constitutive equations for multipoint sections . . . . . . . . . . . . . . 318

22.10 Finite deflections of beams with multipoint sections . . . . . . . . . . . 320

22.11 Plastic hardening. Structural applications . . . . . . . . . . . . . . . 321

23 Limit carrying capacity of a section under bending with normal force

23.1 Plastic interaction curves represented by generalized power series . . . . . 323

23.2 Particular cases of power series . . . . . . . . . . . . . 326

23.3 Power series for typical shapes of the cross-section . . . . . . . . . . . . . 329

23.4 Application of the onefold optimal approximation . . . . . . . . . . 333

23.5 Nonhomogeneity of the material. Structural applications . . . . . . . . 335

24 Biaxial (oblique) bending

24.1 Elastic-plastic biaxial bending with normal force . . . . . . . . . . . 335

24.2 Elastic-plastic biaxial bending of bisymmetric sections . . . . . . . . . . 338

24.3 Limit carrying capacity under biaxial bending with normal force . . . . . . 339

24.4 Limit carrying capacity of a rectangular section under biaxial bending . . . . 340

25 Torsion with shear

25.1 Formulation of the problem ................. 342

25.2 Solution for homogeneous material . . . . . . . . . . . . . . . 345

25.3 Example of material non-homogeneity . . . . . . . . . . . . . . 346

CHAPTER VI Combined loadings at the level $\mathscr{S}$ of a cross-section of a bar; type $\mathscr{P}_{2} / \mathscr{S}_{n}$

26 Introductory remarks and general methods

26.1 Yield condition . . . . . . . . . . . . . . . . . . . . . . . 348

26.2 Lower bounds to the limit carrying capacity . . . . . . . . . . . . . . 349

26.3 Upper bounds to the limit carrying capacity . . . . . . . . . . . . . 350

27 Tension with torsion

27.1 Limit carrying capacity of round cross-sections . . . . . . . . . . . . . 352

27.2 Elastic-plastic range in the case of incompressibility . . . . . . . . . . . . 354

27.3 Influence of elastic compressibility . . . . . . . . . . . . . . . . . 357

27.4 Differential equation in displacements for arbitrary shapes of the cross-section 361

27.5 Perturbation method. Elastic-plastic range . . . . . . . . . . 364

27.6 Plastic tension with torsion of naturally twisted bars . . . . . . . . 366

28 Bending with torsion

28.1 Differential equation in displacements . . . . . . . . . . . . . 372

28.2 Solutions for a circular section by means of the perturbation method . . . . 374

28.3 Approximations and bounds for a circular cross-section . . . . . . . . . . . . 380

28.4 Non-circular sections . . . . . . . . . . . . . . . . . . . 382

28.5 Structural applications and experimental investigations . . . . . . . . . . 384 
29 General case of longitudinal homogeneity of the stress state

29.1 The Handelman-Hill equation . . . . . . . . . . . . . . . . . . . . 385

29.2 Differential equation in displacements . . . . . . . . . . . . . . . . 387

29.3 Straight bending with torsion and tension . . . . . . . . . . . . . . . 388

29.4 Biaxial bending with torsion ... . . . . . . . . . . . . . . . 389

30 Bending with shear

30.1 Introductory remarks . . . . . . . . . . . . . . . . . . . . . . 389

30.2 Basic hypotheses regarding stress distribution . . . . . . . . . . . . . 391

30.3 Limit carrying capacity of a rectangular section . . . . . . . . . . . . . 396

30.4 Thin-walled cross-sections . . . . . . . . . . . . . . . . . . . 399

30.5 Elastic-plastic range, plastic hardening . . . . . . . . . . . . . . . . . 401

31 More general cases of combined loadings

31.1 Bending with tension and shear . . . . . . . . . . . . . 402

31.2 More general cases of combined loadings of thin-walled cross-sections . . . . 404

CHAPTER VII Combined loadings at the level $\mathscr{S}$ of a cross-section of a surface structure

32 Purely membrane states $\mathscr{P}_{3} \rightarrow \mathscr{S}_{3}$, and purely moment states $\mathscr{P}_{3} / \mathscr{S}_{3}$

32.1 General remarks

32.2 Membrane states . . . . . . . . . . . . . . . . 413

32.3 Elastic-plastic moment states ..... . . . . . . . . . . . 416

32.4 Limit moment states . . . . . . . . . . . . . . . . . . . . . 421

33 Combined loadings of the type $\mathscr{P}_{2} / \mathscr{S}_{4}$ in uniform sections

33.1 Limit carrying capacity for the $\mathrm{HMH}$ yield condition . . . . . . . . . . . 424

33.2 Limit carrying capacity for the TG yield condition . . . . . . . . . . . . 427

33.3 Other isotropic yield conditions . . . . . . . . . . . . . . . . . . . 434

33.4 Natural and structural orthotropy . . . . . . . . . . . . . . . . . . . 436

33.5 Elastic-plastic range, plastic hardening . . . . . . . . . . . . . . . . . 437

34 Combined loadings of the type $\mathscr{P}_{2} / \mathscr{S}_{4}$ in sandwich sectipns

34.1 Introductory remarks . . . . . . . . . . . . . . . . . . . . . 441

34.2 Limit carrying capacity for the HMH yield condition . . . . . . . . . . . 442

34.3 Limit carrying capacity for the linear yield conditions . . . . . . . . . . . . 444

34.4 Elastic-plastic range, plastic hardening . . . . . . . . . . . . . . . 445

35 More general combined loadings

35.1 Introductory remarks ... . . . . . . . . . . . . . . . 450

35.2 Uniform section in the case $\mathscr{P}_{3} / \mathscr{P}_{6} \ldots \ldots$. . . . . . . . . . . . . . 451

35.3 Effect of transverse shearing forces . . . . . . . . . . . . . . . . . 457

35.4 Sandwich section . . . . . . . . . . . . . . . . . . 459

35.5 Plastic anisotropy . . . . . . . . . . . . . . . . . 460

CHAPTER VIII Combined loadings at the level $\mathscr{B}$ of the body as a whole

36 Combined loadings of bars and bar systems

36.1 Introductory remarks . . . . . . . . . . . . . . . . . . 462

36.2 Effect of geometry changes . . . . . . . . . . . . . . . . . 467

36.3 Interaction curves (limit curves) for simple models allowing for geometry changes .................. . . 468 
36.4 Combined surface loadings . . . . . . . . . . . . . . . . . . . . . . 478

36.5 Body forces, thermal loadings . . . . . . . . . . . . . . . . . . . . . 481

37 Circularly symmetric combined loadings of cylinders

37.1 Introductory remarks . . . . . . . . . . . . . . . . . . . . . . . . 482

37.2 General circularly symmetric case in thick-walled tubes . . . . . . . . . . 483

37.3 Particular cases of combined surface loadings . . . . . . . . . . . . . . 489

37.4 Body forces and thermal loadings . . . . . . . . . . . . . . . . . . . 496

37.5 Combined loadings of thick-walled spheres . . . . . . . . . . . . . . . 500

37.6 Experimental investigations of thin-walled tubes under combined loadings . . 500

38 Combined loadings of cylinders without circular symmetry

38.1 General case of combined loadings of a thin-walled tube . . . . . . . . . 502

38.2 Particular cases for a thin-walled tube . . . . . . . . . . . . . . . 508

38.3 Effect of circumferential bending of the wall . . . . . . . . . . . . . 510

38.4 General longitudinally homogeneous case of combined loadings of a thickwalled tube . . . . . . . . . . . . . . . . . . . 512

38.5 A perturbation solution for prevailing pressure . . . . . . . . . 515

38.6 A perturbation solution for prevailing torsion . . . . . . . . . . . . 518

38.7 The Hermitian boundary approximation for the plastic interaction surface 521

38.8 Elastic-plastic range. Other cases of non-symmetric combined loadings . . . 523

39 Disks and sheets under combined in-plane loadings

39.1 Annular disks under surface loadings . . . . . . . . . . . . . . . . . . 523

39.2 Rectangular sheets. Sheets with holes . . . . . . . . . . . . . . . . 525

39.3 Disks and sheets under body forces and thermal loadings . . . . . . . . . 527

39.4 Decohesive carrying capacity of an infinite sheet under combined surface and thermal loadings .

40 Combined loadings of plates and shells

40.1 Plates under surface loadings . . . . . . . . . . . . . . . . . . . . 532

40.2 Plates under body forces and thermal loadings . . . . . . . . . . . . . 534

40.3 Shells under surface loadings . . . . . . . . . . . . . . . . . 535

40.4 Shells under body forces and thermal loadings . . . . . . . . . . . . . 537

40.5 Interaction curves for a cylindrical shell under a ring of forces and axial loading 538

40.6 Concluding remarks . . . . . . . . . . . . . . . . 543

\section{References}

Chapter I . . . . . . . . . . . . . . . . . . . . . . . . . . . . . . . 545

Chapter II . . . . . . . . . . . . . . . . . . . . . . ${ }^{546}$

Chapter III . . . . . . . . . . . . . . . . . . . . . . 553

Chapter IV . . . . . . . . . . . . . . . . . . . . . 585

Chapter V . . . . . . . . . . . . . . . . . . . . 608

Chapter VI . . . . . . . . . . . . . . . . . . . . . . . . 617

Chapter VII . . . . . . . . . . . . . . . . . . . . . 627

Chapter VIII . . . . . . . . . . . . . . . . . . . . 643

Index of abbreviations used in bibliography . . . . . . . . . . . . . . 666

Author index . . . . . . . . . . . . . . . . . . . . . 680

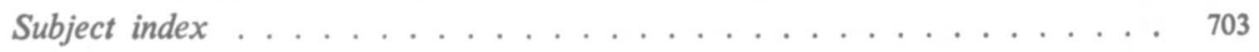




\section{Index of more important symbols}

$\begin{array}{ll}\mathscr{B} & \text { in classification: analysis at the level of the body as a whole } \\ \mathscr{P} & \text { in classification: analysis at the level of a point of the body } \\ \mathscr{S} & \text { in classification: analysis at the level of a cross-section of the body } \\ A & \text { cross-sectional area } \\ A_{\tau} & \text { axiator of the quantity } \tau \\ A^{e} & \text { matrix of elastic moduli (reciprocal moduli) } \\ B & \text { bimoment (warping group) } \\ B & \text { breadth (width) of the cross-section at the centroidal axis } \\ D_{\tau} & \text { deviator of the quantity } \tau \\ E & \text { Young's modulus } \\ E_{i j k l} & \text { components of the tensor of elastic moduli } \\ E_{s} & \text { secant modulus } \\ E_{t} & \text { tangent modulus } \\ E_{1} & \text { modulus of linear hardening } \\ \mathbf{E}_{t}^{e} & \text { matrix of elastic moduli } \\ F_{j} & \text { body forces } \\ F, G, H, L, M, N & \text { Hill's moduli of plastic anisotropy } \\ G & \text { Kirchhoff's modulus } \\ H & \text { half of the thickness of a surface structure } \\ I_{x}, I_{y} & \text { moments of inertia of the cross-section } \\ I_{1 \tau}, I_{2 \tau}, \ldots & \text { basic invariants of the tensor } T_{\tau} \\ I_{1 t}, I_{2 t}, \ldots & \text { basic invariants of the deviator } D_{\tau} \\ I_{e p} & \text { Odqvist's parameter, length of the trajectory in the space of plastic } \\ J_{1 \tau}, J_{2 \tau}, J_{3 \tau} & \text { strains } \\ J_{1 t}, J_{2 t}, J_{3 t} & \text { principal invariants of the tensor } T_{\tau} \\ K & \text { principal invariants of the deviator } D_{\tau} \\ L & \text { bulk modulus } \\ L & \text { length } \\ M_{b}, M_{b y}, M_{b z} & \text { Lagrangian function } \\ M_{\alpha \beta} & \text { bending moments (in bars, beams) } \\ M_{\xi}, M_{\eta} & \text { bending moments in surface structures in engineering notation } \\ M_{\xi \eta}, M_{\eta \xi} & \text { twisting moments in surface structures } \\ & \end{array}$


$M_{t} \quad$ twisting moment (in bars, beams)

$M_{\omega} \quad$ warping moment

$N$

$N_{\alpha \beta}$

$N_{\xi}, N_{\eta}$

$N_{\xi \eta}, N_{\eta \xi}$

normal force (in bars, beams)

$P$

$P_{i}$

membrane forces (in surface structures)

normal forces in surface structures in engineering notation

tangential forces in surface structures

concentrated force

$P_{x}, P_{x \lambda}, P_{\lambda}$

generalized external forces

$P_{M}, P_{M N}, P_{N}$

quadratic forms of generalized strains (in surface structures)

$Q_{i}$

quadratic forms of generalized stresses (in surface structures)

$Q_{\alpha} ; Q_{\xi}, Q_{\eta}$

$R$

generalized stresses

shearing forces (in surface structures)

$R$

reactive force

radius of initial curvature of a circularly curved bar

$R_{\mathrm{z}}$

cylindrical (radial) invariant of the tensor $T_{\tau}$

static moment of the cross-sectional area

$S_{0.5}$

$T$

static moment of a half of the cross-sectional area

temperature

tensor of the quantity $\tau$

volume

$V$

$V_{x}, V_{y}$

$W_{b}$

$W_{0}$

$W^{d}$

$W^{e}$

$W^{0}$

$W^{p}$

$\dot{W}$

shearing forces (in bars, beams)

elastic section modulus in bending

elastic section modulus in torsion

energy dissipated during plastic deformation

recovered energy

energy of residual microstresses

plastic work

power of external loadings

$d W^{*}$

$\delta W$

$X, Y, Z$

elementary excessive work

virtual work

$Z_{\tau}$

material (Lagrangian) coordinates

cylindrical (axial) invariant of the tensor $T_{\mathrm{s}}$

inner radius and outer radius of a cylinder or of a disk cosine of the angle between $x$ and $\xi$

breadth (width) of the rectangular section, current width eccentricity

dimensionless extension of a bar

components of the deviatoric strain in indicial notation

permutation symbols, alternators

function describing the yield condition

function describing the plastic potential

gravitational acceleration

dimensionless unit angle of twist 
$\Delta l$

$m, m_{b}$

$m$

$m_{k}, m_{s}$

$m_{t}$

$m_{\alpha \beta}$

$m_{1}, m_{2}$

$n$

$n$

$n_{\alpha \beta}$

$n_{1}, n_{2}$

$\mathbf{n}_{\boldsymbol{F}}$

$p$

$p$

$p_{n j}$

$q$

$q_{i}$

$r$

$s_{x}, s_{y}, \ldots$

$s_{i j}$

$t$

$t$

$u, v, w$

$u_{r}, u_{\theta}, u_{z}$

$u_{i}$

w

$w$

$x, y, z$

$z_{0}$

$\Delta$

$\Theta$

$\Pi_{i j k l}$

$\Phi$

$\Phi_{i j}$

$\Phi_{f}$

$\Psi$

$\alpha_{i j}$

$\beta=a / b$

$\beta, \tilde{\beta}$ function of plastic hardening

half of the depth of a rectangular section

length

elongation

dimensionless bending moment (in bars, beams)

loading multiplier

kinematically admissible and statically admissible multipliers

dimensionless twisting moment

dimensionless moments (in surface structures)

dimensionless principal moments (in surface structures)

dimensionless normal force (in bars, beams)

normal to a surface

dimensionless membrane forces (in surface structures)

dimensionless principal membrane forces (in surface structures)

unit vector normal to the surface $F$

pressure

dimensionless concentrated force

surface tractions corresponding to the normal $n$

dimensionless normal pressure

generalized strains

radius, cylindrical or polar coordinate; mean radius of a tube

deviatoric stress components in engineering notation

deviatoric stress components in indicial notation

time, time-like parameter

dimensionless tangential pressure

displacements in engineering notation

displacements in cylindrical coordinates

displacements in indicial notation

exertion

dimensionless angular velocity squared

Cartesian coordinates (Eulerian, spatial)

coordinate of the neutral axis

approximation error

dilatation, relative volume change

components of the tensor of moduli of plastic anisotropy

Airy's stress function

components of the Finzi-Krutkov-Blokh stress function tensor

specific distortion energy

Prandtl's stress function

tensorial internal state variables

dimensionless internal radius of a cylinder or of a disk

coefficients of the Bauschinger effect

specific weight of the material 
$\gamma_{x y}, \gamma_{y z}, \gamma_{z x}$ angular strains, shearing strains (in engineering notation)

$\delta$

$\delta_{i j}$

є

$\varepsilon$

$\varepsilon_{i j}$

$\varepsilon_{1}, \varepsilon_{2}, \varepsilon_{3}$

$\varepsilon_{\mathrm{I}}, \varepsilon_{\mathrm{II}}, \varepsilon_{\mathrm{III}}$

$\varepsilon_{e}$

$\varepsilon_{i j}^{E}$

$\varepsilon_{i j}^{x}$

$\varepsilon_{m}$

$\varepsilon^{B}$

$\varepsilon_{0}$

$\theta$

$\vartheta$

$\vartheta_{0}$

$\boldsymbol{x}$

$x_{n}$

$\varkappa_{m}$

$x_{\alpha \beta}$

$x_{1}, x_{2}$

$\Delta x_{1}, \Delta x_{2}$

$\lambda$.

$\lambda, \lambda_{i}$

$\lambda_{\alpha \beta}$

$\lambda_{1}, \lambda_{2}$

$\mu_{\mathrm{r}}$

$\boldsymbol{v}$

$v^{p}$

o

$\varrho$

$\varrho_{n}$

$\sigma$

$\sigma$

$\sigma_{i j}$

$\sigma_{1}, \sigma_{2}, \sigma_{3}$

$\sigma_{\mathrm{l}}, \sigma_{\mathrm{II}}, \sigma_{\mathrm{mI}}$

$\sigma_{e}$

$\sigma_{m}$

$\sigma_{0}$

$\sigma_{0+}, \sigma_{0-}$ wall thickness of a thin-walled section

Kronecker's symbol

vector ascribed to the tensor $T_{e}$

longitudinal strain, relative elongation

components of infinitesimal strain in indicial notation principal strains

principal strains arranged: $\varepsilon_{1} \geqslant \varepsilon_{11} \geqslant \varepsilon_{\mathbf{I I I}}$

effective strain (strain intensity)

components of the Eulerian (Almansi) strain tensor

components of the Lagrangian (Green) strain tensor

mean strain

longitudinal strain in logarithmic measure (Hencky)

extension of the beam axis

angle, cylindrical or polar coordinate

unit angle of twist

initial unit angle of twist

curvature of the beam axis

curvature of the neutral axis

scalar internal state variables

parameters of curvature changes of a surface structure

principal curvatures of a plate

increments of principal curvatures of a shell

function (coefficient of proportionality) in the theory of plastic flow

Lagrangian multipliers

extensions of the middle surface of a surface structure

principal extensions

Lode's parameter for the tensor $T_{\tau}$

Poisson's ratio

coefficient of transverse deformation in the elastic-plastic range radius of curvature of the beam axis

dimensionless radial coordinate

radius of curvature of the neutral axis

vector ascribed to the tensor $T_{\sigma}$

normal stress

stress components in indicial notation

principal stresses

principal stresses arranged: $\sigma_{\mathrm{I}} \geqslant \sigma_{\mathrm{II}} \geqslant \sigma_{\mathrm{II}}$

effective stress (stress intensity)

mean stress

yield-point stress in simple tension

yield-point stresses in tension and in compression (if different) 


\section{CHAPTER I}

\section{Basic concepts of the theory of combined loadings}

\section{Levels of analysis of plastic deformations}

\subsection{Introductory remarks}

The theory of plasticity is concerned with the analysis of permanent (plastic) deformations, characterized by the following two fundamental features:

(1) plastic deformations remain after their causes have been removed; they are associated with a dissipation of energy, and hence the process is history-dependent and irreversible;

(2) they are rate-insensitive, i.e. they are described by constitutive equations invariant with respect to the scale of time used. The effects of rate-sensitivity are termed "viscous" and such effects are beyond the scope of the present book.

The irreversibility of the processes appears to be the most important physical feature of plastic deformations. On the other hand, their main mathematical feature consists in the deviations from linearity of constitutive equations, which is typical for classical elasticity: namely, plastic deformations are governed by non-linear constitutive equations (or, exceptionally, by linear non-homogeneous ones), established on the basis of the results of experimental investigations.

From the point of view of engineering applications the latter feature is often much more importantthan the physical property of irreversibility. In fact, the failure of a structure made of a material which is capable to significant plastic deformations is, as a rule, preceded by such deformations and a more accurate estimation of failure loads requires an application of the equations of the theory of plasticity. We often use these equations merely to describe the process connected with increasing loadings, without being interested in the results of their removal. Of course, there are also cases where the irreversibility of the process is of crucial importance; let us mention, for example, 
(1) the technological processes of forming by means of large plastic deformations;

(2) the estimation of the carrying capacity of structures subject to variable repeated loadings (shakedown problems), or to dynamic loadings,

(3) the estimation of the carrying capacity of structures subject to increasing loadings but resulting in the formation of certain zones in which the energy is no longer dissipated (locally passive zones).

In these problems irreversibility of plastic deformations is of fundamental importance; of course, deviations from physical linearity must be taken into account in any case.

As a result of the nonlinearity of constitutive equations the principle of superposition does not hold and the knowledge for simple (pure) cases is usually insufficient to solve the combined case. Cases of combined loadings must be solved separately, often by means of special methods. It is to such problems of combined loadings, defined more precisely in Sec. 4, that the present book is devoted.

\subsection{Definitions of the levels of analysis and the classification symbolism adopted}

The analysis of plastic deformations usually aims at determining the stress and strain distribution and at estimating the carrying capacity of a body subject to a system of various external loadings. Such an analysis, which may be called "integral", should be preceded, as a rule, by a "local" analysis, referring to the individual points of the body. In the case of certain particular shapes of bodies often used in engineering applications (bars, beams, plates, shells) we also introduce an intermediate level of analysis; namely, we investigate plastic deformations or the carrying capacity of the individual cross-sections.

We shall thus distinguish three levels of analysis of plastic deformations. The first, local level will be connected with the individual points of the body, regarded as a continuous medium; it will be denoted by the symbol $\mathscr{P}$ (as in [1.27)]. The next "semi-integral" level will relate to the cross-section (of the bar, plate, etc.) - we shall denote it by the symbol $\mathscr{S}$. Finally, by the symbol $\mathscr{B}$ we shall denote the last, integral level concerning the body or structure as a whole.

Of course, other levels of analysis might also be introduced. For example, the microscopic or even the molecular level may be considered as the first one. However, making use of the phenomenological theories of plasticity, we omit these levels. Further, for certain structures composed of several elements (e.g. for trusses), a certain intermediate level between $\mathscr{S}$ and $\mathscr{B}$ might also be useful.

To discuss a process at a point or at a cross-section we often have to consider a certain infinitesimal vicinity of that point or cross-section. In the case of a point $P$ we introduce an element whose three dimensions are all infinitesimal and whose shape corresponds to the system of coordinates adopted. In the case of a section of a bar $S$ the element has two finite dimensions and one infinitesimal dimension (along the axis); its shape is unique. Considering cross-sections of surface structures, 
denoted also by $S$, we employ elements with one finite dimension (thickness of the plate or shell) and two infinitesimal dimensions; their shape may depend on the two-dimensional system of coordinates parametrizing the middle surface of the structure. At the integral level $\mathscr{B}$ the problem of infinitesimal vicinity does not occur.

Suppose that the process at any of the above-mentioned levels is controlled by $n$ independent factors of the nature of loadings but understood in a much broader sense (cf. Sec. 1.3-1.6). Making use of the terminology of [1.24], we call any such independent factor (control function) an exertion factor, and this term will uniformly be used at all the three levels $\mathscr{P}, \mathscr{S}$ and $\mathscr{B}$. The essential problem of independence of the exertion factors at the particular levels will be discussed in Sec. 4.3.

Knowledge of the exertion factors as functions of time $t$ determines a process which will be called the deformation process or the loading process. $\left({ }^{1}\right)$ In the theory of plasticity-where we neglect viscous effects-the time scale is, as a rule, unimportant, since the time variable may be eliminated and instead of time we may speak of a time-like parameter. The exceptions are: dynamic processes (with inertia forces taken into account) and processes in which time is a physical parameter (mechanical properties depending on time, heating, irradiation, etc.).

\subsection{Exertion factors at the level of a point of the body (PP)}

Let us now discuss typical exertion factors at the individual levels of analysis. At a given point $P$ of the body the stress components $\sigma_{i j}$ are very often assumed to be the most important exertion factors. Under the assumption of symmetry of the stress tensor (couple stresses being absent) the number of independent stress components in the general case is equal to six. Another possibility is to regard the six strain components $\varepsilon_{i j}$ as independent, i.e. to regard them as exertion factors. In the linearly elastic range the difference between these approaches is almost negligible, whereas in the plastic range it may be essential. For example, testing machines may be controlled either by stresses (soft loading) or by strains (hard loading, R. M. Shneyderovitch [1.17], V. Ya. Yablonko [1.22]). Physical differences between the above two cases are discussed in [1.23].

Besides these extereme cases of the selection of exertion factors, many intermediate cases may occur. Choosing any six of the twelve quantities $\sigma_{i j}, \varepsilon_{i j}$, we obtain $12 ! / 6 ! 6 !=924$ combinations, $\left({ }^{2}\right)$ but of course not all of them are possible, because of certain dependencies. Such a discussion seems to be of purely academic character, but actually it is not. On the contrary, very often the process cannot be described (controlled) either by the six stress components or by the six strain components. For example, perfect plasticity excludes control by the six stresses (since they are not independent in view of the yield condition), and incompressibility of material excludes control by the six strains. In such cases "mixed" control is the most appropriate; Ilyushin suggests here five independent components of the strain

(1) Loading in the broader sense, cf. Sec. 2.2.

(2) A. A. Ilyushin [1.11] suggests here the number 3696 without explanation. 
deviator and mean stress $\sigma_{m}$. In particular cases, however, other combinations are used: for example, if a thin-walled tube is subject to simultaneous tension and torsion in a "hard" testing machine, then the process is controlled by the following six quantitiès (in cylindrical coordinates $r, \theta, z): \varepsilon_{z}=\varepsilon_{z}(t), \gamma_{\theta z}=\gamma_{\theta z}(t), \sigma_{r}=\sigma_{r}(t)$, $\sigma_{\theta}=\sigma_{\theta}(t), \tau_{z r}=\tau_{z r}(t)$ and $\tau_{r \theta}=\tau_{r \theta}(t)$, where $\sigma_{r} \equiv \sigma_{\theta} \equiv \tau_{z r} \equiv \tau_{r \theta} \equiv 0$.

Besides stresses or strains, or their combinations, many other quantities may be regarded as the exertion factors at the level $\mathscr{P}$. The most important of them is temperature, which appears explicitly in the constitutive equations (thermal expansion) and affects the mechanical properties of the material. The corresponding branch of plasticity is called thermoplasticity, the basic equation of which will be discussed in Chapter III.

The influence of other factors on the plastic deformations at the level $\mathscr{P}$ has not been investigated very extensively as yet. Let us mention here the effect of neutron irradiation, magneto-plasticity, humidity of timber, etc. Further, the influence of gradients (stress or strain gradients) may be significant as well, and in those cases gradients should also be considered as exertion factors.

Finally it should be noted that in viscoplasticity strain rates may be regarded as essential exertion factors; in classical plasticity they are in most cases unimportant.

\subsection{Exertion factors at the level of a cross-section $(\mathscr{S})$}

The concept of the cross-section may be introduced for an arbitrarily shaped body; however, it constitutes a particularly useful tool of analysis for structures which may approximately be treated as one-dimensional (one of the dimensions, length, much larger than the remaining two) or two-dimensional (one of the dimensions, thickness, much smaller than the remaining two).

In one-dimensional structures (line structures, more often called bar structures or rods) as the cross-section we understand a section perpendicular to the bar axis. Such a cross-section is two-dimensional and is characterized by its shape, determined, for example, by the function $r=r(\theta)$ describing the contour in polar coordinates $r, \theta$. Of course, in particular cases the Cartesian coordinates may be more suitable than the polar ones. In any case the existing variety of shapes of the crosssections of line structures is of great importance in the theory of plasticity: as a rule, the deformation processes at the level $\mathscr{S}$ must be analysed separately for the individual shapes of the cross-section. Further complications may occur in cases of transverse non-homogeneity of the material.

In the general case of naturally curved and twisted bars the process in a crosssection is affected by the relevant geometrical characteristics of the infinitesimal element, i.e. by the initial curvatures $x_{0 y}$ and $x_{0 z}$ and the initial angle of twist $\vartheta_{0}$, and hence a more accurate description of the cross-section should be completed by these three quantities (components of an antisymmetric tensor or of an associated vector). However, in an engineering approach the results of the analysis for $\varkappa_{0 y}=\varkappa_{0 z}$ $=\vartheta_{0}=0$ are often approximately applied to naturally slightly curved or twisted bars. A similar remark refers to a cross-section of slightly non-prismatic bars. 
Consider the cross-section of a bar with the axis $x$ (if the bar is naturally curved, then $x$ corresponds locally to the direction tangent to the axis), and let the axes $y$ and $z$ coincide with the principal centroidal axes of that section. Resolve the resultant (internal) force in that section into the axial force (or normal force) $N_{x}=N$ and two shear forces (or transverse forces) $V_{y}$ and $V_{z}$, and the resultant (internal) moment into the twisting moment $M_{x}=M_{t}$ and two bending moments $M_{y}$ and $M_{z}$. These six quantities, called generalized stresses (or generalized internal forces), are usually regarded as the exertion factors at the level $\mathscr{S}$. Figure 1 shows them as

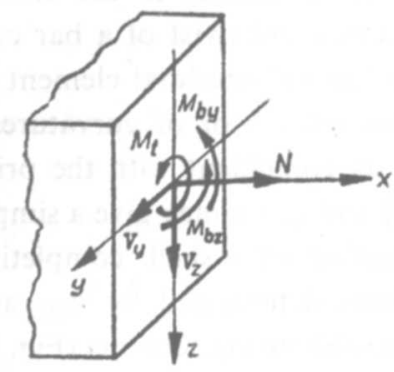

Fig. 1. Generalized stresses in a cross-section of the bar

acting on a face of the infinitesimal element of the bar. The local system of coordinates is in this case uniquely defined (except for sections with infinitely many principal directions), and the problem of transformation and invariance of the constitutive equations is, as a rule, irrelevant here.

The six generalized stresses have six corresponding generalized strains. Usually they are defined in such a way as to be consistent with the generalized stresses in the sense of the principle of virtual work (cf. Sec. 1.6). Thus the generalized strains in the cross-section of the bar or beam are usually interpreted as unit elongation, two transverse shearing strains, unit angle of twist and two curvatures, though, in a more rigorous treatment, their interpretation may be slightly different (e.g. the curvature of the neutral axis instead of the curvature of the centroidal axis of the beam, cf. Sec. 22.5). In the case of naturally curved and twisted bars the interpretation of generalized strains changes into curvature increments and a twist increment (also approximately, cf. Sec. 36.3). The above quantities may also control the process at the level $\mathscr{S}$, and hence they may serve as exertion factors at that level.

In the case of thin-walled bars, besides the above-mentioned six generalized stresses, two other related quantities may appear: the bimoment (warping group, sometimes also called the warping moment) $B$ and warping moment (warping torque) $M_{\omega}$. These quantities may also be treated as independent, and the maximal number of generalized stresses in this case is raised to eight.

The influence of other factors may be more complicated. If we consider the effect of temperature supposed to be constant in the whole cross-section, then the temperature $T$ may also be regarded as an exertion factor at the level $\mathscr{S}$. If the temperature distribution is determined by $n$ parameters, then any of those parameters 
may be treated as an exertion factor. In the general case of arbitrary temperature distribution the number of exertion factors would increase infinitely, complicating the analysis. Similar remarks apply to the distribution of humidity, etc.

Consider now exertion factors at the level $\mathscr{S}$ for a surface structure (plate or shell); some differences with respect to the cross-section of line structures may be observed here. We introduce the concept of a cross-section of a surface structure as follows. The counterpart of a two-dimensional cross-section of a bar is here a one-dimensional object, namely the segment $-H \leqslant z \leqslant H$, where $2 H$ denotes the thickness of a plate or shell, and $z$ is measured perpendicularly to the middle surface. Further, the counterparts of the natural curvatures and twist of a bar correspond here to the curvatures of the middle surface of an infinitesimal element of a shell, $x_{0 x x}, x_{0 y y}, x_{0 x y}$ (components of a two-dimensional tensor of curvatures). Choosing the mutually orthogonal directions $x$ and $y$ as coincident with the principal directions of the tensor of curvatures, denoted by $\xi$ and $\eta$, we may give a simple geometrical interpretation to the concept of a cross-section of a shell, completing the segment $-H \leqslant z \leqslant H$ with two elementary sections determined by $x_{0 \xi \xi}$ and $x_{0 \eta \eta}$, i.e. cut in such a way as to be compatible with the neighbouring sections (Fig. 2).

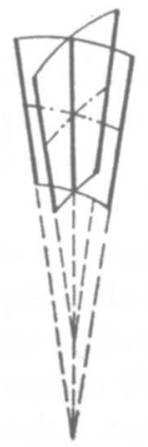

Fig. 2. The concept of a cross-section of a surface structure

Just as in the case of a cross-section of a bar, the simplified engineering approach aims at getting rid of the additional parameters $x_{0}$ and at the application of the analysis carried out for the cross-section of a plate $\left(x_{0 x x}=x_{0 y y}=x_{0 x y}=0\right)$ to slightly curved shells.

In contradistinction to the cross-section of a bar, the cross-section of a surface structure, being one-dimensional, has no shape. However, even here a certain exception may be found. If we consider a sandwich structure, consisting of two working layers and a core transmitting shearing forces (Fig. 3), then the segment defined as the cross-section is approximately reduced to two points. Integration of stresses may be replaced here by simple summation. In certain cases multilayer surface structures are also employed (e.g. P. G. Hodge [1.5]), then the cross-section may be called the multipoint cross-section. Possible transversal non-homogeneity of the material may influence the analysis as in the case of the cross-section of a bar. 


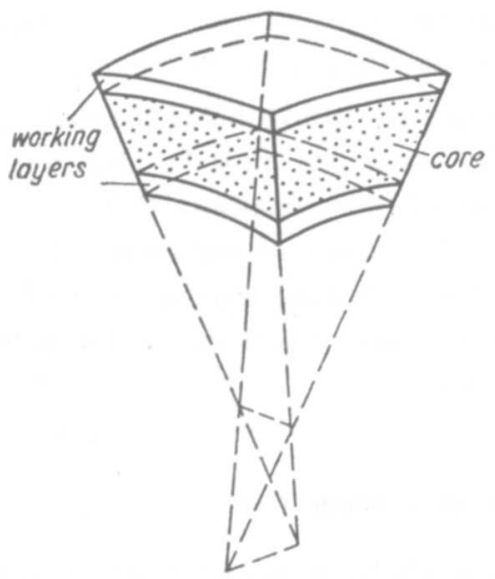

Fig. 3. Element of a sandwich surface structure

In each elementary "component" section of the cross-section of a surface structure, defined above, five generalized stresses (generalized internal forces or sectional forces) may appear: in the classical approach it is assumed that bending moments in the plane tangent to the middle surface vanish. Those generalized stresses are shown in Fig. 4 as acting on the faces of the infinitesimal element of a shell, cut in

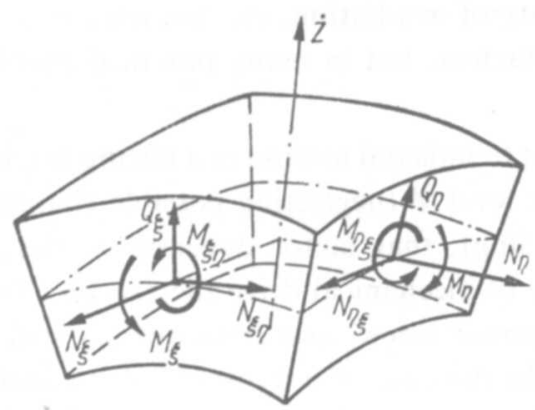

Fig. 4. Generalized stresses acting on the faces of an element of the shell

the principal directions $\xi$ and $\eta$. They are: two bending moments $M_{\xi}$ and $M_{\eta}$, two twisting moments $M_{\xi \eta}$ and $M_{\eta \xi}$, two normal (direct) forces $N_{\xi}$ and $N_{\eta}$, two tangential forces $N_{\xi \eta}$ and $N_{\eta \xi}$, and two shearing forces $Q_{\xi}$ and $Q_{\eta}$. These generalized forces are related to the length of the middle line of the component section, and hence their usual dimensions are divided by the dimension of length. Thus, in the general case in the cross-section of a surface structure there may appear ten exertion factors of type of generalized stresses, or the corresponding ten generalized strains. In the case of a plate (slab) we have $M_{\xi \eta}=M_{\eta \xi}$ and $N_{\xi \eta}=N_{\eta \xi}$ and the above-mentioned number is reduced to eight; if the analysis is confined to small deflections without in-plane loadings, then $N_{\xi}=N_{\eta}=N_{\xi \eta}=0$ and there remain five exertion factors; neglecting the influence of transverse shear, we reduce this number to three; for 
a plate under in-plane loadings only this number also equals three. Other reductions may be due to the rotational symmetry of the loading and of the shape.

In view of the arbitrary choice of the two mutually orthogonal elementary "component" sections, the corresponding constitutive equations must be invariant with respect to the (two-dimensional) transformation of those sections. This invariance is ensured by the tensorial character of moments $M$ and of membrane forces $N$, and by the vectorial character of shearing forces $Q$. Taking into account of other exertion factors (temperature, humidity, etc.) involves difficulties similar to those arising in the case of the cross-section of a bar.

\subsection{Exertion factors at the level of the body as a whole $(\mathscr{B})$}

At the integral level of the body (structure) as a whole, $\mathscr{B}$, the exertion factors are usually identified with independently acting external forces $P_{i}$. Sometimes the external loadings are to a certain degree interdependent, and then it is convenient to express them in terms of the "loading multipliers." For example, if a continuously distributed loading $q(x)$ can be presented in the form $q(x)=\sum_{i} \alpha_{i} f_{i}(x)$, where $f_{i}(x)$ are certain prescribed functions, then the quantities $\alpha_{i}$, called loading multipliers, may be regarded as exertion factors at the level $\mathscr{B}$. Other factors may be connected with the formation of body forces (specific gravity, angular velocity), with the distribution of temperature (heat sources), intensity of irradiation, etc. Sometimes certain displacements may be regarded as exertion factors, but in many practical problems the situation is more complicated.

Consider, for example, a specimen subject to uniaxial tension in a testing machine. Here we have only one exertion factor at the level $\mathscr{B}$ (neglecting possible changes of temperature and the effect of velocity of loading); that factor depends on the construction of the machine. In the simplest case of mechanical drive the exertion factor coincides with the angle of rotation of the power screw, and consequently with the elongation of the specimen (hard loading). In the case of a hydraulic drive such an independent factor may be identified with the amount of oil conveyed to the piston chamber. Under the assumptions of incompressibility of oil, of perfect seal of the chamber and of perfectly rigid structure of the machine, the exertion factor would also correspond to the elongation of the specimen. In fact, however, the machine is not perfectly rigid, and oil is compressible, and so the exertion factor under consideration is connected both with elongation and with oil pressure, i.e. with the force transmitted by the specimen. Even the location of the specimen may be important here: placing it as low as possible, we have the least possible amount of oil under the piston, its compressibility is negligible and the exertion factor is more closely related to the displacement. The extremely opposite case would occur if the fluid were replaced by a gas: under small changes in volume (connected with the elongation of the specimen) gas pressure remains almost constant and so it is the transmitted force that would then be the exertion factor (soft loading). 
Paper [1.26] draws attention to the exertion factors of other types, e.g. the eccentricity of the force compressing a column. Indeed, if such a quantity is independent and can change during the deformation process, then it influences that process in an essential manner. Related exertion factors are: position (coordinate) of the force if moving, say, along the beam; angle of inclination of the force if it can turn round, etc. The characteristic feature of such factors is that they do not cause any deformation process by themselves - it takes place only if at least one other exertion factor is present.

\subsection{Basic exertion factors}

Generalized stresses and generalized strains may be introduced in various ways, but for many reasons it is convenient to base their definitions on energy considerations. Suppose we can find such consistent (or conjugate) systems of: (1) stresses $\sigma_{i j}$ and strains $\varepsilon_{i j}$, (2) generalized stresses $Q_{i}$ and generalized strains $q_{i}$, (3) generalized external forces $P_{i}$ and generalized displacements $u_{i}$, that the virtual work $\delta W$ may be presented

for bar structures in the form

$$
\delta W=\iiint_{V} \sigma_{i j} \delta \varepsilon_{i j} d V=\int_{0}^{l} Q_{i} \delta q_{i} d s=P_{i} \delta u_{i},
$$

and for surface structures in the form

$$
\delta W=\iiint_{V} \sigma_{i j} \delta \varepsilon_{i j} d V=\iint_{A} Q_{i} \delta q_{i} d A=P_{i} \delta u_{i}
$$

in these equations $d s$ denotes the elementary arc of the undeformed axis of a bar, $d A$-the elementary area of the undeformed middle surface of a plate or shell, and the Einstein summation convention holds (whenever the same letter index occurs twice in a term, that index is to be given all possible values and the results added together). Then it is convenient to single out the stresses $\sigma_{i j}$ at the level $\mathscr{P}$, the generalized stresses $Q_{i}$ at the level $\mathscr{S}$, and the generalized external forces $P_{i}$ at the level $\mathscr{B}$; they will be called the basic exertion factors at the individual levels of analysis. Under the restriction to basic exertion factors it is possible to prove many theorems which are no longer valid in the general case, exceeding these factors. The remaining factors will be called the accessory exertion factors, though their influence on the deformation process under consideration may also be essential.

\section{Active and passive processes. Loading and unloading}

\subsection{Definition of plastically active and plastically passive processes}

The theory of plasticity deals, in principle, with irreversible processes; however, certain fragments of those processes may be reversible. Irreversibility is connected 
with the dissipation of the mechanical energy, i.e. with the conversion of that energy into heat. Let us restrict ourselves, for the time being, to the analysis at the level $\mathscr{P}$, of a point of the body. A process (or its fragment) that is irreversible in view of energy dissipation will be called plastically active, or, more briefly active, whereas a process without energy dissipation will be called plastically passive, or passive. These convenient terms were introduced by $\mathrm{H}$. Tresca at the earliest stage of the development of the theory of plasticity; later they were identified with quite different terms, namely "loading" and "unloading", respectively, but such identification has many defects and may lead to certain misunderstandings; therefore in the present book the two sets of terms will be distinguished (Secs. 2.2-2.4). Plastically passive processes are also sometimes called elastic; it seems more convenient to reserve this term for passive processes not preceded by active ones, but in the general case of passive processes we may speak of "elastic changes" (of stresses, strains, loadings, etc.). In the case of passive processes an elastic potential function exists and the final state is independent of the process trajectory.

It is very important to distiguish active and passive processes not only from the physical point of view (different character of the phenomena under discussion), but also from the mathematical point of view, since they are described by different constitutive equations. However, it should also be mentioned here that V. D. Klyushnikov in [1.12] attempted to establish certain universal physical equations which aimed at describing any process in a uniform manner (as an active one, cf. Sec. 15.4).

In principle, there is no difficulty in defining active and passive processes at the level $\mathscr{P}$; difficulties may arise in the formulation of mathematical conditions separating these processes. This problem will be discussed in detail in Secs. 9.5, 11, 12, and 14.

The levels $\mathscr{S}$ and $\mathscr{B}$ furnish a larger variety of processes. As a passive process we understand here a process which is passive at each point of the cross-section or body under consideration. The process which is active at each point will be called fully active or fully plastic. A shorter term, active process (or elastic-plastic process) will be assigned to processes which at individual points are either active or elastic (passive but not preceded by active ones). If the process is active at certain points and, at other points, passive but preceded by an active process, then-referring to the whole section or body-we call that process partly active or active-passive. Such processes are usually the most difficult for analysis: one has to keep track of the formation of locally passive zones. These zones are often called local unloading zones but the latter term does not conform to the definition of loading adopted in the present book, and will be given certain other meaning, cf. Sec. 2.4.

\subsection{Loading and unloading in the case of one exertion factor}

We are now going to define the process of loading (in the narrower sense) and that of unloading. It is true that active processes are usually observed under increasing forces and passive ones-under decreasing forces, but there are many arguments 
against the widely adopted identification of "loading" and "unloading" with "active" and "passive" processes, respectively. To begin with, it should be noted that the concepts of active and passive processes are introduced especially in the theory of plasticity, whereas the much older terms "loading" and "unloading" are traditionally understood as purely technical ones, connected with certain fragments of an experiment or with the type of work of the structure regardless of the properties of the material used. Further, the initial fragment of the deformation process is usually elastic, and hence plastically passive; it would be absurd to call it "unloading", and thus one has to introduce exceptions from the beginning (unloading may be identified with passive processes preceded by active ones). But even here we find further inconsistencies, since certain fragments of unloading may be active (cf. Sec. 8.8), and secondary loading may be passive. Hence we adopt separate definitions of loading and unloading, more traditional and not necessarily connected with the theory of plasticity.

First consider the case of one basic exertion factor at the level $\mathscr{B}$, for example a body under one independent force $P$. It is logically justified to use the term "loading" for the increase of the absolute value of such a factor, and the term "unloading" for its decrease. Further specification may be achieved by the adjectives "secondary" (second loading by the force of the same sense), "reverse" (second loading by the force of the opposite sense), etc. Finally, if the process develops without any change of the exertion factor, it may be called a "spontaneous process", since we have no influence on its course. These terms correspond also to the role of exertion factors as control variables.

Similar definitions can also be introduced for displacement-controlled processes. However, discussing other processes controlled by one accessory exertion factor we realize that it is not always reasonable to distinguish between loading and unloading in the meaning introduced above. For example, consider a beam under a constant-value moving force where the exertion factor is the coordinate of its application point. In the case of a cantilever beam it is justified to apply the term "loading" to the process governed by increasing distance of the force from the clamped end, and the term "unloading" to the process governed by decreasing distance from that end. On the other hand, if we consider simply supported beams, or, particularly, continuous beams under moving forces of constant value, then any division of the process into "loading" and "unloading", conforming to the definitions adopted, seems impossible. We then simply speak of the loading process (or deformation process) understanding "loading" in the broader sense as any change of exertion factors. Similar remarks pertain also to other accessory exertion factors, such as temperature, humidity, irradiation, etc.

Consider now the processes at the levels $\mathscr{P}$ and $\mathscr{S}$, governed by one basic exertion factor. In order to introduce the definitions of loading and unloading similar to those at the level $\mathscr{B}$, we first have to discuss the problem of invariance of the exertion factors at the levels under consideration, e.g. at the level $\mathscr{P}$. The stress components, 
in general, are not invariant since they depend on the system of coordinates adopted. However, if they are exertion factors, i.e. if they control the process at the level $\mathscr{P}$, then they are uniquely defined, and hence invariant. For example, if a cylindrical shell (thin-walled tube) is subject to simultaneous force-controlled tension, torsion and internal pressure, then the process at any point is controlled by axial stress $\sigma_{z}$, circumferential stress $\sigma_{\theta}$ and tangential stress $\tau_{z \theta}$. They are uniquely determined irrespectively of the system of coordinates adopted; they may be interpreted as certain common invariants of the stress tensor and of the tensor of curvatures of the shell (components of the first tensor in the principal directions of the second can). Such a case occurs, first of all, if the stress distribution in the body is statically determinate, or if the strain distribution is geometrically determinate (and such cases are mostly used in experiments). Now, in the case of one exertion factor we can define loading at a point by the increase of the absolute value of that factor, and unloading-by its decrease.

It should be noted, however, that in the general case of an arbitrarily shaped body and of arbitrary external loadings it may be difficult to find the proper exertion factors, controlling the process at an arbitrary point of the body. If the principal directions of stresses or strains at any point of the body are fixed and known, then the principal stresses (or strains) may serve as exertion factors; they are invariant and a distinction between loading and unloading can be introduced. In other, more general cases, if only the stress components in a certain arbitrarily chosen system of coordinates may serve as exertion factors, they are not invariant and in this case loading and unloading at the level $\mathscr{P}$ cannot be distinguished (let us recall, however, that active and passive processes were defined in Sec. 2.1 in a quite general manner).

Before proceeding to more complicated cases let us show a simple example of confrontation of the definition introduced. Consider the process of uniaxial tension-compression of a mild steel specimen, represented by the diagram in Fig. 5.

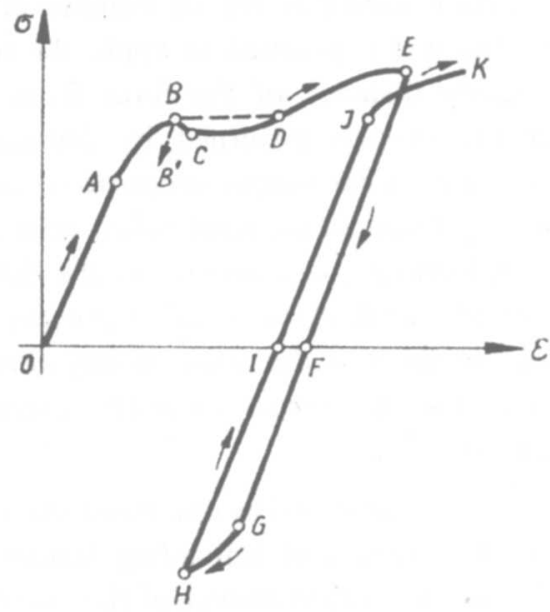

Fig. 5. Active processes versus passive processes; loading versus unloading 
Point $A$ corresponds to the elastic limit, $B$ to the upper yield point, $C$ to the lower yield point. The notions of plastically active and passive processes are defined uniquely (we identify here a plastically active process simply with the deviations from Hooke's law, cf. Secs. 8.5 and 9.5), whereas the terms "loading" and "unloading" depend on the choice of the exertion factor (in fact, on the construction of the testing machine). Two typical cases will be compared: exertion factor $\sigma$ and exertion factor $\varepsilon$. For small plastic deformations of a prismatic, homogeneous specimen those factors are equivalent to the force $P$ (soft loading) and to the elongation $\Delta l$ (hard loading), respectively. The classification of the individual fragments of the processin accordance with the definitions adopted-is given in Table 1.

Table 1

Classification of a tension-compression process

\begin{tabular}{|c|c|c|c|}
\hline \multirow{2}{*}{$\begin{array}{c}\text { fragment } \\
\text { of the process }\end{array}$} & \multirow{2}{*}{$\begin{array}{c}\text { process: } \\
\text { active-passive }\end{array}$} & \multicolumn{2}{|c|}{ process: loading-unloading } \\
\hline & & $\begin{array}{l}\text { exertion } \\
\text { factor } \sigma\end{array}$ & $\begin{array}{l}\text { exertion } \\
\text { factor } \varepsilon\end{array}$ \\
\hline$O A$ & passive & loading & loading \\
\hline$A B$ & active & loading & loading \\
\hline$B C$ & active & & loading \\
\hline$C D$ & active & spontaneous & loading \\
\hline$D E$ & active & loading & loading \\
\hline$E F$ & passive & unloading & unloading \\
\hline$F G$ & passive & $\begin{array}{l}\text { loading } \\
\text { (reverse) }\end{array}$ & unloading \\
\hline$G H$ & $\begin{array}{l}\text { active } \\
\text { (reverse) }\end{array}$ & $\begin{array}{l}\text { loading } \\
\text { (reverse) }\end{array}$ & unloading \\
\hline$H I$ & passive & unloading & $\begin{array}{l}\text { loading } \\
\text { (secondary) }\end{array}$ \\
\hline$I J$ & passive & $\begin{array}{l}\text { loading } \\
\text { (secondary) }\end{array}$ & $\begin{array}{l}\text { loading } \\
\text { (secondary) }\end{array}$ \\
\hline$J K$ & $\begin{array}{l}\text { active } \\
\text { (secondary) }\end{array}$ & $\begin{array}{l}\text { loading } \\
\text { (secondary) }\end{array}$ & $\begin{array}{l}\text { loading } \\
\text { (secondary) }\end{array}$ \\
\hline
\end{tabular}

It may be seen from Table 1 that even in this simple case some misunderstandings may occur and a distinction between an "active process" and "loading" on the one hand and a "passive process" and "unloading" on the other hand seems indispensable. Moreover, it should be noted that the exertion factor (control variable) affects here not only the name but also the process itself: if the process is controlled by $\sigma$, then we obtain the straight segment of a spontaneous process $B D$, whereas $\varepsilon$ as the exertion factor makes it possible to investigate the loading curve $B C D$.

\subsection{Loading and unloading in the case of several exertion factors}

In the case of several basic exertion factors the following definitions seem to constitute a logical extension of the definitions adopted above: loadings occurs if all the 
absolute values of the exertion factors are non-decreasing functions of time (at least one of them increasing); unloading occurs if all the absolute values are nonincreasing functions of time (at least one of them decreasing); a mixed process occurs if some values are increasing and other decreasing; finally, a spontaneous process occurs if at all exertion factors happen to be constant. These definitions are illustrated in Fig. 6 for the case of two exertion factors. Of course, all the difficulties con-

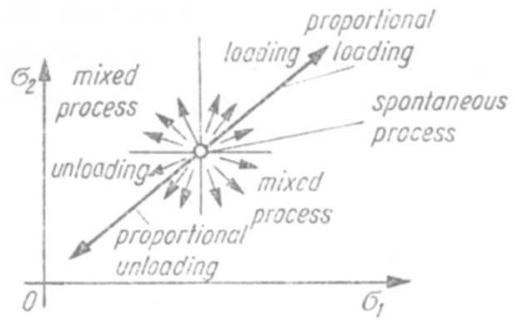

Fig. 6. Loading and unloading in the case of two exertion factors

cerning the accessory exertion factors arise here as well, and a logical distinction between "loading" and "unloading" is not always possible.

An important type of loading takes place if all the absolute values of basic exertion factors increase proportionally to a certain parameter (a monotonically increasing function of time). This process will be called proportional loading, and the case of proportional decrease-proportional unloading. In these specified cases the number of exertion factors is practically reduced to one, since all the other factors depend uniquely on the first one. A related, but slightly more general kind of process, called simple loading, will be defined in Sec. 9.8.

The difference between the definition of active process and that of loading adopted in the present book should be stressed once more. The process of loading (in the narrower sense of the term) may be passive not only in the case where it wholly belongs to the elastic range but even if it starts at the yield point. An example of such a process under plane stress conditions is shown in Fig. 7. The material is

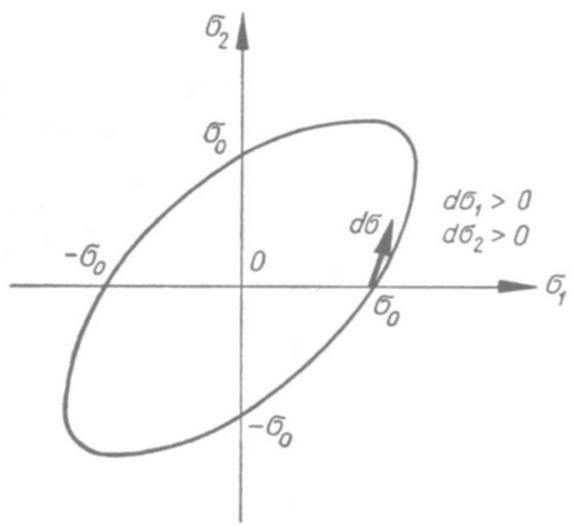

Fig. 7. A passive process corresponding to loading starting from the yield-point stress 
assumed to obey the Huber-Mises-Hencky yield condition. $\left({ }^{1}\right)$ If we take the principal stresses $\sigma_{1}$ and $\sigma_{2}$ as the exertion factors, and start from point $\sigma_{1}=\sigma_{0}$ (yieldpoint stress), $\sigma_{2}=0$, then during the process described by the stress increment vector $d \sigma$ the control variables $\sigma_{1}$ and $\sigma_{2}$ both increase and, according to the definition adopted, loading takes place. On the other hand, according to the HuberMises-Hencky hypothesis, plastic deformations during this process do not change, energy is not dissipated and the process is plastically passive.

\subsection{Interrelation between loading and unloading, active and passive processes at various levels}

The interrelation between active and passive processes at the individual levels of analysis is clearly stated by the definitions adopted. On the other hand, the interrelation between loading and unloading at various levels is more complicated, even in the case of one exertion factor at all levels.

Usually loading at the level $\mathscr{B}$ results in loading also at the levels $\mathscr{S}$ and $\mathscr{P}$, but there are many exceptions. Typical exceptions are connected with the influence of geometry changes, for example in the case of eccentrically compressed slender columns. Then the increase of the force results, in certain zones, in the decrease of stresses (local unloading zones) or even subsequently in the increase of the stresses of opposite sign (zones of reverse loading). If the whole process is elastic, i.e. plastically passive, then these unloadings and secondary loadings are unimportant: they do not influence the constitutive equations at the level $\mathscr{S}$ or $\mathscr{B}$. However, if active processes are followed by partly active ones (with the zones of passive unloading), then the constitutive equations change their form.

A. A. Ilyushin $[1.7,1.8]$ proved that in the simplest case of a proportional loading process at the level $\mathscr{B}$ (by surface tractions and body forces only), the loading is also proportional at the levels $\mathscr{S}$ and $\mathscr{P}$, but even this statement is valid only under very restrictive assumptions of incompressibility and homogeneity of the material, power plastic hardening law, and neglected geometry changes of the body. Thus, under these assumptions, in a body subject to a one-parameter (proportionally increasing) system of forces no unloading occurs. If we reject any of the above-mentioned assumptions, we easily find counterexamples to the theorem.

Typical counterexamples connected with the geometry changes have been known for a long time. The first example of local passive unloading in a system subject to a single increasing force, without geometry changes taken into account, was given by D. C. Drucker [1.3]; he considered a non-homogeneous three-bar system, assuming a rigid-linearly-hardening material. Similar examples were discussed in detail by L. Finzi [1.4] and P. G. Hodge [1.6]. Another simple example is furnished by pure bending of a perfectly elastic-plastic beam with a monosymmetric cross-section: unloading zones are due to the shifting of the neutral axis. If we neglect geometry changes, these effects are usually small (they were studied by I. S. Voronyuk [1.20]),

( ${ }^{1}$ ) A detailed discussion of the yield conditions is given in Secs. 9.3, 11 and 12 . 
but they are clearly visible if geometry changes are taken into account (B. Lücke [1.13], unloading and reverse loading processes at particular points of the body under a monotonically increasing external load).

These complications, shown in very simple cases, illustrate the difficulties encountered in the case of combined loadings. Some more general criteria excluding local passive unloading are given by O. De Donato and G. Maier [1.2].

\section{Concept of the interaction surface (limit surface)}

\subsection{Space of the exertion factors}

A convenient geometrical interpretation of the deformation processes under discussion may be obtained if we introduce the $n$-dimensional Euclidean space of the exertion factors at any level $\mathscr{P}, \mathscr{S}, \mathscr{B}$ [1.24]. Confining our considerations to basic exertion factors, we have $n \leqslant 6$ at the level $\mathscr{P}$, and $n \leqslant 6, n \leqslant 8, n \leqslant 10$ at the level $\mathscr{S}$ for a solid bar, thin-walled bar, and a surface structure, respectively. These numbers follow from the discussion given in Sec. 1 . At the level $\mathscr{B}$ the number of dimensions of the space may be arbitrarily large; the same applies to the levels $\mathscr{P}$ and $\mathscr{S}$ if we take accessory exertion factors into account.

The space of exertion factors might also be called the space of independent variables or of control variables. An arbitrary process may be presented as a line (path, trajectory) in that space.

Similarly, the space of dependent variables may be introduced. For example, if at the level $\mathscr{P}$ the space of independent variables is identified with the stress space, then the strain space may be regarded as the space of dependent variables (or vice versa); such a space has been introduced by $A$. A. Ilyushin $[1.10,1.11]$. In the space of the dependent variables the process (or rather its result) may also be presented by a certain trajectory, corresponding to the given line in the space of the exertion factors. The determination of that resulting line is one of the main problems of the theory of plasticity. It is necessary to know both lines in order to know the whole process. The correspondence of those lines constitutes, from the mathematical point of view, a certain transformation; we look for the value of that transformation.

This type of solution-knowledge of the value of the transformation-may be called solution in the broader sense. In the narrower sense we only look for the coordinates of the point in the space of dependent variables corresponding to the endpoint of the trajectory in the space of exertion factors. Then the result is expressed by the value of a certain functional depending on that trajectory.

The deformation process usually runs over various ranges, differing from each other from the physical and mathematical point of view. Those ranges may be represented by the corresponding domains in the space of exertion factors. The surfaces separating the individual domains, will generally be called interaction surfaces or 
limit surfaces; $\left(^{1}\right)$ in the case of an $n$-dimensional space we may speak about interaction hypersurface, in the case of a plane (two exertion factors)-about interaction curves.

\subsection{Typical interaction surfaces}

We are now going to discuss typical interaction surfaces (limit surfaces) at the levels $\mathscr{P}, \mathscr{S}$, and $\mathscr{B}$. Investigating the deformation process at any these levels, we first have to separate the domain of elastic deformations (passive processes) from the domain in which plastic deformations appear (active processes). However, this problem, simple from the physical point of view, presents some difficulties as regards effective determination of the corresponding interaction surface.

At the level $\mathscr{P}$ this surface should be determined experimentally. The corresponding stress is called elastic limit, and hence the term elastic interaction surface, or more briefly, elastic surface seems to be justified. Practical determination of the elastic limit is usually connected with the measurement of a certain plastic strain, and if the requirements of accuracy are not very sharp, then the elastic limit is identified even with the yield-point stress (cf. Sec. 8.1).

At the levels $\mathscr{S}$ and $\mathscr{B}$ the limits of elastic deformations are as a rule determined theoretically, by using a certain schematization (approximation) of the stress-strain diagram. Here the identification of the elastic limit and the yield-point stress is very common. The limit state in question will be called the elastic carrying capacity and the corresponding interaction surface the elastic surface as at the level $\mathscr{P}$. The corresponding quantities (in the case of a single exertion factor) will be distinguished by a bar, for example $\bar{M}, \bar{x}$, etc.

The second important limit state is connected with perfect yielding: it is characterized by the increments of strains or generalized strains under constant stresses or generalized stresses. The corresponding interaction surface will be called the yield surface and its equation-expressed in stresses-the condition of perfect plasticity, or more briefly, the yield condition. The term yield condition introduced originally at the level $\mathscr{P}$, is now used at the level $\mathscr{S}$ as well, but its application at the level $\mathscr{B}$ may also be justified. At the level $\mathscr{P}$, if we adopt the schematization of a perfectly elastic-plastic body, the yield surface coincides with the elastic surface. At the levels $\mathscr{S}$ and $\mathscr{B}$ such a coincidence does not take place (with some exceptions connected with a homogeneous stress distribution). Perfect yielding is here connected with a certain mechanism of plastic collapse, the limit state in question is called limit carrying capacity or plastic carrying capacity, and the corresponding surface-the plastic interaction surface; however, the general term yield surface may be justified here as well. The corresponding quantities (in the case of a single exertion factor) will be distinguished by a double bar, for example $\overline{\bar{M}}$, $\overline{\bar{\varkappa}}$, etc.

(1) Some authors understand by a limit surface only the surface corresponding to limit carrying capacity (plastic interaction surface), but in the present book it is understood in a quite general sense, as any separating surface in the space of exertion factors. 
During the active part of the process the elastic surfaces are usually subject to changes: they undergo translation, change their dimensions and shape. At the level $\mathscr{P}$ this phenomenon is due to the strain-hardening of the material, whereas at the levels $\mathscr{S}$ and $\mathscr{B}$ it may also take place in a perfectly elastic-plastic body, owing to the redistribution of stresses. Such a surface will be called the modified elastic surface or, particularly at the level $\mathscr{P}$, the neutral surface since the boundary process separating the active and passive processes is called neutral. Other commonly used terms are not compatible with the adopted here definitions: loading surface-because the terms loading and active process are distinguished, and we are speaking here about the separation of active and passive processes; yield surface-because it is better to reserve this name for perfect yielding.

The initial, original elastic surface does not depend on the trajectory of the deformation process. The modified elastic surface (neutral surface) depends on the position of the end-points of the trajectory (or of the active part of the trajectory); moreover, as a result of an active process, it may depend also on the whole trajectory. If the trajectory is not specified, then we usually think of proportional loading.

The elastic surface, the family of neutral surfaces, and the yield surface (if perfect yielding occurs) are the most important interaction surfaces at the level $\mathscr{P}$; sometimes we add here other interaction surfaces, connected with maximal force transmitted (strength), necking (local tensile instability), decohesion, etc. At the levels $\mathscr{S}$ and $\mathscr{B}$ the variety of interaction surfaces is much greater. In addition to all the abovementioned ones, other limit states are determined by the formation of new active zones (for example, in a cross-section subject to bending with tension), new passive zones, etc. In certain cases of boundary conditions the limit carrying capacity (mechanism of plastic collapse) cannot be reached within the frames of the concept of a continuous body: earlier inadmissible discontinuities appear. The corresponding loading parameter was called by K. Szuwalski and M. Życzkowski [1.18] the decohesive carrying capacity, so the respective limit surface will be called the decohesive interaction surface (cf. Sec. 18).

New problems arise if the loading parameter is variable in time: assume that it changes cyclically between its minimal values. Then, even in the case of one loading parameter, two exertion factors may be introduced, for example the mean value, and the amplitude. In this case typical interaction surfaces concern the elastic limit and the limits of the so-called shakedown, replacing here the static collapse mechanism (defined in Chapter IV). Those surfaces are usually introduced at the levels $\mathscr{B}$ and $\mathscr{S}$, but may also be useful at the level $\mathscr{P}$, where they generalize the Haigh fatigue diagram to the low-cycle fatigue.

\subsection{Distance function. Exertion of the material and of the structure}

Consider any interaction surface describing a certain limit state, which may be regarded as dangerous at the discussed level of analysis. The points $Q$ inside the surface represent the states, safe with respect to that limit state; from the engineering 
point of view a certain estimation of the corresponding safety factor is very important. In the case of proportional loading such an estimation may be expressed by the ratio of the segments $O Q$ and $O N_{0}$, Fig. 8. J. Walczak and S. Ziemba [1.21] proposed to call such a ratio at the level $\mathscr{P}$ exertion, $\left({ }^{1}\right)$ namely

$$
w_{0}=\frac{\overline{O Q}}{\overline{O N}_{0}}=1-\frac{\overline{Q N}_{0}}{\overline{O N}_{0}}=1-\frac{1 / r_{o 0}}{1 / r_{Q 0}}, \quad 0 \leqslant w_{0} \leqslant 1 .
$$

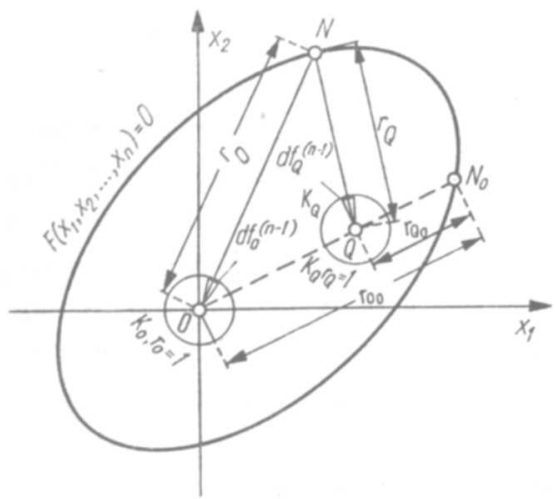

Fig. 8. The concept of exertion of the material or of the structure

Exertion defined in this way is reciprocal to the commonly used safety factor. W. Prager [1.15] mentiones that in the theory of convex surfaces such a ratio is called the distance function and discusses its application in the case of yield surfaces for a rigidplastic body. However, it may also be used in the case of surfaces which are not convex; if the point $N_{0}$ of intersection with the surface is not unique (which may be the case if the loss of stability is considered), then the point nearest to the origin $O$ should be chosen. Of course, some theorems given by Prager for convex surfaces are no longer valid here.

The class of proportional loadings is very narrow, and for other possible deformation processes the expression (1.1) does not give a proper estimation of safety (reciprocal safety). M. Życzkowski generalized (1.1) to the general case of straightline processes starting from the point $Q$; in [1.23] for the level $\mathscr{P}$, and in [1.25] for the remaining levels.

Denote by $X_{i}$ the exertion factors taken into consideration at any of the levels $\mathscr{P}, \mathscr{S}$, and $\mathscr{B}$. They may be of various physical dimensions; it would then be impossible to speak about the distance in the space of exertion factors except for proportional loading. Therefore we first introduce dimensionless exertion factors

$$
x_{i}=\frac{X_{i}}{A_{i}}, \quad i=1,2, \ldots, n,
$$

where $A_{i}$ are certain reference quantities, of the dimensions of $X_{i}$. In the space of $x_{i}$ denote now by $r_{Q}$ the distance between the point $Q$, representing the state in question,

(1) Translated from the Polish wytężenie; the corresponding German term is Anstrengung. 
and an arbitrary point of the limsurfaceit $N$, further by $r_{o}$, the distance $\overline{O N}$, Fig. 8 . Formula (3.1) for exertion was generalized $[1.23,1.26]$ as follows:

$$
w=1-\frac{\mid \mathfrak{M}\left(1 / r_{o}\right)}{\mathfrak{M}\left(1 / r_{Q}\right)} .
$$

The symbol $\mathfrak{M}$ denotes here a certain mean value of the function, namely

$$
\mathfrak{M}\left(1 / r_{Q}\right)=\frac{K_{Q} \mathbf{S S}\left(1 / r_{Q}\right) p d f_{Q}^{(n-1)}}{{\underset{K}{K_{Q}}}_{\mathbf{S}} p d f_{Q}^{(n-1)}},
$$

where $\mathbf{S S}$ is the surface integral over the $(n-1)$-dimensional surface in the Hadamard notation. $K_{Q}$ is the $n$-dimensional unit sphere with the centre at $Q, d f_{Q}^{(n-1)}$ is an element of the surface of that sphere; the mean value is here calculated with the weight function $p$, depending on the direction and expressing the probability of reaching the limit surface in that direction (density of probability). This function may also depend on the coordinates of the point $N$-it should be determined according to the expected working conditions of the structure or its element. The function $p$ need not be normed, since it appears both in the numerator and in the denominator of (3.4) and any constant factor would be cancelled.

If the function $p$ is assumed to be a Dirac function, namely $p=0$ for all directions except the direction $O Q$, for which $p \rightarrow \infty$, then $\mathfrak{M}\left(1 / r_{Q}\right)=1 / r_{Q 0}$, similarly $\mathfrak{M}\left(1 / r_{o}\right)$ $=1 / r_{o 0}$, and we arrive directly at (3.1); thus (3.3) may be considered as a generalization of (3.1). The proposed formula (3.3) meets all the other basic requirements; if $Q$ coincides with $O$, then the denominator is equal to the numerator, and $w=0$; if $Q$ belongs to the limit surface, then the integral in the denominator is divergent and we obtain $w=1$. For certain points $Q$ and certain functions $p$ we may also obtain $w<0$; this means that for the expected working conditions the state $Q$ in question is better than the state $O$. Minimizing $w$, we may determine the optimal prestressing of the structure, etc.

If the weight function $p$ is a function of the direction only (in the space of exertion factors), then the denominators of both mean values in (3.3) are equal and cancelled so that the formula takes the form

$$
w=1-\frac{\underset{K_{0}}{\mathbf{S S}}\left(1 / r_{o}\right) p d f_{o}^{(n-t)}}{\operatorname{SS}_{K_{Q}}\left(1 / r_{Q}\right) p d f_{Q}^{(n-1)}} .
$$

On introducing spherical coordinates with the origin at $Q$ by means of the formulae

$$
\begin{aligned}
& x_{m}=x_{m Q}+r \cos \varphi_{m} \prod_{i=1}^{m-1} \sin \varphi_{i}, \quad m=1,2, \ldots, n-1, \\
& x_{n}=x_{n Q}+r \prod_{i=1}^{n-1} \sin \varphi_{i},
\end{aligned}
$$


after the determination of the function $r=r_{Q}\left(\varphi_{1}, \varphi_{2}, \ldots, \varphi_{n-1}\right)$ (from the equation of the interaction surface) and a subsequent iteration procedure we obtain finally

$$
w=1-\frac{\int_{0}^{\pi} \sin ^{n-2} \varphi_{1} d \varphi_{1} \ldots \int_{0}^{\pi} \sin \varphi_{n-2} d \varphi_{n-2} \int_{0}^{2 \pi} \frac{p\left(\varphi_{1}, \varphi_{2}, \ldots, \varphi_{n-1}\right) d \varphi_{n-1}}{r_{o}\left(\varphi_{1}, \varphi_{2}, \ldots, \varphi_{n-1}\right)}}{\int_{0}^{\pi} \sin ^{n-2} \varphi_{1} d \varphi_{1} \ldots \int_{0}^{\pi} \sin \varphi_{n-2} d \varphi_{n-2} \int_{0}^{2 \pi} \frac{p\left(\varphi_{1}, \varphi_{2}, \ldots, \varphi_{n-1}\right) d \varphi_{n-1}}{r_{Q}\left(\varphi_{1}, \varphi_{2}, \ldots, \varphi_{n-1}\right)}},
$$

The computational details are given in [1.25], and some examples of application in [1.26]. Formula (3.3), generalizing (3.1), furnishes not only a more general and adequate estimation of the structural safety, but in some cases makes it also possible to determine optimal prestressing conditions.

\section{Classification of combined loadings}

\subsection{Symbolism of the classification}

Under the term combined loading we understand the occurrence of more than one exertion factor at any of the levels $\mathscr{P}, \mathscr{S}$, and $\mathscr{B}$. It should be recalled here that by an exertion factor we understand an independent quantity. So, the loading of a beam shown in Fig. $9 \mathrm{a}$ is combined at the level $\mathscr{B}$ if the forces $P_{1}$ and $P_{2}$ are independent;

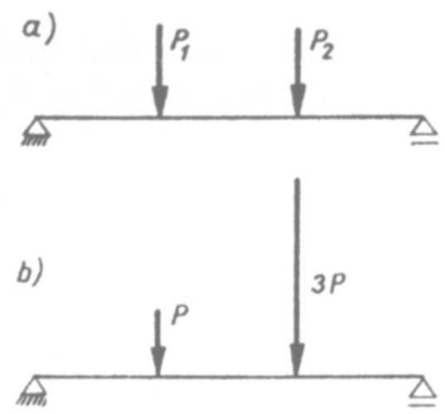

Fig. 9. (a) Combined loading at the level $\mathscr{B}$; (b) loading may be considered as a particular case of combined loading at the level $\mathscr{B}$

if a certain relation between $P_{1}$ and $P_{2}$ is given, as in Fig. $9 \mathrm{~b}$, then the loading is not combined. However, it is sometimes convenient to consider the latter case as a certain particular case of combined loading.

In the case of a combined loading, if more than one exertion factor exists, we may introduce the space of exertion factors and the interaction surfaces in that space. Thus the problems of combined loadings are closely connected with the construction of interaction surfaces. Those interaction surfaces constitute either the final purpose of the analysis (because of their engineering importance, e.g. elastic interaction 
surfaces, plastic interaction surfaces), or simply determine the ranges of validity of the particular constitutive equations.

The term combined loading may be understood in three ways: the loading may be combined at the level $\mathscr{P}, \mathscr{S}$ or $\mathscr{B}$, to a certain degree independently. To avoid confusions we introduce here the classification of combined loadings proposed in [1.27]. To the consecutive symbols $\mathscr{P}, \mathscr{S}$, and $\mathscr{B}$ we add number-indices, showing the number of exertion factors at each level; simultaneously the number of dimensions of the corresponding space will be determined.

Another question very important for the classification concerns the homogeneity or non-homogeneity of the distribution of the exertion factors over the cross-section (transversal) or along the axis or middle surface (longitudinal). The case of homogeneity is of course much more simple to be analysed. We shall denote transversal homogeneity by the symbol $\rightarrow$ between the letters $\mathscr{P}$ and $\mathscr{S}$, and longitudinal homogeneity by the same symbol between $\mathscr{S}$ and $\mathscr{B}$ (since in those cases the transition from the point to the section or from the section to the body is immediate), and non-homogeneity by the symbol " /". These conclusions may be no longer valid in the case of nonhomogeneous or non-prismatic bodies and then " $\rightarrow$ " must often be replaced by"/".

The proposed classification is of course also valid in the linearly elastic range, but in view of the principle of superposition its importance is then limited. On the other hand, it may be useful for the analysis of other physically nonlinear problems, for example in the theory of creep or viscoplasticity.

\subsection{Examples of the classification}

Let us now proceed to some examples. The classical case of a thin-walled circular bar subject to simultaneous tension and torsion (Fig. 10) will be classified as $\mathscr{P}_{2} \rightarrow$

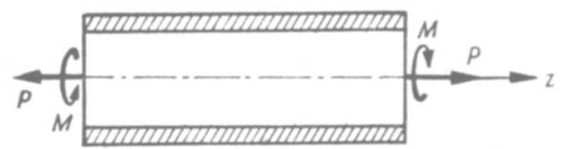

Fig. 10. An example of a homogeneous transition between the levels $\mathscr{P}, \mathscr{S}$, and $\mathscr{B}$

$\mathscr{S}_{2} \rightarrow \mathscr{B}_{2}$ : at any point $\mathscr{P}$ two independent stress components $\sigma_{z}$ and $\tau_{z \theta}$ occur (or two independent strains $\varepsilon_{z}$ and $\gamma_{z \theta}$, since the non-zero transverse strains $\varepsilon_{r}$ and $\varepsilon_{\theta}$ depend on $\varepsilon_{z}$ ), and at any section $\mathscr{S}$-two generalized stresses $N$ and $M_{t}$; finally the body $\mathscr{B}$ is subject to two independent generalized external forces $P$ and $M$. The case is therefore combined at all three levels, but its simplicity consists in homogeneity, transversal as well as longitudinal.

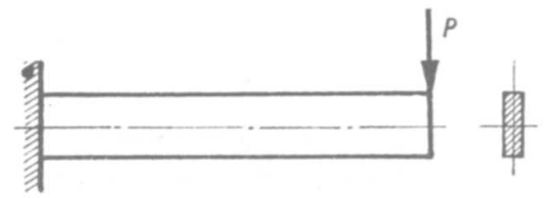

Fig. 11. An example of a non-homogeneous transition between the levels $\mathscr{P}, \mathscr{S}$, and $\mathscr{B}$ 
Bending of a cantilever beam by a transversal concentrated force (Fig. 11), with shear taken into account, will be classified as $\mathscr{P}_{2} / \mathscr{S}_{2} / \mathscr{B}_{1}$; it is much more complex because of the non-homogeneities. A circular plate under a uniform transversal loading $p$ (Fig. 12) will be classified as $\mathscr{P}_{2} / \mathscr{S}_{2} / \mathscr{B}_{1}$ according to the small-deflection
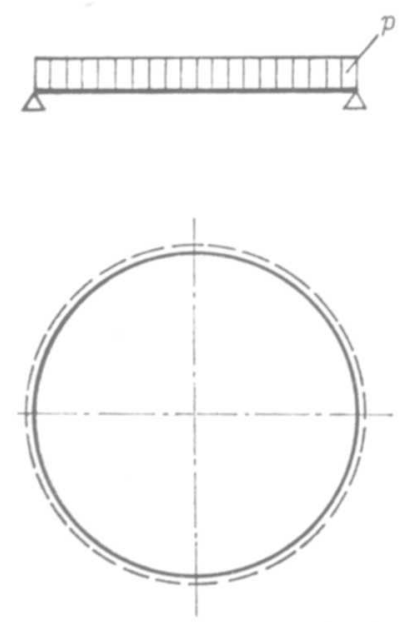

Fig. 12. Classification of a circular plate according to the theory adopted

theory with the shear stresses neglected; it will be classified as $\mathscr{P}_{2} / \mathscr{S}_{4} / \mathscr{B}_{1}$ according to the large-deflection theory (two normal forces must be added here to two bending moments); and finally, as $\mathscr{P}_{3} / \mathscr{S}_{3} / \mathscr{B}_{1}$ according to the small-deflection theory with shear taken into account $\left(\sigma_{r}, \sigma_{\theta}, \tau_{r z} ; M_{r}, M_{\theta}, Q_{r} ; p\right)$. A circular plate under uniform edge couples, $\mathscr{P}_{2} / \mathscr{S}_{2} \rightarrow \mathscr{B}_{1}$ (according to the small-deflection theory) is simpler because of longitudinal homogeneity, Fig. 13. In the case of a thick-walled tube the level $\mathscr{S}$ is often difficult to be defined; a tube subject to the internal pressure $p$ and end load (thrust) $N$ will be classified just as $\mathscr{P}_{3} / \mathscr{B}_{2}\left(\sigma_{r}, \sigma_{\theta}, \sigma_{z} ; p, N\right)$.
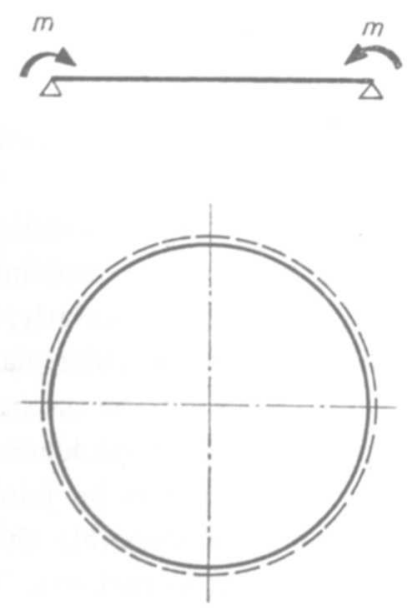

Fig. 13. An example of longitudinal homogeneity of the stress state 
The loading may be combined simultaneously at all three levels, but also at each level separately. It is easy to cite suitable examples. Consider in an exact manner pure bending of an elastic-plastic beam made of a compressible material (Fig. 14); then the assumption of only one non-vanishing stress component $\sigma$ leads to a con-

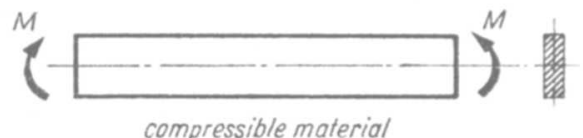

Fig. 14. An example of loading combined only at the level $\mathscr{P}$

tradiction with the compatibility equations. A. A. Ilyushin [1.9] proved that four stress components must appear here and so we arrive at $\mathscr{P}_{4} / S_{1} \rightarrow \mathscr{B}_{1}$; this problem was also treated by J. Mandel [1.14]. An eccentrically compressed column, under the assumption of constant eccentricity, will be classified as $\mathscr{P}_{1} / \mathscr{S}_{2} / \mathscr{B}_{1}$ (Fig. 15).

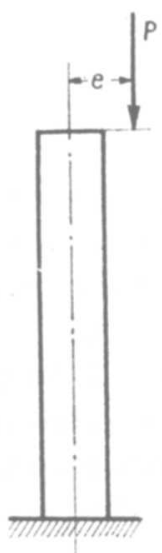

Fig. 15. An example of loading combined only at the level $\mathscr{S}$

Finally, a beam shown in Fig. 9a with shear effects neglected may be classified as $\mathscr{P}_{1} / \mathscr{P}_{1} / \mathscr{B}_{2}$.

It should be noted that some cases are only apparently combined; for example the stresses $\tau_{z x}$ and $\tau_{z y}$ produced by pure torsion may be replaced by their resultant $\tau_{z}$ and there is no need to consider this case as combined at the level $\mathscr{P}$. Similarly, the bending of a circular bar by two bending moments acting in perpendicular planes is only seemingly oblique (although the non-proportional change of the moments in the elastic-plastic range leads to some phenomena typical for combined loading). Sometimes it is also possible to reduce the number of exertion factors by joining them into convenient groups; for example, considering an incompressible thickwalled tube under internal and external pressure in plane strain conditions, it is usually sufficient to take only the pressure difference into account. 
In the above examples we tacitly assumed the homogeneity of the material. In the case of a non-homogeneous body the classification may be essentially changed, and those changes have a clear physical interpretation. For example, if a homogeneous simply connected body is subject to hydrostatic pressure, then at any point uniform three-dimensional compression takes place, which, as a rule, does not result in any plastic effects. The classification $\mathscr{P}_{1} \rightarrow \mathscr{B}_{1}$ may be used here: though at individual points three principal stresses occur, only one of them may be regarded as independent. In the case of a non-homogeneous material the state of stress ceases to be uniform triaxial compression and yielding may take place (J. Rychlewski [1.16]); the classification should be changed here to $\mathscr{P}_{6} / \mathscr{B}_{1}$. The occurrence of a three-dimensional state of stress in a non-homogeneous compressible bar subject to uniaxial tension was investigated by W. Świsterski [1.19].

\subsection{The problem of independence of the exertion factors}

The above example of the eccentric compression of a column, classified as $\mathscr{P}_{1} / \mathscr{S}_{2} / \mathscr{B}_{1}$, will now serve for the discussion of the problem of independence of the exertion factors at the individual levels of analysis. In fact, it is only the exertion factors at the level $\mathscr{B}$, of the body as a whole, that are independent; their program is either prescribed (deterministically or stochastically) in the working conditions of the structure, or may be prescribed-quite arbitrarily, as a rule-in the experimental investigations. The exertion factors at the levels $\mathscr{P}$ and $\mathscr{S}$ are to a certain degree dependent on the factors at the level $\mathscr{B}$; nevertheless, in most cases it is useful to treat them as independent ones.

The problem of the eccentric compression of a column (Fig. 15) may be analysed under the assumption of the (approximate) principle of rigidification $\left(^{1}\right)$ (the influence of geometry changes not being taken into account, theory of the first order), or with the effects of geometry changes taken into account (theories of the second or of the third order, according to the assumed accuracy, E. Chwalla [1.1]). The first approach would involve the classification $\mathscr{P}_{1} / \mathscr{S}_{1} \rightarrow \mathscr{B}_{1}$ since the bending moment at any section is related to the compressive normal force by $M_{b}=N e$; only one quantity is here independent, and moreover, the longitudinal homogeneity of stresses takes place. Just the second, more exact approach corresponds to the classification $\mathscr{P}_{1} / \mathscr{S}_{2} / \mathscr{B}_{1}$; in the individual cross-sections the relation between the bending moment and the normal force is various and it is assumption of independence of $M_{b}$ and $N$ that is necessary for performing the full analysis of the column behaviour and determining its load-carrying capacity.

We should thus speak about independent exertion factors at the level $\mathscr{B}$, and about factors which may be regarded as independent at the levels $\mathscr{P}$ and $\mathscr{S}$.

(1) In Polish zasada zesztywnienia. 


\section{CHAPTER II}

\section{Basic equations of the theory of plasticity}

\section{Systems of invariants of second-order tensors}

\subsection{Number of independent invariants}

Physical laws should be form-invariant to a change of coordinate systems (unless the system is uniquely specified and not subject to any change). This requirement is satisfied by tensorial character of basic physical quantities used in continuum mechanics (stresses, strains, strain rates, etc.). Tensors transform in such a way that if a tensor equation holds in one coordinate system, it holds also in any other system. A second-order tensor transforms (in Cartesian coordinates) according to the rule

$$
\tau_{k l}=a_{t k} a_{j l} \tau_{i j}, \quad i, j=x, y, z ; \quad k, l=x^{\prime}, y^{\prime}, z^{\prime},
$$

where $\tau_{i j}$ denote the components in the "original" reference frame, $x, y, z ; \tau_{k l}$-in the "new" reference frame $x^{\prime}, y^{\prime}, z^{\prime} ; a_{i k}$-the cosines of angles between $x_{i}$ and $x_{k}$ (direction cosines), and the Einstein summation convention is employed. The direction cosines satisfy the following orthogonality relations

$$
a_{i j} a_{i k}=\delta_{j k}= \begin{cases}1 & \text { if } j=k, \\ 0 & \text { if } j \neq k,\end{cases}
$$

where $\delta_{j k}$ denotes the Kronecker symbol.

The tensor formed by $\tau_{i j}$ will be denoted by $T_{t}$. To show effectively the components in the coordinate system $x, y, z$, we use the notation

$$
T_{\tau}=\left\{\begin{array}{lll}
\tau_{x x} & \tau_{x y} & \tau_{x z} \\
\tau_{y x} & \tau_{y y} & \tau_{y z} \\
\tau_{z x} & \tau_{z y} & \tau_{z z}
\end{array}\right\}
$$

The diagonal passing through $\tau_{x x}, \tau_{y y}, \tau_{z z}$ is called the principal diagonal. For the problems of flow and fracture it is very important to distinguish the components on and off the principal diagonal and in the engineering notation even different symbols are being used. 
The above-mentioned requirements of invariance of physical laws to a change of reference frames result in a fundamental role of the invariants of the tensors under consideration. A second-order tensor has infinitely many various invariants, but in the three-dimensional space only six of them may be independent (W. Jaunzemis [2.74]). If we decompose the given tensor into a symmetric and a skew-symmetric part, then, for example, the principal values of the symmetric part and the components of the skew-symmetric part in the principal coordinate system (determined for the symmetric part) constitute such a system of six independent invariants. For a symmetric tensor the maximal number of independent invariants is equal to three.

The classical continuum mechanics excludes the existence of distributed body couples and distributed surface couples. Under this assumption most of the tensors in question are symmetric and here we confine ourselves to the discussion of the systems of three independent invariants of symmetric tensors. The choice of a suitable system of invariants may be very important for the simplicity of formulating the physical relations. Therefore we present here some typical systems of invariants and the relations between the invariants belonging to the particular systems.

The Cosserat continua (with body couples and couple stresses) will not be considered in the present book. It should be mentioned, however, that this branch of plasticity has been widely developed in the last decade (A. E. Green, P. M. Naghdi [2.40], S. Komljenović [2.88], A. Sawczuk, [2.149], A. E. Green, B. C. McInnis, P. M. Naghdi [2.42], H. Lippmann [2.102], V. V. Kolokoltchikov [2.87], N. Coutris, J. Ségura [2.21], Y. Kishino [2.83], J. J. Telega [2.172], M. Hlavaček [2.59], D. Besdo [2.7]).

\subsection{Basic invariants of the tensor}

The simplest way to obtain a system of invariants is to perform consecutive multiplications of the tensor by itself and a complete contraction. The invariants obtained will be called the basic invariants of the tensor $T_{\tau}$ and denoted by $I_{1 \tau}, I_{2 \tau}, \ldots$ :

$$
\begin{aligned}
I_{1 \tau}= & \tau_{i i}=\tau_{x x}+\tau_{y y}+\tau_{z z}, \\
I_{2 \tau}= & \tau_{i j} \tau_{j i}=\tau_{x x}^{2}+\tau_{y y}^{2}+\tau_{z z}^{2}+2 \tau_{x y}^{2}+2 \tau_{y z}^{2}+2 \tau_{z x}^{2}, \\
I_{3 \tau}= & \tau_{i j} \tau_{j k} \tau_{k l}=\tau_{x x}^{3}+\tau_{y y}^{3}+\tau_{z z}^{3}+3 \tau_{x x} \tau_{x y}^{2}+3 \tau_{x x} \tau_{x z}^{2}+ \\
& +3 \tau_{y y} \tau_{y z}^{2}+3 \tau_{y y} \tau_{y x}^{2}+3 \tau_{z z} \tau_{z x}^{2}+3 \tau_{z z} \tau_{z y}^{2}+6 \tau_{x y} \tau_{y z} \tau_{z x} .
\end{aligned}
$$

The expanded notation of these invariants makes use of the assumed symmetry of the tensor $T_{\tau}$. The name "basic invariants" is used, for example, by I. I. Goldenblat [2.38], who also adopts a similar notation. W. Jaunzemis [2.74] calls them "principal moments". Further invariants constructed in this way may be expressed in terms of the three quoted above:

$$
\begin{aligned}
& I_{4 \tau}=\frac{1}{6} I_{1 \tau}^{4}+\frac{1}{2} I_{2 \tau}^{2}-I_{1 \tau}^{2} I_{2 \tau}+\frac{4}{3} I_{1 \tau} I_{3 \tau}, \\
& I_{5 \tau}=\frac{1}{6} I_{1 \tau}^{5}+\frac{5}{6} I_{2 \tau} I_{3 \tau}+\frac{5}{6} I_{1 \tau}^{2} I_{3 \tau}-\frac{5}{6} I_{1 \tau}^{3} I_{2 \tau},
\end{aligned}
$$


Some authors define these invariants with certain coefficients, for example A. M. Freudenthal and H. Geiringer [2.33] introduce the invariants $I_{n \tau} / n$.

The invariants which remain unchanged under a cyclic permutation of the indices

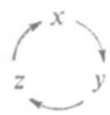

are called symmetric invariants; such invariants are particularly useful for the description of properties of isotropic bodies. It is seen from (5.4) that basic invariants are symmetric.

\subsection{Principal invariants of the tensor}

Another system of invariants is connected with the calculation of the principal values (eigenvalues) of a symmetric tensor. By a principal value we understand (1) the stationary (extremal) value of the diagonal coordinate $\tau_{i j}$ for $j=i$ as a function of the direction, (2) the diagonal coordinate in a direction for which the off-diagonal coordinates $\tau_{i j}, j \neq i$, vanish. It may be proved that both these definitions are equivalent and the principal values are determined by the cubic equation

$$
\tau^{3}-J_{1, \tau} \tau^{2}+J_{2 \tau} \tau-J_{3 \tau}=0
$$

where

$$
\begin{aligned}
& J_{1 \tau}=\tau_{x x}+\tau_{y y}+\tau_{z z}, \\
& J_{2 \tau}=\tau_{x x} \tau_{y y}+\tau_{y y} \tau_{z z}+\tau_{z z} \tau_{x x}-\tau_{x y}^{2}-\tau_{y z}^{2}-\tau_{z x}^{2}, \\
& J_{3 \tau}=\tau_{x x} \tau_{y y} \tau_{z z}+2 \tau_{x y} \tau_{y z} \tau_{z x}-\tau_{x x} \tau_{y z}^{2}-\tau_{y y} \tau_{z x}^{2}-\tau_{z z} \tau_{x y}^{2}=\operatorname{det} T_{\tau} .
\end{aligned}
$$

The symbol det $T_{\tau}$ denotes here the determinant of the tensor components. It may also be proved that there always exist three real roots of this equation, $\tau_{1}, \tau_{2}, \tau_{3}$, and the corresponding directions, called principal directions, are perpendicular to one another (uniquely determined if the roots are distinct). Thus the principal directions may be chosen as a rectangular coordinate system and in this system $T_{\tau}$ is transformed into the diagonal form

$$
T_{\tau}=\left\{\begin{array}{lll}
\tau_{x x} & \tau_{x y} & \tau_{x z} \\
\tau_{y x} & \tau_{y y} & \tau_{y z} \\
\tau_{z x} & \tau_{z y} & \tau_{z z}
\end{array}\right\} \rightarrow\left\{\begin{array}{lll}
\tau_{1} & 0 & 0 \\
0 & \tau_{2} & 0 \\
0 & 0 & \tau_{3}
\end{array}\right\} .
$$

The second definition of the principal values enables us to derive Eq. (5.6) more quickly and easily than the first one; however, from the point of view of flow and fracture the extremality of the principal values of the tensors is more important than the vanishing of the off-diagonal components. The derivation of (5.6) from the extremality conditions may be found in the books by J. Walczak [2.188] (extremum with the auxiliary condition (5.2) for $j=k$ ), A. M. Freudenthal [2.34] (extremum with respect to two independent direction cosines), A. C. Eringen [2.31] and P. G. Hodge 
[2.62]. The formulae determining the principal directions may be found in the book by W. Krzyś and M. Życzkowski [2.91].

The principal values of a tensor are of course invariant, but they are not symmetric: they change with cyclic permutation of the indices. The symmetrization is achieved if the principal values are algebraically ordered-in this case they will be denoted by $\tau_{\mathrm{I}}, \tau_{\mathrm{II}}, \tau_{\mathrm{III}}$, namely $\tau_{\mathrm{I}} \geqslant \tau_{\mathrm{II}} \geqslant \tau_{\mathrm{III}}$. The coefficients of the cubic equation (5.6) are connected with the roots by the relations

$$
\begin{aligned}
& J_{1 \tau}=\tau_{1}+\tau_{2}+\tau_{3}, \\
& J_{2 \tau}=\tau_{1} \tau_{2}+\tau_{2} \tau_{3}+\tau_{3} \tau_{1}, \\
& J_{3 \tau}=\tau_{1} \tau_{2} \tau_{3}
\end{aligned}
$$

and they are symmetric invariants. To distinguish them from the basic invariants we use here the convenient name principal invariants; this term is also used by A. M. Freudenthal and H. Geiringer [2.33], P. Perzyna [2.128], W. Jaunzemis [2.74]. Some authors define $J_{2 \tau}$ with the opposite sign (A. A. Ilyushin [1.8], R. Hill [2.56], W. Prager and P. G. Hodge [2.133], W. Prager [2.136]); the reason will be explained in Sec. 5.5 , but we retain here the classical definition.

The first, linear principal invariant is equal to the first basic invariant of the tensor, and so the adjectives "principal" and "basic" may be omitted here. The further invariants, called quadratic and cubic in the respective systems, are not equal to each other, the relations between them will be given in the table in Sec. 6.2.

Invariant are also the components of the tensor in any coordinates system situated uniquely with respect to the system of principal axes. Symmetric invariants will be obtained if the axis forms equal angles with the three principal directions, $a_{1 x}^{2}=a_{1 y}^{2}$ $=a_{1 z}^{2}=1 / 3$. There exist eight such directions, and the corresponding invariants are called octahedral; they will be discussed in detail, separately for stresses and for strains.

\subsection{Cylindrical invariants of the tensor}

Introduce the three-dimensional space of the principal values $\tau_{1}, \tau_{2}, \tau_{3}$ of a symmetric tensor $T_{\tau}$. In the case of a stress tensor such a space is connected with the names of Haigh [2.50] and Westergaard [2.190] although it was used as far back as the XIX century by H. Hertz (cf. W. Burzyński [2.13]). Every tensor $T_{\tau}$ is then represented by a point in this space. The inverse relation is not unique, since the rotation of the principal axes at constant principal values of the tensor is not reflected in the space under consideration; in many applications, however, this fact is unimportant.

Introduce further a cylindrical system of coordinates in this space as follows: the axis of the system coincides with the equally inclined axis determined by the equation $\tau_{1}=\tau_{2}=\tau_{3}$, and the angle $\omega_{\tau}$ is measured from the plane passing through this axis and $\tau_{1}$, Fig. 16. The cylindrical coordinates of the point $T_{t}$, denoted by 


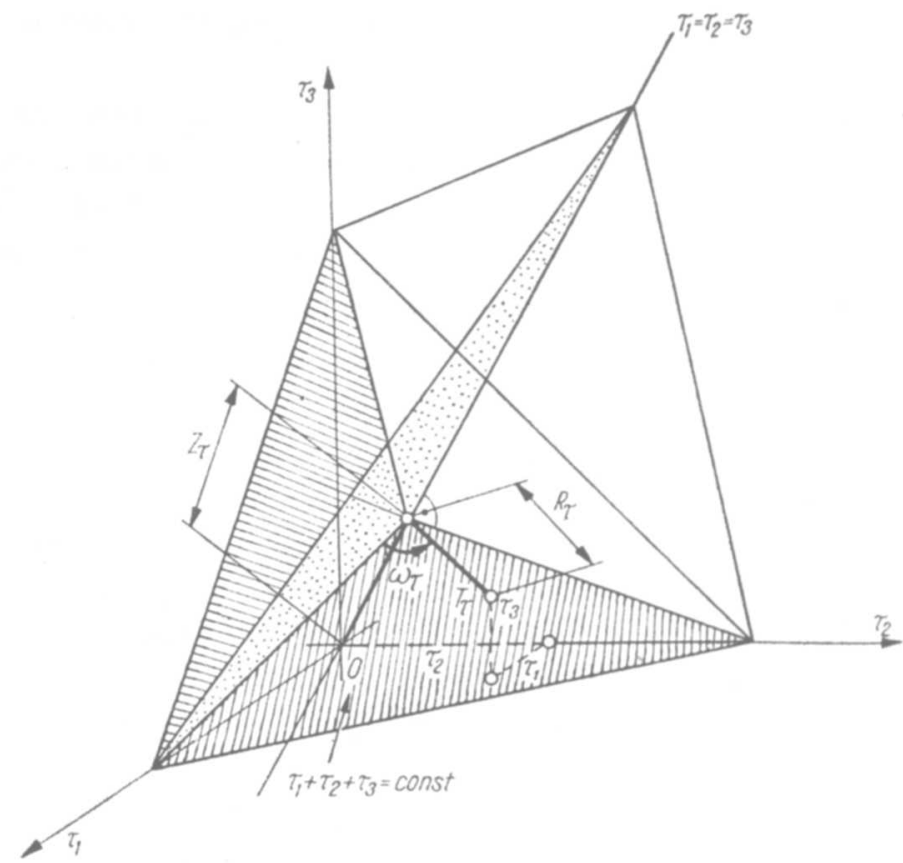

Fig. 16. Cylindrical coordinates $Z_{\tau}, R_{\tau}$ and $\omega_{\tau}$ in the space of principal values of the tensor $T_{\tau}$ $Z_{\tau}, R_{\tau}$, and $\omega_{\tau}$, form also an important system of invariants of the tensor $T_{\tau}$. They may be expressed in terms of the principal values as follows (J. Murzewski [2.116]):

$$
\begin{aligned}
& Z_{\tau}=\sqrt{\frac{1}{3}}\left(\tau_{1}+\tau_{2}+\tau_{3}\right), \\
& R_{\tau}=\sqrt{\frac{1}{3}} \sqrt{\left(\tau_{1}-\tau_{2}\right)^{2}+\left(\tau_{2}-\tau_{3}\right)^{2}+\left(\tau_{3}-\tau_{1}\right)^{2}}, \\
& \omega_{\tau}=\arcsin \frac{\tau_{2}-\tau_{3}}{R_{\tau} \sqrt{2}},
\end{aligned}
$$

and the inverse relations are

$$
\begin{aligned}
& \tau_{1}=\frac{Z_{\tau}}{\sqrt{3}}+\sqrt{\frac{2}{3}} R_{\tau} \cos \omega_{\tau}, \\
& \tau_{2}=\frac{Z_{\tau}}{\sqrt{3}}+\sqrt{\frac{2}{3}} R_{\tau} \cos \left(\omega_{\tau}-\frac{2}{3} \pi\right), \\
& \tau_{3}=\frac{Z_{\tau}}{\sqrt{3}}+\sqrt{\frac{2}{3}} R_{\tau} \cos \left(\omega_{\tau}+\frac{2}{3} \pi\right),
\end{aligned}
$$

The invariants $Z_{\tau}, R_{\tau}$ and $\omega_{\tau}$ will be called cylindrical invariants of the tensor. In what follows we shall use them very often, but with slightly changed coefficients (discussed separately for stresses and for strains). The invariant $\omega_{\tau}$ does not belong to symmetric invariants; however, trigonometric functions of $3 \omega_{\tau}$ are symmetric (cf. (5.23)), and in most cases they occur in physical relations. 


\subsection{Invariants of the axiator and of the deviator}

Any second-order tensor $T_{\tau}$ may be decomposed into an axiator (spherical tensor) $A_{\tau}$ and a deviator $D_{\tau}$ according to the scheme

$$
T_{\tau}=\left\{\begin{array}{lll}
\tau_{x x} & \tau_{x y} & \tau_{x z} \\
\tau_{y x} & \tau_{y y} & \tau_{y z} \\
\tau_{z x} & \tau_{z y} & \tau_{z z}
\end{array}\right\}=\left\{\begin{array}{lll}
\tau_{m} & 0 & 0 \\
0 & \tau_{m} & 0 \\
0 & 0 & \tau_{m}
\end{array}\right\}+\left\{\begin{array}{lll}
\tau_{x x}-\tau_{m} & \tau_{x y} & \tau_{x z} \\
\tau_{y x} & \tau_{y y}-\tau_{m} & \tau_{y z} \\
\tau_{z x} & \tau_{z y} & \tau_{z z}-\tau_{m}
\end{array}\right\} .
$$

The symbol $\tau_{m}$ denotes here the mean value of the diagonal components, proportional to the first invariant

$$
\tau_{m}=\frac{1}{3}\left(\tau_{x x}+\tau_{y y}+\tau_{z z}\right)=\frac{1}{3} I_{1 \tau}=\frac{1}{3} J_{1 \tau} .
$$

Such a decomposition was introduced by M. Kleitz in 1873 (cf. L. E. Malvern [2.108]), and its physical interpretation is clearly visible in the case of a small-strain tensor: the strain axiator corresponds here to the pure volume change (dilatation), and the strain deviator to the pure shape change (distortion). In the isotropic elastic medium this property holds also for the stress tensor, and is usually postulated in plasticity as well. The components of the deviator will be denoted by Roman letters corresponding to the Greek symbols used for the tensor: thus to the letter $\tau$ chosen here quite arbitrarily we ascribe the symbol $t$, writing shortly, instead of (5.12),

$$
t_{i j}=\tau_{i j}-\delta_{i j} \tau_{m} .
$$

In view of the given interpretation of the decomposition (5.12) the invariants of the axiator and of the deviator become particularly important; our discussion will be confined to the symmetric deviators. The first invariant of the axiator is equal to that of the tensor, and thus no new notation will be introduced here. Further invariants of the axiator may be expressed in terms of the first one, which alone is independent.

The basic invariants of the deviator will be denoted by $I_{1 t}, I_{2 t}, I_{3 t}$, and the principal invariants by $J_{1 t}, J_{2 t}, J_{3 t}$. The first invariant is here obviously equal to zero, $I_{1 t}=J_{1 t}=0$, and only the remaining two are independent. The second and the third basic invariant of the deviator may be determined from (5.4); we first obtain

$$
\begin{aligned}
I_{2 t}= & t_{x x}^{2}+t_{y y}^{2}+t_{z z}^{2}+2 t_{x y}^{2}+2 t_{y z}^{2}+2 t_{z x}^{2}=t_{1}^{2}+t_{2}^{2}+t_{3}^{2}=t_{i j} t_{j i}, \\
I_{3 t}= & t_{x x}^{3}+t_{y y}^{3}+t_{z z}^{3}+3 t_{x x} t_{x y}^{2}+3 t_{x x} t_{x z}^{2}+3 t_{y y} t_{y z}^{2}+3 t_{y y} t_{y x}^{2}+ \\
& +3 t_{z z} t_{x x}^{2}+3 t_{z z} t_{z y}^{2}+6 t_{x y} t_{y z} t_{z x}=t_{1}^{3}+t_{2}^{3}+t_{3}^{3}=t_{i j} t_{j k} t_{k i},
\end{aligned}
$$

where $t_{1}, t_{2}, t_{3}$ denote, as usual, the principal values of the deviator. Further, expressing $t_{i j}$ in terms of $\tau_{i j}$, we arrive at

$$
\begin{aligned}
I_{2 t} & =\frac{2}{3}\left[\tau_{x x}^{2}+\tau_{y y}^{2}+\tau_{z z}^{2}-\tau_{x x} \tau_{y y}-\tau_{y y} \tau_{z z}-\tau_{z z} \tau_{x x}+3\left(\tau_{x y}^{2}+\tau_{y z}^{2}+\tau_{z x}^{2}\right)\right] \\
& =\frac{1}{3}\left[\left(\tau_{x x}-\tau_{y y}\right)^{2}+\left(\tau_{y y}-\tau_{z z}\right)^{2}+\left(\tau_{z z}-\tau_{x x}\right)^{2}+6\left(\tau_{x y}^{2}+\tau_{y z}^{2}+\tau_{z x}^{2}\right)\right] \\
& =\frac{1}{3}\left[\left(\tau_{1}-\tau_{2}\right)^{2}+\left(\tau_{2}-\tau_{3}\right)^{2}+\left(\tau_{3}-\tau_{1}\right)^{2}\right],
\end{aligned}
$$




$$
\begin{aligned}
I_{3 t}= & \frac{4}{3} \tau_{x x} \tau_{y y} \tau_{z z}-\frac{1}{3}\left(\tau_{x x}^{2} \tau_{y y}+\tau_{x x}^{2} \tau_{z z}+\tau_{y y}^{2} \tau_{x x}+\tau_{y y}^{2} \tau_{z z}+\tau_{z z}^{2} \tau_{x x}+\tau_{z z}^{2} \tau_{y y}\right)+ \\
& +\frac{2}{9}\left(\tau_{x x}^{3}+\tau_{y y}^{3}+\tau_{z z}^{3}\right)-2\left(\tau_{x x} \tau_{y z}^{2}+\tau_{y y} \tau_{z x}^{2}+\tau_{z z} \tau_{x y}^{2}\right)+ \\
& +\left(\tau_{x x} \tau_{x y}^{2}+\tau_{x x} \tau_{x z}^{2}+\tau_{y y} \tau_{y z}^{2}+\tau_{y y} \tau_{y z}^{2}+\tau_{z z} \tau_{z x}^{2}+\tau_{z z} \tau_{z y}^{2}\right)+6 \tau_{x y} \tau_{y z} \tau_{z x} \\
= & \frac{4}{3} \tau_{1} \tau_{2} \tau_{3}-\frac{1}{3}\left(\tau_{1}^{2} \tau_{2}+\tau_{1}^{2} \tau_{3}+\tau_{2}^{2} \tau_{1}+\tau_{2}^{2} \tau_{3}+\tau_{3}^{2} \tau_{1}+\tau_{3}^{2} \tau_{2}\right)+ \\
& +\frac{2}{9}\left(\tau_{1}^{3}+\tau_{3}^{2}+\tau_{3}^{3}\right)
\end{aligned}
$$

It turns out, moreover, that the principal invariants of the deviator differ from the basic ones only in coefficients, namely

$$
J_{2 t}=-\frac{1}{2} I_{2 t}, \quad J_{3 t}=\frac{1}{3} I_{3 t}
$$

thus, speaking about the deviatoric invariants, we may omit the adjectives "basic" and "principal".

From the first formula (5.16) it follows that the quadratic basic invariant of the deviator is always non-negative, hence the corresponding principal invariant is always non-positive, (5.17). This was the reason for the change in the sign of $J_{2 \tau}$ introduced by some authors mentioned in Sec. 5.3.

It should be noted that the second basic invariant of the deviator $I_{2 t}$ is equal to the cylindrical invariant (radius) $R_{\tau}$ squared. The radius itself, whose dimension is equal to that of the tensor components, is called the intensity of the tensor or the effective value of the tensor (sometimes with other coefficients). The third invariant corresponds to the cylindrical invariant $\omega_{\tau}$; however, the interpretation of $\omega_{\tau}$ is more obvious.

The system of the invariants $I_{1 \tau}-I_{2 t}-I_{3 t}$, commonly used in the theory of plasticity, will be called the system of axiatoric-deviatoric invariants.

\subsection{The Lode parameter}

To estimate the influence of intermediate principal stress on the yielding of metals, W. Lode $[2.104,2.105,2.106]$ introduced a dimensionless parameter which in the general case of a tensor $T_{\tau}$ may be written in the form

$$
\mu_{\tau}=\frac{2 \tau_{\mathrm{II}}-\tau_{\mathrm{I}}-\tau_{\mathrm{III}}}{\tau_{\mathrm{I}}-\tau_{\mathrm{III}}}=\frac{3 t_{\mathrm{II}}}{t_{\mathrm{I}}-t_{\mathrm{III}}},
$$

where the convention of the arrangement $\tau_{\mathbf{I}} \geqslant \tau_{\mathbf{I I}} \geqslant \tau_{\mathrm{III}}$ is used. The values of the parameter $\mu_{\tau}$ are contained within the interval $-1 \leqslant \mu_{\tau} \leqslant 1$. This parameter is directly related to the cylindrical invariant $\omega_{\tau},(5.10),(5.11)$. In the interval $0 \leqslant \omega_{r} \leqslant \pi / 3$, where the arrangement of principal values is such that Roman indices are equal to Arabic ones, making use of (5.11) we obtain

$$
\mu_{\tau}=\sqrt{3} \tan \left(\omega_{\tau}-\frac{1}{6} \pi\right)
$$

in the remaining intervals similar formulae may be derived. Thus the Lode parameter determines the similarity of deviators without ensuring the coincidence of principal directions. 


\subsection{Tensor of directions and similarity}

A symmetric tensor has six independent coordinates, a deviator-five. A. A. Ilyushin [1.8] introduced a convenient auxiliary dimensionless tensor with four independent coordinates: their equality for two given tensors ensures the similarity of deviators and the coincidence of principal directions. Ilyushin called it the "directional tensor"; W. Urbanowski [2.125] proposed the name "tensor of directions and similarity", better expressing its essential nature. The coordinates of this tensor, $t_{i j}^{*}$, are defined as the coordinates of a deviator divided by a certain intensity of that deviator. We assume here the following definition:

$$
t_{i j}^{*}=\frac{t_{i j}}{R_{\tau}}=\frac{t_{i j}}{\sqrt{I_{2 t}}} .
$$

The definitions of A. A. Ilyushin and W. Urbanowski differ from the above (and from each other) in the numerical coefficients.

The tensor thus defined has only one independent invariant, $I_{3 t}^{*}$, or $\omega_{\tau}$, or $\mu_{\tau}$; the other two invariants are $I_{1 t}^{*}=0, I_{2 t}^{*}=1$. The four independent quantities defining this tensor are, for example: the three Euler's angles determining the directions, and the ratios of the principal values of the deviator determining $\omega_{\tau}$. A proportional change of coordinates of the given tensor does not result in any change of the auxiliary tensor of directions and similarity.

\subsection{Calculation of the principal values of the tensor}

The decomposition of the tensor $T_{\tau}$ into the axiator $A_{\tau}$ and the deviator $D_{z}$, and the cylindrical invariant $\omega_{\tau}$ are connected with the calculation of the principal values of the tensor. Namely, the principal values are determined as the roots of the cubic equation (5.6). To solve it, we first reduce it to the canonical form, introducing a new unknown

$$
t=\tau-\frac{1}{3} J_{1 \tau}=\tau-\tau_{m} .
$$

This operation corresponds simply to the above-mentioned decomposition and this unknown, or rather unknows, can be seen to be the principal values of the deviator $D_{\tau}$. Equation (5.6) takes the form

$$
t^{3}+J_{2 t} t-J_{3 t}=0
$$

since $J_{2 t}$ and $J_{3 t}$ denote the principal invariants of the deviator. Further an auxiliary angle $\omega_{\tau}$ is introduced by the

$$
\begin{aligned}
\cos 3 \omega_{\tau} & =\frac{\sqrt{27}}{2} \frac{J_{3 t}}{\left(-J_{2 \tau}\right)^{3 / 2}} \\
& =\frac{27 J_{3 \tau}-9 J_{1 \tau} J_{2 \tau}+2 J_{1 \tau}^{3}}{2\left(J_{1 \tau}^{2}-3 J_{2 \tau}\right)^{3 / 2}}, \quad 0 \leqslant \omega_{\tau} \leqslant \frac{\pi}{3} .
\end{aligned}
$$

It may be proved that this is exactly the angle $\omega_{\tau}$ determined by $(5.10)$. The essential difference between (5.10) and (5.23) consists in the fact that (5.23) is expressed in 
terms of the coordinates, and (5.10) in terms of the principal values (here unknown) only. Computing $3 \omega_{\tau}$ we subsequently evaluate $\omega_{\tau}$; finally, the required principal values of the tensor, $\tau_{1}, \tau_{2}$, and $\tau_{3}$, are determined by (5.11).

T. Pełczyński introduces in [2.127] a certain graphical interpretation of (5.11); it resembles the graphical interpretation of three-phase electric currents since the structure of (5.11) shows here an obvious analogy.

\subsection{Common invariants of several tensors}

Many equations of plasticity use common invariants of two or more tensors. The general theory of such invariants is rather complicated: some of the results have been presented in the books by G. B. Gurevitch [2.48], I. I. Goldenblat [2.38], W. Jaunzemis [2.74] and A. J. M. Spencer [2.164].

We quote here only the results for the simplest case of two symmetric secondorder tensors in the three-dimensional space. The number of independent invariants is here equal to nine. I. I. Goldenblat [2.38] gives a simple explanation of this number: three invariants of the first tensor, three of the second one, and three angles determining the principal directions of the second tensor with respect to the principal directions of the first one.

This does not mean, however, that any of the polynomial invariants of two symmetric tensors $T_{\sigma}$ and $T_{\varepsilon}$ in the "basic" form

$$
I=\sigma_{i j} \sigma_{j k} \ldots \varepsilon_{m n} \varepsilon_{n p} \varepsilon_{p i}
$$

can be expressed as a polynomial of a suitable set of nine basic invariants. The minimal number of "rationally independent" basic invariants is equal here to ten: $I_{1 \sigma}$, $I_{2 \sigma}, I_{3 \sigma}, I_{1 \varepsilon}, I_{2 \varepsilon}, I_{3 \varepsilon}$, and

$$
\begin{aligned}
I_{\sigma \varepsilon} & =\sigma_{i j} \varepsilon_{j i}=\sigma_{x x} \varepsilon_{x x}+\sigma_{y y} \varepsilon_{y y}+\sigma_{z z} \varepsilon_{z z}+2 \sigma_{x y} \varepsilon_{x y}+2 \sigma_{y z} \varepsilon_{y z}+2 \sigma_{z x} \varepsilon_{z x}, \\
I_{\sigma \sigma \varepsilon} & =\sigma_{i j} \sigma_{j k} \varepsilon_{k i}, \\
I_{\sigma \varepsilon \varepsilon} & =\sigma_{l j} \varepsilon_{j k} \varepsilon_{k i}, \\
I_{\sigma \sigma \varepsilon \varepsilon} & =\sigma_{i j} \sigma_{j k} \varepsilon_{k l} \varepsilon_{l i} .
\end{aligned}
$$

The last invariant is functionally dependent on the remaining nine, but its elimination would not be a rational operation and would lead to many-valued results.

The invariant $I_{\sigma \varepsilon}$ often has an energy interpretation. In the case of stresses and strains it is proportional to elastic energy under the assumption of linear elasticity of the material. In the case of stresses and strain rates it is equal to the power of determination without any assumption of physical linearity. Other invariants (5.25) do not have such a simple interpretation.

In the case of three symmetric second-order tensors the number of independent invariants equals 15 and that of rationally independent ones 28 . 


\section{Stress and stress rate}

\subsection{Engineering and indicial notation}

The stress tensor $T_{\sigma}$ with the components $\sigma_{i j}$ may be defined, in general, by an equation of the type (W. Prager [2.136])

$$
d P_{j}=\sigma_{i j} d A_{i}
$$

where $d P_{j}$ denotes the vector of infinitesimal force and $d A_{i}$ the vector of infinitesimal area (perpendicular to that area). Ascribing various meanings to $d P_{j}$ and $d A_{i}$, we define various stress tensors. In the classical small-strain theory we assume the socalled principle of rigidification (neglected influence of geometry changes on the stress distribution, reactive forces, etc.) and do not distinguish $d P_{j}$ and $d A_{i}$ before and after deformation. Taking the geometry changes into account, we may use (6.1) as the definition of the Euler-Cauchy stress tensor in spatial coordinates or, after a suitable modification of $d P_{j}$ and $d A_{i}$ as the definition of the Piola-Kirchhoff stress tensor in material coordinates (these systems of coordinates are defined in Sec. 7). Unless otherwise stated, we understand by $\sigma_{i j}$ the classical stress tensor.

Substituting, in turn, $i, j=x, y, z$, we denote the coordinates of the classical stress tensor by $\sigma_{x x}, \sigma_{x y}, \sigma_{x z}, \sigma_{y x}, \ldots, \sigma_{z z}$. This notation system will be called indicial or mathematical notation: the stresses are denoted consequently by one symbol $\sigma$, the first index corresponding to the direction normal to the area and the second, to the direction of the stress itself. Some authors put $i, j=1,2,3$, but we reserve the numerical indices for the principal components. The indicial notation is convenient in the cases of longer formulae or derivations since it permits the application of the Einstein summation convention.

In most engineering applications, however, it is important to distinguish normal and tangential stresses by separate symbols. Followings Kármán and Timoshenko we denote normal stresses by $\sigma_{x}, \sigma_{y}, \sigma_{z}$, and tangential ones by $\tau_{x y}, \tau_{y z}, \tau_{z x}$. This notation system will be called engineering notation and will be used side by side with the indicial notation. Such dualism of notation, making it possible to take advantage of both systems, is used by W. Johnson and P. B. Mellor [2.76], A. M. Freudenthal [2.34], K. A. Reckling [2.141], and others.

The stress tensor will be decomposed into the axiator $A_{\sigma}$ and the deviator $D_{\sigma}$ according to the scheme $T_{\sigma}=A_{\sigma}+D_{\sigma}$. The only independent component of the axiator will be denoted, according to the general convention assumed, by $\sigma_{m}$ :

$$
\sigma_{m}=\frac{1}{3} \sigma_{u}=\frac{1}{3}\left(\sigma_{x}+\sigma_{y}+\sigma_{z}\right)=\frac{1}{3}\left(\sigma_{1}+\sigma_{2}+\sigma_{3}\right),
$$

where $\sigma_{1}, \sigma_{2}, \sigma_{3}$ denote the principal stresses. Algebraically arranged principal stresses are $\sigma_{1}, \sigma_{1 I}, \sigma_{1 I I}$. The components of the stress deviator will be denoted by $s_{x}, s_{y}, s_{z}, \tau_{x y}, \tau_{y z}, \tau_{z x}$ in engineering notation and by $s_{i j}$ in indicial notation. Components of the tensor of direction and similarity will be denoted by $s_{i j}^{*}$, and the Lode parameter for stresses by $\mu_{\sigma}$. 


\subsection{Transformation of stresses, stress invariants}

Considering the equilibrium of an infinitesimal tetrahedron with the faces normal to $x, y, z$ and a certain inclined direction $n$, we find (in indicial notation with the summation convention)

$$
p_{n i}=\sigma_{i j} a_{n j}
$$

where $p_{n i}$ denote the components of the resultant stress at the inclined face. If the tetrahedron is cut off at the boundary of the body, then $p_{n i}$ are understood as surface tractions, and then (6.3) expresses the stress boundary conditions; if it is cut out of the interior, then (6.3) leads to the tensorial transformation formulae of type (5.1).

Typical systems of invariants will be introduced according to Sec. 5. Basic invariants will be denoted by $I_{1 \sigma}, I_{2 \sigma}, I_{3 \sigma}$, principal invariants by $J_{1 \sigma}, J_{2 \sigma}, J_{3 \sigma}$, and axiatoric-deviatoric invariants by $I_{1 \sigma}, I_{2 s}, I_{3 s}$. Cylindrical invariants, however, will be used with slightly different coefficients. Instead of $Z_{\sigma}$ we shall use the proportional invariant $\sigma_{m}$ (coefficient $1 / 3$ instead of $1 \sqrt{3}$ ) and instead of $R_{\sigma}$-the proportional invariant $\sigma_{e}$,

$$
\begin{aligned}
\sigma_{e} & =\frac{1}{\sqrt{2}} \sqrt{\left(\sigma_{1}-\sigma_{2}\right)^{2}+\left(\sigma_{2}-\sigma_{3}\right)^{2}+\left(\sigma_{3}-\sigma_{1}\right)^{2}}=\sqrt{\frac{3}{2} s_{i j} s_{i j}} \\
& =\sqrt{\sigma_{x}^{2}+\sigma_{y}^{2}+\sigma_{z}^{2}-\sigma_{x} \sigma_{y}-\sigma_{y} \sigma_{z}-\sigma_{z} \sigma_{x}+3\left(\tau_{x y}^{2}+\tau_{y z}^{2}+\tau_{z x}^{2}\right)}
\end{aligned}
$$

(coefficient $1 / \sqrt{2}$ instead of $1 / \sqrt{3}$ ). The invariant $\sigma_{e}$ is called stress intensity or effective stress. The coefficient in (6.3) is chosen in such a way that uniaxial tension gives $\sigma_{e}=\sigma$. Sometimes instead of $\sigma_{e}$ the invariant $\tau_{e}=\sigma_{e} / \sqrt{3}$ is introduced and called shear stress intensity (A. A. Ilyushin [1.8], V. V. Sokolovsky [2.163], symbol $S$ ). The third cylindrical invariant $\omega_{\sigma}$ will be used without any change, (5.10). The system of invariants $\sigma_{m}, \sigma_{e}, \omega_{\sigma}$ is very convenient for applications in plasticity; some advantages are pointed out by G. C. Nayak and O. C. Zienkiewicz [2.120].

Consider now the octahedral stresses $\sigma_{\text {oct }}$ and $\tau_{\text {oct }}$ acting on the face equally inclined to the principal directions. To calculate $\sigma_{\text {oct }}$ we may use the general tensorial transformation formula, starting from the principal directions (vanishing shearing stresses) and substituting $a_{1 n}^{2}=a_{2 n}^{2}=a_{3 n}^{2}=1 / 3$ (the angle being equal to $54^{\circ} 44^{\prime} 07^{\prime \prime}$ ). Finally

$$
\sigma_{\mathrm{oct}}=\frac{1}{3}\left(\sigma_{1}+\sigma_{2}+\sigma_{3}\right)=\sigma_{m}=\frac{1}{\sqrt{3}} Z_{\sigma} .
$$

The tangential octahedral stress $\tau_{\text {oct }}$ will be evaluated as the geometrical difference of the resultant stress $p_{\text {oct }}$, determined by $(6.3)$, and $\sigma_{\mathrm{oct}}$ :

$$
\begin{aligned}
p_{\mathrm{oct}} & =\frac{1}{\sqrt{3}} \sqrt{\sigma_{1}^{2}+\sigma_{2}^{2}+\sigma_{3}^{2}}=\frac{1}{\sqrt{3}} \sqrt{I_{2 \sigma}}, \\
\tau_{\mathrm{oct}} & =\sqrt{p_{\mathrm{oct}}^{2}-\sigma_{\mathrm{oct}}^{2}}=\frac{1}{3} \sqrt{\left(\sigma_{1}-\sigma_{2}\right)^{2}+\left(\sigma_{2}-\sigma_{3}\right)^{2}+\left(\sigma_{3}-\sigma_{1}\right)^{2}} \\
& =\frac{\sqrt{2}}{3} \sigma_{l}=\frac{1}{\sqrt{3}} R_{\sigma}=\frac{1}{\sqrt{3}} \sqrt{I_{2 s}} .
\end{aligned}
$$




\begin{tabular}{|c|c|c|c|c|c|}
\hline 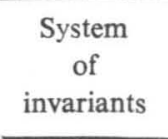 & $\begin{array}{l}\text { Basic invariants } \\
I_{10}, I_{2 \sigma}, L_{3 \sigma} \\
\end{array}$ & $\begin{array}{l}\text { Prinipial invariants } \\
J_{1 \sigma}, J_{2 a}, N_{3 \sigma}\end{array}$ & $\begin{array}{l}\text { Axiatoric-deviatoric invariants } \\
I_{10}, I_{2,}, I_{3 s}\end{array}$ & $\begin{array}{l}\text { Cylindrical invariants } \\
\sigma_{\sigma_{m}, \sigma_{e}, \omega_{\sigma}}\end{array}$ & $\begin{array}{l}\text { Principal streseses } \\
\sigma_{1}, \sigma_{2}, \sigma_{3}\end{array}$ \\
\hline$I_{1 \sigma}=$ & $I_{I \sigma}$ & $J_{1 \sigma}$ & $I_{10}$ & $3 \sigma_{m}$ & $\sigma_{1}+\sigma_{2}+\sigma_{3}$ \\
\hline$I_{2 \sigma}=$ & $I_{2 o}$ & $J_{10}^{2}-2 J_{2 \sigma}$ & $I_{2 \sigma}+\frac{1}{3} I_{1 \sigma}^{2}$ & $\frac{2}{3} \sigma_{\varepsilon}^{2}+3 \sigma_{m}^{2}$ & $\sigma_{1}^{2}+\sigma_{2}^{2}+\sigma_{3}^{2}$ \\
\hline$I_{s o}=$ & $I_{s o}$ & $3 J_{3 \sigma}-3 J_{1 \sigma} J_{2 \sigma}+J_{1 \sigma}^{3}$ & $I_{33}+I_{1 \sigma}+I_{22}+\frac{1}{9} l_{1 \sigma}^{3}$ & $\frac{2}{9} \sigma_{e}^{3} \cos 3 \omega_{\sigma}+2 \sigma_{m} \sigma_{e}^{2}+\sigma_{m}^{3}$ & $\sigma_{1}^{3}+\sigma_{2}^{3}+\sigma_{3}^{3}$ \\
\hline$J_{1 \sigma}=$ & $I_{1 \sigma}$ & $J_{1 \sigma}$ & $I_{i \sigma}$ & $3 \sigma_{m}$ & $\sigma_{1}+\sigma_{2}+\sigma_{3}$ \\
\hline$J_{2 \sigma}=$ & $\frac{1}{2}\left(l_{10}^{2}-I_{2 \sigma}\right)$ & $J_{2 \sigma}$ & $-\frac{1}{2}-I_{25}+\frac{1}{3} I_{1 \sigma}^{2}$ & $3 \sigma_{m}^{2}-\frac{1}{3} \sigma_{e}^{2}$ & $\sigma_{1} \sigma_{2}+\sigma_{2} \sigma_{3}+\sigma_{3} \sigma_{1}$ \\
\hline$J_{s o}=$ & $\frac{1}{3} I_{3 a}-\frac{1}{2} I_{1 \sigma a} I_{\sigma o}+\frac{1}{6} I_{1 \sigma}^{3}$ & $J_{3 \sigma}$ & $\frac{1}{3} I_{3}-\frac{1}{6} I_{16} I_{2}+\frac{1}{27} I_{10}^{3}$ & $\frac{2}{27} \sigma_{e}^{3} \cos 3 \omega_{\sigma}-\frac{1}{3} \sigma_{m} \sigma_{t}^{2}+\sigma_{m}^{3}$ & $\sigma_{1} \sigma_{2} \sigma_{3}$ \\
\hline$I_{1 \sigma}=$ & $I_{1 \sigma}$ & $J_{1 \sigma}$ & $I_{1 \sigma}$ & $3 \sigma_{m}$ & $\sigma_{1}+\sigma_{2}+\sigma_{3}$ \\
\hline$I_{25}=$ & $I_{2 \sigma}-\frac{1}{3} l_{1}^{2} \sigma$ & $-2 J_{2 \sigma}+\frac{2}{3} J_{1 \sigma}^{2}$ & $I_{2 s}$ & $\frac{2}{3} \sigma_{2}^{2}$ & $\frac{2}{3}\left(\sigma_{1}^{2}+\sigma_{2}^{2}+\sigma_{3}^{2}-\sigma_{1} \sigma_{2}-\sigma_{2} \sigma_{3}-\sigma_{3} \sigma_{1}\right)$ \\
\hline$I_{3}=$ & $I_{a_{\sigma}}-I_{\sigma_{\sigma}} I_{2 \sigma}+\frac{2}{9} I_{10}^{2}$ & $3 J_{s o}-J_{10} J_{2 \sigma}+\frac{2}{9} J_{1 \sigma}^{3}$ & $I_{3 s}$ & $\frac{2}{9} \sigma_{c}^{3} \cos 3 \omega_{\sigma}$ & $\frac{4}{3} \sigma_{1} \sigma_{2} \sigma_{3}-\frac{1}{3}\left(\sigma_{1}^{2} \sigma_{2}+\sigma_{1} \sigma_{2}^{2}+\sigma_{2}^{2} \sigma_{3}+\sigma_{2} \sigma_{3}^{2}+\sigma_{3}^{2} \sigma_{1}+\sigma_{3} \sigma_{1}^{2}\right)+\frac{2}{9}\left(\sigma_{1}^{3}+\sigma_{2}^{3}+\sigma_{3}^{3}\right)$ \\
\hline$\sigma_{m}=$ & $\frac{1}{3} I_{10}$ & $\frac{1}{3} J_{1 \sigma}$ & $\frac{1}{3} I_{1 \sigma}$ & $\sigma_{m}$ & $\frac{1}{3}\left(\sigma_{1}+\sigma_{2}+\sigma_{3}\right)$ \\
\hline$\sigma_{e}=$ & $\sqrt{\frac{3}{2} I_{2 \sigma}-\frac{1}{2} I_{10}^{2}}$ & $\sqrt{\sqrt{J_{1 \sigma}^{2}-3 / \sqrt{2 \sigma}}}$ & $\sqrt{\frac{3}{2} I_{2 s}}$ & $\sigma_{e}$ & $\sqrt{\left.\sigma_{1}^{2}+\sigma_{2}^{2}+\sigma_{3}^{2}-\sigma_{1} \sigma_{2}-\sigma_{2} \sigma_{3}-\sigma_{3} \sigma_{1}\right)}$ \\
\hline $\cos 3 \omega_{\sigma}=$ & $\frac{\left(9 I_{3 \sigma}-9 I_{1} I_{2 \sigma}+2 l l_{3}^{3}\right) \sqrt{2}}{\left(3 l_{2 \sigma}-I_{1}^{2}\right)^{3 / 2}}$ & $\frac{27 J_{3 \sigma}-9 J_{1} J_{2 \sigma}+2 J_{\sigma o}^{3}}{2\left(J_{1 \sigma}^{2}-3 J_{J_{\sigma}}\right)^{3 / 2}}$ & $\frac{I_{3} s^{2}}{I_{2 t^{2}}^{2}}$ & $\cos 3 \omega_{\sigma}$ & $\frac{12 \sigma_{1} \sigma_{2} \sigma_{3}-3\left(\sigma_{1}^{2} \sigma_{2}+\sigma_{1} \sigma_{2}^{2}+\sigma_{2}^{2} \sigma_{3}+\sigma_{2} \sigma_{3}^{2}+\sigma_{3}^{2} \sigma_{1}+\sigma_{3} \sigma_{1}^{2}\right)+2\left(\sigma_{1}^{3}+\sigma_{2}^{3}+\sigma_{3}^{3}\right)}{2\left(\sigma_{1}^{2}+\sigma_{2}^{2}+\sigma_{3}^{2}-\sigma_{1} \sigma_{2}-\sigma_{2} \sigma_{3}-\sigma_{3} \sigma_{1}\right)^{3 / 2}}$ \\
\hline$\sigma_{1}=$ & 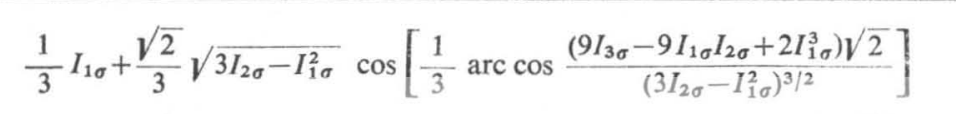 & 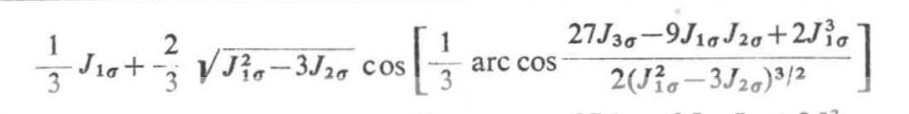 & $\frac{1}{3} I_{10}+\sqrt{\frac{2}{3} I_{22}} \cos \left[\frac{1}{3} \arccos \frac{I_{3} / \sqrt{6}}{I_{2}^{2} 2^{2}}\right]$ & $\sigma_{m}+\frac{2}{3} \sigma_{c} \cos \omega_{\sigma}$ & $\sigma_{1}$ \\
\hline$\sigma_{2}=$ & 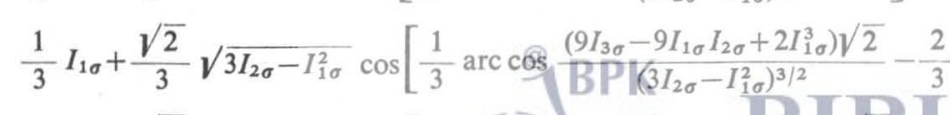 & 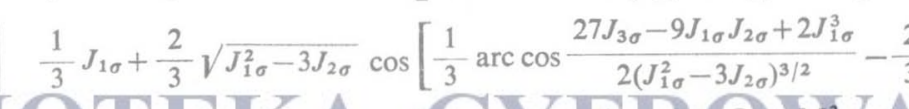 & $\frac{1}{3} I_{10}+\sqrt{\frac{2}{3} I_{25}} \cos \left[\frac{1}{3} \arccos \frac{I_{35} \sqrt{6}}{I_{25}^{212}}-\frac{2}{3}\right.$ & $\sigma_{m}+\frac{2}{3} \sigma_{c} \cos \left(\omega_{\sigma}-\frac{2}{3} \pi\right)$ & $\sigma_{2}$ \\
\hline$\sigma_{3}=$ & 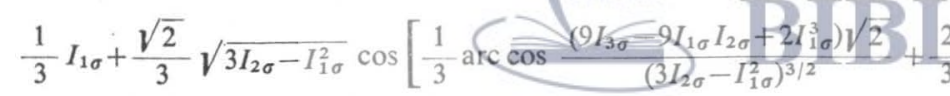 & 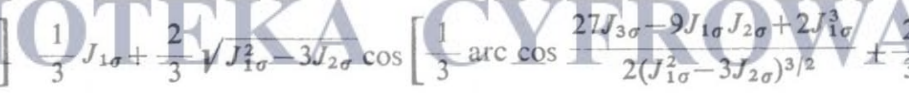 & 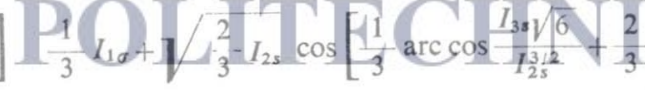 & det $\frac{2}{3} \operatorname{rec} 4\left(\omega_{0}+\frac{2}{3} \pi\right)$ & $N_{\sigma_{3}} \mathrm{SKIIJ}$ \\
\hline
\end{tabular}


So the octahedral stresses are proportional to the first two cylindrical invariants, respectively.

Extremal shearing stresses $\tau$ are also important invariants of the stress tensor. It may be proved that they act at the sections forming the angle $45^{\circ}$ with any two principal sections and are equal to half the differences of the corresponding principal stresses. According to the notation used, the maximal shearing stress equals

$$
\tau_{\max }=\frac{1}{2}\left(\sigma_{I}-\sigma_{\text {III }}\right) \text {. }
$$

Typical systems of the stress invariants are listed in Table 2, which shows the relations between them. Some further relations are given by M. S. Sarkisyan [2.148]; systems of stress invariants are also discussed by T. C. Hsu [2.65].

\subsection{Equations of internal equilibrium}

Figure 17 shows the stresses and body forces (assumed as positive) acting on the infinitesimal parallelepiped cut out of the interior of the body. Each stress component

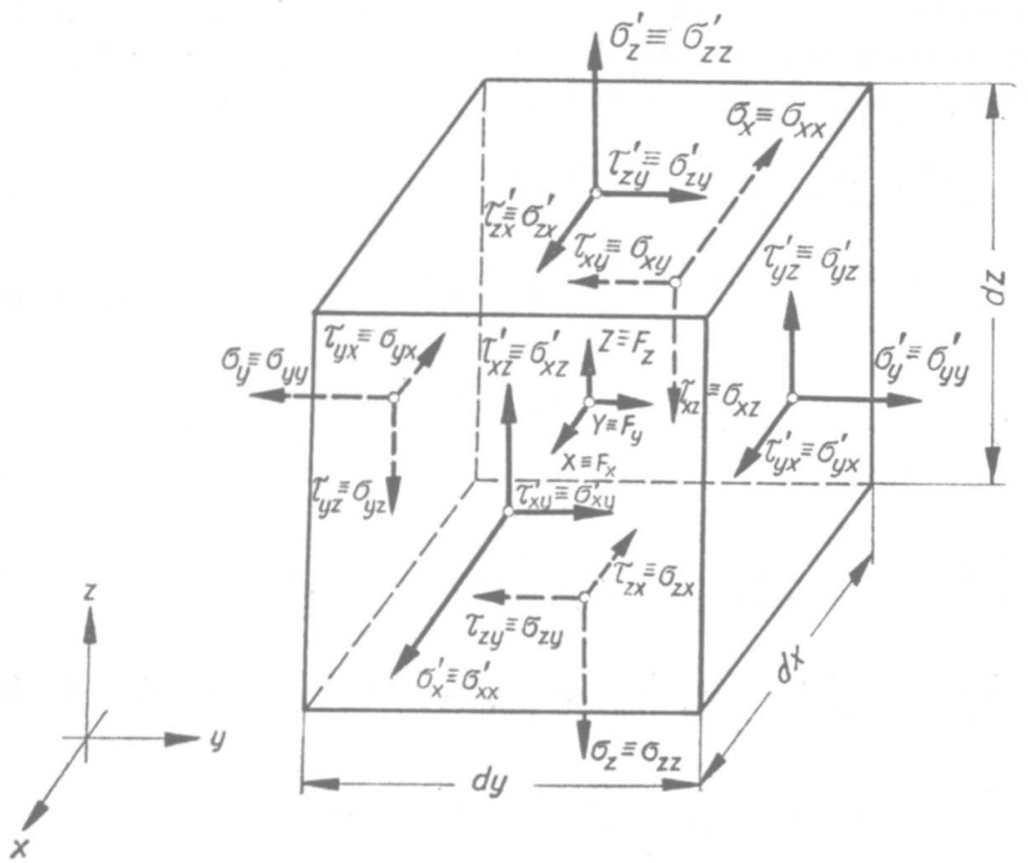

Fig. 17. Engineering and mathematical notation of stresses. Equations of internal equilibrium

is denoted twice, in engineering and in indicial notation. Body forces are denoted by $F_{t}, i=x, y, z$.

In the case of a homogeneous state of stress the corresponding components at opposite faces of the parallelepiped are equal to each other, $\sigma_{x}^{\prime}=\sigma_{x}$, etc. The conditions of equilibrium give vanishing body forces and symmetry of the stress tensor, 
$\tau_{i j}=\tau_{j i}$. In the general non-homogeneous case $\sigma_{x}^{\prime}=\sigma_{x}+\left(\partial \sigma_{x} / \partial x\right) d x$ and the conditions of equilibrium lead to the following equations in Cartesian coordinates

$$
\frac{\partial \sigma_{x}}{\partial x}+\frac{\partial \tau_{y x}}{\partial y}+\frac{\partial \tau_{z x}}{\partial z}+F_{x}=0, \quad \Gamma_{z}^{x} y_{y}
$$

where the symbol of cyclic permutation shows how to obtain the two remaining equations; the relations $\tau_{i j}=\tau_{j i}$ remain without change. In indicial notation we may write shortly

$$
\sigma_{i j, i}+F_{j}=0, \quad i, j=x, y, z,
$$

where the (partial) differentiation is shown by the indices preceded by a comma, and the summation convention applies across the comma. In the case of large strains, formulae (6.9) and (6.10) hold in spatial coordinates, whereas in material coordinates they assume a much more complicated form.

Equations of equilibrium in other systems of coordinates may be derived by considering suitably cut elements or, quite uniformly, by introducing curvilinear tensors and covariant differentiation. Here we quote the results only for the two most commonly used systems: in cylindrical coordinates $r, \theta, z$,

$$
\begin{aligned}
& \frac{\partial \sigma_{r}}{\partial r}+\frac{1}{r} \frac{\partial \tau_{\theta r}}{\partial \theta}+\frac{\partial \tau_{z r}}{\partial z}+\frac{\sigma_{r}-\sigma_{\theta}}{r}+F_{r}=0, \\
& \frac{\partial \tau_{r \theta}}{\partial r}+\frac{1}{r} \frac{\partial \sigma_{\theta}}{\partial \theta}+\frac{\partial \tau_{z \theta}}{\partial z}+2 \frac{\tau_{r \theta}}{r}+F_{\theta}=0, \\
& \frac{\partial \tau_{r z}}{\partial r}+\frac{1}{r} \frac{\partial \tau_{\theta z}}{\partial \theta}+\frac{\partial \sigma_{z}}{\partial z}+\frac{\tau_{r z}}{r}+F_{z}=0,
\end{aligned}
$$

and in spherical coordinates $r, \psi, \theta$

$$
\begin{aligned}
& \frac{\partial \sigma_{r}}{\partial r}+\frac{1}{r} \frac{\partial \tau_{\psi r}}{\partial \psi}+\frac{1}{r \sin \psi} \frac{\partial \tau_{\theta r}}{\partial \theta}+\frac{1}{r}\left[2 \sigma_{r}-\left(\sigma_{\varphi}+\sigma_{\theta}\right)+\tau_{\varphi r} \cot \psi\right]+F_{r}=0, \\
& \frac{\partial \tau_{r \psi}}{\partial r}+\frac{1}{r} \frac{\partial \sigma_{\varphi}}{\partial \psi}+\frac{1}{r \sin \psi} \frac{\partial \tau_{\theta \psi}}{\partial \theta}+\frac{1}{r}\left[3 \tau_{r \varphi}+\left(\sigma_{\varphi}-\sigma_{\theta}\right) \cot \psi\right]+F_{\varphi}=0, \\
& \frac{\partial \tau_{r \theta}}{\partial r}+\frac{1}{r} \frac{\partial \tau_{\varphi \theta}}{\partial \psi}+\frac{1}{r \sin \psi} \frac{\partial \sigma_{\theta}}{\partial \theta}+\frac{1}{r}\left[3 \tau_{r \theta}+2 \tau_{\varphi \theta} \cot \psi\right]+F_{\theta}=0 .
\end{aligned}
$$

The differential equations of equilibrium (6.9), (6.11) or (6.12) hold at regular points, where the stresses are continuous with their first derivatives. In the theory of plasticity discontinuity lines or discontinuity surfaces are investigated as well. Denote by $\xi$ the normal to the discontinuity surface at the point under consideration and by $\eta$ and $\zeta$ two mutually perpendicular tangential directions. The conditions of equilibrium lead here to the continuity of the tractions $\sigma_{\xi}, \tau_{\xi \eta}$, and $\tau_{\xi \xi}$, whereas the remaining stresses $\sigma_{\eta}, \sigma_{\zeta}$, and $\tau_{\eta \zeta}$ may be discontinuous. 
A more detailed analysis of conditions at the discontinuity surfaces is given by R. Hill [2.57, 2.58], T. Y. Thomas [2.173], A. M. Freudenthal, H. M. Geiringer [2.33], W. Prager [2.134] and D. D. Ivlev [2.71, 2.72].

\subsection{Stress rates}

The theory of plasticity uses stress increments $d \sigma$ or stress rates $d \sigma / d t=\dot{\sigma}$; these quantities are equivalent inasmuch as the time factor may usually be eliminated.

In the case of small deformations, where the geometry changes are disregarded, the rates of stress components form a tensor which may be written as

$$
T_{\dot{\sigma}}=\left\{\begin{array}{lll}
\dot{\sigma}_{x} & \dot{\tau}_{x y} & \dot{\tau}_{x z} \\
\dot{\tau}_{y x} & \dot{\sigma}_{y} & \dot{\tau}_{y z} \\
\dot{\tau}_{z x} & \dot{\tau}_{z y} & \dot{\sigma}_{z}
\end{array}\right\}=\left\{\begin{array}{lll}
\dot{\sigma}_{x x} & \dot{\sigma}_{x y} & \dot{\sigma}_{x z} \\
\dot{\sigma}_{y x} & \dot{\sigma}_{y y} & \dot{\sigma}_{y z} \\
\dot{\sigma}_{z x} & \dot{\sigma}_{z y} & \dot{\sigma}_{z z}
\end{array}\right\} .
$$

The invariants of this tensor will be denoted as follows: basic invariants $I_{1}, I_{2} \dot{\sigma}, I_{3 \dot{\sigma}}$; principal invariants $J_{1}, J_{2 \dot{\sigma}}, J_{3 \dot{\sigma}}$, etc. The integral invariant

$$
S_{\sigma}=\int_{t_{A}}^{t_{B}} \sqrt{I_{2 \dot{\sigma}}} d t=\int_{A}^{B} \sqrt{d \sigma_{i j} d \sigma_{i j}}
$$

determines the length of the trajectory in the nine-dimensional stress space between the points $A$ and $B$ (cf. Sec. 10.1).

If the principal directions of the stress tensor are constant, then the principal directions of the stress rate tensor are also constant and these directions coincide; in the general case the coincidence does not occur.

The stress rate tensor may be decomposed according to the scheme $T_{\dot{\sigma}}=A_{\dot{\sigma}}+D_{\dot{\sigma}}$. The components of $T_{\dot{\sigma}}$ must fulfil the conditions of equilibrium of type (6.9).

In the case of finite deformations the definition of the stress rate is not unique. The first proposal is due to S. Zaremba [2.194]; reviews of definitions in use are given by C. Truesdell [2.178], W. Prager [2.135], and Z. H. Guo [2.47].

\section{Strain and strain rate}

\subsection{Displacement vector}

The individual points of a body undergoing deformation are in motion. The Cartesian coordinates of an arbitrary point $P$ of the body $B$ before deformation will be denoted by $X, Y, Z$ or by $X_{i}$ in engineering and in indicial notation, respectively. After deformation the body will be denoted by $b$; point $P$ changes its position to $p$ with the coordinates $x, y, z$ or $x_{i}$ (Fig. 18). The motion may be determined by the functions

$$
x=x(X, Y, Z, t), \quad y=y(X, Y, Z, t), \quad z=z(X, Y, Z, t)
$$

or by the functions

$$
X=X(x, y, z, t), \quad Y=Y(x, y, z, t), \quad Z=Z(x, y, z, t)
$$




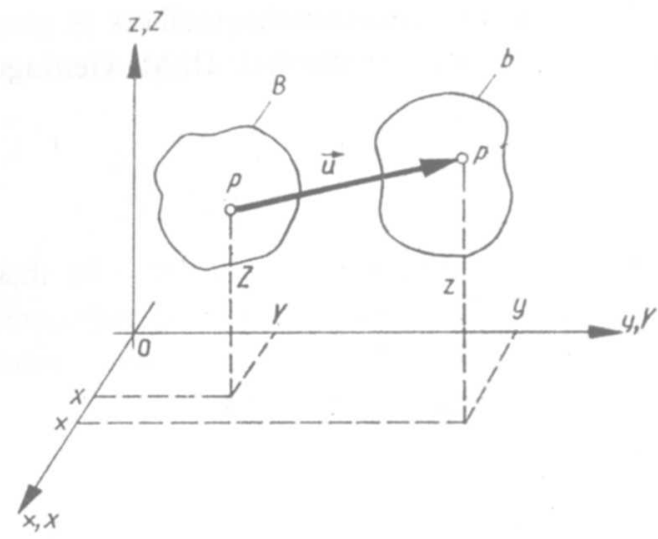

Fig. 18. Material (Lagrangian) and spatial (Eulerian) coordinates

where $t$ denotes time or any time-like parameter. In the theory of elasticity we are often interested not in the course of the process in time but in its final results only; in problems of plasticity the history of the process may be of essential importance.

The coordinates $X, Y, Z$ treated as independent (as in (7.1)) are traditionally called Lagrangian or material coordinates (though actually introduced in 1761 by Euler) and $x, y, z$ treated as independent are called Eulerian or spatial coordinates (though actually introduced in 1752 by d'Alembert; $\mathrm{cf}$. C. Truesdell [2.176, 2.177], V. V. Novozhilov [2.123]). The notation using capitals and lower case was introduced by Truesdell; it is particularly convenient in indicial treatment, since then the capital ndices following a comma denote differentiation with respect to material coordi${ }^{i}$ nates and the small indices-differentiation with respect to spatial ones.

The vector $\overrightarrow{P p}=\mathbf{u}$ will be called the displacement vector. It depends on material or on spatial coordinates, and so it forms a vector field. The components of the displacement vector will be denoted by $U_{K}$ (or $U, V, W$ ) as functions of material coordinates, and by $u_{k}$ (or $\left.u, v, w\right)$ as functions of spatial coordinates:

$$
U_{K}=x_{k}\left(X_{j}, t\right)-X_{K}, \quad u_{k}=x_{k}-X_{K}\left(x_{j}, t\right) .
$$

Very often the dependence on the time $t$ is not shown in the notation.

\subsection{Strain tensors}

There exist many various measures of deformation of the body; at a certain point of the body we characterize deformation by the entity of suitably chosen strain components. The simplest way to obtain tensorial strain components is to consider the change of the squared length of an infinitesimal line element $d S^{2}$ (without squaring we would not obtain a tensorial measure in the general case of finite deformation). Denote the corresponding squared length after deformation by $d s^{2}$ : then, in Cartesian coordinates,

$$
d s^{2}-d S^{2}=d x_{k} d x_{k}-d X_{K} d X_{K}
$$


Now we have to express spatial coordinates in terms of material ones or vice versa. Substituting here (7.3) and the formula for the total differential

$$
d x_{k}=d X_{K}+d U_{K}=d X_{K}+U_{K, J} d X_{J}=\left(\delta_{J K}+U_{K, J}\right) d X_{J},
$$

we may write

$$
d s^{2}-d S^{2}=\left[\left(\delta_{J K}+U_{K, J}\right)\left(\delta_{I K}+U_{K, I}\right)-\delta_{I J}\right] d X_{I} d X_{J}
$$

and finally

$$
d s^{2}-d S^{2}=2 \varepsilon_{I J}^{L} d X_{I} d X_{J}
$$

where

$$
\varepsilon_{I J}^{L}=\frac{1}{2}\left(U_{I, J}+U_{J, I}+U_{K, I} U_{K, J}\right)
$$

is called the Lagrangian (or Green) strain tensor (finite-strain tensor). Its tensorial character is seen from (7.7) since on the left-hand side we have a scalar quantity.

Similarly, substituting into (7.4)

$$
d X_{K}=d x_{k}-d u_{k}=d x_{k}-u_{k, j} d x_{j}=\left(\delta_{j k}-u_{k, j}\right) d x_{j},
$$

we obtain

$$
d s^{2}-d S^{2}=2 \varepsilon_{i j}^{E} d x_{i} d x_{j}
$$

where

$$
\varepsilon_{i j}^{E}=\frac{1}{2}\left(u_{i, j}+u_{j, i}-u_{k, i} u_{k, j}\right)
$$

is called the Eulerian (or Almansi) strain tensor (finite-strain tensor) In the engineering notation we have

$$
\begin{aligned}
& \varepsilon_{x}^{L}=\frac{\partial U}{\partial X}+\frac{1}{2}\left[\left(\frac{\partial U}{\partial X}\right)^{2}+\left(\frac{\partial V}{\partial X}\right)^{2}+\left(\frac{\partial W}{\partial X}\right)^{2}\right] \\
& \varepsilon_{x y}^{L}=\frac{1}{2}\left(\frac{\partial U}{\partial Y}+\frac{\partial V}{\partial X}+\frac{\partial U}{\partial X} \frac{\partial U}{\partial Y}+\frac{\partial V}{\partial X} \frac{\partial V}{\partial Y}+\frac{\partial W}{\partial X} \frac{\partial W}{\partial Y}\right)
\end{aligned}
$$

and

$$
\begin{aligned}
& \varepsilon_{x}^{E}=\frac{\partial u}{\partial x}-\frac{1}{2}\left[\left(\frac{\partial u}{\partial x}\right)^{2}+\left(\frac{\partial v}{\partial x}\right)^{2}+\left(\frac{\partial w}{\partial x}\right)^{2}\right] \\
& \varepsilon_{x y}^{E}=\frac{1}{2}\left(\frac{\partial u}{\partial y}+\frac{\partial v}{\partial x}-\frac{\partial u}{\partial x} \frac{\partial u}{\partial y}-\frac{\partial v}{\partial x} \frac{\partial v}{\partial y}-\frac{\partial w}{\partial x} \frac{\partial w}{\partial y}\right)
\end{aligned}
$$

The components (7.12) and (7.13) do not have a simple geometrical interpretation; however, the changes of the typical segments and angles may be represented 
as functions of those quantities. For example, the infinitesimal segment $d X$ before deformation changes after deformation into $d s_{x}$ with the coordinates $d X+(\partial U / \partial X) d X$, $(\partial V / \partial X) d X,(\partial W / \partial X) d X$, and the unit elongation $\varepsilon_{x}^{* L}$ equals

$$
\begin{aligned}
\varepsilon_{x}^{* L} & \stackrel{\text { def }}{=} \frac{d s_{x}-d X}{d X}=\sqrt{\left(1+\frac{\partial U}{\partial X}\right)^{2}+\left(\frac{\partial V}{\partial X}\right)^{2}+\left(\frac{\partial W}{\partial X}\right)^{2}}-1 \\
& =\sqrt{1+2 \varepsilon_{x}^{L}}-1 .
\end{aligned}
$$

The change $\gamma_{x y}^{* L}$ of the right angle between the segments $d X$ and $d Y$ transformed after deformation into $d s_{x}$ and $d s_{y}$ may be found from the scalar product

$$
\overrightarrow{d s_{x}} \cdot \overrightarrow{d s_{y}}=\left|\overrightarrow{d s}_{x}\right||| \overrightarrow{d s}_{y} \mid \cos \left(\frac{1}{2} \pi-\gamma_{x y}^{* L}\right),
$$

and hence, expressing the scalar product by the coordinates,

$$
\begin{aligned}
& \sin \gamma_{x y}^{* L}=\frac{\overrightarrow{d s}_{x} \cdot \overrightarrow{d s_{3}}}{\left|\overrightarrow{d s_{x}}\right|\left|\overrightarrow{d s_{y}}\right|}, \\
& \gamma_{x y}^{* L}=\arcsin \frac{2 \varepsilon_{x y}^{L}}{\sqrt{\left(1+2 \varepsilon_{x}^{L}\right)\left(1+2 \varepsilon_{y}^{L}\right)}} .
\end{aligned}
$$

In Eulerian coordinates similar formulae may be obtained, but they concern the segment parallel after deformation to $x$, or the angle formed after deformation by $d x$ and $d y$ :

$$
\begin{aligned}
\varepsilon_{x}^{* E} & =\frac{1}{\sqrt{1-2 \varepsilon_{x}^{E}}}-1, \\
\gamma_{x y}^{* E} & =\arcsin \frac{2 \varepsilon_{x y}^{E}}{\sqrt{\left(1-2 \varepsilon_{x}^{E}\right)\left(1-2 \varepsilon_{y}^{E}\right)}} .
\end{aligned}
$$

Formulae (7.8) and (7.11) express the strain tensors in terms of the components of the gradients of the displacement vector $U_{I, J}$ and $u_{i, j}$. If all these components are small with respect to unity, we may neglect the products with respect to linear terms, and then the linearized strain tensors are expressed by the symmetric parts of the gradients of displacement:

$$
\varepsilon_{I J}^{L} \cong \frac{1}{2}\left(U_{I, J}+U_{J, I}\right), \quad \varepsilon_{i j}^{E} \cong \frac{1}{2}\left(u_{i, j}+u_{j, i}\right) .
$$

Skew-symmetric parts determine infinitesimal rotation. It should be noted that in the irrotational case if the principal axes coincide with $x, y, z$, the diagonal components of the linearized tensor $\varepsilon_{I J}^{L}$ are exactly equal to the (finite) unit elongations $\varepsilon_{I J}^{* L},(7.14)$. On the other hand, for the tensor $\varepsilon_{i j}^{E}$ such a coincidence does not occur.

In the general case of small components of $U_{l, J}$ and $u_{i, j}$ the next step of approximation may be made. Namely, if we eliminate rigid translation, then the differences between the Eulerian and Lagrangian coordinates may be neglected, and we write simply, without any superscript,

$$
\varepsilon_{i j}=\frac{1}{2}\left(u_{i, j}+u_{j, i}\right) .
$$


Notation (7.20) corresponds to the Eulerian approach; however, this tensor is usually understood as the limiting case of the Lagrangian approach, since the latter is more commonly used in the mechanics of solids.

Tensor (7.20) is traditionally called the small-strain tensor but it should be remembered that it gives reasonable results only under the assumption that all the components of the gradients of the displacement vector are small; this assumption eliminates large rotations. Without such a limitation (7.20) may lead to false conclusions. For example, if (in the Eulerian approach) $u=y, v=-x, w=0$, then all the components of (7.20) vanish (and so they are undoubtedly small), but it may easily be found from (7.13) that the given displacement field corresponds to a homogeneous biaxial negative stretching.

In what follows we shall mostly use the classical linearized tensor (7.20) assuming both strains and rotations to be sufficiently small. In engineering notation formulae (7.20) are written thus:

$$
\varepsilon_{x}=\frac{\partial u}{\partial x}, \quad \gamma_{x y}=\frac{\partial u}{\partial y}+\frac{\partial v}{\partial x} \cdot \quad \sum_{y}^{x}
$$

Instead of $\varepsilon_{x y}$ we use here $\gamma_{x y}=2 \varepsilon_{x y}$, since in the linearized treatment it follows from (7.17) that $\gamma_{x y}$ directly determines the change of the right angle. The diagonal components $\varepsilon$ determine unit elongations (the first term of the expansion of (7.14)). It should be remembered, however, that the strain tensor is formed by $\varepsilon$ and $\gamma / 2$, and so we write it in the engineering and in indicial notation as follows:

$$
T_{\varepsilon}=\left\{\begin{array}{lll}
\varepsilon_{x} & \frac{1}{2} \gamma_{x y} & \frac{1}{2} \gamma_{x z} \\
\frac{1}{2} \gamma_{y x} & \varepsilon_{x} & \frac{1}{2} \gamma_{y z} \\
\frac{1}{2} \gamma_{z x} & \frac{1}{2} \gamma_{z y} & \varepsilon_{z}
\end{array}\right\}=\left\{\begin{array}{lll}
\varepsilon_{x x} & \varepsilon_{x y} & \varepsilon_{x z} \\
\varepsilon_{y x} & \varepsilon_{y y} & \varepsilon_{y z} \\
\varepsilon_{z x} & \varepsilon_{z y} & \varepsilon_{z z}
\end{array}\right\} .
$$

\subsection{Small strains in cylindrical and spherical coordinates}

Introducing curvilinear tensors and covariant differentiation, we may generalize (7.20) to any system of coordinates. Here we quote just the results for the two most commonly used systems:

In cylindrical coordinates, with the displacements denoted by $u_{r}, u_{\theta}, u_{z}$,

$$
\begin{array}{ll}
\varepsilon_{r}=\frac{\partial u_{r}}{\partial r}, & \gamma_{r \theta}=\frac{\partial u_{\theta}}{\partial r}-\frac{u_{\theta}}{r}+\frac{1}{r} \frac{\partial u_{r}}{\partial \theta}, \\
\varepsilon_{\theta}=\frac{1}{r} \frac{\partial u_{\theta}}{\partial \theta}+\frac{u_{r}}{r}, & \gamma_{\theta z}=\frac{1}{r} \frac{\partial u_{z}}{\partial \theta}+\frac{\partial u_{\theta}}{\partial z}, \\
\varepsilon_{z}=\frac{\partial u_{z}}{\partial z}, & \gamma_{z r}=\frac{\partial u_{r}}{\partial z}+\frac{\partial u_{z}}{\partial r},
\end{array}
$$


and in spherical coordinates, with the displacements denoted by $u_{r}, u_{v}, u_{\theta}$,

$$
\begin{array}{rlrl}
\varepsilon_{r} & =\frac{\partial u_{r}}{\partial r}, & \gamma_{r \psi} & =\frac{\partial u_{\psi}}{\partial r}-\frac{u_{\psi}}{r}+\frac{1}{r} \frac{\partial u_{r}}{\partial \psi}, \\
\varepsilon_{v} & =\frac{1}{r} \frac{\partial u_{\psi}}{\partial \psi}+\frac{u_{r}}{r}, & \gamma_{\psi \theta}=\frac{1}{r} \frac{\partial u_{\theta}}{\partial \psi}+\frac{1}{r \sin \psi} \frac{\partial u_{\psi}}{\partial \theta}-\frac{u_{\theta} \cot \psi}{r} \\
\varepsilon_{\theta}=\frac{1}{r \sin \psi} \frac{\partial u_{\theta}}{\partial \theta}+\frac{u_{\psi} \cot \psi}{r}+\frac{u_{r}}{r}, & \gamma_{\theta r}=\frac{1}{r \sin \psi} \frac{\partial u_{r}}{\partial \theta}+\frac{\partial u_{\theta}}{\partial r}-\frac{u_{\theta}}{r}
\end{array}
$$

Of course, these formulae may also be derived by considering the changes of the appropriate segments and right angles.

\subsection{Decomposition of the strain tensor. Dilatation}

The linearized strain tensor may be decomposed into the axiatoric and the deviatoric part according to the general scheme $T_{\varepsilon}=A_{\varepsilon}+D_{\varepsilon}$. The only independent component of the axiator equals

$$
\varepsilon_{m}=\frac{1}{3}\left(\varepsilon_{x}+\varepsilon_{y}+\varepsilon_{z}\right)
$$

the deviatoric components will be denoted by $e_{x}, e_{y}, e_{z}, \frac{1}{2} \gamma_{x y}, \frac{1}{2} \gamma_{y z}, \frac{1}{2} \gamma_{z x}$ in engineering notation, and by $e_{i j}$ in indicial notation.

This decomposition has an important physical interpretation. Namely, evaluate the unit volume change of an infinitesimal parallelepiped $d x, d y, d z$. Assuming that the system of coordinates coincides with the principal directions of the strain tensor, we obtain, after deformation, a parallelepiped with the sides $d x\left(1+\varepsilon_{1}\right)$, $d y\left(1+\varepsilon_{2}\right), d z\left(1+\varepsilon_{3}\right)$. The unit volume change, called dilatation and denoted by $\Theta$ is

$$
\Theta=\frac{d x\left(1+\varepsilon_{1}\right) d y\left(1+\varepsilon_{2}\right) d z\left(1+\varepsilon_{3}\right)}{d x d y d z}-1
$$

and after dropping of non-linear terms, we obtain

$$
\Theta=\varepsilon_{1}+\varepsilon_{2}+\varepsilon_{3}=3 \varepsilon_{m} .
$$

So the strain axiator presents pure volume change, and the strain deviator-pure shape change. In the case of incompressible bodies the strain axiator vanishes (except thermal expansion). The definition of a strain deviator in the case of finite strains is given, for example, by I. I. Goldenblat [2.38].

\subsection{Invariants of the small-strain tensor}

Typical invariants of the small-strain tensor are denoted as follows: basic invariants by $I_{1 \varepsilon}, I_{2 \varepsilon}, I_{3 \varepsilon}$; principal invariants by $J_{1 \varepsilon}, J_{2 \varepsilon}, J_{3_{\varepsilon}}$; axiatoric-deviatoric invariants by $I_{1 \varepsilon}, I_{2 e}, I_{3 e}$.

Certain differences appear in the definitions of cylindrical invariants. Instead of the invariant $Z_{\varepsilon},(5.10)$, we introduce the proportional invariant $\varepsilon_{m},(7.25)$. The 
definition of $\omega_{\varepsilon},(5.10)$, remains without change, whereas $R_{\varepsilon}$ is usually replaced by a proportional invariant with another numerical coefficient. The formula for $R_{\varepsilon}$ may be written in the form

$$
R_{\varepsilon}=c \sqrt{\left(\varepsilon_{1}-\varepsilon_{2}\right)^{2}+\left(\varepsilon_{2}-\varepsilon_{3}\right)^{2}+\left(\varepsilon_{3}-\varepsilon_{1}\right)^{2}},
$$

where the coefficient $c$ equals $1 / \sqrt{3}$. In the case of stresses we defined the stress intensity $\sigma_{e}$ by replacing in the corresponding formula the coefficient $1 / \sqrt{3}$ by $1 / \sqrt{2}$ and obtaining at uniaxial tension $\sigma_{e}=\sigma$. In the case of strains the proposals are much more diversified. The identical coefficient $c=1 / \sqrt{2}$, which would ensure full analogy to the formulae for stresses, is very seldom assumed (W. Urbanowski [2.125], H. Ford [2.32], E. P. Unksov [2.182]); the direct transition to $\varepsilon_{1}$ in the case $\varepsilon_{2}=\varepsilon_{3}=0$ is of no practical significance, and for incompressible materials such a state of strain is even impossible. N. I. Bezukhov [2.8] suggested the term "strain intensity" (or "effective strain"), $\varepsilon_{e}$, for expression (7.28) with the coefficient $c=$ $1 /(1+v) \sqrt{2}$; then in the elastic range the simplest relation $\sigma_{e}=E \varepsilon_{e}$ holds for incompressible as well as for compressible materials. The disadvantage of this proposal lies in the dependence of strain intensity on the material constant-Poisson's ratio $v$. To avoid this disadvantage most authors assume $v=1 / 2$, as for incompressible materials; such a definition will also be adopted here:

$$
\begin{aligned}
\varepsilon_{e} & =\frac{1}{3} \sqrt{2} \sqrt{\left(\varepsilon_{1}-\varepsilon_{2}\right)^{2}+\left(\varepsilon_{2}-\varepsilon_{3}\right)^{2}+\left(\varepsilon_{3}-\varepsilon_{1}\right)^{2}}=\sqrt{\frac{2}{3} e_{i j} e_{i j}} \\
& =\frac{2}{3} \sqrt{\varepsilon_{x}^{2}+\varepsilon_{y}^{2}+\varepsilon_{z}^{2}-\varepsilon_{x} \varepsilon_{y}-\varepsilon_{y} \varepsilon_{z}-\varepsilon_{z} \varepsilon_{x}+\frac{3}{4}\left(\gamma_{x y}^{2}+\gamma_{y z}^{2}+\gamma_{z x}^{2}\right)} .
\end{aligned}
$$

Some authors make use of the intensity of shearing strains, $\gamma_{e}$, defined by (7.28) with the coefficient either $c=\sqrt{2 / 3}$ (L. M. Kachanov [2.79]), or $c=1 / \sqrt{6}$ (V. V. Sokolovsky [2.163], W. Szczepiński [2.166]), or $c=2 / 3$ (M. Roš, A. Eichinger [2.144], A. A. Ilyushin [1.8], S. D. Ponomarev et al. [2.130]). In the first case we have at pure shear $\gamma_{e}=\gamma$, in the second $\gamma_{e}=\gamma / 2$, whereas the third leads to $\gamma_{e}=\gamma_{\text {oct }}$; namely, octahedral strains are determined by formulae similar to (6.6) and (6.7) but with $\tau$ replaced by $\gamma / 2$ :

$$
\begin{aligned}
& \varepsilon_{\text {oct }}=\frac{1}{3}\left(\varepsilon_{1}+\varepsilon_{2}+\varepsilon_{3}\right), \\
& \gamma_{\text {oct }}=\frac{2}{3} \sqrt{\left(\varepsilon_{1}-\varepsilon_{2}\right)^{2}+\left(\varepsilon_{2}-\varepsilon_{3}\right)^{2}+\left(\varepsilon_{3}-\varepsilon_{1}\right)^{2}} .
\end{aligned}
$$

In the case of an incompressible material $\varepsilon_{1}+\varepsilon_{2}+\varepsilon_{3}=0$ and strain intensity may be determined as a function of two principal strains only, for example $\varepsilon_{1}$ and $\varepsilon_{2}$. We obtain

$$
\varepsilon_{e}=\frac{2}{\sqrt{3}} \sqrt{\varepsilon_{1}^{2}+\varepsilon_{2}^{2}+\varepsilon_{1} \varepsilon_{2}},
$$

whereas $\gamma_{\text {oct }}$ is given by the same formula with the coefficient $2 \sqrt{2 / 3}$.

Extremal shearing strains $\gamma$ correspond to the change of right angles the arms of which form the angle $45^{\circ}$ with the principal directions. Maximal shearing strain equals

$$
\gamma_{\max }=\varepsilon_{\mathrm{I}}-\varepsilon_{\mathrm{III}}
$$


The relations between typical systems of small-strain invariants may be found in Table 2 (on p. 37) with the formal replacement of $\sigma$ and $s$ by $\varepsilon$ and $e$. A certain difference appears only in $\sigma_{e}$, which-in view of the definitions (6.4) and (7.29)should be replaced by $3 \varepsilon_{e} / 2$.

The relations between finite-strain invariants may be found in the books by A. E. Green and W. Zerna [2.39], I. I. Goldenblat [2.38] and A. M. Freudenthal [2.34].

\subsection{Strain rates}

In the case of small strains and small rotations the time derivatives of the strain components form a tensor which may be written as

$$
T_{\dot{\varepsilon}}=\left\{\begin{array}{ccc}
\dot{\varepsilon}_{x} & \frac{\dot{\gamma}_{x y}}{2} & \frac{\dot{\gamma}_{x z}}{2} \\
\frac{\dot{\gamma}_{y x}}{2} & \dot{\varepsilon}_{y} & \frac{\dot{\gamma}_{y z}}{2} \\
\frac{\dot{\gamma}_{z x}}{2} & \frac{\dot{\gamma}_{z y}}{2} & \dot{\varepsilon}_{z}
\end{array}\right\}=\left\{\begin{array}{ccc}
\dot{\varepsilon}_{x x} & \dot{\varepsilon}_{x y} & \dot{\varepsilon}_{x z} \\
\dot{\varepsilon}_{y x} & \dot{\varepsilon}_{y y} & \dot{\varepsilon}_{y z} \\
\dot{\varepsilon}_{z x} & \dot{\varepsilon}_{z y} & \dot{\varepsilon}_{z z}
\end{array}\right\} \text {. }
$$

Differentiating (7.20) with respect to the time $t$, we express $\dot{\varepsilon}_{i j}$ in terms of the velocity components $\dot{u}_{i}$ :

$$
\dot{\varepsilon}_{i j}=\frac{1}{2}\left(\dot{u}_{i, j}+\dot{u}_{j, i}\right) \text {. }
$$

The invariants of the tensor $T_{\dot{\varepsilon}}$ will be denoted by $I_{1 \dot{\varepsilon}}, I_{2 \dot{\varepsilon}}, I_{3_{\varepsilon}}$ (basic invariants), $J_{1 \dot{\varepsilon}}, J_{2 \dot{\varepsilon}}, J_{3 \dot{\varepsilon}}$ (principal invariants), etc. The integral invariant

$$
S_{\varepsilon}=\int_{i_{A}}^{t_{B}} \sqrt{I_{2 \dot{\varepsilon}}} d t=\int_{A}^{B} \sqrt{d \varepsilon_{i j} d \varepsilon_{i j}}
$$

determines the length of the trajectory in the nine-dimensional strain space between the points $A$ and $B$. In order to estimate the influence of intermediate principal strain rate on yielding, the Lode parameter for strain rates (or increments) is often introduced; according to (5.19)

$$
\mu_{\varepsilon}^{*}=\frac{2(d \varepsilon)_{\mathrm{II}}-(d \varepsilon)_{\mathrm{I}}-(d \varepsilon)_{\mathrm{III}}}{(d \varepsilon)_{\mathrm{I}}-(d \varepsilon)_{\mathrm{III}}}=\frac{3(d e)_{\mathrm{II}}}{(d e)_{\mathrm{I}}-(d e)_{\mathrm{III}}} .
$$

The rates are here replaced by the increments after the formal multiplication of numerator and denominator by $d t$. These increments are bracketed since the arrangement does not always lead to $(d \varepsilon)_{\mathbf{1}}=d\left(\varepsilon_{1}\right)$.

The strain rate tensor may be decomposed into the strain rate axiator and the strain rate deviator; for incompressible materials the strain rate axiator $A_{\varepsilon}$ vanishes identically.

In the case of finite strains we first notice that in spatial coordinates the symmetric vart of the finite gradient of the velocity vector is directly connected with defor- 
mation, and the skew-symmetric part with rotation. As a matter of fact, multiplying them by $d t$ we obtain the infinitesimal strain and the infinitesimal rotation respectively (L. I. Sedov [2.152]); thus the decomposition is here simpler than in the case of the finite gradient of the displacement vector.

The symmetric part is called the stretching tensor (or, the rate-of-deformation tensor),

$$
d_{i j}=\frac{1}{2}\left(\dot{u}_{i, j}+\dot{u}_{j, i}\right)
$$

and the skew-symmetric part--the spin tensor

$$
w_{i j}=\frac{1}{2}\left(\dot{u}_{i, j}-\dot{u}_{j, i}\right)
$$

the dots denote here material derivatives $D / D t$, i.e. the time derivatives with the material coordinates hold constant. Hence in material coordinates $D / D t=\partial / \partial t$, and in spatial coordinates $D / D t=\partial / \partial t+\left(\partial X_{K} / \partial t\right)\left(\partial / \partial X_{K}\right)$. L. E. Malvern [2.108] draws attention to the fact that in the case of finite strains (or even finite displacements) there is a difference between (7.35) and (7.38); indeed, (7.38) is consistently calculated in spatial coordinates, whereas (7.35) was obtained by a limiting procedure in material coordinates.

Further, besides (7.38) we may introduce the strain rate tensors, defined as the material derivatives of $\varepsilon_{i j}^{L}$ and $\varepsilon_{i j}^{E}$ :

$$
\begin{aligned}
& \dot{\varepsilon}_{I J}^{L}=\frac{D \varepsilon_{I J}^{L}}{D t}=\frac{\partial \varepsilon_{I J}^{L}}{\partial t}, \\
& \dot{\varepsilon}_{i j}^{E}=\frac{D \varepsilon_{i j}^{E}}{D t}=\frac{\partial \varepsilon_{i j}^{E}}{\partial t}+\frac{\partial \varepsilon_{i j}^{E}}{\partial X_{K}} \frac{\partial X_{K}}{\partial t} ;
\end{aligned}
$$

they are connected with $d_{i j}$ as follows (A. C. Eringen [2.31]):

$$
\begin{aligned}
& \dot{\varepsilon}_{I J}^{L}=d_{k l} x_{k, 1} x_{t, J}, \\
& \dot{\varepsilon}_{i j}^{E}=d_{l j}-\left(\varepsilon_{i k}^{E} \dot{u}_{k, j}+\varepsilon_{j l}^{E} \dot{u}_{l, l}\right) .
\end{aligned}
$$

Many other quantities representing strain rates are also used; an extensive review and a new proposal are given by $Z$. H. Guo [2.47].

\subsection{Logarithmic or natural strain}

Even in the simplest case of uniaxial tension the corresponding (longitudinal) components of the strain tensors $\varepsilon_{x}^{L}, \varepsilon_{x}^{E}$ and $\varepsilon_{x}=\varepsilon_{x}^{*}$ differ from each other. It turns out that all of them are determined by the general formula

$$
\varepsilon_{x}^{(n)}=\frac{1}{n}\left[1-\left(\frac{l_{0}}{l_{1}}\right)^{n}\right],
$$

where $l_{0}=L$ is the length before deformation, and $l_{1}$ is the length after deformation. Namely, for $n=-1$ we obtain the unit elongation $\varepsilon_{x}=\varepsilon_{x}^{*}$, for $n=-2$ the Lagrangian component $\varepsilon_{x}^{L}$, whereas for $n=+2$ the Eulerian component $\varepsilon_{x}^{E}$. The corresponding diagrams are shown in Fig. 19 in terms of $l_{1} / l_{0}$. 


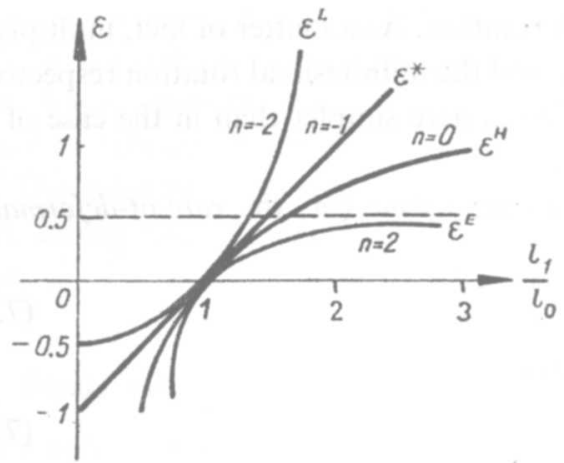

Fig. 19. Various measures of the longitudinal strain

Formula (7.44) was suggested by B. R. Seth $[2.154,2.155,2.156]$ as a generalized strain measure (the $n$th order measure). In fact, measures of deformation are to a certain degree arbitrary, and hence we may obtain a good description of material behaviour by combining a more complicated measure with simpler constitutive relations. This concept was developed by T. C. Hsu, S. R. Davies, and R. Royles [2.64], who described various materials by linear plastic hardening law combined with (7.44). Of course, for an arbitrary value of the exponent $n$ the introduction of a tensorial measure in the general three-dimensional case is more difficult; certain integral formulae were derived by Seth [2.155]. It should also be noted that it may be difficult to find a measure of stress which is consistent with (7.44) in the sense of principle of virtual work (1.1); this problem was not considered by Seth.

A review of the definitions of finite strain is given by A. V. Hershey [2.55], and also by H. Ziegler and D. Mc Vean [2.198].

The classical strain (unit elongation) satisfies a condition of symmetry of the type $\varepsilon\left(l_{1}-l_{0}\right)=-\varepsilon\left(l_{0}-l_{1}\right)$ (bracketed quantities are arguments here); however, none of the above-mentioned strains satisfies the much more justified requirement $\varepsilon\left(l_{1} / l_{0}\right)=-\varepsilon\left(l_{0} / l_{1}\right)$. To obtain such a representation of strain we integrate the length increments related to the current length $l$

$$
\varepsilon^{H}=\int_{l_{0}}^{l_{1}} \frac{d l}{l}=\ln \frac{l_{1}}{l_{0}} .
$$

This quantity was introduced by P. Ludwik [2.107] in 1909 (though a similar proposal was made by A. Mesnager during the physical congress in 1900). More attention to it was paid by H. Hencky [2.54] in 1928, and hence it is called the Hencky strain, $\varepsilon^{H}$. A. Nadai [2.118] introduced the term "natural strain"; other terms in use are "true" or "logarithmic" strain. Another advantage of logarithmic strain lies in the relation between the logarithmic dilatation and the principal strains,

$$
\Theta^{H}=\ln \frac{V_{1}}{V_{0}}=\varepsilon_{1}^{H}+\varepsilon_{2}^{H}+\varepsilon_{3}^{H},
$$


valid for arbitrarily large strains, whereas for unit elongations a similar relation holds only approximately, for small strains, (7.27).

Logarithmic strain may be expressed in terms of unit elongation as follows:

$$
\varepsilon^{H}=\ln (1+\varepsilon)=\varepsilon-\frac{\varepsilon^{2}}{2}+\frac{\varepsilon^{3}}{3}-\ldots,
$$

and in the case of small strains the differences between them are negligible. The relations with the diagonal components of the Lagrangian and Eulerian strain tensors are

$$
\varepsilon^{H}=\frac{1}{2} \ln \left(1+2 \varepsilon^{L}\right)=-\frac{1}{2} \ln \left(1-2 \varepsilon^{E}\right) .
$$

The Hencky strain is also "hidden" in (7.44) and may be obtained by passing to the limit $n \rightarrow 0$; the corresponding diagram is also shown in Fig. 19.

The main difficulties appear in the general, three-dimensional case. Of course, one can always formally construct a tensor whose principal components are $\ln \left(1+\varepsilon_{1}\right)$, $\ln \left(1+\varepsilon_{2}\right), \ln \left(1+\varepsilon_{3}\right)$, or a similar one, related to $\varepsilon_{I J}^{L}$ or $\varepsilon_{i j}^{E}$. Such tensors are sometimes used (J. D. Weber [2.189]), however, their arbitrary components cannot be expressed in terms of displacements by closed-form relations.

Many other proposals, mainly non-tensorial, have been made (J. E. Dorn, A. J. Latter [2.27], A. Gleyzal [2.37], F. D. Murnaghan [2.115], O. Hoffman, G. Sachs [2.63], Y. Yoshimura [2.193], S. I. Gubkin [2.45]). Certain critical remarks were made by H. Richter [2.143] and C. Truesdell [2.176]; Truesdell states that logarithmic strain may be utilized only in irrotational cases, where the principal directions are fixed and known. Nevertheless, in many such cases (rotational symmetry, etc.) the use of logarithmic strain leads to good results, in better agreement with the experiments than conventional strain.

\subsection{Equations of compatibility}

Six components of a symmetric strain tensor may be presented in terms of three components of the displacement vector. If we attempt to reverse the process and to evaluate the displacements while knowing the strains, it turns out that the problem is overdetermined and the strains must satisfy certain additional relations, called the equations of compatibility of de Saint-Venant. Six such relations may be derived, though, in fact, only three of them are independent (L. E. Malvern [2.108]).

In the simplest case of the linearized strain tensor $\varepsilon_{i j}$ the compatibility equations are obtained by direct eliminating $u_{i}$ from (7.20):

$$
\begin{aligned}
& \frac{\partial^{2} \varepsilon_{x}}{\partial y^{2}}+\frac{\partial^{2} \varepsilon_{y}}{\partial x^{2}}=\frac{\partial^{2} \gamma_{x y}}{\partial x \partial y} \\
& \frac{\partial^{2} \gamma_{y z}}{\partial z \partial x}+\frac{\partial^{2} \gamma_{z x}}{\partial y \partial z}-\frac{\partial^{2} \gamma_{x y}}{\partial z^{2}}=2 \frac{\partial^{2} \varepsilon_{z}}{\partial x \partial y} .
\end{aligned}
$$


In indicial notation with the summation convention employed the compatibility equations are usually written in the form

$$
\varepsilon_{i j, k l}+\varepsilon_{k l, i j}-\varepsilon_{i k, j l}-\varepsilon_{j l, i k}=0, \quad i, j, k, l=x, y, z .
$$

However, this form is slightly inconvenient since it describes 81 equations only 6 of which being distinct. To reduce this apparent number of equations we may use another form, with only two free indices,

$$
e_{i k m} e_{j l n} \varepsilon_{k l, m n}=0, \quad i, j, k, l, m, n=x, y, z,
$$

where $e_{i k m}$ denote permutation symbols (alternators): $e_{i k m}=0$ if any two of $i, k, m$ are equal, $e_{i k m}=1$ if $i k m$ are an even permutation of $x, y, \mathrm{z}, e_{i k m}=-1$ for an odd permutation.

In cylindrical coordinates the corresponding system of equations was derived by F. K. G. Odqvist [2.124], and in arbitrary coordinates by V. Z. Vlasov [2.185, $2.186]$. We quote here only the results for cylindrical coordinates, making use of the Vlasov form but changing indicial notation into engineering notation:

$$
\begin{aligned}
& \frac{\partial^{2} \varepsilon_{r}}{\partial z^{2}}+\frac{\partial^{2} \varepsilon_{z}}{\partial r^{2}}-\frac{\partial^{2} \gamma_{z r}}{\partial z \partial r}=0, \\
& \frac{\partial^{2} \varepsilon_{\theta}}{\partial z^{2}}+\frac{1}{r^{2}} \frac{\partial^{2} \varepsilon_{z}}{\partial \theta^{2}}-\frac{1}{r} \frac{\partial^{2} \gamma_{\theta z}}{\partial \theta \partial z}+\frac{1}{r} \frac{\partial \varepsilon_{z}}{\partial r}-\frac{1}{r} \frac{\partial \gamma_{z r}}{\partial z}=0, \\
& \frac{1}{r} \frac{\partial^{2} \varepsilon_{r}}{\partial \theta^{2}}+\frac{1}{r} \frac{\partial}{\partial r}\left(r^{2} \frac{\partial \varepsilon_{\theta}}{\partial r}\right)-\frac{1}{r} \frac{\partial^{2}\left(r \gamma_{r \theta}\right)}{r \partial \partial}-\frac{\partial \varepsilon_{r}}{\partial r}=0, \\
& \frac{\partial^{2} \gamma_{r \theta}}{\partial z^{2}}-r \frac{\partial^{2}}{\partial r \partial z}\left(\frac{\gamma_{\theta z}}{r}\right)-\frac{1}{r} \frac{\partial^{2} \gamma_{z r}}{\partial \theta \partial z}+2 \frac{\partial^{2}}{\partial r \partial \theta}\left(\frac{\varepsilon_{z}}{r}\right)=0, \\
& \frac{1}{r} \frac{\partial^{2}\left(r \gamma_{\theta z}\right)}{\partial r^{2}}-\frac{1}{r^{2}} \frac{\partial^{2}\left(r^{2} \gamma_{r \theta}\right)}{\partial z \partial r}-\frac{\partial^{2}}{\partial r \partial \theta}\left(\frac{\gamma_{z r}}{r}\right)+\frac{2}{r} \frac{\partial^{2} \varepsilon_{r}}{\partial \theta \partial z}-\frac{1}{r^{2}} \frac{\partial\left(r \gamma_{\theta z}\right)}{\partial r}=0, \\
& \frac{1}{r} \frac{\partial^{2} \gamma_{z r}}{\partial \theta^{2}}-\frac{1}{r} \frac{\partial^{2}\left(r \gamma_{\theta z}\right)}{\partial r \partial \theta}-\frac{\partial^{2} \gamma_{r \theta}}{\partial \theta \partial z}+2 \frac{\partial^{2}\left(r \varepsilon_{\theta}\right)}{\partial z \partial r}-2 \frac{\partial \varepsilon_{r}}{\partial z}=0 .
\end{aligned}
$$

For a simply connected body the compatibility equations are necessary and sufficient conditions for the existence and uniqueness of displacements (if the rigid body motion is eliminated). In the case of a multiply connected body we have to determine some constants of integration going back to (7.20). The strain rates (6.13) should satisfy analogical relations.

In the case of finite-strain tensors a similar elimination of $u_{i}$ is very cumbersome. However, making use of the Riemann theorem, we may express the compatibility conditions by the vanishing of the corresponding Riemann-Christoffel curvature tensor. Some explicit results may be found in the books by A. E. Green and W. Zerna [2.39], C. Truesdell and R. Toupin [2.179] and by A. C. Eringen [2.31]; a detailed analysis is given by I. I. Goldenblat [2.38].

For logarithmic strains the general conditions of compatibility are not yet derived. However, in some simple cases such a derivation presents no difficulty. For 
example, in the circularly-symmetric (one-dimensional) case in Lagrangian coordinates we have

$$
\varepsilon_{r}^{H}=\ln \left(1+\frac{d U_{r}}{d R}\right), \quad \varepsilon_{\theta}^{H}=\ln \left(1+\frac{U_{r}}{R}\right),
$$

and hence, after eliminating $U_{r}$,

$$
\varepsilon_{r}^{H}=\varepsilon_{\theta}^{H}+\ln \left(1+R \frac{d \varepsilon_{\theta}^{H}}{d R}\right) .
$$

Similarly, in Eulerian coordinates,

$$
\begin{aligned}
& \varepsilon_{r}^{H}=-\ln \left(1-\frac{d u_{r}}{d r}\right), \quad \varepsilon_{\theta}^{H}=-\ln \left(1-\frac{u_{r}}{r}\right), \\
& \varepsilon_{r}^{H}=\varepsilon_{\theta}^{H}-\ln \left(1-r \frac{d \varepsilon_{\theta}^{H}}{d r}\right) .
\end{aligned}
$$

The equations of compatibility should be satisfied in the regular regions of the body; on the other hand, surfaces of discontinuity may also appear. In a continuous medium discontinuitie sof displacements are inadmissible if we exclude such phenomena as dislocations. Tangential velocities may be discontinuous in the limit state, whereas the discontinuity of velocities normal to the surface would lead in a statical problem to vacancies or overlapping of the material; such a discontinuity is possible in the wave theory (shock waves). A more detailed discussion of discontinuities in strains and strain rates is given by A. M. Freudenthal and H. Geiringer [2.33], C. Truesdell and R. Toupin [2.179], and W. Jaunzemis [2.74]; cf. also Sec. 18.

In the theory of plasticity solutions discontinuous in velocities are mainly connected with slip lines. Numerous papers are devoted to the analysis of the relations along the slip lines; let us mention here T. Y. Thomas [2.173, 2.174], D. D. Ivlev [2.71], G. I. Bykovtsev, D. D. Ivlev, Yu. M. Myasnyankin [2.15, 2.16], W. Johnson, R. Sowerby, J. B. Haddow [2.77], I. S. Degtyarev [2.22, 2.23].

\section{Uniaxial plasticity}

\subsection{True stress-strain diagram}

The simplest and most common basic test in plasticity is the standard tension test. This does not mean that from the theoretical viewpoint this test is the best one: its drawbacks lie in the triaxial state of strain corresponding to uniaxial stress, and in the changing cross-sectional area of the specimen. The pure shear test would be much better here, but the testing technique is more complicated and poses new problems, for example that of the homogeneity of stress state. Thus the tension test remains the basic one. 
a)

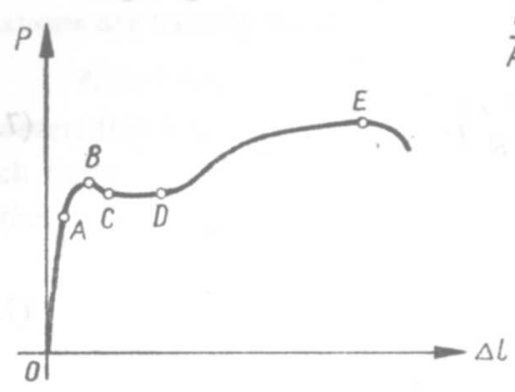

b)

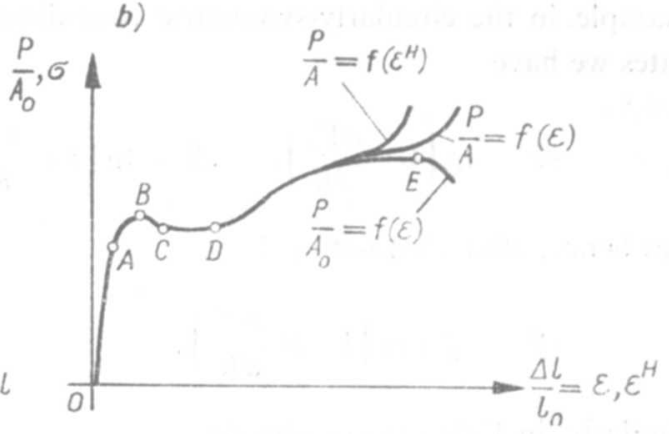

Fig. 20. Stress-strain diagram for a mild steel: (a) in the system $\Delta l-P$, (b) in the system $\varepsilon-\sigma$

Figure 20a shows a typical tension test diagram for low-carbon steel; it is plotted in the coordinate system elongation $\Delta l$ versus force $P$, as delivered by the testing machine. This diagram is converted into the strain-stress variables in order to get rid of the dimensions of the specimen. Under the assumption of proportionality between $\varepsilon$ and $\Delta l$ and between $\sigma$ and $P$ we would obtain in appropriate scales a geometrically similar diagram. Such a situation occurs in the case of conventional (Cauchy) strain and nominal stress $P / A_{0}$, calculated without taking into account geometry changes (principle of rigidification). As a matter of fact, the change of cross-section may be essential for larger deformations and we should make use of the true stress $\sigma=P / A$ (Fig. 20b). Assuming homogeneous strain and plastic incompressibility, and neglecting small elastic strains with respect to plastic ones, we obtain

$$
\sigma=\frac{P}{A}=\frac{P}{A_{0}} \frac{A_{0}}{A}=\frac{P}{A_{0}} \frac{l}{l_{0}}=(1+\varepsilon) \frac{P}{A_{0}},
$$

where $\varepsilon$ is the conventional strain. Further, to obtain the symmetry condition mentioned in Sec. 7.7, the logarithmic strain is often employed; the curve $P / A=f\left(\varepsilon^{H}\right)$ is called the true stress-strain diagram and is also shown in Fig. 20b.

Point $A$ of the diagram corresponds to the elastic limit (usually identified with the proportional limit), point $B$ to the upper yield point, and point $C$ to the lower yield point (these terms were introduced in 1904 by $C$. Bach). The segment $B C$ corresponds to the plastic softening of the material; only certain materials exhibit such a phenomenon, which is connected with material instability. The horizontal segment $C D$ is called the perfectly plastic flow; it corresponds to the lower yield point and this value should be used in further plastic flow calculations.

The segment $D E$ corresponds to the plastic hardening. Actually this hardening goes beyond $E$, but because of the decreasing cross-sectional area $A$ the maximum of the force is reached at $E$ (tensile strength, expressed by nominal stress). The decrease of the force is connected with a certain kind of instability resulting in the "necking" of the specimen.

Many materials do not exhibit perfect yielding. However, for various engineering applications conventional definitions of (apparent) yield-point stress are introduced. 
Various such definitions were compared by R. M. Haythornthwaite [2.52], who distinguished the following criteria: (1) departure from linearity, (2) measurable plastic strain, (3) slope of the diagram equal to a given fraction of the initial slope, (4) intersection of post-yield slope with the stress axis, (5) intersection of post-yield and elastic slopes, (6) offset elastic slope (Fig. 21). Of course, such a variety of

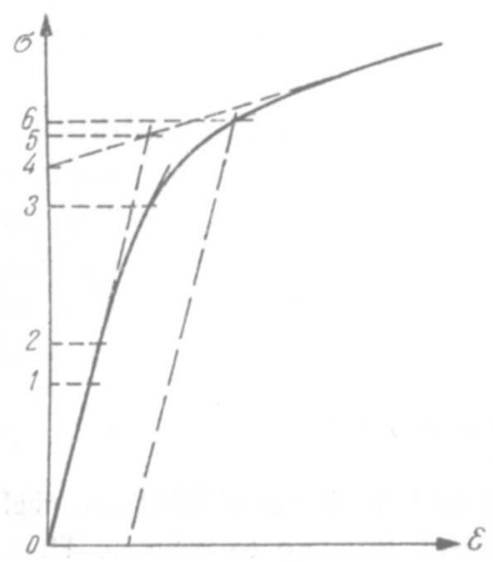

Fig. 21. Various definitions of the conventional yield-point stress

definitions makes the comparison of experimental results very difficult. The differences should be reflected even in the names, although no sharp separation can be made. For example, the first three definitions may be referred to the "conventional elastic limit" and the remaining three to the "conventional yield-point stress".

The stress-strain diagrams depend on the temperature and, sometimes, on the strain rate. The last effect is of rheological type and will not be dealt with here; it will be assumed that the diagrams were obtained at a very small strain rate.

For some applications certain descriptions of the stress-strain diagram other than $\sigma=f(\varepsilon)$ are more convenient. The derivative $d \sigma / d \varepsilon=E_{t}$ will be called the tangent modulus and the ratio $\sigma / \varepsilon=E_{s}$ the secant modulus at the given point of the curve. The stress-strain diagram may also be described by either of the equations $E_{t}=f(\sigma), E_{t}=f(\varepsilon), E_{s}=f(\sigma), E_{s}=f(\varepsilon)$. The first two equations are differential equations with respect to $\sigma=f(\varepsilon)$; particularly the form $E_{t}=f(\sigma)$ is often used in the theory of elastic-plastic stability.

A. A. Ilyushin [1.4] uses the following form of the description of the stress-strain diagram

$$
\sigma=E \varepsilon[1-\omega(\varepsilon)],
$$

where the values of $\omega(\varepsilon)$ satisfy $0 \leqslant \omega<1$; this form belongs to the type $E_{s}=f(\varepsilon)$.

\subsection{Perfectly plastic solids}

Any more exact effective analytical description of the stress-strain diagram presents great difficulties: if an analytical formula is needed, some simple approximations are used. They are called schematizations of the stress-strain diagram. 
For a material with a well-marked segment of perfectly plastic flow and small plastic hardening the schematization shown in Fig. 22 may be used. This diagram is connected with the name of L. Prandtl [2.138], although it was used is some earlier papers, for example by R. Mises [2.112]. It extends the elastic range to the yield

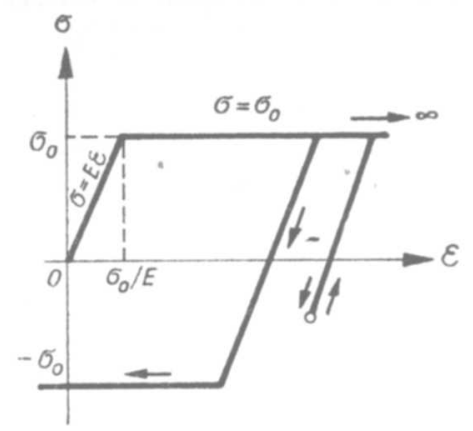

Fig. 22. The stress-strain diagram for a perfectly elastic-plastic body

point, neglects the difference between the upper and the lower yield stress, neglects plastic hardening, but extends the perfectly plastic flow up to infinity. Thus the diagram for loading consists of the segment $\sigma=E \varepsilon$ (perfectly elastic range, plastically passive process) and of the ray $\sigma=\sigma_{0}$ (perfectly plastic range, active process). The symbol $\sigma_{0}$ denotes here the yield-point stress at uniaxial tension, defined by $d \sigma / d \varepsilon=0$. Diagram 22 shows also a schematization of the passive process due to unloading and a reverse active process $\left(\sigma=-\sigma_{0}\right)$. The idealized body, described fully by the Prandtl diagram, is called the perfectly elastic-plastic body, or the elasticperfectly plastic body.

If the plastic strains are large enough, the elastic strains may be neglected with respect to them; this approach corresponds to the limiting case $E \rightarrow \infty$. The perfectly elastic-plastic body then passes into the perfectly rigid-plastic body (Fig. 23). This model is even simpler than the perfectly elastic-plastic one, but it may lead to some doubts. Namely, if it leads-directly used-to results other than those obtained

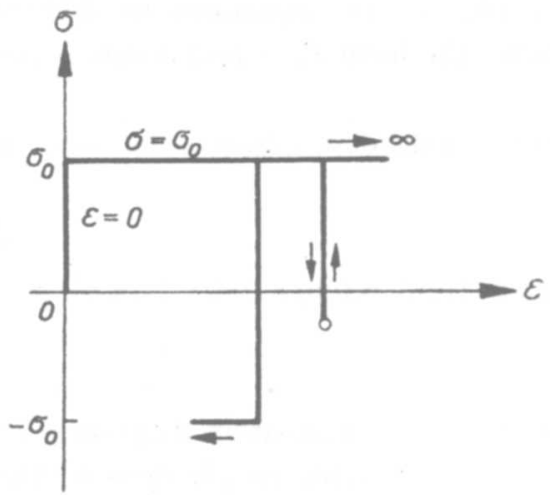

Fig. 23. The stress-strain diagram for a perfectly rigid-plastic body 
in the limiting case $E \rightarrow \infty$, then it is this limiting case that should be regarded as correct. Such problems will be discussed in Sec. 18.

Both models described constitute the basis of the theory of perfect plasticity.

\subsection{Schematizations of plastic hardening}

Before undertaking the approximation of the stress-strain diagram we have to decide which of the diagrams presented in Fig. 20b is to be approximated. In the range of moderate strains the differences are negligible, but for strains larger than 0.1 they become essential. Any diagram may be approximated, but it should later be correspondingly applied: if the change of the cross-section is taken into account, (8.1), it should also be accounted for in further applications. A similar remark applies to the logarithmic strain. The true stress-strain diagram has the widest range of applications.

There exist numerous suggestions for the schematization of plastic hardening; W. R. Osgood [2.126] compares some twenty of them. Various forms of description of the stress-strain curve are discussed by W. Truszkowski [2.180] and P. G. Hodge [2.61]. Here we are going to present only some of the most frequently used equations. The plastic hardening of steel is often described by the linear function (Fig. 24):

$$
\sigma=B+E_{1} \varepsilon, \quad \frac{\sigma_{0}}{E} \leqslant \varepsilon \leqslant \hat{\varepsilon} .
$$

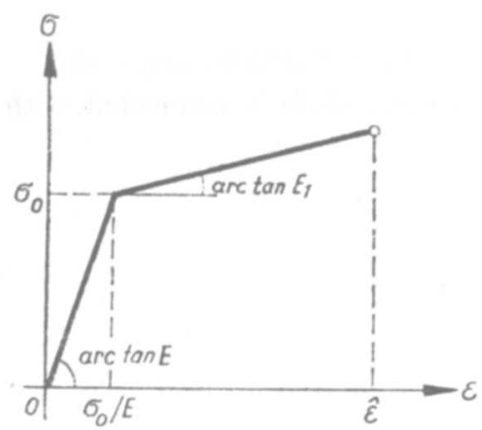

Fig. 24. The stress-strain diagram for a body with linear plastic hardening

The constant $E_{1}$ is called the hardening modulus; practically $0 \leqslant E_{1} \leqslant E / 10$. The straight line should be bounded from above by a certain value of the strain $\hat{\varepsilon}$, since without such a limitation the strength would be infinitely large, in contradiction to reality. The condition of continuity determines the constant $B$ :

$$
B=\left(1-\frac{E_{1}}{E}\right) \sigma_{0} .
$$

In the Ilyushin notation (8.2) we have

$$
\omega(\varepsilon)=\lambda\left(1-\frac{\sigma_{0}}{E \varepsilon}\right), \quad \lambda=\frac{E-E_{1}}{E} .
$$


In the limiting case $E_{1}=0$ we obtain the equations for the perfectly elastic-plastic body, whereas $E \rightarrow \infty$ gives the rigid-plastic body with linear hardening.

Formally, the linear hardening law may be used in connection with definitions of strain other than the conventional one. T. C. Hsu, S. R. Davies and R. Royles [2.64] combined it with the general definition (7.40); L. H. Sjodahl and J. B. Conway [2.162] proved that this proposal is equivalent to an earlier suggestion by $\mathrm{E}$. Voce [2.187].

A more exact approximation may be obtained if several equations of type (8.3) are employed, valid in the successive intervals of $\varepsilon$. This schematization is called piece-wise linear hardening or multiple linear hardening. The difficulties are here connected with the necessity of separation of many subregions in the body and with the application of numerous continuity conditions.

The power plastic hardening schematization,

$$
\sigma=k \varepsilon^{n}, \quad 0 \leqslant \varepsilon \leqslant \hat{\varepsilon},
$$

is free from that drawback. Indeed, this simple monomial is supposed to describe the whole range of variability of $\varepsilon$. To obtain upward convexity $n$ should be chosen from $(0,1)$. In the limiting case $n \rightarrow 1, k=E$, we arrive at Hooke's law. In the opposite limiting case, $n \rightarrow 0$, we obtain the perfectly rigid-plastic schematization; as a matter of fact, writing (8.6) in the form

$$
\frac{\sigma}{\sigma_{0}}=\left(\frac{\varepsilon}{\varepsilon_{0}}\right)^{n},
$$

we state that for $n \rightarrow 0$ it is satisfied by any $\sigma<\sigma_{0}$ at $\varepsilon=0$ and by any $\varepsilon$ at $\sigma=\sigma_{0}$, Fig. 25. On the other hand, the drawback of (8.6) and (8.7) is connected with the

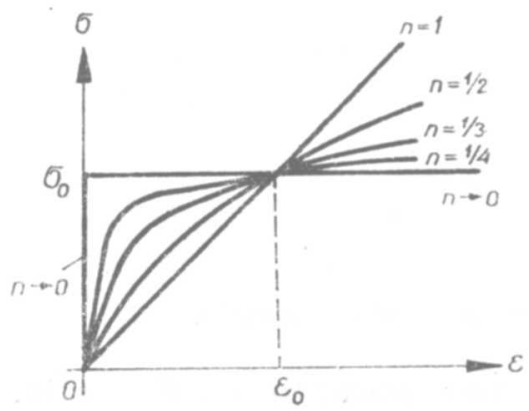

Fig. 25. The stress-strain diagram for bodies with power plastic hardening

infinitely large derivative $d \sigma / d \varepsilon$ at $\varepsilon=0$ (vertical tangent); this property is not shown by any real material, and so these equations should be used for strains that are not too small. In this range they describe quite well the behaviour of copper alloys and aluminium.

The simplest two generalizations of the power plastic hardening law were given by P. Ludwik [2.107]

$$
\sigma=\sigma_{0}+k \varepsilon^{n}
$$


(restricted to rigid-plastic bodies) and by W. Ramberg and W. Osgood [2.140]

$$
\varepsilon=\frac{\sigma}{E}+\left(\frac{\sigma}{k}\right)^{m}
$$

taking elastic strain into account; equation (8.9) with $m=1 / n$ and $E \rightarrow \infty$ turns into (8.6).

\subsection{Special schematizations}

The equations given above pretend to a certain generality and simultaneous simplicity. However, the simplicity of the function $\sigma=f(\varepsilon)$ does not always lead to simple solutions of more complex problems. Therefore it is sometimes better to employ a certain special, apparently more complex schematization, which for a given class of problems leads to simpler results; of course, the error of approximation of the experimental curve should always be kept within reasonable limits.

For example, in the elastic-plastic stability problems (which, incidentally, will not be dealt with in the present book) the dependence $E_{t}=f(\sigma)$ is particularly important. A small error of approximation of $\sigma=f(\varepsilon)$ may result in an essential error in the tangent modulus $E_{t}$, and, consequently, in critical loadings.

The schematization of the perfectly elastic-plastic body is here, as a rule, not sufficiently exact. One has to describe more precisely the segment between the elastic limit and the yield point. A. Ylinen [2.192] suggested here a convenient formula for $E_{t}=f(\sigma)$

$$
E_{t}=\frac{d \sigma}{d \varepsilon}=E \frac{\sigma_{0}-|\sigma|}{\sigma_{0}-c|\sigma|}, \quad 0 \leqslant|\sigma| \leqslant \sigma_{0},
$$

where $c$ is the material constant. Integrating (8.10) as a differential equation with respect to $\sigma=f(\varepsilon)$, we obtain

$$
\varepsilon=\frac{1}{E}\left[c \sigma-(1-c) \sigma_{0} \ln \left(1-\frac{|\sigma|}{\sigma_{0}}\right) \operatorname{sign} \sigma\right], \quad 0 \leqslant|\sigma| \leqslant \sigma_{0} .
$$

This is the proposed schematization of the stress-strain diagram. Equation (8.11), containing three parameters, like (8.8) and (8.9), is presented in a more complicated form, but because of the simplicity of (8.10) leads to simpler results in the stability problems.

The Ylinen equation (8.11) gives $\sigma=\sigma_{0}$ only in the limiting case $\varepsilon \leftrightarrow \infty$. Such behaviour of materials was called asymptotically perfect plasticity by $\mathrm{K}$. Szuwalski and M. Życzkowski in [1.18]. It may also be used in other types of problems, where the perfectly elastic-plastic schematization is too rough. Another well-known approximation of this type was proposed by W. Prager [2.131]

$$
\sigma=\sigma_{0} \tanh \left(\frac{E \varepsilon}{\sigma_{0}}\right), \quad-\infty<\varepsilon<\infty .
$$


Considering the optimal design of thin-walled bars, W. Krzyś [2.92, 2.93] introduced an apparently more complex, three-parameter schematization

$$
E_{t}=E\left[\frac{\left(\sigma_{0}-|\sigma|\right) \sigma^{2}}{\left(\sigma_{0}-\sigma_{p}\right) \sigma_{p}^{2}}\right]^{2 / 3}, \quad \sigma_{p} \leqslant|\sigma| \leqslant \sigma_{0},
$$

where $\sigma_{p}$ denotes the proportional limit.

The optimal design for the material function (8.13) has been found to be the simplest possible, and its accuracy is of the same order as that of other three-parameter schematizations. It has also been applied to other optimization problems by A. Gajewski and M. Życzkowski [2.35].

\subsection{The Bauschinger effect}

The schematization given above describes only the passive and active processes during loading. However, the unloading and reverse loading processes are also very important, not only in view of direct applications, but also for a correct and precise formulation of general physical (constitutive) equations of plasticity.

We shall restrict our considerations to materials which exhibit the same yield stress $\sigma_{0}$ in tension and in compression. Unloading (following active loading) results, at first, in a passive process, which may be described, usually with a sufficient degree of accuracy, by the linear equation

$$
\tilde{\sigma}-\sigma=E(\tilde{\varepsilon}-\varepsilon),
$$

where $\tilde{\varepsilon}$ and $\tilde{\sigma}$ denote the coordinates of the point $\tilde{A}$ at which the unloading starts (Fig. 26). Suppose that the stress $\sigma$ may be treated as the exertion factor. Then,

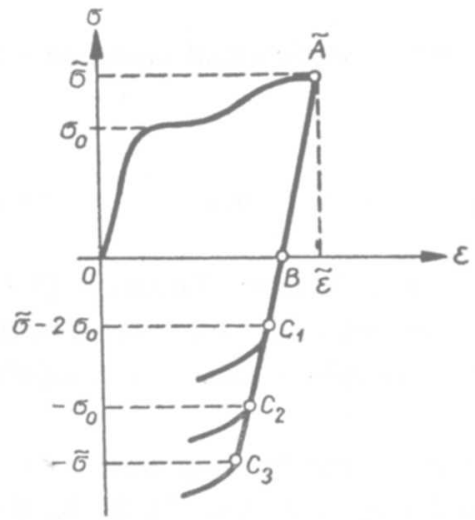

Fig. 26. The Bauschinger effect in uniaxial tension-compression

exceeding the point $B$, we have once more a loading process (reverse). At first, this process is passive and described by $(8.14)$. J. Bauschinger $[2.5,2.6]$ noticed that the initiation of the active process, characterized by any of the points $C_{1}$ on the diagram, occurs under reverse loading earlier than under repeated loading in the 
original sense (direction)-theoretically it would be then the point $\tilde{A}$. This phenomenon is called the Bauschinger effect. We speak of the ideal Bauschinger effect if the coordinate of the point $C_{i}$, denoted by $\tilde{\sigma}_{-}$, equals (point $C_{1}$ )

$$
\tilde{\sigma}_{-}=\tilde{\sigma}-2 \sigma_{0}
$$

such a value would correspond to a full compensation of the increase of the yield point in tension by the decrease in compression. From the physical point of view we should not call this case "plastic hardening" but rather "apparent hardening" and connect it with micro-nonhomogeneity and with residual microstresses at the point $B$ of the diagram, Yu. I. Kadashevitch, V. V. Novozhilov [2.80]. If the active process starts at $C_{3}, \tilde{\sigma}_{-}=-\tilde{\sigma}$, we speak of the lack of the Bauschinger effect - this case might also be called true plastic hardening. A certain intermediate case $\tilde{\sigma}_{-}=-\sigma_{0}$ will be called the stabilization of the yield stress, point $C_{2}$ in Fig. 26. The case of the coincidence of the points $C$ and $B\left(\tilde{\sigma}_{-}=0\right)$ was called the full Bauschinger effect by A. E. Kalatinets [2.81] but such a case seems to be unrealistic (at least for small plastic strains where $\tilde{\sigma}_{-}<0$ ).

Experimental determination of the point $C$ is not possible; practically we only find a substitute $C^{*}$ by using similar approaches to those described in Sec. 8.1; usually the change of plastic strains has a certain conventional value here. W. Szczepiński [2.165] states clearly the fact that the estimation of the Bauschinger effect depends essentially on this value: the smaller is the fixed conventional change, the more evident is the Bauschinger effect.

The first attempt at a theoretical explanation of the Bauschinger effect is due to G. Masing [2.110], who assumed that micro-nonhomogeneity is connected with a random orientation of anisotropic crystals in a polycrystalline body.

Such an explanation corresponds to the ideal Bauschinger effect. Further experimental research was carried out by G. Sachs and H. Shoji [2.146]; the theoretical background is given by $\mathrm{H}$. Shoji [2.160]. Extensive surveys of theoretical and experimental investigations are to be found in the books by V. V. Moskvitin [2.113], R. M. Shneyderovitch [1.17], N. I. Chernyak and D. A. Gavrilov [2.18], and in the survey articles by S. V. Serensen and R. M. Shneyderovitch [2.153] and A. P. Gusenkov [2.49].

Several papers on the Bauschinger effect were published by G. B. Talypov $[2.168,2.169,2.170]$; they were summarized in [2.171]. This effect is characterized there by the ratio $\left|\tilde{\sigma}_{-}\right| /|\tilde{\sigma}|$. Such an approach is inconvenient since if this ratio decreases, then-according to the definition-the Bauschinger effect increases. It would be more convenient to introduce the ratio

$$
\bar{\beta}=1+\frac{\tilde{\sigma}_{-}}{\tilde{\sigma}}=1-\frac{\left|\tilde{\sigma}_{-}\right|}{\tilde{\sigma}}, \quad 0 \leqslant \bar{\beta} \leqslant 2\left(1-\frac{\sigma_{0}}{\sigma}\right),
$$

equal to zero for a vanishing Bauschinger effect, or the ratio

$$
\beta=\frac{\tilde{\sigma}+\tilde{\sigma}_{-}}{2\left(\tilde{\sigma}-\sigma_{0}\right)}=\frac{\tilde{\sigma}-\left|\tilde{\sigma}_{-}\right|}{2\left(\tilde{\sigma}-\sigma_{0}\right)}, \quad 0 \leqslant \beta \leqslant 1,
$$


also equal to zero for a vanishing Bauschinger effect and to unity for the ideal effect. The convenient notation with absolute values is valid only in the case $\tilde{\sigma}_{-} \leqslant 0$.

According to Talypov's investigations, the ratio $\left|\tilde{\sigma}_{-}\right| /|\tilde{\sigma}|$ depends on the strain $\tilde{\varepsilon}$; namely, it first decreases ( $\bar{\beta}$ increases, but $\beta$ decreases, for most materials), and then becomes stabilized. The coefficient $\beta$ for larger plastic strains is of order 0.2 , and thus the Bauschinger effect amounts roughly to one fifth of the ideal one. The Bauschinger effect in shear was examined in [2.167]; the investigations of combined cases will be quoted separately.

Extensive theoretical and experimental studies of the Bauschinger effect were also carried out by A. Abel and H. Muir [2.1, 2.2, 2.3, 2.4]. They described this effect by several parameters, the most important of which coincides with $\bar{\beta},(8.16)$; the other ones are connected with strains and energies.

It should be mentioned that the term Bauschinger effect may lead to certain misunderstandings. Some authors, for example P. M. Naghdi [2.119] and A. C. Eringen [2.31], define by this term the difference between original yield stresses in tension and in compression, i.e. a notion quite different from the generally accepted one. Usually the Bauschinger effect in the common sense is investigated for those materials which do not exhibit the Bauschinger effect in the Naghdi-Eringen sense.

\subsection{Schematizations of cyclic plasticity}

The very simple proposals of schematization quoted in Sec. 8.3 become much more complex if multiple loading-unloading processes are to be uniformly described. These problems are usually called cyclic plasticity although it is not necessarily cyclic processes that are considered.

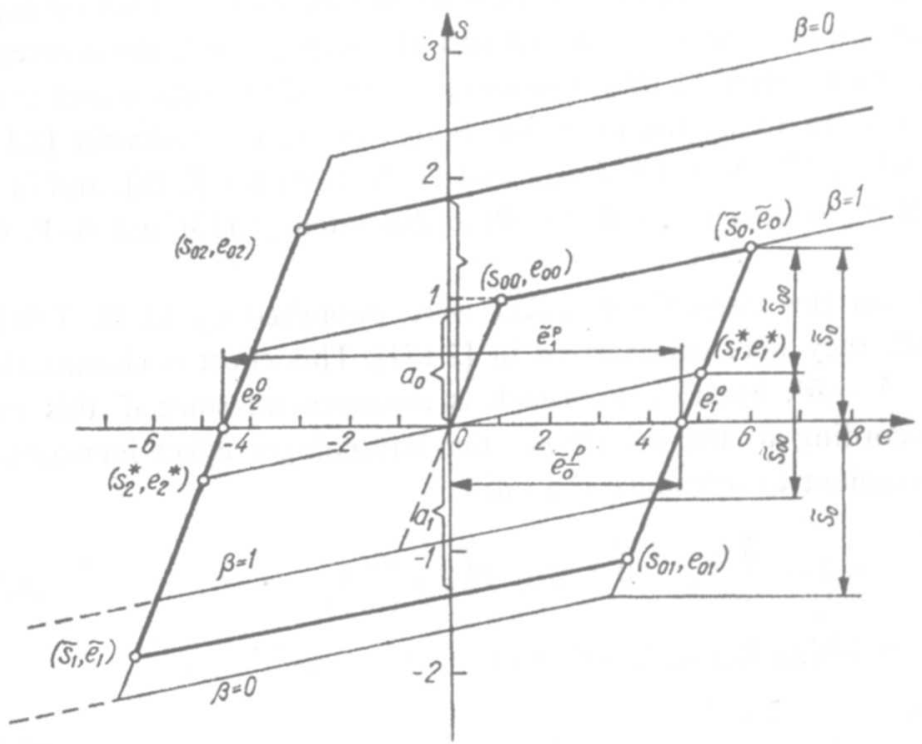

Fig. 27. Hypothesis of a constant share of the Bauschinger effect 
The simplest and most convenient for qualitative analysis are the proposals generalizing linear plastic hardening to the cyclic case. One of such schematizations was proposed by K. Kowalczyk and M. Życzkowski [2.89, 2.90], and will be briefly described here. First generalize the coefficient $\beta$ defined by (8.17) for the first half-cycle, writing for the $n$th half-cycle

$$
\beta_{n}=\frac{\left(\tilde{s}_{n-1}-s_{0 n-1}\right)-\left(\tilde{s}_{n-2}-s_{0 n}\right)}{2\left(\tilde{s}_{n-1}-s_{0 n-1}\right)}, \quad n=1,2, \ldots,
$$

where the dimensionless stress $s$ equals $\sigma / \sigma_{0}$. Further symbols are explained by Fig. 27, $e$ denoting here $e=E \varepsilon / \sigma_{0}$ (and not the deviatoric strain). Indeed, for $n=1$ we obtain $\beta_{1}=\beta,(8.17)$, putting $\tilde{s}_{n-2}=\mp 1$-this value follows from the assumed equal yield stresses in tension and in compression. If we now assume $\beta_{n}$ $=\beta=$ const (a hypothesis of constant share of the Bauschinger effect), then active processes are described by the general equation

$$
s_{n}=a_{n}+(1-\lambda) e_{n},
$$

in which

$$
\begin{aligned}
a_{0}= & \pm \lambda, \\
a_{n}= & \pm \lambda(2 \lambda \beta-2 \lambda-2 \beta+1)^{n}- \\
& -2 \lambda(1-\lambda)(1-\beta) \sum_{i=0}^{n-1} \tilde{e}_{i}(2 \lambda \beta-2 \lambda-2 \beta+1)^{n-1-i} \text { for } n \geqslant 1,
\end{aligned}
$$

and passive process by

$$
s_{n}=e_{n}-e_{n}^{0},
$$

where

$$
\begin{aligned}
e_{0}^{0}= & 0, \quad e_{1}^{0}=\mp \lambda+\lambda \tilde{e}_{0} \\
e_{n}^{0}= & \mp \lambda(2 \lambda \beta-2 \lambda-2 \beta+1)^{n-1}+ \\
& +2 \lambda(1-\lambda)(1-\beta) \sum_{i=0}^{n-2} \tilde{e}_{i}(2 \lambda \beta-2 \lambda-2 \beta+1)^{n-2-i}+ \\
& +\lambda \tilde{e}_{n-1} \quad \text { for } n \geqslant 2 .
\end{aligned}
$$

In these equations $\lambda$ (assumed to be constant) is given by (8.5); the upper signs refer to the process starting with tension, and the lower signs to that starting with compression. The changes need not be strictly cyclic, but passive processes are supposed to be followed by active ones with the same sense of loading. Substituting $\beta=0$, we obtain a schematization without the Bauschinger effect, whereas substituting $\beta=1$ we obtain the ideal Bauschinger effect (isotropic and kinematic hardening, respectively-these terms will be explained in Chapter III).

Linear schematizations of type (8.19) are, as a rule, remote from experimental results: for the cycles with relatively small changes of plastic strains the curved parts of the stress-strain diagram are essential. The first proposal of a curvilinear 
schematization of unloading in the first half-cycle is due to G. Masing [2.110]. It corresponds to the ideal Bauschinger effect and has the form

$$
\tilde{\sigma}-\sigma=2 f\left(\frac{\tilde{\varepsilon}-\varepsilon}{2}\right)
$$

where the symbol $f$ describes the first loading, $\sigma=f(\varepsilon)$. Experimental results confirm (8.23) only for small plastic strains, since for larger strains the Bauschinger effect is much smaller than the ideal one. A better agreement may be achieved by a certain generalization of (8.23), namely

$$
\tilde{\sigma}-\sigma=\alpha_{\sigma} f\left(\frac{\varepsilon-\varepsilon}{\alpha_{\varepsilon}}\right),
$$

proposed by V. V. Moskvitin [2.113]; the coefficients $\alpha_{\sigma}$ and $\alpha_{\varepsilon}$ should be determined here experimentally and may depend on the cycle index. Another generalization of (8.23) was proposed by R. L. Woolley [2.191].

Many other schematizations of cyclic plasticity have been proposed; the references given here are far from complete. Let us mention only some recent suggestions by O. Bruhns [2.11, 2.12] (finite strains), K. Turski [2.181] (generalized power series), T. Kishi and T. Tanabe [2.82] (power functions). Some further proposals (for triaxial cases) will be discussed in Sec. 14.

\subsection{Models of plastic bodies}

Some features of inelastic media are successfully discussed by the use of analogies with mechanical models: stress is here modelled by the force $P$, and strain by the displacement $\Delta$. Such discussion is of course purely qualitative and the conclusions may be overstated.

Perfect plasticity is-as a rule-modelled by Coulomb's dry friction with the coefficient of friction $\mu$ independent of velocity. The first proposals of such models date from the early twenties (C. F. Jenkin [2.75], multiple linear hardening, S. Lees [2.95], model with eccentric mechanism). Certain limitations connected with such

a)
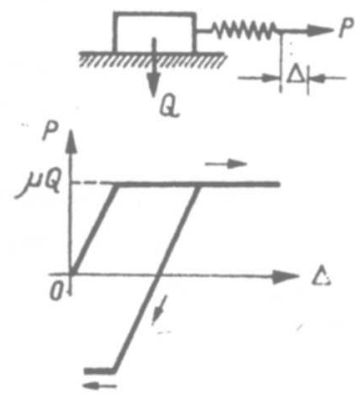

b)
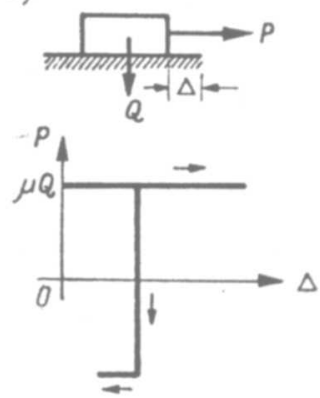

Fig. 28. Models of perfectly plastic bodies: (a) a perfectly elastic-plastic body, (b) a perfectly rigid-plastic body 
modelling were pointed out D. C. Drucker $[2.28,2.29]$, nevertheless the main analogy, based on the energy dissipation during plastic deformation and during motion of a heavy body with friction, is unquestionable. Thus the behaviour of perfectly elastic-plastic and perfectly rigid-plastic bodies may be illustrated by the models shown in Fig. 28; the analogy of unloading and reverse loading is also correct.

There exist various possibilities of modelling plastic hardening. Linear hardening with the ideal Bauschinger effect may be represented by the model shown in Fig. 29; residual microstresses are represented by the internal force in the spring 2.
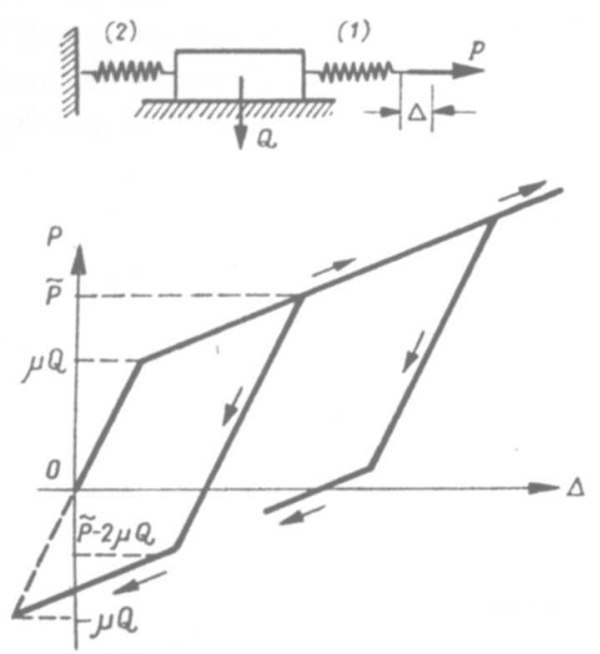

Fig. 29. A model of linear plastic hardening with an ideal Bauschinger effect (kinematic hardening)
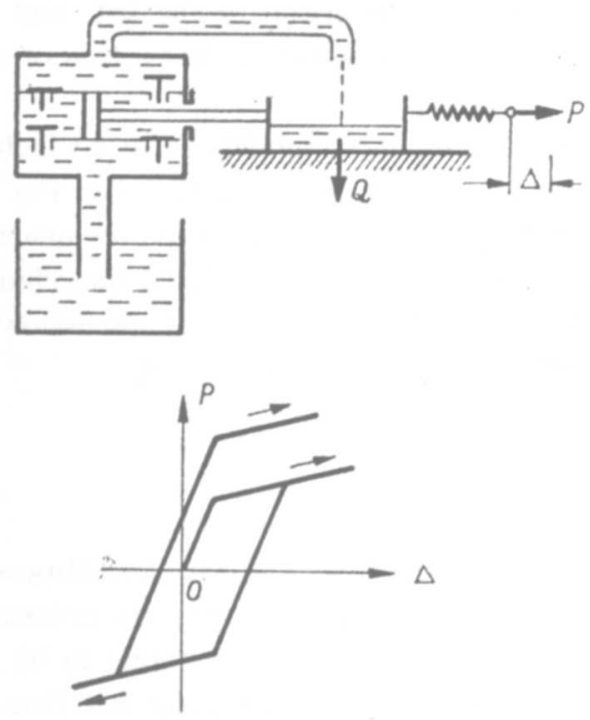

Fig. 30. A model of linear istotropic strain-hardening 
It should be noted that for the maximal tensile force $P$ larger than $2 \mu Q$ the reverse active process takes place even at positive $P$ (tension). The corresponding twodimensional model is discussed by Yu. I. Kadashevitch and V. V. Novozhilov [2.80].

Hardening without the Bauschinger effect (true, isotropic hardening) is more difficult to be modelled. The model proposed by W. Derski and S. Ziemba [2.25] correctly describes loading and passive unloading but leads to softening during active reverse loading; contrary to the requirements.

We propose here two other models of isotropic hardening. The first one, shown in Fig. 30, relates hardening to the change of plastic strains (strain-hardening). The load $Q$, moving with friction, consists here of a fluid-container connected with the piston of the pump supplying the fluid irrespective of the sense of the motion. Accordingly any change of the plastic strain results in the increase of $Q$, and thus in strain hardening.

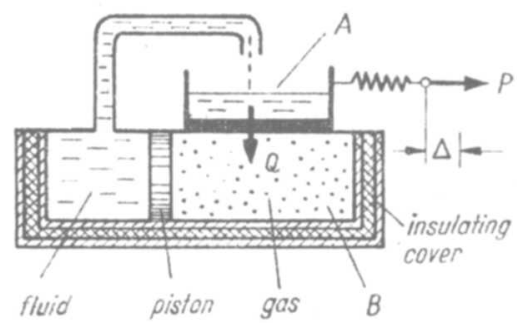

Fig. 31. A model of linear isotropic work-hardening

The second model, shown in Fig. 31, relates hardening to the dissipated work (work-hardening). As a result of friction the temperature of the gas under the load increases, causing an increase of pressure, motion of the piston to the left, and delivery of the fluid to the container (independently of the sense of the motion of that container).

There is no difficulty of course in generalizing these models so as to include the partial Bauschinger effect-it is sufficient to add the second spring as in Fig. 29.

Plastic hardening may also be modelled by certain simple structures made of a perfectly plastic material. Such a model, corresponding to the ideal Bauschinger effect, is considered in detail by K. A. Reckling [2.141]. A general discussion of models of that type is given by H. F. Bohnenblust and P. Duwez [2.9].

\subsection{Plastic strain. Plastic work}

Two characteristic features of plastic strains were mentioned in Sec. 1.1. However, to define plastic strain more precisely, some further discussion may be necessary. Consider the simplest case of uniaxial tension, assume the elastic strains to be governed by Hooke's law and neglect viscous effects; usually it is then assumed that the total strain $\varepsilon$ may be presented as the sum of the elastic strain $\varepsilon^{\boldsymbol{e}}$, vanishing after 
unloading, and the permanent plastic strain $\varepsilon^{p}$. This decomposition is presented by the formula

$$
\varepsilon=\varepsilon^{e}+\varepsilon^{p}, \quad \varepsilon^{p}=\varepsilon-\varepsilon^{e}=\varepsilon-\frac{\sigma}{E},
$$

where $E$ denotes Young's modulus for the undeformed body.

It should be noted, however, that formula (8.25) does not always correspond to the verbal description. The first discrepancy may be connected with the difference between the unloading modulus and Young's modulus $E$. This problem was experimentally investigated by A. M. Zhukov [2.195, 2.196, 2.197], who found a decrease of the unloading modulus with the increasing plastic strains. Particularly large differences (about 20 per cent) were observed when large rotation of principal axes took place, whereas for constant principal directions the differences amounted to less than 10 per cent. However, O. A. Shishmarev and E. Ya. Kuzmin [2.159] found later even smaller differences, and similar results were obtained by J. Litoński and J. Klepaczko for brass and steel [2.103]. Non-linear unloading was considered by O. M. Kochin [2.86].

The second discrepancy may be connected with the strong Bauschinger effect. Figure 32 shows the diagram $\sigma-\varepsilon$ corresponding to the model of the ideal Bau-

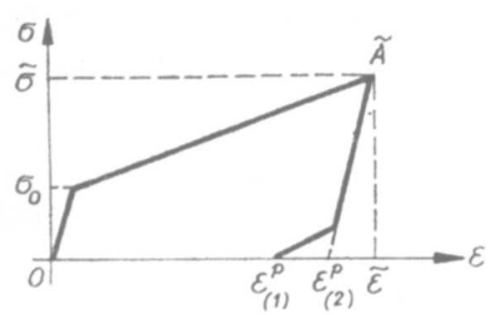

Fig. 32. The two possible definitions of the plastic strain

schinger effect described in Sec. 8.7. The real permanent strain, corresponding to the given strain $\varepsilon=\tilde{\varepsilon}$, is determined by the coordinate $\varepsilon_{(1)}^{p}$, whereas formula (8.25) determines the coordinate $\varepsilon_{(2)}^{p}$. In this situation we must use formula (8.25) to define the plastic strain. If we used $\varepsilon_{(1)}^{p}$ as the definition, then the general idea of decomposition of $\varepsilon$ into the elastic and the plastic parts would fail. Fortunately, the above-mentioned differences are usually small and may be neglected.

More difficult problems arise in the case of finite deformations. An additive decomposition of type (8.25) is used rather rarely, and only in material coordinates (A. E. Green, P. M. Naghdi [2.41], L. I. Sedov [2.151]). In spatial coordinates it may be used with respect to the stretching tensor $d_{i j}$ (Th. Lehmann [2.96]). More often a multiplicative decomposition of displacement gradients with an intermediate non-compatible configuration is postulated (A. E. Green, P. M. Naghdi [2.41], E. H. Lee [2.94], R. J. Clifton [2.19], A. C. Pipkin, R. S. Rivlin [2.129], M. Kleiber [2.84]). 
We are now going to discuss the unit plastic work $W_{0}^{p}$ and the dissipated energy during plastic deformation of a unit element, $W_{0}^{d}$. Usually it is assumed that these quantities coincide; such an approach is justified in cases where the Bauschinger effect is absent (isotropic hardening), whereas the Bauschinger effect results in the appearance of additional stored energy $W_{0}^{0}$, connected with residual microstresses.

The unit plastic work $W_{o}^{p}$ is determined by the area under the stress-strain diagram minus the recoverable elastic energy, Fig. 33:

$$
W_{0}^{p}=\int_{0}^{\tilde{\varepsilon}} \sigma d \varepsilon^{p}=\int_{0}^{\tilde{\varepsilon}} \sigma\left(d \varepsilon-\frac{d \sigma}{E}\right)=\int_{0}^{\tilde{\varepsilon}} \sigma d \varepsilon-\frac{\tilde{\sigma}^{2}}{2 E} .
$$

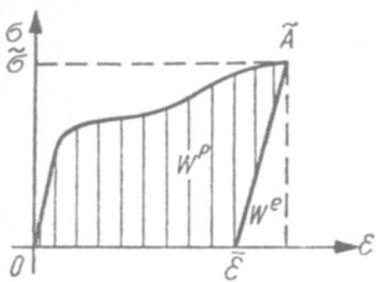

Fig. 33. The plastic work and the recoverable elastic energy

In cases where the Bauschinger effect is absent we may assume $W_{0}^{d}=W_{0}^{p}$. Consider, however, the model of the ideal Bauschinger effect shown in Fig. 29; in this case the dissipated energy is smaller, namely for the model it is $d W_{0}^{d}=\mu Q d \Delta$, and thus for the modelled body

$$
W_{0}^{d}=\int_{0}^{\tilde{\varepsilon}} \sigma_{0} d \varepsilon^{p}=\sigma_{0} \tilde{\varepsilon}-\frac{\sigma_{0} \tilde{\sigma}}{E} .
$$

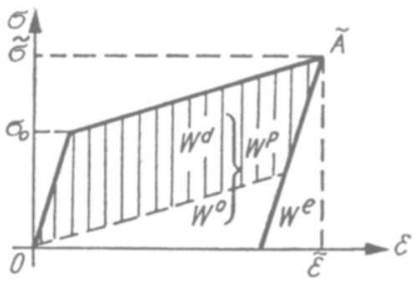

Fig. 34. The plastic work in the case of the ideal Bauschinger effect

The corresponding area, shaded in Fig. 34, is clearly smaller than that determined by (8.26). It should be noted, however, that the Bauschinger effect slightly decreases in time (the "ageing" of the material), and so does the stored energy $W_{0}^{0}$. This energy is discused by T. H. Lin and M. Ito [2.101].

In the general case, when the Bauschinger effect appears but is smaller than the ideal one, the dissipated energy might be determined by the formula

$$
W_{0}^{d}=\int_{0}^{\tilde{\varepsilon}} \sigma^{p} d \varepsilon^{p},
$$


where $\sigma^{p}$ (from the interval $\sigma_{0} \leqslant \sigma^{p} \leqslant \sigma$ ) defines that part of the stress which really works on plastic strains, depending on the Bauschinger effect. A formula of such a type, based on thermodynamic considerations, is derived by $\mathbf{Z}$. Mróz [2.114].

A simple estimation of $\sigma^{p}$, if the Bauschinger coefficients $\bar{\beta}$ or $\beta$ (8.16), (8.17) are known, may be obtained as follows. In both boundary cases the following formula holds:

$$
\sigma^{p}=\frac{\tilde{\sigma}+\left|\tilde{\sigma}_{-}\right|}{2}=\frac{\tilde{\sigma}-\tilde{\sigma}_{-}}{2},
$$

where the tildas denote the stresses as in Sec. 8.5. Assuming this formula to hold in intermediate cases as well, we may eliminate $\tilde{\sigma}_{-}$by $\bar{\beta}$ or $\beta$ :

$$
\tilde{\sigma}_{-}=-(1-\bar{\beta}) \tilde{\sigma}=2 \beta\left(\tilde{\sigma}-\sigma_{0}\right)-\tilde{\sigma} .
$$

Substituting this relation into (8.29) and dropping the now unnecessary tildas, we obtain

$$
\sigma^{p}=\left(1-\frac{1}{2} \bar{\beta}\right) \sigma=\sigma-\beta\left(\sigma-\sigma_{0}\right) .
$$

In the boundary cases we obtain $\sigma^{p}=\sigma$ or $\sigma^{p}=\sigma_{0}$, reducing (8.28) to (8.26) or (8.27), respectively.

In the case of a perfectly elastic-plastic body $\sigma=\sigma_{0}$, the Bauschinger effect does not appear, and the results of all the given formulae coincide.

\section{Classical theories of perfect plasticity}

\subsection{Elastic range, Hooke's law}

In the elastic range, the limits of which will be discussed in detail in Sec. 9.2 and in Chapter III, stresses and strains are interrelated by Hooke's law. The general form of this law for an anisotropic body

$$
\sigma_{l j}=E_{i j k l} \varepsilon_{k l},
$$

where $E_{i j k l}$ denote the components of the fourth-order tensor of elastic moduli and the summation convention is used, is greatly simplified in the case of isotropy. The isotropic fourth-order tensor has only two independent components and may be written thus:

$$
E_{l j k l}=\left[\frac{2 v}{1-2 v} \delta_{l j} \delta_{k l}+\left(\delta_{i k} \delta_{j l}+\delta_{i l} \delta_{j k}\right)\right] G,
$$

where

$$
G=\frac{E}{2(1+v)}
$$


denotes Kirchhoff's modulus, and $v$ Poisson's ratio. Substitution of (9.2) into (9.1) and some simple transformations yield

$$
\sigma_{i j}=2 G\left(\varepsilon_{i j}+\frac{v}{1-2 v} \delta_{i j} \varepsilon_{k k}\right) .
$$

Contraction of tensors leads to the equation

$$
\sigma_{i i}=2 G\left(\varepsilon_{i i}+\frac{3 v}{1-2 v} \varepsilon_{k k}\right)=\frac{E}{1-2 v} \varepsilon_{i i} .
$$

This equation joins the only independent coordinate of the axiator $A_{\sigma}$ with the corresponding coordinate of $A_{\varepsilon}$, and so we may write

$$
A_{\sigma}=\frac{E}{1-2 v} A_{\varepsilon}=3 K A_{\varepsilon},
$$

where $K=E / 3(1-2 v)$ is the bulk modulus. Since $A_{\varepsilon}$ determines the dilatation, equations (9.5) and (9.6) are called the law of dilatation or the law of volume change. In engineering notation it takes the form

$$
\varepsilon_{x}+\varepsilon_{y}+\varepsilon_{z}=\frac{1-2 v}{E}\left(\sigma_{x}+\sigma_{y}+\sigma_{z}\right)
$$

Calculating $\varepsilon_{k k}$ from (9.5) and substituting into (9.4), we solve this system with respect to $\varepsilon_{i j}$

$$
\varepsilon_{i j}=\frac{1}{2 G} \sigma_{i j}-\frac{v}{E} \delta_{i j} \sigma_{k k}
$$

or, in classical engineering notation,

$$
\varepsilon_{x}=\frac{1}{E}\left[\sigma_{x}-v\left(\sigma_{y}+\sigma_{z}\right)\right], \quad \gamma_{x y}=\frac{1}{G} \tau_{x y} . \quad \overbrace{y}^{x}
$$

Subtracting $\varepsilon_{m}=\varepsilon_{k k} / 3$ from both sides of (9.8) and making use of (9.5), we obtain

$$
\varepsilon_{i j}-\delta_{i j} \varepsilon_{m}=\frac{1}{2 G}\left(\sigma_{i j}-\delta_{i j} \sigma_{m}\right)
$$

or

$$
e_{i j}=\frac{1}{2 G} s_{i j}, \quad D_{\varepsilon}=\frac{1}{2 G} D_{\sigma}
$$

Equations (9.10) or (9.11) are called the law of distortion or the law of shape change (in the elastic range). In engineering notation the equations

$$
\varepsilon_{x}-\varepsilon_{m}=\frac{1}{2 G}\left(\sigma_{x}-\sigma_{m}\right)
$$

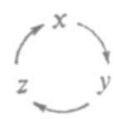


are often used in a different form, which does not contain $\varepsilon_{m}$ and $\sigma_{m}$, namely

$$
\varepsilon_{x}-\varepsilon_{y}=\frac{1}{2 G}\left(\sigma_{x}-\sigma_{y}\right), \quad z_{y}^{x}
$$

obtained from (9.12) by successive subtraction. Among the three relations (9.12) or (9.13) only two are independent; among the six relations (9.10) or (9.11)-only five.

\subsection{Limitation of the elastic range}

In the case of uniaxial tension the elastic range is limited by the proportional limit or elastic limit $\sigma_{p}$; according to most schematizations this limit coincides with the yield stress $\sigma_{0}$. In the case of multiaxial stress or of the influence of auxiliary exertion factors (temperature, humidity, etc.) the elastic range may be presented as a domain inside a certain limit surface (interaction surface), called in Chapter I the neutral surface, or, more precisely, the initial neutral surface. The initial neutral surface does not depend on the loading trajectory, since the elastic processes are reversible; subsequent neutral surfaces may depend on the trajectory.

The determination of the initial neutral surface may be regarded as a problem of the physics of solids or-in the phenomenological approach-of the hypotheses of failure. Our considerations will be restricted only to the phenomenological approach, simpler and more adequate for engineering applications. Two typical variants may be distinguished here. The first variant consists in a hypothetical indication of a physical (invariant) quantity which is responsible for initial yielding and in subsequent experimental verification. Such a quantity was called in [1.23] the criterion of exertion. Conversely, the second variant starts with a series of experiments, followed by an appropriate, purely mathematical approximation, satisfying the requirements of invariance.

The first variant is represented, for example, by the hypothesis of the unit distortion energy, formulated by M. T. Huber [2.66], B. T. Haigh [2.50] and H. Hencky [2.53] and verified experimentally for a wide class of materials. A similar idea was suggested as early as 1856 by J. C. Maxwell in a letter to W. Thomson: an extensive fragment of this letter is quoted by F. K. Van Iterson [2.183]. According to this hypothesis, the elastic range is bounded by a certain constant value of the unit distortion energy $\Phi_{f}$ :

$$
\Phi_{f}=\frac{1}{2} s_{l j} e_{l j}=\text { const }=\frac{1}{2} C,
$$

where the constant $C$ may be determined, for example, in a simple tension test. This hypothesis is thus a one-parameter hypothesis, since one test is sufficient for its effective formulation.

Equation (9.14) does not determine the required neutral surface, since it interrelates twelve quantities (stresses and strains), only six of which may be independent. 
The dependent quantities may be eliminated by using Hooke's law, which is valid up to the initial neutral surface; thus, in view of (9.11), we express this surface either in terms of stresses

$$
s_{i j} s_{i j}=2 G C=\frac{2}{3} \sigma_{0}^{2},
$$

or in terms of strains

$$
e_{i j} e_{i j}=\frac{C}{2 G}=\frac{\sigma_{0}^{2}}{6 G^{2}},
$$

where the constant $C$ is chosen in such a way as to obtain $\sigma=\sigma_{0}$ as the corresponding limit in uniaxial tension. The dependence of the initial neutral surface on auxiliary exertion factors may be expressed here only by means of the dependence of $\sigma_{0}$ on those factors.

The introduction of the concept of "reduced stress" proves useful here. By this term we understand that stress in uniaxial tension $\sigma_{\text {red }}$ which, from the point of view of exertion in proportional loading, is equivalent to the given state of stress. Denote the criterion of exertion by $F$ and express it by means of stresses only; the required equivalence yields

$$
F\left(\sigma_{x}, \sigma_{y}, \ldots, \tau_{z x}\right)=F\left(\sigma_{\text {red }}, 0, \ldots, 0\right),
$$

and hence

$$
\sigma_{\text {red }}=f\left(\sigma_{x}, \sigma_{y}, \ldots, \tau_{z x}\right) .
$$

(In some cases it is effectively possible to express $\sigma_{\text {red }}$ only in terms of principal stresses and not in terms of stress components.) The initial neutral surface is then described by the equation $\sigma_{\text {red }}=\sigma_{0}$, and the elastic range by $\sigma_{\text {red }}<\sigma_{0}$. For the Huber-Haigh-Hencky hypothesis, making use of (9.15), we easily find $\sigma_{\text {red }}=\sigma_{e}$, where $\sigma_{e}$ is the stress intensity (effective stress) defined in Sec. 6.2. So the stress intensity may be considered as a particular case of reduced stress.

The second variant of phenomenological approach, purely approximative, is represented, for example, by the Mises proposal [2.112], which will be discussed in the next section.

\subsection{Conditions of perfect plasticity (yield conditions)}

In the uniaxial tension a perfectly plastic body is characterized by increasing strain at constant stress $\sigma=\sigma_{0}$ during the active part of the process. Such a process is called a perfectly plastic flow. In the general three-axial case the stress components may change but a certain stress invariant must remain constant: the point representing the state of stress remains at the initial neutral surface, which coincides here with the yield surface. For an anisotropic body the stress invariant is replaced by a certain common invariant of the stress tensor and the tensor describing the plastic properties of the material. 
The choice of such an invariant may also be regarded as a problem of the hypotheses of failure. If the criterion of exertion depends on stresses only, then its constancy determines simultaneously the limitation of the elastic range and the yield condition. As an example of such a criterion we may consider the length of the vector of maximal shearing stress $\tau_{\max }$; the equation

$$
\tau_{\max }=\frac{1}{2}\left(\sigma_{1}-\sigma_{\mathrm{III}}\right)=\text { const }=\frac{1}{2} \sigma_{0},
$$

corresponding to the maximal shearing stress hypothesis, was proposed by J. J. Guest [2.46] as the limitation of the elastic range, and even earlier by $\mathbf{H}$. Tresca [2.175] as the condition of perfect plasticity.

A quite different situation arises if the criterion of exertion depends on strains. Consider, for example, the unit distortion energy $\Phi_{f}$; the equation $\Phi_{f}=$ const may describe the limit of the elastic range (which is the subject of Huber's hypothesis) but cannot be regarded as the yield condition, since $\Phi_{f}$ obviously depends on strains. The corresponding yield condition may be obtained by eliminating strains with the use of Hooke's law and then by equating the expression obtained to a constant. Such a procedure performed by $\mathrm{H}$. Hencky resulted in (9.15). The physical sense of the criterion of exertion is then lost, since during the plastic deformation $\Phi_{f}$ is no longer constant. However, to Eq. (9.15) treated as a yield condition another physical interpretation may be ascribed: a constant octahedral shearing stress (M. Roš, A. Eichinger [2.144]), a constant mean square value of shearing stresses in all possible cross-sections passing through the given point (V. V. Novozhilov [2.121]; similar concepts were also published by G. Sachs [2.147] and U. Dehlinger [2.24]), etc.

The second, purely approximative variant of the hypothesis of failure is also used for the formulation of yield conditions. R. Mises in the mentioned paper [2.112] proposed to approximate the yield surface by a sphere in the space of extremal shearing stresses. H. Hencky [2.53] stated that such an approach is equivalent to the hypothesis of distortion energy. The corresponding yield condition will be called here the Huber-Mises-Hencky (HMH) yield condition.

In the case of an approximative approach the derivation of the corresponding formulae for reduced stress may be more complicated than (9.17) and (9.18), since the homogeneity of the resulting expression must be ensured. If the general form of the yield condition is

$$
F\left(\sigma_{i j}, a_{k}\right)=0, \quad k=1,2, \ldots, n,
$$

where $a_{k}$ are the $n$ parameters to be determined, then, at first, we have to express these parameters in terms of yield stresses in various comparative states (uniaxial tension, uniaxial compression, biaxial tension, etc.); subsequently, we express them in terms of $\sigma_{0}$ at uniaxial tension and dimensionless ratios of the yield stresses in the assumed comparative states to $\sigma_{0}$, denoted by $\varkappa_{m}, m=1,2, \ldots, n-1$. Now, if we solve the equation

$$
\bar{F}\left(\sigma_{i j}, \sigma_{0}, x_{m}\right)=0, \quad m=1,2, \ldots, n-1,
$$


with respect to $\sigma_{0}$, namely

$$
\sigma_{0}=f\left(\sigma_{i j}, \varkappa_{m}\right), \quad m=1,2, \ldots, n-1,
$$

then the right-hand side determines the reduced stress $\sigma_{\text {red }}$. Indeed, this expression satisfies the required condition of homogeneity, and the equation $\sigma_{\text {red }}=\sigma_{0}$ represents the corresponding yield condition.

Yield conditions will be discussed in detail in Chapter III. Here we merely draw attention to the fact that in the case of perfect plasticity the condition $\sigma_{\text {red }}<\sigma_{0}$ ensures a passive process, whereas processes satisfying $\sigma_{\text {red }}=\sigma_{0}$ may be either active or passive. Passive processes satisfying the yield condition will be called neutral; in the case of a perfectly elastic-plastic body they cannot be distinguished in the stress space, but are seen in the strain space as boundary processes. Neutral processes should be equivalently described by the equations of active or passive processes; this postulate of continuity of description of neutral processes is not satisfied by all theories of plasticity, and-in the negative case-sharply restricts the applicability of a particular theory.

\subsection{The law of volume change. Coefficient of transversal deformation}

The constitutive (physical) equations, at least for isotropic bodies, are usually presented separately for the volume change and separately for the shape change. Such a presentation, which looks somewhat sophisticated in the theory of elasticity, is quite reasonable in the theory of plasticity, since the volume change and the shape change are governed here by entirely different laws.

Numerous experiments carried out by P. W. Bridgman [2.10] proved that the volume change even in the plastic range is purely elastic and subject to the linear law (9.5), (9.6) or (9.7). It is only in the range of very high hydrostatic pressures $p$ that Bridgman observed deviations from the linear law (9.7) and proposed a quadratic relation; however, in most applications (9.7) is considered as sufficiently exact, or even replacable by the condition of incompressibility,

$$
\varepsilon_{x}+\varepsilon_{y}+\varepsilon_{z}=0 \text {. }
$$

For most materials Eq. (9.23) may be considered only as an approximate one; the approximation errors are often negligible, since in the range of larger plastic deformations the volumetric strains are small as compared to deviatoric strains.

The exact equation of volume change (9.7) makes it possible to determine transversal deformations appearing in uniaxial tension. In the elastic range the ratio of transversal deformations to longitudinal one is constant, namely it is determined by Poisson's ratio:

$$
\frac{\varepsilon_{2}}{\varepsilon_{1}}=\frac{\varepsilon_{3}}{\varepsilon_{1}}=-v \text {. }
$$

In the plastic range this ratio, in general, is no longer constant. Denote by $v^{p}$ the coefficient of transversal deformation, defined by the equations $\varepsilon_{2}=\varepsilon_{3}=-v^{p} \varepsilon_{1}$, 
which are to be valid in uniaxial tension within the whole range of strains (small strains). Substituting these relations into the law of volume change (9.7), we obtain

$$
\left(1-2 v^{p}\right) \varepsilon=\frac{1-2 v}{E} \sigma,
$$

where the indices " 1 " at $\sigma$ and $\varepsilon$ have been dropped. Hence

$$
v^{p}=\frac{1}{2}-\left(\frac{1}{2}-v\right) \frac{\sigma(\varepsilon)}{E \varepsilon},
$$

with the explicit indication that $\sigma$ depends on $\varepsilon$. In the range of Hooke's law $\sigma(\varepsilon)$ $=E \varepsilon$ we obviously obtain $v^{p}=v=$ const, whereas in the general case $v^{p}=v^{p}(\varepsilon)$. Thus for a perfectly plactic body the dependence of $v^{p}$ on $\varepsilon$ is hyperbolic; namely

$$
v^{p}=\frac{1}{2}-\left(\frac{1}{2}-v\right) \frac{\sigma_{0}}{E \varepsilon},
$$

and this function tends asymptotically to $1 / 2$. Only in the case of an incompressible body we have exactly $v^{p}=1 / 2=$ const within the whole range of strains, and this fact results in essential simplifications of the solutions. Moreover, the asymptotic value of (9.27), $v^{p} \rightarrow 1 / 2$, justifies the approximate use of the law of incompressibility (9.23) for compressible materials as well (practically in the range of not too small strains).

The problem of the coefficient of transversal deformation is considered in the papers by G. Gerard and S. Wildhorn [2.36], R. A. Mezhlumyan [2.111], I. Vigness and T. E. Pardue [2.184]. A. K. Sinitsky [2.161] proposed an approximation consisting in the replacement of the law of volume change (9.7) by the hypothesis of a constant coefficient of transversal deformation, $v^{p}=v$; this approach essentially simplifies the calculations; however, the errors may be significant, they were investigated by M. Życzkowski [2.199], Yu. R. Lepik and L. Ya. Luht [2.97]. The influence of compressibility of the material on the solutions of problems in plasticity was considered by V. I. Rozenblyum [2.145] and M. Como [2.20].

There exist certain materials for which the volume change may be elastic-plastic; for the artificial graphite this fact was stated by W. L. Greenstreet and A. Phillips [2.44]. Neglecting these exceptions, however, we may regard the law of volume change (9.7)-or, approximately, (9.23) - as a generally valid law, accepted by most theories of plasticity. The differences between the various proposals appear only in the formulation of the law of shape change: we are now going to quote three classical proposals for this basic constitutive law in the theory of plasticity.

\subsection{The Hencky-Ilyushin theory of small elastic-plastic deformations}

We already mentioned in Sec. 1 that for many engineering applications physical nonlinearity is more important than irreversibility, and dependence on the trajectory. So the simplest theory of plasticity, proposed by H. Hencky in 1924 [2.53] (though applied by A. Nadai [2.117] to the particular problem of torsion one year earlier) 
and developed by A. A. Ilyushin [2.67, 1.7, 2.69], refers to nonlinear elasticity. This theory describes namely active processes by the equations of nonlinear elasticity, but, in contradistinction to non-linear elasticity, it distinguishes also passive processes, described by linear equations of type (8.14). Thus, according to the deformation theory, the final state is independent on the trajectory if the initial passive (elastic) process is followed by an active one; a certain limited dependence on the trajectory (namely on the coordinates of the point $\tilde{A}$ ) appears only in the case of an active process followed by a passive one.

Since in the theory of plasticity a new equation appears, namely the yield condition, the equations of shape change must contain a new unknown so as to equalize the number of equations and unknowns. In the Hencky-Ilyushin theory of small elastic-plastic deformations this unknown is the proportionality coefficient between the stress and strain deviators, denoted by $\varphi$ :

$$
D_{e}=\varphi D_{\sigma}, \quad e_{i j}=\varphi s_{i j} .
$$

Making use of the law of volume change (9.6), we may express strains in terms of stresses as follows:

$$
\varepsilon_{i j}=\varphi \sigma_{i j}+\left(\frac{1}{3 K}-\varphi\right) \sigma_{m} \delta_{i j} .
$$

This notation is valid for compressible materials, or, with the assumption of $1 / 3 K$ $=0$, for incompressible ones.

Substituting $\varphi=1 / 2 G$ and neglecting the yield condition, we may describe by (9.28) or (9.29) the elastic range as well. In the plastic range the function $\varphi$ is to be determined from the yield condition. One way to do it is to multiply each side of (9.28) by itself with full contraction (scalarly)

$$
e_{i j} e_{i j}=\varphi^{2} s_{i j} s_{i j} \text {, }
$$

and hence, in view of the $\mathrm{HMH}$ yield condition and of the definition of the strain intensity (7.29), we obtain

$$
\varphi=\frac{3 \varepsilon_{e}}{2 \sigma_{0}} .
$$

So the function $\varphi$, called sometimes the function of the development of plastic strains, is proportional to the strain intensity. Another way to evaluate $\varphi$ is to multiply (9.28) scalarly by $s_{i j}$; then

$$
\varphi=\frac{3 s_{i j} e_{i j}}{2 \sigma_{0}^{2}} .
$$

In particular cases other methods of the elimination of $\varphi$ may be more useful, for example the division of the successive equations (9.28) side by side. The latter procedure is also convenient in the case of other yield conditions.

It is seen from (9.28) that the Hencky-Ilyushin theory assumes the identity of the tensors of directions and a similarity of stresses and strains, (5.20), namely 
$e_{i j}^{*}=s_{i j}^{*}$, and also the equality of the Lode parameters $\mu_{\varepsilon}=\mu_{\sigma}$. In other words, (9.28) expresses the law of similarity of deviators.

Equations (9.28) form a system of six algebraic equations, only five of them being independent. We present them in form (9.12), or, more often, (9.13):

$$
\left.\varepsilon_{x}-\varepsilon_{y}=\varphi\left(\sigma_{x}-\sigma_{y}\right), \quad \gamma_{x y}=2 \varphi \tau_{x y}, \quad \digamma_{z}^{x}\right]_{y}
$$

If only part of the body is plastic, then the elastic-plastic interface (boundary) is determined by the condition $\varphi=1 / 2 G$ (making use of the solution for the plastic zone), or by $\sigma_{e}=\sigma_{0}$ (approaching from the elastic zone). Along this interface all stress and strain components are continuous (L. M. Kachanov [2.79]).

The above equations describe active processes. Passive processes, according to the Prandtl schematization, are described by equations of type (8.14). In tensorial notation they take the general form

$$
\tilde{D}_{\sigma}-D_{a}=2 G\left(\tilde{D}_{\varepsilon}-D_{\varepsilon}\right),
$$

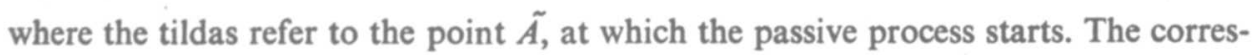
ponding algebraic equations in engineering notation are presented either in a form analogical to (9.33), namely as

$$
\begin{array}{ll}
\tilde{\sigma}_{x}-\tilde{\sigma}_{y}-\sigma_{x}+\sigma_{y}=2 G\left(\tilde{\varepsilon}_{x}-\tilde{\varepsilon}_{y}-\varepsilon_{x}+\varepsilon_{y}\right), & \left.\Gamma^{x}\right) \\
\tilde{\tau}_{x y}-\tau_{x y}=G\left(\tilde{\gamma}_{x y}-\gamma_{x y}\right), &
\end{array}
$$

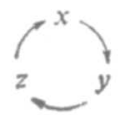

or, with the use of the law of volume change (9.6), in a form corresponding to classical Hooke's law:

$$
\tilde{\varepsilon}_{x}-\varepsilon_{x}=\frac{1}{E}\left[\left(\tilde{\sigma}_{x}-\sigma_{x}\right)-v\left(\tilde{\sigma}_{y}-\sigma_{y}+\tilde{\sigma}_{z}-\sigma_{z}\right)\right] .
$$

The range of applicability of the Hencky-Ilyushin deformation theory is restricted both from the logical point of view and from the point of view of experimental verification; it will be discussed in Sec. 9.8. Nevertheless, this theory may be useful in certain particular cases, where the application of more exact theories is very complicated.

We are now going to establish a criterion separating active and passive processes. Regarding (9.31), one may suppose that an increase of strain intensity results in an active process, and a decrease-in a passive one. However, such a statement, which may be found in many textbooks, is, in general, false, as pointed out by V. V. Novozhilov [2.122].

A correct determination of the neutral surface in the strain space-separating active and passive processes-may be obtained as follows. Consider passive processes, described by (9.34); the final state of such processes is independent of the 
trajectory. The boundary of passive processes is determined by the yield condition satisfied by stresses. Equations (9.34) give

$$
s_{i j}=\tilde{s}_{i j}-2 G\left(\tilde{e}_{i j}-e_{i j}\right),
$$

and the substitution of (9.37) into the HMH yield condition (9.15) leads to

$$
\left[\tilde{s}_{i j}-2 G\left(\tilde{e}_{i j}-e_{i j}\right)\right]\left[\left(\tilde{s}_{i j}-2 G\left(\tilde{e}_{i j}-e_{i j}\right)\right]=\frac{2}{3} \sigma_{0}^{2} .\right.
$$

The stresses $\tilde{s}_{i j}$ obviously fulfil the yield condition; making use of this fact and rearranging the terms, we obtain

$$
G e_{i j} e_{i j}-\left(2 G \tilde{e}_{i j}-\tilde{s}_{i j}\right) e_{i j}+\left(G \tilde{e}_{i j} \tilde{e}_{i j}-\tilde{e}_{i j} \tilde{s}_{i j}\right)=0 \text {. }
$$

This is the equation of the required neutral surface, constructed for an arbitrary point $\tilde{A}$ characterized by $\tilde{s}_{i j}$ and $\tilde{e}_{i j}$. In general, it does not coincide with the condition of constant strain intensity; the differences will be illustrated by a numerical example.

The case of combined loading at the level $\mathscr{P}$-simplest for discussion (although not for experimental realization)-may be obtained if we consider only two nonvanishing stress components $\tau_{z x}$ and $\tau_{z y}$. In this case only two strain components appear, $\gamma_{z x}$ and $\gamma_{z y}$, and we may present the process at the stress plane and at the strain plane (instead of spaces of more dimensions). The HMH yield condition (and any other condition for an isotropic body) is here represented by the circle

$$
\tau_{z x}^{2}+\tau_{z y}^{2}=\tau_{0}^{2},
$$

where $\tau_{0}$ denotes yield stress at pure shear (or torsion). In the case of plane stress the yield condition assumes a more complicated and less general form, and so the case $\tau_{2 x}-\tau_{z y}$ is more convenient for discussion; it was used, for example, by G. I. Bykovtsev, V. V. Dudukalenko and D. D. Ivlev [2.14].

The equation of the neutral surface (9.38) (on substituting $\sigma_{0}^{2}=3 \tau_{0}^{2}$ ) takes here the form

$$
\left[\tilde{\tau}_{z x}-G\left(\tilde{\gamma}_{z x}-\gamma_{z x}\right)\right]^{2}+\left[\tilde{\tau}_{z y}-G\left(\tilde{\gamma}_{z y}-\gamma_{z y}\right)\right]^{2}=\tau_{0}^{2}
$$

and determines in the strain plane a circle with the radius $\tau_{0} / G$, centred at $\tilde{\gamma}_{z x}-\tilde{\tau}_{x x} / G, \tilde{\gamma}_{x y}-\tilde{\tau}_{z y} / G$. The curve of constant strain intensity is also represented by a circle, namely by

$$
\gamma_{z x}^{2}+\gamma_{z y}^{2}=\tilde{\gamma}_{z x}^{2}+\tilde{\gamma}_{z y}^{2}=\text { const, }
$$

but with a larger radius $r=\sqrt{\tilde{\gamma}_{z x}^{2}+\tilde{\gamma}_{z y}^{2}}$, and centrally located. For example, in Fig. 35 it is assumed that $\tilde{\gamma}_{z x}=4 \tau_{0} / G, \tilde{\gamma}_{z y}=0, \tilde{\tau}_{z x}=\tau_{0}, \tilde{\tau}_{z y}=0$, and both circles are shown; the differences are significant.

Consider now the processes starting from the point $\tilde{A}$ and defined in the strain plane by circular trajectories with the radius $\alpha \tau_{0} / G$ :

$$
\gamma_{z x}=\frac{\tau_{0}}{G}[(4-\alpha)+\alpha \cos t], \quad \gamma_{z y}=\frac{\tau_{0}}{G} \alpha \sin t,
$$

where $\alpha$ is constant, and $t$ is the time-like parameter. If $\alpha$ is taken from the interval $1<\alpha<4$, we are dealing with active processes with a decreasing strain intensity. As a matter of fact, the strain intensity $\varepsilon_{e},(7.29)$, equals here

$$
\varepsilon_{e}=\frac{1}{\sqrt{3}} \sqrt{\gamma_{z x}^{2}+\gamma_{z y}^{2}}=\frac{\tau_{0}}{G \sqrt{3}} \sqrt{\left(16-8 \alpha+2 \alpha^{2}\right)+2 \alpha(4-\alpha) \cos t}
$$

and for $1<\alpha<4$ the derivative $d \varepsilon_{e} / d t$ is negative (inside the interval $0<t<\pi$ ). However, if we assumed the process to be passive and substituted, according to (9.37),

$$
\tau_{z x}=\tau_{0}(1-\alpha+\alpha \cos t), \quad \tau_{z y}=\tau_{0} \alpha \sin t,
$$




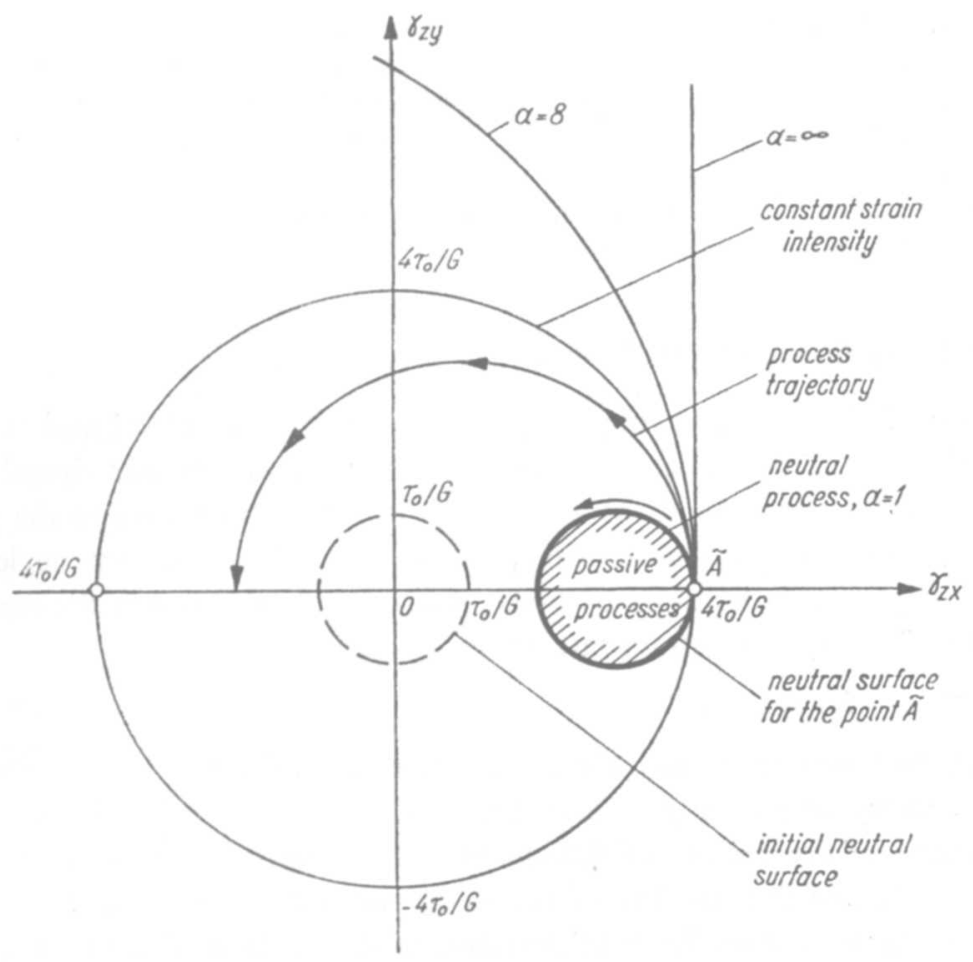

Fig. 35. An example of analysis of the deformation process at a point. A false description of the neutral process by the Hencky-Ilyushin theory

we would obtain

$$
\tau_{z x}^{2}+\tau_{x y}^{2}=\tau_{0}^{2}[1+2 \alpha(\alpha-1)(1-\cos t)]>\tau_{0}^{2},
$$

which is impossible for a perfectly plastic body. Thus the process is active in spite of a decreasing strain intensity.

Making use of the equations of the active process (9.33), we calculate successively

$$
\begin{aligned}
\varphi & =\frac{1}{G} \sqrt{16-8 \alpha+2 \alpha^{2}+2 \alpha(4-\alpha) \cos t,} \\
\tau_{z x} & =\frac{(4-\alpha)+\alpha \cos t}{\sqrt{16-8 \alpha+2 \alpha^{2}+2 \alpha(4-\alpha) \cos t}} \tau_{0}, \\
\tau_{z y} & =\frac{\alpha \sin t}{\sqrt{16-8 \alpha+2 \alpha^{2}+2 \alpha(4-\alpha) \cos t}} \tau_{0} .
\end{aligned}
$$

These results are correct in the sense of the Hencky-Ilyushin theory, but it would be difficult to admit that they adequately describe the processes which really take place. Consider, for example, the limiting case $\alpha=1$ : we approach here the neutral curve from the side of active processes. Equations $9.43)$ an $d(9.49)$ yield

$$
\tau_{z x}=\frac{3+\cos t}{\sqrt{10+6 \cos t}} \tau_{0}, \quad \tau_{z y}=\frac{\sin t}{\sqrt{10+6 \cos t}} \tau_{0}
$$


and for $t=\pi$, or $\gamma_{x x}=2 \tau_{0} / G, \gamma_{x y}=0$, we obtain $\tau_{z x}=+\tau_{0}, \tau_{z y}=0$. On the other hand, the equations of passive processes (9.46), which should also be valid along the boundary curve, give here $\tau_{z x}=-\tau_{0}, \tau_{z y}=0$. Thus, at the boundary, we observe the discontinuity of description of the neutral process, which should not take place and is due to the defects of the Hencky-Ilyushin deformation theory.

Results (9.48) and (9.49) will be compared in Sec. 9.9 with the results of other theories, which describe the process in question more adequately.

\subsection{The Levy-Mises theory of plastic flow}

The oldest theory of plasticity was proposed in 1870 by M. Levy [2.98] and B. de Saint-Venant (the part of the paper containing the basic equations was signed by M. Levy only) and developed by R. Mises [2.112]. In contradistinction to the deformation theory, based on nonlinear elasticity, the Levy-Mises theory neglects elastic strains and describes a rigid-plastic body. According to this theory the deviators of stress and of infinitesimal strain rate are proportional,

$$
D_{\dot{\varepsilon}}=\lambda D_{\sigma}, \quad \dot{e}_{i j}=\lambda s_{i j},
$$

and it resembles, to a certain degree, the constitutive equation of a viscous fluid; hence the terms theory of plastic flow and, for Eqs. (9.51), flow rule. This theory assumes the identity of the tensors of directions and similarity of stresses and of strain rates, $\dot{e}_{i j}^{*}=s_{i j}^{*}$, and the equality of the Lode parameters $\mu_{\dot{i}}=\mu_{\sigma}$. In other words, Eqs. (9.51) express the similarity of deviators of stresses and of strain rates.

The unknown function $\lambda$ should be determined from the yield condition. M. Levy used here the Tresca yield condition (9.19), but R. Mises [2.112] remarked that the use of (9.15) is more convenient. Proceeding as in the case of the Hencky-Ilyushin theory, we arrive either at.

$$
\lambda=\frac{3 \dot{\varepsilon}_{e}}{2 \sigma_{0}}
$$

or at

$$
\lambda=\frac{3 s_{i j} \dot{e}_{i j}}{2 \sigma_{0}^{2}} .
$$

The symbol $\dot{\varepsilon}_{e}$ denotes strain rate intensity (effective strain rate), defined analogically to (7.29). The expression $s_{i j} \dot{e}_{i j}$ in the numerator of (9.53) determines the (unit) power of plastic strains; substituting (9.53) into (9.51) and changing appropriately the notation of indices, we may write

$$
\dot{e}_{i j}=\frac{3 s_{k l} \dot{e}_{k l}}{2 \sigma_{0}^{2}} s_{i j}
$$

or, after a formal multiplication by the time increment $d t$, we obtain the following incremental form with the time factor eliminated:

$$
d e_{i j}=\frac{3 s_{k l} d e_{k l}}{2 \sigma_{0}^{2}} s_{i j} .
$$


The Levy-Mises theory neglects elastic strains, and so in the law of the volume change (9.7) we should consistently substitute $E \rightarrow \infty$ and obtain the incompressibility condition (9.23).

The neutral surface in the stress space is described by the yield condition, whereas in the strain space it reduces to a point: any motion in the strain space means an active process. A passive process corresponds here to the rigid behaviour of the material, and so instead of (9.34) we write simply

$$
\tilde{D}_{\varepsilon}-D_{\varepsilon}=0, \quad D_{\dot{\varepsilon}}=0 .
$$

It should be noted that, in spite of fundamental physical differences between the Hencky-Ilyushin and the Levy-Mises theories, a certain mathematical analogy may be established between them (A. A. Ilyushin [2.68]). Equations (9.51), (9.52), and (9.53) are formally similar to (9.28), (9.31), and (9.32), respectively. As a matter of fact, in some problems it is sufficient to denote by the same symbols either strains or strain rates so as to take both theories jointly into consideration. If these strains or strain rates constitute parameters subject to elimination (as in certain problems of limit carrying capacity), then both theories lead to the same result. However, if the processes are prescribed by strains changing non-proportionally in time, the differences are essential, as is shown in the following example.

Consider, as in Sec. 9.5, the process described by Eqs. (9.43) and Fig. 35. The constant $G$ will now be treated as a certain parameter without any physical meaning. Calculate first the strain rates

$$
\dot{\gamma}_{x x}=-\frac{\tau_{0}}{G} \alpha \sin t, \quad \dot{\gamma}_{z y}=\frac{\tau_{0}}{G} \alpha \cos t,
$$

thus the shearing stresses, which should be proportional to those strain rates and satisfy the yield condition, are determined by the formulae

$$
\tau_{z x}=-\tau_{0} \sin t, \quad \tau_{x y}=\tau_{0} \cos t .
$$

The differences with respect to (9.48) and (9.49) are essential; they will be analyzed in detail in Sec. 9.9. It should only be noted here that any corner on the trajectory in the strain space results in a jump on the corresponding trajectory in the stress space (this fact follows directly from (9.51)). For example, at the point $\tilde{A}$ in Fig. 35 reached along the line $\gamma_{x y}=0$ the stresses were $\tau_{x x}=\tau_{0}$, $\tau_{x y}=0$, but the process considered here starts from $A$ with $\tau_{x x}=0, \tau_{s y}=\tau_{0}$, and so a jump-like change of stresses takes place.

\subsection{The Prandtl-Reuss theory of plastic flow}

L. Prandtl [2.137] (in the particular case of plane stress) and A. Reuss [2.142] improved the accuracy of the Levy-Mises theory by adding elastic strain rates to plastic strain rates. Differentiating the elastic law of shape change (9.11) with respect to time $t$, we may write

$$
D_{\dot{\varepsilon}}^{e}=\frac{1}{2 G} D_{\dot{\sigma}},
$$


and adding this term to $D_{\dot{b}}^{p}$ determined by $(9.51)$ we obtain

$$
D_{\dot{\varepsilon}}=\lambda D_{\sigma}-\frac{1}{2 G} D_{\dot{\sigma}}, \quad \dot{e}_{i j}=\lambda s_{i j}+\frac{1}{2 G} \dot{s}_{i j} .
$$

The physical meaning of these equations does not essentially differ from (9.51), and so we retain here the term theory of plastic flow (the term theory of elastic-plastic flow may also be found). The description of real properties of many materials is much better here; however, certain new mathematical complications arise. The tensors of directions and similarity of stresses and total strains or strain rates are no longer identical; such identity holds separately for elastic strain rates and stress rates, $\dot{e}_{i j}^{e *}=\dot{s}_{i j}^{*}$, and separately for plastic strain rates and stresses, $\dot{e}_{i j}^{p *}=s_{i j}^{*}$. A similar remark applies to the Lode parameters.

The unknown function $\lambda$ may be determined from the yield condition. A formula of type (9.52) is no longer valid, whereas (9.53) remains without change. Namely, multiplying $(9.60)$ by $s_{i j}$, we obtain

$$
s_{i j} \dot{e}_{i j}=\lambda s_{i j} s_{i j}+\frac{1}{2 G} s_{i j} \dot{s}_{i j} ;
$$

further, differentiating the yield condition (9.15), we have

$$
s_{i j} \dot{s}_{i j}=0 \text {; }
$$

hence (9.61) leads to (9.53). The expression $s_{i j} \dot{e}_{i j}$ represents here also the power of plastic strains, though $\dot{e}_{i j}$ are the components of total strain rate; we may write

$$
s_{i j} \dot{e}_{i j}=s_{i j}\left(\dot{e}_{j j}^{p}+\dot{e}_{i j}^{e}\right)=s_{i j} \dot{e}_{i j}^{p}+s_{i j} \frac{\dot{s}_{i j}}{2 G}=s_{i j} \dot{e}_{i j}^{p},
$$

and, in view of (9.62), the second term vanishes.

Substitution of (9.53) into (9.60) and a formal multiplication by $d t$ makes it possible to eliminate the time factor. The resulting equations may be written in engineering notation as

$$
d e_{x}=\frac{3}{2 \sigma_{0}^{2}}\left(s_{x} d e_{x}+s_{y} d e_{y}+s_{z} d e_{z}+\tau_{x y} d \gamma_{x y}+\tau_{y z} d \gamma_{y z}+\tau_{z x} d \gamma_{z x}\right) s_{x}+\frac{1}{2 G} d s_{x},
$$

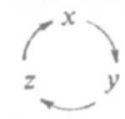

and similarly for the off-diagonal components.

Sometimes it is more convenient to express the Prandtl-Reuss equations in terms of the original tensors, and not in terms of deviators. Subtracting the consecutive equations (9.60) from each other, we eliminate $\dot{\varepsilon}_{m}, \sigma_{m}$, and $\dot{\sigma}_{m}$

$$
\dot{\varepsilon}_{x}-\dot{\varepsilon}_{y}=\lambda\left(\sigma_{x}-\sigma_{y}\right)+\frac{1}{2 G}\left(\dot{\sigma}_{x}-\dot{\sigma}_{y}\right), \quad \frac{\dot{\gamma}_{x y}}{2}=\lambda \tau_{x y}+\frac{1}{2 G} \dot{\tau}_{x y}, \quad \Gamma_{z}^{x} y_{y} .
$$


Further, multiplying the first three equations by $\left(\sigma_{x}-\sigma_{y}\right), \ldots$, the remaining three by $6 \tau_{x y}, \ldots$, adding them together and making use of the $\mathrm{HMH}$ yield condition, we obtain

$$
\begin{aligned}
d \varepsilon_{x}-d \varepsilon_{y}=\frac{1}{2 \sigma_{0}^{2}} & {\left[\left(\sigma_{x}-\sigma_{y}\right)\left(d \varepsilon_{x}-d \varepsilon_{y}\right)+\left(\sigma_{y}-\sigma_{z}\right)\left(d \varepsilon_{y}-d \varepsilon_{z}\right)+\right.} \\
& +\left(\sigma_{z}-\sigma_{x}\right)\left(d \varepsilon_{z}-d \varepsilon_{x}\right)+3 \tau_{x y} d \gamma_{x y}+3 \tau_{y z} d \gamma_{y z}+ \\
& \left.+3 \tau_{z x} d \gamma_{z x}\right]\left(\sigma_{x}-\sigma_{y}\right)+\frac{1}{2 G}\left(d \sigma_{x}-d \sigma_{y}\right)
\end{aligned}
$$

Putting $\lambda=0$ in Eqs. (9.60) or (9.65) and neglecting the yield condition, we may also describe the elastic range by these equations. However, at the elastic-plastic interface the function $\lambda$ is, in general, different from zero, and the easiest way to determine this interface is to put $\sigma_{e}=\sigma_{0}$ in the solution for the elastic zone. The condition $\lambda=0$ together with the yield condition corresponds to a neutral process.

The above equations describe active (or neutral) processes. Passive processes are described by $(9.34),(9.35)$ or $(9.36)$; sometimes the incremental notation is used,

$$
d s_{i j}=2 G d e_{i j},
$$

which determines the process if its starting point is known. The separation of active from passive processes is identical to that described in Sec. 9.5.

As an example apply the Prandtl-Reuss theory to an analysis of the process defined by Eqs. (9.43) and Fig. 35. Equations (9.64) may by written here in the form

$$
\begin{aligned}
& d \gamma_{z x}=\frac{1}{\tau_{0}^{2}}\left(\tau_{z x} d \gamma_{z x}+\tau_{z y} d \gamma_{z y}\right) \tau_{z x}+\frac{1}{G} d \tau_{z x}, \\
& d \gamma_{z y}=\frac{1}{\tau_{0}^{2}}\left(\tau_{z x} d \gamma_{z x}+\tau_{z y} d \gamma_{z y}\right) \tau_{z y}+\frac{1}{G} d \tau_{z y} ;
\end{aligned}
$$

they determine the stresses $\tau_{z x}$ and $\tau_{z y}$ in terms of strains, but are rather complicated and lead to a second-order differential equation. Thus we use a simpler way, namely parametrization of the yield condition (9.40). Substitute

$$
\tau_{z x}=\tau_{0} \cos \psi, \quad \tau_{z y}=\tau_{0} \sin \psi,
$$

replacing two unknows $\tau_{z x}$ and $\tau_{z y}$ by one unknown $\psi$. The initial condition (starting from the point $\tilde{\boldsymbol{A}}$ ) now takes the form $\psi(0)=0$. Equations (9.65) with substituted (9.69) and (9.57) take the form

$$
\begin{aligned}
-\frac{\tau_{0}}{G} \alpha \sin t & =2 \lambda \tau_{0} \cos \psi-\frac{\tau_{0}}{G} \dot{\psi} \sin \psi, \\
\frac{\tau_{0}}{G} \alpha \cos t & =2 \lambda \tau_{0} \sin \psi+\frac{\tau_{0}}{G} \dot{\psi} \cos \psi .
\end{aligned}
$$

Elimination of $\lambda$ may now be performed without any differentiation, simply by multiplying the first equation by $\sin \psi$, the second one by $\cos \psi$ and subtracting them; we obtain

$$
\alpha \cos (\psi-t)=\frac{d \psi}{d t} \text {. }
$$


The substitution $\varphi(t)-t=u(t)$ and the separation of the variables yield

$$
\frac{d u}{\alpha \cos u-1}=d t,
$$

and after integration, the initial condition $\psi(0)=0$ being taken into account, we obtain

$$
t=\frac{1}{\sqrt{\alpha^{2}-1}} \ln \frac{\alpha-1+\sqrt{\alpha^{2}-1} \tan \frac{1}{2} u}{\alpha-1-\sqrt{\alpha^{2}-1} \tan \frac{1}{2} u} .
$$

Inverting relation (9.73) and returning to the unknown $\psi$, we obtain

$$
\psi=t+2 \arctan \left[\sqrt{\frac{\alpha-1}{\alpha+1}} \tanh \left(\frac{\sqrt{\alpha^{2}-1}}{2} t\right)\right] .
$$

This formula together with (9.69) gives the solution; namely, it determines the change of stresses in time. The symbol tanh denotes the hyperbolic tangent; this function often appears in solutions based on the Prandtl-Reuss theory.

A numerical comparison of the results will be given in Sec. 9.9, whereas here we check the continuity of description of the neutral process only. Substituting $\alpha=1$ into (9.74), we describe this process by the equations of active processes; the result is $\psi=t$, and thus $\tau_{z x}=\tau_{0} \cos t, \tau_{z y}$ $=\tau_{0} \sin t$. These results coincide with formulae (9.45), derived under the assumption of a passive process, and so the postulate of a continuous description of the neutral process is satisfied.

\subsection{Coincidence of the deformation and flow theories}

In the general case the theories of a perfectly plastic body described in Sec. $9.5 \div 9.7$ lead to different results. As a result we understand here, according to the remarks given in Sec. 3.1, either the value of a certain transformation (in a broad sense), or the value of a certain functional depending on the trajectory in the space of exertion factors (in a narrow sense); as these exertion factors (independent variables) we usually assume strain or stress components.

Consider now the particular case in which the results of all the three theories of plasticity under discussion coincide. Namely, assume a proportional increase of the components of the strain deviator

$$
e_{i j}=e_{i j}^{(0)} f(t) \text {. }
$$

where $e_{i j}^{(0)}$ are fixed, and $f(t)$ denotes a nondecreasing function of time. The tensor of directions and similarity of the state of strain $e_{i j}^{*},(5.20)$, is then constant during the whole of the process. For sufficiently small values of the function $f(t)$ we are dealing with an elastic process (plastically passive); then

$$
s_{i j}=2 G_{i j}^{(0)} f(t) .
$$

A plastically active process will start when, on the grounds of the HMH hypothesis, we have

$$
s_{i j} s_{i j}=4 G^{2} e_{i j}^{(0)} e_{i j}^{(0)} f^{2}(t)=\frac{2}{3} \sigma_{0}^{2},
$$

i.e., when

$$
f(t)=\frac{\sigma_{0}}{G \sqrt{6 e_{i j}^{(0)} e_{i j}^{(0)}}} .
$$


The corresponding components of the stress deviator (9.76) are then

$$
s_{i j}=\frac{\sigma_{0}}{\sqrt{\frac{3}{2} e_{k l}^{(0)} e_{k l}^{(0)}}} e_{i j}^{(0)}=\sqrt{\frac{2}{3}} \sigma_{0} e_{i j}^{*} .
$$

Assuming further that during the following (active) part of the process the deviatoric components are constant and equal to (9.79), we easily prove that the equations of all the three theories of plasticity are satisfied. As a matter of fact, the HenckyIlyushin equations (9.28) are satisfied if we put

$$
\varphi=\frac{f(t)}{\sigma_{0}} \sqrt{\frac{3}{2} e_{k l}^{(0)} e_{k l}^{(0)}} .
$$

Further, the assumption $s_{i j}=$ const yields $\dot{s}_{i j}=0$, and so the Levy-Mises and the Prandtl-Reuss theories coincide in the case in question; the relevant equations are satisfied if we substitute

$$
\lambda=\frac{\dot{f}(t)}{\sigma_{0}} \sqrt{\frac{3}{2} e_{k l}^{(0)} e_{k l}^{(0)}} .
$$

So in the case under consideration (9.75) the results of all the three theories coincide; namely, they are determined by (9.79). The trajectory in the stress space, corresponding to the active part of the process, degenerates to a point and a "stabilization of stresses" takes place. Such a stabilization may be observed here for an arbitrary yield condition, supposing the constitutive equations to be of the forms (9.28), (9.51) and (9.60) (possible modifications of the flow rule will be discussed in Chapter III).

The process described by Eqs. (9.75) will be called a simple deformation process, or briefly a simple process. Its definition is connected with a proportional increase of deviatoric strain components; formulae (9.76) and (9.79) prove that deviatoric stress components also increase (or non-decrease) proportionally to each other. This proportionality and the coincidence of the results of all the three theories occur also in the case of plastic hardening if such a hardening is described by direct generalizations of (9.28), (9.51) and (9.60). In other descriptions of plastic hardening and in the case of plastic anisotropy the proportional increase of deviatoric strain components does not mean a simultaneous proportional increase of deviatoric stress components; we may then distinguish processes which are simple in strains and processes which are simple in stresses.

A certain particular case of the simple process, namely the case of a proportional increase of all components of the strain tensor (and not merely the strain deviator), will be called proportional in strains; similarly the process proportional in stresses may be defined. A. A. Ilyushin [1.7, 1.8] proved the following important theorem, called the theorem on simple loading: in the case of a proportional increase of all external loadings acting on the body, the process at each point of the body is proportional in strains and in stresses, and so it is a simple process, and the results of all the three classical theories of plasticity coincide. Although this theorem was 
proved under strong assumptions (a power hardening law of type (8.6); incompressibility, isotropy and homogeneity of the material; principle of rigidification, i.e. geometry changes not being taken into account), and does not strictly hold in more general cases, nevertheless it determines fairly well the range of applicability of the Hencky-Ilyushin deformation theory. The conditions of validity of the Ilyushin theorem are discussed in detail by Yu. N. Rabotnov [2.139], and cases of various yield conditions by F. Edelman [2.30]. Other particular cases of the coincidence of the deformation and flow theories are discussed by G. H. Handelman and W. H. Warner [2.51].

In the general case there exist no systems of loadings (except certain trivial ones, e.g. those causing homogeneous stress and homogeneous strain) which would realize a simple process at any point of the body. This problem was investigated by D. D. Ivlev in [2.70] (polynomial schematization of the plastic hardening) and in [2.73] (general schematization), and also by V. D. Klyushnikov [2.85]. L. I. Sedov [2.150] drew attention to the difficulties arising in the case of finite strains.

To sum up, the Hencky-Ilyushin theory of small elastic-plastic deformations may be regarded as correct in the case of simple processes-when its results coincide with those of the Prandtl-Reuss theory; erroneous in the case of neutral and related processes, since then the postulate of continuous description is not satisfied; sufficiently accurate in the case of processes close to simple ones (such processes are analyzed in detail by L. M. Kachanov [2.78]). The Prandtl-Reuss theory may describe the behaviour of the body during arbitrary processes, but its generality does not mean that with reference to a specified material these processes are always described adequately. Certain restrictions on the applicability of flow theories are pointed out by J. Marin and L. W. Hu [2.109].

If we said, however, that the applicability of the Hencky-Ilyushin theory is limited exclusively to the cases in which its results coincide with those of the PrandtlReuss theory, then its importance would undoubtedly be underestimated. In fact, the value of the Hencky-Ilyushin theory is considerably higher. Firstly, even in the case of the coincidence of results, they are so much easier to obtain by using the Hencky-Ilyushin theory that it is convenient to apply that theory and then merely to check the Prandtl-Reuss equations. Secondly, in engineering applications we often deal with processes which are only slightly different from simple ones and then the deformation theory is sufficiently accurate. Thirdly, it should be noted that many experimental results lie between the results of the deformation and the flow theories, though obviously closer to the Prandtl-Reuss theory.

Let us return once more to the phenomenon of stabilization of stresses, (9.79), observed during the active part of a simple process, (9.75). S. D. Leytes [2.99, 2.100], and A. G. Dorfman and S. D. Leytes [2.26] proposed to assume, approximately, a stabilization of stresses even in the case of processes slightly different from a simple one. As compared with the Prandtl-Reuss theory, assumed to be exact, the "hypothesis of stabilization" involves as a rule greater errors than the Hencky-Ilyushin theory; nevertheless, because of its simplicity, it may lead to certain useful results. 


\subsection{Example of a comparison of the classical theories}

In the preceding section we proved that in the case of a simple process the results of all the three above-mentioned theories of a perfectly plastic body coincide. Incidentally, it will be interesting to compare the results in the case of a process departing considerably from a simple one.

Figures 36 and 37 give such a comparison for the processes defined by Eqs. (9.43) and Fig. 35. These figures show the diagrams of stresses $\tau_{z x}$ and $\tau_{z y}$ in terms of the parameter $t$ for $\alpha=8$ (Fig. 36) and $\alpha=\infty$ (Fig. 37, in this case $s=\lim _{\alpha \rightarrow \infty} \alpha t$ ).

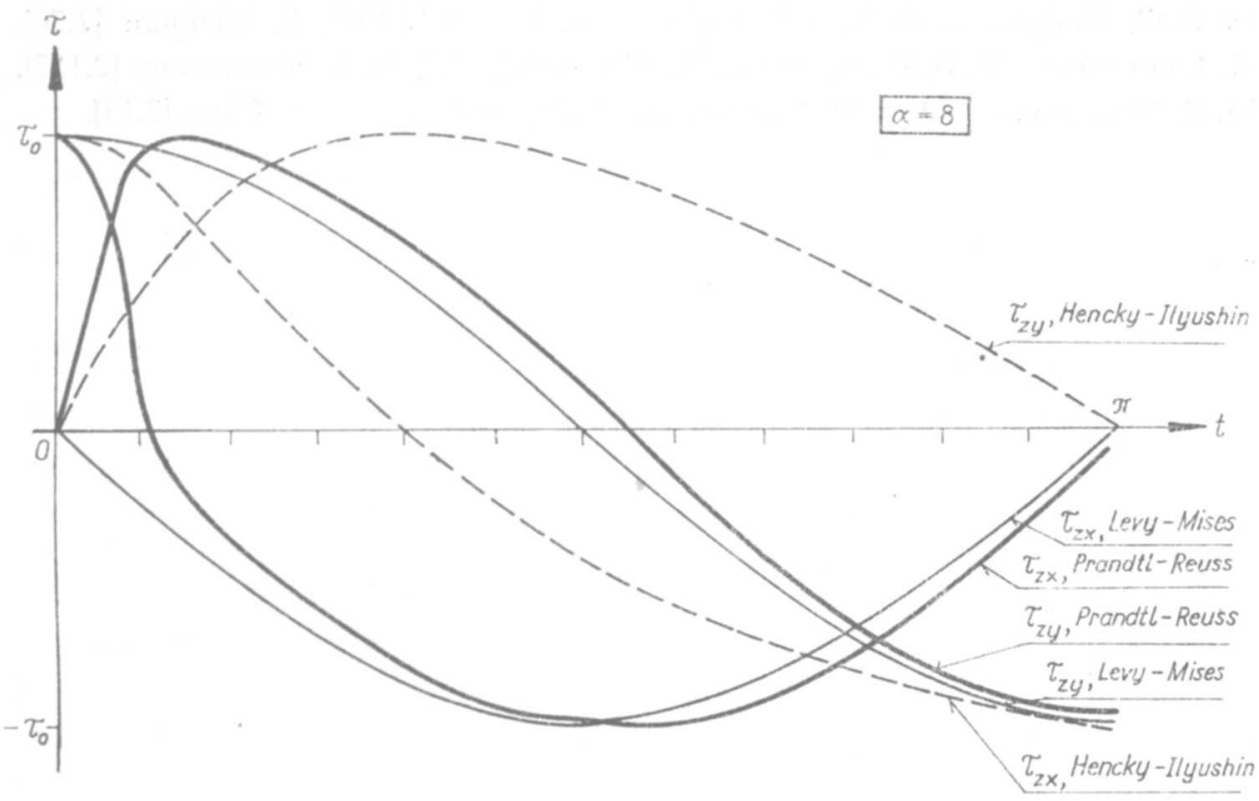

Fig. 36. Variations of stresses for a process shown in Fig. 35 with $\alpha=8$

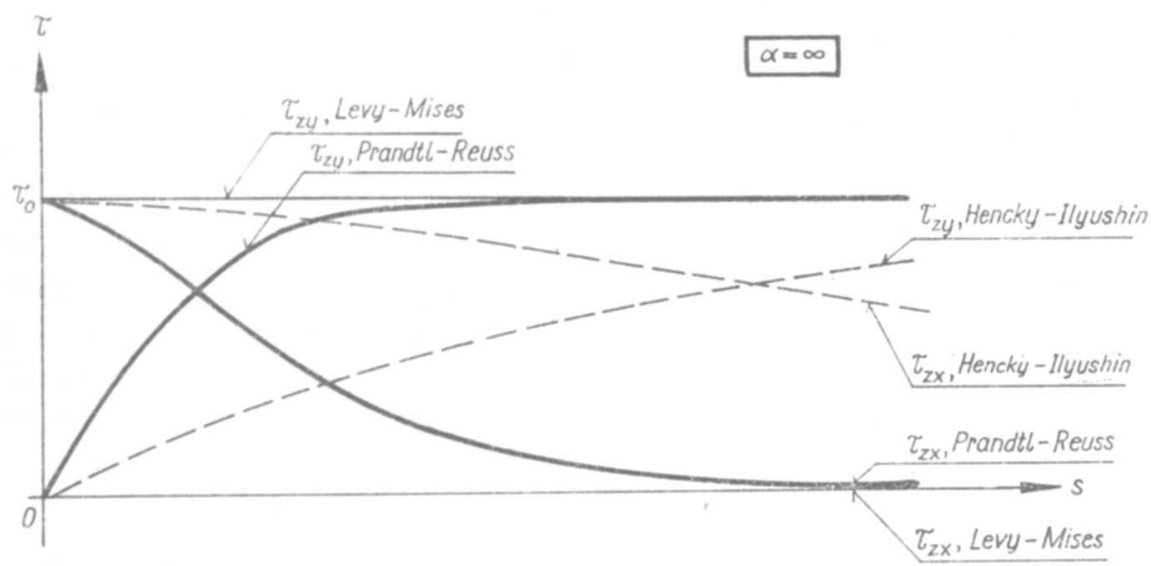

Fig. 37. Variations of stresses for a process shown in Fig. 35 with $\alpha \rightarrow \infty$ 
During the initial stage of the process the Prandtl-Reuss curves are better approximated by the Hencky-Ilyushin theory than by the Levy-Mises theory but with increasing $t$ both flow theories clearly converge and the differences with respect to the deformation theory increase.

In the case of $\alpha=\infty$, when the process is rectilinear (but not simple), all the three lines for each stress component have a common asymptote, according to the Kachanov theorem [2.78]. The numerical differences decrease only for a relatively large strain $\gamma_{z y}$.

Other examples of a comparison of the deformation and flow theories are given by P. G. Hodge and G. N. White [2.60], W. Prager [2.132], F. Edelman [2.30], H. J. Greenberg, W. S. Dorn, and E. H. Wetherell [2.43], M. R. Shammamy [2.157], M. R. Shammamy and O. M. Sidebottom [2.158], and by P. C. T. Chen [2.17]. 


\section{CHAPTER III}

\section{Combined loadings at the level $\mathcal{P}$ of a point of the body}

\section{Auxiliary spaces at the level $\mathscr{P}$}

\subsection{Nine-dimensional spaces}

In Section 1.3 we discussed exertion factors at the level $\mathscr{P}$ and in Section 3.1 we introduced the corresponding space of exertion factors. In most cases it is the stress components that are regarded at that level as exertion factors; we have called them the basic exertion factors. Now we are going to discuss in detail auxiliary spaces related to the level $\mathscr{P}$, and particularly stress spaces.

Any second-order tensor in a three-dimensional space $\tau_{i j}$ may be represented by a certain vector $\tau$ in the auxiliary nine-dimensional space with components equal to the appropriately ordered tensor components. If the addition and multiplication of tensors are transferred to vectors, then the nine-dimensional space satisfies the requirements of a vector space.

Such nine-dimensional spaces for stresses and strains were introduced in 1932 by K. Hohenemser and W. Prager [3.263]; however, their wider application came much later (R. Hill [3.246, 3.247], R. Shield and H. Ziegler [3.721], Z. Mróz [3.553]). They are sometimes called the Prager spaces. The representation of processes in those spaces is quite general, but their graphical interpretation is difficult.

V. V. Novozhilov [3.581, 3.583] draws attention to the fact that such a ninedimensional space has merely an auxiliary character, and a vector in the nine-dimensional space (or, obviously, in a space of fewer dimensions) cannot be equivalent to the given tensor. Indeed, a tensor has three independent invariants whereas an auxiliary vector-only one (length). In the case of a nine-dimensional stress space the length of the vector $\sigma$ is equal to one invariant of the stress tensor, namely

$$
|\sigma|=\sqrt{I_{2 \sigma}} ;
$$

thus the rotation of the physical reference frame changes the vector $\sigma$ but its length remains constant. 
The nine-dimensional spaces under consideration cannot be regarded as spaces of exertion factors. Indeed, as an exertion factor we understand an independent quantity, and the symmetry conditions of the type $\tau_{x y}=\tau_{y x}$ reduce the number of such quantities to six.

\subsection{Six-dimensional spaces}

Making use of the symmetry of the stress and strain tensors, we may consider sixdimensional subspaces (intersections) of the original nine-dimensional spaces. Such six-dimensional spaces were proposed as early as 1930 by H. Fromm [3.188] during the Third Congress of Mechanics in Stockholm, but widely used much later, for example by R. Shield and H. Ziegler [3.721], D. D. Ivlev and G. I. Bykovtsev [3.312], W. Flügge [3.179] and B. Nayroles [3.573]; in the case of stresses they assume successively $\sigma_{x}, \sigma_{y}, \sigma_{z}, \tau_{x y}, \tau_{y z}$ and $\tau_{z x}$ as the coordinates of the auxiliary six-dimensional vectors. The drawback of such an approach is the fact that the length of the vector $\boldsymbol{\sigma}$ is not invariant and that the scalar product $\boldsymbol{\sigma} \boldsymbol{\epsilon}$ has no energy interpretation. These drawbacks may be removed if we replace the coordinates $\tau_{x y}, \tau_{y z}$, and $\tau_{z x}$ by $\tau_{x y} \sqrt{2}, \tau_{y z} \sqrt{2}$, and $\tau_{z x} \sqrt{2}$, and similarly for $\varepsilon_{x y}$, etc.; such a procedure corresponds to the appropriate rotation of the hyperplane of intersection in the ninedimensional space. This idea was introduced already in the above-mentioned paper by K. Hohenemser and W. Prager [3.263] and developed by V. V. Novozhilov [3.583], J. H. Argyris and D. W. Scharpf [3.10]. Sometimes the stress space is retained without change ( $\sigma$ and $\tau$ ) and in the strain space the tensorial components $\varepsilon_{x y}, \varepsilon_{y z}, \varepsilon_{z x}$ are replaced by the engineering ones $\gamma_{x y}, \gamma_{y z}, \gamma_{z x}$ (O. C. Zienkiewicz [3.875], A. A. Čyras, A. E. Borkauskas, R. P. Karkauskas [3.121]); then the vector lengths are not invariant, but the energy interpretation of the scalar product $\boldsymbol{\sigma} \epsilon$ holds.

V. V. Novozhilov [3.583] remarks that the application of six-dimensional vector spaces is limited. Although all the fundamental properties of vector spaces are satisfied, the result of multiplication with a single contraction, e.g.

$$
\sigma_{i k} \varepsilon_{k j}+\sigma_{j k} \varepsilon_{k i}=\vartheta_{i j},
$$

cannot be interpreted in such spaces. He introduces an auxiliary six-dimensional metric tensor space $H_{6}$ with elements (10.2) belonging to that space.

A six-dimensional space may be complemented to a seven-dimensional one by the introduction of temperature as a new independent variable (A. E. Green, P. M. Naghdi [2.41]). Such spaces can usually be regarded as the spaces of exertion factors at the level $\mathscr{P}$. Important exceptions are: perfectly plastic bodies, where the stress components are interrelated by the yield condition, and incompressible bodies, where the strain components are interrelated; in these cases the six stress or the six strain components are not independent and cannot be regarded as the exertion factors. 


\subsection{Five-dimensional spaces}

In many cases the axiatoric part of the stress tensor is without any influence on yielding. On the other hand, in incompressible bodies the axiatoric part of the strain tensor vanishes. It is then sufficient to consider the five-dimensional deviatoric subspaces of the corresponding six-dimensional spaces. In other words, we may regard a six-dimensional space as the Cartesian product of the one-dimensional axiatoric subspace and the five-dimensional deviatoric subspace, and consider the latter only, [3.573].

Such a deviatoric space for an arbitrary symmetric tensor $T_{\tau}$ was introduced by A. A. Ilyushin [1.10]; as the Cartesian coordinates in that space he adopted the following five quantities:

$$
\begin{array}{ll}
\tau_{1}=t_{x x} \sqrt{\frac{3}{2}}, \quad \tau_{3}=t_{x y} \sqrt{2}, \quad \tau_{5}=t_{z x} \sqrt{2}, \\
\tau_{2}=\left(t_{y y}-t_{z z}\right) \frac{\sqrt{2}}{2}, & \tau_{4}=t_{y z} \sqrt{2},
\end{array}
$$

where $t_{i j}$ denote the deviatoric components of the tensor $T_{\tau}$. The length of the corresponding auxiliary five-dimensional vector $\tau$ is proportional to the intensity of the original tensor, and thus it is invariant; e.g. in the case of stresses

$$
|\sigma|=\sigma_{e} \sqrt{\frac{2}{3}} .
$$

Hence the Huber-Mises-Hencky yield condition is represented here by a hypersphere with the equation

$$
|\boldsymbol{\sigma}|=\sigma_{0} \sqrt{\frac{2}{3}}
$$

Ilyushin remarks that such an introduction of a five-dimensional space is not unique. Indeed, we may assume

$$
\begin{aligned}
& \tau_{1}=t_{x x} \sqrt{2} \cos \alpha \pm t_{y y} \sqrt{2} \sin \left(\alpha \pm \frac{1}{6} \pi\right), \\
& \tau_{2}=t_{x x} \sqrt{2} \sin \alpha \mp t_{y y} \sqrt{2} \cos \left(\alpha \pm \frac{1}{6} \pi\right), \\
& \tau_{3}=t_{x y} \sqrt{2}, \quad \tau_{4}=\tau_{y z} \sqrt{2}, \quad \tau_{5}=t_{z x} \sqrt{2}
\end{aligned}
$$

with an arbitrary value of the auxiliary angle $\alpha$ and any system of signs. In formulae (10.3) the value $\alpha=\frac{1}{6} \pi$ and lower signs were adopted; such a choice is convenient for practical applications.

The five-dimensional spaces under consideration, called the Ilyushin spaces, satisfy all the basic conditions of vector spaces. However, as in the case of six-dimensional spaces, elements of type (10.2) cannot be properly interpreted in them; to remove this difficulty, V. V. Novozhilov [3.583] introduced five-dimensional tensor spaces. 


\subsection{Three-dimensional spaces}

Spaces of the principal values of a tensor are the most commonly used threedimensional spaces. A three-dimensional stress space of this kind, introduced by B. T. Haigh and H. M. Westergaard, was mentioned in Sec. 5.4. The advantage of such spaces lies in their simplicity and visual presentation; their drawback is the impossibility of representing a process of changing the principal directions at constant principal values (the whole process is then represented by one fixed pointe). Moreover, in the general case spaces of principal values do not satisfy the requirements for vector spaces: for example, the addition of two tensors does not in general correspond to the addition of their principal values. Such correspondence takes place only in the case of fixed and uniformly numbered principal directions. Nevertheless, spaces of principal values are widely used and in the case of constant principal directions their application is fully justified.

The conditions of perfect plasticity are represented in the three-dimensional Haigh-Westergaard space by certain surfaces (limit surfaces). Photographs of models of such surfaces corresponding to various yield conditions may be found in the papers by W. Burzyński [2.13] and A. Meldahl [3.518].

In contradistinction to the spaces discussed above, the space of principal values of a tensor is an invariant space: limit surfaces in the space of principal stresses do not depend on the assumed system of coordinates in the body. Since among the infinite number of invariants of a symmetric second-order tensor only three may be independent, we may introduce three-dimensional spaces corresponding to an arbitrary system of such invariants, e.g. $I_{1 \sigma}, I_{2 \sigma}, I_{3 \sigma} ; J_{1 \sigma}, J_{2 \sigma}, J_{3 \sigma} ; I_{1 \sigma}, I_{2 s}, I_{3 s}$. To avoid having different dimensions along the individual axes, we may replace the above-mentioned invariants by $\sqrt{\left|I_{2 \sigma}\right|}, \sqrt[3]{I_{3 \sigma}}$, etc. Such invariant spaces are discussed by S. Irmay [3.296]. Symmetric invariants are convenient in the case of isotropic materials, but they do not allow us to represent yield surfaces for anisotropic materials or subsequent neutral surfaces for isotropic materials exhibiting anisotropic hardening. A certain concept of a space of generalized stresses for anisotropic materials was proposed by L. A. Tolokonnikov and N. M. Matchenko [3.811].

The choice of the system of invariants essentially affects the final conclusions. Thus, for example, in the system of axiatoric-deviatoric invariants $I_{1 \sigma}, I_{2 s}, I_{3 s}$, limit surfaces representing yield conditions for many materials do not depend on the first invariant $I_{1 \sigma}$. Analysing this problem in [3.277], L. W. Hu arrives at the paradoxical conclusion that each yield condition must depend on $I_{1 \sigma}$. This conclusion is fully justified and the paradox lies in the fact that it is justified in the system of principal invariants $J_{1 \sigma}, J_{2 \sigma}, J_{3 \sigma}$ (where $J_{1 \sigma}=I_{1 \sigma}$ ) and is not valid in the system $I_{1 \sigma}, I_{2 s}, I_{3 s}$ which is the object of the fundamental discussion.

Non-invariant three-dimensional spaces are also used. For example, V. S. Lensky and I. D. Mashkov [3.438] investigated the behaviour of steel in a three-dimensional 
subspace of the Ilyushin five-dimensional space. The most commonly used is here the space $\sigma_{x}, \sigma_{y}, \tau_{x y}$, describing the plane state of stress with arbitrarily changing principal directions.

\subsection{Two-dimensional spaces, planes}

The representation of the processes taking place at a point of the body is particularly simple if it may be performed in a plane, at least approximately, with a sufficient accuracy. Accordingly, many such planes have been proposed. We present here only some of the more important stress planes; strain planes may be introduced in a similar manner.

If the limit surface in the space of principal stresses is represented by a rotationally symmetric surface with the axis $\sigma_{1}=\sigma_{2}=\sigma_{3}$, then it is uniquely determined by its generator, i.e. by a curve in the plane of cylindrical invariants $Z_{\sigma}, R_{\sigma}$. Instead of this plane we more often use a plane with a changed scale, $\sigma_{m}-\sigma_{e}$ (mean stressstress intensity), where, as we derived in Sec. 5,

$$
\sigma_{m}=Z_{\sigma} / \sqrt{3}, \quad \sigma_{e}=R_{\sigma} \sqrt{3 / 2} .
$$

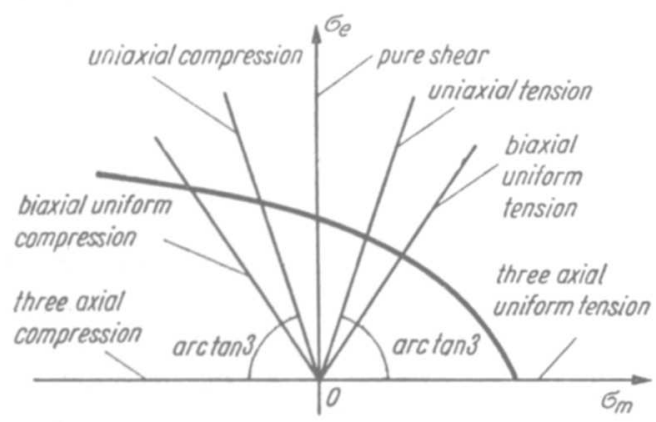

Fig. 38. The Burzyński plane $\sigma_{m}-\sigma_{e}$

In view of $\sigma_{e} \geqslant 0$ it is sufficient to consider only the upper half of the plane (Fig. 38). This plane will be called the Burzyński plane, since Burzyński was the first to use it in 1928, [2.13] (in fact, Burzyński used the coordinates $\sigma_{m}, \sigma_{e} \sqrt{2}$, which are less convenient).

In the coordinates $\sigma_{m}, \sigma_{e}$ uniaxial tension and uniaxial compression are represented by the rays $\sigma_{e}= \pm 3 \sigma_{m}$, biaxial uniform tension and biaxial uniform compression by the rays $\sigma_{e}= \pm \frac{3}{2} \sigma_{m}$, pure shear by the ray $\sigma_{m}=0$, triaxial uniform tension by the ray $\sigma_{e}=0, \sigma_{m}>0$, and triaxial uniform compression by the ray $\sigma_{e}=0, \sigma_{m}<0$.

It should be noted that the Burzyński plane does not satisfy the requirements of a vector space even in the case of constant principal directions. Indeed, if we represent uniaxial tension by a vector and antimetric uniaxial compression by a vector, and add them together as vectors, we do not obtain a zero-vector as should be the case. However, for many applications this deficiency is unimportant. If the limit surface is 
not rotationally symmetric, then it may be presented in the Burzyński plane by a one-parameter family of curves; the most convenient parameters are the third cylindrical invariant $\omega_{\sigma}$ or the Lode parameter $\mu_{\sigma}$.

If the limit surface in the space of principal stresses is represented by a cylindrical surface (non-circular, in general) with the axis $\sigma_{1}=\sigma_{2}=\sigma_{3}$, then it is uniquely determined by the curve of intersection of that cylinder with the plane perpendicular to its axis, namely,

$$
\sigma_{1}+\sigma_{2}+\sigma_{3}=C=\text { const. }
$$

Such a plane is called the deviatoric plane (this term is justified in the case $C=0$ in Eq. (10.8)), or the $\pi$-plane or the Meldahl plane (F. Correia de Araújo [3.115]). The projections of the axes $\sigma_{1}, \sigma_{2}, \sigma_{3}$ on that plane form the angles of $120^{\circ}$. In the case of an isotropic body with a cylindrical limit surface the curve has three axes of symmetry, (its equation contains functions of $3 \omega_{\sigma}$ ) and is fully determined by the segment $0 \leqslant \omega_{\sigma} \leqslant 60^{\circ}$, Fig. 39. If the surface is not cylindrical, then it may be rep-

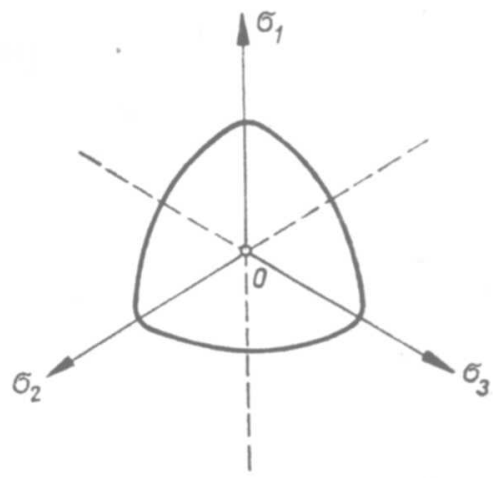

Fig. 39. The deviatoric (Meldahl) plane

resented by a one-parameter family of curves in the Meldahl plane; the parameter is then $C=3 \sigma_{m}$ (equipressure cross-sections, [3.620]), but the picture is not so clear in this case.

The use of a deviatoric Meldahl plane may lead to certain misunderstandings, since the axes denoted by $\sigma_{1}, \sigma_{2}$, and $\sigma_{3}$ are, in fact, only the projections of the original axes on the plane under consideration. Thus, we may lay off along those axes either the principal stresses $\sigma_{1}, \sigma_{2}, \sigma_{3}$, (as was done by Meldahl [3.517]) or their projections, equal to $\sigma_{1} \sqrt{2 / 3}, \sigma_{2} \sqrt{2 / 3}, \sigma_{3} \sqrt{2 / 3}$. Only the latter approach gives the cross-section of the cylindrical limit surface in the correct scale, but for some applications the Meldahl approach is more convenient.

The deviatoric strain plane may be introduced in a similar way. Its range of applications may be even wider than that of the deviatoric stress plane. Indeed, in the case of an incompressible material, we have $\varepsilon_{m}=0$ and each point representing the state of strain in the space of principal strains must remain in the deviatoric plane. The characteristics of various strain paths in the deviatoric plane are discussed 
by T. C. Hsu [3.270]. The concept of a deviatoric plane was developed by $Z$. Marciniak [3.500], who proposed a certain more general plane representation of threedimensional stress and strain states.

Very often limit curves in the plane $\sigma_{1}, \sigma_{2}$ are investigated; this plane may be treated as the intersection of the space of principal stresses by the plane $\sigma_{3}=0$. Such curves do not permit the reproduction of the whole limit surface, but for many applications may be sufficient-they describe the behaviour of a material in the case of plane stress.

The planes described above belong to invariant planes-the limit curves do not depend on the adopted reference frame at a given point of the body. The planes without this property are also employed. For example, analysing combined loadings in the cross-sections of bars we often use the plane $\sigma_{x}, \tau_{x y}$; however, the directions of the axes $x$ and $y$ are then uniquely determined and the problem of transformation does not arise.

It should be reminded here once more that the spaces and planes discussed above may turn out to be insufficient to describe the processes at the level $\mathscr{P}$. Sometimes the stresses and strains must be combined (A. K. Malmeyster [3.488]); and sometimes other axes, corresponding to additional independent factors (temperature, humidity, etc.), should be added.

\subsection{Isotropy and isosensitivity}

The properties of isotropic materials do not depend on the direction; in the plastic range this fact results in some conditions of symmetry described above. Further symmetry conditions appear if those properties are not affected by the simultaneous change of sign of all the stress components. D. D. Ivlev [2.72] introduced here the term "normal isotropy"; however, this property is, to a certain degree, independent of isotropy, and Ivlev's term may be misleading. R. M. W. Frederking and O. M. Sidebottom [3.182] use the terms "even materials" (and "uneven materials"). We propose to call such materials isosensitive, since their properties do not depend on the sense of stress components.

Indeed, any combination of (an-)isotropy and (an-)isosensitivity is possible. For example, mild steel without effects of plastic forming is isotropic and isosensitive; cast iron, concrete and soils are isotropic and anisosensitive; a non-uniformly ribbed steel surface structure furnishes a good model of a material which is anisotropic and isosensitive; finally, timber is anisotropic and anisosensitive. However, such independence of (an-)isotropy and (an-)isosensitivity is restricted to anisosensitivity under normal (principal) stresses. A material which is anisosensitive under tangential (shearing) stresses must be simultaneously anisotropic. Consider, for example, pure shear and assume that for a positive stress the yield-point is reached at $\tau=\tau_{0+}$ and for a negative stress at $\tau=-\tau_{0-}$. Then in the first case the principal stresses are $\sigma_{1}=+\tau_{0+}, \sigma_{2}=0, \sigma_{3}=-\tau_{0+}$, and in the second case we have in the same directions $\sigma_{1}=-\tau_{0-}, \sigma_{2}=0, \sigma_{3}=+\tau_{0-}$. In an isotropic material we may arbitrarily change the indices of the principal stresses, hence it immediately follows 
that $\tau_{0_{+}}=\tau_{0_{-}}$; therefore the case $\tau_{0+} \neq \tau_{0_{-}}$is possible only in anisotropic materials.

If the material is not only isotropic but also isosensitive, further symmetry conditions of the limit surfaces hold. In the space of principal stresses the surface must be symmetrical with respect to the point $O$; in the Burzyński plane the limit curve (generator of the rotationally symmetric surface) must be symmetric with respect to the axis $\sigma_{e}$; in the Meldahl plane the limit curve (cross-section of the cylindrical surface) possesses six symmetry axes (its equation contains the functions of $\left.6 \omega_{\sigma}\right)$ and is fully determined by the segment $0 \leqslant \omega_{\sigma} \leqslant 30^{\circ}$. The corresponding examples are shown in Fig. 40.
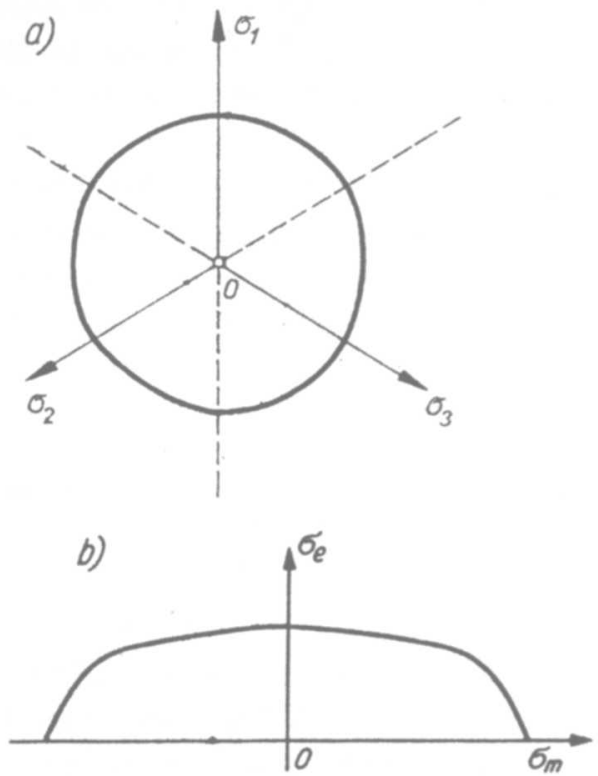

Fig. 40. Isotropic isosensitive material: (a) in the deviatoric plane, (b) in the Burzyński plane

Making use of the definitions adopted above, we may define the Bauschinger effect as acquired anisosensitivity of initially isosensitive materials (due to plastic deformation), or as changed anisosensitivity if the material is anisosensitive in the virgin state. Hence the Bauschinger effect under normal stresses is not necessarily connected with the acquired anisotropy of the material; however, if it occurs under tangential stresses, then the material must become anisotropic as well.

\section{Conditions of perfect plasticity for isotropic bodies}

\subsection{General remarks}

As was mentioned in Sections 9.2 and 9.3, the phenomenological formulation of the condition of perfect plasticity may be regarded as a problem of the hypotheses 
of failure: either a physical criterion of exertion is proposed, or the experimental results constitute the basis for a suitable analytical approximation. Similar approaches are used to somewhat different physical phenomena, such as the limitation of the elastic range, yielding or decohesion, but the form of mathematical description is similar and therefore those phenomena are often treated jointly. There are numerous survey papers describing individual hypotheses and their application; let us mention here the papers by W. Burzyński [2.13], H. Fromm [3.189], J. Marin [3.503], M. T. Huber [3.279], M. M. Filonenko-Boroditch [3.176], T. Pełczyński [3.622], B. Paul [3.620], I. I. Goldenblat and V. A. Kopnov [3.205], and G. S. Pisarenko and A. A. Lebedev [3.643]. The extensive Polish contribution to the development of the failure hypotheses is reviewed by $\mathrm{Z}$. Klębowski [3.352].

We are now going to discuss briefly those hypotheses which are often applied to the formulation of conditions of perfect plasticity (yield conditions) or to the description of initial neutral surfaces in the case of bodies exhibiting plastic hardening. They will be classified according to the shape of the relevant limit surfaces in the space of principal stresses.

\subsection{Cylindrical limit surfaces}

If the yield condition may be written in one of the equivalent forms

$$
f\left(I_{2 s}, I_{3 s}\right)=0 \text { or } f\left(R_{\sigma}, \omega_{\sigma}\right)=0,
$$

i.e., if the equation in question does not depend on the first invariant in the system of axiatoric-deviatoric invariants or in the system of cylindrical invariants, then the relevant limit surface is cylindrical with the axis $\sigma_{1}=\sigma_{2}=\sigma_{3}$. The adoption of such a hypothesis means neglecting the influence of the mean stress $\sigma_{m}$ (hydrostatic pressure) on yielding. This problem was experimentally investigated by $\mathbf{B}$. Crossland [3.117], H. L. D. Pugh and D. Green [3.660]; they found practically no effect of hydrostatic pressure on yielding in ductile metals. On the other hand, S. I. Ratner [3.667] and L. W. Hu [3.273, 3.276] noticed a certain effect, namely the raising of the stress-strain curve. Careful experiments performed by P. K. Fung, D. J. Burns and N. C. Lind [3.191] confirmed the negligibility of the effect of the hydrostatic pressure in metals; they ascribed the apparent rise of the stress-strain curve to friction in the high pressure seals.

It should be stressed that the problem of the influence of hydrostatic pressure on yielding has no direct connection with the isosensitivity of the material: theoretically, cylindrical surfaces may describe anisosensitive materials as well. However, in most practical cases the lack of effect of hydrostatic pressure on yielding results in identical properties in tension and in compression.

The simplest description of a cylindrical limit surface is given by the equation

$$
R_{\sigma}=f\left(\omega_{\sigma}\right) \text { or } \sigma_{e}=f\left(\omega_{\sigma}\right),
$$

which determines the curve in the deviatoric Meldahl plane in polar coordinates, Fig. 39. Equations of the type

$$
J_{2 s}=f\left(J_{3 s}\right) \text {, }
$$


used by W. Prager [3.652] and in many other papers, are less clear; of course, they may be always converted to (11.2) by using the relations given in Table 2 (p. 37).

The limit surface which is simultaneously cylindrical and rotational corresponds to the Huber-Mises-Hencky yield condition. It is described by Eq. (9.15), which will now be rewritten in the form (11.2), i.e.,

$$
\boldsymbol{R}_{\sigma}=\sigma_{0} \sqrt{\frac{2}{3}}=\text { const }
$$

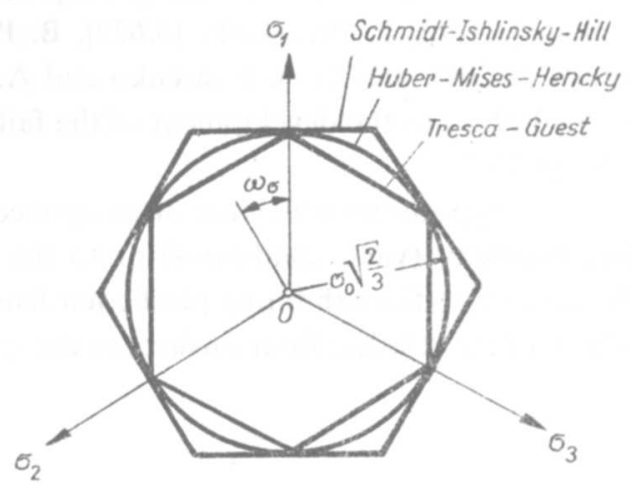

Fig. 41. Intersections of typical cylindrical limit surfaces by the deviatoric plane

(Fig. 41) and in the following two expanded forms, often used in engineering applications:

$$
\begin{aligned}
& \sqrt{\sigma_{x}^{2}+\sigma_{y}^{2}+\sigma_{z}^{2}-\sigma_{x} \sigma_{y}-\sigma_{y} \sigma_{z}-\sigma_{z} \sigma_{x}+3\left(\tau_{x y}^{2}+\tau_{y z}^{2}+\tau_{z x}^{2}\right)}=\sigma_{0}, \\
& \left(\sigma_{x}-\sigma_{y}\right)^{2}+\left(\sigma_{y}-\sigma_{z}\right)^{2}+\left(\sigma_{z}-\sigma_{x}\right)^{2}+6\left(\tau_{x y}^{2}+\tau_{y z}^{2}+\tau_{z x}^{2}\right)=2 \sigma_{0}^{2} .
\end{aligned}
$$

The $\mathrm{HMH}$ yield condition is verified experimentally for a wide class of materials, mainly polycrystalline metals. Let us mention here the experiments of W. Lode [2.104, 2.105, 2.106], M. Roš, A. Eichinger [2.144], G. I. Taylor, H. Quinney [3.791], J. L. M. Morrison [3.538, 3.539], J. M. Lessells, C. W. MacGregor [3.450], A. M. Zhukov [3.864, 3.865, 3.866], L. W. Hu, J. F. Bratt [3.275], G. B. Talypov, V. N.

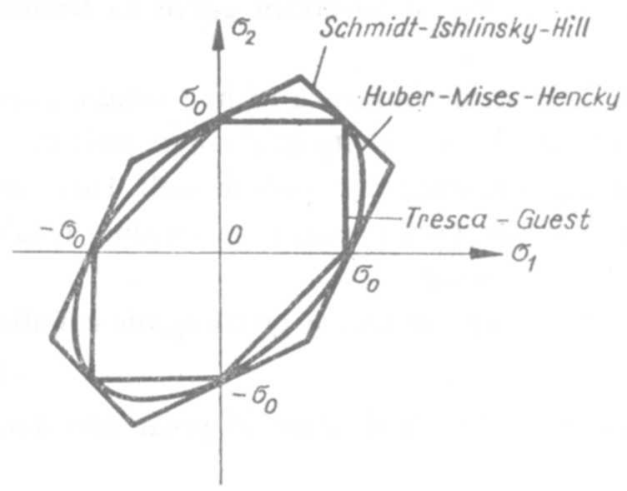

Fig. 42. Intersections of typical cylindrical limit surfaces by the plane $\sigma_{3}=0$ 
Kamentsev [3.783], J. Miastkowski, W. Szczepiński [3.521]. Surveys of experiments are given by I. I. Goldenblat and V. A. Kopnov [3.205], B. Paul [3.620], and by J. F. Bell [3.46]. A certain thermodynamic justification of the $\mathrm{HMH}$ yield condition is given by J. Gouzou and A. Magnée [3.207].

The intersection of the HMH circular cylinder with the plane $\sigma_{3}=0$ is an ellipse with the equation

$$
\sigma_{1}^{2}+\sigma_{2}^{2}-\sigma_{1} \sigma_{2}=\sigma_{0}^{2},
$$

shown in Fig. 42 and describing the limit state in the case of a state of plane stress. Another important particular case is that of a state of plane strain, e.g., $\varepsilon_{z}=\gamma_{z x}$ $=\gamma_{z y}=0$; the final form of the HMH yield condition depends here on the constitutive (physical) equation used. If we assume the Hencky-Ilyushin equation for a compressible body, (9.29), evaluate $\sigma_{z}$ from the condition $\varepsilon_{z}=0$, and substitute into (11.6), then the final result takes a rather complicated form, depending on the value of the function $\varphi$

$$
\left[1+\frac{3}{(6 K \varphi+1)^{2}}\right]\left(\sigma_{x}+\sigma_{y}\right)^{2}-4\left(\sigma_{x} \sigma_{y}-\tau_{x y}^{2}\right)=\frac{4}{3} \sigma_{0}^{2} .
$$

In the case of an incompressible body, $K \rightarrow \infty$, (11.8) is simplified to

$$
\left(\sigma_{x}-\sigma_{y}\right)^{2}+4 \tau_{x y}^{2}=\frac{4}{3} \sigma_{0}^{2},
$$

independent of $\varphi$. Equation (11.9) may also be obtained from the Prandtl-Reuss or the Levy-Mises equations for an incompressible body.

A certain convenient geometrical construction determining the stress intensity $\sigma_{e}$ (reduced stress corresponding to the $\mathrm{HMH}$ yield condition) was proposed by A. Meldahl [3.517] and Z. Marciniak [3.501]. The simple geometrical addition of the vectors $\sigma_{1}, \sigma_{2}$, and $\sigma_{3}$ in the deviatoric plane results in a vector whose length is $\sigma_{e}$,

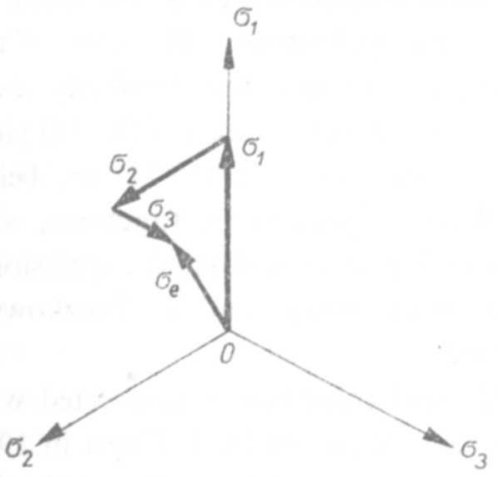

Fig. 43. Graphical determination of the stress intensity in the deviatoric plane

as can easily be proved (Fig. 43). Numerical tables determining $\sigma_{e}$ in combined stress states are given by C. Brutti [3.79].

Another commonly used cylindrical surface corresponds to the Tresca-Guest (TG) yield condition (9.19). An equation of such a type determines a plane in the 
space of principal stresses; in fact, since the symbols $\sigma_{\mathrm{I}}$ and $\sigma_{\mathrm{III}}$ denote the principal stresses algebraically ordered, and there exist six combinations of such an arrangement, (9.19) determines a cylindrical surface, namely a regular hexagonal prism. This prism is inscribed in the HMH circular cylinder; the sections are shown in Figs. 41 and 42. In the important case of a state of plane strain, $\varepsilon_{z}=\gamma_{z x}=\gamma_{z y}=0$, it may be shown (for incompressible bodies) that the principal stress $\sigma_{z}$ has the intermediate value, $\sigma_{z}=\sigma_{\mathrm{II}}$, and the TG yield condition assumes the form

$$
\left(\sigma_{x}-\sigma_{y}\right)^{2}+4 \tau_{x y}^{2}=\sigma_{0}^{2} .
$$

This equation is almost identical with (11.9); if we introduce the yield-point stress in shear $\tau_{0}$, then the two equations coincide:

$$
\left(\sigma_{x}-\sigma_{y}\right)^{2}+4 \tau_{x y}^{2}=4 \tau_{0}^{2} .
$$

The intersection of (9.19) with the deviatoric plane may be described by an equation of the form (11.2) as follows:

$$
R_{\sigma}=\frac{\sigma_{0}}{\sqrt{2} \cos \left(\omega_{\sigma}+\frac{1}{6} \pi-\frac{1}{3} n \pi\right)}, \quad n=1,2, \ldots, 6 .
$$

Another, more uniform but more complicated form of (11.12) was proposed in [1.24]; using the notation adopted here we rewrite it as follows:

$$
\cos 6 \omega_{\sigma}=1-\frac{\left(2 \sigma_{0}^{2}-3 R_{\sigma}^{2}\right)^{2} \sigma_{0}^{2}}{R_{\sigma}^{6}} .
$$

The corresponding equation in the implicit form (11.3) was derived in 1933 by A. Reuss [3.672]:

$$
4 J_{2 s}^{3}+27 J_{3 s}^{2}+\left(3 J_{2 s}+\sigma_{0}^{2}\right)^{2} \sigma_{0}^{2}=0 .
$$

The main advantage of the Tresca-Guest yield condition lies in the linearity and simplicity of the relation (9.19) expressed in principal stresses. However, if the principal directions and the principal stresses are not known, the simplicity mentioned above disappears completely: on the contrary, the expression of the TG yield condition in terms of the stress components in either implicit (11.14) or, being theoretically possible in the explicit form as well (via trigonometric functions, with the use of the formulae given in Table 2), leads to highly complicated expressions. Certain approximative formulae were given by $\mathrm{Z}$. Waszczyszyn and M. Życzkowski [3.841] but even this approximation is complicated.

An even more serious deficiency of the TG yield condition is connected with its poor experimental verification. The experiments carried out by J. Guest in 1900 [2.46] were not sufficiently accurate. Actually no experimental results verify this hypothesis, particularly as regards the existence of corners on the initial neutral surface. This fact is recognized even by the advocates of the TG hypothesis; for example, D. D. Ivlev, who established certain extremal properties of the TG yield condition, [3.302], and supposed accordingly this condition to be generally valid, ascribed better results of the experimental verification of the HMH condition to 
some incidental factors. Several authors found the TG hypothesis useful when investigating the upper yield point (initial yield)-J. L. M. Morrison [3.539], O. A. Shishmarev [3.727], T. H. Lin, M. Ito [3.455], whereas the continuation of the plastic flow was governed by the HMH yield condition. Some authors suppose that the TG yield condition might better hold for monocrystals; however, for most polycrystalline structural materials the superiority of the HMH hypothesis is unquestionable.

It can easily be proved that the numerical differences-measured along the radius-between the results of the HMH and of the TG hypotheses do not exceed 15.5 per cent if referred to TG, or 13.4 per cent if referred to the HMH hypothesis. For some applications these differences are negligible and in the case of known principal directions the TG yield condition is used simply as an approximation. R. Hill [2.56] proposed in this case to replace the yield-point stress $\sigma_{0}$ by $1.077 \sigma_{0}$, and such a procedure reduces the approximation errors by a half. However, it should be remembered that these figures hold only for proportional (radial) loading; in the general case the final results depend on the adopted flow rule and the differences may be much larger. This problem will be discussed in detail in Sec. 13.7.

The generalizations of the TG yield condition to the case of anisosensitive materials, remaining within the class of cylindrical surfaces, were proposed by L. W. Hu [3.274], D. D. Ivlev [3.305] and M. Capurso [3.92]; they will be given together with their anisotropic generalization in Sec. 12. The notation generalizing (11.10) may be found in the paper by M. Sayir [3.700]. Another proposal, defined for plane stress only, was suggested by W. Prager [3.658].

A certain general cylindrical yield condition, including the HMH and the TG conditions as particular cases, was suggested by A. V. Hershey [3.243], and later by E. A. Davis [3.130], B. Paul [3.620] and W. F. Hosford [3.268]. These authors applied to plasticity the Bailey law, well-known in the theory of creep, writing

$$
\left|\sigma_{1}-\sigma_{2}\right|^{n}+\left|\sigma_{2}-\sigma_{3}\right|^{n}+\left|\sigma_{3}-\sigma_{1}\right|^{n}=2 \sigma_{0}^{n} .
$$

Equation (11.15) describes a cylindrical surface which for $n=2$ (and for $n=4$ ) coincides with the circular cylinder $\mathrm{HMH}$, within the interval $2<n<4$ lies outside that cylinder and for $n>4$ lies between the HMH cylinder and the TG prism. In the limiting case $n \rightarrow \infty$ we obtain a transition to the TG yield condition (the largest difference of principal stresses is the only one which remains); the singularities (corners) of the surface appear only in the limiting case $n \rightarrow \infty$. According to A. Hershey, particularly good coincidence with the experimental data may be obtained by assuming $n=6$.

Another hexagonal prism is described by the condition of maximal deviatoric stress

$$
\max \left[\left|\sigma_{1}-\sigma_{m}\right|,\left|\sigma_{2}-\sigma_{m}\right|,\left|\sigma_{3}-\sigma_{m}\right|\right]=\text { const }=\frac{2}{3} \sigma_{0} .
$$

Maximal deviatoric stress as a criterion of exertion was suggested first by R. Schmidt [3.704], 1932, but the yield condition of the form (11.16) was introduced later inde- 
pendently by A. Yu. Ishlinsky [3.297] and R. Hill [3.245], and developed by D. D. Ivlev [3.302] and R. M. Haythornthwaite [3.236]; we call it the Schmidt-IshlinskyHill yield condition. The corresponding limit curves in the deviatoric plane and in the plane $\sigma_{3}=0$ are shown in Figs. 41 and 42 as well. The results of experimental verification of the maximal deviatoric stress yield condition are rather poor, but its value lies in the upper estimation of the HMH condition. As an example of application let us mention the paper by D. D. Ivlev [3.309].

A cylindrical surface described by a linear combination of $J_{2 s}$ and $J_{3 s}$ was investigated by M. Sayir [3.700]. Because of the odd power of $J_{3 s}$ it describes anisosensitive materials. Sayir derived the equation

$$
2\left(\sigma_{0+}^{2}-\sigma_{0+} \sigma_{0-}+\sigma_{0-}^{2}\right) J_{2 s}+9\left(\sigma_{0+}-\sigma_{0-}\right) J_{3 s}=-\frac{2}{3} \sigma_{0+}^{2} \sigma_{0-}^{2}
$$

where $\sigma_{0+}$ and $\sigma_{0-}$ denote the (absolute) yield point stresses under tension and compression, respectively. For $\sigma_{0+}=\sigma_{0-}$ this surface reduces to the HMH circular cylinder. However, there is little chance of describing real plastically anisosensitive materials by cylindrical surfaces, e.g. (11.17), since uniaxial compression is here equivalent to uniform biaxial tension, and uniaxial tension-to uniform biaxial compression; such equivalence is practically never confirmed by experiments.

On the contrary, the cylindrical limit surfaces described by even functions of $J_{3 s}$ are restricted to isosensitive materials only. W. Prager [3.650], D. C. Drucker [3.145], F. D. Stockton and D. C. Drucker [3.769], A. M. Freudenthal and H. Geiringer [2.33] investigated the surfaces

$$
\begin{aligned}
& J_{2 s}^{3}+c_{1} J_{3 s}^{2} \equiv J_{2 s}^{3}\left[1-\frac{5 c_{1}}{27}\left(1+\cos 6 \omega_{\sigma}\right)\right]=A_{1}, \\
& J_{2 s}\left(1+c_{2} \frac{J_{3 s}^{2}}{J_{2 s}^{3}}\right) \equiv J_{2 s}\left[1-\frac{2 c_{2}}{27}\left(1+\cos 6 \omega_{\sigma}\right)\right]=A_{2} .
\end{aligned}
$$

For example, Prager proposed to describe the results of G. I. Taylor and H. Quinney [3.791] by (11.19) with $c_{2}=0.73$.

Other cylindrical limit surfaces were investigated by M. M. Filonenko-Boroditch [3.175], E. Ondraček [3.604], (with concave singularities), Z. Sobotka [3.751], J. L. Swedlow [3.778], A. M. Freudenthal and P. F. Gou [3.185].

\subsection{Rotationally symmetric limit surfaces}

The yield condition for a wide class of materials whose plastic behaviour depends on hydrostatic pressure may be described by equations of rotationally symmetric surfaces with the symmetry axis $\sigma_{1}=\sigma_{2}=\sigma_{3}$.

These equations may alternatively be written in one of the forms

$$
f\left(I_{1 \sigma}, I_{2 s}\right)=0, \quad f\left(Z_{\sigma}, R_{\sigma}\right)=0 \text { or } f\left(\sigma_{m}, \sigma_{e}\right)=0 .
$$

The second of these equations describes the generator of the rotational surfaces (axial section); the last one-the same generator in the Burzyński plane. 
The class of rotationally symmetric surfaces includes the Huber-Mises-Hencky circular cylinder, described above. The oldest hypothesis of the type under consideration was suggested by E. Beltrami (1885); he assumed total elastic energy as the criterion of exertion. This hypothesis has practically no experimental verification and has not been used in plasticity. F. Schleicher [3.701] generalized it, assuming that in the limit state the total elastic energy depends on the mean stress $\sigma_{m}$; the deficiency of Schleicher's proposal is the fact that in the case of isosensitivity it turns into the Beltrami instead of the HMH hypothesis.

The possibility of generalizing the HMH yield condition to the form $\sigma_{e}=f\left(\sigma_{m}\right)$ was first noticed in 1926 by R. Mises in the discussion of the Lode paper [2.104], and then by W. Lode [2.106]. However, the first effective formulation of such a hypothesis in a general form was given by W. Burzyński in 1928 [2.13, 3.87, 3.88]. Taking into account the simplicity of the final formulae, he restricted the general function $\sigma_{e}=f\left(\sigma_{m}\right)$ to a three-parameter condition

$$
A \sigma_{e}^{2}+B \sigma_{m}^{2}+C \sigma_{m}-1=0 .
$$

Assuming that the limit surface is determined by the following three tests: simple tension $\left(\sigma_{0+}\right)$, simple compression $\left(\sigma_{0_{-}}\right)$and simple shear $\left(\tau_{0}\right)$, we may evaluate the constants $A, B, C$; finally

$$
\frac{\sigma_{0+} \sigma_{0-}}{3 \tau_{0}^{2}} \sigma_{e}^{2}+\left(9-\frac{3 \sigma_{0+} \sigma_{0-}}{\tau_{0}^{2}}\right) \sigma_{m}^{2}+3\left(\sigma_{0-}-\sigma_{0+}\right) \sigma_{m}-\sigma_{0+} \sigma_{0-}=0 .
$$

This equation describes in the space of principal stresses an ellipsoid (if $3 \tau_{0}^{2}>\sigma_{0+} \sigma_{0-}$ ) or a hyperboloid (if $3 \tau_{0}^{2}<\sigma_{0+} \sigma_{0-}$ ), but some degenerated cases are also possible. In the boundary case (where the number of independent parameters is reduced to two); namely where

$$
\tau_{0}=\sqrt{\frac{\sigma_{0+} \sigma_{0-}}{3}}
$$

we obtain a paraboloid,

$$
\sigma_{e}^{2}+3\left(\sigma_{0-}-\sigma_{0+}\right) \sigma_{m}-\sigma_{0+} \sigma_{0-}=0,
$$

whereas in another particular case

$$
\tau_{0}=\frac{2}{\sqrt{3}} \frac{\sigma_{0+} \sigma_{0-}}{\sigma_{0+}+\sigma_{0-}},
$$

we obtain (after extracting the root) a circular cone,

$$
\sigma_{e}+3 \frac{\sigma_{0-}-\sigma_{0+}}{\sigma_{0-}+\sigma_{0+}} \sigma_{m}-2 \frac{\sigma_{0+} \sigma_{0-}}{\sigma_{0+}+\sigma_{0-}}=0 .
$$

If $\sigma_{0+}=\sigma_{0-}=\sigma_{0}$ and $\tau_{0}=\sigma_{0} / \sqrt{3}$, then the general equation (11.22) turns into the HMH circular cylinder. The relevant limit curves in the Burzyński plane are shown in Fig. 44. 


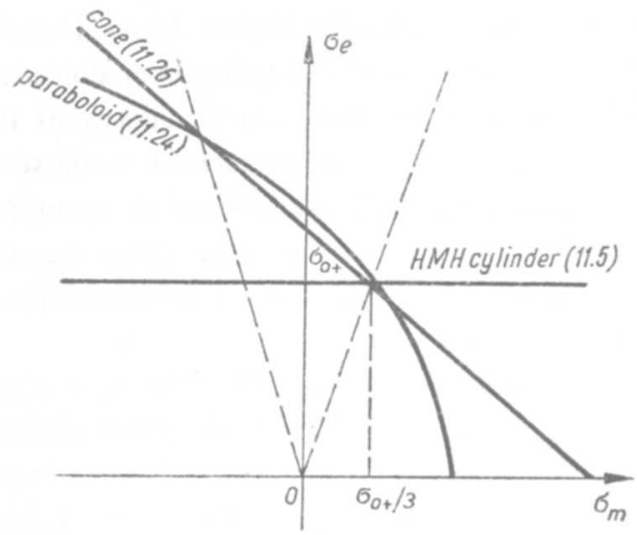

Fig. 44. Particular cases of the Burzyński limit surface

The general equation (11.22) or its particular cases were later proposed-more or less independently - by many authors. Equation (11.22) was suggested in 1931 by Yu. I. Yagn [3.849], in 1938 by L. Rendulic [3.670], in 1954 by M. M. FilonenkoBoroditch [3.175], in 1960 by M. Matsuura [3.511], in 1963 by L. W. Hu and K. D. Pae [3.278] and in 1970 by J. G. Merkle [3.520]. The equation of the paraboloid (11.24) was suggested in 1930 by G. D. Sandel [3.693], in 1937 by P. P. Balandin [3.28], in 1947 by C. Torre [3.812, 3.813, 3.814, 3.815] (partially with reference to Sandel), in 1951 by F. Stassi d'Alia [3.764] (with the additional effect of temperature taken into account), in 1958 by G. A. Genev [3.195], in 1967 by H. H. Le Boiteux [3.414], in 1973 by M. Hartzmann [3.227]. The equation of the cone (11.26) was suggested in 1934 by Z. Klębowski [3.349], in 1938 by N. M. Belyayev (before the USSR Congress on Elasticity and Plasticity), in 1940 by A. I. Botkin [3.74], in 1941 by S. V. Serensen [3.707], in 1952 by D. C. Drucker and W. Prager [3.147], in 1953 by I. N. Mirolyubov [3.530] (who also investigated the general case (11.22)), in 1958 by S. A. Bernshteyn [3.56]. The proposals of H. Hencky [3.242], G. A. Genev [3.194] and Z. Sobotka [3.750] also reduce to particular cases of the Burzyński surface (11.22). Z. Klębowski proposed to approximate the general surface of revolution by two cones and a cylinder [3.351], or by paraboloid (11.22) truncated partially by a cone (11.26), [3.350].

A certain new physical justification of the Burzyński and Schleicher hypotheses was given by J. Zawadzki [3.862]. Experimental verification of Eq. (11.26) for concrete was performed by B. Bresler and K. S. Pister [3.76], and of Eq. (11.24) for cast iron by N. Alberti [3.3]. Many experimental investigations of the yield conditions for soils are presented in the extensive survey by V. N. Nikolayevsky [3.578]. However, in the case of brittle materials one has to be particularly cautious in evaluating experimental data, since some of them refer to yielding and others to various forms of fracture.

The Burzyński quadratic equation (11.22) constitutes the most convenient form of the relation $f\left(\sigma_{m}, \sigma_{e}\right)=0$. Some authors also propose polynomials of a higher 
degree. The parabolic equation, proposed by $\mathrm{R}$. Mises [3.532] and generalized to the three-dimensional states by A. M. Freudenthal and H. Geiringer [2.33], is of the fourth degree. W. Olszak [3.598] proposed a paraboloid of the $n$th degree. K. K. Shkerbelis [3.730] suggested a linear combination of arbitrary powers of $\sigma_{m}$ and $\sigma_{e}$; four parameters are then to be evaluated.

Some authors call the non-cylindrical yield conditions "conditions for plastically compressible bodies". It should be noted, however, that this conclusion is connected with the associated flow rule (defined in Sec. 13), which does not hold in general, and so the non-cylindrical yield conditions may describe plastically incompressible bodies as well.

\subsection{Other limit surfaces in the space of principal stresses}

Limit surfaces corresponding to most classical hypotheses of failure (Galileo, Clebsch, de Saint-Venant, Poncelet) are neither cylindrical nor rotationally symmetric. Those hypotheses, describing rather the strength of brittle bodies, are very seldom used in the theory of plasticity. On the other hand, the hypothesis of C. A. Coulomb and O. Mohr [3.536, 3.537], generalizing that of Tresca-Guest, has been developed in many papers and applied to various problems of plasticity. The original hypothesis (in the linearized version) is represented in the space of principal stresses by a hexagonal pyramide whose cross-sections are symmetric but non-regular hexagons (R. T. Shield [3.720], W. M. Kirkpatrick [3.345]). A pyramide with regular hexagonal cross-sections was introduced by G. D. Sandel [3.692], N. N. Davidenkov [3.127], D. C. Drucker [3.148] and I. I. Tarasenko [3.789]. Other alterations were proposed by A. Leon [3.439], S. D. Volkov [3.836] and Y. Hara [3.224]. A detailed analysis of the cross-sections of the Coulomb-Mohr surface is given by B. Paul [3.620].

A wide class of materials may be described by the limit surfaces of the type

$$
R_{\sigma}=f_{1}\left(Z_{\sigma}\right) f_{2}\left(\omega_{\sigma}\right) \text { or } \sigma_{e}=f_{1}\left(\sigma_{m}\right) f_{2}\left(\omega_{\sigma}\right) .
$$

In the particular case $f_{2}\left(\omega_{\sigma}\right)=$ const we obtain rotationally symmetric surfaces described above, whereas $f_{1}\left(Z_{\sigma}\right)=$ const or $f_{1}\left(\sigma_{m}\right)=$ const leads to cylindrical surfaces. Three-parameter yield conditions of the type (11.27) were proposed by J. Murzewski and Z. Mendera [3.565]

$$
R_{\sigma}=\left(A-B Z_{\sigma}\right)\left(1+C \cos 6 \omega_{\sigma}\right),
$$

by G. Gudehus (with two parameters only) [3.218]

$$
\sigma_{e}=-A \sigma_{m} \sqrt{1-B \cos 3 \omega_{\sigma}}
$$

and by G. A. Genev and V. N. Kissyuk [3.196]

$$
\sigma_{e}^{2}=\left(3 A \sigma_{m}+B\right)\left[1-(1-C)\left(1-\cos 3 \omega_{\sigma}\right)\right] ;
$$

the first and the second generalize a cone and the third a paraboloid. A similar form of the limit surface is also assumed by R. M. Haythorntwaite [3.235] though no analytical description is given. 
Various authors suggest yield conditions whose form is even more complicated than (11.23); let us mention here the five-parameter equation proposed by J. Majer [3.479]

$$
\sigma_{e}=\left(C-\sigma_{m}\right)^{n+p \cos 3 \omega_{\sigma}}\left(A+B \cos 3 \omega_{\sigma}\right) .
$$

Z. Sobotka [3.755, 3.757, 3.758] introduced a tensor called the "material tensor", namely $\sigma_{i j}-\chi \sigma_{k k} \delta_{i j}$ where $\chi$ is a constant, and expressed the yield condition in terms of invariants of that tensor. His quadratic yield condition reduces to (11.22), but conditions of higher degrees introduce the third invariant and are not rotationally symmetric. Other yield conditions joining all the three invariants were proposed by K. Hashiguchi [3.228], M. Maitra, K. Majumdar and A. Das [3.478]. J. B. Haddow and T. M. Hrudey [3.220] derived a yield condition applicable in the case of finite elastic volume change (large hydrostatic pressures).

The problem of yield conditions for non-homogeneous bodies was analysed in detail in the papers by W. Olszak and W. Urbanowski [3.595, 3.596, 3.597], and by W. Olszak, J. Rychlewski and W. Urbanowski [3.599].

\subsection{General form of the "ad hoc" elliptic yield condition}

In most cases of combined loadings of cross-section of bars (tension with torsion, bending with torsion, etc.), the relevant state of stress may be characterized by one normal component $\sigma_{x}=\sigma$ and by one tangential component $\tau_{x y}=\tau$. For such a case the following yield condition is frequently suggested:

$$
\left(\frac{\sigma}{\sigma_{0}}\right)^{2}+\left(\frac{\tau}{\tau_{0}}\right)^{2}=1,
$$

or, briefly,

$$
\sigma^{2}+c \tau^{2}=\sigma_{0}^{2},
$$

where $c=\sigma_{0}^{2} / \tau_{0}^{2}$. These equations may be found in most practically oriented engineering manuals, but are used also in general plasticity (K. Hohenemser, W. Prager [3.263], A. M. Zhukov [3.868], Y. Ohashi, M. Tokuda [3.591]). For the case under consideration this "elliptic" yield condition generalizes the HMH yield condition $(c=3)$ and the TG yield condition $(c=4)$, and may better fit to experimental data; on the other hand, this condition is proposed "ad hoc", for one $\sigma$ and for one $\tau$ only, without any general form valid for the three-dimensional state of stress. It turns out that such a general form is not unique; in what follows we derive a oneparameter family of general yield conditions reducing to (11.33) for one component $\sigma$ and one component $\tau$.

First let us look for a rotationally-symmetric limit surface, expressed in terms of $I_{1 \sigma}$ and $I_{2 s}$. For one $\sigma$ and one $\tau$ we have

$$
I_{1 \sigma}=\sigma, \quad I_{2 s}=\frac{2}{3} \sigma^{2}+2 \tau^{2} ;
$$


inverting these relations with respect to $\sigma$ and $\tau$ and introducing into (11.33), we obtain the required general equation

$$
\frac{1}{2} c I_{2 s}+\left(1-\frac{1}{3} c\right) I_{1 \sigma}^{2}=\sigma_{0}^{2} .
$$

Equation (11.35) describes an ellipsoid or a hyperboloid in the Haigh-Westergaard space; for $c=3$ this surface turns into the $\mathrm{HMH}$ circular cylinder.

The derivation of equation of a cylindrical limit surface, reducing to (11.33), is slightly more difficult. Such an equation will be expressed in terms of $I_{2 s}$ and $I_{3 s}$. For one $\sigma$ and one $\tau$ (with one common index) the third deviatoric invariant equals

$$
I_{3 s}=\frac{2}{9} \sigma^{3}+\sigma \tau^{2},
$$

and the inversion of the formulae for $I_{2 s}$ and $I_{3 s}$ with respect to $\sigma$ and $\tau$ presents difficulties (solution of a cubic equation). Hence we combine the formula for $I_{2 s}$ with (11.33), calculate $\sigma$ and $\tau$,

$$
\sigma^{2}=\frac{3}{c-3}\left(\frac{1}{2} c I_{2 s}-\sigma_{0}^{2}\right), \quad \tau^{2}=\frac{1}{c-3}\left(\sigma_{0}^{2}-\frac{3}{2} I_{2 s}\right),
$$

and later substitute into (11.36), obtaining

$$
3(c-3)^{3} I_{3 s}^{2}=\left(\frac{1}{2} c I_{2 s}-\sigma_{0}^{2}\right)\left[\left(c-\frac{9}{2}\right) I_{2 s}+\sigma_{0}^{2}\right]^{2} .
$$

For $c=3 \mathrm{Eq}$. (11.38) turns into the HMH cylinder (11.4), and for $c=4$ into the TG hexagonal prism (11.14).

To obtain the general form of the yield condition in question we combine (11.37) with the similar solution of the first Eq. (11.34) and (11.33), writing

$$
\begin{aligned}
\sigma^{2} & =\frac{3 \psi}{c-3}\left(\frac{1}{2} c I_{2 s}-\sigma_{0}^{2}\right)+(1-\psi) I_{1 \sigma}^{2}, \\
\tau^{2} & =\frac{\psi}{c-3}\left(\sigma_{0}^{2}-\frac{3}{2} I_{2 s}\right)+(1-\psi) \frac{\sigma_{0}^{2}-I_{1 \sigma}^{2}}{c},
\end{aligned}
$$

where $\psi$ is a parameter with arbitrary real value. Substituting now (11.39) into (11.36) we obtain the required equation of the one-parameter family of limit surfaces, generalizing (11.33) to three-dimensional state of stress:

$$
\begin{aligned}
(c-3)^{3} I_{3 s}^{2}= & {\left[3 \psi\left(\frac{1}{2} c I_{2 s}-\sigma_{0}^{2}\right)+(1-\psi)(c-3) I_{1 \sigma}^{2}\right]\left\{\frac{1}{3} \psi\left[\left(c-\frac{9}{2}\right) I_{2 s}+\sigma_{0}^{2}\right]+\right.} \\
& \left.+\frac{(1-\psi)(c-3)}{c}\left[\left(\frac{2}{9} c-1\right) I_{1 \sigma}^{2}+\sigma_{0}^{2}\right]\right\}^{2} .
\end{aligned}
$$

Indeed, substituting here $\psi=1$ we obtain (11.38), substituting $\psi \rightarrow \infty$ we obtain (11.35), whereas $\psi=0$ gives a combination of $I_{1 \sigma}$ and $I_{3 s}$ (probably the latter case is without any practical value).

\subsection{The influence of accessory exertion factors}

Such quantities as temperature, humidity, intensity of neutron irradiation, etc., called in Sec. 1.6 accessory exertion factors, may essentially affect the yielding of the 
material. In the simplest treatment, writing the yield condition in the form $\sigma_{\text {red }}=\sigma_{0}$, we express the influence of such factors only through the dependence of $\sigma_{0}$ on them. Such an approach may result in considerable errors: for example, a temperature increase usually affects yield stress in tension $\sigma_{0+}$ and yield stress in compression $\sigma_{0-}$ in a different manner (as a rule brings them closer together if they were different at room temperature).

Another approach to the temperature effect was suggested by F. Stassi d'Alia $[3.764,3.765,3.766,3.768]$; he generalized the equation of the Burzyński paraboloid (11.24), introducing into it a certain function of temperature $\alpha(T)$, where $\alpha=1$ at room temperature. The Stassi d'Alia equation will first be written in the form

$$
\sigma_{e}^{2}+3 \alpha\left(\sigma_{0-}-\alpha \sigma_{0+}\right) \sigma_{m}-\alpha^{3} \sigma_{0+} \sigma_{0-}=0,
$$

where $\sigma_{0+}$ and $\sigma_{0-}$ denote the yield stresses at room temperature. Introducing the notation

$$
\frac{\sigma_{0-}}{\sigma_{0+}}=x,
$$

writing briefly ${ }^{\circ} \sigma_{0+}=\sigma_{0}$ and expressing the invariants $\sigma_{m}$ and $\sigma_{e}$ in terms of the stress components, we obtain

$$
\begin{gathered}
\sigma_{x}^{2}+\sigma_{y}^{2}+\sigma_{z}^{2}-\sigma_{x} \sigma_{y}-\sigma_{y} \sigma_{z}-\sigma_{z} \sigma_{x}+3\left(\tau_{x y}^{2}+\tau_{y z}^{2}+\tau_{z x}^{2}\right)+ \\
+\alpha(\varkappa-\alpha) \sigma_{0}\left(\sigma_{x}+\sigma_{y}+\sigma_{z}\right)-\alpha^{3} \varkappa \sigma_{0}^{2}=0 .
\end{gathered}
$$

The above yield condition takes the temperature effect into account in a oneparameter manner, namely, via the function $\alpha(T)$. However, this method is questionable. Namely, considering uniaxial stress states, we arrive at the relation

$$
\sigma^{2}+\alpha(\varkappa-\alpha) \sigma_{0} \sigma-\alpha^{3} \varkappa \sigma_{0}^{2}=0,
$$

and hence, solving this quadratic equation with respect to $\sigma$, we find the following dependence of the yield stresses $\sigma_{0+}(T)$ and $\sigma_{0-}(T)$ on temperature:

$$
\sigma_{0+}(T)=\alpha^{2}(T) \sigma_{0}, \quad \sigma_{0-}(T)=\alpha(T) \varkappa \sigma_{0} .
$$

With rising temperature the function $\alpha(T)$ decreases for most materials and in view of $x>1$ it follows from (11.45) that the ratio $\sigma_{0-}(T) / \sigma_{0+}(T)$ shows an increase instead of the decrease observed in experiments. In the case of the parabolic yield condition under consideration it seems necessary to introduce a two-parameter dependence on temperature. The Stassi d'Alia yield condition was also criticized by A. A. Lebedev [3.413].

An extensive experimental investigation of the yield surface in the space $\sigma-\tau-T$ for aluminium was performed by A. Phillips, C. S. Liu and J. W. Justusson [3.636]. They approximated this surface by a truncated elliptic cone with the axis $T$; isosensitivity was retained at elevated temperature. Other experiments of this type were done by P. M. Ogibalov [3.587], A. A. Lebedev and G. S. Pisarenko [3.411], N. S. Mozharovsky and E. A. Antipov [3.550]. Numerous results of uniaxial experiments at various temperatures are presented in the books by P. M. Ogibalov and I. A. Kiyko 
[3.589], P. F. Koshelev and S. Ye. Belayev [3.377], V. L. Bazhanov, I. I. Goldenblat, N. A. Nikolayenko and A. M. Sinyukov [3.43], and in the paper by J. J. Jonas [3.317].

The effect of neutron irradiation on the plastic behaviour of materials may be very significant. G. V. Dienes [3.134] observed over 17 times greater yield stress in shear for copper monocrystals if subject to an irradiation dose of $6 \times 10^{18}$ neutrons $/ \mathrm{cm}^{2}$; J. C. Wilson and R. G. Berggren [3.847] investigated austenitic steel finding that at $7.8 \times 10^{19}$ neutrons $/ \mathrm{cm}^{2}$ the yield stress is doubled with a simultaneous increase of the difference between the upper and lower yield points. Multiaxial experiments were carried out by T. D. Dudderar and J. Duffy [3.159]. Many experimental results are quoted in the survey papers by V. S. Lensky [3.432], W. Olszak, J. Rychlewski and W. Urbanowski [3.599].

Some authors express their criteria in terms of strains. G. A. Doshtchinsky ascribes the criterion of exertion to the invariant $I_{2 \varepsilon}$ [3.137] or to the linear combination of $I_{1 \varepsilon}$ and $\sqrt{I_{2 \varepsilon}}$ [3.138]; such an approach, when combined with Hooke's law, introduces Poisson's ratio to equations in the space of principal stresses. E. H. Mansfield [3.497] investigates the case of a circle in the plane $\varepsilon_{1}-\varepsilon_{2}$. Many remarks concerning the influence of accessory exertion factors on the plastic behaviour of materials may be found in the books by I. I. Goldenblat and V. A. Kopnov [3.205] and by G. S. Pisarenko and A. A. Lebedev [3.643].

\subsection{The range of applicability of the yield conditions}

In the preceding sections we presented the equations of various yield conditions without stating the limits of their applicability. In fact, very seldom can one assume that they describe yield conditions within the whole domain of determinacy. In certain states of stress, especially in those close to uniform triaxial tension, depending also on the accessory exertion factors, decohesion may precede any significant plastic deformation.

This fact was first noticed by A. J. Becker [3.44], who proposed-in the particular case of plane stress-the limitation of the Tresca-Guest condition by the additional condition of rupture based on the maximal strain hypothesis of de Saint-Venant and Poncelet. H. M. Westergaard [2.190] attempted to generalize this concept to the general three-dimensional case, but a physically correct hypothesis of this type was given only by N. N. Davidenkov and Ya. B. Fridman [3.187]. A similar limitation for the HMH yield condition was proposed by T. Pełczyński [2.127]. The corresponding limit surfaces in the space of principal stresses are then the TG hexagonal prism and the $\mathrm{HMH}$ circular cylinder with cut-offs by three planes of constant elongation (the sharpened pencils hypotheses), Fig. 45.

Several authors propose to perform the cut-offs corresponding to constant normal stresses, and hence they limit the yield conditions by the Galileo hypothesis. D. C. Drucker [3.148] suggests the limitation of the regular hexagonal pyramide 


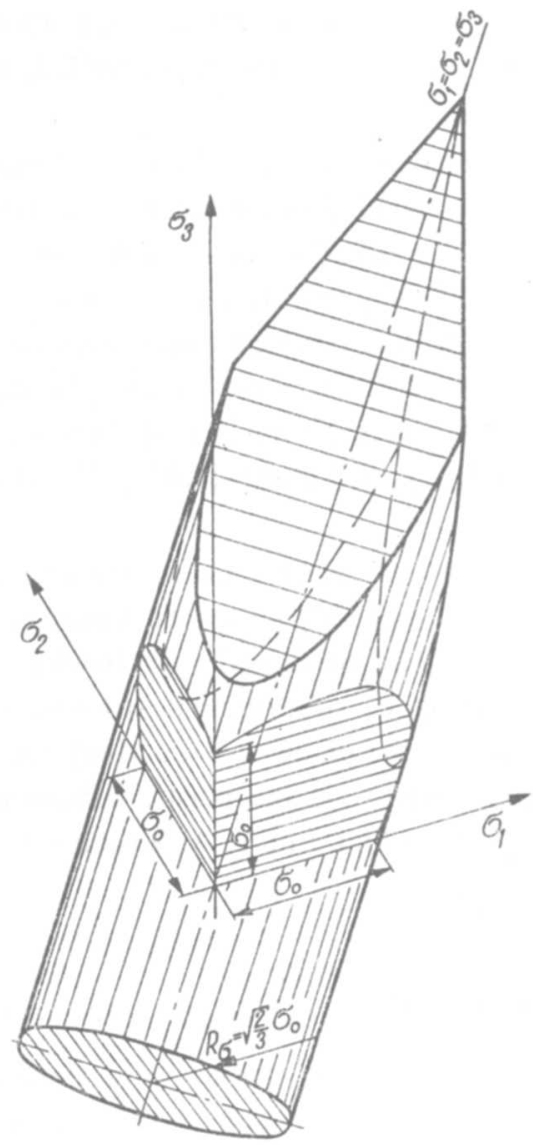

Fig. 45. A cylindrical limit surface with cut-offs (Pelczyński)

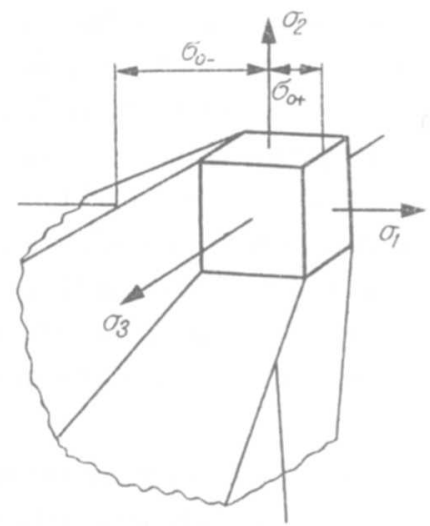

Fig. 46. A pyramidal limit surface with cut-ofts (Paul) 
by zero normal stresses, whereas B. Paul [3.618] admits a certain positive value of $\sigma_{I}$ (Fig. 46).

The above sharp limitations of the range of validity of particular yield conditions seem reasonable for monocrystals. However, for a polycrystalline aggregate the limitations take rather a mild form. So, once more, we may go back to a uniform description of the limit surface part of which is connected predominantly with yielding and part-predominantly with rupture. A hypothesis of this type was suggested by A. A. Lebedev [3.412]; it has the form

$$
A \sigma_{e}^{\alpha}+B \sigma_{I}^{\beta}=1
$$

where the four parameters $A, B, \alpha, \beta$ are to be determined experimentally. Lebedev suggested $\alpha=\beta=2$ for a wide class of materials. This concept was further developed by V. A. Kuz'menko [3.408].

Numerous authors apply a probabilistic approach to the problems of yielding and fracture; we mention here only the papers by S. D. Volkov [3.836] and J. Murzewski [3.564].

In any case it should be remembered that the use of particular yield conditions within their whole domain of determinacy may lead to a risky extrapolation without any experimental evidence.

\section{Conditions of perfect plasticity for anisotropic bodies}

\subsection{Generalizations of the Huber-Mises-Hencky yield condition}

Conditions of perfect plasticity for anisotropic bodies are usually formulated as certain generalizations of the relevant conditions for isotropic bodies. Therefore they will be classified here according to the types of isotropic conditions subject to generalization.

Generalization of the $\mathrm{HMH}$ yield condition may be based either on purely mathematical considerations or on physical (energy) arguments. Following the first way, we look for a general quadratic homogeneous function of stress components, which should be constant in the limit state. The plastic properties of the material depend on the direction, but the expression as a whole must be invariant with respect to the reference frame. Such an expression is obtained by the introduction of the fourth-order tensor of the plastic moduli $\Pi_{i j k l}$, namely

$$
\Pi_{i j k l} \sigma_{i j} \sigma_{k l}=1 \text {, }
$$

where the moduli satisfy the symmetry conditions $\Pi_{i j k l}=\Pi_{k l i j}=\Pi_{j i k l}=\Pi_{i j l k} ; 21$ of such moduli are independent. Equation (12.1) was suggested in 1928 by R. Mises [3.531]. If we require, in addition, the yielding of the material to be independent of the mean stress $\sigma_{m}$, then the number of the moduli $\Pi$ reduces to 15 . In both cases the material under consideration is isosensitive, since the simultaneous change of signs of all the stresses in (12.1) does not change the whole expression. 
The derivation of (12.1) in the case of independence of $\sigma_{m}$, given by R. Mises, was criticized by G. F. Smith [3.744], who proposed a modified form of this condition.

Equation (12.1) takes a much simpler form in the case of orthogonal anisotropy, called briefly orthotropy. Then there exist three mutually perpendicular principal directions of the tensor $\Pi_{i j k l}$. Choosing the reference frame $x y z$ in such a way as to obtain its coincidence with the principal axes of orthotropy, we eliminate 9 terms of the type $\sigma_{x} \tau_{x y}$ and 3 terms of the type $\tau_{x y} \tau_{y z}$ and only the following 9 terms remain:

$$
\begin{aligned}
\Pi_{1111} \sigma_{x}^{2} & +\Pi_{2222} \sigma_{y}^{2}+\Pi_{3333} \sigma_{z}^{2}+2 \Pi_{1122} \sigma_{x} \sigma_{y}+2 \Pi_{2233} \sigma_{y} \sigma_{z}+ \\
& +2 \Pi_{3311} \sigma_{z} \sigma_{x}+4 \Pi_{1212} \tau_{x y}^{2}+4 \Pi_{2323} \tau_{y z}^{2}+4 \Pi_{3131} \tau_{z x}^{2}=1 .
\end{aligned}
$$

Replacing the indicial notation by the engineering notation, we labelled the moduli by the indices $1,2,3$ to point out the principal directions of the tensor $\Pi_{i j k l}$.

If we require the independence of (12.2) of the mean stress $\sigma_{m}$, then the number of plastic moduli reduces to 6. This case was examined by R. Hill [3.244], L. R. Jackson, K. F. Smith and W. T. Lankford [3.314]; in Hill's notation (12.2) takes the form

$$
F\left(\sigma_{y}-\sigma_{z}\right)^{2}+G\left(\sigma_{z}-\sigma_{x}\right)^{2}+H\left(\sigma_{x}-\sigma_{y}\right)^{2}+2 L \tau_{y z}^{2}+2 M \tau_{z x}^{2}+2 N \tau_{x y}^{2}=1 .
$$

The moduli $F, G, H, L, M, N$ may be expressed in terms of the yield-point stresses in tension (or compression) in the directions $x, y, z$, which will be denoted by $\sigma_{0 x}, \sigma_{0 y}$, $\sigma_{0 z}$, and by the yield-point stresses in shear $\tau_{0 x y}, \tau_{0 y z}, \tau_{0 z x}$. One can easily derive the relations

$$
\begin{array}{ll}
F=\frac{1}{2}\left(\frac{1}{\sigma_{0 y}^{2}}+\frac{1}{\sigma_{0 z}^{2}}-\frac{1}{\sigma_{0 x}^{2}}\right), & L=\frac{1}{2 \tau_{0 y z}^{2}}, \\
G=\frac{1}{2}\left(\frac{1}{\sigma_{0 z}^{2}}+\frac{1}{\sigma_{0 x}^{2}}-\frac{1}{\sigma_{0 y}^{2}}\right), & M=\frac{1}{2 \tau_{0 z x}^{2}}, \\
H=\frac{1}{2}\left(\frac{1}{\sigma_{0 x}^{2}}+\frac{1}{\sigma_{0 y}^{2}}-\frac{1}{\sigma_{0 z}^{2}}\right), & N=\frac{1}{2 \tau_{0 x y}^{2}} .
\end{array}
$$

In the particular case of plane stress $\sigma_{z}=\tau_{z x}=\tau_{z y}=0$ we obtain the four-parameter equation

$$
\frac{\sigma_{x}^{2}}{\sigma_{0 x}^{2}}+\frac{\sigma_{y}^{2}}{\sigma_{0 y}^{2}}-\left(\frac{1}{\sigma_{0 x}^{2}}+\frac{1}{\sigma_{0 y}^{2}}-\frac{1}{\sigma_{0 z}^{2}}\right) \sigma_{x} \sigma_{y}+\frac{\tau_{x y}^{2}}{\tau_{0 x y}^{2}}=1 .
$$

In the particular case of plane strain $\varepsilon_{z}=\gamma_{z x}=\gamma_{z y}=0$ the result depends on the physical law; the additional assumption of incompressibility leads-according to most physical laws- to $\sigma_{z}=\frac{1}{2}\left(\sigma_{x}+\sigma_{y}\right)$, and the final result may be written in the two-parameter form

$$
\left(\sigma_{x}-\sigma_{y}\right)^{2}+4(1-c) \tau_{x y}^{2}=4(1-c) \tau_{x y 0}^{2}
$$


where the dimensionless constant $c$ equals

$$
c=1-\frac{1}{\left(\frac{2}{\sigma_{0 x}^{2}}+\frac{2}{\sigma_{\theta y}^{2}}-\frac{1}{\sigma_{0 z}^{2}}\right) \tau_{0 x y}^{2}} .
$$

For the case of plane stress some authors propose conditions simpler than (12.5). Such simplifications are justified if there is a plane of isotropy. For example, if this plane coincides with $x y$, then $\sigma_{0 x}=\sigma_{0 y}=\sigma_{0}$ and instead of (12.5) we may write (W. F. Hosford, W. A. Backofen [3.267], for principal directions)

$$
\sigma_{1}^{2}+\sigma_{2}^{2}-\frac{2 R}{1+R} \sigma_{1} \sigma_{2}=\sigma_{0}^{2} \quad\left(R=-1+2 \sigma_{0 z}^{2} / \sigma_{0}^{2}\right) .
$$

If it coincides with $x z$, then we obtain [3.643],

$$
\frac{\sigma_{x}^{2}}{\sigma_{0 x}^{2}}+\frac{\sigma_{y}^{2}}{\sigma_{0 y}^{2}}-\frac{\sigma_{x} \sigma_{y}}{\sigma_{0 y}^{2}}+\frac{\tau_{x y}^{2}}{\tau_{0 x y}^{2}}=1 .
$$

Another particular case of (12.5) was investigated by L. W. Hu and J. Marin [3.272].

Of course, notation (12.8) does not show any anisotropy of the material; it is hidden, since only a particular case of the stress state is considered. This fact may cause certain misunderstandings (M. Sayir [3.700]).

The generalization of the $\mathrm{HMH}$ yield condition to the case of anisotropic bodies may also be achieved by using energy considerations if we postulate the constancy of the distortion energy at the boundary of the elastic range and then express this energy in terms of stresses. In the most general case of anisotropy the elastic energy cannot be decomposed into the energy of volume change and energy of shape change. This problem was first investigated by W. Burzyński [2.13], who proved that the existence of such a decomposition results in five relations between the elastic moduli, and thus only 16 moduli remain independent. The energy approach to the derivation of quadratic yield conditions for anisotropic bodies was used by I. E. Mileykovsky [3.529], K. Klöppel and M. Yamada [3.354], C. I. Borș [3.73] and Z. Sobotka [3.749, 3.752, 3.754].

The generalization of the $\mathrm{HMH}$ yield condition to non-homogeneous anisotropic materials was given by W. Olszak and W. Urbanowski [3.595, 3.596, 3.603].

The Mises-Hill yield condition was verified experimentally for many materials. A. M. Zhukov [3.863] proved its validity for the metastable aluminium alloy D16T; E. K. Ashkenazi [3.16] found it valid for small anisotropy but observed larger differences in case of essentially anisotropic materials (timber). A. N. Bramley and P. B. Mellor [3.75] determined the Hill constants for four killed steels and J. Klepaczko [3.348] - for pure aluminium sheets; on the other hand, J. Woodthorpe and R. Pearce [3.848] did not confirm the Mises-Hill yield condition for aluminium sheets. F. P. Belyankin, V. F. Yatsenko and G. G. Margolin [3.47] verified (12.5) for plastics (KAST-V). S. P. Yakovlev and V. F. Kuzin [3.852] give the anisotropic constant $c$ in (12.7) for steels 08KP and OCh18N9T, two aluminium alloys and brass, finding $c>0$ in the first case and $c<0$ in the remaining cases. A. Troost and J. Betten 
[3.817] proposed to evaluate the Hill constants by the measurements of the coefficients of transverse deformation. G. S. Kular and M. J. Hillier [3.402] analysed the results of various experiments on anisotropic yielding and proposed a certain general method of evaluation of the Hill constants; however, they stated that for some materials (12.3) is inadequate. Many experimental data are compiled and presented graphically in a handbook by E. K. Ashkenazi and E. V. Ganov [3.17].

Some authors considered the limits of applicability of the Mises-Hill yield condition. A. M. Zhukov [3.863] applied in this case the maximal strain (de SaintVenant) hypothesis, generalizing in this manner the Pełczyński idea. W. Warkenthin [3.839] expressed the limitations by means of maximal normal stresses.

\subsection{Generalizations of the Tresca-Guest yield condition}

The generalization of the Tresca-Guest yield condition to anisotropic materials is very simple but only in a very particular case: if general anisotropy is restricted to orthotropy and the principal directions of the stress state and of orthotropy coincide. The corresponding system of six linear equations, taking into account the possible anisosensitivity of the material, was derived by L. W. Hu [3.274], and then by D. D. Ivlev [3.305] and M. Capurso [3.92]:

$$
\begin{array}{r}
\frac{\sigma_{1}}{\sigma_{01+}}-\left(\frac{1}{\sigma_{01+}}-\frac{1}{\sigma_{03-}}\right) \sigma_{2}-\frac{\sigma_{3}}{\sigma_{03-}}=1, \\
-\frac{\sigma_{1}}{\sigma_{01-}}+\left(\frac{1}{\sigma_{01-}}-\frac{1}{\sigma_{03+}}\right) \sigma_{2}+\frac{\sigma_{3}}{\sigma_{03+}}=1,
\end{array}
$$

Cyclic permutation must be used here both for stresses and for plastic moduli. The symbol $\sigma_{01+}$ denotes yield-point stress in tension in the direction "1", etc. Other generalizations of the TG yield condition to anisotropic bodies may be found in the papers by I. Berman and P. G. Hodge [3.55], A. Sawczuk [3.697], and by B. R. Seth [3.708, 3.709].

The generalization of the TG yield condition to arbitrarily anisotropic but isosensitive body and for arbitrary principal directions of stresses was given by M. Sayir [3.700]. He simply used the symmetric form (11.14) putting instead of $J_{2 s}$ and $J_{3 s}$ the following common invariants of the stress deviator and of the tensors of plastic moduli $\Pi$ :

$$
Q_{2}=\Pi_{l j k l} s_{i j} s_{k l}, \quad Q_{3}=\Pi_{i j k l m n} s_{i j} s_{k l} s_{m n} .
$$

The number of moduli, however, is very large $(15+35+1=51)$, and any practical use of (11.14) with substituted (12.11) seems impossible.

A certain generalization of the Burzyński-Prager-Drucker yield condition (11.26) to plane stress in an anisotropic material was proposed by L. W. Hu and J. Marin [3.272]. Another generalization of a cylindrical limit surface was proposed by L. A. Tolokonnikov, S. P. Yakovlev and V. M. Lyalin [3.809, 3.810]. They investigated the surface

$$
\Pi_{i j k l} s_{i j} s_{k l}=f\left(\cos 3 \omega_{\sigma}\right),
$$


confining the left-hand side to the Hill expression (12.3); the "isotropic" invariant $\omega_{\sigma}$ is defined by $(5.10)$.

The above-mentioned generalizations of the TG yield condition correspond to a phenomenological approach for a polycrystalline material. The relevant theory for a monocrystal, initiated by E. Schmidt and called the "critical shear stress law" $[3.702,3.703]$, was recently developed by Yu. I. Sirotin [3.741].

\subsection{Generalizations of the Burzyński yield condition}

A certain generalization of the rotationally symmetrical Burzyński limit surface (11.21) to the case of anisotropy was proposed in 1955 by I. I. Goldenblat [3.201]. Further generalization by I. I. Goldenblat and V. A. Kopnov [3.203, 3.204, 3.205] resulted in the form

$$
\left(\Pi_{i j} \sigma_{i j}\right)^{\alpha}+\left(\Pi_{i j k l} \sigma_{i j} \sigma_{k l}\right)^{\beta}+\left(\Pi_{i j k l m n} \sigma_{i j} \sigma_{k l} \sigma_{m n}\right)^{\gamma}+\ldots=1,
$$

where $\alpha, \beta, \gamma, \ldots$ are arbitrary exponents, but the authors proposed to assume $\alpha=1, \beta=1 / 2, \gamma=1 / 3, \ldots$ (then the homogeneity of the function on the left-hand side is assured). The yield condition (12.13) in the case of isotropic bodies reduces to an even more general form than (11.21). However, the authors discussed in detail the case

$$
\Pi_{i j} \sigma_{i j}+\sqrt{\Pi_{i j k l} \sigma_{i j} \sigma_{k l}}=1,
$$

which presents a direct generalization of (11.21). The monograph [3.204] gives the method of experimental evaluation of the moduli $\Pi_{i j}$ and $\Pi_{i j k l}$ and many working formulae for particular cases.

Similar but less general suggestions were given by J. Marin [3.508], T. Ota, A. Shindo, H. Fukuoka [3.607, 3.608], V. O. Geogdzhayev [3.198], M. Sh. Mikeladze [3.528], E. K. Ashkenazi [3.16], T. C. Hsu [3.269], H. Neuber [3.575], F. Stassi d'Alia [3.767], Z. Sobotka [3.756], Yu. M. Aryshensky, I. I. Kaluzhsky and V. V. Uvarov [3.15], J. R. Booker and E. H. Davis [3.72] (plane strain only). The case $\alpha=\beta=\gamma=1$ in (12.13) was investigated by A. K. Malmeyster [3.488].

An even more general case than (12.13) was considered by M. Sayir [3.700], who first discussed arbitrary functions of the invariants appearing in (12.13), and then many particular cases. Various anisotropic yield conditions were reviewed by H. Lippmann [3.461]. D. Grzesik [3.217], K. H. Matucha and P. Wincierz [3.512] studied theoretically and experimentally the influence of the texture of the material on the yield condition.

\subsection{Yield conditions in the case of structural anisotropy}

Averaged properties of structurally anisotropic materials (reinforced, ribbed, perforated, etc.) show an even more significant dependence on the direction than naturally anisotropic ones. In the case of structural plastic anisotropy two typical approaches may be distinguished: either a purely formal, phenomenological application of the 
general anisotropic yield conditions, discussed in the previous sections, or a more detailed analysis of the structure combined with an averaging process. However, a sharp separation between those approaches is not always possible.

A certain peculiarity of structurally anisotropic materials should not be forgotten: because of the non-homogeneity of the stress state the material often exhibits apparent plastic hardening even if the constituents are perfectly elastic-plastic. So the limit surfaces under consideration may describe either the initial neutral state, or developed yielding, or even failure due to partial or total fracture.

Following the first approach, V. D. Azzi and S. W. Tsai [3.19] proposed to apply to laminated composites the Mises-Hill yield condition in its particular form (12.9) and verified this condition experimentally. It is restricted to isosensitive materials; in order to take anisosensitivity into account, K. V. Zakharov [3.857] suggested (even earlier) a more general equation, namely a general quadratic yield condition with linear terms included. A similar proposal was made by S. W. Tsai and E. M. Wu [3.820] and a further generalization by I. I. Goldenblat and V. A. Kopnov [3.202]. P. C. Chou, B. M. McNamee and D. K. Chou [3.98] took the anisotropy of the matrix into account. A discussion of particular cases of the Azzi-Tsai approach with thermal effects included may be found in the book by L. R. Calcote [3.91].

The equation derived for laminates by L. Fischer [3.178] may be included in the second group. He applied to the constituents the distortion energy criterion and obtained the general Mises-Hill expression (12.5) but with $\sigma_{0 z}$ related to other plastic moduli through the elastic constants.

Various cases of yield conditions for fibre-reinforced materials are discussed in the book by A. J. M. Spencer [3.762]. It is assumed that the fibres are not subject to yielding and that yielding is unaffected by hydrostatic pressure; hence, Spencer decomposes the stress tensor $\sigma_{i j}$ into a reaction stress $r_{i j}$ and an extra-stress $\mu_{i j}$. For a single family of fibres in an incompressible material

$$
\mu_{i j}=\sigma_{i j}-\frac{1}{2}\left(\sigma_{k k}-a_{r} a_{s} \sigma_{r s}\right) \delta_{i j}+\frac{1}{2}\left(\sigma_{k k}-3 a_{r} a_{s} \sigma_{r s}\right) a_{i} a_{j},
$$

where $a_{i}$ are directional cosines of the (parallel) fibres. In the case without constraints (fibres) we obtain $\mu_{i j}=s_{i j}$, so the extra-stresses generalize deviatoric stresses. The yield condition may now be expressed in terms of three invariants (J. F. Mulhern, T. G. Rogers, A. J. M. Spencer [3.562]):

$$
I_{1}=\mu_{i j} \mu_{i j}, \quad I_{2}=a_{i} a_{j} \mu_{i k} \mu_{j k}, \quad I_{3}=\mu_{i j} \mu_{j k} \mu_{k l} .
$$

A linear combination of the invariants $I_{1}$ and $I_{2}$ (quadratic in stresses) is proposed as the most appropriate effective form of the yield condition

$$
\alpha I_{1}+\beta I_{2}-1=0 \text {. }
$$

The case $\beta=0$ (isotropic matrix, sparcely distributed fibres) was discussed by W. Prager [3.659].

J. D. Helfinstine and R. Lance [3.239], R. Lance and D. N. Robinson [3.409] applied the criterion of maximal shearing stresses to the matrix allowing also for
plastic flow in the direction of fibres. D. R. J. Owen, J. Holbeche and O. C. Zienkie- 
wicz [3.610] investigated the influence of the shape of the cross-section of the fibre on the yield condition. V. Kafka [3.336] analysed the case of a perfectly plastic matrix reinforced by elastic fibres and proposed a method of experimental evaluation of constants. T. H. Lin, D. Salinas and Y. M. Ito [3.458] used the finite-element method to determine the stress distribution (microstresses) in a rectangular array of filaments; G. J. Dvorak, M. S. M. Rao and J. Q. Tarn [3.162, 3.163] applied a similar method to a regular hexagonal array under a triaxial stress state and temperature changes.

In the case of two families of fibres G. E. Smith and A. J. M. Spencer [3.745] expressed the yield condition in terms of six invariants, generalizing (12.16). G. S. Holister, C. Thomas and I. King [3.264] analysed the influence of friction on the plastic behaviour of composites after the failure of some fibres. The case of two families of non-orthogonal fibres was studied in detail by P. V. McLaughlin [3.515]. An arbitrary number of families of parallel fibres was considered by Yu. V. Nemirovsky [3.574]. Yu. N. Rabotnov proposed a general theory of elastic-plastic composite structure [3.662].

The equations of yield surfaces for perforated plates under in-plane loadings, derived by W. J. O'Donnell and J. Porowski [3.585, 3.647] and with A. Sawczuk [3.699], will also be mentioned here. In the case of purely membrane states, composite or perforated plates may be classified either in this section or in the chapter devoted to combined loadings at the level of the cross-section of a surface structure; however, in the case of bending the classification should always be transferred to the level $\mathscr{S}$.

\section{Drucker's definition of a stable plastic material. Constitutive equations of perfect plasticity}

\subsection{Plastic potential}

The components of any second-order tensor $T_{\tau}$ may be presented as partial derivatives at a certain scalar function $g\left(\tau_{i j}\right)$ with respect to $\tau_{i j}$. This function may be identified with the second basic invariant $I_{2 \tau}$ halved, since formula (5.4.2) yields directly

$$
\tau_{i j}=\frac{1}{2} \frac{\partial I_{2 \tau}}{\partial \tau_{i j}}
$$

if the symmetry conditions $\tau_{j i}=\tau_{i j}$ are not taken into account. Hence the HenckyIlyushin equations of small elastic-plastic deformations (9.28) may also be written in the form

$$
e_{i j}=\frac{\varphi}{2} \frac{\partial I_{2 s}}{\partial s_{i j}},
$$


and the Levy-Mises and the Prandtl-Reuss equations of plastic flow, (9.51) and $(9.60)$, in the form

$$
\dot{e}_{i j}^{p}=\frac{\lambda}{2} \frac{\partial I_{2 s}}{\partial s_{i j}}
$$

where $\dot{e}_{i j}^{p}$ denote, as usual, the deviatoric components of plastic strain rates; in the Levy-Mises theory they are identified with total strain rates, whereas in the PrandtlReuss theory elastic strain rates are taken into account as well.

Relations (13.2) may easily be generalized so as to describe a wider class of materials. We first prove that if $I=I\left(\tau_{i j}\right)$ is an arbitrary invariant of the tensor $T_{\tau}$, then the entity

$$
\chi_{i j}=\frac{\partial I\left(\tau_{i j}\right)}{\partial \tau_{i j}}
$$

is a second-order tensor. Indeed, if the Cartesian system of coordinates is subject to rotation, then the invariant $I$ does not change; hence

$$
\chi_{k l}=\frac{\partial I\left(\tau_{k l}\right)}{\partial \tau_{k l}}=\frac{\partial I\left(\tau_{i j}\right)}{\partial \tau_{i j}} \frac{\partial \tau_{i j}}{\partial \tau_{k l}}=\frac{\partial I\left(\tau_{i j}\right)}{\partial \tau_{i j}} a_{i k} a_{j l}=\chi_{i j} a_{i k} a_{j l} .
$$

Making use of the tensorial properties of (13.4), we may write the generalizations of (13.2) and (13.3) in the forms

$$
\varepsilon_{i j}=\varphi \frac{l \partial g\left(\sigma_{i j}\right)}{\partial \sigma_{i j}}
$$

and

$$
\dot{\varepsilon}_{i j}^{p}=\lambda \frac{\partial g\left(\sigma_{i j}\right)}{\partial \sigma_{i j}},
$$

respectively, where $g\left(\sigma_{i j}\right)$ denotes a certain, in principle arbitrary, invariant of the stress tensor. In the case of anisotropy a common invariant of the stress tensor and of the tensor of moduli of plastic anisotropy should be used. Assuming $g\left(\sigma_{i j}\right)$ $=I_{2 s} / 2$, we obtain (13.2) or (13.3), since differentation with respect to $\sigma_{i j}$ or with respect to $s_{i j}$ leads here to identicał results.

The function $g\left(\sigma_{i j}\right)$ is called the plastic potential; this concept was introduced by R. Mises [3.531]. Such a potential exists if the following relations hold:

$$
\frac{\partial \dot{\varepsilon}_{i j}^{p}}{\partial \sigma_{k l}}=\frac{\partial \dot{\varepsilon}_{k l}^{p}}{\partial \sigma_{i j}}
$$

or the corresponding ones for $\varepsilon_{i j}$. Partial differentation is understood here at a constant value of $\varphi$ or $\lambda$; for perfectly plastic bodies these quantities cannot be expressed in terms of stresses in any case. The assumption (13.8) is very weak, and, for example, for an isotropic material relations (13.8) may be treated as obvious.

So, the mere existence of the plastic potential for a wide class of materials is beyond any doubt, but the effective form of the function $g\left(\sigma_{i j}\right)$ is an open question. 
We first notice that, making use of the nine-dimensional auxiliary spaces, defined in Sec. 10.1., we may write-instead of (13.6) and (13.7)--

$$
\epsilon=\varphi \operatorname{grad} g\left(\sigma_{i j}\right)
$$

and

$$
\dot{\boldsymbol{\epsilon}}^{p}=\lambda \operatorname{grad} g\left(\sigma_{i j}\right),
$$

respectively. Hence, if we determine by $g\left(\sigma_{i j}\right)=$ const a certain surface passing through the point $T_{\sigma}$ in the nine-dimensional stress space, then the vector $\boldsymbol{\epsilon}$ or $\dot{\boldsymbol{\epsilon}}^{p}$ is perpendicular to that surface. Further, if these vectors are to represent deviators, then the corresponding surface must be cylindrical (not necessarily circular) with axis $\sigma_{x}=\sigma_{y}=\sigma_{z}$ in the nine-dimensional space, and cylindrical with axis $\sigma_{1}=\sigma_{2}$ $=\sigma_{3}$ in the three-dimensional space of principal stresses. In those cases Eqs. (13.9) or (13.10) describe the change of shape; in the opposite case they determine the shape change as well as the volume change.

Let us write the yield condition in the form

$$
f\left(\sigma_{i j}\right)=\text { const. }
$$

The form is not unique of course, but this fact is here unimportant. In the equations of deformation (total) theories of plasticity the most justified, classical assumption is to identify $g\left(\sigma_{i j}\right)$ with $f_{\mathrm{HMH}}\left(\sigma_{i j}\right)$,

$$
\begin{aligned}
g\left(\sigma_{i j}\right)=f_{\mathrm{HMH}}\left(\sigma_{i j}\right)=\frac{1}{3}[ & \sigma_{x}^{2}+\sigma_{y}^{2}+\sigma_{z}^{2}-\sigma_{x} \sigma_{y}-\sigma_{y} \sigma_{z}-\sigma_{z} \sigma_{x}+ \\
& \left.+\frac{3}{2}\left(\tau_{x y}^{2}+\tau_{y x}^{2}+\tau_{y z}^{2}+\tau_{z y}^{2}+\tau_{z x}^{2}+\tau_{x z}^{2}\right)\right]
\end{aligned}
$$

where $f_{\text {HMH }}\left(\sigma_{i j}\right)$ denotes the function describing the Huber-Mises-Hencky yield condition with an appropriate multiplier, written in a form corresponding to the nine-dimensional space. Equation (13.12) with (13.6) determines the deviatoric components (for any yield condition), whereas the axiatoric ones are given by (9.7). Then and only then the assumption $\varphi=1 / 2 G$ leads to equations of the elastic range; in the opposite case we would not obtain Hooke's law.

We shall now pass to the theory of plastic flow. If the plastic potential $g\left(\sigma_{i j}\right)$ coincides with the left-hand side of the yield condition $f\left(\sigma_{i j}\right),(13.11)$,

$$
g\left(\sigma_{i j}\right)=f\left(\sigma_{i j}\right),
$$

then we speak of an associated flow rule or, more precisely, we say that the flow rule (13.7) is associated with the assumed yield condition (13.11).

In the classical theory of plastic flow, making use of the $\mathrm{HMH}$ yield condition and of the constitutive (physical) equation (13.3), we deal with the associated flow rule, since

$$
g\left(\sigma_{i j}\right)=\frac{1}{2} I_{2 s}\left(\sigma_{i j}\right)=f_{\mathrm{HMH}}\left(\sigma_{i j}\right) .
$$

Such an approach has been fairly well verified experimentally, at least for many metals and for processes with constant principal directions.

In the case of other yield conditions the plastic potential $g\left(\sigma_{i j}\right)$, and, consequently, the flow rule (13.7), should be determined from experiments. If no sufficient 
experimental data are available, then the following two approximate approaches may be suggested: either the flow rule (13.3) may be retained, or it may be replaced by the associated flow rule (13.10) with (13.13). Equation (13.3) expresses the similarity of deviators of stresses and plastic strain rates; it is associated with the HMH yield condition. In many cases this law combined with other yield conditions gives results which are sufficiently accurate (W. Szczepiński [3.782]). Moreover, it should be noted that, in a certain sense, the physical equations of the classical theory of elasticity are associated with the $\mathrm{HMH}$ yield condition, since for the elastic range we may write, instead of (9.11),

$$
e_{i j}=\frac{1}{4 G} \frac{\partial I_{2 s}}{\partial s_{i j}}=\frac{1}{2 G} \frac{\partial f_{\mathrm{HMH}}}{\partial \sigma_{i j}} .
$$

Of course, this relation is not incidental, but is connected with the energy interpretation of the HMH yield condition. The analogy between elastic, plastic and hydrodynamic potentials is discussed by J. Betten [3.59].

Certain arguments motivating the use of associated flow rules will be quoted in Sec. 13.4. In spite of the scarcity of experimental data concerning the plastic flow of materials which are governed by yield conditions other than the HuberMises-Hencky condition, it may be expected that in many cases neither of the approximate approaches described above will be sufficiently accurate. For example, V. N. Nikolayevsky, N. M. Syrnikov and G. M. Shefter [3.579], considering the plastic behaviour of soils subject to the conical yield condition (11.26), propose a certain plastic potential which is far from (11.26), but also far from $f_{\text {нмн }},(13.12)$.

The concept of plastic potential leads to certain indeterminacies if the function $g\left(\sigma_{i j}\right)$ shows singularities, namely, if it is non-differentiable along certain lines or at certain points. In fact, then its gradient is not unique everywhere: an important example is the Tresca-Guest hexagonal prism or hexagon. The corresponding associated flow rule was derived during the thirties by A. Reuss [3.671, 3.672], but a more general theory was only given later by W. Prager [3.654] and W. T. Koiter [3.363, 3.364]. A further generalization is due to J. Mandel [3.493]. Instead of a gradient we sometimes speak here of subdifferential set (a cone of outward normal vectors).

In certain cases quite different approaches to the construction of physical relations are suggested. For example, S. A. Khristyanovitch and E. I. Shemyakin [3.341] complemented the TG yield condition with the following physical law of the deformation theory:

$$
\sigma_{\mathrm{II}}=\frac{3 K}{1+v}\left[v \varepsilon_{m}+(1-2 v) \varepsilon_{\mathrm{II}}\right]
$$

which together with the yield condition (9.19) and with the law of volume change (9.7) fully describes the stress-strain relations. The law (13.16) does not result in a similarity of deviators, the Lode parameters not being equal, and in the authors' opinion-it gives a better description of the experimental data than the law $\mu_{\sigma}=\mu_{\varepsilon}$. 


\subsection{The notions of cycles in the theory of plasticity}

As a cyclic process we usually understand a process which is or may be repeated many times. So we adopt here the following definition of a cycle, coinciding with that used in the problems of fatigue: as a cycle we understand a deformation process at the termination of which both the independent variables (exertion factors) and the dependent variables return to their initial values.

In view of the irreversibility of the processes of plastic deformations the cycles defined above seldom appear in the theory of plasticity. A certain exceptional case is shown in Fig. 47: the return to the initial state is possible, namely, it is due to

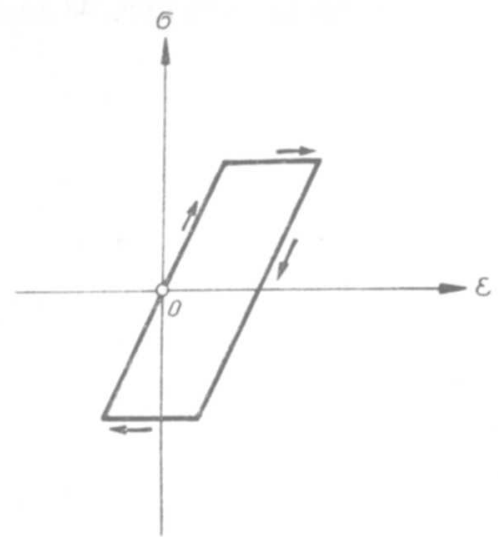

Fig. 47. A cycle in the theory of plasticity

reverse plastic strains. But even here a more precise treatment reveals some deviations from the adopted definition, since the temperature does not return to its initial value.

We introduce therefore a related concept of a quasi-cycle, which may be applied regardless of the reversibility of the process. As a quasi-cycle we understand a deformation process at the termination of which only the independent variables (exertion factors) return to their initial values. Ths dependent variables may behave during each quasi-cycle in a different manner, and so it is difficult to speak of repeatable effects.

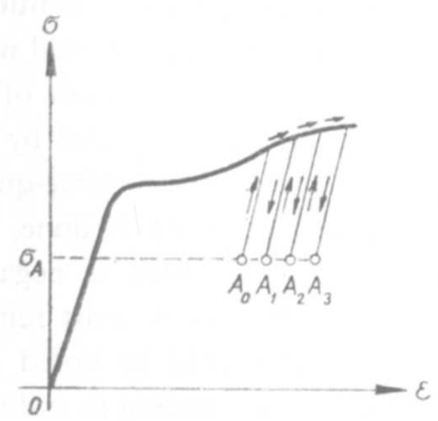

Fig. 48. Stress-quasi-cycles 
Two typical quasi-cycles, depending on the choice of a system of exertion factors, will be distinguished. If the stresses can be treated as the exertion factors, then we shall speak about a stress-quasi-cycle; such a quasi-cycle was used by D. C. Drucker [3.146, 3.149, 3.151] (but he called it a "cycle" and the term caused many misunderstandings). Similarly a strain-quasi-cycle may be introduced (A. A. Ilyushin [3.287]). However, mixed (stress-strain) quasi-cycles may appear as well, according to the general discussion given in Sec. 1.3. In [3.152] D. C. Drucker makes use of a stress-temperature-quasi-cycle.

Typical families of stress-quasi-cycles and strain-quasi-cycles are shown in the case of one exertion factor with successively increasing amplitudes in Figs. 48 and 49 , respectively.

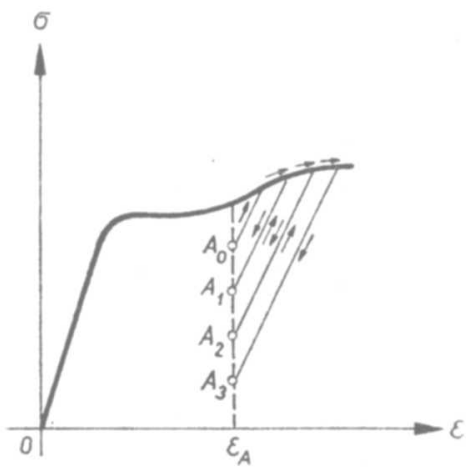

Fig. 49. Strain-quasi-cycles

\subsection{Drucker's definition of a stable plastic material (Drucker's postulate)}

D. C. Drucker $[3.146,3.151]$ proposed a certain criterion of material stability. To illustrate the problem let us first consider the loss of stability of an elastic structure. Namely, consider a shallow spherical elastic shell subject to a force $P$ (Fig. 50). Figure 50 gives also the dependence between the force $P$ and the deflection $f$. Suppose that the shell is loaded by the force $P_{1}$; then an external agency applies an additional force $\triangle P_{1}$ and removes it. A cycle $A B A$ is obtained; during this cycle the total work and the work done by the external agency are equal to zero. The behaviour of the structure is then said to be stable. Suppose further that the shell is loaded by $P_{2}$, and an external agency applies additionally $\Delta P_{2}$ and removes it. A force-quasicycle $C D C_{1}$ is formed; a jump occurs, certain positive total work is done, but the excessive work done by the external agency $\Delta P_{2}$ may be regarded as negative (the shaded area in Fig. 50; if no dissipation occurs, then this work must remain in the form of kinetic energy of vibrations). Incidentally, it should be noted that a further applicaticn of $\Delta P_{2}$ results in the cycle $C_{1} D C_{1}$ corresponding to stability.

Let us new return to plasticity, namely to the analysis of deformation processes 

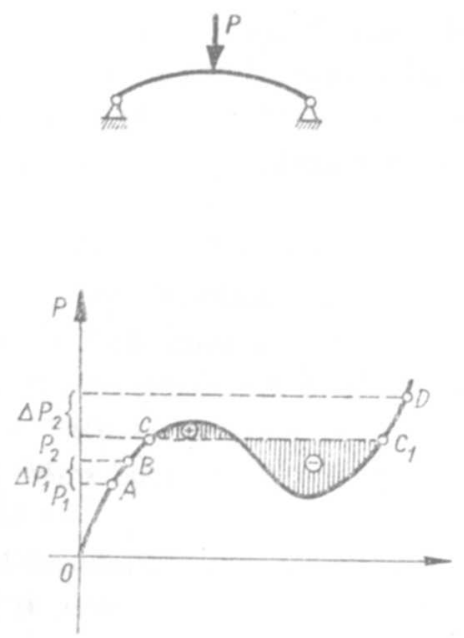

Fig. 50. An elastic shallow spherical shell

at the level of a point of the body $P$. Suppose that there exists a certain state of stress $\sigma_{i j}^{(0)}$; then an external agency applies an additional state of stress $\Delta \sigma_{i j}$ and removes it. If $\sigma_{i j}^{(0)}+\Delta \sigma_{i j}$ and all the intermediate states lie within the neutral surface, then we arrive at a cycle: both the total and the excessive work is equal to zero. If the point representing the stress state reaches the neutral surface at $\sigma_{i j}$ and an infinitesimal plastic strain $d \varepsilon_{i j}^{p}$ takes place, then we have merely a stress-quasi-cycle; the total work is positive, and the excessive work done by the external agency may be positive, negative or equal to zero. For an isothermal deformation process this work is given by

$$
d W^{*}=\left(\sigma_{i j}-\sigma_{i j}^{(0)}\right) d \varepsilon_{i j}^{p}+d \sigma_{i j} d \varepsilon_{i j}^{p}
$$

where the summation convention is employed, and $d \sigma_{i j}$ denotes the stress increment during the active part of the process.

A plastic material is defined by Drucker as stable if for an arbitrary initial state $\sigma_{i j}^{(0)}$ the excessive work $d W^{*}$ is positive: namely, the first term in (13.17) is non-negative and the second one is positive. This strong requirement is possible only for hardening materials, and sometimes the condition $d W^{*}>0$ is even used as a definition of plastic hardening. For perfectly plastic materials only a relaxed definition of stability can be introduced. The material is called indifferently stable if for arbitrary $\sigma_{i j}^{(0)}$ the excessive work is non-negative: namely, the first term in (13.17) is non-negative, and the second one-equal to zero (L. E. Malvern [2.108] uses the term "neutrally stable"). The inequality $d W^{*} \geqslant 0$ is called the Drucker postulate. The second term in (13.17) is of higher order with respect to the first one, however, its sign is of essential importance for the discussion of stability.

There is no reason to assume that all plastic materials are stable or indifferently stable at each stage of the deformation process. Some arguments pointing out the 
impossibility of negative plastic excessive work $d W^{*}$ during each cycle are based on a misunderstanding, since $d W^{*}$ is calculated not during a cycle but during a stressquasi-cycle which, if repeated, no longer gives the same value of $d W^{*}$. On the contrary, unstable plastic materials exist: plastic softening from the upper to the lower yield point may serve as the best example.

In the case of instabilities certain further definitions are useful. If $d W^{*}>0$ only at a certain stage of the deformation process, we call the material temporarily stable. Many materials are only temporarily stable, for example mild steel, whose stress-strain diagram is presented in Fig. 5 (Chapter I), is at first stable and then, in turn, unstable, indifferently stable, and stable again. In some cases $d W^{*}>0$ only for certain $d \varepsilon_{i j}$ or for certain $d \sigma_{i j}$; we may then speak of a material stable in certain directions.

A related requirement $d W^{*} \geqslant 0$ for a strain-quasi-cycle was introduced by A. A. Ilyushin [3.287, 3.288, 3.289, 3.290], and called the plasticity postulate. It is weaker that the Drucker postulate. A certain comparison of those postulates and some generalizations were proposed by R. Hill [3.255, 3.256]. Postulates concerning strain increments during the stress-quasi-cycle and stress increments during the strain-quasi-cycle were formulated by V. I. Malyi [3.490].

\subsection{Implications of the Drucker postulate}

Assuming the validity of the Drucker postulate, i.e., confining the discussion to stable or indifferently stable materials, we prove that for such materials the neutral surfaces must be convex. Indeed, in the auxiliary nine-dimensional space the first term of (13.7) may be interpreted as the scalar product of the vectors $\sigma-\sigma^{(0)}$ and $d \epsilon^{p}$. If the neutral surface were partly concave, then for any $d \epsilon^{p}$ (assumed to be constant) we might find a point $\sigma^{(0)}$ for which the angle between the vectors in question is obtuse and their scalar product is negative contrary to the Drucker postulate (Fig. 51).

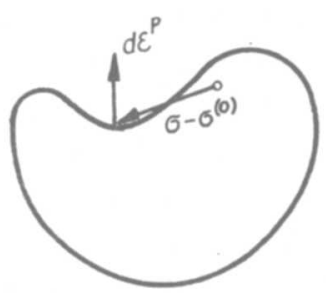

Fig. 51. An example of negative excessive work in the case of a concave limit surface

Further, it may be proved that for stable or indifferently stable materials the vector $d \epsilon^{p}$ is normal to the neutral surface. Indeed, this is the only case in which the scalar product $d \sigma d \epsilon^{p}$ cannot be negative for any $d \sigma$, Fig. 52 (the direction of $d \epsilon^{p}$ is assumed to be independent of the direction of $d \sigma$, cf. 15.3 and 15.4). Therefore 


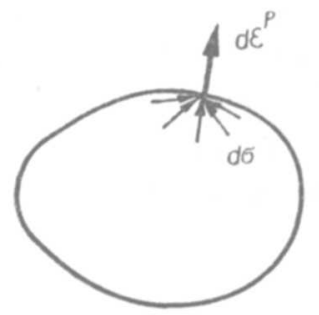

Fig. 52. The normality of the vector $d \epsilon^{p}$ to the limit surface

for the class of materials under consideration the flow rule (13.10) must be associated with the yield condition (or, more generally, with the equation of the neutral surface), i.e., relation (13.13) must hold. This relation is called the normality rule.

The assumption of the validity of the Drucker postulate, i.e. the restriction to stable or indifferently stable materials, has far-reaching consequences, especially important from the theoretical point of view. This postulate furnishes a sufficient condition of the uniqueness of solution of problems with neglected geometry changes (uniqueness is here understood in the incremental sense, and not as the independence of the final state on the trajectory), and simplifies the formulation of variational principles of plasticity. The advantages of the application of associated flow rules were pointed out by G. I. Taylor [3.793], T. Y. Thomas [3.800], D. C. Drucker [3.150], R. Hill [3.249, 3.250], H. Leipholz [3.428], H. J. Buschmann, H. G. Schöpf and P. Stilijanov [3.89].

The assumption of the validity of the Drucker postulate, and, consequently, of the convexity of neutral surfaces, imposes a sharp restriction on the form of the yield condition. Thus, for example, if we consider cylindrical limit surfaces (11.1) for isosensitive materials $\left(\sigma_{0-}=\sigma_{0+}\right)$, then six points in the deviatoric (Meldahl) plane are determined by a simple tension test (Fig. 41), and no admissible limit surface may exceed the Tresca-Guest hexagon from below and the Schmidt-IshlinskyHill hexagon from above (D. D. Ivlev [3.302], R. M. Haythornthwaite [3.236]). Haythornthwaite investigated also the admissible shapes of the limit surfaces in the case of the influence of the first stress invariant, [3.237].

An important theoretical problem is the transposition of convexity from one auxiliary space to another. M. Sayir [3.700] proved such a transposition from the nine-dimensional stress space to the three-dimensional space of principal stresses, and $\mathrm{H}$. Lippmann [3.460]—-from the three-dimensional space to the six-dimensional space. General considerations concerning the convexity of neutral surfaces are due to H. Ziegler, J. Nänni and Ch. Wehrli [3.873, 3.874], A. Troost and J. Betten [3.818].

The form of the Drucker postulate and its basic implications remain without change for an anisotropic material; those implications were discuseed in detail by G. I. Bykovtsev [3.90]. The normality rule in this case was experimentally confirmed, for example, by E. Shiratori and K. Ikegami [3.724]. B. Budiansky [3.80] 
investigated the class of processes which - calculated by using the theory of small elastic-plastic deformations-satisfy the Drucker postulate; according to the author, such an approach may determine the limits of applicability of the deformation theory.

The Ilyushin postulate of plasticity, weaker that that of Drucker, has also weaker implications: the normality rule is retained, bur the convexity of neutral surfaces is not required.

\subsection{Deviations from the Drucker postulate}

Many authors regard the Drucker postulate as a fundamental law of plasticity and the resulting associated flow rule-as obvious and indisputable. In fact, the classical theory of plasticity, making use of the $\mathrm{HMH}$ yield condition and of the LevyMises or Prandtl-Reuss flow rules, satisfies the Drucker postulate. However, real materials may exhibit various deviations from the classical model. This fact was clearly stressed by Drucker himself $[3.151,3.153,3.154]$ who remarked that the definition of a stable inelastic material is merely a definition and not a law of nature.

Some of the arguments supporting the generality of the Drucker postulate are based on a misunderstanding (Sec. 13.3). Its thermodynamic foundation is not general (D. R. Bland [3.66], D. C. Drucker [3.154], A. E. Green, P. M. Naghdi [3.212]). Some authors use this postulate to ensure uniqueness of the solutions. However, as has been pointed out by Z. Mróz [3.551], the Drucker postulate gives a sufficient condition for uniqueness (with geometry changes neglected) but is not necessary: there exists a wider class of materials for which uniqueness of solutions is ensured. This problem was also investigated by G. A. Gemmerling [3.193] and H. H. Bleich [3.67]; Bleich derived certain relations between $f\left(\sigma_{i j}\right)$ and $g\left(\sigma_{i j}\right)$ more general that $f=g$ and sufficient for the uniqueness of solutions.

A typical deviation from the Drucker postulate is, presumably, the phenomenon of plastic softening, but it usually takes place only at a certain stage of plastic deformation. More essential difficulties arise with regard to materials subject to the yield condition depending on the first stress invariant (for example, in the system $I_{1 \sigma}$, $\left.I_{2 s}, I_{3 s}\right)$. Soils and concrete may serve as examples. Associated flow rules lead here, as a rule, to results inconsistent with experiments. Assume, for example, a yield condition in the form (11.24) (a paraboloid) and calculate the plastic strain rates from the associated flow rule

$$
\begin{aligned}
& \dot{\varepsilon}_{x}^{p}=\lambda \frac{\partial f}{\partial \sigma_{x}}=\lambda\left[\left(2 \sigma_{x}-\sigma_{y}-\sigma_{z}\right)+\left(\sigma_{0-}-\sigma_{0+}\right)\right], \\
& \dot{\varepsilon}_{y}^{p}=\lambda \frac{\partial f}{\partial \sigma_{y}}=\lambda\left[\left(2 \sigma_{y}-\sigma_{z}-\sigma_{x}\right)+\left(\sigma_{0-}-\sigma_{0+}\right)\right], \\
& \dot{\varepsilon}_{z}^{p}=\lambda \frac{\partial f}{\partial \sigma_{z}}=\lambda\left[\left(2 \sigma_{z}-\sigma_{x}-\sigma_{y}\right)+\left(\sigma_{0-}-\sigma_{0+}\right)\right] .
\end{aligned}
$$


We do not quote the formulas for shearing strain rates; actually, they coincide with the classical Levy-Mises relations. Let us now consider uniaxial tension of a perfectly plastic material; putting $\sigma_{x}=\sigma_{0+}, \sigma_{y}=\sigma_{z}=0$, we obtain

$$
\begin{aligned}
& \dot{\varepsilon}_{x}^{p}=\lambda\left(\sigma_{0+}+\sigma_{0-}\right), \\
& \dot{\varepsilon}_{y}^{p}=\dot{\varepsilon}_{z}^{p}=-\lambda\left(2 \sigma_{0+}-\sigma_{0-}\right) .
\end{aligned}
$$

In the case $\sigma_{0-}>2 \sigma_{0+}$, often found in applications, we would obtain (with elastic strains neglected) an increase of transverse dimensions of the specimen in tension; this result contradicts most experiments. Considering uniaxial compression, we would obtain an increase of volume (as in any other plastic process, since the vector normal to the curves shown in Fig. 44 is always directed to the right-hand side), and this fact is also not confirmed experimentally, as a rule.

In the cases of non-cylindrical yield conditions which have been considered, the application of associated flow rules (C. Torre [3.814], D. C. Drucker, W. Prager [3.147], T. N. Martynova [3.510]) is more and more often replaced by the application of non-associated flow rules, which furnish a better description of experimental results. In the simplest case the law of similarity of deviators-associated with the $\mathrm{HMH}$ yield condition-may be applied. Then the material is stable only in certain directions (if $\sigma_{m}$ is non-increasing); an example of an unstable process is shown in Fig. 53.

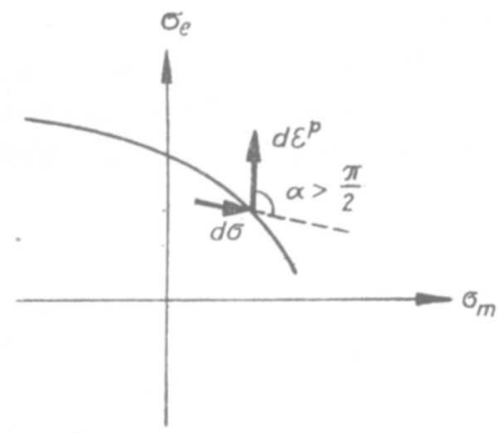

Fig. 53. An example of material instability in the case of the law of similarity of deviators

Deviations from normality of $d \epsilon^{p}$ with respect to the neutral surface in the case of soils were noticed by R. M. Haythornthwaite [3.235], G. de Josselin de Jong [3.318] and many other investigators. The necessity of applying non-associated flow rules was pointed out by D. Radenković [3.663, 3.664], who called the relevant class of materials "non-standard materials". Even some years earlier A. W. Jenike and R. T. Shield [3.315] proposed to combine the Coulomb yield condition with the plastic potential in the Tresca-Guest form. Other, more general proposals were given by G. Gudehus [3.218], R. N. Yong, E. McKyes and V. Silvestri [3.853]; critical reviews were published by V. N. Nikolayevsky [3.578] and A. Drescher [3.144]. A certain comparison of associated and non-associated flow rules was given by D. D. Ivlev and T. N. Martynova [3.308, 3.310]. 
In the case of plastic compressibility even the theoretical aspects of the Drucker postulate are disputable; A. E. Green and P. M. Naghdi [3.212] have concluded that it is impossible to construct a theory of plastically compressible bodies which satisfies all the thermodynamic constraints and the Drucker postulate. The problem of non-necessity of that postulate was also discussed by A. Phillips and M. Eisenberg [3.634].

Deviations from the Drucker postulate appear also in the case of non-convex limit surfaces (neutral surfaces) obtained in experiments. Although the accuracy of experiments may always be questioned and sometimes experiments describe types of failure other than plastic, nevertheless non-convex limit surfaces based on experimental data are quoted in numerous papers (M. M. Filonenko-Boroditch [3.175, 3.176], R. M. Haythornthwaite [3.235], I. N. Mirolubov [3.530], T. C. Hsu [3.271]).

Further discrepancies between the Drucker postulate and experimental results are observed in the case of non-isothermal deformations. Drucker found in [3.152] that the requirement of stability is then connected with an increase of the yield-point stress with temperature. Since usually a decrease is observed, most materials exhibit instability during non-isothermal processes. A different concept of the "postulate of thermoplasticity" was introduced by Yu. M. Shevtchenko and R. G. Terekhov [3.719].

Finally, it should be noted that the Drucker postulate does not always lead to the conclusions presented in Sec. 13.4. These conclusions are based on the assumption that the excessive work of elastic strains during a stress-quasi-cycle is always equal to zero. However, A. A. Ilyushin [3.288] noticed that plastic deformations may cause a change of elastic moduli; then the Drucker postulate results in the relation

$$
\dot{\varepsilon}_{i j}^{p}=\lambda \frac{\partial f}{\partial \sigma_{i j}}-\dot{A}_{i j k l} \sigma_{k l}
$$

where $\dot{A}_{i j k l}$ denotes the rate of elastic moduli. Then the vector $d \epsilon^{p}$ is not normal to the limit surface. The problem of uniqueness of solutions under this assumption was discussed by Yu. M. Bash, R. A. Vasin and K. E. Vega [3.32]. Experimental evaluation of changes in $A_{i j k l}$ due to plastic deformation was done by R. A. Vasin and A. B. Ibragimov [3.835]. The case of a non-linear description of passive processes was investigated by D. M. Kochin [2.86], and that of non-isothermal passive processes-by V. I. Rozenblyum [3.682].

Moreover, the conclusions from the Drucker postulate are much weaker if the direction of the vector $d \epsilon^{p}$ is not constant (cf. Secs. 15.3 and 15.4).

\subsection{Material stability of a perfectly elastic-plastic solid}

Consider a perflettly elastic-plastic solid with a yield surface $f\left(\sigma_{i j}\right)=0$ which is assumed to be convex. Then the material stability in the Drucker sense depends on the sign of the expression $d \sigma_{i j} d \varepsilon_{i j}^{p}$, and this sign will now be investigated. 
The simplest notation is obtained if we represent tensors as auxiliary vectors in the nine-dimensional space. If the (quite arbitrary) plastic potential is denoted by $g\left(\sigma_{i j}\right)$, then the plastic strain increments are

$$
d \mathbf{\epsilon}^{p}=\mathbf{n}_{g} d \lambda,
$$

where the unit vector $\mathbf{n}_{g}$, normal to the surface $g\left(\sigma_{i j}\right)=0$, is defined by

$$
\mathbf{n}_{g}=\frac{\operatorname{grad} g}{|\operatorname{grad} g|}=\frac{\partial g / \partial \sigma_{i j}}{\sqrt{\left(\partial g / \partial \sigma_{k l}\right)\left(\partial g / \partial \sigma_{k l}\right)}}
$$

(this relation should be understood as a purely formal definition of the vector components). For the sake of simplicity we restrict our considerations to regular functions $f$ and $g$, with a uniquely defined normal.

Assume that the increment of total strains $d \boldsymbol{\epsilon}$ is given; in the case of perfect plasticity the process cannot be uniquely determined by stresses. It is also assumed that the increment $d \boldsymbol{\epsilon}$ produces an active process. The corresponding stress increment may be calculated by using Hooke's law as follows:

$$
\begin{aligned}
d \boldsymbol{\sigma} & =d \mathbf{s}+d \boldsymbol{\sigma}_{m}=2 G d \mathbf{e}^{e}+3 K d \boldsymbol{\epsilon}_{m}^{e} \\
& =2 G\left(d \mathbf{e}-d \mathbf{e}^{p}\right)+3 K\left(d \boldsymbol{\epsilon}_{m}-d \boldsymbol{\epsilon}_{m}^{p}\right) .
\end{aligned}
$$

The decomposition into vectors corresponding to deviators and axiators is necessary because of the different forms of Hooke's law for those components. The increments $d \mathbf{e}$ and $d \epsilon_{m}$ are regarded as known, whereas $d \mathrm{e}^{p}$ and $d \epsilon_{m}^{p}$ are calculated as projections of $d \epsilon^{p}$ :

$$
\begin{aligned}
d \mathbf{\epsilon}_{m}^{p} & =\mathbf{n}_{m} \cos \left(\mathbf{n}_{g}, \mathbf{n}_{m}\right) d \lambda, \\
d \mathbf{e}^{p} & =\left[\mathbf{n}_{g}-\mathbf{n}_{m} \cos \left(\mathbf{n}_{g}, \mathbf{n}_{m}\right)\right] d \lambda,
\end{aligned}
$$

where $\mathbf{n}_{m}$ is the unit vector of the axis $\varepsilon_{m}$. The vector $d \boldsymbol{\sigma}$ lies in the hyperplane tangent to the surface $f\left(\sigma_{i j}\right)=0$ (because of the assumed perfect plasticity) and the condition of orthogonality

$$
\begin{aligned}
\mathbf{n}_{f} d \boldsymbol{\sigma}= & 2 G \mathbf{n}_{f}\left\{d \mathbf{e}-\left[\mathbf{n}_{g}-\mathbf{n}_{m} \cos \left(\mathbf{n}_{g}, \mathbf{n}_{m}\right)\right] d \lambda\right\}+ \\
& +3 K \mathbf{n}_{f}\left\{d \boldsymbol{\epsilon}_{m}-\mathbf{n}_{m} \cos \left(\mathbf{n}_{g}, \mathbf{n}_{m}\right) d \lambda\right\}=0
\end{aligned}
$$

determines $d \lambda$ :

$$
d \lambda=\frac{2 G \mathbf{n}_{f} d \mathbf{e}+3 K \mathbf{n}_{f} d \mathbf{\epsilon}_{m}}{2 G \mathbf{n}_{f}\left[\mathbf{n}_{g}-\mathbf{n}_{m} \cos \left(\mathbf{n}_{g}, \mathbf{n}_{m}\right)\right]+3 K \mathbf{n}_{f} \mathbf{n}_{m} \cos \left(\mathbf{n}_{g}, \mathbf{n}_{m}\right)} .
$$

The unit vector $\mathbf{n}_{f}$ is defined analogically to (13.22). Expressing the scalar products by vector lengths and the relevant cosines, we rewrite (13.26) in the form

$$
d \lambda=\frac{(1-2 v)|d \mathbf{e}| \cos \left(\mathbf{n}_{e}, \mathbf{n}_{f}\right)+(1+v)\left|d \boldsymbol{\epsilon}_{m}\right| \cos \left(\mathbf{n}_{f}, \mathbf{n}_{m}\right)}{(1-2 v) \cos \left(\mathbf{n}_{f}, \mathbf{n}_{g}\right)+3 v \cos \left(\mathbf{n}_{f}, \mathbf{n}_{m}\right) \cos \left(\mathbf{n}_{g}, \mathbf{n}_{m}\right)},
$$

where the unit vector $\mathbf{n}_{e}$, corresponding to $d \mathbf{e}$, is assumed to be known. The substitution of (13.24) and (13.27) into (13.23) makes it possible to calculate the excessive work $d \boldsymbol{\sigma} d \epsilon^{p}$ 


$$
\begin{aligned}
& d \boldsymbol{\sigma} d \mathbf{\epsilon}^{p}=2 G \frac{(1-2 v)|d \mathbf{e}| \cos \left(\mathbf{n}_{e}, \mathbf{n}_{f}\right)+(1+v)\left|d \epsilon_{m}\right| \cos \left(\mathbf{n}_{f}, \mathbf{n}_{m}\right)}{\left[(1-2 v) \cos \left(\mathbf{n}_{f}, \mathbf{n}_{g}\right)+3 v \cos \left(\mathbf{n}_{f}, \mathbf{n}_{m}\right) \cos \left(\mathbf{n}_{g}, \mathbf{n}_{m}\right)\right]^{2}} \times \\
& \times\left\{(1-2 v)\left[\cos \left(\mathbf{n}_{e}, \mathbf{n}_{g}\right) \cos \left(\mathbf{n}_{f}, \mathbf{n}_{g}\right)-\cos \left(\mathbf{n}_{e}, \mathbf{n}_{f}\right)\right]|d \mathbf{e}|+\right. \\
& \quad+3 v\left[\cos \left(\mathbf{n}_{e}, \mathbf{n}_{g}\right) \cos \left(\mathbf{n}_{f}, \mathbf{n}_{m}\right)-\right. \\
&\left.\quad-\cos \left(\mathbf{n}_{e}, \mathbf{n}_{f}\right) \cos \left(\mathbf{n}_{g}, \mathbf{n}_{m}\right)\right] \cos \left(\mathbf{n}_{g}, \mathbf{n}_{m}\right)|d \mathbf{e}|+ \\
&\left.\quad+(1+v)\left[\cos \left(\mathbf{n}_{f}, \mathbf{n}_{g}\right) \cos \left(\mathbf{n}_{g}, \mathbf{n}_{m}\right)-\cos \left(\mathbf{n}_{f}, \mathbf{n}_{m}\right)\right]\left|d \epsilon_{m}\right|\right\} .
\end{aligned}
$$

In the case of an associated flow rule, $f=g, \mathbf{n}_{f}=\mathbf{n}_{g}$, we obtain $d \boldsymbol{\sigma} d \epsilon^{p}=0$ and then the material is indifferently stable for all directions of $d \boldsymbol{\epsilon}$. If this coincidence does not occur, then material is stable for certain directions of $d \boldsymbol{\epsilon}$, and unstable for certain other directions.

The discussion is much simpler in the case of a cylindrical yield surface and a cylindrical potential surface, where $\cos \left(\mathbf{n}_{f}, \mathbf{n}_{m}\right)=0$ and $\cos \left(\mathbf{n}_{g}, \mathbf{n}_{m}\right)=0$. Equation (13.28) is then reduced to

$$
d \boldsymbol{\sigma} d \boldsymbol{\epsilon}^{p}=\frac{2 G|d \mathbf{e}|^{2} \cos \left(\mathbf{n}_{e}, \mathbf{n}_{f}\right)}{\cos ^{2}\left(\mathbf{n}_{f}, \mathbf{n}_{g}\right)}\left[\cos \left(\mathbf{n}_{\boldsymbol{e}}, \mathbf{n}_{g}\right) \cos \left(\mathbf{n}_{f}, \mathbf{n}_{g}\right)-\cos \left(\mathbf{n}_{e}, \mathbf{n}_{f}\right)\right] .
$$

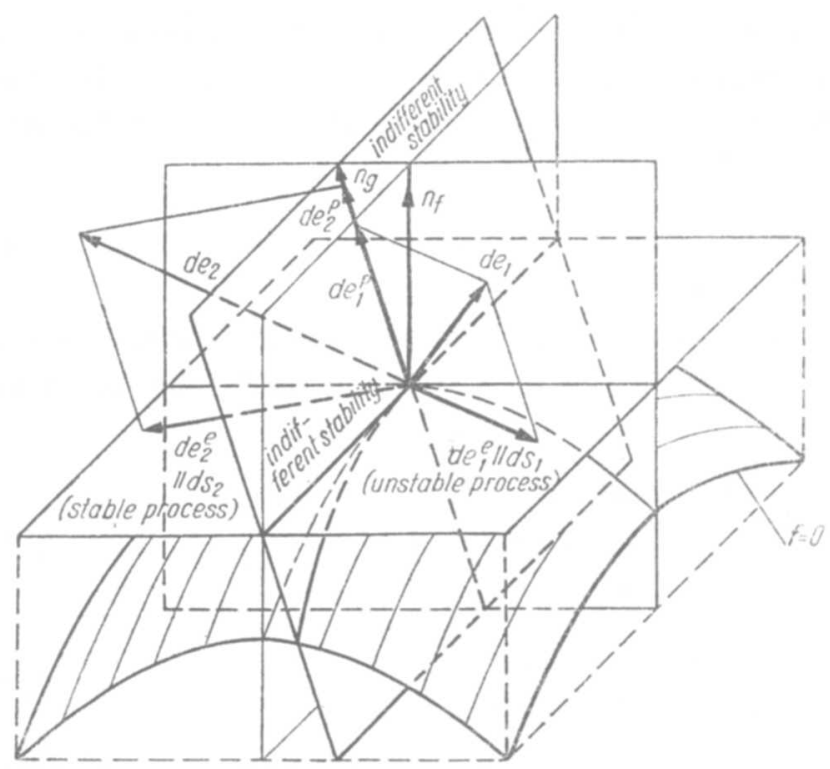

Fig. 54. Material stability in the case of a non-associated flow rule

It can easily be seen that the expression in brackets vanishes if the vector $d \boldsymbol{\epsilon}$ lies in the plane perpendicular to the plane $\mathbf{n}_{g}, \mathbf{n}_{f}$ passing through $\mathbf{n}_{g}$ (Fig. 54). The factor $\cos \left(\mathbf{n}_{e}, \mathbf{n}_{f}\right)$ is positive so as to ensure a process which is plastically active. Hence the processes determined by $d \boldsymbol{\epsilon}$ in this plane are indifferently stable; unstable on one (larger) side of this plane, and stable on the other (smaller) side. Incidentally, 
it should be noted that the inequality $d \sigma_{i j} d \varepsilon_{i j}^{p}>0$ cannot be regarded as a definition of the plastic hardening (as proposed by some authors), since it may also hold in the case of perfect plasticity.

\subsection{The Drucker postulate in the case of approximate yield conditions}

In engineering applications the yield condition which is most suitable for a given material is often replaced by approximate ones, resulting in simpler calculations. Then the choice of a suitable flow rule should be made very carefully. An automatic application of a flow rule associated with the approximate yield condition may lead to essential errors, since small differences in the values of the functions describing the yield condition may be combined with large differences in partial derivatives-and those derivatives appear in the associated flow rule.

The criterion of the choice of an appropriate flow rule should then be connected with a minimization of the approximation errors. Of course, if the exact solution (corresponding to the original, approximated yield condition) is not known, then the error estimation may be difficult, but some suggestions may be obtained from the solution of simpler, related problems, and in certain cases a more general error analysis is available. It turns out very often that more exact results are obtained if the original flow rules (e.g. those associated with the original yield condition) are applied than in the case of flow rules associated with the new, approximate yield condition.

Such a situation appears if we approximate the $\mathrm{HMH}$ yield condition, experimentally better confirmed, by piecewise linear functions, for example by the TG yield condition. In terms of principal stresses linearization is then obtained. Approximation errors, measured along the radius, do not exceed $13.4 \%$ if $\sigma_{0}$ is fixed, or $15.5 \%$ if $\tau_{0}$ is fixed (Fig. 55). However, if the TG yield condition is completed with the associated flow rule $\left(d \epsilon_{(1)}^{p}\right.$ in Fig. 55) instead of the rule of similarity of deviators

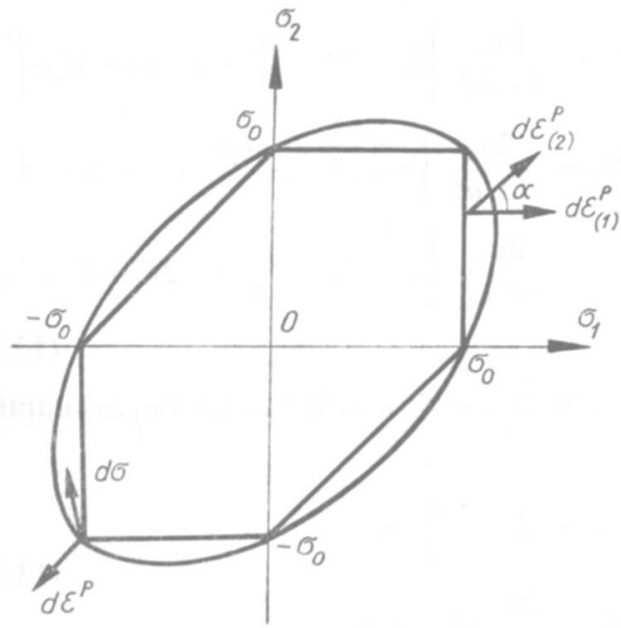

Fig. 55. Errors of approximation of the HMH yield condition by the TG yield condition 
(d $\epsilon_{(2)}^{p}$ in Fig. 55), then the error is much greater, since the angle between those vectors may be as large as $45^{\circ}$. This fact and its consequences were pointed out by V. V. Sokolovsky [3.761]. The problem of errors of linear approximations was also discussed by I. A. Berezhnoy and V. V. Ignatyev [3.48].

Making use of the Tresca yield condition with non-associated flow rule, we admit of course the existence of processes which are unstable in the Drucker sense. An example of such a process (close to the neutral process) is shown in Fig. 55 in the lower left-hand corner; the existence of the vector $d \sigma$ shown in the figure is possible (for a Tresca material) only in the case of plastic hardening. However, this apparent instability of the process has a deeper physical basis. In fact, if we approximate the HMH ellipse by the TG hexagon, then the process shown in Fig. 55 changes its type: this process, actually passive, is approximately treated as an active one. Since such an approximation cannot be regarded as satisfactory even for very modest requirements, one may conclude that the instability of the process means its inadmissibility in the adopted method of approximation.

Using any cylindrical yield condition combined with the law of similarity of deviators and not with the associated flow rule, we always obtain for simple processes in a perfectly plastic body a stabilization of stresses, characteristic for the HMH condition. This qualitative agreement provides an essential argument for the application of the classical Hencky-Ilyushin or Prandtl-Reuss laws instead of associated flow rules, which usually lead to results very distant from stabilization. Such an approach with respect to the Tresca yield condition was initiated by M. Levy and successfully applied to many particular problems (for example by R. Hill [2.56], J. A. König [3.382]).

A general analysis of "local" approximation errors was given in the paper by M. Życzkowski [3.877]. Effective formulae of type (13.23) with substituted (13.24) and (13.26) were subsequently derived (in principal directions) for the HMH yield condition with the Prandtl-Reuss flow rule (similarity of deviators)

$$
\begin{aligned}
& \frac{\dot{\sigma}_{1}}{G}=\frac{1}{1-2 v}\left(\dot{\varepsilon}_{1}+\dot{\varepsilon}_{2}+\dot{\varepsilon}_{3}\right)-\dot{\varepsilon}_{2}+\frac{2 \mu_{\sigma}}{3+\mu_{\sigma}^{2}}\left[\dot{\varepsilon}_{1}-\dot{\varepsilon}_{2}+\frac{\mu_{\sigma}}{3}\left(\dot{\varepsilon}_{1}+\dot{\varepsilon}_{2}-2 \dot{\varepsilon}_{3}\right)\right], \\
& \frac{\dot{\sigma}_{2}}{G}=\frac{2 v}{1-2 v}\left(\dot{\varepsilon}_{1}+\dot{\varepsilon}_{2}+\dot{\varepsilon}_{3}\right)+2 \dot{\varepsilon}_{2}+\frac{2 \mu_{\sigma}}{3+\mu_{\sigma}^{2}}\left[-\dot{\varepsilon}_{1}+\dot{\varepsilon}_{3}+\frac{\mu_{\sigma}}{3}\left(\dot{\varepsilon}_{1}-2 \dot{\varepsilon}_{2}+\dot{\varepsilon}_{3}\right)\right], \\
& \frac{\dot{\sigma}_{3}}{G}=\frac{1}{1-2 v}\left(\dot{\varepsilon}_{1}+\dot{\varepsilon}_{2}+\dot{\varepsilon}_{3}\right)-\dot{\varepsilon}_{2}+\frac{2 \mu_{\sigma}}{3+\mu_{\sigma}^{2}}\left[\dot{\varepsilon}_{2}-\dot{\varepsilon}_{3}+\frac{\mu_{\sigma}}{3}\left(-2 \dot{\varepsilon}_{1}+\dot{\varepsilon}_{2}+\dot{\varepsilon}_{3}\right)\right],
\end{aligned}
$$

for the TG yield condition with the Prandtl-Reuss flow rule (under the assumption $\left.\sigma_{1} \geqslant \sigma_{2} \geqslant \sigma_{3}\right)$

$$
\begin{aligned}
& \frac{\dot{\sigma}_{1}}{G}=\frac{\dot{\sigma}_{3}}{G}=\frac{1}{1-2 v}\left(\dot{\varepsilon}_{1}+\dot{\varepsilon}_{2}+\dot{\varepsilon}_{3}\right)-\dot{\varepsilon}_{2}+\frac{\mu_{\sigma}}{3}\left(\dot{\varepsilon}_{1}-\dot{\varepsilon}_{3}\right), \\
& \frac{\dot{\sigma}_{2}}{G}=\frac{2 v}{1-2 v}\left(\dot{\varepsilon}_{1}+\dot{\varepsilon}_{2}+\dot{\varepsilon}_{3}\right)+2 \dot{\varepsilon}_{2}-\frac{2 \mu_{\sigma}}{3}\left(\dot{\varepsilon}_{1}-\dot{\varepsilon}_{3}\right),
\end{aligned}
$$


and for the TG yield condition with the associated flow rule

$$
\begin{aligned}
& \frac{\dot{\sigma}_{1}}{G}=\frac{\dot{\sigma}_{3}}{G}=\frac{1}{1-2 v}\left(\dot{\varepsilon}_{1}+\dot{\varepsilon}_{2}+\dot{\varepsilon}_{3}\right)-\dot{\varepsilon}_{2}, \\
& \frac{\dot{\sigma}_{2}}{G}=\frac{2 v}{1-2 v}\left(\dot{\varepsilon}_{1}+\dot{\varepsilon}_{2}+\dot{\varepsilon}_{3}\right)+2 \dot{\varepsilon}_{2} .
\end{aligned}
$$

The symbol $\mu_{\sigma}$ denotes here the Lode parameter for stresses, (5.18). The errors of approximation of (13.30) by (13.31) and (13.32) were compared and it was found that in most cases the errors of (13.31) were smaller. The simpler associated flow rule (13.32) should be considered as the next, less exact step of approximation.

To obtain a simple comparison of the two above-mentioned types of approximation let us consider a plate subject to prescribed deformations $\varepsilon_{1}=\varepsilon_{1}(t)$ and $\varepsilon_{2}$ $=\varepsilon_{2}(t)$ in its plane. Since in such a thin plate $\sigma_{3}=\sigma_{3}(t) \equiv 0$, the whole process may be regarded as prescribed in a mixed manner, partly in strains and partly in stresses. Assume incompressibility of material and a proportional increase of the strains:

$$
\varepsilon_{1}=\alpha(t) \frac{\sigma_{0}}{E}, \quad \varepsilon_{2}=m \alpha(t) \frac{\sigma_{0}}{E}, \quad \varepsilon_{3}=-(1+m) \alpha(t) \frac{\sigma_{0}}{E},
$$

where $\alpha(t)$ stands for a certain monotonically increasing function of time, and $m$ taken from the interval $-\frac{1}{2}<m<1$ denotes a constant. This interval of $m$ corresponds to $\sigma_{1}>\sigma_{2}>\sigma_{3}$.

In the elastic range, the stresses are equal to

$$
\begin{aligned}
& \sigma_{1}=\frac{4}{3} E\left(\varepsilon_{1}+\frac{1}{2} \varepsilon_{2}\right)=\frac{4}{3} \alpha \sigma_{0}\left(1+\frac{1}{2} m\right), \\
& \sigma_{2}=\frac{4}{3} E\left(\varepsilon_{2}+\frac{1}{2} \varepsilon_{1}\right)=\frac{4}{3} \alpha \sigma_{0}\left(m+\frac{1}{2}\right),
\end{aligned}
$$

and it can be seen that $\sigma_{1}>\sigma_{2}>\sigma_{3}=0$.

Assume first the $\mathrm{HMH}$ yield condition, treated here as exact. Substituting (13.34) into this condition, we determine the boundary value of $\alpha$, separating the elastic and the plastic range:

$$
\alpha=\bar{\alpha}=\frac{\sqrt{3}}{2\left(1+m+m^{2}\right)},
$$

and the corresponding stresses are equal to

$$
\sigma_{1}=\frac{2+m}{\sqrt{3\left(1+m+m^{2}\right)}} \sigma_{0}, \quad \sigma_{2}=\frac{2 m+1}{\sqrt{3\left(1+m+m^{2}\right)}} \sigma_{0} .
$$

The Prandtl-Reuss equations take the form

$$
\begin{aligned}
& \dot{\varepsilon}_{1}-\dot{\varepsilon}_{3}=\lambda \sigma_{1}+\frac{3}{2 E} \dot{\sigma}_{1}, \\
& \dot{\varepsilon}_{2}-\dot{\varepsilon}_{3}=\lambda \sigma_{2}+\frac{3}{2 E} \dot{\sigma}_{2},
\end{aligned}
$$


and are satisfied by constant values of the stresses (13.26). This stabilization of stresses will be treated as the exact solution.

Now consider the first variant of the Tresca-Guest approximation-combined with the flow rule (13.37). The yield condition gives here simply $\sigma_{1}=\sigma_{0}$, and the boundary value of $\alpha$, separating the elastic and the plastic range, equals

$$
\alpha=\bar{\alpha}=\frac{3}{2(2+m)} .
$$

The corresponding stresses are

$$
\sigma_{1}=\sigma_{0}, \quad \sigma_{2}=\frac{2 m+1}{m+2} \sigma_{0} .
$$

Equations (13.37) are satisfied by constant values of the stresses (13.39); thus during the whole process the numerical differences with respect to (13.36) do not exceed $13.4 \%$ (the largest error corresponds to $m=0$ ).

The second variant of approximation combines the TG yield condition with the associated flow rule, which gives here

$$
\dot{\varepsilon}_{2}^{p}=0 \text {. }
$$

Hence the change of $\varepsilon_{2}$ is purely elastic:

$$
\dot{\varepsilon}_{2}=m \dot{\alpha} \frac{\sigma_{0}}{E}=\frac{\dot{\sigma}_{2}}{E}-\frac{1}{2} \frac{\dot{\sigma}_{1}}{E}=\frac{\dot{\sigma}_{2}}{E},
$$

since $\dot{\sigma}_{1}=0$; this equation determines $\sigma_{2}$ : namely, after integration,

$$
\sigma_{2}=\frac{2 m+1}{m+2} \sigma_{0}+m(\alpha-\bar{\alpha}) \sigma_{0}=\left(\frac{1}{2}+m \alpha\right) \sigma_{0} .
$$

This formula is only valid in a certain interval of time, namely, as long as $0<\sigma_{2}<\sigma_{0}$. The further part of the process depends on the value of the constant $m$.

If $-1 / 2<m<0$, then $\sigma_{2}$ decreases to zero and reaches this value for $\alpha$ $=-1 / 2 \mathrm{~m}$. This point corresponds to the corner of the TG yield hexagon and the flow rule (13.40) is no longer valid at the point: the stresses simply remain constant, $\sigma_{1}=\sigma_{0}, \sigma_{2}=\sigma_{3}=0$. If $0<m<1$, then $\sigma_{2}$ increases to $\sigma_{0}$, reaches this value at $\alpha=1 / 2 m$, and then remains constant. Finally, if $m=0$, then $\sigma_{2}=\sigma_{0} / 2=$ const.

The processes discussed are illustrated in Fig. 56, showing the motion in the plane $\varepsilon_{1}, \varepsilon_{2}$ of the limit curves separating the regions of active and passive processes. In the first two cases, the curves move in the direction of the radius determining the loading path in this plane. In the third case, that of the TG yield condition with the associated flow rule-the curve moves initially in the direction of $\varepsilon_{1}$ and then, in the second period, in the direction of the radius. A certain analogy may be observed here with the concepts of kinematic plastic hardening, which will be discussed in Sec. 14.4: cases (a) and (b) (similarity of deviators) correspond to the Ziegler hardening rule, (14.30), whereas case (c) corresponds to the Melan-Ishlinsky-Prager 

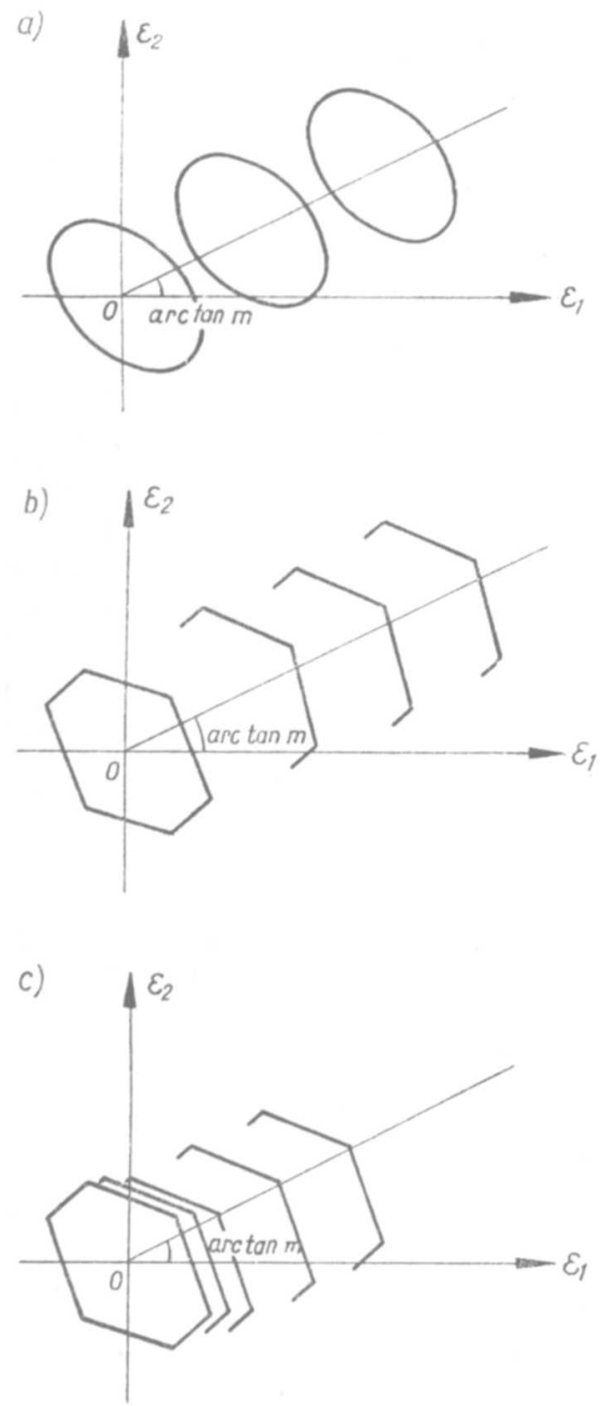

Fig. 56. Subsequent neutral curves in the strain plane: (a) $\mathrm{HMH}$ yield conditon with the law of similarity of deviators, (b) TG yield condition with the law of similarity of deviators, (c) TG yield condition with the associated flow rule

hardening rule (14.26) (similar motion is there observed in the stress space, while in our case the strain space is considered).

Other graphical interpretations of the case (c) (the TG yield condition with the associated flow rule) are shown in Figs. 57 and 58. Figure 57 divides the strain plane $\varepsilon_{1}, \varepsilon_{2}$ into the regions in which the stresses are constant (for simple loading processes) and the regions of varying stresses (the vectors show then the direction of $d \sigma$ ). Figure 58 shows the trajectories of motion (in the stress plane) of the points representing the processes which are simple in the strain plane. These trajectories 


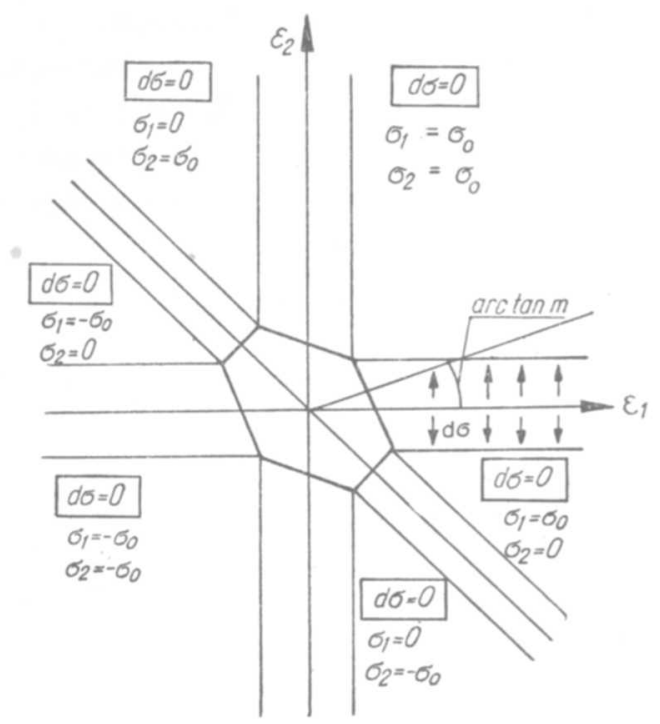

Fig. 57. Division of the strain plane into the subregions

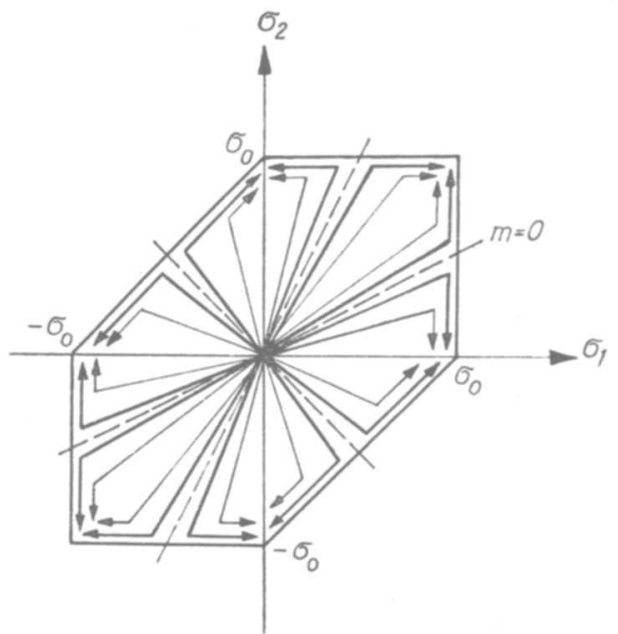

Fig. 58. Trajectories in the stress plane corresponding to simple processes and calculated by using the associated flow rule

are broken lines, running towards the corners. A certain instability in the Lyapunov sense may here to observed: a slight change of the initial condition (of the value $m$, for example in the vicinity of $m=0$ ) may result in a very large change of the trajectory. The lines corresponding to instability are marked by dashed lines. Thus the associated flow rule eliminates instability in Drucker's sense but may cause instability of the trajectory in Lyapunov's sense.

Compare now the numerical results of (13.42) with the result (13.36), treated as exact. The largest error occurs for $m \approx 0$, positive or negative. For a very small 
positive $m$ and for a sufficiently large $\alpha$ we obtain $\sigma_{2}=\sigma_{0}$ instead of $\sigma_{2}=\sigma_{0} / \sqrt{3}$; thus the error is $73 \%$ from above. For a very small negative $m$ and for a sufficiently large $\alpha$ we obtain $\sigma_{2}=0$ instead of $\sigma_{2}=\sigma_{0} / \sqrt{3}$-thus the error may be regarded as $100 \%$ from below. Hence the errors are here much larger than in the first variant of approximation (the TG yield condition with the similarity of deviators).

Recapitulating, we should make a clear distinction between the Tresca material and an approximation by the TG yield condition. In the first case, of little practical importance (probably limited to monocrystals), the associated flow rule might be justified, though the resulting relation $\mu_{\dot{\varepsilon}}=0$, has practically never been confirmed by experiments (J. Mandel [3.494]). In the second, more important case, the associated flow rule usually increases the errors of approximation and should not be used without suitable justification (e.g. further essential simplifications). Incidentally, it should be noted that in arbitrary, non-principal directions the flow rule associated with the TG yield condition is very complicated (M. S. Sarkisyan [2.148]).

\subsection{General constitutive equations of perfect plasticity}

Constitutive equations of classical theories of perfect plasticity, due to HenckyIlyushin (9.28), Levy-Mises (9.51), and Prandtl-Reuss (9.60), are expressed by linear relations between the deviators of strains, strain rates, stresses and stress rates. They may be regarded as particular cases of the general linear relation

$$
c_{1} e_{i j}+c_{2} s_{i j}+c_{3} \dot{e}_{i j}+c_{4} \dot{s}_{i j}=0 \text {. }
$$

This equation was proposed in 1932 by K. Hohenemser and W. Prager [3.263]. The coefficients $c_{n}$ may depend on the invariants of the tensors in question, but in such a way as to ensure the independence of the time scale. They contain a certain unknown function, to be determined from the yield condition; therefore (13.43) means only tensorial linearity whereas the final equations are non-linear with respect to stresses or strains. Moreover, the coefficients $c_{n}$ should satisfy some fundamental postulates (e.g. that of the continuity of description of neutral processes), and so the number of admissible alternatives is considerably limited.

In order to obtain more general constitutive equations, let us assume the plastic potential in a form that is quite general for an isotropic material, namely expressed in terms of the basic invariants:

$$
g=g\left(I_{1 \sigma}, I_{2 \sigma}, I_{3 \sigma}\right) ;
$$

we then obtain the relevant plastic flow rule

$$
\dot{\varepsilon}_{i j}^{p}=\frac{\partial g}{\partial I_{1 \sigma}} \frac{\partial I_{1 \sigma}}{\partial \sigma_{i j}}+\frac{\partial g}{\partial I_{2 \sigma}} \frac{\partial I_{2 \sigma}}{\partial \sigma_{i j}}+\frac{\partial g}{\partial I_{3 \sigma}} \frac{\partial I_{3 \sigma}}{\partial \sigma_{i j}} .
$$

The partial derivatives of the basic invariants (the symmetry conditions not being taken into account) are

$$
\frac{\partial I_{1 \sigma}}{\partial \sigma_{i j}}=\delta_{i j}, \quad \frac{\partial I_{2 \sigma}}{\partial \sigma_{i j}}=2 \sigma_{i j}, \quad \frac{\partial I_{3 \sigma}}{\partial \sigma_{i j}}=3 \sigma_{i k} \sigma_{k j},
$$


and hence

$$
\dot{\varepsilon}_{i j}^{p}=C_{0} \delta_{i j}+C_{1} \sigma_{i j}+C_{2} \sigma_{i k} \sigma_{k j},
$$

where the coefficients $C_{n}$ may depend on the stress invariants and material constants. This general case was investigated by J. L. Dais [3.122], who derived a condition of coincidence of the principal directions of stresses and plastic strain rates.

A slightly different approach, leading to similar results, was initiated in 1945 by M. Reiner [3.669], extended by R. S. Rivlin and J. L. Ericksen [3.675, 3.676] to finite-strain theory and developed by many other authors. It consists in the analysis of general (non-linear) isotropic tensor functions; originally proposed as constitutive, relations of viscoelasticity may also be used in the theory of plasticity. Generalizing the Levy-Mises equations, we may write (for small strains)

$$
\sigma_{i j}=\bar{M}_{0} \delta_{i j}+\bar{M}_{1} \dot{\varepsilon}_{i j}+\bar{M}_{2} \dot{\varepsilon}_{i k} \dot{\varepsilon}_{k j}+\bar{M}_{3} \dot{\varepsilon}_{i k} \dot{\varepsilon}_{k l} \dot{\varepsilon}_{l j}+\ldots
$$

The Cayley-Hamilton theorem yields

$$
\dot{\varepsilon}_{i k} \dot{\varepsilon}_{k l} \dot{\varepsilon}_{l j}=J_{3 \dot{\varepsilon}} \delta_{i j}-J_{2 \dot{\varepsilon}} \dot{\varepsilon}_{i j}+J_{1 \dot{\varepsilon}} \dot{\varepsilon}_{l k} \dot{\varepsilon}_{k j},
$$

and makes it possible to eliminate higher products (powers of the tensor $T_{\dot{\varepsilon}}$ ); finally

$$
\sigma_{i j}=M_{0} \delta_{i j}+M_{1} \dot{\varepsilon}_{i j}+M_{2} \dot{\varepsilon}_{i k} \dot{\varepsilon}_{k j} \text {. }
$$

These relations were studied in detail by P. Stutz [3.772, 3.773], A. Sawczuk and P. Stutz [3.698]. If a perfectly rigid-plastic medium is considered, then $\dot{\varepsilon}_{i j}$ denote total strain rates; if elastic strains are taken into account, then (13.48)-(13.50) describe only plastic strain rates and $\dot{\varepsilon}_{i j}^{p}$ should be substituted. Important generalizations to non-coaxial tensors were given by V. V. Novozhilov [3.583] and K. F. Chernykh [3.95].

Equation (13.47) may of course be derived in the same manner. In the case of an incompressible material, $\dot{\varepsilon}_{i i}=0,(13.47)$ and $(13.50)$ may be reduced to the form

$$
\begin{aligned}
& \dot{e}_{i j}^{p}=\left(C_{1}+\frac{2}{3} C_{2} I_{1 \sigma}\right) s_{i j}+C_{2}\left(s_{i k} s_{k j}-\frac{1}{3} I_{2 s} \delta_{i j}\right), \\
& s_{i j}=M_{1} \dot{e}_{i j}^{p}+M_{2}\left(\dot{e}_{i k}^{p} \dot{e}_{k j}^{p}-\frac{1}{3} I_{2 \dot{e}}^{p} \delta_{i j}\right),
\end{aligned}
$$

where $I_{2 \dot{e}}^{p}$ denotes the second basic invariant of the plastic strain rates (A. M. Freudenthal, H. Geiringer [2.33]). Another approach to the general constitutive equations of perfect plasticity was proposed by N. Fox [3.180], and by Z. Sobotka [3.757, 3.760].

A certain particular problem is connected with flow rules in the plastic corners, appearing, for example in the case of of the Tresca-Guest yield condition; associated flow rules leave here a certain indeterminacy (W. T. Koiter [3.363], W. Prager [3.654]). This problem was discussed by D. D. Ivlev [3.303] and V. I. Rozenblyum [3.681].

Generalizations of the Levy-Mises and Prandtl-Reuss equations to the case of finite strains were proposed by T. Y. Thomas [3.801, 3.802, 2.174]; a detailed discussion may be found in the monographs by W. Olszak, Z. Mróz and P. Perzyna [3.600], and A. C. Eringen [2.31] (cf. also Sec. 15.9). 


\section{Subsequent neutral surfaces during plastic hardening}

\subsection{Hypothesis of a uniform curve of plastic hardening}

We already mentioned in Sec. 8 that the basic information concerning plastic hardening is given by the tension test. Therefore most theories of plastic hardening attempt to employ that information and to "transfer" the stress-strain curve to the general three-dimensional case.

Such an approach is justified, first of all, in the case of materials described by a one-parameter yield condition (initial neutral surface) since only in this case the tension test may determine, for example, the compression test. But even for a oneparameter plastic material various restrictions of such an approach arise. The simplest form of "transferring" of the tension diagram to three-dimensional cases consists in the formulation of a hypothesis which states that for a certain class of deformation processes there exists a unique dependence between certain invariants of the stress tensor and of the strain tensor, reduced stress and reduced strain, namely

$$
\sigma_{\text {red }}=f\left(\varepsilon_{\text {red }}\right) \text {; }
$$

the form of the function $f$ would then be determined by the simple tension test. The problem lies in a suitable choice of the invariants $\sigma_{\text {red }}$ and $\varepsilon_{\text {red }}$ and in experimental verification connected with the limitation of the class of admissible processes.

The most frequently used hypothesis of type (14.1) was formulated in 1926 by M. Roš and A. Eichinger [2.144]; they substituted for $\sigma_{\text {red }}$ and $\varepsilon_{\text {red }}$ the stress intensity and the strain intensity, respectively:

$$
\sigma_{e}=f\left(\varepsilon_{e}\right) \text {. }
$$

In uniaxial tension we have directly $\sigma_{e}=\sigma$, whereas $\varepsilon_{e}=\varepsilon$ only in the case of an incompressible material, (7.29). For such a material the function $f$ is determined directly by the simple tension test. In the case of a compressible material there are some additional complications connected with the change of the coefficient of transversal deformation during the test, (Sec. 9.4), but their practical effect is very small. Larger differences may appear only in the initial phase of the plastic deformations (I. A. Kozlov, A. A. Lebedev, V. L. Akhremenko [3.381]).

R. Schmidt [3.704] compared the results of hypothesis (14.2) with other related hypotheses, namely

$$
\max |s|=f(\max |e|)
$$

(where $\max |s|$ and $\max |e|$ denote the maximal absolute values of the principal deviatoric stresses and strains, respectively), and with

$$
\tau_{\max }=f\left(\gamma_{\max }\right) \text {. }
$$

Relation (14.2) is based on the HMH hypothesis, whereas (14.3) and (14.4) on the maximal deviatoric stress and on the maximal shearing stress, respectively. The hypothesis of the existence of a uniform curve (14.4) was formulated as early as 
1909 by P. Ludwik [2.107], i.e., much earlier than (14.2). Schmidt found that (14.2) gives a better description of the experimental data than (14.3) and (14.4). Similar conclusions were drawn by F. Körber and A. Eichinger [3.384], W. R. Osgood [3.605], A. M. Zhukov [3.864, 3.866, 3.868]. However, some regular deviations from (14.2) were also observed. In order to obtain a more accurate description D. C. Drucker [3.145], F. D. Stockton and D. C. Drucker [3.769] calculated the invariant $\sigma_{\text {red }}$ on the basis of (11.18) or (11.19), leaving $\varepsilon_{\text {red }}$ equal to $\varepsilon_{e}$. A related proposal is due to V. A. Kuz'menko [3.406], whereas L. V. Grebnev [3.209, 3.210] stated that (14.4) gives a better description than (14.2). This statement is probably too extreme, nevertheless a certain intermediate hypothesis between (14.2), and (14.4) seems the most accurate (S. A. Elsufyev [3.167], A. F. Kregers [3.392], N. M. Mitrokhin, Yu. I. Yagn [3.533, 3.534]).

The problem of an appropriate definition of $\varepsilon_{\text {red }}$ corresponding to an adopted $\sigma_{\text {red }}$ may be solved in various ways. The best solution seems to result from Hooke's law applied in the elastic range, since only then the curve (14.1) may hold for any process in that range. J. Christoffersen [3.101] proposed to define $\varepsilon_{\text {red }}$ in such a way as to obtain the increment of unit plastic work determined always by $d W^{p}=\sigma_{\mathrm{red}} d \varepsilon_{\mathrm{red}}^{p}$ (in the case of the HMH hypothesis both definitions coincide); such an approach is also used for anisotropic bodies, cf. Sec. 16.2. On the other hand, J. D. Lubahn and R. P. Felgar [3.467] stated that this problem is not particularly important (in simple loading processes, especially if the described curves are flat), and proposed to assume $\varepsilon_{\text {red }}=\varepsilon_{I}$ (maximal principal strain) for any definition of $\sigma_{\text {red }}$.

The comparison of the two most typical tests, in tension and in compression, was initiated much earlier. P. Ludwik [2.107] stated that for many materials the relevant diagrams $\sigma=f(\varepsilon)$ may be regarded as identical if we understand by $\sigma$ the true stresses (relating to the current cross-sectional area), and by $\varepsilon$ the logarithmic strains. Indeed, this approach makes it possible to extend the ranges of comparison of the diagrams almost up to their end-points (fracture). Further tests were described in the paper by P. Ludwik and L. Scheu [3.470].

In the general three-dimensional case the range of application of the hypothesis (14.2) may also be extended to finite strains if we introduce true stresses and logarithmic strains (H. Hencky [3.241]). Experimental verification of (14.2) understood in this sense was carried out by E. A. Davis [3.128, 3.129], D. M. Cunningham, E. G. Thomsen and J. E. Dorn [3.118], J. Marin [3.507], and L. S. Andreyev [3.4]; relatively small scatter was observed.

The influence of temperature on the concept of a uniform curve of plastic hardening was investigated by G. S. Pisarenko, A. A. Lebedev and V. P. Lamashevsky [3.644], and by V. N. Bastun [3.38]. The former authors found increasing scatter with decreasing temperature (up to $-180^{\circ} \mathrm{C}$ ), whereas Bastun obtained negligible scatter at $+300^{\circ} \mathrm{C}$. A certain physical explanation of this fact was given by V. A. Kuz'menko [3.407, 3.408], who proposed a generalization of Eq. (14.2); it seems sufficiently accurate but difficult in applications.

In the case of anisosensitive materials or other multi-parameter materials, 
the concept of a uniform curve of plastic hardening may also be introduced, but more experiments are needed to determine $\sigma_{\text {red }}$ and $\varepsilon_{\text {red }}$ effectively.

The above-mentioned experimental verifications of hypotheses of type (14.1) refer only to simple loading processes; just in some investigations (Zhukov, Grebnev) certain deviations from simple loading were introduced. In the general case, for example with rotating principal directions, the scatter may be significant (G. N. White, D. C. Drucker [3.844], S. S. Gill, J. Parker [3.199]). The differences in the case of reverse loadings (in torsion) were investigated by $Z$. Marciniak [3.502].

In fact, a hypothesis of form (14.1) has no chances in the general case of combined loading. Suppose that it is generally valid; then increasing $\varepsilon_{\text {red }}$ must always result in a plastically active process as in simple tension (beyond the elastic limit), and decreasing $\varepsilon_{\text {red }}$ should correspond to a passive process. Thus in the general case a hypothesis of type (14.1) gains a new meaning: constancy of the right-hand side should describe the neutral surface at the considered stage of the deformation process. However, in Secs. 9.5-9.7 we investigated a class of processes which were active for a decreasing strain intensity $\varepsilon_{e}$. This has occurred for a perfectly elasticplastic body and may occur for a hardening body as well. The equations $\varepsilon_{\text {red }}=$ const do not, in general, describe neutral surfaces and the postulate of continuous description of neutral processes is violated.

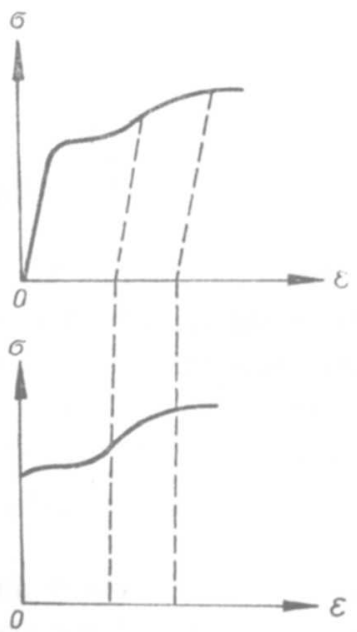

Fig. 59. A graphical construction of the diagram $\sigma=f\left(\varepsilon^{P}\right)$

In order to satisfy the postulate of continuous description we may introduce the intensity of plastic strains, $\varepsilon_{e}^{p}$, by a formula analogous to (7.29), and replace (14.2) by

$$
\sigma_{e}=\bar{f}\left(\varepsilon_{e}^{p}\right) .
$$

The conversion of (14.2) to (14.5) for a tension-test diagram presents no difficulties (Fig. 59). For simple processes the hypothesis of a uniform curve (14.5) is equivalent to (14.2) and vice versa. In fact, making use of the auxiliary nine-dimensional vector 
space, we may interpret the intensity as the length (with an appropriate coefficient) of the vector representing the deviator

$$
\varepsilon_{e}=\sqrt{\frac{2}{3}}|\mathbf{e}| .
$$

The vectorial decomposition of the deviator into its elastic and its plastic part, $\mathbf{e}=\mathbf{e}^{e}+\mathbf{e}^{p}$, gives for the lengths the relation

$$
|\mathbf{e}|^{2}=\left|\mathbf{e}^{e}\right|^{2}+\left|\mathbf{e}^{p}\right|^{2}+2\left|\mathbf{e}^{e}\right|\left|\mathbf{e}^{p}\right| \cos \left(\mathbf{e}^{e}, \mathbf{e}^{p}\right) \text {. }
$$

For a simple loading process governed by the Prandtl-Reuss equations (or their generalization to plastic hardening, described in detail in Sec. 15), the corresponding elastic and plastic strain components are proportional to each other; hence the angle $\left(\mathbf{e}^{e}, \mathbf{e}^{p}\right)=0$, and

$$
|\mathbf{e}|=\left|\mathbf{e}^{e}\right|+\left|\mathbf{e}^{p}\right|, \quad \varepsilon_{e}=\varepsilon_{e}^{e}+\varepsilon_{e}^{p} .
$$

This formula (in the second form) was given by A. A. Ilyushin [1.8], but without the reservation that it is valid for simple loadings only. For other processes we may obtain for example $\varepsilon_{e}=0$ at $\varepsilon_{e}^{e}>0$ and $\varepsilon_{e}^{p}>0$, which contradicts (14.8), but is possible in view of (14.7).

In the case of simple loading, making use of (14.8) and of the relation

$$
\varepsilon_{e}^{e}=\frac{\sigma_{e}}{3 G},
$$

(easy to derive if we define $\varepsilon_{e}$ by (7.29)), we obtain from (14.2)

$$
\sigma_{e}=f\left(\frac{\sigma_{e}}{3 G}+\varepsilon_{e}^{p}\right) .
$$

A formal solution of (14.10) with respect to $\sigma_{e}$ leads to (14.5).

Hypothesis (14.5), which does not contradict the postulate of continuous description of neutral processes, has therefore greater chances in the general case of combined loading than (14.2). It is sometimes used (D. D. Ivlev, G. I. Bykovtsev [3.312]), but the gain is not very large: for simple processes it reduces to (14.2), and the general case is still excluded, since even the plastic strain intensity, $\varepsilon_{e}^{p}$, may decrease during an active process.

We are going to show an example of decreasing plastic strain intensity during an active process. Making use of the Prandtl-Reuss equations (for a perfectly elasticplastic body) and passing to the vectorial notation, we calculate

$$
\frac{d}{d t}\left[\left(\varepsilon_{e}^{p}\right)^{2}\right]=\frac{4}{3} e_{i j}^{p} \dot{e}_{i j}^{p}=\frac{4}{3} \lambda s_{i j} e_{i j}^{p}=\frac{4}{3} \lambda \mathbf{s e}^{p}=\frac{4}{3} \lambda|\mathbf{s}|\left|\mathbf{e}^{p}\right| \cos \left(\mathbf{s}, \mathbf{e}^{p}\right) .
$$

Suppose that a simple process is followed by a neutral one: during this process $\lambda=0$, the plastic strains are constant, $\varepsilon_{e}^{p}=$ const, whereas the stresses $\sigma_{i j}$ are subject to variation. Continuing the neutral process up to the point at which the angle between $\mathbf{s}$ and $\mathrm{e}^{p}$ is obtuse and then starting a new active process, we observe 
a decrease of the plastic strain intensity $\varepsilon_{e}^{p}$. Therefore the curve (14.5) cannot describe such a process correctly.

In reference to the example analysed in Secs. 9.5-9.7 the above process was illustrated in the planes $\tau_{z x}-\tau_{z y}$ and $\gamma_{z x}-\gamma_{z y}$ in Fig 60. For the point $B$ reached
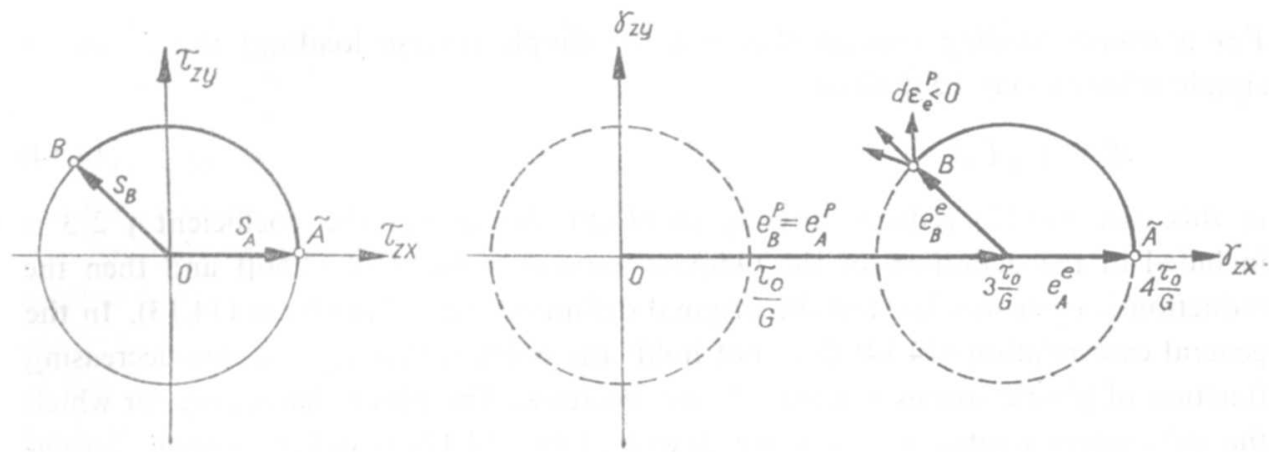

Fig. 60. An example of decreasing stress intensity during an active process

as a result of a neutral process, the vectors $\mathbf{e}_{B}^{p}$ and $\mathbf{e}_{A}^{p}$ are equal whereas the vector $\mathbf{s}_{B}$ is essentially changed with respect to $\mathbf{s}_{A}$ and the angle between $\mathbf{e}_{B}^{p}$ and $s_{B}$ is obtuse.

A sufficiently general extension of the concept of a uniform curve of plastic hardening (14.1) or (14.2) to arbitrary non-proportional processes must therefore go further and use a non-decreasing function of plastic strains as the argument of the right-hand side. Such proposals are described in the following section.

\subsection{Hypotheses of isotropic hardening}

A full description of plastic hardening consists of two types of equations: constitutive (physical) equations for active processes, and equations of subsequent neutral surfaces determining the range of validity of those physical equations. The second group is more significant for the type of hardening, and the relevant hypotheses with their experimental verification will be discussed in this section, whereas constitutive equations will be given separately in Sec. 15. Such an approach is demonstrative and clear, but one has to remember that the considerations of Sec. 14 alone are to a certain degree ineffective, since the plastic strains appearing here should be calculated with the use of the equations given in Sec. 15.

The most frequently used non-decreasing scalar functions of plastic strains, giving a possible correct generalization of (14.5) to arbitrary non-proportional processes, are connected with length of the trajectory in the plastic strain space, or with plastic work.

The simpler form (though historically later) was proposed in 1933 by F. K. G. Odqvist [3.586]; it constitutes a direct generalization of (14.5):

$$
\sigma_{e}=f_{1}\left(I_{\varepsilon p}\right)
$$


The Odqvist parameter $I_{e p}$ is here understood simply as the length of the trajectory in the plastic strain space

$$
I_{\varepsilon p}=\int_{0}^{\tilde{t}} \sqrt{\dot{\varepsilon}_{i j}^{p} \dot{\varepsilon}_{i j}^{p}} d t=\int_{0}^{\tilde{t}} \sqrt{\dot{e}_{i j}^{p} \dot{e}_{i j}^{p}} d t=\int_{0}^{\tilde{e}^{p}} \sqrt{d e_{i j}^{p} d e_{i j}^{p}} .
$$

For a simple loading process (but not for simple reverse loading) the following simple relation may be derived:

$$
\varepsilon_{e}^{p}=\sqrt{\frac{2}{3}} I_{\varepsilon p} ;
$$

in this case (14.12) reduces directly to (14.5). Sometimes the coefficient $\sqrt{2 / 3}$ is included in the definition of the Odqvist parameter ( $\mathrm{R}$. Hill [2.56]) and then the reduction is even simpler, but the original definition was of the form (14.13). In the general case relation (14.14) does not hold; the point is that $I_{\varepsilon p}$ is a non-decreasing function of plastic strains whereas $\varepsilon_{\boldsymbol{e}}^{p}$ may decrease. The plastic hardening for which the subsequent neutral surfaces are described by (14.12) is called isotropic strainhardening, or briefly strain hardening.

The geometrical interpretation of (14.12) is very simple. For each $I_{e p}$ we have a geometrically similar, centrally located neutral surface; their dimensions increase monotonically with $I_{\varepsilon p}$ (Fig. 61). An isotropic material remains isotropic and hence

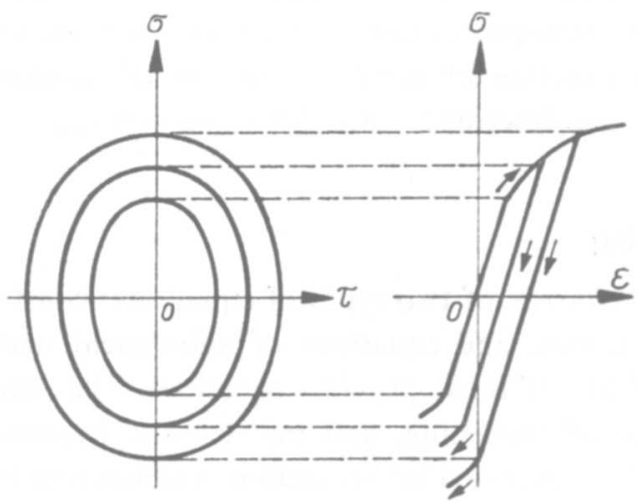

Fig. 61. Isotropic plastic hardening

the name. In the uniaxial case the material behaves similarly to the model shown in Fig. 30, Sec. 8.7.

Special attention should be paid to the symbols $d \varepsilon$ or $d e$. If such an increment is understood as always related to the initial length, then the integration leads to the classical Cauchy definition of strain; if, however, it is understood as related to the actual length, then we arrive at the logarithmic (Hencky) strain. The latter approach may be regarded as correct only in the case of a process described in constant principal directions (the difficulties arising in the more general case were discussed in Sec. 7.7). 
If a restriction to small strains is made, then (14.12) may correctly describe the process even with changing principal directions. A certain experimental evidence of that was given by Yu. I. Yagn and L. V. Grebnev [3.851], S. A. Elsufyev and I. N. Izotov [3.168].

It should be noted that in his original definition Odqvist substituted total strain rates into (14.13) but with a clear statement that elastic strain rates are neglected. Some authors attempted to use (14.13), substituting total strain rates even if elastic strain rates were not neglected (A. A. Ilyushin [1.10], V. I. Malyi [3.492], A. S. Kravtchuk [3.390]), but the range of applicability of such an approach is very narrow and for processes close to a neutral one the results may be misleading.

Another non-decreasing scalar function of plastic strains, without direct connection to (14.5), was suggested as a tool of correct description of isotropic hardening by G. I. Taylor and H. Quinney [3.791] in 1931, and independently by R. Schmidt [3.704] in 1932. They proposed to express plastic hardening as a function of plastic work $W^{p}$, or dissipated energy $W^{d}$, namely,

$$
\sigma_{e}=f_{2}\left(W^{p}\right)=f_{2}\left(W^{d}\right) .
$$

In Sec. 8.8 we pointed to the differences between $W^{p}$ and $W^{d}$. They are connected mainly with microstresses, i.e. with the Bauschinger effect, whereas for isotropic hardening neglecting that effect we may substitute

$$
W^{p}=W^{d}=\int_{0}^{\tilde{e}^{p}} \sigma_{i j} d \varepsilon_{i j}^{p}=\int_{0}^{\tilde{e}^{p}} \sigma_{i j} d e_{i j}^{p}=\int_{0}^{\tilde{e}^{p}} s_{i j} d e_{i j}^{p} .
$$

The plastic hardening described by (14.15) is called isotropic work hardening or briefly work hardening. A corresponding mechanical model was shown in Fig. 31, Sec. 8.7. D. R. Bland [3.65] proved that Eqs. (14.12) and (14.15) are equivalent, and further, that their generalizations making use of any quadratic yield condition are respectively equivalent. This class of equivalence was extended by K. Hellan [3.240].

The generalization of (14.12) or (14.15) to other yield conditions presents no difficulties, at least on the left-hand side: stress intensity may be replaced by any other reduced stress calculated on the basis of the relevant failure hypothesis. Assuming, as usual, that $\sigma_{\text {red }}$ is a homogeneous function of the first degree with respect to the stress components, we obtain as before a family of geometrically similar concentric surfaces. The problem of the right-hand side is then open: one may retain the corresponding expressions (R. Hill [2.56]) or introduce new non-decreasing scalar functions which seem to be more appropriate. Such functions, generalizing the Odqvist parameter, were proposed by C. A. Berg [3.53] and probably will be particularly useful for anisosensitive materials. For isosensitive materials the retention of the right-hand sides of (14.12) and (14.15) usually gives a better description; for example, O. M. Sidebottom [3.737] compared the equation

$$
\tau_{\max }=f_{1}\left(\int d \gamma_{\max }^{p}\right)
$$


with $\tau_{\max }=f_{1}\left(I_{\varepsilon p}\right)$ finding that the latter is in closer agreement with experimental results. Similar results were obtained by G. N. White and D. C. Drucker [3.844]. Another proposal, based on the Tresca-Guest hypothesis, was given by D. D. Ivlev [3.304]. A general theory of piece-wise linear isotropic hardening was proposed by P. G. Hodge [3.259].

\subsection{Generalized isotropic hardening}

The subsequent neutral surfaces, described in Sec. 14.2, were concentric and geometrically similar to each other, and so the initial isotropy of the material was retained. However, the possible class of materials whose subsequent neutral surfaces preserve initial isotropy is much broader, and, for example, may describe a certain type of the Bauschinger effect as well. Such types of plastic hardening will be called generalized isotropic hardening in order to retain the traditional name "isotropic hardening" for the classical case of geometrically similar concentric surfaces.

Theoretically, any function of the type

$$
F\left(I_{1 \sigma}, I_{2 \sigma}, I_{3 \sigma} ; I_{1 \varepsilon}^{p}, I_{2 \varepsilon}^{p}, I_{3 \varepsilon}^{p}, k_{i}\right)=0
$$

may describe in the stress space a family of subsequent neutral surfaces which retain the isotropy of the material. This type of functions was proposed by D. D. Ivlev and G. I. Bykovtsev [3.312], who regarded $k_{i}$ as constants; in fact, those functions may include also integral invariants, such as, for example, the Odqvist parameter or plastic work. Any other system of symmetric invariants may also be introduced nto (14.18).

A further specification of generalized isotropic hardening seems useful. If the material is initially isosensitive, then by (14.18) the isosensitivity may be retained or not. In the first case we may speak of isosensitive hardening (the classical isotropic hardening belongs of course to this type); in the second case-of anisosensitive isotropic hardening, allowing for the Bauschinger effect.'

It is reasonable to confine isosensitive hardening to the non-decreasing functions of plastic deformations, for example

$$
F\left(I_{1 \sigma}, I_{2 \sigma}, I_{3 \sigma} ; I_{s p}, k_{i}\right)=0 \text {. }
$$

This equation may be regarded as a further generalization of (14.12). As an example of isosensitive generalized linear strain hardening let us consider the function

$$
\left(1-\psi I_{\varepsilon p}\right) \sigma_{e}+\psi I_{\varepsilon p}\left(\sigma_{I}-\sigma_{I I I}\right)=\sigma_{0}+\sqrt{\frac{2}{3}} E_{1} I_{\varepsilon p},
$$

where $E_{1}$ denotes the hardening modulus and $\psi$ is a constant; Eq. (14.20) gradually transforms-in the course of plastic deformations-the $\mathrm{HMH}$ yield condition into the TG one.

In the case of anisosensitive isotropic hardening it is justified to admit also the decreasing functions of plastic deformations, i.e. to use the general form of (14.18). For example, any function of the type

$$
\sigma_{e}^{2}+f_{1}\left(I_{1 \varepsilon}^{p}, I_{2 \varepsilon}^{p}, I_{3 \varepsilon}^{p}\right) \sigma_{m}=\sigma_{0}^{2}\left[1+f_{2}\left(I_{1 \varepsilon}^{p}, I_{2 \varepsilon}^{p}, I_{3 \varepsilon}^{p}\right)\right]
$$


where $f_{1}(0,0,0)=f_{2}(0,0,0)=0$, transforms the HMH cylinder into a Burzyński paraboloid, and hence it creates anisosensitivity (describes the Bauschinger effect), leaving initial isotropy. A related equation of subsequent neutral surfaces for concrete was suggested by $\mathbf{Z}$. Mróz [3.558].

One should be extremely cautious when suggesting any particular form of (14.18). These functions bring often unexpected phenomena, far from the behaviour of real materials; some of them were mentioned by Ivlev and Bykovtsev. The Bauschinger effect is usually (though not necessarily) connected with induced anisotropy and one cannot expect to describe it properly by (14.18) or, in particular, by (14.21). However, the value of such equations is not only theoretical; they may be practically useful when combined with kinematic hardening, described in Sec. 14.4. For example, kinematic hardening assumes the same Bauschinger effect for normal and for shearing stresses, whereas (14.21) describes the Bauschinger effect for normal stresses only (cf. Sec. 10.6). Hence, if that effect is different for normal and tangential stresses, a combination of (14.21) with kinematic hardening may be appropriate.

Finally, it should be noted that Y. C. Fung [3.190] ascribes to the functions proposed by F. Edelman and D. C. Drucker [3.164] the Bauschinger effect with preserved isotropy. Actually, isotropy is not preserved there and those functions will be discussed in Sec. 14.8.

\subsection{Hypotheses of kinematic hardening}

The simplest type of anisotropic plastic hardening corresponding to the ideal Bauschinger effect $(\beta=1$, Sec. 8.5$)$ is called kinematic hardening. Its dynamical model was shown in Fig. 29, but its kinematic model is even simpler. In uniaxial tensioncompression the ideal Bauschinger effect is obtained in the case of constant length of the segment of passive processes and may be modelled by a rigid translation of that segment along the axis of stresses. A natural generalization consists in a hypothesis of rigid translation of the initial neutral surface in the nine-dimensional auxiliary stress space. If the initial surface is described by the equation

$$
F\left(\sigma_{i j}\right)=C,
$$

then as a result of plastic hardening (or rather apparent hardening, Sec. 8.5) it takes the form

$$
F\left(\sigma_{i j}-\alpha_{i j}\right)=C .
$$

Therefore such a hypothetical behaviour of the material is called kinematic hardening. The tensor $\alpha_{i j}$ describes the location of the centre of subsequent neutral surfaces; an effective formulation of the hypothesis of kinematic hardening requires-besides the function $F$-a precise specification of the dependence of $\alpha_{i j}$ on $\varepsilon_{i j}^{p}$ or $\sigma_{i j}$.

The first concept of kinematic hardening is due to A. Reuss [3.673], 1934. W. Prager [3.649] combined it in 1935 with isotropic hardening, but the proposed expression did not have an invariant character (the entity $\alpha_{i j}$ was not a tensor) and found no application. If $\alpha_{i j}$ is a tensor and the function $F$ is invariant, then 
(14.23) is of course invariant; we may easily derive the formulae for basic invariants of the difference of two tensors

$$
\begin{aligned}
& I_{1(\sigma-\alpha)}=I_{1 \sigma}^{\gamma \beta}-I_{1 \alpha}, \\
& I_{2(\sigma-\alpha)}=I_{2 \sigma}-2 I_{\sigma \alpha}^{\sigma \alpha}+I_{2 \alpha}, \\
& I_{3(\sigma-\alpha)}=I_{3 \sigma}-3 I_{\sigma \sigma \alpha}+3 I_{\sigma \alpha \alpha}-I_{3 \alpha},
\end{aligned}
$$

where the "common" invariants are defined by (5.25). Hence (14.23) belongs to the invariant functions of the type

$$
F\left(I_{1 \sigma}, I_{2 \sigma}, I_{3 \sigma}, I_{1 \alpha}, I_{2 \alpha}, I_{3 \alpha}, I_{\sigma \alpha}, I_{\sigma \sigma \alpha}, I_{\sigma \alpha \alpha}\right)=C,
$$

which will be discussed in detail in Sec. 14.8.

The first correctly invariant, effective hypothesis of kinematic hardening was formulated in 1938 by E. Melan [3.516]. He considered two variants of the expression for of $\alpha_{i j}$ : either

$$
d \alpha_{i j}=c d \varepsilon_{i j}^{p}, \quad c=\text { const },
$$

(this case is limited to linear plastic hardening) or

$$
d \alpha_{i j}=d \sigma_{i j}
$$

(this proposal may apply to any curve of plastic hardening but does not give a continuous description of the neutral process and hence its use is subject to restrictions). The Melan proposals were not reflected in world literature for a considerable time. It was only the papers of A. Yu. Ishlinsky [3.298], 1954, and W. Prager [3.655, 3.656], 1955-1956, that gained wider recognition. Prager proposed the name "kinematic hardening", connected with his kinematic models. Ishlinsky and Prager determine the tensor $\alpha_{i j}$ by Eq. (14.26), Ishlinsky for the initial HMH yield condition, and Prager for the general one. If the associated flow rule is additionally postulated, then the direction of motion of the neutral surface does not depend on $d \sigma$ (contrary to the Melan hypothesis (14.27)) and is determined by the normal to that surface at the point representing the actual stress state.

Yu. I. Kadashevitch and V. V. Novozhilov $[3.322,2.80]$ gave a physical interpretation of $\alpha_{i j}$ calling it the "tensor of residual microstresses" and generalized (14.26) to the form

$$
\alpha_{i j}=2 g \varepsilon_{i j}^{p} ;
$$

in [3.322] $g$ was supposed to be a function of plastic strain intensity, and in [2.80] a function of invariants of the tensor $\alpha_{i j}$. Such an approach was criticized by M. A. Eisenberg and A. Phillips [3.165], who proposed to express $g$ in (14.28) as a function of the Odqvist parameter (14.13).

Kadashevitch and Novozhilov proposed also a mechanical model generalizing that given in Fig. 29 to two dimensions. Plastic deformations are modelled, as usual, by dry friction. A further development of that model was given by Yu. I. Kadashevitch and N. V. Kirsanova [3.328], and a related model, consisting of many springs and friction elements, by C. H. Wells and P. R. Paslay [3.843]. V. V. Novozh- 
ilov [3.584] presented microstresses as the sum of elastic microstresses vanishing with vanishing plastic strains and friction-like microstresses which need not vanish:

$$
\alpha_{i j}=\frac{\partial \psi}{\partial \varepsilon_{i j}^{p}}+\int \varepsilon_{i j}^{p} d \chi,
$$

where $\psi=\psi\left(\varepsilon_{i j}^{p}\right)$ and $\chi$ is a function of intensity of the tensor $\sigma_{i j}-\alpha_{i j}$. The further development of the concept of microstresses is due to Yu. I. Kadashevitch and V. V. Novozhilov [3.325, 3.326, 3.327], A. A. Vakulenko [3.826] (based on thermodynamical considerations), A. A. Vakulenko and M. L. Kachanov [3.828], V. Kafka [3.331, 3.332, 3.334, 3.335], V. Kafka and R. Novotný [3.333] Y. R. Rashid [3.666], and D. A. Gokhfeld, I. A. Ivanov, O. S. Sadakov [3.200] (cyclic loading). A probabilistic treatment of microstresses was suggested by G. I. Barenblatt and V. A. Gorodtsov [3.31] (analogy to turbulent motion), and Yu. I. Kadashevitch [3.330].

A different but related physical background of kinematic hardening is connected with the macro- or micrononhomogeneity of the material, stress concentration around holes and inclusions, etc. Such concepts were proposed by D. C. Drucker [3.155], W. D. Iwan [3.313], V. V. Dudukalenko and V. A. Minayev [3.161], I. A. Ivanov [3.299], J. F. Williams and N. L. Svensson [3.846]. I. A. Berezhnoi, D. D. Ivlev and E. V. Makarov [3.49] proposed a model of kinematic hardening without making use of the concept of microstresses. The influence of time effects on kinematic hardening was considered by A. Berio, L. Bortolotti, P. Manca and A. Paglietti [3.54].

A certain deficiency of the basic concept (14.26) lies in the fact that-in generalif it holds in the nine-dimensional Prager space, then it does not hold in a subspace of fewer dimensions: instead of rigid translation we obtain there a certain deformation of the neutral surface. This is a logical deficiency and causes certain difficulties in applications. This problem was studied in detail by R. Shield and H. Ziegler [3.721], C. Clavout and H. Ziegler [3.106], and by N. Perrone and P. G. Hodge [3.623]. They stated that the problem is not essential in the case of the Huber-MisesHencky hypothesis, but significant differences appear between the behaviour of neutral surfaces in the nine-dimensional space and in subspaces if other failure hypotheses are considered, e.g. that of Tresca-Guest. Perrone and Hodge introduced the terms "complete kinematic hardening" if it takes place in the complete ninedimensional space, and "direct kinematic hardening" if it is assumed formally in a subspace. The latter concept may be regarded as an approximation of complete kinematic hardening. Perrone and Hodge transferred that concept also to the level $\mathscr{S}$ of analysis (a section of a bar or of a surface structure).

Another approach was suggested by $\mathrm{H}$. Ziegler [3.870], who proposed a certain modification of the kinematic hardening hypothesis. Instead of the commonly used law (14.26), he described translatory motion of the neutral surface by

$$
d \alpha_{i j}=\left(\sigma_{i j}-\alpha_{i j}\right) d \mu .
$$

In this case translatory motion without deformation of the surface is transferred from the original space to any subspace: it takes place in the radial direction deter- 
mined by the vector $\sigma-\alpha$. The proportionality coefficient $d \mu$ may be found from the postulate that after an active process the point representing the stress state must remain on the neutral surface (called the "consistency condition" by W. Prager [3.653], cf. Sec. 15.3).

$$
d F=\frac{\partial F}{\partial \sigma_{k l}} d \sigma_{k l}+\frac{\partial F}{\partial \alpha_{k l}} d \alpha_{k l}=0,
$$

and hence, in view of (14.30) and $\partial F / \partial \alpha_{k l}=-\partial F / \partial \sigma_{k l}$,

$$
d \mu=\frac{\frac{\partial F}{\partial \sigma_{k l}} d \sigma_{k l}}{\frac{\partial F}{\partial \sigma_{m n}}\left(\sigma_{m n}-\alpha_{m n}\right)}
$$

However, even in the case of the Ziegler kinematic hardening rule certain objections may be raised: N. K. Das Talukder [3.126] shows that translatory motion without deformation of the neutral surface is not transferred to the subspaces if a "new" active process is preceded by a passive one and takes place in a direction different from the "old" one.

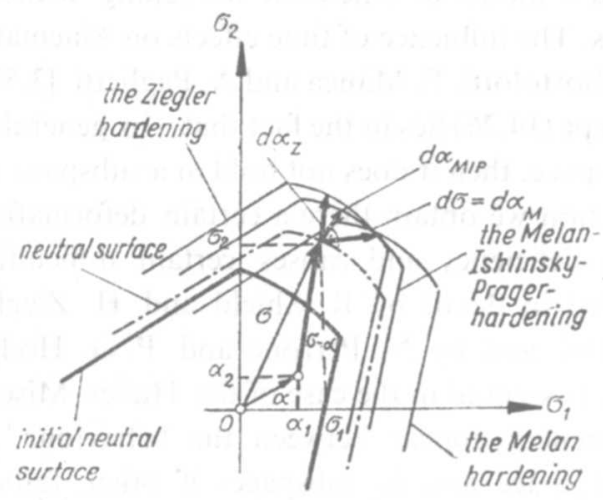

Fig. 62. Various concepts of kinematic hardening rules

Various concepts of kinematic hardening rules are shown in Fig. 62: the MelanIshlinsky-Prager rule (14.26) combined with associated flow rule, the Melan rule (14.27) and the Ziegler rule (14.30). To simplify the picture, the possible deformation of the neutral surface in the plane treated as a subspace of the nine-dimensional space has been disregarded.

The Ziegler hardening rule may also be presented in another way. Namely, one can retain (14.26) without change, but replace the associated flow rule by the rule

$$
d \varepsilon_{i j}^{p}=\frac{1}{c}\left(\sigma_{i j}-\alpha_{i j}\right) d \mu
$$


constituting a certain counterpart of the Levy-Mises or Prandtl-Reuss law of similarity of deviators. Translatory motion of the neutral surface in the stress space then remains without change, (14.30), whereas the corresponding plastic strains change. A similar approach may be found in paper [3.13] by R. A. Arutyunyan and A. A. Vakulenko. An adaptation of the Ziegler rule to the effective calculation of structures subject to constant or cyclic loadings was given by H. Armen, G. Isakson and A. Pifko [3.11], who prepared the relevant computer programs. V. V. Moskvitin and Zh. I. Tairova [3.545] mentioned certain simplifications in applying the MelanIshlinsky-Prager hardening rule combined with the Tresca-Guest hypothesis to the calculation of cyclic loading processes; however, the authors assume no attitude to the critical remarks of Shield, Clavout and Ziegler [3.721, 3.106] concerning such an approach.

\subsection{A triple analogy for kinematic hardening}

The term kinematic hardening was introduced by Prager with reference to the kinematic analogy - translatory motion of a rigid frame. On the other hand, in Sec. 13.7 we observed a similar motion of neutral surfaces for a perfectly elastic-plastic material in the strain space. Hence-under certain restrictions - a triple analogy between kinematics, kinematic plastic hardening and perfect plasticity may be stated (M. Życzkowski [3.878]). We are now going to formulate the conditions of validity of three variants of that analogy, put together in Table 3.

\section{Variant 1}

A. Consider the motion of a rigid frame given by the equation $F\left(y_{i}\right)=F\left(x_{i}-\xi_{i}\right)$ $=0, i=1,2, \ldots, 9$, led by a frictionless pin in a nine-dimensional space. Assume a "forcing" increment of the pin trajectory $d x$ : then the increment of the frame centre trajectory is $d \xi$, in the direction normal to the surface at $\times$ (Fig. 63):

$$
d \xi_{i}=|d \mathbf{x}| \cos \varphi n_{F i}=\left(\mathbf{n}_{F} d \mathbf{x}\right) n_{F i}
$$

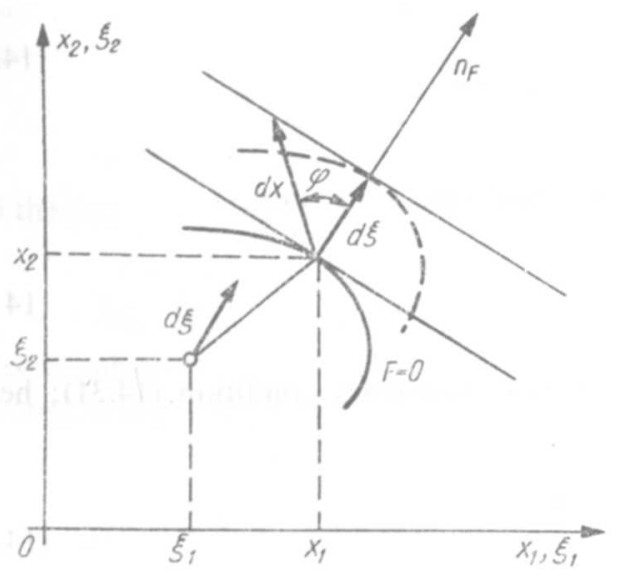

Fig. 63. The Melan-Ishlinsky-Prager kinematic hardening rule 
Table 3

Variants of a triple analogy for kinematic hardening
A. Kinematics, translatory motion
B. Plastic hardening, stress space
C. Perfect plasticity, strain space

1. Frame led by a frictionless pin

The Melan-Ishlinsky-Prager

Associated flow rule hardening (14.26) with the associated flow rule

2. Frame led in actual radial direction

The Ziegler hardening (14.30)

3. Frame led by a glued pin The Melan hardening (14.27) The Leytes stabilization hypothesis $d \sigma_{\imath \jmath}=0$

Corresponding quantities, $i=1,2, \ldots, 9$

a. Absolute coordinates

"Total"

Total

$x_{i}$

stresses $\sigma_{t}$

strains $\varepsilon_{i}$

b. Base coordinates, locations
of the centre $\xi_{l}$
Residual microstresses $\alpha_{i}$
Plastic strains $\varepsilon_{t}^{P}$
c. Relative coordinates
"Free" stresses
Elastic strains
$y_{l}=x_{l}-\xi_{l}$
$\tau_{l}=\sigma_{t}-\alpha_{l}$
$\varepsilon_{i}^{e}=\varepsilon_{i}-\varepsilon_{i}^{P}$

where $\mathbf{n}_{F}$ denotes the unit vector normal to $F$ at $\mathbf{x}$, and $n_{F i}$ its coordinates. Expressing these coordinates by formulae of the type (13.22), we obtain for the frame motion

$$
d \xi_{i}=\frac{\frac{\partial F}{\partial\left(x_{j}-\xi_{j}\right)} d x_{j}}{\frac{\partial F}{\partial\left(x_{k}-\xi_{k}\right)} \frac{\partial F}{\partial\left(x_{k}-\xi_{k}\right)}} \frac{\partial F}{\partial\left(x_{i}-\xi_{i}\right)} .
$$

B. Combining (14.26) with the associated flow rule, we obtain

$$
d \alpha_{i j}=c_{1} \frac{\partial F}{\partial \sigma_{i j}} .
$$

The constant $c_{1}$ may be determined from the consistency condition (14.31); hence

$$
d \alpha_{i j}=\frac{\frac{\partial F}{\partial \sigma_{k l}} d \sigma_{k l}}{\frac{\partial F}{\partial \sigma_{m n}} \frac{\partial F}{\partial \sigma_{m n}}} \frac{\partial F}{\partial \sigma_{i j}}
$$


Finally, in view of $\partial F / \partial \sigma_{k l}=\partial F / \partial\left(\sigma_{k l}-\alpha_{k l}\right)$, we obtain

$$
d \alpha_{i j}=\frac{\frac{\partial F}{\partial\left(\sigma_{k l}-\alpha_{k l}\right)} d \sigma_{k l}}{\frac{\partial F}{\partial\left(\sigma_{m n}-\alpha_{m n}\right)}-\frac{\partial F}{\partial\left(\sigma_{m n}-\alpha_{m n}\right)}} \frac{\partial F}{\partial\left(\sigma_{i j}-\alpha_{i j}\right)},
$$

and in the auxiliary nine-dimensional stress space the Prager analogy between (14.35) and (14.38) is proved.

C. For a perfectly elastic-plastic body with the yield condition $F\left(\sigma_{i j}\right)=0$, the associated flow rule gives

$$
d \varepsilon_{i j}^{p}=\frac{\partial F}{\partial \sigma_{i j}} d \lambda
$$

Multiplication by $\partial F / \partial \sigma_{i j}$ makes it formally possible to eliminate $d \lambda$ (though the yield condition is not yet used) and to write

$$
d \varepsilon_{i j}^{p}=\frac{\frac{\partial F}{\partial \sigma_{k l}} d \varepsilon_{k l}^{p}}{\frac{\partial F}{\partial \sigma_{m n}} \frac{\partial F}{\partial \sigma_{m n}}} \frac{\partial F}{\partial \sigma_{i j}} .
$$

In order to obtain an analogy with (14.35) and (14.38) we have to introduce differentiation with respect to elastic strains, Table 3. Under the restriction to isotropic bodies we write a counterpart to $(9.4)$ in the form

$$
\varepsilon_{i j}^{e}=\left(\delta_{i k} \delta_{j l}-\frac{v}{1+\nu} \delta_{i j} \delta_{k l}\right) \frac{\sigma_{k l}}{2 G} ;
$$

hence, expressing $F$ in terms of $\varepsilon_{i j}^{e}$,

$$
\frac{\partial F}{\partial \sigma_{i j}}=\frac{\partial F}{\partial \varepsilon_{k l}^{e}} \frac{\partial \varepsilon_{k l}^{e}}{\partial \sigma_{i j}}=\frac{1}{2 G} \frac{\partial F}{\partial \varepsilon_{i j}^{e}}-\frac{v}{1+\nu} \frac{\delta_{i j} \delta_{k l}}{2 G} \frac{\partial F}{\partial \varepsilon_{k l}^{e}} .
$$

The analogy will be obtained if the second term in (14.42) vanishes. This occurs in two cases: (1) if the yield surface is cylindrical, since then $\delta_{k l} \partial F / \partial \varepsilon_{\boldsymbol{k} l}^{e}=0$, and (2) if $v=0$. Under either of those assumptions we may write

$$
\frac{\partial F}{\partial \sigma_{i j}}=\frac{1}{2 G} \frac{\partial F}{\partial \varepsilon_{i j}^{e}},
$$

and the yield condition expressed in terms of $\varepsilon_{i j}^{e}$ gives

$$
\frac{\partial F}{\partial \varepsilon_{i j}^{e}} d \varepsilon_{i j}^{e}=0 \text {. }
$$

Indeed, substituting into (14.40) the relation (14.43), putting on the right-hand side $d \varepsilon_{k l}^{p}=d \varepsilon_{k l}-d \varepsilon_{k l}^{e}$ and making use of (14.44), we obtain

$$
d \varepsilon_{i j}^{p}=\frac{\frac{\partial F}{\partial \varepsilon_{k l}^{e}} d \varepsilon_{k l}}{\frac{\partial F}{\partial \varepsilon_{m n}^{e}} \frac{\partial F}{\partial \varepsilon_{m n}^{e}}} \frac{\partial F}{\partial \varepsilon_{i j}^{e}},
$$


and the analogy with (14.35) and (14.38) can be seen. We have thus proved the first variant of the triple analogy, provided that for the perfectly elastic-plastic body either the yield surface is cylindrical or Poisson's ratio vanishes.

\section{Variant 2}

A. The motion of the rigid frame in a "radial" direction is presented in Fig. 64 . We now have

$$
d \xi_{i}=|d \mathbf{x}| \frac{\cos \varphi}{\cos \psi} \frac{x_{i}-\xi_{i}}{|\mathbf{x}-\xi|} .
$$

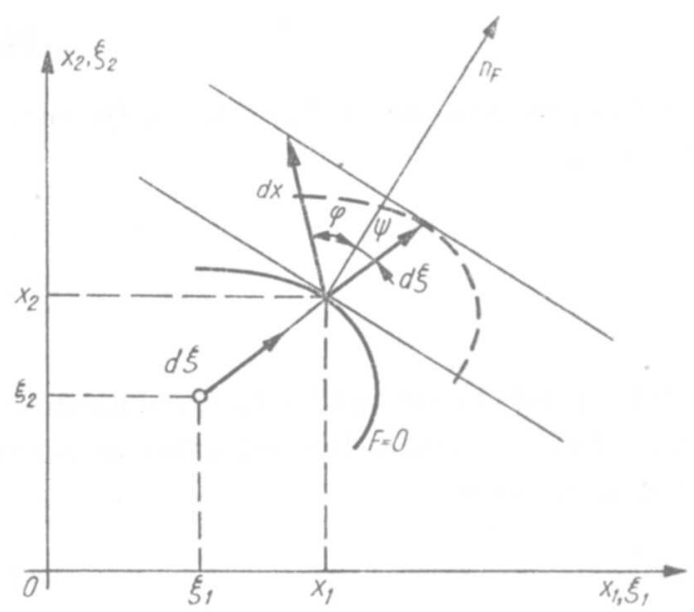

Fig. 64. The Ziegler kinematic hardening rule

The cosines will now be expressed by means of scalar products of vectors

$$
\cos \varphi=\frac{\mathbf{n}_{F} d \mathbf{x}}{|d \mathbf{x}|}, \quad \cos \psi=\frac{\mathbf{n}_{F}(\mathbf{x}-\xi)}{|\mathbf{x}-\xi|},
$$

and finally, in terms of coordinates,

$$
d \xi_{i}=\frac{\mathbf{n}_{F} d \mathbf{x}}{\mathbf{n}_{F}(\mathbf{x}-\xi)}\left(x_{i}-\xi_{i}\right)=\frac{\frac{\partial F}{\partial\left(x_{j}-\xi_{j}\right)} d x_{j}}{\frac{\partial F}{\partial\left(x_{k}-\xi_{k}\right)}\left(x_{k}-\xi_{k}\right)}\left(x_{i}-\xi_{i}\right) .
$$

B. The Ziegler kinematic hardening rule is determined by (14.30) with the substitution of (14.32):

$$
d \alpha_{i j}=\frac{\frac{\partial F}{\partial \sigma_{k l}} d \sigma_{k l}}{\frac{\partial F}{\partial \sigma_{m n}}\left(\sigma_{m n}-\alpha_{m n}\right)}\left(\sigma_{i j}-\alpha_{i j}\right) .
$$


Writing this equation in the equivalent form

$$
d \alpha_{i j}=\frac{\frac{\partial F}{\partial\left(\sigma_{k l}-\alpha_{k l}\right)} d \sigma_{k l}}{\frac{\partial F}{\partial\left(\sigma_{m n}-\alpha_{m n}\right)}\left(\sigma_{m n}-\alpha_{m n}\right)}\left(\sigma_{i j}-\alpha_{i j}\right),
$$

we obtain in the auxiliary nine-dimensional stress space a full analogy with (14.48).

C. The law of similarity of deviators for a perfectly elastic-plastic body (PrandtlReuss) may be written as $\left({ }^{1}\right)$

$$
d e_{i j}^{p}=s_{i j} d \lambda .
$$

Multiplication of both sides by $\partial F / \partial \sigma_{i j}$ makes it formally possible to eliminate $d \lambda$ and to write

$$
d e_{i j}^{p}=\frac{\frac{\partial F}{\partial \sigma_{k l}} d e_{k l}^{p}}{\frac{\partial F}{\partial \sigma_{n q}} s_{n q}} s_{i j} .
$$

The analogy with (14.48) and (14.50) will take place if (14.52) can be reduced to the form

$$
d \varepsilon_{i j}^{p}=\frac{\frac{\partial F}{\partial \varepsilon_{k l}^{e}} d \varepsilon_{k l}}{\frac{\partial F}{\partial \varepsilon_{n q}^{e}} \varepsilon_{n q}^{e}} \varepsilon_{i j}^{e} .
$$

In order to obtain such a form we substitute on the right-hand side of (14.52) $d e_{k l}^{p}$ $=d \varepsilon_{k l}^{p}=d \varepsilon_{k l}-d \varepsilon_{k l}^{e}$ and confine our considerations to cylindrical yield surfaces. In view of (14.43) and (14.44) we arrive at

$$
d \varepsilon_{i j}^{p}=\frac{\frac{\partial F}{\partial \varepsilon_{k l}^{e}} d \varepsilon_{k l}}{\frac{\partial F}{\partial \varepsilon_{n q}^{e}} s_{n q}} s_{i j} .
$$

Hooke's law will now be written in the form

$$
s_{l j}=2 G e_{i j}^{e}=2 G\left(\varepsilon_{i j}^{e}-\delta_{l j} \varepsilon_{m}^{e}\right),
$$

where $\varepsilon_{m}^{e}$ denotes the mean elastic normal strain. The substitution of (14.55) into (14.54) does not lead to (14.53) as yet. However, if we assume additionally the elastic incompressibility, $\varepsilon_{m}^{e}=0$, then (14.54) is reduced to (14.53) and the analogy is proved. Recapitulating, Variant 2 of the triple analogy takes place if the perfectly elastic-plastic body under consideration is assumed to be incompressible and described by a cylindrical yield surface.

$\left.{ }^{1}\right)$ Symbol $\lambda$ used here denotes a function slightly different from that used in Sec. 9 (they are interrelated by a differentiation). 


\section{Variant 3}

This variant of the analogy is quite obvious and requires no additional calculations. Indeed, if the pin is glued to the frame (for active processes only), then its relative coordinates are constant, $d y_{i}=0, d \xi_{i}=d x_{i}$. This behaviour corresponds exactly to the second Melan kinematic hardening hypothesis $d \alpha_{i j}=d \sigma_{i j}$. Finally, if we adopt the Leytes stabilization hypothesis $[2.99,2.100]$ to any yield condition for a perfectly elastic-plastic body, then $d \sigma_{i j}=0$; hence $d \varepsilon_{i j}^{e}=0, d \varepsilon_{i j}^{p}=d \varepsilon_{i j}$ and the analogy is proved.

We have to stress once more here that the range of application of both the Melan second kinematic hardening hypothesis and the Leytes stabilization hypothesis is sharply limited to processes close to a simple process.

\subsection{Hypotheses of mixed hardening}

The classical hypotheses of isotropic plastic hardening do not describe the Bauschinger effect, whereas the hypotheses of kinematic hardening correspond to the ideal Bauschinger effect. Real materials usually exhibit a certain intermediate behaviour and hence a more accurate description may be obtained if we combine both the above hypotheses, assuming simultaneous rigid translation with a proportional increase of the neutral surface. Such an idea was initiated by W. Prager in 1935 in the already mentioned paper [3.649]. This hypothesis of plastic hardening will be called mixed hardening.

Combining the general concept of kinematic hardening (14.23) with isotropic strain-hardening (14.12) leads to the equation

$$
F\left(\sigma_{i j}-\alpha_{i j}\right)=f_{1}\left(I_{e p}\right),
$$

where the components of the tensor $\alpha_{i j}$ may be determined by any of the formulae given in Sec. 14.4. Basing ourselves on the HMH hypothesis, we may reduce (14.56) to the form

$$
\left(s_{i j}-\alpha_{i j}^{\prime}\right)\left(s_{i j}-\alpha_{i j}^{\prime}\right)=f_{1}\left(I_{e p}\right),
$$

where $\alpha_{i j}^{\prime}$ denote the deviatoric components of the tensor $\alpha_{i j}$; usually one may assume $\alpha_{i j}^{\prime}=\alpha_{i j}$. If we know the coefficient of the Bauschinger effect (8.17), then both the function $f_{1}$ and the parameter $c$ in (14.26) should depend on this coefficient; for $\beta=0$ we have $c=0$, and for $\beta=1$ (the ideal Bauschinger effect) $f_{1}=$ const $=\frac{2}{3} \sigma_{0}^{2}$. The methods of optimal experimental evaluation of these quantities were proposed by V. N. Bastun and N. I. Chernyak [3.36] (the method of least squares), E. V. Bashkirov [3.33], V. N. Bastun and V. Ya. Bash [3.39] (a method based on the concept of a uniform curve of plastic hardening).

The concept of mixed hardening (14.56), based on the Tresca-Guest hypothesis, was developed by P. G. Hodge [3.260] and Khuan Ke-Chzhi [3.344]. Equation (14.59), mentioned in paper [2.80] by Yu. I. Kadashevitch and V. V. Novozhilov, was developed by G. B. Talypov [2.170, 3.785, 2.171], who additionally takes into 
account the dependence of the Bauschinger effect on the Lode parameter. K. F. Kovalov [3.380] proposed to measure the Bauschinger effect by the translation of the centre of the surface (14.56), i.e. by the quantity $\Delta=\sqrt{\alpha_{i j} \alpha_{i j}}$. R. A. Arutyunyan [3.14] discussed mixed plastic hardening in the case of plastically compressible bodies.

An adaptation of the hypothesis of mixed plastic hardening to the analysis of cyclic loadings was the aim of the paper [3.13] by R. A. Arutyunyan and A. A. Vakulenko. They stated that for most materials the function $f_{1}\left(I_{\varepsilon p}\right)$ has a horizontal asymptote and for a large number of loading cycles (or rather stress-quasi-cycles shown in Fig. 48) $f_{1}=$ const may be assumed. Further investigations in this line were carried out by R. G. Vos and W. H. Armstrong [3.837].

\subsection{Problem of the formation of plastic corners}

The hypotheses of plastic hardening described above transformed subsequent neutral surface without introducing any qualitative changes; for example, the $\mathrm{HMH}$ circular cylinder is always transformed into a regular surface. However, some physical considerations, e.g. the slip theory of S. B. Batdorf and B. Budiansky [3.40, 3.41] and some structural models of plastic hardening (Yu. N. Rabotnov [3.661]) predict the formation of the so-called plastic corners at the points corresponding to the given stress state in the course of an active process (Fig. 65). In what follows such

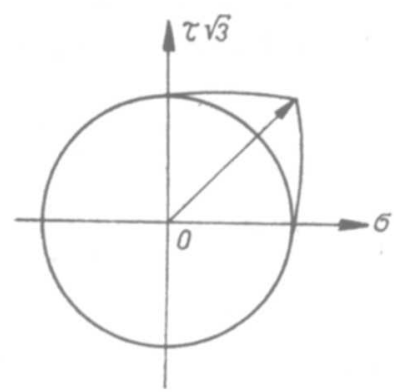

Fig. 65. Hypothesis of formation of a plastic corner

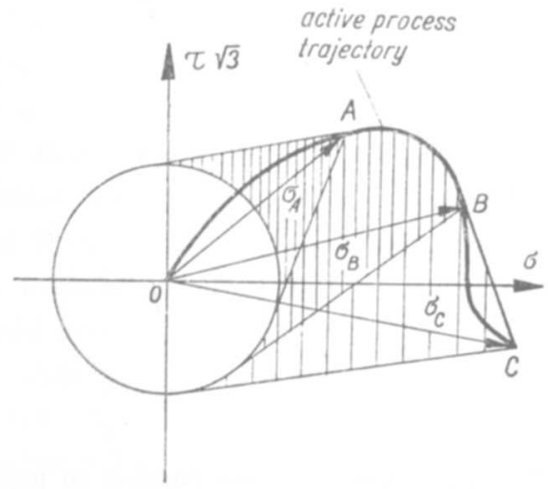

Fig. 66. The Batdorf-Budiansky hardening hypothesis 
points will be called the control points; the term generic stress points is also used. According to the original Batdorf-Budiansky concept the actual neutral curve in the stress plane consists of segments of the initial neutral curve, some tangential segments and some (convex) segments of the process trajectory. (Figure 66 shows subsequent neutral curves for the control points $A, B, C$.) According to that concept the domain of passive processes would be monotonically non-decreasing, similarly as predicted by the hypothesis of isotropic hardening, which requires an even more significant increase of this domain.

Numerous theories describe the formation of the plastic corners. We mention here the theories based on physical considerations, e.g. those proposed by S. B. Batdorf and B. Budiansky [3.40], T. H. Lin [3.451], Y. Yoshimura [3.854], H. Payne [3.621], M. Como and A. Grimaldi [3.108], A. F. Kregers [3.395, 3.396], as well as some phenomenological theories, suggested by J. L. Sanders [3.694], V. D. Klyushnikov [3.358, 3.359], N. Cristescu [3.116]. Some general considerations concerning formation of the plastic corners may be found in the papers by A. Phillips [3.631] and W. Olszak [3.602].

The formation of the corners can also be explained by means of certain structural models of plastic hardening. The Rabotnov model [3.661] compared plastic hardening to the increase of mutually perpendicular bending moments acting on a perfectly elastic-plastic thin-walled tube (pseudo-oblique bending); the initial neutral curve in the moment plane is a circle, but subsequent neutral curves exhibit corners. Further models were given by V. S. Zarubin and A. A. Polyakov [3.861], I. A. Berezhnoi and V. I. Tseyler [3.51, 3.52].

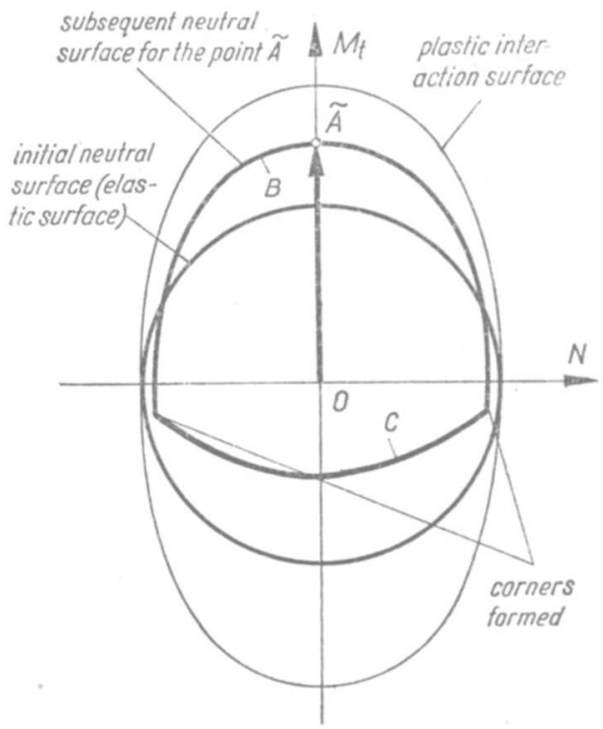

Fig. 67. Formation of plastic corners on subsequent neutral surfaces corresponding to tension with torsion 
One has to be cautious, however, when analysing such structural models: some features have no counterparts in the behaviour of real materials. For example, the Rabotnov model shows corners not only at the control point, but also at two or four other points. Analysing subsequent neutral surfaces for simultaneous tension and torsion for a solid circular perfectly elastic-plastic bar, M. Stodulski [3.771] found no corner at the control point $\tilde{A}$ corresponding to loading by pure torque, but only at two other points (Fig. 67). This feature is connected with the fact that secondary plastification in the combined case may start either at the points $B$ or at the points $C$ of the cross-section (Fig. 68); on the other hand, if we had considered

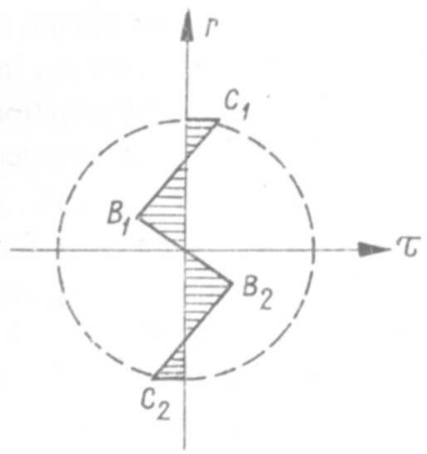

Fig. 68. Stress distribution in the cross-section of a circular bar under elastic-plastic torsion

this combined case as a model of behaviour of real materials at a point, then no experiments would have confirmed such a hypothesis. Three plastic corners are predicted by the slip theory of M. Como, S. D'Agostino [3.107], M. Como and A. Grimaldi [3.108], but even the verification of corners at the control points is not quite certain; some experimental results will be described in Sec. 14.11.

\subsection{Hypotheses of general anisotropic hardening}

The hypotheses of isotropic, kinematic or mixed plastic hardening assume a gradually decreasing or a constant curvature of subsequent neutral surfaces. On the other hand, the hypotheses of plastic corners predict infinite increase of that curvature at the control point. Many experimental results show an intermediate behaviour of real materials, namely an essentially increasing, but finite curvature of subsequent neutral surfaces. Such behaviour must be described by more general hypotheses than that of mixed hardening. Since in most cases of plastic hardening the anisotropy of the material is also induced, the relevant proposals of analytical description are called hypotheses of general anisotropic hardening.

Another problem is connected with the proper form of the description of subsequent neutral surfaces. From the thermodynamic point of view, they should be described by state variables and depend on the history of the process. Theoretically, plastic strain components cannot be regarded as appropriate state variables, 
since they do not describe the history of the process: the same plastic strains may determine various neutral surfaces, if those strains are reached along various trajectories (J. Kestin [3.339], T. Lehmann [2.96, 3.423]). One should either employ certain functionals or introduce new variables, related to plastic strains by means of differential (rate-type) equations. Both these approaches are to a certain degree equivalent, but the second is usually more convenient. Such new variables are called internal state variables (hidden parameters) and are widely used in physics, particularly in quantum mechanics and chemical physics. Usually they are scalar or tensorial quantities, but in auxiliary spaces they may also be presented as vectors (or matrices).

Actually, such parameters were introduced in the theory of plasticity in the early thirties, since one can include here the Odqvist parameter $I_{\varepsilon p}$ or plastic work $W^{p}$ (scalars, pseudo-state variables, T. Lehmann [3.423]), and the tensor $\alpha_{i j}$ in the kinematic hardening hypothesis (14.23). However, a more formal introduction of internal state variables based on thermodynamic considerations was initiated in the fifties by P. W. Bridgman [3.77] and consequently performed by A. A. Vakulenko $[3.824,3.825]$. Those variables may be introduced either in a purely formal way without any physical interpretation or with an interpretation based on the analysis of physical mechanisms of plastic flow (E. Kröner [3.398], P. Perzyna and W. Wojno [3.625], J. Kratochvil and O. W. Dillon [3.385], J. Kestin and J. R. Rice [3.340] and others) or with an interpretation based on statistical physics (E. Kröner [3.399]). An extensive review of papers on the description of plasticity using internal state variables is given by P. Perzyna [3.628].

Making use of internal state variables, we may describe subsequent neutral surfaces as

$$
F\left(\sigma_{i j}, \alpha_{(k) i j}, x_{m}\right)=0,
$$

where $\alpha_{(k)}, k=1,2, \ldots, k_{n}$, and $\varkappa_{m}, m=1,2, \ldots, m_{n}$, are tensorial and scalar internal state variables, respectively. The differential equations joining $\alpha_{(k) i j}, x_{m}, \varepsilon_{i j}^{p}$ and $\sigma_{i j}$ are called the evolution laws and will be discussed briefly in Sec. 15.4. In order to simplify (14.58), the evolution laws are often assumed, as an approximation, as finite (holonomic) relations instead of differential ones, i.e. plastic strains are treated as pseudo-state variables, and then (14.61) may be reduced either to the form

$$
F\left(\sigma_{i j}, \varepsilon_{i j}^{p}, \varkappa_{m}\right)=0
$$

or even to a form without scalar parameters $\varkappa_{m}$. Such a form does not show the invariance of the function $F$. An invariant form of (14.59) was proposed by D. D. Ivlev and G. I. Bykovtsev [3.312]:

$$
F\left(I_{1 \sigma}, I_{2 \sigma}, I_{3 \sigma}, I_{1 \varepsilon p}, I_{2 \varepsilon p}, I_{3 \varepsilon p}, I_{\sigma \varepsilon p}, I_{\sigma \sigma \varepsilon p}, I_{\sigma \varepsilon p e p}, k_{m}\right)=0,
$$

where the common invariants of stresses and plastic strains are defined by (5.25). Ivlev and Bykovtsev regarded $k_{m}$ as constants, but in order to describe a more general class of materials we have to introduce here at least the above-mentioned parameters (functionals) $\varkappa_{m}$. Of course, (14.58) may also be presented in an in- 
variant form, but in the case of several tensors $\alpha_{(k)}$ such notation may be very lengthy; in the case of one tensor $\alpha_{(k)}$ it was used by H. P. Shrivastava and R. N. Dubey [3.733].

Probably the oldest hypothesis of anisotropic plastic hardening of type (14.59), more general than those described by mixed hardening, was proposed by F. Edelman and D. C. Drucker [3.164] in 1951:

$$
F\left(I_{2 s}, I_{3 s}\right)-\left[P\left(I_{2 s}, I_{3 s}\right) s_{i j}+Q\left(I_{2 s}, I_{3 s}\right) t_{i j}\right] \varepsilon_{i j}^{p}=f
$$

where

$$
t_{i j}=s_{i k} s_{k j}-\frac{1}{3} I_{2 s} \delta_{i j}
$$

are the deviatoric components of the stress deviator squared, and $f$ is a function of plastic strains to be determined from the simple tension test. Hypothesis (14.61) belongs to the class (14.60). The authors used a particular form of (14.61) to describe analytically the experiments carried out by S. I. Liu and G. Sachs [3.462]. The basic features of (14.61) were described by D. C. Drucker in [3.153].

Though very general, (14.61) has some drawbacks; they were studied by $\mathrm{T}$. Kurtyka [3.405]. Consider for example linear plastic hardening in the simplest case where the functions in (14.61) do not depend on $I_{3 s}$. Equation (14.61) takes then the form

$$
\sigma_{e}\left(\sigma_{e}-\sigma_{0}\right)-E_{1} s_{i j} \varepsilon_{i j}^{p}=0 ;
$$

indeed, in the uniaxial case this equation turns into $\sigma= \pm \sigma_{0}+E_{1} \varepsilon^{p}$ according to the assumption and $f \equiv 0$ in (14.61). In the simplest combined case of two shearing stress components and two shearing strain components we obtain

$$
\sqrt{\tau_{z x}^{2}+\tau_{z y}^{2}}\left(\sqrt{\tau_{z x}^{2}+\tau_{z y}^{2}}-\tau_{0}\right)-B\left(\tau_{z x} \gamma_{z x}^{p}+\tau_{z y} \gamma_{z y}^{p}\right)=0
$$

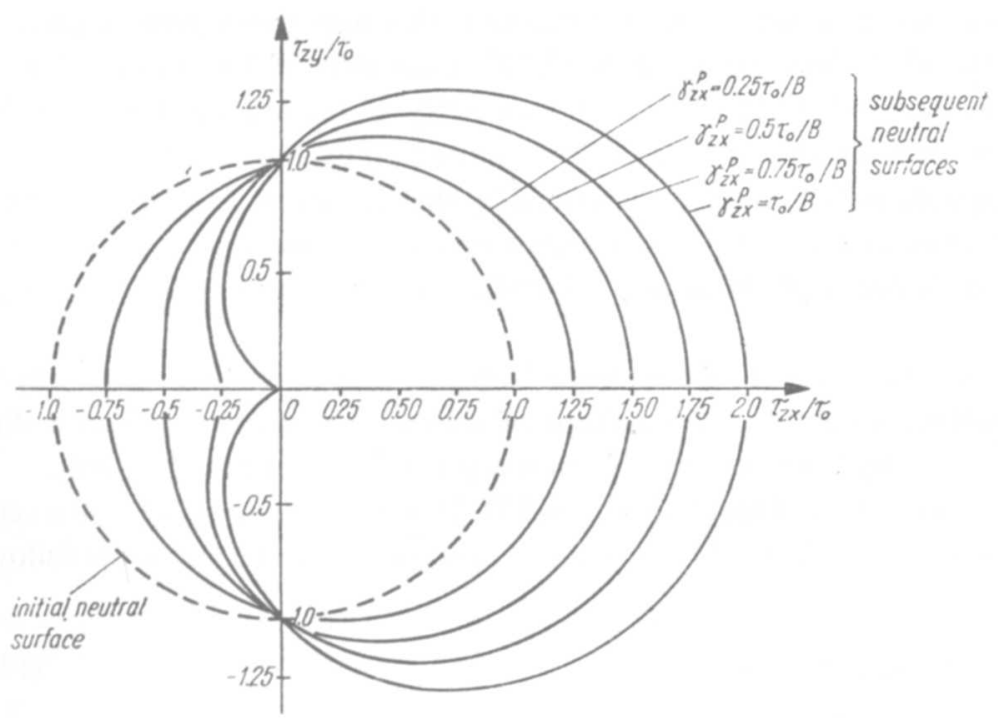

Fig. 69. An example of subsequent neutral curves according to the Edelman-Drucker hardening rule 
where $B=E_{1} / 3$. Subsequent neutral surfaces for $\gamma_{z y}^{p}=0$ are shown in Fig. 69 . It turns out that at the control point the curvature of this family of surfaces does not increase (as expected) whereas at the opposite point $\left(\tau_{z x}<0, \tau_{z y}=0\right)$ the curvature decreases very quickly, for $\gamma_{z x}^{p}>0.5 \tau_{0} / B$ it is negative and for $\gamma_{z x}^{p}=\tau_{0} / B$ a concave singular point is formed. Such phenomena are not confirmed by experiments, and therefore (14.63) is limited to slight plastic hardening. However, in most cases the ideal Bauschinger effect is described even more accurately by the hypothesis of kinematic hardening than by (14.63).

Another early proposal of anisotropic hardening is due to I. F. Besseling [3.58]. The material is considered as consisting of subelements gradually joining the plastic flow; hence the Bauschinger effect is connected with micrononhomogeneity.

More attention will be paid to the hypothesis of A. Baltov and A. Sawczuk [3.30]. The authors consider a material which is initially described by the HMH yield surface which-as a result of plastic deformation - turns into a general quadratic Mises surface for an anisotropic body with simultaneous translation

$$
N_{i j k l}\left(s_{i j}-\alpha_{l j}\right)\left(s_{k l}-\alpha_{k l}\right)-1=0,
$$

where $N_{i j k l}$ denotes the fourth-order tensor of the plastic moduli, depending on the plastic strain tensor and some hardening parameters (functionals). Assuming $N_{i j k l}=\delta_{i k} \delta_{j l} f\left(I_{e p}\right)$, we obtain the hypothesis of mixed plastic hardening, and in more particular cases-isotropic or kinematic hardening. As a result of a detailed analysis the following more specified form of (14.65) is proposed

$$
s_{i j} s_{i j}-2 s_{i j} \alpha_{i j}+A \alpha_{i j} \alpha_{k l}\left(s_{i j}-\alpha_{i j}\right)\left(s_{k l}-\alpha_{k l}\right)-\frac{2}{3} \sigma_{0}^{2}=0,
$$

where the tensor components $\alpha_{i j}$ are determined by (14.26). Though the isotropic hardening component is not taken into account, this hypothesis gives a better description of the H. J. Ivey experiments [3.300] than that of kinematic hardening. The main deficiency of (14.66) lies in the symmetry of the second-degree surfaces: a simultaneous increase of curvature at the control point and a decrease of curvature at the opposite point cannot be described by that equation. A related hypothesis transforming an isotropic body into an orthotropic one, governed by the Mises-Hill yield condition, is due to R. Mansurov [3.498], and its further development is given in [3.499].

Another general concept of combined isotropic and kinematic hardening with a certain simultaneous change of the shape of subsequent neutral surfaces is due to G. B. Talypov [3.786], but its effective form was given for simple loading only.

G. I. Bykovtsev, V. V. Dudukalenko and D. D. Ivlev proposed in [2.14] a certain alteration of the general form (14.60), namely expressing it in terms of the following nine invariants:

$$
F\left(\sigma_{j}, \varepsilon_{j}^{p}, \alpha, \beta, \gamma\right)=0,
$$

where $\sigma_{j}$ and $\varepsilon_{j}^{p}$ stand for principal stresses and principal plastic strains respectively, whereas $\alpha, \beta$, and $\gamma$ denote Euler's angles between the corresponding principal 
directions. The problem of uniqueness of solutions in this case was considered by V. V. Dudukalenko [3.160].

An alteration of the Edelman-Drucker hardening hypothesis, belonging directly to the class (14.60), was suggested by Y. Yoshimura and Y. Takenaka [3.855]. A very general, multiparameter hypothesis of anisotropic hardening was suggested by J. F. Williams and N. L. Svensson [3.845], but effective evaluation of many anisotropic moduli seems difficult. A relatively simpler expression of that type, proposed by M. Tanaka and Y. Miyagawa [3.787], may be written in the form

$$
\left[\left(\delta_{i k} \delta_{j l}+\delta_{i l} \delta_{j k}\right)+A\left(\varepsilon_{i k}^{p} \varepsilon_{j l}^{p}+\varepsilon_{i l}^{p} \varepsilon_{j k}^{p}\right)\right]\left(s_{i j}-a_{i j}\right)\left(s_{k l}-a_{k l}\right)-K=0,
$$

where $a_{i j}$ denote the deviatoric parts of $\alpha_{i j}$, determined by (14.26) or (14.30), and $A$ and $K$ depend on the Odqvist parameter. The general form (14.61) with internal state variables was investigated by H. P. Shrivastava, Z. Mróz and R. N. Dubey [3.731, 3.732, 3.733], who proposed also a certain particular expression (quadratic function of invariants). The problem of analytical treatment of experimental investigations of subsequent neutral surfaces was discussed by H. D. Bui and J. Zarka [3.86].

G. Backhaus $[3.22,3.23,3.24,3.25]$ suggested a different approach to anisotropic hardening. He presented the deviatoric stress increment as the sum of the following three terms

$$
d s_{i j}=C s_{i j}^{(0)} d I_{\varepsilon p}+B d \varepsilon_{i j}^{p}+A s_{k l}^{(0)} \frac{d \varepsilon_{k l}^{p}}{d I_{\varepsilon p}} d \varepsilon_{i j}^{p},
$$

where $s_{i j}^{(0)}$ denote the deviatoric stresses at the beginning of an active process, whereas $A, B$ and $C$ are certain functions of the Odqvist parameter to be determined experimentally. The first term corresponds to isotropic hardening, the second to kinematic hardening of the Melan-Ishlinsky-Prager type, and the third to general anisotropic hardening. Integrating along the trajectory in the plastic strain space, we obtain

$$
s_{i j}=s_{i j}^{(0)}+\int d s_{i j}
$$

calculating from this equation $s_{i j}^{(0)}$ and substituting into the yield condition $s_{i j}^{(0)} s_{i j}^{(0)}$ $=2 \sigma_{0}^{2} / 3$, we obtain the equation of the actual neutral surface. A certain concept close both to the Baltov-Sawczuk hypothesis (14.65) and to the Backhaus equation (14.69) was proposed by V. L. Danilov [3.123, 3.124].

P. G. Hodge [3.261] formulated a general theory of anisotropic hardening based on the Tresca-Guest hypothesis. It is relatively simple if the principal directions are constant and known; then it is sufficient to discuss the transformation of the initial regular hexagon in the deviatoric Meldahl plane. Hodge assumed that plastic hardening results in a parallel translation of all sides of the hexagon. If the translation forced by the control point is equal to $a$, then the translation of two adjacent sides may be described as $\alpha a$, of the further two sides as $\beta a$, finally of the opposite side-as $\gamma a$; Fig. 70. An effective formulation of the hardening hypothesis requires the knowledge of the function $a=a\left(\varepsilon^{p}\right)$ (determined by the simple tension test; Hodge considered piece-wise linear hardening) and the choice of the coef- 

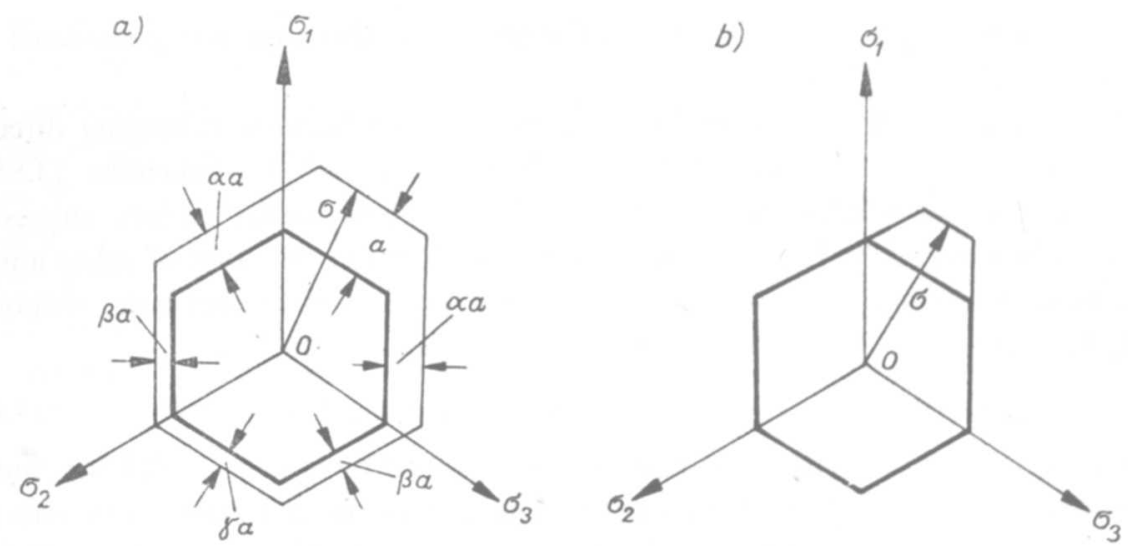

Fig. 70. The Hodge hardening hypothesis as applied to the TG yield condition: (a) general case, (b) the case of independent mechanisms $\alpha=\beta=\gamma=0$

ficients $\alpha, \beta$ and $\gamma$. Mixed plastic hardening, discussed in Sec. 14.6, may be obtained as a particular case of the proposed general hypothesis, namely if the following relations hold:

$$
\beta=3 \alpha-2, \quad \gamma=4 \alpha-3,
$$

and the number of "degrees of freedom" is reduced from three to one. Further specification is connected with the choice of one parameter, say $\alpha$, only. For $\alpha=1$ we have $\beta=\gamma=1$, and this case describes isotropic hardening. Assuming $\alpha=1 / 2$, we obtain $\beta=-1 / 2, \gamma=-1$, a rigid translation of the hexagon takes place and we arrive at kinematic hardening in the deviatoric plane.

The type of plastic hardening shown in Fig. 70a may be defined as an interaction of individual segments of the neutral surface, corresponding to various mechanisms of the plastic flow. Sometimes the case of independent mechanisms is also considered (J. L. Sanders [3.694] P. M. Naghdi [2.119], Fig. 70b); it may be treated as a particular case of the Hodge theory, obtained if $\alpha=\beta=\gamma=0$.

The further development of piece-wise linear anisotropic plastic hardening is due to K. Saito, H. Igaki and M. Sugimoto [3.691], A. F. Akhmerov and Yu. B. Tyurin [3.2]. The interaction of various mechanisms of plastic flow was considered by J. Mandel [3.493], and H. D. Bui [3.85].

The above-mentioned hypotheses of general anisotropic hardening give a multiparameter, often very complicated description of subsequent neutral surfaces. However, in most cases, in spite of that general and complicated description, the main goal is not achieved: an increase of the curvature in the vicinity of the control point with the simultaneously decreasing curvature at the opposite side of the neutral surface, observed in experiments, is not reflected in the equations.

Further complications are connected with accessory exertion factors, first of all with variable temperature. Temperature as an independent factor was first included in the hardening hypothesis (14.59) by W. Prager [3.657], but very few 
effective forms of such a hypothesis were suggested; many papers, based on thermodynamics, end with general theoretical conclusions. An extension of the MelanIshlinsky-Prager kinematic hardening to thermoplasticity was proposed by I. Z . Palley [3.613] and T. Y. Chang [3.94] and of mixed hardening — by S. Cuomo [3.119] and S. M. Belevitch [3.45]. T. Inoue and K. Tanaka [3.294] generalize the Yoshimura anisotropic hardening rule and use it for the description of thermocyclic processes. An extension of the Baltov-Sawczuk hypothesis to temperature effects is given in the book by D. Kolarov, A. Baltov and N. Bontcheva [3.366], where a more general discussion is also included. Time effects at elevated temperatures were studied by D. C. Drucker [3.156]. A. Phillips with his collaborators J. L. Tang [3.637], R. Kasper [3.638] and G. Weng [3.642] proposed a description of anisotropic hardening with possible temperature effects. It was based on numerous experiments and consists of kinematic hardening close to the second Melan concept (14.27) and of a deformation of the neutral surface.

A general method of constructing of subsequent neutral surfaces in the case of various accessory exertion factors (influence of various physical fields) was suggested by I. V. Demyanushko and Yu. M. Temis [3.132]. J. A. König and W. Olszak [3.383] proposed a hardening hypothesis additionally taking into account stress and strain gradients.

\subsection{Concept of double neutral surfaces}

The classical theory of plastic hardening is based on the concept of a neutral surface inside which all the processes are passive (elastic, reversible) and outside which at least the initial part of the process is active (plastic, irreversible). Experiments, in general, verify this concept if very slight symptoms of irreversibility (very small changes of plastic strains) may be neglected. The sharper is the definition of a passive process, the smaller is the corresponding neutral surface and the greater the Bauschinger effect. Going further in this direction, we finally arrive at V. D. Klyushnikov's concept of a uniform analytical theory of plasticity, where all processes are active except simple unloading [1.12]. Hence the classical concept of a neutral surface (which in the case of relaxed requirements as regards the definition of a passive process may be called differently, for example the yield surface, as used by many authors) and Klyushnikov's concept of almost exclusively active processes constitute two extremal formulations. The first is practically oriented, whereas the second is of a rather theoretical character. In many engineering applications a relaxed definition of a passive process (with very small changes of plastic strains admitted) is justified, but in some cases (quasi-cyclic processes, stability, etc.) such an approach may cause too essential errors and a more accurate approach is necessary. A certain intermediate concept was proposed by A. Phillips and R. L. Sierakowski [3.633] and will be briefly described here.

Phillips and Sierakowski aimed at the description of the well-known but usually forgotten fact that the length of passive part of reloading is smaller than that of 


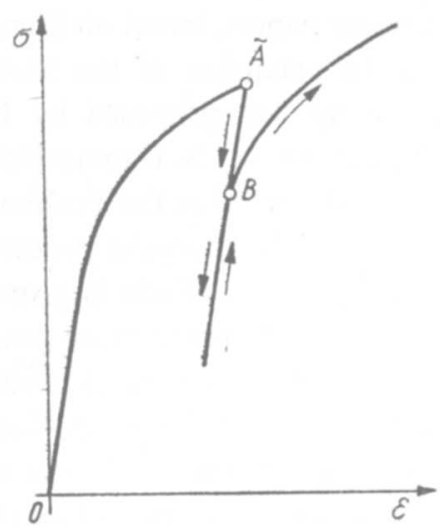

Fig. 71. Unloading and reloading

passive unloading (Fig. 71). This fact, described in detail by J. D. Lubahn and R. P. Felgar [3.647], contradicts the classical concept of a neutral surface passing through the point $\tilde{A}$. Thus they introduced two surfaces: one passing through $\tilde{A}$ and called the loading surface and the other passing through $B$ and called the yield surface. To conform to out terminology, we call them the outer neutral surface and the inner neutral surface, respectively. The control point is in contact with the outer neutral surface during active processes and during passive processes between $\tilde{A}$ and $B$. During such processes the outer neutral surface shrinks in size and coincides with the inner neutral surface if the control point is inside the latter. Within the inner neutral surface all the processes are passive without restrictions whereas between the inner and the outer surface they are also passive if they go inside; since then the outer neutral surface shrinks in size, any outside direction causes an active

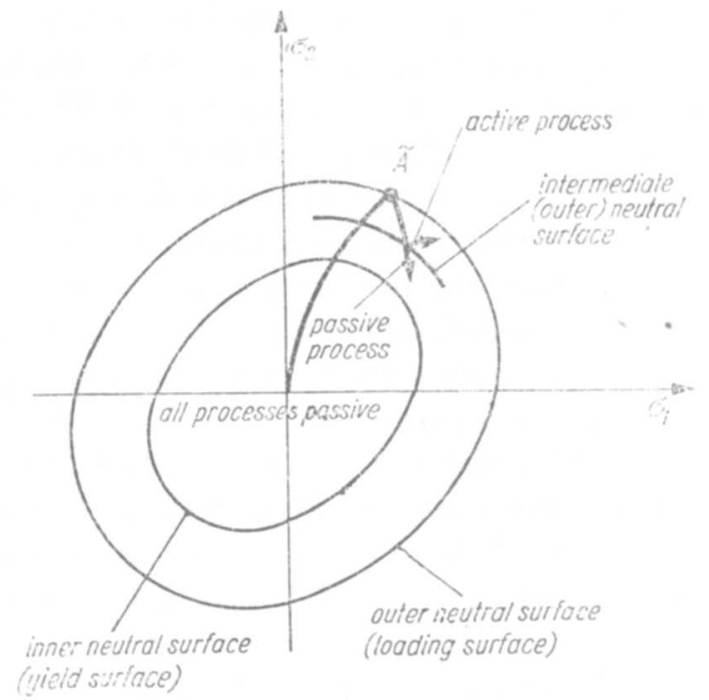

Fig. 72. Hypothesis of outer and inner neutral surfaces (Phillips-Sierakowski) 
process (Fig. 72). During active processes both neutral surfaces change (increase, as a rule), whereas during passive ones the inner surface does not change and the outer either decreases or coincides with the inner one.

J. W. Justusson and A. Phillips [3.319] investigated material stability in the Drucker sense in the case of double neutral surfaces. A certain modification of the Drucker postulate implied the convexity of the outer neutral surface. Quasi-cyclic processes were investigated by R. L. Sierakowski and A. Phillips [3.740]. Further development of this theory is due to M. A. Eisenberg and A. Phillips [3.166]; the authors restricted their considerations to isotropic hardening, but announced further generalizations in a subsequent paper.

A certain related hypothesis of plastic hardening was proposed by $Z$. Mróz [3.554]; in the stress space his model consists of a sequence of neutral surfaces corresponding to boundaries of particular regions of piece-wise linear hardening. In [3.555] this hypothesis was used for the description of quasi-cyclic processes; further proposals were published in [3.556. 3.557. 3.560. 3.561], partly with C. Goss and N. C. Lind.

\subsection{Neutral surfaces in the strain space}

The type of plastic hardening is usually defined by the behaviour of neutral surfaces in the stress space. However, for certain applications, if the strain components may be treated as exertion factors (for example, with reference to the construction of most testing machines and for the description of plastic softening), an analysis of neutral surfaces in the strain space is also important. This problem is mentioned, e.g., by W. M. Baldwin [3.29], I. F. Besseling [3.58], D. Trifan [3.816], and A. Kammerer [3.337].

The behaviour of neutral surfaces in the strain space in the case of perfectly elastic-plastic bodies was considered in Secs. 9.5, 13.7 and 14.5; an analogy with kinematic hardening in the stress space can be seen here: the surface is subject to translation as a rigid body. Similarly, in the case of a body subject to kinematic
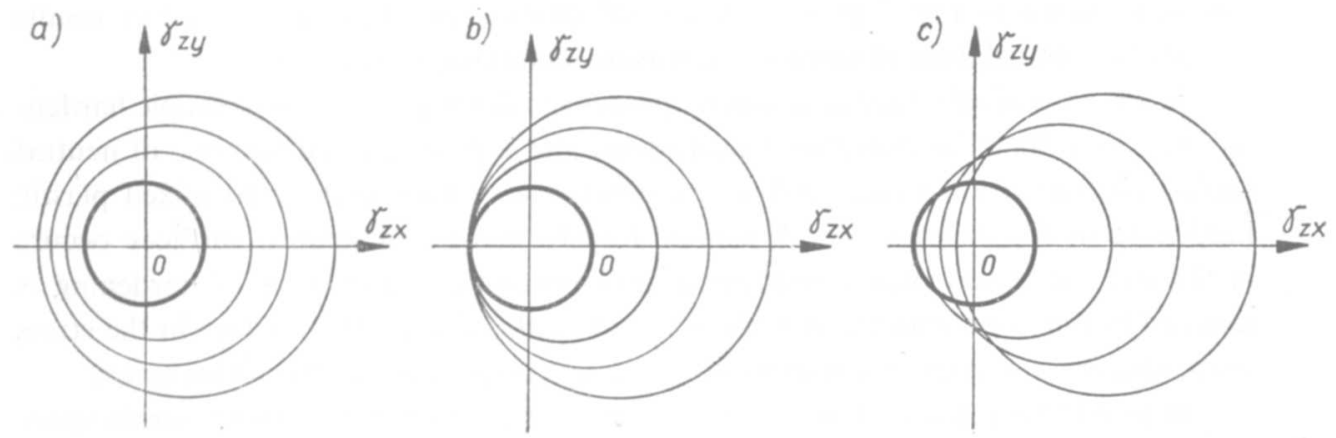

Fig. 73. Subsequent neutral surfaces in the strain space corresponding to isotropic hardening 
hardening translation in the strain space takes place, but the motion may be regarded as slower in comparison with perfect plasticity. The direction of this motion is determined by the physical law adopted.

The situation changes in the case of isotropic hardening. Let us consider, for the sake of simplicity, the plane $\gamma_{z x}-\gamma_{z y}$ as in Sec. 9.5, and confine ourselves to simple processes $\left(\gamma_{z x}\right.$ is the only component to increase). Theoretically all the three cases of behaviour of neutral surfaces shown in Fig. 73 are possible. The boundary case (b) corresponds to the onset of a reverse active process at a constant value of strain, i.e. to the stress-strain diagram shown in Fig. 74.

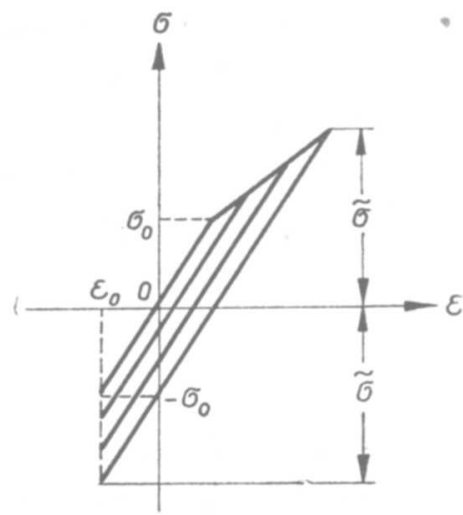

Fig. 74. The boundary case of linear isotropic hardening

Taking into account the identity of the segments $\tilde{\sigma}$, required by the hypothesis of isotropic hardening, we may easily derive the corresponding equation of the hardening curve,

$$
\sigma=\frac{\sigma_{0}}{2}+\frac{E}{2} \varepsilon
$$

hence we arrive at linear plastic hardening with the modulus $E_{1}=E / 2$. Real materials show, as a rule, a much smaller hardening modulus, and thus usually the situation shown in Fig. 73c is observed. Of course, any Bauschinger effect results in "quicker" translation of surfaces increasing simultaneously in size.

In the case of arbitrary non-simple processes, the hypothesis of isotropic hardening gives simultaneous curvilinear translation and a proportional increase of neutral surfaces in the strain space, and so we observe a certain analogy to mixed plastic hardening in the stress space. A similar transformation of neutral surfaces occurs in the case of mixed plastic hardening, and hence the latter type of hardening is mapped in the same manner in both spaces. Finally, if a neutral surface in the stress space changes its shape, a corresponding change is observed in the strain space.

It should be noted that incompressibility of the material reduces the strain space to a certain subspace, e.g. the three-dimensional space of principal strains to the 
plane $\varepsilon_{1}+\varepsilon_{2}+\varepsilon_{3}=0$. The cross-section of the HMH cylinder is then a circle with the radius $\sigma_{0} / G \sqrt{6}$; however, very often we draw the limit curve as an ellipse in the plane $\varepsilon_{1} \varepsilon_{1}$ shown in Fig. 56a. This is not a section of the cylinder by the plane $\varepsilon_{1} \varepsilon_{2}$ but a projection of the circular section by the plane $\varepsilon_{1}+\varepsilon_{2}+\varepsilon_{3}=0$ on the plane $\varepsilon_{1} \varepsilon_{2}$.

Let us also mention an apparently similar concept of the space of plastic strains, introduced by I. A. Berezhnoi, V. V. Dudukalenko and D. D. Ivlev [3.50]. Actually there is an essential difference between the total strain space and the plastic strain space, since in the latter any neutral surface reduces to a point: each motion in that space corresponds to an active process. Hence the authors used the space of plastic strains for a different purpose.

\subsection{Experimental investigations of neutral surfaces and difficulties in their interpretation}

Hypotheses concerning the transformation of subsequent neutral surfaces are extremely difficult for experimental verification. Theoretically, such a surface should correspond to neutral processes, i.e. to boundary processes in the course of which the change of plastic strains (and the dissipated energy) tends to zero. However, it is difficult to determine such a process experimentally: usually, if we observe lack of change of plastic strains, then the process is passive, lying inside the neutral surface. On the other hand, the onset of a change of plastic strains is stated by the measurement of a certain conventional value of those strain changes, and hence we deal with an active process. Practically neutral processes are very seldom investigated, but neutral surfaces are determined by the end-points of passive processes, where those end-points correspond to a conventional value of the change of plastic strains. This value may be expressed, for example, by the intensity of finite increments of plastic strains, $\left(\Delta \varepsilon^{p}\right)_{e}$ or, in brief notation, $\Delta \varepsilon_{e}^{p}$, calculated similarly to $(7.29)$.

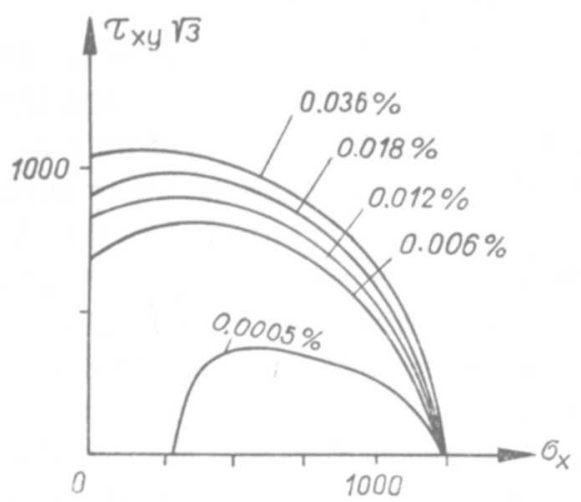

Fig. 75. Dependence of the limit curves on the conventional value of plastic strain 
Many authors draw attention to the essential dependence of experimentally determined neutral surfaces on the adopted value of $\Delta \varepsilon_{e}^{p}$ (Yu. I. Yagn, O. A. Shishmarev [3.850], W. Szczepiński [2.162, 3.779], O. A. Shishmarev [3.727], I. Hayashi, K. Kawaguchi, H. Fukuda [3.234], M. Como, S. D'Agostino, A. Grimaldi [3.114]). Figure 75 shows the dependence of the limit curves for simultaneous tension and tension of a thin-walled tube, experimentally determined by Yagn and Shishmarev [3.850], on the conventional value of the change of plastic strains; the nickel tube was subject to initial tension with $\varepsilon_{x}^{p}=1.1$ per cent (control point). Very sharp criteria of $\Delta \varepsilon_{e}^{p}$ result in small domains of passive (elastic) processes, and we could even speak of an "overideal" Bauschinger effect or "overkinematic" plastic hardening. Usually such sharp criteria are unnecessary, but they may be essential in quasicyclic processes, where even small amounts of dissipated energy quickly cumulate. In the case of such processes one may obtain a more accurate description by using the Phillips-Sierakowski concept of double neutral surfaces or similar hypotheses.

Thus, a comparison of the results of various authors and drawing more general conclusions presents certain difficulties. The same experiments may be interpreted as a verification of isotropic hardening (if we adopt a larger value $\Delta \varepsilon_{\boldsymbol{e}}^{p}$ ) or as a verification of kinematic hardening (for smaller $\Delta \varepsilon_{e}^{p}$ ). In the limiting case $\Delta \varepsilon_{e}^{p} \rightarrow 0$ we may arrive at the Klyushnikov concept of almost exclusive active processes (the domain of passive processes reduced to a segment), [1.12]. A certain standardization of $\Delta \varepsilon_{e}^{p}$ may be useful for comparing the results of various experiments; J. Miastkowski [3.523] proposed to assume it equal to the intensity of the accompanying elastic strains $\varepsilon_{e}^{e}$. L. M. Kachanov [3.320, 3.321] gave an extrapolation method of determining the theoretical neutral surface corresponding to $\Delta \varepsilon_{e}^{p}=0$. However, G. B. Talypov [3.786] raised some arguments against too small allowances $\Delta \varepsilon_{e}^{p}$ and advised to go no further than half of the width of the hysteresis loop. Further difficulties are connected with the time effects, since many materials exhibit slightly viscoplastic instead of purely plastic properties-these effects were discussed by D. C. Drucker [3.156], who remarked that primary creep and plastic deformations are practically indistinguishable, and introduced the concept of a rate-of-strain surface; further research is due to J. Rogan and A. Shelton [3.677, 3.678].

Only a few older papers verified the hypothesis of isotropic hardening: D. M. Cunningham, E. G. Thomsen, J. E. Dorn [3.118] J. Parker, M. B. Bassett [3.617]. Many investigators regarded the hypothesis of mixed plastic hardening as sufficiently accurate, but with a varying share of the two components: under relatively small plastic strains it was rather close to kinematic hardening, whereas larger plastic strains produced a remarkable isotropic component (W. Szczepiński [2.162], J. Miastkowski and W. Szczepiński [3.521], J. Miastkowski [3.522, 3.523, 3.524] V. M. Bastun, N. I. Chernyak [3.34], A. M. Koreneva and G. A. Doshtchinsky [3.372], K. Turski [3.821]). In a series of experiments [3.139, 3.140, 3.141, 3.481], G. A. Doshtchinsky, V. I. Maksak and A. M. Koreneva found an increase of strain intensity in the course of the process $\sigma_{e}=$ const; this fact testified to the share of kinematic hardening, since under isotropic hardening the process $\sigma_{e}=$ const should 
be neutral and, similarly to perfect plasticity (Secs. 9.5-9.7), the total strain intensity should decrease. They also proposed an analytical description of subsequent neutral surfaces based on the assumption of a constant angle between the stress vector and the plastic strain rate vector during the circumferential stress trajectory, [3.142]. On the other hand, in earlier investigations of the processes $\sigma_{e}=$ const, J. Marin and L. W. Hu $[3.504,3.506]$ noted first an increase and later a decrease of $\varepsilon_{e}$; probably their experiments covered a larger range of variability of strains.

Many investigators treated even the hypothesis of mixed plastic hardening as insufficiently accurate and verified various hypotheses of general anisotropic hardening. For example, W. M. Mair and H. Pugh [3.476], J. Miastkowski and W. Szczepiński [3.523], E. Shiratori, K. Ikegami and H. Okano [3.722] M. L. Gorb, D. M. Karpinos and A. A. Ostrovsky [3.206] observed a certain rotation of the neutral surface which can be explained on the basis of general anisotropic hardening only. K. Turski [3.822] verified the Baltov-Sawczuk hardening hypothesis. N. G. Sverdlova [3.777] verified the symmetry of the initially symmetrical (circular) subsequent neutral surface with respect to the loading path (under simple loading). The influence of the non-radial loading path on subsequent neutral surfaces was investigated by W. Szczepiński and J. Miastkowski [3.780], A. Phillips and J. L. Tang [3.637], K. R. Johnson and O. M. Sidebottom [3.316], E. Shiratori, K. Ikegami and K. Kaneko [3.726]; in most cases the initial symmetry of the neutral surface disappeared. An impressive picture of subsequent neutral surfaces showing at first symmetry (simple loading) and then loss of symmetry is quoted after A. Phillips and J. L. Tang [3.637], Fig. 76.

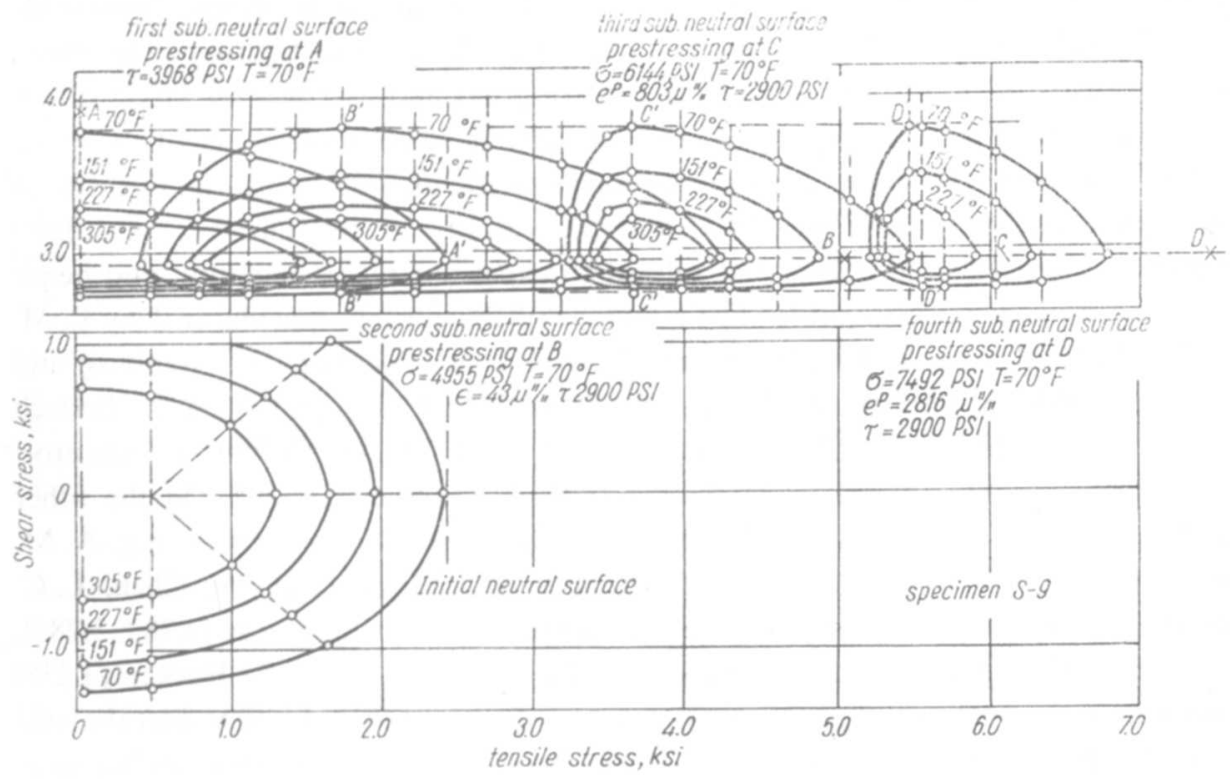

Fig. 76. Subsequent neutral curves determined experimentally (Phillips-Tang) 
An important feature of particular hypotheses of plastic hardening is connected with the so-called "cross effects", i.e. the behaviour of subsequent yield surfaces in the direction perpendicular to the (straight) loading path.

It should be noted that the term "cross effects" may cause some misunderstandings, since one may be interested either in transversal dimensions of the neutral surface along the initial transversal axis, or in maximal transversal dimensions. According to the isotropic hardening hypothesis, the dimensions of subsequent yield surfaces in transverse direction should always increase, but according to kinematic hardening the maximal transversal dimensions remain constant, whereas the dimensions measured along the original orthogonal axis decrease in most cases. The slip theories predict as a rule no cross effects, and the hypotheses of mixed and general anisotropic hardening admit all the possibilities. In fact, experimental investigations show either an increase of the transversal dimensions of subsequent neutral surfaces (P. S. Theocaris, C. R. Hazell [3.799], R. A. Arutyunyan [3.12], J. Miastkowski [3.523] A. I. Chistyakov [3.97]-with several stress-quasi-cycles applied) or no cross effects (P. M. Naghdi, F. Essenburg, W. Koff [3.568] along the initial orthogonal axis; H. J. Ivey [3.300], A. Phillips, G. Weng [3.642], if maximal width is considered), or a decrease of transversal dimensions (L. W. Hu, J. F. Bratt [3.275], J. Miastkowski, W. Szczepiński [3.521] N. G. Suturina [3.775], M. J. Michno, W. N. Findley [3.527]). A. B. Ibragimov and I. D. Mashkov [3.283] proposed to describe cross-effects and induced anisotropy by two parameters. Of course, all the above-mentioned experimental results depend on the material, on the plastic strains determining the particular neutral surfaces and on the assumed value $\Delta \varepsilon_{e}^{p}$, Fig. 75 .

Experimental verification of the hypothesis of the existence of plastic corners (pointed vertices) presents a problem in itself. Practically, it is almost impossible to verify such a hypothesis in an experimental way. Many investigators determined the increments of plastic strain near the control point and ascribed the larger differences in the direction of those increments for various stress increments to the existence of a corner. However, such an approach tacitly assumed tensorial linearity of constitutive equations (cf. Sec. 15.3) and the validity of the Drucker postulate; neither is generally valid and is just subject to experimental verification, and hence the conclusions are questionable. The existence of the corners was claimed by P. M. Naghdi, J. C. Rowley and C. W. Beadle [3.567], P. M. Naghdi, F. Essenburg and W. Koff [3.568], A. Phillips and G. A. Gray [3.632], H. J. Ivey [3.300], B. K. Bertsch and W. N. Findley [3.57], P. S. Theocaris and C. R. Hazell [3.799], E. Shiratori and K. Ikegami [3.723], M. J. Michno and W. N. Findley [3.525]. On the other hand, many special experiments denied the existence of plastic corners, e.g. A. M. Zhukov [3.867], Yu. I. Yagn, O. A. Shishmarev [3.850], B. Paul, W. Chen, L. C. Lee [3.619], W. Szczepiński [2.162], G. B. Talypov, V. V. Kamentsev [3.783, 3.784], A. I. Chistyakov [3.96], V. N. Bastun, N. I. Chernyak [3.34]. S. S. Hecker [3.238] investigated the problem of plastic corners with reference to the Phillips-Sierakowski concept of double neutral surfaces; he concluded that the outer neutral surface may exhibit a vertex whereas the inner one is always regular. The dependence of con- 
clusions as regards the existence of corners on the assumed criterion $\Delta \varepsilon_{e}^{p}$ determining the neutral surface was discussed by V. D. Klyushnikov [3.360].

The most probable, compromising hypothesis predicts an essential increase of curvature of the neutral surface at the control point with a simultaneous decrease of curvature at the opposite side. Such a behaviour was observed and discussed by A. Phillips [3.630, 3.631], W. M. Mair [3.477], E. Shiratori, K. Ikegami, K. Kaneko [3.725], M. J. Michno and W. N. Findley [3.526, 3.527]. In the case of a general, non-simple loading, H. D. Bui [3.83, 3.84] observed the maximal curvature of the neutral surface at a certain point different from the control point; such a behaviour may easily be explained by the cumulation of curvature at current changing control points.

A particularly sharp verification of the plastic hardening hypotheses is furnished by cyclic or quasi-cyclic tests, since small effects are then subject to cumulation. According to the hypotheses of kinematic or mixed plastic hardening with holonomic relations between $\alpha_{i j}$ and $\varepsilon_{i j}^{p}$, the induced anisotropy should vanish after a full cycle, when $\sigma_{i j}, \varepsilon_{i j}$ and $\varepsilon_{i j}^{p}$ are simultaneously reduced to zero; however, the relevant tests did not confirm this conclusion (R. A. Arutyunyan [3.12]); hence more general hypotheses with internal state variables are here necessary. Similarly, the experiments carried out by K. R. Kenk [3.338] in cyclic torsion showed essential discrepancies with regard to the hypotheses of mixed hardening; on the other hand, experimental investigations of cyclic torsion superimposed on a constant normal force, carried out by A. M. Freudenthal [3.184], could be interpreted in the light of the concept of mixed plastic hardening.

A. Phillips and R. L. Sierakowski verified their concept of double neutral surfaces in the case of variable repeated loading in [3.740]. R. Marjanović and W. Szczepiński [3.509] evaluated experimentally subsequent neutral curves after several non-simple stress-quasi-cycles; the results were close to the predictions of the kinematic hardening hypothesis.

Numerous experiments on plastic hardening with taking account of temperature effects were performed by A. Phillips, partly with C. S. Liu, J. W. Justusson, J. L. Tang and M. Ricciuti [3.635, 3.636, 3.637, 3.639, 3.640]; some typical results are shown in Fig. 76. These cross-sections are convex, but the perpendicular crosssections show concavities, [3.641]. Experimental tests on quasi-cyclic processes at elevated temperatures were carried out by $\mathrm{T}$. Inoue, $\mathrm{K}$. Tanaka and T. Ishizaki [3.293, 3.294].

An alternative method of description of plastic hardening was proposed by W. Szczepiński and K. Turski [3.781]. They prestrained tubular brass specimens along various simple loading paths up to a certain constant value of stress intensity, then evaluated a conventional yield point in uniaxial tension and plotted the measured values along the radius of prestraining. Such "lines of influence" may be useful in calculating coldworking processes. In general, the results were in agreement with the kinematic hardening hypothesis. 


\section{Constitutive equations of plastic hardening}

\subsection{General remarks}

Equations of subsequent neutral surfaces discussed in Sec. 14, may be treated in two ways. First, they are doubtlessly physical equations, depending on the material considered. Secondly, they simultaneously describe the limit surfaces separating the ranges of applicability of the basic physical equations, namely constitutive equations, valid for plastically active processes and valid for plastically passive ones. Further, they are very closely connected with the equation of active processes: an unknown function appearing in those equations should be evaluated from the equation of the actual neutral surface, and if we additionally assume the associated flow rule, then the constitutive equations of active processes are fully determined.

A more detailed discussion of constitutive equations joining stresses and strains will be given in the present section. Some of them do not formally differ from the classical equations of perfect plasticity, e.g. (9.28), (9.51), or (9.60); the differences only appear after the elimination of $\varphi$ or $\lambda$. However, those differences are of qualitative character: stress increments, as a rule, determine here strain increments in a unique manner, whereas in the case of perfect plasticity strain increments are determined with the accuracy of a constant factor. Certain physical equations are adjusted to the adopted hypothesis of plastic hardening; they will be discussed in detail and possible generalizations will also be mentioned.

\subsection{Total (deformation, holonomic) theories of plasticity}

H. Hencky [2.53] proposed his equations (9.29) in principle for perfectly plastic materials, but their adjustment to the description of plastic hardening presents no difficulties (A. Nadai [2.118], A. A. Ilyushin [2.67]). Since those equations may describe only simple processes (or, approximately, processes close to simple ones), the hypothesis of hardening (isotropic, kinematic, etc.) is almost meaningless-it merely determines the range of application of the equations of passive processes. It is sufficient to adopt the hypothesis of a uniform curve of plastic hardening (14.2); one obtains then

$$
\varphi=\frac{3 \varepsilon_{e}}{2 \sigma_{e}}=\frac{3 \varepsilon_{e}}{2 f\left(\varepsilon_{e}\right)},
$$

and after substitution into $(9.28)$

$$
e_{i j}=\frac{3 \varepsilon_{e}}{2 f\left(\varepsilon_{e}\right)} s_{i j} .
$$

Inverting Eq. (14.2), we may express strains in terms of stresses alone; instead of $\sigma_{e}$ the invariant $I_{2 s}=2 \sigma_{e}^{2} / 3$ is often introduced here, namely

$$
e_{i j}=f_{1}\left(I_{2 s}\right) s_{i j} \text {. }
$$


The criterion of the active process is here of the form $d \varepsilon_{e}>0$, which is equivalent to $d \sigma_{e}>0$ or $d I_{2 s}>0$ if plastic softening and perfect plasticity are excluded; as we stated in Sec. 14.2, this criterion is not quite general, but sufficient in the case of simple processes described on the basis of the HMH hypothesis.

A generalization of the law (15.3) was proposed by W. Prager [3.651]. Assuming that the stress-strains curve in uniaxial tension may be described by the following power series

$$
e=c_{1} s+c_{3} s^{3}+c_{5} s^{5}+\ldots
$$

(even powers disappear for isosensitive materials), replacing $e$ by $e_{i j}$ and $s$ by $s_{i j}$ and eliminating higher powers of the deviators by using the Cayley-Hamilton theorem, he obtained an equation of the type

$$
e_{i j}=f_{1}\left(I_{2 s}, I_{3 s}^{2}\right) s_{i j}+f_{2}\left(I_{2 s}, I_{3 s}^{2}\right) I_{3 s}\left(s_{i k} s_{k j}-\frac{1}{3} I_{2 s} \delta_{i j}\right) \text {. }
$$

The functions $f_{1}$ and $f_{2}$ should be chosen in such a way as to obtain the ratios of principal strains depending on the ratios of principal stresses only (the ratio $f_{1} / f_{2}$ should be a homogeneous function of the fourth degree of the stress components). Instead of $I_{3 s}$ we may introduce into (15.5) the invariant $\omega_{\sigma}$ or the Lode parameter $\mu_{\sigma}$.

A particular form of (15.5) was used by D. C. Drucker [3.145], but increasing accuracy is connected here with complicated formulas. J. Christoffersen [3.101] pointed out that (15.5) may also be written in the form

$$
e_{i j} d \sigma_{i j}=\frac{1}{2} f_{1}\left(I_{2 s}, I_{3 s}^{2}\right) d I_{2 s}+\frac{1}{3} f_{2}\left(I_{2 s}, I_{3 s}^{2}\right) d I_{3 s}
$$

and that the differential on the right-hand side is, in general, not an exact differential, and hence such a theory is not identical with non-linear elasticity. Further general considerations are due to V. V. Novozhilov [3.580], K. F. Chernykh [3.95] and M. S. Sarkisyan [2.148], whereas an effective form based on experiments on mild steal was proposed by Y. Ohashi, M. Tokuda and S. Mizuno [3.592]. Equations of type (15.5) make it possible to describe practically any relation of the Lode parameters $\mu_{e}=f\left(\mu_{\sigma}\right)$, whereas the Hencky-Ilyushin law (15.2) results in $\mu_{\varepsilon}=\mu_{\sigma}$; however, G. A. Doshtchinsky [3.143] stated that for many materials the law $\mu_{\varepsilon}=\mu_{\sigma}$, expressing similarity of deviators, is sufficiently accurate and that the observed deviations are often connected with an improper interpretation of experimental results.

Theories of type (15.5) relate total strains to total stresses, and hence they are often called total theories of plasticity. They are much simpler than the incremental theories (of plastic flow), but the essential problem is connected with their range of application, which, unfortunately, is rather narrow. The problem is how to determine such a class of processes for which the results of total theory are still sufficiently accurate. This question was studied from the positions of more accurate theories by A. A. Ilyushin [2.67], B. Budiansky [3.80], D. D. Ivlev [3.311, 2.73], V. D. Klyushnikov [3.359], B. A. Rytchkov and N. Yu. Shvayko [3.690], K. S. Havner [3.229], M. Como and A. Grimaldi [3.112], Ya. I. Rudayev [3.684]. Z. Mróz [3.552] deter- 
mined a class of processes for which uniqueness of solutions is ensured. A. S. Kravtchuk [3.389] determined such a program of loading of a thick-walled tube under tension, torsion and internal pressure which realizes simple processes at any point of the tube in the case of linear plastic hardening (in general such a program does not exist for schematizations other than the power hardening law, D. D. Ivlev [2.70]). Some other related papers were mentioned in Secs. 9.8 and 9.9. After a suitable modification the total (deformation) theory of plasticity may also describe reverse and quasi-repeated simple processes. Such a modification was introduced by V. V. Moskvitin [3.541, 3.542, 3.546]. Assume that a passive process due to unloading starts at $\tilde{A}$ (Fig. 77); in the uniaxial case it may be described by the equation

$$
\Delta \varepsilon=\frac{1}{E} \Delta \sigma,
$$

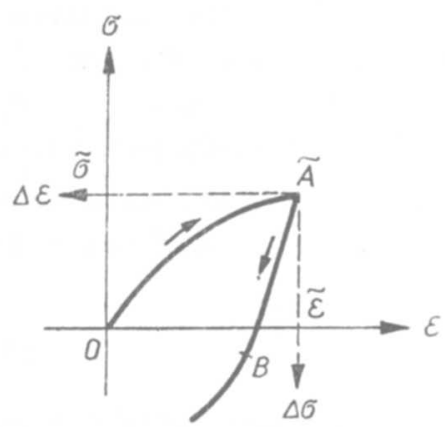

Fig. 77. Reverse active processes in the deformation theory

where $\Delta \varepsilon$ and $\Delta \sigma$ may be regarded as coordinates in the new system. A new reverse active process starts at $B$; the ideal Bauschinger effect predicts $\Delta \sigma_{B}=2 \sigma_{0}$, whereas usually $\Delta \sigma_{B}>2 \sigma_{0}$. Moskvitin proposed to describe this process in the coordinates $\Delta \varepsilon, \Delta \sigma$ similarly as the first, original active process was described in the coordinates $\varepsilon, \sigma$. Hence for a three-dimensional case we may write the equations

$$
\Delta e_{i j}=\varphi \Delta s_{i j},
$$

which must be completed by the relation

$$
(\Delta \sigma)_{e}=f\left[(\Delta \varepsilon)_{e}\right],
$$

obtained from experiments. According to the Masing hypothesis, described in Sec. 8.6 , such a relation is readily determined by the simple tension test, but in many cases Eq. (8.23) is not sufficiently accurate.

The modification described above served as a basis for extensive monographs by V. V. Moskvitin [2.113] and R. M. Shneyderovitch [1.17]. Conditions ensuring simple processes in the case under consideration were investigated by V. V. Moskvitin [3.543] and by the same author and E. Palne [3.544]. 


\subsection{Incremental (flow, anholonomic) theories of plasticity}

A general, logically sound description of arbitrary processes of plastic hardening in the case of combined loadings at a point of the body may be provided only by theories generalizing the theories of perfectly plastic flow, (9.51) or (9.60). They relate stress and strain increments and are called incremental theories; L. Finzi [3.177] proposed here the appropriate term "anholonomic theories" (total theories may then be called "holonomic"), but these terms are not yet in common use. On the other hand, the commonly used term "theories of plastic flow" seems inadequate in the case of plastic hardening, since under "plastic flow" we understand an increase of strains under constant stresses, which, in general, is not the case here (some exceptional cases were investigated by $R$. Hill [3.253]).

The basic postulates of classical incremental theories of the plastic hardening were summarized by W. Prager [3.653] in the following four points:

(1) continuous description of neutral processes (the same description by the equations of active and of passive processes, the continuity condition);

(2) during active processes the control point remains on the subsequent neutral surfaces (the consistency condition);

(3) positiveness of the expression $\dot{\sigma}_{i j} \dot{\varepsilon}_{i j}^{p}$ during any active process (the uniqueness condition, conforming to the Drucker postulate);

(4) non-negativeness of the increment of plastic work, $\sigma_{i j} d \varepsilon_{i j}^{p} \geqslant 0$ (the irreversibility condition).

An additional postulate (5) assumes linear relations between stress rates and strain rates,

$$
\dot{\varepsilon}_{i j}^{p}=A_{i j k l} \dot{\sigma}_{k l},
$$

where $A_{i j k l}$ do not depend on stress rates. Such an assumption is sufficient but not necessary to eliminate the time factor; if the time factor cannot be eliminated, then the materials are said to be viscoplastic, but such materials will not be considered in the present book.

Almost all these postulates were discussed by various authors and either partially relaxed or strengthened. In particular, the consistency condition (2) was strengthened in the already mentioned papers by A. Phillips, R. L. Sierakowski and M. A. Eisenberg [3.663, 3.166], so as to hold during certain passive processes as well. The uniqueness condition (3) leads to associated incremental laws (with some exceptions, discussed in Sec. 13); it is too restrictive for many materials and will be used only in part of this section. The discussion of postulate (4) will be given below. Further, (15.10) gives a sufficient but non-necessary condition of elimination of time, and linear functions are sometimes replaced by more general homogeneous functions of the first degree.

Discussion of the basic postulates may be found e.g. in the papers by R. Hill [3.250], A. A. Ilyushin [3.289], A. C. Pipkin and R. S. Rivlin [2.129], K. C. Valanis [3.830], B. Nayroles [3.572]. 
Incremental theories of the plastic hardening have been connected from the very beginning with the concept of the plastic potential introduced by R. Mises [3.531] for perfect plasticity. Assume the equation of subsequent neutral surfaces in a particular form of (14.62), namely

$$
F\left(\sigma_{i j}, \varepsilon_{i j}^{p}, I_{s p}\right)=0 \text {, }
$$

where the sign of $F$ is chosen in such a way as to give $F(0,0,0)<0$. Hypothesis (15.11) is not quite general but comprises most particular hypotheses described in Sec. 14. Denote by $d_{\sigma} F$ the quasi-total differential of the function $F$, temporarily regarded as a function of stresses only, at fixed plastic strains:

$$
d_{\sigma} F=\frac{\partial F}{\partial \sigma_{i j}} d \sigma_{i j} .
$$

Any process $d_{\sigma} F=0$ is, by definition, a neutral one and should not result in any change of plastic strains; to satisfy this requirement and to obtain a correct tensorial form of the physical law, we replace (13.7) by

$$
d \varepsilon_{i j}^{p}=\frac{1}{C} \frac{\partial g\left(\sigma_{i j}, \varepsilon_{i j}^{p}, I_{e p}\right)}{\partial \sigma_{i j}} d_{\sigma} F
$$

where $g\left(\sigma_{i j}, \varepsilon_{i j}^{p}, I_{e p}\right)$ denotes the plastic potential, and $C$ a scalar function of invariants, characterizing plastic hardening. The case $C \rightarrow 0$ corresponds to the transition to perfect plasticity (though in many particular cases such a transition may involve various difficulties, cf. R. Hill [3.248]); then simultaneously $d_{\sigma} F \rightarrow 0$ and

$$
\lim _{C \rightarrow 0} \frac{d_{\sigma} F}{C}=\lambda d t \text {. }
$$

The incremental law (15.13) was proposed for the first time probably by W. Prager and H. Geiringer in 1934 (cf. E. Melan [3.516]). R. Hill [2.56] with reference to Melan introduced slightly different notation

$$
d \varepsilon_{i j}^{p}=h \frac{\partial g}{\partial \sigma_{i j}} d_{\sigma} F=h \frac{\partial g}{\partial \sigma_{l j}} \frac{\partial F}{\partial \sigma_{k l}} d \sigma_{k l} .
$$

In the limiting case of perfect plasticity the function $h=1 / C$ increases infinitely; however, in his later papers, e.g. [3.247, 3.248], Hill uses the notation $h=C$ instead of $h=1 / C$ as in (15.15).

The law (15.13) or (15.15) is valid only in the case of an active process, if $d_{\sigma} F>0$; in the case of a passive process, if $d_{\sigma} F \leqslant 0$, we obviously have $d \varepsilon_{i j}^{p}=0$. Both cases may be described jointly in the following manner (P. G. Hodge, W. Prager [3.258], D. C. Drucker [2.28]):

$$
d \varepsilon_{i j}^{p}=\frac{1}{2} h \frac{\partial g}{\partial \sigma_{i j}}\left(d_{\sigma} F+\left|d_{\sigma} F\right|\right) .
$$

In what follows, however, our considerations will be limited to the description of active processes. Increments of total strains may be determined by the addition of elastic strain increments $d \varepsilon_{i j}^{e}$, calculated from Hooke's law. 
If we assume, as usual, that plastic strains do not result in any volume change, then the plastic potential $g$ must satisfy the relation

$$
\frac{\partial g}{\partial \sigma_{l i}}=0
$$

this means that the surface $g=0$ must be a cylindrical surface equally inclined in the principal stress space. Making no restriction to associated incremental laws (associated flow rules), we may at the same time consider the equations of neutral surfaces depending on $\sigma_{m}$ in the system of invariants $\sigma_{m}, \sigma_{e}, \omega_{\sigma}$.

The "consistency condition" (2), makes it possible to determine the scalar function $h$. We are now going to derive such a formula for a more general case (15.11). The condition of remaining of the control point on subsequent neutral surfaces means that the total differential of the function $F$, treated as a function of all its arguments, must vanish. Making use of the definition of the Odqvist parameter (14.13), we may write

$$
d_{\sigma} F+\frac{\partial F}{\partial \varepsilon_{i j}^{p}} d \varepsilon_{i j}^{p}+\frac{\partial F}{\partial I_{\varepsilon p}} \sqrt{d \varepsilon_{i j}^{p} d \varepsilon_{i j}^{p}}=0 .
$$

In view of (15.15) we obtain

$$
d_{\sigma} F+\frac{\partial F}{\partial \varepsilon_{i j}^{p}} h \frac{\partial g}{\partial \sigma_{i j}} d_{\sigma} F+\frac{\partial F}{\partial I_{\varepsilon p}} h d_{\sigma} F \sqrt{\frac{\partial g}{\partial \sigma_{i j}} \frac{\partial g}{\partial \sigma_{i j}}}=0
$$

and after cancelling $d_{\sigma} F$

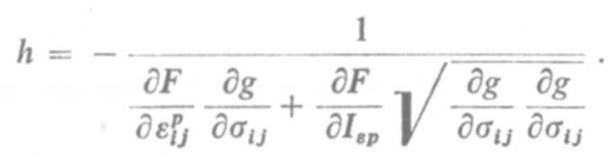

The substitution of (15.20) into (15.15) leads to physical equations in their final form

$$
d \varepsilon_{i j}^{p}=-\frac{\frac{\partial g}{\partial \sigma_{i j}} \frac{\partial F}{\partial \sigma_{k l}}}{\frac{\partial F}{\partial \varepsilon_{m n}^{p}} \frac{\partial g}{\partial \sigma_{m n}}+\frac{\partial F}{\partial I_{e p}} \sqrt{\frac{\partial g}{\partial \sigma_{m n}} \frac{\partial g}{\partial \sigma_{m n}}}} d \sigma_{k l} .
$$

In general, this formula is still a "quasi-explicit" one, since the derivatives depend on plastic strains $\varepsilon_{i j}^{p}$ and direct integration is not possible.

A similar formula for the case of the Odqvist parameter $I_{s p}$ in (15.11) being replaced by plastic work $W^{p}$ was derived by W. Prager [3.657]. However, such an approach, almost identical in the case of isotropic hardening, may result in essential qualitative differences in the case of a large Bauschinger effect. In fact, $I_{\varepsilon p}$ is always a non-decreasing function of plastic strains, whereas $W^{p}$ defined by (14.16) may decrease if $\sigma_{i j} d \varepsilon_{i j}^{p}<0$, i.e. if the origin of the reference frame in the stress space is not embraced by a subsequent neutral surface. Prager assumed $\sigma_{i j} d \varepsilon_{i j}^{p} \geqslant 0$ as a postulate, (4), and H. Ziegler [3.872] showed that under certain assumptions this 
inequality even may be proved. However, certain experiments did not confirm this postulate (H. J. Ivey [3.300]); A. Phillips and M. Eisenberg [3.634] showed that thermodynamic considerations lead to a weaker inequality and $\sigma_{i j} d \varepsilon_{i j}^{p} \geqslant 0$ is not necessarily valid. A deviation from $\sigma_{i j} d \varepsilon_{i j}^{p} \geqslant 0$ is shown in Fig. 32 based on the model of kinematic hardening, Fig. 29.

The uniqueness condition (3), or the Drucker postulate, leads to associated incremental laws, $g \equiv F$,

$$
d \varepsilon_{i j}^{p}=h \frac{\partial F}{\partial \sigma_{i j}} d_{\sigma} F=h \frac{\partial F}{\partial \sigma_{i j}} \frac{\partial F}{\partial \sigma_{k l}} d \sigma_{k l} .
$$

E. Melan [3.516] used such a law in connection with his concepts of kinematic hardening as early as 1938 , more than ten years before the Drucker postulate was published.

The assumption of isotropic hardening combined with the Huber-Mises-Hencky hypothesis (independent of the Lode parameter $\mu_{\sigma}$ ) is a counterpart of the LevyMises or the Prandtl-Reuss equations, namely, it results in the following particular form of (15.22):

$$
d \varepsilon_{i j}^{p}=d e_{i j}^{p}=h\left(I_{2 s}\right) s_{i j} s_{k l} d s_{k l},
$$

where all the numerical coefficients are included in the function $h$. Equation (15.23) was proposed in 1938 by W. Prager [2.131], and in 1943 by J. H. Laning [3.410]. It may be regarded as the classical incremental constitutive law of isotropic hardening. If we write (14.12) in the form

$$
F=g=\frac{1}{2} s_{i j} s_{i j}-f\left(I_{\varepsilon p}\right)=0,
$$

and introduce consistently deviatoric stresses and strains in (15.21), then this equation yields

$$
d e_{i j}^{p}=\frac{s_{i j} s_{k l}}{f^{\prime}\left(I_{\varepsilon p}\right) \sqrt{s_{m n} s_{m n}}} d s_{k l}=\frac{s_{i j} s_{k l}}{f^{\prime}\left(I_{e p}\right) \sqrt{2 f\left(I_{e p}\right)}} d s_{k l} .
$$

The function $f\left(I_{e p}\right)$ is determined by one basic test, for example the simple tension test. Using this test, we present experimental results in the form

$$
\frac{1}{3} \sigma^{2}-f\left(\sqrt{\frac{3}{2}} \varepsilon^{p}\right)=0 ;
$$

hence the function $f$ may be regarded as known. Equation (15.25) takes then the form

$$
d \varepsilon_{i j}^{p}=\frac{3}{2} \frac{s_{i j} d \sigma_{e}}{\sigma_{e} \sigma_{e}^{\prime}}
$$

(R. Hill [2.56], A. Mendelson [3.519]), but one has to be cautious when using (15.27): the symbol $\sigma_{e}^{\prime}$ denotes here the derivative with respect to $\varepsilon^{p}$ not for the argument $\varepsilon_{e}^{p}$ but for the argument $\sqrt{\frac{2}{3}} I_{s p}$ (and these values only coincide for simple loading). Equations (15.27) are sometimes called the Reuss equations (S. D. Klyatchko [3.355], A. Mendelson [3.519]), but Reuss applied his equations to perfect plasticity only; it is thus more justified to connect them with the names of Prager and Laning. 
For some theoretical considerations, but also for some applications, it is convenient to present physical relations in vectorial notation. Introduce an auxiliary vector space satisfying the requirements discussed in Sec. 10.1. It may be the ninedimensional Prager space, or, if we restrict our considerations to deviators, the five-dimensional Ilyushin space; as a matter of fact, in the case of deviators even the nine-dimensional space is reduced to a certain five-dimensional subspace, since the following four relations hold: $\tau_{x y}=\tau_{y x}, \tau_{y z}=\tau_{z y}, \tau_{z x}=\tau_{x z}, s_{x x}+s_{y y}+s_{z z}=0$. We introduce such spaces for stresses, strains and plastic strains.

Introducing the unit normal vector by formula (13.22), we may write (15.15) in the form

$$
d \epsilon^{p}=\bar{h}\left(\mathbf{n}_{F} d \boldsymbol{\sigma}\right) \mathbf{n}_{g},
$$

where the scalar product of vectors is bracketed and

$$
\bar{h}=h \sqrt{\frac{\partial F}{\partial \sigma_{k l}} \frac{\partial F}{\partial \sigma_{k l}} \frac{\partial g}{\partial \sigma_{p q}} \frac{\partial g}{\partial \sigma_{p q}}}
$$

is a certain function of invariants. Notation (15.28) is used, for example, by Z. Mróz $[3.553,2.114]$, and in the case of an associated incremental law, with $g \equiv F, \mathbf{n}_{g}$ $=\mathbf{n}_{F}=\mathbf{n}$, by R. Hill [3.248, 3.250]. Expressing the scalar product by vector lengths and the angle, and bearing in mind that $|\mathbf{n}|=1$, we obtain in the latter case

$$
d \boldsymbol{\epsilon}^{p}=\vec{h} \mathbf{n}|d \boldsymbol{\sigma}| \cos (\mathbf{n}, d \boldsymbol{\sigma}) \quad \text { if } \cos (\mathbf{n}, d \boldsymbol{\sigma}) \geqslant 0 .
$$

Notation (15.30) obviously describes also the Prager-Laning law (15.23), but it is possible to present this law without introduction of the vector $\mathbf{n}$. Namely, Eq. (15.23) assumes, in vectorial notation, the form

$$
d \mathbf{e}^{p}=h(\mathbf{s} d \mathbf{s}) \mathbf{s}=h \mathbf{s}|\mathbf{s}||d \mathbf{s}| \cos (\mathbf{s}, d \mathbf{s})=\tilde{h} \mathbf{s} \cos (\mathbf{s}, d \mathbf{s})|d \mathbf{s}|,
$$

the invariant $|\mathbf{s}|$ being included in the function $\tilde{h}$. In order to eliminate $h$, i.e. to use form (15.27), we first notice that

and finally

$$
\sigma_{e}=\sqrt{\frac{3}{2}}|\mathbf{s}|, \quad \varepsilon_{e}=\sqrt{\frac{2}{3}}|\mathbf{e}|,
$$

$$
d \mathbf{e}^{p}=\frac{\mathbf{s} d|\mathbf{s}|}{|\mathbf{s}||\mathbf{s}|^{\prime}}=\frac{|\mathbf{e}|^{\prime}}{|\mathbf{s}|} \mathbf{s} d|\mathbf{s}|,
$$

where the derivative $|\mathbf{s}|^{\prime}=d|\mathbf{s}| / d|\mathbf{e}|$ determined from the tension test should be taken for the argument $I_{\varepsilon p}$ and not for $\left|\mathbf{e}^{p}\right|$. The law (15.33), based on the hypothesis of isotropic hardening, holds if $d|\mathbf{s}| \geqslant 0$.

Let us return once more to the general notation (15.28). The increments of total strains may be obtained by adding the increments of elastic strains:

$$
d \boldsymbol{\epsilon}=\mathbf{A}^{e} d \boldsymbol{\sigma}+\bar{h}\left(\mathbf{n}_{F} d \boldsymbol{\sigma}\right) \mathbf{n}_{g},
$$

where $\mathbf{A}^{e}$ denotes the matrix of elastic moduli (with the dimensions $\mathrm{cm}^{2} / \mathrm{kG}$ ). Formally, this law may be inverted so as to express $d \boldsymbol{\sigma}$. Namely, denote by $\mathbf{E}^{e}$ the matrix of elastic moduli with the dimensions $\mathrm{kG} / \mathrm{cm}^{2}$, inverse with respect to $\mathbf{A}^{\boldsymbol{e}}$; hence

$$
d \boldsymbol{\sigma}=\mathbf{E}^{e}\left[d \boldsymbol{\epsilon}-\bar{h}\left(\mathbf{n}_{F} d \boldsymbol{\sigma}\right) \mathbf{n}_{g}\right] .
$$


To eliminate the scalar product $\mathbf{n}_{\boldsymbol{F}} d \boldsymbol{\sigma}$, which is unknown here, we multiply both sides of (15.35) scalarly by $\mathbf{n}_{F}$, so as to obtain (Z. Mróz [2.114])

$$
\mathbf{n}_{\boldsymbol{F}} d \boldsymbol{\sigma}=\mathbf{n}_{\boldsymbol{F}}\left(\mathbf{E}^{e} d \boldsymbol{\epsilon}\right)-\bar{h}\left(\mathbf{n}_{\boldsymbol{F}} d \boldsymbol{\sigma}\right) \mathbf{n}_{\boldsymbol{F}}\left(\mathbf{E}^{e} \mathbf{n}_{\boldsymbol{g}}\right) ;
$$

hence

$$
\mathbf{n}_{F} d \boldsymbol{\sigma}=\frac{\mathbf{n}_{F}\left(\mathbf{E}^{e} d \epsilon\right)}{1+\bar{h}\left[\mathbf{n}_{F}\left(\mathbf{E}^{e} \mathbf{n}_{g}\right)\right]}
$$

and finally

$$
d \boldsymbol{\sigma}=\mathbf{E}^{e}\left\{d \boldsymbol{\epsilon}-\frac{\bar{h}\left[\mathbf{n}_{F}\left(\mathbf{E}^{e} d \boldsymbol{\epsilon}\right)\right] \mathbf{n}_{g}}{1+\bar{h}\left[\mathbf{n}_{F}\left(\mathbf{E}^{e} \mathbf{n}_{g}\right)\right]}\right\}=\left\{\mathbf{E}^{e}-\frac{\bar{h}\left(\mathbf{E}^{e} \mathbf{n}_{\boldsymbol{F}}\right) \otimes\left(\mathbf{E}^{e} \mathbf{n}_{g}\right)}{1+\bar{h}\left[\mathbf{n}_{F}\left(\mathbf{E}^{e} \mathbf{n}_{g}\right)\right]}\right\} d \boldsymbol{\epsilon} .
$$

The symbol $\otimes$ denotes here a dyadic product of two vectors (such notation is used, for example, by C. Truesdell), and hence the second bracketed term [in the second form of notation of (15.38)] is also a matrix. In the case of the isotropic body under consideration, the matrix $\mathbf{E}^{e}$ is determined by formulae (9.2). The law (15.38) is valid in the case of active processes, $\mathbf{n}_{F} d \boldsymbol{\sigma}>0$, and in view of (15.37)

$$
\mathbf{n}_{F}\left(\mathbf{E}^{e} d \boldsymbol{\epsilon}\right)>0 \text {; }
$$

in the opposite case a passive process takes place and we have $d \boldsymbol{\sigma}=\mathbf{E}^{e} d \boldsymbol{\epsilon}$.

Formula (15.38) becomes effective only after expressing the hardening function $\bar{h}$ and the coordinates of the vectors $\mathbf{n}$ in terms of strains, and such an expression may cause difficulties. This problem was considered in detail by D. Trifan [3.816], who derived very general formulae of type (15.38) and by Yu. I. Kadashevitch [3.323] in relation to the theory of residual microstresses. Formula (15.38) cannot be directly used in the limiting case of a rigid-plastic body, since then some of the components of $\mathbf{E}^{e}$ tend to infinity; this limiting case was discussed by R. N. Dubey and Z. Mróz [3.158].

The Prager-Laning equations (15.23) were experimentally tested by various investigators: J. L. Morrison, W. M. Shepherd [3.540], H. D. Bui, J. Mandel (cf. [3.494]), S. D. Klyatchko [3.355], O. A. Shishmarev [3.729]; on the one hand, they confirmed the better accuracy of the incremental theories as compared with the total theories (contrary to the earlier experiments of K. Hohenemser [3.262], who, however, neglected plastic hardening in calculations), but, on the other hand, those experiments showed also 'some deviations, 'connected probably with the hypothesis of isotropic hardening, included in (15.23) and not confirmed for most materials.

Effective forms of constitutive equations associated with subsequent neutral surfaces corresponding to the hypotheses of kinematic or mixed plastic hardening were derived and investigated by Yu. I. Kadashevitch and V. V. Novozhilov [2.80], M. A. Eisenberg and A. Phillips [3.126], G. B. Talypov [2.168], and M. Tanaka [3.788].

Finally, let us quote two important general forms of incremental laws, (15.15) or (15.28). Assuming isotropic hardening in connection with the HMH hypothesis, 
G. H. Handelman, C. C. Lin and W. Prager [3.223] formulated in 1947 the following non-associated incremental law:

$$
d \varepsilon_{i j}^{p}=\left[P\left(I_{2 s}, I_{3 s}\right) s_{i j}+Q\left(I_{2 s}, I_{3 s}\right)\left(s_{i k} s_{k j}-\frac{1}{3} I_{2 s} \delta_{i j}\right)\right] d I_{2 s},
$$

where the condition of an active process (and of the applicability of that law) is $d I_{2 s}>0$. For any initial yield condition and isotropic hardening we may write, instead of (15.40),

$$
d \varepsilon_{i j}^{p}=\frac{1}{2}\left[P\left(I_{2 s}, I_{3 s}\right) s_{i j}+Q\left(I_{2 s}, I_{3 s}\right)\left(s_{i k} s_{k j}-\frac{1}{3} I_{2 s} \delta_{i j}\right)\right]\left(d_{\sigma} F+\left|d_{\sigma} F\right|\right),
$$

describing in this way both active and passive processes. The law (15.41) was suggested by P. G. Hodge and W. Prager [3.258].

The laws (15.40) and (15.41) are tensorially non-linear with respect to $T_{\sigma}$ (though still linear in $T_{\dot{\sigma}}$ ). Such laws introduce the so-called second-order effects, such as e.g. the Poynting effect (change in length of a bar subject to torsion). Those effects were studied in detail by A. M. Freudenthal with M. Ronay [3.183] and with P. F. Gou [3.185], and by T. Lehmann [3.424].

\subsection{Generalizations of incremental theories}

Generalizations of the basic incremental law of plastic hardening (15.23) are so varied that it is even difficult to classify them. Nevertheless, several principal directions may be distinguished.

The first direction involves more general linear deviatoric laws. Following the idea of K. Hohenemser and W. Prager [3.263], A. A. Ilyushin [1.8] proposed in 1949 the following general law:

$$
L_{1}\left[D_{\sigma}\right]=L_{2}\left[D_{e}\right],
$$

where the linear integro-differential operator $L_{1}$ is of the form

$$
L_{1}\left[D_{o}\right]=A_{1} D_{\sigma}+B_{1} D_{\dot{\sigma}}+\int_{0}^{t} C_{1} D_{\sigma} d \tau+\ldots,
$$

the operator $L_{2}$ is analogical, and the coefficients $A, B, C, \ldots$ are certain functions of invariants. The law (15.42) includes all the classical theories of plasticity, but it does not contain tensorially non-linear laws, such as (15.5) or (15.40). This law was studied in detail by D. D. Ivlev [3.301]; Yu. I. Kadashevitch [3.324] stated that in the case of plastic hardening the orders of the two operators $L_{1}$ and $L_{2}$ should be equal, whereas for perfectly plastic bodies the order of $L_{2}$ should be higher by one than the order of $L_{1}$. Further related proposals were made by C. Hartung and T. Lehmann [3.226], Yu. I. Kadashevitch [3.329] (higher-order derivatives) and V. M. Panferow, R. Kh. Ismagilov, L. M. Ilyin, E. Z. Korol and V. F. Tyurin [3.616] (for constant principal directions only).

The second direction of the generalization of incremental laws, initiated by W. T. Koiter [3.363], is connected with the concept of plastic corners or the formation of such corners in the course of plastic hardening. If we assume that the plastic 
potential function has such a corner, then the normal is not unique in it and its direction may lie inside a certain cone (a certain angle in the two-dimensional case). Then the associated incremental law (15.22) may be replaced by

$$
d \varepsilon_{i j}^{p}=\sum_{\alpha=1}^{m} h_{\alpha} \frac{\partial F_{\alpha}}{\partial \sigma_{i j}} \frac{\partial F_{\alpha}}{\partial \sigma_{k l}} d \sigma_{k l},
$$

where

$$
\begin{aligned}
& h_{\alpha} \geqslant 0 \quad \text { for } \quad \frac{\partial F_{\alpha}}{\partial \sigma_{k l}} d \sigma_{k l} \geqslant 0, \\
& h_{\alpha}=0 \quad \text { for } \quad \frac{\partial F_{\alpha}}{\partial \sigma_{k l}} d \sigma_{k l}<0 .
\end{aligned}
$$

Such a law in the case of $n=6$ terms was studied in detail by W. H. Warner and G. H. Handelman [3.840]. Linear functions $F_{\alpha}\left(\sigma_{i j}\right)$ were investigated by W. Prager [3.654], J. L. Sanders [3.694], P. G. Hodge [3.259, 3.260, 3.261]. Further proposals are due to J. Mandel [3.493] (dependent mechanisms of plastic flow), J. Christoffersen $[3.100,3.102]$ and M. J. Sewell [3.710].

Tensorially non-linear laws (with respect to the tensors $T_{\dot{\varepsilon}}$ and $T_{\dot{\sigma}}$ ) will be classified in the third group of generalizations. Discussing the basic assumptions of the incremental theories of plasticity in Sec. 15.3, we noticed that linear relations between $\dot{\varepsilon}_{i j}^{p}$ and $\dot{\sigma}_{i j},(5)$, are sufficient to eliminate the time factor, but they are not necessary: linear functions may be replaced by more general homogeneous functions of the first degree. Such a possibility was pointed out by R. Hill [3.250] and W. T. Koiter [3.365], but a detailed discussion is due to Z. Mróz [3.553, 2.114]. Assuming that the increment $d \varepsilon_{i j}^{p}$ depends only on the increment $d s_{i j}$ and on the direction of the normal $\mathbf{n}$ to the actual (regular) neutral surface, Mróz derived the following sevenparameter law

$$
\begin{aligned}
d \varepsilon_{i j}^{p} & =\left(\alpha_{1} n_{l j}+\alpha_{2} n_{i k} n_{k j}\right) n_{p q} d s_{p q}+ \\
& +\left[\alpha_{3} d s_{i j}+\alpha_{4}\left(n_{i k} d s_{k j}+n_{k j} d s_{i k}\right)+\alpha_{5}\left(n_{i k} n_{k l} d s_{l j}+n_{k l} n_{l j} d s_{i k}\right)\right] \frac{n_{p q} d s_{p q}}{\sqrt{d s_{u v} d s_{u v}}}+ \\
& +\left[\alpha_{6} d s_{i k} d s_{k j}+\alpha_{7}\left(d s_{i k} d s_{k l} n_{l j}+d s_{k l} d s_{l j} n_{i k}\right)\right] \frac{n_{p q} d s_{p q}}{d s_{u v} d s_{u v}},
\end{aligned}
$$

where the coefficients $\alpha_{1}, \ldots, \alpha_{7}$ may depend on common invariants of the tensors $n_{i j}, s_{i j}$ and $d s_{i j}$, homogeneous of degree zero with respect to $d s_{i j}$. This law may be regarded as a generalization of (15.28) which is obtained if only $\alpha_{1}=\bar{h}$ is different from zero. Paper [2.114] analyses in detail the case of two non-vanishing coefficients, $\alpha_{1}$ and $\alpha_{3}$. A certain essential difference with respect to tensorially linear laws is seen: vector $d \epsilon^{p}$ (in an auxiliary vector space) may change its direction depending on vector $d \mathbf{s}$, whereas in the case of linear laws the direction of $d \mathbf{\epsilon}^{p}$ was constant; namely, it coincided with the normal $\mathbf{n}_{\mathbf{g}}$. Certain other proposals, e.g. that of V. D. 
Klyushnikov [3.356] and R. A. Vasin [3.833] also belong to the type under discussion; tensorially non-linear laws of A. A. Ilyushin will be described in Sec. 15.5. Such an approach may be interpreted as the introduction of $d s_{i j}$ into the plastic potential $g$, (15.13); indeed, $g$ may be a homogeneous function of the zeroth degree of $d s_{i j}$.

The fourth type of generalizations may be called semi-empirical. Since simple (radial) deformation processes are described, as a rule, with a sufficiently high accuracy, one attempts to describe experimental results concerning orthogonal (circumferential) processes more accurately than by (15.23); usually the influence of the third invariant, $\omega_{\sigma}$, is also taken into account. Such proposals are due to S. A. Elsufyev [3.169], I. M. Korovin [3.376], S. A. Khristyanovitch and E. I. Shemyakin [3.342], S. Smith and B. O. Almroth [3.746], and V. I. Maksak [3.482]. For example, Elsufyev [3.169] used (15.23) with the stress deviator replaced by a certain other coaxial deviator, determined by the general formulae of V. V. Novozhilov [3.580], where two functions joining the invariants of both deviators are to be determined.

Theories consistently using the internal state variables (besides $I_{\varepsilon p}$ and $W^{p}$ ) will be classified in the fifth group. We mentioned in Sec. 14.8 that in this case the basic constitutive equations must be completed by the evolution laws determining $\alpha_{(k) i j}$ and $\varkappa_{m}$. Retaining (15.15) with the plastic potential $g$ depending on $\alpha_{(k) l j}$ and $\varkappa_{m}$, we may write the simplest evolution laws in the tensorially linear form

$$
\begin{aligned}
& \dot{\alpha}_{(k) i j}=A_{(k) i j l q} \dot{\varepsilon}_{q q}^{p}, \\
& \dot{\varkappa}_{m}=B_{(m) t q} \dot{\varepsilon}_{l q}^{p},
\end{aligned}
$$

where $A_{(k)}$ and $B_{(m)}$ depend on stress components and internal state variables. However, even one of the oldest evolution laws, that for the Odqvist parameter, namely

$$
\dot{I}_{e p}=\sqrt{\dot{\varepsilon}_{i j}^{p} \dot{\varepsilon}_{i j}^{p}}
$$

does not conform to (15.47), and it turns out that the linear expressions on the right-hand side of (15.47) should be replaced by more general homogeneous functions of the first degree (such homogeneity is necessary to eliminate the time factor). Let us mention here the papers bv Q. S. Nguyen and H. D. Bui [3.576, 3.577] and by Z. Mróz [3.559]; other papers on this subject concern finite strains and will be discussed in Sec. 15.9.

The sixth type is an alteration (rather than generalization) of (15.23) consisting in introduction of maximal shearing stresses and of maximal shearing strains into constitutive equations. It is claimed by S. A. Khristyanovitch [3.343], J. Gouzou and R. D'Haeyer [3.208] that such an approach describes experimental results better than the Prager-Laning equations do.

The "analytical" proposal of V. D. Klyushnikov [1.12], mentioned several times in the preceding sections, may also be treated as a generalization of the PragerLaning law (15.23). Namely, the law (15.23) may be written in the form

$$
d \mathbf{e}^{p}=\tilde{h} \mathbf{s}|d \mathbf{s}| f(\alpha),
$$


where $\alpha$ denotes the angle between the vectors $\mathbf{s}$ and $d \mathbf{s}$; further

$$
f(\alpha)=\left\{\begin{array}{cll}
\cos \alpha & \text { if } 0 \leqslant \alpha \leqslant \frac{1}{2} \pi, \\
0 & \text { if } \frac{1}{2} \pi \leqslant \alpha \leqslant \pi .
\end{array}\right.
$$

In fact, as Klyushnikov remarks, an active process takes place for obtuse angles $\alpha$ as well, but then the changes of plastic strains are very small. Abandoning the distinction of passive processes, i.e. assuming that each process is to certain degree active, we may describe the function $f(\alpha)$ in a uniform analytical manner instead of (15.50); Klyushnikov proposes, for example,

$$
f(\alpha)=\left(\frac{1+\cos \alpha}{2}\right)^{\omega}=\left(\cos \frac{\alpha}{2}\right)^{2 \omega}
$$

with the recommendation of $\omega=2$ as a good description of experimental data. A comparison of (15.50) and (15.49) for $\omega=2$ is shown in Fig. 78. Though the

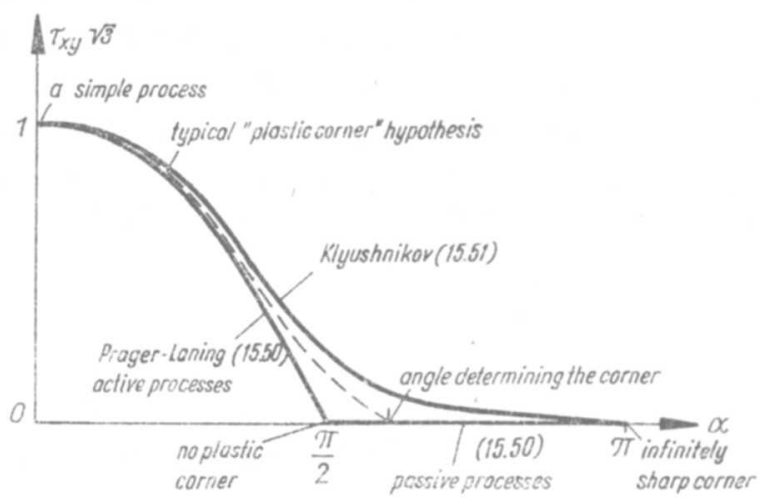

Fig. 78. A comparison of the Prager-Laning and the Klyushnikov hardening rules

Prager-Laning function (15.50) cannot be regarded as a particular fcase of (15.51), nevertheless one can choose such a family of differentiable functions as to obtain the non-differentiable function (15.50) as their limit. A typical "plastic corner" hypothesis is also shown in Fig. 78. The Klyushnikov proposal (15.51) may be called the "hypothesis of an infinitely sharp plastic corner".

Let us also mention several related papers. H. Ziegler [3.871] investigated the problem of coincidence of principal axes of stresses and strain rates in the case of anisotropic hardening. W. Olszak and P. Perzyna [3.601] discussed neutral surfaces depending on time as a parameter; such a situation may take place, for example, as a result of changing temperature or humidity of the body. V. V. Moskvitin [3.547, 3.548] analysed the influence of microcracks on the constitutive equations of plasticity. G. Brinkmann [3.78] considered second-order constitutive laws, expressing plastic strain increments in terms of stresses and total strains. R. J. Green [3.216] derived constitutive equations for porous solids. J. Bahuaud and M. Boivin [3.27] discussed the influence of plastic compressibility on variously defined coefficients of transversal deformation. P. Mazilu [3.513, 3.514] combined (15.23) with a non- 
linear description of elastic deformations. The results of calculations based on various constitutive equations were compared by $\mathrm{K}$. Sh. Babamuradov and $\mathrm{Zh}$. Kultursynov [3.20] on the example of a thin-walled tube subject to combined loadings. Many proposals of constitutive equations were discussed in survey papers by $\mathrm{T}$. Lehmann and K. Thermann [3.422], A. A. Vakulenko and L. M. Kachanov [3.829].

\subsection{The Ilyushin geometrical theory of plasticity}

A distinct phenomenological approach to the basic concepts of the theory of plasticity was suggested in 1954 by A. A. Ilyushin [1.10]. He introduced auxiliary fivedimensional vector spaces, described by us in Sec. 10.3, and made use of them in a much more essential manner than had been done with similar spaces before; namely, he made use of geometrical invariants of trajectories in such spaces. Therefore we call his (second) theory the geometrical theory of plasticity. The main postulates and basic equations of this theory were presented in papers [1.10, 3.287, 3.288, $3.289,1.11,3.292]$.

Consider a process prescribed in strains. Ilyushin assumed that $\varepsilon_{m}$ has no influence on the process, and hence the five independent coordinates of the strain deviator may be regarded as the exertion factors. Instead of those "non-symmetrical" quantities, five "symmetrical" components $e_{i}$ of the vector e may be introduced by formulae of type (10.3). This symmetrization procedure is an essential point of the theory, since only then the geometrical invariants of the trajectory are also invariant to rotation of the physical reference frame.

The functions $e_{i}=e_{i}(t), i=1,2,3,4,5$, considered as known, determine the trajectory in the auxiliary five-dimensional strain space. This trajectory is characterized by five independent geometrical invariants, for example

$$
|\mathrm{e}|=\sqrt{e_{i} e_{i}}=\sqrt{e_{k l} e_{k l}}, \quad x_{n}=\left|\frac{d^{n+1} \mathrm{e}}{d s_{e}^{n+1}}\right|, \quad n=1,2,3,4,
$$

where $s_{e}$ denotes the current length of the strain trajectory

$$
s_{e}=\int_{0}^{t} \sqrt{\frac{d e_{l}}{d \tau} \frac{d e_{i}}{d \tau}} d \tau .
$$

This quantity is close to the Odqvist parameter, (14.13), but plastic strains are replaced here by total strains. Further, $|\mathbf{e}|$ is proportional to the strain intensity, and $\varkappa_{1}$ determines the curvature of the trajectory. It may be proved that the following five unit vectors

$$
\mathbf{p}_{1}=\frac{d \mathrm{e}}{d s_{e}}, \quad \mathbf{p}_{2}=\frac{1}{\chi_{1}} \frac{d^{2} \mathrm{e}}{d s_{e}^{2}}, \quad \ldots, \quad \mathbf{p}_{5}=\frac{1}{\varkappa_{4}} \frac{d^{5} \mathrm{e}}{d s_{e}^{5}}
$$

are mutually orthogonal and may be assumed as a local reference frame in the five-dimensional strain space. In the case of a three-dimensional space the relevant directions would coincide with the directions of the tangent, the normal and the binormal, respectively. 
Denote now by $\mathbf{s}$ the auxiliary five-dimensional vector in the stress space, corresponding to $\mathbf{e}$; it represents the dependent quantities (the sixth quantity $\sigma_{m}$ either may be found from the law of volume change, or, in the case of incompressibility, must be regarded as known). Formally, each vector $\mathbf{s}$ may be decomposed in the reference frame (15.54) as follows:

$$
\mathbf{s}=S_{n} \mathbf{p}_{n} .
$$

Now, the central idea of the Ilyushin geometrical theory is called by him the postulate of isotropy. This postulate may be expressed as follows [3.431]: for a deformation of an originally quasi-isotropic solid from its virgin state $(e=0)$, the image in the stress space is invariant with respect to the transformations of the strain trajectory by means of rotation or reflection. In other words, the functions $S_{n}$ in (15.55) depend only on the intrinsic geometry of the strain trajectory and not on its position (initial direction) in the strain space. The constitutive equations of plasticity become effective if we evaluate (experimentally, as a rule) the functions $S_{n}$.

The postulate of isotropy may be considered as consisting of two parts. First, if we change (rotate) the physical reference frame, then the deviatoric components $e_{i j}$ change, the auxiliary vector components $e_{i}$ also change, and so does the trajectory $e_{i}(t)$. However, Ilyushin proved that such a change corresponds to a rigid rotation of the five-dimensional reference frame, and hence the geometrical invariants (15.52) remain unchanged. Conversely, the second aspect of the postulate of isotropy cannot be proved mathematically, but only verified experimentally, since rotations and reflections of the trajectory in the five-dimensional space describe a much wider class of processes than the identical processes described in the rotated physical reference frame. Whereas the first aspect of the postulate of isotropy holds for any material (isotropic or anisotropic), the second aspect is in the Ilyushin space limited not only to isotropy but to a restricted class of isotropic bodies. For example, this

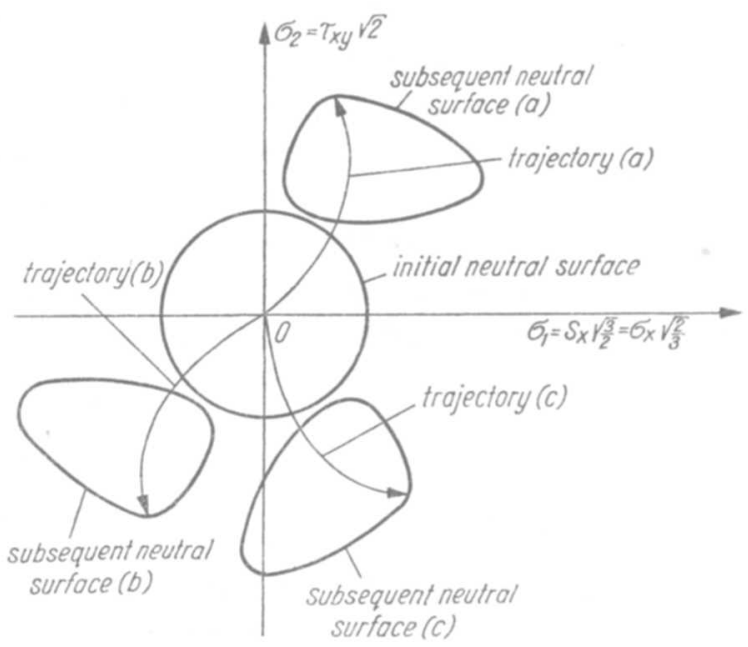

Fig. 79. The Ilyushin postulate of isotropy 
postulate requires first plastification to be determined only by the length of the straight trajectory, and hence the material must be governed by the Huber-MisesHencky initial yield condition (mapped by a hypersphere). Further, this postulate implies for rotated trajectories an appropriate rigid rotation of subsequent neutral surfaces (the same deformation of those surfaces at the corresponding points of the trajectories), Fig. 79.

Assuming, for example, $S_{2}=S_{3}=S_{4}=S_{5}=0$, we obtain

$$
\mathbf{s}=S_{1} \mathbf{p}_{1}=S_{1} \frac{d \mathbf{e}}{d s_{e}},
$$

and, after contraction and evaluation of $S_{1}$,

$$
d e_{i j}=\frac{\sqrt{d e_{k l} d e_{k l}}}{|\mathbf{s}|} s_{i j} .
$$

Equation (15.57) is reminiscent of the incremental theory, and for simple loading coincides with the Hencky-Ilyushin total strain theory; the important difference consists in the fact that plastic strain increments are replaced in it by total strain increments. If we retain $S_{n}, n \geqslant 2$, in (15.55), then higher-order derivatives with respect to time are introduced into the constitutive equations. The invariant functions $S_{n}$ should be determined experimentally.

The law (15.55) is effective at regular points of the trajectory; at singular points (corners of the trajectory), the quantities $\varkappa_{n}$ increase infinitely. For such cases Ilyushin [3.289] derives the constitutive equation

$$
d \mathbf{e}=\frac{1}{N} d \mathbf{s}+\frac{N-P}{N P} \frac{(\mathbf{s} d \mathbf{s})}{\mathbf{s}^{2}} \mathbf{s},
$$

where $N=2 G$ in the virgin state, but may also depend on the actual deformation (change of elastic constants resulting from plastic deformation). This law may be regarded as a direct generalization of the Prager-Laning law in the form (15.31); the only difference is that $\tilde{h}$ in (15.31) does not depend on $d \mathbf{s}$, whereas Ilyushin assumes that $P$ in (15.58) depends on $d \mathbf{s}$, being a homogeneous function of the zero order of that increment (the tensorially non-linear law).

The first experimental verification of the postulate of isotropy was performed by V. S. Lensky $[3.429,3.430,3.286,3.431,3.433]$. Some results for copper specimens subject to a combined tension-torsion test [3.431], are encouraging indeed: the vectors show stresses at subsequent points of the strain trajectories and the differences for a reflected trajectory are of the order of 10 per cent, Fig. 80 . Of course, for the materials not governed by the $\mathrm{HMH}$ hypothesis the postulate of isotropy has no chances (without appropriate generalizations); hence its name is too broad and does not correspond to general isotropy. Probably the term postulate of $I_{2}$-isotropy (where $I_{2}$ is understood as $I_{2 e}$ or $I_{2 s}$ ) would be more adequate. This lack of generality and some other features of the theory were criticized by D. D. Ivlev [3.306, 3.307], followed by a longer discussion between V. S. Lensky [3.434], A. A. Ilyushin [3.290, 3.291] and V. V. Novozhilov [3.581, 3.582]. The replacement of 


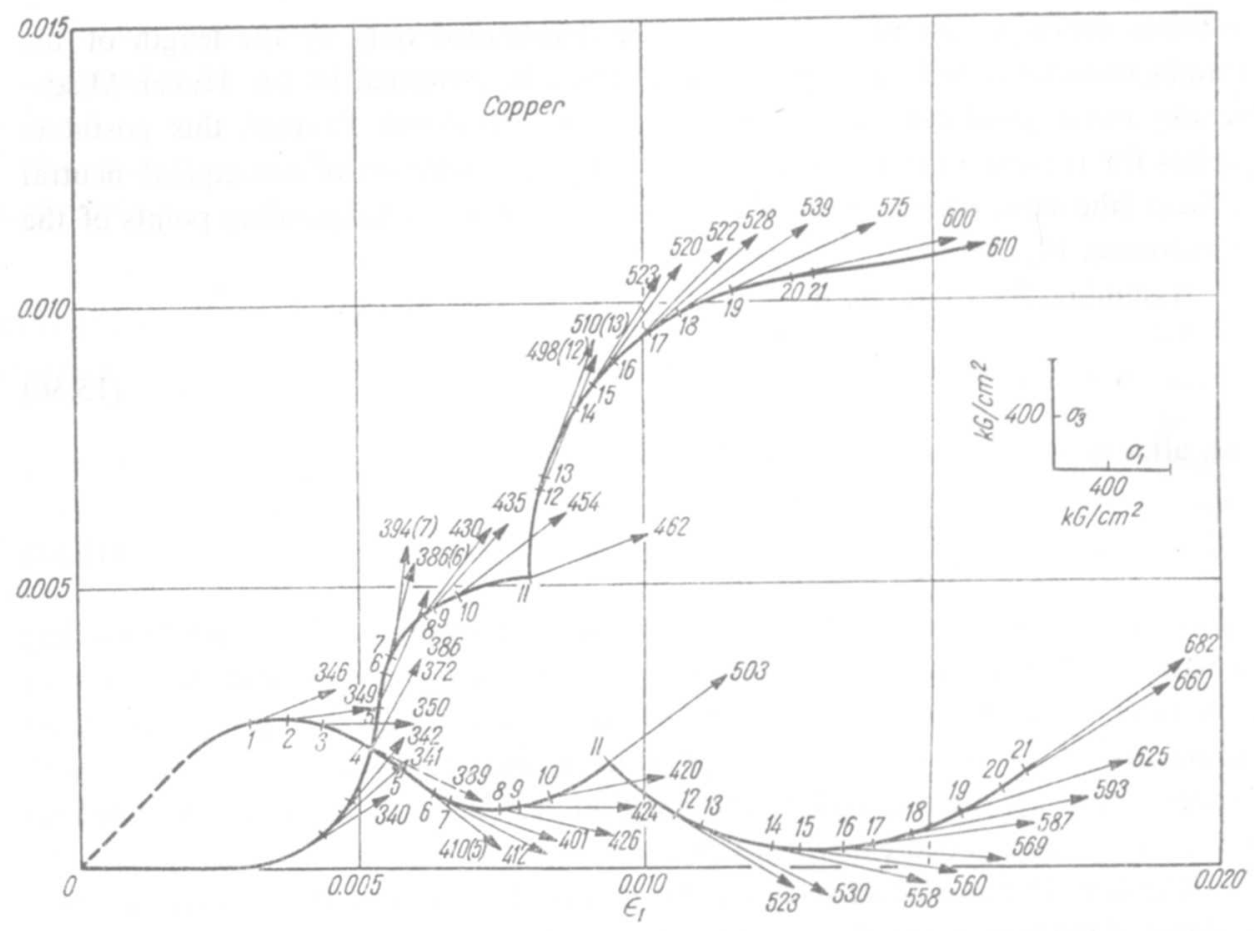

Fig. 80. Lensky's verification of the postulate of isotropy

the Odqvist parameter by (15.53) seems also inconvenient for the effective formulation of the constitutive equations.

Many further series of experiments confirmed the postulate of isotropy for various materials and various types of loading. This postulate, originally formulated for the strain space, may also be applied to the trajectories in the stress space. Such a formulation was experimentally verified by L. S. Andreyev for steel 40 [3.5, 3.7, 3.8] and for brass L68 [3.6], by B. D. Annin and B. P. Rusov [3.9] for copper, by O. A. Shishmarev [3.728] for mild steel. Yu. N. Shevtchenko and R. G. Terekhov confirmed the postulate of isotropy for various types of tests [3.716] (tension with internal pressure was compared to tension with torsion) and for changing principal directions [3.795]. The experiments of T. Ota, A. Shindo and H. Fukuoka [3.607] on mild steel tubes subject to tension and torsion may-to a certain degree-serve as a verification of the postulate of isotropy as well (symmetry of subsequent neutral surfaces). It may be concluded that if the material obeys the $\mathrm{HMH}$ hypothesis, it obeys also the postulate of isotropy (with the accuracy of the order of 10 per cent).

The differences may become larger in the case of variable repeated loading. Y. Ohashi, M. Tokuda and H. Yamashita [3.591, 3.593] verified in this case the postulate of isotropy for mild steel, found the influence of the third deviatoric invariant more essential and proposed a method of eliminating that influence. 
A certain simplified variant of the geometrical theory of plasticity, called the hypothesis of local determinacy was introduced by V. S. Lensky [3.429, 3.435, 3.436]. Instead of (15.55) he writes the constitutive equations in the form

$$
\frac{\partial \vartheta_{i}}{\partial s_{e}}=f_{i}\left(\vartheta_{1}, \vartheta_{2}, \ldots, \vartheta_{5}, s_{e}\right),
$$

where

$$
\cos \vartheta_{i}=\frac{\mathbf{s p}_{i}}{|\mathbf{s}|}
$$

and $\mathbf{p}_{i}$ are defined by (15.54). In the simplest case (15.59) may be reduced to (15.58). Experimental verification of (15.59), besides of Lensky, was performed by Z. B. Dao [3.125]. Other simplified variants of the geometrical theory were given by V. I. Malyi [3.491, 3.492] (developments in power series of a small parameter) and by R. A. Vasin [3.834]. A general survey paper on the basic assumptions, simplified variants and experimental verifications of the Ilyushin geometrical theory of plasticity was published by P. M. Ogibalov [3.590].

Let us also mention another direction of the theory of plasticity, essentially based on geometrical considerations and proposed by K. Kondo [3.367, 3.368, $3.369,3.370]$. He introduced a non-holonomic six-dimensional space of averaged coordinates of individual particles and of their fluctuations, and presented plastic deformations as a transformation of a three-dimensional subspace of that space into a non-Riemannian one.

\subsection{Theories based on the concept of slip}

The constitutive equations of plastic hardening described above are typically phenomenological, convenient for engineering applications but with a rather weak physical background. Physically, plastic deformations in metals may be presented as resulting from slips in single crystals. Hence, side by side with the purely phenomenological theories, the theories based on the concept of slip were also developed.

The first proposals of the description of slips in single crystals are due to G. I. Taylor and C. F. Elam [3.790, 3.792], and to E. Schmid [3.702]. Schmid established the law of critical shear stress, stating that slip takes place when the resolved shear stress reaches a critical value and that this value is unaffected by the magnitude of the normal stress on the slip plane.

S. B. Batdorf and B. Budiansky $[3.40,3.41]$ extended the slip concept to real metals treated as polycrystalline aggregates. The aggregate is considered as composed of randomly oriented crystals and after an averaging process the assumptions of homogeneity and isotropy in macroscale are justified. Further, it is assumed that each crystal in the aggregate has only one slip system and there is no interaction of individual crystals.

Consider an elementary cross-sectional area $d \Omega$ in a plane with the normal $\xi$. Slip in the direction $\eta$ in this plane takes place if the shearing stress $\tau_{\xi \eta}$ exceeds 


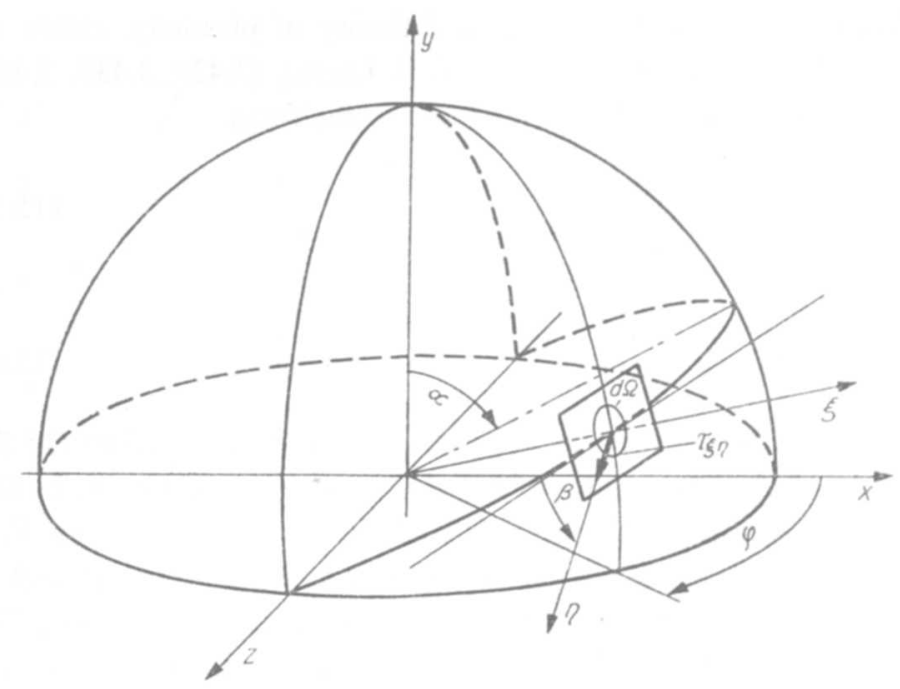

Fig. 81. The Batdorf-Budiansky theory of slip

the corresponding yield-point stress $\tau_{0}$, Fig. 81. The resulting plastic shearing strain $\gamma_{\xi \eta}^{p}$ is assumed to be a function of $\tau_{\xi \eta}$ only; namely, $\gamma_{\xi \eta}^{p}=F\left(\tau_{\xi \eta}\right)$, where the function $F$ determines plastic hardening and should be evaluated experimentally, from the simple tension test. This part of the theory is of phenomenological character. Batdorf and Budiansky propose to assume the function $F$ in the form of the following series or of the polynomial

$$
\gamma_{\xi \eta}^{p}=F\left(\tau_{\xi \eta}\right)=\left\{\begin{array}{lll}
0 & \text { if } & \tau_{\xi \eta} \leqslant \tau_{0}, \\
\sum_{n} b_{n}\left(\frac{\tau_{\xi \eta}}{\tau_{0}}-1\right)^{n} & \text { if } & \tau_{\xi \eta} \geqslant \tau_{0} .
\end{array}\right.
$$

Any strain component $\varepsilon_{i j}^{p}$ in the Cartesian system $x y z$ corresponding to the deformation of a single crystal may be calculated from the transformation formulae in which only $\gamma_{\xi \eta}^{p}$ is retained:

$$
\varepsilon_{i j}=\frac{1}{2}\left(a_{i \xi} a_{j \eta}+a_{i \eta} a_{j \xi}\right) \gamma_{\xi \eta} .
$$

This formula is of course understood without summation over $\xi$ and $\eta$. Now we have to take into account various crystal orientations and various slip planes. To this end, (15.62) is integrated over all directions $\beta$ in the slip plane and over all planes passing through the point under consideration (over the unit hemisphere $\Omega$ shown in Fig. 81):

$$
\varepsilon_{i j}^{p}=\frac{1}{2} \int_{\Omega} d \Omega \int_{-\pi / 2}^{\pi / 2}\left(a_{i \xi} a_{j \eta}+a_{i \eta} a_{j \xi}\right) F\left(\tau_{\xi \eta}\right) d \beta .
$$

The integration over $\Omega$ may be performed in spherical coordinates $\alpha, \varphi$; the relevant formulae for the cosines $a_{i \xi}, a_{i \eta}, a_{j \xi}, a_{j \eta}$ are given in [3.40]; namely, 


$$
\begin{array}{ll}
a_{x \xi}=\sin \alpha \cos \varphi, & a_{x \eta}=\cos \alpha \sin \beta-\sin \alpha \cos \beta \sin \varphi, \\
a_{y \xi}=\cos \alpha \cos \varphi, & a_{y \eta}=-\sin \alpha \sin \beta-\cos \alpha \cos \beta \sin \varphi, \\
a_{z \xi}=\sin \varphi, & a_{z \eta}=\cos \beta \sin \varphi .
\end{array}
$$

A further development of the above theory is due to P. Cicala [3.104, 3.105], B. Budiansky, Z. Hashin and J. L. Sanders [3.81]. J. F. W. Bishop and R. Hill [3.63, 3.64] proved the normality of the plastic strain increment vector to the neutral surface for a rigid-plastic polycrystalline aggregate; the proof was based on the principle of maximal plastic work. W. T. Koiter [3.363] noticed that the slip theory may be regarded as an incremental theory with infinitely many intersecting segments of neutral surfaces.

Although certain experiments indicated the superiority of the slip theory, e.g. over the hypothesis of isotropic hardening (F. D. Stockton [3.770], J. Marin and L. W. Hu [3.506]), nevertheless essential deviations from experimental results were observed as well, and some theoretical deficiencies were also pointed out. Y. Yoshimura [3.854] remarked that $F\left(\tau_{\xi \eta}\right)$ in (15.63) cannot be regarded as a material function, but, in general, depends on the type of the stress state. According to I. Knets [3.361] these critical remarks were based merely on approximation errors of empirical curves, but I. D. Rogozin [3.679, 3.680] supported Yoshimura's attitude, and stated that the function $F$ depends essentially on the Lode parameter $\mu_{\sigma}$. Hence many alterations of the slip theories were proposed.

T. H. Lin [3.451, 3.452, 3.453] proposed a slip theory taking into account the possibility of three slip directions instead of one. He assumes that the resolved shearing stress depends on the sum of the three slips on the crystal plane, $\tau=f\left(\gamma_{1}+\right.$ $+\gamma_{2}+\gamma_{3}$ ). A further development of this concept is due to H. Payne [3.621]. T. H. Lin and M. Ito [3.454, 3.455] proposed a method of calculating subsequent neutral surfaces and deriving constitutive equations for an aggregate from the single-crystal characteristics, but the final formulae are lengthy and not convenient for engineering applications. These investigations were summarized in the book [3.465] and in the survey paper [3.457], whereas the paper by T. H. Lin, Y. M. Ito and C. L. Yu [3.459] presents a new, more accurate variant of the slip theory of plasticity.

Another variant of the slip theory, proposed by A. K. Malmeyster [3.484, $3.485,3.486,3.487,3.489$ ], was called the theory of locality of deformations. Shearing strains along any local plane of slip or twinning were regarded as mechanisms of plastic deformation. Making use of N. N. Afanasyev's averaging process, [3.1], Malmeyster derived the formula

$$
\varepsilon_{i j}^{p}=\frac{1}{8 \pi} \int_{\Omega} F\left(\tau_{\xi \eta}\right)\left(a_{i \xi} a_{j \eta}+a_{i \eta} a_{j \xi}\right) d \Omega
$$

where $\gamma_{z x}^{p}=F\left(\tau_{z x}\right)$ denotes shearing strain in the local plane, and $\Omega$ the surface of the unit sphere. Comparing this with the Batdorf-Budiansky proposal (15.63), we find no integration over $\beta$, which is unjustified in Malmeyster's opinion. A further 
development of the theory of locality of deformations is due to A. F. Zilauts [3.876] (derivation based on tensor analysis), A. F. Kregers [3.391, 3.394] (application to tension with torsion, plastic compressibility), G. A. Teters [3.797, 3.798] (a new, generalized variant and its application to the shell theory), and V. Ya. Sausin [3.695, 3.696] (theory of locality analysed in the Ilyushin space). A detailed discussion of many variants of the theory of locality of plastic deformations may be found in the surveys by I. V. Knets [3.361, 3.362].

M. Ya. Leonov and N. Yu. Shvayko [3.440, 3.441] proposed another theory based on the concept of slips, called the "theory of anisotropically hardening planeplastic medium". The "plane-plastic medium" is understood as a coincidence of plane stress with plane strain, which is typical for pure shear states. This theory, which may be regarded as a generalization of the Batdorf-Budiansky concept, was extensively developed, mainly in Frunze. Let us mention the papers by M. Ya. Leonov [3.442, 3.443, 3.444, 3.445, 3.448], the same author with E. I. Blinov [3.446], with V. Ya. Molotnikov and B. A. Rytchkov [3.447, 3.449], with B. Bayterekov [3.42]; K. N. Rusinko [3.685, 3.686]; Rusinko with E. I. Blinov [3.687, 3.68], with V. I. Kuneyev [3.688, 3.403], with A. E. Kalatinets and S, S. Dreval [3.689]; N. Yu. Shvayko [3.736], Shvayko with Yu. V. Klyshevitch [3.734], with Ya. I. Rudayev [3.735], with L. B. Zagovaylov [3.856]; Ya. I. Rudayev [3.683], N. N. Kudryashov and B. A. Rytchkov [3.401], E. I. Blinov [3.69, 3.70], A. E. Kalatinets [2.81], and V. I. Kuneyev [3.404]. Particular attention was paid to trajectories with corners, to the description of the Bauschinger effect and to the deviations from the Ilyushin postulate of isotropy.

M. Como and S. D'Agostino [3.108] introduced the Bauschinger effect into the Batdorf-Budiansky theory and presented the stress-strain relations in incremental form. A paper [3.110] by M. Como and A. Grimaldi introduces the "plane body plastic model", to a certain degree similar to that of Leonov and Shvayko. These concepts were developed in [3.109, 3.111, 3.113].

E. Kröner [3.397] proposed a model of a polycrystalline metal which took grain interactions into account and was limited to small plastic strains. B. Budiansky and T. T. Wu [3.82] and J. Hutchinson [3.280] used this model for calculating the overall stress-strain behaviour of the polycrystal. A similar model proposed by R. Hill [3.251, 3.252] used another method for calculating the stresses and strains in individual grains; a further development of this concept is due to J. Hutchinson [3.281], and to K. S. Havner [3.230, 3.231]. A statistical approach to the description of grain interactions was suggested by A. G. Kostyuk [3.379]. Y. Hara [3.225] combined the Batdorf-Budiansky concept with the HMH yield condition. J. Cerny [3.120] suggested a model taking account of normal stresses and strains in the slip plane as well.

As we mentioned in Sec. 14.7, the slip theories predict the formation of a corner at the control point in the stress space. This problem was discussed in detail by V. D. Klyushnikov [3.357, 3.358, 3.359], who compared the results of various theories and models. 


\subsection{Plastic deformations at variable temperature}

In Sec. 1.6 we classified temperature as an accessory exertion factor; indeed, in many cases it may be regarded as a certain fixed parameter. However, if in the course of a process essential changes of temperature take place, then we have to regard temperature as a new independent variable, i.e. to increase the number of dimensions of the space of exertion factors. The influence of temperature changes may be significant: for example, a proportional decrease of the stress components may cause an active process, if it is combined with a simultaneous sufficient increase of temperature.

Though several simple thermoplastic problems were solved as early as the forties, the first general description of anisothermal plastic deformations-with reference to the incremental theory-is due to W. Prager [3.657], 1958. He assumed the equation of subsequent neutral surfaces in the form

$$
F\left(\sigma_{l j}, \varepsilon_{i j}^{p}, W^{p}, T\right)=0 .
$$

This equation may be regarded as a certain generalization of (15.11) to the case of variable temperature with the simultaneous replacement of the Odqvist parameter by plastic work $W^{p}$. A neutral process, separating active and passive processes, is here defined as follows: plastic work $W^{p}$ and plastic strains $\varepsilon_{i j}^{p}$ do not change, but the equation $F=0$ should hold as before; hence

$$
\frac{\partial F}{\partial \sigma_{i j}} d \sigma_{i j}+\frac{\partial F}{\partial T} d T=0
$$

In the case of plastic hardening a positive value of the left-hand side of (15.67) determines an active process, whereas a negative value determines a passive process. Thus the generalization of the incremental law (15.15) satisfying the requirement of a continuous description of neutral processes may be written in the form

$$
d \varepsilon_{i j}^{p}=h \frac{\partial g}{\partial \sigma_{i j}}\left(\frac{\partial F}{\partial \sigma_{k l}} d \sigma_{k l}+\frac{\partial F}{\partial T} d T\right)
$$

(Prager applied the associated incremental law, assuming $g \equiv F$ ).

Consider a plastically active process. Following the way described in Sec. 15.3, we write

$$
\frac{\partial F}{\partial \sigma_{k l}} d \sigma_{k l}+\frac{\partial F}{\partial T} d T+\frac{\partial F}{\partial \varepsilon_{i j}^{p}} d \varepsilon_{i j}^{p}+\frac{\partial F}{\partial W^{p}} \sigma_{i j} d \varepsilon_{i j}^{p}=0 .
$$

substituting (15.68) and cancelling by the expression in the bracket, we obtain

$$
1+\left(\frac{\partial F}{\partial \varepsilon_{i j}^{p}}+\frac{\partial F}{\partial W^{p}} \dot{\sigma}_{l j}\right) h \frac{\partial g}{\partial \sigma_{i j}}=0
$$


Calculating the function $h$ and substituting it into (15.66), we may write the final equations of the incremental theory in the form

$$
d \varepsilon_{i j}^{p}=-\frac{\frac{\partial g}{\partial \sigma_{t j}}\left(\frac{\partial F}{\partial \sigma_{k l}} d \sigma_{k l}+\frac{\partial F}{\partial T} d T\right)}{\frac{\partial g}{\partial \sigma_{m n}}\left(\frac{\partial F}{\partial \varepsilon_{m n}^{p}}+\frac{\partial F}{\partial W^{p}} \sigma_{m n}\right)} .
$$

This notation, general (but without a consistent use of internal state variables), comprises most hypotheses of plastic hardening described in Sec. 14. Various particular cases were investigated by B. A. Boley and J. H. Weiner [3.71], P. M. Naghdi [2.119], I. Z. Palley and E. A. Konyayev [3.613, 3.371, 3.614] (generalization of the Kadashevitch-Novozhilov theory of residual microstresses), A. A. Vakulenko and I. Z. Palley [3.827] (effect of strain rate on the deformation process), I. A. Birger and I. V. Demyanushko [3.60, 3.62], Yu. G. Korotkikh and S. M. Belevitch [3.373, 3.374] (dynamical problems), A. G. Ugodchikov and Yu. G. Korotkikh [3.823]. T. Y. Chang [3.94] and T. Tokuoka [3.806] presented constitutive equations in a form solved with respect to stress rate components. A general theory of thermoplasticity based on extensive experimental investigations was proposed by by $\mathrm{A}$. Phillips and R. Kasper [3.638].

The total (deformation) theory of plasticity was also generalized to the case of anisothermal processes, even earlier than the incremental theory. The key problem is here the definition of a simple process, and, consequently, the ranges of applicability of the theory. This problem was first discussed in 1950 by P. M. Ogibalov [3.588] (material constants not depending on temperature), then by A. G. Zhuravlev [3.869], I. A. Birger [3.61], V. N. Churakov [3.103], V. V. Moskvitin [3.549], Yu. N. Shevtchenko [3.713, 3.714], V. S. Lensky and V. A. Lomakin [3.437] (constants depending on temperature). Yu. N. Shevtchenko proved in [3.711] two theorems concerning passive processes and reverse active processes, and in [3.712] discussed changes of elastic moduli resulting from thermoplastic deformations. A theory of passive processes with elastic moduli depending on temperature was developed by V. I. Rozenblyum [3.682]. Yu. N. Shevtchenko with R. G. Terekhov [3.718, 3.796] postulated the existence of a thermomechanical surface $\varepsilon=f(\sigma, T)$, any anisothermal deformation process being presented by a line on that surface, and verified this postulate experimentally. I. A. Birger and I. V. Demyanuskho [3.62] derived the equations of a deformation theory of thermoplasticity in incremental form.

Theories of thermoplasticity using internal state variables were initiated in the papers by A. A. Vakulenko [3.824, 3.825, 3.826]. Cyclic thermoplastic processes were described in this way by Z. Mróz [3.559]. The basic equations of coupled thermoplasticity (taking account of the thermal effects of plastic deformations) were derived and applied by B. Raniecki and A. Sawczuk [3.665]; they used one scalar internal state variable. The papers dealing with finite thermoplastic strains will be listed in Sec. 15.9. 
D. C. Drucker in paper [3.152] extended his stability postulate to anisothermal processes; however, the class of stable materials is very narrow in this case, since the stability postulate requires, as a rule, that the yield-point stress should increase with temperature, which contradicts most experimental results.

Finally let us mention several related papers. V. S. Zarubin and A. A. Polyakov $[3.858,3.859,3.860,3.861]$ developed a theory of thermoplasticity based on the concept of slip. V. P. Demutsky and R. V. Polovin [3.131] derived the fundamental equations of magnetoplasticity based on thermodynamic considerations. The problems of quenching and phase transitions were considered by T. Mura [3.563], V. A. Lomakin [3.464], P. Perzyna and A. Sawczuk [3.627], T. Inoue and K. Tanaka [3.295]. The basic equations of plasticity of irradiated materials were derived by V. S. Lensky [3.432] (total theory), T. Y. Thomas [3.803], Yu. N. Shevtchenko [3.715] (incremental theory) and P. Perzyna [3.626] (internal state variables theory). W. L. Greenstreet and A. Phillips [2.44] described thermoplastic processes in artificial graphite.

\subsection{Theories of plastic softening}

Certain peculiarities appear in the description of plastic softening, observed, for example, between the upper and the lower yield points in the case of steel specimens. For other materials this phenomenon may be even more important; it corresponds to material instability, since the Drucker definition of stability does not hold at the stage of a falling stress-strain curve. Investigations of plastic softening are also essential for the construction of a more general uniform theory valid for the levels $\mathscr{S}$ and $\mathscr{B}$ as well, where material instability may be complemented or replaced by structural instability.

The first investigations are due here to G. Maier and D. C. Drucker [3.471], 1966. Further papers were written by the same authors with A. C. Palmer [3.615], and by G. Maier [3.472, 3.473, 3.474, 3.475]. The authors found that neutral surfaces for plastically softening bodies may be concave and examined the magnitude of that concavity. The case of cyclic work-softening was discussed by W. R. Powell [3.648].

Stress components treated as exertion factors given in the case of plastic softening no unique description of the process, since, for example, decreasing stresses may correspond either to a passive or to an active process. On the other hand, uniqueness is obtained if we prescribe the process in strains (total strains). Such an approach will be presented here after V. A. Ibragimov [3.284] with slight modifications.

Our considerations will be restricted to deviators, and hence we rewrite the equation of a subsequent neutral surface (15.11) in the form

$$
F\left(s_{i j}, e_{i j}^{p}, I_{\varepsilon p}\right)=0 .
$$


Eliminating stress components by using Hooke's law for an isotropic body, we may write

$$
F\left[2 G\left(e_{i j}-e_{i j}^{p}\right), e_{i j}^{p}, I_{\varepsilon p}\right]=0 .
$$

It can be seen that at constant plastic strains $\partial F / \partial e_{i j}=2 G \partial F / \partial s_{i j}$, and hence (15.14) takes the form

$$
d e_{i j}^{p}=\frac{h}{2 G} \frac{\partial g}{\partial e_{i j}} \frac{\partial F}{\partial e_{k l}}\left(d e_{k l}-d e_{k l}^{p}\right)
$$

where $g$ denotes the plastic potential (Ibragimov assumed $g \equiv F$ ). The solution of (15.74) with respect to $d e_{i j}^{p_{j}}$ presents some difficulties, and we may postulate another, but related law

$$
d e_{j}^{p}=\bar{h} \frac{\partial \bar{g}}{\partial e_{i j}} \frac{\partial \bar{F}}{\partial e_{k l}} d e_{k l},
$$

which also satisfies the requirement of a continuous description of a neutral process. Hence

$$
d s_{i j}=2 G d e_{i j}^{e}=2 G d e_{i j}-2 G \bar{h} \frac{\partial \bar{g}}{\partial e_{i j}} \frac{\partial \bar{F}}{\partial e_{k l}} d e_{k l} .
$$

This constitutive equation describes plastic softening if the values of the function $\bar{h}$ are sufficiently large. Namely, multiplying both sides of (15.76) by $\partial \bar{F} / \partial e_{i j}$, we obtain

$$
\frac{\partial \bar{F}}{\partial e_{i j}} d s_{i j}=2 G \frac{\partial \bar{F}}{\partial e_{i j}} d e_{i j}\left(1-\bar{h} \frac{\partial \bar{F}}{\partial e_{k l}} \frac{\partial \bar{g}}{\partial e_{k l}}\right),
$$

and for a sufficiently large $\bar{h}$ we obtain the inequalities $\left(\partial \bar{F} / \partial e_{i j}\right) d s_{i j}<0$ at $\left(\partial \bar{F} / \partial e_{i j}\right) d e_{i j}>0$, typical for plastic softening.

Q. S. Nguyen and H. D. Bui [3.577] proposed another method of description of plastic softening, using the space $\sigma \times \boldsymbol{\alpha}$, where $\boldsymbol{\alpha}_{i}$ are internal state parameters. V. A. Ibragimov and V. D. Klyushnikov [3.282] investigated types of differential equations appearing in the theory of plastically softening bodies.

\subsection{Theories of finite plastic strains}

Finite-strain theories are, in principle, beyond the scope of this book, nevertheless we quote here several basic references. The following differences, mainly complicating, arise here in comparison to small-strain theories: (1) influence of geometry changes of the element, resulting in differences between true stresses and nominal stresses; (2) classical measure of strain is inadequate and often should be replaced by logarithmic strain (cf. Sec. 7.7); (3) Eulerian and Lagrangian coordinates are clearly distinguished; (4) decomposition of finite strains into their elastic and plastic parts presents difficulties (cf. Sec. 8.8); (5) finite rotations of the element if present, result in even more serious complications than finite strains.

In the case of constant principal directions, where the problem of rotations is practically eliminated, the simplest theory of finite plastic strains, proposed in 1937 by A. Nadai and E. A. Davis [2.118, 3.128, 3.129, 3.566], may be useful. They pos- 
tulated a simple extension of the law of similarity of deviators to true principal stresses $\sigma_{J}$ (related to the actual cross-sectional area) and to logarithmic plastic strain increments $d \varepsilon_{J}^{H}$; namely, for constant material coordinates $X_{i}$,

$$
d e_{J}^{H}=s_{J} d \Phi, \quad J=1,2,3 .
$$

The function $\Phi$ is given by

$$
d \Phi=\sqrt{\frac{d e_{J}^{H} d e_{J}^{H}}{s_{K} s_{K}}}=\sqrt{\frac{3}{2}} \frac{d I_{e_{p}}^{H}}{\sigma_{e}},
$$

and should be determined by one test, for example the simple tension test; $d I_{\varepsilon p}^{H}$ stands here for the increment of the Odqvist parameter for logarithmic (Hencky) strains, and $\sigma_{e}$ denotes the intensity of true stresses. If the ratios of principal stresses are kept constant during the process, then (15.78) may be integrated, and we obtain an even simpler form of the relations

$$
e_{J}^{H}=\Phi_{s_{j}}=\frac{3}{2} \frac{\varepsilon_{e}^{H}}{\sigma_{e}} s_{J},
$$

resembling the Hencky-Ilyushin equations (9.28) and (15.2). Of course, the range of application of (15.78), and particularly of (15.80) is sharply restricted, nevertheless these equations describe the two main features of finite strains: (1) influence of geometry changes, i.e. resignation from the principle of rigidification, (2) correct correlation (roughly speaking) between the results of tension and compression tests, where the logarithmic strain measure gives much more adequate results than the conventional one. Further investigations in this direction are due to G. A. SmirnovAlyayev and V. M. Rozenberg [3.742, 3.743], F. Körber and A. Eichinger [3.384], J. E. Dorn and A. J. Latter [2.27], and V. A. Lomakin [3.463].

In some problems principal directions are materially fixed, being variable in spatial coordinates (e.g. in rotationally symmetric shells); in these cases Eq. (15.78) may also lead to satisfactory results. On the other hand, the problem of applicability of integration of (15.78) to the form (15.80) is much more difficult than in the case of small strains: L. I. Sedov [2.150] showed that the Ilyushin theorem on simple loading is no longer valid here and that one cannot find a type of loading for which (15.78) can be simultaneously integrated at each point of the body (except the trivial cases of homogeneous stress). B. E. Pobedrya [3.645] introduced for finite strains the notion of "simple loading in a broader sense", which takes place if

$$
\varepsilon_{i j}(t)=\lambda(t) B_{i j}+\mu(t) C_{i j},
$$

where $\varepsilon_{t}$, stands for a characteristic tensor, for example the Langrangian strain tensor $\varepsilon_{I J}^{L},(7.8)$. This notion may be useful in considering the ranges of applicability of (15.78) and (15.80). This problem was also discussed and experimentally verified by R. K. Mittal [3.535], Yu. N. Shevtchenko, V. G. Savtchenko, R. G. Terekhov and A. I. Borisyuk [3.717].

Theories accounting for finite strains and finite rotations were initiated in the fifties and were at first connected with general rheology (J. G. Oldroyd [3.594]) or hypo-elasticity (C. Truesdell [3.819], A. E. Green [3.211]). A related proposal 
is due to R. Hill [3.249, 3.250], and further results in this direction were obtained by T. Tokuoka [3.805, 3.807].

Another direction of development of the theory consists in straight generalizations of the existing geometrically linearized theories of plasticity; in most cases, however, elastic strains are assumed here to remain small or even are neglected. It was T. Y. Thomas [3.801, 3.802, 2.171], who first generalized the Levy-Mises and the Prandtl-Reuss equations for a perfectly plastic body. These equations are discussed and compared to those based on hypo-elasticity by A. C. Eringen [2.31], W. Olszak, Z. Mróz and P. Perzyna [3.600]. Constitutive equations describing isotropic plastic hardening were extended to finite strains by T. Tokuoka [3.804] and N. Fox [3.181], and those describing anisotropic hardening by T. Lehmann [3.419, 3.420, 3.423, 3.424], L. H. N. Lee and J. T. Horng [3.418]. Generalizations of the slip theory were given for monocrystals by J. Christoffersen [3.99], and for polycrystalline aggregates by J. R. Rice [3.674], and K. S. Havner [3.232, 3.233].

In the case of finite strains and finite rotations, particularly analysed in spatial coordinates, the notion of an increment of a tensorial quantity is not unique, and instead of increments various forms of material derivatives are employed (W. Prager [2.135], L. I. Sedov [3.705], R. Hill [3.254], A. J. M. Spencer and J. E. Ferrier [3.763], cf. also Secs. 6.4 and 7.6); hence it is more adequate to use the term rate type theories for generalizations of the relevant "incremental" ones. That term was introduced by A. E. Green and P. M. Naghdi [2.41] in order to distinguish such theories from an alternative, functional approach to constitutive equations.

Theories considering both elastic and plastic strains as finite ones (cf. Sec. 8.8) belong to the third group of papers. Though in most applications elastic strains are small, such theories have the virtue of being general and uniform and may be used in some exceptional cases, e.g. if the body is subject to explosive or impact loadings. The first proposal is due here to M. E. Backman [3.26], and a general theory was given by A. E. Green and P. M. Naghdi in the above-mentioned paper [2.41], 1965, and in [3.213]; their equations describe the initial anisotropy of the material and also anisothermal processes. An independent, more specific approach was proposed almost simultaneously by E. H. Lee and D. T. Liu [3.415, 3.416, 2.94]; the relations between these theories and some controversial points were discussed by Green and Naghdi [3.214, 3.215], K. A. Holsapple [3.266], F. Sidoroff [3.738], E. H. Lee and P. Germain [3.417]. Further proposals of rate-type constitutive equations for finite elastic and plastic strains are due to L. B. Freund [3.186], J. B. Haddow and T. M. Hrudey [3.220], H. Kitagawa and Y. Tomita [3.346], H. T. Hahn and W. Jaunzemis [3.221, 3.222], J. R. Osias and J. L. Swedlow [3.606], T. Lehmann [3.426], M. Kleiber [3.347] (perfect plasticity), P. M. Naghdi and J. A. Trapp [3.569, 3.570, 3.571]. Let us also mention here some related papers: T. Lehmann [2.96], Yu. G. Korotkikh [3.375], T. Lehmann and G. Zander [3.427] analysed in detail large thermoplastic strains, and $\mathrm{O}$. Bruhns $[2.11,2.12]$ quasi-cyclic processes.

The functional theories of finite plastic strains explicitly introduce the history of the process into the constitutive equations. This direction was initiated by A. C. 
Pipkin and R. S. Rivlin [2.129], who treated elastic-plastic materials as a particular case of rate-independent materials with memory. P. Perzyna [3.624] and K. A. Holsapple [3.265] considered elastic-plastic materials as a particular case of simple materials, defined in general rheology. Further related concepts are due to D. R. Owen [3.609, 3.611], K. C. Valanis [3.831, 3.832], and J. Lubliner [3.468, 3.469].

An alternative method of taking the history of the process into account is based on the consistent introduction of internal state variables. Such an approach for finite strains, mentioned by L. I. Sedov and M. E. Eglit [3.706, 2.151], was developed by P. Perzyna and W. Wojno [3.625], who extended the theory of B. D. Coleman and M. E. Gurtin [3.107] to plasticity and considered elastic-plastic material as a particular case of a visco-plastic one. As internal variables they assumed the components of a certain tensor, called the "inelastic deformation tensor" and determined by the solution of the initial-value problem for an ordinary first-order differential equation. Further proposals are due to J. Kratochvil and O. W. Dillon [3.385, 3.135, 3.387], C. Teodosiu and E. Kröner [3.794, 3.400], J. R. Rice [3.674] (internal variables identified with local shearing strains or with dislocation glides), R. Hill and J. R. Rice [3.257], Y. S. Wang [3.838], J. Mandel [3.495, 3.496], F. Fardshisheh and E. T. Onat [3.173], F. Sidoroff [3.739]. The above-mentioned paper by Valanis [3.831] also made use of internal state variables. The conditions of equivalence of descriptions of materials by means of functionals and with the aid of internal state variables were investigated by W. Kosiński and W. Wojno [3.378].

Let us also mention the approach of M. I. Estrin [3.172], who applied operators of the Lie algebra to the derivation of constitutive equations of plasticity.

A difficult problem is the fitting of a general constitutive equation to experimental data available for the material under consideration. A certain "inverse" method was suggested here by J. Kratochvil and N. Zárubová [3.388] in connection with the finite-strain, internal-variable constitutive equations of thermoplasticity proposed by J. Kratochvil [3.386].

There exist states of finite deformation which can be realized for any constitutive equations describing a certain class of materials. J. L. Ericksen [3.170, 3.171] proved that for an elastic material those states must be homogeneous, but $\mathrm{A}$. $\mathrm{Pa}$ glietti [3.612] noticed that in thermoplasticity the class of such finite ("universal") deformations is much broader.

\section{Constitutive equations of the theory of plastically anisotropic materials}

\subsection{Flow rules for perfectly plastic anisotropic bodies}

The theory of perfect plasticity of anisotropic bodies was initiated in $1928^{\circ}$ by R. Mises [3.531]. Assuming the hypothesis of the plastic potential identified with the left-hand side of the yield condition (12.1), namely

$$
g\left(\sigma_{i j}\right)=\frac{1}{2} \Pi_{i j k l} \sigma_{l j} \sigma_{k l}
$$


(where the coefficient $\frac{1}{2}$ was introduced for the convenience of notation: it has no influence on the final form of constitutive equations), he proposed the flow rule

$$
\dot{\varepsilon}_{i j}^{p}=\lambda \frac{\partial g}{\partial \sigma_{i j}} .
$$

The number of independent moduli $\Pi_{i j k l}$ in (16.1) equals 21 , as we mentioned in Sec. 12.1, and the additional assumption of the independence of yielding on the mean stress $\sigma_{m}$ reduces this number to 15. In the latter case Eqs. (16.2) in expanded engineering notation take the form

$$
\begin{aligned}
& \dot{\varepsilon}_{x}^{p}=\lambda\left[K_{12}\left(\sigma_{x}-\sigma_{y}\right)+K_{31}\left(\sigma_{x}-\sigma_{z}\right)-\left(K_{24}+K_{34}\right) \tau_{y z}+K_{15} \tau_{z x}+K_{16} \tau_{x y}\right], \\
& \cdots \cdots \cdots \cdots \cdots \cdots \cdots \cdots \cdots \cdots \cdots \cdots \cdots \cdots \cdots \cdots \cdots \cdots \cdots \cdots \cdots \cdots \cdots \cdots \cdots \cdots \cdots \cdots \cdots \cdots \\
& \frac{\dot{\gamma}_{y z}^{p}}{2}=\lambda\left[-K_{24}\left(\sigma_{x}-\sigma_{y}\right)-K_{34}\left(\sigma_{x}-\sigma_{z}\right)+K_{44} \tau_{y z}+K_{45} \tau_{z x}+K_{64} \tau_{x y}\right],
\end{aligned}
$$

We used here a notation close to that applied by W. Olszak and W. Urbanowski [3.595, 3.596], who generalized (16.1) to the case of non-homogeneity and curvilinear anisotropy; paper [3.596] gives the relations between the moduli $K_{l j}$ and $\Pi_{i j k l}$ (denoted by $H_{i j k l}$ ).

The case of orthotropy was investigated in detai! by E. V. Makhover [3.480] and R. Hill [3.244]. We have then $K_{24}=K_{34}=K_{35}=K_{15}=K_{16}=K_{26}=K_{45}$ $=K_{56}=K_{64}=0$ and in Eqs. (16.3) only six independent moduli remain. An experimental verification of (16.3) in this case was carried out by L. A. Tolokonnikov, V. V. Shevelev and S. P. Yakovlev [3.808]; they obtained fairly good agreement for five materials investigated.

A certain distinct approach to the case of general anisotropy was suggested by Z. Sobotka [3.747, 3.748, 3.753]. He introduced the tensor of transformed stresses

$$
\omega_{k l}=A_{i j k l} \sigma_{i j}
$$

with the principal directions different, in general, from the principal directions of $\sigma_{i j}$, and proposed the law of similarity of deviators of strain rates and transformed stresses:

$$
\dot{\varepsilon}_{i j}-\delta_{i j} \dot{\varepsilon}_{m}=\lambda\left(\omega_{i j}-\delta_{i j} \omega_{m}\right) \text {. }
$$

In [3.759] this concept was generalized to anisosensitive materials.

The above-mentioned constitutive equations are connected with the generalization of the HMH yield condition to anisotropic bodies. However, the concept of associated flow rules (16.2) may formally be extended also to other yield conditions for anisotropic bodies. I. I. Goldenblat and V. A. Kopnov [3.204, 3.205] applied it to (12.14) and derived the equations

$$
\dot{\varepsilon}_{i j}^{p}=\lambda\left[\Pi_{i j}+\frac{\Pi_{i j k l} \sigma_{k l}}{2 \sqrt{\Pi_{p q r s} \sigma_{p q} \sigma_{r s}}}\right] .
$$

The case of orthotropy was discussed in detail in [3.204, 3.205] and in the papers by T. C. Hsu [3.269], Yu. M. Aryshensky, I. I. Kaluzhsky and V. V. Uvarov [3.15]. 
The flow rules associated with the generalized Tresca-Guest yield condition, e.g. (12.10), were derived by L. W. Hu [3.274] and A. Sawczuk [3.697]. L. A. Tolokonnikov, S. P. Yakovlev and V. M. Lyalin [3.809, 3.810] derived the flow rule associated with their yield condition (12.12), taking into account the invariant $\omega_{\sigma}$. Experimental data concerning flow rules in the case of yield conditions more general than (12.2) are very scarce and practically the associated flow rules are not sufficiently confirmed, and effective non-associated flow rules are not suggested, either.

In cases of structural anisotropy (e.g. reinforcement), associated flow rules are also the most frequently used (A. J. M. Spencer [3.762]).

\subsection{Hypothesis of a uniform curve of plastic hardening in the case of anisotropy}

In Sec. 14.1 we discussed the difficulties connected with the introduction of the concept of a uniform curve of plastic hardening for isotropic bodies. In the case of anisotropy further complications occur-in the postulated relation of type (14.1) the stress and strain invariants must be replaced by certain common invariants of any of those tensors and of the tensor of moduli of anisotropy. Nevertheless, the hypotheses of existence of a certain uniform curve of plastic hardening are proposed as the simplest method of description of hardening of anisotropic materials.

A certain general approach is due to V. O. Geogdzhayev [3.197]. For the case of general anisotropy he introduced generalized stress intensity and generalized strain intensity by the formulae

$$
\begin{gathered}
\sigma_{\mathrm{red}}^{2}=\left(K^{*} b_{i j k l}-\alpha_{i j} \alpha_{k l}\right) \sigma_{i j} \sigma_{k l}, \\
\varepsilon_{\mathrm{red}}^{2}=\left(\frac{E_{i j k l}}{K^{*}}-\beta_{i j} \beta_{k l}\right) \varepsilon_{i j} \varepsilon_{k l}
\end{gathered}
$$

and postulated the existence of a uniform relation $\sigma_{\text {red }}=f\left(\varepsilon_{\text {red }}\right)$, at least for simple loadings. In those formulas $E_{i j k l}$ stands for the tensor of elastic moduli as in (9.1), $b_{i j k l}$ for the tensor of the moduli in the inverse relation, $\alpha_{i j}$ and $\beta_{i j}$ for the dimensionless tensors of plastic anisotropy which are to be determined experimentally, and finally $K^{*}$ for the generalized bulk modulus:

$$
K^{*}=E_{l j k l} \alpha_{i j} \alpha_{k l} \text {. }
$$

In the simpler case of an orthotropic body the concept of a uniform curve of plastic hardening was developed in the years 1948-1950 by L. J. Klinger and G. Sachs [3.353], L. R. Jackson, K. F. Smith, W. T. Lankford [3.314], J. E. Dorn [3.136] and R. Hill [2.56]. Generalizing his yields condition (12.3), Hill derived at first the following formula for the reduced stress (generalized stress intensity):

$$
\begin{aligned}
\sigma_{\mathrm{red}}^{2} & =\frac{3}{2\left(F_{0}+G_{0}+H_{0}\right)}\left[F_{0}\left(\sigma_{y}-\sigma_{z}\right)^{2}+G_{0}\left(\sigma_{z}-\sigma_{x}\right)^{2}+\right. \\
& \left.+H_{0}\left(\sigma_{x}-\sigma_{y}\right)^{2}+2 L_{0} \tau_{y z}^{2}+2 M_{0} \tau_{z x}^{2}+2 N_{0} \tau_{x y}^{2}\right]
\end{aligned}
$$


where $f_{0}, G_{0}, \ldots$ denote the moduli (12.3) in the initial yield condition. Further, the generalized plastic strain intensity $\varepsilon_{\mathrm{red}}^{p}$ was defined in such a way as to obtain for plastic work

$$
d W^{p}=\sigma_{\mathrm{red}} d \varepsilon_{\mathrm{red}}^{p} .
$$

Finally

$$
\begin{aligned}
d \varepsilon_{\mathrm{red}}^{p} & =\sqrt{\frac{2}{3}\left(F_{0}+G_{0}+H_{0}\right)}\left\{\frac { 1 } { ( F _ { 0 } G _ { 0 } + G _ { 0 } H _ { 0 } + H _ { 0 } F _ { 0 } ) ^ { 2 } } \left[F_{0}\left(G_{0} d \varepsilon_{y}^{p}-H_{0} d \varepsilon_{z}^{p}\right)^{2}+\right.\right. \\
& \left.+G_{0}\left(H_{0} d \varepsilon_{z}^{p}-F_{0} d \varepsilon_{x}^{p}\right)^{2}+H_{0}\left(F_{0} d \varepsilon_{x}^{p}-G_{0} d \varepsilon_{y}^{p}\right)^{2}\right]+ \\
& \left.+2\left[\frac{\left(d \gamma_{y z}^{p}\right)^{2}}{L_{0}}+\frac{\left(d \gamma_{z x}^{p}\right)^{2}}{M_{0}}+\frac{\left(d \gamma_{x y}^{p}\right)^{2}}{N_{0}}\right]\right\}^{1 / 2},
\end{aligned}
$$

and the hypothesis of a uniform curve may be written in the form $\sigma_{\text {red }}=f\left(\varepsilon_{\text {red }}^{p}\right)$. Particular forms of this dependence were studied by K. A. Reckling [2.141, 3.668], whereas F. J. Fava [3.174] proposed a certain alteration of that hypothesis.

Experimental verifications of the hypothesis of a uniform curve for anisotropic bodies are very scarce. H. W. Babel, D. A. Eitman and R. W. McIver [3.21] verified the Hill hypothesis, obtaining good results for textured titanium; K. A. Reckling [3.668] obtained good results for sheets of aluminium alloy AlMg 3, whereas for steel sheets larger deviations were observed. R. M. W. Frederking and O. M. Sidebottom [3.182] described the simple-loading experiments on steel SAE 1020, copper alloy 360 and aluminium alloy 2024-T351 by means of the Hill hypothesis and by the appropriate generalization of the Ota-Shindo-Fukuoka yield condition [3.608] to the case of hardening, finding the latter more accurate, but also more complicated. A. N. Bramley and P. B. Mellor [3.75] verified the Hill hypothesis by means of a series of uniaxial tests, using specimens cut in various directions. Another description of similar tests was used by V. N. Bastun [3.35], who employed the formulae for stress and strain intensity as defined for isotropic materials and investigated the dependence of the secant modulus $E_{s}=\sigma_{e} / \varepsilon_{e}$ on the direction of deformation.

\subsection{Subsequent neutral surfaces for anisotropic bodies}

The generalization of the hypothesis of isotropic plastic hardening to anisotropic bodies presents formally no difficulties. For example, making use of the Hill expression (16.12) and integrating it along the trajectory, we may write

$$
\sigma_{\text {red }}=f\left(\int d \varepsilon_{\text {red }}^{p}\right)
$$

or, in alternative form,

$$
\sigma_{\text {red }}=f\left(\boldsymbol{W}^{p}\right),
$$

regarding these equations as describing subsequent neutral surfaces. In both cases the surfaces expand proportionally, and the term proportional hardening seems more justified here than "isotropic hardening". Equation (16.14), describing work-harden- 
ing, was proposed in the above-mentioned papers [3.314, 3.136]; R. Hill [2.56] proved that the strain-hardening concept (16.13) in the case of his quadratic yield condition is equivalent to (16.14). For real materials, however, the hypotheses (16.13) and (16.14) give various accuracies of the description of experimental data: J. A. Chakrabarty [3.93] found (16.13) more accurate than (16.14).

It should be noted that the hypothesis of proportional plastic hardening has even smaller chances for anisotropic materials than in the case of isotropy. Indeed, plastic anisotropy is very often due to preceding plastic deformations, and then it can hardly be expected that such anisotropy will not change during the process under consideration. Hence various hypotheses of non-proportional plastic hardening were also proposed.

The first hypothesis of non-proportional hardening of an anisotropic material is due to F. Edelman and D. C. Drucker [3.164] and to N. L. Svensson [3.776]; it may be regarded as a counterpart to the Baltov-Sawczuk equation (14.68). Namely, the authors postulated the following equation of subsequent neutral surfaces

$$
N_{i j k l}\left(s_{i j}-m e_{i j}^{p}\right)\left(s_{k l}-m e_{k l}^{p}\right)-1=0,
$$

$N_{i j k l}$ being anisotropic from the beginning of the process. Svensson applied this equation to cold-rolled aluminium (acquired anisotropy); further experimental evidence was presented by E. Shiratori and K. Ikegami [3.724, 3.285]. A similar hardening hypothesis was proposed by R. N. Dubey and M. J. Hillier [3.157]. I. I. Goldenblat and V. I. Kopnov [3.204, 3.205] extended the Melan-IshlinskyPrager hypothesis of kinematic hardening to their yield condition (12.14) and determined subsequent neutral surfaces by the equation

$$
\Pi_{i j}\left(\sigma_{i j}-\alpha_{i j}\right)+\sqrt{\Pi_{i j k l}\left(\sigma_{i j}-\alpha_{i j}\right)\left(\sigma_{k l}-\alpha_{k l}\right)}-1=0,
$$

where the tensor $\alpha_{i j}$ is proportional to $\varepsilon_{i j}^{p}$. The authors mention also some other variants of the hardening hypothesis. T. Lehmann [3.425] noticed that an orthotropic body loses its orthotropy in the course of a general plastic deformation process and becomes generally anisotropic; an adequate mathematical description of this phenomenon was given. Further investigations are due to V. N. Bastun [3.37], T. Kishi and T. Tanabe [2.82].

\subsection{Total (deformation) theories of anisotropic plasticity}

The first formulation of a deformation theory of anisotropic plasticity, generalizing the Hencky-Ilyushin theory for isotropic bodies, is due to P. P. Petrishtchev [3.629]; further generalizations were given by I. I. Goldenblat [3.201], V. O. Geogdzhayev [3.197] and V. A. Lomakin [3.465]. Petrishtchev assumed that the law of volume change for an isotropic body $\sigma_{m}=K \varepsilon_{m}$ remains also valid in the case of anisotropy; Geogdzhayev noticed that such an assumption is, in general, not justified, and proposed the following more general law:

$$
\alpha_{i j} \sigma_{i j}=\alpha_{i j} E_{i j k l} \varepsilon_{k l},
$$


where the symbols were explained in Sec. 16.2. Further, integrating the Mises flow rule for a simple deformation process, Geogdzhayev derived the remaining constitutive equations

$$
s_{i j}^{*}=\sigma_{i j}-\beta_{i j} \alpha_{k l} \sigma_{k l}, \quad e_{i j}^{*}=\varepsilon_{i j}-\alpha_{i j} \beta_{k l} \varepsilon_{k l} .
$$

where the "anisotropic" deviatoric components are given by

$$
s_{i j}^{*}=\frac{\sigma_{\mathrm{red}}}{K^{*} \varepsilon_{\mathrm{red}}} E_{i j k l} e_{k l}^{*},
$$

and $\sigma_{\text {red }}, \varepsilon_{\text {red }}$ and $K^{*}$ are defined by $(16.7) \div(16.9)$. Equations (16.18) give only 5 independent equations; they are complemented by (16.15) and the hypothesis of a uniform hardening curve $\sigma_{\text {red }}=f\left(\varepsilon_{\text {red }}\right)$.

In contradistinction to isotropic bodies, where the deformation theory is very simple, particularly in the case of incompressibility, the Geogdzhayev theory is much more complicated and its application involves many difficulties.

\subsection{Incremental theories of anisotropic plasticity}

Similarly to the case of perfect anisotropic plasticity, most incremental constitutive equations of plastic hardening are assumed to be associated with the equations of subsequent neutral surfaces, usually without sufficient experimental verification. The first equations of this type, connected with the hypothesis of proportional hardening, were derived for orthotropic materials by R. Hill [2.56], and analysed in detail by V. Diaconiţa [3.133] and N. N. Malinin [3.483]. A generalization to general anisotropy is due to V. A. Lomakin [3.466]. Also the hypotheses of non-proportional hardening were combined, as a rule, with associated incremental laws (I. I. Goldenblat, V. A. Kopnov [3.204, 3.205]).

The possible forms of the plastic potential for anisotropic bodies were discussed by H. Neuber [3.575]. G. Augusti, J. B. Martin and W. Prager [3.18] analysed the cases of rigid-plastic anisotropic bodies which may be described by constitutive equations written separately for deviators and separately for axiators. A certain very general non-associated incremental law was proposed by T. Lehmann [3.421]: he assumed the equation of initial neutral surface in the form (12.13), and to the associated law added a certain term depending on the loading history. The Malmeyster theory of locality of deformations was generalized to orthotropic bodies by A. F. Kregers [3.393].

A distinct approach to constitutive equations of hardening anisotropic bodies is due to I. Berman and P. G. Hodge [3.55]. They developed a general theory of linear anisotropic hardening of materials described by piece-wise linear subsequent neutral surfaces; the linearity was assumed in the space of principal stresses and principal strains and the coincidence of principal directions for stresses and strains was assumed. Generalizations of the Tresca-Guest yield condition were considered as a particular case. A related theory was proposed by M. Sugimoto, H. Igaki and K. Saito [3.774]. 
A description of finite élastic-plastic strains in anisotropic bodies was included in the general theory of A. E. Green and P. M. Naghdi [2.41], who also discussed non-isothermal processes. More extensive investigations of anisotropic thermoplasticity are due to Z. Gabryszewski [3.192], F. C. Weiler [3.842], and T. Tokuoka [3.806].

Once more it should be observed that experimental verification of the abovementioned constitutive equations is very scarce and most of them are of a purely formal character. 


\section{CHAPTER IV}

\section{Transition from the local level $\mathcal{P}$ to the integral levels $\delta$ and $\$$}

\section{.17 Limit carrying capacity}

\subsection{Concept of the limit carrying capacity $\left({ }^{1}\right)$}

The traditional estimation of the load-carrying capacity of a structure under static loading was based on the "local" condition

$$
\sup _{x, y, z} \sigma_{\text {red }}=\bar{\sigma},
$$

where $\sigma_{\text {red }}$ denotes a certain reduced stress according to the assumed failure hypothesis, calculated as for a perfectly elastic body, and $\bar{\sigma}$ a certain material constant.

Such an approach may be justified as a first approximation for elastic-brittle bodies, but in most cases it gives no adequate estimation for ductile ones, capable of significant plastic deformations. Replacing $\bar{\sigma}$ by $\sigma_{0}$, we may determine by (17.1) merely the elastic carrying capacity of a structure or of a cross-section (cf. Sec. 3.2).

More realistic approaches must take plastic deformation into account. The simplest estimation of the load-carrying capacity is furnished by the concept of the limit carrying capacity. Though the related ideas date back to the 18th century (cf. A. A. Gvozdev [4.196], W. Prager [4.472]), it was G. Kazinczy in Hungary [4.275] who first calculated in 1914 the limit carrying capacity of a clamped beam and verified his theory experimentally; in 1917 N. C. Kist introduced this concept independently in his inaugural lecture at the Technical University of Delft [4.281, 4.282]. Limit analysis of plates was initiated several years later by A. Ingerslev [4.244].

The limit carrying capacity may be defined in two ways. As the first approach we consider the definition used by R. Hill [4.211, 4.212]: the limit load or limit carrying capacity is identified with the yield-point load for a rigid-plastic-hardening

(1) Abbreviation of the term "limit load-carrying capacity". 
structure, i.e. a load (system of loadings) which causes an infinitesimal deformation of that structure (or cross-section). In Hill's definition plastic hardening is inessential, the limit carrying capacity is determined by the stress initiating plastic deformation, which may be denoted by $\sigma_{0}$. Since up to the plastic deformation the structure remains rigid, there are no geometry changes at the limit state and the principle of rigidification is automatically satisfied.

The second, even older approach pertains to a quite different, namely perfectly elastic-plastic schematization of the material. The limit carrying capacity is defined as a system of loadings which results in uncontained plastic flow, i.e. in an increase of deformations at constant values of those loadings, under the additional assumption of the principle of rigidification. This definition is used by W. Prager [4.466, 4.467] and his collaborators, side by side with a "compromising" definition based on the schematization of a rigid-perfectly-plastic body; the latter may be regarded as the most "classical" one, but it is often ineffective if used without appropriate limiting procedures, $\mathrm{cf}$. Sec. 18.

Denote by $Q_{i}, i=1,2, \ldots, n$, the generalized forces: the generalized external forces for the body as a whole, $\mathscr{B}$, or generalized internal forces (generalized stresses) for the cross-section, $\mathscr{S}$. Further, denote by $\dot{q}_{i}$ the corresponding generalized plastic strain rates, defined in such a way that the rate of dissipated work, $D=\dot{W}^{p}$, equals

$$
D=\dot{W}^{p}=Q_{i} \dot{q}_{i} .
$$

Sometimes this definition is used with a certain coefficient on the right, for example to have the quantities $Q_{i}$ and $\dot{q}_{i}$ non-dimensionalized (P. G. Hodge [4.221]). For external forces Prager [4.468] uses distinct notations, $P_{x}$ and $\dot{p}_{x}$, but it is our aim to unify the approach.

In both approaches the limit carrying capacity may be described by the equation

$$
f\left(Q_{1}, Q_{2}, \ldots, Q_{n}\right)=0 .
$$

In Prager's approach we have moreover

$$
d Q_{i}=0, \quad i=1,2, \ldots, n,
$$

for at least one combination $\dot{q}_{i} \dot{q}_{i}>0$, which determines the relevant "mechanism of plastic collapse"; in Hill's approach Eqs. (17.4) are, in general, not satisfied, unless a rigid-hardening material is replaced by a perfectly rigid-plastic one. The surface (17.3) is one of the most important limit surfaces at the levels $\mathscr{S}$ and $\mathscr{B}$ and was called in Sec. 3.2 the plastic interaction surface or the yield surface.

An essential problem is connected with the equivalence of the definitions given above. Hill's definition is more consistent, but makes use of a very rough schematization of the stress-strain diagram (rigid-plastic). Prager's definition is closer to the real behaviour of the materials, since elastic strains do exist, but it makes use of the principle of rigidification, which in a more rigorous treatment is a contradiction in itself; though this principle is widely used in the classical strength of materials and theory of elasticity, its consequences in the theory of limit analysis may be 
much more significant, since the displacements are here larger or even infinite. In most cases both definitions lead to coinciding results (17.3), but there appear also some differences. They were discussed by D. C. Drucker, H. J. Greenberg, E. H. Lee and W. Prager [4.118, 4.119, 4.120], E. H. Lee [4.321], R. Hill [4.213, 3.246] and J. F. W. Bishop [4.40]. Although W. Prager [4.468] states that those differences are insignificant, and P. S. Symonds [4.564] treats the two approaches as identical, it will be shown in Sec. 18 that in some cases the differences are remarkable and even of qualitative character (the limiting procedure may lead to different results).

Rejecting the contradiction in Prager's approach and considering the geometry changes preceding uncontained flow-which is the most desirable-we often arrive at a quite different formulation of the problem, which will be discussed in Sec. 17.3.

In the simplest case of the material which is neutrally stable in the sense of Drucker, and of geometry changes neglected, W. Prager [4.466, 4.467] showed that at the level $\mathscr{S}$ the surface (17.3) is convex and that the generalized plastic strain rates are governed by the association flow rules, as at the level $\mathscr{P}$, namely

$$
\dot{q}_{i}=\lambda \frac{\partial f}{\partial Q_{i}}
$$

(the normality rule). A. A. Gvozdev [4.195, 4.196] proved these theorems for a system of elements, and P. G. Hodge and C. K. Sun [4.225] for the general case at the level $\mathscr{B}$.

In cases of uniaxial stress throughout a perfectly elastic-plastic body the Drucker postulate is automatically satisfied and the deviations from convexity may be connected with geometry changes only; such changes are particularly significant at the level $\mathscr{B}$, but may take place at the level $\mathscr{S}$ as well (thin-walled bars with a deformable cross-section, sandwich shells with a soft core not preserving the distance between the carrying layers, etc.).

The important problem of uniqueness of the plastic interaction surface (17.3) may be considered in at least four aspects: (1) uniqueness of solution for the given loading program, (2) dependence of the plastic interaction surface on the trajectory in the space of exertion factors, (3) dependence on the geometric gradients, (4) dependence on the static gradients (a fifth aspect will be discussed additionally in Secs. 17.2 and 18). Uniqueness for the given loading program (simple loading, as a rule) was proved by R. Hill [4.211], D. C. Drucker, W. Prager and H. J. Greenberg [4.120] for bounded regions under the assumption of an associated flow rule, but for unbounded regions these theorems, in general, do not hold (E. M. Shoemaker, W. F. Chen [4.535]). Uniqueness in the sense of the plastic interaction surface at the level $\mathscr{S}$ being independent of the trajectory was proved by W. Prager [4.466, 4.467], but this does not mean that the actual carrying capacity of a body or of a cross-section is path-independent, even if perfect plasticity is assumed; for example, alternating loads may lead to a low-cycle fatigue before reaching the plastic interaction surface at any instant. The further two above-mentioned aspects concern the 
level $\mathscr{S}$ only. Theoretically, the gradients introduce non-uniqueness of the plastic interaction surface: in fact, this surface may slightly differ for the cross-section of a prismatic bar, and for the same cross-section of a non-prismatic bar; for a straight bar and for a curved bar; for a plate and for a shell (particularly if the curvatures are not very small). Similarly, plastic interaction surfaces may be influenced by the gradients of the generalized internal forces, e.g. in the case of bending with shear, where the gradient of the bending moment practically cannot vanish. In most cases, however, those effects are insignificant, and plastic interaction surfaces calculated for the simplest cases constitute a useful tool of further analysis even under more complicated conditions.

The number of papers devoted to problems of the limit carrying capacity of various structures, mainly beams, frames and plates, is enormous. Let us mention here just the monographs written by K. W. Johansen [4.263], J. A. Van den Broek [4.592], A. A. Gvozdev [4.196], A. R. Rzhanitsyn [4.492], Z. Sobotka [4.544, 4.545] B. G. Neal [4.413], J. F. Baker, M. R. Horne and J. Heyman [4.17], L. S. Beedle [4.25], J. Heyman [4.205], R. H. Wood [4.609], H. Haase [4.198], D. Niepostyn [4.420, 4.421], A. Sawczuk and T. Jaeger [4.513], P. G. Hodge [4.223], A. Sawczuk [4.514], M. Janas [4.257], M. A. Save and C. E. Massonnet [4.508], L. Dietrich, J. Miastkowski and W. Szczepiński [4.113, 4.567]. A statistical approach to limit analysis in the case of random non-homogeneity of the material has been applied by G. Sacchi [4.497].

A fundamental problem is posed by the relation of the limit carrying capacity defined above to the real carrying capacity of engineering structures. We are going to discuss the influence of the following three factors: elastic strains, geometry changes, and plastic hardening. The fourth factor, namely the limited ductility of the material, will be discussed in Sec. 18.

\subsection{Influence of elastic strains}

As was mentioned above, the influence of elastic strains on the limit carrying capacity is usually assumed to be inessential if the geometry changes are not taken into account. Typical diagrams: generalized force $Q$ versus generalized displacement $q$ for a perfectly elastic-plastic material are shown in Figs. 82 and 83. In the case shown in Fig. 82 we observe an elastic range limited by the parameters of the elastic carrying capacity $\bar{Q}$ and $\bar{q}$, then an elastic-plastic range, $\bar{Q}<Q<\bar{Q}, \bar{q}<q<\overline{\bar{q}}$,

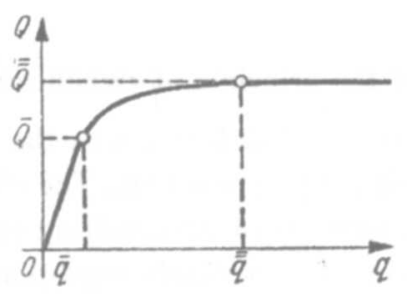

Fig. 82. Limit carrying capacity reached at a finite generalized strain 
and finally a purely plastic range in which Eq. (17.4) is satisfied for the loading parameter $Q=\overline{\bar{Q}}$. An example is provided here by the torsion of a bar with an annular cross-section: the generalized displacement $q$ is then understood as the unit angle of twist (or the total angle of twist) and the generalized force as the torque. A thick-walled tube under internal pressure serves as another example.

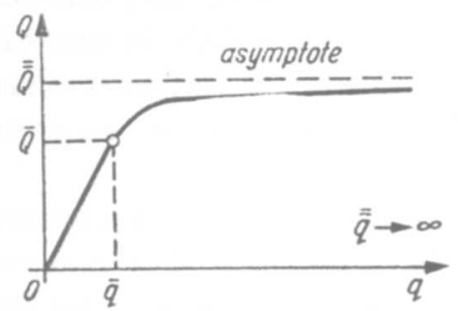

Fig. 83. Limit carrying capacity reached at an infinitely large generalized strain

In the case shown in Fig. 83, Eq. (17.4) is satisfied for infinitely large $q$ only; the generalized force $Q$ tends to $\overline{\bar{Q}}$ just in the limit, and this fact explains to a certain degree the terms "limit carrying capacity" and "limit analysis". Pure torsion of a solid bar or pure bending may serve as examples of such behaviour. In both cases under consideration the limit carrying capacity is the same for perfectly rigid-plastic and for perfectly elastic-plastic bodies.

As a rule, the same values of the limit carrying capacity are also obtained if we consider asymptotically perfect plasticity (cf. Sec. 8.4), but then $Q \rightarrow \overline{\bar{Q}}$ may occur only in the limiting case $q \rightarrow \infty$. J. Betten [4.31] analysed several examples, making use of the stress-strain relation

$$
\sigma=\frac{E \varepsilon}{\left[1+\left(\frac{E \varepsilon}{\sigma_{0}}\right)^{n}\right]^{1 / n}}
$$

(the particular case $n=2$ was suggested earlier by H. Neuber [4.417]) and found that in all cases the limit carrying capacity coincided with that for a perfectly plastic body. Hence a new aspect of the uniqueness of the limit load may be formulated: uniqueness understood as the independence of that load of the assumed stress-strain diagram belonging to the class of asymptotically perfect plasticity. Such independence may be observed in many cases, but there are also some exceptions; they will be discussed in Sec. 18.

\subsection{Influence of geometry changes (abandonment of the principle of rigidification)}

Changes of geometry of a structure may introduce essential differences in the behaviour of that structure and in the concept of the limit carrying capacity. The effect of geometry changes may be stabilizing (geometric hardening) or destabilizing (geometric softening). Assume, at first, the schematization of a perfectly rigid-plastic material; typical cases of the geometric effects are shown in Figs 84-86. 

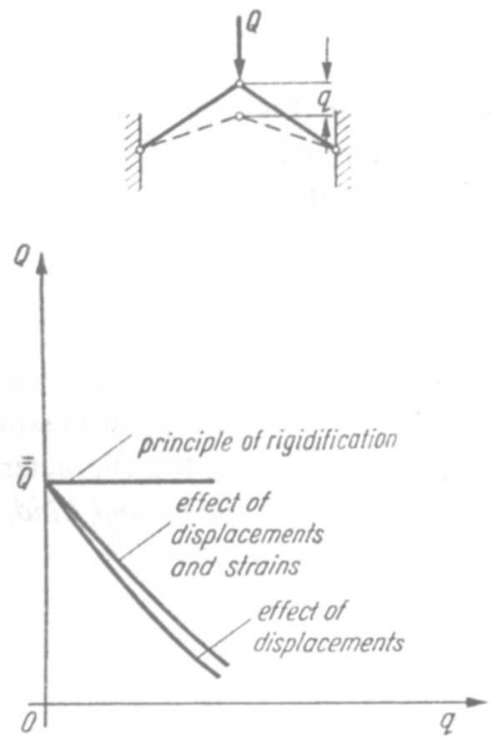

Fig. 84. Destabilizing geometric effects in a rigid-plastic structure

In the case of a truss shown in Fig. 84 (the Mises truss [4.393]) the force $\overline{\bar{Q}}$ calculated by using the principle of rigidification is, simultaneously, the maximal force for the structure under consideration (the range after the "jump" of the truss is not considered). A condition of type (17.4) is not satisfied at any point of the diagram $Q=f(q)$ (which would take place for $q=0$ under the simplifying assumption of the principle of rigidification); nevertheless, the force $\overline{\bar{Q}}$ may be called the limit carrying capacity in the Hill sense. However, the geometrical effects, which are destabilizing here, necessitate a cautious application of $\overline{\bar{Q}}$ in engineering calculations;

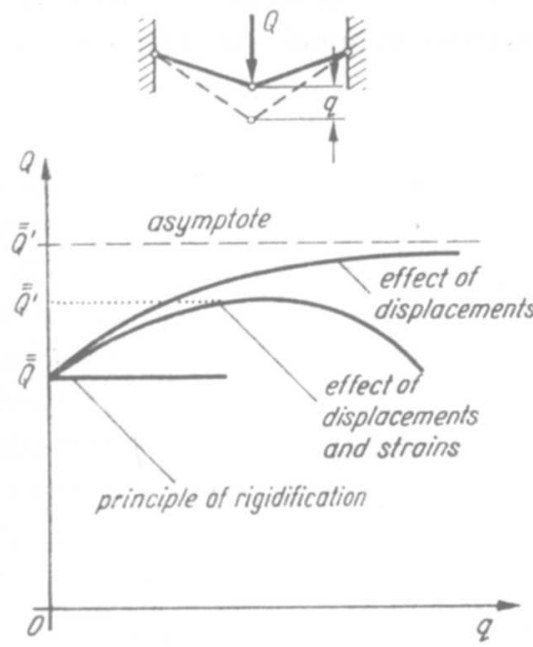

Fig. 85. Stabilizing geometric effects in a rigid-plastic structure 
in case of compressed bars buckling might occur and result in a further lowering of the characteristic diagram.

A quite opposite behaviour is shown by the truss in Fig. 85. In this case the effect of displacement is stabilizing, and if we consider only this effect combined with the strains governed by the principle of rigidification (constant cross-sectional area during the process; such an approach may be justified in the case of very slender bars), then the characteristic diagram $Q=f(q)$ has a horizontal asymptote $Q=\overline{\bar{Q}}^{\prime}$. A more accurate and consistent approach rejects the principle of rigidification also for strains and takes into account the changes of the cross-sectional area. The latter effect is destabilizing and the resulting curve has a local maximum $\overline{\bar{Q}}^{\prime \prime}$ at a finite displacement $q$. Any of the loads $\overline{Q^{\prime}},{\overline{Q^{\prime \prime}}}^{\prime \prime}$, satisfying the characteristic condition (17.4), may be called the limit carrying capacity of the second kind, but various other terms are used for these loads; they will be listed below.

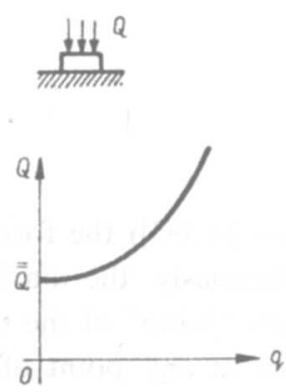

Fig. 86. Infinitely large maximal carrying capacity

In the extreme case of a short bar subject to compression (Fig. 86) the limit carrying capacity of the second kind increases infinitely. Indeed, if we assume an infinite diagram $\sigma=f(\varepsilon)$ with the yield condition $\sigma=\sigma_{0}$ written for true stresses, and take into consideration the increase of the cross-sectional area, then the diagram $Q=f(q)$ has a vertical asymptote.

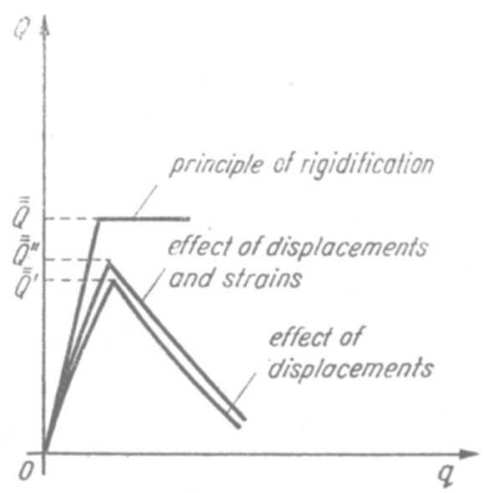

Fig. 87. Destabilizing geometric effects in an elastic-plastic structure 
The problem is more complicated if we take into account both elastic strains and geometry changes. Two typical diagrams $Q=f(q)$ in this case are shown in Figs 87 and 88; they correspond to Figs 84 and 85 , respectively, if elastic strains are accounted for. In these cases the relation of the diagram to the classical limit carrying

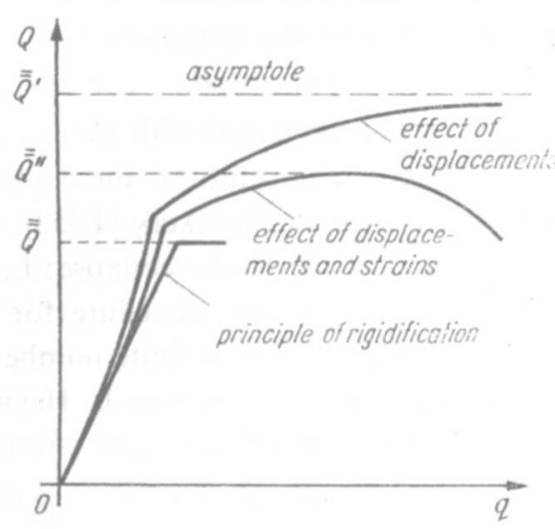

Fig. 88. Stabilizing geometric effects in an elastic-plastic structure

capacity $\overline{\bar{Q}}$ is no longer clear: particularly, in the case shown in Fig. 87 the maximal force may be much lower than $\overline{\bar{Q}}$. Hence, in cases of a significant influence of geometry changes the evaluation or estimation of the maximal values of $Q$ may be of considerable interest.

The first investigations of maximal load for an elastic-plastic structure accounting for geometry changes concerned eccentrically compressed columns. Various approximate approaches for strain-hardening materials have been proposed since the beginning of the twentieth century (cf. Sec. 17.4), but it was K. Ježek [4.261, 4.262] who gave in 1934 the first analytical solutions for perfectly elastic-plastic columns and determined the maximal compressive forces. He called such forces the "loadcarrying capacity" (Tragfähigkeit) or the "critical forces"; indeed, any maximal value of the force is supposed to separate the stable from the unstable behaviour of a structure. S. D. Leytes [4.329, 4.330] and A. A. Pikovsky [4.458], who have extended Ježek's theory, use for columns a more precise term "critical force of the second kind"; such precision is particularly important in cases of central compression of straight columns (or, in general, of certain perfect structures), where a well-known critical force of the first kind exists which determines equilibrium branching. Considering beam-column effects in shells, B. Paul and P. G. Hodge [4.447] used the terms "elastic-plastic carrying capacity" in parallel with "collapse load"; other common terms are the "maximum carrying capacity", and the "ultimate load" (L. S. Beedle [4.25]), the "failure load" (M. R. Horne [4.237]), or the "ultimate strength" (P. B. Mellor [4.382]). In what follows we shall use the term maximal carrying capacity.

The first experimental investigations of the load-carrying capacity of circular arches, carried out by C. Dyrbye and P. Lange Hansen [4.134], indicated significant 
effects of geometry changes: the maximal carrying capacity was much larger in the case of the force going radially outwards (geometric hardening) than in the case of its going inwards (geometric softening). Similar effects of geometric hardening were observed by R. M. Cooper and G. A. Shifrin [4.91], J. Foulkes and E. T. Onat [4.143], and by R. Hooke [4.233], who found substantial influences of membrane forces due to geometry changes in circular plates; though the materials used were very close to being perfectly plastic, no "platform" $d Q=0$ occurred.

The evaluation of the maximal carrying capacity is connected with the analysis of the stability of the structure and of the uniqueness of the solution; these aspects were discussed by R. Hill [3.249], E. T. Onat [4.434] and B. Storåkers [4.553]. Geometry changes may result in new types of mechanisms of the plastic collapse; L. M. Belenky [4.26] analysed this problem for frames. An iterative procedure for the evaluation of the maximal carrying capacity for a structure with a finite number of degrees of freedom was proposed by A. M. Protsenko [4.475], whereas J. Heyman [4.207] proposed a simple engineering method of estimation of geometric effects.

In many complicated cases, where the theoretical evaluation of the maximal carrying capacity would be very difficult, a certain estimation of the behaviour of the structure is furnished by a number of terms of the power series expansion or by the initial slope of the curve $Q=f(q)$ for a rigid-plastic structure (initial post-yield behaviour, determining the stability). Such an approach was used, for example, by E. T. Onat and R. M. Haythornthwaite [4.431, 4.432, 4.201], Yu. R. Lepik [4.326, 4.327], A. Sawczuk [4.515, 4.516] and M. Duszek [4.129, 4.130, 4.132].

The influence of geometry changes is also significant in the case of rotating structural elements, since radial displacement of an element of the body results in increasing centrifugal force. This problem has been analysed by $\mathrm{M}$. $\mathrm{H}$. Lee $\mathrm{Wu}$ [4.613] (rotating disks) and by W. Szczepiński [4.565] (rotating cylinders).

\subsection{Influence of plastic hardening}

The classical concept of the limit carrying capacity was introduced in Sec. 17.1 in two ways: first, for a perfectly elastic-plastic material, and then it determined also the maximal carrying capacity if geometry changes could be neglected; second, it was introduced for rigid-plastic materials with arbitrary hardening, and in this case it determined the initiation of motion of the structure. For an elastic-plastichardening structure the concept of the limit carrying capacity may also be useful if hardening is preceded by a perfectly plastic behaviour and if the non-homogeneity of the stress state is not too strong: than a "platform" or at least a point of inflection of the curve $Q=f(q)$ at the levels $\mathscr{S}$ or $\mathscr{B}$ may be observed (P. G. Hodge [4.222]). In opposite cases this concept may be almost useless: the curve $Q=f(q)$ has neither a "platform" nor an inflection point and may well exceed the classical limit carrying capacity calculated for a conventional yield-point stress.

In contradistinction to perfect plasticity, the maximal carrying capacity for 
a structure made of a plastic hardening material does not exist, unless (1) geometry changes are taken into account, or (2) the diagram $\sigma=f(\varepsilon)$ has a horizontal asymptote or plastic hardening is followed by plastic softening, or (3) the diagram is limited by a certain criterion of decohesion. Conditions (1) and (2) may be insufficient (cf. Fig. 86), but in most practical cases a certain maximal carrying capacity can be determined. A horizontal asymptote (second yield point) was discussed by $\mathrm{P}$. V. Mc Laughlin [4.377] and D. I. Shakhnovsky [4.525], and the maximal carrying capacity for a beam cross-section in the case of plastic softening was analysed by V. A. Ibragimov and V. D. Klyushnikov [3.282], S. V. Gavrilenkov and S. Kerimbayev [4.157], G. Maier, A. Zavelani and J. C. Dotreppe [4.353]. Criteria of decohesion will be discussed in Sec. 18.

If geometry changes are taken into account, then the maximal carrying capacity may exist not only in the case of plastic hardening but also for perfectly elastic structures. The Mises truss (Fig. 84) may serve here as a good example: maximal force causes the "jump" of the truss. Hence if geometry changes are taken into account, then the basic differences between perfect plasticity and plastic hardening disappear, and, as a rule, the maximal carrying capacity may be determined in both cases.

The earliest investigations of the maximal carrying capacity of plastic hardening structures concern eccentrically loaded columns. T. Kármán [4.272] considered in 1910 the maximal carrying capacity of columns under the simplifying assumption of small eccentricity. Further approximate solutions are due to R. Krohn [4.309], M. Roš and J. Brunner [4.486], but it was E. Chwalla [4.77, 4.78] who was the first to give, in 1928, an exact solution in closed integral form. Let us also mention here the related problems of slender structures, e.g. the bending of straight thin-walled tubes (L. G. Afendik, M. F. Kolesnitchenko [4.2, 4.3]), or curved tubes (R. M. Mello, D. S. Griffin [4.381], C. R. Calladine [4.58]), where the existence of the maximal carrying capacity is due to the ovalization of the cross-section. In some cases several local maxima of loading may exist; such a phenomenon was observed by J. Ledziński and Z. Waszczyszyn [4.319], who investigated the Mises truss made of a material exhibiting piece-wise linear plastic hardening. A general theory of subsequent neutral surfaces for structures with geometry changes taken into account was given by G. Maier and D. C. Drucker [4.354]. On the other hand, the theory of limit surfaces describing the maximal carrying capacity of structures has hardly been studied at all as yet.

Geometry changes are very important in the case of pressure vessels, particularly those made of highly ductile materials: an increase of dimensions results here in an increase of the resultant force with a simultaneous decrease of wall thickness. A thickwalled circular cylinder was considered under these assumptions by E. T. Onat [4.433], and multilayer initially expanded cylinders by M. Życzkowski and M. Galos [4.634, 4.152]. A general finite-deflection, finite-strain membrane theory of rotationally symmetrical pressure vessels, proposed by A. S. Grigorev [4.186, 4.187, 4.188], was developed by J. Orkisz [4.435, 4.436, 4.437]. 


\subsection{Maximal carrying capacity resulting in necking or bulging}

The part of the diagram $Q=f(q)$ beyond the maximal carrying capacity corresponds, as a rule, to various forms of instability. In the case of compressed elements this instability is due to increasing displacements (deflections), whereas in the case of tension to increasing strains (necking) or strains and displacements (bulging of pressure vessels).

Though necking of a bar in tension was classified as a form of instability as early as 1909 by P. Ludwik [2.107], more accurate theories of necking were developed in the forties (A. Yu. Ishlinsky [4.249], P. W. Bridgman [4.51], E. Siebel [4.538], N. N. Davidenkov, N. I. Spiridonova [4.103], G. Sachs, J. D. Lubahn [4.499], A. M. Zhukov [4.621], A. Nadai [3.566], W. Truszkowski [4.587]). These theories of engineering type assumed simply $d P=0$ as the criterion of necking. Writing $P=A \sigma=V \sigma / l$, where $V$ denotes the volume of the bar, we easily derive for an incompressible material ( $V=$ const) the condition

$$
E_{t} \equiv \frac{d \sigma}{d \varepsilon}=\sigma
$$

where $\varepsilon=\varepsilon^{H}$ denotes the logarithmic strain and $\sigma$ the true stress. If the (elastic) compressibility of the material is taken into account, then (17.7) takes the form (W. Johnson, P. B. Mellor [2.76], J. Mandel [3.494])

$$
E_{t} \equiv \frac{d \sigma}{d \varepsilon}=\frac{\sigma}{1+\sigma / 3 K} .
$$

Further research in this direction is due to N. N. Malinin and Zh. L. Petrosyan [4.359] (distribution of stresses in the smallest cross-section), P. F. Thomason [4.584], M. A. Kaplan [4.270] (parabolic profile of the neck), H. J. Kleemola and M. A. Nieminen [4.284] (various schematizations of plastic hardening). Non-isothermal and rate-dependent necking problems were analysed by E. W. Hart [4.200] and J. Klepaczko [4.289, 4.290].

In the case of a pressure vessel (straight or curved tube, etc.), instability beyond the maximal carrying capacity results in bulging: the diameter locally increases whereas in the remaining part of the tube passive processes take place (due to decreasing pressure) and the strain energy is there returned. This problem was investigated by G. Espey in 1946 [4.137]; further papers consider simultaneous pressure with axial force (W. T. Lankford, E. Saibel [4.316], H. W. Swift [4.560], Z. Marciniak [4.366], P. B. Mellor [4.383], M. J. Hillier [4.215, 4.216], G. Sines [4.539], V. I. Maksak, V. I. Chernomortchenko [4.358], N. N. Malinin [4.360], V. N. Bastun [4.22]). B. Storåkers [4.550] took into account the additional effect of torsion. Necking in sheets under plane stress conditions was investigated by P. P. Bijlaard [4.32] R. Hill [3.244], Z. Marciniak [4.368], M. L. Gorb, A. A. Ostrovsky and N. I. Chernyak [4.179]. A. M. Vinogradova [4.595, 4.596] considered necking in cylindrical shells subject to axial tension (both theoretically and experimentally), while V. O. Vakorin and A. S. Fedorov [4.590] dealt with necking in the case of pure 
bending of beams. Numerous experiments were reported by I. N. Izotov and A. G. Mityukov [4.255].

More exact analyses of necking, bulging and related phenomena are based on the general criterion of uniqueness and stability formulated by R. Hill: for rigid-plastic solids in [3.248, 4.214], and for elastic-plastic solids in [3.249]. In both cases geometry changes were taken into account. Though in the simplest case of uniaxial tension of an incompressible specimen this criterion results in (17.7), nevertheless it has permitted a proper formulation of more complicated problems. G. R. Cowper and E. T. Onat [4.96] studied necking in rectangular bars in plane strain conditions, while R. N. Dubey and S. T. Ariaratnam [4.125, 4.126] in plane stress conditions. J. P. Miles [4.387], J. Hutchinson and J. P. Miles [4.239] showed that Hill's sufficient condition for uniqueness provides a lower bound for the necking problem, and gave for cylindrical bars a necking solution using the Bessel functions. A close but much simpler solution was obtained by J. Christoffersen [4.76]. A. Needleman [4.415] suggested a numerical method, using finite elements, suitable for the analysis of necking problems based on Hill's criterion; other procedures are due to S. Tang [4.576], and W. H. Chen [4.68] (the Kantorovitch method). J. P. Miles [4.388] investigated the influence of the fluid pressure on necking of a cylindrical bar in tension. More details may be found in the survey papers by M. J. Sewell [4.524] and B. Storåkers [4.552, 4.553].

Hill's criterion has also been used in certain more complicated cases, e.g. for the stability analysis of thick-walled pressure vessels. B. Storåkers [4.551] found that plastic instability of a cylindrical tube may result in non-symmetrical collapse modes, determined earlier by J. Skrzypek and M. Życzkowski [4.542]. A similar analysis for thick-walled spherical vessels was given by $\mathrm{H}$. Strifors and B. Storåkers [4.556].

\subsection{Limit carrying capacity and full plastification of the body}

It is well known that the limit carrying capacity of a perfectly plastic body may be exhausted without full plastification of that body: the collapse mechanism of a prismatic cantilever beam loaded by a concentrated force at the free end may serve as a good example. On the contrary, full plastification in the limit state may be regarded rather as an exception. Of course, the material in the zones remaining rigid or elastic at collapse it not fully utilized and the first step towards optimal design in the plastic range is to eliminate such zones, if possible. In some cases this criterion is sufficient to determine the corresponding shapes of the structural elements (Z. Kordas [4.297, 4.298, 4.299], partly with M. Życzkowski and W. Skraba).

On the other hand, it is very often assumed that $f . l l$ plastification of the body furnishes a sufficient condition of exhaustion of the limit carrying capacity of that body. In fact, in most cases such coincidence does take place, but certain exceptions also appear: the limit carrying capacity is not exhausted before a certain collapse 
mechanism is formed (possible increments of generalized strains without increments of generalized stresses), hence the above statement cannot be regarded as general.

We are now going to present a simple example of full plastification of a body which does not result in the exhaustion of its limit carrying capacity. $\left({ }^{1}\right)$ Consider a thin-walled circular cylinder the wall of which is made of two different materials permanently joined to each other (Fig. 89). The elastic properties of both materials

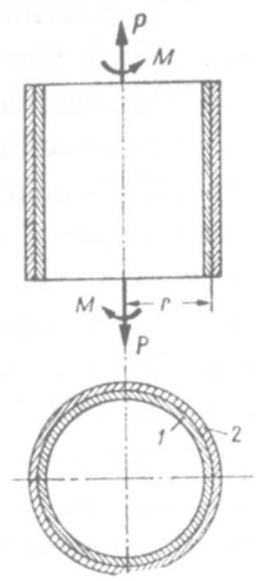

Fig. 89. An example of full plastification before the limit carrying capacity is exhausted

are assumed, for the sake of simplicity, to be identical (incompressible materials, $E=3 G$ ), whereas the yield-point stress of the first material $\sigma_{01}$ is smaller than that of the second material $\sigma_{02}$; the ratio $\sigma_{02} / \sigma_{01}$ will be fixed effectively later, in order to simplify the calculations.

The cross-sectional areas of the two constituents are the same and equal $A$; we assume that the wall is so thin that the radius $r$ may be regarded as the mean radius for both constituents. The problem of wall stability is not considered.

At the first stage the cylinder is subject to the axial force $P$ equal to $P=3 \sigma_{01} A$; if we assume $\sigma_{02}>2 \sigma_{01}$, then the stresses are $\sigma_{1}=\sigma_{01}$ (plastic), $\sigma_{2}=2 \sigma_{01}$ (elastic). At the second stage the cylinder is subject to additional torsion under the constant axial force $P$. Then in the first cylinder constituent an active process will take place, and we describe it by the Prandtl-Reuss equations, whereas in the second constituent there will be a passive process described by Hooke's law. The HMH yield condition for the first cylinder will be parametrized as follows:

$$
\sigma_{1}=\sigma_{01} \cos \psi_{1}, \quad \tau_{1}=\frac{\sigma_{01}}{\sqrt{3}} \sin \psi_{1} .
$$

The Prandtl-Reuss equations may be written as

$$
\frac{d \varepsilon-d \sigma_{1} / 3 G}{d \gamma-d \tau_{1} / G}=\frac{\sigma_{1}}{3 \tau_{1}}
$$

(1) The central idea of this example is due to $Z$. Mróz. 
(the increments $d \varepsilon$ and $d \gamma$ are without indices since the strains in both cylinders are assumed to be equal), and on substituting (17.9) we obtain

$$
d \varepsilon+\frac{\sigma_{01}}{3 G} \sin \psi_{1} d \varphi_{1}=\left(d \gamma-\frac{\sigma_{01}}{G \sqrt{3}} \cos \psi_{1} d \psi_{1}\right) \frac{\cot \varphi_{1}}{\sqrt{3}}
$$

Hooke's law for the second cylinder gives

$$
d \varepsilon=\frac{d \sigma_{2}}{3 G}=-\frac{d \sigma_{1}}{3 G}, \quad d \gamma=\frac{d \tau_{2}}{G},
$$

since the assumption of constant axial force yields $d \sigma_{1}+d \sigma_{2}=0$. Substitution and simple rearrangements result in the following differential equation of the first order:

$$
d \tau_{2}=\frac{2 \sigma_{01}}{\sqrt{3}} \frac{d \psi_{1}}{\cos \psi_{1}}-\frac{\sigma_{01}}{v^{\overline{3}}} \cos \psi_{1} d \psi_{1} .
$$

Integrating and making use of the initial condition $\tau_{2}(0)=0$, we obtain

$$
\tau_{2}=\frac{2 \sigma_{01}}{\sqrt{3}} \ln \tan \left(\frac{\psi_{1}}{2}+\frac{\pi}{4}\right)-\frac{\sigma_{01}}{\sqrt{3}} \sin \psi_{1} .
$$

The imposed condition of constant axial force determines $\sigma_{2}$ :

$$
\sigma_{2}=3 \sigma_{01}-\sigma_{1}=3 \sigma_{01}-\sigma_{01} \cos \psi_{1}
$$

and Eqs. (17.9), (17.14) and (17.15) describe the second stage of the process by means of the functions of the parameter $\psi_{1}$.

The termination of the second stage and the beginning of full plastification is determined by the yield condition being satisfied by $\sigma_{2}$ and $\tau_{2}$ :

$$
\left(3 \sigma_{01}-\dot{\sigma}_{01} \cos \psi_{1}\right)^{2}+\left[2 \sigma_{01} \ln \tan \left(\frac{\psi_{1}}{2}+\frac{\pi}{4}\right)-\sigma_{01} \sin \psi_{1}\right]^{2}=\sigma_{02}^{2} \text {. }
$$

This is a transcendental equation with respect to the parameter $\psi_{1}$. To simplify the calculations, however, we have not yet fixed $\sigma_{02}$; following an inverse procedure, we now assume for example $\psi_{1}=\pi / 6=30^{\circ}$, and evaluate the relevant value of $\sigma_{02}$ :

$$
\frac{\sigma_{02}}{\sigma_{01}}=\sqrt{10-3 \sqrt{3}-\ln 3+\ln ^{2} 3}=2.216
$$

Hence, at the end of the second stage the stresses are: $\sigma_{1}=0.866 \sigma_{01}, \tau_{1}=0.289 \sigma_{01}$, $\sigma_{2}=2.134 \sigma_{01}=0.963 \sigma_{02}, \tau_{2}=0.346 \sigma_{01}=0.156 \sigma_{02}$. A parametrization of the yield condition for the second constituent cylinder, analogous to (17.9), results in the following value of $\psi_{2}$, corresponding to the initiation of full plastification: $\psi_{2}=\arctan \tau_{2} \sqrt{3} / \sigma_{2}=0.273=15^{\circ} 38^{\prime}$.

The above stresses result in full plastification of the cylinder as a whole, but its limit carrying capacity is not yet exhausted. Indeed, for the constant force $P$ $=3 \sigma_{0} A$ and for the constant torque $M_{t}=\left(\tau_{1}+\tau_{2}\right) A r=0.635 \sigma_{01} A r$ the possibility of increase of $\gamma$ and $\varepsilon$ does not exist, i.e. no mechanism of plastic collapse can be 
found. Any further increase of $\gamma$ results in an increase of the torque. This third stage of the deformation process will be described by the Prandtl-Reuss equations for both constituent cylinders. Introducing a parametrization of type (17.9) for both cylinders, we obtain after simple rearrangements the system of equations

$$
\begin{aligned}
& \sin \psi_{1} d \varepsilon-\frac{1}{\sqrt{3}} \cos \psi_{1} d \gamma=-\frac{\sigma_{01}}{3 G} d \psi_{1}, \\
& \sin \psi_{2} d \varepsilon-\frac{1}{\sqrt{3}} \cos \psi_{2} d \gamma=-\frac{\sigma_{02}}{3 G} d \psi_{2},
\end{aligned}
$$

which together with the condition of the constant axial force

$$
\sigma_{01} \cos \psi_{1}+\sigma_{02} \cos \psi_{2}=\frac{P}{A}=3 \sigma_{01}
$$

determines all the three unknown functions, e.g. $\psi_{1}=\psi_{1}(\gamma), \psi_{2}=\psi_{2}(\gamma)$ and $\varepsilon=\varepsilon(\gamma)$.

The integration of the above system presents some difficulties, but the determination of the limit carrying capacity of the cylinder is much simpler. Regard Eqs. (17.18) as a system with unknowns $d \varepsilon$ and $d \gamma$; the principal determinant of the system equals

$$
\Delta=-\frac{1}{\sqrt{3}} \sin \left(\psi_{1}-\psi_{2}\right)
$$

and vanishes for $\psi_{1}=\psi_{2}$ (other roots are of no interest). If $\psi_{1}=\psi_{2}=$ const, then $d \psi_{1}=d \psi_{2}=0$ and the system (17.18) becomes indeterminate; in this case $d \varepsilon$ and $d \gamma$ may increase without a simultaneous increase of generalized forces. Hence $\psi_{1}=\psi_{2}=$ const determines a certain collapse mechanism and the limit

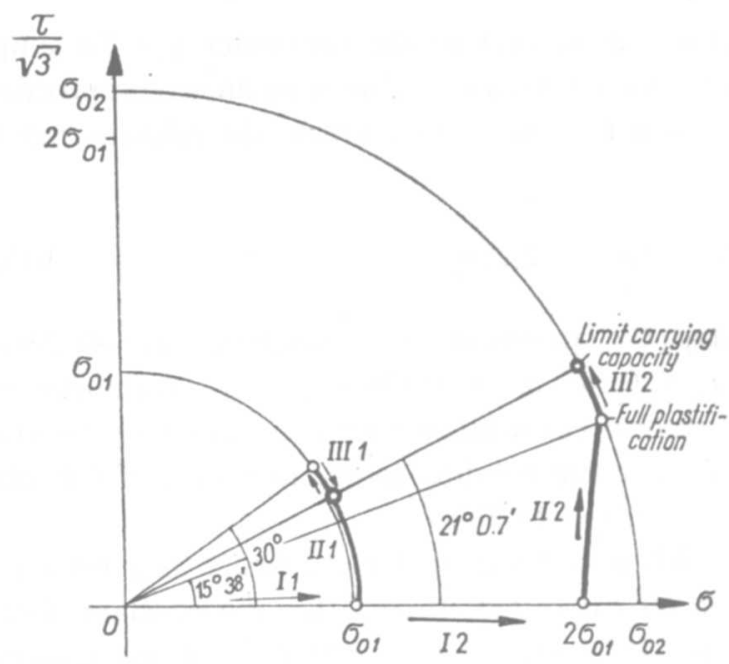

Fig. 90. Trajectories in the stress space for a tube shown in Fig. 89 
carrying capacity of the cylinder is exhausted. The auxiliary angle $\psi_{1}=\psi_{2}=\psi$ may be evaluated from Eq. (17.19):

$$
\cos \psi=\frac{3 \sigma_{01}}{\sigma_{01}+\sigma_{02}}=0.9328, \quad \psi=0.369=21^{\circ} 07^{\prime} ;
$$

it takes a certain intermediate value between $\psi_{1}$ and $\psi_{2}$ at the beginning of the third stage of the process. The relevant torque equals

$$
\bar{M}=\frac{1}{\sqrt{3}}\left(\sigma_{01}+\sigma_{02}\right) A r \sin \psi=0.676 \sigma_{01} A r
$$

and is by 7 per cent higher than that at the beginning of full plastification.

The course of the particular stages (I, II, III) of the process is illustrated by the diagram $\sigma-\tau \sqrt{3}$ in Fig. 90. The limit carrying capacity of the cylinder as a whole is not exhausted before the points representing stress states in the two constituent cylinders reach a common radius.

\section{Decohesive carrying capacity}

18.1 Cases of non-existence of solutions in perfect plasticity and the concept of decohesive carrying capacity

In real materials the process of plastic deformations, if continued, usually leads to decohesion. Decohesion is a physical phenomenon governed by separate equations; some of them were mentioned in Sec. 11.6, and more attention will be devoted to this problem in Secs. 18.7 and 18.8. However, even if we assume an infinitely long stress-strain diagram without any particular physical criterion of decohesion (e.g. in perfect plasticity), then in some cases of non-homogeneities (of stresses or strains or of the material) the continuous solution ceases to exist, inadmissible discontinuities appear, and the diagram $Q=f(q)$ cannot be extended to infinity but ends at a certain point. The corresponding loading parameter was called by K. Szuwalski and M. Życzkowski [1.18] the decohesive carrying capacity. This "mathematical" criterion of decohesion, connected with the formation of inadmissible discontinuities, may be regarded as an upper bound for all physical criteria of decohesion.

The classical theory of plastic limit analysis assumes that there exists at least one mechanism of plastic collapse (infinitesimal motion at a constant loading parameter), and that if it exists for a rigid-plastic body, then it may be attained by an elastic-perfectly plastic structure as well (coincidence of the two definitions of limit carrying capacity, given in Sec. 17.1). In many cases both these assumptions are justified; however, some exceptions prove that they are not quite obvious. In general, no mechanism of plastic collapse may exist, or, if it exists for a rigid-plastic body, it may be unreachable by an elastic-plastic structure without violating the required 
continuity conditions. The existence theorem for elastic-perfectly plastic bodies fails (G. Del Piero [4.110]), and the papers by K. Szuwalski and M. Życzkowski $[1.18,4.570]$ show several examples of the non-existence of any mechanism of plastic collapse: earlier, in the elastic-plastic range, inadmissible discontinuities appear and the decohesive carrying capacity is exhausted.

Indeed, there is no mathematical or physical reason to assume that a limit state of an elastic-plastic structure, defined as in Sec. 17.1, always exists. On the contrary, the concept of decohesive carrying capacity makes it possible to clarify many paradoxes observed in the theory of perfect plasticity. Such paradoxes were pointed out as early as 1935 by F. Stüssi and C. F. Kollbrunner [4.557], and in 1955 by H. Craemer [4.97]. A solution for a notched bar in tension, involving partial decohesion, was given in 1952 by E. H. Lee [4.322]. Cases of the non-existence of the limit carrying capacity for certain slabs (plates) were shown by R. H. Wood [4.610]. The problem of a half-plane loaded by a semi-infinite uniform normal pressure in plane strain conditions was discussed by S. S. Golushkevitch [4.178] and V. O. Geogdzhayev [4.162]; they evaluated the decohesive carrying capacity, but called it the "limit carrying capacity". Numerous problems of the non-existence of solutions and of inadmissible discontinuities were discussed by E. M. Shoemaker $[4.533,4.534,4.536,4.537]$. The concept of decohesive carrying capacity is also useful in thermoplasticity, since under purely thermal loadings usually no mechanism of plastic collapse can be found, whereas a certain decohesive carrying capacity does exist, [4.642].

The admissibility of discontinuities is a problem requiring subtle discussion. In classical continuum mechanics any discontinuity of stresses, displacements or velocities normal to the discontinuity surface (line) is inadmissible. On the other hand, in the engineering approach to limit analysis the strains or strain rates are often assumed in the form of a distribution (generalized function, e.g. of the Dirac type); then the displacements or velocities are discontinuous as the Heaviside functions. An attempt to form the theoretical background for such an approach was made by R. Hill [2.57], who proposed a "relaxed" treatment of discontinuities, regarding jumps in normal velocities in plane stress problems as admissible, leading to necking. In fact, such jumps might be treated as admissible at the end of the process, but if the process is to be continued, then they lead to inadmissible discontinuities in displacements, at least within the framework of the small-strain theory. However, since the strains increase infinitely, a more rigorous treatment requires here the application of a finite-strain theory. Then in most cases it turns out that the jumps due to positive normal strains remain inadmissible and result in a certain decohesive carrying capacity, whereas the jumps due to negative strains disappear in view of the increasing cross-sectional area, and in the latter case the process may be continued.

In a statically determinate problem decohesion always results in a full collapse, but in the case of statical indeterminacy a structure may work even after partial decohesion; the relevant discussion is given in $[1.18,4.569,4.570]$. 
The present chapter gives several examples of decohesive carrying capacity, mainly based on the small-strain theory, but some comparisons with the finite-strain theory will also be given.

\subsection{Bars and bar systems}

Consider first the influence of non-homogeneities on the behaviour of a simple bar in tension. Assume an asymptotically perfectly plastic material characterized by the function

$$
\sigma=f(\varepsilon) \quad \text { or } \quad \varepsilon=g(\sigma), \quad 0 \leqslant \varepsilon<\infty, \quad 0 \leqslant \sigma<\sigma_{0} .
$$

If the bar is homogeneous and prismatic, no body forces are present, and the smallstrain theory is applied, then the "integral" diagram $Q=f(q)$ is (in an appropriate scale) identical to $\sigma=f(\varepsilon)$; by the generalized force $Q$ we understand here the force $P$, and by the generalized displacement $q$ the elongation $\Delta l$.

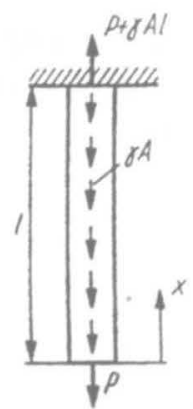

Fig. 91. A bar under non-homogeneous tension

Introduce now a certain non-homogeneity, for example due to an arbitrarily small own weight of the bar (Fig. 91). The elongation of the bar equals in this case

$$
q=\Delta l=\int_{0}^{l} \varepsilon d x=\int_{\sigma(0)}^{\sigma(l)} \frac{g(\sigma)}{d \sigma / d x} d \sigma=\frac{1}{\gamma} \int_{\sigma(0)}^{\sigma(l)} g(\sigma) d \sigma,
$$

since

$$
\sigma=\frac{P}{A}+\gamma x,
$$

where $\gamma$ stands for the specific weight of the material of the bar; the symbols $\sigma(0)$ and $\sigma(l)$ denote the stresses at $x=0$ and $x=l$, respectively. The maximal elongation $q$ is reached for $\sigma(l)=\sigma_{0}$; hence

$$
\hat{q}=\hat{\Delta l}=\frac{1}{\gamma} \int_{\sigma(0)}^{\sigma_{0}} g(\sigma) d \sigma .
$$


The integral (18.3) is improper, since for asymptotically perfect plasticity $g\left(\sigma_{0}\right) \rightarrow \infty$, and may be convergent or divergent. In the first case the maximal elongation $\hat{q}$ is finite and the decohesive carrying capacity $\hat{Q}$ is then reached (Fig. 92, (a), whereas in the second case $\hat{q}$ increases infinitely and we may speak of the limit carrying

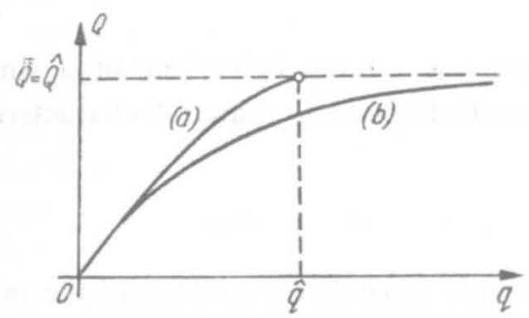

Fig. 92. Decohesive carrying capacity (a) and limit carrying capacity (b)

capacity $\overline{\bar{Q}}$, Fig. 92 , (b) (in this case $\overline{\bar{Q}}=\hat{Q}$, but in general such coincidence does not take place).

The integral (18.3) has a simple physical interpretation: it is proportional to a part of the complementary specific energy, Fig. 93. Hence decohesion takes

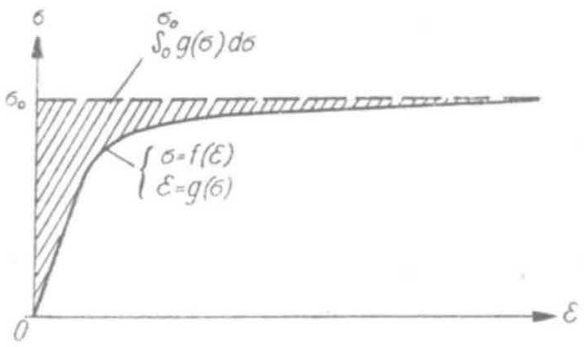

Fig. 93. Interpretation of the improper integral (18.3)

place if that complementary energy is finite. For example, if we consider the most frequently used laws of asymptotically perfect plasticity, that of A. Ylinen (8.11) or that of W. Prager (8.12), then the integral (18.3) is convergent, decohesion occurs and the "integral" behaviour of the bar is quite-brittle. Of course, this statement also holds for a perfectly elastic-plastic material.

To avoid decohesion we have to consider the stress-strain curves approaching the yield stress $\sigma_{0}$ less rapidly. For example, analysing the functions of the type

$$
\varepsilon=g(\sigma)=\frac{\sigma}{E\left(1-\sigma / \sigma_{0}\right)^{n}},
$$

we find that for $n \geqslant 1$ the integral (18.3) is divergent, the elongations may increase infinitely, and there is no qualitative difference between the diagrams $\sigma=f(\varepsilon)$ and $Q=f(q)$.

The above analysis was based on the small-strain theory; however, the assumed criterion of decohesion $\varepsilon=d u / d x \rightarrow \infty$ is inconsistent with this theory and a re- 
examination of results, based on a finite-strain theory, is required. Of course, various degrees of accuracy of such theories are possible. Here we use a relatively simple theory, using true stresses and logarithmic strains, but preserving the simplifying assumption of a uniaxial stress state; the material is regarded as incompressible.

Using the Lagrangian coordinate $X, 0 \leqslant X \leqslant l_{0}$, we may write now instead of (18.2)

$$
\sigma=\frac{P}{A(X)}+\frac{\gamma X A_{0}}{A(X)},
$$

where $\sigma$ stands for the true stress, $A_{0}$ for the initial cross-sectional area, $A(X)$ for the actual cross-sectional area, $l_{0}$ for the initial length of the bar. Making use of the incompressibility condition, we find

$$
A=A_{0} e^{-\varepsilon},
$$

where $\varepsilon$ denotes logarithmic axial strain, and hence

$$
\sigma=\left(\frac{P}{A_{0}}+\gamma X\right) e^{\varepsilon}=f(\varepsilon) .
$$

The function $f(\varepsilon)$, describing the material, is assumed to be known; thus (18.7) may be regarded as a relation between $\varepsilon$ and $X$. The maximal $\sigma$ is reached for $X$ $=I_{0}$

$$
\sup \sigma=\left(\frac{P}{A_{0}}+\gamma l_{0}\right) e^{e\left(l_{0}\right)}=f\left[\varepsilon\left(l_{0}\right)\right],
$$

and hence the force $P$ may be expressed as the following function of the logarithmic strain $\varepsilon\left(l_{0}\right)$ :

$$
P=A_{0} e^{-\varepsilon\left(l_{0}\right)} f\left[\varepsilon\left(l_{0}\right)\right]-\gamma A_{0} l_{0} .
$$

The maximal value of the force is determined by $d P / d\left[\varepsilon\left(l_{0}\right)\right]=0$; this condition leads to

$$
f\left[\varepsilon\left(l_{0}\right)\right]=\frac{d f\left[\varepsilon\left(l_{0}\right)\right]}{d\left[\varepsilon\left(l_{0}\right)\right]},
$$

and this condition is identical with the condition of necking for a homogeneous case, (17.7). In the non-homogeneous case under consideration, (18.10) ends the process: indeed, a decreasing force would result in passive processes at any point except $X=l_{0}$, and an active process at $X=l_{0}$ cannot result in any elongation of the whole bar. The maximal length of the bar at decohesion $l$ is given by

$$
l=\int_{0}^{l_{0}} e^{\varepsilon} d X=\frac{1}{\gamma} \int_{\varepsilon(0)}^{\varepsilon\left(l_{0}\right)}\left[f^{\prime}(\varepsilon)-f(\varepsilon)\right] d \varepsilon
$$

with substituted (18.10), since the function $X=X(\varepsilon)$ is determined by (18.7). 
It should be noted that in the above finite-strain approach the former criterion of decohesion $d u / d x \rightarrow \infty$ is replaced by $d \sigma / d X \rightarrow \infty$. In fact,

$$
\frac{d \sigma}{d X}=\frac{d \sigma / d \varepsilon}{d X / d \varepsilon}
$$

and the denominator of (18.12) vanishes at $X=l_{0}$ in view of (18.7) and (18.10). Further, $d A / d X \rightarrow \infty$ at $X=l_{0}$, and hence in a more rigorous treatment a triaxial state of stress should be taken into account. Such an analysis will not be given here, but probably in most cases the qualitative result will be retained: a limited elongation of the bar as a whole, resulting in decohesion.

We now return to the simpler small-strain theory, but consider statically indeterminate bar systems instead of single bars: the systems are more interesting, since the differences between the limit carrying capacity and the decohesive carrying capacity are then much more evident.

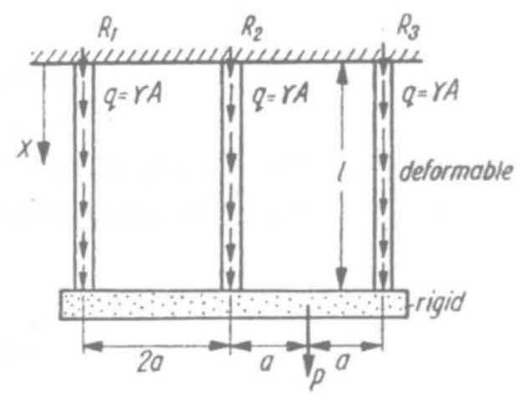

Fig. 94. Decohesive carrying capacity and limit carrying capacity of a bar system

Consider the system shown in Fig. 94 and assume a perfectly elastic-plastic material of the bars. In the elastic range the reactive forces are qual to

$$
R_{1}=\frac{1}{12} P+\gamma A l, \quad R_{2}=\frac{1}{3} P+\gamma A l, \quad R_{3}=\frac{7}{12} P+\gamma A l,
$$

if the weight of the rigid horizontal beam is disregarded; this would introduce no change to the discussion. The largest stress occurs at the fixed cross-section under the reactive force $R_{3}$ and equals $R_{3} / A$. Thus the equation

$$
\frac{7}{12} \frac{P}{A}+\gamma l=\sigma_{0}
$$

describes the elastic carrying capacity of the system (we assume no collapse under its own weight alone, $\gamma l<\sigma_{0}$ ).

The further behaviour of the system depends on $\gamma$. If $\gamma=0$, then (18.14) determines the onset of plastification of the whole bar " 3 ". Under the assumption of perfect plasticity a redistribution of stresses takes place until the bar " 2 " becomes plastic. Then a mechanism of plastic collapse is formed (infinitesimal rotation about a hinge at the lower end the bar " 1 ") and the corresponding limit carrying capacity equals $\overline{\bar{P}}=2 A \sigma_{0}$. 
If the weight of the bar is taken into account, $\gamma \neq 0$, the force

$$
\bar{P}=\hat{P}=\frac{12}{7} A\left(\sigma_{0}-\gamma l\right)
$$

determines simultaneously the elastic carrying capacity and the decohesive carrying capacity of the system. Indeed, the force carried by the bar " 3 " under the assumption of perfect plasticity cannot increase; on the other hand, for the force (18.15) the whole bar " 3 " remains elastic except its upper cross-section, and its further elongation, resulting in a redistribution of stresses, is impossible. Hence the force (18.15) cannot be exceeded, and the decohesive carrying capacity is exhausted. In the limiting case $\gamma \rightarrow 0$ we obtain $\hat{P} \rightarrow \frac{12}{7} A \sigma_{0}$, but in this case the necessity of decohesion disappears, and a much larger limit carrying capacity $\overline{\bar{P}}=2 A \sigma_{0}$ holds; thus for $\gamma \rightarrow 0$ we observe a discontinuity of solution.

To obtain an even more conspicuous and suggestive picture let us replace perfect plasticity by asymptotically perfect plasticity. Assume the Ylinen law (8.11); it is convenient for limiting procedures, since for $c \rightarrow 1$ the material becomes perfectly elastic-plastic. Substituting (8.11) into (18.1), we determine the elongation of the bar with its own weight taken into account by the formula

$$
\Delta l=\left.\frac{1}{E \gamma}\left\{\frac{c \sigma^{2}}{2}+(1-c) \sigma_{0}\left(\sigma_{0}-\sigma\right)\left[\ln \left(1-\frac{\sigma}{\sigma_{0}}\right)-1\right]\right\}\right|_{\sigma(0)} ^{\sigma(l)} .
$$

The maximal elongation $\Delta \hat{l}$, (18.3), is reached for $\sigma(l)=\sigma_{0}$, and for $\gamma \neq 0$ it is finite; a simple limiting procedure leads to the result

$$
\hat{\Delta l}=\frac{l}{E}\left[\left(\sigma_{0}-\frac{\gamma l}{2}\right) c+(1-c) \sigma_{0}\left(1-\ln \frac{\gamma l}{\sigma_{0}}\right)\right] .
$$

Apply now (18.16) and (18.17) to the bar system shown in Fig. 94. To shorten the notation introduce dimensionless loading, dimensionless reactive forces and elongations

$$
p=\frac{P}{A \sigma_{0}}, \quad r_{i}=\frac{R_{l}}{A \sigma_{0}}, \quad \lambda_{i}=\frac{\Delta l_{i}}{l}, \quad i=1,2,3,
$$

and the dimensionless parameters

$$
\mu=\frac{\gamma l}{\sigma_{0}}, \quad s=\frac{\sigma_{0}}{E} .
$$

The basic equations of statics and geometry take the form

$$
\begin{gathered}
r_{1}+r_{2}+r_{3}-3 \mu-p=0, \\
3 r_{1}+r_{2}-r_{3}-3 \mu=0, \\
\lambda_{1}+\lambda_{3}-2 \lambda_{2}=0,
\end{gathered}
$$

and together with (18.16) describe the functions $r_{i}=r_{i}(p)$. To determine the decohesive carrying capacity of the system we put $r_{3}=1$ (then $\varepsilon \rightarrow \infty$ at the upper 
cross-section of the bar " 3 "), and use for this bar (18.17) instead of (18.16). From the first two equations (18.20) we find $\hat{p}$,

$$
\hat{p}=2-2 r_{1}
$$

and the third Eq. (18.20) may be reduced to a transcendental equation with respect to the reactive force $r_{1}$ :

$$
\begin{array}{r}
\left(1+6 \mu-7 r_{1}\right) c+\left[\ln \frac{\mu\left(1+\mu-r_{1}\right)}{\left(3 r_{1}-2 \mu\right)^{2}}+\frac{1-r_{1}}{\mu} \ln \frac{1+\mu-r_{1}}{1-r_{1}}-\right. \\
\left.-6 \frac{r_{1}-\mu}{\mu} \ln \frac{3 r_{1}-2 \mu}{3\left(r_{1}-\mu\right)}\right](1-c)=0 .
\end{array}
$$

This equation can easily be solved with respect to the material constant $c$, and such an inverse procedure makes it possible to present $p$ as a function of two parameters: $\mu$ (influence of own weight of the bars), and $\vartheta=1-c$ (deviations from a perfectly elastic-plastic material), Fig. 95.

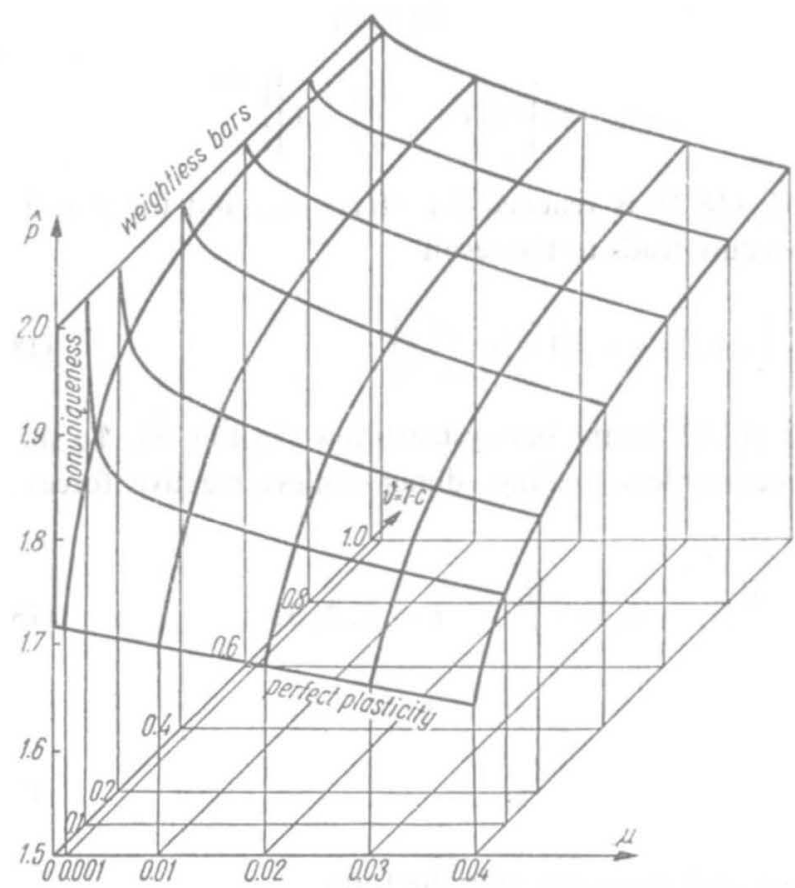

Fig. 95. Decohesive carrying capacity of a bar system in the case of asymptotically perfect plasticity

The point $\mu=\vartheta=0$ (weightless, perfectly elastic-plastic system) is a singular point, and the value of $\hat{p}$ at this point depends on the limiting procedure. Indeed, if we assume at first $\mu=0$, then for any $\vartheta$ the limit carrying capacity $\hat{p}=\overline{\bar{p}}=2$ is reached. Conversely, if we assume at first $\vartheta=0$, then for any $\gamma$ the decohesive carrying capacity is given by (18.15), and for $\gamma \rightarrow 0$ we obtain $\hat{p} \rightarrow 12 / 7$. 
To investigate the singularity, let us derive power series expansions of (18.21) in the vicinity of $\vartheta=0$ and in the vicinity of $\mu=0$. The first expansion may be presented in the form of an ordinary power series of $\vartheta$; we finally obtain [4.569],

$$
\begin{aligned}
r_{1}=\frac{1+6 \mu}{7} & +\frac{1}{7}\left[\ln \frac{7 \mu(6+\mu)}{(3+4 \mu)^{2}}+\frac{6(1-\mu)}{7 \mu} \ln \frac{6+\mu}{2(3+4 \mu)}\right]\{\vartheta+ \\
+ & {\left.\left[1+\frac{1}{7 \mu} \ln \frac{6(1-\mu)}{6+\mu}+\frac{6}{7 \mu} \ln \frac{3(1-\mu)}{3+4 \mu}\right] \vartheta^{2}+\ldots\right\} . }
\end{aligned}
$$

To obtain the second expansion, we must assume a generalized power series of $\mu$ the first term of which is proportional to $\sqrt{\mu}$; longer calculations lead to

$$
r_{1}=\left(\frac{1}{3} \exp \frac{1-2 \vartheta}{\vartheta}\right) \sqrt{\mu}+\left[\frac{5}{6}+\left(\frac{1}{3}-\frac{1}{18 \vartheta}\right) \exp \frac{1-2 \vartheta}{\vartheta}\right] \mu+\ldots
$$

Putting $\vartheta=0$ in (18.22), we obtain $r_{1}=\frac{1}{7}(1+6 \mu)$, and hence $\hat{p}=\frac{12}{7}(1-\mu)$, whereas putting $\mu=0$ in (18.23), we obtain $r_{1}=0, \hat{p}=2$; however, for small $\mu$ series (18.22) becomes divergent, and for small $\vartheta$ series (18.23) becomes divergent. Such divergencies are typical in the vicinity of a singular point.

K. Szuwalski investigated in [4.569] also another type of non-homogeneity of the stress state, connected with a slight change of the cross-section of the bars. The results of his investigations do not differ from those given above: the point of vanishing deviations from the constant cross-section and of vanishing deviations from perfect plasticity is a singular point similar to that shown in Fig. 95.

\subsection{The Stiissi-Kollbrunner paradox}

F. Stüssi and C. F. Kollbrunner [4.557] analysed in 1935 the limit carrying capacity of statically indeterminate beams and in the case of the beam shown in Fig. 96 pointed out the following paradox: the limit load $\overline{\bar{P}}$ equals $\overline{\bar{P}}=8 \overline{\bar{M}} / l_{2}$ (where $\bar{M}$

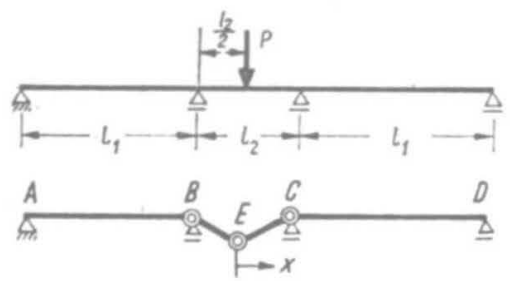

Fig. 96. The Stüssi-Kollbrunner paradox

is the limit bending moment for the cross-section) and is independent of the ratio of the span lengths $l_{1} / l_{2}$. However, for $l_{1} \rightarrow \infty$ the middle span is practically unaffected by the outer spans and we shoul dobtain $\overline{\bar{P}}=4 \overline{\bar{M}} / l_{2}$ as for a simply supported beam. Hence the case $l_{1} \rightarrow \infty$ leads to a certain discontinuity of the result, which is not justified from the physical point of view.

The experimental tests carried out by Stüssi and Kollbrunner [4.557], and also by H. Maier-Leibnitz [4.357], do not agree with the classical limit analysis: the 
real carrying capacity depends on the ratio $l_{1} / l_{2}$. A. M. Freudenthal [4.146] noticed that these results lie between the elastic and the limit carrying capacities, $\bar{P}$ and $\overline{\bar{P}}$ respectively, and proposed to assume-quite arbitrarily - the arithmetic mean of $\bar{P}$ and $\overline{\bar{P}}$ as the real carrying capacity of the beam.

Several attempts have been made to clarify the Stüssi-Kollbrunner paradox. P. S. Symonds and B. G. Neal [4.563] calculated the deflections by assuming an ideal I-section and found that the central deflection increases infinitely in the limiting case $l_{1} \rightarrow \infty$. Their calculations were extended by $\mathrm{K}$. A. Reckling [2.141], who tried to introduce a "distributed plastic hinge" with the length $0.1 l_{2}$. However, those calculations admitted a finite jump of the rotation angle $\alpha$ under the force, which contradicts the continuity requirements. It turns out that the paradox under discussion disappears if we consistently adopt the assumptions of a continuous medium and perform the calculations only until the formation of the first inadmissible discontinuity.

Such a discontinuity is introduced by the plastic hinge, if it is achieved at finite deflection of the beam; according to A. R. Rzhanitsyn [4.492] this case occurs if the bending moment reaches its strong maximum with a simultaneous jump in its derivative, under concentrated force. Indeed, the plastic hinge may be understood as corresponding to the limit carrying capacity of the cross-section: an infinitesimal rotation at a constant bending moment. For a rigid-plastic multi-span beam three such infinitesimal rotations describe a certain mechanism of plastic collapse (Fig. 96), but, in general, this mechanism will not be reached by an elastic-plastic beam, since the formation of the first hinge under the concentrated force will terminate the process and the decohesive carrying capacity is reached. Namely, any finite rotation angle $\alpha$ in the hinge is impossible, since it cannot be described by a displacement field which is continuous or contains admissible discontinuities. It leads to vacancies on the tensile side and to overlapping of the material on the compressive side of the beam, Fig. 97.

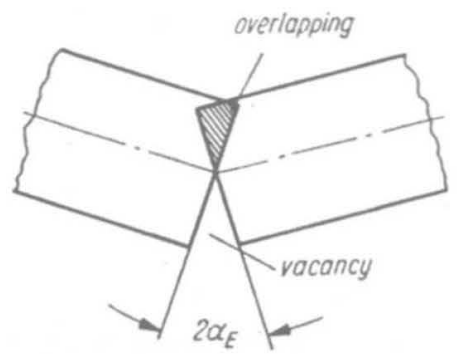

Fig. 97. Inadmissible discontinuities in a plastic hinge with a finite angle of mutual rotation

Following the paper [4.586] by Tran-le Binh and M. Życzkowski, we calculate the decohesive carrying capacity of the Stüssi-Kollbrunner beam. This quantity depends continuously on the length of the outer span $l_{1}$, and in this statement of the problem no paradox occurs. 
Because of the symmetry of the beam, consider only its right-hand side, Fig. 96. The bending moment under the force will be equal to the limit carrying capacity of the cross-section, $\bar{M}$. Hence, assuming no rotation angle at this point, we determine the decohesive carrying capacity, whereas admitting rotation and assuming the subsequent maximal moment $\left|M_{C}\right|$ equal to $\bar{M}$, we estimate the inadmissible discontinuity determined by the magnitude of $\alpha$ under the force, corresponding to the classical mechanism of plastic collapse.

For a beam of a rectangular cross-section $b \times h$ we introduce a dimensionless force $p$, a dimensionless coordinate $\xi$, and a dimensionless deflection $v$ :

$$
\begin{aligned}
& p=\frac{l_{2}}{\overline{\bar{M}}} P=\frac{4 l_{2}}{b h^{2} \sigma_{0}} P, \\
& \xi=\frac{x}{L}=\frac{x}{l_{2} / 2+l_{1}}, \\
& v=\frac{E h}{3 \sigma_{0} l_{2}^{2}} w,
\end{aligned}
$$

where $w$ denotes physical deflection. The geometry of the beam will be characterized by the ratio

$$
k=\frac{l_{2}}{2 L}=\frac{l_{2}}{l_{2}+2 l_{1}}, \quad 0<k<1 .
$$

The dimensionless bending moments in the inner span, $m_{1}$, and in the outer span, $m_{11}$, equal

$$
\begin{aligned}
& m_{1}=\frac{M_{1}}{\bar{M}}=1-\frac{p}{4 k} \xi, \quad 0 \leqslant \xi \leqslant k, \\
& m_{\mathrm{II}}=\frac{M_{\mathrm{II}}}{\overline{\bar{M}}}=-\frac{p-4}{4(1-k)}(1-\xi), \quad k \leqslant \xi \leqslant 1 .
\end{aligned}
$$

Integrating the corresponding differential equation of bending within the elastic range (without the assumption $m(0)=1$ ), we easily determine the elastic carrying capacity of the beam:

$$
\bar{p}=\frac{16}{3} \frac{1+2 k}{2+k} .
$$

In the elastic-plastic range the number of intervals for integration equals three and may increase to five if at the support $C$ the elastic carrying capacity of the crosssection is exceeded, $|m(k)|>2 / 3$. Substituting $m(k)=-2 / 3$ into (18.26) or (18.27), we find $p=20 / 3$ as the boundary value of the force, separating the range of three intervals for integration from the range of five intervals (in the state of decohesive carrying capacity or of limit carrying capacity).

In the case of five intervals the differential equations of bending are 


$$
\begin{array}{ll}
v_{1}^{\prime \prime}(\xi)=-\frac{4 \sqrt{k}}{3 \sqrt{3} \sqrt{p \xi}}, & 0 \leqslant \xi \leqslant \xi_{1}, \\
v_{2}^{\prime \prime}(\xi)=\frac{p \xi}{4 k}-1 . & \xi_{1} \leqslant \xi \leqslant \xi_{2}, \\
v_{3}^{\prime \prime}(\xi)=\frac{4 \sqrt{k}}{3 \sqrt{3} \sqrt{8 k-p \xi},} & \xi_{2} \leqslant \xi \leqslant k, \\
v_{4}^{\prime \prime}(\xi)=\frac{4 \sqrt{1-k}}{3 \sqrt{3} \sqrt{(p-4) \xi+8-4 k-p}}, & k \leqslant \xi \leqslant \xi_{3} \\
v_{5}^{\prime \prime}(\xi)=-\frac{p-4}{4(1-k)}(\xi-1), & \xi_{3} \leqslant \xi \leqslant 1,
\end{array}
$$

where the boundary coordinates are

$$
\xi_{1}=\frac{4 k}{3 p}, \quad \xi_{2}=\frac{20 k}{3 p}, \quad \xi_{3}=\frac{3 p+8 k-20}{3(p-4)}
$$

The general integrals of (18.29) are

$$
\begin{aligned}
& v_{1}(\xi)=-\frac{16 \sqrt{k \xi^{3}}}{9 \sqrt{3 p}}+A_{1} \xi+A_{2}, \\
& v_{2}(\xi)=\frac{p \xi^{3}}{24 k}-\frac{\xi^{2}}{2}+B_{1} \xi+B_{2}, \\
& v_{3}(\xi)=\frac{16 \sqrt{k(8 k-p \xi)^{3}}}{9 \sqrt{3} p^{2}}+C_{1} \xi+C_{2}, \\
& v_{4}(\xi)=\frac{16 \sqrt{1-k} \sqrt{[(p-4) \xi+8-4 k-p]^{3}}}{9 \sqrt{3}(p-4)^{2}}+D_{1} \xi+D_{2}, \\
& v_{5}(\xi)=-\frac{p-4}{8(1-k)}\left(\frac{\xi^{3}}{3}-\xi^{2}\right)+E_{1} \xi+E_{2} .
\end{aligned}
$$

The boundary conditions $v(k)=0$, and $v(1)=0$, the eight continuity conditions, and the additional condition (symmetry) $v_{1}^{\prime}(0)=0$, make it possible to determine ten integration constants and the load parameter, i.e. the decohesive carrying capacity $\hat{p}$. The final equation takes the form

$$
3 \sqrt{3(8-\hat{p})}\left[(1+2 k) \hat{p}^{2}+(4-28 k) \hat{p}+48 k\right]-80(1-k) \hat{p}=0 .
$$

It determines $\hat{p}$ in the range of five intervals under consideration, $\hat{p}>20 / 3$. Substituting this boundary value into (18.32), we find the corresponding boundary value of $k$, namely $k=5 / 11$, and hence $(18.32)$ is valid if $5 / 11 \leqslant k \leqslant 1$. For $k=1$ (clamped beam) we obtain $\hat{p}=8$, and here $\hat{p}=\overline{\bar{p}}$.

If $0<k<5 / 11$, then the beam should be divided into three intervals only, (1), (2), and (5), since the elastic-plastic intervals (3) and (4) disappear. In this case the decohesive carrying capacity is determined by an explicit equation 


$$
\hat{p}=\frac{4}{2+k}[1+2 k+\sqrt{(1+k)(1+5 k)}] .
$$

In the limiting case $k \rightarrow 0$ we obtain $\hat{p}=4$ and this result corresponds to the classical limit load for a simply supported beam. Inside the interval $0 \leqslant k \leqslant 1$ the dependence $\hat{p}=\hat{p}(k)$ is continuous and no paradox appears. This dependence is shown in Fig. 98 , together with $\bar{p}=\bar{p}(k)$ and the paradoxical $\overline{\bar{p}}=\overline{\bar{p}}(k)$.

Paper [4.586] gives also an estimation of inadmissible discontinuities in the classical limit state of the Stiussi-Kollbrunner beam. They are characterized by the

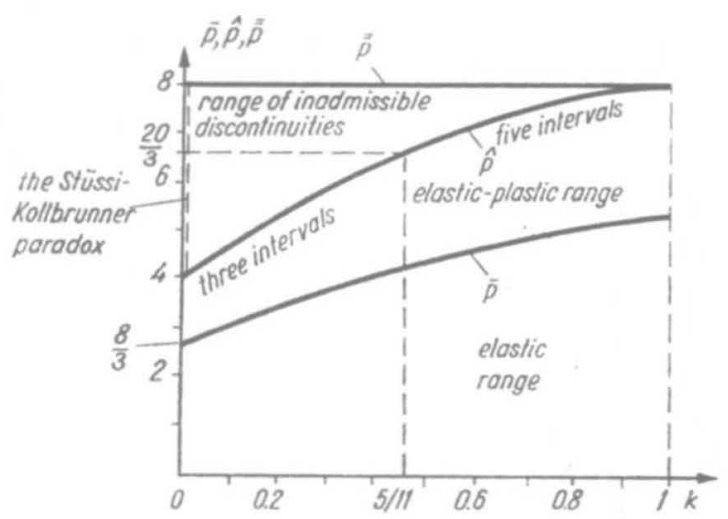

Fig. 98. Elastic, decohesive and limit carrying capacities of the Stüssi-Kollbrunner beam

angle of mutual rotation of the two adjacent sections in the hinge. For example, if $\sigma_{0} / E=0.001, l_{2} / h=50, l_{1}=l_{2}$, then this inadmissible angle equals 0.0658 $=3^{\circ} 46^{\prime}$.

The decohesive carrying capacity of several other statically indeterminate beams was evaluated by K. Szuwalski and Tran-Le Binh in [4.568].

Of course, the above conclusions do not mean that the classical theory of the limit carrying capacity of beams is useless. In most cases it makes use of "relaxed" continuity conditions, exceeding the basic requirements of the continuum mechanics; on the other hand, in most cases of real materials an even small plastic hardening justifies such a "relaxation" and the picture experimentally observed is not very far from that predicted by the theory of plastic hinges with finite rotations. However, the theory making use of the concept of decohesive carrying capacity is more consistent and can explain many paradoxes encountered in the classical limit analysis.

It should also be noted that Hill's definition of the limit carrying capacity, using other limiting procedures (infinitesimal motion of a rigid-hardening structure), may lead to different conclusions as regards the existence of solutions and the decohesive carrying capacity (E. N. Fox [4.144]); this fact stresses once more the possibility of discrepancies between the two definitions of the limit carrying capacity discussed in Sec. 17.1. 


\subsection{Decohesive carrying capacity of disks and infinite circularly-symmetric sheets}

An analysis of circularly-symmetric disks loaded in their plane results in the simplest plane-stress problems, since partial differential equations are here reduced to ordinary differential equations. Usually the elastic-plastic range for such perfectly elasticplastic disks may be extended up to their limit carrying capacity, but in some cases the process terminates with the decohesive carrying capacity.

As an example consider an annular disk with rigid circular inclusion (e.g. permanently joined at inner radius $r=a$ with a rigid shaft), subject to normal

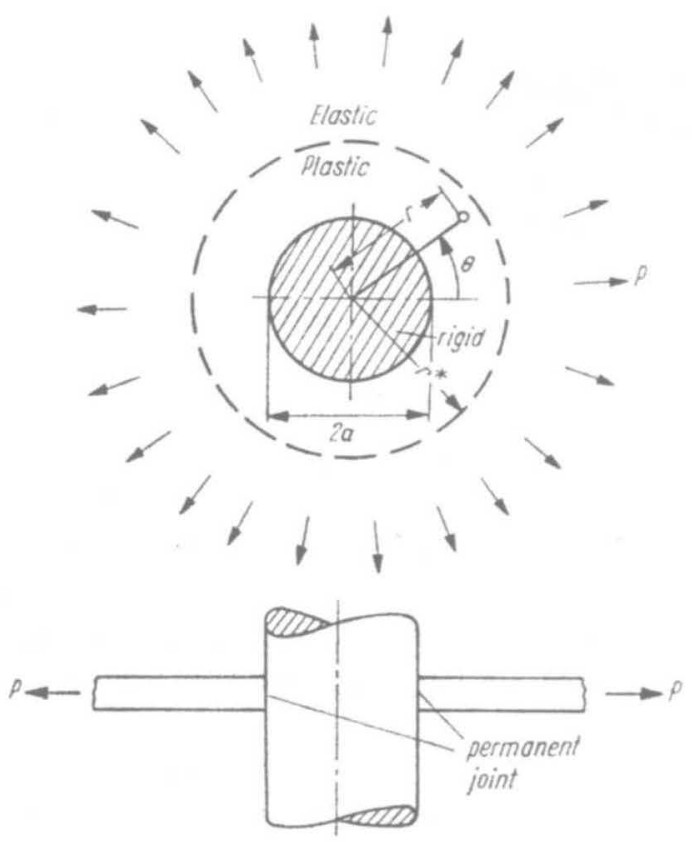

Fig. 99. An infinite sheet with a rigid circular inclusion

tractions $p$ at the outer radius $r=b$. To simplify the analysis we quote here only the results for the limiting case $b \rightarrow \infty$ (infinite sheet, Fig. 99); the finite value of $b$ was analysed in detail by K. Szuwalski [4.570].

For small values of the traction $p$ the whole sheet will be elastic and the general expressions for the stresses and radial displacement are

$$
\sigma_{r}=A+\frac{B}{r^{2}}, \quad \sigma_{\theta}=A-\frac{B}{r^{2}}, \quad u=\frac{1}{E}\left[(1-v) A r-(1+v) \frac{B}{r}\right] .
$$

The boundary conditions

$$
u(a)=0, \quad \sigma_{r}(\infty)=p,
$$

yield

$$
A=p, \quad B=\frac{1-v}{1+v} p a^{2}
$$


The maximal stress intensity occurs at $r=a$, and making use of the Huber-MisesHencky failure hypothesis, we determine the elastic carrying capacity of the sheet $\bar{p}$ by the formula

$$
\bar{p}=\frac{1+\nu}{2 \sqrt{1-\nu+\nu^{2}}} \sigma_{0} .
$$

We have assumed here an arbitrary elastic compressibility of the material; it turns out that this compressibility has an essential influence on the decohesive carrying capacity of the sheet.

For $p>\vec{p}$ a plastic zone $a<r<r^{*}$ appears. The distribution of stresses in the plastic zone is statically determinate (up to a certain constant) and may be determined regardless of any particular theory of plasticity. Use the Nadai-Sokolovsky parametrization of the yield condition (cf. Sec. 19.4; for the disks we change the symbol $\omega$ into $\zeta$ to avoid confusion with angular velocity),

$$
\sigma_{r}=\frac{2}{\sqrt{3}} \sigma_{0} \sin \zeta, \quad \sigma_{\theta}=\frac{2}{\sqrt{3}} \sigma_{0} \sin \left(\zeta+\frac{\pi}{3}\right),
$$

where $\zeta$ is a parameter, the distribution of which $\zeta=\zeta(r)$ should be found. The stresses $\sigma_{r}$ and $\sigma_{\theta}$ are both non-negative here and it turns out that $\sigma_{r} \geqslant \sigma_{\theta}$, and thus $\pi / 3 \leqslant \zeta \leqslant 2 \pi / 3$. Substituting (18.38) into the condition of equilibrium in polar coordinates, we obtain the equation

$$
\frac{d \zeta}{d r} \cos \zeta+\frac{1}{r}\left[\sin \zeta-\sin \left(\zeta+\frac{\pi}{3}\right)\right]=0,
$$

which may be solved with respect to $r$, thus determining the function inverse with respect to $\zeta=\zeta(r)$ :

$$
r=\frac{C_{1} \exp (\sqrt{3} \zeta / 2)}{\sqrt{|\sin (\zeta-\pi / 3)|}},
$$

$C_{1}$ being the constant of integration. The boundary condition for the plastic zone, at $r=a$, refers to the displacements (or velocities) and not to the stresses, and thus the constant $C_{1}$ cannot be evaluated without considering the distribution of displacements.

To determine displacements we use the Hencky-Ilyushin deformation theory. The Prandtl-Reuss theory was applied to that problem in [1.18]; it resulted in much more complicated formulae, involving non-elementary integrals, but the qualitative discussion remained unchanged.

Equations (9.29) take here the form

$$
\begin{aligned}
& \varepsilon_{r}=\frac{1}{3 K} \sigma_{m}+\varphi\left(\sigma_{r}-\sigma_{m}\right), \\
& \varepsilon_{\theta}=\frac{1}{3 K} \sigma_{m}+\varphi\left(\sigma_{\theta}-\sigma_{m}\right) .
\end{aligned}
$$


Elimination of the unknown function $\varphi$ leads to the equation

$$
\left(2 \sigma_{\theta}-\sigma_{r}\right) \varepsilon_{r}-\left(2 \sigma_{r}-\sigma_{\theta}\right) \varepsilon_{\theta}=\frac{1}{3 K}\left(\sigma_{\theta}^{2}-\sigma_{r}^{2}\right) .
$$

The general solution of this equation with respect to the radial displacement $u$ and the strains $\varepsilon_{\boldsymbol{r}}$ and $\varepsilon$ was given in paper [4.630]. Here it is more convenient to express the stresses by means of the parameter $\zeta(18.38)$, not introduced in [4.630]. Using the compatibility condition

$$
\varepsilon_{r}=\varepsilon_{\theta}+r \frac{d \varepsilon_{\theta}}{d r}
$$

which may be deduced for the circularly-symmetric states from (7.51) (the third equation), or derived directly from the strain-displacement relations, we obtain

$$
r \cos \zeta \frac{d \varepsilon_{\theta}}{d r}+\sqrt{3} \cos \left(\zeta+\frac{1}{6} \pi\right) \varepsilon_{\theta}=\frac{2 \sigma_{0}}{3 K \sqrt{3}} \sin \left(\zeta+\frac{1}{6} \pi\right) \cos \left(\zeta+\frac{1}{6} \pi\right),
$$

and eliminating $r$ by using (18.39), we have

$$
\frac{d \varepsilon_{\theta}}{d \zeta}+\sqrt{3} \varepsilon_{\theta}=\frac{2 \sigma_{0}}{3 K \sqrt{3}} \sin \left(\zeta+\frac{1}{6} \pi\right) \text {. }
$$

The general solution of this linear ordinary differential equation may be written in the form

$$
\varepsilon_{\theta}=\frac{\sigma_{0}}{3 K \sqrt{3}} \sin \zeta+C_{2} \exp (-\sqrt{3} \zeta),
$$

where $C_{2}$ denotes the constant of integration.

Substituting $u=r \varepsilon_{\theta}$ with $r$ determined by (18.40), we express the radial displacement in terms of the parameter $\zeta$ as follows:

$$
u=\frac{C_{1} \sigma_{0} \sin \zeta \exp (\sqrt{3} \zeta / 2)}{3 K \sqrt{\left|3 \sin \left(\zeta-\frac{1}{3} \pi\right)\right|}}+C_{1} C_{2} \frac{\exp (-\sqrt{3} \zeta / 2)}{\sqrt{\left|\sin \left(\zeta-\frac{1}{3} \pi\right)\right|}} .
$$

The radial strain $\varepsilon_{r}$ equals

$$
\varepsilon_{r}=\frac{d u}{d r}=\frac{d u}{d \zeta} \frac{d \zeta}{d r}
$$

and having performed the differentiations and several simple rearrangements of the trigonometric functions, we finally obtain

$$
\varepsilon_{r}=\frac{\sigma_{0}}{3 K \sqrt{3}} \sin \left(\zeta+\frac{1}{3} \pi\right)+\frac{C_{2}}{\cos \zeta} \sin \left(\zeta-\frac{1}{6} \pi\right) \exp (-\sqrt{3} \zeta) .
$$

Now we may combine the general solution for the elastic zone, (18.34), denoted henceforth by the superscript (e), with the general solution for the plastic zone, (18.38), (18.40), (18.46), (18.47) and (18.49), denoted by (p). We have to evaluate four constants of integration, $A, B, C_{1}$ and $C_{2}$, the radius separating the 
zones $r^{*}$, and the values of the parameter $\zeta_{\mathbf{a}}$ and $\zeta^{*}$, corresponding to $a$ and $r^{*}$, i.e. seven unknowns in all. To this aim we have the following seven boundary conditions:

$$
\begin{aligned}
& u^{(p)}=0 \text { and } \zeta=\zeta_{a} \quad \text { for } r=a, \\
& u^{(p)}=u^{(e)}, \quad \sigma_{r}^{(p)}=\sigma_{r}^{(e)}, \quad \sigma_{e}^{(e)}=\sigma_{0} \text { and } \zeta=\zeta^{*} \\
& \sigma_{r}^{(e)}=p \quad \text { for } r \rightarrow \infty,
\end{aligned}
$$

where $\sigma_{e}^{(e)}$ denotes the stress intensity in the elastic zone. Solving these equations successively, we may express six unknowns in terms of the remaining one, $\zeta_{a}$, as follows:

$$
\begin{aligned}
& A=p=q \sigma_{0} \\
& \left.B=\frac{\sigma_{0} a^{2}}{\sqrt{3}} \sin \left(\zeta_{a}-\frac{1}{3} \pi\right) \exp \mid \sqrt{3}\left(\frac{1}{3} \pi+\arccos q-\zeta_{a}\right)\right] \\
& C_{1}=a \exp \left(-\frac{\sqrt{3}}{2} \zeta_{a}\right) \sqrt{\left|\sin \left(\zeta_{a}-\frac{1}{3} \pi\right)\right|} \\
& C_{2}=-\frac{\sigma_{0}}{3 K \sqrt{3}} \sin \zeta_{a} \exp \left(\sqrt{3} \zeta_{a}\right) \\
& r^{*}=\frac{a}{\sqrt[4]{1-q^{2}}} \sqrt{\left|\sin \left(\zeta_{a}-\frac{1}{3} \pi\right)\right|} \exp \left[\frac{\sqrt{3}}{2}\left(\frac{1}{3} \pi+\arccos q-\zeta_{a}\right)\right] \\
& \zeta^{*}=\pi-\arcsin \left(\frac{1}{2}\left(\sqrt{1-q^{2}}+q \sqrt{3}\right)\right)=\frac{1}{3} \pi+\arccos q,
\end{aligned}
$$

where $q=p / \sigma_{0}$ denotes the dimensionless traction at infinity. The last unknown, $\zeta_{a s}$ is determined by a transcendental equation, $f\left(\zeta_{a}, q, v\right)=0$. However, this equation may be solved with respect to Poisson's ratio $v$, and an inverse procedure may be used:

$$
v=\frac{1}{2}-\frac{3 \sqrt{1-q^{2}}-q \sqrt{3}}{4 \sin \zeta_{a}} \exp \left[\sqrt{3}\left(\frac{1}{3} \pi+\arccos q-\zeta_{a}\right)\right] .
$$

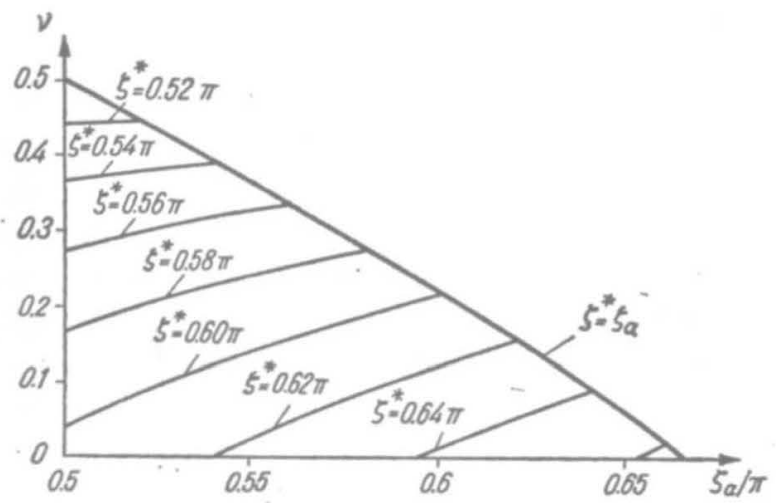

Fig. 100. Determination of the stress distribution in an elastic-plastic sheet 
Figure 100 presents the diagram of the even simpler function $v=\left(\zeta_{a}, \zeta^{*}\right)$, namely

$$
v=\frac{1}{2}+\frac{\sqrt{3}}{2} \frac{\cos \zeta^{*}}{\sin \zeta_{a}} \exp \left[\sqrt{3}\left(\zeta^{*}-\zeta_{a}\right)\right],
$$

and makes it possible to find $\zeta_{a}=\zeta_{a}\left(\zeta^{*}, v\right)$ in a graphical way. The corresponding value of $q$ may then be found from the last formula of (18.51) inverted:

$$
q=\cos \left(\zeta^{*}-\frac{1}{3} \pi\right)
$$

the whole solution is then presented in parametrical form with $\zeta^{*}$ as the parameter. Figure 100 shows also the propagation of plastic deformations. The plastic zone starts when $\zeta^{*}=\zeta_{a}$ (limiting line). With an increasing load both $\zeta_{a}$ and $\zeta^{*}$ decrease (motion along $v=$ const to the left). This process ends for $\zeta_{a}=\frac{1}{2} \pi$, as it will be shown below.

Indeed, substituting (18.51) into (18.49), we determine the radial strain $\varepsilon_{r}^{(p)}$ by the formula

$$
\varepsilon_{r}^{(p)}=\frac{\sigma_{0}}{3 K \sqrt{3}}\left\{\sin \left(\zeta+\frac{1}{3} \pi\right)-\frac{\sin \zeta_{a} \sin \left(\zeta-\frac{1}{6} \pi\right)}{\cos \zeta} \exp \left[\sqrt{3}\left(\zeta_{a}-\zeta\right)\right]\right\} .
$$

The maximal value of $\varepsilon_{r}^{(p)}$ occurs at $r=a$ and equals

$$
\varepsilon_{r a}^{(p)}=\frac{\sigma_{0}}{3 K \sqrt{3}} \frac{\cos \left(2 \zeta_{a}-\frac{1}{6} \pi\right)}{\cos \zeta_{a}} .
$$

For $\zeta_{a}$ approaching $\frac{1}{2} \pi$ the strain $\varepsilon_{r a}^{(p)}$ increases infinitely, which leads to an inadmissible discontinuity and terminates the process. The corresponding decohesive

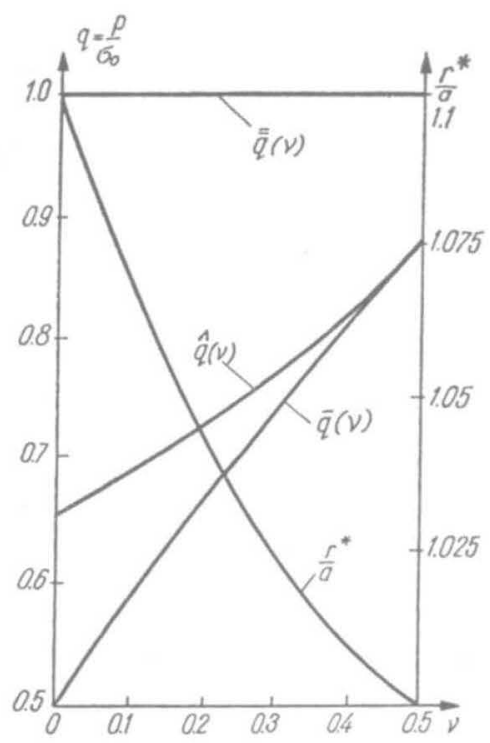

Fig. 101. Elastic, decohesive and limit carrying capacities of a sheet with rigid circular inclusion 
carrying capacity $\hat{q}$ is determined by (18.54), where $\zeta^{*}$ is to be found from Fig. 100 for $\zeta_{a}=\frac{1}{2} \pi$ and for the given value of Poisson's ratio $v$. Thus $\hat{q}$ depends on $v$; this dependence is shown in Fig. 101 together with the plots $\bar{q}=\bar{q}(v),(18.37)$, and with the limit carrying capacity $\overline{\bar{q}}$, determined for the sheet after decohesion. For an incompressible material, $v=1 / 2$, the plastic zone starts at $\zeta_{a}=\frac{1}{2} \pi$, and the decohesion occurs immediately, $\hat{q}=\bar{q}$.

An example of the distribution of stresses and strains at the moment of decohesion is presented in Fig. 102. Poisson's ratio is assumed to be $v=0.3$ and the radius

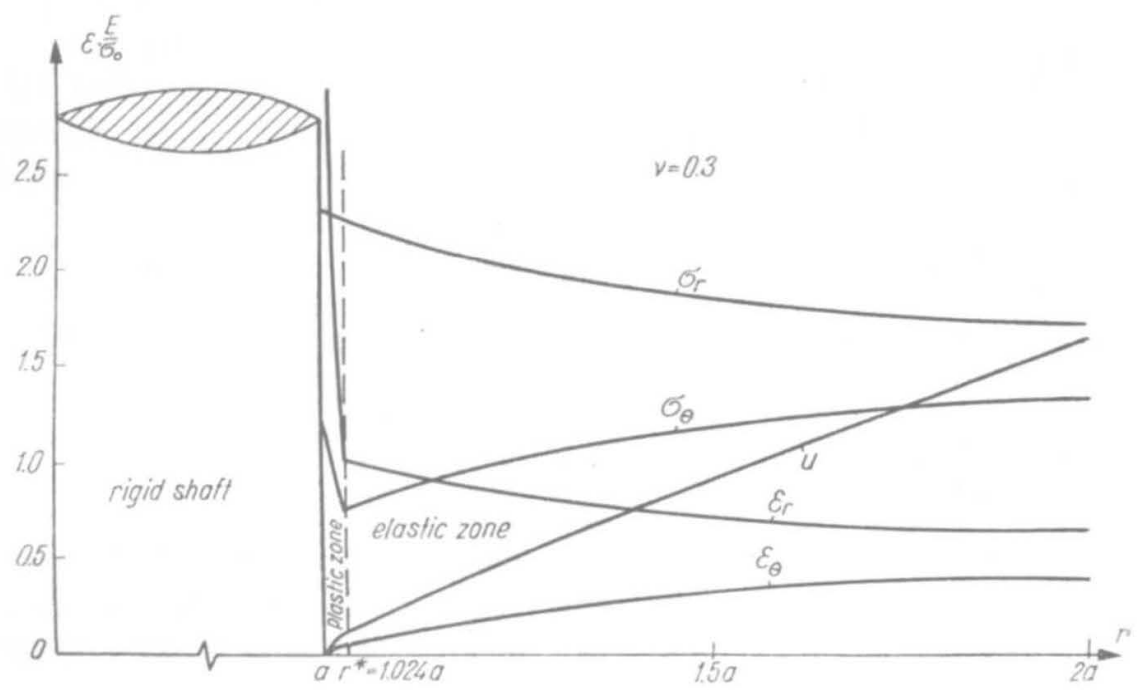

Fig. 102. Stress and strain distributions corresponding to decohesive carrying capacity

$r^{*}$, determined by (18.51), equals $r^{*}=1.024 a$; thus the decohesion corresponds in this case to a small but finite plastic zone. In the similar thermoplastic problem, [4.642], the plastic zone is much larger, namely $r^{*}=1.751 a$.

Other cases of the decohesive carrying capacity of disks were discussed by K. Szuwalski: asymptotically perfect plasticity [4.571], variable thickness [4.572], non-homogeneity of the material of rotating disks [4.573]. A full homogeneous disk of constant thickness will always deform up to its limit carrying capacity (if no separate criteria of decohesion are introduced), but changes in thickness or nonhomogeneity may result in decohesive carrying capacity even without rigid inclusion.

On the other hand, J. Lenard and J. B. Haddow [4.324] determined the limit carrying capacity of a rotating disk with rigid inclusion, making use of "relaxed" continuity conditions permitting the discontinuity of the displacement $u$. Such an approach is hardly acceptable from the theoretical point of view because of the large value of that discontinuity, but the result may be practically useful if we bear in mind plastic hardening of real materials. 


\subsection{Decohesive carrying capacity of disks and sheets in the light of finite-strain theory}

As in the case of bars, the assumed criterion of decohesion $\varepsilon_{r}=d u / d r \rightarrow \infty$ is inconsistent with the small-strain theory; it determines certain limits of applicability of this theory, but further analysis based on a finite-strain theory is desirable.

Following the paper [4.643] by K. Szuwalski and M. Życzkowski we present here such a solution, based on the simplest finite-strain theory (Nadai-Davis in finite form, (15.80)). This theory uses true stresses and logarithmic strains, and hence the basic features of a finite-strain theory are taken into account; however, some simplifications are connected with the assumption of plane stress, since the components $\sigma_{z}, \tau_{z r}$ and $\tau_{z \theta}$ will be neglected, and the stress distribution will be assumed to be uniform throughout the thickness. Further, the condition of a permanent joint with rigid inclusion will be written in the form $U(a)=0$ without considering the axial displacements $U_{z}$, which are assumed to be free.

Consider once more the simplest circularly-symmetrical case of an infinite sheet with rigid circular inclusion, subject to traction $p$ at infinity, Fig. 99. In the polar Lagrangian coordinates $R, \Theta$ the equation of internal equilibrium takes now the form

$$
\frac{1}{h} \frac{d}{d R}\left(h \sigma_{r}\right)+\frac{\sigma_{r}-\sigma_{\theta}}{R+U}\left(1+\frac{d U}{d R}\right)=0,
$$

where $h=h(R)$ denotes current thickness subject to changes during the deformation process, and $U=U(R)$ is the radial displacement. Geometrical equations take the form (7.52) and (7.53) (the compatibility condition), and the third principal strain $\varepsilon_{z}$, equals

$$
\varepsilon_{z}=\ln \frac{h}{h_{0}} .
$$

It turns out that the solution for the plastic zone may be obtained independently and later complemented with the solution for the elastic zone. Assume perfect plasticity related to the true stresses $\sigma_{r}$ and $\sigma_{\theta}$ and parametrize the HMH yield condition by (18.38). The Nadai-Davis equations (15.80) take here the form

$$
\frac{\varepsilon_{r}-\varepsilon_{m}}{\varepsilon_{\theta}-\varepsilon_{m}}=\frac{\sigma_{r}-\sigma_{m}}{\sigma_{\theta}-\sigma_{m}},
$$

where $\varepsilon$ denote logarithmic strains and $\sigma$ true stresses. The law of volume change will be assumed in the classical form (9.7), formally extended to logarithmic strains and true stresses.

The system of eight equations (18.57), (7.52), (18.58), (18.59) and (9.7) contains the following eight unknowns: $\sigma_{r}, \sigma_{\theta}, \zeta, \varepsilon_{r}, \varepsilon_{\theta}, \varepsilon_{z}, U, h$. It can be simplified by introducing the dimensionless logarithmic radius

$$
\Xi=\ln \frac{R}{a},
$$


where $a$ denotes the radius of rigid inclusion. Successive elimination of unknowns reduces that system to the following two first-order differential equations:

$$
\begin{aligned}
& \frac{d \varepsilon_{0}}{d \Xi}=\exp \left\{\frac{\sin \left(\zeta-\frac{1}{3} \pi\right)}{\cos \zeta}\left[\sqrt{3} \varepsilon_{\theta}-\frac{2}{\sqrt{3}} \frac{\sigma_{0}}{E}(1-2 v) \cos \left(\zeta-\frac{1}{3} \pi\right)\right]\right\}-1, \\
& \frac{d \zeta}{d \Xi}=\frac{\frac{d \varepsilon_{\theta}}{d \Xi} \frac{\sqrt{3}}{2} \cos \zeta-\sin \left(\zeta-\frac{1}{3} \pi\right) \cos ^{2} \zeta}{\cos ^{3} \zeta-\frac{\sqrt{3}}{2} \varepsilon_{\theta} \sin \zeta+\frac{2}{\sqrt{3}}(1-2 v) \frac{\sigma_{0}}{E} \sin \zeta\left(\frac{\sqrt{3}}{4} \sin \zeta+\cos ^{3} \zeta\right)} .
\end{aligned}
$$

This system, convenient for numerical integration, requires two initial conditions. To the physical condition $\varepsilon_{\theta}=0$ for $\Xi=0$ we may add $\zeta=\zeta_{a}$ for $\Xi=0$, regarding $\zeta_{a}$ as a parameter; then the external loading $p$ may be evaluated in terms of this parameter.

A decrease of $\zeta_{a}$ results in an increase of loading $p$ as in the small-strain theory, Fig. 100 and formula (18.54). However, the decrease of $\zeta_{a}$ down to $\zeta_{a}=\frac{1}{2} \pi$ as before, is in general impossible. The denominator of (18.61) at the point $\Xi=0$, $\varepsilon_{\theta}=0$, vanishes earlier, hence $d \zeta / d \Xi \rightarrow \infty$ and $d \sigma_{r} / d \Xi \rightarrow \infty$ terminating the process and furnishing the criterion of decohesion in the case under consideration. Indeed, further increase of $p$ is impossible without inadmissible discontinuity. Denote the corresponding value of $\zeta_{a}$ by $\zeta_{a 0}$; then $\zeta_{a 0}$ is determined by the following trigonometric equation

$$
\cos ^{3} \zeta_{a 0}+\frac{2 s}{\sqrt{3}}(1-2 v) \sin \zeta_{a 0}\left(\frac{\sqrt{3}}{4} \sin \zeta_{a 0}+\cos ^{3} \zeta_{a 0}\right)=0,
$$

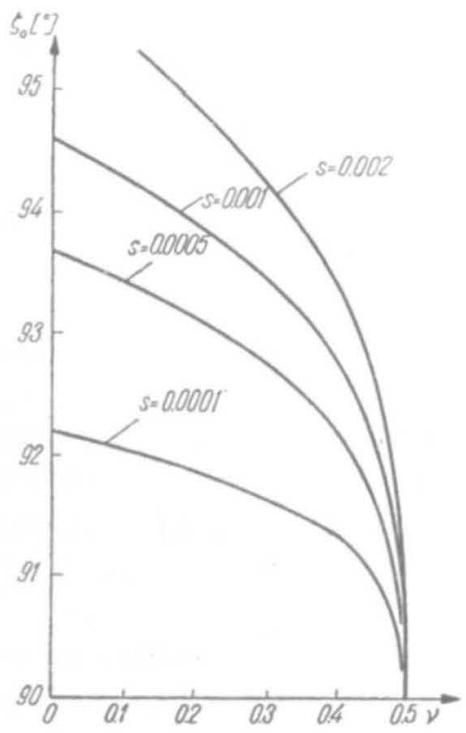

Fig. 103. Solutions of Eq. (18.62) (finite strains in a sheet) 
where $s=\sigma_{0} / E$ is a dimensionless material constant. The solution of (18.62), $\zeta_{a 0}$ $=\zeta_{a 0}(v, s)$, is presented in Fig. 103.

To find the corresponding decohesive carrying capacity $\hat{q}=\hat{p} / \sigma_{0}$ we have to integrate (18.61) numerically. This integration runs over the plastic zone up to the boundary coordinate $\Xi^{*}$ determined by the equation

$$
\varphi=\frac{\varepsilon_{r}-\varepsilon_{\theta}}{\sigma_{r}-\sigma_{\theta}}=\frac{1}{2 G}=\frac{1+\nu}{E} .
$$

Namely, without larger errors, the solution of (18.61) may be combined with the small-strain solution for the elastic zone (18.34), since elastic strains are very small if a not too high value of $s=\sigma_{0} / E$ is assumed. Denoting $\zeta\left(\Xi^{*}\right)=\zeta^{*}$ we find $\hat{q}$ from (18.54). The resulting diagram is shown in Fig. 104. It is very close to that

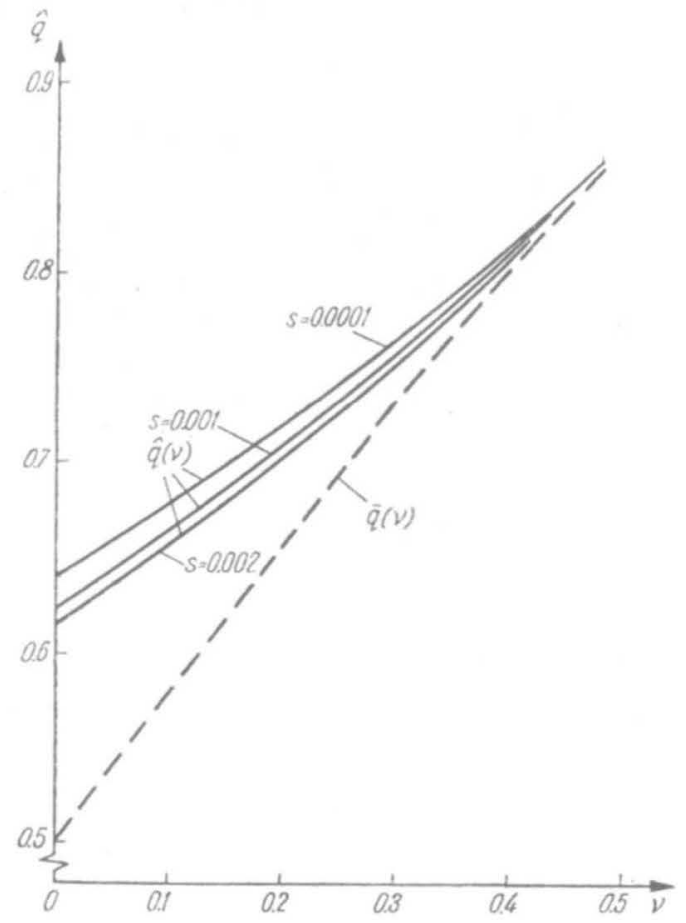

Fig. 104. Elastic and decohesive carrying capacities of a sheet based on a finite-strain theory

obtained from the small-strain theory, Fig. 101, though it is based on a changed criterion of decohesion $d \sigma_{r} / d r \rightarrow \infty$. The values of $\hat{q}$ in Fig. 104 are smaller than those in Fig. 101, and hence we may conclude that in the case under consideration the finite-strain theory predicts an even earlier decohesion than the small-strain theory.

A further step towards increasing accuracy of the analysis would be to regard the disk or sheet as a spatial problem rather than a plane-stress one; this step seems even more important than the application of a physically sounder finite-strain 
theory. Indeed, it may be proved [4.643] that at the stage of decohesion the above solution gives $d h / d r \rightarrow \infty$ for $r=a$, and hence the stresses $\sigma_{z}$ and $\tau_{z r}$ become essential at least in the vicinity of $r=a$. Such a formulation of the problem is highly complicated; in the elastic range a spatial approach for disks was initiated by $\mathrm{A}$. S. Kobayashi and P. R. Trumpler [4.292], and for a Ramberg-Osgood solid a simplified spatial solution was obtained by $\mathrm{H}$. Abé [4.1], but for perfectly elastic-plastic solid a more exact finite-strain analysis of that type is lacking. However, comparing the solutions obtained in Secs. 18.4 and 18.5, one may suppose that in the case of a disk with rigid inclusion or in related problems even a more rigorous treatment results in a certain decohesive carrying capacity connected with the termination of the existence of the solution.

\subsection{Stages of typical deformation processes at the levels $\mathscr{S}$ and $\mathscr{B}$}

Summarizing the considerations of Sec. 17 and Sec. 18, we may delineate successive stages of typical deformation processes at the levels $\mathscr{S}$ or $\mathscr{B}$. Table 4 presents such stages without allowing for geometry changes, and Table 5-with geometry changes taken into account.

\section{Table 4}

Deformation processes in a perfectly elastic-plastic body without allowing for geometry changes

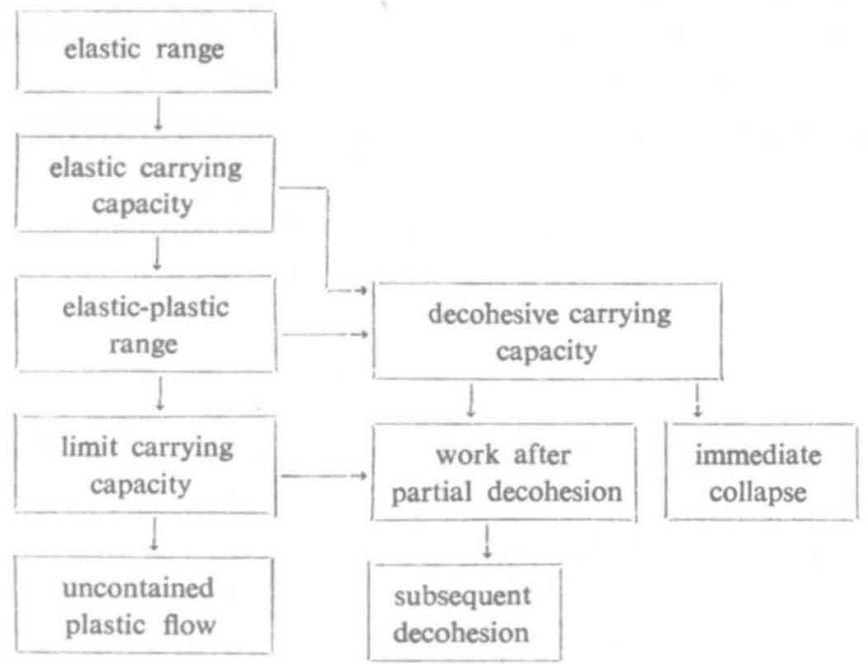

Of course, various deviations from the schemes given in Table 4 and Table 5 are also possible. For example, the problem of simultaneous tension and torsion, discussed in Sec. 17.6, shows that the limit carrying capacity may be preceded by a purely plastic range. 


\section{Table 5}

Deformation processes in a perfectly elastic-plastic body with geometry changes taken into account

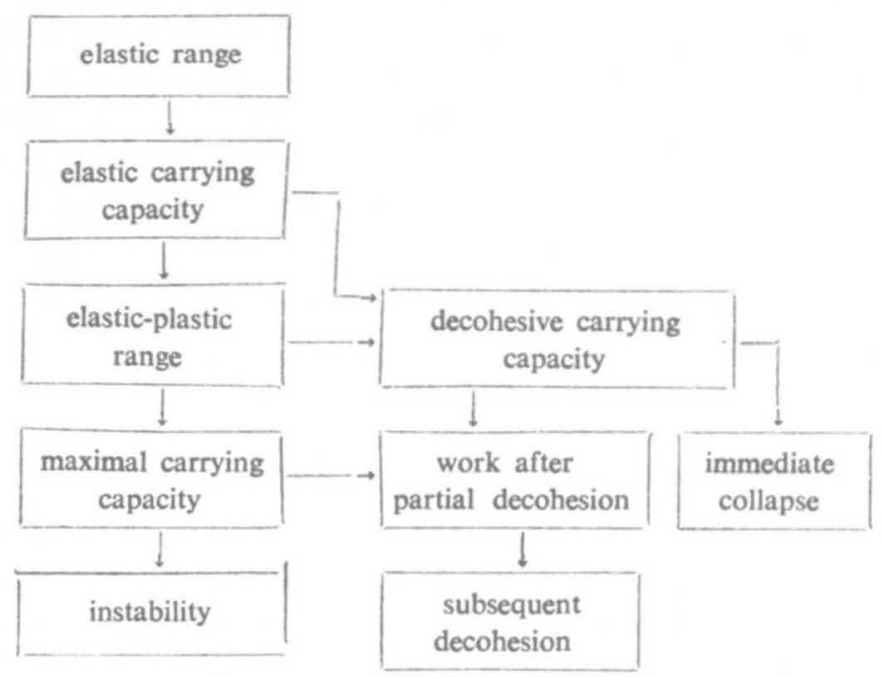

In the case of materials exhibiting plastic hardening, Table 4 would be subject to essential changes since no limit carrying capacity is observed and the decohesive carrying capacity must be described by a separate equation. On the other hand, if such an equation is introduced, then Table 5 may hold unchanged, because the maximal carrying capacity may be observed for any type of the stress-strain diagram.

\subsection{Physical criteria of decohesion under static (monotonic) loadings}

Assuming the schematization of perfect plasticity, we usually identify the yield surface in the stress space with the limit surface of strength, whereas in the strain space no limitation is introduced (no separate criterion of decohesion is formulated); as a rule, such simplification results in negligible errors. On the other hand, taking plastic hardening into account, we have to introduce certain physical criteria of decohesion, so as to avoid infinite strength and obtain a more realistic description of the behaviour of the material. Of course, such criteria may also be employed in the theory of perfect plasticity, but then they result in essential changes and the classical theory must be reformulated.

Various phenomenological criteria of decohesion (fracture, rupture) have been proposed; sometimes they describe different forms of decohesion. To a certain degree, the classical failure hypotheses may also be included here (Galileo, de SaintVenant-Poncelet, etc.), but their effective use in the theory of plasticity is rather limited. J. Datsko and C. T. Yang [4.101, 4.102] proposed a simple criterion which may be expressed in the form

$$
\varepsilon_{I}=\text { const }=\hat{\varepsilon} \text {; }
$$


it resembles that of de Saint-Venant, but is not transferred to the stress space by using Hooke's law, which usually loses its validity well before decohesion. The criterion suggested by V. Panc [4.442] is expressed in stresses and corresponds to a cylinder truncated by another rotationally symmetric surface.

Some authors express the criteria of decohesion in terms of stresses and strains. For example, A. A. Labutin [4.314] described the experiments on steel specimens by the equation

$$
\hat{\varepsilon}_{e}=A+B \frac{\sigma_{m}}{\sigma_{e}},
$$

where $\hat{\varepsilon}_{e}$ denotes the logarithmic strain intensity at decohesion, $A$ and $B$ are material constants. This hypothesis was developed in [4.315], where the influence of the type of stress state on $\hat{\varepsilon}_{e}$ was more thoroughly investigated.

Criteria presented in integral form are more difficult to apply. For example, M. Cockroft and A. Latham [4.80] suggested the equation

$$
\int_{0}^{\hat{\varepsilon}} \sigma^{*} d \varepsilon=\text { const, }
$$

where $\sigma^{*}$ denotes a certain reduced tensile stress.

Z. Marciniak [4.369] proposed the condition

$$
\int_{0}^{\hat{\varepsilon}} \Phi\left(\frac{\sigma_{m}}{\sigma_{e}}\right) d \varepsilon=\text { const, }
$$

and its generalization to anisotropic bodies ( $\sigma_{e}$ being replaced by the corresponding $\left.\sigma_{\text {red }}\right) ; \Phi$ stands here for a material function, for example

$$
\Phi\left(\frac{\sigma_{m}}{\sigma_{e}}\right)=\left(\frac{\sigma_{m}}{\sigma_{e}}-a\right)^{s} .
$$

T. Y. Thomas [4.583, 2.173, 2.174] identified surfaces of fracture with surfaces of instability, i.e. with surfaces for which the tangential discontinuity of velocity does not decrease to zero and remains finite during the deformation process. Related concepts were developed and applied by J. G. Lenard [4.323], K. Ohji and M. Saito [4.428], G. Gaudel, M. Boivin and J. Bahuaud [4.181].

Z. Marciniak and K. Kuczyński [4.367] connected decohesion in a sheet with necking due to non-uniform thickness: indeed, localized deformation results in a rapid increase of strains and of dissipated energy, leading in most cases to decohesion. This concept is to a certain degree similar to that described in Secs. 18.118.5. It was developed by Z. Marciniak, K. Kuczyński and T. Pokora in [4.370], and further research is due to A. K. Tadros and P. B. Mellor [4.574], H. van Minh, R. Sowerby and J. L. Duncan [4.390, 4.391] (probabilistic approach). Localized deformation resulting in decohesion was also considered by P. B. Bowden [4.47] and J. Gouzou [4.182]. 
Another approach to problems of decohesion of ductile (plastic) materials consists in the application of Griffith's crack theory to plasticity: in contradistinction to the above-mentioned approaches, most of which are purely phenomenological, this approach may be called "semi-phenomenological". The number of papers is very great here; let us mention only the monographs and survey papers written by G. R. Irwin [4.248], F. A. McClintock and A. S. Argon [4.375], T. Yokobori [4.617], B. Paul [3.620], D. C. Drucker and J. R. Rice [4.124], L. M. Kachanov [4.266], and G. P. Cherepanov [4.70].

Though various physical criteria and theories of decohesion have been developed, their application to the estimation of the decohesive carrying capacity of elastic-plastic structures is not very common. Plastic bending of beams with decohesion taken into account was considered by G. Maier [4.344], V. Ya. Bachinsky and I. N. Tkachenko [4.16], and V. A. Kiselev [4.280]. Z. Marciniak applied his theory of limit strains to various problems of plastic forming in [4.369].

\subsection{Decohesion as a result of variable repeated loadings}

The peculiarity of variable repeated loadings consists in the cumulation of even very small effects, e.g. dissipated energy, and hence an even small multiple exceeding of the neutral surface may lead to decohesion. On the other hand, in most cases the initial neutral surface, if exceeded, is then subject to subsequent modifications (transformations), and cyclic loading may result in processes which are not cyclic but quasi-cyclic (cf. Sec. 13.2). For an infinitely large number of loading cycles the total dissipated energy may be limited or not. In the first case (Fig. 105)

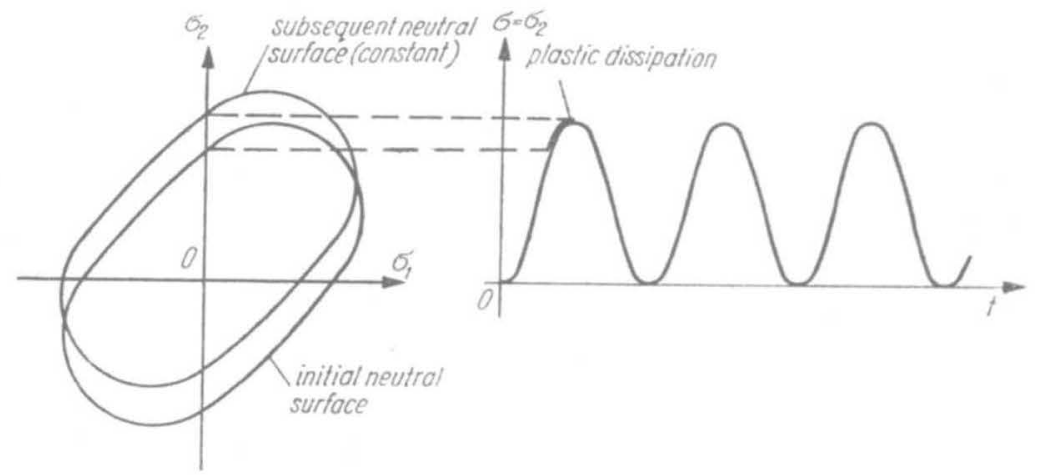

Fig. 105. Plastic shakedown under variable loadings

decohesion usually does not occur (if the static strength is not exceeded); at the level $\mathscr{P}$ we then speak of loading programs which are contained within the fatigue limits or within the fatigue limit surface, and at the levels $\mathscr{S}$ and $\mathscr{B}$-of the plastic shakedown of a cross-section or structure under the given loading program. In the case of unbounded dissipation energy decohesion does occur (Fig. 106); at all 


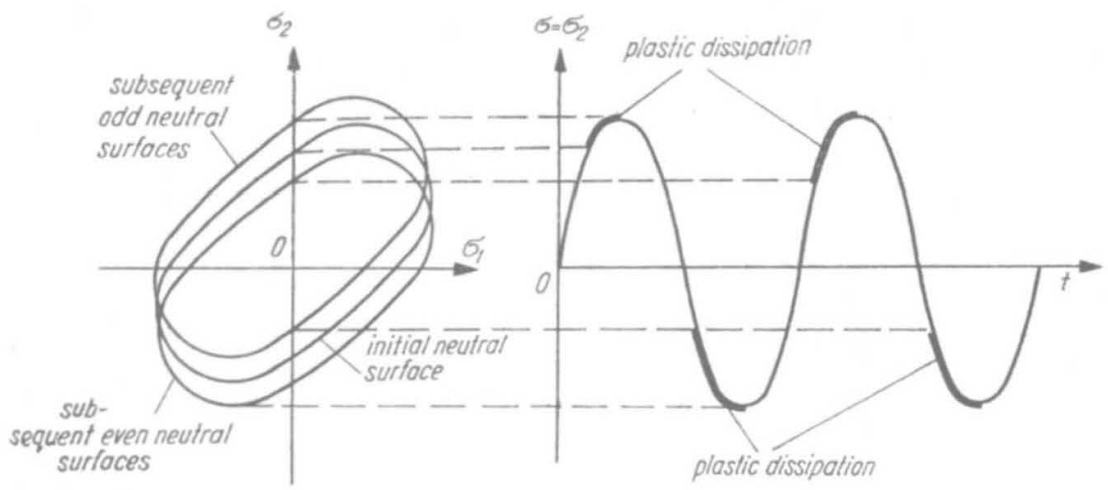

Fig. 106. Plastic non-shakedown (inadaptation) under variable loadings

levels we call this case fatigue, mostly low-cycle fatigue, and at the levels $\mathscr{S}$ and $\mathscr{B}$ the term inadaptation or non-shakedown may also be used. In uniaxial cases nonshakedown may further be specified as incremental collapse or unbounded ratcheting, due to increments of plastic strains with a constant sense, and alternating plasticity (M. R. Horne [4.236]) or cyclic collapse (P. G. Hodge [1.6]), due to increments with an alternating sense, but in the general multiaxial case such a specification is usually no longer possible.

Traditionally the behaviour under cyclic loading is analysed separately at the level $\mathscr{P}$ (fatigue or low-cycle fatigue problems) and at the levels $\mathscr{S}$ and $\mathscr{B}$ (shakedown problems). However, some features are common to all the three levels and, moreover, a more realistic analysis at the levels $\mathscr{S}$ and $\mathscr{B}$ should take into account the results of analysis at the level $\mathscr{P}$.

At the level $\mathscr{P}$ a modification of the initial neutral surface takes place as a result of plastic hardening; for perfectly elastic-plastic bodies we observe no modification in the stress space, but it may occur in the strain space (strain-controlled loading, hard loading). Suppose that a material exhibits an ideal Bauschinger effect (kinematic hardening); then the modification of the neutral surface reduces to its rigid translation. If we construct for such a material the limit curve $\sigma_{x m}-\sigma_{x a}$ (mean stress of the cycle - amplitude), called the Haigh diagram, then it takes the form shown in Fig. 107. The fatigue limit $\sigma_{-1}$ for an isosensitive material is in this case equal to the theoretical elastic limit (no modification of the initial neutral surface); this

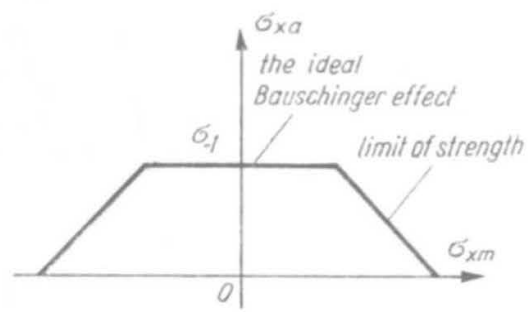

Fig. 107. Diagram of the Haigh type for low-cycle fatigue in the case of kinematic hardening 
approach is due to J. Bauschinger. On the other hand, isotropic plastic hardening would result in an essential modification of the initial neutral surface and the Haigh diagram would take the form shown in Fig. 108 (limited by static strength); however, this form is not confirmed experimentally, which points to the importance

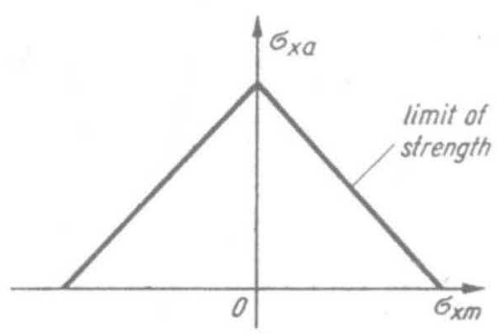

Fig. 108. Diagram of the Haigh type for low-cycle fatigue in the case of isotropic hardening

of the Bauschinger effect. For most materials a certain intermediate behaviour is observed and $\sigma_{-1}$ may be slightly higher than the theoretical elastic limit; E. Plenard [4.539] proposed to regard it as an independent material constant.

If the Haigh diagram is exceeded, then decohesion takes place after a finite number of loading cycles (the endurance limit). This phenomenon was first investigated by $\mathrm{A}$. Wöhler in the eighteen sixties. He proposed the empirical relation

$$
\hat{\sigma}=A-B \log N, \quad N \leqslant N_{0},
$$

where $N_{0}=10^{7}$ for most materials, and $\hat{\sigma}=$ const for $N \geqslant N_{0}$. Many similar formulae were suggested but it turns out that the relations joining the changes of plastic deformations $\Delta \varepsilon^{p}$ (width of the hysteresis loop) instead of maximal stress and the number of loading cycles $N$ better hold for a wide range of $N$ : they may uniformly describe classical fatigue, low-cycle fatigue and even simple static loading. R. Miner [4.389] and L. F. Coffin [4.82, 4.83] suggested the formula

$$
\left(\Delta \varepsilon^{p}\right) N^{1 / m}=\text { const. }
$$

F. R. Shanley [4.527] assumed a similar formula with $m=1$, but J. F. Tavernelli and L. F. Coffin [4.577], and S. S. Manson [4.361] found that $m=2$ gives a better approximation for most materials. The authors claim also a good description of simple loading if $N=1 / 4$ is substituted; indeed, this fraction of the typical cycle corresponds to static loading, though the first quasi-cycle is not typical and the changes of plastic deformations are smaller in comparison with further cycles (cf. Fig. 106). Equation (18.70) was investigated in detail by S. V. Serensen [4.521].

The Miner-Coffin concept was generalized to multiaxial stress states and arbitrary loading programs by V. V. Novozhilov and O. G. Rybakina [4.422, 4.423, 4.424] as follows:

$$
\sqrt{\frac{2}{3} \varepsilon_{i j}^{p} \varepsilon_{i j}^{p}} \int_{0}^{t} \sqrt{\overline{\dot{\varepsilon}_{k l}^{p}} \dot{\varepsilon}_{k l}^{p}} d t \equiv I_{e p} \varepsilon_{e}^{p}=\text { const. }
$$


The authors considered their general approach as the first step towards a description of this complicated phenomenon and suggested further research. Let us also mention the application of the concept of deterioration to alternating plasticity (V. V. Moskvitin [3.547, 3.548]), the proposal of S. V. Serensen and R. M. Shneyderovitch [4.522], and numerous papers on fracture mechanics applied to fatigue problems (cf. G. P. Cherepanov [4.70]).

Decohesion may also occur as a result of cyclic changes of temperature under constant stresses (thermal fatigue). Theoretical and experimental investigations of this phenomenon were initiated in the fifties (L. F. Coffin [4.81], S. V. Serensen and P. I. Kotov [4.520], I. A. Oding and Yu. V. Kostotchkin [4.427]). We mention here only the relevant monographs written by N. N. Davidenkov and V. A. Likhatchev [4.104], S. S. Manson [4.362], Yu. F. Balandin [4.18], and R. A. Dulnev [4.128]. Thermal ratcheting was investigated in detail by $T$. Inoue, K. Tanaka and M. Aoki [4.245]. More attention is paid to relevant problems at the level $\mathscr{B}$, where thermal ratcheting results in more visible effects.

At the levels $\mathscr{S}$ and $\mathscr{B}$ modification of the initial neutral surface may take place also as a result of plastic hardening, similarly to the level $\mathscr{P}$, but even without hardening the modifications are due to a redistribution of internal forces and to the appearance of self-stresses, namely of residual stresses. The latter effect is often more important than plastic hardening, and the theory of variable repeated loadings at the levels $\mathscr{S}$ and $\mathscr{B}$ was developed (just as the shakedown theory) primarily for perfectly elastic-plastic bodies, without direct connection with the relevant theory at the level $\mathscr{P}$.

The problem of plastic shakedown (of beams) was first formulated by M. Grüning [4.189] in 1926; further related papers, partially experimental, are due to G. Kazinczy [4.276], J. Fritzsche [4.147], E. O. Paton and B. N. Gorbunov [4.446]. H. Bleich [4.41] and E. Melan [4.379] proved the first general theorem for bar systems, and E. Melan [4.380] - for the three-dimensional continuum. A simpler proof of the Melan theorem (which will be quoted in Sec. 20) was given by P. S. Symonds [4.562] and W. T. Koiter [4.293]. Further investigations were carried out by J. Dutheil [4.133], M. R. Horne [4.236], B. G. Neal [4.412] (with plastic hardening taken into account), P. S. Symonds and W. Prager [4.561].

The second general theorem (on non-shakedown) was proved in 1956 by W. T. Koiter [4.294]. J. A. König [4.303] expressed shakedown theorems in terms of generalized stresses.

Mathematical criteria of shakedown may be expressed in various forms; they were compared by J. A. König [4.306]. Usually it is assumed that the specific plastic work is finite at any point of the body

$$
\sup _{x, y, z} W^{p}=\sup _{x, y, z} \int_{0}^{t} \sigma_{l j} \dot{\varepsilon}_{i j}^{p} d t<\infty
$$


for an arbitrary duration period $t$, and even for $t \rightarrow \infty$. J. Rychlewski [4.491] proposed a less stringent criterion, namely

$$
\bar{W}^{p}=\frac{1}{V} \iiint_{V}\left(\int_{0}^{t} \sigma_{i j} \dot{\varepsilon}_{i j}^{p} d t\right) d V<W_{0},
$$

where $W_{0}$ is a constant; if the volume of the body $V$ is finite and the integrand has no singularities, then the above two criteria are equivalent. The Bleich-Melan shakedown theorem, adopted to criterion (18.73), was proved by L. Konieczny [4.295]. Another criterion, expressed in terms of plastic strains only, was introduced and applied by $\mathrm{G}$. Maier [4.355].

General and survey papers on plastic shakedown problems were published by P. G. Hodge [4.219], W. T. Koiter [3.365], J. A. König [4.305], J. M. Davies [4.105], J. Heyman [4.206], A. Sawczuk [4.517], D. A. Gokhfeld and O. F. Chernyavsky [4.177] and B. N. Kuznetsov [4.313]. Simple models illustrating the cases of shakedown and non-shakedown were proposed by S. S. Gill [4.164], C. Ruiz [4.490], E. Chianese [4.74] (localized incremental collapse), T. M. Mulcahy [4.399], A. R. Belyakov and O. F. Chernyavsky [4.27]. V. M. Moskvitin [3.543] formulated the conditions of a simple deformation process in the case of variable repeated loadings; many particular cases are discussed in his book [2.113]. R. M. Kirakosyan [4.278] considered arbitrary plastic hardening, C. Gavarini and G. Beolchini [4.155] and G. Maier [4.351, 4.355], considered the shakedown of structures with geometrical or physical instabilities. R. M. Shneyderovitch, O. A. Levin, N. A. Makhutov and M. D. Novopashin [4.532] worked out experimental methods appropriate for shakedown investigations. The problem of shakedown of rigidhardening structures was formulated by W. Prager [4.473].

The analysis of plastic shakedown under dynamic loads and under forced vibrations was initiated by G. Ceradini [4.65] and C. Gavarini [4.154]; further papers are due to H.S. Ho [4.217], Z. Mróz [3.556], G. Maier and E. Vitiello [4.356] (plastic hardening).

The problem of plastic shakedown is also very important in the case of thermal loadings (e.g. in moulds, chemical plants, aircraft structures, etc.). Theoretical treatment of this problem, mentioned in 1954 by E. W. Parkes [4.445], is due to W. Prager [4.469, 4.470], and to V. I. Rozenblyum [4.487, 4.488, 4.489]. Extensive investigations of this topic were carried out by D. A. Gokhfeld [4.166, 4.167, 4.168, 4.170, 4.171, 4.173], P. I. Ermakov, A. S. Nesmeyanov, B. E. Dorofeyev and V. I. Morozov [4.135] (influence of various factors), J. A. König [4.304] (dependence of $E$ on temperature), N. S. Mozharovsky, E. A. Antipov and G. I. Gontcharov [4.395]. Monographs on plastic shakedown under thermal loadings were published by D. A. Gokhfeld [4.174] and Yu. N. Shevtchenko [4.528].

In the problems of combined loadings there exist various possibilities of presentation of results. The simplest form is to describe the rectangular shakedown domains by the inequalities 


$$
Q_{i}^{-}\left(\alpha_{j}\right) \leqslant Q_{i} \leqslant Q_{i}^{+}\left(\alpha_{j}\right)
$$

where $\alpha_{j}$ denote parameters determining the families of rectangular domains. However, for some applications it is important to know more general, larger domains bounded by curved surfaces, namely the modified elastic domains

$$
f\left(Q_{i}, \alpha_{j}\right) \leqslant 0
$$

determined by condition (17.1) for stress distributions with certain residual stresses taken into account. Residual stresses may by determined here as resulting from arbitrary processes exceeding the initial neutral surface; in a paper by K. Kowalczyk and M. Życzkowski [2.90] such processes were called "control cycles", and $\alpha_{j}$ in (18.75) were understood as parameters of those cycles.

In contradistinction to the level $\mathscr{P}$, in the case of non-shakedown at the levels $\mathscr{S}$ and $\mathscr{B}$ the relevant "endurance limit" has hardly been investigated so far (if we disregard the trivial cases of homogeneous stresses where the transition is immediate). Fatigue tests on notched bars may be included here, but the connections of the theory of such tests with the theory of plasticity are rather loose. On the other hand, the theory of plasticity makes it possible to estimate bounds for displacements in the case of limited ratcheting followed by shakedown; such problems were investigated by D. G. Eyre and T. V. Galambos [4.138] A. R. S. Ponter [4.461], E. Vitiello [4.597], R. Brzeziński and J. A. König [4.54, 4.55], M. Capurso [4.62], V. A. Ikrin and V. V. Filippov [4.240], and S. Dorosz [4.117].

It seems that the connections between "local" analysis at the level $\mathscr{P}$ and "integral" analysis at the levels $\mathscr{S}$ and $\mathscr{B}$ are not strong enough in shakedown problems. In particular, the schematizations commonly used at the levels $\mathscr{S}$ and $\mathscr{B}$ (perfectly elastic-plastic, elastic with linear hardening, etc.) may be insufficient in shakedown problems since even small deviations from perfect elasticity under the yield-point stress may result in large dissipated energy and in subsequent decohesion.

\section{Methods of reduction of the number of plasticity equations}

\subsection{List of the equations of the classical theory of perfect plasticity}

The system of equations of the classical theory of perfect plasticity in the general case consists of 16 equations. Nine of these equations are common to all branches of the mechanics of solids: three equations of internal equilibrium, which in the Cartesian coordinates take the form

$$
\sigma_{i j, i}+F_{j}=0
$$

and six equations joining strains and displacements, the linearized form of which is

$$
\varepsilon_{i j}=\frac{1}{2}\left(u_{i, j}+u_{j, i}\right) .
$$


These equations may be replaced by the compatibility conditions. Moreover, according to experimental results, the elastic law of volume change

$$
\varepsilon_{m}=\frac{1-2 v}{E} \sigma_{m} .
$$

holds also in the theory of plasticity as the tenth equation. The differences with respect to the theory of elasticity are seen only in the law of shape change: such laws contain a new unknown function, $\lambda$ or $\varphi$. According to Hencky-Ilyushin, for an isotropic body

$$
e_{i j}=\varphi s_{i j}
$$

according to Levy-Mises

$$
\dot{e}_{i j}=\lambda s_{i j} ;
$$

and according to Prandtl-Reuss

$$
\dot{e}_{i j}=\lambda s_{i j}+\frac{1}{2 G} \dot{s}_{i j}
$$

Each of the systems of equations (19.4)-(19.6) contains five independent algebraic equations. The condition of perfect plasticity

$$
\sigma_{\mathrm{red}}=\sigma_{0}
$$

is the sixteenth equation.

In other systems of coordinates the differential equations (19.1) and (19.2) are the only ones subject to change, whereas in locally orthogonal systems a formal change of indices in the remaining equations is sufficient.

The above equations determine six components of the stress tensor, six components of the strain tensor, three components of the displacement vector, and the function $\varphi$ or $\lambda$. Boundary conditions determine, as a rule, three stresses (tractions) or three displacements or velocities at the boundary of the body, $S$. Usually one can distinguish a part of the boundary subject to stress boundary conditions, $S_{T}$,

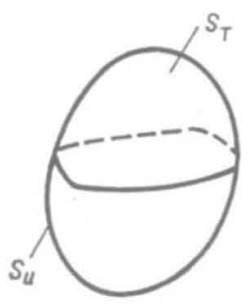

Fig. 109. Boundary conditions in stresses and in displacements (or velocities)

and another part subject to displacement boundary conditions $S_{U}$, Fig. 109. In an elastic-plastic problem we have to determine the plastic zones in which $\lambda>0$ or $\varphi>1 / 2 G$ and all the sixteen unknowns are to be found, and the elastic zones in 
which $\lambda=0$ or $\varphi=1 / 2 G, \sigma_{\text {red }}<\sigma_{0}$ and the number of unknowns is fifteen. In a problem of evaluating the elastic carrying capacity, the limit carrying capacity or the decohesive carrying capacity, we assume that the exertion factors are determined up to a certain multiplier; in the simplest case we assume

$$
\mathbf{p}_{n}=m \mathbf{p}_{n 0}
$$

and the corresponding values of the multiplier $m$, namely $\bar{m}, \overline{\bar{m}}$ or $\hat{m}$ have to be found.

In view of the large number of equations and unknowns the methods of reducing that number become particularly important; such reduction is essential, above all, when combined with analytical methods of solution, but it may also be useful in connection with some numerical methods and with the computer technique. Reduction of the number of equations can be achieved as a result of elimination of unknowns or of the parametrization of certain equations.

\subsection{Displacement equations of the theory of plasticity}

One of the simplest and most effective methods of elimination of unknowns in the theory of elasticity consists in expressing stresses in terms of strains, and subsequently in terms of displacements, and in substituting the resulting formulae into the equilibrium equations. The resulting three equations with three unknown displacements (for compressible bodies) are known as the Lamé equations.

In the theory of plasticity such an approach is also possible if we use the HenckyIlyushin or the Levy-Mises equations, whereas the application of the PrandtlReuss equations involves serious complications. The central idea of derivation of such equations is due to A. A. Ilyushin [1.8] (the Hencky-Ilyushin theory), H. Geiringer [4.161] and T. Y. Thomas [2.174] (the Levy-Mises theory). The equations derived below in the expanded form will be valid for both theories. They will be restricted to Cartesian coordinates; a similar derivation for general curvilinear coordinates was given in [4.637].

The case of an incompressible body and the case of an elastically compressible body must be considered separately. For an incompressible body a system of four equations containing four unknowns $u_{l}(i=x, y, z)$ and $\sigma_{m}$ will be derived, whereas in the case of compressibility three equations with three unknowns $u_{i}$ are sufficient. To shorten the notation the summation convention will be used and dummy indices will be denoted by Greek letters.

In the case of incompressible bodies we use the equilibrium equations (19.1), the Cauchy relations (19.2), the condition of incompressibility

$$
u_{\beta, \beta}=0 \text {, }
$$

the law of shape change (19.4) (which may be interpreted either as the HenckyIlyushin law if $e_{i j}$ stand for deviatoric strains, or as the Levy-Mises law if $e_{i j}$ denote, quite formally, deviatoric strain rates), and the HMH yield condition (9.15). In 
view of the identity $e_{i j}=\varepsilon_{i j}$ in this case, we express the function $\varphi$ be using $(9.30)$ in terms of $\varepsilon_{i j}$ as follows:

$$
\varphi=\frac{1}{\sigma_{0}} \sqrt{\frac{3}{2} \varepsilon_{x \lambda} \varepsilon_{x \lambda}} .
$$

The stress components $\sigma_{i j}$ are then given by

$$
\sigma_{i j}=s_{i j}+\delta_{i j} \sigma_{m}=\frac{\varepsilon_{i j}}{\varphi}+\delta_{i j} \sigma_{m} .
$$

(this step presents the main difficulty when using the Prandtl-Reuss theory). Substituting now (19.11) into (19.1) and using (19.2), we obtain the required system of equations. The first step of substitution may be written thus:

$$
\frac{\varepsilon_{\alpha j, \alpha} \varphi-\varphi, \alpha}{\varphi^{2}}+\sigma_{m, \alpha} \delta_{\alpha j}+F_{j}=0 .
$$

The derivative $\varphi, \alpha$ equals

$$
\varphi_{, \alpha}=\frac{1}{\sigma_{0}} \sqrt{\frac{3}{2}} \frac{\varepsilon_{\mu v, \alpha} \varepsilon_{\mu \nu}}{\sqrt{\varepsilon_{x \lambda} \varepsilon_{\times \lambda}}},
$$

and hence (19.12) may be rewritten in the form

$$
\frac{1}{\sigma_{0}} \sqrt{\frac{3}{2}} \varepsilon_{x \lambda} \varepsilon_{x \lambda} \varepsilon_{\alpha j, \alpha}-\frac{1}{\sigma_{0}} \sqrt{\frac{3}{2}} \frac{\varepsilon_{\mu \nu, \alpha} \varepsilon_{\mu \nu}}{\sqrt{\varepsilon_{x \lambda} \varepsilon_{x \lambda}}} \varepsilon_{\alpha j}+\left(\sigma_{m, j}+F_{j}\right) \frac{3}{2 \sigma_{0}^{2}} \varepsilon_{x \lambda} \varepsilon_{x \lambda}=0 \text {. }
$$

Finally, making use of (19.2) and rearranging the terms, we obtain the system of non-linear partial differential equations of the second order, homogeneous of the third degree with respect to $u_{j}$

$$
\begin{aligned}
u_{x, \lambda}\left(u_{x, \lambda}+u_{\lambda, \kappa}\right) \nabla^{2} u_{j}-\left(u_{x, \lambda \alpha} u_{x, \lambda} u_{j, \alpha}+u_{x, \lambda \alpha} u_{\lambda, x} u_{j, \alpha}+u_{x, \lambda \alpha} u_{x, \lambda} u_{\alpha, j}+\right. \\
\left.+u_{x, \lambda \alpha} u_{\lambda, x} u_{\alpha, j}\right)+\frac{\sqrt{3}}{\sigma_{0}}\left(\sigma_{m, j}+F_{j}\right)\left[u_{x, \lambda}\left(u_{x, \lambda}+u_{\lambda, x}\right)\right]^{3 / 2}=0
\end{aligned}
$$

which is completed by the linear equation (19.9). The unknowns are in this case: three displacements (or velocities) $u_{j}$, and the mean stress $\sigma_{m}$.

In the case of compressible bodies (with reference only to the Hencky-Ilyushin theory), instead of (19.10) we may write

$$
\varphi=\frac{1}{\sigma_{0}} \sqrt{\frac{3}{2}\left(\varepsilon_{x \lambda}-\frac{1}{3} \varepsilon_{\beta \beta} \delta_{x \lambda}\right)\left(\varepsilon_{x \lambda}-\frac{1}{3} \varepsilon_{\mu \mu} \delta_{x \lambda}\right)},
$$

and, instead of (19.11),

$$
\sigma_{i j}=\frac{\varepsilon_{i j}-\frac{1}{3} \varepsilon_{\beta \beta} \delta_{i j}}{\varphi}+\frac{E}{3(1-2 v)} \varepsilon_{\beta \beta} \delta_{i j} .
$$

Substitution into the equilibrium equations (19.1) gives

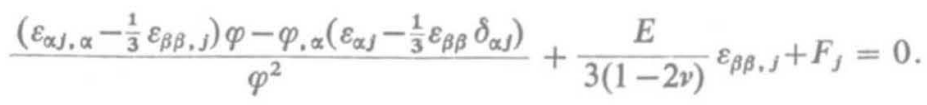


Finally, making use of the Cauchy equations (19.2), we may present the resulting three partial differential equations in the form

$$
\begin{aligned}
\left(3 u_{x, \lambda} u_{x, \lambda}+3 u_{x, \lambda} u_{\lambda, x}-2 u_{x, x} u_{\lambda, \lambda}\right)\left(u_{\alpha, j \alpha}+3 u_{j, \beta \beta}\right)- \\
-\left(3 u_{x, \lambda \alpha} u_{x, \lambda}+3 u_{x, \lambda \alpha} u_{\lambda, \alpha}-2 u_{x, \alpha} u_{\lambda, \lambda \alpha}\right)\left(3 u_{\alpha, j}+3 u_{j, \alpha}-2 u_{\beta, \beta} \delta_{\alpha j}\right)+ \\
\quad+\frac{3}{\sigma_{0}}\left[\frac{E}{3(1-2 v)} u_{\beta, \beta j}+F_{j}\right]\left(3 u_{x, \lambda} u_{x, \lambda}+3 u_{x, \lambda} u_{\lambda, x}-2 u_{x, \alpha} u_{\lambda, \lambda}\right)^{3 / 2}=0 .
\end{aligned}
$$

The main adventage of Eqs. (19.15) or (19.19) results from the fact of relatively small influence of strains on stresses in the plastic range; hence, even if those equations are integrated numerically with relatively poor accuracy, the resulting errors in stress distribution and in the value of the limit carrying capacity are usually very small.

Equations of perfect plasticity expressed in velocities and based on various yield conditions (Tresca-Guest, Schmidt-Ishlinsky-Hill) were derived by D. D. Ivlev and A. D. Chernyshov [4.252], and displacement equations of thermoplasticity were obtained and applied by Yu. G. Korotkikh and A. A. Kravtchenko [4.301].

\subsection{Stress functions}

Another method of reduction of number of equations consists in parametrization of the equilibrium equations. Parametrizing functions are called the stress functions. Since the equilibrium equations are identical in all branches of the mechanics of solids, the stress functions are also introduced in an identical manner; the differences are seen only in the final govering equations. The required reduction takes place if the number of stress functions equals the number of unknown stress components minus the number of equilibrium equations, and hence in the general triaxial case the number of stress functions should be three.

Numerous particular cases of stress functions have been introduced since 1862 (G. B. Airy), but it was B. Finzi [4.141] who in 1934 proposed a general approach to the problem of parametrization of equations of internal equilibrium. He introduced the tensor of stress functions $T_{\Phi}$ by the formula

$$
\sigma_{i j}=e_{i \alpha \mu} e_{j \beta \nu} \Phi_{\alpha \beta, \mu \nu}-\delta_{i j} U,
$$

where $e_{i \alpha \mu}$ and $e_{j \beta v}$ are the permutation symbols (alternators), and $U$ denotes the potential of body forces (non-conservative body forces are excluded in this approach).

In Cartesian coordinates the effective formulae for $\sigma_{i j}$ in terms of $\Phi_{i j}$ in the case of vanishing body forces may be written thus:

$$
\begin{aligned}
\sigma_{x} & =\frac{\partial^{2} \Phi_{z z}}{\partial y^{2}}+\frac{\partial^{2} \Phi_{y y}}{\partial z^{2}}-2 \frac{\partial^{2} \Phi_{y z}}{\partial y d z}, \\
\tau_{y z} & =\frac{\partial}{\partial x}\left(-\frac{\partial \Phi_{y z}}{\partial x}+\frac{\partial \Phi_{z x}}{\partial y}+\frac{\partial \Phi_{x y}}{\partial z}\right)-\frac{\partial^{2} \Phi_{x x}}{\partial y \partial z}
\end{aligned}
$$


Formulae (19.21) were derived independently, and studied in detail from the standpoint of the theory of elasticity by Yu. A. Krutkov [4.310] and V. I. Blokh $[4.42,4.43]$. It turns out that the required reduction takes place if only three components of the stress function tensor are retained; the remaining three may be assumed to be equal to zero. There are $\left(\begin{array}{c}6 \\ 3\end{array}\right)=20$ combinations of such choice of three stress functions out of six, but three of these combinations are not sufficiently general (e.g. assuming $\Phi_{y y} \equiv \Phi_{z z} \equiv \Phi_{y z} \equiv 0$, we would obtain $\sigma_{x} \equiv 0$ and the generality is lost), and hence 17 general combinations remain. The best known general systems are:

the Maxwell stress functions $\Phi_{x x}, \Phi_{y y}, \Phi_{z z}$ (if $\Phi_{x y} \equiv \Phi_{y z} \equiv \Phi_{z x} \equiv 0$ )

$$
\left.\sigma_{x}=\frac{\partial^{2} \Phi_{z z}}{\partial y^{2}}+\frac{\partial^{2} \Phi_{y y}}{\partial z^{2}}, \quad \tau_{y z}=-\frac{\partial^{2} \Phi_{x x}}{\partial y \partial z}, \quad \succ_{z}^{x}\right\rangle_{y}
$$

and the Morera stress functions $\Phi_{x y}, \Phi_{y z}, \Phi_{z x}$ (if $\Phi_{x x} \equiv \Phi_{y y} \equiv \Phi_{z z} \equiv 0$ )

$$
\sigma_{x}=-2 \frac{\partial^{2} \Phi_{y z}}{\partial y \partial z}, \quad \tau_{y z}=\frac{\partial}{\partial x}\left(-\frac{\partial \Phi_{y z}}{\partial x}+\frac{\partial \Phi_{z x}}{\partial y}+\frac{\partial \Phi_{x y}}{\partial z}\right) .
$$

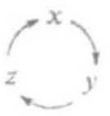

In the case of plane stress in the plane $x y$ it is sufficient to employ the function $\Phi_{z z}$ $=\Phi_{z z}(x, y)$; this oldest stress function is called the Airy function. In the case of free torsion of a prismatic bar with the axis $z$, if-following the de Saint-Venant assumption-we can put $\sigma_{x} \equiv \sigma_{y} \equiv \sigma_{z} \equiv \tau_{x y} \equiv 0$, and the remaining two stresses depend only on the variables $x$ and $y$, then we may use the function

$$
\Psi=\frac{\partial \Phi_{y z}}{\partial x}-\frac{\partial \Phi_{z x}}{\partial y},
$$

which is called the Prandtl stress function.

It should be mentioned that the system of stress functions (19.20), though very general, is not complete. It turns out that they describe totally self-equilibrated stress fields and the resultant force and moment must vanish on any closed surface (W. Jaunzemis [3.74]). If the boundary of the body consists of several separate closed surfaces (e.g. in a hollow sphere), then more general external loadings are also possible. The relevant generalization of system (19.20) was proposed by M. E. Gurtin [4.192].

Following paper [4.505] by H. Sanecki and M. Życzkowski, we now derive the basic system of equations of perfect plasticity expressed in terms of general stress functions (19.20). The HMH yield condition will be valid for any theory of plasticity, whereas the remaining equations will be derived for each theory (HenckyIlyushin, Levy-Mises, Prandtl-Reuss) separately. To use form (9.14) we first determine the mean stress

$$
\sigma_{m}=\frac{1}{3} \sigma_{\gamma \nu}=\frac{1}{3} e_{\gamma \alpha \mu} e_{\gamma \beta \nu} \Phi_{\alpha \beta, \mu \nu}-U
$$


making use of the identity

$$
e_{\gamma \alpha \mu} e_{\gamma \beta \nu}=\delta_{\alpha \beta} \delta_{\mu \nu}-\delta_{\alpha \nu} \delta_{\beta \mu},
$$

we finally obtain

$$
\sigma_{m}=\frac{1}{3}\left(\Phi_{\alpha \alpha, \beta \beta}-\Phi_{\alpha \beta, \alpha \beta}\right)-U \text {. }
$$

The deviatoric stresses can now be expressed as follows:

$$
s_{i j}=\sigma_{i j}-\delta_{i j} \sigma_{m}=e_{i \alpha \mu} e_{j \beta \nu} \Phi_{\alpha \beta, \mu \nu}-\frac{1}{3} \delta_{i j}\left(\Phi_{\alpha \alpha, \beta \beta}-\Phi_{\alpha \beta, \alpha \beta}\right) \text {. }
$$

Substituting (19.28) into the HMH yield condition (9.14), we obtain, after contraction and some rearrangements, its final form

$$
3 \Phi_{\alpha \beta, \mu v}\left(\Phi_{\alpha \beta, \mu v}+\Phi_{\mu v, \alpha \beta}-\Phi_{\alpha \mu, \beta v}-\Phi_{\beta v, \alpha \mu}\right)-\left(\Phi_{\alpha \alpha, \beta \beta}-\Phi_{\alpha \beta, \alpha \beta}\right)^{2}=2 \sigma_{0}^{2} .
$$

If the problem under consideration is internally statically determinate, then Eq. (19.29) is sufficient for the solution. For example, for the Airy stress function $\Phi_{z z}=\Phi_{z z}(x, y)$, putting in (19.29) other stress functions equal to zero, we obtain one equation with one unknown

$$
\left(\frac{\partial^{2} \Phi_{z z}}{\partial x^{2}}\right)^{2}+\left(\frac{\partial^{2} \Phi_{z z}}{\partial y^{2}}\right)^{2}-\frac{\partial^{2} \Phi_{z z}}{\partial x^{2}} \frac{\partial^{2} \Phi_{z z}}{\partial y^{2}}+3\left(\frac{\partial^{2} \Phi_{z z}}{\partial x \partial y}\right)^{2}=\sigma_{0}^{2} .
$$

Leaving in (19.29) only two stress functions $\Phi_{x z}=\Phi_{x z}(x, y)$ and $\Phi_{y z}=\Phi_{y z}(x, y)$, we obtain

$$
3\left(\frac{\partial \Phi_{y z}}{\partial x^{2}}-\frac{\partial \Phi_{x z}}{\partial x \partial y}\right)+3\left(\frac{\partial^{2} \Phi_{y z}}{\partial x \partial y}-\frac{\partial^{2} \Phi_{x z}}{\partial y^{2}}\right)^{2}=\sigma_{0}^{2},
$$

and hence, for the Prandtl function $\Psi$, (19.24), we derive the well-known equation of the surface of constant slope

$$
3\left(\frac{\partial \Psi}{\partial x}\right)^{2}+3\left(\frac{\partial Y^{\prime}}{\partial y}\right)^{2}=\sigma_{0}^{2} \text {. }
$$

In the case of an internally statically indeterminate problem we have to take the relations between strains and stresses into account. Using these relations and substituting the resulting expressions for strains into the Cauchy formulae (19.2), we obtain a system with 7 unknowns, whereas the substitution into the compatibility equations leads to a system with only 4 unknowns. We follow here the latter way, though in certain cases it is impossible to evaluate all the integration constants without an effective calculation of displacements or velocities.

We first apply the Prandtl-Reuss equations, as the most complicated ones. Making use of the law of volume change (19.3), we present them in the form

$$
\dot{\varepsilon}_{i j}=\lambda\left(\sigma_{i j}-\delta_{i j} \sigma_{m}\right)+\frac{1}{2 G} \dot{\sigma}_{i j}-\left(\frac{1}{2 G}-\frac{1}{3 K}\right) \delta_{i j} \dot{\sigma}_{m} .
$$

Substituting here (19.20) and (19.27), we may write

$$
\begin{aligned}
\dot{\varepsilon}_{i j}= & \lambda\left[e_{i \alpha \mu} e_{j \beta \nu} \Phi_{\alpha \beta, \mu \nu}-\frac{1}{3} \delta_{i j}\left(\Phi_{\alpha \alpha, \beta \beta}-\Phi_{\alpha \beta, \alpha \beta}\right)\right]+ \\
& +\frac{1}{2 G} e_{i \alpha \mu} e_{j \beta \nu} \dot{\Phi}_{\alpha \beta, \mu \nu}-\frac{\nu}{E} \delta_{i j}\left(\dot{\Phi}_{\alpha \alpha, \beta \beta}-\dot{\Phi}_{\alpha \beta, \alpha \beta}\right)+\frac{1}{3 K} \delta_{i j} \dot{U},
\end{aligned}
$$


where $v$ as a multiplier denotes Poisson's ratio and should not be confused with $v$ as a dummy index. Finally, substituting (19.34) into the compatibility equations in the form (7.51), we obtain the following system of equations for the Prandt1-Reuss theory:

$$
\begin{aligned}
& 3\left[\lambda_{, \alpha \beta}+\lambda_{, \alpha} \partial_{\beta}+\lambda_{, \beta} \partial_{\alpha}+\left(\lambda+\frac{1}{2 G} \frac{d}{d t}\right) \partial_{\alpha \beta}^{2}\right]\left(\Phi_{i j, \alpha \beta}+\Phi_{\alpha \beta, i j}-\right. \\
& \left.-\Phi_{i \alpha, j \beta}-\Phi_{j \beta, i \alpha}\right)+\left[\lambda_{, i j}-\delta_{i j}\left(\nabla^{2} \lambda+2 \lambda_{, \mu} \partial_{\mu}\right)+\lambda_{, l} \partial_{j}+\lambda_{, j} \partial_{l}+\right. \\
& \left.+\left(\lambda+\frac{3 v}{E} \frac{d}{d t}\right)\left(\partial_{i j}^{2}-\delta_{i j} \nabla^{2}\right)\right]\left(\nabla^{2} \Phi_{\alpha \alpha}-\Phi_{\alpha \beta, \alpha \beta}\right)+ \\
& \quad+\frac{1}{K}\left(\dot{U}_{. i j}-\delta_{i j} \nabla^{2} \dot{U}\right)=0,
\end{aligned}
$$

where the operator $\partial_{\alpha}$ denotes partial differentiation with respect to the coordinate $\alpha(\alpha=x, y, z)$.

The corresponding equations for the Levy-Mises theory may be derived from (19.35) if we substitute $1 / G=1 / E=1 / K=0$. For the Hencky-Ilyushin theory one obtains

$$
\begin{aligned}
& 3\left(\varphi_{. \alpha \beta}+\varphi_{, \alpha} \partial_{\beta}+\varphi_{, \beta} \partial_{\alpha}+\varphi \partial_{\alpha \beta}^{2}\right)\left(\Phi_{i j, \alpha \beta}+\Phi_{\alpha \beta, i j}-\Phi_{i \alpha, j \beta}-\Phi_{j \beta, i \alpha}\right)+ \\
& \quad+\left[\varphi_{. i j}-\delta_{i j}\left(\nabla^{2} \varphi+2 \varphi_{, \mu} \partial_{\mu}\right)+\varphi_{, i} \partial_{j}+\varphi_{. j} \partial_{i}+\right. \\
& \left.+\left(\varphi-\frac{1}{3 K}\right)\left(\partial_{i j}^{2}-\delta_{i j} \nabla^{2}\right)\right]\left(\nabla^{2} \Phi_{\alpha \alpha}-\Phi_{\alpha \beta, \alpha \beta}\right)+ \\
& \quad+\frac{1}{K}\left(U_{. i j}-\delta_{i j} \nabla^{2} U\right)=0 .
\end{aligned}
$$

We mentioned already in Sec. 7.8 that only three compatibility equations are really independent, and hence the system of (19.29) with (19.35) or with (19.36) contains four independent equations. The number of unknowns is also four: three stress functions (chosen out of six), and the function $\lambda$ or $\varphi$. In their general form those equations are difficult to apply, but in some particular cases they will be applied in Chapter VIII to the problems of thick-walled tubes under combined loadings.

In other systems of coordinates one can derive the corresponding equations by using covariant differentiation or by appropriate transformations of stresses, stress functions and coordinates. According to V. I. Blokh [4.43], in the general case formulated in cylindrical coordinates only 18 systems of stress functions remain, and for rotational symmetry-only 10 . One of such systems is given by M. M. Filonenko-Boroditch [4.140].

We quote here the formulae only for some particular cases. If the stresses depend only on the coordinates $r$ and $\theta$, then in the three equilibrium equations (6.11) 
merely 5 stress components remain ( $\sigma_{z}$ disappears). These equations may be parametrized by the following two functions $\Phi_{z z}$ and $\Psi$ of two variables $r$ and $\theta$ :

$$
\begin{array}{rlrl}
\sigma_{r} & =\frac{1}{r} \frac{\partial \Phi_{z z}}{\partial r}+\frac{1}{r^{2}} \frac{\partial^{2} \Phi_{z z}}{\partial \theta^{2}}, & \tau_{z r}=\frac{1}{r} \frac{\partial \Psi}{\partial \theta}, \\
\sigma_{\theta}=\frac{\partial^{2} \Phi_{z z}}{\partial r^{2}}, & \tau_{\theta z}=-\frac{\partial \Psi}{\partial r} . \\
\tau_{r \theta}=-\frac{1}{r} \frac{\partial^{2} \Phi_{z z}}{\partial r \partial \theta}+\frac{1}{r^{2}} \frac{\partial \Phi_{z z}}{\partial \theta}, &
\end{array}
$$

On the other hand, if the stresses depend only on the coordinates $r$ and $z$ (rotational symmetry), then all the stresses appear in (6.11), since $\sigma_{\theta}$ remains in the first equation. The required reduction is obtained if we introduce, say, three stress functions $\Phi, \chi$ and $\Psi$ of two variables $r$ and $z$ as follows (M. M. FilonenkoBoroditch [4.140]):

$$
\begin{aligned}
\sigma_{r} & =\frac{\Phi}{r}, & \tau_{\theta z} & =-\frac{1}{r^{2}} \frac{\partial \Psi}{\partial r}, \\
\sigma_{\theta} & =\frac{\partial \Phi}{\partial r}+\frac{\partial^{2} \chi}{\partial z^{2}}, & \tau_{z r} & =\frac{1}{r} \frac{\partial \chi}{\partial z}, \\
\sigma_{z} & =-\frac{1}{r} \frac{\partial \chi}{\partial r}, & \tau_{r \theta} & =\frac{1}{r^{2}} \frac{\partial \Psi}{\partial z} .
\end{aligned}
$$

In the particular case of $\tau_{\theta z} \equiv \tau_{r \theta} \equiv 0$, i.e. if the direction $\theta$ is a principal direction, then $\Psi \equiv 0$. Sometimes just this case is called the rotational symmetry; we prefer to use the term rotational symmetry in a narrower sense. When considering the torsion of circular non-prismatic bars or of circularly curved bars, we may assume $\Phi \equiv \chi \equiv 0$, and $\Psi=\Psi(r, z)$ is the only non-vanishing stress function.

The governing equations of plasticity for the stress functions in cylindrical coordinates may be derived in the same way as that shown for Cartesian coordinates; some of them will be given in Chapter VIII.

As we mentioned before, stress functions may be introduced by identical formulae in the theories of elasticity, plasticity, and in rheology. However, in the theory of plasticity it is convenient to introduce a certain coefficient with the dimension of stress, e.g. $\sigma_{0}$ or $\tau_{0}$; then the stress functions themselves are dimensionless (if simultaneously dimensionless coordinates are used).

In the theory of elasticity the term "stress functions" is sometimes used for functions which not only parametrize the equilibrium equations but also uncouple the resulting compatibility equations. Such functions are of course introduced by separate formulae for particular types of solids (e.g. anisotropic materials); to avoid misunderstandings, S. Kaliski [4.268] proposed the term "stress bifunctions" for those functions. In the theory of plasticity uncoupling of equations of type (19.35) or (19.36) by means of bifunctions is, in general, not possible. 


\subsection{Parametrization of the yield condition}

The above-mentioned methods of reduction were identical or similar to those used in the theory of elasticity. A distinct method, typical for the theory of plasticity, consists in the parametrization of the yield condition. In the general triaxial case one condition of perfect plasticity interrelates six stress components, and hence it is necessary to express stresses in terms of five appropriately introduced parameters.

The parametrization of the Huber-Mises-Hencky yield condition in the general case was proposed by V. V. Sokolovsky [4.546, 2.163]. This equation will be satisfied identically if we put

$$
\begin{aligned}
\sigma_{x} & =\frac{2}{3} \sigma_{0}\left[l_{1}^{2} \cos \omega+l_{2}^{2} \cos \left(\omega-\frac{2}{3} \pi\right)+l_{3}^{2} \cos \left(\omega+\frac{2}{3} \pi\right)\right]+\sigma_{m}, \\
\sigma_{y} & =\frac{2}{3} \sigma_{0}\left[m_{1}^{2} \cos \omega+m_{2}^{2} \cos \left(\omega-\frac{2}{3} \pi\right)+m_{3}^{2} \cos \left(\omega+\frac{2}{3} \pi\right)\right]+\sigma_{m}, \\
\sigma_{z} & =\frac{2}{3} \sigma_{0}\left[n_{1}^{2} \cos \omega+n_{2}^{2} \cos \left(\omega-\frac{2}{3} \pi\right)+n_{3}^{2} \cos \left(\omega+\frac{2}{3} \pi\right)\right]+\sigma_{m}, \\
\tau_{y z} & =\frac{2}{3} \sigma_{0}\left[m_{1} n_{1} \cos \omega+m_{2} n_{2} \cos \left(\omega-\frac{2}{3} \pi\right)+m_{3} n_{3} \cos \left(\omega+\frac{2}{3} \pi\right)\right], \\
\tau_{z x} & =\frac{2}{3} \sigma_{0}\left[n_{1} l_{1} \cos \omega+n_{2} l_{2} \cos \left(\omega-\frac{2}{3} \pi\right)+n_{3} l_{3} \cos \left(\omega+\frac{2}{3} \pi\right)\right], \\
\tau_{x y} & =\frac{2}{3} \sigma_{0}\left[l_{1} m_{1} \cos \omega+l_{2} m_{2} \cos \left(\omega-\frac{2}{3} \pi\right)+l_{3} m_{3} \cos \left(\omega+\frac{2}{3} \pi\right)\right],
\end{aligned}
$$

where $l_{i}, m_{i}$, and $n_{i}$ are the directional cosines of the principal axes; they are interrelated by six equations of the type $\sum l_{i}^{2}=1, \sum l_{i} m_{i}=0$, and may be expressed by Euler's angles $\alpha, \beta, \gamma$, e.g. by means of the formulae

$$
\begin{array}{rlrl}
l_{1} & =\cos \alpha \cos \beta-\sin \alpha \sin \beta \cos \gamma, & & \\
l_{2} & =-\cos \alpha \sin \beta-\sin \alpha \cos \beta \cos \gamma, & & \\
l_{3} & =\sin \alpha \sin \gamma, & & \\
m_{1} & =\sin \alpha \cos \beta+\cos \alpha \sin \beta \cos \gamma, & n_{1}=\sin \beta \sin \gamma, \\
m_{2} & =-\sin \alpha \sin \beta+\cos \alpha \cos \beta \cos \gamma, & n_{2}=\cos \beta \sin \gamma, \\
m_{3}=-\cos \alpha \sin \gamma, & n_{3}=\cos \gamma .
\end{array}
$$

Thus six unknown stress components (in any locally orthogonal system of coordinates) are replaced by five parameters: $\sigma_{m}, \omega, \alpha, \beta, \gamma$. If the directions of the system coincide with the principal directions, then we may assume, say, $\alpha=\beta=\gamma=0$; hence $l_{1}=m_{2}=n_{3}=1, l_{2}=l_{3}=m_{1}=m_{3}=n_{1}=n_{2}=0$, and three unknowns, $\sigma_{1}, \sigma_{2}, \sigma_{3}$, are replaced by two parameters $\sigma_{m}$ and $\omega$. The physical sense of these unknown parameters is connected with the cylindrical invariants of the stress tensor, Eqs. (5.11) and Table 2.

In the general case formulae (19.39) and (19.40) are applied very seldom in view of their complicated form and the reduction of one unknown only. On the other hand, they are used much more widely in the case of known principal directions, and particularly in plane stress problems, for example if $\sigma_{3}=0$. Then

$$
\begin{aligned}
& \sigma_{1}=\frac{2}{3} \sigma_{0} \cos \omega+\sigma_{m}, \\
& \sigma_{2}=\frac{2}{3} \sigma_{0} \cos \left(\omega-\frac{2}{3} \pi\right)+\sigma_{m}, \\
& \sigma_{3}=\frac{2}{3} \sigma_{0} \cos \left(\omega+\frac{2}{3} \pi\right)+\sigma_{m}=0 ;
\end{aligned}
$$


calculating $\sigma_{m}$ from the third equation and substituting it into the two first equations, we obtain

$$
\begin{aligned}
& \sigma_{1}=\frac{2}{\sqrt{3}} \sigma_{0} \sin \left(\omega+\frac{1}{3} \pi\right), \\
& \sigma_{2}=\frac{2}{\sqrt{3}} \sigma_{0} \sin \omega .
\end{aligned}
$$

This parametrization was already introduced by A. Nadai in 1931, [4.403] (with a somewhat changed angle $\omega$ ). It permits replacing two unknowns, $\sigma_{1}$ and $\sigma_{2}$, by one unknown $\omega$, and is particularly useful in cylindrical coordinates, cf. Sec. 18.4.

A simple generalization of (19.41) makes it possible to parametrize a much wider class of yields conditions. Suppose that the condition of perfect plasticity is of the form

$$
\sigma_{e}=f\left(\sigma_{m}, \omega_{\sigma}\right) ;
$$

this form describes cylindrical limit surfaces (11.1) and rotationally symmetric limit surfaces (11.20) as particular cases. It can be seen that the formulae

$$
\begin{aligned}
& \sigma_{1}=\frac{2}{3} f\left(\sigma_{m}, \omega\right) \cos \omega+\sigma_{m}, \\
& \sigma_{2}=\frac{2}{3} f\left(\sigma_{m}, \omega\right) \cos \left(\omega-\frac{2}{3} \pi\right)+\sigma_{m}, \\
& \sigma_{3}=\frac{2}{3} f\left(\sigma_{m}, \omega\right) \cos \left(\omega+\frac{2}{3} \pi\right)+\sigma_{m},
\end{aligned}
$$

parametrize (19.43) (cf. Table 2; the index of $\omega_{\sigma}$ has been dropped). Returning to the form (19.39), we may also derive the relevant formulae for the general case of arbitrary principal directions.

A transition to the case of plane stress is here simple only for cylindrical surfaces, $\sigma_{e}=f\left(\omega_{\sigma}\right)$; namely

$$
\begin{aligned}
& \sigma_{1}=\frac{2}{\sqrt{3}} f(\omega) \sin \left(\omega+\frac{1}{3} \pi\right), \\
& \sigma_{2}=\frac{2}{\sqrt{3}} f(\omega) \sin \omega .
\end{aligned}
$$

However, in some particular cases of rotationally symmetric limit surfaces a similar parametrization is also possible. For example, the Burzyński-Stassi d'Alia parabolic yield condition (11.24) in the case of plane stress with known principal directions was parametrized by T. Liszka and M. Życzkowski [4.337] as follows:

$$
\begin{aligned}
& \sigma_{1}=\left[\frac{2}{\sqrt{3}} \sqrt{x^{2}-x+1} \sin \left(\omega+\frac{1}{3} \pi\right)+(x-1)\right] \sigma_{0+}, \\
& \sigma_{2}=\left[\frac{2}{\sqrt{3}} \sqrt{x^{2}-x+1} \sin \omega+(x-1)\right] \sigma_{0+},
\end{aligned}
$$

where $x=\sigma_{0-} / \sigma_{0+}$. 
The "elliptic" yield condition (11.32) is obviously parametrized by

$$
\begin{aligned}
& \sigma=\sigma_{0} \cos \psi, \\
& \tau=\tau_{0} \sin \psi=\frac{\sigma_{0}}{\sqrt{c}} \sin \psi ;
\end{aligned}
$$

a parametrization of this type was already used in Sec. 17.6.

Finally, it should be noted that the oldest parametrization in the theory of plasticity was applied to the case of plane strain of an incompressible body. The relevant yield condition, if written in the form (11.11), holds for the Tresca-Guest as well as for the Huber-Mises-Hencky hypothesis. M. Levy [4.328] parametrized this condition in 1871 as follows:

$$
\begin{aligned}
\sigma_{x} & =\tau_{0}(2 \chi+\cos 2 \varphi), \\
\sigma_{y} & =\tau_{0}(2 \chi-\cos 2 \varphi), \\
\tau_{x y} & =\tau_{0} \sin 2 \varphi ;
\end{aligned}
$$

he thus replaced three unknowns, $\sigma_{x}, \sigma_{y}, \tau_{x y}$, by two, $\chi$ and $\varphi$. The geometric interpretation of (19.48) is clear if we use Mohr's circle; though Mohr proposed his construction for the triaxial case in 1882, the circle for the plane case has been known since 1866 (K. Culmann). The parametrization (19.48) is widely employed in the method of characteristics, particularly effective in plane strain problems.

\section{Analytical methods of solution}

\subsection{General remarks. Geometrical hypotheses}

The difficulties in solving plasticity problems are considerable and of diverse character (non-linearity, unknown elastic-plastic interface, dependence on the history of the process, etc.), and hence various appropriate, mainly approximate methods of solution have been developed. We give here merely a key to the extensive bibliography and a short description of the methods which are most frequently used in the problems of combined loadings. The methods will be divided into two groups: analytical methods (Sec. 20) and numerical, mainly computer-oriented methods (Sec. 21), although a sharp division between these groups is not possible and certain methods may be classified in either of the two sections. More extensive surveys of methods of solution of plasticity problems were published by P. G. Hodge [4.226, 4.230], G. P. Cherepanov [4.69], Q. S. Nguyen and J. Zarka [4.418], and H. G. Hopkins [4.235].

For certain particular shapes of the bodies the solution may be greatly simplified if an appropriate system of coordinates is chosen, making it possible to write the boundary conditions in a simpler form, or even to reduce the number of independent variables. This fact stresses the advantages of formulating the basic equations of plasticity in general curvilinear coordinates and of developing appropriate 
methods of solutions. Curvilinear coordinates were introduced into the theory of plasticity comparatively early (H. Hencky [4.204]), and the relevant basic equations may be found even in the earliest monograph on plasticity by $\mathrm{H}$. Mierzejewski [4.386].

The solution of many problems is essentially simplified if it can be based on certain geometrical hypotheses, such as for example the Bernoulli hypothesis of plane cross-sections in the case of bars, or the Love-Kirchhoff hypothesis of straight normals in the case of surface structures. Such solutions may be either exact or merely approximate if the hypothesis adopted does not permit to satisfy all the basic equations of plasticity. In any case it should be noted that the assumption of geometrical hypotheses usually leads to the results presented in parametrical form. For example, the plastic interaction surface is then mostly determined by the equations

$$
Q_{i}=Q_{i}\left(\tilde{q}_{j}\right), \quad i=1,2, \ldots, n ; j=1,2, \ldots, n-1,
$$

where $Q_{i}$ denote the generalized forces (internal at the level $\mathscr{S}$ or external at the level $\mathscr{B}$ ), and $\tilde{q}_{j}$ denote the ratios of geometrical parameters, e.g. of curvature to unit elongation, or the ratios of their rates. The number of such geometrical parameters usually is also $n$, and hence the number of independent ratios equals $n-1$. If an explicit (or even implicit) equation of the plastic interaction curve is required, then we have to eliminate $\tilde{q}_{j}$, i.e. to deparametrize (20.1). Such a procedure is not always effectively possible, and then the parametrical form (20.1) must be used.

The geometrical hypotheses may be regarded as a particular case of general constraints imposed on continuous media. Such a theory of constrained continuous media was developed by C. Woźniak [4.611] and applied to plasticity by M. Kleiber [4.285], who derived a general integral expression for the plastic interaction surfaces at the level $\mathscr{S},(20.1)$. A related general method of construction of (20.1) was proposed by H. S. Ho [4.218].

\subsection{Statically admissible solutions}

In Sec. 19.1 we formulated the general problem of the theory of perfect plasticity as a problem of solving 16 equations with 16 unknowns. However, much simpler approximate solutions may be obtained if only some of those equations-appropriately chosen with physical interpretation-are sa isfied; then the number of the equations is smaller than that of the unknowns and some "degrees of freedom" remain in the solution. Two basic approaches of this kind, used mainly for estimation of the limit carrying capacity of a structure, are called statically admissible solutions and kinematically admissible solutions. The basic theorems of limit analysis determine in both cases the sign of the approximation error, and hence the lower and the upper bounds to the limit carrying capactity are obtained.

A stress field $\sigma_{i j}^{*}$ is called statically admissible if it satisfies the equilibrium equations (19.1), the yield condition in the form of a weak inequality,

$$
\sigma_{\text {red }}^{*} \leqslant \sigma_{0},
$$


and stress boundary conditions on $S_{T}$ up to the multiplier $m$ in (19.8) (H. J. Greenberg, W. Prager [4.185]). This stress field may be continuous or exhibit admissible discontinuities, discussed in Sec. 6.3. The first basic theorem of limit analysis states that, under the assumptions of material stability in Drucker's sense and of neglected geometry changes, the approximate value of the limit carrying capacity calculated by using any statically admissible stress field is not larger than the exact value.

The proof of this theorem is based on the principle of virtual work, stating that

$$
\iint_{S_{T}} p_{n j} \dot{u}_{j} d S+\iiint_{V} F_{j} \dot{u}_{j} d V=\iiint_{V} \sigma_{i j} \dot{\varepsilon}_{i j} d V,
$$

i.e. the rates of external work and of internal work are equal for any statically admissible stress field $\sigma_{i j}=\sigma_{i j}^{*}$ and for any kinematically admissible velocity field $\dot{u}_{J}=\dot{u}_{j}^{* * *}$ (which will be defined in Sec. 20.3), not necessarily related to $\sigma_{i j}^{*}$ (P.G. Hodge [2.62], T. H. Lin [3.456], L. E. Malvern [2.108]). In the case of discontinuous velocity fields $\dot{u}_{j}^{* *}$, the integration should be performed according to the rules for generalized functions (distributions). For the sake of simplicity we omit now the body forces $F_{j}$, present the surface tractions in the form (19.8), replace $\dot{\varepsilon}_{i j}$ by $\dot{\varepsilon}_{i j}^{p}$ (for a rigid-plastic body), and write (20.3) twice, for exact $\sigma_{i j}$ and $\dot{u}_{j}$ and for any statically admissible $\sigma_{i j}^{*}$ with unchanged $\dot{u}_{j}$. In the first case the multiplier $m$ of external loadings has its exact value, whereas in the second case a certain other value, $m_{s}$ (statically admissible multiplier), is used:

$$
\begin{aligned}
& m \iint_{S_{T}} p_{n j 0} \dot{u}_{j} d S=\iiint_{V} \sigma_{i j} \dot{\varepsilon}_{i j}^{p} d V, \\
& m_{s} \iint_{S_{T}} p_{n j 0} \dot{u}_{j} d S=\iiint_{V} \sigma_{i j}^{*} \dot{\varepsilon}_{i j}^{p_{j}} d V .
\end{aligned}
$$

Subtracting these equations, we obtain

$$
\left(m-m_{s}\right) \iint_{S_{T}} p_{n j 0} \dot{u}_{j} d S=\iiint_{V}\left(\sigma_{i j}-\sigma_{i j}^{*}\right) \dot{\varepsilon}_{i j}^{p} d V .
$$

In view of the assumed material stability, (13.17), we find that the integral on the right is non-negative throughout the body, and since the surface integral on the left is positive, we prove that

$$
m-m_{s} \geqslant 0, \quad m_{s} \leqslant m .
$$

Hence we have proved that the "static approach" gives a lower bound to the actual limit carrying capacity of the structure. Such approximate solutions are of particular interest for engineering applications, since the approximation error cannot decrease the safety factor. The statically admissible multiplier $m_{s}$ is a functional depending on the stress field $\sigma_{i j}^{*}$; since the exact stress field is also statically admissible, thus the maximum of lower bounds $m_{s}$ is equal to the exact value $m$.

The construction of a statically admissible stress field leaves, in general, two 
"degrees of freedom" inside the body, since six functions $\sigma_{i j}^{*}$ have to satisfy four equations (19.1) and (20.2), even if we regard (20.2) as an equation and not as an inequality. Additional constraints result from the stress boundary conditions on $S_{T}$, nevertheless the search for a statically admissible solution is usually much simpler than the search for the exact solution. In particular cases the number of "degrees of freedom" may be reduced to one or even to zero (statically internally determinate problems). In the latter case, if the solution of the relevant equations is unique, then there exists only one statically admissible stress field corresponding to the exact solution. However, in view of the non-linearity of the plasticity equations, even in this case several statically admissible solutions may exist and then the maximal value of $m_{s}$ furnishes the smallest approximation error.

A certain general method of constructing statically admissible stress fields was proposed by H. Gajewski [4.148]. Many problems can be successfully solved by using statically admissible stress fields with jump-like non-homogeneity (homogeneous stress fields in individual subdomains). W. Szczepiński [4.566] applied this concept to the optimal design of numerous machine elements. Statically admissible solutions are also useful in the problem of transition from the level $\mathscr{S}$ to the level $\mathscr{B}$; then we may introduce statically admissible fields of generalized stresses (internal forces) and a similar theorem holds (W. Prager [4.468], P. G. Hodge [4.221]).

The lower bound theorem was proved in the particular case of statically indeterminate beams by G. Kazinczy [4.275]; further papers were published by A. A. Gvozdev [4.193, 4.194, 4.195], J. A. Van den Broek [4.592] and S. M. Feynberg [4.139], who formulated this theorem for the general case. A complete proof of the theorem was given by D. C. Drucker, H. J. Greenberg and W. Prager in [4.118] for the case of plane strain, and in [4.120] for the general case, but under the assumption of a regular limit surface; W. T. Koiter [3.363] and W. Prager [3.654] extended this theorem also to the case of limit surfaces with singularities '(e.g. corresponding to the Tresca-Guest yield condition).

The lower bound theorem leads to the following related theorems (D. C. Drucker [4.121]): the addition of weightless material cannot lower the limit carrying capacity of the structure; an increase of the yield-point stress of the material in any subregion cannot lower the limit carrying capacity. However, one has to be cautious when applying these theorems. First, they are valid only in relation to the Hill definition of the limit carrying capacity for rigid-plastic materials, whereas for perfectly elastic-plastic materials the limit carrying capacity is then usually replaced by the decohesive carrying capacity, which may be much smaller (Sec. 18). Second, for real materials (elastic-plastic-hardening with a physical criterion of decohesion) an inappropriate addition of material obviously leads to a decrease of the actual carrying capacity because of a worse redistribution of internal forces; this statement was confirmed by some simple experiments on bar systems carried out at the Technical University of Cracow.

For the shakedown problems E. Melan [4.380] formulated a theorem which may be regarded as an extension of the lower bound theorem for static loadings. 
This theorem states that the structure will shake down if there exists any time-independent distribution of self-stresses $\varrho_{i j}(x)$ such that

$$
f\left[\sigma_{i j}^{e}(x, t)+\varrho_{i j}(x)\right]<\sigma_{\mathrm{EL}}
$$

for any time $t$, where $\sigma_{i j}^{e}$ denote stresses representing a perfectly elastic response to the given loading $P(t)$ (varying within prescribed limits), $f$ denotes the reduced stress according to the assumed failure hypothesis, and $\sigma_{\mathrm{EL}}$-the elastic limit (in simplest schematizations identified with the yield-point stress $\sigma_{0}$ ). In other words, the structure will shake down if the loading program is contained within a certain constant modified initial neutral surface (elastic surface modified by the addition of self-stresses, determined by (20.8) regarded as an equation). Usually it is assumed that shakedown and a purely elastic response will take place after a finite number of loading cycles if the function $P(t)$ is periodic or quasi-periodic; however, this is by no means necessary, and an example of shakedown in the sense of (18.12) with dissipations of energy up to $t \rightarrow \infty$ is shown in Fig. 110 .

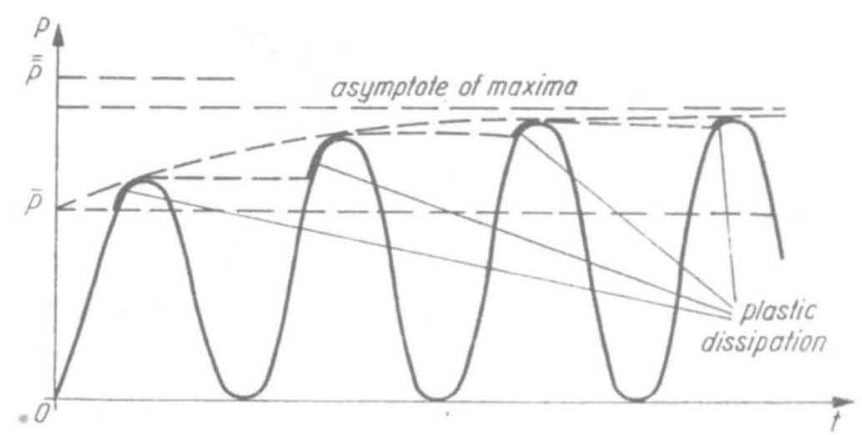

Fig. 110. An example of shakedown in spite of plastic dissipation up to $t \rightarrow \infty$

The Melan theorem provides the most commonly used tool of shakedown analysis. Some methods of application of this theorem to an effective determination of safe shakedown limits were proposed by V. P. Kryzhanovsky, L. G. Lantukh and A. V. Perelmuter [4.312], V. I. Lyubarov [4.339], R. J. Melosh and R. Levy [4.384]. Extensions of the Melan theorem to thermal loadings were mentioned in Sec. 18.8; a certain theorem connected with a step-by-step calculation method was suggested for this case by Zh. I. Tairova [4.575].

\subsection{Kinematically admissible solutions}

A velocity field $i_{i}^{* * *}=v_{i}^{* * *}$ is called kinematically admissible if it satisfies the displacement boundary conditions on $S_{U}$, the incompressibility condition inside the body

$$
v_{i, i}^{* *}=0
$$


(in relation to the rigid-plastic schematization) and the condition of positiveness of power of external loadings

$$
\dot{W}=\iint_{S_{U}} p_{n j} \dot{u}_{j}^{* *} d S+\iiint_{V} F_{j} \dot{u}_{j}^{* *} d V>0 .
$$

The second basic theorem of limit analysis states that under the assumptions of material stability in Drucker's sense and of neglected geometry changes, the limit carrying capacity calculated by using any kinematically admissible velocity field is not smaller than the exact value.

Indeed, let us apply the principle of virtual work (20.3) to the chosen kinematically admissible velocity field $\dot{u}_{j}^{* *}$ combined with the exact stress distribution $\sigma_{i j}$ (with the exact loading multiplier $m$ )

$$
m \iint_{S_{T}} p_{n j 0} \dot{u}_{j}^{* *} d S=\iiint_{V} \sigma_{i j} \dot{\varepsilon}_{j}^{* * p} d V,
$$

(the body forces are omitted) and define the kinematically admissible multiplier $m_{k}$ by the equation

$$
m_{k} \iint_{S_{T}} p_{n j 0} \dot{u}_{j}^{* *} d S=\iiint_{V} \sigma_{i j}^{* *} \dot{\varepsilon}_{i j}^{* * p} d V,
$$

where $\sigma_{i j}^{* *}$ correspond to $\dot{\varepsilon}_{i j}^{* *}$ and are calculated from the physical equations and the yield condition (with an accuracy up to $\sigma_{m}^{* *}$, which is irrelevant here). Subtracting (20.11) from (20.12), we obtain

$$
\left(m_{k}-m\right) \iint_{S_{T}} p_{n j 0} \dot{u}_{j}^{* *} d S=\iiint_{V}\left(\sigma_{i j}^{* *}-\sigma_{i j}\right) \dot{\varepsilon}_{i j}^{* * p} d V .
$$

Now, in view of (13.17) the integrand on the right is non-negative, since in this case we apply the Drucker postulate to the point $\sigma_{i j}^{* *}$; in view of the assumption (20.10) the integral on the left is positive, and we find that

$$
m_{k}-m \geqslant 0, \quad m_{k} \geqslant m \text {. }
$$

We have thus proved that the "kinematic approach" gives the upper bound to the actual limit carrying capacity of the structure. The approximation error goes here against safety, but combining (20.14) with (20.7) we may write

$$
m_{s} \leqslant m \leqslant m_{k}
$$

and taking the arithmetic mean value $m \approx\left(m_{s}+m_{k}\right) / 2$ as the next approximation, we reduce the error and estimate it by $\left(m_{k}-m_{s}\right) / 2$. The kinematically admissible multiplier is a functional depending on $\dot{u}_{j}^{* *}$, defined by (20.12); the minimal value of this functional is equal to the exact value $m$.

The construction of a kinematically admissible velocity field also leaves, in general, two "degrees of freedom", as in the static approach, since three functions $\dot{u}_{j}^{* *}$ are interrelated inside the body by only one equation (20.9). In particular cases the number of "degrees of freedom" may even be reduced to zero (geometrically or kin- 
ematically internally determinate problems, e.g. thick-walled circular cylinders under pressure in plane strain conditions). Kinematically admissible velocity fields may exhibit admissible discontinuities, tangential to the discontinuity surface. However, normal discontinuities are also assumed in many engineering applications (velocity fields expressed by generalized functions); in the case of plates and shells they were investigated by M. Janas [4.258], and in the general case by M. Sayir and H. Ziegler [4.518]. Such an approach may be justified in relation to the Hill definition of the limit carrying capacity (beginning of motion of rigid-plastic-hardening body), but in the case of a perfectly elastic-plastic body they lead to inadmissible discontinuities in displacements and to decohesion.

In the classical approach to the basic theorems of limit analysis one assumes a simple loading process, (19.8). However, this assumption is not essential: more general multiparameter loadings were discussed by V. Franciosi [4.145], P. G. Hodge [4.227] and J. J. Telega [4.579]; of course, "too complicated" loading programs must be considered within the frames of the shakedown theory.

In the case of materials not conforming to the Drucker definition of material stability, inequality (20.15) does not necessarily hold. Certain simple counterexamples were given by W. T. Koiter [3.364] and D. C. Drucker [4.122], and another example of $m_{s}>m_{k}$, concerning plasticity of soils, was discussed in detail by J. Salençon [4.504]. For non-standard materials (with the plastic potential not coinciding with the yield condition, but under the assumption of convexity of both surfaces) D. Radenković [3.663, 3.664] proved some weaker basic theorems of limit analysis; further research in this direction is due to A. C. Palmer [4.440], G. Sacchi and M. Save [4.495], and J. Salençon [4.501, 4.502].

I. F. Collins [4.85] discussed the upper bound theorem in the case of friction loadings. The extension of limit theorems to composite materials is due to P. V. Mc Laughlin and S. C. Batterman [4.376].

T. Mura and S. L. Lee [4.400] derived statically admissible solutions and kinematically admissible solutions from a single functional under various constraints. This concept was developed and applied by T. Mura, S. L. Lee, R. H. Bryant and W. H. Rimawi [4.401, 4.402]; related proposals are due to G. Sacchi and M. Save [4.496], M. J. Sewell [4.523], D. Radenković and Q. S. Nguyen [4.480], and J. J. Telega [4.580] (for the Sawczuk-Stutz constitutive equations (13.50)).

The theorem of W. T. Koiter [4.294] may be regarded as an extension of the upper bound theorem to shakedown problems. This theorem states that the structure cannot shake down if there exists any kinematically admissible cyclic velocity field $\dot{u}_{j}^{*}{ }^{*}(t)$ and such a cyclic loading $P_{n j}(t)$, belonging to the prescribed loading domain, that

$$
\int_{0}^{T} d t\left(\iint_{S_{T}} p_{n j} \dot{u}_{j}^{* * *} d S+\iiint_{V} F_{j} \dot{u}_{j}^{* * *} d V\right)>\int_{0}^{T} d t \iiint_{V} \sigma_{i j}^{* *} \dot{\varepsilon}_{j j}^{* * p} d V,
$$

where $T$ denotes the loading period for variable repeated loadings. The proof of 'this theorem is given by W. T. Koiter [4.294, 3.365]. 
The generalizations of the Koiter theorem to the case of thermal cycles and to non-associated flow rules are due to G. Maier [4.347] (linear programming method), O. De Donato [4.107], and R. M. Kirakosyan [4.279]; the generalization to dynamic loadings was proposed by L. Corradi and G. Maier [4.92, 4.93]. Non-proportional changes of various exertion factors were discussed by A. Gjelsvik [4.165].

\subsection{Statically partially admissible solutions}

Some authors derived approximate formulae for the limit carrying capacity of various structures or cross-sections using stress fields satisfying even weaker assumptions than those defined in Sec. 20.2. Such fields will be called here statically partially admissible stress fields. Particularly often one can find solutions based on stress fields which satisfy the yield condition and the stress boundary conditions but do not satisfy the equations of internal equilibrium. Let us mention here, for example, A. A. Gvozdev's solution for a thin-walled circular section subject to simultaneous tension, torsion, bending, and shear, [4.196], pp. 249-254; the equilibrium equation (or the circulation theorem) requires the independence of shearing stresses $\tau_{z \theta}$ of the angle $\theta$ at least for a vanishing shear force, whereas the author admits also the functions $\tau_{z \theta}=f(\theta)$.

In such a case one cannot state that the solution obtained presents a lower bound to the exact solution. On the contrary, if we evaluate the approximate value of the loading multiplier $m$ by means of the calculus of variations, looking for the maximal value of this functional, then we usually arrive at an upper bound, lowering the safety factor. Indeed, if the maximum of the functional $m_{s}$ under additional constraints (equilibrium equations) equals $m$, then the unconstrained maximum of that functional is not smaller than the exact value $m$, and usually larger than $m$.

It turns out that the above-mentioned Gvozdev solution (e.g. for bending with torsion) corresponds to a kinematically admissible velocity field, based on the assumption of no warping of the cross-section, and in view of the unsatisfied equilibrium equations we prove that it gives an upper bound to the exact solution. A similar remark applies to many other solutions determining the limit carrying capacity of a cross-section where the equilibrium equations are not considered; some of them will be quoted in Chapters VI, VII, and VIII.

Another case of statically partially admissible stress fields occurs if the equilibrium equations are satisfied, but the stress boundary conditions are not. Then the loading multiplier gives a lower bound, though not to the problem under consideration, but to another one, with external loadings corresponding through the boundary conditions to the stress field assumed. The sign of the approximation error for the primary problem remains, as a rule, unknown. For example, assuming for a square bar subject to pure torsion the stress field

$$
\tau_{z \theta}=\tau_{0},
$$


we satisfy both the equilibrium equations and the yield condition, but the boundary conditions at the lateral surface are not satisfied (Fig. 111). Disregarding this fact and treating (20.17) as a statically partially admissible stress field for the given problem of pure torsion, we obtained the approximate limit value of the torque with an error from above.

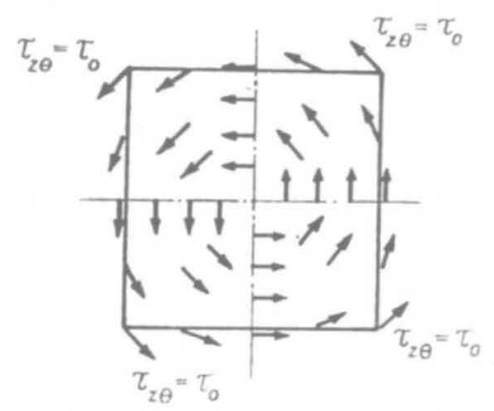

Fig. 111. A statically partially admissible stress field (the boundary conditions left unsatisfied)

The boundary conditions are not satisfied e.g. by the stress field, proposed by A. R. Rzhanitsyn [4.492] for the problem of the limit carrying capacity of a section under simultaneous bending and shear. Hence it is difficult to state whether the approximation error is from below or from above; probably the exact value is not unique but depends on the gradients of the generalized internal forces along the axis of the beam.

The statically partially admissible solutions under discussion are not as valuable as the statically admissible solutions, since they do not permit error estimation; however, in many cases the error is not too large and the basic advantage of such solutions lies in their simplicity.

\subsection{Variational methods}

Statically or kinematically admissible solutions of the problems of the limit carrying capacity may be obtained without direct use of the calculus of variations. In fact, the relavant proofs of the theorems are based on the calculus of variations; moreover, if the statically or kinematically admissible fields are assumed with an accuracy up to a certain function, then the optimal choice of that function is determined by the requirement of an extremum of the functional (loading multiplier), and so it consti utes a problem of the calculus of variations. The connection of statically partially admissible solutions with the calculus of variations is less evident.

Hence the construction of statically or kinematically admissible solutions belongs to variational methods of the theory of plasticity. Similar methods may also be used in the elastic-plastic range and for plastic hardening materials. In the theory of elasticity variational principles are used to derive equations, to derive exact solutions in the form of infinite series, and to obtain approximate solutions; in the 
theory of plasticity only the third direction seems to be effective as a method (P. G. Hodge [4.230]); moreover, variational principles are also often used in the proofs of uniqueness theorems. We are now going to present a short historical outline of variational principles in plasticity; detailed survey papers were published by R. Hill [4.210], D. C. Drucker [4.123], W. T. Koiter [3.365], P. G. Hodge [4.221, 4.226], H. Lippmann [4.336], and the books by R. Hill [2.56], L. M. Kachanov [2.79], W. Olszak, Z. Mróz and P. Perzyna [3.600] and K. Washizu [4.601] also pay considerable attention to this topic.

The oldest variational principle in the theory of plasticity is due to A. Haar and T. Kármán [4.197], 1909. This principle generalized the Castigliano principle and states that the actual stresses $\sigma_{i j}$ minimize the elastic energy of the body; for an isotropic body

$$
\iiint_{V}\left(\frac{\sigma_{e}^{2}}{6 G}+\frac{\sigma_{m}^{2}}{2 K}\right) d V=\min ,
$$

under the constraints of internal equilibrium, boundary conditions, and the yield condition. Haar and Kármán assumed the Tresca-Guest yield condition, employed the Lagrange equations and in this way derived the first (inconvenient) physical equations of the deformation theory. It was H. Hencky [2.53] who combined (20.18) with the $\mathrm{HMH}$ yield condition and derived the classical equations of the deformation theory. The principle (20.18) was proved in 1949 by H. Greenberg [4.184] under the assumption that no unloading occurred at any stage of the deformation process. W. Prager and P. S. Symonds [4.465] expressed the Haar-Kármán principle in terms of residual stresses; further variational principles for residual stresses were proposed by G. Ceradini [4.64], A. R. S. Ponter [4.460] and A. M. Protsenko [4.478]. Greenberg's proof was based on the Hencky-Ilyushin theory; W. Prager [4.468] found several examples of the validity of this principle in connection with flow theories and changing stresses, but W. Koiter [3.365] claimed several counterexamples and stressed the limitations of validity.

The second variational principle in plasticity, due to G. Colonnetti [4.86], 1918, [4.87, 4.88, 4.89], and H. Reissner [4.483], is also connected with the deformation theory and may be regarded as another generalization of the Castigliano principle. This principle states that the actual stresses minimize the expression

$$
\iiint_{V}\left(\frac{\sigma_{e}^{2}}{6 G}+\frac{\sigma_{m}^{2}}{2 K}+\sigma_{i j} \varepsilon_{i j}^{p}\right) d V=\min ,
$$

if the distribution of the plastic strains $\varepsilon_{i j}^{p}(x)$ is known. Further generalizations of the Castigliano principle are due to L. M. Kachanov [4.264, 4.265] and V. M. Panferov [4.444].

R. Mises [3.531] formulated in 1928 for a perfectly plastic body the principle of maximal power with respect to the variations of stresses at constant strain rates; this principle is connected with the concept of plastic potential, introduced in the same paper, though Mises remarks that the derivation of his principle cannot be 
regarded as strictly mathematical. A related heuristic principle of maximal plastic resistance was proposed in 1943 by M. A. Sadowsky [4.500]. It was supported and extended by G. H. Handelman [4.199], but is not generally valid; the conditions of its validity were established by A. H. Philippidis [4.451] (general proof) and R. Hill [4.209]. Hill introduced the principle of maximal plastic work,

$$
\iint_{S_{U}} \sigma_{i j}^{*} n_{j} \dot{u}_{i} d S=\max ,
$$

if $\sigma_{i j}^{*}$ denotes any statically admissible stress field. A complementary principle,

$$
\sigma_{0} \iiint_{V} \sqrt{\frac{2}{3} \dot{\varepsilon}_{i j}^{* *} \dot{\varepsilon}_{i j}^{* * *}} d V-\iint_{S_{T}} p_{n j} \dot{u}_{j}^{* *} d S=\min ,
$$

for kinematically admissible velocity fields was introduced by A. A. Markov [4.371] and developed by R. Hill [4.210]. A general principle, including Hill's and Markov's principles as particular cases, was proposed by J. Schroeder and A. N. Sherbourne [4.519].

The first variational principles for stress rates and strain rates in elastic-plastichardening bodies with regular neutral surfaces were formulated by P. G. Hodge and W. Prager [3.258], and extended by H. J. Greenberg [4.183] and Y. Yamamoto [4.615]. W. T. Koiter [3.363] derived similar principles for singular neutral surfaces and P. G. Hodge [4.220] developed this concept for general piece-wise linear plasticity. Generalizations to non-homogeneous and anisotropic bodies are due to W. Olszak and P. Perzyna [4.429, 4.430]. H. Lippmann [4.335] considered ranges of validity of the rate theorems; he presented a counter-example to the kinematic theorem in its original version and proposed a suitable modification.

Complementary variational principles for piece-wise linear hardening described by the deformation (total) theory of plasticity were proposed by G. Maier [4.343, $4.345,4.346]$; further development is due to A. R. S. Ponter and J. B. Martin [4.462, $4.372,4.373$ ], who determined in this way a class of admissible loading paths in the deformation theory of plasticity.

Variational principles for thermoplastic problems were suggested by A. J. Wang and W. Prager [4.600] (valid for a yield condition independent of temperature, [4.481]), H. Ziegler [4.622] E. P. Brumfitt and J. Schroeder [4.53], B. Raniecki [4.481, 4.482], Z. Mróz and B. Raniecki [4.398].

The first formulation of variational principles for finite strains is due to A. Phillips [4.452] and A. Nádai [4.404]. R. Hill [3.248, 4.214] derived a variational principle and discussed uniqueness in the case of rigid-plastic-hardening body with geometry changes taken into account. Further proposals are due to M. Capurso [4.59], O. De Donato [4.108], G. Maier [4.350], K. W. Neale [4.414], M. Duszek [4.131], L. Corradi and G. Maier [4.95].

Most variational principles cease to be valid if plastic softening takes place. The relevant principles were formulated by G. Maier [4.340, 3.475, 4.349] and O. De Donato [4.109]. 
Variational principles are widely used for obtaining approximate analytical solutions of various plasticity problems, but their importance for numerical methods is also rapidly increasing; some of these methods will be discussed in Sec. 21 .

\subsection{Perturbation (small parameter) method}

The perturbation method, developed by $\mathrm{H}$. Poincaré, consists in the expansion of the solution into the power series of a certain parameter $\psi$; it is called the small parameter, since its value is limited by the radius of convergence of the series obtained, and practically by a sufficiently rapid convergence of these series. The "classical" (regular) perturbation method may be applied if the solution can be presented in the form of a power series, but some more general cases will also be discussed below. Moreover, from the practical point of view, one requires the simplicity of the solution for $\psi=0$, since this solution constitutes the zeroth approximation for the perturbed problem, $\psi \neq 0$. So, in [4.628, 2.198] M. Życzkowski used the perturbation method to take into account the compressibility of the material when the solutions for an incompressible material were relatively simple; D. D. Ivlev and L. V. Ershov $[4.250,4.251,4.136]$ accounted for the deviations from simple shapes of the bodies considered; A. J. M. Spencer in the series of papers [4.547] considered the problems of small non-homogeneity, deviations from simple shapes and small body forces; M. Życzkowski and M. Galos [4.641] and A. Dudek and M. Życzkowski [4.127] regarded as a small parameter the unit angle of twist of naturally uniformly twisted bars.

In the simplest case the solution of the problem under consideration is presented in the form

$$
X_{i}=\sum_{j=0}^{\infty} X_{i j} \psi^{j},
$$

where the general symbol $X_{i}$ denotes unknown quantities, e.g. stresses, strains, displacements, the functions $\varphi$ or $\lambda$, etc. Substituting (20.22) into the basic equations of the theory of plasticity (or after a reduction of the unknowns) and equating the coefficients of the successive powers of the parameter $\psi$, one obtains the equations for $X_{i j}$. As a rule, these equations are much simpler than those for $X_{i}$ (linearized, uncoupled, etc.).

The perturbation method may be particularly useful in problems of combined loadings: the small parameter may describe a "new" type of loading superposed on the "basic" loading, for which the solution is well known and simple. Of course, one can also consider the influence of several additional loadings introducing several small parameters. In this way a certain fragment of the limit surface in the neighbourhood of the basic loading is determined. Such an approach was used in the papers by S. Piechnik [4.453], S. Piechnik and M. Życzkowski [4.454], M. Wnuk [4.605, 4.607], M. Życzkowski [4.636, 4.638], J. Skrzypek [4.541], J. Skrzypek and M. Życzkowski [4.543]. This manner of application of the perturbation method 
also proved useful in the related problem of creep under combined loadings (S. Piechnik [4.542, 4.543, 4.544], M. Życzkowski and J. Skrzypek [4.639, 4.640]).

The basic element of the perturbation technique consists in operations on power series: multiplication, division, raising to any power, inversion, substitution into another power series and deparametrization. The relevant formulae and tables are given in [4.631, 4.632, 4.635]; since in some applications one has to employ generalized power series of the type

$$
X_{i}=\sum_{j=0}^{\infty} X_{i j} \psi^{\mu+v j}
$$

(e.g. in the analysis of combined plastic bending with tension [4.636]), thus the above-mentioned papers refer to a broader class od series, (20.23): The symbols $\mu$ und $y \neq 0$ in (20.23) stand for arbitrary real numbers.

We quote here only the formulae for raising power series to any power; the other operations listed above may be reduced to this basic operation. The problem is to calculate the coefficients $A_{j, \xi}$ in the equation

$$
[y(\psi)]^{\xi}=\left(\sum_{j=0}^{\infty} a_{j} \psi^{\mu+v j}\right)^{\xi}=\sum_{j=0}^{\infty} A_{j, \xi} \psi^{\mu \xi+v j},
$$

where $\xi$ is an arbitrary real number. In the notation used, the index preceded by a comma does not denote differentiation.

The coefficients $A_{j, \xi}$ are determined by the general formula

$$
A_{j, \xi}=\sum_{s=1}^{S(j)} \frac{\xi(\xi-1) \ldots(\xi-m+1)}{k_{1 s} ! k_{2 s} ! \ldots k_{j s} !} a_{0}^{\xi-m} a_{1}^{k} 1 s a_{2}^{k_{2 s}} \ldots a_{j}^{k j s},
$$

where $k_{i s}$ denote successive solutions of the equation

$$
k_{1}+2 k_{2}+3 k_{3}+\ldots+j k_{j}=j,
$$

in non-negative integers, $s$ denotes the ordinal number of the solution, $s=1,2, \ldots$ $\ldots, S(j), S$ denotes the total number of such solutions, and finally

$$
m=k_{1}+k_{2}+k_{3}+\ldots+k_{j}=m(s) .
$$

Thus the first terms $A_{j, \xi}$ are determined by the formulae, [4.631],

$$
\begin{aligned}
A_{0, \xi}= & a_{0}^{\xi}, \\
A_{1, \xi}= & \xi a_{0}^{\xi-1} a_{1}, \\
A_{2, \xi}= & \frac{\xi(\xi-1)}{2 !} a_{0}^{\xi-2} a_{1}^{2}+\frac{\xi}{1 !} a_{0}^{\xi-1} a_{2}, \\
A_{3, \xi}= & \frac{\xi(\xi-1)(\xi-2)}{3 !} a_{0}^{-3} a_{1}^{3}+\frac{\xi(\xi-1)}{1 ! 1 !} a_{0}^{\xi-2} a_{1} a_{2}+\frac{\xi}{1 !} a_{0}^{\xi-1} a_{3}, \\
A_{4, \xi}= & \frac{\xi(\xi-1)(\xi-2)(\xi-3)}{4 !} a_{0}^{\xi-4} a_{1}^{4}+\frac{\xi(\xi-1)(\xi-2)}{2 ! 1 !} a_{0}^{\xi-3} a_{1}^{2} a_{2}+ \\
& +\frac{\xi(\xi-1)}{2 !} a_{0}^{\xi-2} a_{2}^{2}+\frac{\xi(\xi-1)}{1 ! 1 !} a_{0}^{\xi-2} a_{1} a_{3}+\frac{\xi}{1 !} a_{0}^{\xi-1} a_{4} .
\end{aligned}
$$


The tables of $A_{j, \xi}$ for the most often used exponents $\xi$ are given in [4.632]. The problem of operations on power series from the combinatorial point of view is treated by L. Comtet in [4.90], where also numerous auxiliary tables are enclosed.

The regular perturbation method described above cannot be applied if the unknown function $X_{i}$ has a discontinuity at a point (or along a line) depending on the parameter $\psi$; such cases often appear in the plasticity problems.

In fact, if for $\psi=\psi_{1}$ discontinuity occurs at $x_{1}$, and for $\psi=\psi_{2}$ at $x_{2}$, then the change of the function $X_{i}$ at a certain intermediate point $\bar{x}$ is big even for a very

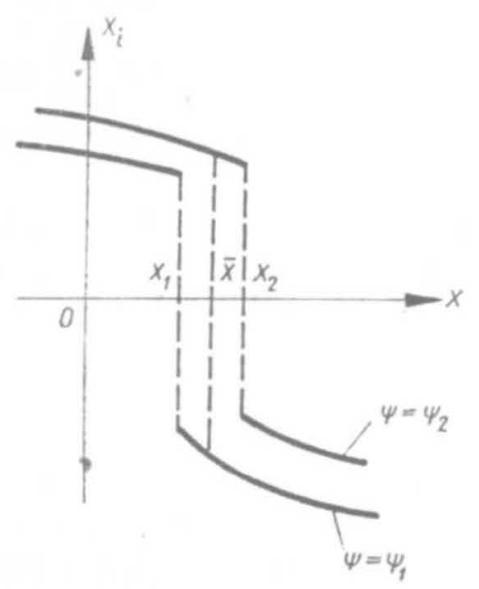

Fig. 112. Discontinuity of a function depending on a parameter

small change of the parameter $\psi$ (Fig. 112). Thus a series of type (20.22) is divergent at least in the vicinity of the discontinuity.

In most such cases a certain modified perturbation method may be applied. This method, mentioned by Poincaré, was developed in 1949 by M. J. Lighthill [4.331], and is called the method of strained coordinates (M. Van Dyke [4.593], A. Nayfeh [4.409]) or the PLK method (Poincaré-Lighthill-Kuo, H.S. Tsien [4.588]). The basic idea is to expand into power series not only the unknown functions but also the independent variables, making use of Lighthill's principle: "Higher approximations shall be no more singular than the first." This procedure, relatively simple in the case of ordinary differential equations, becomes complicated for partial differential equations. Moreover, it was found to be inappropriate for elliptic and parabolic equations and hence in paper [4.332] Lighthill advises his method only for hyperbolic equations.

Numerous mathematical papers are devoted to the problem of convergence of the perturbation method, but in most cases they are far from applications. Let us only mention the monographs by T. Kato [4.273] and H. Baumgärtel [4.23], where numerous references can be found. The problem of convergence of the PLK method was discussed by W. Wasow [4.602]. 


\subsection{Approximation of the Hermitian type}

The perturbation method makes it possible to determine certain fragments of the limit surface by means of power series; in other words, we know at certain points the value of the function itself and of several first derivatives. These conditions are sufficient for the approximate determination of the limit surface in the whole domain under consideration by the use of the Hermitian approximation or of a certain generalization of this procedure.

$\mathrm{Ch}$. Hermite formulated the following problem of interpolative approximation: he sought the polynomial $H(x)$, which at $n$ points (nodes) $x_{i}$ coincides with the given function $y(x)$ up to the derivative $y^{\left(x_{i}-1\right)}$ inclusively. Hence the total number of conditions imposed on $H(x)$ is $\sum_{i=0}^{n} \alpha_{i}$; such a polynomial of degree $\left(\sum_{i=0}^{n} \alpha_{i}\right)-1$ exists and is unique (cf. I. P. Natanson [4.407], where the error estimation is also given).

Suppose now that we are interested in an approximate presentation of a limit curve, e.g. the plastic interaction curve $X_{2}=X_{2}\left(X_{1}\right)$, where $X_{1}$ and $X_{2}$ are two arbitrary exertion factors, and that we know the power series expansions at $X_{1}=0$ (then $X_{2}=\overline{\bar{X}}_{2}$, neighbourhood of pure $X_{2}$ ) and at $X_{2}=0$ (then $X_{1}=\overline{\bar{X}}_{1}$, neighbourhood of pure $X_{1}$ ). Then, in principle, we might use Hermite's approximation with multiple conditions of coincidence at two nodes $X_{1}=0$ and $X_{1}=\overline{\bar{X}}_{1}$. This type of approximation will be called Hermite's boundary approximation; it is also termed the two-point Taylor interpolation (P. J. Davis [4.106]). Effective formulae for the coefficients of such polynomials are given in [4.629].

However, in most cases under consideration all the derivatives of $X_{2}=X_{2}\left(X_{1}\right)$ at $X_{1}=X_{1}$ increase infinitely (Fig. 113), and the perturbation method determines

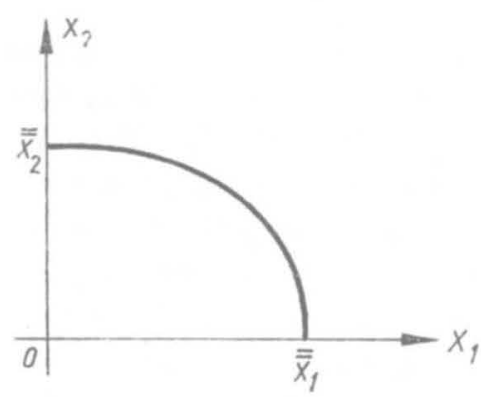

Fig. 113. The generalized Hermite boundary approximation

at this point the derivatives of the inverse function, $X_{1}=X_{1}\left(X_{2}\right)$. The polynomial approximation cannot then be used, but usually one can suggest an appropriate implicit function $f\left(X_{1}, X_{2}\right)=0$ which shows the required coincidence at $X_{1}=0$ and at $X_{2}=0$. Such an approximation will be called the generalized Hermite boundary approximation; it was applied by S. Piechnik and M. Życzkowski [4.454], 
M. Wnuk [4.606, 4.608], M. Mrowiec and M. Życzkowski [4.397], J. Skrzypek [4.540].

Further complications arise in multidimensional cases if we intend to describe limit surfaces or hypersurfaces in this way. Then the coincidence of several consecutive partial derivatives at individual points is required. A certain example of the application of such approximation in the four-dimensional space of exertion factors was given by J. Skrzypek and M. Życzkowski [4.543].

In general, the applicability of Hermite's boundary approximation is limited to regular curves or surfaces, showing no singularity inside the interval. However in some cases good results may be obtained even if the approximated function shows weak singularities, e.g. the discontinuity of the second derivative; such a case was discussed in [4.636].

\subsection{Onefold optimal approximation}

The Hermite boundary approximation discussed above shows, as a rule, good accuracy in the neighbourhood of both boundary points, but may result in larger errors inside the interval. On the contrary, the Chebyshev "best" or "optimal" approximation shows a possibly small error in the whole interval but does not coincide with the given function at boundary points, which are usually important because of their physical interpretation. A certain intermediate type of approximation was proposed by M. Życzkowski in [4.633] and called the onefold optimal approximation. It uses the conditions of $k$-fold coincidence at the left-hand boundary point, $(n-k-1)$-fold coincidence at the right-hand boundary point, and leaves one more free parameter to be determined from the Chebyshev condition of minimal distance between the given function and the approximating function. Practically, this condition can only be satisfied in a numerical way.

The onefold optimal approximation was applied in [4.636] to an analytical, explicit and uniform description of plastic interaction curves for simultaneous bending and tension of bars with various shapes of the cross-section.

\section{Numerical methods in plasticity}

\subsection{General remarks}

Numerical methods used in continuum mechanics usually concern the operations of differentiation and integration. The replacement of these operations by simple algebraic operations may result in poor accuracy unless a sufficiently large number of algebraic operations is introduced, and hence the development and effective application of numerical methods is closely bound with the development of computers. 
Usually, the first step is here to choose a number of discrete points of the body and look for the values of the solution only at those points (nodes). Such discretization procedures are performed in various ways, depending on the numerical method used. Further, basic equations are reformulated so as to describe relations between the unknowns at the nodes. The matrix notation is here the most effective and suitable for computer technique. J. F. Besseling [4.30] points out the analogy between matrix equations and the continuous field equations of structural analysis.

Surveys of numerical methods in plasticity were published by P. G. Hodge [4.230] and I. A. Birger [4.37]. In what follows we only give a short description of the most important numerical methods and quote the basic references.

\subsection{Direct integration method. Integration along characteristics}

In the case of ordinary differential equations with all boundary conditions at one end (initial-value-problem) we may replace them by systems of first-order equations and simply integrate by any direct, step-by-step numerical technique. First such a procedure was suggested by Euler over 200 years ago, but the much more accurate Runge-Kutta procedure is now in common use. The interval of integration is divided into a certain number of equal subintervals (equally distributed nodes), but in the case of insufficient accuracy a further subdivision is automatically introduced.

However, in most cases boundary conditions are imposed at both ends or even at certain mid-points of the interval (boundary-value problem). Then we have to assume the initial values as some unknown parameters and a certain iterative procedure is necessary to satisfy the remaining boundary conditions. Traditionally, such a procedure is called the Newton-Raphson method without suitable justification; in fact, in most cases we cannot evaluate the derivatives with respect to the parameters required in the Newton-Raphson method, and practically we apply the falsi rule, extended by K. Gauss to systems of equations (A. Ostrowski [4.438]).

In the case of partial differential equations direct integration is not always possible. However, many equations of plasticity are of hyperbolic type, and then direct integration is possible along certain lines, called characteristics. We present this procedure briefly for the general case of a system of first-order equations of two independent variables and for plane strain problems of perfect plasticity as an example.

Consider a system of $n$ quasi-linear partial differential equations

$$
P_{i k}\left(x, y, u_{j}\right) u_{k, x}+Q_{i k}\left(x, y, u_{j}\right) u_{k, y}+R_{i}\left(x, y, u_{j}\right)=0,
$$

where $i, j, k=1,2, \ldots, n$, and the summation convention holds. Equations (21.1) contain partial derivatives with respect to $x$ and $y$; the central idea of the method of characteristics is to introduce such a transformation of independent variables as to obtain the derivatives with respect to a single variable (H. G. Hopkins [4.234]) and in this way to replace (21.1) by ordinary differential equations, ready for direct integration. 
Introduce two new independent variables, $s$ and $\alpha$, and substitute them into (21.1):

$$
u_{k, x}=\frac{u_{k, s} y_{\alpha}^{\prime}-u_{k, \alpha} y_{, s}}{x_{, s} y_{, \alpha}-x_{, \alpha} y_{, s}}, \quad u_{k, y}=\frac{-u_{k, s} x_{, \alpha}+u_{k, \alpha}{ }^{\prime \prime} x_{, s}}{x_{, s} y_{, \alpha}-x_{, \alpha} y_{, s}} .
$$

We obtain then

$$
P_{i k}\left(u_{k, s} y_{, \alpha}-u_{k, \alpha} y_{, s}\right)-Q_{i k}\left(u_{k, s} x_{, \alpha}-u_{k, \alpha} x_{, s}\right)+R_{i}\left(x_{, s} y_{, \alpha}-x_{, \alpha} y_{, s}\right)=0,
$$

where the arguments of $P, Q$, and $R$ are dropped. Rewrite now (21.3) in the form

$$
\begin{aligned}
\left(P_{i k} y_{, s}-Q_{i k} x_{, s}\right) u_{k, \alpha}+\left(Q_{i k} u_{k, s}+R_{i} y_{, s}\right) x_{, \alpha}- & \\
& -\left(P_{i k} u_{k, s}+R_{l} x_{, s}\right) y_{, \alpha}=0
\end{aligned}
$$

and require that the derivatives with respect to $s$ (characteristic direction) should not depend on the auxiliary, quite arbitrary, direction $\alpha$. Such a situation may take place if all the $n$th order determinants formed from the matrix of the coefficients of $u_{k, \alpha}, x_{, \alpha}, y_{, \alpha}^{\prime \prime}$ in (21.4) vanish. This rectangular $n \times(n+2)$ matrix $\mathbf{M}$ has the form

$$
\mathbf{M}=\left[\begin{array}{ccccc}
P_{11} y_{, s}-Q_{11} x_{, s} & P_{12} y_{, s}-Q_{12} x, s & \ldots & Q_{1 k} u_{k, s}+R_{1} y_{, s} & -P_{1 k} u_{k, s}-R_{1} x, s \\
P_{21} y_{,}-Q_{21} x_{, s} & P_{22} y_{, s}-Q_{22} x_{, s} & \ldots & Q_{2 k} u_{k, s}+R_{2} y_{, s} & -P_{2 k} u_{k, s}-R_{2} x, s \\
\vdots & \vdots & & \vdots & \vdots \\
P_{n 1} y_{, s}-Q_{n 1} x_{, s} & P_{n 2} y_{, s}-Q_{n 2} x_{, s} & \ldots & Q_{n k} u_{k, s}+R_{n} y_{, s} & -P_{n k} u_{k, s}-R_{n} x_{, s}
\end{array}\right]
$$

It can be seen that the last column of (21.5) is not independent of the others: it may be obtained by the multiplication of the first $n$ columns by $\left(-u_{k, s} / y_{, s}\right)$, $k=1,2, \ldots, n$, the multiplication of the $(n+1)$ th column by $\left(-x_{s}, y_{, s}\right)$, and addition. In this case, if the system under consideration (21.1) is non-singular, it is sufficient to require that any two $n$th order determinants formed from (21.5) should vanish. Taking the first $n$ columns and introducing the symbol det for a determinant, we may write briefly

$$
\operatorname{det}\left(P_{i k} y_{, s}-Q_{i k} x_{, s}\right)=0
$$

as the equation determining the characteristic directions. It gives an $n$th degree equation with respect to

$$
\frac{d y}{d x}=\frac{y_{, s}}{x_{, s}}
$$

and for a totally hyperbolic system (21.1) it has $n$ distinct roots. For example, in the case of two equations (21.1) we obtain

$$
\begin{aligned}
\left(P_{11} P_{22}-P_{12} P_{21}\right)\left(\frac{d y}{d x}\right)^{2}-\left(P_{11} Q_{22}+\right. & \left.P_{22} Q_{11}-P_{12} Q_{21}-P_{21} Q_{12}\right) \frac{d y}{d x}+ \\
+ & \left(Q_{11} Q_{22}-Q_{12} Q_{21}\right)=0 .
\end{aligned}
$$

If at least one root of (21.6) is real, system (21.1) is called non-elliptic, and only then the method of characteristics may be used. 
The vanishing of the second determinant gives the relations along the characteristics which are to be integrated. Taking the last two columns in (21.5), we would obtain an inconvenient equation, quadratic with respect to unknown derivatives $u_{k, s}$; to obtain a linear equation we may take the $(n+1)$ th column instead of the $n$th one in (21.6):

$$
\left|\begin{array}{cccc}
P_{11} y_{, s}-Q_{11} x_{, s} & \ldots & P_{1(n-1)} y_{, s}-Q_{1(n-1)} x_{, s} & Q_{1 k} u_{k, s}+R_{1} y_{, s} \\
P_{21} y_{, s}-Q_{21} x_{, s} & \ldots & P_{2(n-1)} y_{, s}-Q_{2(n-1)} x_{, s} & Q_{2 k} u_{k, s}+R_{2} y_{, s} \\
\vdots & & \vdots & \vdots \\
P_{n 1} y_{, s}-Q_{n 1} x_{, s} & \ldots & P_{n(n-1)} y_{, s}-Q_{n(n-1)} x_{, s} & Q_{n k} u_{k, s}+R_{n} y_{, s}
\end{array}\right|=0 .
$$

If Eqs. (21.1) are linear, i.e. do not depend on $u_{j}$, then (21.6) determine the characteristics effectively; in the case of quasi-linear equations the characteristics may be determined only together with the unknown functions $u_{j}$, and (21.6) must be integrated simultaneously with (21.9), in most cases numerically, step-by-step. Such a method of integration was developed by J. Massau [4.374] at the end of the XIXth century. A more detailed discussion of the general case (21.1), presented in matrix notation, is given by H. G. Hopkins [4.235].

As an example consider the problems of plane strain in an incompressible body. Under this assumption such problems become pseudo-statically-determinate; in fact, using the physical equations (similarity of deviators), we eliminate $\sigma_{z}$ and reduce the yield condition (HMH or TG) to the form (11.11), and then two equations of equilibrium together with (11.11) determine just three unknown stresses. Parametrizing (11.11) by (19.48) and substituting into the first two equations (6.9) (body forces being neglected), we obtain

$$
\begin{aligned}
& \frac{\partial \chi}{\partial x}-\sin 2 \varphi \frac{\partial \varphi}{\partial x}+\cos 2 \varphi \frac{\partial \varphi}{\partial y}=0, \\
& \frac{\partial \chi}{\partial y}+\cos 2 \varphi \frac{\partial \varphi}{\partial x}+\sin 2 \varphi \frac{\partial \varphi}{\partial y}=0,
\end{aligned}
$$

and hence the equation of characteristics (21.8) takes the form

$$
\left(\frac{d y}{d x}\right)^{2}-2 \tan 2 \varphi\left(\frac{d x}{d y}\right)-1=0
$$

with two solutions, determining two families of characteristics,

$$
\frac{d y}{d x}=\frac{\sin 2 \varphi \pm 1}{\cos 2 \varphi}
$$

or after some rearrangements,

$$
\frac{d y}{d x}=\tan \left(\varphi \pm \frac{1}{4} \pi\right) \text {. }
$$

Equation (21.9) yields

$$
y_{, s}\left(\chi_{, s}+\sin 2 \varphi \varphi_{, s}\right)+\cos 2 \varphi x_{, s} \varphi_{. s}=0,
$$


and making use of (21.12) we obtain

$$
d \varphi \pm d \chi=0, \quad \varphi \pm \chi=\text { const }
$$

where the upper signs and the lower signs in (21.13) and (21.15) correspond to each other.

In a typical problem of plasticity the boundary conditions determine $\chi$ and $\varphi$ along a non-characteristic curve. Then the relatively simple relations (21.15) may be integrated along the characteristics (21.13) analytically, or, in more complicated cases, numerically. The nets of characteristics (21.13) are called the Hencky-Prandtl nets.

The method of characteristics in the theory of plasticity has an extensive literature. Let us only mention here some classical papers by H. Hencky [4.203], L. Prandtl [4.474], H. Geiringer [4.158, 4.160], H. Geiringer and W. Prager [4.159], and a more detailed treatment in the monographs by V. V. Sokolovsky [2.163], D. D. Ivlev [2.72], J. Rychlewski [2.125], E. G. Thomsen, C. T. Yang and S. Kobayashi [4.585], W. Szczepiński [2.166], W. Johnson, R. Sowerby and J. B. Haddow [2.77] and J. Salençon [4.503].

The method of characteristics was adapted to axially-symmetric problems of perfect plasticity by R. T. Shield [4.531]. It is also commonly used in dynamic problems of plasticity (H. G. Hopkins [4.234]). C. C. Lin [4.333] derived the equations of characteristics subject to perturbations; in fact, this is the PLK (modified) perturbation method applied to hyperbolic equations. Perturbed characteristics are employed in the plasticity problems by A. J. M. Spencer [4.548], O. Richmond and H. L. Morrison [4.485] and R. J. Izbicki [4.254]. P. Dewhurst and I. F. Collins [4.112] introduced a matrix technique based on characteristics. D. D. Ivlev [4.253] used the method of characteristics to the determination of displacements in elasticplastic problems. The method of the Riemann function may be regarded as an extension of the method of characteristics; it was applied to plasticity problems by J. Kravtchenko and J. Lanier [4.307]. Behaviour of characteristics in the vicinity of singular points was studied in detail by J.-L. Auriault, J. Kravtchenko, R. Negre and $\mathrm{R}$. Sibille [4.15].

\subsection{Finite difference method}

The finite difference method is to a certain degree similar to that of direct integration: it replaces derivatives by finite difference ratios but formulates the resulting algebraic equations at once for the whole domain and, in principle, in linear cases no iteration is necessary. This advantage of the method is connected with a large number of equations to be solved simultaneously. In the case of linear differential equations we have to solve a system of linear algebraic equations and the application of computers makes that possible even for a large number of unknowns. In the pre-computer period the Southwell relaxation method was very helpful in this respect, since it facilitates the solution by iterations: it was first appled to plasticity problems by D. N. Allen and R. V. Southwell [4.6] and by J. A. Jacobs [4.256]. 
In the cases of non-linear ${ }_{2}$ differential equations, typical for plasticity, the system of non-linear algebraic equations must practically be solved by iterations, and in such cases the relaxation technique is still in use.

For a one-dimensional continuum the typical discretization procedure of the finite difference method consists in choosing equispaced nodes and expressing finite differences in terms of the nodal values. For a two-dimensional case equispaced grid lines with rectangular meshes are introduced; this procedure may be applied also to elliptic differential equations, in contradistinction to the method of characteristics. Such an approach is convenient for rectangular domains, and those which may easily be transformed into rectangular ones (e.g. circular or annular domains), but for irregular shapes the method has some disadvantages. Firstly, for such domains the approximation of boundary conditions is difficult and inaccurate. Secondly, P. G. Hodge [4.229, 4.230] remarked that in this case the principle of virtual work does not necessarily hold, and proposed a suitable modification of the finite difference method. But even in the case of a rectangular domain the application of the finite difference method to plasticity may involve difficulties connected with discontinuities appearing in many solutions. Nevertheless, in some plasticity problems the method under consideration proved very useful (J. G. Crose, A. H. S. Ang [4.98], finite deflections of plates; Yu. G. Korotkikh, A. K. Lyubimov [4.300], cyclic loadings; Yu. G. Korotkikh, S. M. Belevitch [4.302], thermoplasticity).

To obtain valuable solutions for irregular domains the finite difference method has to be extended to grids with irregular meshes. This direction was initiated by P. S. Jensen [4.260], N. Perrone and R. Kao [4.449], Z. Kączkowski and R. Tribiłło [4.277], T. Liszka and J. Orkisz [4.338]; such an approach may compete with the finite element method, where the irregularities of shape do not result in essential difficulties.

\subsection{Finite element method}

One of the most general and flexible numerical methods of structural analysis, applicable to various plasticity problems, is the finite element method. It consists in dividing the body into a number of compatible finite elements, interconnected at nodal points situated on their boundaries. In its classical, "displacement" version, the displacements within each finite element are expressed in terms of nodal displacements by means of certain approximating functions (shape functions); such an approach determines the strain distribution, the stress distribution may then be found from the constitutive equations, and the equivalent nodal forces are determined by the principle of virtual work. The equilibrium equations for all the nodes and the boundary conditions are sufficient to determine all the unknowns. Applying the principle of virtual work to the body as a whole, and not to individual elements, we may eliminate the "interelement forces" and in this way reduce the number of unknowns. 
In the simplest two-dimensional case the boundary is approximated by a polygon, finite elements are assumed in the form of triangles, and the displacements are assumed to change linearly within the elements (Fig. 114):

$$
\mathbf{u}(x, y)=\frac{1}{2 \Delta} \sum_{i=j, k, l}\left(a_{i}+b_{i} x+c_{i} y\right) \mathbf{u}_{i},
$$

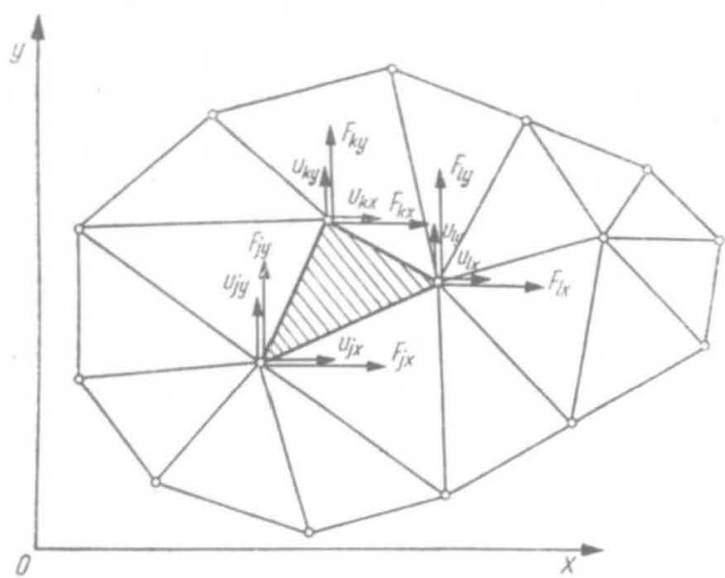

Fig. 114. Finite elements in a two-dimensional case

where $\mathbf{u}_{l}(i=j, k, l)$ stand for nodal displacements with two components denoted by $u$ and $v$, and $\Delta$ is equal to the area of the triangle. Then the strains are constant in each element and in a homogeneous body the stresses are also constant. We may write in matrix form

$$
\epsilon=\mathbf{B} \delta
$$

where $\delta$ is a six-vector whose components are nodal displacements, the compatibility matrix $\mathbf{B}$ depends on the geometry of the element, and $\boldsymbol{\epsilon}$ is a three-vector with the components $\varepsilon_{x}, \varepsilon_{y}, \gamma_{x y}$ (or $\gamma_{x y} / \sqrt{2}$, cf. Sec. 10.2). In the small-strain theory $\mathbf{B}$ is independent of the displacements and we obtain also

$$
d \mathbf{\epsilon}=\mathbf{B} d \boldsymbol{\delta} .
$$

For the linear distribution (21.16) in triangular elements one obtains

$$
\left\{\begin{array}{l}
\varepsilon_{x} \\
\varepsilon_{y} \\
\gamma_{x y}
\end{array}\right\}=\frac{1}{2 \Delta}\left[\begin{array}{llllll}
b_{j} & 0 & b_{k} & 0 & b_{l} & 0 \\
0 & c_{j} & 0 & c_{k} & 0 & c_{l} \\
c_{j} & b_{j} & c_{k} & b_{k} & c_{l} & b_{l}
\end{array}\right]\left[\begin{array}{l}
u_{j} \\
v_{j} \\
u_{k} \\
v_{k} \\
u_{l} \\
v_{l}
\end{array}\right\} .
$$


Constitutive relations of plasticity may be expressed in various forms, as was discussed in Chapter III, but in most cases we may write them in the following concise form:

$$
d \boldsymbol{\sigma}=\mathbf{D}_{\mathrm{ep}} d \boldsymbol{\epsilon}
$$

where the elastic-plastic (tangent) stiffness matrix $\mathbf{D}_{\mathrm{ep}}$ may depend on stresses, plastic strains, internal state variables, etc. Particular forms of such matrices were derived by Y. Yamada, N. Yoshimura and T. Sakurai [4.614], O. G. Zienkiewicz, S. Valliappan and I. P. King [4.623], G. Maier [4.348] and O. De Donato [4.109] (piecewise linear hardening), R. G. Venkateswara and A. V. Krishna Murty [4.594] (the Ramberg-Osgood schematization). Using the deformation (total) theory of plasticity, we may write

$$
\boldsymbol{\epsilon}=\boldsymbol{\Phi}_{\mathrm{ep}} \boldsymbol{\sigma},
$$

where $\Phi_{\text {ep }}$ is the elastic-plastic (secant, inverse) stiffness matrix, corresponding to $\varphi$ in the Hencky-Ilyushin equations, determined from the uniform stress-strain curve. The effective evaluation of stresses by means of (21.20) or (21.21) requires iterations, or step-by-step integration (even in the case of (21.21) because of possible passive processes), or both.

The six-vector of equivalent nodal forces $\mathbf{F}$ may be determined by means of the matrix B introduced above. Indeed, write the principle of virtual work in the form

$$
d \boldsymbol{\delta}^{\mathrm{T}} \mathbf{F}=d \boldsymbol{\epsilon}^{\mathrm{T}} \boldsymbol{\sigma}
$$

where the superscripts $\mathrm{T}$ denote transposition of the matrix or of the vector. Of course, on the right-hand side one has to use either $\gamma$ and $\tau$, or $\gamma / \sqrt{2}$ and $\tau \sqrt{2}$. The substitution of (21.18) and the rule for a transposed matrix product yields

$$
d \boldsymbol{\delta}^{\mathrm{T}} \mathbf{F}=d \boldsymbol{\delta}^{\mathrm{T}} \mathbf{B}^{\mathrm{T}} \boldsymbol{\sigma},
$$

and hence

$$
\mathbf{F}=\mathbf{B}^{\mathrm{T}} \boldsymbol{\sigma} .
$$

The number of equilibrium equations for the forces $\mathbf{F}$ at each node is $2 N$ for the plane case under consideration (where $N$ stands for the number of nodes), and equals the number of unknown displacements (or velocities) $\delta$.

The formal presentation of the finite element method is due to M. J. Turner, R. W. Clough, H. C. Martin and L. P. Topp [4.589], 1956, though similar ideas were published in the forties (Courant, Prager and Synge). The term "finite elements" was introduced by R. W. Clough in 1960, [4.79]. J. R. Whiteman [4.604] compiled over 2000 references to the finite element method; let us only mention here the monographs published by O. C. Zienkiewicz [3.875], J. T. Oden [4.425], C. S. Desai and J. F. Abel [4.111] and the survey papers by O. C. Zienkiewicz [4.624] (engineering aspects), and J. T. Oden [4.426] (mathematical aspects).

The first applications of finite elements to plasticity problems are due to R. H. Gallagher, J. Padlog, and P. P. Bijlaard [4.151], J. H. Argyris [4.9] and G. G. Pope [4.463]. Further fundamental papers (besides those mentioned above) 
were published by J. H. Argyris, D. W. Scharpf and J. B. Spooner [4.10, 4.11, 4.12], P. V. Marcal and I. P. King [4.363], R. M. Richards and J. R. Blacklock [4.484], P. G. Hodge [4.228], S. C. Anand, S. L. Lee and E. C. Rossow [4.7], G. Bartelds [4.21], S. Valliappan [4.591]. J. H. Argyris and A. S. L. Chan [4.13] introduced finite elements in space and time, particularly appropriate for dynamic problems in plasticity. Basic equations of plasticity of discretized bodies, derived on the basis of variational theorems, were discussed by B. Nayroles [4.410], I. G. Teregulov [4.581], D. H. Nguyen [4.419] and M. Kleiber [4.286, 4.287]. More refined shapes of finite elements were used by J. H. Percy [4.448] (quadrilateral), C. H. Lee and S. Kobayashi [4.320] (quadrilateral), J. C. Nagtegaal, D. M. Parks and J. R. Rice [4.405] (numerically accurate special shapes), D. R. J. Owen and E. M. Salonen [4.439] (hexahedrons).

The application of finite elements to problems of large displacements in plasticity was initiated by J. H. Argyris, D. W. Scharpf and J. B. Spooner [4.12], P. V. Marcal [4.365], G. Maier and D. C. Drucker [4.354], and generalized to finite strains by H. D. Hibbitt, P. V. Marcal and J. R. Rice [4.208], L. D. Hofmeister, G. A. Greenbaum and D. A. Evensen [4.232], E. H. Wu and E. A. Witmer [4.612], M. Kawahara and K. Horii [4.274], J. S. Gunasekera and J. M. Alexander [4.191], H. Kitagawa and Y. Tomita [4.283], P. K. Larsen and E. P. Popov [4.318], M. Kleiber [4.288], R. M. Mc Meeking and J. R. Rice [4.378]. Related problems of plastic softening were discussed by G. Maier [4.350], G. C. Nayak and O. C. Zienkiewicz [4.408].

After suitable modifications the finite element method may be applied directly to one-dimensional or two-dimensional structural elements; stresses and strains are then usually replaced by generalized stresses and generalized strains. Such an adaptation to elastic-plastic plates was made by T. Belytschko and M. Velebit [4.28], S. A. Kapustin, L. K. Kiselev and V. A. Trubitsyn [4.271] and A. W. Wegmuller [4.603], to elastic-plastic shells by E. P. Popov and P. Sharifi [4.464], A. Hoffmann, M. Livolant and R. Roche [4.231], J. Bäcklund and H. Wennerström [4.24], to frames by G. Thierauf [4.582]. The adaptation of the finite element method to shakedown problems is due to G. Maier [4.347], H. Armen, H. Isakson, and A. Pifko [4.14], T. Belytschko [4.29] and V. A. Petushkov [4.450], and the adaptation to thermoplasticity was proposed by A. P. Goryachev and E. I. Sankov [4.180], D. Radaj [4.479], J. Gerij [4.163], T. Inoue and K. Tanaka [4.246].

Various computer systems of structural analysis based on finite element method allow for plastic deformations and are more or less adapted to the calculations of combined loading problems. Let us mention here the following systems: ASKA (Automatic System for Kinematic Analysis, Stuttgart), described from the viewpoint of plasticity by H. Balmer, J. St. Doltsinis and M. König [4.19], FESS (Finite Element Solutions Swansea), [3.875], NASTRAN (NAsa STructural ANalysis), [4.111], and DYPLAS (DYnamic PLASticity), Z. Zudans, M. M. Reddi and H. C. Tsai [4.627]. 


\subsection{Iteration and incremental (step-by-step) procedures}

An effective use of constitutive equations of plasticity requires in most cases iteration procedures, even in the case of deformation theories. Rewrite (21.21) in two alternative, obviously equivalent forms:

$$
\begin{aligned}
& \boldsymbol{\sigma}=\mathbf{E} \varepsilon+\left(\boldsymbol{\Phi}_{\mathrm{ep}}^{-1}-\mathbf{E}\right) \in \stackrel{\text { def }}{=} \mathbf{E} \varepsilon+\boldsymbol{\sigma}_{(0)}, \\
& \boldsymbol{\epsilon}=\mathbf{E}^{-1} \boldsymbol{\sigma}+\left(\boldsymbol{\Phi}_{\mathrm{ep}}-\mathbf{E}^{-1}\right) \boldsymbol{\sigma} \stackrel{\text { def }}{=} \mathbf{E}^{-1} \boldsymbol{\sigma}+\boldsymbol{\epsilon}_{(0)},
\end{aligned}
$$

where $\boldsymbol{\sigma}_{(0)}$ and $\boldsymbol{\epsilon}_{(0)}$ are defined by these equations. Three forms of constitutive equations, (21.21), (21.25), and (21.26), give rise to three basic iterative procedures: one may iterate $\boldsymbol{\Phi}_{\mathrm{ep}}$, or $\boldsymbol{\sigma}_{(0)}$, or $\boldsymbol{\epsilon}_{(0)}$. Procedures of the first type are termed variable stiffness methods, whereas the remaining procedures use elastic stiffness for iterations and are called methods of successive elastic solutions.

The first method of elastic solutions, proposed by A. A. Ilyushin in 1943, [2.67], designed for general continuum problems and not for the finite element method, expressed stresses in terms of strains, (21.25), and was later more precisely called the "method of supplementary loadings". The central idea of this method is as follows: Equations (21.25) are introduced into the equilibrium equations with the terms of type $\sigma_{(0)}$ left on the right-hand side, and strains are expressed in terms of displacements. Hence we obtain non-homogeneous "elastic" Lamé equations for displacements, where the right-hand side terms may be interpreted as supplementary body forces and tractions and are assumed to be known. Namely, in the zeroth approximation we put those forces equal to zero, solve the elastic problem, determine the strain intensity as a function of $x_{i}$, calculate $\boldsymbol{\Phi}_{\text {ep }}^{-1}$ for that strain intensity, and then find the next approximation of the supplementary loadings. The convergence of this method in a certain particular case was proved by V. M. Panferov [4.443]; P. P. Vorovitch and Yu. P. Krasovsky [4.599] showed that under some weak assumptions its convergence in the general case is determined by a geometrical progression. Further results are due to S. O. Sarkisyan [4.506, 4.507]. Basic equations in an "expanded" form may be found in the monograph [2.130] by S. D. Ponomarev et al.

V. D. Klyushnikov [4.291] adjusted the method of supplementary loadings to the incremental theories of plasticity, combining it with power series. A comparison of the Klyushnikov and of the original method was given by L. L. Boyko [4.48, 4.49]. Another modification of the method of supplementary loadings was proposed by D. L. Bykov and V. A. Shatchnev [4.56]. G. L. Brovko and V. S. Lensky [4.325, 4.52] proposed a related method for non-homogeneous bodies starting the iterations from a homogeneous body; thermal loadings are also allowed for. Another applications to thermoplasticity are due to Yu. N. Shevtchenko $[4.529,4.530]$. A. N. Chizhevsky [4.75] claimed better convergence of the method if Young's modulus $E$ is replaced by a certain appropriately chosen fraction of that modulus.

The Ilyushin method was proposed primarily for analytical applications, but in most cases only a numerical approach is effective. A counterpart of this iteration 
procedure, designed especially for the finite element method, was introduced by O. C. Zienkiewicz, S. Valliappan and I. P. King [4.623] and called the "initial stress process". It was also generalized so as to be applicable to incremental theories of plasticity; then it has to be combined with the incremental, step-by-step procedure. A refined, self-correcting incremental procedure was suggested by J. A. Stricklin, W. E. Haisler and W. A. Riesemann [4.554].

The second method of successive elastic solutions, proposed for continuum problems by I. A. Birger [4.33] in 1951, makes use of (21.26) for subsequent iterations, and was called the "method of supplementary strains". Equations (21.26) are substituted into the compatibility equations with the terms of type $\boldsymbol{\epsilon}_{(0)}$ left on the right-hand side. Using additionally the equilibrium conditions, one has to solve non-homogeneous elastic equations, where the right-hand side corresponds to plastic strains, but may also be interpreted as supplementary strains, e.g. due to a fictive temperature distribution. Such a problem of elasticity with supplementary strains was discussed by $\mathrm{H}$. Reissner as early as 1931 [4.483]. In the zeroth approximation we put these strains equal to zero and solve the "unperturbed" elastic problem. Then the crucial point is to evaluate the plastic strains to be allowed for in the next approximation.

Two typical approaches to this problem have been suggested. In Birger's original version the "constant stress approach" was used (Fig. 115): the function

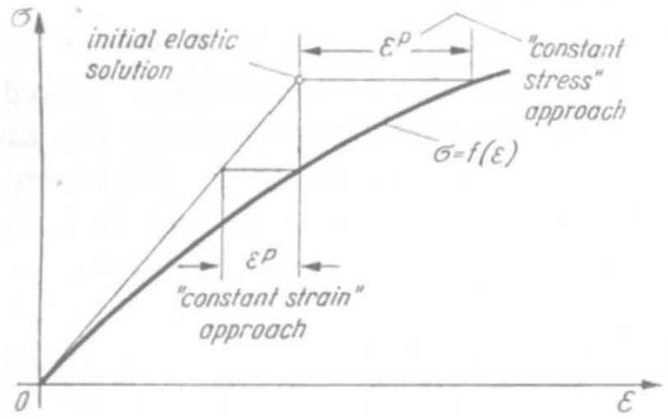

Fig. 115. The "constant stress" approach and the "constant strain" approach

$\varphi$ (or the matrix $\boldsymbol{\Phi}_{\mathrm{ep}}$ ) is simply calculated for the stress intensity evaluated in the former step. This procedure is very good for statically determinate cases (it then gives the exact solution after the first step), but may be divergent for statically indeterminate problems, particularly for very small plastic hardening, and is, in general, inapplicable to perfect plasticity. The reason is that the stress-strain curve is used here to increase strains at constant stresses and not to decrease stresses at constant strains. Hence in [4.34] I. A. Birger proposed a modified version of supplementary strains: the function $\varphi$ is calculated not for the stress intensity but for the strain intensity evaluated in the former step. This procedure is particularly suitable for geometrically determinate problems, under displacement-type loadings. It resembles the procedure used in the method of supplementary loadings, but-accord- 
ing to Birger-the problems of elasticity with supplementary strains are simpler to solve than those with supplementary loadings. A similar procedure for incremental theories of plasticity was proposed even earlier by A. Mendelson 'and S. S. Manson [4.385, 3.519], and then by I. A. Birger [4.35], and S. Nemat-Nasser [4.416] (reduced to integral equations); a combination with the step-by-step procedure is then necessary. A related thermoelastic analogy was proposed by T. H. Lin [4.334, 3.456].

A counterpart of the method of supplementary strains, designed especially for the finite element method, was proposed by R. H. Gallagher, J. Padlog and P. P. Bijlaard [4.151], and by J. H. Argyris [4.9], and called the "initial strain process". W. Lansing, W. R. Jensen and W. Falby [4.317] compared the constant-stress approach and the constant-strain approach in this procedure (Fig. 115).

The third fundamental iterative procedure is based either on the form (21.20) or on the form (21.21) of the constitutive equations, and consists in iterations of the matrices $\boldsymbol{\Phi}_{\text {ep }}$ or $\mathbf{D}_{\text {ep }}$. This procedure, called the "method of variable stiffnes" or the "method of variable elastic moduli", was proposed for continuum problems by I. A. Birger [4.33, 4.36], and in relation to the finite element method by G. G. Pope [4.463], P. Marcal and I. P. King [4.363]. A simplified version of this procedure was suggested by S. S. Hecker, C. H. Hamilton and L. J. Ebert [4.202]. The convergence of the variable stiffness method was investigated by V.V. Bokov and E. A. Sidorov [4.44], and by Y. Yamamoto [4.616].

Comparisons of various iteration procedures are given by P. Marcal [4.364], J. H. Argyris and D. W. Scharpf [3.10] (where also some "combined" procedures are suggested), I. N. Ananev and Yu. I. Nyashin [4.8] (they pointed out the advantages of the variable stiffness method), J. A. Stricklin, W. E. Haisler and W. A. Riesemann [4.555], R. Kao [4.269] (geometrical non-linearity), and D. L. Bykov [4.57]. A. S. Kravtchuk [4.308] compared various iteration procedures for the Ilyushin geometrical theory of plasticity. T. R. Hsu and A. W. M. Bertels [4.238] proposed an approximation 'of the stress-strain relationship facilitating the incremental variable-stiffness procedure. O. C. Zienkiewicz and I. C. Cormeau [4.625, 4.626] remarked that a convenient procedure for obtaining the plastic solution is to perform the limiting process for the relevant visco-plastic problem. V. I. Chernov, O. N. Ivanchenko and V. P. Bardzilovsky [4.72] proposed to start the iterations from the purely plastic solution instead of the purely elastic solution.

In some cases the convergence of the iteration processes is very slow and its acceleration is of primary importance. A very convenient, powerful method of acceleration was proposed by A. C. Aitken [4.4] (convergence of series) and J. F. Steffensen [4.549] (convergence of iterations). Denote the results of three consecutive iterations or three consecutive partial sums of a series by ${ }^{1} x_{1}, x_{2}, x_{3}$. Assuming that they approach their unknown limit $x$ as a geometrical series, i.e. that

$$
\frac{x_{1}-x}{x_{2}-x}=\frac{x_{2}-x}{x_{3}-x}
$$


we obtain the following approximation of the required limit:

$$
x=\frac{x_{1} x_{3}-x_{2}^{2}}{x_{1}-2 x_{2}+x_{3}}=x_{3}-\frac{\left(x_{3}-x_{2}\right)^{2}}{x_{1}-2 x_{2}+x_{3}} \stackrel{\text { def }}{=} x_{2}^{(1)} .
$$

The second form of this formula is more convenient for numerical calculations. If the value (21.28) is not accurate enough, one may form the sequence $x_{2}^{(1)}, x_{3}^{(1)}$, $x_{4}^{(1)}, \ldots$ and calculate from $(21.28)$ the next approximation $x=x_{3}^{(2)}$, etc.

For some irregularly convergent series or irregular iterations the AitkenSteffenson formula (21.28) may be useful after a simple modification. For example, considering the Fourier series, we may obtain good acceleration by taking every fifth or every tenth partial sum instead of consecutive ones.

Procedure (21.18) not only gives essential acceleration for convergent series or convergent iteration processes but also may lead to the correct result "hidden" in the divergent cases. For example, D. Shanks [4.526] considered the divergent series

$$
\ln 3=2-\frac{2^{2}}{2}+\frac{2^{3}}{3}-.
$$

and applied the Aitken-Steffensen accelerator (21.28) five times. Using 12 terms of the original series, he obtained in this way an impressive 8-figure accuracy though the accuracy of the twelfth original term could not be discussed at all. This fact may be interpreted as the convergence of (21.29) in the opposite direction, and (21.28) acts irrespective of the direction. Such a transformation of divergent series or iterations into convergent ones may be very important for practical applications. V. I. Krylov, V. V. Bobkov and P. I. Monastyrnyi [4.311] give a theoretical treatment of the Aitken-Steffensen accelerator and conclude that it extends the radius of convergence of the series up to the next pole in the complex plane.

The Aitken-Steffensen accelerator was applied to iterations in the finite element method by B. M. Irons and R. C. Tuck [4.247], E. F. Boyle and A. Jennings [4.50].

Convergence of iterations is analysed mainly by the methods of functional analysis. Recently, the functional analysis is more and more commonly applied to plasticity problems; let us mention here a monograph by G. Dincă [4.114], and papers by H. Gajewski [4.148], B. Nayroles [4.411, 3.573], J. J. Moreau [3.394] and A. M. Protsenko [4.476, 4.477].

\subsection{Mathematical programming methods}

A numerical realization of variational principles of plasticity is often reduced to mathematical programming. Consider, for example, the static theorem of limit analysis and, instead of searching for an exact maximum of the relevant functional, assume the solution in the Ritz approximate form

$$
\sigma_{i j}=\sum_{n=1}^{N} \alpha_{n} \sigma_{i j n},
$$


where $\sigma_{i j n}\left(x_{k}\right)$ satisfy equilibrium equations and the stress boundary conditions. Then the functional becomes simply a function of the parameters $\alpha_{n}$ and the most accurate solution is obtained for the maximal value of this function, subject to the inequality constraints resulting from the yield condition. This is a problem of mathematical programming, in general non-linear programming; if the yield condition is approximated by linear functions, then in most cases the problem may be reduced to linear programming.

The first paper applying linear programming methods to limit analysis was published in 1951 by A. Charnes and H. Greenberg [4.66]. Almost simultaneously linear programming was applied to the problems of optimal plastic design (J. Foulkes [4.142]). Further fundamental papers were published by A. Charnes, C. E. Lemke and O. C. Zienkiewicz [4.67], D. C. A. Koopman and W. H. Lance [4.296], G. Ceradini and C. Gavarini [4.63, 4.153] and M. Capurso [4.61]. One of the basic problems of the application of linear programming to limit analysis consists in replacing the non-linear yield condition by a piece-wise linear function. For plane stress $\left(\sigma_{x}, \sigma_{y}, \tau_{x y}\right)$ this problem was studied in detail by A. Zavelani-Rossi [4.618, 4.619, 4.620], L. Corradi and A. Zavelani [4.94]; they proposed to replace the Huber-MisesHencky ellipsoid by various inscribed polyhedrons, with $6,12,18$ and 20 vertices, recommending either an irregular icosahedron with 12 vertices and 20 faces (Fig. $116)$ or a polyhedron with 18 vertices and 14 faces (Fig. 117).

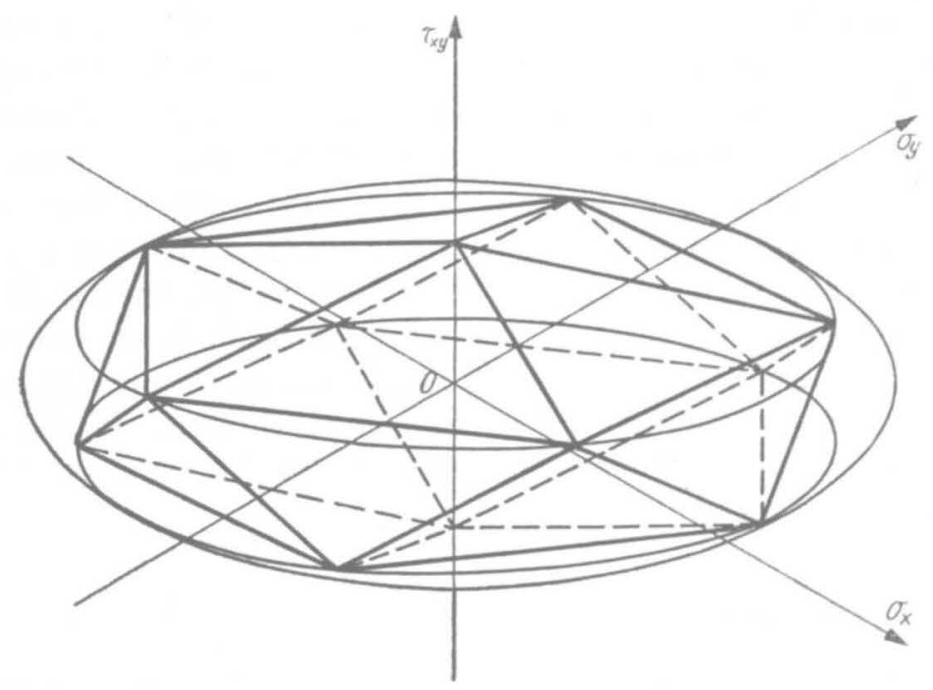

Fig. 116. The HMH ellipsoid approximated by a polyhedron with 12 vertices and 20 faces

The linear programming method was adopted to plastic analysis of plates by W. Prager [4.471], G. Sacchi and G. Buzzi Ferraris [4.494], G. Ceradini and C. Gavarini [4.63], A. Borkauskas and A. Čyras [4.45], A. E. Borkauskas and R. P. Karkauskas [4.46]. Plastic analysis of shells using linear programming was initiated 


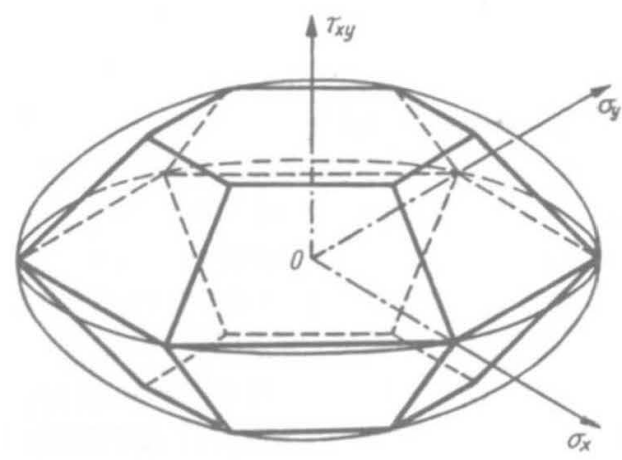

Fig. 117. The HMH ellipsoid approximated by a polyhedron with 18 vertices and 14 faces

by A. R. Rzhanitsyn [4.493]; further papers are due to B. Yu. Mirzabekyan and M. I. Reitman [4.392], Yu. A. Nagyavitchius and A. I. Čyras [4.406], K. S. Dinno and S. S. Gill [4.115] (thick-walled shells).

The method of non-linear programming, more accurate but also more difficult, was first applied to limit analysis by P. G. Hodge [4.224], 1964, published 1966. He used the Sequential Unconstrained Minimization Technique (SUMT), with penalty functions, for beams with axial forces and for arches. G. Maier [4.341, 4.342], M. Capurso and G. Maier [4.60], developed the quadratic programming method. Non-linear programming is particularly convenient as a method of the limit analysis of shells (A. Biron and P. G. Hodge [4 38], A. Biron and U. S. Chawla [4.39], S. A. Kalanta, Yu. A. Nagyavitchius, and A. A. Cyras [4.267], K. S. Dinno and S. S. Gill [4.116]); A. C. Palmer [4.441] applied to similar problems the method of dynamic programming, and O.F. Chernyavsky [4.73] - the Pontryagin maximum principle.

The methods of mathematical programming are also very useful in shakedown problems. Linear programming was first applied by D. A. Gokhfeld and O. F. Chernyavsky $[4.169,4.172,4.175,4.176], 1966$, then widely developed by G. Maier [4.347, 4.348]; further papers are due to M. Z. Cohn, S. K. Ghosh and S. R. Parimi [4.84], M. Save [4.509], L. Corradi and A. Zavelani [4.94]. Problems of movable loads are considered by G. Sacchi, G. Maier and M. Save [4.498].

A. M. Protsenko [4.75] applied non-linear programming to the evaluation of the maximal carrying capacity of systems with a finite number of degrees of fieedom subject to large displacements.

A monographic treatment of linear programming as applied to elastic-plastic analysis is given by A. A. Cyras [4.99]; mathematical programming applied to optimization problems in plasticity is discussed in detail in the books by A. A. Cyras [4.100], A. A. Cyras, A. E. Borkauskas and R. P. Karkauskas [3.121]. Survey papers are due to J. J. Telega [4.578] (linear programming), C. Gavarini [4.156] and G. Maier [4.352] (general mathematical programming). 


\subsection{Other methods}

Many elastic-plastic problems of strain concentration around holes in plane-strain conditions may be solved by means of analytic functions. Determination of the plastic zone around a hole constitutes a statically determinate problem, and then the solution for the elastic zone (but with the elastic-plastic interface unknown) may be solved by means of the Kolosov-Muskhelishvili approach. This method was suggested by L. A. Galin [4.150], and applied to several combined loading problems by G. N. Savin and O. S. Parasyuk [4.510, 4.511, 4.512].

L. F. Chernous'ko and N. V. Banichuk [4.20, 4.71] applied to elastic-plastic problems the method of local variations. The methods of integral equations were used by J. L. Swedlow and T. A. Cruse [4.558, 4.559]. I. S. Akhmedyanov and A. V. Kireyev [4.5] applied Fourier series combined with iterations to the analysis of the general loading of an elastic-plastic spherical shell.

Many problems of combined loadings require a strong experimental background. A. A. Ilyushin developed a general method, called SN-EVM (testing machine for combined loadings-computer), making it possible to determine the constitutive equations in five-dimensional auxiliary spaces in an experimental way, [4.241, $4.242,4.243]$. Some problems may be analysed experimentally by means of the photoplastic method; let us only mention here the monographs written by S. I. Gubkin, S. I. Dobrovolsky and B. B. Boyko [4.190], V. K. Vorontsov and P. I. Polukhin [4.598], J. Javornicky [4.259], and the survey paper by E. Mönch [4.396].

The concept of a direct coupling of testing machines with computers seems to be highly perspective for future research. 


\section{CHAPTER V}

\section{Combined loadings at the level $\delta$ of a cross-section of a bar; type $\mathscr{P}_{1} / \delta_{n}$}

\section{Elastic-plastic bending with normal force}

\subsection{General remarks}

According to the definition given in Sec. 4.1 we use the term combined loading of a cross-section if that section is subject to more than one exertion factor. In the case of a line structure (bar) with the axis $x$, one usually assumes generalized stresses (or, in other words, generalized internal forces) as those exertion factors: normal force $N$, two mutually perpendicular shear forces $V_{y}$ and $V_{z}$, twisting moment $M_{t}$ and two bending moments $M_{b y}$ and $M_{b z}$. The above six exertion factors have been called basic exertion factors. In the case of thin-walled bars the bimoment $B$ and the warping moment $M_{\omega}$ may also be important. Other possible exertion factors were mentioned in Sec. 1.4.

The level $\mathscr{S}$ has an integral character in relation to the level $\mathscr{P}$. Hence the difficulties of analysis depend here in an essential manner on the situation at level $\mathscr{P}$. The simplest combined cases were classified in Sec. 4.1 as $\mathscr{P}_{1} / \mathscr{S}_{n}$; at the individual points of the cross-section only one exertion factor appears and the analysis does not require any specific failure hypothesis. The present chapter is devoted to such simpler cases, whereas combined loadings of the type $\mathscr{P}_{m} / \mathscr{S}_{n}, m \geqslant 2$, will be discussed in Chapter VI.

The investigations of combined loadings of a cross-section may be divided into two basic groups. The first group aims at the construction of interaction surfaces (limit surfaces), separating particular ranges of work of the section. The plastic interaction surface, corresponding to the limit carrying capacity, is particularly important here. Such surfaces are presented in an appropriate space of exertion factors. Dimensionless exertion factors are often introduced: the physical quantities are related either to the elastic carrying capacity or to the limit carrying capacity. Both approaches have certain advantages: the first gives a direct esti- 
mation of gains due to the plastic properties of the material in relation to a perfectly elastic material (but with limited strength). The second refers rather to the schematization of a rigid-plastic body and does not require any elastic analysis. In the present book both types of dimensionless quantities will be used according to the problem under discussion.

The second basic group of investigations pertains to states represented by points lying inside individual regions determined by limit surfaces. For example in the case of bending with normal force, we analyse the states of one-side plastification, two-side plastification, etc. Such analysis 'aims at the derivation of constitutive equations for the cross-section, joining exertion factors with dependent variables. Constitutive equations serve as a starting point for further analysis at the level $\mathscr{B}$ (calculation of deflections, limit carrying /capacity of the body as a whole, etc.).

It should be remarked that the notion of the limit carrying capacity of a crosssection, frequently used in engineering applications, may not be defined uniquely. Two different approaches to such a definition, due to Hill and to Prager, were discussed in Sec. 17.1. But even if we use a particular definition for a given section subject to a system of proportionally increasing generalized stresses, the limit carrying capacity of that section may, to a certain degree, depend on the gradients of generalized stresses, gradients of the cross-sectional area, initial curvature of the axis of the bar, etc. Such a situation is not new as compared with the level $\mathscr{P}$-it was found experimentally that plastification at a point may also depend on stress gradients. Nevertheless, the influence of above-mentioned factors on the limit carrying capacity of a cross-section is insignificant as a rule, and this notion, being (approximately) uniquely defined, is widely used in engineering applications, e.g. when discussing collapse mechanisms for bar systems.

General considerations concerning plastic interaction surfaces for a crosssection are due to W. Prager [4.466, 4.467] and H. Ziegler [5.208, 5.209, 5.210]. They discussed only the basic exertion factors for the cross-section. Prager introduced the notion of plastic reaction, i.e. a generalized stress which does not appear in the expression for dissipated work (for example the circumferential bending moment in a cylindrical shell). M. Save [5.160] proved that such plastic reaction does not appear in the relevant equation of the plastic interaction surface (yield condition for the cross-section). Ziegler analysed constitutive equations (flow rules) for the cross-section, stating that under the assumption of an associated flow rule at individual points of the cross-section, the "integral" flow rule is also associated: generalized strain rates are proportional to partial derivatives of the yield condition for the cross-section with respect to the corresponding generalized stresses. J. A. König [4.303] considered the general problem of plastic shakedown under variable loadings at the level $\mathscr{S}$.

Let us also mention that the analysis of pure (single) loadings of the crosssection of bars in the elastic-plastic range was initiated at the very beginning of the development of theory of plasticity. As early as in 1871 B. de Saint-Venant [5.158] 
solved the problem of elastic-plastic torsion of a bar with a circular cross-section and elastic-plastic bending of a bar with a rectangular cross-section. A more general theory of bending was developed in the thirties (J. Fritzsche [5.49]). We mention also the papers by $\mathrm{Ch}$. Massonnet and $\mathrm{M}$. Save [5.120, 5.121] which investigate the influence of the deformation of a thin-walled cross-section on its maximal carrying capacity. From among numerous papers on plastic torsion we note the one by A. Nádai [5.130], who established the sand-hill analogy and the roof analogy, and the paper by L. A. Galin [5.50] (elastic-plastic torsion of bars with an arbitrary cross-section). General analytical formulae for the limit carrying capacity of bars in torsion were derived by M. Galos and M. Życzkowski [5.51]. On the other hand, pure shear in a cross-section is almost without practical meaning, since it is usually accompanied by bending. Finally, the case of pure tension is trivial except for the problem of necking, discussed in Sec. 17.5.

\subsection{Constitutive equations of perfectly elastic-plastic bending with tension or} compression of bars with an arbitrary cross-section

Bending with tension or compression presents the simplest combined loading of the type $\mathscr{P}_{1} / \mathscr{S}_{2}$. The assumption of uniaxial stress state at individual points is generally adopted, though it is not quite rigorous in the case of compressible materials. This problem was investigated by A. A. Ilyushin [1.9], who found that even in pure bending four stress components must appear. However, the stresses $\sigma_{y}, \sigma_{z}$, and $\tau_{y z}$, necessary to satisfy the compatibility equations, are practically very small compared with the axial stress $\sigma_{x}$, and may be neglected.

The theory of elastic-plastic bending with normal force was developed mainly in connection with the analysis of maximal carrying capacity of eccentrically compressed columns and calculation of finite deflections of beams. Some historical notes were given in Secs. 17.3 and 17.4.

We present here an analysis close to that given by K. Ježek [4.261, 4.262], but with stretchability of the axis taken into account. Consider a monosymmetric cross-section, subject to bending with normal force. Under the assumptions of a positive bending moment $M_{b}$ (convexity in positive $z$-direction), a perfectly elastic-plastic material and no plastically passive processes following active ones,
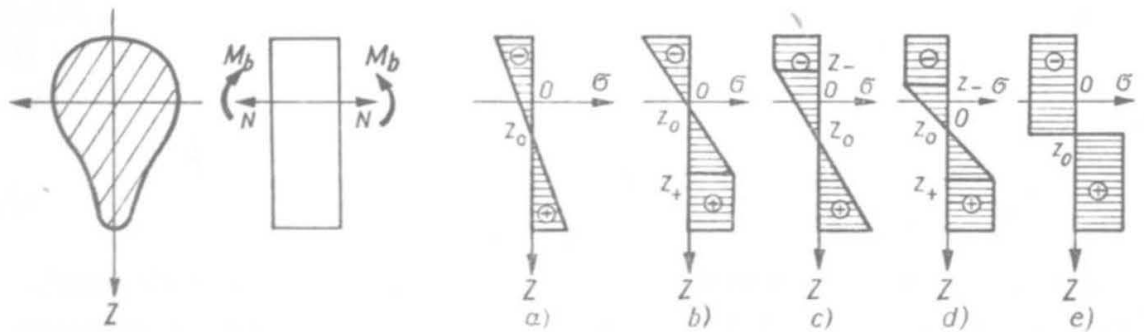

Fig. 118. Stress distributions under elastic-plastic bending with tension or compression 
five various types of stress distributions may occur (Fig. 118). They will be analysed in detail; in the case of $M_{b}<0$ the differences are inessential.

If active processes are followed by passive ones, then curvilinear stress distributions may appear. This problem, important in analysing eccentric compression for example, will not be dealt with here.

Assume the Bernoulli hypothesis of plane cross-sections. The Lagrangian coordinate measured along the undeformed axis of the beam will be denoted by $X$, and in the perpendicular direction-by $Y$ and $Z$. An elementary segment of the straight beam $d X$ will turn after deformation into a segment of an arc with the radius of curvature $\varrho+Z$, and hence the length of the segment corresponding to the variable $Z$ is equal to $(\varrho+Z) d \varphi$, Fig. 119. The coordinate $X$ will be treated as
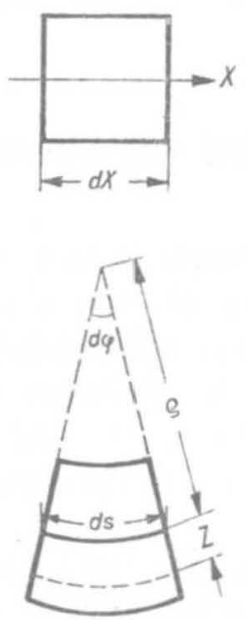

Fig. 119. Deformation of an element of a bar (beam)

an independent variable, whereas the deflection angle $\varphi$ and the length of the deformed axis of the beam as dependent variables. The distribution of strains is given by

$$
\varepsilon_{z}=\varepsilon=\frac{(\varrho+Z) d \varphi}{d X}-1=\frac{d \varphi}{d X} Z-\left(1-\varrho \frac{d \varphi}{d X}\right)
$$

and, in view of $\varrho d \varphi=d s$, we may write

$$
\varepsilon=\frac{d \varphi}{d X}\left[Z-\left(\frac{d X}{d \varphi}-\frac{d s}{d \varphi}\right)\right]=\frac{d \varphi}{d X}\left(Z-Z_{0}\right),
$$

where

$$
Z_{0}=\frac{d X}{d \varphi}-\frac{d s}{d \varphi}=\frac{1-d s / d X}{d \varphi / d X}
$$

denotes the coordinate of the neutral axis (Fig. 118). The linear strain distribution (22.2) explains the stress distributions shown in Fig. 118; they simply correspond to the stress-strain diagram for a perfectly elastic-plastic body. Integrating the stresses, 
we may calculate the bending moment $M_{b}$ and normal force $N$, and hence derive the relations between generalized stresses and generalized strains (constitutive equations for the cross-section, usually written in the form $d \varphi / d X=f\left(M_{b}, N\right)$, $\left.d s / d X=f\left(M_{b}, N\right)\right)$.

In the case under consideration the cross-section may be divided into three zones: the elastic zone (the corresponding quantities will be denoted by the subscript $e$ ), the plastic zone at tensile side (subscript + ), and the plastic zone at compressive side (subscript -). In these zones the stresses are given by

$$
\begin{array}{ll}
\sigma=-\sigma_{0} & \text { on the area } A_{-}, \\
\sigma=E \frac{d \varphi}{d X}\left(Z-Z_{0}\right) & \text { on the area } A_{e}, \\
\sigma=+\sigma_{0} & \text { on the area } A_{+} .
\end{array}
$$

The differences between the cases (a)-(e), shown in Fig. 118, are due only to the appearance of particular zones.

In the elastic range, (a), where the whole cross-sectional area $A$ reduces to $A_{e}$, integrations over this area yield

$$
N=-E A \frac{d \varphi}{d X} Z_{0}, \quad M_{b}=E I \frac{d \varphi}{d X},
$$

with $I=I_{Y}$ denoting the moment of inertia about the axis $Y$. In view of (22.3) we may write the basic (constitutive) equations in the form

$$
\frac{d \varphi}{d X}=\frac{M_{b}}{E I}, \quad \frac{d s}{d X}=1+\frac{N}{E A} .
$$

In the case of one-side plastification at the tensile side, (b), integrating over appropriate areas we obtain

$$
\begin{aligned}
& N=\sigma_{0} A_{+}+E \frac{d \varphi}{d X}\left(S_{e}-Z_{0} A_{e}\right), \\
& M_{b}=\sigma_{0} S_{+}+E \frac{d \varphi}{d X}\left(I_{e}-Z_{0} S_{e}\right),
\end{aligned}
$$

where $S$ with a corresponding subscript denotes the static moment of the area under consideration with respect to the axis $Y$. Further, denoting by $Z_{+}$the boundary coordinate between the elastic zone and the plastified tensile zone,] we obtain from the condition of continuity of stresses

$$
E \frac{d \varphi}{d X}\left(Z_{+}-Z_{0}\right)=\sigma_{0} \text {. }
$$

The system of three equations (22.7) and (22.8) with $Z_{0}$ eliminated by means of (22.3) determines the derivatives $d \varphi / d X, d s / d X$, and the coordinate $Z_{+}$. However, in the general case we may express effectively only the above derivatives and derive 
an equation with respect to $Z_{+}$. Making use of the obvious relations $A_{+}+A_{e}=A$, $S_{+}+S_{e}=0$, we arrive at

$$
\begin{aligned}
\frac{d \varphi}{d X} & =\frac{M_{b}}{E\left(I_{e}-S_{e} Z_{+}\right)}, \\
\frac{d s}{d X} & =1+\frac{\left(\sigma_{0} A_{+}-N\right) Z_{+}+\sigma_{0} S_{e}}{E\left(S_{e}-A_{e} Z_{+}\right)}
\end{aligned}
$$

with the additional equation

$$
\left[M_{b} A_{e}+\left(\sigma_{0} A-N\right) S_{e}\right] Z_{+}-\left[M_{b} S_{e}+\left(\sigma_{0} A-N\right) I_{e}\right]=0 .
$$

Of course, this equation is not linear with respect to $Z_{+}$as it may seem to be, since the coordinate $Z_{+}$is also hidden in the geometrical quantities $A_{+}, S_{+}, A_{e}, S_{e}$, and $I_{e}$, depending on the shape of the cross-section. Integrating (22.9), we may derive exact formulae for displacements (deflections), but one has to remember that the influence of the shearing force was tacitly neglected.

In the case of one-side plastification at the compressive side, (c), denoting by $Z_{\text {_ }}$ the boundary coordinate, we obtain similarly

$$
\begin{aligned}
\frac{d \varphi}{d X} & =\frac{M_{b}}{E\left(I_{e}-S_{e} Z_{-}\right)}, \\
\frac{d s}{d X} & =1-\frac{\left(\sigma_{0} A_{-}+N\right) Z_{-}+\sigma_{0} S_{e}}{E\left(S_{e}-A_{e} Z_{-}\right)},
\end{aligned}
$$

and the equation for the coordinate $Z_{\text {- }}$

$$
\left[M_{b} A_{e}-\left(\sigma_{0} A+N\right) S_{e}\right] Z_{-}-\left[M_{b} S_{e}-\left(\sigma_{0} A+N\right) I_{e}\right]=0 .
$$

In the case of two-side plastification, (d), we have, after integrations,

$$
\begin{aligned}
& N=\sigma_{0} A_{+}+E \frac{d \varphi}{d X}\left(S_{e}-Z_{0} A_{e}\right)-\sigma_{0} A_{-}, \\
& M_{b}=\sigma_{0} S_{+}+E \frac{d \varphi}{d X}\left(I_{e}-Z_{0} S_{e}\right)-\sigma_{0} S_{-},
\end{aligned}
$$

whereas the continuity conditions lead to two further equations

$$
E \frac{d \varphi}{d X}\left(Z_{+}-Z_{0}\right)=\sigma_{0}, \quad E \frac{d \varphi}{d X}\left(Z_{-}-Z_{0}\right)=-\sigma_{0} .
$$

The system of equations (22.13) and (22.14) with $Z_{0}$ eliminated from (22.3) determines the derivatives $d \varphi / d X$ and $d s / d X$ and the coordinates $Z_{+}$and $Z_{-}$. As in preceding cases, we may express effectively only the derivatives in terms of $Z_{+}$and $Z_{-}$ and derive two further equations for these coordinates. Namely, Eqs. (22.14) and (22.3) give

$$
\frac{d \varphi}{d X}=\frac{2 \sigma_{0}}{E\left(Z_{+}-Z_{-}\right)}, \quad \frac{d s}{d X}=1-\frac{\left(Z_{+}+Z_{-}\right) \sigma_{0}}{\left(Z_{+}-Z_{-}\right) E},
$$


and the substitution of (22.15) into (22.13) yields

$$
\begin{aligned}
& {\left[2 S_{e}+\left(2 A_{+}-A\right) Z_{+}+\left(2 A_{-}-A\right) Z_{-}\right] \sigma_{0}-\left(Z_{+}-Z_{-}\right) N=0,} \\
& 2\left(I_{e}+S_{+} Z_{+}+S_{-} Z_{-}\right) \sigma_{0}-\left(Z_{+}-Z_{-}\right) M_{b}=0 .
\end{aligned}
$$

Having determined $Z_{+}$and $Z_{-}$from (22.16) for the given cross-section and substituted these quantities in (22.15), we obtain effective constitutive equations in the case under consideration.

In the limiting case (e), corresponding to the limit carrying capacity of the cross-section, the elastic zone vanishes and (22.13) reduce to

$$
N=\sigma_{0}\left(A_{+}-A_{-}\right), \quad M_{b}=\sigma_{0}\left(S_{+}-S_{-}\right) .
$$

These equations determine the boundary coordinate $Z_{+}=Z_{0}=Z_{-}$and the plastic interaction curve for simultaneous bending with tension, $M_{b}=f(N)$ (yield condition for the cross-section). Such curves for various shapes of the cross-section will be discussed in detail in Sec. 23.

\subsection{Approximate assumption of unstretchability of the axis}

We mentioned above that the constitutive equations derived for particular ranges may serve as a basis for the calculation of large deflections of beams, and, after suitable simplifications, for the calculation of small deflections. However, even when analysing finite deflections, one often introduces an approximation neglecting stretchability of the axis; this was already done by Euler in deriving the classical equations of the elastica. In the elastic range such an approximation corresponds to disregard of the effect of normal forces; in fact, dropping $N$ in (22.6), we obtain $d s=d X$. In the elastic-plastic range this problem is more important, since an approximatel assumption $N=0$ does not lead to $d s=d X$. Nevertheless, in some cases the errors of approximation may be small, and hence we pay some attention to the approximate assumption of unstretchability of the axis.

Disregard of stretchability consists in the identification of the variables $s$ and $X$, dropping !Eq. (22.3) and treating the coordinate $Z_{0}$ as a parameter which is to be eliminated. So the system of Eqs. (22.7) and (22.8) with $d X$ replaced by $d s$ may be treated as a system with unknowns $d \varphi / d s, Z_{+}$and $Z_{0}$. We note that in this system the derivative $d s / d X$ does not appear (it appears only in Eq. (22.3), ?which is neglected here), and hence the resulting equation is again of the form (22.9) with $d X$ replaced by $d s$ :

$$
\frac{d \varphi}{d s}=\frac{M_{b}}{E\left(I_{e}-S_{e} Z_{+}\right)},
$$

where the coordinate $Z_{+}$is determined by $(22.10)$ as before. Similarly we obtain the first equation (22.11) with $d X$ replaced by $d s$. Of course, in effective solutions the differences appear since the expressions for $M_{b}$ are different according to whether stretchability of the axis is neglected or allowed for. 
It should be stressed, however, that the approximate disregard of stretchability of the axis leads to a certain contradiction within the theory. In fact, assuming $d s=d X$, we always obtain from (22.3) $Z_{0}=0$, whereas when eliminating $Z_{0}$ from the relevant system of equations, e.g. (22.7) and (22.8), we obtain as a rule $Z_{0} \neq 0$. This contradiction leads in most cases to small errors and might be accepted if the results were simpler than those obtained from exact equations. It turns out, rather surprisingly, that exact constitutive equations often lead to simpler results than those assuming unstretchability of the axis and then the approximate assumption should be regarded as useless.

\subsection{Constitutive equations of perfect plasticity for a rectangular cross-section}

In the case of the combined loadings under consideration the rectangular crosssection leads to particularly simple results, important for engineering applications. Denote the width of the cross-section by $b$ and the depth (height) by $2 h-$ such a notation is more convenient here than the traditional $h$, Fig. 120 . In this case the
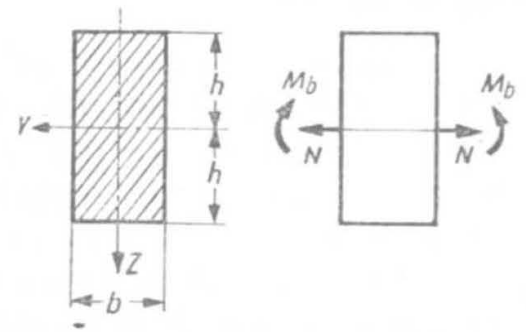

Fig. 120. Beam with a rectangular cross-section

general equations derived in Sec. 22.2 for perfectly elastic-plastic material may be reduced to an explicit form and integrated effectively.

The elastic range (a) will not be dealt with here since the general equations (22.6) are already sufficiently effective.

In the case of one-side plastification at the tensile side, (b), we substitute

$$
\begin{array}{ll}
A=2 b h, & S_{e}=-\frac{b}{2}\left(h^{2}-Z_{+}^{2}\right), \\
A_{e}=b\left(h+Z_{+}\right), & I_{e}=\frac{b}{12}\left(h+Z_{+}\right)^{3}+\frac{b}{4}\left(h+Z_{+}\right)\left(h-Z_{+}\right)^{2} .
\end{array}
$$

Equation (22.10) reduces to a cubic equation with respect to the coordinate $Z_{+}$. However, we may check that it has a double root $Z_{+}=-h$, which is of no interesi in the case under consideration; dividing this equation by $\left(Z_{+}+h\right)^{2}$, we obtain a linear equation with the root

$$
Z_{+}=2 h-\frac{3 M_{b}}{2 b h \sigma_{0}-N} .
$$


To simplify the notation we now introduce dimensionless generalized stresses (exertion factors), relating them to the limit carrying capacities in pure bending and pure tension, respectively:-

$$
m=\frac{M_{b}}{\overline{\bar{M}}_{b}}=\frac{M_{b}}{b h^{2} \sigma_{0}}, \quad n=\frac{N}{\overline{\bar{N}}}=\frac{N}{2 b h \sigma_{0}} .
$$

Equation (22.20) now takes the form

$$
\frac{Z_{+}}{h}=2-\frac{3 m}{2(1-n)} \text {. }
$$

Substituting here the functions $m=m(X)$ and $n=n(X)$ (equations of bending moment and normal force; they may also depend on deflections), we may determine the distribution of plastified zones along the beam. On the other hand, substitution of (22.21) into (22.9) makes it possible to write the basic differential equations for one-side plastification of rectangular cross-section in the dimensionless form

$$
\begin{aligned}
h \frac{d \varphi}{d X} & =\frac{16}{9} a^{2} \frac{(1-n)^{3}}{(2-2 n-m)^{2}}, \\
\frac{d s}{d X} & =1+\left[1-\frac{8}{9} \frac{(4-4 n-3 m)(1-n)^{2}}{(2-2 n-m)^{2}}\right] a^{2},
\end{aligned}
$$

where $a^{2}$ denotes the following positive material constant:

$$
a^{2}=\frac{\sigma_{0}}{E} \text {. }
$$

In the case of one-side plastification at the compressive side, (c), we obtain similarly

$$
\frac{Z_{-}}{h}=-2+\frac{3 m}{2(1+n)}
$$

and the system of basic differential equations

$$
\begin{aligned}
h \frac{d \varphi}{d X} & =\frac{16}{9} a^{2} \frac{(1+n)^{3}}{(2+2 n-m)^{2}}, \\
\frac{d s}{d X} & =1-\left[1-\frac{8}{9} \frac{(4+4 n-3 m)(1+n)^{2}}{(2+2 n-m)^{2}}\right] a^{2} .
\end{aligned}
$$

In the case of bisymmetric cross-sections, and hence also in the case of a rectangular cross-section, one-side plastification at the tensile side may take place only for $n>0$, and one-side plastification at the compressive side - only for $n<0$. Taking this fact into account and introducing the signs of absolute value, we may jointly write (22.23) and (22.26) in a uniform manner; moreover, considering the cases $m<0, n>0$ and $m<0, n<0$, we arrive at the following general form of basic 
differential equations, valid in the case of one-side plastification for arbitrary signs of bending moment and normal force:

$$
\begin{aligned}
h \frac{d \varphi}{d X} & =\frac{16}{9} a^{2} \frac{(1-|n|)^{3}}{(2-2|n|-|m|)^{2}} \operatorname{sign} m, \\
\frac{d s}{d X} & =1+\left[1-\frac{8}{9} \frac{(4-4|n|-3|m|)(1-|n|)^{2}}{(2-2|n|-|m|)^{2}}\right] a^{2} \operatorname{sign} n,
\end{aligned}
$$

where sign $m=+1$ for $m>0$, sign $m=-1$ for $m<0$, and similarly sign $n$.

Let us now proceed to the case of two-side plastification. We substitute here

$$
\begin{aligned}
& A_{e}=b\left(Z_{+}-Z_{-}\right), \quad A_{+}=b\left(h-Z_{+}\right), \quad A_{-}=b\left(h+Z_{-}\right), \\
& S_{e}=\frac{b}{2}\left(Z_{+}^{2}-Z_{-}^{2}\right), \quad S_{+}=\frac{b}{2}\left(h^{2}-Z_{+}^{2}\right), \quad S_{-}=-\frac{b}{2}\left(h^{2}-Z_{-}^{2}\right), \\
& I_{e}=\frac{b}{12}\left(Z_{+}-Z_{-}\right)^{3}+\frac{b}{4}\left(Z_{+}-Z_{-}\right)\left(Z_{+}+Z_{-}\right)^{2} .
\end{aligned}
$$

The system of equations (22.16), determining $Z_{+}$and $Z_{-}$, consists in this case of one quadratic and one cubic equation. However, it turns out that both equations may be divided by the factor $\left(Z_{+}-Z_{-}\right)$, different from zero, and hence we obtain a simplified system which can easily be solved:

$$
\frac{Z_{+}}{h}=-n+\sqrt{3} \sqrt{1-n^{2}-m}, \quad \frac{Z_{-}}{h}=-n-\sqrt{3} \sqrt{1-n^{2}-m} .
$$

Substituting here the functions $m=m(X)$ and $n=n(X)$, we may determine the distribution of plastified zones along the beam. On the other hand, substitution of (22.29) into (22.15) yields the basic differential equations for two-side plastification. We quote them here in the dimensionless form, valid also for $m<0$ :

$$
\begin{aligned}
h \frac{d \varphi}{d X} & =\frac{a^{2}}{\sqrt{3} \sqrt{1-n^{2}-|m|}} \operatorname{sign} m, \\
\frac{d s}{d X} & =1+\frac{a^{2} n}{\sqrt{3} \sqrt{1-n^{2}-|m|}} .
\end{aligned}
$$

The above-mentioned approximate disregard of stretchability of the axis reduces here to: (1) replacement of $d X$ by $d s$ in the first equations in systems (22.27) and (22.30), (2) dropping the second equations in these systems and (3) substitution of the functions $m=m(s)$ and $n=n(s)$, usually different from the functions $m=m(X)$ and $n=n(X)$.

\subsection{Limit curves}

We now derive the equations of limit (interaction) curves, separating in the plane $m-n$ the domains of particular phases of work of the cross-section. The boundary 
between the elastic range and one-side plastification will be obtained by equating the coordinate $Z_{+}(22.22)$ with half the depth $h$; we arrive at the straight line

$$
m=\frac{2}{3}(1-n),
$$

the segment $0<n<1$ of which presents the solution. Comparing $Z_{-}(22.25)$ with $(-h)$ and repeating this procedure for negative $m$, we obtain four segments of straight lines, which form a diamond-shaped elastic domain. All the four lines may be presented jointly by the equation

$$
|m|=\frac{2}{3}(1-|n|) \text {. }
$$

The boundary between one-side and two-side plastification may be determined by equating $Z_{+}$with $h$ and $Z_{-}$with $-h$ in (22.29). We obtain two parabolas

$$
\begin{aligned}
& m=\frac{2}{3}(1+n)(1-2 n), \\
& m=\frac{2}{3}(1-n)(1+2 n) .
\end{aligned}
$$

For negative bending moments a simple change of sign at the right-hand side takes place.

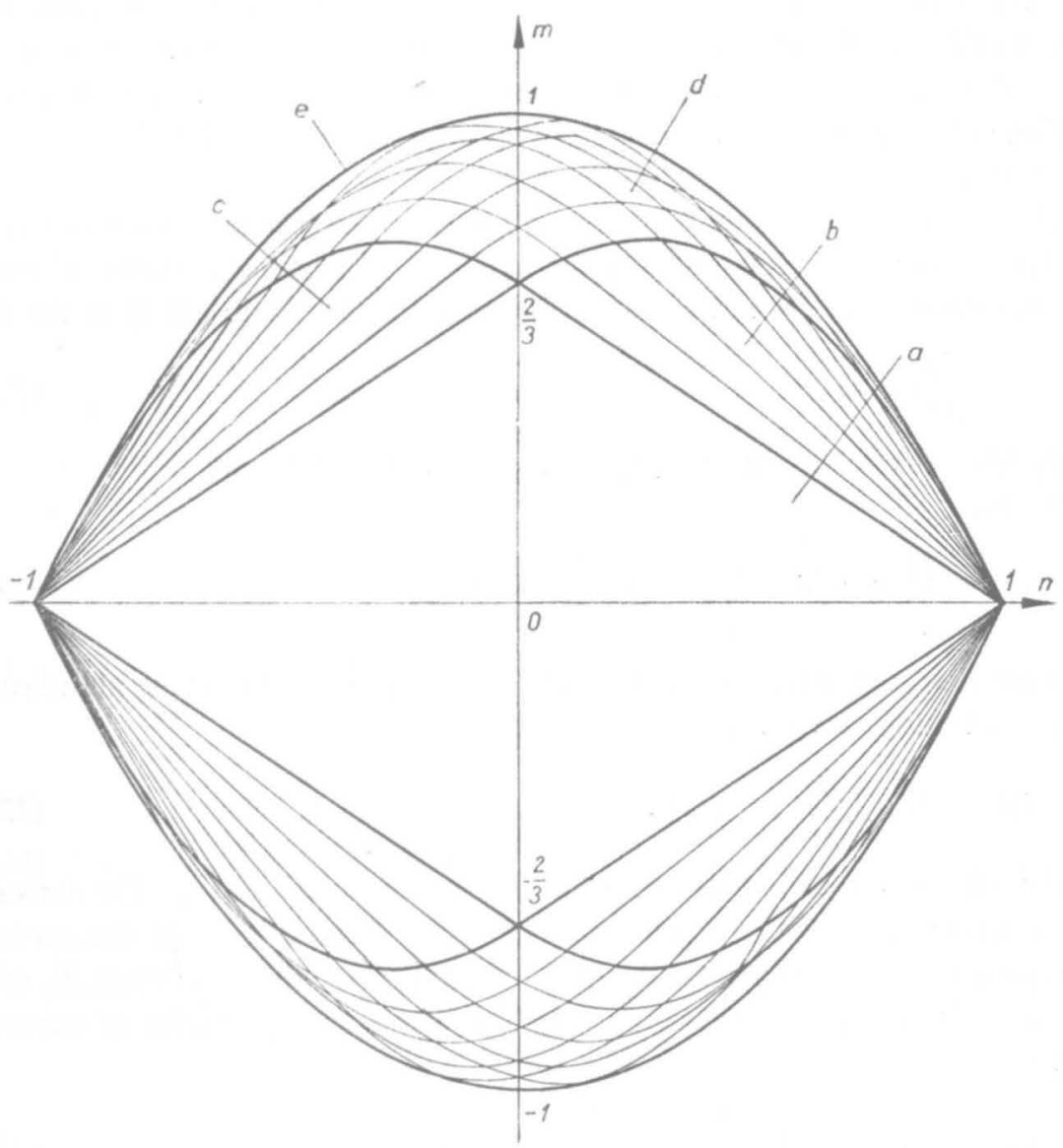

Fig. 121. Interaction curves for a rectangular section under bending with normal force 
Finally, the equation of the curve of limit carrying capacity (plastic interaction curve) may be obtained from (22.29) by assuming $Z_{+}=Z_{-}$. The equation of the parabola

$$
m=1-n^{2}
$$

was first derived by K. Girkmann [5.61] in 1931 .

A complete picture of limit curves in the plane $m-n$ is shown in Fig. 121; subdomains are denoted by the letters conforming to Fig. 118. Further, Fig. 121 gives the lines of constant $Z_{+} / h$ and $Z_{-} / h$, making it possible to determine the ranges of applicability of the formulae derived above. Namely, we exclude passive processes following active ones; such processes will not take place if the coordinate $Z_{+}$is a non-increasing and $Z_{-}$is a non-decreasing function of time. Geometrically one can say that the loading trajectory must cut the lines of both families $\left(Z_{+}=\right.$const and $Z_{-}=$const $)$in the outward direction; in the opposite case locally passive processes will take place.

Figure 121 presents limit curves for a rectangular cross-section if generalized stresses are assumed as exertion factors (independent variables). In some cases other quantities should be regarded as independent, e.g. generalized strains. Determination of limit curves in the plane of generalized strains is even easier than it is in the plane of generalized stresses; moreover, they are identical for all bisymmetric cross-sections.

In the case of simultaneous bending with tension one usually assumes curvature of the beam axis $\varkappa$ and elongation of the axis $\varepsilon_{0}$ as generalized strains. However, energy considerations lead to somewhat different results. Write (22.1) in the form

$$
\varepsilon=\frac{d \varphi}{d X} Z+\varepsilon_{0}
$$

(resulting from the definition of $\varepsilon_{0}$ ) and calculate the virtual work in the case under consideration

$$
\delta W=\iint_{A} \sigma \delta \varepsilon d A=\iint_{A} \sigma\left[z \delta\left(\frac{d \varphi}{d X}\right)+\delta \varepsilon_{0}\right] d A .
$$

After integration, the result of which does not depend on the possible plastification of the cross-section, we have

$$
\delta W=M_{b} \delta\left(\frac{d \varphi}{d X}\right)+N \delta \varepsilon_{0} .
$$

Hence the role of generalized strains is played here by $d \varphi / d X$ and $\varepsilon_{0}$. The derivative $d \varphi / d X$, however, does not represent the curvature of the axis $x$ but the curvature of the neutral layer, which will be denoted by $\varkappa_{n}$. Indeed, the coordinate $Z_{0}$ of the neutral layer is determined by (22.3), and the corresponding radius of curvature $\varrho_{n}$ equals

$$
\varrho_{n}=\varrho+z_{0}=\frac{d s}{d \varphi}+\frac{d X}{d \varphi}-\frac{d s}{d \varphi}=\frac{d X}{d \varphi} .
$$


The differences between $x$ and $x_{n}$ are small as a rule, but may become important for large absolute values of normal forces. Those quantities are interrelated by means of the equation

$$
x_{n}=\varkappa\left(1+\varepsilon_{0}\right) \text {. }
$$

Let us now derive the equations of limit curves in the planes $\varepsilon_{0}-\varkappa_{n}$ and $\varepsilon_{0}-\varkappa$. Such curves will be determined by the conditions $Z_{+}=h$ or $Z_{-}=-h$, where $h$ denotes half the depth of an arbitrary bisymmetric cross-section. Making use of the general relation (22.8) and substituting (22.3), we write the first of those conditions in the form

$$
E \frac{d \varphi}{d X}\left(h-\frac{d X}{d \varphi}+\frac{d s}{d \varphi}\right)=\sigma_{0},
$$

and hence

$$
x_{n} h+\varepsilon_{0}=\frac{\sigma_{0}}{E}=a^{2} .
$$

From the condition $Z_{-}=-h$ and two other conditions for the opposite sense of the bending moment we obtain four equations of straight lines, which may jointly be written thus:

$$
\pm x_{n} h \pm \varepsilon_{0}=a^{2} .
$$

These limit curves are shown in Fig. 122.

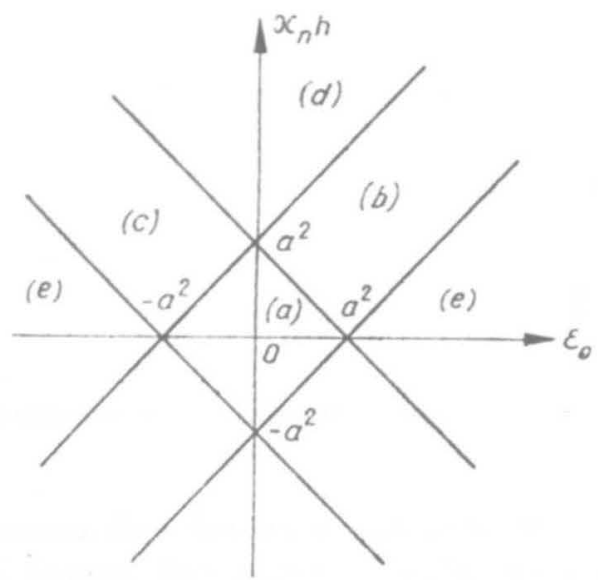

Fig. 122. Interaction curves in the plane: elongation of the axis-curvature of the neutral layer

Substitution of (22.40) into (22.43) makes it possible to determine directly the limit curves in the plane $\varepsilon_{0}-\varkappa$ (elongation of the axis-curvature of the axis). We arrive at four hyperbolas with the equations

$$
x h= \pm \frac{a^{2} \pm \varepsilon_{0}}{1+\varepsilon_{0}} .
$$

They are shown in Fig. 123. 


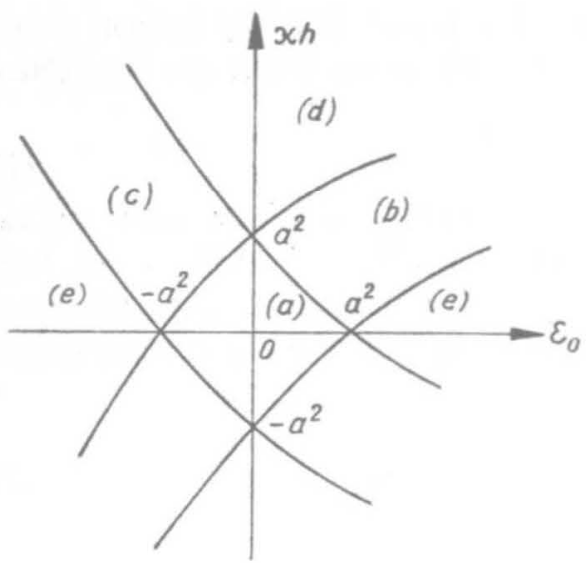

Fig. 123. Interaction curves in the plane: elongation of the axis-curvature of the axis

The relations between $m, n, \varepsilon_{0}$ and $\varkappa$ or $\varkappa_{n}$ may be represented by certain surfaces in appropriately chosen three-dimensional spaces. Examples of such surfaces may be found e.g. in the papers by L. K. Stevens [5.174] and I. L. Dikovitch [5.39]; Fig. 124 quoted after Dikovitch, presents the function $\varkappa_{n}=f\left(M_{b}, N\right)$.

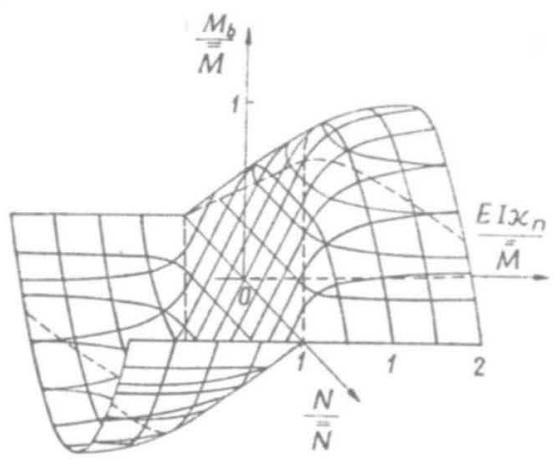

Fig. 124. Curvature of the neutral layer in terms of the bending moment and normal force (after Dikovitch)

A slightly different approach to constitutive equations and limit curves for a rectangular cross-section subject to elastic-plastic bending with normal force is given by K. Ingerle [5.77]. N. F. Ershov [5.44] and M. V. Pankova [5.141] treated the problem as a two-dimensional problem of plasticity (for a straight bar and for a curved bar respectively). A. J. Barrett [5.11], J. M. Frankland and R. E. Roach [5.46] investigated the effect of change of width during the deformation process.

\subsection{Subsequent neutral curves. Shakedown problems}

The initial domain of passive processes (elastic domain) is a tetragon bounded by four lines (22.32). If the process trajectory exceeds any of these four lines, then 
plastic deformations introduce certain residual stresses changing the onset of yielding and a subsequent domain of passive processes is formed. The boundaries of such domains will be called subsequent neutral curves (surfaces, in general), similarly to the terminology used in Chapter III at the level $\mathscr{P}$; the term modified elastic curves is also appropriate. According to the Melan theorem, any domain inside subsequent neutral curves is always a shakedown domain and hence the analysis of subsequent neutral curves is closely connected with the shakedown analysis.

Modified elastic curves for simultaneous bending with tension of a perfectly elastic-plastic rectangular cross-section were analysed in detail by E. Cegielski [5.26]. We quote here the most important results of that paper.

Consider a point $\tilde{B}(\tilde{m}, \tilde{n})$ within the range of one-side plastification at the tensile side, and the subsequent neutral curve corresponding to that point. The point $\tilde{B}$ will be called here the control point for that curve, as at the level $\mathscr{P}$, Sec. 14.7. The corresponding curvature of the neutral layer and the elongation of the axis are denoted by $\tilde{x}_{n}$ and $\tilde{\varepsilon}_{0}$, respectively; they are related to $\tilde{m}$ and $\tilde{n}$ by means of (22.23) with substituted (22.39). To determine the subsequent neutral curve we have to superpose passive (elastic) processes on the stresses in $\tilde{B}$ and discuss the onset of secondary plastification. It turns out that besides the four conditions

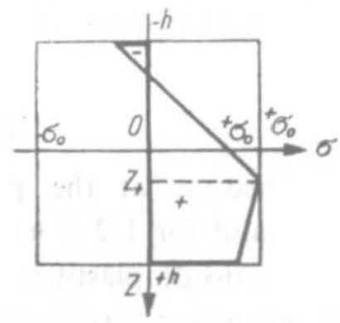

Fig. 125. The fifth variant of onset of plastification if residual stresses are allowed for

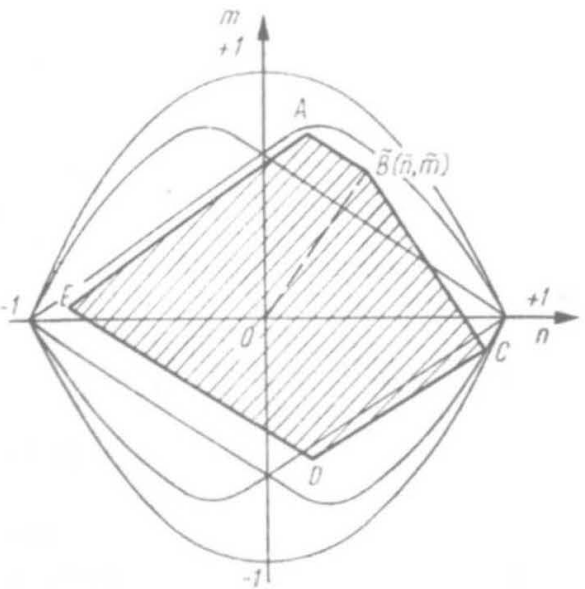

Fig. 126. Transition of the initial tetragon into a pentagon 
$\sigma( \pm h)= \pm \sigma_{0}$, as for initial yielding, the fifth condition $\sigma\left(Z_{+}\right)=\sigma_{0}$ may also take place (Fig. 125), and hence the initial tetragon is truncated so as to form a pentagon (Fig. 126). The equations of the particular sides of that pentagon are as follows:

$$
\begin{aligned}
& A \tilde{B}: \quad m-\tilde{m}+\frac{2}{3}(n-\tilde{n})=0, \\
& \tilde{B} C: \quad m-\tilde{m}+\frac{4(1-\tilde{n})}{3(4-4 \tilde{n}-3 \tilde{m})}(n-\tilde{n})=0, \\
& C D: \quad m-\tilde{m}-\frac{2}{3}(n-\tilde{n})=-\frac{16}{9} \frac{(1-\tilde{n})^{2}}{2-2 \tilde{n}-\tilde{m}}, \\
& D E: \quad m-\tilde{m}+\frac{2}{3}(n-\tilde{n})=-\frac{4}{3}, \\
& E A: \quad m-\tilde{m}-\frac{2}{3}(n-\tilde{n})=-\frac{16}{9} \frac{(1-\tilde{n})^{2}}{2-2 \tilde{n}-\tilde{m}}+\frac{4}{3} .
\end{aligned}
$$

At the control point $\tilde{B}$ a new corner is formed, as in the slip theories at the level $\mathscr{P}$. The rest of the initial tetragon is subject to rigid translation.

If the points $C$ and $D$ coincide, then the pentagon is reduced to a tetragon (smaller than the initial one). The relevant boundary line in the plane $m-n$ is given by

$$
\tilde{m}=\frac{2}{3}(1-\tilde{n})[4-\tilde{n}-\sqrt{(5-\tilde{n})(1-\tilde{n})}] .
$$

It intersects the parabola (22.34), limiting one-side plastification, at the point $n=1 / 2, m=2 / 3$, and hence the boundary line (22.46) is valid for $1 / 2 \leqslant n \leqslant 1$.

Consider now the control point $\tilde{B}$ inside the domain of two-side plastification. Then the following two new conditions may describe the secondary plastification: $\sigma\left(Z_{+}\right)=\sigma_{0}, \sigma\left(Z_{-}\right)=-\sigma_{0}$; however, two of the original conditions $\sigma(h)=\sigma_{0}$

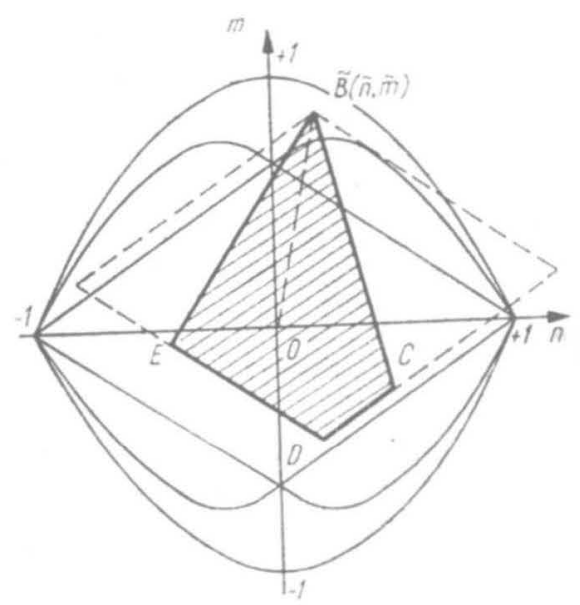

Fig. 127. Doubly truncated tetragon 
and $\sigma(-h)=-\sigma_{0}$ cannot be satisfied except at $\tilde{B}$. Hence the initial tetragon is doubly truncated to form a new tetragon (Fig. 127). The equations are:

$$
\begin{array}{ll}
\tilde{B} C: & m-\tilde{m}+\frac{2}{3\left(\sqrt{3} \sqrt{\left.1-\tilde{m}-\tilde{n}^{2}-\tilde{n}\right)}\right.}(n-\tilde{n})=0, \\
C D: & m-\tilde{m}-\frac{2}{3}(n-\tilde{n})=-\frac{4}{3}, \\
D E: & m-\tilde{m}+\frac{2}{3}(n-\tilde{n})=-\frac{4}{3}, \\
E \tilde{B}: & m-\tilde{m}-\frac{2}{3\left(\sqrt{3} \sqrt{1-\tilde{m}-\tilde{n}^{2}}-\tilde{n}\right)}(n-\tilde{n})=0 .
\end{array}
$$

The new corner at the control point $\tilde{B}$ is "more acute" here than in the preceding case.

If the points $C$ and $D$ coincide, then the tetragon is reduced to a triangle. The relevant boundary line is a parabola

$$
\tilde{m}=1-\frac{4}{3} \tilde{n}^{2} .
$$

It intersects (22.34) also at the point $n=1 / 2, m=2 / 3$; hence (22.48) is valid for $0<\tilde{n}<1 / 2$. The full system of boundary lines, separating the domains where subsequent neutral curves have the form of pentagons, tetragons, and triangles,

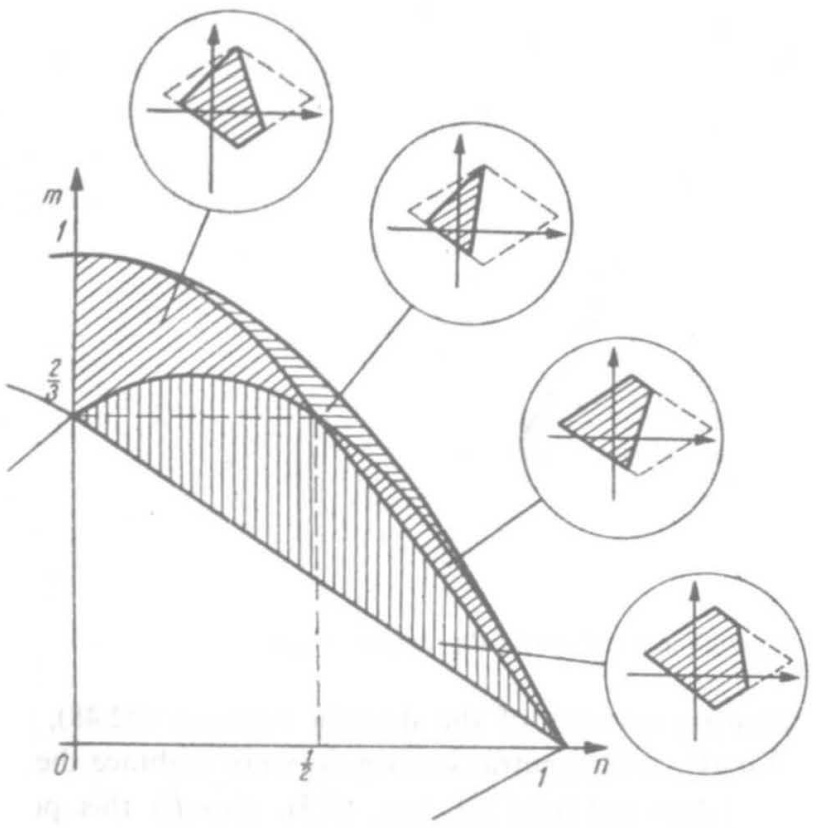

Fig. 128. Division of the plane $m-n$ into the subregions

is shown in Fig. 128. If the control point reaches the outer parabola (22.35), then the modified elastic domains are degenerated to segments of straight lines (J. A. König [5.92]). 
In most cases subsequent neutral curves comprise the origin of the reference frame $m=n=0$. Physically, this means that full unloading is then purely passive (elastic). However, there exist certain zones close to the curve of limit carrying capacity (22.35) where full unloading causes secondary plastic strains. To determine the boundary of those zones we require that the point $m=n=0$ should satisfy either the equation of the line $E A$ in (22.45), or $\tilde{B} C$ or $E \tilde{B}$ in (22.47). For one-side plastification we obtain

$$
\tilde{m}=\frac{1}{3}(1-2 \tilde{n})+\sqrt{1-\frac{8}{9}} \tilde{n} .
$$

This line intersects the parabola (22.34) at the point $\tilde{n}=\sqrt{2} / 2, \tilde{m}=\sqrt{2} / 3$, and hence (22.49) is valid for $\sqrt{2} / 2 \leqslant \tilde{n} \leqslant 1$. Both conditions for two-side plastification lead to a cubic equation with respect to $\tilde{m}$, but easy to be solved for $\tilde{n}$ :

$$
\tilde{n}=\frac{3}{2} \tilde{m} \sqrt{\frac{3(1-\tilde{m})}{9 \tilde{m}^{2}-3 \tilde{m}+1}}
$$

valid for $0 \leqslant \tilde{n} \leqslant \sqrt{2} / 2$, or $\sqrt{2} / 3 \leqslant \tilde{m} \leqslant 1$. The boundary lines (22.49) and (22.50) are shown in Fig. 129.

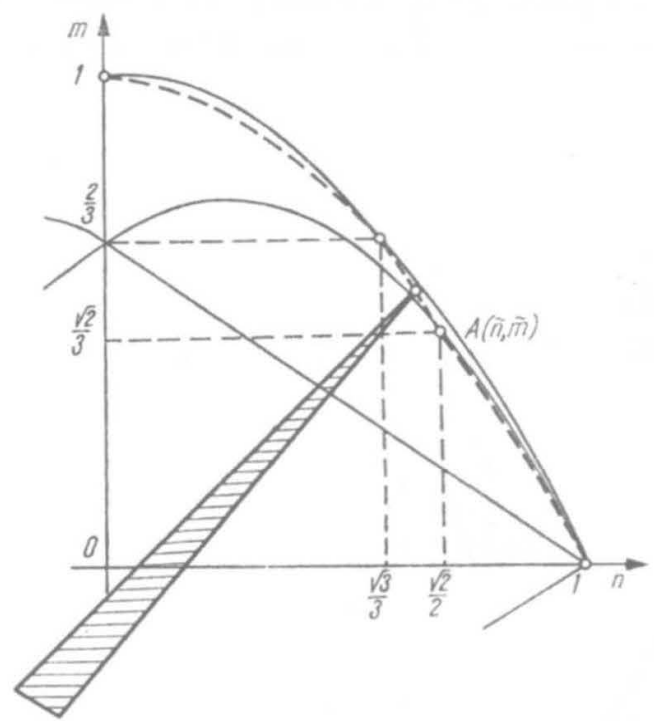

Fig. 129. Full unloading corresponding to a plastically active process

Let us note two interesting features of the domain between (22.48), (22.49) and the parabola (22.35). Firstly, if the neutral surface does not embrace the origin, then the Prager postulate (4) does not hold (cf. Sec. 15.3). Though this postulate was formulated for the level $\mathscr{P}$, it holds also in most cases at the levels $\mathscr{S}$; the case under consideration is an exception here. Secondly, in this domain we have to distinguish self-stresses from residual stresses which actually occur after unloading; self-stresses due to non-uniform plastic deformation would have been equal to residual stresses only if full unloading had been passive (elastic). 
Unloading processes were also analysed by Y. Tozawa [5.187], H. Sudzuki, E. Takano and H. Negisi [5.178].

The family of subsequent neutral surfaces, corresponding to individual control points, is also the family of shakedown domains. However, for some applications it is convenient to know rectangular shakedown domains, e.g. $0<m<m_{1}$, $0<n<n_{1}$, or to know the maximal admissible cycles $-m_{1}<m<m_{1}$ for a given value of $n$. Hence various approaches to shakedown problems of simultaneous bending with tension have been proposed; we mention here the papers by B. I. Lyubarov [5.112, 5.113, 5.114], A. V. Gemmerling and B. N. Kuznetsov [5.55, 5.98, 5.99], P. Grundy [5.65], D. A. Gokhfeld [4.173, 4.174] (shakedown domains for symmetric cycles of $m$ under constant $n$ ), J. A. König [5.92] (applications to frames and arches), M. Yamada and K. Shirakawa [5.204], K. Yoshimura [5.206] (seismic loading), C. Cichoń [5.34] (numerical analysis for linear plastic hardening).

\subsection{Finite deflections of beams with a rectangular cross-section}

Constitutive equations, derived in Sec. 22.4, serve as a basis for the calculation of deflections of beams under bending with tension. The most rigorous results are obtained if we retain an exact expression for the curvature and take into account the stretchability of the axis; of course, even in this case some errors are due to the neglected influence of shear forces. Further steps of approximation consist in disregarding the stretchability of the axis and in a restriction to small deflections.

First we quote the solution obtained by Z. Waszczyszyn and M. Życzkowski [5.196], rigorously taking into account the stretchability of the axis. Suppose that the beam is loaded by concentrated forces and concentrated couples only and

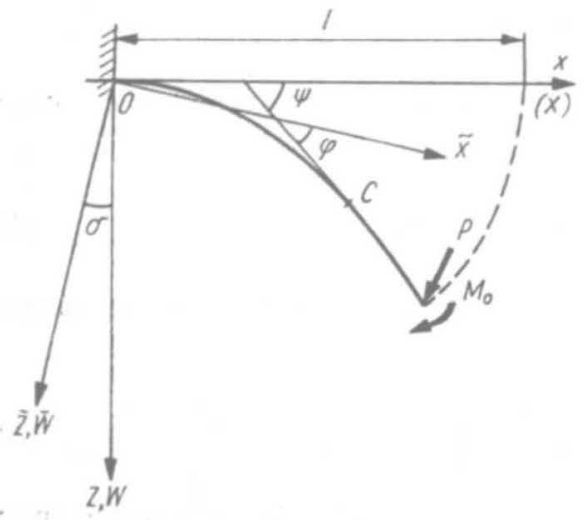

Fig. 130. Loading of the beam

consider a certain interval of this beam (Fig. 130). We introduce a new rotated reference frame $\tilde{x} \tilde{z}$ with the direction $\tilde{z}$ coinciding with the direction of the force $P$ (force at the end or the resultant force acting on the cut off portion of the beam). 
The deflection angle $\varphi$ will now be measured from the axis $\tilde{x}$. Hence the bending moment $M_{b}$ and the normal force $N$ at an arbitrary point $C$ of the axis are

$$
\begin{aligned}
M_{b} & =P \int_{s}^{s_{t}} d s \cos \varphi+M_{0}=P \int_{x}^{t} \frac{d s}{d X} \cos \varphi d X+M_{0}, \\
N & =P \sin \varphi,
\end{aligned}
$$

where $s$ denotes the length of the arc after deformation. We have introduced no new symbol for the variable of integration in (22.51) to keep the notation as simple as possible. Moreover, the sign convention for the bending moment is opposite to that normally used; the positive moment corresponds here to the positive derivative $d \varphi / d X$, and this fact simplifies further calculations. Let us introduce dimensionless loading parameters

$$
p=\frac{P}{\overline{\bar{N}}}=\frac{P}{2 b h \sigma_{0}}, \quad m_{0}=\frac{M_{0}}{\overline{\bar{M}}}=\frac{M_{0}}{b h^{2} \sigma_{0}}
$$

and rewrite (22.51) in the dimensionless form

$$
m=\frac{2 p}{h} \int_{X}^{l} \frac{d s}{d X} \cos \varphi d X+m_{0}, \quad n=p \sin \varphi .
$$

Consider first the case of one-side plastification (b) or (c). Substitution of (22.53) into (22.27) leads to a system of integro-differential equations with respect to $\varphi$ and $s$. However, this system may easily be reduced to one differential equation: one has to calculate $m$ from the first Eq. (22.27), differentiate and compare to the differentiated first equation (22.53). Equation (22.27) yields (under the assumptions of $m>0, n>0$ )

$$
m=2-2 n-\frac{4}{3} \frac{a}{\sqrt{h}}(1-n)^{3 / 2}\left(\frac{d \varphi}{d X}\right)^{-1 / 2} .
$$

Substituting (22.54) into the second Eq. (22.27), we express the derivative $d s / d X$ in terms of $d \varphi / d X$ and $n$ :

$$
\frac{d s}{d X}=1+a^{2}-h \frac{d \varphi}{d X}\left(2 a \sqrt{\frac{1-n}{h d \varphi / d X}}-1\right) .
$$

Differentiating now the first Eq. (22.53) and substituting (22.55), we determine the derivative $d m / d X$ :

$$
\frac{d m}{d X}=-\frac{2 p}{h} \cos \varphi\left[1+a^{2}-h \frac{d \varphi}{d X}\left(2 a \sqrt{\frac{1-n}{h d \varphi / d X}}-1\right)\right],
$$

Comparison of this expression with the differentiated (22.54) leads to the required differential equation with the unknown function $\varphi=\varphi(X)$

$$
(1-p \sin \varphi)^{3 / 2} a \varphi^{\prime \prime}-3 a p(1-p \sin \varphi)^{1 / 2} \cos \varphi \varphi^{\prime 2}+\frac{3\left(1+a^{2}\right)}{\sqrt{h}} p \cos \varphi \varphi^{\prime 3 / 2}=0 .
$$


where primes denote differentiation with respect to the Lagrangian coordinate $X$. It should be noted that Eq. (22.57) contained also the terms with $\varphi^{\prime 5 / 2}$, but they were reduced, and this fact essentially simplifies integration. Namely, we introduce a new dependent variable $u$ by the formulae

$$
\varphi^{\prime}=u^{2}(\varphi), \quad \varphi^{\prime \prime}=2 u u^{\prime} \varphi^{\prime}=2 u^{3} u^{\prime},
$$

and, dividing (22.57) by $u^{3}$, we obtain a linear first-order equation

$$
2(1-p \sin \varphi)^{3 / 2} a u^{\prime}-3 a p(1-p \sin \varphi)^{1 / 2} \cos \varphi u+\frac{3\left(1+a^{2}\right)}{\sqrt{h}} p \cos \varphi=0 .
$$

The general integral of (22.59) may be written in the form

$$
u=\sqrt{\frac{d \varphi}{d X}}=\frac{C_{1}+k(1-p \sin \varphi)}{(1-p \sin \varphi)^{3 / 2}},
$$

where $C_{1}$ denotes the integration constant, and $k$ the constant

$$
k=\frac{3\left(1+a^{2}\right)}{2 a \sqrt{h}} .
$$

Now it is convenient to perform further integration in parametrical form since Eq. (22.60) - though integrable in elementary functions-cannot then be solved with respect to $\varphi$. In view of obvious relations (Fig. 130)

$$
\begin{aligned}
& d x=d s \cos (\varphi+\delta)=\frac{s^{\prime}}{\varphi^{\prime}} \cos (\varphi+\delta) d \varphi, \\
& d w=d s \sin (\varphi+\delta)=\frac{s^{\prime}}{\varphi^{\prime}} \sin (\varphi+\delta) d \varphi,
\end{aligned}
$$

with (22.55) substituted for $s^{\prime}$ and (22.60) for $\varphi^{\prime}$, we obtain after formal integration the following parametrical equations of the deflection line:

$$
\begin{array}{r}
x=x_{0}+\int\left\{\left(1+a^{2}\right) \frac{(1-p \sin \varphi)^{3}}{\left[C_{1}+k(1-p \sin \varphi)\right]^{2}}-\right. \\
\left.-2 a \sqrt{h} \frac{(1-p \sin \varphi)^{2}}{C_{1}+k(1-p \sin \varphi)}+h\right\} \cos (\varphi+\delta) d \varphi, \\
w=w_{0}+\int\left\{\left(1+a^{2}\right) \frac{(1-p \sin \varphi)^{3}}{\left[C_{1}+k(1-p \sin \varphi)\right]^{2}}-\right. \\
\left.-2 a \sqrt{h} \frac{(1-p \sin \varphi)^{2}}{C_{1}+k(1-p \sin \varphi)}+h\right\} \sin (\varphi+\delta) d \varphi .
\end{array}
$$

The integration constants $C_{1}, w_{0}$ and $x_{0}$ should be evaluated from boundary conditions. 
Let us now proceed to the case of two-side plastification, (d), which is even simpler than the preceding one. The procedure is quite similar here. First Eq. (22.30) solved with respect to $m$ (under the assumption of $m>0$ ) yields

$$
m=1-n^{2}-\frac{a^{4}}{3 h^{2} \varphi^{\prime}},
$$

where the primes denote differentation with respect to $X$, as before. Substituting (22.64) into the second Eq. (22.30), we obtain

$$
s^{\prime}=1+h n \varphi^{\prime} \text {. }
$$

Further, substitution of (22.65) into the first Eq. (22.53), differentiation and equating to the differentiated (22.64) results in the equation

$$
a^{4} \varphi^{\prime \prime}+3 p h \cos \varphi \varphi^{\prime 3}=0,
$$

where the terms with $\varphi^{\prime 4}$ are reduced and vanish. Substituting $\varphi^{\prime}=u(\varphi)$, we arrive at the equation with separated variables

$$
a^{4} u^{\prime}+3 p h \cos \varphi u^{2}=0,
$$

and after integration

$$
\frac{1}{u}=\frac{d X}{d \varphi}=\frac{3 p h}{a^{4}} \sin \varphi+D_{1} .
$$

This equation may easily be integrated once more

$$
X=-\frac{3 p h}{a^{4}} \cos \varphi+D_{1} \varphi+D_{2},
$$

but this relation cannot be solved effectively with respect to $\varphi$ and we have to present the final relations in parametrical form, as in the preceding case. Namely, in view of (22.62), (22.65) and (22.68), we obtain

$$
\begin{aligned}
& x=x_{0}+\int\left[\left(1+\frac{3}{a^{4}}\right) p h \sin \varphi+D_{1}\right] \cos (\varphi+\delta) d \varphi, \\
& w=w_{0}+\int\left[\left(1+\frac{3}{a^{4}}\right) p h \sin \varphi+D_{1}\right] \sin (\varphi+\delta) d \varphi .
\end{aligned}
$$

Equations (22.63) and (22.70) together with the equations of Euler's elastica taking into account the stretchability of the axis (Z. Waszczyszyn, M. Życzkowski [5.195]) present the solution for the problem under consideration. Some difficulties may be encountered when "joining" the integrals for particular zones by means of continuity conditions; a certain example is given in [5.196]. Moreover, Z. Waszczyszyn [5.197, $5.198,5.199$ ] obtained some approximate solutions by using the collocation method, took into account plastic hardening and presented an experimental verification of the theory.

For the sake of comparison we present also the solution based on the approximate assumption of an unstretchable axis. Instead of the systems of equations 
(22.27) and (22.30) we shall obtain only one equation for any of the cases under consideration; nevertheless, simplification is only apparent since, substituting the expressions for $m$ and $n$, we shall arrive at equations more complicated than (22.57) and (22.66), respectively.

In the case of one-side plasticification (b) or (c) we now write instead of (22.26)

$$
h \frac{d \varphi}{d s}=\frac{16}{9} a^{2} \frac{(1-n)^{3}}{(2-2 n-m)^{2}},
$$

and instead of the first Eq. (22.53)

$$
m=\frac{2 p}{h} \int_{s}^{l} \cos \varphi d s+m_{0} \text {. }
$$

Calculating $m$ from (22.71), we again obtain (22.54) in which, however, the derivative $d \varphi / d X$ should be replaced by $d \varphi / d s$. Differentiating this equation with respect to $s$ and comparing with the differentiated (22.72), we obtain the equation

$$
\begin{aligned}
& (1-p \sin \varphi)^{3 / 2} a \varphi^{\prime \prime}-3 p \sqrt{h} \cos \varphi \varphi^{5 / 2}+ \\
& +3 a p(1-p \sin \varphi)^{1 / 2} \cos \varphi \varphi^{\prime 2}+3 \frac{p}{\sqrt{h}} \cos \varphi \varphi^{3 / 2}=0,
\end{aligned}
$$

where the primes denote differentiation with respect to the (unchangeable) length of the arc $s$. Comparing (22.73) with (22.57), we observe an important difference: the terms with $\varphi^{5 / 2}$ are not reduced here. This fact results in essential complications in integration: instead of the linear equation (22.59) one obtains a non-linear equation of the Riccati type which may be integrated only by means of Bessel functions. Details of calculations may be found in [5.211].

Similar complications arise in the case of two-side plastification (d). Writing instead of (22.30) (under the assumption $m>0$ ) the equation

$$
h \frac{d \varphi}{d s}=\frac{a^{2}}{\sqrt{3} \sqrt{1-n^{2}-m}},
$$

we again determine $m$ by (22.64), where the primes denote now differentiation with respect to $s$. Differentiating this function with respect to $s$ and comparing with the differentiated (22.72), we obtain

$$
a^{4} \varphi^{\prime \prime}-3 p^{2} h^{2} \sin \varphi \cos \varphi \varphi^{\prime 4}+3 p h \cos \varphi \varphi^{\prime 3}=0 .
$$

So, the terms with $\varphi^{\prime 4}$ are not reduced in this case and $(22.75)$ is more complicated than (22.66). The substitution $\varphi^{\prime}=u(\varphi)$ transforms this equation into an Abel equation. Though the latter equation may be integrated in a closed form, [5.211], the results are more intricate than (22.70). Only the combination with Euler's elastica is somewhat simpler here.

S. D. Leytes [4.329, 5.107], analysing finite deflections of perfectly elastic-plastic eccentrically compressed bars of a rectangular cross-section, adopted the approximate assumption of an unstretchable axis. On the other hand, L. O. Kolesnikov [5.88] and A. M. Kochetkov [5.87], who considered plastic hardening, took into 
account the stretchability of the axis in an exact or in an approximate manner. G. Prathap and T. K. Varadan [5.148] based their analysis on the Ramberg-Osgood law and neglected the stretchability.

The above considerations concerned finite deflections of beams. Under the restriction to small deflections the disregard of stretchability is as a rule, fully justified, and, moreover, the expressions for curvature are subject to linearization. Assuming $d X \approx d s \approx d x$ and substituting $\varphi \approx-d w / d x$ into the first equations of the systems (22.27) and (22.30), we obtain (under the usual sign convention for the bending moment):

for one-side plastification

$$
\frac{d^{2} w}{d x^{2}}=-\frac{16}{9} \frac{a^{2}}{h} \frac{(1-|n|)^{3}}{(2-2|n|-|m|)^{2}} \operatorname{sign} m,
$$

for two-side plastification

$$
\frac{d^{2} w}{d x^{2}}=-\frac{a^{2}}{h \sqrt{3} \sqrt{1-n^{2}-|m|}} \operatorname{sign} m .
$$

Solutions of these equations in the case of $n=$ const, $m=\alpha+\beta w$ were given by K. Ježek [4.262]; they were studied in detail by S. D. Leytes [4.330]. Further solutions were obtained by A. Z. Lokshin and V. A. Tulin [5.109]. One has to be very cautious when applying (22.76) and (22.77) to the calculation of deflections under eccentric compression; in this case active processes are often followed by passive ones, and then the equations derived here are no longer valid. Some results for this case were obtained by I. S. Voronyuk [1.20].

The rectangular cross-section discussed above belongs to the simplest sections in the problems of combined bending with tension. Sections consisting of rectangles ( $T, I$, box-section, channel-section) present the next degree of difficulty. On the other hand, solutions for curvilinear contours are very rare; purely numerical procedures were proposed by B. P. Makarov [5.117] (finite deflections of beams) and S. D. Leytes [5.106] (maximal carrying capacities of columns). Some further related papers will be quoted in Sec. 22.11, devoted to plastic hardening and applications.

Effective solutions for perfectly elastic-plastic sections consisting of rectangles were given by K. Ježek [4.262], F. B. Seely and J. O. Smith [5.163], L. K. Stevens [5.174], A. G. Dorfman and S. D. Leytes [5.41], P. I. Sikalo [5.168]. A detailed analysis of all possible ranges of work was presented by Yu. N. Aleshinsky [5.3, 5.4, 5.5]. F. B. Seely and J. O. Smith [5.163] analysed the circular cross-section; T. J. Dwyer, T. V. Galambos [5.43] and S. D. Leytes [4.330] calculated elasticplastic deflections of columns with an annular section.

\subsection{Multipoint substitutive cross-sections}

A convenient analytical approximate method of analysis of elastic-plastic bending with tension of beams with an arbitrary section consists in replacement of that section by an equivalent multipoint one (with concentrated areas), Fig. 131. In the 


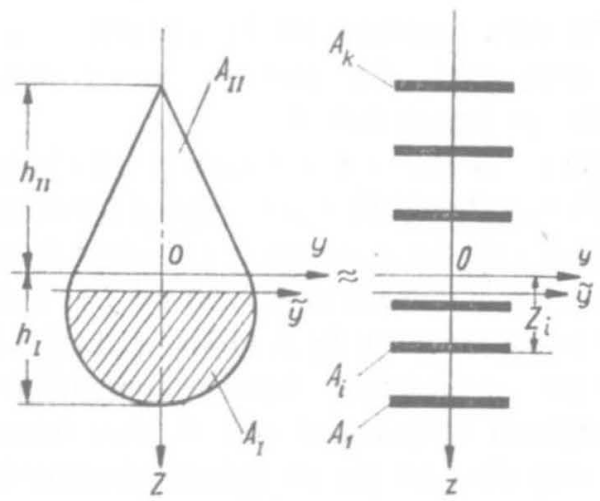

Fig. 131. A multipoint substitutive cross-section

case of simple bending with tension the term "equivalent multilayer cross-sections" is also adequate. Equivalence is understood here as the coincidence of a certain number of basic geometrical characteristics. One then obtains uniform constitutive equations, valid for all ranges of work of the cross-section (with differences only in coefficients), and the relevant integrals have a relatively simple form, The difficulties are connected with the large number of particular ranges and the necessary "joining" of solutions for the respective zones.

The principles of optimal choice of equivalent multipoint substitutive crosssections were first discussed by J. Orkisz [5.135]. He considered approximation of monosymmetric sections by multipoint sections. Assuming $k$ concentrated areas, we have $2 k$ free parameters to be determined: $k$ areas $A_{i}$ and $k$ respective coordinates $Z_{i}$. In view of the applications to elastic-plastic bending with normal force, we should first require the coincidence of elastic stiffnesses in bending and in tension (separately), of elastic carrying capacities and of limit carrying capacities. Making use of the following six conditions

$$
\sum_{i}^{k_{1,1}} A_{i} Z_{i}^{j}=\iint_{A_{1, \mathrm{II}}} Z^{j} d A, \quad j=0,1,2,
$$

where $A_{1}$ and $A_{\mathrm{II}}$ denote the areas below and above the central axis $y$ respectively, $k_{\mathrm{I}}$ and $k_{\mathrm{II}}-$ the numbers of concentrated areas $A_{i}$ below and above $y\left(A_{1}+A_{\mathrm{II}}\right.$ $=A, k_{1}+k_{11}=k$ ), we obtain the coincidence required in all cases except elastic carrying capacity in pure bending. This coincidence takes place if, moreover, the depths of the cross-sections are equal, $Z_{1}=h_{1}, Z_{k}=-h_{\mathrm{II}}$.

The conditions listed above, totalling eight, are sufficient to determine a fourpoint substitutive section. The system of eight equations is split into two systems of four equations and one obtains

$$
\begin{array}{ll}
A_{1}=\frac{A_{\mathrm{I}} I_{\mathrm{I} y}-S_{\mathrm{Iy}}^{2}}{I_{\mathrm{I} y}-2 S_{\mathrm{Iy}} h_{\mathrm{I}}+A_{\mathrm{I}} h_{\mathrm{I}}^{2}}, & Z_{1}=h_{\mathrm{I}}, \\
A_{2}=\frac{\left(S_{\mathrm{I} y}-A_{\mathrm{I}} h_{\mathrm{I}}\right)^{2}}{I_{\mathrm{I} y}-2 S_{1 y} h_{\mathrm{I}}+A_{1} h_{1}^{2}}, & Z_{2}=\frac{I_{\mathrm{I} y}-S_{\mathrm{Iy}} h_{\mathrm{I}}}{S_{\mathrm{I} y}-A_{1} h_{\mathrm{I}}},
\end{array}
$$


where $A_{\mathrm{I}}, S_{\mathrm{I} y}$ and $I_{\mathrm{I} y}$ denote the area, the static moment and the moment of inertia about $y$ of part I of the given section respectively. The areas $A_{3}$ and $A_{4}$ and the coordinates $Z_{3}$ and $Z_{4}$ are determined by analogous formulae.

The four-point equivalent cross-section is the simplest section satisfying all the basic conditions of coincidence and hence should be recommended for practical applications. A higher degree of accuracy may be achieved if we adopt a larger number of concentrated areas $k$. Then the requirement of $(22.78)$ for $j \geqslant 3$ seems to be justified; the relevant effective formulae are given by J. Orkisz [5.135], where also a proof of convergence of the procedure is presented. Another approach to the optimal choice of parameters of a multipoint substitutive sections was proposed by J. Krużelecki and W. Krzyś [5.93]; they dropped the conditions of coincidence of cross-sectional areas $A$ and of elastic carrying capacities $\overline{M_{b}}$, requiring, in return, a better agreement of the characteristics $M_{b}=f(x)$ in pure bending.

\subsection{Constitutive equations for multipoint sections}

As we mentioned above, constitutive equations for multipoint cross-sections under bending with normal force have a simple uniform structure. Consider first a relatively general case of multiple linear plastic hardening

$$
\sigma=\sigma_{\alpha}+E_{\alpha}\left(\varepsilon-\varepsilon_{\alpha}\right) \text { for } \varepsilon_{\alpha} \leqslant \varepsilon \leqslant \varepsilon_{\alpha+1} .
$$

Substituting here the strain distribution (22.36), we obtain

$$
\sigma=\sigma_{\alpha}+E_{\alpha}\left(\frac{d \varphi}{d X} Z+\varepsilon_{0}-\varepsilon_{\alpha}\right) .
$$

To the individual concentrated areas $A_{i}$ we ascribe the stresses (22.81) for $Z=Z_{i}$. Hence the normal force $N$ and the bending moment $M_{b}$ will be determined by finite sums

$$
\begin{aligned}
& N=\sum_{\alpha} \sum_{i}\left[\sigma_{\alpha}+E_{\alpha}\left(\frac{d \varphi}{d X} Z_{i}+\varepsilon_{0}-\varepsilon_{\alpha}\right)\right] A_{i}, \\
& M_{b}=\sum_{\alpha} \sum_{i}\left[\sigma_{\alpha}+E_{\alpha}\left(\frac{d \varphi}{d X} Z_{i}+\varepsilon_{0}-\varepsilon_{\alpha}\right)\right] A_{i} Z_{i} .
\end{aligned}
$$

Summation is to be performed over all segments $(\alpha)$ of the piece-wise linear stressstrain diagram which actually appear and over all concentrated areas (i) lying inside the segment under consideration. We may write briefly

$$
N=B_{0}+C_{0} \varepsilon_{0}+C_{1} \frac{d \varphi}{d X}, \quad M_{b}=B_{1}+C_{1} \varepsilon_{0}+C_{2} \frac{d \varphi}{d X},
$$

where the following notation has been introduced

$$
\begin{array}{rlr}
B_{j} & =\sum_{\alpha} \sum_{i}\left(\sigma_{\alpha}-E_{\alpha} \varepsilon_{\alpha}\right) A_{i} Z_{i}^{j}, & \\
C_{J} & =\sum_{\alpha} \sum_{i} E_{\alpha} A_{i} Z_{i}^{j}, & \text { for } j=0,1,2 .
\end{array}
$$


The constants $B_{j}$ and $C_{j}$ depend on material constants, on geometrical characteristics of the multipoint section and on the range of work of the section (the numbers of points corresponding to the particular segments of the stress-strain diagram). Hence for particular ranges we obtained linear relations between $N$ and $M_{b}$ on the one hand, $\varepsilon_{0}$ and $d \varphi / d X$ on the other hand. Under the assumption $C_{1}^{2}-C_{0} C_{2} \neq 0$ those relations may be inverted with respect to $\varepsilon_{0}$ and $d \varphi / d X$ :

$$
\begin{aligned}
\frac{d \varphi}{d X} & =b_{1} M_{b}+b_{2} N+b_{3}, \\
\varepsilon_{0} & =\frac{d s}{d X}-1=c_{1} M_{b}+c_{2} N+c_{3},
\end{aligned}
$$

where the coefficients $b_{i}$ and $c_{i}$ are equal

$$
\begin{aligned}
b_{1} & =\frac{C_{0}}{C_{0} C_{2}-C_{1}^{2}}, & b_{2}=c_{1}=-\frac{C_{1}}{C_{0} C_{2}-C_{1}^{2}}, \\
b_{3} & =\frac{B_{0} C_{1}-B_{1} C_{0}}{C_{0} C_{2}-C_{1}^{2}}, & c_{2}=\frac{C_{2}}{C_{0} C_{2}-C_{1}^{2}}, \\
c_{3} & =\frac{B_{1} C_{1}-B_{0} C_{2}}{C_{0} C_{2}-C_{1}^{2}} . &
\end{aligned}
$$

In the particular case of a perfectly elastic-plastic material Eqs. (22.85) are, of course, valid, but the coefficients $b_{i}$ and $c_{i}$ may be expressed in a more detailed form. Equations (22.4) hold in this case and the coordinate $Z_{0}$ is determined by (22.3). The normal force $N$ and the bending moment $M_{b}$ are again given by (22.13) where

$$
A_{+}=\sum_{i_{+}} A_{i}, \quad S_{+}=\sum_{i_{+}} A_{i} Z_{i}, \quad I_{+}=\sum_{i_{+}} A_{i} Z_{i}^{2},
$$

and similarly $A_{e}, S_{e}, I_{e}, A_{-}, S_{-}$and $I_{-}$. The symbols $i_{+}, i_{e}$ and $i_{-}$refer to all the concentrated areas lying inside $A_{+}, A_{e}$ and $A_{-}$, respectively. In the case of one-side plastification one simply has to assume $A_{+}=S_{+}=I_{+}=0$ or $A_{-}=S_{-}=I_{-}=0$. The continuity conditions (22.14) are not necessary in the case under consideration, since the boundary coordinates $Z_{+}$and $Z_{-}$do not appear in (23.13) either effectively or via $A, S$ and $I$ (for a multipoint section); hence (22.3) and (22.13) fully determine $d \varphi / d X, d s / d X$ and $Z_{0}$ here. The main problem consists in determining the range of work of the section: how many points lie in the zones $A_{+}, A_{e}$ and $A_{-}$. To solve this problem we have to know the limit curves in the plane $M_{b}-N$. For multipoint sections such curves consist of segments of straight lines; they were studied in detail by J. Orkisz [5.136].

Eliminating from (22.3) and (22.13) the coordinate $Z_{0}$ and solving the system thus obtained with respect to $d \varphi / d X$ and $d s / d X$, we obtain linear relations of type (22.85) where the constants $b_{i}$ and $c_{i}$ may now be written in the form 


$$
\begin{aligned}
& b_{1}=\frac{A_{e}}{E\left(A_{e} I_{e}-S_{e}^{2}\right)}, \quad b_{2}=c_{1}=-\frac{S_{e}}{E\left(A_{e} I_{e}-S_{e}^{2}\right)}, \\
& c_{2}=\frac{I_{e}}{E\left(A_{e} I_{e}-S_{e}^{2}\right)}, \quad b_{3}=\frac{\sigma_{0}\left[\left(S_{-}-S_{+}\right) A_{e}-\left(A_{-}-A_{+}\right) S_{e}\right]}{E\left(A_{e} I_{e}-S_{e}^{2}\right)}, \\
& c_{3}=\frac{\left.\sigma_{0}\left[S_{+}-S_{-}\right) S_{e}-\left(A_{+}-A_{-}\right) I_{e}\right]}{E\left(A_{e} I_{e}-S_{e}^{2}\right)} .
\end{aligned}
$$

In particular cases (22.88) are reduced to a simpler form. For example, if the section is bisymmetric and the number of plastified areas on the tensile side is equal to that on the compressive side (this always occurs for $N=0$ but may also take place for $N \neq 0$ ), then $S_{e}=0, A_{+}=A_{-}$, and $b_{2}=c_{1}=c_{3}=0$.

\subsection{Finite deflections of beams with multipoint sections}

Equations (22.85) may be applied to the calculation of deflections of beams; various degrees of approximation, described in Sec. 22.7, may be employed also in this case. Consider for example the beam shown in Fig. 130.

First we take into account the stretchability of the axis in a rigorous manner, integrating both equations (22.85). Eliminating from that system the bending moment, we express $d s / d X$ in terms of $N$

$$
\frac{d s}{d X}=\frac{c_{1}}{b_{1}} \frac{d \varphi}{d X}+\left(c_{2}-\frac{c_{1} b_{2}}{b_{1}}\right) N+1+c_{3}-\frac{c_{1} b_{3}}{b_{1}} .
$$

Substitution of the second Eq. (22.51) into (22.89) makes it possible to express $d s / d X$ in terms of the angle $\varphi$ only; differentiating (22.51) with respect to $X$, we obtain

$$
\frac{d M_{b}}{d X}=-P \frac{d s}{d X} \cos \varphi, \quad \frac{d N}{d X}=P \frac{d \varphi}{d X} \cos \varphi ;
$$

hence both these derivatives may also be expressed in terms of $\varphi$ only. Substitution of those formulae into the differentiated first Eq. (22.85) leads to the required basic differential equation of deflections of the beam

$$
\begin{aligned}
\varphi^{\prime \prime} & +P\left(c_{1}-b_{2}\right) \cos \varphi \varphi^{\prime}+P\left(b_{1}+b_{1} c_{3}-b_{3} c_{1}\right) \cos \varphi+ \\
& +P^{2}\left(b_{1} c_{2}-b_{2} c_{1}\right) \sin \varphi \cos \varphi=0,
\end{aligned}
$$

where the primes denote differentiation with respect to $X$. We note, however, that $c_{1}=b_{2}$; hence the second term in (22.91) is subject to reduction and this fact results in essential simplification of integration. Substituting (22.86), we obtain the following equation for arbitrary linear plastic hardening:

$$
\varphi^{\prime \prime}+\frac{\left(C_{0}-B_{0}\right) P}{C_{0} C_{2}-C_{1}^{2}} \cos \varphi+\frac{P^{2}}{C_{0} C_{2}-C_{1}^{2}} \sin \varphi \cos \varphi=0 .
$$

This equation is analogous to the equation of finite elastic deflections of beams with a stretchable axis. The substitution $\sin \varphi=v$ leads to the equation

$$
v^{\prime \prime}\left(1-v^{2}\right)+v v^{\prime 2}+D_{1}\left(1-v^{2}\right)^{2}+D_{2} v\left(1-v^{2}\right)^{2}=0,
$$


where $D_{1}$ and $D_{2}$ denote the coefficients appearing in (22.92). The independent variable $X$ does not appear in (22.93), and hence this equation may be integrated at least once; we obtain

$$
\frac{d v}{d X}=\sqrt{\left(1-v^{2}\right)\left(2 C-2 D_{1} v-D_{2} v^{2}\right)},
$$

where $C$ denotes an integration constant. Further integration leads to elliptic integrals; the details may be found in the paper by $Z$. Waszczyszyn [5.200].

Compare the integration presented above with the approximate assumption of an unstretchable axis. We now put $d X=d s$ and drop the second of Eqs. (22.85). Instead of (22.92) we obtain

$$
\varphi^{\prime \prime}+\frac{C_{1} P}{C_{0} C_{2}-C_{1}^{2}} \cos \varphi \varphi^{\prime}+\frac{C_{0} P}{C_{0} C_{2}-C_{1}^{2}} \cos \varphi=0,
$$

where the term with $\cos \varphi \varphi^{\prime}$ is, in general, not subject to reduction. The substitution $\varphi^{\prime}=u(\varphi)$ makes it possible to perform the first integration; the second integration, however, cannot be expressed in terms of elementary or known non-elementary functions. Only in the case of $C_{1}=0$, corresponding for example to symmetric plastification of a bisymmetric section, the solution is relatively simple and the deflection line is of the type of Euler's elastica. The details of integration may be found in the paper by J. Orkisz and M. Życzkowski [5.139].

The method of substitutive multipoint sections is even more effective in the calculation of small deflections of beams, under the assumption $d X \approx d s \approx d x$. The solutions obtained are close to those for elastic, slightly curved beams; numerous examples may be found in [5.137]. Moreover, J. Orkisz [5.138] calculated small elastic-plastic deflections of initially slightly curved beams with multipoint sections, finding no essential complications in comparison with initially straight beams.

\subsection{Plastic hardening. Structural applications}

The simplest form of constitutive equations and effective solutions in the case of elastic-plastic-hardening bending with normal force is obtained if we assume linear hardening. General considerations for an arbitrary section may be found in the monographs by V. V. Pinadzhyan [5.145, 5.146] who applied the formulae derived to the calculation of the maximal carrying capacity of columns. A more detailed analysis for a trapeziform section and for a circular one was given by S. V. Serensen, V. P. Kogayev and R. M. Shneyderovitch [5.164] (also for curved bars), and for an annular section - by B. S. Bilobran [5.18]. Rigid-linear-hardening beams are considered by I. L. Dikovitch in the monograph [5.39]; the author derives the general equations and gives tables for more important cases as well as examples of deflection calculations. Yu. I. Lepik [5.105] investigated deflections of prestressed columns. V. Iannelli $[5.75,5.76]$ discussed passive processes due to deflections of columns subject to monotonic loading; T. Ohta and T. Yamasaki [5.132] investigated the influence of residual stresses on elastic-plastic deflections. 
In the case of arbitrary plastic hardening much attention has been paid to numerical methods and the application of computers. Such methods were first proposed in the thirties by E. Chwalla [4.77, 4.78] and F. Hartmann [5.70, 5.71]. Further papers are due to R. M. Shneyderovitch [5.167], who applied to this case the "method of variable stiffness" (proposed by I. A. Birger [4.33, 4.36]), L. O. Kolesnikov [5.88], who calculated finite deflections of beams with an arbitrary crosssection, M. G. Lay [5.101] (effects of material instability between the upper and the lower yield-point stress), and J. Bäcklund [5.14] (finite strains, matrix approach). A certain numerical method appropriate for reinforced concrete sections was suggested by G. Gurfinkel and A. Robinson [5.66].

A. A. Makarevitch [5.115] discussed arbitrary plastic hardening but restricted his considerations to a rectangular section. A. F. Akhmerov [5.2] considered piecewise linear and piece-wise parabolic hardening with passive processes taken into account. F. Baron and M. S. Venkatesan [5.9], M. K. Shen and G. Thierauf [5.166] considered various loading histories with secondary yielding and the Bauschinger effect. N. Kh. Suyunshkaliyev [5.179] constructed the surfaces of constant dissipated work for a thin-walled circular section, assuming the power hardening law. Ya. Z. Skomorovsky [5.169] considered technological problems of bending with tension of tube elements with residual stresses taken into account. B. A. Boley [5.20] investigated bending with tension at elevated temperatures with thermal stresses and melting effects. A. R. Rzhanitsyn [5.156] gave a method of determining the maximal bending moment for an arbitrary stress-strain diagram $\sigma=f(\varepsilon)$, different in tension and in compression.

L. Finzi [5.45] established an analogy between elastic-plastic bending with axial force and a certain problem of dynamics of a rigid body; N. L. Moshensky [5.127] proposed an electric analogy. G. E. Belsky [5.15] considered qualitative aspects of elastic-plastic stability of bars with cross-section of an arbitrary shape under bending and compression. A. R. Rzhanitsyn [5.157] introduced the concept of a matrix of momentary stiffnesses of the cross-section, $\partial M / \partial x, \partial M / \partial \varepsilon_{0}, \partial N / \partial x$, $\partial N / \partial \varepsilon_{0}$. A similar approach was proposed independently by V.P. Kolomiets [5.89], whereas A. V. Gemmerling [5.56] developed and widely applied to bar systems a modified version of the original, Rzhanitsyn's concept.

The analysis of large elastic-plastic deflections of beams on immovable supports (or with constrained movability) is a typical application field of the case of combined loadings at the level $\mathscr{S}$ under consideration. Such an analysis was initiated by R. M. Haythornthwaite $[5.72,4.201]$; much attention was paid to that problem by Z. Waszczyszyn [5.197, 5.198, 5.199], who proposed certain approximate methods and gave an experimental verification of the theory. Further papers were published in the seventies by N. Jones [5.82, 5.83], S. S. Gill [5.60] (distributed load), A. Gürkök and H. G. Hopkins [5.67], T. I. Campbell and T. M. Charlton [5.24] (numerical and experimental approach) and P. G. Hodge [5.74] (partial end fixity). H. U. Bilek [5.17] calculated maximal carrying capacity of a beam with finite deflections. 
Elastic-plastic deflections of columns were also investigated by K. K. Mukhanov and Yu. V. Sobolev [5.129] (non-prismatic columns, experimental verification), B. V. Terentev [5.182] and F. E. Monasa [5.124] (influence of deflections on maximal carrying capacity), M. P. Kamat, G. W. Swift and D. E. Killian [5.84] (numerical procedure). Elastic-plastic deflections of frames were analysed by K. A. Amariei and B. T. Kolundžija [5.6] (matrix approach), G. Thierauf [5.183] (maximal carrying capacity) and Y. Luscouarch [5.110] (program for IBM 370).

Certain differences may be encountered in analysis of bending with normal force in a cross-section of a curved bar. If the initial curvature is considerable, then the hyperbolic strain distribution occurs, and in the case of highly curved bars the radial stresses $\sigma_{r}$ may become important. In highly curved thin-walled bars the contour line may be subject to deformation. The analysis of rectangular sections of curved bars under bending with normal force was initiated by W. Swida [5.180]; arbitrary shape of the cross-section was discussed by A. Phillips [5.143] and J. Wölfel [5.203].

The analysis of a cross-section of a curved bar is particularly often applied to calculations of elastic-plastic deflections and of stability of arches. We mention here the papers by L. K. Stevens [5.173], G. I. Rozenblat [5.155], F. A. Pivnev [5.147], E. Jõgi [5.80, 5.81], L. A. Lebedinets and N. A. Lebedinets [5.102, 5.104] Z. Waszczyszyn, C. Cichoń and M. Radwańska [5.201, 5.33, 5.150], A. M. Lokoshtchenko and S. A. Shesterikov [5.108], and J. Bäcklund [5.14].

\section{Limit carrying capacity of a section under bending with normal force}

\subsection{Plastic interaction curves represented by generalized power series}

In the case of simultaneous bending with tension the limit carrying capacity of a cross-section corresponds to full plastification of that section; some exceptions

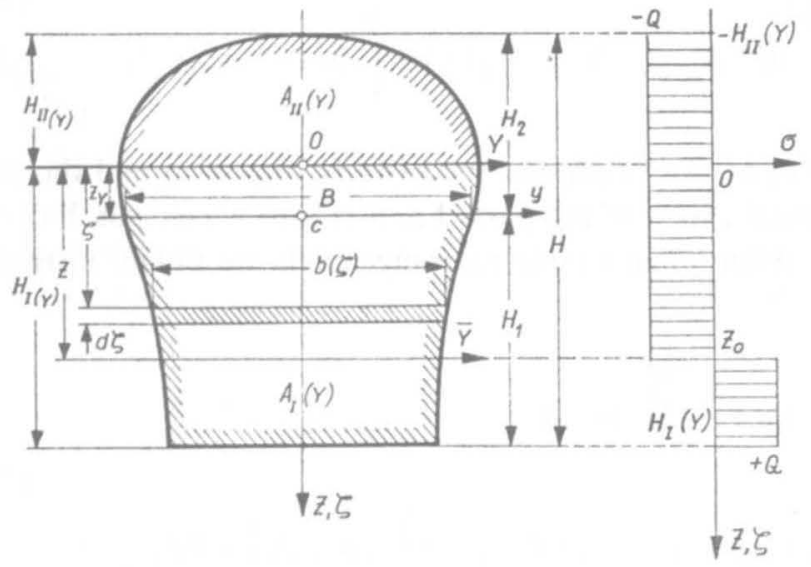

Fig. 132. Full plastification of a monosymmetric section 
may take place if we discuss thin-walled, considerably deformable profiles, but such problems will not be dealt with here. Generalized stresses $N$ and $M_{b}$ in the limit state are determined by relatively simple, parametrical general formulae (22.17), where the parameter $Z_{0}$ denotes the coordinate separating tensile and compressive zones. This parameter occurs in the formulae for $A_{+}, A_{-}, S_{+}$and $S_{-}$ usually in a rather complicated form and cannot be effectively eliminated, and then we cannot determine the limit curve $M_{b}=f(N)$ by an explicit formula. On the other hand, that parameter may be eliminated by applying generalized power series and operations on these series; we present here such an approach, taken from the present author's paper [4.636].

Consider a monosymmetric cross-section and assume a system of coordinates $Y Z$ as shown in Fig. 132. The origin of the system is located at an arbitrary point at the symmetry axis; the $Y$-axis will be called the starting axis. In what follows, we shall obtain expansions of the function $M_{b}=f(N)$ into generalized power series with the centre at the point corresponding to the coincidence of the discontinuity axis (neutral axis) $\bar{Y}$ with the starting axis $Y$. Thus, assuming the $Y$-axis to divide the cross-sectional area $A$ into two equal parts, we shall arrive at the expansion in the neighbourhood of pure bending; assuming the $Y$-axis as tangent to the boundary -in the neighbourhood of pure compression and of pure tension, etc.

Denote the variable of integration by $\zeta$, the variable width (breadth) of the cross-section by $b=b(\zeta)$, the width of the section along the centroidal axis $y$ by $B$, the coordinate $Z$ of the centroidal axis $y$ by $Z_{y}$, the coordinate $Z$ of the discontinuity axis $\bar{Y}$ by $Z_{0}$, the height of the section by $H$, where $H=H_{\mathrm{I}}(Y)+H_{\mathrm{II}}(Y)$ (Fig. 132). We now express the quantities appearing in (22.17) in such a form as to begin the integration always from $\zeta=0$. Namely, we substitute

$$
\begin{array}{ll}
A_{+}=A_{\mathrm{I}}(Y)-\int_{0}^{Z_{0}} b(\zeta) d \zeta, & S_{+}=S_{\mathrm{I}}(Y)-\int_{0}^{Z_{0}} b(\zeta)\left(\zeta-Z_{Y}\right) d \zeta, \\
A_{-}=A_{\mathrm{II}}(Y)+\int_{0}^{Z_{0}} b(\zeta) d \zeta, & S_{-}=S_{\mathrm{II}}(Y)+\int_{0}^{Z_{0}} b(\zeta)\left(\zeta-Z_{Y}\right) d \zeta,
\end{array}
$$

where $A_{1}(Y), A_{11}(Y), S_{1}(Y)$ and $S_{\mathrm{II}}(Y)$ denote, successively, the areas and the static moments about the centroidal axis $y$ of the parts I and II into which axis $Y$ divides the whole of the area $A$. After some simple rearrangements we obtain instead of (22.17)

$$
\begin{aligned}
& N=\sigma_{0}\left[A_{\mathrm{I}}(Y)-A_{\mathrm{II}}(Y)-2 \int_{0}^{Z_{\mathrm{o}}} b(\zeta) d \zeta\right], \\
& M_{\mathrm{b}}=\sigma_{0}\left[2 S_{\mathrm{I}}(Y)+A_{\mathrm{I}}(Y) Z_{Y}-A_{\mathrm{II}}(Y) Z_{Y}-2 \int_{0}^{Z_{0}} \zeta b(\zeta) d \zeta\right]-N Z_{Y}
\end{aligned}
$$


or, in dimensionless notation,

$$
\begin{aligned}
& m_{b}=\vartheta_{1}(Y)-\vartheta_{2}(Y) n-\psi_{2} \int_{0}^{z} u \beta(u) d u, \\
& n=\vartheta_{3}(Y)-\psi_{0} \int_{0}^{z} \beta(u) d u .
\end{aligned}
$$

The symbols $m_{b}$ and $n$ stand here for $M_{b} / W_{b} \sigma_{0}$ and $N / A \sigma_{0}$, respectively, hence for the physical quantities referred to the elastic carrying capacities $\bar{M}_{b}$ and $\bar{N}\left(m_{b}\right.$ is defined in a different way from that introduced in Sec. 22); $W_{b}$ denotes the elastic section modulus for bending. Dimensionless variables are defined by the formulae $z=Z_{0} / H, u=\zeta / H, \beta=b / B$, and $\vartheta_{i}(Y)$ denote the following functions depending on the position of the starting axis $Y$ :

$$
\begin{aligned}
& \vartheta_{1}(Y)=\frac{1}{W_{b}}\left[2 S_{1}(Y)+A_{1}(Y) Z_{Y}-A_{1 I}(Y) Z_{Y}\right], \\
& \vartheta_{2}(Y)=\frac{A Z_{Y}}{W_{b}}, \quad \vartheta_{3}(Y)=\frac{1}{A}\left[A_{1}(Y)-A_{\mathrm{II}}(Y)\right] ;
\end{aligned}
$$

finally, $\psi_{0}$ and $\psi_{2}$ are certain dimensionless characteristics of the shape of the crosssection:

$$
\psi_{0}=\frac{2 B H}{A}, \quad \psi_{2}=\frac{2 B H^{2}}{W_{b}} .
$$

Equations (23.3), similarly to (22.17), determine the plastic interaction curve in parametrical form. To eliminate the parameter $z$ we assume that the function $\beta=\beta(u)$ (width of the cross-section) may in a certain neighbourhood of the point 0 (starting position of the $Y$-axis) be expanded into a generalized power series

$$
\beta(u)=\sum_{j=0}^{\infty} \beta_{j} u^{\mu+v j}
$$

where $\mu>-1$ and $\nu>0$ may be arbitrary real numbers. In general, we have to assume here $u \geqslant 0$; this assumption corresponds to the shifting of the discontinuity axis in the direction of the positive $Z$-axis, and, consequently, to the decrease of the normal force. Small alternations permitting the derivation of the expansions valid in the opposite direction will be mentioned later.

Two definite integrals, appearing in (23.3), we denote by $s$ and $a$; hence

$$
\begin{aligned}
& s=\int_{0}^{z} u \beta(u) d u=\sum_{j=0}^{\infty} \frac{\beta_{j}}{\mu+2+v j} z^{\mu+2+v j}, \\
& a=\int_{0}^{z} \beta(u) d u=\sum_{j=0}^{\infty} \frac{\beta_{j}}{\mu+1+v j} z^{\mu+1+v j} .
\end{aligned}
$$


and the problem is reduced to deparametrization of the function $s=s(z), a=a(z)$, so as to obtain an explicit form $s=s(a)$, which yields at once $m_{b}=m_{b}(n)$. This operation consists of two elements: inversion of the second series (23.7) and substitution into the first one. Both these operations may be performed by using the formulae for raising a power series to an arbitrary power (20.25), (20.28) and equating the coefficients at both sides of the resulting equations; however, [4.635] [gives also ready formulae for the compound operation of deparametrization. We finally obtain

$$
s=s(a)=\sum_{p=0}^{\infty} c_{p} a^{\frac{\mu+2}{\mu+1}+\frac{\nu}{\mu+1} p},
$$

where the coefficients $c_{p}$ depend on the coefficients of expansion of the contour equation $\beta_{j}$ and on the exponents $\mu$ and $v$ as follows:

$$
\begin{aligned}
& c_{0}=\frac{(\mu+1)^{\frac{\mu+2}{\mu+1}}}{\mu+2} \beta_{0}^{-\frac{1}{\mu+1}}, \\
& c_{1}=-\frac{(\mu+1)^{\frac{\mu+\nu+2}{\mu+1}}}{(\mu+\nu+1)(\mu+v+2)} \beta_{0}^{-\frac{\mu+v+2}{\mu+1}} \beta_{1}, \\
& c_{2}=\frac{1}{2}(\mu+1)^{\frac{\mu+\nu+2}{\mu+1}} \beta_{0}^{-\frac{2 \mu+2 v+3}{\mu+1}}\left[\frac{\beta_{1}^{2}}{(\mu+\nu+1)^{2}}-\frac{2 \beta_{0} \beta_{2}}{(\mu+2 v+1)(\mu+2 v+2)}\right],
\end{aligned}
$$

Hence, by virtue of (23.3), we finally arrive at the equation of the plastic interaction curve for simultaneous bending and tension in the form of a generalized power series

$$
m_{b}=\vartheta_{1}(Y)-\vartheta_{2}(Y) n-\psi_{2} \sum_{p=0}^{\infty} c_{p}\left[\frac{\vartheta_{3}(Y)-n}{\psi_{0}}\right]^{\frac{\mu+2}{\mu+1}+\frac{\nu}{\mu+1} p} .
$$

As already indicated, the expansions valid in the direction of increasing normal forces $n$ may be slightly different. We then obtain

$$
m_{b}=\vartheta_{1}(Y)-\vartheta_{2}(Y) n-\psi_{2} \sum_{p=0}^{\infty} c_{p}\left[\frac{n-\vartheta_{3}(Y)}{\psi_{0}}\right]^{\mu+2}+\frac{\nu}{\mu+1} p
$$

where the coefficients $\beta_{j}$ (and, consequently, $c_{p}$ ) should be calculated by assuming the $Z$-axis in the opposite direction to that shown in Fig. 132 (upwards). In most cases, however, the differences between (23.10) and (23.11) are purely formal.

\subsection{Particular cases of power series}

Assuming the starting axis $Y$ to coincide with the centroidal axis $y$, we obtain the expansion of the plastic interaction curve in the neighbourhood of the maximal bending moment. Denote the areas of corresponding parts of the section by $A_{1}$ and $A_{2}$, 
and the static moment of the area $A_{1}$ about axis $Y=y$ by $S_{1}$, and introduce the following notations of dimensionless sectional characteristics

$$
\frac{2 S_{1}}{W_{b}}=\psi_{1}, \quad \frac{A_{1}-A_{2}}{A}=\delta_{1} .
$$

We have here $Z_{Y}=0, \vartheta_{1}(Y)=\psi_{1}, \vartheta_{2}(Y)=0, \vartheta_{3}(Y)=\delta_{1}$, and, moreover, $\mu=0$ since in the sections appearing in applications the width along the centroidal axis is different from zero (an exception may be found only in theoretical thin-walled sections). Thus (23.10) assumes here a simpler form

$$
m_{b}=\psi_{1}-\psi_{2} \sum_{p=0}^{\infty} c_{p}\left(\frac{\delta_{1}-n}{\psi_{0}}\right)^{2+y p},
$$

where the coefficients $c_{p}$ are determined by (23.9) with $\mu=0$. In this important and frequent case, these formulae assume a much simpler form:

$$
\begin{aligned}
& c_{0}=\frac{1}{2} \beta_{0}^{-1}, \\
& c_{1}=-\frac{1}{(v+1)(v+2)} \beta_{0}^{-v-2} \beta_{1}, \\
& c_{2}=\frac{1}{2} \beta_{0}^{-2 v-3}\left[\frac{\beta_{1}^{2}}{(v+1)^{2}}-\frac{\beta_{0} \beta_{2}}{(v+1)(2 v+1)}\right],
\end{aligned}
$$

If $\nu$ is an integer, the generalized power series (23.13) becomes here an ordinary power series. Moreover, by virtue of the definition of $\beta$ we may put here $\beta_{0}$ $=b(0) / B=1$, and this fact results in further simplification; nevertheless, we have retained $\beta_{0}$ in (23.14), since in what follows these formulae will also be used in more general cases.

The assumption that the axis $Y$ divides the area $A$ into two equal parts obviously corresponds to the expansion of the plastic interaction curve in the neighbourhood of pure bending. We have here $A_{1}(Y)=A_{\mathrm{II}}(Y)=A / 2, Z_{Y}=Z_{c}$ (change of position of the neutral axis in pure bending); denoting the corresponding static moment $S_{\mathrm{I}}(Y)$ by $S_{0.5}$ and

$$
\frac{S_{0.5}}{W_{b}}=\psi_{0.5}, \quad \frac{Z_{c} A}{W_{b}}=\delta_{2},
$$

we obtain $\vartheta_{1}(Y)=\psi_{0.5}, \vartheta_{2}(Y)=\delta_{2}, \vartheta_{3}(Y)=0, \mu=0$, and

$$
m_{b}=\psi_{0.5}-\delta_{2} n-\psi_{2} \sum_{p=0}^{\infty} c_{p}\left(-\frac{n}{\psi_{0}}\right)^{2+v p},
$$

where the coefficients $c_{p}$ are determined by (23.14). This expansion is valid for negative $n$; for positive $n$ we have, as a rule, to change the sign inside the brackets, changing simultaneously the direction of the $Z$-axis when calculating $\beta_{j}$. 
In the case of bisymmetric sections, both the preceding variants coincide. Namely, we have $Z_{c}=0, \delta_{1}=\delta_{2}=0, \psi_{0.5}=\psi_{1}$, and

$$
m_{b}=\psi_{1}-\psi_{2} \sum_{p=0}^{\infty} c_{p}\left(\frac{n}{\psi_{0}}\right)^{2+v p} .
$$

Further simplifications may be obtained if the contour at the symmetry axis has no singularity (as, for example, in a diamond section) but is regular. Then we have $v=2$ (or $I v=4,6, \ldots$, but this alteration is without meaning), and instead of (23.17) we get an ordinary power series

$$
m_{b}=\psi_{1}-\psi_{2} \sum_{p=0}^{\infty} c_{p}\left(\frac{n^{2}}{\psi_{0}^{2}}\right)^{1+p}
$$

valid, of course, in both directions. The coefficients $c_{p}$, with substituted $v=2$, $\beta_{0}=1$, are equal to

$$
\begin{aligned}
& c_{0}=\frac{1}{2}, \quad c_{1}=-\frac{1}{12} \beta_{1}, \\
& c_{2}=\frac{1}{90}\left(5 \beta_{1}^{2}-3 \beta_{2}\right), \quad c_{3}=-\frac{1}{2520}\left(140 \beta_{1}^{3}-168 \beta_{1} \beta_{2}+45 \beta_{3}\right),
\end{aligned}
$$

Assuming the $Y$-axis to pass through the upper boundary point of the contour, we arrive at the expansion of the plastic interaction curve in the neighbourhood of pure tension. Let us introduce the dimensionless sectional characteristics

$$
\frac{H_{1} A}{W_{b}}=\eta_{1}, \quad \frac{H_{2} A}{W_{b}}=\eta_{2},
$$

(where $H_{1}$ and $H_{2}$ are as in Fig. 132), then $Z_{Y}=H_{2}, A_{1}(Y)=A, A_{\mathrm{II}}(Y)=0$, $S_{1}(Y)=0, \vartheta_{1}(Y)=\vartheta_{2}(Y)=\eta_{2}, \vartheta_{3}(Y)=1$, and hence

$$
m_{b}=\eta_{2}(1-n)-\psi_{2} \sum_{p=0}^{\infty} c_{p}\left(\frac{1-n}{\psi_{0}}\right)^{\frac{\mu+2}{\mu+1}+\frac{\nu}{\mu+1} p} .
$$

This series is valid, of course, in the direction of decreasing $n$. The parameter $\mu$ (exponent) is often different from zero here. Typical cases are $\mu=1$ (diamond, apex of a triangle) and $\mu=1 / 2$ (circle, ellipse, parabola). In these two cases, the generalized power series (23.21) cannot be reduced to an ordinary power series.

If the $Y$-axis passes through the lower boundary point of the contour, we obtain the expansion in the neighbourhood of pure compression, close to (23.21)

$$
m_{b}=\eta_{1}(1+n)-\psi_{2} \sum_{p=0}^{\infty} c_{p}\left(\frac{1+n}{\psi_{0}}\right)^{\frac{\mu+2}{\mu+1}+\frac{\nu}{\mu+1} p}
$$

valid in the direction of increasing normal forces $n$. 
The expansions of the plastic interaction curve $m_{b}=f(n)$ (23.13), (23.16), (23.21) and (23.22) are, as a rule, quite sufficient to determine this curve if the contour is regular or if the singularities occur only at the centroidal axis $y$. However, in the case of other singularities (e.g. jump-like change of width of the crosssection in the case of an I-Section), additional location of $Y$-axis in the places of such singularities may be necessary; one has to apply then the general formulae (23.10) and (23.11). In [4.636] it is shown that the singularities of the contour equation are reflected in the equation of the limit curve via the singularities in derivatives of order higher by two: thus a jump in the width causes the discontinuity of $d^{2} m_{b} / d n^{2}$, a jump in the derivative $d b / d Z$ causes the discontinuity of $d^{3} m_{b} / d n^{3}$, etc.

\subsection{Power series for typical shapes of the cross-section}

We now give several examples of power series expansions of the plastic interaction curve $m_{b}=m_{b}(n)$.

In the case of a rectangle we substitute $\beta_{0}=1, \beta_{j}=0$ for $j \geqslant 1$ (at any position of the starting axis $Y$ ), further $\psi_{0}=2, \psi_{1}=3 / 2, \psi_{2}=12, \eta_{1}=\eta_{2}=3$, $\mu=0, c_{0}=1 / 2, c_{p}=0$ for $p \geqslant 1$, and arrive at the Girkmann formula in a closed form, [5.61]

$$
m_{b}=\frac{3}{2}-\frac{3}{2} n^{2}
$$

The difference with respect to (22.35) results from a different definition of $m_{b}$ here, namely, in the present section $m_{b}=M_{b} / \bar{M}_{b}$.

Circular and elliptical sections may be treated jointly since in dimensionless coordinates $\beta=\beta(\mu)$ their equations are identical. We have here

$$
\varphi_{0}=\frac{8}{\pi}, \quad \psi_{1}=\frac{16}{3 \pi}, \quad \psi_{2}=\frac{64}{\pi}, \quad \eta_{1}=\eta_{2}=4 .
$$

Assuming the $Y$-axis to be the symmetry axis, we write the equation of the contour in the form

$$
\beta=\sqrt{1-4 u^{2}}=1-2 u^{2}-2 u^{4}-4 u^{6}-\ldots,
$$

because $H=2 R$ and $\zeta=R$ corresponds to $u=1 / 2$. The parameters $\mu$ and $v$ for the series (23.24) are $\mu=0, v=2$; hence substitution of $\beta_{j}$ taken from (23.24) into (23.18) and (23.19) gives

$$
\begin{aligned}
& m_{b}=\frac{16}{3 \pi}-\frac{\pi}{2} n^{2}-\frac{\pi^{3}}{384} n^{4}-\frac{13 \pi^{5}}{184320} n^{6}-\ldots \\
& m_{b}=1.69765-1.57080 n^{2}-0.08075 n^{4}-0.02158 n^{6}-\ldots
\end{aligned}
$$

Assuming now the $Y$-axis to be the upper boundary axis, we write the equation of the contour in the form (which may be obtained from (23.24) by substituting $u-1 / 2$ for $u)$

$$
\beta=2 \sqrt{u-u^{2}}=2 u^{1 / 2}-u^{3 / 2}-\frac{1}{4} u^{5 / 2}-\frac{1}{8} u^{7 / 2}-\ldots
$$


Here $\mu=1 / 2, v=1$; making use of (23.9) and (23.21) we finally obtain

$$
\begin{aligned}
m_{b}= & 4(1-n)-\frac{3^{5 / 3} \pi^{2 / 3}}{5 \cdot 2^{1 / 3}}(1-n)^{5 / 3}-\frac{3^{7 / 3} \pi^{4 / 3}}{35 \cdot 2^{11 / 3}}(1-n)^{7 / 3}- \\
& -\frac{33 \pi^{2}}{11200}(1-n)^{3}-\ldots, \\
m_{b}= & 4(1-n)-2.12482(1-n)^{5 / 3}-0.13437(1-n)^{7 / 3}-0.02908(1-n)^{3}-\ldots
\end{aligned}
$$

This series converges better than (23.25). Indeed, $n= \pm 1$ is a singular point of the curve (the type of singularity is clearly presented by (23.27)), and the series (23.25) has the radius of convergence $R=1$ whereas the radius of convergence of (23.27) may be equal to $R=2$. The third important position of the $Y$-axis, passing through the lower boundary point, gives once more (23.27) with $(1+n)$ instead of $(1-n)$.

An annular section (thick-walled tube) is more complicated. Denote the inner radius by $r$, the outer radius by $R$, and $r / R=\varphi$. The dimensionless parameters $\varphi$ and $\eta$ are

$$
\begin{array}{ll}
\psi_{0}=\frac{8}{\pi(1+\varphi)}, & \psi_{1}=\frac{16\left(1+\varphi+\varphi^{2}\right)}{3 \pi(1+\varphi)\left(1+\varphi^{2}\right)}, \\
\psi_{2}=\frac{64}{\pi(1+\varphi)\left(1+\varphi^{2}\right)}, & \eta_{1}=\eta_{2}=\frac{4}{1+\varphi^{2}} .
\end{array}
$$

The width of the cross-section is determined here by two formulae; assuming the $Y$-axis to coincide with the symmetry axis, we have

$$
\begin{array}{ll}
b=2\left(\sqrt{R^{2}-\zeta^{2}}-\sqrt{r^{2}-\zeta^{2}}\right) & \text { for } \zeta \leqslant r, \\
b=2 \sqrt{R^{2}-\zeta^{2}} & \text { for } \zeta \geqslant r .
\end{array}
$$

Expanding in the neighbourhood of pure bending, we have to use (23.29). Since $B=2(R-r)$, the dimensionless width $\beta$ is given by

$$
\begin{aligned}
\beta= & \frac{\sqrt{1-4 u^{2}}-\sqrt{\varphi^{2}-4 u^{2}}}{1-\varphi}=1+\frac{2}{\varphi} u^{2}+ \\
& +\frac{2\left(1+\varphi+\varphi^{2}\right)}{\varphi^{3}} u^{4}+\frac{4\left(1+\varphi+\varphi^{2}+\varphi^{3}+\varphi^{4}\right)}{\varphi^{5}} u^{6}+\ldots
\end{aligned}
$$

Substituting the values $\beta_{j}$ into (23.18) and (23.19), we obtain

$$
\begin{aligned}
m_{b}= & \frac{16\left(1+\varphi+\varphi^{2}\right)}{3 \pi(1+\varphi)\left(1+\varphi^{2}\right)}-\frac{\pi(1+\varphi)}{2\left(1+\varphi^{2}\right)} n^{2}+\frac{\pi^{3}(1+\varphi)^{3}}{384 \varphi\left(1+\varphi^{2}\right)} n^{4}+ \\
& +\frac{\pi^{5}\left(3-7 \varphi+3 \varphi^{2}\right)(1+\varphi)^{5}}{184320 \varphi^{3}\left(1+\varphi^{2}\right)} n^{6}+\ldots
\end{aligned}
$$

Putting $\varphi=0$, we obtain a divergent series and not the series $(23.25)$; indeed, the interval of validity of (23.29) tends here to zero, and the series (23.32) is convergent at only one point, $n=0$. In general, the series (23.32) determines the plastic inter- 
action curve inside the interval $|n| \leqslant n^{*}$ where $n^{*}$ corresponds to the discontinuity axis tangent to the inner circle and is equal to

$$
n^{*}=1-\frac{2 \arccos \varphi-2 \varphi \sqrt{1-\varphi^{2}}}{\pi\left(1-\varphi^{2}\right)} .
$$

The corresponding bending moment $m_{b}^{*}$ equals

$$
m_{b}^{*}=\frac{16 \sqrt{1-\varphi^{2}}}{3 \pi\left(1+\varphi^{2}\right)} .
$$

To obtain the expansion in the neighbourhood of pure tension, we use (23.30) with $R-\zeta$ substituted for $\zeta$. Thus

$$
\beta=\frac{2 \sqrt{u-u^{2}}}{1-\varphi}=\frac{2}{1-\varphi} u^{1 / 2}-\frac{1}{1-\varphi} u^{3 / 2}-\frac{1}{4(1-\varphi)} u^{5 / 2}-\ldots
$$

and (23.9) with (23.28) give

$$
\begin{aligned}
m_{b}= & \frac{4}{1+\varphi^{2}}(1-n)-\frac{3^{5 / 3} \pi^{2 / 3}\left(1-\varphi^{2}\right)^{2 / 3}}{5 \cdot 2^{1 / 3}\left(1+\varphi^{2}\right)}(1-n)^{5 / 3}- \\
& -\frac{3^{7 / 3} \pi^{4 / 3}\left(1-\varphi^{2}\right)^{4 / 3}}{35 \cdot 2^{11 / 3}\left(1+\varphi^{2}\right)}(1-n)^{7 / 3}-\frac{33 \pi^{2}\left(1-\varphi^{2}\right)^{2}}{11200\left(1+\varphi^{2}\right)}(1-n)^{3}+\ldots
\end{aligned}
$$

Assuming $\varphi \rightarrow 1$ in the expansion (23.32), we at once obtain the solution for a thin-walled tube

$$
m_{b}=\frac{4}{\pi}-\frac{\pi}{2} n^{2}+\frac{\pi^{3}}{96} n^{4}-\frac{\pi^{5}}{11520} n^{6}+\ldots
$$

The sum of this series is equal to the exact solution in a closed form

$$
m_{b}=\frac{4}{\pi} \cos \frac{\pi}{2} n
$$

given by A. A. Gvozdev [4.196] in somewhat different notation. The series (23.36) cannot be used for a similar limiting procedure since its range of validity would then decrease to zero.

In some cases the generalized power series lead to exact formulae in closed form. Thus, for a diamond section we obtain

$$
m_{b}=6(1-n)-4(1-n)^{3 / 2} ;
$$

and for an isosceles triangular section

$$
m_{b}=8(1+n)-4 \sqrt{2}(1+n)^{3 / 2} .
$$

Other examples of closed-form equations, expansions into generalized power series and numerical tables may be found in [4.636]. A table of closed-form equations for thin-walled profiles is given by I. L. Dikovitch [5.39]. I-sections were studied in 
$332 \mathrm{~V}$ LOADINGS AT LEVEL $\mathscr{S}$ OF A CROSS-SECTION OF A BAR; TYPE $\mathscr{P}_{1} / \mathscr{S}_{n}$

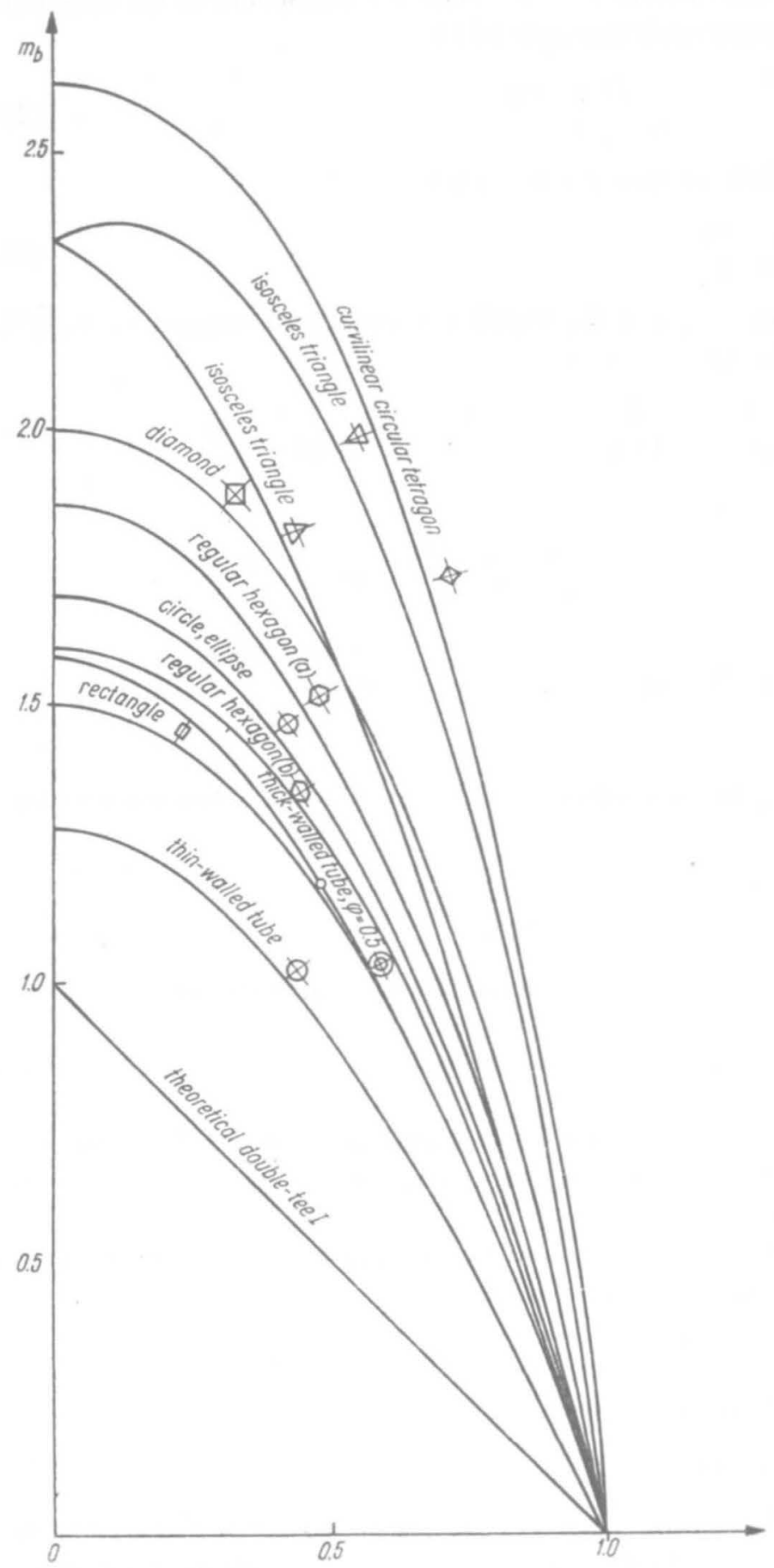

Fig. 133. Plastic interaction curves $m=f(n)$ for some shapes of the cross-section 
detail by L. S. Beedle [4.25] and M. Como [5.36]; annular sections by J. C. Wilhoit and J. E. Merwin [5.202] (ovalization of the cross-section) and by D. Goldner [5.62].

Plastic interaction curves $m_{b}=f(n)$ for various typical shapes of cross-section are shown in Fig. 133, [4.636].

\subsection{Application of the onefold optimal approximation}

For certain applications it is especially convenient to have the plastic interaction curve given neither by series nor by a numerical table but by a simple approximative formula; polynomial approximation is here preferred. Coefficients of such polynomials may be chosen in various ways; particularly convenient in this case is the onefold optimal approximation (Sec. 20.8), making use of conditions of coincidence at the boundaries of the interval and minimizing simultaneously the error inside the interval.

For bisymmetric sections, where it is sufficient to consider the interval $0 \leqslant n \leqslant 1$ only, the following approximate equation has been proposed in [4.636]:

$$
m_{b}=a+b n^{2}+c n^{4} \text {. }
$$

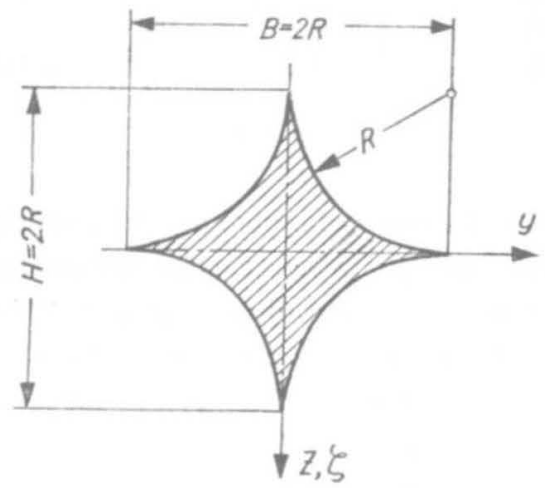

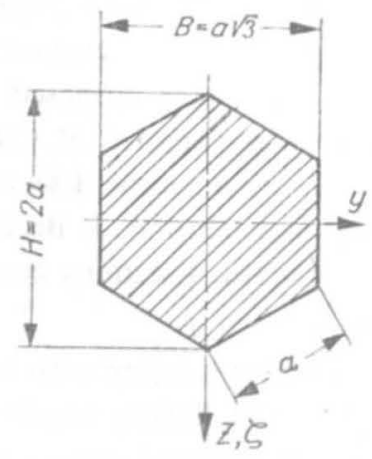

a)

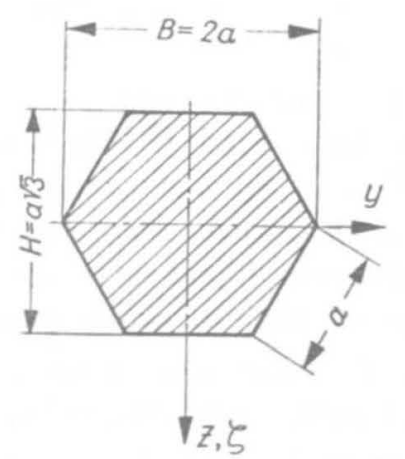

b)

Fig. 134. Some shapes of the cross-section included in Table 6 
The coefficients $a, b$, and $c$ are determined from the conditions of agreement of functions at both ends and from the condition of minimization of absolute deviation; moreover, the agreement of the derivatives $d m_{b} / d n$ at the left-hand end $n=0$ is satisfied automatically. Hence, according to the notation used in [4.633], this onefold optimal approximation is of the type $2+1+1$. The coefficients $a, b, c$, and the maximal absolute deviation of $m_{b}$, denoted by $\Delta$, are given in Table 6 ; the three relevant shapes of the section are shown in Fig. 134.

Table 6

Plastic interaction curves determined by means of onefold optimum approximation $m=a+b n^{2}+c n^{4}$

\begin{tabular}{l|c|r|r|r}
\hline Shape of the cross-section & $a$ & $b$ & \multicolumn{1}{c}{$c$} & $|\Delta| \leqslant$ \\
\hline Circle, ellipse & 1.6977 & -1.5294 & -0.1683 & 0.0053 \\
Thick-walled tube, $\varphi=0.5$ & 1.5845 & -1.7563 & 0.1718 & 0.0150 \\
Thin-walled tube & 1.2732 & -1.5584 & 0.2852 & 0.0012 \\
Curvilinear circular tetragon & 2.6263 & -1.3375 & -1.2888 & 0.0371 \\
Diamond & 2.0000 & -1.4710 & -0.5290 & 0.0139 \\
Regular hexagon (a) & 1.8667 & -1.7060 & -0.1607 & 0.0137 \\
Regular hexagon (b) & 1.6000 & -1.4004 & -0.1996 & 0.0012 \\
\hline
\end{tabular}

The approximation errors, given in the last column, may be considered very small, though the classical error estimations are in most cases useless here, since certain derivatives of the approximated functions are unbounded within the interval $0<n<1$, as may be seen from (23.21) for $\mu \neq 0$. Similar approximations for monosymmetric sections are also possible, but then the whole interval $-1 \leqslant n \leqslant 1$ should be considered.

Other types of approximation, particularly for I-sections, were proposed by T. R. Higgins [5.73], B. G. Neal [4.413] Ch. Massonnet and M. Save [5.119], B. L. Srivastava and S. C. Goyal [5.172]. Certain graphical methods of evaluation of the limit carrying capacity in the case under consideration were proposed by L. L. Valter [5.188] and C. V. Rabinovitch [5.149]. P. I. Valukonis and A. P. Čižas [5.189] considered the problem of optimal design of an unsymmetric I-section with respect to the limit carrying capacity under bending with tension. J. Bródka and M. Kwaśniewski [5.23] determined limit curves for symmetric I-sections corresponding to a constant value of maximal plastic strain $\hat{\varepsilon}$.

Plastic interaction curves for I-section of curved bars under bending with tension were investigated by D. Lamblin and G. Guerlement [5.100]. Strictly speaking, such problems may no longer be classified as $\mathscr{P}_{1} / \mathscr{S}_{n}$ since the second stress component (radial stress) becomes important here and a certain failure hypothesis must be introduced. 


\subsection{Non-homogeneity of the material. Structural applications}

The limit carrying capacity of non-homogeneous sections was investigated mainly in connection with reinforced concrete structures. Much attention to such problems was paid by A. A. Gvozdev [4.196]; we mention also the papers by B. Barbarito [5.7], G. Giannelli [5.59], A. Raithel and B. Barbarito [5.151], B. Barbarito and S. Aiello [5.8], A. A. Makarevitch [5.116]. A survey of Italian papers on this topic was given by E. Giangreco [5.58].

Application of the concept of limit carrying capacity of a cross-section under bending with tension to the limit analysis of frames was initiated by E. T. Onat and W. Prager in [5.133], and to the limit analysis of arches-by the same authors in [5.134]. We mention also some further papers on the limit analysis of frames, published by H. Tanaka [5.181], T. R. Higgins [5.73], L. S. Beedle [4.25], M. Z. Cohn and T. Rafay [5.35]. The problem of optimal design of frames with respect to their limit carrying capacity was discussed by A. R. Toakley [5.184], V. N. Kissyuk [5.85], S. C. Batterman and L. P. Felton [5.12], P. I. Valukonis and A. P. Čižas [5.190], Y. Yokoo, T. Nakamura and M. Keii [5.205], I. Konishi, N. Shiraishi, T. Taniguchi and H. Furuta [5.90]. Further papers on limit analysis of arches are due to A. Sawczuk and M. Janas [5.161, 4.257], M. Mrowiec [5.128] (non-homogeneous arch modelling cross-section of a pipe-line), V. G. Sebeshev [5.162], D. A. Da Deppo and R. Schmidt [5.37] (with finite deflections taken into account). Experimental verification of the limit analysis of arches was performed by T. Kozłowski [5.91]. Optimal design of arches with respect to their limit carrying capacity was discussed by R. V. Vebra and A. A. Cyras [5.191], L. N. Lebedinets and N. A. Lebedinets [5.103], S. C. Batterman and L. P. Felton [5.12].

We mention also some related problems. H. Dietmann [5.38] and J. Mielniczuk [5.123] considered the limit carrying capacity of bars with notches and cutouts. The influence of normal force on the technological process of plastic bending was considered by A. F. Akhmerov [5.1], J. M. Roberts and T. Sheppard [5.154] and A. D. Tomlenov [5.185]. T. Nonaka [5.131] investigated the influence of normal force on plastic bending due to impact loadings.

\section{Biaxial (oblique) bending}

\subsection{Elastic-plastic biaxial bending with normal force}

It is justified to regard biaxial bending in the theory of plasticity as a combined loading at the level $\mathscr{S}$; indeed, we observe here limit curves and constitutive equations typical for a combined case. Even in the case of a circular or annular cross-section where bending in any plane is in fact a simple bending, one can find some advantage in regarding this problem as apparently biaxial bending; such an ap- 
proach was used by Yu. N. Rabotnov [3.661], who proposed a certain model of plastic hardening illustrating the formation of plastic corners.

To begin with, consider biaxial bending with tension of perfectly elastic-plastic bars with an arbitrary cross-section, Fig. 135. Introducing notation similar to that
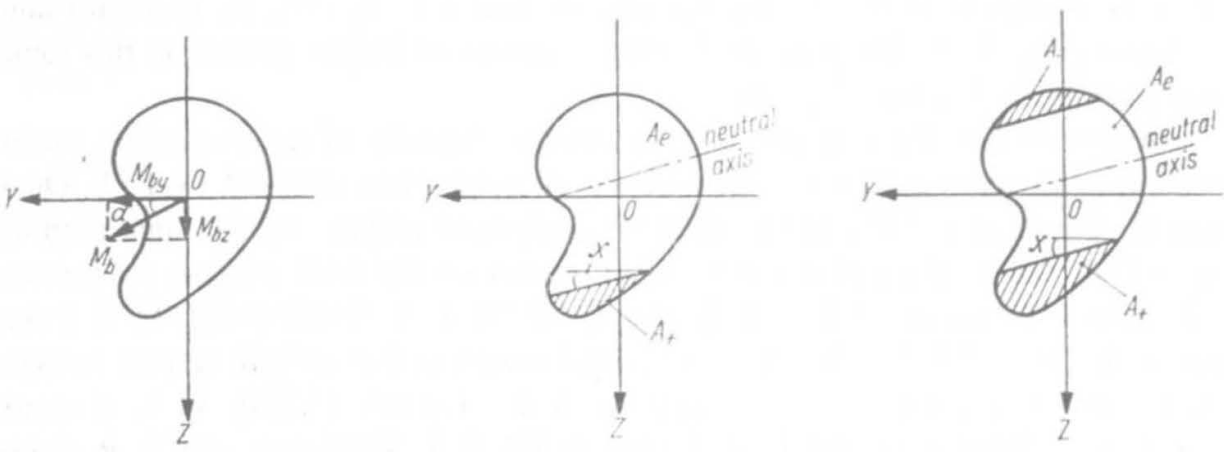

Fig. 135. Plastification under biaxial bending with normal force

of Sec. 22.2 and assuming the hypothesis of plane cross-sections, we may describe the stress distribution by means of the formulae

$$
\sigma= \begin{cases}-\sigma_{0} & \text { on the area } A_{-}, \\ B_{1} Y+B_{2} Z+C & \text { on the area } A_{e}, \\ +\sigma_{0} & \text { on the area } A_{+} .\end{cases}
$$

Integrating over the cross-sectional area $A$, we obtain

$$
\begin{aligned}
& N=\iint_{A} \sigma d A=-\sigma_{0} A_{-}+B_{1} S_{z e}+B_{2} S_{y e}+C A_{e}+\sigma_{0} A_{+}, \\
& M_{b y}=M_{b} \cos \alpha=\iint_{A} \sigma Z d A=-\sigma_{0} S_{y-}+B_{1} D_{y z e}+B_{2} I_{y e}+C S_{y e}+\sigma_{0} S_{y+}, \\
& M_{b z}=M_{b} \sin \alpha=\iint_{A} \sigma Y d A=-\sigma_{0} S_{z-}+B_{1} I_{z e}+B_{2} D_{y z e}+C S_{z e}+\sigma_{0} S_{z+},
\end{aligned}
$$

where $S_{y}, S_{z}, I_{y}, I_{z}$ and $D_{y z}$ with additional subscripts denote static moments, moments of inertia and moments of deviation of the respective zones of the crosssection. Moreover, the conditions of continuity of stresses determine the straight lines separating the subdomains $A_{+}$and $A_{e}$ :

$$
B_{1} Y+B_{2} Z+C=\sigma_{0}
$$

and the subdomains $A_{e}$ and $A_{-}$:

$$
B_{1} Y+B_{2} Z+C=-\sigma_{0} .
$$

In the case of two-side plastification Eqs. (24.2) may be regarded as a system 
of three equations with three unknowns $B_{1}, B_{2}$ and $C$. These equations, however, are non-linear with respect to $B_{1}, B_{2}$, and $C$, since, by virtue of (24.3) and (24.4), these unknowns occur also in the integration limits, namely in the quantities $A, S, I$ and $D$. Hence, even in particular cases an analytical solution may be difficult and numerical procedures should be applied.

Having determined $B_{1}, B_{2}$ and $C$, we may evaluate the inclination angle $\chi$ (equal for the neutral axis and for both elastic-plastic interfaces, Fig. 135),

$$
\chi=\arctan \frac{B_{1}}{B_{2}} ;
$$

this angle is, in general, not only different from the inclination angle $\alpha$ of the moment $M_{b}$, but also changing with the increasing moment. It should be noted that such a change of the angle $\chi$ takes place also in straight (uniaxial) elastic-plastic bending of non-symmetric sections. The neutral axis is, of course, determined by the equation

$$
B_{1} Y+B_{2} Z+C=0 .
$$

The coefficients $B_{1}$ and $B_{2}$ are sufficient to determine the constitutive equations, since (under the assumption of an unstretchable axis) the curvature in the plane $X Y$ is $x_{z}=B_{1} / E$, and in the plane $X Z$ it is $\varkappa_{y}=B_{2} / E$.

In the case of one-side plastification, e.g. at the tensile side, we have to substitute $A_{-}=S_{y_{-}}=S_{z_{-}}=0$ in (24.2) and omit the continuity condition (24.4). Nevertheless, the system of three equations (24.2) with three unknowns, $B_{1}, B_{2}$ and $C$, remains.

Biaxial bending in elastic-plastic range was first considered by I. N. Kudryavtsev [5.94], who also took into account the possible difference between yield-point stress in tension $\sigma_{0+}$ and in compression $\sigma_{0-}$. Further investigations are due to A. Ormerod [5.140]. Particular cases of non-symmetrical sections were considered by A. I. Strelbitskaya (or O. I. Strelbitska in Ukrainian)-paper [5.175] gives a theoretical analysis, and paper [5.177] written jointly with Yu. L. Biryukovitch and E. O. Pryadko-the results of experimental investigations of channel sections. S. D. Kudryavtsev [5.95, 5.96] gave a detailed analysis of the ranges of work of unsymmetric I-sections under biaxial bending and proposed an approximate method of calculation of elastic-plastic deflections.

Biaxial bending in the case of the power hardening law $(8.6)$ was considered by A. Jakubowicz [5.79]; the simplicity of analysis lies here in the fact that for a constant angle of inclination of the bending moment $a$ the angle $\chi$ of the neutral axis is also constant, independently of the value of moment $M_{b}$.

The theory of elastic-plastic biaxial bending with normal force was developed mainly for the sake of the analysis of eccentric compression of columns with biaxial eccentricity. V. V. Pinadzhyan [5.144] considered this problem for an I-section, J. Dutheil [5.42] and F. Campus [5.25] for a broader class of shapes of the crosssection. D. H. Garbaccio [5.54] took into account linear plastic hardening and gave 
an analysis for rectangular, I and elliptic sections. Further papers are due to C. Birnstiel and J. Michalos [5.19] (maximal carrying capacity for I-section columns), G. A. Harstead, C. Birnstiel and K. C. Leu [5.69] (second-order torsional effects), D. Pantchovski [5.142] (a grapho-analytical method of calculation of deflections), A. R. Gent and H. R. Milner [5.57] (elastic clamping, experimental verification), J. F. McDonough [5.122] (iterative method for various shapes of the cross-section), P. J. Marshall and J. S. Ellis [5.118] (maximal carrying capacity for box-section columns, experimental verification), H. Djalaly [5.40] (plastic hardening, effect of residual stresses), W. F. Chen and T. Atsuta [5.28] (maximal carrying capacity for I-section columns), W. F. Chen [5.29] (kinematic hardening, repeated loading), S. Vinnakota and Y. Aoshima [5.192] (maximal carrying capacity) and R. A. Skripnikova [5.170] (arbitrary plastic hardening, I-section columns). Many particular solutions are quoted in the monograph by W. F. Chen and T. Atsuta [5.30].

\subsection{Elastic-plastic biaxial bending of bisymmetric sections}

If we restrict our considerations to bisymmetric sections under biaxial bending without normal force, then the equations derived in Sec. 24.1 are considerably simplified. The neutral axis always passes through the centre of gravity of the cross-section, one-side plastification is excluded and the areas $A_{+}$and $A_{-}$are equal to each other. Figure 136 shows an example of distribution of zones for a rectangular section.

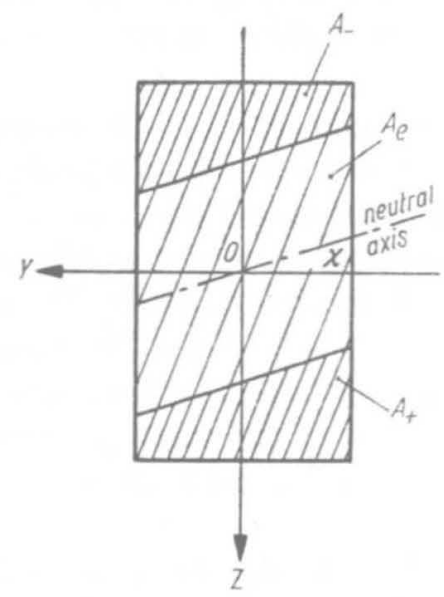

Fig. 136. Elastic-plastic biaxial bending of a rectangular section

Because of symmetry it is sufficient to consider half of the section, e.g. that determined by the condition $\sigma>0$. The first Eq. (24.1) is satisfied identically, and the remaining two equations yield

$$
\begin{aligned}
& M_{b y}=2\left(B_{1} D_{y z e}^{\prime}+B_{2} I_{y e}^{\prime}+\sigma_{0} S_{y+}\right), \\
& M_{b z}=2\left(B_{1} I_{y e}^{\prime}+B_{2} D_{y z e}^{\prime}+\sigma_{0} S_{z+}\right),
\end{aligned}
$$


where primes denote the quantities related to half of the elastic zone. These equations together with an equation of continuity

$$
B_{1} Y+B_{2} Z=\sigma_{0}
$$

determine $B_{1}$ and $B_{2}$, and subsequently the angle $\chi,(24.5)$.

Besides the above-mentioned general paper by I. N. Kudryavtsev [5.94], we quote here the paper by A. I. Strelbitskaya [5.175] concerning a symmetric I-section; E. R. Chorna and M. L. Chernov [5.31] determined theoretically and experimentally the deflections and the distribution of plastic zones on I-beams; S. Santathadaporn and W. F. Chen [5.159] proposed a numerical method for I-beams under biaxial bending.

A detailed analysis of elastic-plastic biaxial bending of a rectangular beam was carried out by D.S. Brooks [5.21]. In spite of a relative simplicity of Eqs. (24.7) in this case, a computer was used for effective calculations. The relation $\chi=\chi\left(M_{b}\right)$ was investigated for a constant angle $\alpha$; the author states that this angle may change by $7^{\circ}$ for a square section, and by $13^{\circ}$ for a rectangular section with the side ratio 1:4. Paper [5.21] gives also an analysis of deflections.

Biaxial bending of a rectangular beam with plastic hardening taken into account was considered by A. J. Barrett [5.10], O. V. Luzhin [5.111], N. I. Bezukhov and O. V. Luzhin [5.16].

N. L. Chernov and S. D. Kudryavtsev [5.32, 5.97] investigated theoretically and experimentally elastic-plastic deflections under moving loads, whereas B. I. Lyubarov [5.113] formulated the problem of plastic shake-down of a section under biaxial bending.

\subsection{Limit carrying capacity under biaxial bending with normal force}

The limit carrying capacity of the section is exhausted if the elastic zone $A_{e}$ vanishes. Equations (24.2) then assume the form

$$
\begin{aligned}
& N=\sigma_{0}\left(A_{+}-A_{-}\right), \\
& M_{b y}=2 \sigma_{0} S_{y+}, \\
& M_{z y}=2 \sigma_{0} S_{z+},
\end{aligned}
$$

where the conditions of the type $S_{+}+S_{-}=0$ are already introduced. These equations determine two parameters, e.g. $B_{1} / C$ and $B_{2} / C$, in the equation of the neutral axis (discontinuity axis) (24.6), and the plastic interaction surface $f\left(N, M_{b y}, M_{b z}\right)=0$.

The form of Eqs. (24.9) is very simple, but effective calculations may be rather complicated. This problem was investigated in detail by A. A. Gvozdev [4.196], who derived the final equations for an elliptic section, a theoretical I-section with zero-section of the web, and for a three-point section. For an elliptic section the relevant equation of the plastic interaction surface may be written in parametrical form 


$$
\begin{aligned}
& \left(\frac{M_{b y}}{a}\right)^{2}+\left(\frac{M_{b z}}{b}\right)^{2}=\frac{16}{9} a^{2} b^{2}\left(1-\beta^{2}\right)^{3} \sigma_{0}^{2}, \\
& N=a b \sigma_{0}\left[\pi-2\left(\arccos \beta-\beta \sqrt{1-\beta^{2}}\right)\right],
\end{aligned}
$$

where $\beta$ denotes a parameter, and $a$ and $b$ the semiaxes of the ellipse.

The limit carrying capacity of a rectangular section under biaxial bending with normal force was investigated by A. J. Barrett [5.10] and G. Ritter [5.153], whereas some other sections were analysed by Z. Bažant [5.13]. G. A. Morris and S. J. Fenves [5.125] proposed linearized plastic interaction surfaces for rectangular, circular, I and box-sections; their approximations furnish lower bounds to the actual limit carrying capacity of the section. More exact analysis for those sections was given by W. F. Chen and T. Atsuta [5.27]. Experimental verification of the theory for rectangular and box-sections was carried out by $\mathrm{H}$. Shakir-Khalil and G. S. Tadros [5.165]. The problem of biaxial bending with tension from the point of view of the technological theory of plasticity was discussed by M. I. Razumikhin and Yu. B. Drobot [5.152].

\subsection{Limit carrying capacity of a rectangular section under biaxial bending}

In the case of bisymmetric sections subject to biaxial bending without normal force, the neutral axis always passes through the centre of gravity and we have $C=0$ in the equation of the neutral (discontinuity) axis (24.6). The first equation (24.9) is satisfied automatically, whereas the remaining two equations determine the parameter $B_{1} / B_{2}$ in (24.6) and the plastic interaction curve.

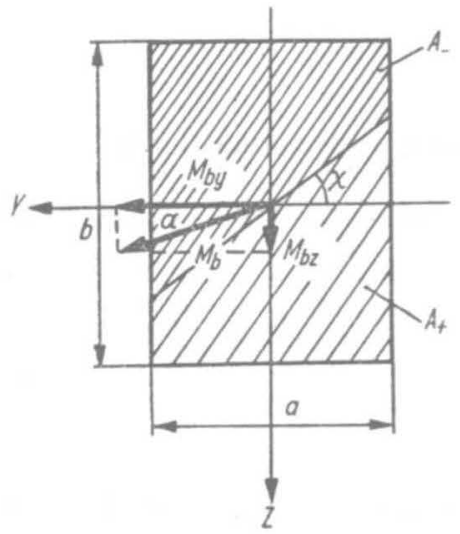

Fig. 137. Full plastification of a rectangular section under biaxial bending

Consider for example a rectangular cross-section (Fig. 137). Substituting into (24.9) the static moments $S_{y_{+}}$and $S_{z+}$ in terms of the angle $\chi$, we obtain in this case (under the assumption of $\chi \leqslant \arctan b / a$ )

$$
\begin{aligned}
& M_{b y}=\frac{1}{4} a b^{2} \sigma_{0}-\frac{1}{12} a^{3} \sigma_{0} \tan ^{2} \chi, \quad M_{b z}=\frac{1}{6} a^{3} \sigma_{0} \tan \chi, \\
& M_{b y}=\frac{1}{4} a b^{2} \sigma_{0}-3 \frac{M_{b z}^{2}}{a^{3} \sigma_{0}},
\end{aligned}
$$


or, in a dimensionless form,

where

$$
m_{b y}=1-\frac{3}{4} m_{b z}^{2},
$$

$$
m_{b y}=\frac{M_{b y}}{\overline{\bar{M}}_{b y}}=\frac{4 M_{b y}}{a b^{2} \sigma_{0}}, \quad m_{b z}=\frac{M_{b z}}{\overline{\bar{M}}_{b z}}=\frac{4 M_{b z}}{a^{2} b \sigma_{0}} .
$$

The angle of inclination of the bending moment, $\alpha$, is determined by the formula

$$
\tan \alpha=\frac{M_{b z}}{M_{b y}}=\frac{\tan \chi}{\frac{3}{2} \frac{b^{2}}{a^{2}}-\frac{1}{2} \tan ^{2} \chi} .
$$

In engineering applications, as a rule, the angle $\alpha$ is given and we look for the angle $\chi$; the solution of $(24.15)$ with respect to $\psi$ may be written in the form

$$
\tan \chi=\frac{3 b^{2} \tan \alpha}{a^{2}\left(1+\sqrt{1+3 \frac{b^{2}}{a^{2}} \tan ^{2} \alpha}\right)} .
$$

In the boundary case $\chi=\arctan b / a$ we obtain $M_{b y}=a b^{2} \sigma_{0} / 6, M_{b z}=a^{2} b \sigma_{0} / 6$; thus $m_{b y}=m_{b z}=2 / 3$ and $\alpha=\arctan a / b=(\pi / 2)-\chi$. If $\alpha$ and $\chi$ are larger than these values, the positions of the directions $Y$ and $Z$ are interchanged and instead of the parabola (24.13) we obtain

$$
m_{b z}=1-{ }_{4}^{3} m_{b y}^{2} .
$$

A quadrant of the plastic interaction curve for a rectangular section is shown in Fig. 138; the remaining quadrants show full symmetry.

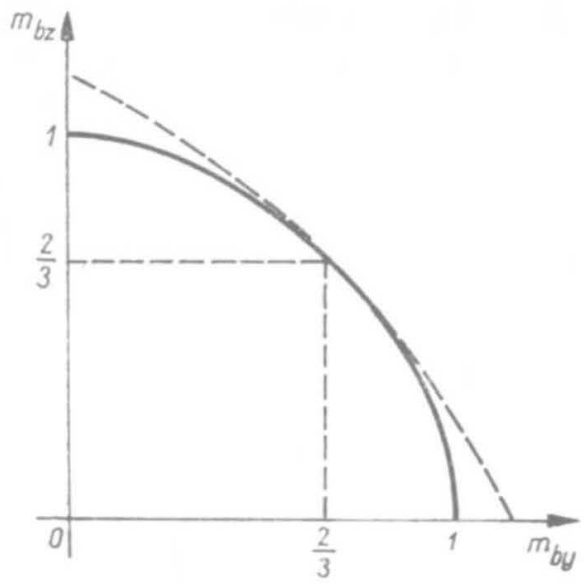

Fig. 138. Plastic interaction curve for a rectangular section under biaxial bending

The analysis of the limit carrying capacity of a cross-section under biaxial bending is simpler than the analysis of the elastic-plastic range, and hence the earliest papers were restricted to that problem. We mention here the first paper by B. N. Gor- 
bunov and V. G. Chudnovsky [5.63], published in 1935, and by B. N. Gorbunov and S. A. Palchevsky [5.64], concerning a rectangular and an I-section. A detailed analysis for an I and for a channel section and the relevant numerical tables were given by O. I. Strelbitska [5.176]. O. N. Totsky [5.186] and E. H. Brown [5.22] investigated the locus of gravity centres of halves of the cross-section for various directions $\chi$; these gravity centres effectively determine the limit carrying capacity of the cross-section. Totsky stated that such loci are very close to ellipses and proposed approximate equations, whereas Brown introduced the notion of a "centroidal curve" and investigated the basic properties of these curves.

Theoretical and experimental investigations of the limit carrying capacity of statically indeterminate beams with a rectangular cross-section subject to biaxial bending were carried out by H. B. Harrison [5.68].

\section{Torsion with shear}

\subsection{Formulation of the problem}

The case of simultaneous torsion with shear may also be classified as $\mathscr{P}_{1} / \mathscr{P}_{2}$; though, as a rule, at individual points of the cross-section two components of the shearing stress occur, these components may always be added geometrically and we may analyse the relevant problems without any specified failure hypothesis. Moreover, the stress distribution in the case of perfect plasticity may be determined without the strain analysis (a statically internally determined problem).

The problem under consideration is of no particular importance in analysing cross-sections of straight bars, since shear is then accompanied by bending (at least in neighbouring sections) and we should consider rather a multiply combined

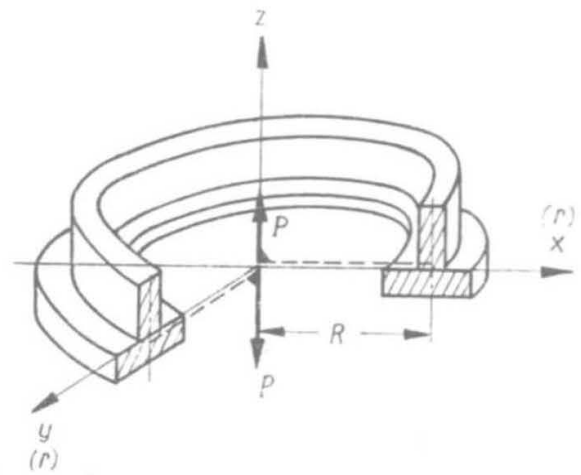

Fig. 139. Torsion with shear of a circularly curved bar

case. On the other hand, torsion with shear without bending may occur in circularly curved bars (Fig. 139) and this case will be discussed in the present chapter (M. Galos and M. Życzkowski [5.53]). 
Let us introduce a cylindrical system of coordinates $r, \theta, z$ (Eulerian and Lagrangian coordinates are not distinguished here) and first determine the distribution of stresses $\tau_{\theta r}$ and $\tau_{\theta z}$. In the case under consideration the derivatives of stresses with respect to the variable $\theta$ vanish, and hence the second equation of equilibrium (6.11) (the only one that is not satisfied indentically) yields

$$
\frac{\partial \tau_{\theta r}}{\partial r}+\frac{\partial \tau_{\theta z}}{\partial z}+2 \frac{\tau_{\theta r}}{r}=0
$$

The condition of perfect plasticity may be written in the form $|\tau|=\tau_{0}$, i.e.

$$
\tau_{\theta r}^{2}+\tau_{\theta z}^{2}=\tau_{0}^{2}
$$

Hence in the case of full plastification (or in plastic zones in the elastic-plastic range) the stress distribution is determined by the system of equations (25.1) and (25.2). The most convenient way to reduce this system to one equation is to introduce a stress function $\Psi$ by means of formulae (19.38). We obtain the following first-order partial differential equation

$$
\left(\frac{\partial \Psi}{\partial z}\right)^{2}+\left(\frac{\partial \Psi}{\partial r}\right)^{2}=r^{4} \tau_{0}^{2}=\bar{\tau}_{0}^{2}(r, z),
$$

where $\bar{\tau}_{0}=r^{2} \tau_{0}$ may be regarded as a certain "reduced" yield-point stress in shear. If $\tau_{0}$ depends on $r$ and $z$, then we consider a non-homogeneous material, which, after all, does not introduce any essential complications to the analysis. Instead of (25.3) we may write

$$
\left|\operatorname{grad} \Psi^{\prime}\right|=\bar{\tau}_{0}(r, z) .
$$

This equation is identical with the equation of plastic torsion of a non-homogeneous prismatic bar. The function $\vec{\tau}_{0}(r, z)=r^{2} \tau_{0}$ may be called a substitutive non-homogeneity. Equation (25.4) may be interpreted as determining the maximal slope of the function $\Psi$. Lines of maximal slope (or, strictly speaking, their projections on the basic plane) are determined in the non-homogeneous case by the following ordinary differential equation (M. Galos [5.52]):

$$
z^{\prime} \frac{\partial \vec{\tau}_{0}}{\partial r}-\frac{\partial \vec{\tau}_{0}}{\partial z}=-\frac{z^{\prime \prime} \bar{\tau}_{0}}{1+z^{\prime 2}},
$$

where $z=z(r)$ is the equation of the line of maximal slope. If we parametrize the yield condition (25.2) by the formulae of the type (9.69) and substitute them into (25.1) we obtain a quasi-linear equation with respect to the parameter $\psi$; the characteristics for this equation are determined again by (25.5) (I. A. Kiyko [5.86]), and hence the lines of maximal slope are simply called the characteristics.

The only boundary condition, following from (6.3), results in the requirement that the lines of maximal slope should be orthogonal to the contour. Having deter- 
mined a one-parameter family of these lines, we may evaluate the function $\Psi$, since $\Psi=0$ along the (simply connected) contour and

$$
\left|\frac{d \Psi}{d m}\right|=\bar{\tau}_{0}(r, z)
$$

along any line of maximal slope, where $\mathrm{dm}$ denotes an element of that line.

Equation (25.6) determines the function $\Psi$ inside individual subdomains of the cross-sectional area. In the case of full plastification, these subdomains are separated by the lines of stress discontinuities; the discontinuity lines may be found from the conditions of equal values of the function $\Psi$ in neighbouring subdomains (this requirement satisfies all the continuity conditions discussed in Sec. 6.3). Namely, denote by $l$ the parameter of a line of maximal slope (corresponding to a certain parametrization of the contour equation), and by $s$ the parameter ascribed to individual points of that line; then the discontinuity line separating for example the zones "1" and "2" may be determined as follows (Fig. 140):

$$
\begin{gathered}
\Psi_{1}\left(s_{1}, l_{1}\right)=\Psi_{2}\left(s_{2}, l_{2}\right), \\
r_{1}\left(s_{1}, l_{1}\right)=r_{2}\left(s_{2}, l_{2}\right), \\
z_{1}\left(s_{1}, l_{1}\right)=z_{2}\left(s_{2}, l_{2}\right) .
\end{gathered}
$$

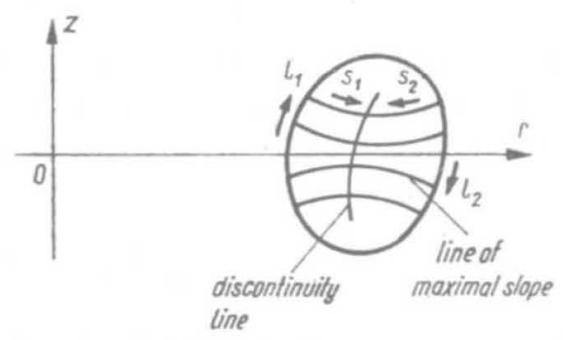

Fig. 140. Determination of the lines of stress discontinuity

These equations make it possible to express three parameters in terms of the fourth one, for example $l_{1}$, and finally the discontinuity line may be determined by a parametrical equation $r=r\left(l_{1}\right), z=z\left(l_{1}\right)$.

Knowing the function $\Psi$ in individual subdomains, we can determine the limit carrying capacity of the cross-section, namely the twisting moment $M_{t}$ and the shearing force $V$. An infinitesimal moment $d M_{t}$ equals

$$
d M_{t}=\left[\tau_{\theta z}(r-R)-\tau_{\theta r} z\right] d A,
$$

where $R$ denotes the radius corresponding to the centre of gravity of the crosssection, Fig. 139. Substitution of the formulae for $\tau_{\theta z}$ and $\tau_{\theta r},(19.38)$, iteration, integration per partes and the subsequent return to a double integral lead to the following formula:

$$
M_{t}=2 R \iint_{A} \frac{\Psi(r, z)}{r^{3}} d A
$$


Of course, the relevant shearing force equals

$$
V=P=2 \iint_{A} \frac{\Psi(r, z)}{r^{3}} d A
$$

Because of the simple relation $M_{t}=V R$, it seems that formulae (25.9) and (25.10), applied to a fully plastic section, do not determine any limit curve in the plane of generalized stresses $M_{t}-V$. Indeed, for a given radius $R$ the quantities $M_{t}$ and $V$ are not independent; nevertheless, regarding $R$ as a parameter, we may consider (25.9) and (25.10) as parametrical equations of the limit curve ( $R$ is also hidden in the integration limits).

The formulae derived are still ineffective, since practically we know $\Psi=\Psi(s, l)$ (where $s$ and $l$ are the parameters introduced above) and not directly the function $\Psi=\Psi(r, z)$. However, introducing an appropriate Jacobian determinant of transformation, we may write instead of (25.9)

$$
M_{t}=2 R \iint_{A} \frac{\Psi(s, l)}{r^{3}(s, l)}\left|\left(\frac{\partial r}{\partial s} \frac{\partial z}{\partial l}-\frac{\partial r}{\partial l} \frac{\partial z}{\partial s}\right)\right| d s d l,
$$

and this notation is already adjusted to the method used. A similar formula determines the shearing force $V$.

\subsection{Solution for homogeneous material}

In the case of homogeneous material we have $\bar{\tau}_{0}=\bar{\tau}_{0}(r)=r^{2} \tau_{0}$, where $\tau_{0}$ is a constant. The differential equation of lines of maximal slope, (25.5), assumes the form

$$
2 z^{\prime} r=-\frac{z^{\prime \prime}}{1+z^{\prime 2}},
$$

and its general solution may be written thus:

$$
z= \pm \int \frac{C d r}{\sqrt{r^{4}-C^{2}}}+D
$$

where $C$ and $D$ are the integration constants. Denote by $M$ a current contour point, describe the contour by the parametrical equations $z_{M}=z_{M}(l), r_{M}=r_{M}(l)$ and assume the parameter $l$ to be also the parameter of individual lines of maximal slope. The condition of orthogonality of those lines to the contour yields (under the assumption that the lower integration limit is zero)

$$
C=\frac{r_{M}^{2} z_{M}^{\prime}}{\sqrt{r_{M}^{\prime 2}+z_{M}^{\prime 2}}}, \quad D=z_{M},
$$

where the primes denote the derivatives with respect to the parameter $l$. The integral (25.13) is an elliptic integral. Reducing it to the normal form, we obtain

$$
z= \pm \sqrt{\frac{C}{2}}\left[F\left(\arccos \frac{\sqrt{C}}{r}, \frac{\sqrt{2}}{2}\right)-F\left(\arccos \frac{\sqrt{C}}{r_{M}}, \frac{\sqrt{2}}{2}\right)\right]+D
$$


Further, making use of the relation (25.6) and of the boundary condition $\Psi=0$ along the contour, we determine the values of $\Psi$ along a line of maximal slope by the formula

$$
\begin{gathered}
\Psi=\tau_{0}\left\{\sqrt { \frac { C ^ { 3 } } { 1 2 } } \left[F\left(\arccos \frac{\sqrt{C}}{r}, \frac{\sqrt{2}}{2}\right)-F\left(\arccos \frac{\sqrt{C}}{r_{M}}, \frac{\sqrt{2}}{2}\right)+\right.\right. \\
\left.+\frac{r}{3} \sqrt{r^{4}-C^{2}}-\frac{r_{M}}{3} \sqrt{r_{M}^{4}-C^{2}}\right\} .
\end{gathered}
$$

This equation is of the type $\Psi=\Psi(s, l)$, where $l$ is the contour parameter (appearing in $r_{M}$ ) and the parameter $s$ may be identified with $r$ (the line $r=$ const is excluded here). Having determined for the given contour the discontinuity lines (limits of integration), we may substitute (25.15) and (25.16) in (25.11) and evaluate the limit carrying capacity in the case of torsion with shear which is here considered.

The problem of the limit carrying capacity of a circular section (important because of application in helical springs), subject to torsion with shear, was first considered in 1955 by A. J. Wang and W. Prager [5.194]. Further papers are due to W. Freiberger and W. Prager [5.47] (annular sections) and W. Freiberger [5.48] (arbitrary sections); these papers give also an approximate graphical method of evaluating the limit carrying capacity. Application to helical springs is given by A. M. Wahl [5.193], three-dimensional helical curve was considered by D. D. Ivlev [5.78]; he took into account the geometry of such a curve, but neglected the influence of the bending moment and normal force occurring in this case. Ivlev derived the equations of characteristics for the basic equations parametrized by (9.69), but did not consider directly the limit carrying capacity. Approximate equations of the plastic interaction curve based on simple statically partially admissible stress fields for various cross-sections were derived by Z. Sobotka [5.171].

A. Mioduchowski [5.124] considered elastic-plastic torsion with shear of a circularly curved bar; he used a variational approach combined with the method of local variations. Arbitrary plastic hardening was studied by M. A. Zadoyan [5.207].

\subsection{Example of material non-homogeneity}

In the general case of transversal non-homogeneity $\tau_{0}=\tau_{0}(r, z)$ the differential equation of lines of maximal slope (25.5) cannot be integrated in closed form. However, if the yield-point stress $\tau_{0}$ depends on $r$ only, then $\bar{\tau}_{0}=r^{2} \tau_{0}$ is also a function of $r$, the integration is relatively simple and we obtain a generalization of (25.13)

$$
z= \pm \int \frac{C d r}{\sqrt{\bar{\tau}_{0}^{2}-C^{2}}}+D
$$

The simplest particular case of non-homogeneity occurs if

$$
\tau_{0}=\frac{K R^{2}}{r^{2}}
$$


where $K$ is a constant of the dimension of stress, since then substitutive non-homogeneity corresponds to homogeneity: $\bar{\tau}_{0}=\tau_{0} r^{2}=K R^{2}=$ const. Thus (25.17) may be written in the form

$$
z=B r+D,
$$

and the lines of maximal slope are straight lines.

Consider, for example, a circular cross-section with the radius $r_{0}$; the radius of curvature of the axis will be denoted by $R$, as before. The contour equation will be written in parametrical form

$$
r_{M}=R+r_{0} \cos l, \quad z_{M}=-r_{0} \sin l .
$$

with $l$ as a parameter. Using the orthogonality condition to determine $B$ and $D$ in (25.19), we obtain the following equation of the family of lines of maximal slope

$$
z=(R-r) \tan l .
$$

As the parameter $s$ determining individual points of these lines assume the distance from the contour. Then

$$
r=R+\left(r_{0}-s\right) \cos l, \quad z=-\left(r_{0}-s\right) \sin l,
$$

and the values of the function $\Psi$, determined by (25.6), are $\Psi=K R^{2} s$.

The limit carrying capacity of the cross-section is determined by (25.11) and by a similar formula for the shearing force $V$. The Jacobian equals here $|J|=r_{0}-s$; the discontinuity line reduces to a point $s=r_{0}$, and hence the integration may be taken over one domain $0 \leqslant l \leqslant 2 \pi, 0 \leqslant s \leqslant r_{0}$. After integration with respect to $l$ we obtain

$$
M_{t}=2 \pi K R^{3} \int_{0}^{r} \frac{\left[2 R^{2}+\left(r_{0}-s\right)^{2}\right] s\left(r_{0}-s\right) d s}{\left[R^{2}-\left(r_{0}-s\right)^{2}\right]^{5 / 2}} .
$$

This integral may be expressed in terms of elementary functions, but the final formula is rather complicated and will not be quoted here. On the other hand, the result is simple if we present it in the form of a power series of the small parameter $\alpha=r_{0} / R$ :

$$
\begin{aligned}
M_{t} & =2 \pi K r_{0}^{3}\left(\frac{1}{3}+\frac{3}{10} \alpha^{2}+\frac{45}{168} \alpha^{4}+\ldots\right), \\
V & =2 \pi K r_{0}^{2}\left(\frac{1}{3} \alpha+\frac{3}{10} \alpha^{3}+\frac{45}{168} \alpha^{5}+\ldots\right) .
\end{aligned}
$$

The formulae (25.24) determine the limit carrying capacity of the cross-section in the case under consideration. However, one cannot regard them as parametrical equations of a certain plastic interaction curve, since the change of parameter $\alpha$ results in simultaneous change of material non-homogeneity. 


\section{CHAPTER VI}

\section{Combined loadings at the level $\delta$ of a cross-section of a bar; type $\mathscr{P}_{2} / \delta_{n}$}

\section{Introductory remarks and general methods}

\subsection{Yield condition}

In the elementary theory of bars one assumes at most three stress components to occur (e.g., $\sigma_{x}, \tau_{x y}, \tau_{x z}$ ); however, the two shearing stresses may be added geometrically and the cases under consideration are then classified, in general, as $\mathscr{P}_{2} / \mathscr{S}_{n}$. More rigorously, other stress components may also appear, particularly as a result of compressibility of the material (A. A. Ilyushin [1.9], M. Życzkowski [4.628]), but the influence of compressibility on the quantitative aspects of the phenomena, for example on the limit surfaces, is usually very small.

If we restrict our considerations to the elementary theory of isotropic bars, then the use of the "ad hoc" elliptic yield condition (11.33) is in most cases justified. In an arbitrary locally orthogonal system of coordinates $x, \alpha, \beta$ (where $x$ is measured along the axis of the bar) this condition may be written as

$$
\sigma_{x}^{2}+c\left(\tau_{x \alpha}^{2}+\tau_{x \beta}^{2}\right)=\sigma_{0}^{2} .
$$

It generalizes the HMH yield condition $(c=3)$ and the TG yield condition $(c=4)$; a certain other value of the parameter $c$ may better fit experimental data. In some cases, particularly in cylindrical coordinates, we shall denote the axial coordinate by $z$ instead of $x$.

If we use the general form of the yield condition (26.1), then the problem arises of an appropriate choice of the physical equations (constitutive equations at the level $\mathscr{P}$ ). The law of similarity of deviators of stresses and strains or plastic strain rates is associated with (26.1) for $c=3$ only. If $c \neq 3$, then the physical equations should be subject to a more precise but difficult experimental verification. In what follows we restrict our considerations almost exclusively to $c=3$ (HMH hypothesis) and to the law of similarity of deviators. 


\subsection{Lower bounds to the limit carrying capacity}

Exact solutions of problems of the type $\mathscr{P}_{2} / \mathscr{S}_{n}$, even in the purely plastic state, without elastic zones, may be very complicated. On the other hand, making use of statically admissible stress fields, based on the solutions given in Chapter V, we can derive lower bounds to the limit carrying capacity of the cross-section with an accuracy sufficient for most engineering applications.

Suppose we know the exact equation of the plastic interaction surface for simultaneous biaxial bending with tension (a problem of the type $\mathscr{P}_{1} / \mathscr{S}_{3}$ )

$$
f_{1}\left(M_{b y}, M_{b z}, N\right)=0
$$

and for simultaneous torsion with shear

$$
f_{2}\left(V_{y}, V_{z}, M_{t}\right)=0
$$

or for any of the more particular cases of (26.2) or (26.3). In the case of biaxial bending with tension we have a certain distribution of normal stresses $\sigma=\sigma_{1}(y, z)$, where $|\sigma| \leqslant \sigma_{0}$ and in the case of torsion with shear $\tau=\tau_{2}(y, z)$, where $|\tau| \leqslant \tau_{0}$. Hence the stress field

$$
\sigma=\varphi \sigma_{1}(y, z), \quad \tau=\chi \tau_{2}(y, z),
$$

where $\psi$ and $\chi$ are certain constants, satisfies the equations of equilibrium, stress boundary conditions and the required conditions of continuity (equilibrium) along the discontinuity lines if such lines occur. Moreover, the yield condition (11.32) will be satisfied as a weak inequality if

$$
\psi^{2}+\chi^{2}=1 \text {. }
$$

Thus the stress field

$$
\sigma=\psi \sigma_{1}(y, z), \quad \tau=\sqrt{1-\psi^{2}} \tau_{2}(y, z)
$$

is statically admissible and corresponds to the general case under consideration. The relevant generalized stresses are then smaller than those in (26.2) or (26.3), namely are multiplied by $\psi<1$ or $\sqrt{1-\psi^{2}}$, respectively. The equations

$$
\begin{aligned}
& f_{1}\left(\frac{M_{b y}}{\psi}, \frac{M_{b z}}{\psi}, \frac{N}{\psi}\right)=0, \\
& f_{2}\left(\frac{V_{y}}{\sqrt{1-\psi^{2}}}, \frac{V_{z}}{\sqrt{1-\psi^{2}}}, \frac{M_{t}}{\sqrt{1-\psi^{2}}}\right)=0
\end{aligned}
$$

may be regarded as a parametrical presentation of the plastic interaction surface in the general case. If $\psi$ can be eliminated effectively, then we obtain an equation of the type $f\left(M_{b y}, M_{b z}, N, V_{y}, V_{z}, M_{t}\right)=0$. In the case of thin-walled bars, if a bimoment $B$ and a warping moment $M_{\omega}$ occur, they may be included in the first group and in the second group of generalized stresses, respectively.

If Eqs. (26.2) and (26.3) are not exact but determine a certain lower bound, then (26.7) describe also a lower bound; if they correspond to a certain upper bound, then the direction of error of (26.7) is unknown. 
The general method given above is an extension of the concept of N. I. Bezukhov [6.25], who proposed equation of the plastic interaction surface in the form

$$
\left(\frac{M_{b}}{\overline{\bar{M}}_{b}^{*}}\right)^{2}+\left(\frac{M_{t}}{\overline{\bar{M}}_{t}^{*}}\right)^{2}=1,
$$

where $\overline{\bar{M}}_{b}^{*}$ denotes the limit bending moment reduced with respect to the presence of axial force, and $\overline{\bar{M}}_{t}^{*}$ the limit twisting moment reduced with respect to the presence of shear. Another approach to the derivation of lower bounds to the limit carrying capacity of a cross-section was suggested by M. I. Erkhov [6.63].

In the cases of only two generalized stresses $Q_{1}$ and $Q_{2}$, one corresponding to $\sigma$ and another one to $\tau$, the equation of a lower bound to the plastic interaction curve describes a circle in dimensionless coordinates or an ellipse in physical coordinates:

$$
\left(\frac{Q_{1}}{\overline{\bar{Q}_{1}}}\right)^{2}+\left(\frac{Q_{2}}{\overline{\bar{Q}_{2}}}\right)^{2}=1 .
$$

Hence the exact plastic interaction curve lies here outside the circle. It should be noted, however, that for two generalized stresses both corresponding to $\sigma$ or both corresponding to $\tau$ (as discussed in Chapter V) this statement is no longer valid: for example, a segment of the parabola (22.35) lies inside the circle (26.9).

\subsection{Upper bounds to the limit carrying capacity}

From the engineering viewpoint, knowing the lower bounds to the limit carrying capacity is more important than knowing the upper bounds: indeed, the application of the lower bounds results in increased safety. On the other hand, the upper bounds may be used for the estimation of errors of the lower bounds.

According to the considerations given in Sec. 20.4 we now derive the general upper bounds to the limit carrying capacity combining statically partially admissible stress fields with the variational approach. The yield condition (26.1) will be satisfied at any point of the cross-section, whereas the equilibrium equations will not be taken into account at all.

Parametrize the yield condition (11.33) or (26.1) in Cartesian coordinates as follows:

$$
\sigma_{x}=\sigma_{0} \cos \chi, \quad \tau_{x y}=\tau_{0} \sin \chi \cos \psi, \quad \tau_{x z}=\tau_{0} \sin \chi \sin \psi,
$$

where $\chi=\chi(y, z)$ and $\psi=\psi(y, z)$ are certain parameters; their distribution is to be determined. Generalized stresses will now be presented as the functionals

$$
\begin{aligned}
N & =\sigma_{0} \iint_{A} \cos \chi d A, & V_{y} & =\tau_{0} \iint_{A} \sin \chi \cos \psi d A, \\
M \Xi & =\sigma_{0} \iint_{A} z \cos \chi d A, & V_{z} & =\tau_{0} \iint_{A} \sin \chi \sin \psi d A, \\
M_{b z} & =\sigma_{0} \iint_{A} y \cos \chi d A, & M_{t} & =\tau_{0} \iint_{A} \sin \chi(z \cos \psi-y \sin \psi) d A .
\end{aligned}
$$


We now look for the maximal value of any of these functionals under the constraints of constant values of the remaining ones. Write the Langrangian function in the form

$$
\begin{aligned}
L= & \lambda_{1} \sigma_{0} \cos \chi+\lambda_{2} \sigma_{0} z \cos \chi+\lambda_{3} \sigma_{0} y \cos \chi+\lambda_{4} \tau_{0} \sin \chi \cos \psi+ \\
& +\lambda_{5} \tau_{0} \sin \chi \sin \psi+\lambda_{6} \tau_{0} \sin \chi(z \cos \varphi-y \sin \psi) .
\end{aligned}
$$

One of the Langrangian multipliers $\lambda_{i}$ is not necessary here (being equal to unity); however, all multipliers are introduced for the sake of symmetry of notation, and in the final formulae only the ratios of those multipliers will occur.

The Euler-Lagrange conditions with respect to the functions $\psi(y, z)$ and $\chi(y, z)$ lead to the system of equations

$$
\begin{aligned}
& -\lambda_{4} \sin \psi+\lambda_{5} \cos \psi-\lambda_{6} z \sin \psi-\lambda_{6} y \cos \psi=0, \\
& -\left(\lambda_{1}+\lambda_{2} z+\lambda_{3} y\right) \sigma_{0} \sin \chi+\left(\lambda_{4} \cos \psi+\lambda_{5} \sin \psi+\right. \\
& \left.+\lambda_{6} z \cos \psi-\lambda_{6} y \sin \psi\right) \tau_{0} \cos \chi=0,
\end{aligned}
$$

and hence

$$
\tan \psi=\frac{\lambda_{5}-\lambda_{6} y}{\lambda_{4}+\lambda_{6} z}, \quad \tan \chi=\frac{\sqrt{\left(\lambda_{5}-\lambda_{6} y\right)^{2}+\left(\lambda_{4}+\lambda_{6} z\right)^{2}}}{\left(\lambda_{1}+\lambda_{2} z+\lambda_{3} y\right) \sqrt{c} .} .
$$

Substitution of (26.14) into (26.10) determines the stress distribution. The Equilibrium equations and the boundary conditions are, in general, not satisfied, and hence the maximum of the functional under consideration exceeds the exact value equal to a constrained maximum. Finally the upper bound to the plastic interaction surface is determined by the following integral formulae:

$$
\begin{aligned}
& N=\sigma_{0} \sqrt{c} \iint_{A} \frac{\left(\lambda_{1}+\lambda_{2} z+\lambda_{3} y\right) d A}{\sqrt{\left(\lambda_{5}-\lambda_{6} y\right)^{2}+\left(\lambda_{4}+\lambda_{6} z\right)^{2}+c\left(\lambda_{1}+\lambda_{2} z+\lambda_{3} y\right)^{2}},} \\
& M_{b y}=\sigma_{0} \sqrt{c} \iint_{A} \frac{\left(\lambda_{1}+\lambda_{2} z+\lambda_{3} y\right) z d A}{\sqrt{\left(\lambda_{5}-\lambda_{6} y\right)^{2}+\left(\lambda_{4}+\lambda_{6} z\right)^{2}+c\left(\lambda_{1}+\lambda_{2} z+\lambda_{3} y\right)^{2}}}, \\
& M_{b z}=\sigma_{0} \sqrt{c} \iint_{A} \frac{\left(\lambda_{1}+\lambda_{2} z+\lambda_{3} y\right) y d A}{\sqrt{\left(\lambda_{5}-\lambda_{6} y\right)^{2}+\left(\lambda_{4}+\lambda_{6} z\right)^{2}+c\left(\lambda_{1}+\lambda_{2} z+\lambda_{3} y\right)^{2}}}, \\
& V_{y}=\tau_{0} \iint_{A} \frac{\left(\lambda_{4}+\lambda_{6} z\right) d A}{\sqrt{\left(\lambda_{5}-\lambda_{6} y\right)^{2}+\left(\lambda_{4}+\lambda_{6} z\right)^{2}+c\left(\lambda_{1}+\lambda_{2} z+\lambda_{3} y\right)^{2}},} \\
& V_{z}=\tau_{0} \iint_{A} \frac{\left(\lambda_{5}-\lambda_{6} y\right) d A}{\sqrt{\left(\lambda_{5}-\lambda_{6} y\right)^{2}+\left(\lambda_{4}+\lambda_{6} z\right)^{2}+c\left(\lambda_{1}+\lambda_{2} z+\lambda_{3} y\right)^{2}}}, \\
& M_{t}=\tau_{0} \iint_{A} \frac{\left[\left(\lambda_{4}+\lambda_{6} z\right) z-\left(\lambda_{5}-\lambda_{6} y\right) y\right] d A}{\sqrt{\left(\lambda_{5}-\lambda_{6} y\right)^{2}+\left(\lambda_{4}+\lambda_{6} z\right)^{2}+c\left(\lambda_{1}+\lambda_{2} z+\lambda_{3} y\right)^{2}}} .
\end{aligned}
$$

These equations describe the plastic interaction surface in parametrical form: six generalized stresses are expressed in terms of five parameters, namely of five independent ratios of Lagrangian multipliers $\lambda_{i} / \lambda_{j}$. 
In the case of thin-walled bars the approximation errors of (26.15) may be reduced. Namely, if we assume the shearing stresses $\tau$ to be directed tangentially to the middle line of the profile, then we reduce two parametrizing functions to one only. The boundary conditions are then satisfied, and this fact results in improved accuracy of the relevant formulae. Such an approach for a thin-walled circular tube was suggested by A. A. Gvozdev [4.196]; it will be quoted in Chapter VIII in a generalized form allowing for a simultaneous action of internal pressure on the tube.

\section{Tension with torsion}

\subsection{Limit carrying capacity of round cross-sections}

Consider an element of a bar with a round cross-section (circular or annular), subject to simultaneous tension with torsion (Fig. 141). The limit carrying capacity

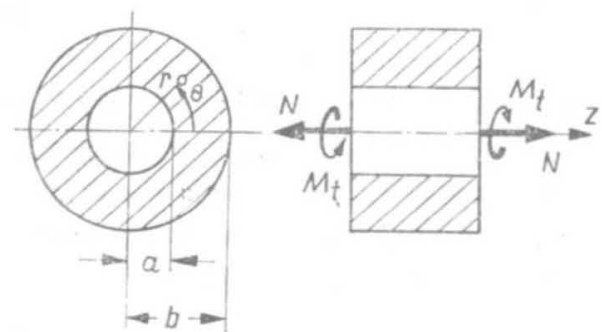

Fig. 141. Tension with torsion of a bar with a round cross-section

of the cross-section in such a case was first determined by A. Nadai in 1931 [4.403]. Namely, if we make use of the hypothesis of plane sections and substitute, in cylindrical coordinates,

$$
\varepsilon_{z}=\varepsilon=\text { const }, \quad \gamma_{z \theta}=\vartheta r,
$$

where $\vartheta$ denotes the unit angle of twist, then the compatibility conditions are of course satisfied. Further, assume incompressibility of material; the influence of compressibility will be discussed later. Under this assumption $\varepsilon_{r}=\varepsilon_{\theta}=-\varepsilon / 2$; the Hencky-Ilyushin or the Levy-Mises equations and the boundary condition $\sigma_{r}=0$ at $r=b$ yield $\sigma_{r}=\sigma_{0}=0$ in the whole domain under consideration. The non-vanishing stresses $\sigma_{z}$ and $\tau_{\theta z}$ are determined by

$$
\sigma_{z}=\frac{3}{2 \varphi} \varepsilon_{z}=\frac{3}{2 \varphi} \varepsilon, \quad \tau_{z \theta}=\frac{1}{2 \varphi} \gamma_{z \theta}=\frac{\vartheta r}{2 \varphi} .
$$

Equations (27.2) are understood as the Hencky-Ilyushin equations; however, they may also be interpreted as the Levy-Mises equations if we regard $\varepsilon$ as the axial strain rate, and $\vartheta$ as the rate of unit angle of twist, and denote the function $\lambda$ formally 
by $\varphi$. Substituting $c=3$ in the yield condition (11.33) (Huber-Mises-Hencky), we determine the function $\varphi$ :

$$
\varphi=\frac{1}{2 \sigma_{0}} \sqrt{9 \varepsilon^{2}+3 \vartheta^{2} r^{2}}
$$

thus the stress distribution is finally determined by

$$
\sigma_{z}=\frac{3 \varepsilon \sigma_{0}}{\sqrt{9 \varepsilon^{2}+3 \vartheta^{2} r^{2}}}, \quad \tau_{z \theta}=\frac{\vartheta r \sigma_{0}}{\sqrt{9 \varepsilon^{2}+3 \vartheta^{2} r^{2}}} .
$$

It may easily be checked that the equilibrium equations (6.11) and the boundary conditions at lateral surfaces are identically satisfied, and hence the solution obtained under the assumption (27.1) is exact.

The twisting moment $M_{t}$ and the normal force $N$ are, in the case of a circular cross-section, given by

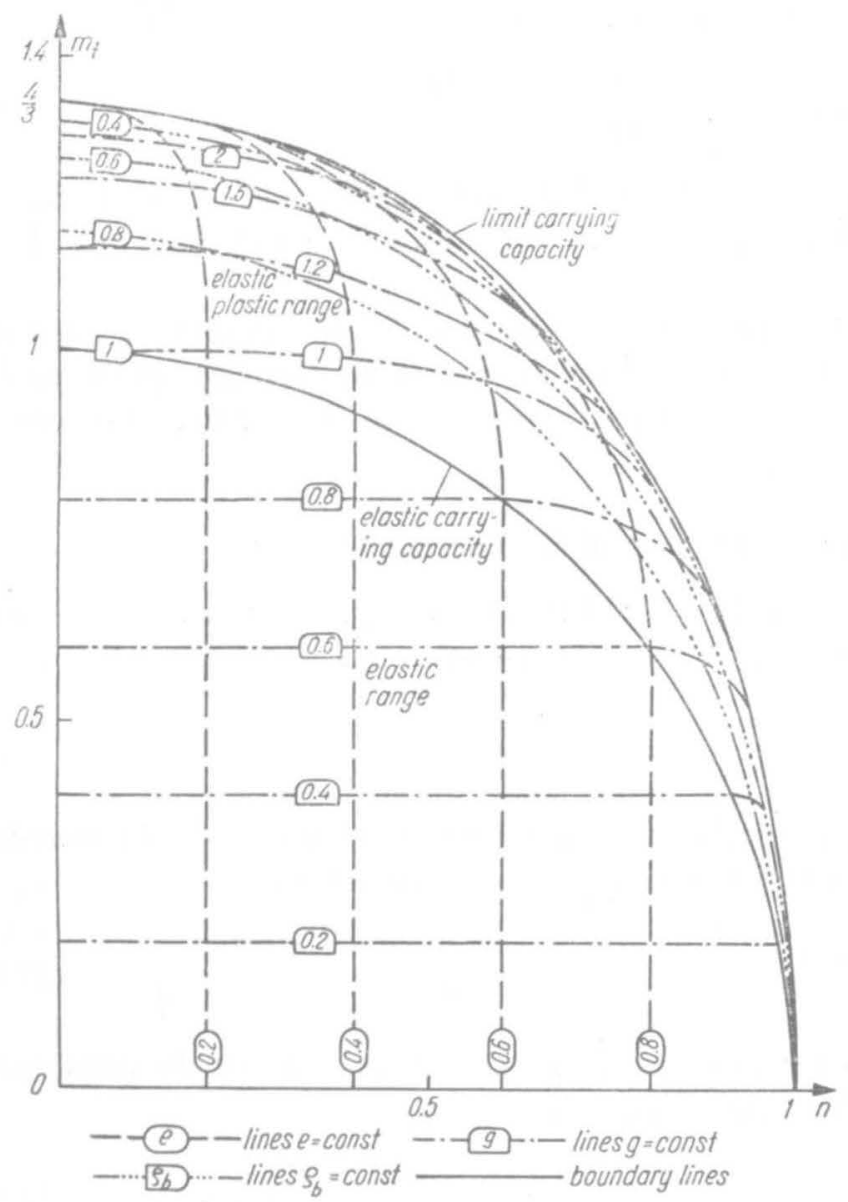

Fig. 142. Interaction curves for a circular section under tension with torsion 


$$
\begin{aligned}
& M_{t}=2 \pi \int_{0}^{b} \tau_{z \theta} r^{2} d r=\frac{2 \pi \sigma_{0}}{9 \vartheta^{3}}\left[\left(\vartheta^{2} b^{2}-6 \varepsilon^{2}\right) \sqrt{9 \varepsilon^{2}+3 \vartheta^{2} b^{2}}+18 \varepsilon^{3}\right], \\
& N=2 \pi \int_{0}^{b} \sigma_{z} r d r=\frac{2 \pi \varepsilon \sigma_{0}}{\vartheta^{2}}\left(\sqrt{9 \varepsilon^{2}+3 \vartheta^{2} b^{2}}-3 \varepsilon\right) .
\end{aligned}
$$

In the case of an annular section the integration should be taken from $a$ to $b$, and this fact results in a certain complication of the final formulae.

The generalized stresses $M_{t}$ and $N$ depend here on the dimensionless geometrical parameter $\varepsilon / b \vartheta$. Thus Eqs. (27.5) and (27.6) may be treated as parametrical equations of the plastic interaction curve. However, the parameter $\varepsilon / b \vartheta$ may be eliminated; the final formula will be written in the form

$$
m_{t}=\frac{2}{3}(2+n) \sqrt{1-n}=\frac{4}{3} \sqrt{1-\frac{3}{4} n^{2}-\frac{1}{4} n^{3}},
$$

where dimensionless generalized stresses are introduced by the formulae

$$
n=\frac{N}{\bar{N}}=\frac{N}{\pi b^{2} \sigma_{0}}, \quad m_{t}=\frac{M_{t}}{\bar{M}_{t}}=\frac{2 \sqrt{3} M_{t}}{\pi b^{3} \sigma_{0}} .
$$

The curve (27.7) is close to an ellipse of the type (26.9); it is shown in Fig. 142 together with other curves, describing the behaviour of a section in the elasticplatic range.

B. V. Panov [6.180] investigated the influence of cold working on the plastic interaction curve $M_{t}=f(N)$. V. N. Gorelov and A. S. Neymark [6.80] applied the Hill yield condition (12.3) taking the orthotropy of the material into account.

\subsection{Elastic-plastic range in the case of incompressibility}

In the case of an incompressible material the stress distribution in the plastic zone $r_{b}<r<b$ is determined by (27.4), and in the elastic zone $a<r<r_{b}$ or $0<r<r_{b}$ by Hooke's law

$$
\sigma_{z}=E \varepsilon, \quad \tau_{z \theta}=G \vartheta r .
$$

The boundary radius $r_{b}$ separates the elastic from the plastic zone. We introduce dimensionless parameters of deformation (generalized strains)

$$
\frac{E}{\sigma_{0}} \varepsilon=e, \quad \frac{G b \sqrt{3}}{\sigma_{0}} \vartheta=g
$$

and, because of $E=3 G$ for an incompressible body, we represent the stress distribution as follows: in the elastic zone

$$
\sigma_{z}=\sigma_{0} e, \quad \tau_{z \theta}=\frac{\sigma_{0}}{\sqrt{3}} g \varrho, \quad 0<\varrho<\varrho_{b} \quad \text { or } \quad \beta<\varrho<\varrho_{b},
$$


and in the plastic zone

$$
\sigma_{z}=\frac{\sigma_{0} e}{\sqrt{e^{2}+g^{2} \varrho^{2}}}, \quad \tau_{z \theta}=\frac{\sigma_{0} g \varrho}{\sqrt{3} \sqrt{e^{2}+g^{2} \varrho^{2}}}, \quad \varrho_{b}<\varrho<1,
$$

where $\varrho=r / b, \varrho_{b}=r_{b} / b, \beta=a / b$. Formulae (27.12) are now clearly connected with the Hencky-Ilyushin theory, and thus with simple loading, since the LevyMises theory cannot be applied to elastic-plastic problems.

Now we restrict our considerations to circular cross-sections, $0 \leqslant \varrho \leqslant 1$. Introducing dimensionless, generalized stresses by (27.8) and integrating over the respective zones, we obtain

$$
\begin{aligned}
& n=2 e \int_{0}^{e_{b}} \varrho d \varrho+2 e \int_{Q_{b}}^{1} \frac{\varrho d \varrho}{\sqrt{e^{2}+g^{2} \varrho^{2}}}, \\
& m_{t}=4 g \int_{0}^{e_{b}} \varrho^{3} d \varrho+4 g \int_{e_{b}}^{1} \frac{\varrho^{3} d \varrho}{\sqrt{e^{2}+g^{2} \varrho^{2}}} ;
\end{aligned}
$$

or, after integration,

$$
\begin{aligned}
n= & \varrho_{b}^{2} e+\frac{2 e}{g^{2}} \sqrt{e^{2}+g^{2}}-\frac{2 e}{g^{2}} \sqrt{e^{2}+g^{2} \varrho_{b}^{2}}, \\
m_{t}=\varrho_{b}^{4} g & +\frac{4}{g} \sqrt{e^{2}+g^{2}}-\frac{8}{3 g^{3}}\left(e^{2}+g^{2}\right)^{3 / 2}-\frac{4 \varrho_{b}^{2}}{g} \sqrt{e^{2}+g^{2} \varrho_{b}^{2}}+ \\
& +\frac{8}{3 g^{3}}\left(e^{2}+g^{2} \varrho_{b}^{2}\right)^{3 / 2} .
\end{aligned}
$$

The dimensionless boundary radius $\varrho_{b}$ is determined by the condition of continuity of stresses (or, in other words, by the condition $\varphi=1 / 2 G$ ), namely

$$
e^{2}+g^{2} \varrho_{b}^{2}=1, \quad \varrho_{b}=\frac{\sqrt{1-e^{2}}}{|g|} .
$$

So, the five quantities $n, m_{t}, e, g$ and $\varrho_{b}$ are interrelated by the three equations (27.14) and (27.15). Theoretically, these equations make it possible to express any three quantities in terms of the remaining two. Thirty formulae of this type may be derived; however, only twelve of them can be expressed effectively in terms of elementary functions, [4.628]. The relations which are most important for applications, namely $e=f\left(m_{t}, n\right)$ and $g=f\left(m_{t}, n\right)$ (generalized strains in terms of generalized stresses), cannot be expressed effectively, but paper [4.628] gives three-figure tables and diagrams of those functions.

The elastic carrying capacity of the cross-section is determined by the condition $\varrho_{b}=1$, i.e.

$$
e^{2}+g^{2}=1 \text {. }
$$


Formulae (27.14) then express generalized stresses in terms of one parameter only, for example $e$; eliminating this parameter, we obtain the equation of the elastic carrying capacity in terms of generalized stresses, namely

$$
m_{t}^{2}+n^{2}=1 \text {. }
$$

The curves (27.7) and (27.17) are shown in Fig. 142 together with the curves $e=$ const, $g=$ const, and $\varrho_{b}=$ const in the first quadrant of the plane $n-m_{t}$.

The above solution is based on the Hencky-Ilyushin deformation theory, valid for simple processes. The conditions of a simple process are realized here if the generalized strains $\varepsilon$ and $\vartheta$ increase proportionally; in the elastic-plastic range this requirement does not correspond exactly to a proportional increase of the generalized stresses $N$ and $M_{t}$ though the differences are rather small. Various loading paths were analysed by F. A. Gaydon [6.73], who applied the Prandtl-Reuss theory. A comparison of the results of various theories in the case under consideration was given by M. R. Shammamy [2.157] and this author together with O. M. Sidebottom [2.158]. A. G. Dorfman and S. D. Leytes [2.26, 6.140, 2.100] analysed various loading paths, using the Leytes hypothesis of stabilization (cf. Sec. 9.8). B. C. Hoskin [6.100] determined the torsional rigidity of a circular section following plastic deformations due to tension.

The first papers considering plastic hardening in round cross-sections under tension with torsion were based on the hypothesis of isotropic hardening $(\mathrm{H}$. Mii [6.156], S. W. Serensen, W. P. Kogayev and R. M. Shneyderovitch [5.164]). D. S. Brooks [6.32] applied the Ramberg-Osgood schematization of the stressstrain curve, (8.9), whereas J. Betten [6.20] considered asymptotically perfect plasticity. I. P. Renne, V. A. Ogorodnikov and V. G. Nakhaytchuk [6.201] discussed analytical treatment of experimental data. Numerous papers apply slip theories of plasticity to the case under consideration: A. F. Kregers [3.391] used Malmeyster's theory of locality of deformations, introducing here the concept of a compliance tensor; Payne's theory was applied by H. Payne and S. J. Czyzak [6.182]; the Leonov-Shvayko theory was used by N. Yu. Shvayko [6.220], this author together with Ya. I. Rudayev [3.735] and by Ya. I. Rudayev [3.683], [6.204], who discussed the problem of applicability of the deformation theory to non-proportional loading paths. I. M. Korovin [6.121] applied the Ilyushin geometrical theory of plasticity and discussed various loading paths. The range of finite strains was investigated by C. Hartung [6.88]. S. N. Makky [6.148] applied the Thomas theory and determined the surfaces of instability corresponding to initiation of fracture.

Many papers considered variable (cyclic) loadings and shakedown problems in this relatively simple combined case. The first paper is due to P. S. Symonds [4,562], whereas V. I. Rozenblyum [4.488] took also the possibility of variable temperature into account. A. I. Kuznetsov [6.133] applied the Arutyunyan-Vakulenko theory [3.13], considering cyclic torsion under the constraints $N=$ const or $\varepsilon=$ const, and observing the increase of $\varepsilon$ or decrease of $N$, respectively; similar effects were investigated by H. P. Shrivastava, Z. Mróz and R. N. Dubey [3.731]. 
A. P. Gusenkov and M. P. Gavrilov [6.86] analysed the conditions of applicability of the deformation theory to cyclic non-proportional loadings. Experimental investigations were carried out by G. Mets [6.152], M. Ohnami and K. Shiozawa [6.174], G. Schröder and R. Zeller [6.209], and by V. M. Radhakrishnan [6.185, 6.186].

The simplest shape of the cross-section, namely a thin-walled annular section, ensures practically full homogeneity of stresses, $\mathscr{P}_{2} \rightarrow \mathscr{S}_{2} \rightarrow \mathscr{B}_{2}$, and has frequently been used to experimental verification of the basic equations of plasticity. The first experiments of that type were carried out by K. Hohenemser [3.262] in 1931. Numerous further papers will be quoted and classified in Sec. 37.6 devoted to thin-walled tubes.

The relative simplicity of the case of simultaneous tension with torsion of a thin-walled annular section permitted also the development of the theory of plastic waves in this case. The basic equations of the theory were derived in 1960 by R. J. Clifton [6.49]. Further theoretical papers were published by the same author $[6.50,6.51]$, H. Fukuoka $[6.68,6.69]$ T. C. T. Ting $[6.252,6.253]$, R. P. Goel and L. E. Malvern [6.77], C. D. Myers and M. A. Eisenberg [6.167], H. C. Wu and H. C. Lin [6.266]. Experimental verification of the theory was carried out by J. Lipkin and R. J. Clifton [6.143, 6.144], U. S. Lindholm [6.141], H. Fukuoka and T. Masui [6.70]; the agreement of theoretical and experimental results, however, was not always satisfactory.

\subsection{Influence of elastic compressibility}

The influence of compressibility of the material results in some qualitative differences in comparison with the incompressible solution. The coefficient of transversal deformation in the plastic zone (external) becomes larger than Poisson's ratio $v$ and that zone acts compressively on the internal, elastic zone. Hence new stress components $\sigma_{r}$ and $\sigma_{\theta}$ appear and the case under consideration may be classified as $\mathscr{P}_{4} / \mathscr{S}_{2}$.

Under the restriction to a simple loading process we may use the HenckyIlyushin equations, which for a compressible material take the form (9.29). The assumption of the strain distribution (27.1) is here also justified, and the distribution of $\varepsilon_{r}$ and $\varepsilon_{\theta}$ is to be determined. Substituting for $\tau_{z \theta}$ the formula (27.2) (the relevant formula for $\sigma_{z}$, however, being no longer valid), we may describe the problem by the following system of basic equations:

$$
\begin{aligned}
& \varepsilon_{r}=\varphi \sigma_{r}+\left(\frac{1}{9 K}-\frac{\varphi}{3}\right)\left(\sigma_{r}+\sigma_{\theta}+\sigma_{z}\right), \\
& \varepsilon_{\theta}=\varphi \sigma_{\theta}+\left(\frac{1}{9 K}-\frac{\varphi}{3}\right)\left(\sigma_{r}+\sigma_{\theta}+\sigma_{z}\right), \\
& \varepsilon=\varphi \sigma_{z}+\left(\frac{1}{9 K}-\frac{\varphi}{3}\right)\left(\sigma_{r}+\sigma_{\theta}+\sigma_{z}\right), \quad \varepsilon_{r}-\varepsilon_{\theta}-r \frac{d \varepsilon_{\theta}}{d r}=0, \\
& \sigma_{\theta}-\sigma_{r}-r \frac{d \sigma_{r}}{d r}=0, \quad \sigma_{r}^{2}+\sigma_{\theta}^{2}+\sigma_{z}^{2}-\sigma_{r} \sigma_{\theta}-\sigma_{\theta} \sigma_{z}-\sigma_{z} \sigma_{r}+\frac{3 \vartheta^{2} r^{2}}{4 \varphi^{2}}=\sigma_{0}^{2} .
\end{aligned}
$$


This system contains six unknown functions: $\varepsilon_{r}, \varepsilon_{\theta}, \sigma_{r}, \sigma_{\theta}, \sigma_{z}$ and $\varphi$. Eliminating $\varepsilon_{r}$ nad $\varepsilon_{\theta}$ from the first two equations, $\sigma_{\theta}$ from the fifth and $\sigma_{z}$ from the third, we arrive at a system of two equations with two unknowns $\sigma_{r}$ and $\varphi$. Following the present author's paper [4.628], we write this system in a dimensionless form as follows:

$$
\begin{gathered}
\left(4 \psi^{2} \varrho p^{3}+5 \psi \varrho p^{2}+\varrho p\right) s_{r}^{\prime \prime}+\left(-4 \psi^{2} \varrho p^{2} p^{\prime}+12 \psi^{2} p^{3}-2 \psi \varrho p p^{\prime}+15 \psi p^{2}-\right. \\
\left.-\varrho p^{\prime}+3 p\right) s_{r}^{\prime}-6 \psi^{2} p^{2} p^{\prime} s_{r}+2 \psi(1+\psi) e p^{2} p^{\prime}=0, \\
\left(12 \psi^{2} \varrho^{2} p^{2}+6 \psi \varrho^{2} p+3 \varrho^{2}\right) s_{r}^{\prime 2}+36 \psi^{2} \varrho p^{2} s_{r} s_{r}^{\prime}+36 \psi^{2} p^{2} s_{r}^{2}- \\
-12 \psi(1+\psi) e p^{2}\left(2 s_{r}+\varrho s_{r}^{\prime}\right)+4 g^{2} \psi^{2} \varrho^{2} p^{4}+8 g^{2} \psi \varrho^{2} p^{3}+ \\
+\left[4 g^{2} \varrho^{2}-4 \psi^{2}+4(1+\psi)^{2} e^{2}\right] p^{2}-8 \psi p-4=0 .
\end{gathered}
$$

The following symbols are introduced in those equations:

$$
s_{r}=\frac{\sigma_{r}}{\sigma_{0}}, \quad p=\frac{1}{2 G \varphi}, \quad \psi=\frac{1-2 \nu}{2(1+\nu)} ;
$$

hence $s_{r}$ denotes the dimensionless radial stress (and not, as usual, the deviatoric component), $p$ the dimensionless secant modulus, $\psi$ a dimensionless material constant, equal to zero for an incompressible material and equal to $1 / 2$ for $\nu=0$. Moreover, $e$ and $g$ are introduced by formulae (27.10), and primes denote differentiation with respect to the dimensionless radius $\varrho=r / b$.

We quoted system (27.19) in order to demonstrate the complications introduced to the theory of plasticity by the phenomenon of compressibility of material. A computer solution for given values of $e, g$, and $\psi$ presents no difficulties, but lack of a closed-form solution makes a general analysis more difficult. To obtain an analytical solution, in [4.628] the perturbation method was applied with the material constant $\psi,(27.20)$, as a small parameter. Indeed, $\psi=0$ corresponds to an incompressible material and thus to a simple solution (27.12). Assuming the solution of system (27.19) in the form of power series

$$
s_{r}=\sum_{i=0}^{\infty} s_{r i}(\varrho) \psi^{i}, \quad p=\sum_{i=0}^{\infty} p_{i}(\varrho) \psi^{i},
$$

substituting these series in (27.19) and equating the coefficients of the successive powers of $\psi$, we obtain a sequence of systems of equations with respect to the functions $s_{r i}$ and $p_{i}$. For the zeroth approximation we obtain the system

$$
\begin{aligned}
& \varrho p_{0} s_{r 0}^{\prime \prime}+\left(-\varrho p_{0}^{\prime}+3 p_{0}\right) s_{r 0}^{\prime}=0, \\
& 3 \varrho^{2} s_{r 0}^{\prime 2}+4 g^{2} \varrho^{2} p_{0}^{2}+4 e^{2} p_{0}^{2}-4=0
\end{aligned}
$$

with the solution

$$
s_{r 0} \equiv 0, \quad p_{0}=\frac{1}{\sqrt{e^{2}+g^{2} \varrho^{2}}} .
$$

This solution satisfies of course the boundary condition $s_{r 0}(1)=0$; making use of the remaining equations of the basic system (27.18), we finally arrive at formulae (27.12) derived earlier in a simpler way. 
The first approximation will be determined by equating the coefficients of $\psi_{1}$ on both sides of equations (27.19); we arrive at the system

$$
\begin{aligned}
& \varrho\left(e^{2}+g^{2} \varrho^{2}\right)^{2} s_{r 1}^{\prime \prime}+\left(3 e^{2}+4 g^{2} \varrho^{2}\right)\left(e^{2}+g^{2} \varrho^{2}\right) s_{r 1}^{\prime}=2 e g^{2} \varrho, \\
& \left(e^{2}+g^{2} \varrho^{2}\right)^{2} p_{1}+e^{2} \sqrt{e^{2}+g^{2} \varrho^{2}}-e^{2}=0 .
\end{aligned}
$$

The second equation determines directly the first correction for the secant modulus

$$
p_{1}=-\frac{e^{2}\left(\sqrt{e^{2}+g^{2} \varrho^{2}}-1\right)}{\left(e^{2}+g^{2} \varrho^{2}\right)^{2}},
$$

whereas the general integral of the first equation has the form

$$
s_{r 1}=\frac{1}{e} \ln \frac{e^{2}+g^{2} \varrho^{2}}{\varrho^{2}}-\frac{2 e}{g^{2} \varrho^{2}}+C_{1}\left(\frac{g^{2}}{e^{3}} \ln \frac{\sqrt{e^{2}+g^{2} \varrho^{2}}+e}{\varrho}-\frac{e^{2}+g^{2} \varrho^{2}}{e^{2} \varrho^{2}}\right)+C_{2} .
$$

The constants of integration $C_{1}$ and $C_{2}$ depend on whether we consider the elastic-plastic range or full plastification of the cross-section. In the elastic-plastic range we first have to determine the boundary radius $\varrho_{b}$; equating the expression $p_{0}+\psi p_{1}$ to unity, we again arrive at formula (27.15), and hence the compressibility of the material has no influence on that radius (with the accuracy of the first approximation). Further, we have to determine the stress distribution in the elastic zone. It may easily be found that there occurs a homogeneous state of stress, namely uniform compression in the radial and the circumferential directions and tension in the axial direction (for $e>0$ ). Since $\sigma_{r}=\sigma_{\theta}$ in the elastic zone, the equilibrium

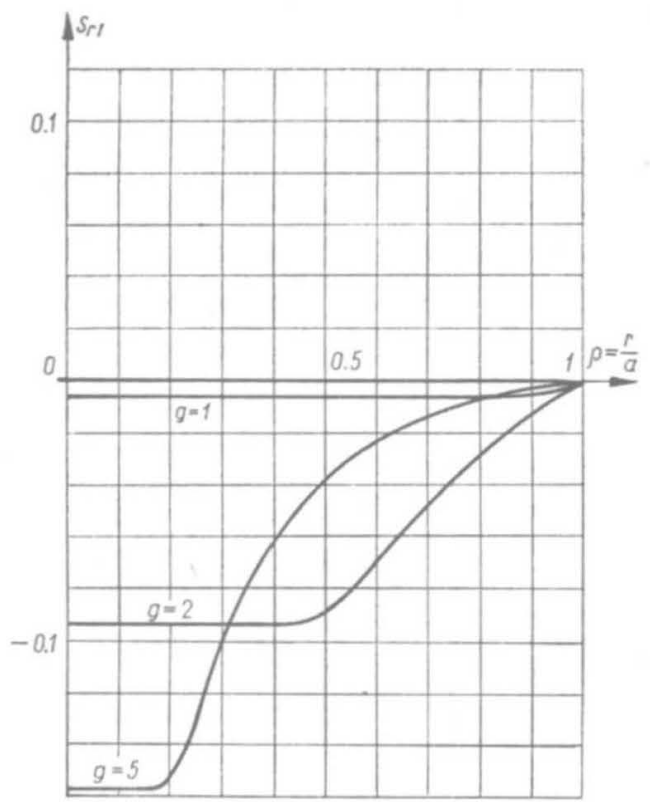

Fig. 143. Distribution of radial stresses in a circular section under tension with torsion in the case of a compressible material 
equation yields the boundary condition $s_{r 1}^{\prime}=0$ for $\varrho=\varrho_{b}$. The second boundary condition obviously has the form $s_{r 1}=0$ for $\varrho=1$; hence, having determined the integration constants, in view of (27.15), we finally obtain

$$
\begin{aligned}
s_{r 1}=- & \left\{\frac{1+e^{2}}{e^{2}} \ln \frac{\sqrt{e^{2}+g^{2} \varrho^{2}}+e}{\varrho\left(\sqrt{e^{2}+g^{2}}+e\right)}-\frac{1}{e} \ln \frac{e^{2}+g^{2} \varrho^{2}}{\left(e^{2}+g^{2}\right) \varrho^{2}}+\right. \\
& \left.+\frac{2 e}{g^{2}}\left(\frac{1}{\varrho^{2}}-1\right)-\frac{\left(1+e^{2}\right)\left(\sqrt{e^{2}+g^{2} \varrho^{2}}-\varrho^{2} \sqrt{e^{2}+g^{2}}\right)}{e g^{2} \varrho^{2}}\right\} .
\end{aligned}
$$

Paper [4.628] gives also the formulae for $\sigma_{\theta 1}$ and $\sigma_{z 1}$. The diagrams of the stresses $\sigma_{r}$ and $\sigma_{0}$ for $e=0.5$ and the various values of the unit angle of twist $g$ are shown in Figs. 143 and 144.

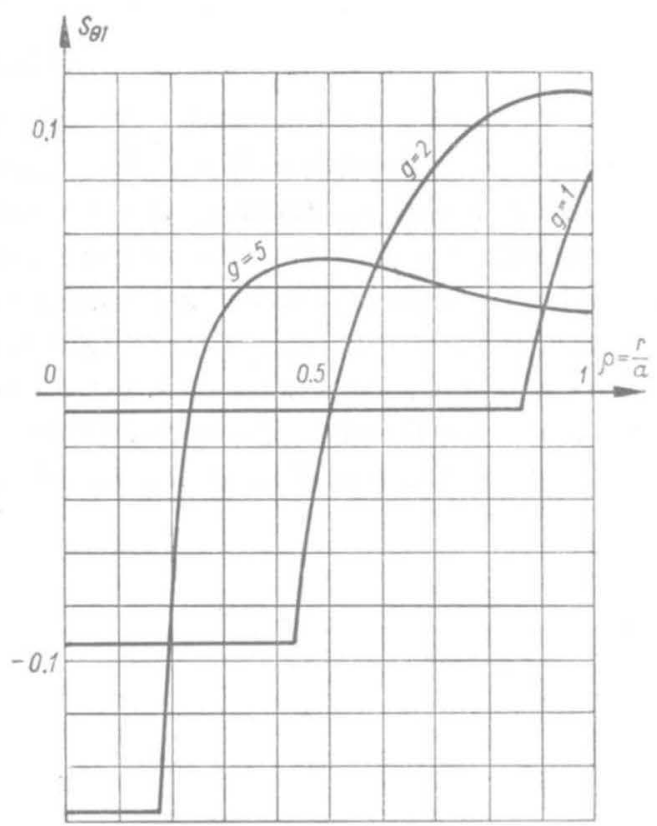

Fig. 144. Distribution of circumferential stresses in a circular section under tension with torsion in the case of a compressible material

In the case of a fully plastic section the boundary condition $s_{r 1}(1)=0$ remains, whereas the second integration constant may be found from the condition of boundedness of the function $s_{r 1}$ at the singular point $\varrho=0$. Finally one obtains

$$
\begin{aligned}
S_{r 1}=- & {\left[\frac{2}{e} \ln \frac{\left(\sqrt{e^{2}+g^{2} \varrho^{2}}+e\right) \sqrt{e^{2}+g^{2}}}{\left(\sqrt{e^{2}+g^{2}}+e\right) \sqrt{e^{2}+g^{2} \varrho^{2}}}+\frac{2 e}{g^{2}}\left(\frac{1}{\varrho^{2}}-1\right)-\right.} \\
& \left.-\frac{2}{g^{2} \varrho^{2}}\left(\sqrt{e^{2}+g^{2} \varrho^{2}}-\varrho^{2} \sqrt{e^{2}+g^{2}}\right)\right] .
\end{aligned}
$$

Diagrams of stresses in this case may be found in [4.628]. Also in this case generalized stresses can be expressed in terms of generalized strains by means of elementary 
functions; however, the formulae are very lengthy, while the numerical differences with respect to incompressibility are of the order of a few per cent.

The problem of simultaneous tension and torsion of circular bars in the case of a compressible material was first considered by V. V. Sokolovsky [6.225] in 1946; he proposed an iterative method of solution. The Prandtl-Reuss equations for non-simple processes were applied by G. Svéd and D. S. Brooks [6.249]; they derived a system of four hyperbolic partial differential equations and obtained a solution for increasing normal force at a constant torque. Even here the numerical differences with respect to the case of incompressibility are very small. Linear plastic hardening with compressibility taken into account was considered in this case by J. O. Smith and O. M. Sidebottom [6.221].

\subsection{Differential equation in displacements for arbitrary shapes of the cross-section}

In the case of torsion of non-round bars the section is subject to warping regardless of the elasticity or inelasticity of deformation. Additional tension does not change the situation, and hence the strain distribution (27.1), based on the hypothesis of plane cross-sections, cannot be accepted.

To obtain an exact solution of the problem under consideration we may use one of the two basic approaches described in Sec. 19: either the introduction of an appropriate stress function or reduction to a displacement equation. Historically, a stress function was first to be used: the Prandtl function is suitable here. This function was introduced in 1944 by G. H. Handelman [4.199] and later by R. Hill [4.209, 2.56], for a more general case of plastic tension with torsion and bending; this approach will be discussed in Sec. 29.1. However, from the viewpoint of numerical calculations, the introduction of a stress function has some drawbacks: even small errors in the values of the stress function may result in much larger errors in stresses which are expressed in terms of partial derivatives of that function.

The displacement approach does not exhibit this drawback. On the contrary, even relatively large errors in displacements result, as a rule, in small errors in stresses (though partial derivatives of displacements also occur in the final formulae) and in even smaller errors in generalized stresses. Hence we now quote the derivation of the displacement equation, given by M. Wnuk [4.605].

Assume a Cartesian system of coordinates with the $z$-axis coincident with the axis of the bar (as in Sec. 27.2). Under the assumption of material incompressibility we may write, as in the classical de Saint-Venant problem,

$$
u=\vartheta y z-\frac{1}{2} \varepsilon x, \quad v=-\vartheta x z-\frac{1}{2} \varepsilon y, \quad w=\vartheta w_{0}(x, y)+\varepsilon z,
$$

where the warping function $w_{0}(x, y)$ will be treated as an unknown. As in Sec. 27.2 the symbols $\varepsilon$ and $\vartheta$ denote the unit elongation and the unit angle of twist, respectively; when discussing the limit carrying capacity of the cross-section, we may formally regard them as the corresponding rates, in connection with the Levy-Mises 
theory. Making use of the law of similarity of deviators, we obtain for the stresses the following expressions:

$$
\begin{aligned}
& \sigma_{x}=\sigma_{y}=\tau_{x y}=0, \quad \sigma_{z}=\frac{3 \varepsilon}{2 \varphi}, \\
& \tau_{x z}=\frac{\vartheta}{2 \varphi}\left(y+\frac{\partial w_{0}}{\partial x}\right), \quad \tau_{z y}=\frac{\vartheta}{2 \varphi}\left(-x+\frac{\partial w_{0}}{\partial y}\right),
\end{aligned}
$$

where $\varphi$ is a modulus in the Hencky-Ilyushin or in the Levy-Mises equations. Further, making use of the HMH yield condition, (26.1) with $c=3$, we determine the function $\varphi$ by the formula

$$
\varphi=\frac{\sqrt{3}}{2 \sigma_{0}} \sqrt{3 \varepsilon^{2}+\vartheta^{2}\left[\left(y+\frac{\partial w_{0}}{\partial x}\right)^{2}+\left(-x+\frac{\partial w_{0}}{\partial y}\right)^{2}\right]},
$$

and the non-vanishing stresses by the formulae

$$
\begin{gathered}
\sigma_{z}=\frac{\varepsilon \sigma_{\varepsilon^{2}+\frac{\vartheta^{2}}{3}\left[\left(y+\frac{\partial w_{0}}{\partial x}\right)^{2}+\left(-x+\frac{\partial w_{0}}{\partial y}\right)^{2}\right]}}{\tau_{z x}=\frac{\vartheta \sigma_{0}\left(y+\frac{\partial w_{0}}{\partial x}\right)}{3 \sqrt{\varepsilon^{2}+\frac{\vartheta^{2}}{3}\left[\left(y+\frac{\partial w_{0}}{\partial x}\right)^{2}+\left(-x+\frac{\partial w_{0}}{\partial y}\right)^{2}\right]}},} \\
\tau_{z y}=\frac{\vartheta \sigma_{0}\left(-x+\frac{\partial w_{0}}{\partial y}\right)}{\sqrt[3]{\varepsilon^{2}+\frac{\vartheta^{2}}{3}\left[\left(y+\frac{\partial w_{0}}{\partial x}\right)^{2}+\left(-x+\frac{\partial w_{0}}{\partial y}\right)^{2}\right]}} .
\end{gathered}
$$

Substitution of these formulae into the only equilibrium equation which is not identically satisfied, yields the following governing equation for the problem under consideration:

$$
\begin{gathered}
\nabla^{2} w_{0}+\frac{\vartheta^{2}}{3 \varepsilon^{2}}\left[\left(y+\frac{\partial w_{0}}{\partial x}\right)^{2} \frac{\partial^{2} w_{0}}{\partial y^{2}}-2\left(y+\frac{\partial w_{0}}{\partial x}\right)\left(-x+\frac{\partial w_{0}}{\partial y}\right) \frac{\partial^{2} w_{0}}{\partial x \partial y}+\right. \\
\left.+\left(-x+\frac{\partial w_{0}}{\partial y}\right)^{2} \frac{\partial^{2} w_{0}}{\partial x^{2}}\right]=0 .
\end{gathered}
$$

This non-linear second-order partial differential equation may be interpreted as a certain particular case of general displacement equations for an incompressible medium, (19.15). The boundary condition for this equation, expressing the tangency of shearing stresses to the contour has the form

$$
\left(y+\frac{\partial w_{0}}{\partial x}\right) d y-\left(-x+\frac{\partial w_{0}}{\partial y}\right) d x=0 .
$$


Having solved Eq. (27.33) with the boundary condition (27.34) for the given shape of the contour, we may effectively determine the stresses (27.32), and then the axial force $N$ and the twisting moment $M_{t}$ in the limit state, corresponding to full plastification of the cross-section. These quantities are determined by the integrals

$$
\begin{aligned}
& N=\sigma_{0} \operatorname{sign} \varepsilon \iint_{A} \frac{d A}{\sqrt{1+\frac{\vartheta^{2}}{3 \varepsilon^{2}}\left[\left(y+\frac{\partial w_{0}}{\partial x}\right)^{2}+\left(-x+\frac{\partial w_{0}}{\partial y}\right)^{2}\right]}}, \\
& M_{t}=\frac{\vartheta \sigma_{0}}{3|\varepsilon|} \iint_{A} \frac{\left[\left(y+\frac{\partial w_{0}}{\partial x}\right) y-\left(-x+\frac{\partial w_{0}}{\partial y}\right) x\right] d A}{\frac{1+\frac{\vartheta^{2}}{3 \varepsilon^{2}}\left[\left(y+\frac{\partial w_{0}}{\partial x}\right)^{2}+\left(-x+\frac{\partial w_{0}}{\partial y}\right)^{2}\right]}{\sqrt{1+(y)}}},
\end{aligned}
$$

Eliminating from these equations the parameter $\vartheta / \varepsilon$, we may determine-at least theoretically - the plastic interaction curve in an explicit form. For a circular crosssection, assuming $w_{0}=0$, we indeed obtain the exact equation (27.7). For other shapes of the cross-section we usually have to apply numerical or approximate analytical methods of integration of (27.33).

As an example let us consider a very narrow rectangular cross-section. It may easily be checked that the function $w_{0}=x y$ satisfies exactly Eq. (27.33). Substituting this function into the boundary condition (27.34), we obtain $d y=0$; hence the segments of the contour can only be parallel to the $x$-axis. Strictly speaking, this boundary condition corresponds to an infinite strip parallel to the $x$-axis, Fig. 145,

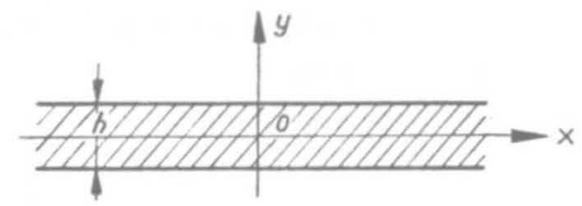

Fig. 145. A narrow rectangular cross-section

however, this shape of the cross-section is of little practical importance. On the other hand, this simple solution may be regarded as an approximate solution for a narrow rectangular cross-section $b \times h$, where $h \ll b$. Formulae (27.32) give in this case

$$
\sigma_{z}=\frac{\varepsilon \sigma_{0}}{\sqrt{\varepsilon^{2}+\frac{4}{3} \vartheta^{2} y^{2}}}, \quad \tau_{z x}=\frac{2 \vartheta \sigma_{0} y}{3 \sqrt{\varepsilon^{2}+\frac{4}{3} \vartheta^{2} y^{2}}}, \quad \tau_{z y} \cong 0 .
$$

These formulae were first obtained by A. R. Rzhanitsyn [4.492] in some other way. For a rectangular cross-section with a finite dimension $b$ along the $x$-axis the solution (27.36) can hardly be regarded as an exact one; the "trickiness" of approximation consists in the fact that-according to Rzhanitsyn-the twisting moment produced by the stresses $\tau_{z y}$ (small stresses, large arm) is, in fact, equal to that pro- 
duced by $\tau_{z x}$ (large stresses, small arm), and hence if we integrate $\tau_{z x}$ only, we have to multiply the result by 2 . The results of integration are as follows:

$$
\begin{gathered}
N=b \int_{-h / 2}^{h / 2} \sigma_{z} d y=\sqrt{3} \frac{\varepsilon}{\vartheta} b \sigma_{0} \ln \left(\frac{\sqrt{3}}{3} \frac{\vartheta}{\varepsilon} h+\sqrt{1+\frac{1}{3} \frac{\vartheta^{2}}{\varepsilon^{2}} h^{2}}\right), \\
M_{t}=2 b \int_{-h / 2}^{h / 2} \tau_{z x} y d y=-\frac{b h \sigma_{0}}{2} \frac{\varepsilon}{\vartheta} \sqrt{1+\frac{1}{3} \frac{\vartheta^{2}}{\varepsilon^{2}} h^{2}-} \\
-\frac{\sqrt{3}}{2} b \sigma_{0} \frac{\varepsilon^{2}}{\vartheta^{2}} \ln \left(\frac{\sqrt{3}}{3} \frac{\vartheta}{\varepsilon} h+\sqrt{1+\frac{1}{3} \frac{\vartheta^{2}}{\varepsilon^{2}} h^{2}}\right) .
\end{gathered}
$$

These equations may jointly be regarded as a parametrical presentation of the plastic interaction curve with $\varepsilon / \vartheta$ as a parameter (we have assumed here $\varepsilon>0$ and $\vartheta>0$ ). Eliminating this parameter, we may derive an implicit equation of the limit curve; an explicit form, however, cannot be expressed in terms of elementary functions.

\subsection{Perturbation method. Elastic-plastic range}

Two basic variants of the perturbation method may be applied to Eq. (27.33): either the ratio $\vartheta / \varepsilon$ or the ratio $\varepsilon / \vartheta$ may be regarded as a small parameter. In the first case the zeroth approximation pertains to pure tension, and in the second case to pure torsion. The solution in the first case is much easier: firstly, pure tension does not exhibit any singularities and the convergence of the perturbation method may be expected to be good; secondly, putting in Eq. (27.33) $\vartheta / \varepsilon=0$, we obtain not the zeroth but the first approximation, since the function $w_{0}$ itself is multiplied by $\vartheta$, (27.29). Hence we give here only the solution for small torsion superposed on tension, regarding the parameter $\vartheta / \varepsilon$ as small.

If we assume the solution of (27.33) in the form of the power series

$$
w_{0}=\sum_{i=0}^{\infty} w_{0 i}\left(\frac{\vartheta}{\varepsilon}\right)^{2 i}=w_{00}+w_{01}\left(\frac{\vartheta}{\varepsilon}\right)^{2}+w_{02}\left(\frac{\vartheta}{\varepsilon}\right)^{4}+\ldots .
$$

then the successive "corrections" $w_{0 i}$ will be determined by the equations

$$
\begin{aligned}
\nabla^{2} w_{00}=0, & \nabla^{2} w_{01}=-\frac{1}{3}\left[\left(y+\frac{\partial w_{00}}{\partial x}\right)^{2} \frac{\partial^{2} w_{00}}{\partial y^{2}}+\left(-x+\frac{\partial w_{00}}{\partial y}\right)^{2} \frac{\partial^{2} w_{00}}{\partial x^{2}}-\right. \\
& \left.-2\left(y+\frac{\partial w_{00}}{\partial x}\right)\left(-x+\frac{\partial w_{00}}{\partial y}\right) \frac{\partial^{2} w_{00}}{\partial x \partial y}\right],
\end{aligned}
$$

with the non-homogeneous boundary condition $(27.34)$ for the function $w_{00}$ and the homogeneous boundary conditions

$$
\frac{\partial w_{0 i}}{\partial x} d y-\frac{\partial w_{0 i}}{\partial y} d x=0
$$


for the remaining functions $w_{0 i}$. The first equation of the system (27.40) is the Laplace equation with respect to the function $w_{00}$ and together with the boundary condition of the type (27.34) describes the process of elastic torsion of a bar with given shape of the cross-section. Particular integrals of that equation for many cross-section shapes are well known. The subsequent equations, also linear, are of Poisson's type, in any case much simpler than the original non-linear equation (27.33).

The determination even of the harmonic function $w_{00}$ is sufficient to obtain one term of the series for $M_{t}$ and two terms of the series for $N$. Namely, in the already mentioned paper by $M$. Wnuk [4.605] the following formulae were derived:

$$
\begin{aligned}
& M_{t}=\frac{1}{3} \frac{\vartheta}{\varepsilon} \sigma_{0} \iint_{A}\left[\left(y+\frac{\partial w_{00}}{\partial x}\right) y-\left(-x+\frac{\partial w_{00}}{\partial y}\right) x\right] d A+\ldots, \\
& N=\sigma_{0} A-\frac{\sigma_{0}}{6}\left(\frac{\vartheta}{\varepsilon}\right)^{2} \iint_{A}\left[\left(y+\frac{\partial w_{00}}{\partial x}\right)^{2}+\left(-x+\frac{\partial w_{00}}{\partial y}\right)^{2}\right] d A+\ldots,
\end{aligned}
$$

and it was shown that the values of the integrals appearing in (27.42) are equal to each other and equal to the doubled volume of the Prandtl hill for the corresponding problem of elastic torsion. Moreover, M. Wnuk proposed for the plastic interaction curve Hermite's boundary approximation of the type

$$
A M_{t}^{2}+B N^{2}+C N^{3}=1,
$$

making use of the coincidence of the first two terms of the power series expansions of (27.42) and (27.43) and of the coincidence of the limit carrying capacity in pure torsion. The form of Eq. (27.43) was chosen in such a way as to obtain for a circular section the exact solution (27.7). Paper [4.606] gives examples of expansions (27.42) and of approximations (27.43) for various shapes of the cross-section, paper [6.264] is devoted to a direct design of the cross-section with respect to limit carrying capacity and [4.608] gives a comparison of the elastic carrying capacity and the limit carrying capacity.

Some papers propose lower bounds and upper bounds to the limit carrying capacity in the case under consideration. E. A. Beylin [6.23] proposed for a rectangular section a statically admissible stress field, assuming a sinusoidal distribution of shearing stresses along the shorter side. A simpler approach of the type (26.4) was used by K. A. Reckling [2.141]. More accurate lower and upper bounds were obtained by M. Wnuk [6.263], who proposed, for example, for an upper bound the distribution of displacements (27.29) with $w_{0}$ corresponding to warping in pure plastic torsion. Approximate equations of the plastic interaction curve based on simple statically partially admissible stress fields for various cross-sections were derived by Z. Sobotka [5.171].

Considering tension with torsion of bars with an arbitrary cross-section in the elastic-plastic range, we have to satisfy (under the assumptions of incompressibility and of a simple loading process) (27.33) in the plastic zone, the harmonic equation $\nabla^{2} w=0$ in the elastic zone, to determine the elastic-plastic interface, to satisfy the 
continuity conditions along that interface and to satisfy the boundary condition (27.34). This difficult problem is considerably simplified if we apply the perturbation method, since in the first approximation the displacement equation for the plastic zone is identical with that for the elastic zone and the continuity conditions are satisfied automatically.

If we know the solution of the equation $\nabla^{2} w=0$ with the boundary condition (27.34) for the given shape of the cross-section, we just have to determine the elastic-plastic interface and generalized stresses by means of appropriate integrations. The elastic-plastic interface may be determined from the condition stating that the stresses in the elastic zone have to satisfy the yield condition of the type (26.1); hence we obtain

$$
\left(\frac{E \varepsilon}{\sigma_{0}}\right)^{2}+3\left(\frac{G \vartheta}{\sigma_{0}}\right)^{2}\left[\left(y+\frac{\partial w^{e}}{\partial x}\right)^{2}+\left(-x+\frac{\partial w^{e}}{\partial y}\right)^{2}\right]=1 .
$$

M. Wnuk [4.607] determined this line effectively for the following shapes: ellipse, equilateral triangle and wing-type profile; moreover, for the elliptic cross-section the relations between generalized stresses and generalized strains were derived.

Elastic-plastic tension with torsion of a bar with a narrow rectangular crosssection was considered by V. A. Kolgadin [6.117].

Isotropic plastic hardening in the case under consideration was investigated by S. I. Ivanov [6.109], who applied the method of elastic solutions combined with finite differences. A similar method was applied by B. P. Kishkin [6.113] to the problem of elastic-plastic stress concentration in a hyperbolic notch. S. Komatsu and T. Sakimoto [6.120] discussed the elastic-plastic-hardening tension with torsion of box-sections. I. M. Korovin [6.121] investigated the influence of the loading path on the behaviour of a bar with a narrow rectangular section in the plastic hardening range. C. Clavuot [6.48] considered arbitrary convex cross-sections in the case of a rigid-plastic material with hardening of the Ziegler type, (14.30), giving a detailed solution for circular and annular sections.

\subsection{Plastic tension with torsion of naturally twisted bars}

By a naturally uniformly twisted bar we understand a bar formed by simultaneous shift and proportional rotation of an arbitrary cross-section about a straight axis (Fig. 146). The corresponding coefficient of proportionality $\vartheta_{0}$ will be called the unit angle of natural twist. Naturally twisted bars often appear in engineering applications (spiral drills, air screws, etc.); following the papers by M. Życzkowski and M. Galos [4.641], A. Dudek and M. Życzkowski [4.127], we now consider the problem of fully plastic tension with torsion in such bars.

Introduce the following system of curvilinear coordinates (Fig. 146):

$$
\xi=x \cos \vartheta_{0} z+y \sin \vartheta_{0} z, \quad \eta=-x \sin \vartheta_{0} z+y \cos \vartheta_{0} z, \quad \zeta=z .
$$




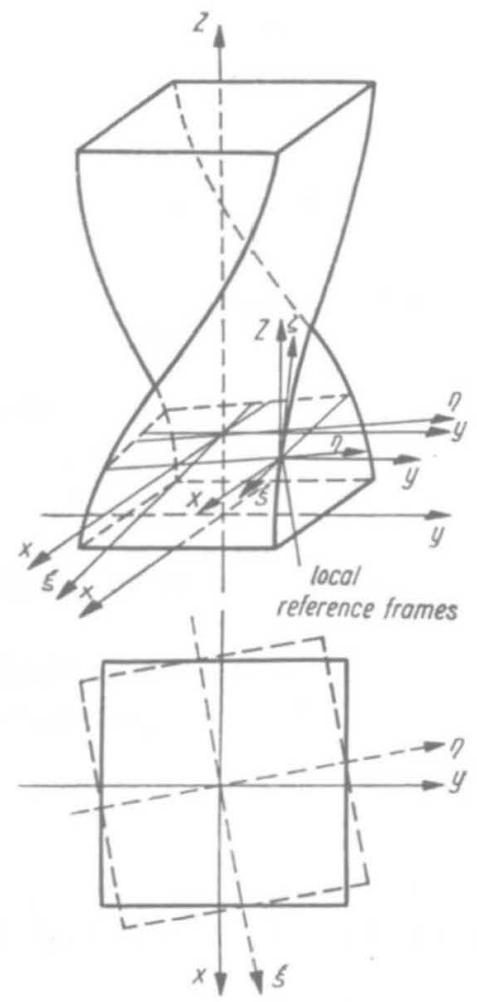

Fig. 146. A naturally uniformly twisted bar

In these coordinates the problem of tension with torsion is reduced to the twodimensional case, since all the derivatives of stresses and strains with respect to $\zeta$ vanish. Conversely, the assumption $\partial / \partial \zeta=0$, corresponding to the general longitudinal homogeneity of stresses and strains, leads here to tension and torsion only, since the bending of naturally twisted bars does not conform to this assumption. The lines $\xi, \eta, \zeta$ (described subsequently by the equations $\eta=$ const, $\zeta=$ const; $\zeta=$ const, $\xi=$ const; $\xi=$ const, $\eta=$ const) are not locally orthogonal, but we refer the stresses and strains to the locally orthogonal system $\xi, \eta, z$.

To derive the basic equations of the theory of plasticity in the coordinates $\xi, \eta, \zeta$ we transform the stresses, strains and displacements to the system $\xi, \eta, z$ and transform differentiation with respect to $x, y, z$ into differentiation with respect to $\xi, \eta, \zeta$. Such an approach, used for example by G. Yu. Dzhanelidze and A. I. Lurie $[6.60,6.61]$, seems to be the simplest in the case under consideration. The transformation of stresses and strains (or strain rates) is a two-dimensional tensorial one:

$$
\begin{aligned}
& \sigma_{x}=\sigma_{\xi} \cos ^{2} \vartheta_{0} \zeta+\sigma_{\eta} \sin ^{2} \vartheta_{0} \zeta-2 \tau_{\xi \eta} \sin \vartheta_{0} \zeta \cos \vartheta_{0} \zeta, \\
& \cdots \cdots \cdots \cdots \cdots \cdots \cdots \cdots \cdots \cdots \cdots \cdots \cdots \cdots \cdots \cdots \cdots \cdots \cdots \cdots \cdots \cdots \cdots \cdots \cdots \cdots \\
& \sigma_{z}=\sigma_{z},
\end{aligned}
$$


and the displacements (or velocities) are transformed as vectors:

$$
\begin{aligned}
& u_{x}=u_{\xi} \cos \vartheta_{0} \zeta-u_{\eta} \sin \vartheta_{0} \zeta, \\
& u_{y}=u_{\xi} \sin \vartheta_{0} \zeta+u_{\eta} \cos \vartheta_{0} \zeta, \\
& u_{z}=u_{z} .
\end{aligned}
$$

The differentiation is transformed as follows:

$$
\begin{aligned}
& \frac{\partial}{\partial x}=\frac{\partial}{\partial \xi} \frac{\partial \xi}{\partial x}+\frac{\partial}{\partial \eta} \frac{\partial \eta}{\partial x}+\frac{\partial}{\partial \zeta} \frac{\partial \zeta}{\partial x}=\cos \vartheta_{0} \zeta \frac{\partial}{\partial \xi}-\sin \vartheta_{0} \zeta \frac{\partial}{\partial \eta} \\
& \frac{\partial}{\partial y}=\sin \vartheta_{0} \zeta \frac{\partial}{\partial \xi}+\cos \vartheta_{0} \zeta \frac{\partial}{\partial \eta} \\
& \frac{\partial}{\partial z}=\vartheta_{0} \eta \frac{\partial}{\partial \xi}-\vartheta_{0} \xi \frac{\partial}{\partial \eta}+\frac{\partial}{\partial \zeta} .
\end{aligned}
$$

In what follows, the derivatives of stresses and strains with respect to $\zeta$ will be omitted.

Substituting (27.46) and (27.48) into the equations of internal equilibrium without body forces (6.9), we may write the first two equations in the general form

$$
\begin{aligned}
& \Omega_{1}\left[\sigma_{i j}\right] \sin \vartheta_{0} \zeta+\Omega_{2}\left[\sigma_{i j}\right] \cos \vartheta_{0} \zeta=0, \\
& \Omega_{1}\left[\sigma_{i j}\right] \cos \vartheta_{0} \zeta-\Omega_{2}\left[\sigma_{i j}\right] \sin \vartheta_{0} \zeta=0,
\end{aligned}
$$

where $\Omega_{1}$ and $\Omega_{2}$ are certain differential operators. Hence $\Omega_{1}\left[\sigma_{i j}\right]=\Omega_{2}\left[\sigma_{i j}\right]=0$ and finally, with the third equation added, we arrive at the following system of equilibrium equations:

$$
\begin{aligned}
& \frac{\partial \sigma_{\xi}}{\partial \xi}+\frac{\partial \tau_{\xi \eta}}{\partial \eta}+\vartheta_{0}\left(\eta \frac{\partial \tau_{\xi z}}{\partial \xi}-\xi \frac{\partial \tau_{\xi z}}{\partial \eta}-\tau_{\eta z}\right)=0, \\
& \frac{\partial \tau_{\xi \eta}}{\partial \xi}+\frac{\partial \sigma_{\eta}}{\partial \eta}+\vartheta_{0}\left(\eta \frac{\partial \tau_{\eta z}}{\partial \xi}-\xi \frac{\partial \tau_{\eta z}}{\partial \eta}+\tau_{\xi z}\right)=0, \\
& \frac{\partial \tau_{\xi z}}{\partial \xi}+\frac{\partial \tau_{\eta z}}{\partial \eta}+\vartheta_{0}\left(\eta \frac{\partial \sigma_{z}}{\partial \xi}-\xi \frac{\partial \sigma_{z}}{\partial \eta}\right)=0 .
\end{aligned}
$$

Similarly, substituting formulae analogous to (27.46) for strains, (27.47) for displacements, and (27.48) into the Cauchy equations (7.21), we compare the coefficients of the corresponding trigonometrical functions on both sides of the subsequent equations and obtain

$$
\begin{aligned}
& \varepsilon_{\xi}=\frac{\partial u_{\xi}}{\partial \xi}, \quad \varepsilon_{\eta}=\frac{\partial u_{\eta}}{\partial \eta}, \quad \gamma_{\xi \eta}=\frac{\partial u_{\xi}}{\partial \eta}+\frac{\partial u_{\eta}}{\partial \xi}, \\
& \varepsilon_{z}=\frac{\partial u_{z}}{\partial \zeta}+\vartheta_{0}\left(\eta \frac{\partial u_{z}}{\partial \xi}-\xi \frac{\partial u_{z}}{\partial \eta}\right), \\
& \gamma_{\xi z}=\frac{\partial u_{z}}{\partial \xi}+\frac{\partial u_{\xi}}{\partial \zeta}+\vartheta_{0}\left(\eta \frac{\partial u_{\xi}}{\partial \xi}-\xi \frac{\partial u_{\xi}}{\partial \eta}-u_{\eta}\right), \\
& \gamma_{\eta z}=\frac{\partial u_{z}}{\partial \eta}+\frac{\partial u_{\eta}}{\partial \zeta}+\vartheta_{0}\left(\eta \frac{\partial u_{\eta}}{\partial \xi}-\xi \frac{\partial u_{\eta}}{\partial \eta}+u_{\xi}\right) .
\end{aligned}
$$


Further, we transform the compatibility conditions (7.49) into the form (the derivatives with respect to $\zeta$ being omitted)

$$
\begin{aligned}
& \frac{\partial^{2} \varepsilon_{\xi}}{\partial \eta^{2}}+\frac{\partial^{2} \varepsilon_{\eta}}{\partial \xi^{2}}=\frac{\partial^{2} \gamma_{\xi \eta}}{\partial \xi \partial \eta} \\
& \frac{\partial^{2} \varepsilon_{z}}{\partial \xi^{2}}=\vartheta_{0}\left\{A\left[\gamma_{\xi z}\right]-\frac{\partial \gamma_{\eta z}}{\partial \xi}\right\}- \\
& -\vartheta_{0}^{2}\left\{\eta A\left[\varepsilon_{\xi}\right]-\xi B\left[\varepsilon_{\eta}\right]-2\left(\eta \frac{\partial \gamma_{\xi \eta}}{\partial \xi}-\xi \frac{\partial \gamma_{\xi \eta}}{\partial \eta}+\varepsilon_{\xi}-\varepsilon_{\eta}\right)\right\}, \\
& \frac{\partial^{2} \varepsilon_{z}}{\partial \eta^{2}}=\vartheta_{0}\left\{B\left[\gamma_{\eta z}\right]+\frac{\partial \gamma_{\xi z}}{\partial \eta}\right\}- \\
& -\vartheta_{0}^{2}\left\{\eta A\left[\varepsilon_{\eta}\right]-\xi B\left[\varepsilon_{\xi}\right]+2\left(\eta \frac{\partial \gamma_{\xi \eta}}{\partial \xi}-\xi \frac{\partial \gamma_{\xi \eta}}{\partial \eta}+\varepsilon_{\xi}-\varepsilon_{\eta}\right)\right\}, \\
& 2 \frac{\partial^{2} \varepsilon_{z}}{\partial \xi \partial \eta}=\vartheta_{0}\left\{A\left[\gamma_{\eta z}\right]+B\left[\gamma_{\xi z}\right]-\frac{\partial \gamma_{\eta z}}{\partial \eta}+\frac{\partial \gamma_{\xi z}}{\partial \xi}\right\}- \\
& -\vartheta_{0}^{2}\left\{\eta A\left[\gamma_{\xi \eta}\right]-\xi B\left[\gamma_{\xi \eta}\right]+4 \eta\left(\frac{\partial \varepsilon_{\xi}}{\partial \xi}-\frac{\partial \varepsilon_{\eta}}{\partial \xi}\right)-\right. \\
& \left.-4 \xi\left(\frac{\partial \varepsilon_{\xi}}{\partial \eta}-\frac{\partial \varepsilon_{\eta}}{\partial \eta}\right)-4 \gamma_{\xi \eta}\right\} \\
& \frac{\partial}{\partial \xi}\left(\frac{\partial \gamma_{\eta z}}{\partial \xi}-\frac{\partial \gamma_{\xi z}}{\partial \eta}\right)=\vartheta_{0}\left\{-2 B\left[\varepsilon_{\xi}\right]+A\left[\gamma_{\xi \eta}\right]+2 \frac{\partial \varepsilon_{\xi}}{\partial \xi}-2 \frac{\partial \varepsilon_{\eta}}{\partial \xi}+2 \frac{\partial \gamma_{\xi \eta}}{\partial \eta}\right\} \text {, } \\
& \frac{\partial}{\partial \eta}\left(\frac{\partial \gamma_{\eta z}}{\partial \xi}-\frac{\partial \gamma_{\xi z}}{\partial \eta}\right)=\vartheta_{0}\left\{2 A\left[\varepsilon_{\eta}\right]-B\left[\gamma_{\xi \eta}\right]-2 \frac{\partial \varepsilon_{\xi}}{\partial \eta}+2 \frac{\partial \varepsilon_{\eta}}{\partial \eta}+2 \frac{\partial \gamma_{\xi \eta}}{\partial \xi}\right\},
\end{aligned}
$$

where the operators $A$ and $B$ are as follows:

$$
A=\frac{\partial}{\partial \xi}\left(\eta \frac{\partial}{\partial \xi}-\xi \frac{\partial}{\partial \eta}\right), \quad B=\frac{\partial}{\partial \eta}\left(\eta \frac{\partial}{\partial \xi}-\xi \frac{\partial}{\partial \eta}\right) .
$$

For small angles of natural twist $\vartheta_{0}$, the first compatibility condition refers to the two-dimensional problem in the plane $\xi \eta$, the next three conditions - to the elongation $\varepsilon_{z}$, and the last two-to the problem of torsion.

Let us now transform the boundary conditions. If the boundary surface of the body is described by the equation $F(x, y, z)=0$, then the stress boundary conditions (6.3) may be written in Cartesian coordinates as follows (W. Krzyś, M. Życzkowski [2.91]):

$$
\pm p_{n i} \sqrt{F_{, j} F_{, j}}=\sigma_{i k} F_{, k},
$$

where $p_{n i}$ denote surface tractions, $i=x, y, z$. Transforming these tractions according to (27.47), the stresses according to (27.46) and the derivatives as indicated by (27.48) and bearing in mind that $\partial F / \partial \zeta=0$, we arrive at 


$$
\begin{aligned}
& \pm p_{n \xi} \sqrt{F_{\xi}^{\prime 2}+F_{\eta}^{\prime 2}+\vartheta_{0}^{2}\left(\eta F_{\xi}^{\prime}-\xi F_{\eta}^{\prime}\right)^{2}}=\sigma_{\xi} F_{\xi}^{\prime}+\tau_{\xi \eta} F_{\eta}^{\prime}+\vartheta_{0}\left(\eta F_{\xi}^{\prime}-\xi F_{\eta}^{\prime}\right) \tau_{\xi x}, \\
& \pm p_{n \eta} \sqrt{F_{\xi}^{\prime 2}+F_{\eta}^{\prime 2}+\vartheta_{0}^{2}\left(\eta F_{\xi}^{\prime}-\xi F_{\eta}^{\prime}\right)^{2}}=\tau_{\xi \eta} F_{\xi}^{\prime}+\sigma_{\eta} F_{\eta}^{\prime}+\vartheta_{0}\left(\eta F_{\xi}^{\prime}-\xi F_{\eta}^{\prime}\right) \tau_{\eta z}, \\
& \pm p_{n z} \sqrt{F_{\xi}^{\prime 2}+F_{\eta}^{\prime 2}+\vartheta_{0}^{2}\left(\eta F_{\xi}^{\prime}-\xi F_{\eta}^{\prime}\right)^{2}}=\tau_{\xi z} F_{\xi}^{\prime}+\tau_{\eta z} F_{\eta}^{\prime}+\vartheta_{0}\left(\eta F_{\xi}^{\prime}-\xi F_{\eta}^{\prime}\right) \sigma_{z} .
\end{aligned}
$$

Hence for a free surface the expressions on the right-hand side of these equations are equal to zero. The conditions along the discontinuity lines, possible under pure torsion, were discussed in [4.641].

Since the stresses and strains are expressed in a locally orthogonal system, the Hencky-Ilyushin physical equations have the form (9.28); these equations may also be understood as the Levy-Mises ones if we formally treat $e_{i j}$ as strain rates. Finally, we assume incompressibility, (9.23), and the $\mathrm{HMH}$ yield condition in the form (11.6).

Thus we have derived the whole system of 16 equations, namely three equilibrium equations (27.50), six geometrical equations (27.51) or (27.52), six physical equations (9.28) and (9.23) and the yield condition (11.6). These equations contain also 16 unknowns: stresses, strains or strain rates, displacements or velocities and the function $\varphi$. In the limit state it is assumed that these equations hold for the body as a whole.

The solution of the problem under consideration is much simpler if we apply the perturbation method with $\vartheta_{0}$ as a small parameter: then the zeroth-order approximation refers to a prismatic bar. The solutions of the problems of pure tension and pure torsion are well known here; the problem of combined tension with torsion was discussed in Sec. 27.4.

We present the solution in the form

$$
\begin{array}{rlrl}
\sigma_{i j} & =\sum_{n=0}^{\infty} \sigma_{i j n} \vartheta_{0}^{n}, & \varepsilon_{i j} & =\sum_{n=0}^{\infty} \varepsilon_{i j n} \vartheta_{0}^{n}, \\
u_{i} & =\sum_{n=0}^{\infty} u_{i n} \vartheta_{0}^{n}, & \varphi=\sum_{n=0}^{\infty} \varphi_{n} \vartheta_{0}^{n} .
\end{array}
$$

In the zeroth-order approximation, $\sigma_{\xi 0} \equiv \sigma_{\eta 0} \equiv \tau_{\xi \eta 0} \equiv \gamma_{\xi \eta 0} \equiv 0$; the equations may be reduced here to the displacement equation (27.33). For the higher-order approximations $n \geqslant 1$ we obtain the following system of equations, linear with respect to the successive unknowns:

$$
\begin{aligned}
& \frac{\partial \sigma_{\xi n}}{\partial \xi}+\frac{\partial \tau_{\xi \eta n}}{\partial \eta}=-\eta \frac{\partial \tau_{\xi z(n-1)}}{\partial \xi}+\xi \frac{\partial \tau_{\xi z(n-1)}}{\partial \eta}+\tau_{\eta z(n-1)}, \\
& \frac{\partial \tau_{\xi \eta n}}{\partial \xi}+\frac{\partial \sigma_{\eta n}}{\partial \eta}=-\eta \frac{\partial \tau_{\eta z(n-1)}}{\partial \xi}+\xi \frac{\partial \tau_{\eta z(n-1)}}{\partial \eta}-\tau_{\xi z(n-1)}, \\
& \frac{\partial \tau_{\xi z n}}{\partial \xi}+\frac{\partial \tau_{\eta z n}}{\partial \eta}=-\eta \frac{\partial \sigma_{z(n-1)}}{\partial \xi}+\xi \frac{\partial \sigma_{z(n-1)}}{\partial \eta}
\end{aligned}
$$




$$
\begin{aligned}
\frac{\partial^{2} \varepsilon_{\xi n}}{\partial \eta^{2}}+ & \frac{\partial^{2} \varepsilon_{\eta n}}{\partial \xi^{2}}-\frac{\partial^{2} \gamma_{\xi \eta n}}{\partial \xi \partial \eta}=0, \\
\frac{\partial^{2} \varepsilon_{z n}}{\partial \xi^{2}}= & A\left[\gamma_{\xi z(n-1)}\right]-\frac{\partial \gamma_{\eta z(n-1)}}{\partial \xi}-\eta A\left[\varepsilon_{\xi(n-2)}\right]+\xi B\left[\varepsilon_{\xi(n-2)}\right]+ \\
& +2\left(\eta \frac{\partial \gamma_{\xi \eta(n-2)}}{\partial \xi}-\xi \frac{\partial \gamma_{\xi \eta(n-2)}}{\partial \eta}+\varepsilon_{\xi(n-2)}-\varepsilon_{\eta(n-2)}\right), \\
\frac{\partial^{2} \varepsilon_{z n}}{\partial \eta^{2}}= & \left.B\left[\gamma_{\eta z(n-1)}\right]+\frac{\partial \gamma_{\xi z(n-1)}}{\partial \eta}-\eta A\left[\varepsilon_{\eta(n-2)}\right]+\xi B\left[\varepsilon_{\eta(n-2)}\right)\right]- \\
& -2\left(\eta \frac{\partial \gamma_{\xi \eta(n-2)}}{\partial \xi}-\xi \frac{\partial \gamma_{\xi \eta(n-2)}}{\partial \eta}+\varepsilon_{\xi(n-2)}-\varepsilon_{\eta(n-2)}\right),
\end{aligned}
$$$$
2 \frac{\partial^{2} \varepsilon_{z n}}{\partial \xi \partial \eta}=A\left[\gamma_{\eta z(n-1)}\right]+B\left[\gamma_{\xi z(n-1)}\right]-\frac{\partial \gamma_{\eta z(n-1)}}{\partial \eta}+\frac{\partial \gamma_{\xi z(n-1)}}{\partial \xi}-
$$$$
-\eta A\left[\gamma_{\xi \eta(n-2)}\right]+\xi B\left[\gamma_{\xi \eta(n-2)}\right]-4 \eta\left(\frac{\partial \varepsilon_{\xi(n-2)}}{\partial \xi}-\frac{\partial \varepsilon_{\eta(n-2)}}{\partial \xi}\right)-
$$$$
-4 \xi\left(\frac{\partial \varepsilon_{\xi(n-2)}}{\partial \eta}-\frac{\partial \varepsilon_{\eta(n-2)}}{\partial \eta}\right)+4 \gamma_{\xi \eta(n-2)},
$$

$$
\begin{aligned}
\frac{\partial^{2} \gamma_{\eta z n}}{\partial \xi^{2}}-\frac{\partial^{2} \gamma_{\xi z n}}{\partial \xi \partial \eta}= & -2 B\left[\varepsilon_{\xi(n-1)}\right]+A\left[\gamma_{\xi(n-1)}\right]+ \\
& +2\left(\frac{\partial \varepsilon_{\xi(n-1)}}{\partial \xi}-\frac{\partial \varepsilon_{\eta(n-1)}}{\partial \xi}+\frac{\partial \gamma_{\eta \xi(n-1)}}{\partial \eta}\right),
\end{aligned}
$$

$$
\begin{aligned}
\frac{\partial^{2} \gamma_{\eta z n}}{\partial \xi \partial \eta}-\frac{\partial^{2} \gamma_{\xi z n}}{\partial \eta^{2}} & =2 A\left[\varepsilon_{\eta(n-1)}\right]-B\left[\gamma_{\xi \eta(n-1)}\right]- \\
& -2\left(\frac{\partial \varepsilon_{\xi(n-1)}}{\partial \eta}-\frac{\partial \varepsilon_{\eta(n-1)}}{\partial \eta}-\frac{\partial \gamma_{\xi(n-1)}}{\partial \xi}\right),
\end{aligned}
$$

$\varepsilon_{\xi n}+\varepsilon_{\eta n}+\varepsilon_{z n}=0$,

$\varepsilon_{z n}-\varepsilon_{\xi n}-\varphi_{0}\left(\sigma_{z n}-\sigma_{\xi n}\right)-\varphi_{n} \sigma_{z 0}=\sum_{i=1}^{n-1} \varphi_{i}\left[\sigma_{z(n-i)}-\sigma_{\xi(n-i)}\right]$,

$\varepsilon_{z n}-\varepsilon_{\eta n}-\varphi_{0}\left(\sigma_{z n}-\sigma_{\eta n}\right)-\varphi_{n} \sigma_{z 0}=\sum_{i=1}^{n-1} \varphi_{i}\left[\sigma_{z(n-i)}-\sigma_{\eta(n-i)}\right]$,

$\gamma_{\xi \eta n}-2 \varphi_{0} \tau_{\xi \eta n}=2 \sum_{i=1}^{n-1} \varphi_{i} \tau_{\xi \eta(n-i)}$

$\gamma_{\eta z n}-2\left(\varphi_{0} \tau_{\eta_{z n}}+\varphi_{n} \tau_{\eta z 0}\right)=2 \sum_{i=1}^{n-1} \varphi_{i} \tau_{\eta z(n-i)}$,

$\gamma_{\xi z n}-2\left(\varphi_{0} \tau_{\xi \geq n}+\varphi_{n} \tau_{\xi=0}^{*}\right)=2 \sum_{i=1}^{n-1} \varphi_{i} \tau_{\xi z(n-i)}$, 


$$
\begin{aligned}
& \sigma_{z 0}\left(2 \sigma_{z n}-\sigma_{\xi n}-\sigma_{\eta n}\right)+6 \tau_{\xi z 0} \tau_{\xi z n}+6 \tau_{\eta z 0} \tau_{\eta z n} \\
&=-\sum_{i=1}^{n-1}\left[\sigma_{\xi i} \sigma_{\xi(n-i)}+\sigma_{\eta i} \sigma_{\eta(n-i)}+\sigma_{z i} \sigma_{z(n-i)}-\sigma_{\xi l} \sigma_{\eta(n-i)}-\sigma_{\eta i} \sigma_{z(n-i)}-\right. \\
&\left.-\sigma_{z i} \sigma_{\xi(n-i)}+3 \tau_{\xi \eta l} \tau_{\xi \eta(n-i)}+3 \tau_{\xi z i} \tau_{\xi z(n-i)}+3 \tau_{\eta x i} \tau_{\eta z(n-i)}^{-}\right]
\end{aligned}
$$

The boundary conditions (27.55) at the free lateral surface of the bar now take the form

$$
\begin{aligned}
& \sigma_{\xi n} F_{\xi}^{\prime}+\tau_{\xi \eta n} F_{\eta}^{\prime}=-\left(\eta F_{\xi}^{\prime}-\xi F_{\eta}^{\prime}\right) \tau_{\xi z(n-1)}, \\
& \tau_{\xi \eta n} F_{\xi}^{\prime}+\sigma_{\eta n} F_{\eta}^{\prime}=-\left(\eta F_{\xi}^{\prime}-\xi F_{\eta}^{\prime}\right) \tau_{\eta z(n-1)}, \\
& \tau_{\xi z n} F_{\xi}^{\prime}+\tau_{\eta z n} F_{\eta}^{\prime}=-\left(\eta F_{\xi}^{\prime}-\xi F_{\eta}^{\prime}\right) \sigma_{z(n-1)} .
\end{aligned}
$$

External loadings, determining the limit carrying capacity (plastic interaction curve in the combined case) are given by the formulae

$$
\begin{aligned}
& N=\sum_{n=0}^{\infty} \vartheta_{0}^{n} \iint_{A} \sigma_{z n} d A, \\
& M_{t}=\sum_{n=0}^{\infty} \vartheta_{0}^{n} \iint_{A}\left(\tau_{\eta_{z n}} \xi-\tau_{\xi z n} \eta\right) d A,
\end{aligned}
$$

$A$ being the cross-sectional area of the bar.

Papers [4.641] and [4.127] give no effective solution for the combined case of torsion with tension. On the other hand, in [4.641] an example of pure torsion and of pure tension for a square cross-section was considered, whereas [4.127] gives several examples of pure tension. In the latter case the situation is much simpler since, instead of 16 equations at each step of the approximation, we have only 5 equations for any of the odd "corrections" and only 11 equations for any of the even "corrections". Effective solutions were given for an elliptic cross-section and for a circular cross-section with a small circular notch.

\section{Bending with torsion}

\subsection{Differential equation in displacements}

Bending with torsion may often be encountered in various engineering applications both in mechanical and in building structures. This case does not exhibit circular symmetry and hence the problem of formulation for non-circular sections is not more difficult than for circular ones.

Basic approaches to the problem are similar to those applied to tension with torsion of non-circular sections. The first papers by G. H. Handelman [4.199] and R. Hill [4.209, 2.56] used the Prandtl stress function; however, the displacement approach is more convenient in many cases, particularly when we look for approximate solutions. Such an approach was proposed by S. Piechnik and M. Życzkowski 
in $[4.453,4.454]$; moreover, $\mathrm{S}$. Piechnik analysed in [4.455] the non-linear creep of a bar under bending with torsion.

In extreme cases both of pure bending and of pure torsion of a circular bar, the Bernoulli hypothesis of plane sections leads to exact results since no warping of the section appears. It turns out, however, that in the inelastic range this hypothesis contradicts the equations" of internal equilibrium, and hence-it may furnish only an upper bound to the actual limit carrying capacity of the cross-section. Such bounds were derived by R. Hill and M. P. L. Siebel [6.95], A. R. Rzhanitsyn [6.207], I. N. Mirolyubov [6.162], and J. Mandel [3.494].

To obtain an exact formulation of the problem we allow for the warping of the section and assume-for an incompressible material-the displacement distribution in Cartesian coordinates in the form

$$
\begin{aligned}
u & =\varkappa x z+\vartheta u_{0}(y, z), \\
v & =-\frac{1}{2} x y z+\vartheta x z, \\
w & =-\frac{1}{4} x\left(2 x^{2}-y^{2}+z^{2}\right)-\vartheta x y .
\end{aligned}
$$

This distribution corresponds to the moments $M_{b}$ and $M_{t}$ constant along the axis $x$. The bar axis is denoted here by $x$ as in Chapter $\mathrm{V}$ and in contradistinction to Sec. 27, where the notation $z$ was more convenient. The symbols $x$ and $\theta$ denote the curvature and the unit angle of twist, respectively; when using the Levy-Mises theory of plasticity, we may regard $x$ and $\vartheta$ as the relevant rates, but then the elastic-plastic range cannot be analysed.

Equations (28.1) result in the following distribution of strains:

$$
\begin{array}{ll}
\varepsilon_{x}=x z, & \gamma_{y z}=0, \\
\varepsilon_{y}=-\frac{1}{2} x z, & \gamma_{z x}=\vartheta\left(\frac{\partial u_{0}}{\partial z}-y\right), \\
\varepsilon_{z}=-\frac{1}{2} x z, & \gamma_{x y}=\vartheta\left(\frac{\partial u_{0}}{\partial y}+z\right) ;
\end{array}
$$

the Hencky-Ilyushin equations or the Levy-Mises equations lead to the following stress distribution:

$$
\begin{array}{rlrl}
\sigma_{x} & =\frac{3}{2 \varphi} x z, & \tau_{y z}=0, \\
\sigma_{y}=0, & \tau_{z x}=\frac{\vartheta}{2 \varphi}\left(\frac{\partial u_{0}}{\partial z}-y\right), \\
\sigma_{z}=0, & \tau_{x y}=\frac{\vartheta}{2 \varphi}\left(\frac{\partial u_{0}}{\partial y}+z\right) .
\end{array}
$$

The normal stresses $\sigma$ might here be changed by an arbitrary but equal value of the mean stress increment $\Delta \sigma_{m}$, but the boundary conditions result in $\Delta \sigma_{m}=0$.

Two further equations must be satisfied: the first equation of internal equlibrium and the yield condition of the type (26.1). The Handelman-Hill approach consists 
in the elimination of the equilibrium equation by means of the Prandtl stress function; this function is then governed by the yield condition. Conversely, using the displacement approach, we first eliminate $\varphi$ from the yield condition. Namely, substituting (28.3) into (26.1) and assuming $c=3$ (HMH condition), we obtain

$$
\varphi=\frac{1}{2 \sigma_{0}} \sqrt{9 x^{2} z^{2}+3 \vartheta^{2}\left[\left(\frac{\partial u_{0}}{\partial y}+z\right)^{2}+\left(\frac{\partial u_{0}}{\partial z}-y\right)^{2}\right]} .
$$

The succesive substitution of (28.4) into (28.3) and into the equilibrium equation results in the following differential equation with respect to the warping function $u_{0}$ :

$$
\begin{aligned}
3 x^{2} z\left(z \nabla^{2} u_{0}\right. & \left.-\frac{\partial u_{0}}{\partial z}\right)+\vartheta^{2}\left[\left(\frac{\partial u_{0}}{\partial z}-y\right)^{2} \frac{\partial^{2} u_{0}}{\partial y^{2}}-2\left(\frac{\partial u_{0}}{\partial z}-y\right) \times\right. \\
& \left.\times\left(\frac{\partial u_{0}}{\partial y}+z\right) \frac{\partial^{2} u_{0}}{\partial y \partial z}+\left(\frac{\partial u_{0}}{\partial y}+z\right)^{2} \frac{\partial^{2} u_{0}}{\partial z^{2}}\right]=-3 x^{2} y z .
\end{aligned}
$$

This second-order equation is non-homogeneous, and the function $u_{0} \equiv 0$ is not an integral of this equation except for $x=0$, that is, in the case without bending. Moreover, warping is also equal to zero for zero torsion, since we have denoted warping by $\vartheta u_{0}$. The relevant boundary condition is analogous to (27.34), namely

$$
\left(\frac{\partial u_{0}}{\partial z}-y\right) d y-\left(\frac{\partial u_{0}}{\partial y}+z\right) d z=0 .
$$

Having solved Eq. (28.5) with the boundary condition (28.6), i.e. having determined the warping function $u_{0}$, we may calculate the bending moment $M_{b}$ and the twisting moment $M_{t}$, and determine the parametrical equations of the plastic interaction curve. These moments are given by the formulae

$$
\begin{aligned}
& M_{b}=\iint_{A} \sigma_{x} z d A=3 x \sigma_{0} \iint_{A} \frac{z^{2} d A}{\sqrt{9 x^{2} z^{2}+3 \vartheta^{2}\left[\left(\frac{\partial u_{0}}{\partial y}+z\right)^{2}+\left(\frac{\partial u_{0}}{\partial z}-y\right)^{2}\right]}}, \\
& M_{t}=\iint_{A}\left(\tau_{x y} z-\tau_{x z} y\right) d A=\vartheta \sigma_{0} \iint_{A} \frac{\left[\left(\frac{\partial u_{0}}{\partial y}+z\right) z-\left(\frac{\partial u_{0}}{\partial z}-y\right) y\right] d A}{\sqrt{9 x^{2} z^{2}+3 \vartheta^{2}\left[\left(\frac{\partial u_{0}}{\partial y}+z\right)^{2}+\left(\frac{\partial u_{0}}{\partial z}-y\right)^{2}\right]}} .
\end{aligned}
$$

\subsection{Solutions for a circular section by means of the perturbation method}

For a circular section the boundary condition (28.6) takes a particularly simple form in cylindrical coordinates. Namely, introducing the dimensionless coordinates

$$
\varrho=\frac{1}{b} \sqrt{y^{2}+z^{2}}, \quad \theta=\arctan \frac{z}{y},
$$


where $b$ stands for the radius of the circle (of the cross-section), we obtain

$$
\begin{aligned}
& 3 x^{2} b^{4} \varrho^{3} \sin \theta\left[\left(\varrho^{2} \frac{\partial^{2} u_{0}}{\partial \varrho^{2}}+\frac{\partial^{2} u_{0}}{\partial \theta^{2}}\right) \sin \theta-\frac{\partial u_{0}}{\partial \theta} \cos \theta\right]+ \\
& +\vartheta^{2}\left[\varrho \frac{\partial^{2} u_{0}}{\partial \varrho^{2}}\left(\frac{\partial u_{0}}{\partial \theta}-b^{2} \varrho^{2}\right)^{2}-2 \frac{\partial u_{0}}{\partial \varrho}\left(\varrho \frac{\partial^{2} u_{0}}{\partial \varrho \partial \theta}-\frac{\partial u_{0}}{\partial \theta}\right)\left(\frac{\partial u_{0}}{\partial \theta}-b^{2} \varrho^{2}\right)+\right. \\
& \left.+\varrho\left(\frac{\partial u_{0}}{\partial \varrho}\right)^{2}\left(\varrho \frac{\partial u_{0}}{\partial \varrho}+\frac{\partial^{2} u_{0}}{\partial \theta^{2}}\right)\right]=-3 x^{2} b^{6} \varrho^{5} \sin \theta \cos \theta
\end{aligned}
$$

with the boundary condition $\partial u_{0} / \partial \varrho=0$ for $\varrho=1$. The non-linear partial differential equation (28.9) suggests two basic variants of the perturbation method: either the ratio $x / \vartheta$ may be regarded as small, or the ratio $\vartheta / \varkappa$ may be regarded as small. In the first variant we determine the influence of bending on the limit carrying capacity in torsion, and in the second variant-the influence of torsion on bending. Both variants will be presented briefly after the papers by S. Piechnik [4.453], S. Piechnik and M. Życzkowski [4.454].

Assuming the ratio $x / \vartheta$ to be small, we introduce the dimensionless quantities

$$
\frac{x^{2}}{\vartheta^{2}}=\psi, \quad \frac{u_{0}}{b^{2}}=t,
$$

and rewrite (28.9) in the dimensionless form

$$
\begin{aligned}
& 3 \psi \varrho^{3} \sin \theta {\left[\left(\varrho^{2} t_{\rho \varrho}^{\prime \prime}+t_{\theta \theta}^{\prime \prime}\right) \sin \theta-t_{\theta}^{\prime} \cos \theta\right]+\varrho t_{e \varrho}^{\prime \prime}\left(t_{\theta}^{\prime}-\varrho^{2}\right)^{2}-} \\
&-2 t_{\rho}^{\prime}\left(\varrho t_{\varrho}^{\prime \prime}-t_{\theta}^{\prime}\right)\left(t_{\theta}^{\prime}-\varrho^{2}\right)+\varrho t_{\varrho}^{\prime 2}\left(\varrho t_{e}^{\prime}+t_{\theta \theta}^{\prime \prime}\right)=-3 \psi \varrho^{5} \sin \theta \cos \theta,
\end{aligned}
$$

where the primes denote differentiation with respect to the corresponding variables. The zeroth approximation (for $\psi=0$ ) equals zero; hence we substitute $t=t_{1} \psi+$ $+t_{2} \psi^{2}+\ldots$ and obtain, for the successive approximations $t_{i}(\varrho, \theta)$, the following sequence of relatively simple linear differential equations:

$$
\begin{aligned}
t_{1 e \varrho}^{\prime \prime}= & -3 \sin \theta \cos \theta, \\
\varrho^{3} t_{2 \varrho}^{\prime \prime}= & -3 \varrho \sin ^{2} \theta\left(\varrho^{2} t_{1 \varrho \varrho}^{\prime \prime}+t_{1 \theta \theta}^{\prime}\right)+ \\
& +3 \varrho \sin \theta \cos \theta t_{1 \theta}^{\prime}+2 \varrho t_{1 \varrho \ell}^{\prime \prime} t_{1 \theta}^{\prime}-2 \varrho t_{1 \varrho}^{\prime} t_{1 \varrho \theta}^{\prime \prime}+2 t_{1 \varrho}^{\prime} t_{1 \theta}^{\prime},
\end{aligned}
$$

Integration and application of the boundary conditions yields in turn

$$
\begin{aligned}
& t_{1}=3\left(\varrho-\frac{\varrho^{2}}{2}\right) \sin \theta \cos \theta, \\
& t_{2}=9\left[4 \varrho(\ln \varrho-1) \sin ^{3} \theta \cos \theta+\frac{1}{2}\left(\varrho-\frac{\varrho^{2}}{2}\right) \sin \theta \cos \theta\right],
\end{aligned}
$$

and hence

$$
t=3 \varrho \sin \theta \cos \theta\left\{\left(1-\frac{\varrho}{2}\right) \psi+3\left[4(\ln \varrho-1) \sin ^{2} \theta+\frac{1}{2}\left(1-\frac{\varrho}{2}\right)\right] \psi^{2}+\ldots\right\} .
$$


Calculating the function $\varphi$ from (28.4) and substituting into (28.3), we may determine the stress distribution! in the form of a power series of the small parameter $\psi$. However, a better convegence of the series may be obtained if we do not perform all the operations on the series but retain the form

$$
\begin{aligned}
\sigma_{x}= & \frac{\sigma_{0} \sqrt{3}}{\Omega} \varrho \sin \theta \sqrt{\psi}, \\
\tau_{x y}= & -\frac{\sigma_{0} \cos \theta}{\Omega \sqrt{3}}\left\{-\varrho+\frac{3}{2}\left(-\varrho+2 \cos ^{2} \theta\right) \psi+\frac{9}{4}[-\varrho+\right. \\
& \left.\left.+\left(16 \sin ^{2} \theta+2 \cos ^{2} \theta\right)+48(\ln \varrho-1) \sin ^{2} \theta \cos ^{2} \theta\right] \psi^{2}+\ldots\right\}, \\
\tau_{x z}= & \frac{\sigma_{0} \sin \theta}{\Omega \sqrt{3}}\left\{\varrho+\frac{3}{2}\left(-\varrho+2 \sin ^{2} \theta\right) \psi+\frac{9}{4}\left[-\varrho+48 \sin ^{2} \theta \cos ^{2} \theta-\right.\right. \\
& \left.\left.-16 \sin ^{4} \theta+2 \sin ^{2} \theta+16 \ln \varrho\left(\sin ^{4} \theta-2 \sin ^{2} \theta \cos ^{2} \theta\right)\right] \psi^{2}+\ldots\right\},
\end{aligned}
$$

where $\Omega$ denotes the square root of the series

$$
\begin{aligned}
\Omega^{2}= & \varrho^{3}+3\left[\varrho^{2} \cos ^{2} \theta-2 \varrho\left(\cos ^{2} \theta-\sin ^{2} \theta\right)\right] \psi+ \\
& +\frac{9}{4}\left[\left(3-4 \sin ^{2} \theta\right) \varrho+8\left(13 \sin ^{2} \theta \cos ^{2} \theta-4 \sin ^{4} \theta-\cos ^{2} \theta\right)-\right. \\
& \left.-32 \varrho \ln \varrho\left(3 \sin ^{2} \theta \cos ^{2} \theta-\sin ^{4} \theta\right)+4\left(1-3 \sin ^{2} \theta \cos ^{2} \theta\right)\right] \psi^{2}+\ldots
\end{aligned}
$$

These formulae make it possible to evaluate effectively the stresses in the crosssection; the resulting diagrams may be found in [4.453].

However, in order to calculate the moments (28.7) we have to expand the expression for $\Omega$ (to take the square root of the series) and perform division on the series. The convergence of the series detoriorates, but is then improved by integration. Finally one obtains

$$
\begin{aligned}
& M_{b}=\pi b^{3} \sigma_{0} \sqrt{3 \psi}\left(\frac{1}{3}-\frac{7}{8} \psi+\frac{639}{64} \psi^{2}-\ldots\right), \\
& M_{t}=\frac{\pi b^{3} \sigma_{0}}{\sqrt{3}}\left(\frac{2}{3}-\frac{1}{2} \psi+\frac{63}{32} \psi^{2}+\ldots\right) .
\end{aligned}
$$

Introducing dimensionless moments by the formulae $m_{b}=M_{b} / M_{b}$ and $m_{t}=M_{t} / \bar{M}_{t}$, we rewrite these equations as follows:

$$
\begin{aligned}
& m_{b}=1.3603 \sqrt{\psi}-3.5708 \psi^{3 / 2}+40.7452 \psi^{5 / 2}+\ldots, \\
& m_{t}=1-0.7500 \psi+2.9297 \psi^{2}+\ldots,
\end{aligned}
$$

and on eliminating the parameter $\psi$

$$
m_{t}=1-0.4053 m_{b}^{2}-0.1943 m_{b}^{4}+\ldots
$$

This series determines the plastic interaction curve $m_{t}=f\left(m_{b}\right)$ in a certain neighbourhood of the point corresponding to pure torsion. 
We now pass to the second variant of the perturbation method. Assume the ratio $\vartheta / x$ in (28.9) to be small; denote this ratio by $\lambda$ and introduce here a dimensionless warping function $t$ by the formula

$$
t=\frac{\vartheta u_{0}}{x b^{2}} \quad \text { or } \quad u_{0}=\frac{x}{\vartheta} b^{2} t
$$

Substituting (28.21) into (28.9), we rewrite the basic displacement equation in the form

$$
\begin{aligned}
& 3 \sin \theta\left[\left(\varrho^{2} t_{\varrho Q}^{\prime \prime}+t_{\theta \theta}^{\prime \prime}\right) \sin \theta-t_{\theta}^{\prime} \cos \theta\right]+\frac{1}{\varrho^{2}} t_{\varrho \varrho}^{\prime \prime}\left(t_{\theta}^{\prime}-\lambda \varrho^{2}\right)^{2}- \\
& -\frac{2}{\varrho^{3}} t_{\varrho}^{\prime}\left(\varrho t_{\varrho \theta}^{\prime \prime}-t_{\theta}^{\prime}\right)\left(t_{\theta}^{\prime}-\lambda \varrho^{2}\right)+\frac{1}{\varrho^{2}} t_{\varrho}^{\prime 2}\left(\varrho t_{\varrho}^{\prime}+t_{\theta \theta}^{\prime \prime}\right)=-3 \lambda \varrho^{2} \sin \theta \cos \theta .
\end{aligned}
$$

The warping function $t$ must be an odd function of the parameter $\lambda$, and hence we substitute $t=t_{1} \lambda+t_{2} \lambda^{3}+\ldots$; the successive corrections $t_{i}$ are determined by the following sequence of equations

$$
\begin{aligned}
& \varrho^{2} \sin \theta t_{1 \varrho \varrho}^{\prime \prime}+\sin \theta t_{1 \theta \theta}^{\prime \prime}-\cos \theta t_{1 \theta}^{\prime}=-\varrho^{2} \cos \theta, \\
& \varrho^{2} \sin \theta t_{2 \varrho \varrho}^{\prime \prime}+\sin \theta t_{2 \theta \theta}^{\prime \prime}-\cos \theta t_{2 \theta}^{\prime}=-\frac{1}{3 \sin \theta}\left[t_{1 \varrho \varrho}^{\prime \prime}\left(\frac{1}{\varrho} t_{1 \theta}^{\prime}-\varrho^{2}\right)^{2}-\right. \\
& \left.\quad-2 t_{1 \varrho}^{\prime}\left(t_{1 \varrho \theta}^{\prime \prime}-\frac{1}{\varrho} t_{1 \theta}^{\prime}\right)\left(\frac{1}{\varrho^{2}} t_{1 \theta}^{\prime}-1\right)+\frac{1}{\varrho} t_{1 \varrho}^{\prime 2}\left(t_{1 \varrho}^{\prime}+\frac{1}{\varrho} t_{1 \theta \theta}^{\prime \prime}\right)\right],
\end{aligned}
$$

These linear partial differential equations are of the elliptic type, much more difficult for integration than the parabolic equations (28.12). Their form suggests the application of Fourier series of the type

$$
t_{i}(\varrho, \theta)=\sum_{n=1}^{\infty} g_{i, n}(\varrho) \sin 2 n \theta,
$$

which lead to exact solutions, though no longer in a closed form. The lack of the sines of odd multiples of the angle $\theta$ and the lack of the cosines follow from the fact that warping becomes zero for $\theta=n \pi$ and from the symmetry conditions of the profile.

The system of equations (28.23) can now be written in the compact form

$$
\left(\varrho^{2} t_{i \varrho Q}^{\prime \prime}+t_{i \theta \theta}^{\prime \prime}\right) \sin \theta-t_{i 0}^{\prime} \cos \theta=\sum_{n=1}^{\infty} H_{t, n}(\varrho) \cos (2 n-1) \theta .
$$

The possibility of representing the right-hand side of these equations in the form of Fourier series in which only odd cosine terms appear follows also from the symmetry condition. For example, for $i=1$ we have $H_{1,1}(\varrho)=-\varrho^{2}, H_{1, n}(\varrho)=0$ for $n \geqslant 2$. Substituting (28.24) into (28.25) and changing the notation of some indices so as to obtain the argument of all cosines equal to $(2 n-1) \theta$, we arrive 
at the following system of ordinary differential equations with respect to the functions $g_{i, n}$ :

$$
\varrho^{2} g_{i, n}^{\prime \prime}-2 n(2 n+1) g_{i, n}=2 H_{i, n}+\varrho^{2} g_{i, n-1}^{\prime \prime}-2(n-1)(2 n-3) g_{l, n-1},
$$

$i=1,2, \ldots, n=1,2, \ldots$, where by $g_{i, 0}$ we understand a function identically equal to zero. Equations (28.26) are linear non-homogeneous equations of the Euler type. Denote their right-hand sides by $K_{i, n}=K_{i, n}\left(\varrho, g_{i, 1}, \ldots, g_{i, n-1}\right)$; then the integrals of (28.26) satisfying the boundary conditions $g_{i, n}^{\prime}(1)=0$ and $g_{i, n}$ bounded for $\varrho=0$ may be presented in the form

$$
\begin{aligned}
g_{i, n}= & -\frac{1}{4 n+1} \varrho^{-2 n} \int_{0}^{\varrho} \xi^{2 n-1} K_{i, n}(\xi) d \xi- \\
& -\frac{2 n}{(2 n+1)(4 n+1)} \varrho^{2 n+1} \int_{0}^{1} \xi^{2 n-1} K_{i, n}(\xi) d \xi- \\
& -\frac{1}{4 n+1} \varrho^{2 n+1} \int_{\rho}^{1} \xi^{-2 n-2} K_{i, n}(\xi) d \xi .
\end{aligned}
$$

In the most important case $i=1$, representing the first approximation to the warping distribution in the cross-section, we obtain very simple formulae

$$
\begin{aligned}
& g_{1,1}=\frac{1}{2} \varrho^{2}-\frac{1}{3} \varrho^{3}, \\
& g_{1,2}=\frac{2}{3 \cdot 7} \varrho^{3}-\frac{2}{5 \cdot 7} \varrho^{5}, \\
& g_{1,3}=\frac{1}{7 \cdot 9} \varrho^{3}+\frac{8}{5 \cdot 7 \cdot 11} \varrho^{5}-\frac{5}{3 \cdot 7 \cdot 11} \varrho^{7},
\end{aligned}
$$

and hence this approximation may be presented in the form of the following Fourier series

$$
\begin{aligned}
t_{1}(\varrho, \theta)= & \left(\frac{1}{2} \varrho^{2}-\frac{1}{3} \varrho^{3}\right) \sin 2 \theta+\left(\frac{2}{21} \varrho^{3}-\frac{2}{35} \varrho^{5}\right) \sin 4 \theta+ \\
& +\left(\frac{1}{63} \varrho^{3}+\frac{8}{385} \varrho^{5}-\frac{5}{231} \varrho^{7}\right) \sin 6 \theta+\ldots
\end{aligned}
$$

Paper [4.454] gives also some details of the evaluation of the second approximation, $i=2$, but the calculations are very tedious in this case.

To evaluate the stress distribution we have to determine the function $\varphi$, (28.4). Making use of (28.29) we may present this function squared in the form

$$
\varphi^{2}=\frac{3 b^{2} x^{2}}{4 \sigma_{0}^{2}} \sum_{j=0}^{\infty} \sum_{n=0}^{\infty} \alpha_{j, n}(\varrho) \lambda^{2 j} \cos 2 n \theta ;
$$


the functions $\alpha_{\mathrm{j}, \mathrm{n}}$ for $j=1$ are given in the form of the following series:

$$
\begin{aligned}
& \alpha_{1,0}=2 \varrho^{2}-1.667 \varrho^{3}+0.8423 \varrho^{4}-0.1500 \varrho^{6}+0.0651 \varrho^{8}+\ldots, \\
& \alpha_{1,1}=-2 \varrho^{2}+2 \varrho^{3}-0.4862 \varrho^{4}-0.4668 \varrho^{5}+0.3942 \varrho^{6}-0.0948 \varrho^{8}+\ldots, \\
& \alpha_{1,2}=0.0924 \varrho^{3}-0.3654 \varrho^{4}+0.4570 \varrho^{5}-0.1857 \varrho^{6}-0.2813 \varrho^{7}+\ldots,
\end{aligned}
$$

Substituting (28.30) into (28.3), we present the stresses as follows:

$$
\begin{aligned}
\sigma_{x} & =\sigma_{0} \sqrt{3} \frac{\varrho \sin \theta}{\sqrt{3 \varrho^{2} \sin ^{2} \theta+\left(\alpha_{1,0}+\alpha_{1,1} \cos 2 \theta+\alpha_{1,2} \cos 4 \theta+\ldots\right) \lambda^{2}+\ldots}} \\
\tau_{x z} & =\frac{\sigma_{0}}{\sqrt{3}} \frac{\left(t_{1 \rho}^{\prime} \sin \theta+\frac{1}{\varrho} t_{1 \theta}^{\prime} \cos \theta-\varrho \cos \theta\right) \lambda+\left(t_{2 \rho}^{\prime} \sin \theta+\frac{1}{\varrho} t_{2 \theta}^{\prime} \cos \theta\right) \lambda^{3}+\ldots}{\sqrt{3 \varrho^{2} \sin ^{2} \theta+\left(\alpha_{1,0}+\alpha_{1,1} \cos 2 \theta+\alpha_{1,2} \cos 4 \theta+\ldots\right) \lambda^{2}+\ldots}} . \\
\tau_{x y} & =\frac{\sigma_{0}}{\sqrt{3}} \frac{\left(t_{1 \rho}^{\prime} \cos \theta-\frac{1}{\varrho} t_{1 \theta}^{\prime} \sin \theta+\varrho \sin \theta\right) \lambda+\left(t_{2 \rho}^{\prime} \cos \theta-\frac{1}{\varrho} t_{2 \theta}^{\prime} \sin \theta\right) \lambda^{3}+\ldots}{\sqrt{3 \varrho^{2} \sin ^{2} \theta+\left(\alpha_{1,0}+\alpha_{1,1} \cos 2 \theta+\alpha_{1,2} \cos 4 \theta+\ldots\right) \lambda^{2}+\ldots}} .
\end{aligned}
$$

The stress distribution for $\theta=\pi / 2$ (maximal influence of bending) is shown in Fig. 147; further diagrams may be found in [4.454].
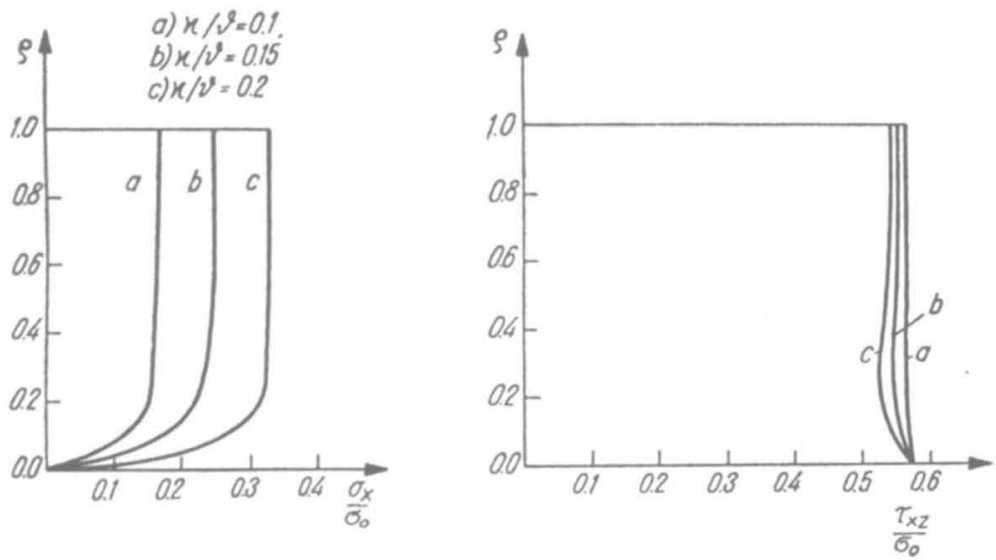

Fig. 147. Stress distribution under fully plastic bending with torsion of a circular section

The series (28.32), when the indicated operations are performed, become divergent in the vicinity of $\theta=0$ and $\theta=\pi$; in fact, the lines $\theta=0$ and $\theta=\pi$ are the discontinuity lines for the zeroth approximation here (pure bending). Nevertheless, the bending moment and the twisting moment (28.7) may be calculated by using numerical integration in the vicinity of $\theta=0$ and $\theta=\pi$. The results obtained in [4.454] are

$$
\begin{aligned}
& M_{b}=\sigma_{0} b^{3}\left(1.3339-0.6870 \lambda^{2}+\ldots\right), \\
& M_{t}=\frac{\sigma_{0} b^{3}}{\sqrt{3}}(2.3655 \lambda+\ldots),
\end{aligned}
$$


or, in a dimensionless form with parameter $\lambda$ eliminated,

$$
m_{b}=1-0.4036 m_{t}^{2}-\ldots
$$

The series (28.34) determines the plastic interaction curve in a certain neighbourhood of the point corresponding to pure bending.

\subsection{Approximations and bounds for a circular cross-section}

The series (28.20) determines the value of the function $m_{t}=f\left(m_{b}\right)$ and of its several derivatives at the point $m_{b}=0$, whereas the series (28.34) determines the value of the function $m_{b}=f\left(m_{t}\right)$ and of its several derivatives at the point $m_{t}=0$. Such information is sufficient for constructing a Hermitian boundary approximation for the whole interval under consideration. However, the situation is more complicated here since all the derivatives of the function $m_{t}=f\left(m_{b}\right)$ tend to infinity at the point $m_{b}=1$ and all the derivatives of the function $m_{b}=f\left(m_{t}\right)$ tend to infinity at the point $m_{t}=1$. Hence we apply a certain generalization of the Hermitian boundary approximation, assuming the equation of the approximating curve in an implicit form and making use of the conditions of agreement after having solved that equation either with respect to $m_{t}$ or with respect to $m_{b}$.

In view of the expected form of the plastic interaction curve, its equation will be assumed in the form

$$
A m_{t}^{2}+B m_{b}^{2}+C m_{t}^{4}+D m_{b}^{4}=1,
$$

which may easily be solved with respect to $m_{t}$ and $m_{b}$; (28.35) satisfies all the symmetry conditions required. Solving this equation with respect to $m_{t}^{2}$, we obtain

$$
m_{t}^{2}=\frac{1}{2 C}\left[-A+\sqrt{A^{2}+4 C\left(1-B m_{b}^{2}-D m_{b}^{4}\right)}\right],
$$

and, after expanding it into the power series of $m_{b}$

$$
m_{t}^{2}=\frac{1}{2 C}\left[-A+\sqrt{A^{2}+4 C}\left(1-\frac{2 B C m_{b}^{2}}{A^{2}+4 C}+\ldots\right)\right) .
$$

The Hermitian conditions of coincidence will be satisfied if we compare (28.37) with the series (28.20) squared. Namely, comparing two coefficients, we obtain the system of equations

$$
A+C=1, \quad B=0.8106(2-A),
$$

ensuring the coincidence of the function itself and of the second derivatives respectively; the coincidence of the first derivative (equal to zero) is satisfied automatically in view of the form of the approximating function (28.35).

Similarly, solving (28.35) with respect to $m_{b}^{2}$, expanding the expression obtained into the power series of the variable $m_{t}$ and comparing with the series (28.34) squared, we obtain the system of equations

$$
B+D=1, \quad A=0.8072(2-B) .
$$


Solving the system of linear equations (28.38) and (28.39) we determine all the constants $A, B, C, D$, and finally the approximation of the plastic interaction curve takes the form

$$
0.8845 m_{t}^{2}+0.9042 m_{b}^{2}+0.1155 m_{t}^{4}+0.0958 m_{b}^{4}=1 .
$$

The curve (28.40) is shown in Fig. 148 together with an upper bound and with a lower bound derived below.

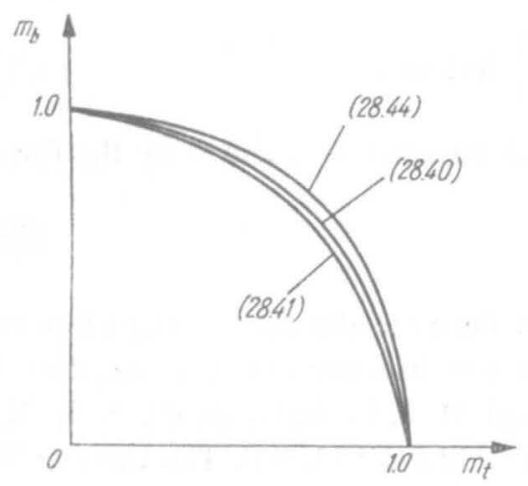

Fig. 148. Plastic interaction curve for a circular section under bending with torsion

An exact solution of Eq. (28.5) or of the corresponding equation for the stress function is-as has been seen-rather complicated, whereas the derivation of a lower bound (a statically admissible solution) or of an upper bound (a kinematically admissible solution) is relatively simple in the case under consideration.

A lower bound is given directly by (26.9); using the notation adopted, we may present it in the form

$$
m_{t}^{2}+m_{b}^{2}=1 \text {. }
$$

To obtain an upper bound we apply the displacement field (or the velocity field) (28.1) with substituted $u_{0} \equiv 0$ (hypothesis of plane cross-sections). The bending moment and the twisting moment are determined by formulae (28.7) in which $u_{0}=0$; introducing cylindrical coordinates (28.8) with an appropriate Jacobian and integrating over $\varrho$ we obtain

$$
\begin{aligned}
& M_{b}=\varkappa \sigma_{0} b^{3} \int_{0}^{2 \pi} \frac{\sin ^{2} \theta d \theta}{\sqrt{9 x^{2} \sin ^{2} \theta+3 \vartheta^{2}}}, \\
& M_{t}=\frac{\theta \sigma_{0} b^{3}}{3} \int_{0}^{2 \pi} \frac{d \theta}{\sqrt{9 x^{2} \sin ^{2} \theta+3 \vartheta^{2}}} .
\end{aligned}
$$

Thus we have arrived at elliptic integrals; the second one may be reduced to a complete elliptic integral of the first kind whereas the first one reduces to a combination of complete elliptic integrals both of the first and of the second kind. The simplest 
notation is obtained if we present the elliptic integrals not as a function of the modulus $k$, as usual, but as a function of an auxiliary angle $\alpha$, namely

$$
\mathbf{K}(\alpha)=\int_{0}^{\pi / 2} \frac{d \theta}{\sqrt{1-\sin ^{2} \alpha \sin ^{2} \theta}},
$$

and similarly $\mathbf{E}(\alpha)$. Having reduced the integrals $(28.42)$ to the normal form, one obtains

$$
m_{b}=\frac{\mathbf{E}(\alpha)-\mathbf{K}(\alpha) \cos ^{2} \alpha}{\sin \alpha}, \quad m_{t}=\frac{2}{\pi} \mathbf{K}(\alpha) \cos \alpha,
$$

where the parameter $\alpha$ is connected with the parameters $x$ and $\vartheta$ by the formula

$$
\sin \alpha=\frac{x}{\sqrt{\varkappa^{2}+\frac{1}{3} \vartheta^{2}}} .
$$

The formulae (28.44) determine an upper bound to the plastic interaction curve given in a parametrical form. This upper bound was derived in some other way in the earlier mentioned papers by R. Hill and M. P. L. Siebel [6.95], A. R. Rzhanitsyn [6.207], I. N. Mirolyubov [6.162] and J. Mandel [3.494]. The curves (28.41) and (28.44) are shown also in Fig. 148. An estimation of the accuracy of the approximate assumption of plane sections (28.44) was given by $\mathrm{Yu}$. A. Rakovshtchik [6.190], who found that the errors with respect to the exact solution do not exceed 4 per cent. Experimental investigation of the limit carrying capacity in the case under consideration was carried out by P. G. Bytchkov [6.36], who admitted various loading paths including reverse loading.

The elastic-plastic range for tubes under bending with torsion was analysed by T. Ohta, T. Nakazawa and K. Saito [6.175]. M. A. Zadoyan [6.273] considered arbitrary plastic hardening for a thick-walled tube, using the displacement approach. Relatively simple approximate solutions for linear plastic hardening were given by R. M. Shneyderovitch and M. B. Groman [6.217, 6.218, 6.219]; convenient diagrams for engineering applications may be found in a book by S. V. Serensen, V. P. Kogayev and R. M. Shneyderovitch [5.164].

\subsection{Non-circular sections}

Equation (28.5) is valid for any shape of the cross-section, but its solution for noncircular sections is even more difficult than that given above. We quote here only one exceptionally simple example.

It may easily be checked that the function $u_{0}=y z$ satisfies exactly the differential equation (28.5), just as the function $w_{0}=x y$ satisfied the corresponding equation for tension with torsion (27.33). The boundary condition (28.6) will be satisfied only if $d z=0$; this condition determines an infinite strip shown in Fig. 145, where the symbols $x$ and $y$ should now be replaced by $y$ and $z$, respectively. Just as in Sec. 27.4, this solution may approximately be applied to a narrow rectangu- 
lar section with finite dimensions $b$ and $h$, where $b \ll h$. Bending occurs here in the plane of smaller stiffness and, unfortunately, this fact restricts applicability to engineering practice.

The stress distribution (28.3) with substituted (28.4) takes here the form

$$
\begin{aligned}
& \sigma_{x}=\frac{3 \sigma_{0} x \operatorname{sign} z}{\sqrt{9 x^{2}+12 \vartheta^{2}}}, \quad \tau_{x y}=\frac{2 \sigma_{0} \vartheta \operatorname{sign} z}{\sqrt{9 x^{2}+12 \vartheta^{2}}}, \\
& \sigma_{y}=\sigma_{z}=\tau_{x z}=\tau_{y z}=0 .
\end{aligned}
$$

Calculating the moments $M_{b}$ and $M_{t}$ by means of formulae (28.7), multiplying $M_{t}$ by 2 (as in Sec. 27.4), and eliminating the parameter $x / \vartheta$, we obtain the following equation of the plastic interaction curve:

$$
16 M_{b}^{2}+12 M_{t}^{2}=\sigma_{0}^{2} b^{2} h^{4} \text {. }
$$

This equation was first derived by A. R. Rzhanitsyn [4.492] in some other way. It coincides with the general form of lower bounds (26.9), since in the case under consideration the exact stress distribution is of the type (26.6).

The first numerical integration was performed by M. C. Steele [6.226]; he used the stress-function approach and obtained the solution for a square section. E. O. Imegwu [6.103, 6.104] carried out similar integrations for circular and triangular equilateral sections, making use of the Southwell relaxation method.

P. M. Miller and L. E. Malvern [6.159, 6.160, 6.161] drew attention to the fact that numerical integration of the displacement equation (28.5) gives a much better accuracy of results than the equation for the stress function. A general analysis of this problem is given in [6.159], where also an equation for velocities in a rigidplastic-hardening material is derived. Paper [6.160] contains numerical integration of (28.5) for a square section and paper [6.161]-integration of the corresponding equation for plastic hardening; the results of the deformation theory of plasticity and of the incremental theory are compared. M. A. Zadoyan and L. A. Levonyan [6.275] considered plastic hardening in the case of a rectangular section, whereas P. V. Galptchian [6.72] discussed circular and annular sectors. The RambergOsgood law of plastic hardening was applied to several shapes of the cross-section by $\mathrm{H}$. Ishikawa $[6.105,6.107,6.108]$. T. Yamasaki and O. Tosyaki [6.269] considered the elastic-plastic range for a square section under constant torque and increasing bending moment. A. Shindo, Y. Seguchi, O. Shinmura and H. Kojima [6.216] employed the Ramberg-Osgood and the Prager law and took material compressibility into account.

Analytical approximations for the problem of plastic bending with torsion were initiated by R. Hill and M. P. L. Siebel [6.94], E. T. Onat and R. T. Shield [6.176]; they analysed thin-walled sections. A subsequent paper by R. Hill and M. P. L. Siebel [6.95], quoted earlier, gave a general method of deriving of lower and upper bounds for solid sections. Their idea was developed by F. A. Gaydon and H. Nuttall [6.74], who proposed certain upper bounds for rectangular, I and box-sections taking into account warping of the cross-section. Further approximate solutions 
were given by V. I. Batsikadze [6.12], O. I. Greste [6.85], L. P. Vinokurov and A. G. Yurev [6.258]. C. D. Goode and M. A. Helmy [6.79] derived a simple approximation for the limit carrying capacity of a reinforced rectangular section. This problem was widely developed by P. Lampert [6.134].

An original concept of a statically admissible stress field was suggested by K. A. Reckling [2.141]: he assumed the distribution of $\sigma$ corresponding to pure elastic-plastic bending and the distribution of $\tau$ resulting from the yield condition (and hence $\tau \neq 0$ only in the elastic zones of pure bending). However, an exact determination of the shearing stress distribution may be difficult even in this case and a certain further step of approximation is proposed. In any case, the results should be more accurate than the lower bound (26.9).

Some authors determine the plastic interaction curve in a purely approximative manner, making use - to a certain degree-of known solutions. R. Sankaranarayanan and P. C. Hodge [6.208] approximated this curve by octagons, whereas Le-Wu Lu [6.146] proposed hexadecagons for various shapes of the cross-section, including thin-walled profiles. Such an approach is of particular interest for piece-wise linear limit analysis in the case under consideration.

Simultaneous inelastic bending with torsion of thin-walled bars was investigated in detail by A. I. Strelbitskaya within the framework of more general combined loadings of such bars. Her first paper on this particular topic [6.228] was devoted to the limit carrying capacity, whereas [6.230] to plastic hardening. Paper [6.231] analysed the elastic-plastic range in the case of a channel section, paper [6.240]channel and I-sections in the case of different materials of the web and of the flanges. Further papers are due to E. A. Rayevskaya [6.194, 6.195], K. S. Dinno and W. Merchant [6.57].

We also mention several papers on plastic shakedown of a cross-section under variable bending with torsion. G. S. Trofimov [6.255] analysed a channel section under constant torque and cyclic bending and extended his analysis to optimal design of such section. B. I. Lyubarov [5.113] determined the boundary coefficients of asymmetry of cycles separating shakedown and non-shakedown regions.

\subsection{Structural applications and experimental investigations}

Limit analysis and elastic-plastic analysis of bending with torsion in a cross-section serves as a basis for the calculation of the limit carrying capacity and inelastic deflections of shafts, eccentrically loaded beams, spatial frames, arches loaded perpendicularly to their plane, etc. The first papers on limit design of frames and grids taking account of torsion are due to J. Heyman [6.90, 6.91], 1951. Some examples of piece-wise linear approach to such problems may be found in the book by P. G. Hodge [1.6]. O. I. Strelbitska [6.233, 6.234, 6.239] investigated the influence of torsion on the limit carrying capacity of frames under eccentric loading; these results were summed up in the monograph [6.244]. Further papers on grids are due to P. F. Walsh [6.260], M. Kurata and K. Sonoda [6.128] (optimal design), 
R. Askari [6.5], B. Boni and M. Kleiber [6.27]. Calculation of bents and arches taking account of torsion was developed by N. S. Boulton and B. Boonsukha [6.28], J. B. Martin [6.150] and P. Seetharam [6.212]. Influence of torsion on I-beams under eccentric transverse loading was analysed by J. Bródka [6.35] and A. Z. Zarifyan [6.276].

Experimental investigations of the limit carrying capacity and of the elasticplastic range in the case of bending with torsion were carried out mainly on thinwalled sections, important in view of applications to building structures. In the first experiments, 1950, A. V. Gemmerling [6.75] loaded simply supported I-beams by an eccentric force at the centre; he measured the limit carrying capacity and did not observe any loss of stability. H. Mii [6.157] investigated beams made of light alloys. Further studies on the limit carrying capacity were carried out by P. G. Bytchkov [6.37] (rectangular, triangular and I-cross-sections), E. A. Rayevskaya [6.196], O. I. Strelbitska, E. O. Pryadko and G. I. Evseyenko [6.237, 6.238, 6.247], S. S. Gill and J. K. G. Boucher [6.76] (rectangular cross-section).

P. C. Massey [6.151] investigated torsional rigidity of I-beams subject to plastic bending. B. Fontaine [6.66] carried out experiments on bending and torsion of thinwalled tubes with cutouts (e.g. for windows); he proposed for the limit carrying capacity in this case a simple approximate formula of the type (26.9).

\section{General case of longitudinal homogeneity of the stress state}

\subsection{The Handelman-Hill equation}

The most general case of longitudinal homogeneity of stresses in a homogeneous prismatic bar corresponds to tension, torsion and biaxial bending: it may be classified as $\mathscr{P}_{3} / \mathscr{S}_{4} \rightarrow \mathscr{B}_{n}$. The stress distribution in a fully plastic cross-section may then be determined in a comparatively simple manner up to one unknown function: either the Prandtl stress function or the warping function. These functions are governed by non-linear second-order partial differential equations.

Such an equation for the Prandtl stress function was first derived by G. H. Handelman [4.199] and R. Hill [4.209, 2.56]. Superposing the displacement (or velocities) corresponding to individual loadings $M_{b y}, M_{b z}, M_{t}$ and $N$, they assumed the following displacement distribution:

$$
\begin{aligned}
u & =x_{y} x z+x_{z} x y+\varepsilon x+\vartheta u_{0}(y, z), \\
v & =-\frac{1}{2} x_{y} y z-\frac{1}{4} x_{z}\left(2 x^{2}+y^{2}-z^{2}\right)-\frac{1}{2} \varepsilon y+\vartheta x z, \\
w & =-\frac{1}{2} x_{z} y z-\frac{1}{4} x_{y}\left(2 x^{2}-y^{2}+z^{2}\right)-\frac{1}{2} \varepsilon z-\vartheta x y .
\end{aligned}
$$

Of course, such a superposition of displacements should not be confused with the superposition of effects of particular loadings, which does not hold here. The symbols $\varkappa_{y}, \varkappa_{z}, \varepsilon$ and $\vartheta$ denote here either the curvatures of the axis, the elongation and the unit angle of twist or the rates of the respective quantities. Handelman and 
Hill assumed the second interpretation. Under the first interpretation some of the above parameters may increase infinitely and only their ratios remain finite.

Using (29.1) to calculate the strain distribution and then evaluating the stress distribution from the law of similarity of deviators, we obtain

$$
\begin{aligned}
\sigma_{x} & =\frac{3}{2 \varphi}\left(\varkappa_{y} z+x_{z} y+\varepsilon\right), & \sigma_{y} & =\sigma_{z}=\tau_{y z}=0, \\
\tau_{x y} & =\frac{\vartheta}{2 \varphi}\left(\frac{\partial u_{0}}{\partial y}+z\right), & \tau_{x z} & =\frac{\vartheta}{2 \varphi}\left(\frac{\partial u_{0}}{\partial z}-y\right),
\end{aligned}
$$

where $\varphi$ denotes the (variable) modulus in the Hencky-Ilyushin or in the Levy-Mises equations. Further, we have to satisfy one equilibrium equation (the first one) and the yield condition. Handelman and Hill satisfied the equilibrium equation, introducing a stress function of the Prandtl type

$$
\tau_{x y}=-\tau_{0} \frac{\partial \Psi}{\partial z}, \quad \tau_{x z}=\tau_{0} \frac{\partial \Psi}{\partial y} .
$$

The HMH yield condition now determines the distribution of normal stresses

$$
\sigma_{x}= \pm \sigma_{0} \sqrt{1-\left(\frac{\partial \Psi}{\partial y}\right)^{2}-\left(\frac{\partial \Psi}{\partial z}\right)^{2}}
$$

comparing the expression with the first formula (29.2), we may evaluate the function $\varphi$ :

$$
\varphi= \pm \frac{3\left(x_{y} z+x_{z} y+\varepsilon\right)}{2 \sigma_{0} \sqrt{1-\left(\frac{\partial \Psi}{\partial y}\right)^{2}-\left(\frac{\partial \Psi}{\partial z}\right)^{2}}} .
$$

The differential equation for the stress function $\Psi$ will be obtained by eliminating the function $u_{0}$. Equating (29.2) and (29.3), we obtain

$$
\frac{\partial u_{0}}{\partial y}=-z-\frac{2 \varphi \tau_{0}}{\vartheta} \frac{\partial \Psi}{\partial z}, \quad \frac{\partial u_{0}}{\partial z}=y+\frac{2 \varphi \tau_{0}}{\vartheta}-\frac{\partial \Psi}{\partial y},
$$

and equating subsequently the mixed derivatives evaluated in turn from both these formulae, we arrive at the following partial differential equation of the elliptic type:

$$
\begin{aligned}
& \left(x_{y} z+x_{z} y+\varepsilon\right)\left(\nabla^{2} \Psi-\Psi_{z}^{\prime 2} \Psi_{y y}^{\prime \prime}+2 \Psi_{y}^{\prime} \Psi_{z}^{\prime} \Psi_{y z}^{\prime \prime}-\Psi_{y}^{\prime \prime 2} \Psi_{z z}^{\prime \prime}\right)+ \\
& \quad+\left(1-\Psi_{y}^{\prime 2}-\Psi_{z}^{\prime 2}\right)\left(x_{y} \Psi_{z}^{\prime}+x_{z} \Psi_{y}^{\prime}\right) \pm \frac{2}{\sqrt{3}} \vartheta\left(1-\Psi_{y}^{\prime 2}-\Psi_{z}^{\prime 2}\right)^{3 / 2}=0,
\end{aligned}
$$

where a shortened notation for partial derivatives has been introduced. The boundary condition for Eq. (29.7) has a particularly simple shape; as usual, when introducing a stress function by formulae (29.3), we obtain

$$
\Psi=\text { const }
$$

along the contour, and for simply connected cross-sections this constant may be assumed to be equal to zero. 
Having solved Eq. (29.7) with the boundary condition (29.8) for the given shape of the cross-section, we may evaluate the corresponding generalized stresses causing full plasticification of that section. Such formulae are relatively simple: the twisting moment is expressed in terms of the Prandtl function by the well-known formula

$$
M_{t}=2 \tau_{0} \iint_{A} \Psi d A,
$$

whereas the bending moments and the normal force are determined by simple integration of the stresses $\sigma_{x},(29.4)$,

$$
\begin{aligned}
M_{b y} & = \pm \sigma_{0} \iint_{A} z \sqrt{1-\Psi_{y}^{\prime 2}-\Psi_{z}^{\prime 2}} d A, \\
M_{b z} & = \pm \sigma_{0} \iint_{A} y \sqrt{1-\Psi_{y}^{\prime 2}-\Psi_{z}^{\prime 2}} d A, \\
N & = \pm \sigma_{0} \iint_{A} \sqrt{1-\Psi_{y}^{\prime 2}-\Psi_{z}^{\prime 2}} d A .
\end{aligned}
$$

The sign of integrals in (29.10) should coincide with the sign of the expression $x_{y} z+x_{z} y+\varepsilon$; in fact, in most cases we have to divide the cross-sectional area into two parts separated by the straight line

$$
x_{y} z+x_{z} y+\varepsilon=0,
$$

integrate over these parts separately and add the results of integration.

As was mentioned above, the Handelman-Hill equation (29.7) has been integrated numerically in various particular cases. However, the simplicity of that equation and of formulae (29.9) and (29.10) is rather apparent; the integration over two areas in (29.10) is inconvenient. Even by using the perturbation method, S. Piechnik [4.453] found that the displacement approach leads to more convenient solutions.

\subsection{Differential equation in displacements}

Making use of (29.2) in another way, we now eliminate the function $\varphi$ from the HMH yield condition

$$
\varphi=\frac{1}{2 \sigma_{0}} \sqrt{9\left(x_{y} z+x_{z} y+\varepsilon\right)^{2}+3 \vartheta^{2}\left[\left(\frac{\partial u_{0}}{\partial y}+z\right)^{2}+\left(\frac{\partial u_{0}}{\partial z}-y\right)^{2}\right]} .
$$

Substituting the expression into (29.2) and into the equilibrium equation, we finally obtain the following partial differential equation for the function $u_{0}=u_{0}(y, z)$, determining the warping of the cross-section

$$
\begin{aligned}
& 3 \nabla^{2} u_{0}\left(x_{y} z+x_{z} y+\varepsilon\right)^{2}-3\left(x_{y} z+x_{z} y+\varepsilon\right)\left(x_{z} u_{0 y}^{\prime}+x_{y} u_{0 z}^{\prime}\right)+ \\
& \quad+\vartheta^{2}\left[\left(u_{0 z}^{\prime}-y\right)^{2} u_{0 y y}^{\prime \prime}-2\left(u_{0 z}^{\prime}-y\right)\left(u_{0 y}^{\prime}+z\right) u_{0 y z}^{\prime \prime}+\left(u_{0 y}^{\prime}+z\right)^{2} u_{0 z z}^{\prime \prime}\right] \\
& \quad=-3\left(x_{y} z+x_{z} y+\varepsilon\right)\left(y x_{y}-z x_{z}\right),
\end{aligned}
$$


where also a shortened notation of partial derivatives has been introduced. The corresponding boundary condition is of the form (28.6), more complicated than (29.8). Similarly, the formulae for generalized stresses are longer than (29.9) and (29.10):

$$
\begin{aligned}
& M_{t}=\vartheta \sigma_{0} \iint_{A} \frac{\left[\left(u_{0 y}^{\prime}+z\right) z-\left(u_{0 z}^{\prime}-y\right) y\right] d A}{\left.\sqrt{9\left(x_{y} z+x_{z} y+\varepsilon\right)^{2}+3 \vartheta^{2}\left[\left(u_{0 y}^{\prime}+z\right)^{2}+\left(u_{0 z}^{\prime}-y\right)^{2}\right.}\right]}, \\
& M_{b y}=3 \sigma_{0} \iint_{A} \frac{\left(x_{y} z+x_{z} y+\varepsilon\right) z d A}{\sqrt{9\left(\varkappa_{y} z+x_{z} y+\varepsilon\right)^{2}+3 \vartheta^{2}\left[\left(u_{0 y}^{\prime}+z\right)^{2}+\left(u_{0 z}^{\prime}-y\right)^{2}\right]}}, \\
& M_{b z}=3 \sigma_{0} \iint_{A} \frac{\left(\varkappa_{y} z+\varkappa_{z} y+\varepsilon\right) y d A}{\sqrt{9\left(x_{y} z+\varkappa_{z} y+\varepsilon\right)^{2}+3 \vartheta^{2}\left[\left(u_{0 y}^{\prime}+z\right)^{2}+\left(u_{0 z}^{\prime}-y\right)^{2}\right]}} \text {, } \\
& N=3 \sigma_{0} \iint_{A} \frac{\left(x_{y} z+x_{z} y+\varepsilon\right) d A}{\sqrt{9\left(x_{y} z+x_{z} y+\varepsilon\right)^{2}+3 \vartheta^{2}\left[\left(u_{0 y}^{\prime}+z\right)^{2}+\left(u_{0 z}^{\prime}-y\right)^{2}\right]}},
\end{aligned}
$$

but these formulae are effective without separating any subdomains as in (29.10), and are less sensitive to errors of numerical integration of the governing equation (29.13).

\subsection{Straight bending with torsion and tension}

Assuming, say, $x_{z}=0$ in (29.7) or (29.13), we do not obtain any significant simplification. The only simple exact solution may be obtained for a narrow rectangular section; it was derived by M. A. Zadoyan [6.272].

For an arbitrary rectangular section a simple lower bound may be obtained from (26.8) combined with the Girkmann parabola (22.35); it has the form

$$
m_{b} \sqrt{1-m_{t}^{2}}+m_{t}^{2}+n^{2}=1 \text {. }
$$

where the dimensionless generalized stresses are referred to the corresponding limit values. C. R. Calladine [6.38] proposed a slightly different lower bound

$$
\left(|n|+\left|m_{b}\right|\right)^{2}+m_{t}^{2}=1 \text {, }
$$

which is better justified for I-sections. A more detailed analysis of the limit carrying capacity of thin-walled sections in the case under consideration was carried out by O. I. Strelbitska [6.232]. O. I. Ibrayev investigated the effect of the loadings path and stated that this effect is small both in the elastic-plastic range, [6.101], and in the plastic hardening range [6.102]. Several effective approximate solutions for the plastic interaction surface were derived by $Z$. Bažant [6.17]. J. Lindner [6.142] applied the solutions for a cross-section to the approximate determination of the limit carrying capacity of beams under various loadings.

The analysis of elastic-plastic behaviour in the case of bending, torsion and normal force is particularly important because of the application to stability problems under torsion with compression. This problem was considered by L. M. Kachanov [6.112]. Certain simple approximate solutions were given by N. A. Smolyago [6.222] (without stability considerations). 


\subsection{Biaxial bending with torsion}

The first effective solution for fully plastic biaxial bending with torsion was given by P. G. Hodge and R. Sankaranarayanan [6.97]; for a square section they derived a lower bound and proposed a method of derivation of an upper bound (namely of Eq. (26.15) in the case $\lambda_{1}=\lambda_{4}=\lambda_{5}=0$ ). The lower bound results from (26.8) and from the equation of the plastic interaction curve for biaxial bending, (24.13),

$$
m_{b y} \sqrt{1-m_{t}^{2}}+\frac{3}{4} m_{b z}^{2}+m_{t}^{2}=1, \quad\left|m_{b z}\right|<m_{b y},
$$

and similarly for other ranges of $m_{b y}$ and $m_{b z}$. In the monograph [1.6] P. G. Hodge applied a piece-wise linear approximation of the curve $(29.17)$ to the limit analysis of a spatially loaded frame.

The limit carrying capacity of thin-walled cross-sections under biaxial bending with torsion was investigated in detail by O. I. Strelbitska [6.242, 6.243]; she carried out experimental verification jointly with G. I. Evseyenko [6.241, 6.245]. Further experiments were carried out by E. R. Chernaya [6.43]. I. V. Davidov [6.54] noted that the limit carrying capacity of an I-section may be exhaused without full plastification of the section and proposed a method of calculation in this case.

Elastic-plastic range in thin-walled sections under biaxial bending with torsion was investigated by V.A. Kolgadin: in [6.116] he proposed a general theory and gave an application to a channel section, and in [6.118] he considered an I-section. A more simplified approach to this case is due to E. R. Chernaya [6.42], who calculated elastic-plastic deflection of an I-beam loaded by an oblique concentrated force acting at the mid-point of a flange. N. L. Chernov and E. R. Chernaya investigated elastic-plastic deflections under a moving oblique force, in [6.44] (theory), and in [6.46] (experiment).

Effective solutions for the general case of biaxial bending with torsion and tension were obtained by P. Lampert [6.134] (homogeneous and reinforced crosssections), Y. Yamada and K. Takatsuka [6.268] (finite element method, slightly compressible material) and M. A. Zadoyan [6.274] (plastic hardening, perturbation method). Experimental verification of the theory was carried out by $\mathrm{H}$. Shakir-Khalil and G. S. Tadros [5.165] and H. Shakir-Khalil [6.213] (box sections). G. A. Morris and S. J. Fenves [6.165] applied linearized plastic interaction surfaces to elastic-plastic analyses of frames, taking into account also the passive processes following full plastification.

\section{Bending with shear}

\subsection{Introductory remarks}

Bending with shear doubtlessly constitutes the most difficult case of the type $\mathscr{P}_{2} / \mathscr{S}_{2}$. Firstly, almost all the other cases showed longitudinal homogeneity and could be classified as $\mathscr{P}_{2} / \mathscr{S}_{2} \rightarrow \mathscr{B}_{2}$, whereas the case under consideration shows, 
because of the Schwedler-Zhuravsky theorem $V=d M_{b} / d x$, an "automatic" longitudinal non-homogeneity $\mathscr{P}_{2} / \mathscr{S}_{2} / \mathscr{B}_{n}$. Sometimes we have to use even the classification $\mathscr{P}_{4} / \mathscr{S}_{2} / \mathscr{B}_{n}$, since in certain (mainly thin-walled) sections the stresses $\sigma_{z}$ and $\tau_{x y}$, resulting from equilibrium equations, cannot be neglected. Only when considering some non-prismatic bars, we may, somewhat artificially, assume the classification $\mathscr{P}_{n} / \mathscr{S}_{2} \rightarrow \mathscr{B}_{1}$; e.g. in an infinite bar shown in Fig. 149, treated as a
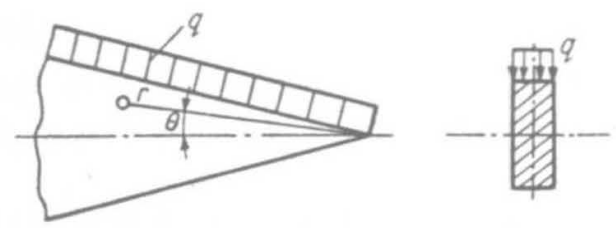

Fig. 149. An infinite wedge under bending with shear

wedge, the stresses in the plane polar coordinates $r \theta$ do not depend on $r$, and hence we may regard any cross-section (subject to bending and shear) in an identical manner.

Further, because of the longitudinal non-homogeneity of stresses the notion of the limit carrying capacity of a cross-section under bending with shear may be non-unique: namely, this capacity may depend on the gradient of generalized stresses. This fact has been pointed out by many authors, e.g. by D. C. Drucker [6.58] and B. G. Neal [6.168]. A more accurate analysis of stress distribution in a cross-section requires, strictly speaking, an analysis of stress distribution in the beam as a whole (and this fact reduces to a certain degree the advantages of the analysis at the level of a cross-section); on the other hand, however, the quantitative influence of the gradients on the plastic interaction curve for the crosssection is, as a rule, almost negligible.

The next difficulty is connected with the type of the stress state at individual points; as was mentioned above, we often have to take into account not only the "basic" stresses $\sigma_{x}$ and $\tau_{x z}$ but also $\sigma_{z}$ and $\tau_{x y}$ (the latter, for example, in flanges of I-sections before full plastification). This fact may seriously complicate the yield condition: in the case $\sigma_{z} \neq 0$ the form (26.1) is no longer sufficient and the differences between the HMH and the TG yield conditions may become essential.

Finally, it is difficult to estimate the limit carrying capacity of a cross-section under pure shearing force $V$. There exist very few cases of loading of beams where the plastic collapse may be due to pure shear. One of such beams is shown in Fig. 150. If the length $l$ of the beam is sufficiently small with respect to the depth $h$, the clamped cross-section (in which $M_{b}=0$ ) might be the dangerous cross-section; on the other hand, in this case the applicability of the elementary theory of beams is doubtful. In any case, it is important to have an estimation of the limit carrying capacity $\overline{\bar{V}}$, at least for the construction of the lower bound (26.9). The most likely structure of such a formula,

$$
\overline{\bar{V}}=\tau_{0} A,
$$



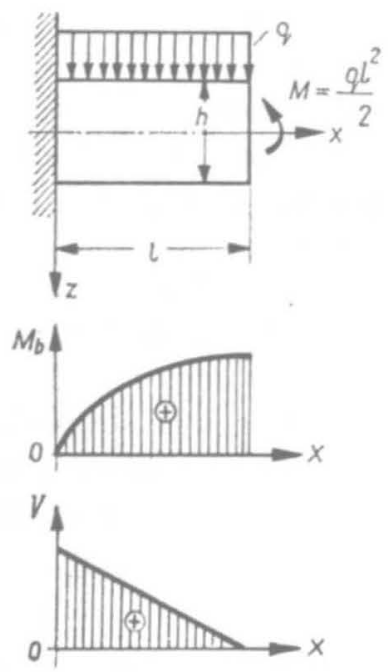

Fig. 150. A beam for which the dangerous section may correspond to pure shear

doubtlessly determines an upper bound, probably never coinciding with the exact solution: in fact, formula (30.1) gives an upper bound to the functional

$$
V=\iint_{A} \tau_{x z} d A
$$

in a class of functions broader than the class of admissible functions, namely satisfying only the yield condition and, in general, not satisfying either the equilibrium equations or the boundary conditions (statically partially admissible functions). In the case of a rectangular section the error of (30.1) may be fairly small, but in thin-walled sections this error may be essential and then a more cautions approach should be applied (e.g. the area $A$ may be understood as the web area). In general one can write

$$
\overline{\bar{V}}=\mu \tau_{0} A,
$$

where the numerical coefficient $\mu$ depends on the shape of the cross-section, but also on the distribution of shearing forces along the beam (at least on the gradient $d V / d x)$. The determination of the coefficient $\mu$ presents, in general, a difficult theoretical problem.

We may assume that the substitution of (30.3) with an appropriately evaluated coefficient $\mu$ into (26.9) gives in the combined case under consideration a lower bound of the plastic interaction curve.

\subsection{Basic hypotheses regarding stress distribution}

Since it is difficult to obtain any exact solutions of the problem of plastic bending with shear and because of the above-mentioned non-uniqueness of the limit carrying capacity in the case under consideration, many approximate appoaches were pro- 
posed. These approaches formulate, as a rule, certain hypotheses regarding stress distribution in the limit state. Such hypotheses usually describe only the distribution of $\sigma_{x}$ and $\tau_{x z}$; the stresses $\sigma_{z}$ and $\tau_{x y}$ are taken into account only in more accurate considerations. Though these approaches are purely static, the resulting equations give either lower bounds or upper bounds to the actual plastic interaction curves, since not all stress distributions conform to the requirements of static admissibility.

The first hypothesis for stress distribution was stated in 1931 by F. Stüssi [6.248], but the stresses did not satisfy the yield condition. It was N. I. Bezukhov [6.24] who first proposed, in 1936, a fully statically admissible stress field. Namely, Bezukhov assumed that yielding in the outer zones is due to normal stresses only, $\sigma_{x}= \pm \sigma_{0}$, in the inner zone there exists a linear distribution of normal stresses, and the distribution of shearing stresses results from the equilibrium equation,

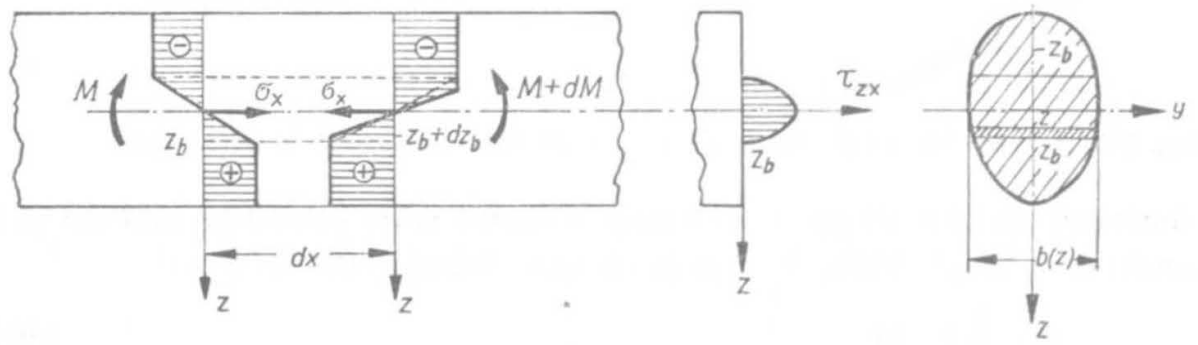

Fig. 151. Bezukhov's hypothesis as regards the stress distribution under elastic-plastic bending with shear

Fig. 151 (e.g. in a rectangular section this distribution is parabolic). The shearing stresses are then determined by the formula

$$
\tau_{x z}=\frac{V S_{y}^{(e)}(z)}{I_{y}^{(e)} b(z)},
$$

where $S_{y}^{(e)}(z)$ denotes static moment about the axis $y$ of the part of the elastic zone cut at height $z$ (densely shaded in Fig. 151), $I_{y}^{(e)}$ the moment of inertia of the elastic zone, and $b(z)$ the current width of the cross-section. It is assumed that in the inner zone yielding does not occur, and at $\operatorname{most} \max \tau_{z x}=\tau_{0}$. A certain related approach was suggested by A. N. Mitinsky [6.163], and later by M. R. Horne [6.98], who allowed also for the stresses $\sigma_{z}$ resulting from the equilibrium equations. A slight modification is due to T. van Langendonck [6.138], who proposed for the inner zone the stress distribution

$$
\sigma_{x}=\sigma_{0} \sin \frac{\pi z}{2 z_{b}}, \quad \tau_{x z}=\tau_{0} \cos \frac{\pi z}{2 z_{b}} .
$$

This distribution satisfies at each point the yield condition and "raises" the plastic interaction curve, but is no longer statically admissible since the equilibrium equations are not satisfied here.

The vanishing of the shearing stresses $\tau_{x z}$ in the whole outer zones of prismatic beams may be proved by using the yield condition, boundary conditions and equi- 
librium equations. Such proof was given by W. Prager and P. G. Hodge [2.133] and by M. R. Horne [6.98] under the assumption that only $\sigma_{x}$ and $\tau_{x z}$ occur. We present that proof here in a more general form, allowing for the existence of $\sigma_{z}$ and based on a general yield condition in the form $\sigma_{\text {red }}=f\left(\sigma_{x}, \sigma_{z}, \tau_{x z}\right)=\sigma_{0}$.

The boundary conditions at the free surface $z=h$ of a prismatic bar will be written thus:

$$
\sigma_{z}(x, h)=0, \quad \tau_{x z}(x, h)=0, \quad \text { and hence } \quad \frac{\partial \tau_{x z}}{\partial x}(x, h)=0 ;
$$

so the yield condition on the tensile side gives

$$
\sigma_{x}(x, h)=\sigma_{0}, \quad \frac{\partial \sigma_{x}}{\partial x}(x, h)=0 .
$$

The equilibrium equations (with the derivatives $\partial / \partial y$ omitted) now lead to the conclusions

$$
\frac{\partial \tau_{x z}}{\partial z}(x, h)=0, \quad \frac{\partial \sigma_{z}}{\partial z}(x, h)=0,
$$

and the differentiated yield condition

$$
\frac{\partial f}{\partial \sigma_{x}} \frac{\partial \sigma_{x}}{\partial z}+\frac{\partial f}{\partial \sigma_{z}} \frac{\partial \sigma_{z}}{\partial z}+\frac{\partial f}{\partial \tau_{x z}} \frac{\partial \tau_{x z}}{\partial z}=0
$$

results in the equation

$$
\frac{\partial \sigma_{x}}{\partial z}(x, h)=0
$$

Since this equation leads in turn to the conclusion $\partial^{2} \sigma_{x} / \partial x \partial z=0$, the differentiated equilibrium equation gives $\partial^{2} \tau_{z x} / \partial z^{2}=0$; in this way we prove step by step that all the derivatives of the stresses $\sigma_{x}, \sigma_{z}, \tau_{x z}$ with respect to $z$ are zero along the line $z=h$. Cautiously speaking, this conclusion is not identical with the proof that within the whole outer zone we have $\sigma_{x}=\sigma_{0}, \sigma_{z}=\tau_{x z}=0$ (this fact is not mentioned by the authors), since, for example, the function

$$
\tau_{x z}=A(x) e^{-\frac{c(x)}{\left(z^{2}-h^{2}\right)^{2}}}, \quad c(x)>0,
$$

has, along the lines $z=h$ and $z=-h$, all the derivatives with respect to $z$ equal to zero while it is itself not identically equal to zero; however, the stress distribution of the type (30.11) is of little probability, and the distribution $\sigma_{x}=\sigma_{0}, \sigma_{z}=\tau_{x z}=0$ leads to much simpler results. In any case, the above consideration gives a strong argument for the Bezukhov hypothesis.

The second basic approach looks for an upper bound (in the sense of the calculus of variations) of partially statically admissible solutions, satisfying only the yield condition. This approach was first suggested by P. F. Papkowitch in 1939, [6.181], who tried to consider simultaneously the boundary conditions and the equilibrium equations. He arrived at a very complicated isoperimetric problem of 
the calculus of variations and did not obtain any effective solution. A similar idea was independently developed in 1949 by A. R. Rzhanitsyn [4.492], and in 1957 by P. G. Hodge [6.96]; they restricted the auxiliary conditions to the yield condition. Namely, if we present the shearing force $V$ by the integral (30.2), then-making use of the HMH yield condition, supposed to hold at any point of the section, and neglecting the stresses $\tau_{x y}$ and $\sigma_{z}$-we may express the bending moment as follows:

$$
M_{b}=\iint_{A} z \sqrt{\sigma_{0}^{2}-3 \tau_{x z}^{2}} d A .
$$

The requirement of the maximal bending moment under a constant value of the shearing force leads to an isoperimetric problem of the calculus of variations; the relevant Lagrangian function

$$
L\left[\tau_{x z}(z)\right]=z \sqrt{\sigma_{0}^{2}-3 \tau_{x z}^{2}}+\lambda \tau_{x z}
$$

does not contain any derivatives of the function $\tau_{x z}=\tau_{x z}(z)$, and hence no boundary conditions may be satisfied. From the condition of extremum we obtain only an algebraic equation

$$
\frac{-3 z \tau_{x z}}{\sqrt{\sigma_{0}^{2}-3 \tau_{x z}^{2}}}+\lambda=0,
$$

and hence, choosing the sign "+" when taking the root, we have

$$
\tau_{x z}=\frac{\lambda \sigma_{0}}{\sqrt{3} \sqrt{3 z^{2}+\lambda^{2}}}, \quad \sigma_{x}=\frac{\sqrt{3} \sigma_{0} z}{\sqrt{3 z^{2}+\lambda^{2}}} .
$$

The stress distribution (30.15) is shown in Fig. 152; it can be seen that the boundary condition (30.6) is not satisfied.
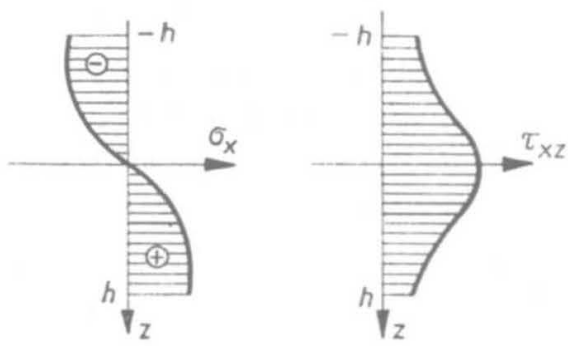

Fig. 152. Stress distribution corresponding to the upper bound of statically partially admissible stress fields (yield condition is the only equation satisfied)

The plastic interaction curve is determined here by the following parametrical equations

$$
V=\frac{\lambda \sigma_{0}}{\sqrt{3}} \iint_{A} \frac{d A}{\sqrt{3 z^{2}+\lambda^{2}}}, \quad M_{b}=\sqrt{3} \sigma_{0} \iint_{A} \frac{z^{2} d A}{\sqrt{3 z^{2}+\lambda^{2}}} .
$$

Moreover, Rzhanitsyn showed (in the second edition, 1954), that Papkovitch's approach may also be reduced to (30.15). Equations (30.16) obtained from the 
variational approach applied to the class of functions broader than the admissible class provide of course an upper bound to the actual plastic interaction curve (though this fact is not widely known); the corresponding kinematically admissible velocity field was also derived by A. R. Rzhanitsyn [4.492] and by P. G. Hodge [6.96]. Effective results of integration for the sections: rectangular, I, circular, annular, tee, diamond and triangular, are given in the monograph [5.39] by I. L. Dikovitch, who also carried out the elimination of the parameter $\lambda$.

It should be stressed here that in the case of cross-sections which are not bisymmetric, the stress distribution (30.15) may correspond not only to the shearing force $V=V_{z}$ and to the bending moment $M_{b}=M_{b y}$ but also to some other generalized stresses. Thus, in the case of a triangular section located as in Fig. 153a, a cer-
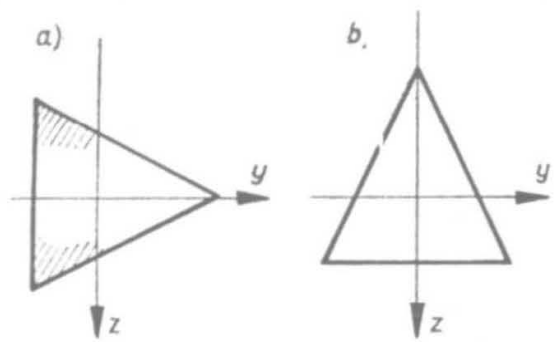

Fig. 153. Formation of additional generalized stresses in a monosymmetric cross-section

tain twisting moment will appear, due to a decrease of shearing stresses in the shaded areas, lying on the same side of the centre of gravity. In the case of a triangular section as shown in Fig. 153b we obtain a certain normal force even at vanishing shear, since there is no shift of the neutral axis required in such monosymmetric sections. This fact causes additional complications (a new element of approximation, usually per excess) if we apply (30.16) to bending with shear without any other generalized stresses. This element of approximation is usually left unnoticed.

Further, the boundary conditions for shearing stresses, left unsatisfied here, are more important than it is supposed. In fact, they lead to a violation of the equilibrium equations expressed in generalized stresses. For example, for a rectangular section $b \times 2 h$ we have

$$
\begin{aligned}
\frac{d M}{d x} & =b \int_{-h}^{h} \frac{\partial \sigma_{x}}{\partial x} z d z=-b \int_{-h}^{h} \frac{\partial \tau_{x z}}{\partial z} \cdot z d z \\
& =-\left.b \tau_{x z} z\right|_{-h} ^{h}+b \int_{-h}^{h} \tau_{x z} d z=-2 b h \tau_{x z 0}+V,
\end{aligned}
$$

where $\tau_{x z 0}$ denotes the value of shearing stresses $\tau_{x z}$ at the boundary points $(z=h$, and the same for $z=-h$ ). Thus, the Schwedler-Zhuravsky equilibrium equation $d M / d x=V$ is unsatisfied; this conclusion shows once more that (30.16) furnishes 
only an upper bound to the plastic interaction curve, and not the exact solution, as supposed by many authors.

The third basic hypothesis regarding stress distribution in the limit state is due to S. A. Palchevsky [6.179]; it assumes a distribution similar to that in bending with normal force (Fig. 154). This stress distribution is also only partially statically admiss-

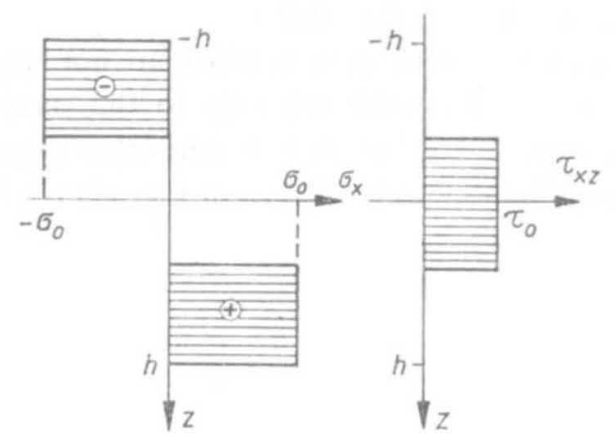

Fig. 154. Palchevsky's hypothesis as regards the stress distribution in the fully plastic state

ible, since the shearing stress $\tau_{z x}$ shows an inadmissible discontinuity; nevertheless, in most cases this leads to a lower bound of the plastic interaction curve. Only in the range of large shearing forces $V$ the errors are per excess. Because of the analogy to bending with tension, one can apply here without major changes all the expansions into generalized power series and the approximation discussed in Sec. 23. The hypothesis under consideration, of a rather poor accuracy, is to a certain degree convenient when discussing simultaneous bending, tension and shear (cf. Sec. 31) because of uniformity of approach. Moreover, all the critical remarks concerning the appearance of additional generalized stresses, mentioned in the previous discussion, are applicable here as well.

Let us also mention here two other proposals of a general type. N. K. Snitko [6.223] suggested keeping the ratio $\sigma_{x} / \tau_{x z}$ identical to that in the elastic range with the values of $\sigma$ and $\tau$ determined by the yield condition which is to be satisfied throughout the section. The boundary conditions are then satisfied, but the equilibrium equations are not. Z. Sobotka [4.544] proposed to assume $\sigma=$ const and $\tau=$ const in the whole section; this proposal would lead to a lower bound of type (26.9) if the state $\tau=\tau_{0}$ were statically admissible. However, this is not the case, and $\tau=\tau_{0}$ gives an upper bound. As was mentioned above, a lower bound of type (26.9) may be obtained if we understand by $\overline{\bar{V}}$ the value determined by (30.3).

\subsection{Limit carrying capacity of a rectangular section}

In the combined case under consideration a rectangular cross-section is most frequently analysed both because of its simplicity and because of its engineering appli- 
cations. We shall now quote, for a rectangular section, results based on hypotheses discussed in Sec. 30.2 and some other investigations.

Assuming Bezukhov's hypothesis [6.24], we determine the bending moment for a rectangular section with depth $2 h$ and width $b$ by the formula corresponding to pure elastic-plastic bending

$$
M_{b}=\sigma_{0} b\left(h^{2}-\frac{1}{3} z_{b}^{2}\right),
$$

where $z_{b}$ denotes the boundary coordinate; the distribution of shearing stresses $\tau_{x z}$ is parabolic with the maximal stress at the axis being equal to the yield point in shear:

$$
\tau_{x z}=\tau_{0}\left(1-\frac{z^{2}}{z_{b}^{2}}\right) .
$$

The shearing force is then equal to

$$
V=\frac{3}{4} \tau_{0} b z_{b} .
$$

Eliminating the parameter $z_{b}$ from (30.18) and (30.20), we may write the final result in a dimensionless form as follows:

$$
m_{b}=1-\frac{3}{4} v^{2},
$$

where $m_{b}=M_{b} / \bar{M}_{b}=M_{b} / \sigma_{0} b h^{2}$, and the shearing force $V$ is referred not to the exact value of the limit carrying capacity $\overline{\bar{V}}$, difficult to be evaluated, but to its upper bound (30.1): $v=V / 2 \tau_{0} b h$. Formula $(30.21)$ is valid for $|v| \leqslant 2 / 3$, i.e. $m_{b} \geqslant 2 / 3$; the value of the shearing force $v=2 / 3$ is regarded as maximal, causing collapse due to slip.

Rzhanitsyn's variational approach [4.492] leads to integral formulae (30.16), which for a rectangular section may easily be presented effectively. Namely, we obtain

$$
\begin{aligned}
& V=\frac{2}{3} \lambda \sigma_{0} b \ln \frac{h \sqrt{3}+\sqrt{3 h^{2}+\lambda^{2}}}{\lambda}, \\
& M_{b}=\frac{1}{\sqrt{3}} \sigma_{0} b\left(h \sqrt{3 h^{2}+\lambda^{2}}-\frac{\lambda^{2}}{\sqrt{3}} \ln \frac{h \sqrt{3}+\sqrt{3 h^{2}+\lambda^{2}}}{\lambda}\right) .
\end{aligned}
$$

The curve (30.21) determining an upper bound exceeds only very slightly the circle

$$
m_{b}^{2}+v^{2}=1
$$

proposed by Z. Sobotka [4.544].

Palchevsky's proposal [6.179] leads in the case of a rectangular section to the equation of a parabola

$$
m_{b}=1-v^{2}
$$

analogous to the Girkmann parabola (22.35). 
Several papers give a continuation or modifications of Bezukhov's concept. M. R. Horne [6.98] took the component $\sigma_{z}$ into account and obtained the equation

$$
m_{b}=1-0.44 v^{2} ; \quad v \leqslant 0.79 \text {. }
$$

D. C. Drucker [6.58] derived a formula which may be written in the form

$$
m_{b}=0.98\left(1-v^{4}\right) \text {. }
$$

S. D. Leytes $[2.99,6.140]$ applied his "stabilization hypothesis" (cf. Sec. 9.8). Further related papers are due to F. E. Klimenko [6.114], E. A. Beylin [6.21], W. P. Chen and E. M. Shoemaker [6.41] and R. Windels [6.262]. The curves (30.21) $\div(30.26)$ are shown in Fig. 155 .

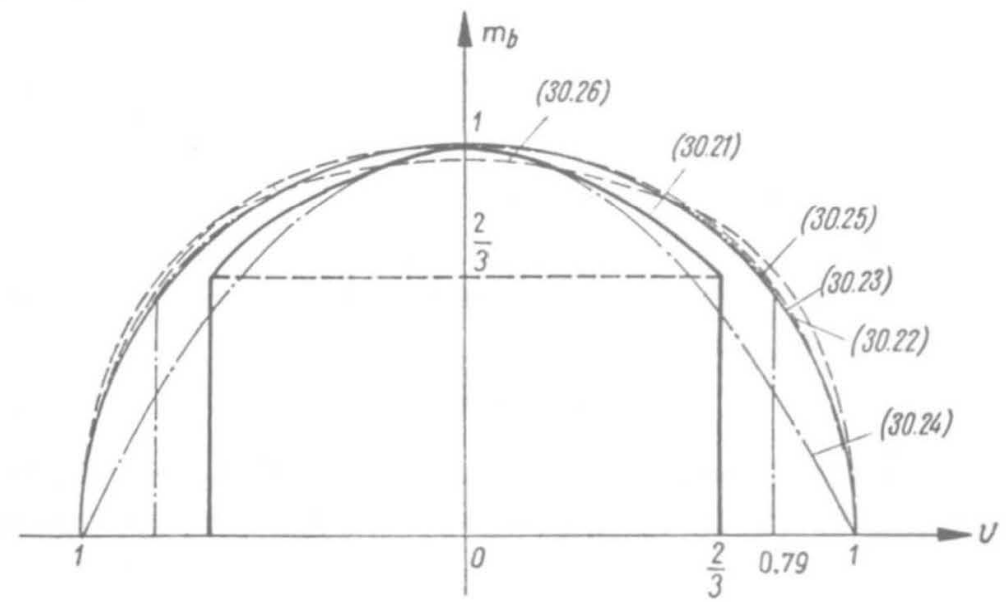

Fig. 155. Interaction curves for a rectangular section under bending with shear

Many authors are aware of the non-uniqueness of the plastic interaction curve in the case under consideration, namely of the dependence of that curve on the variability of $M_{b}$ and $V$ along the axis, and consider the problem as a two-dimensional problem of plasticity; in the simplest approach of that type the stresses $\sigma_{z}$ are neglected. The first two-dimensional analysis is due to B. M. Broude [6.33, 6.34], who discussed in detail a simply supported beam loaded by a concentrated force $P$. Broude's approach was generalized to non-prismatic and statically indeterminate beams by L. P. Kunitsky [6.126]. A detailed analysis of kinematically admissible fields in a rigid-plastic beam, leading to upper bounds, was carried out by A. P. Green [6.83]. A similar but elastic-plastic approach is due to V. N. Kukudzhanov [6.125], who considered three zones in the cross-section: elastic, plastic due to bending and plastic due to shear. A further increase of accuracy may be achieved if we introduce "second" normal stresses $\sigma_{z}$-those stresses were accounted for by M. Capurso [6.40], B. G. Neal [6.171] and K. A. Reckling [2.141, 6.197, 6.199]. Neal carried out a numerical optimization of lower bounds, considering the maximal limit carrying capacity as the objective function. Reckling applied power series of the variable $z$ to obtain elastic-plastic solutions; his plastic interaction curve (after 
a suitable change of notation) may be reduced to (30.21), hence, in the first approximation, the stress $\sigma_{z}$ has no influence on that curve. An extension of the planestress solutions to tapered beams is due to A. S. Ranchi, N. R. Chitkara and W. Johnson [6.191].

The introduction of $\sigma_{z}$ into the considerations results in essential differences between plane stress solutions and plane strain solutions; the latter correspond to wide beams. Under the additional assumption of material incompressibility, the HMH yield condition for plane strain (11.9) has an even simpler form than that for plane stress. Simultaneous bending with shear in plane strain conditions was first considered by E. T. Onat and R. T. Shield [6.177] and A. P. Green [6.83]. More detailed studies are due to G. S. Shapiro [6.214], J. Oudin and Y. Ravalard [6.178].

The limit carrying capacity of a reinforced concrete section was considered by M. Como [6.52]; he assumed the schematization of a perfectly plastic-brittle body. N. I. Bezukhov [6.25] investigated the influence of the loading path on the limit carrying capacity of a cross-section and found this influence to be very small.

Many papers are devoted to applications of a simplified theory of the limit carrying capacity of a cross-section under bending with shear to the analysis of a beam as a whole. As a rule, small influence of shear was stated in statically determinate beams, whereas this influence was considerable in the case of statical indeterminacy. To begin with, let us mention here a paper by A. Galli and V. Franciosi [6.71], who, for a beam with both ends clamped, found a decrease of the carrying capacity of the order 30 per cent; further applications may be found in monographs by B. G. Neal [4.413] and I. L. Dikovitch [5.39]. L. M. Belenky [6.18] investigated the influence of the degree of loading concentration on the limit carrying capacity of a beam: he stated that dangerous cross-sections of a simple supported beam do not correspond to the maximal bending moment but to the boundary points of the loading (regarded as uniformly distributed along a small portion of the beam).

Application of the plastic interaction curves for bending with shear to the optimal design of beams with a rectangular section of variable depth may be found in a paper by G. M. Nosov [6.173]. Yu. V. Nemirovsky [6.172] considered dynamic bending of beams taking account of shear. The rectangular cross-section of a curved beam under bending with shear was investigated by G. F. Malikov [6.149].

Most experimental investigations on plastic bending with shear were carried out on I-beams; rectangular beams were investigated by A. I. Kuzin [6.130, 6.131, 6.132], who proposed simple empirical formulae for the limit carrying capacity of beams taking account of shear.

\subsection{Thin-walled cross-sections}

Most papers on simultaneous bending and shear of thin-walled cross-sections analyse symmetrical I-sections; however, the results may easily be applied to related sections, e.g. channel or box-sections. 
In the first paper (1950) A. I. Strelbitskaya [6.227] applied Palchevsky's hypothesis, to I-sections, assuming that yielding due to shear may take place both in the web and in the flanges. Such an approach leads to considerable errors from above, since for thin-walled sections in the limiting case $M_{b} \rightarrow 0$ formula (30.1) largely overestimates the limit shearing force $\bar{V}$. Doubtlessly the assumption

$$
\bar{V}=\tau_{0} A_{w},
$$

where $A_{w}$ denotes the web area only, is better justified. In her further papers, summarized in the monograph [6.235], Strelbitskaya assumed no shear, stresses in the flanges (at least no $\tau_{x z}$ components appearing in the integral formula for the shearing force) and discussed some further hypotheses regarding the stress distribution in the limit state, [6.236].

M. R. Horne [6.98] applied a certain modification of Bezukhov's hypothesis, taking the stresses $\sigma_{z}$ into account; assuming shearing stresses in the web only, he derived a formula which may be written in the form

$$
m_{b}=1-0.44 \frac{A-A_{f}}{A+A_{f}} v^{2}, \quad v \leqslant 0.79,
$$

where $m_{b}=M_{b} / \bar{M}_{b}, v=V / \bar{V}, \bar{V}$ is determined by (30.27), and $A_{f}$ denotes the crosssectional area of both flanges. Similar formulae were also proposed by A. Galli and V. Franciosi [6.71), K. A. Reckling [2.141, 6.198], I. V. Davidov and P. I. Zaytsev [6.53] (various materials of the flanges and of the web).

J. Heyman and V. L. Dutton [6.92] assumed a uniform stress distribution $\sigma_{x}= \pm \sigma_{0}$ in the flanges and a uniform distribution of $\tau_{z x}$ and of $\sigma_{x}=\sqrt{\sigma_{0}^{2}-3 \tau_{z x}^{2}}$ in the web; the platic interaction curve is then determined by the equation

$$
m_{b}=1-\frac{A-A_{f}}{A+A_{f}}\left(1-\sqrt{1-v^{2}}\right) .
$$

A detailed analysis of the limit carrying capacity of I-sections was prefaced by $B$. M. Broude $[6.33,6.34]$ by a definition of that carrying capacity from the engineering point of view. He stated that in most cases the real carrying capacity is exhausted before full plastification. The results of his analysis were represented by semi-empirical equations of the type

$$
m_{b}^{2}+v^{2}-\alpha m_{b}^{2} v^{2}=1,
$$

where the coefficient $\alpha$ depends on the geometry of the cross-section. B. B. Lampsi [6.136] extended Broude's theory, taking $\sigma_{z}$ into account.

From among the numerous other theoretical investigations let us mention here papers by A. P. Green [6.83], who discussed kinematically admissible fields; C. F. A. Leth [6.139] (upper bound); B. G. Neal [6.168] (lower bound, analysis of a cantilever as a whole); V. V. Kukudzhanov [6.125] discussed arbitrary bisymmetric thin-walled sections; S. C. Goyal [6.82] proposed both an upper and a lower bound; B. Goschy [6.81] assumed a sinusoidal distribution of $\sigma$ and a cosinusoidal distribution of $\tau$ in the web; a similar proposal was made by Z. M. Rakhimbekova [6.189]; G. Albrecht [6.1] discussed the reduced width of the flanges due to elastic- 
plastic deflections. J. Heyman [6.93] drew attention to the fact that $M_{b}$ and $V$ are not independent (because $V=d M_{b} / d x$ ), and this fact may introduce some modifications into the classical limit analysis. B. F. Botchkov ${ }_{i=1}[6.26]$ calculated the limit carrying capacity of non-symmetric I-sections, with various flanges. C. R. Calladine [6.39] extended the theory to plate girders. W. J. Worley [6.265], J. E. Bower [6.30, 6.31], E. Amstutz [6.4], A. S. Ranshi, N. R. Chitkara fand W. Johnson [6.192] considered the influence of various cutouts in the web on the limit carrying capacity of the section. G. I. N. Rozvany [6.202, 6.203] discussed the optimal plastic design of I-beams taking account of shear.

Experimental investigations of $I$-sections under bending with shear are also very extensive. The results are diversified, depending on the cross-sectional shape, length and mode of loading of beams and on the material used. The investigators observed either a stabilization of loading, typical for a plastic hinge with negligible hardening and geometrical effects (B. M. Broude [6.34], J. Heyman, V. L. Dutton [6.92]; E. Longbottom and J. Heyman [6.145],) or monotonically increasing loading with reduced slope (J. F. Baker, J. W. Roderick [6.11]; A. W. Hendry [6.89]). Further series of experiments were carried out by J. Mutermilch, E. OIszewski and M. Lubiński [6.166], C. Berardi [6.19], A. P. Green and B. B. Hundy [6.84], B. B. Lampsi [6.135] M. Škaloud [6.250] and P. Juhás [6.111]; most authors summarized their results in the form of empirical or semi-empirical formulae for the plastic interaction curve.

\subsection{Elastic-plastic range, plastic hardening}

Because of longitudinal non-homogeneity of stresses in the case of combined bending with shear, all more rigorous approaches to limit analysis must be based on elastic-plastic analysis and the relevant papers were discussed in the preceding sections. Hence we mention here only the papers devoted to the calculation of deflections, shakedown problems and plastic hardening.

The influence of shear on elastic-plastic deflections of a rectangular beam was analysed by S. A. Ambartsumyan and M. A. Zadoyan [6.3], who bosed their considerations on the Bezukhov-Prager-Hodge approach to stress distribution. A similar problem for an I-section was considered by A. H. Hall and N. M. Newmark [6.87]; in general, a slight influence of shear on deflections was stated.

Plastic shakedown of a cross-section under bending with shear was analysed by B. I. Lyubarov [5.113]. Shear effects in elastic-plastic beams under moving loads were investigated by L. P. Kunitsky [6.127] (rectangular section), N. A. Kravtsov [6.123], N. L. Chernov [6.45], N. L. Chernov and S. D. Kudryavtsev [6.70] (I-section).

Several papers analyse bending with shear in the range of plastic hardening. Linear plastic hardening was considered by V. I. Davydov [6.55], and then by Z. M. Rakhimbekova [6.187], Z. M. Rakhimbekova and L. V. Bozhkova [6.188], who gave a method of calculating deflections in this case. The power law of plastic 
hardening was applied by I. P. Renne [6.200] and A. Phillips [6.183]; D. Valalas [6.257] calculated the deflections taking account of shear. It should be noted, however, that some of these papers are based on the quasi-power-hardening law: the authors assume that (8.6) holds for $\sigma$ and $\varepsilon$ instead of $\sigma_{e}$ and $\varepsilon_{e}$, and shearing stresses are found from the equilibrium equations. Then the interaction between bending and shear is almost ignored.

A general formula for the distribution of shearing stresses for any dependence $\sigma=f(\varepsilon)$ was derived by M. Mikkola [6.158], and shearing deflections in the general case were calculated by D. G. Eyre [6.64]. Experimental verification of the plastic shearing deflections was carried out by V. I. Dunayevsky, V. S. Sviderskaya and I. Z. Entin [6.59]. A three-dimensional approach to the problem of plastic bending with shear was proposed by H. Ishikawa [6.106]. L. M. Belenky [6.18] noted that the influence of concentration of loading is particularly important in the case of plastic hardening.

\section{More general cases of combined loadings}

\subsection{Bending with tension and shear}

Most papers devoted to bending with tension and shear constitute a direct generalization of the solutions discussed in Sec. 30. The first paper is due to N. I. Bezukhov [6.24], who assumed the stress distribution shown in Fig. 151, but with the neu-

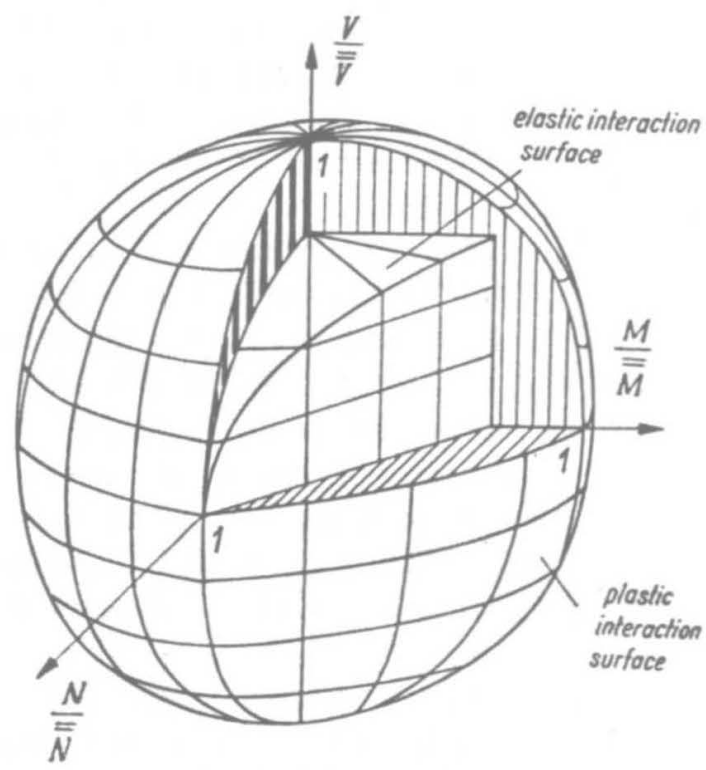

Fig. 156. Elastic and plastic interaction surfaces for a rectangular section under bending with tension and shear (after Dikovitch) 
tral axis appropriately shifted. For a rectangular cross-section one then obtains the equation of the plastic interaction surface

$$
m_{b}=1-n^{2}-\frac{3}{4} v^{2}, \quad|v| \leqslant \frac{2}{3},
$$

which is a "combination" of Eqs. (22.35) and (30.21). This equation determines a lower bound to the actual limit carrying capacity. An upper bound, following from (26.15) with substituted $\lambda_{3}=\lambda_{4}=\lambda_{6}=0$, was discussed in detail by I. L. Dikovitch [5.39]; the relevant plastic interaction surface for a rectangular section is shown in Fig. 156, quoted after Dikovitch.

V. Franciosi and E. Giangreco [6.67] derived a solution close to that of Bezukhov but based on various yield conditions. Z. Sobotka [4.544] proposed a uniform stress distribution in the cross-section, $\sigma= \pm \psi \sigma_{0}, \tau=\tau_{0} \sqrt{1-\psi^{2}}$; such an approximate assumption results in the following equation of the plastic interaction surface

$$
m_{b} \sqrt{1-v^{2}}+n^{2}+v^{2}=1 .
$$

Other solutions for the rectangular section based on various approximate hypotheses, are due to A. P. Green [6.83], who constructed slip-line fields; W. Johnson, N. R. Chitkara and A. S. Ranshi [6.110] (extension of Green's solution); K. Klöppel and M. Yamada [6.115]; E. A. Beylin [6.22], who assumed a sinusoidal distribution of $\sigma$ and a cosinusoidal distribution of $\tau$; B. G. Neal [6.170]; S. Smirak [6.251]; R. Vukotié [6.259] and R. Windels [6.261]. Z. Bažant [6.13, 6.14, 6.15] considered biaxial bending with tension and biaxial shear. T. Kusuda [6.129] proposed a certain lower bound and carried out experimental verification.

Yu. N. Aleshinsky [6.2] investigated the limit carrying capacity of a section of non-prismatic beams, and J. Wölfel [5.203] - of curved beams. A. Sawczuk and M. Janas [5.161], R. H. Lance and D. N. Robinson [6.137] considered reinforced sections. F. Ellyin and R. Deloin [6.62], V. A. Monakhov and V. I. Sebekina [6.164] applied the results for the combined case under consideration to the limit analysis of arches. Optimal design of arches taking account of shear was considered by Yu. B. Goldshteyn and M. A. Solomeshtch [6.78], V. G. Sebeshev and I. A. Chaplinsky [6.210].

The influence of normal and shearing forces on elastic-plastic deflections of beams was considered by Xu Bing-Hang [6.267], who derived a system of three differential equations and gave/ some numerical solutions. A. Ylinen [6.270] investigated a related problem of influence of shearing forces on the elastic-plastic buckling of columns. S. M. Yufin [6.271] found an important effect of normal and shearing forces on the plastic shakedown of beams.

In the case of thin-walled sections, particularly I, box, and channel-sections, the flanges practically carry no shearing stresses (at least those perpendicular to the middle line of the profile), and this fact must be taken into account in calculations. In accordance with the above statement, A. I. Strelbitskaya [6.235] assumed the stress distributions shown in Fig. 157: shearing stresses in flanges do occur, but 

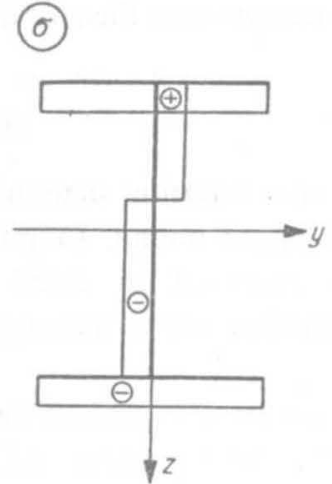

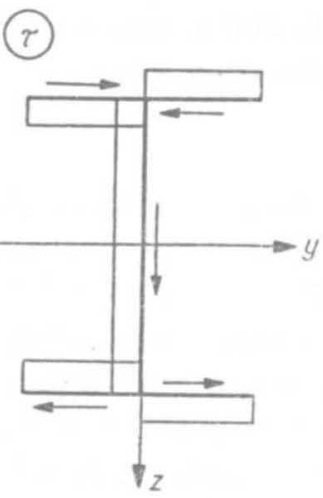

Fig. 157. Strelbitskaya's hypothesis as regards the stress distribution in the fully plastic state under bending with tension and shear

have no influence on the value of the shearing force $V$. Expressing the generalized stresses $M_{b}$ and $N$ by the parameter $z_{0}$, subsequently eliminating this parameter and making use of the yield condition, one arrives at the following equation of the plastic interaction surface:

$$
\frac{1}{2}\left[m_{b}^{2}+m_{b} \sqrt{m_{b}^{2}+\frac{\left(2 A-A_{f}\right)^{2}}{A^{2}-A_{f}^{2}} n^{2}}\right]+\frac{\left(2 A-A_{f}\right)^{2}}{4\left(A^{2}-A_{f}^{2}\right)} n^{2}+v^{2}=1,
$$

where $A_{f}$ denotes the cross-sectional area of both flanges, the dimensionless normal force is referred to the total area $A$, the dimensionless shearing force $v$ to the web area $A-A_{f}$, and the dimensionless bending moment $m_{b}$ to the limit carrying capacity in bending. A related approach is due to Z. Sobotka [6.224]; however, he neglected shear in flanges. Moreover, Sobotka derived an upper bound of the type (26.15) and gave an application to the limit analysis of statically indeterminate beams.

Simple approximate formulae for the limit carrying capacity of an I-section under bending, tension and shear were derived by M. R. Horne [6.99], who noticed the possible influence of web buckling, K. Klöppel and M. Yamada [6.115] and B. G. Neal [6.169]. A. S. Ranchi, N. R. Chitkara and W. Johnson [6.192] derived a slip line field solution. Castellated beams were discussed by A. N. Sherbourne and J. van Oostrom [6.215]. An application of the theory to the limit analysis of I-sectioned arches was given by V. G. Sebeshev and I. A. Chaplinsky [6.211], who also carried out an experimental verification.

The influence of normal and shearing forces on elastic-plastic deflections of an I-beam was considered by V. V. Kozlyakov [6.122]; he assumed linear plastic hardening, discussed subsequent! stages of yielding and applied the method of elastic solutions.

\subsection{More general cases of combined loadings of thin-walled cross-sections}

As was mentioned in Sec. 1.4, in the case of thin-walled sections we may regard the bimoment (warping group) $B$ and the warping moment $M_{w}$ as independent, and 
hence the total number of generalized stresses may here be raised to eight. Four of these generalized stresses are connected with normal stresses $\sigma\left(N, M_{b y}, M_{b z}, B\right)$, and four-with shearing stresses $\tau\left(V_{y}, V_{z}, M_{t}, M_{\omega}\right)$.

The first more general approach to the limit carrying capacity of thin-walled bars is due to A. R. Rzhanitsyn [6.206], 1941. He considered simuitaneous bending with torsion of an I-section and of a channel section taking the bimoment into account.

The general case of eight generalized stresses was investigated in detail by A. 1. Strelbitskaya [6.227, 6.231, 6.235]. She assumed almost-uniform stress distribution with possible jumps due to change of sign, namely, in the whole cross-section $\sigma= \pm \psi \sigma_{0}, \tau= \pm \sqrt{1-\psi^{2}} \tau_{0}$. The stresses $\sigma$ are assumed to be constant across the wall, whereas $\tau$ may change sign also along a "longitudinal" line; such an assumption is necessary, for example, even when we consider pure torsion of open thinwalled profiles. Moreover, it is assumed that the contour as a whole is nondeformable and no buckling occurs.

Under these assumptions, the system of generalized stresses $N, M_{b y}, M_{b z}, B$ may be considered lindependently of the system $\mid V_{y}, V_{z}, M_{t}, M_{\omega}$; they are interrelated only via the parameter $\psi$. Consequently, we obtain usually a lower bound to the limit carrying capacity proceeding as was shown in Sec. 26.2. Certain exceptions (an upper bound instead of a lower bound) may occur if the stress fields assumed do not satisfy all the requirements of static admissibility.

Strelbitskaya noted that the analysis of the distribution of normal stresses $\sigma$ involves more difficulties than that of shearing stresses $\tau$. Namely, eliminating the coordinates of jump-like discontinuities of $\sigma$, i.e. expressing these coordinates in terms of generalized stresses, we arrive at algebraic equations of a rather high degree. In the general case that equation with respect to $\sigma$ is of the eighth degree. If only

(6)

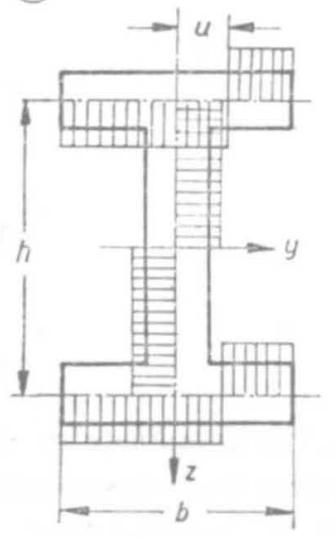

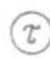

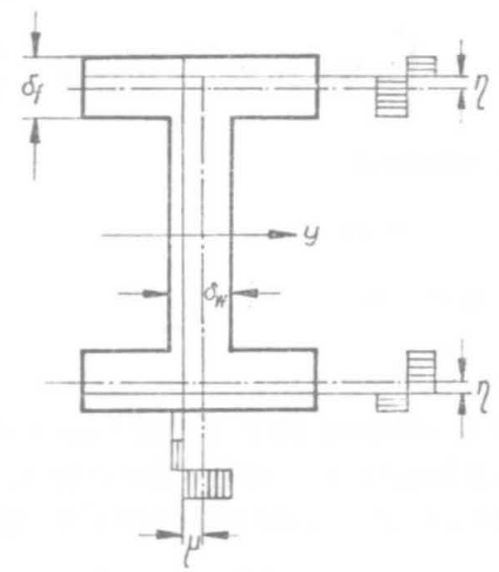

Fig. 158. Strelbitskaya's hypothesis as regards the stress distribution in the fully plastic state under five generalized stresses 
two points of discontinuity appear, we obtain a quadratic equation, and if only one discontinuity takes place-as was shown in Fig. 157-we have only a quadratic equation. A similar procedure in the case of shearing stresses usually leads to quadratic equations for $\tau$.

As an example we quote here the solution derived by Strelbitskaya [6.235] for a thin-walled section under five generalized stresses: $M_{b y}, B ; V_{z}, M_{t}, M_{\omega}$; this case may be classified as $\mathscr{P}_{2} / \mathscr{S}_{5}$. Because of the restriction of the first system to two generalized stresses, $M_{b y}$ and $B$ only, the solution is relatively simple.

Consider for example an I-section and assume the stress distribution shown in Fig. 158. The distribution of $\sigma$ corresponds to a bending moment

$$
M_{b y}=\left(2 \delta_{f} h u+\delta_{w} \frac{h^{2}}{4}\right)
$$

and to a bimoment

$$
B=\sigma \delta_{w} h\left(\frac{b^{2}}{4}-u^{2}\right)
$$

where the symbols $\delta_{f}, \delta_{w}, h, b, u$ are shown in Fig. 158. Eliminating $u$, we obtain a quadratic equation with respect to $\sigma$. The solution of that equation may be written in the following general form, valid for a fairly broad class of thin-walled sections:

$$
\sigma=\left[\frac{B-D M_{b y}}{2 \bar{B} K}+\sqrt{\frac{\left(B-D M_{b y}\right)^{2}}{4 \bar{B}^{2} K^{2}}+\frac{M_{b y}^{2}}{4 C \overline{\bar{B}} K \sigma_{0}}}\right] \sigma_{0},
$$

where $\overline{\bar{B}}$ denotes the limit bimoment for the cross-section, $C, D$, and $K$-constants depending on the geometry of the section.

The distribution of shearing stresses $\tau$ corresponds to a twisting moment (de Saint-Venant moment)

$$
M_{t}=\tau\left[b\left(\delta_{f}^{2}-4 \eta^{2}\right)+\frac{h}{2}\left(\delta_{w}^{2}-4 \mu^{2}\right)\right],
$$

to a warping moment

$$
M_{\omega}=2 \tau h\left(b \eta+a_{y} \mu\right)
$$

and to a shearing force

$$
V=2 \tau h \mu \text {. }
$$

The symbol $a_{y}$ denotes here, for the sake of generality, the coordinate of shear centre; for an I-section we may substitute $a_{y}=0$. Eliminating from these equations the parameters $\mu$ and $\eta$, we arrive at the following equation, quadratic in $\tau$ :

$$
\overline{\bar{M}}_{t} \tau^{2}-M_{t} \tau_{0} \tau-\frac{V^{2} \tau_{0}}{2 h}-\frac{\left(M_{\omega}-V a_{y}\right)^{2} \tau_{0}}{b h^{2}}=0,
$$


where

$$
\overline{\bar{M}}_{t}=\alpha\left(b \delta_{f}^{2}+\frac{h}{2} \delta_{w}^{2}\right) \tau_{0}
$$

is the limit twisting (de Saint-Venant) moment, $\alpha$ denotes a coefficient depending on the shape of the section. The solution of (31.10) may be written thus:

$$
\tau=\left\{\frac{M_{t}}{2 \overline{\bar{M}}_{t}}+\sqrt{\left.\frac{M_{t}^{2}}{4 \overline{\bar{M}}_{t}^{2}}+\frac{1}{\overline{\bar{M}}_{t} \tau_{0}}\left[\frac{V^{2}}{2 h}+\frac{\left(M_{\omega}-V a_{y}\right)^{2}}{b h^{2}}\right]\right\}} \tau_{0} .\right.
$$

Substituting (31.6) and (31.12) into the elliptic yield condition (11.33), we obtain the following equation of the limit surface (hypersurface in five-dimensional space):

$$
\begin{aligned}
& \frac{\left(B-D M_{b y}\right)^{2}}{2 \bar{B}^{2} K^{2}}+\sqrt{\frac{\left(B-D M_{b y}\right)^{4}}{4 \overline{B^{4}} K^{4}}+\frac{M_{b y}^{2}\left(B-D M_{b y}\right)^{2}}{4 C \bar{B}^{2} K^{3} \sigma_{0}}}+ \\
& +\frac{M_{b y}^{2}}{4 C \overline{\bar{B}} K \sigma_{0}}+\frac{M_{t}^{2}}{2 \overline{M_{t}^{2}}} \sqrt{\frac{M_{t}^{4}}{4 \overline{M_{t}^{4}}}+\frac{M_{t}^{2}}{\bar{M}_{t}^{3} \tau_{0}}\left[\frac{V^{2}}{2 h}+\frac{\left(M_{\omega}-V a_{y}\right)^{2}}{b h^{2}}\right]}+ \\
& +\frac{1}{\overline{\bar{M}}_{t} \tau_{0}}\left[\frac{V^{2}}{2 h}+\frac{\left(M_{\omega}-V a_{y}\right)^{2}}{b h^{2}}\right]=1 .
\end{aligned}
$$

For an I-section the constants are

$$
\begin{aligned}
& C=\delta_{f} h, \quad D=\frac{1}{8} \frac{\delta_{w}}{\delta_{f}} h, \quad K=1-\frac{\delta_{w}^{2} h^{2}}{16 \delta_{f}^{2} b^{2}}, \\
& a_{y}=0, \quad \bar{B}=\frac{1}{4} \delta_{f} h b^{2} \sigma_{0},
\end{aligned}
$$

Strelbitskaya gives also the relevant values for a channel section and discusses in detail particular cases of (31.13).

Biaxial bending and additional normal force would essentially complicate (31.14). A very simple approximation for such a case was proposed by B. Åkesson and J. Băcklund [6.10]; in fact, convexity of the plastic interaction surface ensures that an equation of the type

$$
\sum \frac{\left|Q_{i}\right|}{\overline{\bar{Q}}_{i}}=1
$$

furnishes a lower bound to the actual surface. The authors take $Q_{i}$ to be equal in turn to $N, M_{b y}, M_{b z}, M_{t}$ and $B$, but $V_{y}, V_{z}$ and $M_{\omega}$ may be included here as well. A more accurate approximation for the general case may be obtained if we combine (31.15) with the approach discussed in Sec. 26.2; it has the form

$$
\left(\sum \frac{\left|Q_{l}\right|}{\bar{Q}_{l}}\right)^{2}+\left(\sum \frac{\left|Q_{j}\right|}{\overline{\bar{Q}}_{j}}\right)^{2}=1,
$$


where $Q_{i}=N, M_{b y}, M_{b z}, B$, whereas $Q_{j}=V_{y}, V_{z}, M_{t}, M_{\omega}$. Another approach to the general case was proposed by G. M. Pinsker [6.184], but only some more particular cases were analysed in detail.

The limit analysis of I-beams under restricted torsion, taking account of the warping moment and the bimoment, was considered by E. A. Rayevskaya [6.193], J. Rutecki [6.205] and G. Augusti [6.6,6.7]. Particular attention was paid to plastic bending combined with restricted torsion; we mention here papers by V. Křupka [6.124] (with experimental verification), E. A. Rayevskaya [6.195] P. G. Bytchkov [6.37], N. S. Boulton [6.29], S. S. Gill and J. K. G. Boucher [6.76] (experimental), K. S. Dinno and S.S. Gill [6.56], K. S. Dinno and W. Merchant [6.57], G. Augusti and R. Scuderi [6.8], G. Augusti [6.9]. Additional shearing force was considered by Z. Bažant and S. Šmiřak [6.16], additional normal force--by T. Usami [6.256]. L. E. Farmer and P. M. Ferguson [6.65] investigated the limit carrying capacity of reinforced concrete tee-sections under bending with restricted torsion.

The elastic-plastic range of thin-walled sections under various combinations of loadings was also considered by A. I. Strelbitskaya [6.235]. Moreover, paper [6.229] is devoted to linear and power plastic hardening. Plastic hardening in thinwalled section under bending with tension and restricted torsion was considered in detail by R. A. Mezhlumyan [6.153, 6.154, 6.155]. M. Tochacek [6.254] applied to the elastic-plastic analysis of open thin-walled sections the method of elastic solutions. An open cross-section under the bimoment and the warping moment was analysed in the elastic-plastic range by K. Lundgren [6.147]. Many effective elasticplastic solutions may be found in the book by C. F. Kollbrunner and N. Hajdin [6.119].

Extensive experimental investigations of the inelastic behaviour of thin-walled bars under combined loadings were presented in papers by A. I. Strelbitskaya and G. I. Evseyenko [6.245, 6.246] and summarized in their monograph [6.247]. 


\section{CHAPTER VII}

\section{Combined loadings at the level of a cross- section of a surface structure}

32 Purely membrane states $\mathscr{P}_{3} \rightarrow \mathscr{S}_{3}$, and purely moment states $\mathscr{P}_{3} / \mathscr{S}_{3}$

\subsection{General remarks}

We defined in Sec. 1.4 the cross-section of a surface structure as a segment $-H$ $\leqslant z \leqslant H$ with two mutually perpendicular elementary cross-sections cut in such a way as to be compatible with the neighbouring sections, $\left({ }^{1}\right)$ Fig. 2 . In the case of a plate the directions $x$ and $y$, normal to those elementary sections, may be chosen arbitrarily, but in considering shells it is convenient to choose these directions coinciding with the lines of principal initial curvatures (along which the natural twist is zero). Such lines, $\xi=$ const and $\eta=$ const (Gaussian coordinates), always exist and are mutually perpendicular, though not always they are unique. Integrating the stresses $\sigma_{\alpha \beta}$ and $\sigma_{\alpha z}(\alpha, \beta=\xi, \eta)$ over the relevant elementary cross-sectional areas (curvilinear rectangles) and referring the results to the elementary arcs of the middle surface, we express the ten generalized stresses (stress resultants, sectional forces) as follows:

membrane forces

$$
N_{\alpha \beta}=\int_{-H}^{H} \sigma_{\alpha \beta}\left(1+\frac{z}{R_{\gamma}}\right) d z,
$$

bending and twisting moments

$$
M_{\alpha \beta}=\int_{-H}^{H} \sigma_{\alpha \beta} z\left(1+\frac{z}{R_{\gamma}}\right) d z,
$$

(1) The traditional notation $z$ instead of a more consistent $Z$ is used in this chapter. 
and shearing forces

$$
Q_{\alpha}=\int_{-H}^{H} \sigma_{\alpha z}\left(1+\frac{z}{R_{\gamma}}\right) d z,
$$

where $\alpha, \beta, \gamma=\xi, \eta$ and $\gamma \neq \alpha(\gamma$ is complementary to $\alpha)$; the symbols $R_{y}$ denote the principal initial radii of curvature of the shell $\left(R_{\gamma} \rightarrow \infty\right.$ for a plate). In the case of sandwich surface structures whose layers are assumed to be in membrane state, the integration in (32.1)-(32.3) is replaced by simple summation.

Most theories of surface structures are based on the Love-Kirchhoff hypothesis of straight normals, which after deformation remain straight and normal to the deformed middle surface. This assumption leads to the following distribution of strains in the cross-section (V. V. Novozhilov [7.232]):

$$
\varepsilon_{\alpha \beta}(z)=\frac{1}{2}\left(\frac{1}{1+z / R_{\gamma}}+\frac{1}{1+z / R_{\theta}}\right)\left(\lambda_{\alpha \beta}+\varkappa_{\alpha \beta} z\right),
$$

where, in turn, $\alpha, \beta=\xi, \eta$; further, in all cases covered by (32.4), $\gamma=\alpha$ and $\vartheta=\beta$, but these symbols have been introduced to avoid summation here and to facilitate the notation of subsequent formulae.

The quantities $\lambda_{\alpha \beta}$ in (32.4) denote strain components at the middle surface, $z=0$, (extensions including in-plane shear) whereas the geometrical interpretation of $\chi_{\alpha \beta}$ is more complicated. In the case of a plate they denote the actual curvatures of the respective strain-neutral layers (where $\varepsilon_{\alpha \beta}(z)=0$, cf. (22.39)). In the case of a shell the quantities $\varkappa_{\alpha \beta}$ are usually interpreted as the increments of curvatures of the middle surface; this interpretation is not exact. To derive the exact formula for $x_{\xi \xi}$, for example, we make use of the definition of small strain (Fig. 159)

$$
\varepsilon_{\xi \xi}(z)=\frac{\left(\varrho_{\xi}+z\right) d \varphi-\left(R_{\xi}+z\right) d \varphi_{0}}{\left(R_{\xi}+z\right) d \varphi_{0}}
$$

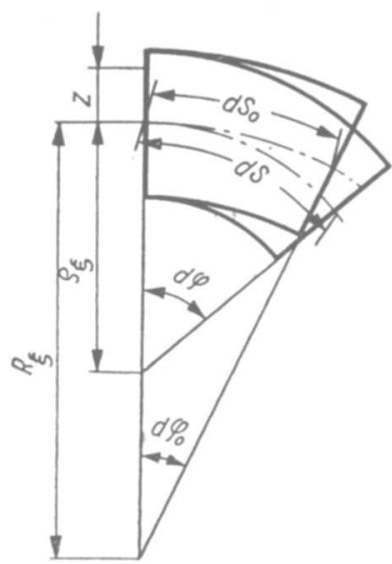

Fig. 159. Deformation of an element of a shell 
and by virtue of

$$
\varrho_{\xi} d \varphi=\left(1+\lambda_{\xi \xi}\right) R_{\xi} d \varphi_{0}
$$

we obtain the following expression for the coefficient of $z$ in the numerator of (32.4)

$$
x_{\xi \xi}=\frac{1+\lambda_{\xi \xi}}{\varrho_{\xi}}-\frac{1}{R_{\xi}}=\left(1+\lambda_{\xi \xi}\right) \Delta x_{\xi \xi}+\frac{\lambda_{\xi \xi}}{R_{\xi}},
$$

where $\varrho_{\xi}$ denotes the radius of curvature after deformation and $\Delta x_{\xi \xi}=\left(1 / \varrho_{\xi}\right)-$ $-\left(1 / R_{\xi}\right)$ stands for the increment of curvature. It can be seen that $x_{\xi \xi}=\Delta x_{\xi \xi}$ if and only if $\lambda_{\xi \xi}=0$, and in shells this is rather exceptional. The quantity $x_{\xi \xi}$ should rather be interpreted as the increment of the elementary angle referred to the respective elementary arc of the middle surface: in fact (Fig. 159),

$$
\frac{d \varphi-d \varphi_{0}}{d s_{0}}=\frac{d s}{\varrho_{\xi} d s_{0}}-\frac{1}{R_{\xi}}=\frac{1+\lambda_{\xi \xi}}{\varrho_{\xi}}-\frac{1}{R_{\xi}}=\chi_{\xi \xi} .
$$

Such an increment can be different from zero even if $\Delta x_{\xi \xi}=0,(32.7)$.

It should be noted that the Love-Kirchhoff hypothesis of straight normals implies (32.4), but the reverse is not true: these strain distributions do not exclude warping of the cross-section or deviations from normality (cf. (29.1) and (29.2) for bars). They are sometimes combined, for example, with shearing strains $\varepsilon_{\alpha z}$, exceeding the Love-Kirchhoff assumptions (G.S. Shapiro [7.332]).

The generalized stresses $N_{\alpha \beta}$ and $M_{\alpha \beta}$ and the generalized strains $\lambda_{\alpha \beta}$ and $x_{\alpha \beta}$ are consistent as far as the virtual work is concerned. Indeed, the element of volume of a shell may be represented by the formula

$$
d V=\left(1+\frac{z}{R_{\xi}}\right)\left(1+\frac{z}{R_{\eta}}\right) d A d z
$$

where $d A$ is the elementary area of the middle surface; the virtual work (1.2) with substituted (32.4) and neglected shearing stresses $\sigma_{\alpha z}$ may be represented as follows:

$$
\begin{aligned}
\delta W= & \iiint_{V} \sigma_{\alpha \beta} \delta \varepsilon_{\alpha \beta} d V \\
= & \frac{1}{2} \iint_{A} d A \int_{-H}^{H} \sigma_{\alpha \beta}\left(\frac{1}{1+z / R_{\gamma}}+\frac{1}{1+z / R_{\theta}}\right)\left(1+\frac{z}{R_{\xi}}\right)\left(1+\frac{z}{R_{\eta}}\right) \times \\
& \times\left(\delta \lambda_{\alpha \beta}+\delta x_{\alpha \beta} z\right) d z,
\end{aligned}
$$

where $\gamma=\alpha$ and $\vartheta=\beta$ for any $\alpha$ and $\beta$ and the "plane" or "two-dimensional" summation convention holds. Cancelling brackets, we may write

$$
\delta W=\frac{1}{2} \iint_{A} d A \int_{-H}^{H} \sigma_{\alpha \beta}\left[\left(1+\frac{z}{R_{\gamma}}\right)+\left(1+\frac{z}{R_{\theta}}\right)\right]\left(\delta \lambda_{\alpha \beta}+\delta x_{\alpha \beta} z\right) d z,
$$


where now $\gamma \neq \alpha$ and $\vartheta \neq \beta$ ( $\gamma$ and $\vartheta$ are complementary to $\alpha$ and to $\beta$, respectively). Finally, making use of the definitions (32.1) and (32.2) and of the symmetry of $\lambda_{\alpha \beta}$ and $\varkappa_{\alpha \beta}$, we obtain

$$
\delta W=\iint_{A}\left(N_{\alpha \beta} \delta \lambda_{\alpha \beta}+M_{\alpha \beta} \delta \varkappa_{\alpha \beta}\right) d A .
$$

Hence, according to the definition adopted in Sec. 1.6, the quantities $N_{\alpha \beta}$ and $M_{\alpha \beta}$ may be regarded as basic exertion factors at the level $\mathscr{S}$ under consideration and limit surfaces may be constructed, first of all, in the space of those exertion factors. However, in most cases only the cross-sections of plates are analysed in this manner, and in the case of thin shells certain simplifications are introduced.

To begin with, definitions $(32.1) \div(32.3)$ have some drawbacks; for example, assuming $\sigma=$ const, we obtain from (32.2) $M \neq 0$ (even in the transverse cross-section of a cylindrical shell), since the moments are calculated not about the centre of gravity of the curvilinear rectangle. Thus, in most cases formulae $(32.1) \div(32.3)$ are replaced by the formulae, symmetric with respect to the indices $\alpha$ and $\beta$,

$$
\begin{aligned}
N_{\alpha \beta} & =\int_{-H}^{H} \sigma_{\alpha \beta} d z, \\
M_{\alpha \beta} & =\int_{-H}^{H} \sigma_{\alpha \beta} z d z, \\
Q_{\alpha} & =\int_{-H}^{H} \sigma_{\alpha z} d z ;
\end{aligned}
$$

they are exact in the case of plates and are sufficiently accurate for thin shells, $\max \left(H / R_{y}\right) \ll 1$. Further, it is convenient to linearize the hyperbolic strain distri$\gamma=\xi, \eta$

butions (32.4). Simply neglecting $\left(z / R_{\gamma}\right)$ and $\left(z / R_{\theta}\right)$ in the denominators we may write

$$
\varepsilon_{\alpha \beta}(z)=\lambda_{\alpha \beta}+\varkappa_{\alpha \beta} z .
$$

Other approaches to linearization are of course also possible. For example expanding (32.4) into a power series of $z$ and retaining all the linear terms, we obtain in the formula for $\varepsilon_{\xi \xi}$ the following coefficient of $z$ :

$$
x_{\xi \xi}^{*}=x_{\xi \xi}-\frac{\lambda_{\xi \xi}}{R_{\xi}}=\left(1+\lambda_{\xi \xi}\right) \Delta x_{\xi \xi} .
$$

However, formula (32.16) has the following advantage: if combined with the definitions (32.13) and (32.14) and with (32.9), in which the terms $\left(z / R_{\xi}\right)$ and $\left(z / R_{\eta}\right)$ are neglected, it leads to the expression for virtual work (32.12), and hence the system of generalized stresses and strains remains consistent. In what follows we shall use (32.16), even without a precise definition of $\varkappa_{\alpha \beta}$; as a rule, such a definition 
is not necessary at the level $\mathscr{S}$ and must be introduced only when the analysis at the level $\mathscr{S}$ is applied to the shell as a whole.

In view of the state of stress under consideration, which under the usual assumption $\sigma_{z z}=0$ is two-dimensional, or even three-dimensional if $\sigma_{\alpha z}$ are taken into account, the influence of the yield condition adopted is much more essential here than in the case of bars. For ductile materials the HMH yield condition with the associated flow rule (similarity of deviators) is best verified by experiments. On the other hand, if the principal directions of stresses are constant during the process and coincide with the lines of principal curvatures of the shell, then the TG yield condition with the associated flow rule leads to much simpler results. If we consider this approach only as an approximation of the $\mathrm{HMH}$ yield condition, then the errors of approximation may be quite considerable (cf. Sec. 13.7). Sometimes the TG yield condition may be combined with the law of similarity of deviators (V. V. Sokolovsky [7.344], J. A. König [3.382]), and then in most cases the errors are reduced.

There exist many monographs and survey papers dealing wholly or partly with limit surfaces for a cross-section of surface structures, with constitutive equations valid in particular ranges, and with the application of those equations to the calculation of elastic-plastic deflections and of the limit carrying capacity of a structure as whole. Let us mention here the monographs published by A. A. Ilyushin [1.8], P. G. Hodge [1.6, 4.223], R. H. Wood [4.609], D. Niepostyn [4.420, 4.421], H. Haase [4.198], Ch. Massonnet and M. Save [5.119, 4.508], M. Sh. Mikeladze [3.528, 7.199], A. Sawczuk and Th. Jaeger [4.513], collective work edited by W. Olszak, P. Perzyna and A. Sawczuk [2.125], the monographs by W. Olszak and A. Sawczuk [7.250], M. Janas, J. A. König and A. Sawczuk [7.113, 7.312], T. H. Lin [3.456], A. I. Strelbitskaya, V. A. Kolgadin and S. I. Matoshko [7.354], V. I. Korolev [7.136], Z. Sobotka [4.545], R. Valid [7.377], M. I. Erkhov [7.55], and A. Sawczuk [7.313]. Survey papers were published by A. Sawczuk and W. Olszak [7.302, 7.304], Yu. R. Lepik [7.156], G. S. Shapiro [7.333], A. I. Strelbitskaya [7.349, 7.358], L. M. Kachanov [7.122], J. Rychlewski and G. S. Shapiro [7.284], A. A. Gvozdev and A. M. Protsenko [7.80], M. Janas and A. Sawczuk [7.114, 7.309], N. Jones [5.82], and M. Save [7.295].

In comparison with the scope of the above monographs and survey papers the present chapter will give a relatively concise treatment, but with a comprehensive guide to bibliography.

\subsection{Membrane states}

Suppose that all the moments $M_{\alpha \beta}$ determined by (32.14) and the shearing forces (32.19) vanish; then the state in the cross-section of a surface structure is called the membrane state. Membrane states occur, first of all, if in a cross-section of a surface structure whose material is at least transversally homogeneous, the strains $\varepsilon_{\alpha \beta}(z)$ are constant and $\varepsilon_{\alpha z}=0$. Indeed, then the stresses $\sigma_{\alpha \beta}(z)$ are also constant, 
and all the integrals in (32.14) vanish; further, $\sigma_{\alpha z}=0$ and (32.15) vanish. In the linearized treatment (32.16) the strains are constant if $\varkappa_{\alpha \beta}=0$; in a more rigorous approach (32.4) the condition $x_{\alpha \beta}=0$ is, in general, not sufficient to eliminate the moments.

However, under the assumption of perfect plasticity the range of applicability of membrane theory is slightly broader. Consider a circular cylindrical shell of moderate thickness, for which formulae (32.4) may be regarded as adequate; the shell is subject to uniform radial pressure $p$ (Fig. 160). If the deflections are not

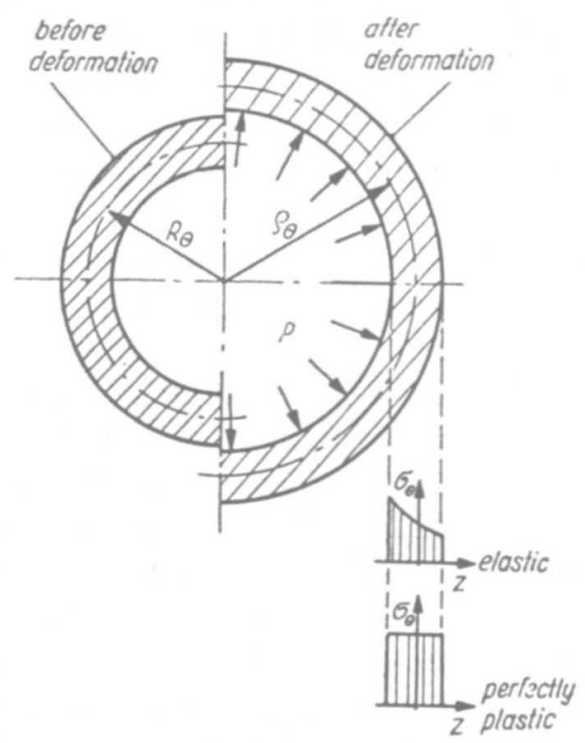

Fig. 160. An example of $x_{\theta}=0$ in spite of the change of curvature of a shell

restricted so as to be very small, then the circumferential curvature changes. Introduce the engineering notation; though the parameter $\varkappa_{\theta}$ is equal to zero [since the angles do not change, $\left(1+\lambda_{\theta}\right) R_{\theta}=\varrho_{\theta}$, formula (32.4) gives a hyperbolic distribution of the strains $\varepsilon_{\theta}(z)$. In the elastic range the distribution of the stresses $\sigma_{\theta}(z)$ is also hyperbolic, and $M_{\theta} \neq 0$. In the plastic range, if $\sigma_{z}=0$, then the stresses $\sigma_{\theta}$ become uniform, formula (32.14) or even (32.2) gives $M_{\theta}=0$, and the conditions of a membrane state are exactly satisfied.

In what follows, we assume constant stresses in a cross-section in the membrane state. Then the integral formulae (32.13) lead to the following simple results:

$$
N_{\xi}=2 \sigma_{\xi} H, \quad N_{\eta}=2 \sigma_{\eta} H, \quad N_{\xi \eta}=2 \tau_{\xi \eta} H,
$$

and hence the combined case under consideration may be classified as $\mathscr{P}_{3} \rightarrow \mathscr{S}_{3}$. The membrane forces (32.18) form a symmetric two-dimensional tensor, since $N_{\eta \xi}=N_{\xi \eta}$ (the definitions (32.1) would lead, in general, to $N_{\eta \xi} \neq N_{\xi \eta}$ ). If we present 
the yield condition in a form close to (9.22), namely homogeneous of the first degree in $\sigma_{i j}$,

$$
\frac{\sigma_{\text {red }}}{\sigma_{0}}=f\left(\frac{\sigma_{\xi}}{\sigma_{0}}, \frac{\sigma_{\eta}}{\sigma_{0}}, \frac{\tau_{\xi \eta}}{\sigma_{0}}\right)=1,
$$

and substitute (32.18), then the analogous equation

$$
f\left(\frac{N_{\xi}}{2 \sigma_{0} H}, \frac{N_{\eta}}{2 \sigma_{0} H}, \frac{N_{\xi \eta}}{2 \sigma_{0} H}\right)=1
$$

determines simultaneously the elastic interaction surface and the plastic interaction surface for a cross-section, constructed in the space of generalized stresses. The elastic-plastic range in the case under consideration does not occur (unless the membrane plastic state was preceded by a hyperbolic stress distribution in the elastic range). If we introduce dimensionless membrane forces by the formulae

$$
\frac{N_{\xi}}{2 \sigma_{0} H}=n_{\xi}, \quad \frac{N_{\eta}}{2 \sigma_{0} H}=n_{\eta}, \quad \frac{N_{\xi \eta}}{2 \sigma_{0} H}=n_{\xi \eta},
$$

then Eq. (32.20) may be written in the following concise form:

$$
n \stackrel{\text { def }}{=} f\left(n_{\xi}, n_{\eta}, n_{\xi \eta}\right)=1,
$$

The geometrical interpretation of (32.22) coincides of course with the interpretation of the yield condition (32.19). For some reasons it would be more convenient to define the dimensionless tangential force $n_{\xi \eta}$ as $N_{\xi \eta} / 2 \tau_{0} H$, so as to obtain $n_{\xi \eta}=1$ in the limit state under pure in-plane shear, but the definition (32.21) is generally adopted.

We now quote some of the most important equations of the plastic interaction surfaces for the membrane state. The $\mathrm{HMH}$ yield condition results in

$$
n_{\xi}^{2}-n_{\xi} n_{\eta}+n_{\eta}^{2}+3 n_{\xi \eta}^{2}=1 \text {. }
$$

The Tresca-Guest yield condition expressed in principal directions of stresses may be written thus

$$
\max \left(\left|n_{1}\right|,\left|n_{2}\right|,\left|n_{1}-n_{2}\right|\right)=1 .
$$

The principal membrane forces may be expressed by arbitrary components as follows:

$$
n_{1,2}=\frac{1}{2}\left(n_{\xi}+n_{\eta}\right) \pm \frac{1}{2} \sqrt{\left(n_{\xi}-n_{\eta}\right)^{2}+4 n_{\xi \eta}^{2}},
$$

and hence (32.24) determines three surfaces,

$$
\begin{aligned}
n_{\xi}+n_{\eta}-n_{\xi} n_{\eta}+n_{\xi \eta}^{2} & =1, \\
-n_{\xi}-n_{\eta}-n_{\xi} n_{\eta}+n_{\xi \eta}^{2} & =1, \\
n_{\xi}^{2}+n_{\eta}^{2}-2 n_{\xi} n_{\eta}+4 n_{\xi \eta}^{2} & =1 .
\end{aligned}
$$

The resulting plastic interaction surface is formed by those parts of (32.26) which are closest to the origin of coordinates in the space of generalized stresses. 
In view of the similarity of Eqs. (32.19) and (32.22), one very often simply uses the stress components instead of the membrane forces. More essential differences occur only in the case of variable thickness $2 H$ (in equilibrium equations) and in the case of non-homogeneity of the material. M.P. Nielsen [7.228] derived equations of the plastic interaction surface for a cross-section of reinforced concrete shells (transversal non-homogeneity).

The membrane states under consideration are mainly applied to plates under in-plane loading. We mention here the papers by R. P. Nordgren and P. M. Naghdi [7.230, 7.231] dealing with an infinite plate with a circular hole loaded by normal and tangential pressure; J. M. Chern and S. Nemat-Nasser [7.28] considered a similar problem for a variable-thickness plate using a finite-strain theory. The general circularly-symmetric case of small strains in a disk was considered by M. Życzkowski [4.630]. It should be noted that perfectly plastic membrane states are statically determinate: the two equilibrium equations and the yield condition determine three membrane forces $N_{\alpha \beta}$.

Apparent anisotropy occurs if we apply an averaging approach to isotropic perforated plates. Such a theory was developed by W. J. O'Donnell and J. Porowski [3.585, 3.647] who obtained lower bounds to plastic interaction surfaces by using stress fields with jump-like discontinuities; hexagonal and square arrays of circular holes were considered.

P. G. Hodge and J. Panarelli [7.103] investigated the general case of a uniform plastic membrane state in a circular cylindrical shell; moreover, they took into account some boundary bending effects. M. I. Erkhov [7.53] considered membrane limit states in shells under non-symmetric loadings. Conditions of plastic membrane states in shells were discussed by Yu. V. Nemirovsky [7.227]. V. R. Terrovere [7.368] analysed membrane states in shells taking into account finite deflections. Finite plastic deflections of membranes were considered by N. N. Malinin and Zh. L. Petrosyan [7.178].

It should be noted that in view of the lack of the superposition principle, which, in general, is no longer valid in the plastic range, a combination of several membrane states (at least one of which is longitudinally non-homogeneous) may result in bending effects. Such bending effects were analysed by M. Mrowiec and M. Życzkowski $[7.206,4.397]$ in a circular cylindrical shell under simultaneous pressure and bending as a whole.

\subsection{Elastic-plastic moment states}

Consider now purely moment states in a cross-section of an elastic-plastic surface structure. For a plate we can always transform the system of coordinates $\xi-\eta$ so as to obtain coincidence with the principal directions of moments; then the twisting moment vanishes and we may discuss the reduced case $\mathscr{P}_{2} / \mathscr{S}_{2}$ instead of $\mathscr{P}_{3} / \mathscr{S}_{3}$. For a shell we have defined the directions $\xi$ and $\eta$ as those of the principal initial curvatures of the shell and, in general, we would have to consider the case $\mathscr{P}_{3} / \mathscr{S}_{3}$ 
(or even $\mathscr{P}_{3} / \mathscr{S}_{4}$ if, more rigorously, definitions (32.2) are employed and $M_{\xi \eta} \neq M_{\eta \xi}$ ). However, in what follows we confine our considerations to the case of coincidence of the principal directions of moments and of initial curvatures, $\mathscr{P}_{2} / \mathscr{S}_{2}$; hence the axes $\xi, \eta$ will be denoted by 1 and 2 .

In the case of uniform homogeneous surface structures (plates and thin shells) made of isosensitive materials the vanishing of all membrane forces $N_{\alpha \beta}$ corresponds to the vanishing of all extensions $\lambda_{\alpha \beta}$. Strictly speaking, the inextensional deformation of plates and shells takes place very seldom. The Gaussian curvature should remain constant; Love derives the corresponding condition in the linearized form, valid under the assumption of small deflections,

$$
\frac{x_{1}}{R_{2}}+\frac{x_{2}}{R_{1}}=0
$$

where, in the case under consideration, $\lambda_{1}=\lambda_{2}=0$, and $x_{1}=\Delta x_{1}, x_{2}=\Delta x_{2}$, (32.7) or (32.17). Condition (32.27) is always satisfied for plates, but it holds only if small deflections are considered; in the case of large deflections a non-linear term should be included into (32.27), and, practically, the extensions and the membrane forces appear. In the case of shells condition (32.27) should rather be regarded as exceptional; hence the following considerations will refer mainly to plates in the range of small deflections.

We first determine the stress distribution in a cross-section in the elastic-plastic range. In perfectly elastic-plastic beams a "bitrapeziform" stress distribution occurs. A similar distribution of stresses $\sigma_{1}$ and $\sigma_{2}$ is also often assumed in the cross-section of plates; then the relationship between bending moments and curvatures is particularly simple. However, it turns out that such distributions occur only in incompressible materials; elastic compressibility results in curvilinear stress distributions, and the bitrapeziform diagrams may be regarded only as certain approximations. We present here an analysis based on paper [2.199].

Assume the linear strain distribution (32.16) with $\lambda_{x \beta}=0$ and write for the principal directions

$$
\varepsilon_{1}=\varkappa_{1} z, \quad \varepsilon_{2}=\varkappa_{2} z .
$$

Under the usual assumption $\sigma_{z}=\sigma_{3}=0$ we may present the Hencky-Ilyushin equations (for proportional changes of curvatures) as follows:

$$
\frac{\sigma_{1}}{\sigma_{2}}=\frac{\varepsilon_{1}-\varepsilon_{3}}{\varepsilon_{2}-\varepsilon_{3}} \text {. }
$$

Combining (32.29) with the law of volume change

$$
\sigma_{1}+\sigma_{2}=3 K\left(\varepsilon_{1}+\varepsilon_{2}+\varepsilon_{3}\right),
$$

we eliminate $\varepsilon_{3}$ and obtain

$$
\left(2 \varepsilon_{2}+\varepsilon_{1}\right) \sigma_{1}-\left(2 \varepsilon_{1}+\varepsilon_{2}\right) \sigma_{2}-\frac{1}{3 K}\left(\sigma_{1}^{2}-\sigma_{2}^{2}\right)=0 .
$$


Equation (32.31) together with the $\mathrm{HMH}$ yield condition (11.7) determines the distribution of $\sigma_{1}$ and $\sigma_{2}$ in a perfectly plastic zone. Both equations are quadratic in $\sigma_{1}$ and $\sigma_{2}$. The HMH ellipse may be reduced to normal form if we introduce new unknowns

$$
x=\frac{\sigma_{1}+\sigma_{2}}{2}, \quad y=\frac{\sigma_{1}-\sigma_{2}}{2} .
$$

Namely, we obtain

$$
x^{2}+3 y^{2}=\sigma_{0}^{2}, \quad\left(\varepsilon_{2}-\varepsilon_{1}\right) x+3\left(\varepsilon_{1}+\varepsilon_{2}\right) y-\frac{4}{3 K} x y=0 .
$$

Now the second equation is linear in $x$; eliminating this unknown, we obtain the following equation of the fourth degree with respect to $y$ :

$$
\begin{gathered}
48 y^{4}+72 K\left(\varepsilon_{1}-\varepsilon_{2}\right) y^{3}+\left[108 K^{2}\left(\varepsilon_{1}^{2}+\varepsilon_{1} \varepsilon_{2}+\varepsilon_{2}^{2}\right)-16 \sigma_{0}^{2}\right] y^{2}- \\
-24 \sigma_{0}^{2} K\left(\varepsilon_{1}-\varepsilon_{2}\right) y-9 \sigma_{0}^{2} K^{2}\left(\varepsilon_{1}-\varepsilon_{2}\right)^{2}=0 .
\end{gathered}
$$

Equation (32.34), with variable coefficients $\varepsilon=\varepsilon(z)$, is easy to be solved only in particular cases. In the case $\varepsilon_{1}=\varepsilon_{2}=\varepsilon$ (spherical bending) we obtain the only real root $y=0$, and hence $\sigma_{1}=\sigma_{2}= \pm \sigma_{0}=$ const; the stress distribution is then bitrapeziform. In the case $K \rightarrow \infty$ (incompressible material) we obtain a quadratic equation

$$
12\left(\varepsilon_{1}^{2}+\varepsilon_{1} \varepsilon_{2}+\varepsilon_{2}^{2}\right) y^{2}-\left(\varepsilon_{1}-\varepsilon_{2}\right)^{2} \sigma_{0}^{2}=0,
$$

and after solution and substitution of (32.28)

$$
y= \pm \frac{x_{1}-x_{2}}{2 \sqrt{3} \sqrt{x_{1}^{2}+x_{1} x_{2}+x_{2}^{2}}} \sigma_{0}=\text { const }
$$

hence also in this case the stress distribution is bitrapeziform.

In the general case Eq. (32.34) was solved in [2.199] by means of the perturbation method. Assuming the dimensionless quantity $(1-2 v)$ as a small parameter, equal to zero for an incompressible material, we obtain

$$
\begin{aligned}
\sigma_{1} & =\frac{\left(2 \varepsilon_{1}+\varepsilon_{2}\right) \sigma_{0}}{\sqrt{3} \sqrt{\varepsilon_{1}^{2}+\varepsilon_{1} \varepsilon_{2}+\varepsilon_{2}^{2}}}+\frac{\left(\varepsilon_{1}^{2}-\varepsilon_{2}^{2}\right) \varepsilon_{2} \sigma_{0}^{2}}{2\left(\varepsilon_{1}^{2}+\varepsilon_{1} \varepsilon_{2}+\varepsilon_{2}^{2}\right)^{2} E}(1-2 \nu)+ \\
& +\frac{\left(\varepsilon_{1}^{2}-\varepsilon_{2}^{2}\right)\left(-2 \varepsilon_{1}^{3}+3 \varepsilon_{1}^{2} \varepsilon_{2}+18 \varepsilon_{1} \varepsilon_{2}^{2}+5 \varepsilon_{2}^{3}\right) \sigma_{0}^{3}}{8 \sqrt{3}\left(\varepsilon_{1}^{2}+\varepsilon_{1} \varepsilon_{2}+\varepsilon_{2}^{2}\right)^{7 / 2} E^{2}}(1-2 \nu)^{2}+\ldots
\end{aligned}
$$

The formula for $\sigma_{2}$ follows from (32.37) with interchanged indices. Formula (32.37) is of general character for plastic plane stress states. In the case of a cross-section of a surface structure under consideration we substitute (32.28), obtaining for $z>0$

$$
\begin{aligned}
\sigma_{1} & =\frac{\left(2 x_{1}+x_{2}\right) \sigma_{0}}{\sqrt{3}} \frac{\left(x_{1}^{2}-x_{2}^{2}\right) x_{2} \sigma_{0}^{2}}{\sqrt{x_{1}^{2}+x_{1} x_{2}+x_{2}^{2}}}+\frac{1-2 v}{2\left(x_{1}^{2}+x_{1} x_{2}+x_{2}^{2}\right)^{2} E} \frac{1}{z}+ \\
& +\frac{\left(x_{1}^{2}-x_{2}^{2}\right)\left(-2 x_{1}^{3}+3 x_{1}^{2} x_{2}+18 x_{1} x_{2}^{2}+5 x_{2}^{3}\right) \sigma_{0}^{3}}{8 \sqrt{3}\left(x_{1}^{2}+x_{1} x_{2}+x_{2}^{2}\right)^{7 / 2} E^{2}} \frac{(1-2 v)^{2}}{z^{2}}+\ldots
\end{aligned}
$$


and a similar formula for $\sigma_{2}$. For negative $z$ the two stresses change only their signs. Formula (32.38) is valid in the plastic zone $z_{b}<z<H$, where the boundary coordinate $z_{b}$ is determined by the series, [2.199],

$$
\begin{aligned}
z_{b}=\frac{\sigma_{0} \sqrt{3}}{2 E \sqrt{x_{1}^{2}+x_{1} x_{2}+x_{2}^{2}}}[ & 1+\frac{2 x_{1}^{2}+5 x_{1} x_{2}+2 x_{2}^{2}}{3\left(x_{1}^{2}+x_{1} x_{2}+x_{2}^{2}\right)}(1-2 v)- \\
& \left.-\frac{\left(x_{1}^{2}-x_{2}^{2}\right)^{2}}{2\left(x_{1}^{2}+x_{1} x_{2}+x_{2}^{2}\right)^{2}}(1-2 v)^{2}+\ldots\right] .
\end{aligned}
$$

Hence the stress distribution in a compressible material is hyperbolic in the plastic zones. These hyperbolae turn into straight lines in the cases $x_{1}=x_{2}$ (spherical bending) and $x_{1}=-x_{2}$. With increasing $x_{1}$ and $x_{2}$ the hyperbolic terms decrease, since the powers of $x$ are higher in the denominators than in the numerators.

Paper [2.199] derives also the relationships between bending moments and curvatures in the elastic-plastic range for a compressible material. With the accuracy of linear terms in $(1-2 v)$ the bending moment $M_{1}$ is determined by the formula

$$
\begin{aligned}
M_{1}=\frac{\left(2 x_{1}+x_{2}\right) \sigma_{0} H^{2}}{\sqrt{3}} \sqrt{\sqrt{x_{1}^{2}+x_{1} x_{2}+x_{2}^{2}}}\left[1-\frac{\sigma_{0}^{2}}{4 E^{2} H^{2}\left(x_{1}^{2}+x_{1} x_{2}+x_{2}^{2}\right)}\right]+ \\
+\frac{\sigma_{0}^{2}}{E\left(x_{1}^{2}+x_{1} x_{2}+x_{2}^{2}\right)^{2}}\left[\left(x_{1}^{2}-x_{2}^{2}\right) x_{2} H-\right. \\
\left.-\frac{\left(4 x_{1}^{3}+18 x_{1}^{2} x_{2}+9 x_{1} x_{2}^{2}-4 x_{2}^{3}\right) \sigma_{0}}{6 \sqrt{3} E \sqrt{x_{1}^{2}+x_{1} x_{2}+x_{2}^{2}}}\right](1-2 v) ;
\end{aligned}
$$

the moment $\mathrm{M}_{2}$ is of course determined by the same formula with interchanged indices.

Approximate bitrapeziform stress distributions in the case of compressible materials may be obtained in various ways. One approach consists in approximate neglection of compressibility; if we adopt this assumption in plastic zones only, then at the elastic-plastic interface discontinuities appear. On the other hand, this assumption applied also to elastic zones results in much larger approximation errors. Another possibility is to adopt the hypothesis of a constant coefficient of transversal deformation also in the plastic zones, $v^{p}=v$; this hypothesis was used for example by W. Olszak and J. Murzewski [7.247] who analysed elastic-plastic bending of non-homogeneous orthotropic circular plates, and by V. A. Kolgadin and A. I. Strelbitskaya [7.133] (rectangular isotropic plates).

Examples of elastic-plastic stress distributions (for $z>0$ ) in a cross-section of inextensible surface structures are shown in Fig. 161. Poisson's ratio is assumed $v=0.3$; further $\varkappa_{1}=-2 \varkappa_{2}$, and in turn $\varkappa_{2}=\sigma_{0} / E H$ and $\varkappa_{2}=2 \sigma_{0} / E H$. The diagrams show the exact stress distribution [solution of Eq. (32.34)], approximation by the first two terms of the series (32.38), approximate assumption of incompress- 

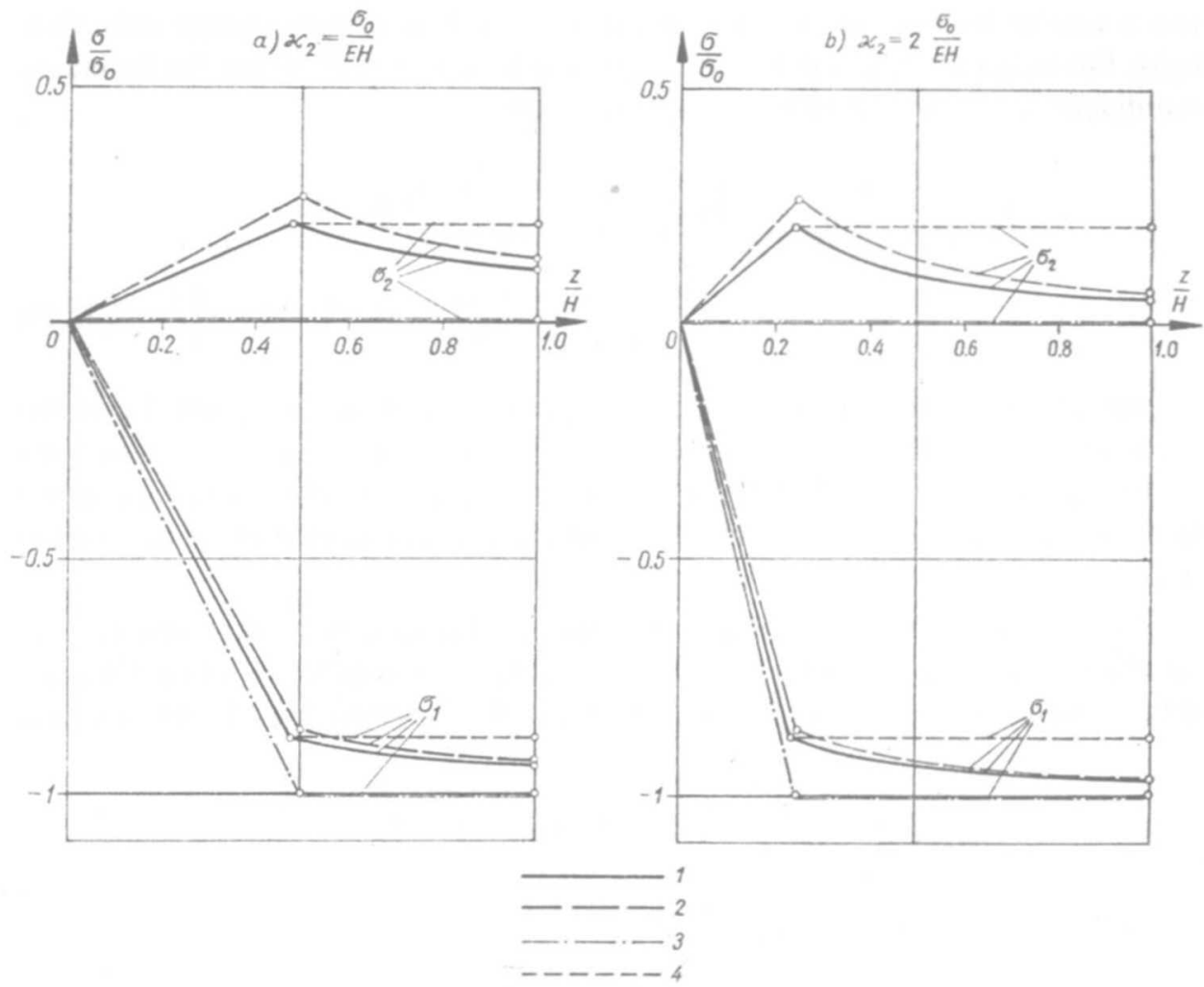

Fig. 161. Stress distributions in a cross-section of a surface structure in the case $\varkappa_{1}=-2 \varkappa_{2}$, $v=0.3$ ( 1 , exact solution $v=0.3 ; 2$, two-terms series approximation; 3 , incompressible material; 4 , hypothesis of constant coefficient of transversal deformation)

ibility and approximate hypothesis of a constant coefficient of transversal deformation in the plastic range.

The influence of material compressibility on the elastic-plastic behaviour of a cross-section was analysed also by A. S. Grigorev [7.75], Yu. R. Lepik and L. Ya. Luht [2.97].

Relationships of type (32.40) or similar ones, based on various simplifying assumptions, constitute the basis for the calculation of small elastic-plastic deflections of plates. Let us mention here the first general results obtained by A. A. Ilyushin [1.8] (circular and rectangular plates), further investigations on circularly symmetric plates by V. V. Sokolovsky [7.343], A. S. Grigorev [7.75, 7.76], R. M. Haythornthwaite [7.91], I. V. Shirko [7.339], B. Tekinalp [7.365] (the Prandtl-Reuss theory for a sandwich plate), G. S. Shapiro [7.331], G. Eason [7.47] and a very extensive investigation by Y. Ohashi and S. Murakami [7.239]; elastic-plastic deflections of rectangular plates were analysed by A. L. Strelbitskaya and her collaborators, V. A. Kolgadin and S. I. Matoshko [7.348, 7.350, 7.353] and with R. I. Rybakova [7.351], by R. Dobrev [7.35] and in some other papers. 


\subsection{Limit moment states}

In purely moment states the limit carrying capacity of an elastic-plastic cross-section is exhausted at infinitely large curvatures. In fact, denoting the ratio $\varkappa_{1} / x_{2}$ by $\psi$ (which should be constant if the Hencky-Ilyushin theory is applied) we may rewrite (32.40) in the more convenient form

$$
\begin{aligned}
& M_{1}=\frac{(2 \psi+1) \sigma_{0} H^{2}}{\sqrt{3} \sqrt{\psi^{2}+\varphi+1}}\left[1-\frac{\sigma_{0}^{2}}{4 \varkappa_{2}^{2} E^{2} H^{2}\left(\psi^{2}+\varphi+1\right)}\right]+ \\
& +\frac{\sigma_{0}^{2}}{E \varkappa_{2}\left(\psi^{2}+\varphi+1\right)}\left[\left(\psi^{2}-1\right) H-\frac{\left(4 \psi^{3}+18 \psi^{2}+9 \psi-4\right) \sigma_{0}}{6 \sqrt{3} E \varkappa_{2} \sqrt{\psi^{2}+\psi+1}}\right](1-2 v) .
\end{aligned}
$$

If $\varkappa_{2}$ increases infinitely at a constant value $\psi$, then the boundary coordinate (32.39) tends to zero and the bending moment $M_{1}$ approaches its limit value

$$
M_{1}=\frac{(2 \psi+1) \sigma_{0} H^{2}}{\sqrt{3} \sqrt{\psi^{2}+\psi+1}}
$$

regardless of the compressibility of the material; a similar formula holds for $M_{2}$. Eliminating from these relations the parameter $\psi$, we arrive at the equation of the plastic interaction curve for the cross-section

$$
M_{1}^{2}-M_{1} M_{2}+M_{2}^{2}=\sigma_{0}^{2} H^{4} .
$$

Introducing dimensionless bending moments

$$
\frac{M_{1}}{\bar{M}}=\frac{M_{1}}{\sigma_{0} H^{2}}=m_{1}, \quad \frac{M_{2}}{\overline{\bar{M}}}=\frac{M_{2}}{\sigma_{0} H^{2}}=m_{2},
$$

we may rewrite $(32.43)$ in a simpler form

$$
m_{1}^{2}-m_{1} m_{2}+m_{2}^{2}=1 \text {. }
$$

This form is analogous to the form of the yield condition adopted, (11.7), In fact, under the assumption of transversal homogeneity of the material we obtain "birectangular" stress diagrams regardless of compressibility, hence the yield condition adopted is transformed directly into the equation of the plastic interaction curve for moments. In general, if we assume a yield condition for plane stress states in form (32.19), then, introducing the dimensionless bending moments $m_{\xi}$ and $m_{\eta}$ by formulae of type (32.44) and the dimensionless twisting moment by the formula

$$
\frac{M_{\xi \eta}}{\sigma_{0} H^{2}}=m_{\xi \eta},
$$

we may present the equation of the plastic interaction surface for a section under biaxial bending and twisting in the form

$$
m \stackrel{\text { def }}{=} f\left(m_{\xi}, m_{\eta}, m_{\xi \eta}\right)=1 .
$$

Hence the geometrical interpretation of this equation coincides with the interpretation of (32.19). The combined case under consideration may be classified, in general, as $\mathscr{P}_{3} / \mathscr{S}_{3}$. 
Form (32.47) is obvious for isosensitive materials. In the case of anisosensitivity, $\sigma_{0+} \neq \sigma_{0-}$, the neutral layer is translated and Eq. (32.47) is different from (32.19), namely, is subject to symmetrization.

In the case of the HMH yield condition, Eq. (32.47) presents an ellipsoid

$$
m_{\xi}^{2}-m_{\xi} m_{\eta}+m_{\eta}^{2}+3 m_{\xi \eta}^{2}=1 \text {, }
$$

and assuming the TG yield condition, we obtain the plastic interaction surface consisting of three parts, analogous to (32.26) (two elliptic cones and an elliptic cylinder):

$$
\begin{aligned}
m_{\xi}+m_{\eta}-m_{\xi} m_{\eta}+m_{\xi \eta}^{2} & =1, \\
-m_{\xi}-m_{\eta}-m_{\xi} m_{\eta}+m_{\xi \eta}^{2} & =1, \\
m_{\xi}^{2}+m_{\eta}^{2}-2 m_{\xi} m_{\eta}+4 m_{\xi \eta}^{2} & =1 .
\end{aligned}
$$

The surfaces (32.48) and (32.49) are shown in Fig. 162.

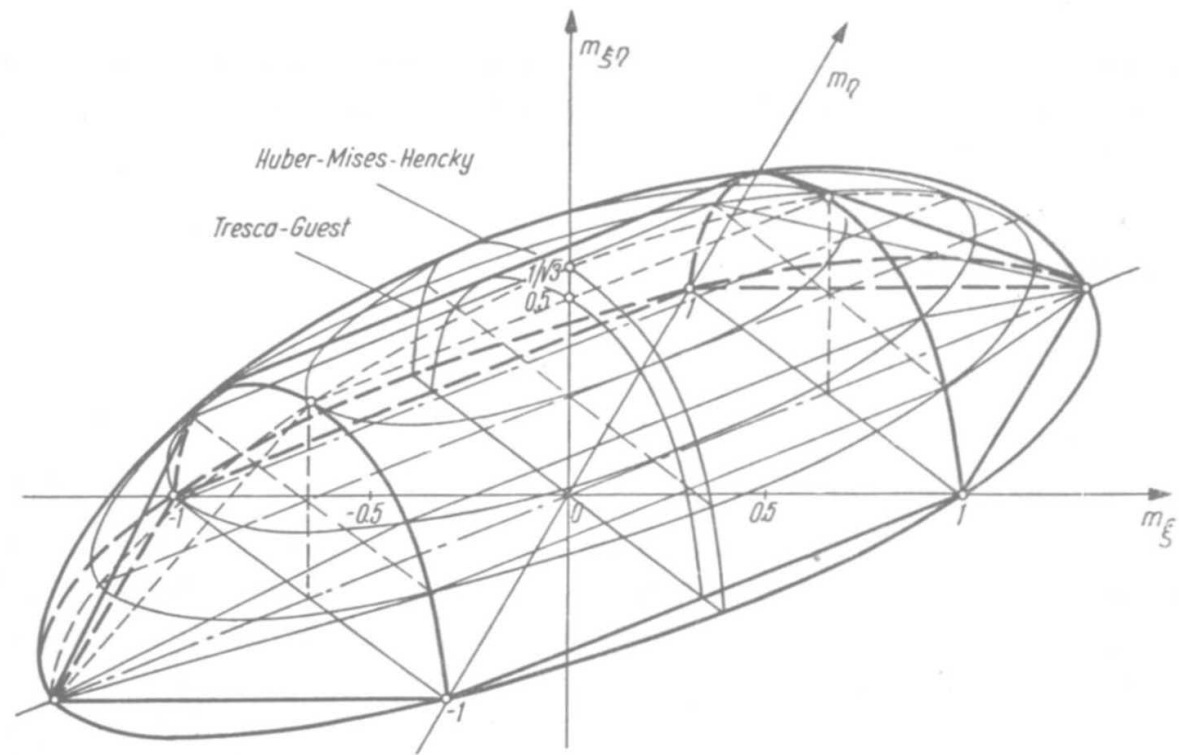

Fig. 162. Plastic interaction surfaces for purely moment states in a cross-section of surface structures

The equation of the plastic interaction surface based on the Burzyński-Stassi d'Alia yield condition in the case $\mathscr{P}_{2} / \mathscr{S}_{2}$ was derived and applied to circular plates by G. Menditto [7.188, 7.189, 7.190], and in the case $\mathscr{P}_{3} / \mathscr{S}_{3}$-by M. Capurso [7.21]. Natural orthotropy of the material or orthotropy due to reinforcement or ribs was considered by A. Sawczuk [7.296, 7.300], T. Mura, J. S. Kao and S. L. Lee [7.212] (circular plate whose orthotropy does not conform to circular symmetry), K. O. Kemp [7.127], M. W. Kwieciński [7.141], C. T. Morley [7.204] and M. Save [7.294] (all the four papers deal with reinforced concrete slabs), J. A. König and J. Rychlewski [7.139] (orthotropy and jump-like non-homogeneity), M. Capurso 
and A. Gandolfi [7.25] (influence of ribs), A Sawczuk [7.311] (general anisotropy). Apparent anisotropy resulting from an averaging process applied to isotropic perforated plates was considered by A. Sawczuk, W. J. O'Donnell and J. Porowski [3.699]. Experimental investigations on the yield conditions for the cross-section of a plate were carried out by P. S. Theocaris and C. R. Hazell [3.799].

Effective application of the plastic interaction surfaces for the cross-section to the limit analysis of a surface structure as a whole requires in most cases knowledge of the constitutive equations joining curvatures (or their rates) and moments. Usually rigid-plastic schematization is then applied, though the results do not always coincide with those obtained for elastic-plastic structures (G. S. Shapiro [7.331]). It has been shown that if the flow rule is associated at the level $\mathscr{P}$, then it is also associated at the level $\mathscr{S}$; namely, if we write the equation of the plastic interaction surface in the form $f\left(M_{\xi}, M_{\eta}, M_{\xi \eta}\right)=1$,

$$
\dot{x}_{\xi}=\lambda \frac{\partial f}{\partial M_{\xi}}, \quad \dot{x}_{\eta}=\lambda \frac{\partial f}{\partial M_{\eta}}, \quad \dot{x}_{\xi \eta}=\lambda \frac{\partial f}{\partial M_{\xi \eta}},
$$

(H. Ziegler [5.208, 5.209, 5.210], A. Sawczuk and J. Rychlewski [7.298], M. Save [5.160]). In the case of the $\mathrm{HMH}$ yield condition relations (32.50) may not be questioned, but the situation is quite different if we apply an approximate yield condition at the level $\mathscr{P}$, e.g. if we replace, for the sake of simplicity, the HMH by the TG yield condition. Then the application of (32.50) associated with the approximate yield condition results, as a rule, in an increase of the error of approximation.

A more general approach to rigid-plastic constitutive equations in the case under consideration is due to A. Sawczuk [7.306]. He stated that they may be written in the general form

$$
\dot{x}_{i j}=A M_{i j}-B \delta_{i j}, \quad i, j=\xi, \eta,
$$

where $A$ and $B$ are certain functions of invariants.

Let us also mention some typical applications of the plastic interaction surface for the cross-section to limit analysis, mainly to plates with small deflections. The limit carrying capacity of a circular plate was analysed by H. G. Hopkins and A. J. Wang [7.104] for an arbitrary yield condition. Antisymmetric loading of such plate was discussed by V. N. Alpert [7.5]. Methods of derivation of upper and lower bounds to the limit carrying capacity of plates were discussed in detail by A. Zavelani-Rossi [7.393, 7.394], W. Gutkowski and W. Szczepiński [7.78, 7.362]. Many particular solutions for plates were given in the monographs quoted in Sec. 32.1 .

The problem of discontinuities of moments and of curvature rates in the limit state of rigid-perfectly-plastic plates was discussed by Z. Mróz and A. Sawczuk [7.207], A. Sawczuk and P. G. Hodge [7.307]. However, according to the considerations given in Sec. 18.1, the approaches from the side of rigid-plastic-hardening plates and from the side of elastic-perfectly-plastic plates may lead here to different 
results; in the latter case discontinuities of moments and of curvature rates normal to discontinuity lines are not admissible.

Plastic hardening in plates with small deflections was considered by E. Faccioli [7.57] (rigid-plastic material with isotropic and kinematic hardening), A. I. Strelbitskaya and V.A. Kolgadin [7.353] (elastic-linearly-hardening), Yu. M. Voltchkov, G. V. Ivanov and V. N. Solodovnikov [7.382] (elastic-plastic material with isotropic hardening, deformation and flow theories), F. Laudiero and A. Tralli [7.147] (finite elements applied to elastic-plastic plates with linear hardening).

\section{Combined loadings of the type $\mathscr{P}_{2} / \mathscr{S}_{4}$ in uniform sections}

\subsection{Limit carrying capacity for the HMH yield condition}

When considering shells or large deflections of plates, we usually have to take into account the interaction of moments and membrane forces. The general case of moments, membrane forces and shearing forces in the cross-section of a surface structure has been classified as $\mathscr{P}_{5} / \mathscr{S}_{10}$. However, a much simpler case occurs if (1) we adopt the definitions (32.13) and (32.14) and discuss symmetric tensors, (2) the principal directions of the initial curvatures, of moments and of membrane forces coincide, and (3) the effect of shearing forces may be neglected. This case may be classified as $\mathscr{P}_{2} / \mathscr{S}_{4}$ : the two principal moments $M_{1}$ and $M_{2}$ and the two principal membrane forces $N_{1}$ and $N_{2}$ represent generalized stresses in the section. Essential simplifications take place if the TG yield condition or related yield conditions are employed, but the HMH yield condition also leads to simpler results. The applications of the case under consideration are very broad, e.g. when rotational symmetry of structures and of loadings is considered.

Assume the linearized distribution of principal strains (or strain rates) $\varepsilon_{1}$, $\varepsilon_{2}$ according to (32.16). The assumption of incompressibility, fully justified in the limit state, determines the third strain

$$
\varepsilon_{3}=\varepsilon_{z}=-\left(x_{1}+x_{2}\right) z-\left(\lambda_{1}+\lambda_{2}\right) \text {. }
$$

The law of similarity of deviators (Hencky-Ilyushin or Levy-Mises) combined with the usual assumption $\sigma_{3}=\sigma_{z}=0$ results in

$$
\sigma_{1}=\frac{1}{\varphi}\left(\varepsilon_{1}-\varepsilon_{3}\right), \quad \sigma_{2}=\frac{1}{\varphi}\left(\varepsilon_{2}-\varepsilon_{3}\right),
$$

The difference between the Hencky-Ilyushin theory and the Levy-Mises theory is purely formal here. Substituting (32.16), (33.1) and (33.2) into the HMH yield condition, we determine the function $\varphi$ :

$$
\begin{aligned}
\varphi=\frac{\sqrt{3}}{\sigma_{0}} & {\left[\left(x_{1}^{2}+x_{1} x_{2}+x_{2}^{2}\right) z^{2}+\left(2 x_{1} \lambda_{1}+2 x_{2} \lambda_{2}+x_{1} \lambda_{2}+x_{2} \lambda_{1}\right) z+\right.} \\
& \left.+\left(\lambda_{1}^{2}+\lambda_{1} \lambda_{2}+\lambda_{2}^{2}\right)\right]^{1 / 2}
\end{aligned}
$$


Substitution of (33.3) into (33.2) results in effective evaluation of the stress distribution. Making use of the definitions of membrane forces (32.13) and of moments (33.14), we may present these generalized stresses in the form

$$
\begin{aligned}
& N_{1}=\left(2 \varkappa_{1}+\varkappa_{2}\right) J_{1}+\left(2 \lambda_{1}+\lambda_{2}\right) J_{0}, \\
& N_{2}=\left(2 \varkappa_{2}+\varkappa_{1}\right) J_{1}+\left(2 \lambda_{2}+\lambda_{1}\right) J_{0}, \\
& M_{1}=\left(2 \varkappa_{1}+\varkappa_{2}\right) J_{2}+\left(2 \lambda_{1}+\lambda_{2}\right) J_{1}, \\
& M_{2}=\left(2 \varkappa_{2}+\varkappa_{1}\right) J_{2}+\left(2 \lambda_{2}+\lambda_{1}\right) J_{1},
\end{aligned}
$$

where $J_{k}$ denote the integrals

$$
J_{k}=\int_{-H}^{H} \frac{z^{k}}{\varphi} d z, \quad k=0,1,2 .
$$

and the function $\varphi$ is given by (33.3). The expressions (33.4) are homogeneous functions of the zeroth degree of the arguments $x_{1}, x_{2}, \lambda_{1}, \lambda_{2}$, and hence they may be presented in terms of their ratios, e.g. $x_{2} / x_{1}, \lambda_{1} / x_{1}$ and $\lambda_{2} / x_{1}$. Thus Eqs. (33.4) determine the plastic interaction hypersurface in the parametrical form. The integrals (33.5) belong to elementary integrals, but their evaluation is cumbersome and the final form depends on the values of the parameters $x$ and $\lambda$. More important cases were analysed by A. A. Ilyushin [7.106, 1.8] (who first derived the equations of the limit hypersurface in the more general case $\left.\mathscr{P}_{3} / \mathscr{S}_{6}\right)$, V. V. Rozhdestven"sky [7.281], A. Sawczuk and J. Rychlewski [7.298].

Equations (33.4) take a particularly simple form in the case

$$
2 \varkappa_{1} \lambda_{1}+2 \varkappa_{2} \lambda_{2}+\varkappa_{1} \lambda_{2}+\varkappa_{2} \lambda_{1}=0
$$

which was called by Ilyushin [1.8] the simpler combined case. Then the function $\varphi$ is an even function of the variable $z$, the integrals $J_{1}$ vanish, and the remaining integrals may be expressed in terms of the logarithmic function. Condition (33.6) is of course satisfied in purely membrane and in purely moment states, and, moreover, in the important cases

$$
\varkappa_{1}=0, \quad \lambda_{1}=-2 \lambda_{2}, \quad \text { and } \quad \lambda_{1}=0, \quad \varkappa_{1}=-2 \varkappa_{2},
$$

and in the corresponding cases with interchanged indices.

Consider for example a circular cylindrical shell under symmetric radial loading without end loads. Then, in cylindrical coordinates $r, \theta, x$ (we reserved the symbol $z$ for the coordinate perpendicular to the middle surface of shell) we have $\varkappa_{\theta}=0$ [exactly, cf. (32.8)], and $N_{x}=0$. It can be shown that in this case (33.6) holds. Indeed, Eqs. (33.4) give the relation

$$
2 x_{x} J_{1}+\left(2 \lambda_{x}+\lambda_{\theta}\right) J_{0}=0,
$$

and hence the coefficient of $z$ in (33.3) may be reduced to the form $\left(-2 x_{x}^{2} J_{1} / J_{0}\right)$. If this coefficient were zero, (33.5) would give $J_{1}=0$. Thus, in fact, $J_{1}=0,(33.7)$ 
yields $2 \lambda_{x}+\lambda_{\theta}=0$, and (33.6) is satisfied. Evaluating the remaining integrals, we finally obtain

$$
\begin{aligned}
& n_{\theta}=\frac{\chi}{2} \ln \frac{\sqrt{1+\chi^{2}}+1}{\sqrt{1+\chi^{2}}-1}, \\
& m_{x}=2 m_{\theta}=\frac{2}{\sqrt{3}}\left(\sqrt{1+\chi^{2}}-\frac{\chi^{2}}{2} \ln \frac{\sqrt{1+\chi^{2}}+1}{\sqrt{1+\chi^{2}}-1}\right),
\end{aligned}
$$

where $\chi$ denotes the parameter

$$
\chi=\frac{\sqrt{3}}{2} \frac{\lambda_{\theta} H}{\varkappa_{x}}
$$

and the dimensionless generalized stresses are introduced by (32.21) and (32.44). The plastic interaction curve (33.8) is shown in Fig. 163; it exceeds only very slightly the ellipse marked for comparison by a dashed line. This ellipse

$$
n_{\theta}^{2}+\frac{3}{4} m_{x}^{2}=1 \text {, }
$$

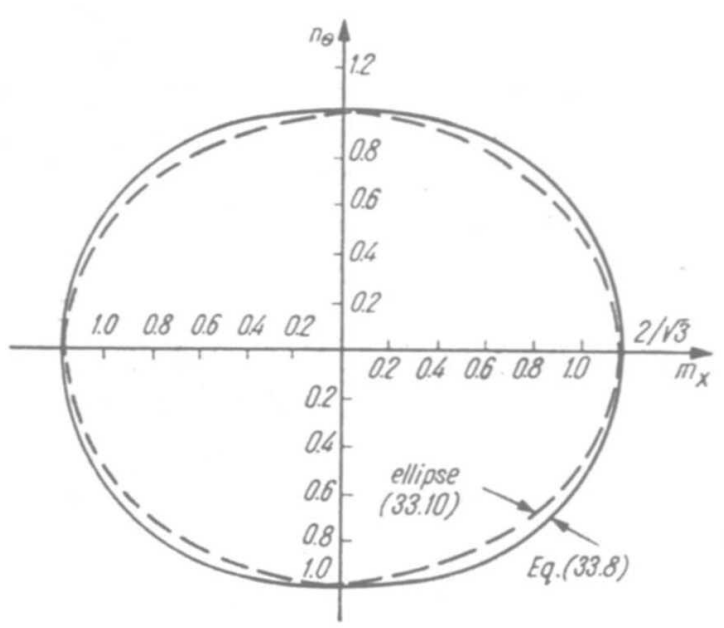

Fig. 163. Plastic interaction curve $n_{\theta}=f\left(m_{x}\right)$

which constitutes an exact yield condition for a sandwich cross-section (cf. Sec. 34.2), is often used as a good approximation for uniform sections (C. R. Calladine [4.58], limit analysis of curved tubes under bending).

Another parametrization of Eqs. (33.4) has been proposed by P. G. Hodge $[7.100,7.101,4.223]$. Introducing the Nadai-Sokolovsky parametrization for the intensity of strains (or of strain rates), calculating the stresses and performing the integrations (32.13) and (32.14), he arrives at the following parametrical equations of the limit hypersurface in the four-dimensional space $n_{1}, n_{2}, m_{1}, m_{2}$ : 


$$
\begin{aligned}
& n_{1,2}=\frac{1}{\sqrt{3} \sin (p-q)}\left[\cos p \cos q \cos \left(r \mp \frac{\pi}{6}\right) \ln \frac{(1+\sin q)(1-\sin p)}{(1-\sin q)(1+\sin p)}+\right. \\
&\left.+2 \sin \left(r \mp \frac{\pi}{6}\right)(\cos p-\cos q)\right], \\
& m_{1,2}=\frac{2 \cos p \cos q}{\sqrt{3} \sin ^{2}(p-q)}\left\{[ \operatorname { l n } \frac { ( 1 + \operatorname { s i n } q ) ( 1 - \operatorname { s i n } p ) } { ( 1 - \operatorname { s i n } q ) ( 1 + \operatorname { s i n } p ) } ] \left[\sin (p+q) \cos \left(r \mp \frac{\pi}{6}\right)+\right.\right. \\
&\left.\left.+\cos p \cos q \sin \left(r \mp \frac{\pi}{6}\right)\right]\right\}- \\
&-4 \cos \left(r \mp \frac{\pi}{6}\right)(\cos p-\cos q)+2 \sin \left(r \mp \frac{\pi}{6}\right)(\sin p-\sin q) .
\end{aligned}
$$

In these equations the upper signs refer to $n_{1}$ and $m_{1}$, the lower signs to $n_{2}$ and $m_{2}$; $p, q$ and $r$ are the parameters whose domains were investigated by Hodge.

Variational derivations of the plastic interaction surfaces are due to S. M. Feynberg [4.139], V. V. Rozhdestvensky [7.281] and T. H. H. Pian [7.261]. Material non-homogeneity induced by elevated temperature and melting was considered by E. Friedman and B. A. Boley [7.61].

Methods of linearization of the $\mathrm{HMH}$ limit surfaces for cylindrical shells were discussed by Yu. A. Nagyavitchius and A. A. Cyras [7.216].

\subsection{Limit carrying capacity for the TG yield condition}

In the case of coinciding principal directions of the initial curvatures, of moments and of membrane forces, the Tresca-Guest yield condition results as a rule in much simpler equations of the plastic interaction surfaces at the level $\mathscr{S}$. This simplification is particularly conspicuous if we also assume the associated flow rule, though the differences with respect to the $\mathrm{HMH}$ yield condition are, in general, subject to a further increase.

The basic form of the TG yield condition, (9.19), may be expressed in the case of plane stress, which we are now considering, by the following six equations, corresponding to various possible arrangements of principal stresses:

$$
\begin{aligned}
& \Phi_{1}=\sigma_{1}-\sigma_{0} \quad=0, \quad \Phi_{4}=-\sigma_{1}-\sigma_{0} \quad=0, \\
& \Phi_{2}=\sigma_{2}-\sigma_{0} \quad=0, \quad \Phi_{5}=-\sigma_{2}-\sigma_{0} \quad=0, \\
& \Phi_{3}=\sigma_{2}-\sigma_{1}-\sigma_{0}=0, \quad \Phi_{6}=-\sigma_{2}+\sigma_{1}-\sigma_{0}=0 .
\end{aligned}
$$

The oldest approach to the Tresca-Guest yield condition as applied to shells combined it with a very specific flow rule: S. M. Feynberg [4.139, 7.58], 1948, actually replaced that rule by the hypothesis that there exists a physical neutral surface $z=z_{0}$, common for all the stresses, and that the stresses are constant in the two subintervals separated by the coordinate $z_{0}$. Feynberg applied this concept to the limit analysis of shallow spherical caps. 
Assume now that the stresses are related to strains or strain rates by the law of similarity of deviators (33.2). Substituting the linearized expressions for the strains (32.16) and eliminating the function $\varphi$, we obtain the relationship

$$
\frac{\sigma_{1}}{\sigma_{2}}=\frac{\left(2 \varkappa_{1}+\varkappa_{2}\right) z+\left(2 \lambda_{1}+\lambda_{2}\right)}{\left(2 \varkappa_{2}+\varkappa_{1}\right) z+\left(2 \lambda_{2}+\lambda_{1}\right)} .
$$

Now, if we assume the first equation (33.12) $\sigma_{1}=\sigma_{0}$, then the distribution of $\sigma_{2}$ is hyperbolic:

$$
\sigma_{2}=\frac{\left(2 \varkappa_{2}+\varkappa_{1}\right) z+\left(2 \lambda_{2}+\lambda_{1}\right)}{\left(2 \varkappa_{1}+\varkappa_{2}\right) z+\left(2 \lambda_{1}+\lambda_{2}\right)} \sigma_{0} .
$$

Conversely, in the case of the second equation (33.12), $\sigma_{2}=\sigma_{0}$, the distribution of $\sigma_{1}$ is hyperbolic:

$$
\sigma_{1}=\frac{\left(2 \varkappa_{1}+x_{2}\right) z+\left(2 \lambda_{1}+\lambda_{2}\right)}{\left(2 x_{2}+x_{1}\right) z+\left(2 \lambda_{2}+\lambda_{1}\right)} \sigma_{0} .
$$

The boundary coordinate $z_{b}$, separating the regions of applicability of (33.14) and (33.15), is determined by the condition $\sigma_{1}=\sigma_{2}$ :

$$
z_{b}=-\frac{\lambda_{1}-\lambda_{2}}{x_{1}-x_{2}} .
$$

Of course, (33.16) is effective only if $-H<z_{b}<H$.

Making use of the third equation (33.12) we obtain hyperbolic distributions of both stresses $\sigma_{1}$ and $\sigma_{2}$ :

$$
\begin{aligned}
& \sigma_{1}=\frac{\left(2 x_{1}+x_{2}\right) z+\left(2 \lambda_{1}+\lambda_{2}\right)}{\left(x_{2}-x_{1}\right) z+\left(\lambda_{2}-\lambda_{1}\right)} \sigma_{0}, \\
& \sigma_{2}=\frac{\left(2 \varkappa_{2}+x_{1}\right) z+\left(2 \lambda_{2}+\lambda_{1}\right)}{\left(x_{2}-x_{1}\right) z+\left(\lambda_{2}-\lambda_{1}\right)} \sigma_{0} .
\end{aligned}
$$

Similarly we can determine the stress distributions corresponding to the remaining equations of (33.12) and the boundary coordinates. The membrane forces and moments may be determined from (32.14) and (32.15) splitting the interval $-H<z<H$ into the appropriate subintervals. The integrals are elementary and may be expressed by the logarithmic functions; the final formulae are rather complicated, though simpler than in the case of the $\mathrm{HMH}$ yield condition.

Much simpler results are obtained if the associated flow rule is employed. This approach, suggested earlier by D. C. Drucker [7.36], was worked out in detail by E. T. Onat and W. Prager [7.251, 7.252]. The simplification consists in the occurrence of only the stress states corresponding to the vertices of the Tresca-Guest hexagon; hence the stresses $\sigma$ are piece-wise constant and equal to $\pm \sigma_{0}$ or 0 . Namely, the associated flow rule requires the vector $\dot{\boldsymbol{\epsilon}}$ to be orthogonal to the surface (curve) $\Phi=0$; in the case of the TG hexagon shown in Fig. 164, the ratio $\dot{\varepsilon}_{1} / \dot{\varepsilon}_{2}$ for the successive sides $\Phi_{1}$ to $\Phi_{6}$ should be equal to $\infty, 0,-1, \infty, 0,-1$, respectively. 


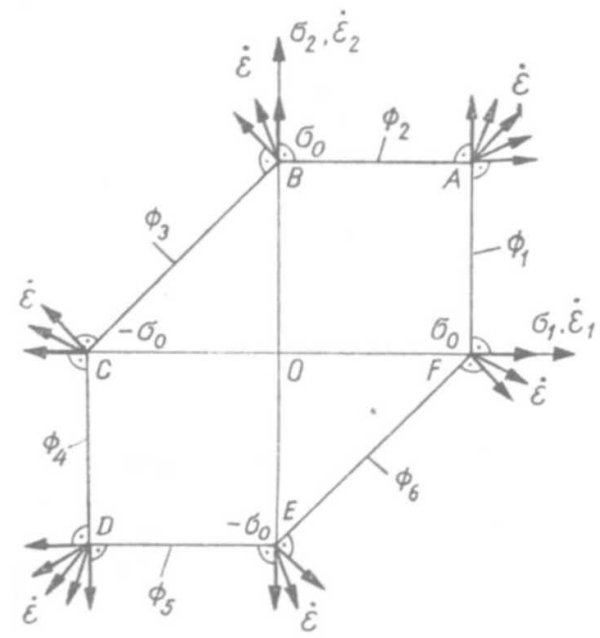

Fig. 164. The TG yield condition with the associated flow rule as applied to limit analysis of a crosssection of surface structures

The principal strain rates determined by formulae of type (32.16) do not in general satisfy this condition, and other values of the ratio $\dot{\varepsilon}_{1} / \dot{\varepsilon}_{2}$ can occur only at the vertices $A-F$, where the direction of the vector $\dot{\boldsymbol{\varepsilon}}$ is not uniquely determined. Hence the plane $\dot{\varepsilon}_{1} / \dot{\varepsilon}_{2}$ is divided into six subregions corresponding to the individual points $A-F$ (Fig. 165) and the stresses $\sigma_{1}$ and $\sigma_{2}$ are piecewise constant.

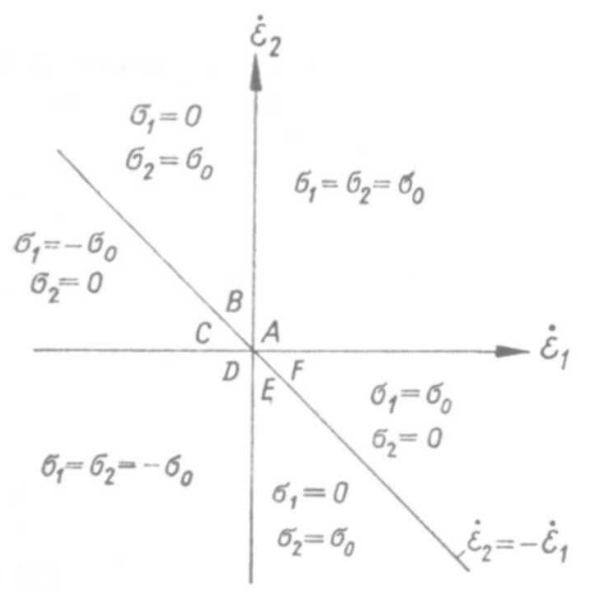

Fig. 165. Division of the plane $\dot{\varepsilon}_{1}-\dot{\varepsilon}_{2}$ into the subregions

Figure 166 shows an exemplary distribution of principal strains (or strain rates, $\dot{\varepsilon}_{1}$ and $\dot{\varepsilon}_{2}$ ), and the corresponding distributions of principal stresses $\sigma_{1}$ and $\sigma_{2}$, determined in turn (a) from the HMH yield condition and the law of similarity of deviators; (b) from the TG yield condition and the law of similarity, $(33.13) \div(33.17)$; (c) from the TG yield condition with the associated flow rule. 

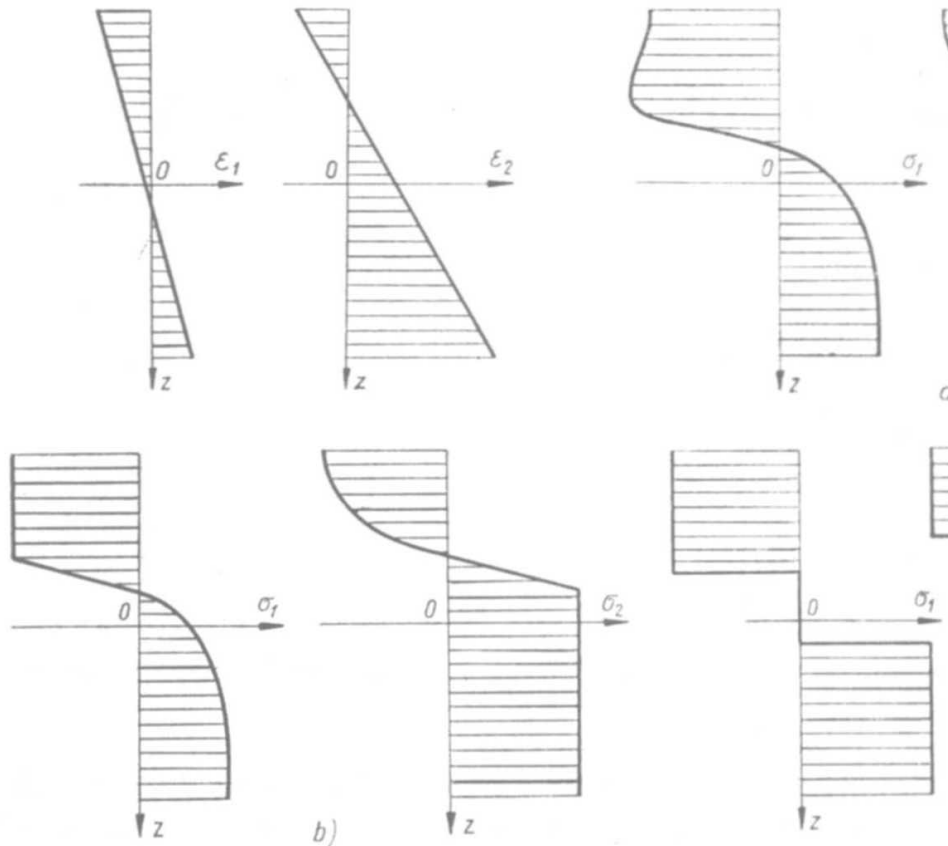
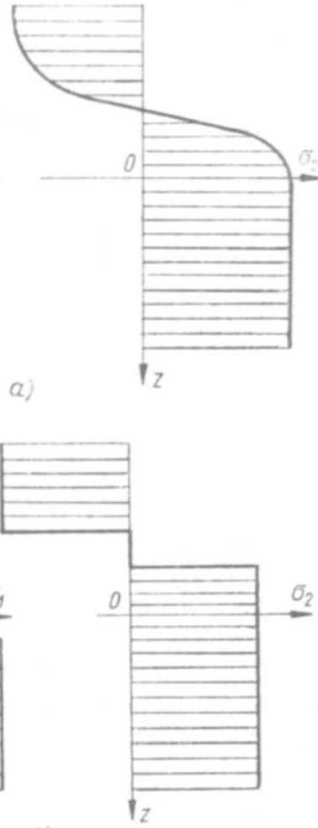

Fig. 166. Three variants of stress distribution for a given strain distribution

Regarding the last two cases as approximations of the first one, we see larger errors introduced by the associated flow rule, but the simplicity of the diagrams (c) is also obvious.

In the case (c), of the TG yield condition combined with the associated flow rule, the calculation of the membrane forces $N_{1}, N_{2}$, and the moments $M_{1}, M_{2}$ presents no difficulties; they may be expressed in terms of three appropriately chosen parameters. Onat and Prager [7.251, 7.252, 4.468] suggested assuming for those parameters the coordinates of vanishing strains (or strain rates) $\varepsilon_{1}, \varepsilon_{2}, \varepsilon_{3}$, divided by the thickness $2 H$. Denote the respective coordinates by $z_{1}, z_{2}, z_{3}$, then formulae (32.16) and (33.1) give

$$
z_{1}=-\frac{\lambda_{1}}{x_{1}}, \quad z_{2}=-\frac{\lambda_{2}}{x_{2}}, \quad z_{3}=-\frac{\lambda_{1}+\lambda_{2}}{x_{1}+x_{2}} .
$$

Thus the Onat-Prager parameters are

$$
p=-\frac{\lambda_{1}}{2 H \varkappa_{1}}, \quad r=-\frac{\lambda_{2}}{2 H \varkappa_{2}}, \quad q=-\frac{\lambda_{1}+\lambda_{2}}{2 H\left(\varkappa_{1}+\varkappa_{2}\right)} .
$$

The calculation of membrane forces and moments now depends on the algebraic arrangement of the parameters $p, q, r$. The six possible arrangements are listed in Table 7, where dimensionless forces $n_{j}$ and dimensionless moments $m_{j}$ have been introduced by formulae of types (32.21) and (32.44), respectively. If any of the parameters $p, r, q$ exceeds $\pm \frac{1}{2}$, it must be replaced by $\pm \frac{1}{2}$, since the root $z_{i}$ lying outside the cross-section does not influence the stress distribution. 
Table 7

Non-cylindrical parts of the Tresca-Guest yield hypersurface

\begin{tabular}{|c|c|c|c|c|c|}
\hline $\begin{array}{c}\text { Parameter } \\
\text { of intermediate } \\
\text { value }\end{array}$ & $n_{1}$ & & $n_{2}$ & $m_{1}$ & $m_{2}$ \\
\hline$p$ & $\mp(p+q)$ & • & $\mp(q-r)$ & $\pm 1 \mp 2\left(p^{2}+q^{2}\right)$ & $\pm 2\left(r^{2}-q^{2}\right)$ \\
\hline$q$ & $\mp(p+q)$ & & $\mp(q+r)$ & $\pm 1 \mp 2\left(p^{2}+q^{2}\right)$ & $\pm 1 \mp 2\left(q^{2}+r^{2}\right)$ \\
\hline$r$ & $\mp(q-p)$ & & $\mp(q+r)$ & $\pm 2\left(p^{2}-q^{2}\right)$ & $\pm 1 \mp 2\left(q^{2}+r^{2}\right)$ \\
\hline
\end{tabular}

Cases of plane strain must be considered separately. In fact, if for example $\lambda_{1}=\varkappa_{1}=0$ (i.e. $\varepsilon_{1} \equiv 0$ ), then the parameters $r$ and $q$ coincide and $p$ is indefinite. These cases do not conform to the vertices but to the sides of the TG hexagon and lead to the equations of six parabolic hypercylinders

$$
\pm m_{1}+n_{1}^{2}=1, \quad \pm m_{2}+n_{2}^{2}=1, \quad \pm\left(m_{1}-m_{2}\right)+\left(n_{1}-n_{2}\right)^{2}=1 \text {. }
$$

Hence the whole plastic interaction hypersurface corresponding to the TG yield condition with the associated flow rule consists of six non-cylindrical parts $G_{1}^{ \pm}, G_{12}^{ \pm}$, $G_{2}^{ \pm}$described by Table 7, and six parabolic hypercylinders $H_{1}^{ \pm}, H_{12}^{ \pm}, H_{2}^{ \pm},(33.20)$. The intersections of this four-dimensional hypersurface by the hyperplanes $n_{2}$ = const are shown in Fig. 167 (quoted after T. Nakamura [7.217], W. Flügge and T. Nakamura [7.59]).

It can be seen that the hypercylinders $H(33.20)$ form an overwhelming part of the hypersurface under consideration. On the other hand, Eqs. (33.20) are much simpler than the parametrical equations listed in Table 7. Hence T. Nakamura [7.217], and independently Z Z. Mróz and Xu Bing-Ye [7.209] suggested circumscribing the exact hypersurface by these hypercylinders only. This approximate hypersurface furnishes an upper bound; Nakamura has shown that the largest error along the radius is $(1 / 0.851)-1=17.5$ per cent. Thus, if the approximate hypersurface is shrunk by the factor 0.851 , it is fully inscribed in the exact surface and gives a lower bound. The intersection of the approximate hypersurface by the plane $n_{2}=0$ is shown in Fig. 168.

The Tresca-Guest hypersurface is considerably simplified in particular cases. In the most important particular case $\varkappa_{2}=\varkappa_{\theta}=0$, corresponding to rotationally symmetric cylindrical shells, the circumferential moment $m_{\theta}$ may be eliminated and the resulting three-dimensional surface (P. G. Hodge [7.93], E. T. Onat [7.252]) consists of four planes

$$
n_{\theta}= \pm 1, \quad n_{\theta}-n_{x}= \pm 1,
$$

two parabolic cylinders

$$
m_{x}= \pm\left(1-n_{x}^{2}\right),
$$

and four paraboloids

$$
m_{x}= \pm\left[1-\frac{1}{2}\left(2 n_{\theta} \pm 1\right)^{2}-\frac{1}{2}\left(2 n_{\theta}-2 n_{x} \pm 1\right)^{2}\right],
$$



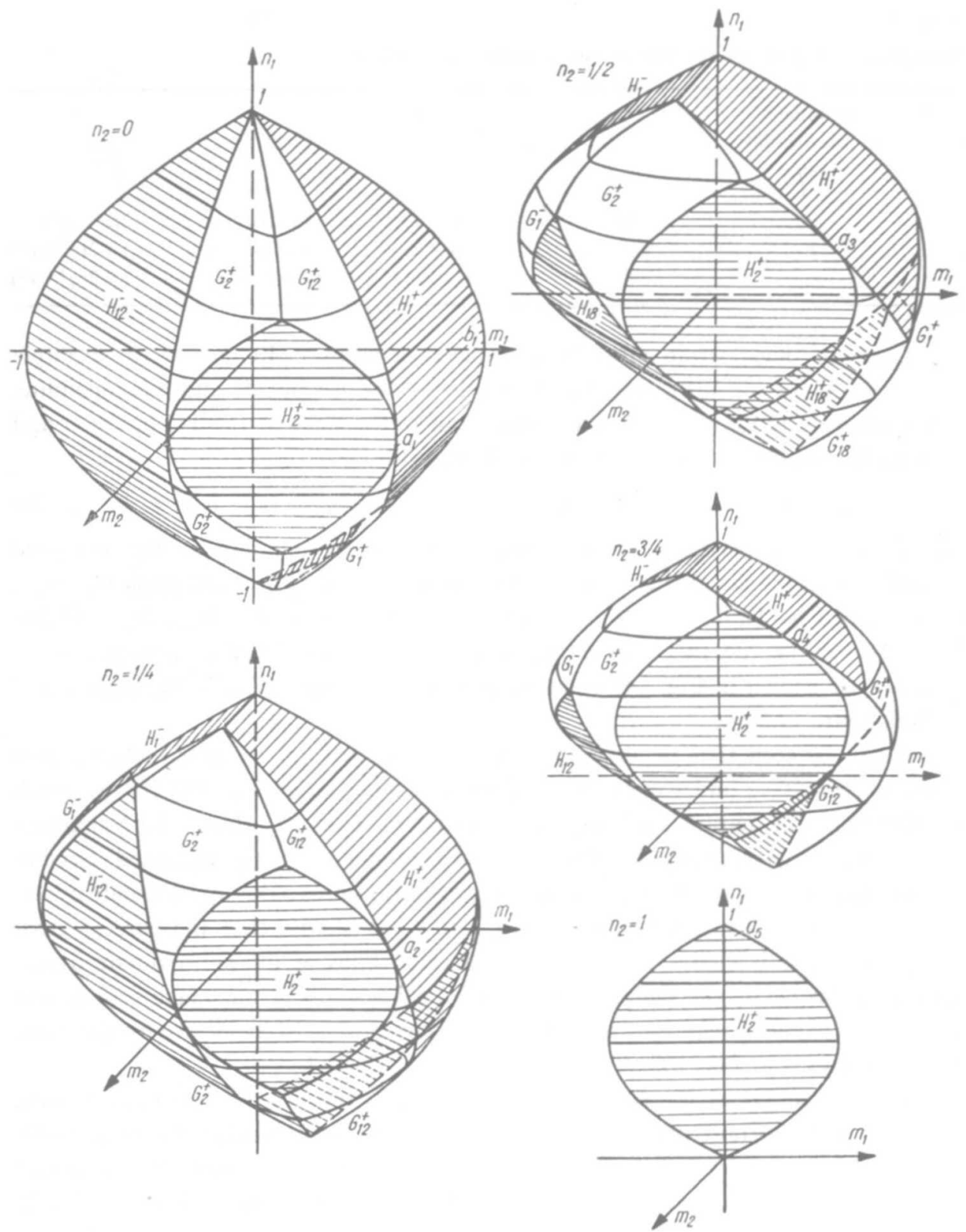

Fig. 167. Three-dimensional sections of the four-dimensional TG plastic interaction surface (after Nakamura)

where the signs inside the round brackets should be chosen equal to each other. The resulting surface is shown in Fig. 169. D. C. Drucker and R. T. Shield [7.37] suggested applying Eqs. $(33.21) \div(33.23)$ approximately for arbitrary shells of 


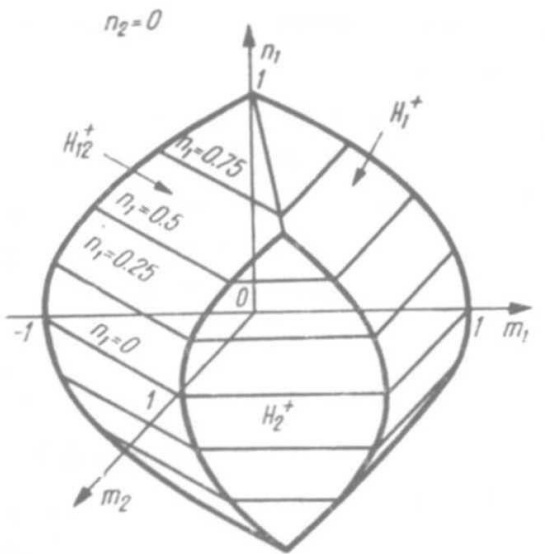

Fig. 168. The approximate TG plastic interaction surface

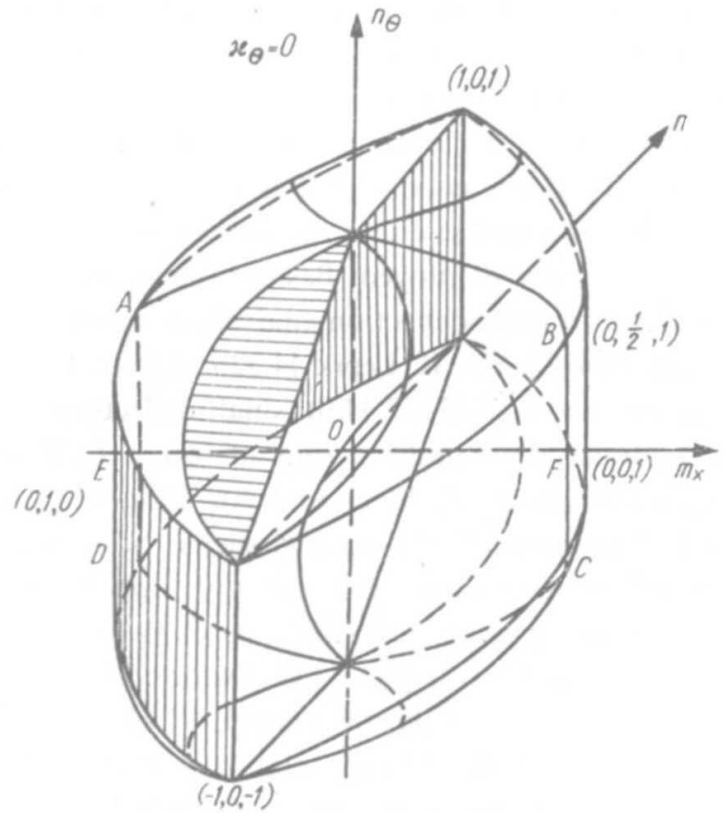

Fig. 169. The TG plastic interaction surface in the case $x_{\theta}=0$

revolution. C. R. Calladine [7.20] derived the surface shown in Fig. 169 in a purely geometrical way suggested for frames by W. Prager [4.468].

Transversal non-homogeneity of material was dealt with by W. Olszak and A. Sawczuk [7.248], Yu. R. Lepik [7.155], Yu. P. Listrova and M. A. Rudis [7.164] and by R. Sankaranarayanan [7.292]; non-homogeneity due to non-uniform temperature distribution was considered by Yu. P. Listrova and M. A. Rudis [7.165].

G. I. Bykovtșev, Yu. P. Listrova and G. A. Murlina [7.18] generalized the Tresca-Guest hypersurface for moderate-thickness shells; they assumed the hyper- 
bolic strain rate distribution (32.4) and calculated membrane forces and moments by means of formulae (32.1) and (32.2), respectively.

Though formulae $(33.20)$ or $(33.21) \div(33.23)$ describe much simpler surfaces than the original one, many further going approximations have been proposed. The replacement of uniform shells by sandwich shells will be discussed in Sec. 34 . An even simpler concept, of neglected circumferential moment $m_{\theta}$ and no interaction between membrane stresses and moments, was suggested by R.T. Shield and D. C. Drucker [7.337]:

$$
\max \left(\left|n_{x}\right|,\left|n_{\theta}\right|,\left|n_{x}-n_{\theta}\right|,\left|m_{x}\right|\right)=1 \text {. }
$$

A more exact, two-moment limited interaction surface was proposed by P.G. Hodge [7.99]; it may be used for any principal directions:

$$
\max \left(\left|n_{1}\right|,\left|n_{2}\right|,\left|n_{1}-n_{2}\right|,\left|m_{1}\right|,\left|m_{2}\right|,\left|m_{1}-m_{2}\right|\right)=1 \text {. }
$$

Hodge estimated also the errors of approximation.

It should be remembered that (33.25) furnishes an upper bound to the plastic interaction surface, and hence the direction of errors of lower bounds based on (33.25) (R. F. Gabbasov [7.63]) cannot be determined.

The plastic interaction hypersurface for a cross-section of surface structures, based on the Tresca-Guest yield condition and associated flow rule, was very often applied to the plastic analysis of plates and shells: either its original form was used or the above-mentioned approximations were employed. Besides the papers quoted above, let us mention here applications to large-deflection analysis of circular plates (E. T. Onat, R. M. Haythornthwaite [4.432], Yu. R. Lepik [4.326, 7.154], N. Jones [7.117], A. N. Sherbourne, N. K. Srivastava [7.334, 7.346]), small-deflection analysis of shells (G. Eason [7.46], cylindrical shell under a ring of force; E. T. Onat [7.255] and P. G. Hodge [7.98], conical shells; Z. Mróz and Xu Bing-Ye [7.209], spherical shells; W. Flügge and T. Nakamura [7.59], conical shells; S. L. Lee and B. J. Thorn [7.148], general method of derivation of lower and upper bounds; M. Sayir [7.314, 7.315], general integrals for cylindrical shells; A. C. Palmer [4.441], dynamic programming), large-deflection analysis of shells (M. Duszek [4.129, 7.38], M. Duszek and A. Sawczuk [4.130], cylindrical shells; M. Duszek [7.40, 7.41, 7.43], spherical shells; K. A. Koba and O. N. Shabliy [7.132], clamped shallow shells) and to optimal plastic design of shells (E. T. Onat, W. Prager [7.253], W. Freiberger [7.60], V. R. Terrovere [7.366, 7.367]).

D. A. Gokhfeld and O. F. Chernyavsky [4.175] introduced fictitious TG plastic interaction surfaces, describing shakedown limits for surface structures.

\subsection{Other isotropic yield conditions}

The yield condition of maximal normal stress (Galileo-Clebsch-Rankine) may be regarded as a further simplification of the TG failure hypothesis. Indeed, it is described by the same equations (33.12) where $\Phi_{3}=1$ and $\Phi_{6}=0$ are omitted, and is represented by a square in the $\sigma_{1}-\sigma_{2}$ plane. On the other hand, the maximal 
normal stress criterion deviates so much from the $\mathrm{HMH}$ hypothesis that regarding it as an approximation we would arrive at too large approximation errors; this hypothesis is used, for example, in reinforced structures.

Combining the yield condition under consideration with the law of similarity of deviators, we obtain the stress distributions (33.14) and (33.15), and these equations are not particularly simple. Essential simplifications are obtained just by combining the yield condition with the associated flow rule; this approach is fully justified in the case of reinforced or ribbed structures. It turns out that the stress distributions must then correspond to the vertices of the square, $\sigma_{1}= \pm \sigma_{0}$, $\sigma_{2}= \pm \sigma_{0}$, except some particular combinations of $x_{1}, x_{2}, \lambda_{1}$ and $\lambda_{2}$. Hence the stress distribution at least in one direction coincides with that in the limit state of a rectangular bar cross-section under bending with tension, and the plastic interaction hypersurface consists of two pairs of parabolic hypercylinders

$$
m_{1}= \pm\left(1-n_{1}^{2}\right), \quad m_{2}= \pm\left(1-n_{2}^{2}\right)
$$

Equations (33.26) give a counterpart of the Girkmann parabola (22.35) in the limit analysis of surface structures.

Equations (32.26) are used in connection with the concept of yield lines (concentrated deformations, justified if we consider a rigid-perfectly-plastic body as a limiting case of rigid-plastic-hardening materials. A. R. Rzhanitsyn [6.207], A. Sawczuk [4.516] and C. R. Calladine [7.19] applied (33.26) to the calculation of finite deflections of plates. Relations between limit analysis and yield line theory were discussed by A. Sawczuk and P. G. Hodge [7.307]. Generalizations of the concept of yield lines to shell problems were suggested by G. Kazinczy [7.126] and K. W. Johansen [7.116] and developed by A. R. Rzhanitsyn [7.286, 7.287] and M. Janas [7.110].

Plastic interaction surfaces for the case $\mathscr{P}_{2} / \mathscr{S}_{4}$ under consideration were also derived for various generalizations of the Galileo and of the Tresca-Guest yield condition to anisosensitive isotropic materials. The earliest papers concern sandwich structures (cf. Sec. 34.3); the uniform cross-section was first considered by R. Sankaranarayanan [7.289] (generalized maximal normal stress conditions). Generalizations of the Tresca-Guest yield condition were employed by Yu. P. Listrova and N. I. Mokashova [7.166] Yu. P. Listrova and G. A. Murlina [7.169] (Coulomb yield condition), Yu. R. Lepik [7.160], Yu. V. Nemirovsky, O. N. Shabliy and M. S. Mikhalishin [7.226, 7.325] (various linearizations of the Burzyński-Balandin yield condition) and by V.S. Popov [7.267]. Transversal non-homogeneity was additionally considered by R. Sankaranarayanan and W. Olszak [7.289, 7.290], Yu. P. Listrova, V. N. Potapov and M. A. Rudis [7.167, 7.168].

Limited interaction surfaces for anisosensitive materials were considered by Yu. A. Lellep and Yu. R. Lepik [7.150]; anisosensitivity introduces a certain complication, since the limit curve for moments is subject to symmetrization.

D. D. Ivlev [3.309] and Yu. P. Listrova [7.163] derived the equations of the plastic interaction surface for the yield condition of maximal deviatoric stress, 
(11.16). These equations, together with the equations of the Tresca-Guest surface, provide for the HMH plastic interaction surface an upper and a lower bound, respectively.

\subsection{Natural and structural orthotropy}

We have defined the combined case $\mathscr{P}_{2} / \mathscr{S}_{4}$ as that in which the principal directions of initial curvatures, of moments and of membrane forces coincide. It remains relatively simple if the principal directions of orthotropy of material also coincide; most investigators then use various orthotropic generalizations of the Tresca-Guest yield condition.

The first paper on this topic was published by D. Niepostyn [7.229], 1956, who considered an orthotropic circular cylinder, though the Hill condition (12.3) was applied to an even more general case, $\mathscr{P}_{3} / \mathscr{S}_{6}$, by M. Sh. Mikeladze [7.191] two years earlier (cf. Sec. 35). A general approach to anisosensitive orthotropic materials, described by the Hu-Ivlev-Capurso yield condition (12.10) was used for a uniform cross-section by G. I. Bykovtsev [7.17] and V. I. Sebekina [7.320]. Further investigations were carried out by J. Chakrabarty [7.27]. Anisotropic, anisosensitive non-homogeneous shells were considered by R. Sankaranarayanan and W. Olszak [7.291, 7.293].

Structural orthotropy may be due to densely spaced ribs or dense reinforcement by stringers, fibres, etc., analysed by an averaging approach. The load-carrying capacity of such structures can be raised without material volume increase.

In the earliest paper on the limit carrying capacity of externally ribbed circular cylinders, M. E. Lunchick [7.175] applied the HMH yield condition and the simplified hypothesis of a common physical neutral surface (coincident with the Feynberg approach [7.58]). Complete limit interaction surfaces for a cross-section of ribbed surface structures were first derived and analysed in detail by Yu. V. Nemirovsky

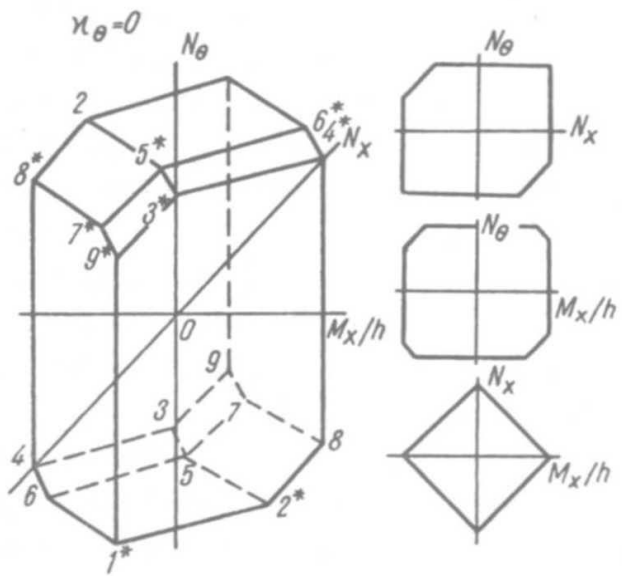

Fig. 170. Plastic interaction surface for a cross-section of a ribbed shell (after Nemirovsky-Rabotnov) 
and Yu. N. Rabotnov [7.219, 7.220] for cylindrical shells and generalized to arbitrary shells of revolution by D. D. Ivlev, Yu. I. Listrova and Yu. V. Nemirovsky [7.109]: the Tresca-Guest and the maximal deviatoric stress yield conditions were employed. A typical TG plastic interaction surface for a cross-section of a symmetrically ribbed circular cylindrical shell is shown in Fig. 170, [7.219]. Further investigations are due to A. Biron and A. Sawczuk [7.9, 7.10].

The theory of reinforced plates and shells has been developed mainly in connection with reinforced concrete structures. The earliest solutions obtained for reinforced concrete shells employed the simplified kinematical approach (A. L. L. Baker [7.8], 1950, N. V. Akhvledyani and V. N. Shayshmelashvili [7.3, 7.4], A. R. Rzhanitsyn [7.285]). Complete limit surfaces for cross-sections of reinforced concrete structures in the case $\mathscr{P}_{2} / \mathscr{S}_{4}$ were derived by Z. Mróz [7.208], A. Sawczuk and W. Olszak [7.301] and E. T. Onat [7.257]. Double reinforcements were considered by M. Janas and J. A. König [4.258, 7.113]. Further papers are due to M. Capurso and A. Gandolfi [7.22, 7.23], M. W. Braestrup [7.13], and Yu. V. Nemirovsky [7.223, 7.224]. V. V. Vasilev [7.379] considered metal shells reinforced by glass fibres; R. H. Lance and D. N. Robinson [7.143] applied the plastic interaction surface to the limit analysis of a fluid-filled cylindrical shell. Large plastic deflections of reinforced concrete plates were analysed by M. Janas [7.112, 7.115]. Optimal design of reinforced concerte shells in the case under consideration was investigated by Z. Mróz [7.210], S. Kaliszky [7.123], R. E. Melchers and G. I. N. Rozvany [7.187], M. I. Reitman and L. I. Yarin [7.274].

\subsection{Elastic-plastic range, plastic hardening}

The generalization of the Hencky-Ilyushin deformation theory of perfectly plastic plates and shells to the elastic-plastic range and plastic hardening is straightforward (A. A. Ilyushin [7.105], 1944). Indeed, under the assumption of incompressibility of the material, Eqs. (33.2) hold; now, for a given relation $\sigma_{e}=\sigma_{e}\left(\varepsilon_{e}\right)$, the function $\varphi$ is determined by $(9.30)$ and (7.29)

$$
\varphi=\frac{3 \varepsilon_{e}}{2 \sigma_{e}\left(\varepsilon_{e}\right)}
$$

with substituted strain intensity, (7.32),

$$
\begin{array}{r}
\varepsilon_{e}=\frac{2}{\sqrt{3}}\left[\left(x_{1}^{2}+x_{1} x_{2}+x_{2}^{2}\right) z^{2}+\left(2 x_{1} \lambda_{1}+2 x_{2} \lambda_{2}+x_{1} \lambda_{2}+x_{2} \lambda_{1}\right) z+\right. \\
\left.+\left(\lambda_{1}^{2}+\lambda_{1} \lambda_{2}+\lambda_{2}^{2}\right)\right]^{1 / 2}
\end{array}
$$

Equations (33.4) hold; these constitutive equations in the case under consideration are usually presented in a matrix form

$$
\left\{\begin{array}{l}
N_{1} \\
N_{2} \\
M_{1} \\
M_{2}
\end{array}\right\}=\left[\begin{array}{rrrr}
2 J_{0} & J_{0} & 2 J_{1} & J_{1} \\
J_{0} & 2 J_{0} & J_{1} & 2 J_{1} \\
2 J_{1} & J_{1} & 2 J_{2} & J_{2} \\
J_{1} & 2 J_{1} & J_{2} & 2 J_{2}
\end{array}\right]\left\{\begin{array}{l}
\lambda_{1} \\
\lambda_{2} \\
x_{1} \\
x_{2}
\end{array}\right\},
$$


or briefly,

$$
\mathbf{Q}=\mathbf{D}_{d} \mathbf{q} .
$$

In the Levy-Mises theory of plastic flow, pertaining to rigid-plastic bodies, instead of (33.30) one obtains

$$
\mathbf{Q}=\mathbf{D}_{f} \dot{\mathbf{q}},
$$

and in the incremental theories (Prandtl-Reuss, Prager-Laning) one may derive the incremental equations of the general form

$$
d \mathbf{Q}=\mathbf{D}_{i} d \mathbf{q} .
$$

Hence, the central point of the theory reduces to the calculation of the elements of "stiffness matrices" $\mathbf{D}_{d}, \mathbf{D}_{f}$, or $\mathbf{D}_{i}$. In the simplest case they are reduced to the integrals of the type (33.5) with substituted (33.27) and (33.28), but they are often much more complicated, e.g. when compressibility of the material is allowed for, or if plastically passive processes follow the active ones.

Even in the simplest case the integrals (33.5) can seldom be evaluated analytically. Some results were obtained by Ilyushin; analytical formulae for linear plastic hardening were derived by Yu. R. Lepik [7.151, 7.152], and for elastic-perfectlyplastic material by O. M. Paliy and V. S. Chuvikovsky [7.259]. Much more effective are numerical methods, based mainly on the conceptual division of a given uniform structure into a multilayer structure and replacement of integration by summation over sections of individual layers (multipoint section).

Plastic interaction surfaces for cross-sections of multilayer surface structures were analysed by P. G. Hodge [1.5, 7.102] and Yu. V. Nemirovsky [7.221], but the idea of such multilayer substitutive structures is particularly useful in analysing elastic-plastic deformations. This concept was introduced by J. A. Stricklin, PaoTan Hsu and T. H. H. Pian [7.361] and P. Marcal [7.180] and developed by E. P. Popov, M. Khojasteh-Bakht and P. Sharifi [7.266], J. C. Gerdeen, F. A. Simonen and D. T. Hunter [7.64, 7.65], P. V. Marcal [7.182], S. Yaghmai and E. P. Popov [7.391]. Replacement of uniform sections by multipoint sections may of course be considered as an application of a simple numerical quadrature formula (trapezoidal rule). One can also apply more refined quadrature formulae: $\mathrm{Z}$. Waszczyszyn [7.386] employed the Simpson rule for mixed plastic hardening and orthotropy.

The calculation of large elastic-plastic deflections of circularly-symmetrical plates constitutes a typical application of the case under consideration. This problem was initiated by P. M. Naghdi [7.215] in 1952; further research is due to R. M. Cooper and G. A. Shifrin [4.91] (experimental evidence of an essential effect of membrane forces), R. M. Haythornthwaite and E. T. Onat [7.92], Yu. R. Lepik [7.151, $7.152,7.153]$, but it was $\mathrm{Y}$. Ohashi with his group, who considered this problem in detail. Y. Ohashi and S. Murakami [7.233, 7.235, 7.236] introduced a trigonometric representation of extensions and curvatures. If we substitute for an incompressible material 


$$
\begin{aligned}
& \varkappa_{1,2}= \pm \frac{2}{\sqrt{3}} \varkappa_{e} \sin \left(\omega_{x} \pm \frac{1}{6} \pi\right), \\
& \lambda_{1,2}= \pm \frac{2}{\sqrt{3}} \lambda_{e} \sin \left(\omega_{\lambda} \pm \frac{1}{6} \pi\right),
\end{aligned}
$$

thus replacing the four parameters $x_{1}, x_{2}, \lambda_{1}, \lambda_{2}$ by other four parameters, $x_{e}$, $\lambda_{e}, \omega_{x}, \omega_{\lambda}$, then the expression for strain intensity (33.28) assumes a much simpler form,

$$
\varepsilon_{e}=\frac{2}{\sqrt{3}}\left[\varkappa_{e}^{2} z^{2}+2 \varkappa_{e} \lambda_{e} z \cos \left(\omega_{\lambda}-\omega_{x}\right)+\lambda_{e}^{2}\right]^{1 / 2},
$$

and the new parameters are convenient for numerical calculations. Formulae (33.33) represent an extension of the Sokolovsky substitution for small deflections of plates [7.343]. The problem becomes much more complicated for compressible bodies, since then $\varepsilon_{e}$ is no longer a unique function of $\varkappa_{1}, \varkappa_{2}, \lambda_{1}, \lambda_{2}$; it depends also on material constants resulting from the elimination of $\varepsilon_{z}$ by virtue of the equation $\sigma_{z}=0$. The corresponding formulae of the type (33.33) for compressible bodies were introduced for the elastic-plastic range [7.236], locally passive processes [7.238] and cyclic loading [7.244]. Y. Ohashi and S. Murakami [7.234] considered finite deflections of a perfectly elastic-plastic clamped circular plate, Y. Ohashi, S. Murakami and A. Endo [7.237] - a perfectly elastic-plastic annular plate, Y. Ohashi, S. Murakami and K. Kawashima [7.238, 7.241, 7.242, 7.243]-circular plates with locally passive processes and total unloading, Y. Ohashi and N. Kamiya [7.240]-a circular plate with non-linear plastic hardening, Y. Ohashi and K. Kawashima [7.244]a circular plate under cyclic loading. Locally passive processes accompanying large deflections of circular plates were also analysed by E. P. Popov, M. KhojastehBakht and S. Yaghmai [7.265].

Further investigations concerning large inelastic deflections of plates were published by Gu Guo-ji and Gu Qiu-Lin [7.77] (perturbation method), E. Aarend, Yu. Lepik and L. Luht [7.1] (linear hardening, variational approach), A. Z. Lokshin and V.I. Mamay [7.171] (influence of compressibility), Z. Waszczyszyn [7.384] (various circularly-symmetric loadings), M. Capurso and R. Ramasco [7.26], M. Dacko [7.33], V. A. Lukin and I. V. Shirko [7.174] (perfectly elasticplastic with TG yield condition), J. Myszkowski [7.213, 7.214] (simplified treatment based on bitrapeziform stress distributions, arbitrary boundary conditions), M. S. Mikhalishin and O. N. Shabliy [7.200] (anisosensitive rigid-plastic material), P. Ladevèze and R. Valid [7.142] (clamped plate), A. N. Sherbourne and N. K. Srivastava [7.334, 7.346] (hypothesis of limited interaction), Zh. V. Kachalov, Yu. P. Listrova and V. N. Potapov [7.121] (the Schmidt-Ishlinsky-Hill maximal deviatoric stress yield condition), A. Shindo, Y. Seguchi, T. Shirai and K. Denpo [7.338] (linear plastic hardening, finite difference method), O. N. Shabliy and K. A. Koba [7.324] (rigid-plastic-hardening, various yield conditions), M. Hamada and M. Tanaka [7.82, 7.82a, 7.363] (mixed plastic hardening of Ziegler type, finite difference method). R. M. Kirakosyan [7.130] considered small deflections of a plate under 
in-plane and perpendicular loadings. Large deflections of an orthotropic plate were discussed by M. Pignataro [7.262].

Another field of application of the combined case $\mathscr{P}_{2} / \mathscr{S}_{4}$ under consideration is that of elastic-plastic rotationally symmetric shells. The original Ilyushin approach combined with the iterative method of elastic solutions was developed by I. S. Tsurkov [7.374, 7.375], V. V. Vasilev [7.378], V. I. Korolev [7.135] (combined with the perturbation method), I. S. Chernyshenko [7.29, 7.30] (finite deflections) and [7.31] (variable thickness), A. N. Guz', I. S. Chernyshenko and K. I. Shnerenko [7.79] (spherical shells with cutouts), V. A. Gorlatch [7.73, 7.74] (compressible material and finite deflections), A. N. Frolov [7.62] (an improved algorithm for finite deflections), and K. Soonets [7.345]. A numerical procedure for cylindrical shells was suggested by P. Klement [7.131]. The Hencky-Ilyushin deformation theory with parametrization (33.33) was also applied to cylindrical shells by Y. Ohashi and T. Okouchi [7.245].

A quite different, much simpler approach to elastic-plastic-hardening shells is due to P. G. Hodge. He used piece-wise linear theory of plasticity, namely the limited interaction TG yield condition or the TG yield condition for sandwich shells combined with linear plastic hardening and assumed that a shell cross-section is either fully elastic or fully plastic. P. G. Hodge and F. Romano [7.95, 7.96] considered a cylindrical shell under radial internal distributed and ring-shaped pressures, whereas P. G. Hodge and S. V. Nardo [7.97], B. Paul and P. G. Hodge [4.447, 7.260] calculated deflections and the maximal carrying capacity of a cylindrical shell under external hydrostatic pressure. Further applications of the TG yield condition may be found in papers by E. T. Onat [7.254] (isotropic and kinematic hardening), B. J. Thorn, J. S. Kao and S. L. Lee [7.369] (analysis of stability of displacements), N. Jones [7.118] (finite deflections under dynamic loadings), O. Lomacky and B. Hyman [7.172] (finite deflections), O, N. Shabliy [7.321, 7.322] (rigid-plastic shells with linear isotropic hardening) and [7.323] (generalization of the TG yield condition to orthotropy), O. N. Shabliy and K. A. Koba [7.326] (finite deflections).

The Prandtl-Reuss equations and their generalizations to plastic hardening were applied to shell problems mainly in connection with a numerical approach. P. V. Marcal and C. E. Turner [7.179] considered small and finite deflections of rotationally symmetric shells, using direct numerical integration; their method was developed by P. V. Marcal and W. R. Pilgrim [6.180, 6.181] ("local stiffnesses"). The finite difference method was applied by P. Montague and M. R. Horne [7.203], J. C. Gerdeen, F. A. Simonen and D. T. Hunter [7.64, 7.65], V. V. Vasilev [7.380] I. V. Shirko and V. L. Yakushev [7.340]. The incremental theories of plasticity combined with the finite element method were applied to shell problems by E. P. Popov, M. Khojasteh-Bakht and P. Sharifi [7.266], P. Marcal [7.182], Y. Ando, K. Iida, T. Kawai, G. Yagawa and F. Kikuchi [7.7]. Z. Waszczyszyn [7.385, 7.386] considered finite deflections of orthotropic shells using deformation and incremental theories of plasticity combined with various numerical methods. 
The elastic-plastic stress distributions in a cross-section of surface structures may be influenced by thermal effects (thermal expansion and the dependence of mechanical properties of the material on temperature). The earliest papers analysed uniform biaxial stress distribution $\sigma_{1}=\sigma_{2}=\sigma$ in a cross-section of a plate (J. H. Weiner [7.389], H. G. Landau and J. H. Weiner [7.144], H. Yüksel [7.392], periodic loading). General biaxial stress states were analysed by Yu. R. Lepik [7.157], V. V. Piskun [7.263], A. I. Borisyuk [7.11], V. I. Makhnenko and E. A. Velikoivanenko [7.177], A. V. Kolgadin [7.134], I, V. Prokhorenko [7.269], Yu. P. Listrova, G. A. Murlina and T. D. Semykina [7.170], Yu. G. Korotkikh and S. A. Kapustin [7.137] and Yu. N. Shevtchenko [7.336]. E. Friedman and B. A. Boley [7.61] considered the effect of partial melting of plates. More papers on thermal stresses in plates and shells will be discussed in Sec. 40 , devoted to combined loadings at the level $\mathscr{B}$.

The shakedown problems at the level $\mathscr{S}$ of plates and shells were considered by J. A. König [4.303, 7.140], A. Sawczuk [7.305, 7.308], A. K. Lyubimov [7.176], Y. Ohashi and K. Kawashima [7.244]. Thermal effects in shakedown problems were analysed mainly by D. A. Gokhfeld [7.68] and his group: P. I. Ermakov and I. M. Plagov [7.67], A. G. Laptevsky, O. F. Chernyavsky and E. F. Chernyayev [7.69, $7.70,7.71,7.72]$.

\section{Combined loadings of the type $\mathscr{P}_{2} / \mathscr{S}_{4}$ in sandwich sections}

\subsection{Introductory remarks}

We mentioned in Sec. 33.5 the concept of multilayer substitutive surface structures which approximately replace the original uniform structure. The simplest case is obtained if we reduce the number of working layers to two thin external layers, separated by a core which transmits shear only (sandwich structure, Fig. 3). In a sandwich section (two-point section) the stress distribution is statically determinate. Indeed, in the general case of loading by moments $M_{\alpha \beta}$ and membrane forces $N_{\alpha \beta}$ we have-under the restrictions to (1) a symmetric section, (2) no bending resistance of individual layers and (3) the accuracy of formulae (32.13) and (32.14)-

$$
N_{\alpha \beta}=\left(\sigma_{\alpha \beta}^{+}+\sigma_{\alpha \beta}^{-}\right) h, \quad M_{\alpha \beta}=\left(\sigma_{\alpha \beta}^{+}-\sigma_{\alpha \beta}^{-}\right) H h,
$$

where $h$ denotes the layer thickness and $2 H$ the distance between the middle surfaces of the layers. The superscripts \pm refer to the layers $z= \pm H$, respectively. Equations (34.1) can simply be solved with respect to the stresses $\sigma_{\alpha \beta}^{+}$and $\sigma_{\alpha \beta}^{-}$:

$$
\sigma_{\alpha \beta}^{+}=\left(n_{\alpha \beta}+m_{\alpha \beta}\right) \sigma_{0}, \quad \sigma_{\alpha \beta}^{-}=\left(n_{\alpha \beta}-m_{\alpha \beta}\right) \sigma_{0},
$$

where dimensionless membrane forces $n_{\alpha \beta}$ and dimensionless moments $m_{\alpha \beta}$ are introduced by the formulae

$$
n_{\alpha \beta}=\frac{N_{\alpha \beta}}{\overline{\bar{N}}}=\frac{N_{\alpha \beta}}{2 \sigma_{0} h}, \quad m_{\alpha \beta}=\frac{M_{\alpha \beta}}{\overline{\bar{M}}}=\frac{M_{\alpha \beta}}{2 \sigma_{0} H h} .
$$


Comparing (34.3) with (32.21) and (32.44), i.e. requiring the same limit carrying capacity in tension and in bending respectively, we may determine the dimensions of the substitutive sandwich section

$$
2\left(\sigma_{0} H h\right)_{s}=\sigma_{0} H^{2}, \quad 2\left(\sigma_{0} h\right)_{s}=2 \sigma_{0} H,
$$

where the subscripts $s$ refer to the sandwich section. Hence

$$
\sigma_{0 s} h_{s}=\sigma_{0} H, \quad H_{s}=H / 2 ;
$$

we deliberately have admitted here different yield-point stresses $\sigma_{0}$ for the two structures (P. G. Hodge [4.223]), since equal values of $\sigma_{0}$ would lead to $h_{s}=2 H_{s}$, and such a result would contradict the basic assumption of a sandwich structure $h_{s} \ll H_{s}$.

According to (32.16) the strains in the layers $z= \pm H$ are

$$
\varepsilon_{\alpha \beta}^{+}=\lambda_{\alpha \beta}+x_{\alpha \beta} H, \quad \varepsilon_{\alpha \beta}^{-}=\lambda_{\alpha \beta}-\varkappa_{\alpha \beta} H .
$$

The present section is confined to the coincidence of the principal directions of initial curvatures, of moments and of membrane forces, and instead of arbitrary $\sigma_{\alpha \beta}$ and $\varepsilon_{\alpha \beta}$ we substitute here $\sigma_{1}, \sigma_{2}, \varepsilon_{1}, \varepsilon_{2}$, respectively.

We have introduced sandwich surface structures as a tool of approximate analysis of uniform plates and shells, but sandwich structures themselves are also widely employed in engineering applications. They are particularly advisable in the case of prevailing bending states, since then the inner layers of a uniform structure contribute only very little to the carrying capacity of the cross-section and may be replaced without loss by a lighter core.

\subsection{Limit carrying capacity for the HMH yield condition}

The limit carrying capacity of a sandwich section is exhausted even if only one layer becomes plastic: indeed, an infinitesimal rotation is then possible about the second layer, which has no bending resistance. Considering the layer $z=+H$ and substituting $\sigma_{1}^{+}$and $\sigma_{2}^{+},(34.2)$, into the $\mathrm{HMH}$ yield condition (11.7), we obtain the equation of one portion of the plastic interaction hypersurface

$$
\left(n_{1}+m_{1}\right)^{2}-\left(n_{1}+m_{1}\right)\left(n_{2}+m_{2}\right)+\left(n_{2}+m_{2}\right)^{2}=1 .
$$

Similarly, plastification of the layer $z=-H$ results in the equation

$$
\left(n_{1}-m_{1}\right)^{2}-\left(n_{1}-m_{1}\right)\left(n_{2}-m_{2}\right)+\left(n_{2}-m_{2}\right)^{2}=1 .
$$

Assuming a rigid-perfectly-plastic material governed by the associated flow rule (Levy-Mises), we may present generalized strain rates corresponding to (34.7) as follows:

$$
\begin{array}{ll}
\dot{x}_{1}=\frac{\Lambda}{H}\left(2 n_{1}+2 m_{1}-n_{2}-m_{2}\right), & \dot{x}_{2}=\frac{\Lambda}{H}\left(2 n_{2}+2 m_{2}-n_{1}-m_{1}\right), \\
\dot{\lambda}_{1}=\Lambda\left(2 n_{1}+2 m_{1}-n_{2}-m_{2}\right), & \dot{\lambda}_{2}=\Lambda\left(2 n_{2}+2 m_{2}-n_{1}-m_{1}\right) .
\end{array}
$$


The constant $H$ results from the chain rule, namely from multiplying by $d m / d M$, (34.3); other constants are included into the multiplier $A$. It can be seen that we have here $H \varkappa_{j}=\lambda_{j}, j=1,2$; indeed, this result follows also from (34.6) if we substitute $\varepsilon_{\alpha \beta}^{-}=0$ (rigid layer). Such a relation is very seldom kinematically admissible; in most cases both layers must be plastic in the limit state and Eqs. (34.7) and (34.8) must be satisfied simultaneously. The generalized strain rates may then be presented as linear combinations of (34.9) and of similar formulae corresponding to (34.8).

Consider for example a cylindrical shell under circularly-symmetric loading. The generalized strain $\varkappa_{\theta}$ is then equal to zero. Assuming one-side plastification only, we obtain also $\lambda_{\theta}=0$; (34.9) then gives

$$
m_{\theta}=\frac{n_{x}+m_{x}}{2}-n_{\theta}
$$

and substituting this relation into (34.7), we obtain

$$
\left|n_{x} \pm m_{x}\right|=\frac{2}{\sqrt{3}} .
$$

The lower sign corresponds here to plastification of the layer $z=-H,(34.8)$.

In the case of two-side yielding the corresponding geometrical condition

$$
\dot{x}_{\theta}=\frac{A_{1}}{H}\left(2 n_{\theta}+2 m_{\theta}-n_{x}-m_{x}\right)+\frac{A_{2}}{H}\left(2 n_{\theta}-2 m_{\theta}-n_{x}+m_{x}\right)=0
$$

determines only the ratio $\Lambda_{1} / \Lambda_{2}$; however, the bending moment $m_{\theta}$ may simply be eliminated from (34.7) and (34.8). Subtracting these equations side by side we obtain a linear equation with respect to $m_{\theta}$ and hence

$$
m_{\theta}=\frac{n_{\theta}-2 n_{x}}{2 n_{\theta}-n_{x}} m_{x} .
$$

Substitution of (34.13) into Eqs. (34.7) and (34.8) added together gives finally

$$
\left(n_{x}^{2}-n_{x} n_{\theta}+n_{\theta}^{2}\right)\left[1+\frac{3 m_{x}^{2}}{\left(2 n_{\theta}-n_{x}\right)^{2}}\right]=1 .
$$

Equations (34.11) and (34.14) determine the plastic interactions surface in the space $n_{x}-n_{\theta}-m_{x}$ for the case under consideration. It was analysed in detail by A. Sawczuk and J. Rychlewski [7.298], A. Sawczuk and P. G. Hodge [7.299, 7.100]. For the important case $n_{x}=0$ we obtain the ellipse (33.10) shown in Fig. 163.

Equations of the plastic interaction surface for a sandwich section, based on the HMH yield condition, were derived by Yu. N. Rabotnov [7.272] and V. I. Rozenblyum [7.278] for an even more general case $\mathscr{P}_{3} / \mathscr{S}_{6}$, which will be discussed in Sec. 35 .

Analytical applications of the equations derived are rather difficult because of their non-linearity. Some numerical procedures were proposed, e.g. A. Biron and U. S. Chawla [4.39] employed the non-linear programming methods (cf. also Sec. 21).

Generalization to the Burzyński-Stassi d'Alia yield condition for anisosensitive materials is due to C. Mazzarella [7.184]. 


\subsection{Limit carrying capacity for the linear yield conditions}

The yield conditions which are linear in principal stresses lead-in the case $\mathscr{P}_{2} / \mathscr{S}_{4}$ under consideration - to linear equations of the plastic interaction surfaces regardless of the flow rules employed: the hypersurface consists of hyperplanes only.

Assuming the Tresca-Guest yield condition and substituting $\sigma_{1}^{+}$and $\sigma_{2}^{+}$, and, in turn, $\sigma_{1}^{-}$and $\sigma_{2}^{-}$into the six equations (33.12), we obtain 12 equations of hyperplanes, namely

$$
\begin{array}{ll}
n_{1}+m_{1}=1, & n_{1}-m_{1}=1, \\
n_{2}+m_{2}=1, & n_{2}-m_{2}=1, \\
n_{2}+m_{2}-n_{1}-m_{1}=1, & n_{2}-m_{2}-n_{1}+m_{1}=1, \\
-n_{1}-m_{1}=1, & -n_{1}+m_{1}=1, \\
-n_{2}-m_{2}=1, & -n_{2}+m_{2}=1, \\
-n_{2}-m_{2}+n_{1}+m_{1}=1, & -n_{2}+m_{2}+n_{1}-m_{1}=1 .
\end{array}
$$

The resulting supersurface is formed by those parts of (34.15) which are closest to the origin of the system of coordinates in the space of generalized stresses. Equations (34.15) were derived by P. G. Hodge [7.93] and E. T. Onat [7.252].

In the case of a cylindrical shell, where $x_{\theta}=0$, this condition does not introduce the elimination of $m_{\theta}$ directly. However, assuming two-side yielding, we may simply eliminate $m_{\theta}$ from one equation of the left column and one equation of the right column of (34.15). Excluding different signs of $\sigma_{\theta}^{+}$and $\sigma_{\theta}^{-}$which are impossible here, we obtain a system of 12 equations (four of (34.15), which do not contain $m_{\theta}$, and eight as a result of eliminations):
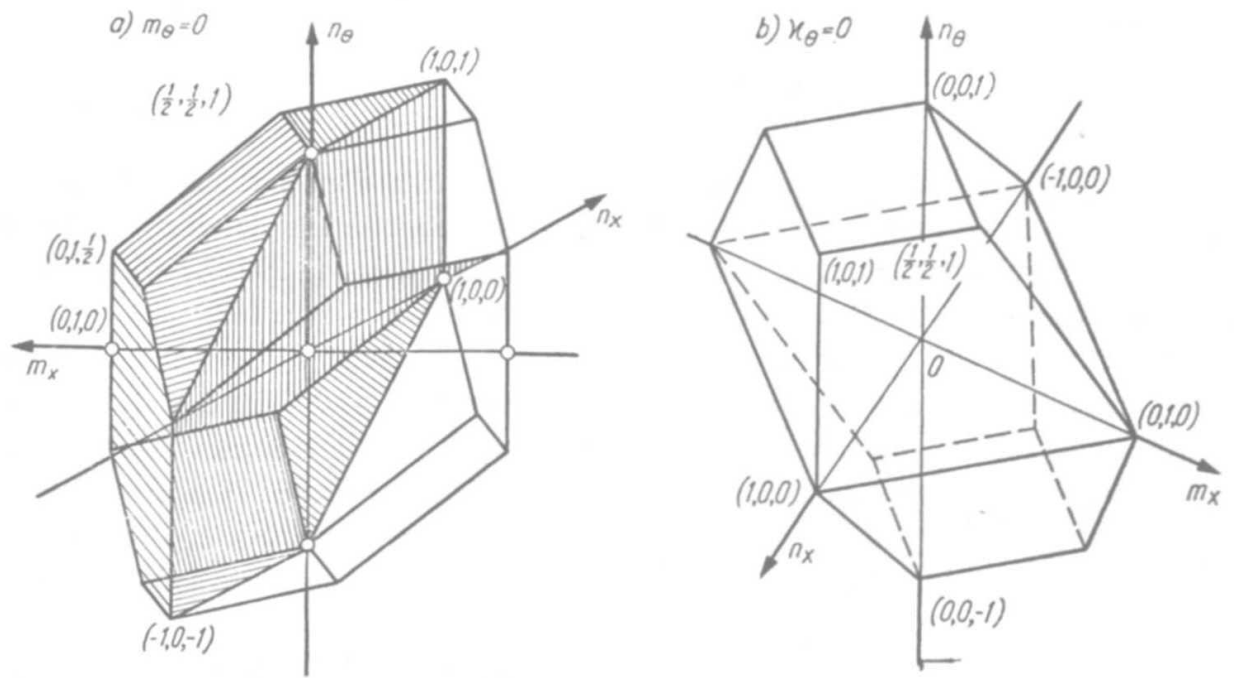

Fig. 171. Two particular cases of the TG plastic interaction surface for a sandwich cross-section 


$$
\begin{array}{ll}
\quad n_{1} \pm m_{1}=1, & -n_{1} \pm m_{1}=1, \\
\pm n_{2}=1, & \pm n_{2} \mp n_{1}=1, \\
2 n_{2}-n_{1} \pm m_{1}=2, & -2 n_{2}+n_{1} \pm m_{1}=2 .
\end{array}
$$

The result of the elimination of $m_{\theta},(34.16)$, and the intersection of (34.15) with the plane $m_{2}=m_{\theta}=0$, are shown for comparison in Fig. 171, [7.298].

The main advantage of Eqs. (34.15) lies in their linearity. Linear equations of the general form

$$
a_{1} m_{1}+a_{2} m_{2}+b_{1} n_{1}+b_{2} n_{2}=1
$$

may determine lower or upper bounds to more complicated limit interaction surfaces. This problem was studied by P. G. Hodge [7.101] in relation to the HMH and TG yield conditions for a uniform section of surface structures. Linearization of both these yield conditions and of the Hill condition for orthotropic materials was used by W. Olszak and A. Sawczuk [7.249] to the limit analysis of cylindrical shells. A detailed comparison of plastic interaction surfaces for uniform sections and for sandwich sections was given by A. Sawczuk and P. G. Hodge [7.299]. Linearization problems were also studied by Yu. A. Nagyavitchius and A. A. Cyras [7.216], and the subsequent application to optimization problems was given by A. A. Cyras, A. E. Borkauskaus and R. P. Karkauskas [7.32]. Linear equations of the type (34.17), and particularly (34.15) and (34.16), simplest in the theory of plastic surface structures, were often applied to the analysis of limit state and postyield behaviour of plates and shells. We mention here the application to finite deflections of rigid-plastic plates (Yu. R. Lepik [4.327]) and to various shell problems (P. G. Hodge [7.94], M. I. Erkhov [7.52], B. W. Shaffer, M. Senator [7.330], Yu. R. Lepik [7.158, 7.159], M. Duszek [7.39], F. Ellyin [7.48, 7.49]).

Numerous generalization of Eqs. (34.15) have been proposed. Orthotropic isosensitive materials were analysed in detail by A. Sawczuk [3.697]; further investigations are due to N. K. Das Talukder and R. K. Chakrabarti [7.34]. Isotropic anisosensitive materials $\left(\sigma_{0+} \neq \sigma_{0_{-}}\right)$were first analysed by W. Prager [3.658] and then by Ya. A. Lellep [7.149] and V. S. Popov [7.268]. Non-symmetric layers were considered by L. I. Polyakov, Yu. T. Polyakov and M. A. Rudis [7.264] (plates) and T. Nakamura [7.218] (shells). Bimetallic shells were investigated by Yu. V. Nemirovsky [7.225], M. S. Mikhalishin, Yu. V. Nemirovsky and O. N. Shabliy [7.201] (anisosensitive materials). Multilayer orthotropic shells were discussed by Yu. V. Nemirovsky [7.221, 7.222]. Thermal effects were considered by E. T. Onat and S. Yamantürk [7.256] (cylindrical shells) and by V. N. Stelmashuk [7.347], who generalized Eqs. (34.17).

\subsection{Elastic-plastic range, plastic hardening}

The typical direction of analysis: point $\rightarrow$ section $\rightarrow$ body as a whole, cannot in general be inverted, since the transition to subsequent levels requires some integral operations. However, sandwich structures admit a certain inverse procedure: in the 
general case $\mathscr{P}_{3} / \mathscr{L}_{6}$ six generalized stresses (34.1) are linear functions of six stress components $\sigma_{\alpha \beta}^{+}$and $\sigma_{\alpha \beta}^{-}, \alpha, \beta=\xi, \eta$ and the inversion of these relations is immediate, (34.2). Of course, the same conclusion holds also for the case $\mathscr{P}_{2} / \mathscr{S}_{4}$ under consideration. Particularly, if the yield condition is non-linear, or if a more accurate description of plastic hardening is employed, then it is usually convenient to go "back to the point", i.e. to retain the stresses as dependent variables and to express generalized stresses in terms of stresses, (34.1). Namely, it is more convenient to keep the non-linear equations as simple as possible and to complicate the linear equations (equilibrium conditions and geometrical equations if linearized) instead.

As an example consider a perfectly elastic-plastic sandwich cylindrical shell, subject to rotationally-symmetric normal internal pressure $q_{r}^{*}=q_{r}^{*}(x)$ and to tensile axial end load $p_{x}^{*}$ per unit length of the perimeter, Fig. 172 (Tran-le Binh

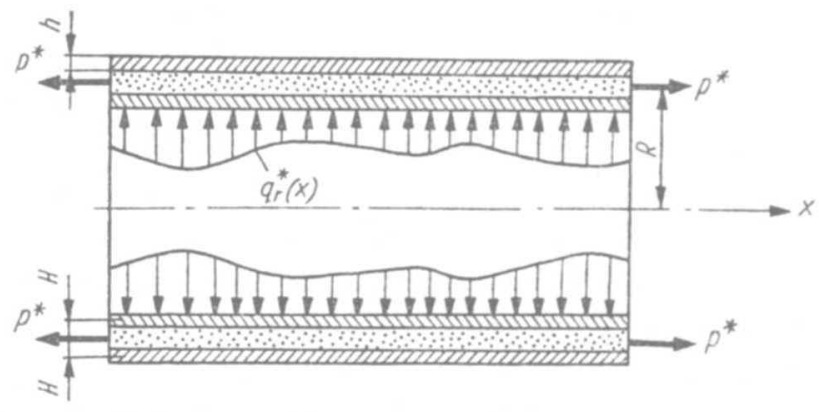

Fig. 172. Rotationally symmetric loading of a sandwich cylindrical shell

and M. Życzkowski [7.371, 7.373]). Assume incompressibility of the material, the HMH yield condition and the Hencky-Ilyushin deformation theory of plasticity, and derive the governing equations in the ranges of one-side plastification and of two-side plastification.

To make our equations consistent with the Timoshenko solution for the elastic range, assume now the sign convention used in [7.370]; namely, $z$ and $w$ will be directed inwards, and positive moments will be opposite to those shown in Fig. 4. Retain, however, the superscript "+" for the outer layer and "-" for the inner layer, as before. Then the strains are

$$
\begin{aligned}
& \varepsilon_{x}^{+}=\lambda_{x}-\varkappa_{x} H=\frac{d u^{*}}{d x^{*}}+H \frac{d^{2} w^{*}}{d x^{* 2}}, \\
& \varepsilon_{x}^{-}=\lambda_{x}+\varkappa_{x} H=\frac{d u^{*}}{d x^{*}}-H \frac{d^{2} w^{*}}{d x^{* 2}}, \\
& \varepsilon_{\theta}^{+}=\varepsilon_{\theta}^{-}=\lambda_{\theta}=-\frac{w^{*}}{R},
\end{aligned}
$$

where the dimensional variables are starred, so as to retain the usual symbols for dimensionless quantities; $R$ denotes the mean radius of the shell, and the ex- 
pressions for $x$ and $\lambda$ are substituted in linearized form. Now introduce dimensionless variables,

$$
x=\frac{x^{*}}{\sqrt{R H}}, \quad u=\frac{E u^{*}}{\sigma_{0} \sqrt{R H}}, \quad w=\frac{E w^{*}}{\sigma_{0} R},
$$

and rewrite (34.18) as follows:

$$
\varepsilon_{x}^{+}=\left(u^{\prime}+w^{\prime \prime}\right) \frac{\sigma_{0}}{E}, \quad \varepsilon_{x}^{-}=\left(u^{\prime}-w^{\prime \prime}\right) \frac{\sigma_{0}}{E}, \quad \varepsilon_{\theta}^{+}=\varepsilon_{\theta}^{-}=-w \frac{\sigma_{0}}{E},
$$

where the primes denote differentiation with respect to $x$.

Because of the changed sign convention for moments, instead of (34.1) we have here

$$
M_{x}=\left(\sigma_{x}^{-}-\sigma_{x}^{+}\right) H h, \quad M_{\theta}=\left(\sigma_{0}^{-}-\sigma_{\theta}^{+}\right) H h .
$$

The equilibrium equations where (34.19) is introduced and the beam-column effect is neglected, take the form

$$
\begin{aligned}
& N_{x}^{\prime}=0, \quad N_{x}=p_{x}^{*}=\text { const }, \\
& M_{x}^{\prime \prime}+H N_{\theta}=R h q_{r}^{*} .
\end{aligned}
$$

Expressing now $M$ and $N$ in terms of stresses, we obtain

$$
\begin{aligned}
& \sigma_{x}^{-}+\sigma_{x}^{+}=2 \sigma_{0} p, \\
& \left(\sigma_{x}^{-}-\sigma_{x}^{+}\right)^{\prime \prime}+\left(\sigma_{\theta}^{-}+\sigma_{\theta}^{+}\right)=2 \sigma_{0} q_{r},
\end{aligned}
$$

where the dimensionless loadings

$$
p=\frac{p_{x}^{*}}{2 \sigma_{0} h}, \quad q_{r}=\frac{R q_{r}^{*}}{2 \sigma_{0} h}
$$

are introduced in such a way as to give $p=1$ and $q_{r}=1$, corresponding to plastification under pure axial tension and pure constant internal pressure, respectively.

Further equations depend on the range of work of the cross-section under consideration. We first discuss one-side plastification, of the outer layer only. Then the stresses $\sigma_{\theta}^{+}$and $\sigma_{x}^{+}$must satisfy the $\mathrm{HMH}$ yield condition (11.7); it is convenient to parametrize this condition by a substitution of the type (19.42), namely

$$
\sigma_{i}=\sigma_{\theta}^{+}=\frac{2}{\sqrt{3}} \sigma_{0} \sin \left(\omega_{+}+\frac{1}{3} \pi\right), \quad \sigma_{2}=\sigma_{x}^{+}=\frac{2}{\sqrt{3}} \sigma_{0} \sin \omega_{+},
$$

thus replacing two unknowns, $\sigma_{\theta}^{+}$and $\sigma_{x}^{+}$, by one unknown $\omega_{+}$only. The first equation of (34.23) determines now the stress $\sigma_{x}^{-}$,

$$
\sigma_{x}^{-}=2 \sigma_{0} p-\frac{2}{\sqrt{3}} \sigma_{0} \sin \omega_{+} .
$$

The Hencky-Ilyushin equation of shape change will be written in the form

$$
\varepsilon_{x}^{+}=\frac{2 \sigma_{x}^{+}-\sigma_{\theta}^{+}}{2 \sigma_{\theta}^{+}-\sigma_{x}^{+}} \varepsilon_{\theta}^{+} .
$$


Hence, after the substitution of (34.20) and (34.25), we obtain the following formula for the derivative $u^{\prime}$ :

$$
u^{\prime}=\frac{w}{2}-\frac{\sqrt{3}}{2} w \tan \omega_{+}-w^{\prime \prime} .
$$

Finally, to the elastic inner layer we apply Hooke's law for an incompressible body

$$
\begin{aligned}
& \sigma_{x}^{-}=\frac{4}{3} E\left(\varepsilon_{x}^{-}+\frac{1}{2} \varepsilon_{\theta}^{-}\right)=\frac{4}{3} \sigma_{0}\left(u^{\prime}-w^{\prime \prime}-\frac{w}{2}\right), \\
& \sigma_{\theta}^{-}=\frac{4}{3} E\left(\varepsilon_{\theta}^{-}+\frac{1}{2} \varepsilon_{x}^{-}\right)=\frac{4}{3} \sigma_{0}\left(-w+\frac{u^{\prime}}{2}-\frac{w^{\prime \prime}}{2}\right) .
\end{aligned}
$$

Comparing the first equation of (34.29), where (34.28) is substituted-with (34.26), we obtain

$$
w^{\prime \prime}+\frac{\sqrt{3}}{4} w \tan \omega_{+}=\frac{\sqrt{3}}{4} \sin \omega_{+}-\frac{3}{4} p .
$$

Substitution of (34.25), (34.26) and (34.29) into the second equilibrium equation (34.23) yields

$$
\begin{aligned}
w^{\prime \prime} & +\frac{\sqrt{3}}{4} w \tan \omega_{+}+\frac{3}{4} w=\left(\omega_{+}^{\prime 2} \sin \omega_{+}-\omega_{+}^{\prime \prime} \cos \omega_{+}\right) \sqrt{3}+ \\
& +\frac{\sqrt{3}}{4} \sin \omega_{+}+\frac{3}{4} \cos \omega_{+}-\frac{3}{2} q_{r} .
\end{aligned}
$$

Equations (34.30) and (34.31) are linear in the unknown $w$ and non-linear in the unknown $\omega_{+}$; hence $w$ may easily be eliminated. Subtracting (34.30) from (34.31), we obtain

$$
w=\frac{4}{\sqrt{3}}\left(\omega_{+}^{\prime 2} \sin \omega_{+}-\omega_{+}^{\prime \prime} \cos \omega_{+}\right)+\cos \omega_{+}+p-2 q_{r},
$$

and the substitution of (34.32) into either (34.30) or (34.31) yields the governing equation for $\omega_{+}$:

$$
\begin{aligned}
\omega_{+}^{\mathrm{Iv}} & =\frac{\sqrt{3}}{4} \omega_{+}^{\prime 2} \tan ^{2} \omega_{+}+\left(4 \omega_{+}^{\prime} \omega_{+}^{\prime \prime \prime}+3 \omega_{+}^{\prime \prime 2}-\frac{\sqrt{3}}{2} \omega_{+}^{\prime \prime}-\omega_{+}^{\prime 4}\right) \tan \omega_{+}+ \\
& +6 \omega_{+}^{\prime 2} \omega_{+}^{\prime \prime}-\frac{\sqrt{3}}{4} \omega_{+}^{\prime 2}+\frac{3 p}{16 \cos \omega_{+}}\left(\tan \omega_{+}+\sqrt{3}\right)- \\
& -\frac{\sqrt{3}}{8 \cos \omega_{+}}\left(q_{r} \sqrt{3} \tan \omega_{+}+4 q_{r}^{\prime \prime}\right) .
\end{aligned}
$$

The solution of (34.33) with the appropiate boundary conditions makes it possible to determine the distribution of stresses, strains and displacements in the shell (cf. Sec. 40).

One-side plastification of the inner layer does not introduce major changes, [7.372]. Now the stresses $\sigma_{\hat{\theta}}^{-}$and $\sigma_{x_{x}}^{-}$are parametrized by formulae of the type 
(34.25) with $\omega_{-}$as a parameter, and Hooke's law (34.29) is applied to the outer layer. Instead of $(34.28)$ one obtains

$$
u^{\prime}=\frac{w}{2}-\frac{\sqrt{3}}{2} w \tan \omega_{-}+w^{\prime \prime}
$$

instead of (34.32) we have

$$
w=-\frac{4}{\sqrt{3}}\left(\omega_{-}^{\prime 2} \sin \omega_{-}-\omega_{-}^{\prime \prime} \cos \omega_{-}\right)+\cos \omega_{-}+p-2 q_{r},
$$

and the final governing equation for $\omega_{-}$takes the form

$$
\begin{aligned}
\omega_{-}^{\mathrm{IV}} & =-\frac{\sqrt{3}}{4} \omega_{-}^{\prime 2} \tan ^{2} \omega_{-}+\left(4 \omega_{-}^{\prime} \omega_{-}^{\prime \prime \prime}+3 \omega_{-}^{\prime \prime 2}+\frac{\sqrt{3}}{2} \omega_{-}^{\prime \prime}-\omega_{-}^{\prime 4}\right) \tan \omega_{-}+ \\
& +6 \omega_{-}^{\prime 2} \omega_{-}^{\prime \prime}+\frac{\sqrt{3}}{4} \omega_{-}^{\prime 2}+\frac{3 p}{16 \cos \omega_{-}}\left(\tan \omega_{-}+\sqrt{3}\right)- \\
& -\frac{\sqrt{3}}{8 \cos \omega_{-}}\left(q_{r} \sqrt{3} \tan \omega_{-}-4 q_{r}^{\prime \prime}\right) .
\end{aligned}
$$

Two-side plastification introduces some qualitative changes. Now the stresses in both layers are parametrized by formulae of type (34.25). The Hencky-Ilyushin equations for both layers lead to (34.28) and (34.34); comparing these equations, we arrive at

$$
w^{\prime \prime}+\frac{\sqrt{3}}{4} w\left(\tan \omega_{+}-\tan \omega_{-}\right)=0 .
$$

The equilibrium equations (34.23) yield

$$
\begin{aligned}
& \sin \omega_{+}+\sin \omega_{-}=p \sqrt{3}, \\
& \left(\sin \omega_{-}-\sin \omega_{+}\right)^{\prime \prime}+\sin \left(\omega_{+}+\frac{1}{3} \pi\right)+\sin \left(\omega_{-}+\frac{1}{3} \pi\right)=q_{r} \sqrt{3} .
\end{aligned}
$$

Equations (34.37) and (34.38) determine three unknown functions, $\omega_{+}, \omega_{-}$and $w$. However, (34.38) do not contain $w$, and hence the system of equations is partly uncoupled. We may eliminate $\omega_{-}$,

$$
\sin \omega_{-}=p \sqrt{3}-\sin \omega_{+},
$$

and substituting this expression into the second equation of (34.38), we obtain the following second-order equation for $\omega_{+}$

$$
\begin{aligned}
\omega_{+}^{\prime \prime} & =\omega_{+}^{\prime 2} \tan \omega_{+}+ \\
& +\frac{\sqrt{3}}{4 \cos \omega_{+}}\left(\cos \omega_{+} \pm \sqrt{\cos ^{2} \omega_{+}+2 \sqrt{3} p \sin \omega_{+}-3 p^{2}}+p-2 q_{r}\right),
\end{aligned}
$$

where the sign before the root depends on the range of $\omega_{-}:$one should choose "+" for $\omega_{-}<\frac{1}{2} \pi$ and "-" for $\omega_{-}>\frac{1}{2} \pi$. In most practical cases the sign "+" 
should be chosen. Further, substituting (34.39) into (34.37), we obtain a secondorder equation for $w$ :

$$
w^{\prime \prime}=\frac{\sqrt{3}}{4} w\left( \pm \frac{\sqrt{3} p-\sin \omega_{+}}{\sqrt{\cos ^{2} \omega_{+}+2 \sqrt{3} p \sin \omega_{+}-3 p^{2}}}-\tan \omega_{+}\right) .
$$

Partial uncoupling of (34.40) and (34.41) facilitates the solution. Equation (34.41), in contradistinction to (34.30), is homogeneous with respect to $w$ and hence the magnitude of the deflections can be determined here only by non-homogeneous boundary conditions or by continuity conditions.

Elastic-plastic deflections of sandwich shells, based on the HMH yield condition, were first studied by Yu. N. Rabotnov [7.272] and A. R. Rzhanitsyn [7.286]. P. Klement [7.131] solved the problem of a cylindrical sandwich shell under a ring of radial forces. J. A. König [7.138] considered cylindrical shells employing the Prandtl-Reuss theory of plastic flow, but approximately assuming that the whole cross-section is either fully elastic or fully plastic. More exact applications of PrandtlReuss equations are due to H. F. Muensterer and F. P. J. Rimrott [7.211] (with experimental verifications) and to N. A. Shoeb and W. C. Schnobrich [7.341] (finite element method). Parametrization of the yield condition may also be useful when analysing finite deflections or finite strains in rigid-plastic shells (J. Skrzypek [7.342], toroidal shells).

The analysis of plastic hardening in sandwich shells was initiated by P. G. Hodge and his group [7.95, 7.96, 7.97], who based their considerations on the TG yield condition and regarded the sandwich section as an approximation to the uniform section. Plastic hardening in isotropic and orthotropic plates and shells at large deflections was given much attention by $\mathrm{Z}$. Waszczyszyn [7.386, 7.387, 7.388].

\section{More general combined loadings}

\subsection{Introductory remarks}

As has been mentioned above, the general case of loading in a cross-section of a surface structure may be classified as $\mathscr{P}_{5} / \mathscr{S}_{10}$ (if auxiliary exertion factors, e.g. temperature, are not taken into account). However, most considerations are based on some simplifying assumptions: if we assume $M_{\xi \eta}=M_{\eta \xi}$ and $N_{\xi \eta}=N_{\eta \xi}$ (or perform an appropriate symmetrization) and neglect transverse shear, then the relevant classification reduces to $\mathscr{P}_{3} / \mathscr{S}_{6}$.

The generalization of the analysis based on the HMH yield condition to the case $\mathscr{P}_{3} / \mathscr{S}_{6}$-if the principal directions of initial curvatures and of stresses do not coincide or if the analysis in principal directions is not convenient-is relatively simple and was given by A. A. Ilyushin as early as the forties [7.105, 7.106, 1.8]. Hence it is the HMH yield condition that will mainly be used in the present chapter. 
The analysis based on the TG yield condition, on the other hand, is here much more complicated, and comparatively simple only for a sandwich section. General considerations concerning intersections and projections of plastic interaction hypersurfaces in multidimensional spaces are due to A. Sawczuk and J. 'Rychlewski [7.298].

\subsection{Uniform section in the case $\mathscr{P}_{3} / \mathscr{P}_{6}$}

Assume the directions $\xi, \eta$ coinciding with the lines of principal initial curvatures and let the strain distribution be given by the linear relations (32.16). For an incompressible material we can determine the third normal strain as follows:

$$
\varepsilon_{z}=-\varkappa_{\alpha \alpha} z-\lambda_{\alpha \alpha}{ }^{\prime},
$$

where the "plane" summation convention was employed. The law of similarity of deviators (Hencky-Ilyushin or Levy-Mises, if $\lambda$ and $x$ stand for the respective rates) yields

$$
\sigma_{i j}=\frac{1}{\varphi}\left(\varepsilon_{i j}-\delta_{i j} \varepsilon_{z}\right)=\frac{1}{\varphi}\left[\left(\varkappa_{i j}+\delta_{i j} \varkappa_{\alpha \alpha}\right) z+\left(\lambda_{i j}+\delta_{i j} \lambda_{\alpha \alpha}\right)\right] .
$$

The HMH yield condition for plane stress state with the "plane" summation convention adopted may be written thus

$$
3 \sigma_{\alpha \beta} \sigma_{\alpha \beta}-\sigma_{\alpha \alpha} \sigma_{\beta \beta}=2 \sigma_{0}^{2} \text {. }
$$

Substitution of (35.2) and some rearrangements make it possible to determine the function $\varphi$ :

$$
\varphi=\frac{1}{\sigma_{0}} \sqrt{\frac{3}{2}\left(\varepsilon_{\alpha \beta} \varepsilon_{\alpha \beta}+\varepsilon_{\alpha \alpha} \varepsilon_{\beta \beta}\right)} .
$$

Making use of (32.16) we may express $\varphi$ in terms of the variable $z$; the result will be written in the form suggested by Ilyushin, namely

$$
\varphi=\varphi(z)=\frac{\sqrt{3}}{\sigma_{0}} \sqrt{P_{\lambda}+2 P_{x \lambda} z+P_{x} z^{2}},
$$

where $P_{\lambda}, P_{x \lambda}$ and $P_{x}$ are the following quadratic forms in $\lambda_{i j}$ and $\varkappa_{i j}$ :

$$
\begin{aligned}
& P_{\lambda}=\frac{1}{2}\left(\lambda_{\alpha \beta} \lambda_{\alpha \beta}+\lambda_{\alpha \alpha} \lambda_{\beta \beta}\right)=\lambda_{\xi \xi}^{2}+\lambda_{\xi \xi} \lambda_{\eta \eta}+\lambda_{\eta \eta}^{2}+\lambda_{\xi \eta}^{2}, \\
& P_{x}=\frac{1}{2}\left(x_{\alpha \beta} x_{\alpha \beta}+x_{\alpha \alpha} \varkappa_{\beta \beta}\right)=x_{\xi \xi}^{2}+x_{\xi \xi} x_{\eta \eta}+x_{\eta \eta}^{2}+x_{\xi \eta}^{2}, \\
& P_{x \lambda}=\frac{1}{2}\left(\lambda_{\alpha \beta} x_{\alpha \beta}+\lambda_{\alpha \alpha} x_{\beta \beta}\right)=\lambda_{\xi \xi} x_{\xi \xi}+\lambda_{\eta \eta} x_{\eta \eta}+\frac{1}{2} \lambda_{\xi \xi} x_{\eta \eta}+\frac{1}{2} \lambda_{\eta \eta} x_{\xi \xi}+\lambda_{\xi \eta} x_{\xi \eta} .
\end{aligned}
$$

The symbols $\lambda_{\xi \eta}$ and $x_{\xi \eta}$ are understood here as Itensorial components; the corresponding "engineering" symbols would denote here quantities twice as large, and hence in engineering notation we should substitute $\lambda_{\xi \eta} / 2$ and $x_{\xi \eta} / 2$ (such notation is used for example by G. S. Shapiro [7.332]). Finally, the stress components are determined by the following formulae:

$$
\sigma_{i j}=\frac{\sigma_{0}}{\sqrt{3}} \frac{\left(\varkappa_{i j}+\delta_{i j} x_{\alpha \alpha}\right) z+\left(\lambda_{i j}+\delta_{i j} \lambda_{\alpha \alpha}\right)}{\sqrt{P_{\lambda}+2 P_{x \lambda} z+P_{x} z^{2}}}, \quad i, j=\xi, \eta .
$$


The stress distribution (35.7), based on (32.16), satisfies the boundary conditions for $z= \pm H$ and leads to exact results if the equations on internal equilibrium are also satisfied; in the opposite case we would obtain an upper bound to the plastic interaction surface.

Integrating (35.7) with respect to $z$, we may express the generalized stresses in terms of the generalized strains $\varkappa_{i j}$ and $\lambda_{i j}$. The result will be written in a form analogous to (33.4), namely

$$
\begin{aligned}
& N_{i j}=\left(\varkappa_{i j}+\delta_{i j} \varkappa_{\alpha \alpha}\right) J_{1}+\left(\lambda_{i j}+\delta_{i j} \lambda_{\alpha \alpha}\right) J_{0}, \\
& M_{i j}=\left(\varkappa_{i j}+\delta_{i j} \varkappa_{\alpha \alpha}\right) J_{2}+\left(\lambda_{i j}+\delta_{i j} \lambda_{\alpha \alpha}\right) J_{1},
\end{aligned}
$$

where $J_{k}$ denote the integrals (33.5) where (35.5) is substituted. Equations (35.8) determine the plastic interaction hypersurface in the six-dimensional space in terms of five parameters, namely ratios of generalized strains (or strain rates). The results of integration depend on the values of the quadratic forms (35.6); the relevant discussion is given by A. A. Ilyushin [1.8].

Formulae (35.8) may formally be inverted with respect to $\lambda_{i j}$ and $x_{i j}$ (with the accuracy of a constant multiplier); finally we obtain, [1.8, 7.298]

$$
\begin{aligned}
& \lambda_{i j}=\frac{1}{\Delta}\left[\left(N_{i j}-\frac{1}{3} \delta_{i j} N_{\alpha \alpha}\right) J_{2}-\left(M_{i j}-\frac{1}{3} \delta_{i j} M_{\alpha \alpha}\right) J_{1}\right], \\
& \chi_{i j}=\frac{1}{\Delta}\left[-\left(N_{i j}-\frac{1}{3} \delta_{i j} N_{\alpha \alpha}\right) J_{1}+\left(M_{i j}-\frac{1}{3} \delta_{i j} M_{\alpha \alpha}\right) J_{0}\right],
\end{aligned}
$$

where $\Delta$ stands for the principal determinant of the system,

$$
\Delta=J_{0} J_{2}-J_{1}^{2} \text {. }
$$

However, formulae (35.9) are not effective, since the integrals $J_{k}$ depend on $x_{i j}$ and $\lambda_{i j}$. On the other hand, using (35.8) or (35.9) we may derive the following relations joining the quadratic forms of generalized stresses and the quadratic forms of generalized strains:

$$
\begin{aligned}
P_{N} & =3\left(J_{0}^{2} P_{\lambda}+2 J_{0} J_{1} P_{x \lambda}+J_{1}^{2} P_{\varkappa}\right), \\
P_{M N} & =3\left[J_{0} J_{1} P_{\lambda}+\left(J_{0} J_{2}+J_{1}^{2}\right) P_{\varkappa \lambda}+J_{1} J_{2} P_{\varkappa}\right], \\
P_{M} & =3\left(J_{1}^{2} P_{\lambda}+2 J_{1} J_{2} P_{\varkappa \lambda}+J_{2}^{2} P_{\varkappa}\right),
\end{aligned}
$$

where the forms $P_{N}, P_{M N}$ and $P_{M}$ are introduced by the formulae

$$
\begin{aligned}
P_{N} & =\frac{3}{2} N_{\alpha \beta} N_{\alpha \beta}-\frac{1}{2} N_{\alpha \alpha} N_{\beta \beta}, \\
P_{M N} & =\frac{3}{2} M_{\alpha \beta} N_{\alpha \beta}-\frac{1}{2} M_{\alpha \alpha} N_{\beta \beta}, \\
P_{M} & =\frac{3}{2} M_{\alpha \beta} M_{\alpha \beta}-\frac{1}{2} M_{\alpha \alpha} M_{\beta \beta} .
\end{aligned}
$$

Equations (35.11) are of fundamental importance for the analysis of the plastic interaction surface, since they make it possible to reduce the number of dimensions of the space. In fact, Eqs. (35.11) may be regarded as parametrical equations of a certain surface in the three-dimensional space $P_{N}, P_{M N}, P_{M}$, the parameters being for example $P_{\lambda} / P_{x}$ and $P_{x \lambda} / P_{k}$; namely, the integrals $J_{k}$ are homogeneous functions. 
of the degree $(-1 / 2)$ of the quadratic forms $P_{x}, P_{\lambda}$ and $P_{x \lambda}$, and hence the functions (35.11) are homogeneous of order zero with respect to these forms. This fact facilitates approximate descriptions of the plastic interaction surface.

Introduce dimensionless membrane forces (32.21), dimensionless moments (32.44) and (32.46), and dimensionless quadratic forms $P_{n}, P_{m n}$ and $P_{n}$, analogous to $(35.12)$; in expanded notation

$$
\begin{aligned}
P_{n} & =n_{\xi \xi}^{2}-n_{\xi \xi} n_{\eta \eta}+n_{\eta \eta}^{2}+3 n_{\xi \eta}^{2}, \\
P_{m n} & =m_{\xi \xi} n_{\xi \xi}-\frac{1}{2} m_{\xi \xi} n_{\eta \eta}-\frac{1}{2} m_{\eta \eta} n_{\xi \xi}+m_{\eta \eta} n_{\eta \eta}+3 m_{\xi \eta} n_{\xi \eta}, \\
P_{m} & =m_{\xi \xi}^{2}-m_{\xi \xi} m_{\eta \eta}+m_{\eta \eta}^{2}+3 m_{\xi \eta}^{2} .
\end{aligned}
$$

Thus the equation of the plastic interaction surface may formally be written in the implicit form

$$
f\left(P_{n}, P_{m n}, P_{m}\right)=0 .
$$

though the effective reduction of (35.11) to (35.14) is not always possible.

Consider some particular cases. The case $P_{x \lambda}=0$ was called by Ilyushin the "simpler combined case"; certain particular examples of this case were discussed in Sec. 33.1. Then $J_{1}=0$ and also $P_{m n}=0$. The quadratic forms $P_{m}$ and $P_{n}$ are interrelated by the parametrical equations, [1.8],

$$
\begin{aligned}
& P_{n}=\frac{\mu^{2}}{1-\mu^{2}} \ln ^{2} \frac{1+\sqrt{1-\mu^{2}}}{\mu}, \\
& P_{m}=\left(\frac{\mu^{2}}{1-\mu^{2}} \ln \frac{1+\sqrt{1-\mu^{2}}}{\mu}-\frac{1}{\sqrt{1-\mu^{2}}}\right)^{2},
\end{aligned}
$$

where $\mu$ is a parameter of the curve. Equations (35.15) may be reduced to the implicit form $f\left(P_{n}, P_{m}\right)=0$ [by eliminating the logarithm, calculating $\mu$ and then substituting $\mu$ into either equation of (35.15)], but the final form is rather complicated. Ilyushin [1.8] gave a table of the function $P_{m}=f\left(P_{n}\right)$ and proposed a simple approximation

$$
P_{m}+P_{n}=1
$$

stating that the errors of (35.16) do not exceed 9 per cent. Ch. Massonnet [7.183] remarked that the approximation

$$
P_{m}+P_{n}=c
$$

with $c=1.045$ reduces the errors by half though it furnishes no longer a lower bound to (35.15) (Fig. 173). M. I. Erkhov [7.50, 7.51, 7.54] found that $c=1.09$ in (35.17) gives an upper bound and $c=0.75$-a lower bound to the exact equation (35.11) in the general case $P_{m n} \neq 0$.

Another relatively simple case occurs if

$$
P_{m n}^{2}=P_{m} P_{n} ;
$$

Ilyushin found that this case is possible only if

$$
\frac{n_{\xi \xi}}{m_{\xi \xi}}=\frac{n_{\eta \eta}}{m_{\eta \eta}}=\frac{n_{\xi \eta}}{m_{\xi \eta}} \text {. }
$$




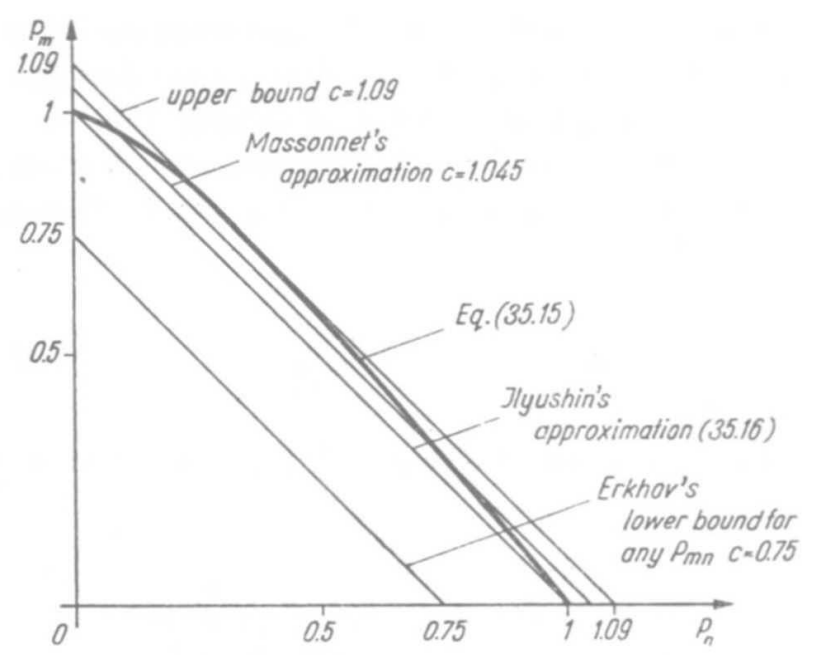

Fig. 173. Approximations for the simpler combined case

The equation of the plastic interaction surface (or rather the plastic interaction curve) may then be written in the form

$$
P_{m}=\left(1-P_{n}\right)^{2}, \quad\left|P_{m n}\right|=\left(1-P_{n}\right) \sqrt{P_{n}} .
$$

The general combined case was split by Ilyushin into two subcases. Analysing the function $\varphi,(35.5)$, we find that it attains its minimum at the point $z_{0}=-P_{x \lambda} / P_{x}$; if $\left|z_{0}\right|<H$, then bending states prevail, and if $\left|z_{0}\right|>H$, then membrane forces are predominant. For the subcase of prevailing bending Ilyushin proposed a simple linear approximation (i.e. an approximation which is homogeneous quadratic in generalized stresses)

$$
\dot{P}_{m}+P_{n}+\frac{1}{\sqrt{3}}\left|P_{m n}\right|=1,
$$

giving a generalization of (35.16). Errors of (35.21) were analysed in detail by M. Robinson [7.275], who found that they do not exceed 6 per cent on the safe side and 3.5 per cent against safety.

A certain more accurate approximation of (35.11) was suggested by G. V. Ivanov [7.108]. From among the surfaces described by the equation

$$
P_{n}+\frac{1}{2} P_{m}-\frac{\alpha\left(P_{m} P_{n}-P_{m n}^{2}\right)}{P_{n}+\beta P_{m}}+\frac{1}{2} \sqrt{P_{m}^{2}+4 P_{m n}^{2}}=1
$$

he proposed to choose the surface determined by the parameters $\alpha=\beta=0$, or, more accurately, $\alpha=0.25, \beta=0.48$; the latter suggestion is based on a numerical analysis by a computer. In [7.108a] Ivanov derived the corresponding approximate constitutive equations of the Prandtl-Reuss type. Namely, it was shown by E. T. Onat [7.257] that though Ilyushin's original considerations were based on the deformation theory of plasticity, his results may also be used in the theory of plastic flow. 
Various simpler approximate equations of the plastic interaction surface have been proposed. Many authors develop the concept of limited interaction surfaces: it consists in approximate replacement of a surface in the three-dimensional space (35.14) by a curve

$$
\psi(m, n)=1
$$

where $m$ and $n$ are defined by (32.22) and (32.47). In the case of the HMH yield condition and the analysis based on (32.13), (32.14) and (32.16) we have $m \equiv$ $\sqrt{P_{m}}, n \equiv \sqrt{P_{n}}$. If we assume no interaction between membrane forces and moments (Drucker-Shield-Hodge), then (35.23) has the form

$$
m=1, \quad n=1
$$

and determines a square in the plane $m-n$. However, this simple approximation gives relatively large errors per excess. A certain lower bound was proposed by S. Qu $[7.270,7.271]$

$$
n^{2}+m=1 \text {; }
$$

for the HMH yield condition it coincides with (35.20) (the influence of $P_{m n}$ being neglected), whereas when applied to the TG yield condition it resembles the Nakamura-Mróz-Xu approximation (33.20), though it includes also some other combinations, e.g. $n_{1}^{2}+m_{2}=1$.

V. I. Rozenblyum [7.277, 7.278, 7.279] introduced an averaged yield condition, assuming linear stress distribution (which is not justified in the plastic range) and postulating the following averaging procedure:

$$
\int_{-H}^{H} \sigma_{\mathrm{red}}^{2} d z=2 H \sigma_{0}^{2} .
$$

In the case of the HMH yield condition (35.26) leads to

$$
n^{2}+\frac{3}{4} m^{2}=1
$$

and gives errors per excess, particularly in the range of prevailing bending. Equation (35.17) may be rewritten here in the similar form

$$
n^{2}+m^{2}=c \text {; }
$$

the Ilyushin approximation $c=1$ corresponds to a sandwich section (cf. Sec. 35.4). Rozenblyum [7.278] proposed also a simple lower bound

$$
n+m=1 \text {. }
$$

A comparison of approximate equations $(35.24) \div(35.29) ?$ is 'shown in Fig. 174 .

Many papers generalize the Ilyushin theory of plastic plates and shells to compressible materials. Then, apart from the integrals $J_{k}$, (33.5), some slightly modified integrals must be introduced. A. N. Vozyanov [7.383] remarks that compressibility may result in an increase of deflections of the order of magnitude of 15 per cent. Further investigations are due to V. A. Gorlatch [7.73], Yu. M. Shevtchenko and O. I. Borisyuk [7.335, 7.12], who also considered the temperature changes. 


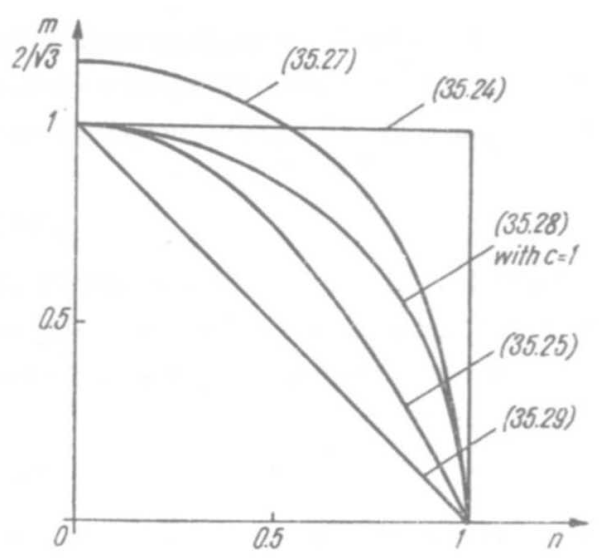

Fig. 174. Approximations for the general combined case

V. I. Rozenblyum [7.280] generalized the Ilyushin theory to the case in which the ratios $H / R_{i}$ are not very small in comparison with unity. Making use of the Lurie-Novozhilov theory of moderate-thickness shells, based on (32.1), (32.2) and (32.4), he found that the quadratic forms (35.13) should be replaced by

$$
\begin{aligned}
P_{n} & =n_{\xi \xi}^{2}-n_{\xi \xi} n_{\eta \eta}+n_{\eta \eta}^{2}+\frac{3}{4}\left(n_{\xi \eta}+n_{\eta \xi}-\frac{H}{2 R_{1}} m_{\xi \eta}-\frac{H}{2 R_{2}} m_{\eta \xi}\right)^{2}, \\
P_{m n} & =m_{\xi \xi} n_{\xi \xi}-\frac{1}{2} m_{\xi \xi} n_{\eta \eta}-\frac{1}{2} m_{\eta \eta} n_{\xi \xi}+m_{\eta \eta} n_{\eta \eta}+ \\
& +\frac{3}{4}\left(m_{\xi \eta}+m_{\eta \xi}\right)\left(n_{\xi \eta}+n_{\eta \xi}-\frac{H}{2 R_{1}} m_{\xi \eta}-\frac{H}{2 R_{2}} m_{\eta \xi}\right), \\
P_{m} & =m_{\xi \xi}^{2}-m_{\xi \xi} m_{\eta \eta}+m_{\eta \eta}^{2}+\frac{3}{4}\left(m_{\xi \eta}+m_{\eta \xi}\right)^{2} .
\end{aligned}
$$

It is assumed that the directions $\xi$ and $\eta$ coincide with the principal directions of initial curvatures of the shell. A further generalization, valid for arbitrary directions $x, y$, was given by $\mathrm{J}$. Rychlewski [7.282, 7.283]. The problem of symmetrization of $m_{\xi \eta}$ and $n_{\xi \eta}$ was discussed in detail by M. Duszek and A. Sawczuk [7.42]. Theories more general than that based on the Love-Kirchhoff hypothesis of straight normals were developed by $H$. Abe [7.2] and M. Duszek [7.44, 7.45].

A generalization of the Ilyushin theory to non-homogeneous surface structures is due to A. I. Strelbitskaya, V. A. Kolgadin and S. I. Matoshko [7.353] and to T. P. Vinogradova [7.381] (who considered also thermal stresses). H. M. Haydl and A. N. Sherbourne [7.89] showed that plastic analysis of irradiated shells may be reduced to that of variable-thickness shells. The influence of a non-proportional loading path on final stress distribution in a cross-section was studied by S. A. Kapustin [7.125]; stress distribution under variable loading was considered by T. Buriyev [7.16]. P. V. McLaughlin and S. C. Batterman [7.185] constructed a plastic interaction hypersurface in a six-dimensional space for fibrous composite plates. 
Equations derived for the case $\mathscr{P}_{3} / \mathscr{S}_{6}$ under consideration have mainly been applied to the calculation of large plastic deflections of plates and to the calculation of small or large plastic deflections of shells without rotational symmetry. Finite deflections of non-circular plates were first analysed by Yu. R. Lepik [7.151] (linear plastic hardening); further papers are due to A. Langenbach [7.146] (methods of functional analysis), P. A. Lukash [7.173] (arbitrary plastic hardening), M. F. Ershov [7.56] (plastic deformations following elastic loss of stability), A. Sawczuk [4.515] (initiation of membrane forces), M. Capurso [7.24] (rigid-plastic plates of arbitrary shape), Ch. Massonnet [7.183] (finite difference method combined with iterations), N. Jones and R. M. Walters [7.119, 7.120] (rigid-plastic clamped rectangular plates), A. I. Strelbitskaya, partly with S. A. Goloborodko and R. I. Rybakova [7.355, 7.356, 7.357] (finite differences, method of elastic solutions, linear hardening following perfect plasticity), E. Saareste and K. Soonets [7.288] (square plate, finite differences), T. H. Lin, S. R. Lin and B. Mazelsky [7.162] (clamped plate, Prandtl-Reuss theory), H. Okamura and K. Yoshida [7.246] (ribbed rectangular plates), F. Kikuchi and Y. Ando [7.129] (finite element method).

Small [plastic deflections of shells |withouti rotational symmetry |were considered, for example, by V. N. Ionov] [7.107] (zero Gaussian 'curvature, linear plastic hardening), M. I. Reitman [7.273] (general discussion of characteristics for perfect plasticity), S. A. Kapustin [7.124] (finite difference method applied to displacement equations), J. Schroeder and P. Roy [7.318] (influence of twisting moments on the limit carrying capacity) and [7.319] (pipe branching), O. Bruhns [7.15] (general equations for the incremental theory with mixed hardening), R. Yu. Kerimov [7.128] (stress concentration around cutouts), A. I. Strelbitskaya [7.359, 7.360] (linear hardening, finite differences combined with the method of elastic solutions). Large plastic deflections were analysed by I. S. Tsurkov [7.376] (rectangular panel), P. A. Lukash [7.173] (arbitrary plastic hardening), M. F. Ershov [7.56], A. Sawczuk [7.310] (consistent Lagrangian formulation), F. Kikuchi and Y. Ando [7.129] (finite element method) and by S. C. Tang [7.364] (application to the analysis of car crashes).

\subsection{Effect of transverse shearing forces}

In surface structures the influence of shearing forces may be even more essential than in the case of beams. In fact, shearing forces under an external concentrated force tend to infinity, hence also under a load distributed on a small area they are very large. Dynamic distributed loads may even result in infinitely large shearing forces (G. S. Shapiro [7.332]).

The problem of the influence of shearing forces on the limit carrying capacity of a circular plate was first analysed by J. F. Brotchie [7.14], 1960, who based his approximate analysis on the TG yield condition and on a certain assumed stress distribution. A general treatment is due to G. S. Shapiro [7.332]; he employed the 
kinematic approach, assuming the distribution of strain rates in the form (32.16), and additionally $\varepsilon_{\xi z}=\gamma_{\xi z} / 2=$ const and $\varepsilon_{\eta z}=\gamma_{\eta z} / 2=$ const. The case under consideration may be classified as $\mathscr{P}_{5} / \mathscr{S}_{8}$. Making use of the HMH yield condition, one obtains formulae of the type (35.8) for $N_{i j}$ and $M_{i j}$, and additionally the transverse shearing forces are determined by the formulae

$$
Q_{j}=\varepsilon_{\jmath z} J_{0}, \quad j=\xi, \eta \text {. }
$$

The integral $J_{0}$ is determined here by the general formula (33.5) with substituted (35.5) but with an extended quadratic form $P_{2}$; instead of (35.6) one should substitute

$$
P_{\lambda}=\lambda_{\xi \xi}^{2}+\lambda_{\xi \xi} \lambda_{\eta \eta}^{p}+\lambda_{\eta \eta}^{2}+\lambda_{\xi \eta}^{2}+\varepsilon_{\xi z}^{2}+\varepsilon_{\eta z}^{2},
$$

whereas the quadratic forms $P_{x}^{\dagger}$ and $P_{x \lambda}$ remain without change, (35.6).

Equations (35.8) and (35.31) express eight generalized stresses in terms of seven parameters (the ratios of $\lambda_{i j}, \varkappa_{i j}$ and $\varepsilon_{j z}$ ); hence they determine the plastic interaction hypersurface in a parametrical form. In fact, they furnish an upper bound to the actual limit carrying capacity of the section; the shearing stresses $\tau_{\xi z}$ and $\tau_{\dot{\eta} z}$ do not satisfy, in general, either the boundary condition $\tau=0$ for $z= \pm H$ or the equations of internal equilibrium. The same result was obtained by Shapiro [7.332] when applying the variational approach to evaluate the limit carrying capacity based on statically partially admissible stress fields; in view of the considerations given in Sec. 20.4, this approach leads also to an upper bound for the limit carrying capacity, and not to a lower bound as mentioned by the author. Shapiro proposed also some methods of approximate description of the plastic interaction hypersurface. G. Landgraf [7.145] derived a similar upper bound applying the variational approach to statically partially admissible stress fields, and analysed in detail the cases of cross-sections of a plate and of a cylindrical shell. An alternative derivation was given by V. N. Alpert [7.6]. J. Schroeder and A. N. Sherbourne $[7.316,7.317]$ allowed additionally for the stresses $\sigma_{z}$. M. Robinson [7.276] proposed a method of formal replacement of problems with transverse shear by certain reduced problems without shear. A simple approximation, based in fact on a sandwich representation of uniform structures, was derived by H. M. Haydl and A. N. Sherbourne [7.84], who also gave a comparison of various known approximations.

A quite different approach, based on the TG yield condition, was suggested by N. P. Zhuk and O. N. Shabliy [7.395]. They combined the TG hypothesis with the elliptic approximation (11.33) and replaced the yield surface in the space $\sigma_{r}$, $\sigma_{\theta}, \tau_{r z}$ by the following inscribed dodecahedron:

$$
\max \left(\left|\sigma_{r}-\sigma_{\theta}\right|+\left|\tau_{r z} \sqrt{c}\right|,\left|\sigma_{r}\right|+\left|\tau_{r z} \sqrt{c}\right|,\left|\sigma_{\theta}\right|+\left|\tau_{r z} \sqrt{c}\right|\right)=\sigma_{0}
$$

(a consistent application of the TG yield condition would require $c=4$ ). They derived the plastic interaction surface for a cross-section, generalizing that given by Table 7 and Eqs. (33.20) and solved a simple example.

Equations allowing for transverse shear were applied mainly to the limit analysis of circularly symmetric plates. A. Sawczuk and M. Duszek [7.303] evaluated 
effectively the integrals $J_{k}$, proposed a linearization of the equation of the limit surface and solved several examples. Possible mechanisms of plastic collapse of such plates allowing for shearing forces were considered in detail by A. Oschatz [7.258]. The effect of shear on the onset of yielding of a plate was discussed by A. J. Morris [7.205], J. B. Haddow [7.81] investigated the effects of transverse shear and membrane forces in plates. Theoretical and experimental investigations of reinforced concrete plates under concentrated loads were carried out by $\mathrm{H}$. Gesund and Y. P. Kaushik [7.66], whereas fiber-reinforced plates were analysed by R. H. Lance and D. N. Robinson [6.137]. Further papers are due to R. H. Wood [7.390], H. M. Haydl and A. N. Sherbourne [7.85, 7.87] (variable thickness), M. M. Mohaghegh and M. D. Coon [7.202] (linearization of the HMH yield condition), Z. Sobotka [4.545] (a simple piece-wise constant stress distribution), N. P. Zhuk and O. N. Shabliy [7.396] (analytical solutions based on the linearized TG yield condition).

The effect of shear in cylindrical shells was considered by H. M. Haydl and A. N. Sherbourne [7.83, 7.90], O. N. Shabliy and N. P. Zhuk [7.327]; in spherical shells-by Haydl and Sherbourne [7.86, 7.88]. O. N. Shabliy and K. A. Koba [7.328, 7.329] analysed large deflections of plates and shells including shear effects. Optimal design of rotationally symmetric shells allowing for transverse shear was initiated by Yu. R. Lepik [7.161].

\subsection{Sandwich section}

Consider the limit carrying capacity of a sandwich section in the general case $\mathscr{P}_{3} / \mathscr{S}_{6}$. To begin with, assume the HMH yield condition in the form (35.3); substituting the stresses $\sigma_{\alpha \beta}^{+},(34.2)$, into (35.3), we obtain the following conditionj of plastification of the outer layer $z=+H$

$$
3\left(n_{\alpha \beta}+m_{\alpha \beta}\right)\left(n_{\alpha \beta}+m_{\alpha \beta}\right)-\left(n_{\alpha \alpha}^{\alpha-}+m_{\alpha \alpha}^{\prime c}\right)\left(n_{\beta \beta}+m_{\beta \beta}\right)=2 \text {, }
$$

Introducing the notation of dimensionless quadratic forms (35.13) we may write

$$
P_{m}+2\left|P_{m n}\right|+P_{n}=1 \text {. }
$$

Considering the layer $z=-H$ we obtain only the change of sign of $P_{m n}$, and this fact has already been introduced into the notation (35.35). In view of the larger coefficient of $P_{m n}$ in (35.35) in comparison with the Ilyushin approximation for a uniform section (35.21) we realize that the surface (35.35) lies inside the surface (35.21); this conclusion is typical for sandwich sections when compared to uniform ones.

If both layers are plastified, then both variants of (35.35) hold simultaneously. Hence, subtracting these equations we arrive at a simpler system of equations

$$
P_{m n}=0, \quad P_{m}+P_{n}=1 ;
$$

this case coincides with the Ilyushin approximation (35.16).

Equations (35.34) or (35.35) were first derived by Yu. N. Rabotnov [7.272]; a more detailed analysis is given by A. Sawczuk and J. Rychlewski [7.298]. 
P. V. McLaughlin [7.186] considered moderately thick shells with the terms of order $H / R$ retained; his considerations were based on various yield conditions.

Employing the Tresca-Guest yield condition, we may simply substitute (34.2) into any one of the three equations of type (32.36); if fact, we assume individual layers of a sandwich structure to be in a purely membrane state. For example, substituting (34.2) into the first form of yielding of the layer $z=+H$

$$
\sigma_{\xi \xi}^{+} \sigma_{0}+\sigma_{\eta \eta}^{+} \sigma_{0}-\sigma_{\xi \xi}^{+} \sigma_{\eta \eta}^{+}+\left(\sigma_{\xi \eta}^{+}\right)^{2}-\sigma_{0}^{2}=0
$$

we obtain'

$$
n_{\xi \xi}+m_{\xi \xi}+n_{\eta \eta}+m_{\eta \eta}-\left(n_{\xi \xi}+m_{\xi \xi}\right)\left(n_{\eta \eta}+m_{\eta \eta}\right)+\left(n_{\xi \eta}+m_{\xi \eta}\right)^{2}=1 .
$$

Finally, the complete system of equations of the plastic interaction surface has the form

$$
\begin{aligned}
n_{\xi \xi} \pm m_{\xi \xi}+n_{\eta \eta} \pm m_{\eta \eta}-\left(n_{\xi \xi} \pm m_{\xi \xi}\right)\left(n_{\eta \eta} \pm m_{\eta \eta}^{-}\right)+\left(n_{\xi \eta} \pm m_{\xi \eta}\right)^{2} & =1, \\
-n_{\xi \xi} \mp m_{\xi \xi}-n_{\eta \eta} \mp m_{\eta \eta}-\left(n_{\xi \xi} \pm m_{\xi \xi}\right)\left(n_{\eta \eta} \pm m_{\eta \eta}\right)+\left(n_{\xi \eta} \pm m_{\xi \eta}\right)^{2} & =1, \\
\left(n_{\xi \xi} \pm m_{\xi \xi}\right)^{2}+\left(n_{\eta \eta} \pm m_{\eta \eta}\right)^{2}-2\left(n_{\xi \xi} \pm m_{\xi \xi}\right)\left(n_{\eta \eta} \pm m_{\eta \eta}\right)+4\left(n_{\xi \eta} \pm m_{\xi \eta}\right)^{2} & =1,
\end{aligned}
$$

where the upper signs refer to the plastification of the layer $z=+H$, and the lower signs - to the layer $z=-H$. If both layers are plastified, then a combination of one equation for $z=+H$ and one equation for $z=-H$ holds simultaneously.

Equations (35.39) are doubtlessly much more complicated than (35.35) and the application of the TG hypothesis to the general case under consideration seems useless.

Equations (35.39) describe also the plastic interaction surface for the Galileo condition of maximal normal stress: simply the two equations written in the last line should be omitted.

A generalization of the TG yield condition to anisosensitive materials was considered by M. Janas [7.111].

\subsection{Plastic anisotropy}

In the case $\mathscr{P}_{3} / \mathscr{S}_{6}$ under consideration the extension of analysis to anisotropic materials is also simpler if based on generalizations of the HMH yield condition. A generalization of the Ilyushin theory to orthotropic materials governed by the Hill yield condition was given by M. Sh. Mikeladze [7.191, 7.193, 7.195, 3.528], 1954. Under the usual assumption $\sigma_{z}=0$ we may write the Hill yield condition (12.3) in the form

$$
(G+H) \sigma_{\xi}^{2}-2 H \sigma_{\xi} \sigma_{\eta}^{2}+(H+F) \sigma_{\eta}^{2}+2 N \tau_{\xi \eta}^{2}=1
$$

or, introducing symbols of type (12.4),

$$
\frac{\sigma_{\xi}^{2}}{\sigma_{0 \xi}^{2}}-\left(\frac{\sigma_{0 \eta}^{2}}{\sigma_{0 \xi}}+\frac{\sigma_{0 \xi}}{\sigma_{0 \eta}}-\frac{\sigma_{0 \xi} \sigma_{0 \eta}^{7}}{\sigma_{0 z}^{2}}\right) \frac{\sigma_{\xi}}{\sigma_{0 \xi}} \frac{\sigma_{\eta}}{\sigma_{0 \eta}}+\frac{\sigma_{\eta}^{2}}{\sigma_{0 \eta}^{2}}+\frac{\tau_{\xi \eta}^{2}}{\tau_{0 \xi \eta}^{2}}=1 .
$$

The constitutive equations have been assumed in the form associated with (35.41). Mikeladze investigated in detail the "simpler combined cases", in which the equa- 
tion of the plastic interaction surface may be reduced to (35.15). Such a possibility occurs in four cases:

$$
\begin{aligned}
& (H+F) \lambda_{\xi}+H \lambda_{\eta}=x_{\eta}=\lambda_{\xi \eta}=0, \text { hence } N_{\xi}=N_{\xi \eta}=M_{\eta}=0, \\
& P_{n}=\frac{H+F}{4 h^{2}} N_{\eta}^{2}, \quad P_{m}=\frac{G H+H F+F G}{(H+F) h^{4}} M_{\xi}^{2}+\frac{2 N}{h^{4}} M_{\xi \eta}^{2} ; \\
& (H+F) \lambda_{\xi}+H \lambda_{\eta}=x_{\eta}=x_{\xi \eta}=0, \text { hence } N_{\eta}=M_{\xi \eta}=M_{\eta}=0, \\
& P_{n}=\frac{H+F}{4 h^{2}} N_{\xi}^{2}+\frac{N}{2 h^{2}} N_{\xi \eta}^{2}, \quad P_{m}=\frac{G H+H F+F G}{(H+F) h^{4}} M_{\xi}^{2} ; \\
& (H+F) x_{\xi}+H x_{\eta}=\lambda_{\eta}=x_{\xi \eta}=0, \text { hence } N_{\eta}=M_{\xi \eta}=M_{\xi}=0, \\
& P_{n}=\frac{G H+H F+F G}{4(H+F) h^{2}} N_{\xi}^{2}+\frac{N}{2 h^{2}} N_{\xi \eta}^{2}, \quad P_{m}=\frac{H+F}{h^{4}} M_{\eta}^{2} ; \\
& (H+F) x_{\xi}+H x_{\eta}=\lambda_{\eta}=\lambda_{\xi \eta}=0, \quad \text { hence } N_{\eta}=N_{\xi \eta}=M_{\xi}=0, \\
& P_{n}=\frac{G H+H F+F G}{4(H+F) h^{2}} N_{\xi}^{2}, \quad P_{m}=\frac{H+F}{h^{4}} M_{\eta}^{2}+\frac{2 N}{h^{4}} M_{\xi \eta}^{2} .
\end{aligned}
$$

In all these cases the quadratic forms $P_{n}$ and $P_{m}$ defined above satisfy the parametrical equations of the plastic interaction curve (surface), (35.15). The symbol $h$ denotes here half of the shell thickness, so as to retain the symbol $H$ for the plastic modulus. Further, $N$ denotes a plastic modulus and $N_{\alpha \beta}$ the membrane forces, so, in spite of the similarity of symbols, there is no fear of a misunderstanding.

Equation (35.41) is subject to essential simplification if the coefficient in the bracket is equal to unity. This particular case of the Hill yield condition was verified experimentally by L. W. Hu and J. Marin [3.272]; most of Mikeladze's book [3.528] is devoted to this case. In [7.192] Mikeladze considered multilayar sections, in [7.194]-sandwich sections, in [7.196, 7.197]-optimal design of orthotropic shells with respect to their limit carrying capacity, and in [7.199]-a simplified technical theory of anisotropic plastic shells.

A generalization of the Mikeladze theory to an arbitrary anisotropy, described by the Mises yield condition (12.1), is due to A. Sawczuk [7.297, 7.300], who considered both uniform and sandwich cross-sections. 


\section{CHAPTER VIII}

\section{Combined loadings at the level 93 of the body as a whole}

\section{Combined loadings of bars and bar systems}

\subsection{Introductory remarks}

In contradistinction to the analysis of combined loadings at the levels $\mathscr{P}$ and $\mathscr{S}$, where the number of exertion factors was to a certain degree limited, at the level $\mathscr{B}$ that number may be arbitrarily large. As was mentioned in Sec. 1.5, the process may be controlled here not only by $n$ independent external (surface) forces $P_{i}$, and their positions if they are movable or without a uniquely specified point of application, but also by displacements, parameters responsible for body forces, parameters characterizing the temperature distribution, intensity of irradiation, etc.

There exist of course very many solutions to problems of elastic-plastic or rigidplastic structures under a system of forces. However, according to the definition adopted in Sec. 4.1, we do not assign these solutions to combined loadings if all the forces increase proportionally to each other and the factors of proportionality are fixed a priori (Fig. 9b). The essential feature of combined loadings is the independence of individual loading parameters (Fig. 9a), and the corresponding construction of limit surfaces (interaction surfaces) in appropriate spaces.

The general theory of limit surfaces at the level $\mathscr{B}$ is much less developed that at the levels $\mathscr{P}$ and $\mathscr{S}$. P.G. Hodge and C. K. Sun [4.225] investigated plastic interaction surfaces under the assumption of a rigid-perfectly-plastic material; in that case the plastic interaction surface describes the initiation of deformation of the body and hence neglecting geometry changes is justified. Further, Drucker's postulate was assumed and exertion factors were restricted to external forces (basic exertion factors). Under these assumptions it was proved that the plastic interaction surfaces are convex and that the vector of plastic flow $\delta u_{i}$, defined by the equation of virtual work (1.1), is normal to such a surface.

As a simple example, consider the homogeneous statically indeterminate bar with the cross-sectional area $A$ shown in Fig. 175. The bar is subject to two inde- 


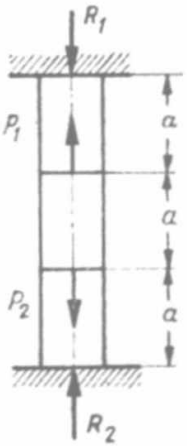

Fig. 175. A statically indeterminate bar under two independent forces

pendent external forces $P_{1}$ and $P_{2}$, positive if their senses coincide with those shown in Fig. 175. We derive the equations of the elastic interaction curve and of the plastic interaction curve; in the case of a perfectly rigid-plastic material the elastic inter action curve does not occur and should be omitted, whereas the plastic interaction curve does not change (since in the example under consideration the geometry changes are neglected). Introduction of elastic properties makes it also possible to discuss the problem of shakedown under variable forces $P_{1}=P_{1}(t)$ and $P_{1}=P_{2}(t)$

In the elastic range the reactive forces are

$$
R_{1}=\frac{2}{3} P_{1}-\frac{1}{3} P_{2}, \quad R_{2}=\frac{2}{3} P_{2}-\frac{1}{3} P_{1} .
$$

Calculating longitudinal forces in particular intervals and equating them to $\pm \sigma_{0} A$, we obtain the equations of six straight lines, determining the elastic interaction curve in the plane $P_{1}-P_{2}$ (Fig. 176):

$$
2 P_{1}-P_{2}= \pm 3 \sigma_{0} A, \quad P_{1}+P_{2}= \pm 3 \sigma_{0} A, \quad 2 P_{2}-P_{1}= \pm 3 \sigma_{0} A .
$$

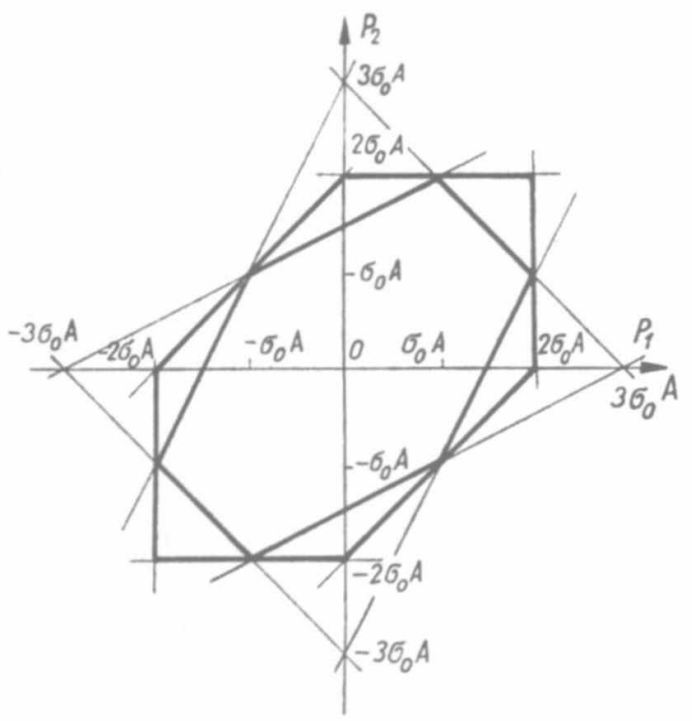

Fig. 176. Elastic and plastic interaction curves for the bar shown in Fig. 175. 
The limit carrying capacity is exhausted if one interval is plastified by tension and a certain other interval-by compression. The relevant conditions determine the reactive forces and the relations between $P_{1}$ and $P_{2}$, i.e. the plastic interaction curve. Six various combinations of yielding result in six equations

$$
P_{1}= \pm 2 \sigma_{0} A, \quad P_{2}= \pm 2 \sigma_{0} A, \quad P_{1}-P_{2}= \pm 2 \sigma_{0} A \text {. }
$$

The plastic interaction curve is also shown in Fig. 176. It may be noted that this curve is analogous to a graphical interpretation of the Tresca-Guest yield condition, whereas the elastic interaction curve corresponds to the Schmidt-IshlinskyHill yield condition.

Both curves shown in Fig. 176 are convex and it is easy to check the normality of the vector of plastic flow to the plastic interaction curve. The virtual work may be expressed by external forces as follows:

$$
\delta W=P_{1} \delta u_{1}+P_{2} \delta u_{2}
$$

where $u_{1}$ and $u_{2}$ denote axial displacements under the forces, positive if their senses coincide with the senses of $P_{1}$ and $P_{2}$ shown in Fig. 175. Hence $u_{1}$ and $u_{2}$ are generalized displacements consistent with the forces $P_{1}$ and $P_{2}$. If only the upper two intervals are plastic, then $u_{2}=0, \delta u_{1}>0$ for plastification of the upper interval by compression, and the vector $\delta \mathrm{u}$ in normal to the side $P_{1}=2 \sigma_{0} A$ of the hexagon (for an elastic-perfectly plastic material we have to write $u_{2}^{p}=0$ instead of $u_{2}=0$ ). If the upper interval is plastified by compression and the lower one by tension, then $\delta u_{1}=-\delta u_{2}$ and the vector $\delta \mathrm{u}$ is normal to the side $P_{1}-P_{2}=2 \sigma_{0} A$. Similar arguments hold for other combinations of yielding.

An arbitrary program of loading $P_{1}=P_{1}(t)$ and $P_{2}=P_{2}(t)$ lying inside the elastic curve corresponds, of course, to the plastic shakedown of the structure, since in that case plastic deformations will not occur at all. Moreover, according to Melan's theorem, shakedown will take place if the program lies inside any fixed modified elastic curve constructed for an arbitrary system of self-stresses superposed.

In the case under consideration such a system of self-stresses may be interpreted as due to heating or cooling of the whole bar; we then have simply $\sigma=\sigma_{s}=$ const,

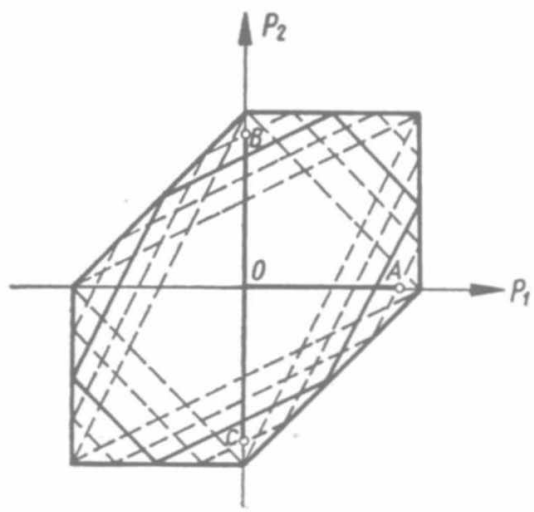

Fig. 177. A family of shakedown domains as determined by Melan's theorem 
the same in all intervals. Equating in particular intervals, the stresses with superposed $\sigma_{s}$ to $\pm \sigma_{0}$, we obtain the following equations of a one-parameter family of modified elastic curves:

$$
\begin{aligned}
2 P_{1}-P_{2} & = \pm 3 \sigma_{0} A+3 \sigma_{s} A, \\
P_{1}+P_{2} & = \pm 3 \sigma_{0} A-3 \sigma_{s} A, \\
2 P_{2}-P_{1} & = \pm 3 \sigma_{0} A+3 \sigma_{s} A .
\end{aligned}
$$

They are shown in Fig. 177. In the limit case the hexagons turn into triangles (further increase of $\left|\sigma_{s}\right|$ would only diminish those triangles).

An arbitrary program of loading lying inside any fixed modified elastic curve corresponds also to plastic shakedown of the structure. For example, the cyclic program $0 A 0 B 0 \ldots$ lies inside one of the triangles and will result in shakedown. On the other hand, the cyclic program $0 A 0 C 0 \ldots$ does not lie inside any of the modified elastic curves, and in this case shakedown will not take place; it may be shown by simple calculations that plastic strains will change in every cycle and that the dissipated energy increases infinitely.

For some applications it is more convenient to know the rectangular shakedown domains. They are smaller than the domains bounded by modified elastic curves, but may be derived from those curves. For example, consider the rectangles $0 \leqslant P_{1} \leqslant P_{1}^{+}, 0 \leqslant P_{2} \leqslant P_{2}^{+}$; they will be determined by the function $P_{2}^{+}=f\left(P_{1}^{+}\right)$. First suppose $P_{2}^{+}>P_{1}^{+}$; then for a given $P_{2}^{+}$we may find from Fig. 177 the maximal value of $P_{1}^{+}$so as to obtain the rectangle inscribed in the hexagon. The point $P_{1}=0$, $P_{2}=P_{2}^{+}$determines $\sigma_{s}$ in the last equation (36.5), and for this $\sigma_{s}$ and $P_{2}=P_{2}^{+}$ we find $P_{1}^{+}$from the second equation (36.5). Finally, one obtains

$$
P_{2}^{+}=2 \sigma_{0} A-\frac{1}{3} P_{1}^{+} \text {. }
$$

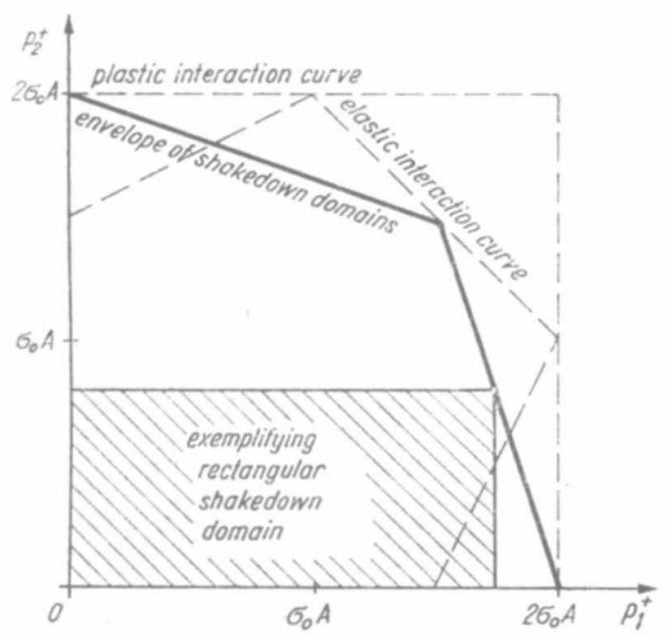

Fig. 178. Envelope of rectangular shakedown domains 
A similar equation with interchanged subscripts holds for $P_{1}^{+}>P_{2}^{+}$. The result is shown in Fig. 178: any rectangle inscribed in the tetragon is a shakedown domain. Larger rectangles may result in non-shakedown due to alternating plasticity.

Let us also mention the paper by G. Backhaus [8.25], discussing the engineering approach to plastic design under constant or variable combined loadings.

Exceeding the Hodge-Sun assumptions, we may arrive at different conclusions: in particular, the surfaces may show concavities. For example, if the material does not conform to Drucker's postulate, then the surfaces may be concave even at the level $\mathscr{P}$, and a homogeneous transition to the levels $\mathscr{S}$ and $\mathscr{B}$ preserves these concavities. However, in most cases the concavities are due to the effect of geometry changes, and this effect will be discussed separately in Sec. 36.2.

Concavities may also occur if we take into consideration accessory exertion factors at the level $\mathscr{B}$. Consider, for example, the eccentrically compressed column shown in Fig. 179, and regard as exertion factors the force $P$ and the eccentricity $e$.

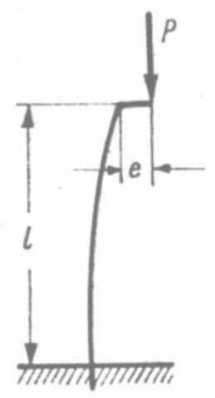

Fig. 179. Eccentricity of loading as an exertion factor

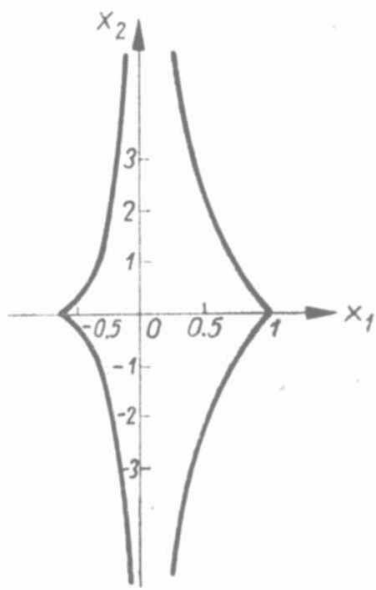

Fig. 180. Elastic interaction curve for the bar shown in Fig. 179 with variable eccentricity of loading 
Figure 180, quoted after [1.26], shows the corresponding elastic interaction curve. The dimensionless exertion factors are defined here as follows:

$$
x_{1}=\frac{1}{\sigma_{0} A} P, \quad x_{2}=\frac{A}{W_{b}} e,
$$

where $W_{b}$ denotes the elastic section modulus for bending. This curve shows concavities both for tensile and for compressive forces $P$. The geometry changes have been taken into account but even if they were neglected, the concavities would remain.

Eccentric compression is formally equivalent to the loading by a force $P$ and a moment $M=P e$. However, the limit curve in the plane $P-M$ is different from that shown in Fig. 180, namely, it may be obtained by transformation $P=P$, $M=P e$. The use of this or that interaction curve should be based on a careful checking which factors are really independent in the case under consideration. Even if the values of $P$ and $e$, or $P$ and $M=P e$ are given, the proper estimation of the probability of a collapse of the structure depends essentially on the appropriate choice of independent quantities.

Concavities of limit surfaces at the level $\mathscr{B}$ may also be encountered in analysing the surfaces of decohesive carrying capacity (cf. Secs. 39 and 40), or even in the case of elastic interaction surfaces if thermal loadings are analysed and the material constants depend on temperature.

Because of the large variety of problems of combined loadings at the level $\mathscr{B}$, the solutions obtained in the literature will by reviewed here rather briefly. More attention will only be paid to thick-walled cylinders, where the introduction of the level $\mathscr{S}$ may be difficult, and that is why they were not discussed in preceding chapters.

\subsection{Effect of geometry changes}

Though the effect of geometry changes may be observed at the levels $\mathscr{P}$ (finite strains) and $\mathscr{S}$ (highly deformable sections), it is at the level $\mathscr{B}$ that they are most visible, even within the small-strain theory. The analysis of geometric effects in the theory of plasticity presents difficulties in the general case of a continuum, but many qualitative conclusions may be obtained from the study of systems with a finite number of degrees of freedom. Such considerations are due to G. Maier and D. C. Drucker [4.354]; we shall briefly present here their approach and results which apply directly to pin-joined trusses and to finite element models of a continuum.

The equations given in Sec. 21.4 will now be rewritten in a somewhat more general form, allowing for geometry changes. Since the present considerations are not necessarily connected with the finite element method, the notation will also be slightly changed to make it compatible with [4.354]. Denote by $\mathbf{P}$ the vector of generalized external forces, by $\mathbf{u}$ the vector of generalized displacements, by $\boldsymbol{\sigma}$ the vector of stresses (or generalized stresses), and by $\epsilon$ the vector of strains (or generalized strains), consistent in the sense of the principle of virtual work:

$$
\delta W=\boldsymbol{\sigma}^{T} \delta \boldsymbol{\epsilon}=\mathbf{P}^{T} \delta \mathbf{u} .
$$


Because of the geometric effects the incremental form (21.18) will serve as the basic form of compatibility equations:

$$
\delta \boldsymbol{\epsilon}=\mathbf{B} \delta \mathbf{u} .
$$

Now the compatibility matrix $\mathbf{B}$ depends on the displacements $\mathbf{u}$ (or on the strains $\boldsymbol{\epsilon}$ ). The equilibrium equations (21.24) hold,

$$
\mathbf{P}=\mathbf{B}^{T} \boldsymbol{\sigma},
$$

and their incremental form is as follows:

$$
\delta \mathbf{P}=\mathbf{B}^{T} \delta \boldsymbol{\sigma}+\delta \mathbf{B}^{T} \boldsymbol{\sigma} .
$$

The matrix $\delta \mathbf{B}^{T}$ depends linearly on $\delta \mathbf{u}$, and hence we may write

$$
\delta \mathbf{B}^{T} \boldsymbol{\sigma}=\mathbf{G} \delta \mathbf{u},
$$

where the $n \times n$ matrix $\mathbf{G}$ is called the geometric stiffness matrix; it depends linearly on $\boldsymbol{\sigma}$ and non-linearly on $\mathbf{u}$. Maier and Drucker proved that $\mathbf{G}$ is symmetric. Substitution of (36.12) into (36.11) gives the equation of equilibrium in incremental form,

$$
\delta \mathbf{P}=\mathbf{B}^{T} \delta \boldsymbol{\sigma}+\mathbf{G} \delta \mathbf{u} .
$$

Making use (36.13) and of the incremental constitutive relations

$$
\delta \boldsymbol{\epsilon}=\mathbf{L} \delta \boldsymbol{\sigma}+\mathbf{M} \delta \boldsymbol{\lambda},
$$

Maier and Drucker investigated the essential features of the system, namely the normality, uniqueness, stability and convexity of modified elastic surfaces. They conclude that normality carries over from the level $\mathscr{P}$ to the level $\mathscr{B}$, but convexity, in general does not; stability carries over to the system if the matrix $\mathbf{G}$ is positive semidefinite (this condition is sufficient but not necessary). Instability due to softening was analysed by G. Maier, A. Zavelani and J. C. Dotreppe [4.353].

\subsection{Interaction curves (limit curves) for simple models allowing for geometry changes}

As an example illustrating the Maier-Drucker theory consider the column shown in Fig. 181 (K. Kowalczyk [8.263, 8.265]). It consists of one short deformable element and of one long rigid element, and is subject to two independent forces $P_{1}$ and $P_{2}$; then $U_{1}$ and $U_{2}$ are the generalized displacements consistent with $P_{1}$ and $P_{2}$, respectively (small letters are reserved for dimensionless quantities). A similar model was used by C. Calladine [8.64] and by M. Życzkowski and A. Zaborski [8.578] to the analysis of creep buckling; however, the present model is more general because of the initial curvature of the deformable part. In view of the small length of that part, the curvature is assumed to be constant along the length: it is equal to $1 / R$ before deformation and 1/ $\varrho$ after deformation. The model under consideration is relatively simple but involves non-homogeneous stress distribution. 


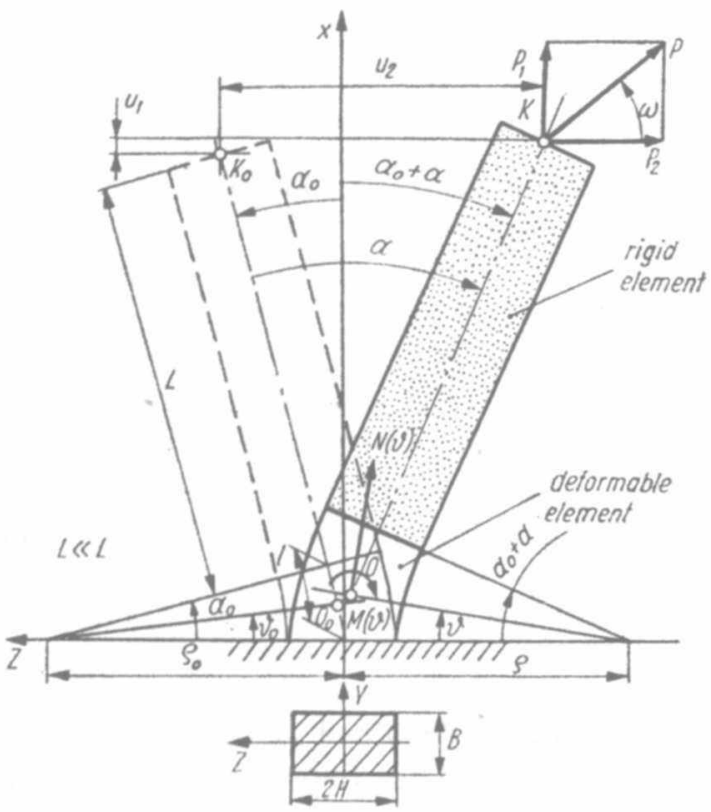

Fig. 181. Model of a column with initial curvature

To define consistent generalized stresses $\boldsymbol{\sigma}$ and generalized strains $\boldsymbol{\epsilon}$ we assume a uniaxial stress state and consider the virtual work

$$
\delta W=\iiint_{V_{0}} \sigma \delta \varepsilon d V_{0}
$$

where $\sigma$ and $\varepsilon$ are the axial (or, more exactly, the circumferential in cylindrical coordinates) stresses and strains respectively, and $V_{0}$ denotes the volume of the deformable part of the column. The Bernoulli hypothesis of plane sections applied to a curved bar results in a hyperbolic strain distribution of the type (32.4), namely

$$
\varepsilon(Z)=\frac{1}{1+Z / R}\left(\varepsilon_{0}+x Z\right)
$$

where, according to (32.7) and (32.8), $\varkappa$ determines the unit angle increment

$$
x=\frac{1+\varepsilon_{0}}{\varrho}-\frac{1}{R}=\frac{\alpha}{l},
$$

$l=R \alpha_{0}$ denotes the initial length of the deformable part of the column, and $\varepsilon_{0}$ is the axial extension. Substituting (36.16) and $d V_{0}=(R+Z) d \vartheta_{0}$ into (36.15) and making use of the definitions of the bending moment $M(\vartheta)$ and of the normal force $N(\vartheta)$, we obtain

$$
\delta W=\int_{0}^{\alpha_{0}} R\left[N(\vartheta) \delta \varepsilon_{0}+M(\vartheta) \delta \varkappa\right] d \vartheta_{0} .
$$


Finally, substituting

$$
d \vartheta_{0}=\frac{\alpha_{0}}{\alpha_{0}+\alpha} d \vartheta
$$

(the sense of $\alpha$ is assumed to be opposite to that of $\alpha_{0}$, Fig. 181), and performing integration in the deformed state, we may write

$$
\delta W=\left(N \delta \varepsilon_{0}+M \delta x\right) l,
$$

where $N$ and $M$ are defined by the formulae

$$
\begin{aligned}
& N=\frac{1}{\alpha_{0}+\alpha} \int_{0}^{\alpha_{0}+\alpha} N(\vartheta) d \vartheta, \\
& M=\frac{1}{\alpha_{0}+\alpha} \int_{0}^{\alpha_{0}+\alpha} M(\vartheta) d \vartheta,
\end{aligned}
$$

and may be interpreted as the mean values of $N(\vartheta)$ and $M(\vartheta)$.

In order to introduce dimensionless quantities (more convenient in further calculation) which would also be consistent in the sense of virtual work, we restrict our considerations to the rectangular cross-section of the deformable part $\mathrm{B} \times 2 \mathrm{H}$ and present the virtual work in the form

$$
\delta W=\sigma_{0} A l\left(p_{1} \delta u_{1}+p_{2} \delta u_{2}\right)=\sigma_{0} A l\left(n \delta e_{0}+m \delta k\right),
$$

where

$$
\begin{array}{ll}
p_{1}=\frac{1}{\sigma_{0} A} P_{1}, & p_{2}=\frac{3 L}{\sigma_{0} A H} P_{2}, \\
u_{1}=\frac{1}{l} U_{1}, & u_{2}=\frac{H}{3 L l} U_{2}, \\
n=\frac{1}{E A} N, & m=\frac{1}{E A H} M, \\
e_{0}=\frac{E}{\sigma_{0}} \varepsilon_{0}, & k=\frac{E H}{\sigma_{0}} x,
\end{array}
$$

$A=2 B H$ denotes the cross-sectional area and $L$-the length of the rigid part of the column. The dimensionless forces $p_{1}$ and $p_{2}$ are determined in such a way as to obtain $p_{1}=1$ and $p_{2}=1$ for the elastic carrying capacity under pure action of the corresponding force only; similar definitions are introduced for $e_{0}$ and $k$, whereas the remaining definitions follow from (36.22). Finally, the vectors introduced in Sec. 36.2 take here the form

$$
\mathbf{P}=\left\{\begin{array}{l}
p_{1} \\
p_{2}
\end{array}\right\}, \quad \mathbf{u}=\left\{\begin{array}{l}
u_{1} \\
u_{2}
\end{array}\right\}, \quad \boldsymbol{\sigma}=\left\{\begin{array}{l}
n \\
m
\end{array}\right\}, \quad \boldsymbol{\epsilon}=\left\{\begin{array}{l}
e_{0} \\
k
\end{array}\right\} .
$$


Let us now derive the geometric relations (compatibility conditions). Generalized displacements may be expressed as follows:

$$
\begin{aligned}
& U_{1}=L\left[\cos \left(\alpha_{0}+\alpha\right)-\cos \alpha_{0}\right]+\varrho \sin \left(\alpha_{0}+\alpha\right)-R \sin \alpha_{0}, \\
& U_{2}=L\left[\sin \left(\alpha_{0}+\alpha\right)-\sin \alpha_{0}\right]+\varrho\left[1-\cos \left(\alpha_{0}+\alpha\right)\right]-R\left(1-\cos \alpha_{0}\right) .
\end{aligned}
$$

Introducing dimensionless displacements (36.24), strains (36.26), and dimensionless material and geometric parameters

$$
\mu=\frac{\sigma_{0}}{E}, \quad \eta=\frac{l}{H}, \quad \varphi=\frac{L}{H},
$$

we rewrite $(36.28)$ in the form

$$
\begin{aligned}
u_{1}= & \frac{1+\mu e_{0}}{\alpha_{0}+\mu \eta k} \sin \left(\alpha_{0}+\mu \eta k\right)-\frac{2 \varphi}{\eta} \sin \left(\alpha_{0}+\frac{\mu \eta k}{2}\right) \sin \frac{\mu \eta k}{2}-\frac{\sin \alpha_{0}}{\alpha_{0}}, \\
u_{2}= & \frac{1+\mu e_{0}}{3 \varphi\left(\alpha_{0}+\mu \eta k\right)}\left[1-\cos \left(\alpha_{0}+\mu \eta k\right)\right]+ \\
& +\frac{2}{3 \eta} \cos \left(\alpha_{0}+\frac{\mu \eta k}{2}\right) \sin \frac{\mu \eta k}{2}-\frac{1-\cos \alpha_{0}}{3 \varphi \alpha_{0}} .
\end{aligned}
$$

Differentiating (36.30) with respect to $e_{0}$ and $k$ and inverting the relations obtained, we arrive at the incremental form of compatibility conditions (36.9), with the following components of the matrix $\mathbf{B}$

$$
\begin{aligned}
B_{11}=\frac{1}{C}\{ & \varphi\left(\alpha_{0}+\mu \eta k\right)^{2} \cos \left(\alpha_{0}+\mu \eta k\right)+\eta\left(1+\mu e_{0}\right)\left[\left(\alpha_{0}+\mu \eta k\right) \sin \left(\alpha_{0}+\mu \eta k\right)+\right. \\
& \left.\left.\quad+\cos \left(\alpha_{0}+\mu \eta k\right)-1\right]\right\}, \\
B_{12}=\frac{3 \varphi}{C}\left\{\varphi\left(\alpha_{0}+\mu \eta k\right)^{2} \sin \left(\alpha_{0}+\mu \eta k\right)+\right. & \\
& \left.\quad+\eta\left(1+\mu e_{0}\right)\left[\sin \left(\alpha_{0}+\mu \eta k\right)-\left(\alpha_{0}+\mu \eta k\right) \cos \left(\alpha_{0}+\mu \eta k\right)\right]\right\}, \\
B_{21}= & -\frac{2}{C}\left(\alpha_{0}+\mu \eta k\right) \sin ^{2} \frac{\alpha_{0}+\mu \eta k}{2}, \\
B_{22}= & \frac{6 \varphi}{C}\left(\alpha_{0}+\mu \eta k\right) \sin \frac{\alpha_{0}+\mu \eta k}{2} \cos \frac{\alpha_{0}+\mu \eta k}{2},
\end{aligned}
$$

where

$$
C=2 \mu \sin \frac{\alpha_{0}+\mu \eta k}{2}\left[\varphi\left(\alpha_{0}+\mu \eta k\right) \cos \frac{\alpha_{0}+\mu \eta k}{2}+\eta\left(1+\mu e_{0}\right) \sin \frac{\alpha_{0}+\mu \eta k}{2}\right] .
$$

To derive the equilibrium equations we first calculate the functions $N(\vartheta)$ and $M(\vartheta)$,

$$
\begin{aligned}
N(\vartheta)= & P_{1} \cos \vartheta+P_{2} \sin \vartheta \\
M(\vartheta)= & -P_{1} L \sin \left(\alpha_{0}+\alpha\right)-P_{1} \varrho\left[\cos \vartheta-\cos \left(\alpha_{0}+\alpha\right)\right]+ \\
& +P_{2} L \cos \left(\alpha_{0}+\alpha\right)+P_{2} \varrho\left[\sin \left(\alpha_{0}+\alpha\right)-\sin \vartheta\right]
\end{aligned}
$$


Making use of the definitions (36.21) and introducing dimensionless quantities, we obtain

$$
\begin{aligned}
& n=\frac{\mu \sin \left(\alpha_{0}+\mu \eta k\right)}{\alpha_{0}+\mu \eta k} p_{1}+\frac{\mu\left[1-\cos \left(\alpha_{0}+\mu \eta k\right)\right]}{3 \varphi\left(\alpha_{0}+\mu \eta k\right)} p_{2}, \\
& m=-\mu\left\{\varphi \sin \left(\alpha_{0}+\mu \eta k\right)-\frac{\eta\left(1+\mu e_{0}\right)}{\alpha_{0}+\mu \eta k}\left[\cos \left(\alpha_{0}+\mu \eta k\right)-\right.\right. \\
& \left.\left.\quad-\frac{\sin \left(\alpha_{0}+\mu \eta k\right)}{\alpha_{0}+\mu \eta k}\right]\right\} p_{1}+\frac{\mu}{3 \varphi}\left\{\varphi \cos \left(\alpha_{0}+\mu \eta k\right)+\right. \\
& \left.\quad+\frac{\eta\left(1+\mu e_{0}\right)}{\alpha_{0}+\mu \eta k}\left[\sin \left(\alpha_{0}+\mu \eta k\right)-\frac{1-\cos \left(\alpha_{0}+\mu \eta k\right)}{\alpha_{0}+\mu \eta k}\right]\right\} p_{2} .
\end{aligned}
$$

Solving (36.34) with respect to $p_{1}$ and $p_{2}$, we arrive at the form (36.10) and prove that these relations are also expressed by the components $B_{i j}$, (36.31).

The results we have hitherto obtained hold irrespective of the constitutive equations adopted. Now we specify the material as subject to linear plastic hardening (8.3). Introducing dimensionless strains and dimensionless stresses

$$
e=\frac{E}{\sigma_{0}} \varepsilon, \quad s=\frac{1}{\sigma_{0}} \sigma,
$$

and the Ilyushin hardening parameter $\lambda$, (8.5), we may write

$$
s=\left\{\begin{array}{cc}
-\lambda+(1-\lambda) e & e \leqslant-1, \\
e, & -1 \leqslant e \leqslant 1, \\
\lambda+(1-\lambda) e, & e \geqslant 1 .
\end{array}\right.
$$

In what follows we neglect the hyperbolic term in the strain distribution and substitute $e=e_{0}+k z$, where $z=Z / H$ and $-1 \leqslant z \leqslant 1$. Integrating the stresses over the cross-sectional area, we obtain the following constitutive equations for the level $\mathscr{S}$ : elastic range

$$
n=\mu e_{0}, \quad m=\frac{\mu}{3} k
$$

one-side plastification $z>z_{+}$

$$
\begin{aligned}
& n=\mu\left[e_{0}-\frac{\lambda}{4 k}\left(e_{0}+k-1\right)^{2}\right], \\
& m=\frac{\mu}{6}\left\{\frac{\lambda}{2}\left(1-e_{0}\right)\left[3-\left(\frac{1-e_{0}}{k}\right)^{2}\right]+k(2-\lambda)\right\} ;
\end{aligned}
$$

one-side plastification $z<z_{-}$

$$
\begin{aligned}
& n=\mu\left[e_{0}+\frac{\lambda}{4 k}\left(e_{0}-k+1\right)^{2}\right], \\
& m=\frac{\mu}{6}\left\{\frac{\lambda}{2}\left(1+e_{0}\right)\left[3-\left(\frac{1+e_{0}}{k}\right)^{2}\right]+k(2-\lambda)\right\} ;
\end{aligned}
$$


two-side plastification $z<z_{-}$and $z>z_{+}$

$$
\begin{aligned}
& n=\mu\left(1-\lambda+\frac{\lambda}{k}\right) e_{0}, \\
& m=\frac{\mu}{3}\left[\frac{\lambda}{2}\left(3-\frac{1+3 e_{0}^{2}}{k^{2}}\right)+k(1-\lambda)\right],
\end{aligned}
$$

where

$$
z_{+}=\frac{1-e_{0}}{k}, \quad z_{-}=-\frac{1+e_{0}}{k} .
$$

Equations $(36.38) \div(36.40)$ hold only for positive bending moments, but the equations for negative moments are quite analogous.

We begin the discussion of interaction curves in the plane $p_{1}-p_{2}$ with the initial neutral curves (elastic curves). They are determined by the conditions

$$
s( \pm 1)=e_{0} \pm k= \pm 1 \text {. }
$$

Substituting (36.37) into (36.42) and then into (36.34), we obtain equations of the type $f\left(p_{1}, p_{2} ; \alpha_{0} ; \mu, \eta, \varphi\right)=0$ (or their counterparts in parametrical form). The

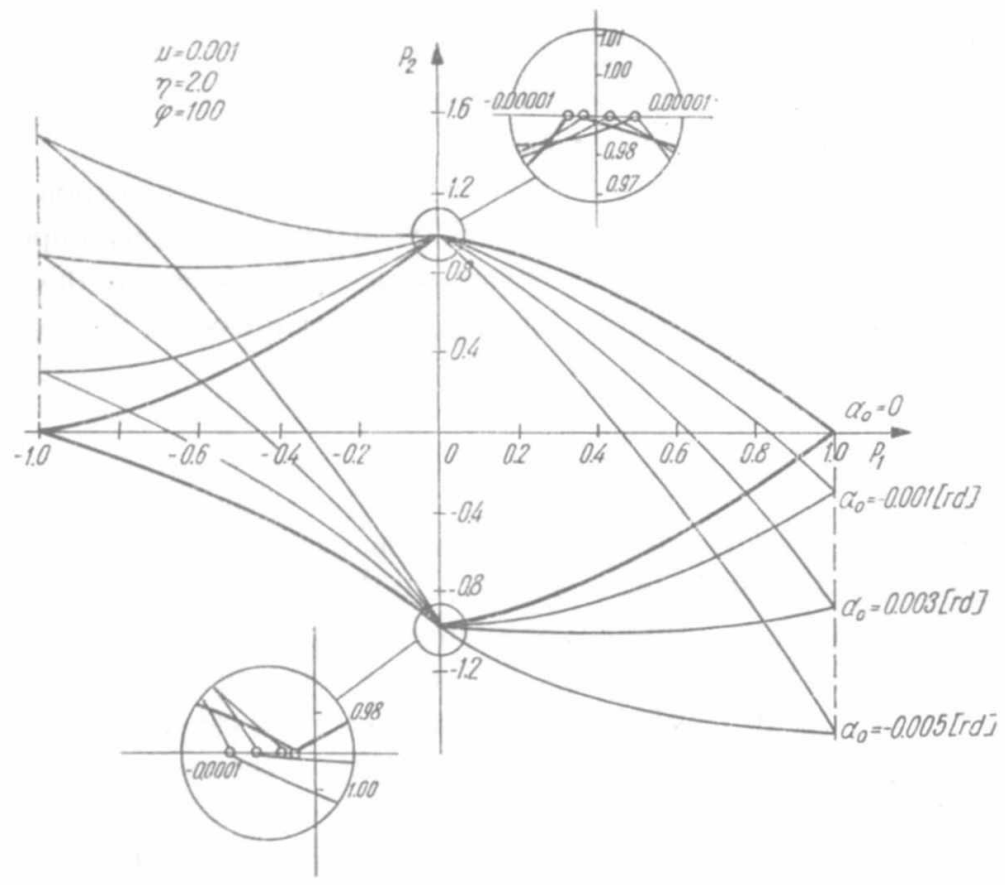

Fig. 182. Elastic interaction curves for the model shown in Fig. 181 (small slenderness)

initial neutral curves are curvilinear tetragons with two convex and two concave sides. Figure 182 shows these tetragons for relatively small slenderness ratio $\varphi=100$. Greater slenderness results in elastic buckling and then the point $p_{1}=-1$ practi- 


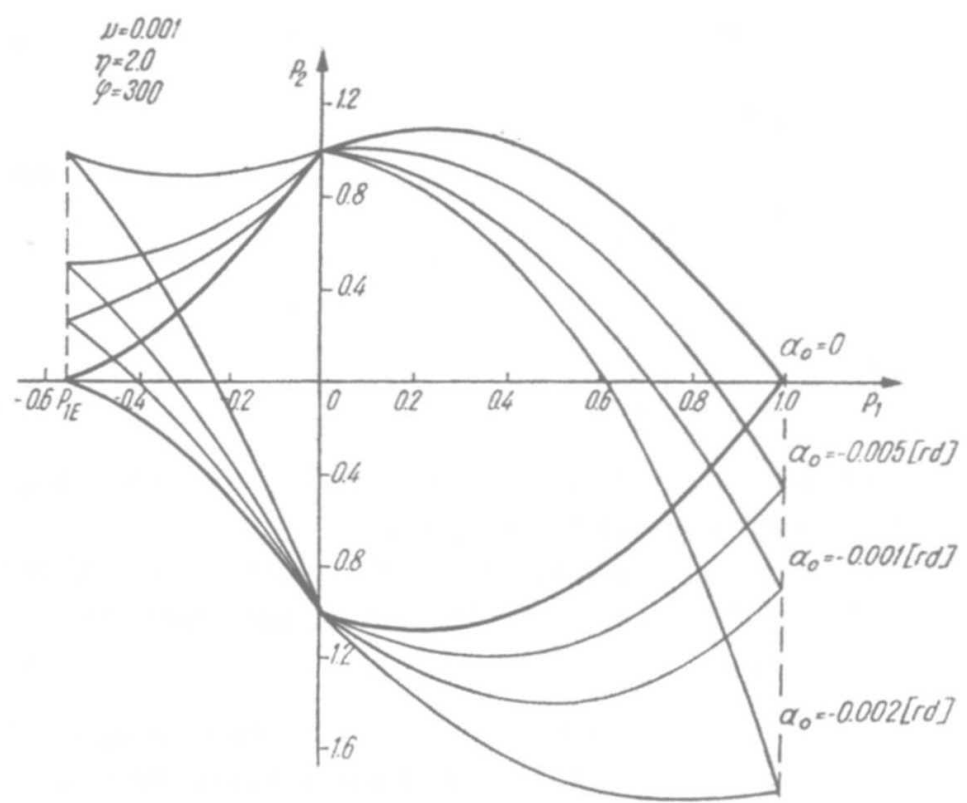

Fig. 183. Elastic interaction curves for the model shown in Fig. 181 (large slenderness)

cally cannot be reached. Figure 183 shows the elastic curves for $\varphi=300$; then the corresponding Euler force for a straight bar is $p_{1 E}=-0.56$.

The curves separating one-side plastification and two-side plastification result from the same condition (36.42) applied to (36.38) or (36.39). A very important limit curve corresponds to the maximal carrying capacity. To determine this curve we confine our considerations to simple loading processes, introduce the auxiliary parameter $\omega$,

$$
\omega=\arctan \frac{P_{1}}{P_{2}}=\arctan \frac{3 \varphi p_{1}}{p_{2}},
$$

express $p_{1}$ in terms of $p_{2}$ and $\omega$, and require the maximal value of $p_{2}$ at a constant $\omega$ (simple loading), $d\left|p_{2}\right| / d k=0$. In the case of $p_{2}=0$ the relevant maximal carrying capacity may be evaluated by a limiting procedure. Beyond the maximal carrying capacity the process may be continued (e.g. if controlled by displacements) but is no longer stable. On the side of tensile $P_{1}$ the maximal carrying capacity, in general, does not exist (within the framework of the small-strain theory adopted here). Hence, in order to obtain certain bounds to the solution, a simple physical criterion of decohesion (18.64) has been introduced with $\hat{e}=20$, i.e. $\hat{\varepsilon}=20 \sigma_{0} / E$. All the limit curves are shown in Fig. 184, together with the lines $z_{+}=$const and $z_{-}$ $=$ const.

Paper [8.264] by K. Kowalczyk and M. Życzkowski analyses modified elastic curves (shakedown domains) for the model under consideration. Some results are also shown in Fig. 184, namely the regions in which these curves are triangles, tetra- 


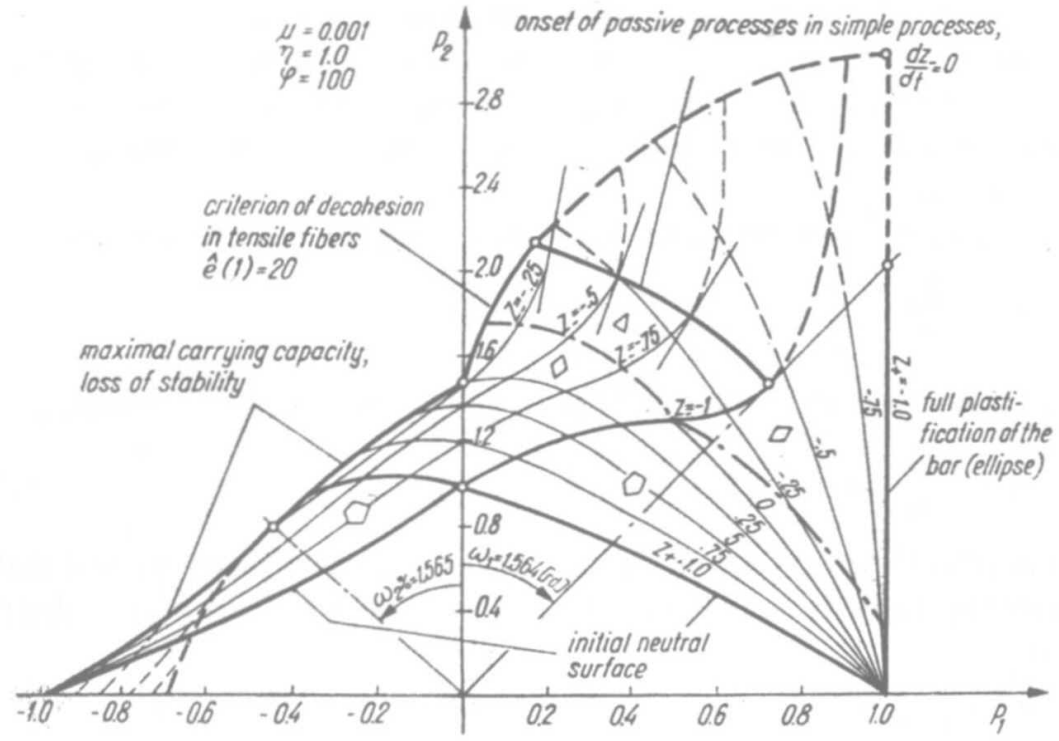

Fig. 184. Various interaction curves for the model shown in Fig. 181

gons and pentagons. The regions depend on the Bauschinger effect, which has been characterized by the coefficient $\beta$, (8.17). K. Kowalczyk [8.266] used also the model under consideration to an approximate analysis of shakedown of leaf springs.

Many interesting effects of geometry changes may also be studied on a similar but much simpler model shown in Fig. 185 (M. Życzkowski [8.577]); it consists of a rigid part and two deformable bars and extends the Ryder-Shanley model

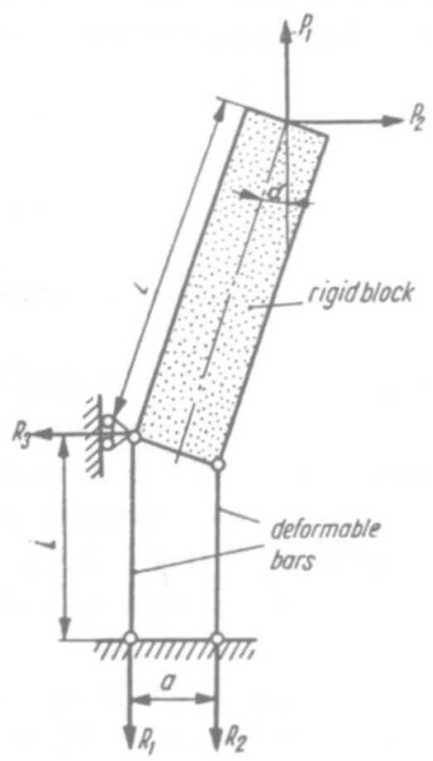

Fig. 185. A simple model of the column 
to the action of two independent forces. The system is externally and internally statically determinate, and, for example, the classical Melan theorem practically cannot be applied to it since the residual stresses simply cannot develop. On the other hand, because of the geometric effects, a certain family of shakedown domains does exist.

First, let us disregard the geometric effects; then the reactive forces are

$$
R_{1}=\frac{P_{1}}{2}+\frac{L}{a} P_{2}, \quad R_{2}=\frac{P_{1}}{2}-\frac{L}{a} P_{2} .
$$

Introducing dimensionless forces by formulae analogous to (36.23), namely by

$$
p_{1}=\frac{1}{2 \sigma_{0} A} P_{1}, \quad p_{2}=\frac{L}{a \sigma_{0} A} P_{2},
$$

where $A$ denotes the cross-sectional area of any of deformable bars, we find that the elastic carrying capacity (equal here to the limit carrying capacity) is described by a square

$$
p_{2}= \pm\left(p_{1} \pm 1\right) \text {. }
$$

Let us now consider the geometry changes, but assume small-deflection theory, $\sin \alpha \approx \alpha, \cos \alpha \approx 1$. The equilibrium equations are now

$$
R_{1}+R_{2}=P_{1}, \quad R_{2} a=-P_{2} L+P_{1}(a / 2+\alpha L)
$$

and, together with Hooke's law applied to both bars,

$$
\left(R_{1}-R_{2}\right) \frac{L}{E A}=a \alpha,
$$

they determine the reactive forces and the angle $\alpha$ in the elastic range. The conditions $R_{1}= \pm \sigma_{0} A$ and $R_{2}= \pm \sigma_{0} A$ determine the initial elastic curve as follows:

$$
p_{2}= \pm\left(p_{1} \pm 1\right)\left(\chi p_{1}+1\right) \text {, }
$$

where

$$
\chi=\frac{4 L l \sigma_{0}}{a^{2} E}
$$

is a parameter connected with the slenderness and elasticity of the system; it encloses all the three parameters (36.29).

Assumption $\chi=0$ corresponds to a rigid-plastic theory, $E \rightarrow \infty$; then and only then the curve (36.49) is convex. The range $0<\chi<1$ corresponds to plastic buckling under the compressive force $P_{1}$ alone; within the range $\chi>1$ we have elastic buckling before plastification. The signs in (36.49) may be arbitrarily combined. Figure 186 shows the initial elastic curves (36.49) for $\chi=0, \chi=0.5, \chi=1$ and $\chi=2$. These curves are partly concave and practically coincide with those shown in Figs. 182 and 183 for $\alpha_{0}=0$.

Consider now the shakedown domains. Residual stresses cannot exist, but residual strains are possible. They result in a residual mean elongation $\Delta l_{0}$ of the deformable bars and in a residual deflection angle $\alpha_{0}$ of the rigid part of the column. 


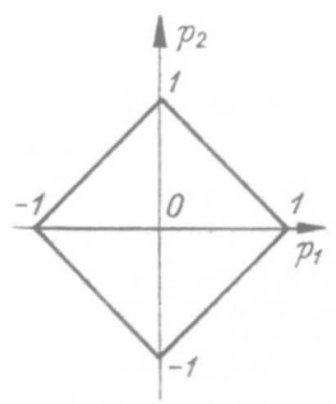

$x=0$

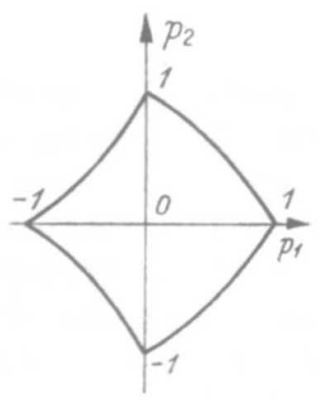

$\chi=0.5$

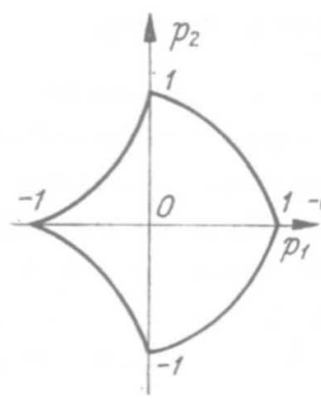

$x=1$

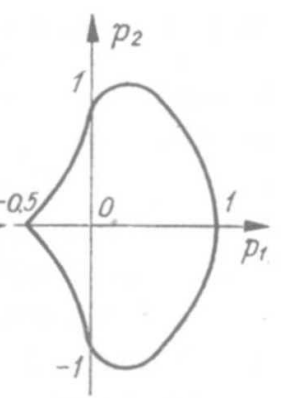

$\chi=2$

Fig. 186. Elastic interaction curves for the model shown in Fig. 185 with various slenderness ratios

The quantity $\Delta l_{0}$ has no influence on the further behaviour of the system, but $\alpha_{0}$ appears in the modified Eq. (36.48) of subsequent elastic changes, namely

$$
\left(R_{1}-R_{2}\right) \frac{l}{E A}=a\left(\alpha-\alpha_{0}\right) \text {. }
$$

For a perfectly elastic-plastic material the modified elastic curves, determining the shakedown domains, are

$$
p_{2}= \pm\left(p_{1} \pm 1\right)\left(\chi p_{1}+1\right)+\varphi p_{1},
$$

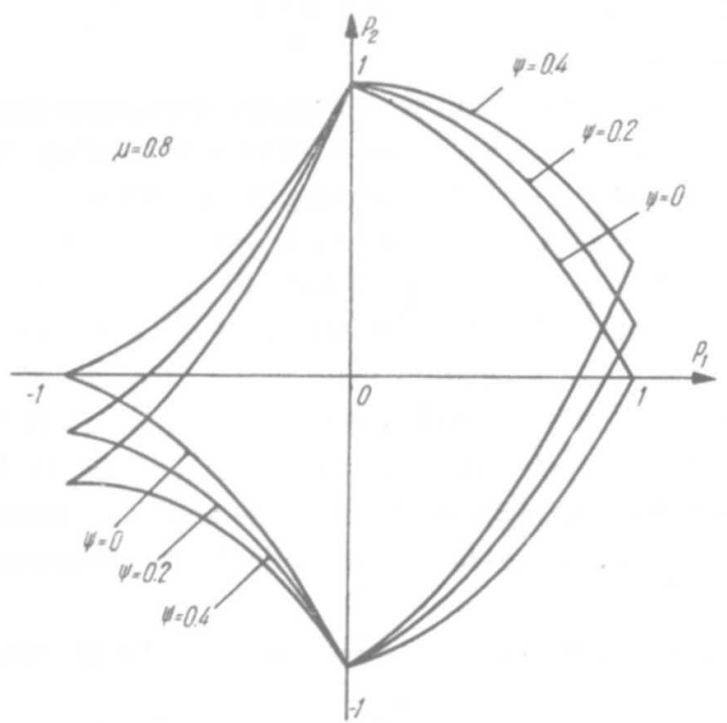

Fig. 187. A family of shakedown domains without residual stresses

where the parameter of the family $\psi$ equals $\psi=(2 L / a) \alpha_{0}$; they are shown in Fig. 187. Thus the geometric effects make it possible to discuss the family of shakedown domains in the problem, which is quite trivial if these effects are not taken into account. 


\subsection{Combined surface loadings}

Particular attention has been paid to the plastic behaviour of beam-columns loaded simultaneously by forces parallel and perpendicular to the axis. The first solutions for a perfectly elastic-plastic material were obtained by K. Ježek [4.261, 8.217], 1934, and by E. Chwalla [8.86]; they used the linearized expression for the curvature of the beam. Further results are due to J. A. Van den Broek [8.540] and S. D. Leytes [4.330]. Finite-deflection-theory was used by I. G. Grechukho [8.178], who evaluated the maximal carrying capacity! for the axial forces under a constant transverse loading. Impulsive loadings were investigated by $\mathrm{H}$. Maeda and $\mathrm{H}$. Namikawa [8.315]. N. L. Moshensky [5.127] considered plastic hardening and applied the method of elastic solutions. Independent action of two eccentric forces (one at the end and one at the mid-span) was investigated theoretically and experimentally by K. K. Mukhanov and Yu. V. Sobolev [5.129]. Le-Wu Lu and H. Kamalvand [8.312] considered I-sectioned beam-columns under several loadings. A. N. Bogdanov [8.51] discussed an initially slightly curved cantilever under transverse and axial forces at the free end. Yu. A. Rakovshtchik [8.421] determined residual deflections of beam-columns. J. M. Aribert and P. Jouve [8.17] replaced the beamcolumn by elastic finite elements with plastic deformations concentrated in the hinges; a simpler approach, with rigid finite elements, is due to L. M. Belenky [8.37, 8.38]. An extensive analysis of inelastic beam-columns was carried out by W. F. Chen and his collaborators, S. Santathadaporn and T. Atsuta $[8.69,8.70,5.30]$; a survey has been given by C. E. Massonnet [8.331].

A certain specific approach to a beam under distributed transverse loading is due to B. B. Lampsi [8.293]. He proposed an equation of the limit surface in the space bending moment-shearing force-distributed loading in the form of an ellipsoid. The first two quantities belong to generalized stresses, and the third one-to generalized external forces. The generalized stresses depend of course on the external forces, but the author wanted to take additionally into account the stresses $\sigma_{z}$ (usually neglected in the theory of plastic bending with shear) and the gradient $d V / d x$. A similar approach for $I$-beams was developed by A. I. Kuzin [8.283].

The problem of spatial deflections of beam-columns was considered by S. D. Leytes [8.306]. H. B. Harrison [5.68] investigated, theoretically and experimentally, biaxial plastic bending of a rectangular beam under two independent forces acting in different planes.

Optimal design of beams for multiple loading was considered by R. Mayeda and W. Prager [8.332].

Of the numerous papers discussing combined loading of frames, let us mention here those by C. G. Schilling, F. W. Schutz and L. S. Beedle [8.448], J. Heyman [4.205], M. Wakabayashi, T. Nonaka and S. Morino [8.551] (experimental), R. J. Alvarez and C. Birnstiel [8.9] (allowing for the effects of longitudinal forces and deflections), E. W. Wright [8.560] (dead weight and wind load), G. Augusti and A. Baratta [8.20] (probabilistic approach), M. Duszek and A. Sawczuk [8.121, 8.122] 
(stability of post-yield behaviour). The optimal plastic design of frames was initiated by H. J. Greenberg and W. Prager [4.185], J. Heyman [8.191, 8.192] and J. Foulkes [8.151, 8.152]; the case of several loadings was discussed in detail by $\mathbf{R} . \mathbf{K}$. Livesley [8.309], L. P. Felton and R. B. Nelson [8.141]. The limit analysis of trusses under combined loadings was developed by N. V. Akhvledyani and G. I. Lezhava [8.4], whereas J. C. Nagtegaal and W. Prager [8.358, 8.359] and C. Polizzotto [8.411, 8.412] considered the optimal plastic design of trusses under multiple loads.

A general approach to plastic analysis of statically indeterminate bar systems under combined loadings was given by G. Ceradini [8.66] and H. Lippmann [8.308].

The problems of plastic shakedown of bars and bar systems under variable loadings present the next, generally more difficult, step of the analysis. The relevant theory was initiated by M. Grüning [4.189] in 1926; the classical papers were briefly reviewed in Sec. 18.8. In the present chapter we discuss some more recent investigations.

Shakedown of beams was considered by A. F. M. Ghani and A. Roushan [8.165] (non-prismatic beams), L. Konieczny [4.295] (adaptation of the BleichMelan theorem to the criterion (18.73) and some related theorems), C. Dyrbye [8.123] (continuous beams under distributed loadings), M. M. Ghamian, S. G. Krishnasamy and A. N. Sherbourne [8.271, 8.164] (displacement-controlled loading, effect of lateral buckling), J. D. Meyer and K. H. Gerstle [8.338] (essential influence of plastic hardening), P. K. Das, D. C. Chandler and B. K. Foster [8.110] (nonshakedown of beams), D. G. Eyre and T. V. Galambos [8.137] (continuous beams under extreme concentrated loads). The problem of estimation of shakedown deflections of beams was mentioned in Sec. 18.8; we add here only the papers by B. N. Kuznetsov [8.288] (shakedown deflections of continuous beams), P. Grundy and F. Tin Loi [8.182] (initial strain method applied to deflection analysis).

Shakedown of bars under variable torque was discussed by A. Mioduchowski and K. Thermann [8.346]; they applied a finite-strain theory to the analysis of the Poynting effect. The influence of the Bauschinger effect on the shakedown domains for a bar shown in Fig. 175 was investigated by K. Kowalczyk and M. Życzkowski [8.262].

Numerous papers have dealt with shakedown of columns under constant compression and variable bending; this type of loading is particularly important in seismic analysis. We mention here the papers by B. I. Lyubarov [5.112], U. Säverin [8.447] (multispan columns), B. Kato and H. Akiyama [8.231], C. Amariei [8.14], C. Gavarini and G. Beolchini [4.155] (second-order effects), M. Yamada and K. Shirakawa [5.204], G. Thierauf [8.525], S. Igarashi, C. Matsui and K. Yoshimura [8.207] (experimental), A. B. Higginbotham and R. D. Hanson [8.196], L. F. Kahn and R. D. Hanson [8.225] (experimental). Biaxial variable bending with normal force was considered by W. F. Chen [5.29], whereas T. Nonaka [8.366] analysed a possible loss of stability under repeated axial loading.

Plastic shakedown of trusses was considered in detail by N. S. Streletsky [8.508], A. R. Rzhanitsyn [4.492], P. S. Symonds and W. Prager [4.561]. A. Zingali and 
F. Braga [8.573] considered shakedown of trusses in the case of large displacements; J. A. Karczewski [8.230] allowed in his shakedown analysis for the possible buckling of compressed bars of a truss.

The theory of plastic shakedown of frames was initiated by P. S. Symonds and B. G. Neal [8.513, 8.360, 8.361, 8.514], M. R. Horne [4.236, 8.205], and J. Heyman [4.205]; experimental verification of the theory was carried out by P. S. Symonds [8.515]. Further papers are due to V. V. Kupriyanov [8.281] (influence of longitudinal and transverse forces), J. M. Davies [8.111] (a matrix method allowing for possible buckling), J. A. König [5.92] (application of [4.303], arbitrary cross-section), K. Inoue and K. Ogawa [8.213] (finite element method, kinematic hardening), and B. N. Kuznetsov $[8.286,8.287]$ (a simple approximate approach). A related theory of shakedown of arches was developed by V. Franciosi, G. Augusti and R. Sparacio [8.154], Y. Fukumoto and H. Yoshida [8.159], P. G. Hodge and A. J. Kalinowski [8.202], and J. A. König [5.92].

Optimal design of bar structures with respect to plastic shakedown under variable repeated loadings was first considered by J. Heyman [8.193], 1958; he discussed optimization of frames. Further papers on frame optimization in shakedown conditions are due to A. A. Cyras [8.97], W. Prager [8.416, 8.417], V. N. Kissyuk [8.246], M. Z. Cohn and D. E. Grierson [8.89], M. Z. Cohn and S. R. Parimi [8.90], R. P. Baronas [8.29], C. Polizzotto, C. Mazzarella and T. Panzeca [8.413]. Optimal beams were considered by A. Zavelani-Rossi [8.568] (reinforced concrete), and optimal trusses-by V. N. Kissyuk [8.245]. A more general approach to optimization in shakedown conditions is due to A. A. Cyras [8.98, 8.99], Yu. M. Pochtman and Z. I. Pyatigorsky [8.404, 8.405, 8.406, 8.407, 8.410]. V. A. Ikrin [8.209] considered the problem of optimal loading trajectories for beams and frames under multiparameter loading.

The plastic analysis of structures under moving loadings is closely related to shakedown problems. In this case the positions of individual forces may also be considered as exertion factors. The earliest investigations of beams under moving loads are due to L. I. Malament [8.322], 1940, J. F. Baker [8.26] and N. D. Zhudin [8.570, 8.571]. Particular attention was paid to that problem by N. L. Chernov (or M. L. Chernov in Ukrainian): in [8.75] he investigated theoretically and experimentally the carrying capacity of beams under a system of moving forces, in [8.76] - deflections under a system of moving forces, in [8.77] he considered plastic hardening, in [6.45] he estimated residual deflections after a series of cycles, in [6.44] (with E. R. Chernaya) he considered moving forces causing oblique bending with torsion, in [8.78] (with V. L. Tarasenko) he investigated beams on elastic supports, in [6.47] (with S. D. Kudryavtsev) he dealt with shear effects; in [8.79] Chernov discussed the relation of the case of moving forces to Bleich's shakedown theorem, in [8.80] he considered contact stresses and in [8.81] he investigated theoretically and experimentally the spreading of plastic zones in the beam. Further papers on plastic beams under moving loads are due to J. F. Baker, M. R. Horne and L. G. Johnson [8.27], L. P. Kunitsky [6.127] (shear effects), A. Kolař [8.251, 8.252], R. M. Kira- 
kosyan [8.239, 8.240] (distributed loading), S. D. Kudryavtsev [5.97] (biaxial bending), D. G. Eyre and T. V. Galambos [4.138], and B. N. Kuznetsov [8.285] (deflection analysis). V. P. Vikulenkov [8.544] considered forces with variable positions and directions. Experimental investigations on beams under moving forces were carried out by T. G. Toridis and R. K. Wen [8.532], A. Mrázik [8.351], Y. Fukumoto and $\mathrm{H}$. Yoshida [8.158]. Shakedown of reinforced concrete arches under moving loads was analysed by R. Sparacio [8.503]. A numerical procedure suitable for elastic-plastic arches under moving loads was proposed by C. Cichoń [8.87].

Optimal plastic design of bar systems under moving loads was first tackled by M. R. Horne [8.204], who considered a beam clamped at both ends. A more general approach was proposed by O. Gross and W. Prager [8.181], M. Save and W. Prager [8.442]. Further papers are due to V. N. Kissyuk [8.244] (beams and frames), G. Sacchi and A. Zavelani-Rossi [8.432], D. Lamblin and M. A. Save [8.291, 8.292], G. Sacchi, G. Maier and M. Save [8.433] (frames designed by linear programming), A. Čyras and K. Vislavičius [8.100] (convex programming methods).

\subsection{Body forces, thermal loadings}

When we consider bodies in motion, particularly rotating elements, and also if the weight of the body itself is considerable, the relevant body forces may essentially influence the plastic deformations and the limit carrying capacity. These forces may usually be described by several parameters (e.g. the angular velocity $\omega$, the specific weight $w$ ), which may be regarded as separate exertion factors at the level $\mathscr{B}$. Approximate methods of limit analysis in the presence of body forces were discussed by A. D. Cox and A. J. M. Spencer [8.92]: they compared the perturbation method with the energy methods.

Elastic-plastic bending of rotating bars of a circular section was first analysed in a dissertation by D. D. Savvin [8.445]. The problem was then considered by I. N. Danilova [8.107], who accounted also for non-uniform heating of the bar, V. E. Voronkov [8.548, 8.549] (bending followed by rotation) and R. M. Kirakosyan [8.241]. V. E. Voronkov and V. V. Moskvitin [8.547] investigated a thinwalled circular tube under bending and rotation in the case of linear plastic hardening.

N. S. Kurdin [8.282] considered elastic-plastic torsion of a rotating circular shaft. He found that the onset of yielding may take place at the axis or at the outer surface, but cannot start from any other point of the cross-section of the shaft.

As was mentioned in Sec. $1.3 \div 1.5$, the temperature may affect plastic deformations in various ways. Even if constant throughout the body, it may influence material properties, and-in statically indeterminate cases-may result in thermal stresses. Then this constant temperature may be regarded as an exertion factor. In more complicated cases of variable temperature it may usually be described by several parameters, and sometimes one may regard the intensities of heat sources as exertion factors.

B. V. Lopatin [8.311] considered the formation of plastic hinges in a rectangular 
or an I-beam in the case of non-uniform temperature distribution; the dependence of material constants on temperature was taken into account. Gh. Buzdugan [8.63] and S. Cuomo [8.96] analysed the behaviour of beams at elevated uniform temperature; the first paper discussed the influence of temperature on the formation of plastic zones in a beam clamped at both ends, and the second one investigated the influence of changed mechanical properties on the elastic-plastic deflections of the beam. Zh. G. Ovanesova and V. V. Abramov [8.378, 8.379] considered multi-span composite beams under combined surface and thermal loadings.

L. A. Shapovalov [8.459] investigated the elastic-plastic buckling of a column clamped at both ends, due to elevated temperature, which was assumed constant along the axis and linearly variable in the transverse direction. For a perfectly elastic-plastic material and for a linearly hardening material he obtained closed-form solutions. S. R. Bodner [8.50] analysed a similar problem by means of the energy method.

An extensive literature is devoted to the analysis of plastic shakedown of bars and bar systems under variable thermal loadings. Such an analysis is relatively simple and in most cases leads to qualitative conclusions of a general character: in his first paper on thermal shakedown, W. Prager [4.470] analysed a beam as a suitable example. The phenomenon of non-shakedown, observed in many experiments and in real structures, is due here mainly to thermal ratcheting resulting in incremental collapse.

Tension-compression bar systems were investigated in detail by D. A. Gokhfeld: one-parameter systems in [4.166], and multiparameter systems in [8.172]; experimental verification is given in [8.168]. Further papers are due to E. H. Mansfield [8.326] (linear plastic hardening), L. I. Fridman [8.157] (shakedown of turbine blades), T. M. Mulcahy [8.353] (thermal ratcheting of a model of nuclear reactor elements), A. R. Brunsvold, H. V. Ahmed and C. C. Stone [8.60] (a similar model with unequal areas and properties). Ratcheting of buckling type was analysed by D. A. Gokhfeld and A. E. Kharitontchik [8.176], and by D. Burgreen [8.62]. Problems of thermal shakedown of beams were discussed, besides Prager, by B. E. Gatewood and R. W. Gehring [8.161] (cyclic bending, compression and temperature), L. I. Fridman [8.155] (non-uniform temperature distribution), and by K. B. Ayers [8.22] (cyclic bending and temperature, yield-point stress linearly decreasing with temperature). The above-mentioned papers on shakedown analysis of reinforced concrete beams by R. Sparacio [8.503] and by A. Zavelani-Rossi [8.568] allowed also for possible cyclic temperature changes.

\section{Circularly symmetric combined loadings of cylinders}

\subsection{Introductory remarks}

The classification of combined loadings in thick-walled or thin-walled tubes, straight or curved, presents certain difficulties. If a thick-walled tube is not subject to internal 
or external pressure, then it may be regarded as a bar or beam with a doubly-connected cross-section: the introduction of appropriate generalized stresses (normal and shear forces, bending and twisting moments) presents no difficulties and we may use limit surfaces constructed in the space of those exertion factors. However, in most applications the tubes are subject to pressure, which influences the work of the tube, or even of a cross-section of the tube, and does not have the character of a generalized stress. A thin-walled tube may either be analysed by methods of the theory of thin-walled closed-section bars (if not subject to pressure), or by methods of the theory of shells; in the latter case the concept of a cross-section and of generalized stresses may obviously be introduced, but in a quite different manner (a more "local" approach than for the bars).

In order to obtain a more uniform approach we regard all the loadings as generalized external forces and classify them at the level $\mathscr{B}$ only. Such a treatment presents no difficulties inasmuch as bending, torsion and tension in a straight tube are concerned, since then we may assume (exactly or approximately) the longitudinal homogeneity of stresses and regard those generalized stresses as being equal to the corresponding generalized external forces acting on the ends of the tube. Some problems arise if shearing force is discussed: then longitudinal homogeneity is excluded (except in curved tubes, cf. Sec. 25). In this case the relevant interaction surfaces must be regarded as constructed in a space of partly internal and partly external generalized forces. Nevertheless, such an approach is in most cases sufficient for an approximate analysis of shear effects in pipe-lines.

Particular attention should be paid to thin-walled tubes under simultaneous tension, torsion and internal pressure. In this case practically a complete homogeneity of stresses and strains occurs (in a homogeneous material and before the loss of stability), and hence the relevant classification is $\mathscr{P}_{3} \rightarrow \mathscr{B}_{3}$. In view of that homogeneity the case under consideration (or its subcases) was frequently used in experimental investigations of combined loadings at the level $\mathscr{P}$, i.e. in experimental investigations of the material behaviour (yield conditions, flow rules, etc.). Here belong the classical experiments by J. J. Guest [2.46], 1900, W. Lode [2.104, 2.105], M. Roš and A. Eichinger [2.144], K. Hohenemser [3.262], G. I. Taylor and H. Quinney [3.791]; some more important recent experimental investigations of this type will briefly be reviewed in Sec. 37.6.

\subsection{General circularly symmetric case in thick-walled tubes}

As a circularly symmetric case we understand such a case of loading and of geometry of the body in which the stresses and strains depend only on the coordinate $r$ (radius) in cylindrical coordinates; the displacements (or velocities), however, may depend on all the three coordinates $r, \theta, z$.

Following paper [8.575], we assume circular symmetry and solve the corresponding system of equations (for a perfectly plastic material) and then determine 
the loading occurring in the case under consideration. We assume no body forces and no thermal loadings; they will be discussed in Sec. 37.4.

Under the assumption adopted, the equilibrium equations in cylindrical coordinates (6.11) take the form

$$
\frac{d \sigma_{r}}{d r}+\frac{\sigma_{r}-\sigma_{\theta}}{r}=0, \quad \frac{d \tau_{r \theta}}{d r}+2 \frac{\tau_{r \theta}}{r}=0, \quad \frac{d \tau_{r z}}{d r}+\frac{\tau_{r z}}{r}=0 .
$$

The strain-displacement relations will be rewritten in the form (7.23), where the dependence of strains on only the variable $r$ is effectively marked

$$
\begin{array}{ll}
\varepsilon_{r}=\frac{\partial u_{r}}{\partial r}=f_{1}(r), & \gamma_{\theta z}=\frac{1}{r} \frac{\partial u_{z}}{\partial \theta}+\frac{\partial u_{\theta}}{\partial z}=f_{4}(r), \\
\varepsilon_{\theta}=\frac{1}{r} \frac{\partial u_{\theta}}{\partial \theta}+\frac{u_{r}}{r}=f_{2}(r), & \gamma_{z r}=\frac{\partial u_{z}}{\partial r}+\frac{\partial u_{r}}{\partial z}=f_{5}(r), \\
\varepsilon_{z}=\frac{\partial u_{z}}{\partial z}=f_{3}(r), & \gamma_{r \theta}=\frac{\partial u_{\theta}}{\partial r}-\frac{u_{\theta}}{r}+\frac{1}{r} \frac{\partial u_{r}}{\partial \theta}=f_{6}(r) .
\end{array}
$$

The functions $f_{i}(r), i=1,2, \ldots, 6$, will be regarded as unknown. Instead of (37.2) the compatibility equations (7.52) may also be employed. The HMH yield condition will be written in the form

$$
\left(\sigma_{r}-\sigma_{\theta}\right)^{2}+\left(\sigma_{\theta}-\sigma_{z}\right)^{2}+\left(\sigma_{z}-\sigma_{r}\right)^{2}+6\left(\tau_{r \theta}^{2}+\tau_{\theta z}^{2}+\tau_{z r}^{2}\right)=2 \sigma_{0}^{2} ;
$$

we admit here $\sigma_{0}=\sigma_{0}(r)$, i.e. circularly symmetric plastic non-homogeneity. Moreover, we assume plastic incompressibility

$$
\varepsilon_{r}+\varepsilon_{\theta}+\varepsilon_{z}=0
$$

and the law of similarity of deviators (9.28) (Hencky-Ilyushin or Levy-Mises if $\varepsilon_{i j}$ are regarded as strain rates and $u_{i}$ as velocities).

We begin solving the above system of equations with the analysis of (37.2). Integrating the first of these equations, we obtain

$$
u_{r}=\int f_{1}(r) d r+F_{1}(\theta, z),
$$

where $F_{1}(\theta, z)$ denotes a function which is unknown as yet. Substitution of (37.5) into the second equation (37.2) and integration with respect to $\theta$ makes it possible to determine the displacement $u_{\theta}$ by the formula

$$
u_{\theta}=\theta r f_{2}(r)-\theta \int f_{1}(r) d r-\int F_{1}(\theta, z) d \theta+F_{2}(r, z),
$$

and the third equation of (37.3) results in

$$
u_{z}=z f_{3}(r)+F_{3}(r, \theta) \text {. }
$$

The functions $F_{i}$, resulting from integration, will now be determined from the remaining equations of (37.2) and from the boundary conditions for displacements. Namely, substituting $(37.5) \div(37.7)$ into the remaining equations of $(37.2)$, we 
obtain the following system of equations, which is to be satisfied as a system of identities:

$$
\begin{aligned}
& \frac{1}{r} \frac{\partial F_{3}(r, \theta)}{\partial \theta}-\int \frac{\partial F_{1}(\theta, z)}{\partial z} d \theta+\frac{\partial F_{2}(r, z)}{\partial z}=f_{4}(r) \\
& z f_{3}^{\prime}(r)+\frac{\partial F_{3}(r, \theta)}{\partial r}+\frac{\partial F_{1}(\theta, z)}{\partial \theta}=f_{5}(r) \\
& \theta r f_{2}^{\prime}(r)-\theta f_{1}(r)+\frac{\partial F_{2}(r, z)}{\partial r}+\frac{\theta}{r} \int f_{1}(r) d r+ \\
& \quad+\frac{1}{r} \int F_{1}(\theta, z) d \theta-\frac{1}{r} F_{2}(r, z)+\frac{1}{r} \frac{\partial F_{1}(\theta, z)}{\partial \theta}=f_{6}(r) .
\end{aligned}
$$

The second of these equations yields, to begin with,

$$
f_{3}^{\prime}(r)=C_{1}=\text { const }, \quad f_{3}(r)=C_{1} r+\varepsilon ;
$$

in fact, the product of the functions of $r$ and of $z$ must be excluded, since no other erm contains such a product. The constant of integration is here denoted by $\varepsilon$ because of its physical interpretation (the unit elongation). Further, the second equation of (37.8) yields two more relations,

$$
\frac{\partial F_{1}(\theta, z)}{\partial z}=-C_{1} z+F_{4}(\theta), \quad \frac{\partial F_{3}(r, \theta)}{\partial r}+F_{4}(\theta)=f_{5}(r),
$$

where $F_{4}(\theta)$ is a new unknown. Integrating these equations we express the functions $F_{1}(\theta, z)$ and $F_{3}(r, \theta)$ in terms of new unknowns-the functions of one variable $F_{4}(\theta), F_{5}(\theta)$ and $F_{6}(\theta)$. Substituting these expressions into the first and third equations of (37.8), we determine $F_{2}(r, z), F_{4}(\theta), F_{s}(\theta)$ and $F_{6}(\theta)$ (the details of calculations are given in [8.575]). Finally one obtains

$$
\begin{aligned}
u_{r}= & \int f_{1}(r) d r+\left(C_{4} \sin \theta+C_{5} \cos \theta\right) z+C_{6} \sin \theta+C_{7} \cos \theta, \\
u_{0}= & \vartheta r z+r \int \frac{f_{6}(r)}{r} d r+\left(C_{4} \cos \theta-C_{5} \sin \theta\right) z+ \\
& +C_{6} \cos \theta-C_{7} \sin \theta+C_{8} r, \\
u_{z}= & \varepsilon z+\int f_{5}(r) d r-\left(C_{4} \sin \theta+C_{5} \cos \theta\right) r+C_{2} \theta+C_{3},
\end{aligned}
$$

where $C_{i}$ are constants; $\vartheta$ is also a constant, denoted differently because of its physical interpretation (the unit angle of twist). Moreover, Eqs. (37.8) lead to the result

$$
f_{1}(r)=\frac{d}{d r}\left[r f_{2}(r)\right]
$$

which represents the well-known compatibility equation for circularly symmetric states. 
In order to exclude the rigid-body motion, we put $C_{3}=C_{6}=C_{7}=0$ (elimination of translations) and $C_{4}=C_{5}=C_{8}=0$ (elimination of rotations). Further, the function $u_{z}$ must be a periodic function of $\theta$, and hence $C_{2}=0$. So the displacement distribution is determined up to the accuracy of three functions, $f_{6}, f_{5}$, and $f_{1}$ or $f_{2}$. Returning to the strains (37.2), we obtain

$$
\varepsilon_{z}=\varepsilon=\text { const }, \quad \gamma_{\theta z}=\vartheta r,
$$

whereas other strain components still remain undetermined. However, $\varepsilon_{r}$ and $\varepsilon_{\theta}$ are interrelated by (37.12) and, making use of the incompressibility equation (37.4), we arrive at

$$
\varepsilon_{r}=-\frac{C_{r}}{r^{2}}-\frac{\varepsilon}{2}, \quad \varepsilon_{\theta}=\frac{C_{r}}{r^{2}}-\frac{\varepsilon}{2},
$$

where $C_{r}$ denotes an integration constant.

Let us now proceed to the remaining equations, (37.1), (37.3) and (9.28), totalling nine. They determine nine unknowns, namely six stress components, two strain components $\gamma_{z r}$ and $\gamma_{r \theta}$ and the function $\varphi$. The second and the third of the equilibrium equations (37.1) are uncoupled and we obtain directly

$$
\tau_{r \theta}=\frac{C_{\theta}}{r^{2}}, \quad \tau_{z r}=\frac{C_{z}}{r},
$$

where $C_{\theta}$ and $C_{z}$ are integration constants. Further, substituting into the yield condition (37.3) the formulae (37.15) and the remaining stresses, expressed in terms of strains and of the function $\varphi$, we obtain one equation with one unknown $\varphi$ and hence

$$
\varphi=\frac{\sqrt{3}}{2} \sqrt{\frac{\vartheta^{2} r^{6}+3 \varepsilon^{2} r^{4}+4 C_{r}^{2}}{\sigma_{0}^{2} r^{4}-3 C_{z}^{2} r^{2}-3 C_{\theta}^{2}}} .
$$

Knowing the function $\varphi$, we calculate subsequently

$$
\begin{aligned}
& \gamma_{z r}=f_{5}(r)=\frac{C_{z} \sqrt{3}}{r} \sqrt{\frac{\vartheta^{2} r^{6}+3 \varepsilon^{2} r^{4}+4 C_{r}^{2}}{\sigma_{0}^{2} r^{4}-3 C_{z}^{2} r^{2}-3 C_{\theta}^{2}}}, \\
& \gamma_{r \theta}=f_{6}(r)=\frac{C_{\theta} \sqrt{3}}{r^{2}} \sqrt{\frac{\vartheta^{2} r^{6}+3 \varepsilon^{2} r^{4}+4 C_{r}^{2}}{\sigma_{0}^{2} r^{4}-3 C_{z}^{2} r^{2}-3 C_{\theta}^{2}}}, \\
& \tau_{\theta z}=\frac{\vartheta r}{\sqrt{3}} \sqrt{\frac{\sigma_{0}^{2} r^{4}-3 C_{z}^{2} r^{2}-3 C_{\theta}^{2}}{\vartheta^{2} r^{6}+3 \varepsilon^{2} r^{4}+4 C_{r}^{2}}} .
\end{aligned}
$$

To determine the distribution of normal stresses we use the first of the equilibrium equations (37.1):

$$
\frac{d \sigma_{r}}{d r}=-\frac{\sigma_{r}-\sigma_{\theta}}{r}=-\frac{\varepsilon_{r}-\varepsilon_{\theta}}{\varphi r}=\frac{2 C_{r}}{\varphi r^{3}} .
$$


Assume the normal pressure at the inner radius $r=a$ to be $p_{a r}$ and write the integral of (37.18) in the form

$$
\sigma_{r}=-p_{a r}+2 C_{r} \int_{a}^{r} \frac{d \xi}{\xi^{3} \varphi(\xi)},
$$

where $\xi$ denotes the variable of integration. The remaining normal stresses are determined by the strain distribution (37.13) and (37.14) and by the law of similarity of deviators (9.28); finally

$$
\begin{aligned}
& \sigma_{\theta}=-p_{a r}+\frac{2 C_{r}}{\varphi r^{2}}+2 C_{r} \int_{a}^{r} \frac{d \xi}{\xi^{3} \varphi(\xi)}, \\
& \sigma_{z}=-p_{a r}+\frac{1}{\varphi}\left(\frac{3}{2} \varepsilon+\frac{C_{r}}{r^{2}}\right)+2 C_{r} \int_{a}^{r} \frac{d \xi}{\xi^{3} \varphi(\xi)} .
\end{aligned}
$$

In view of the form of the function $\varphi=\varphi(\xi)$, (37.16), with $r$ replaced by $\xi$, the above integrals are hyperelliptic (for a homogeneous material). Hence the strain distribution and the distribution of shearing stresses is determined by elementary functions, and the distribution of normal stresses-by non-elementary functions. However, in more important particular cases these integrals reduce to elliptic or even to elementary ones, and the determination of stress distribution and of the plastic interaction surface is facilitated. Similar formulae were also obtained by M. A. Zadoyan [8.564] in some other way.

It should be mentioned that certain types of plastic non-homogeneity may result in a simplification of the solution. For example, non-homogeneities of the

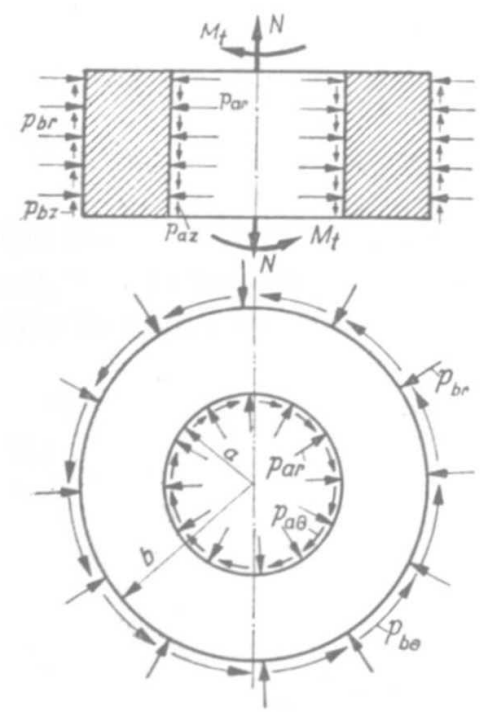

Fig. 188. Loadings of a thick-walled cylinder in the general circularly symmetric case 
type $\sigma_{0}=B / r^{2}$ or $\sigma_{0}=B / r^{4}$ reduce the hyperelliptic integrals to elliptic ones. However, in what follows we confine our considerations to homogeneous cylinders.

The stress distribution determined here holds throughout the body in the limit state (with some exceptions discussed in [8.575] if large tangential pressures result in decohesive carrying capacity rather than in limit carrying capacity) or in the plastic zones of an elastic-plastic tube. In what follows we consider in detail only the limit state of the cylinder.

Making use of the boundary conditions at the bottoms and at the lateral surfaces we find that the general case under consideration corresponds to five types of loadings: normal force $N$, twisting moment $M_{t}$, normal pressures $p_{a r}$ and $p_{b r}$, tangential pressures with circumferential direction $p_{a \theta}$ and $p_{b \theta}$, and tangential pressures with axial direction $p_{a z}$ and $p_{b z}$ (Fig. 188). Hence this general circularly symmetric case may be classified as $\mathscr{P}_{6} / \mathscr{B}_{5}$. The loadings listed above are determined by the formulae

$$
\begin{aligned}
& N=2 \pi \int_{a}^{b} \sigma_{z} r d r, \quad M_{t}=2 \pi \int_{a}^{b} \tau_{\theta z} r^{2} d r, \quad p_{b r}=-\sigma_{r}(b), \\
& p_{a \theta}=\frac{C_{\theta}}{a^{2}}, \quad p_{b \theta}=\frac{C_{\theta}}{b^{2}}, \quad p_{a z}=\frac{C_{z}}{a}, \quad p_{b z}=\frac{C_{z}}{b} ;
\end{aligned}
$$

the pressure $p_{a r}$ has already been accounted for in formulae (37.19) and (37.20). First consider the external normal pressure $p_{b r}$, determined by the third equation (37.21). In view of (37.19) we may write

$$
p_{b r}=p_{a r}-2 C_{r} \int_{a}^{b} \frac{d \xi}{\xi^{3} \varphi(\xi)} .
$$

In what follows we introduce dimensionless notation

$$
\Delta q=q_{a r}-q_{b r}=\frac{c_{r}}{\sqrt{3}} \int_{\beta^{2}}^{1} \frac{p(x)}{x^{2}} d x,
$$

where $q$ denote the dimensionless pressures $q_{a r}=p_{a r} / \sigma_{0}, q_{b r}=p_{b r} / \sigma_{0}, x$ the dimensionless variable of integration $x=\xi^{2} / b^{2}$ belonging to the interval $\beta^{2} \leqslant x \leqslant 1$, where $\beta=a / b$; the function $p(x)$, being the dimensionless reciprocal of $\varphi(\xi)$, is given by the formula

$$
p(x)=\frac{1}{2 G \varphi}=\sqrt{\frac{x^{2}-\beta^{2} c_{z}^{2} x-\beta^{4} c_{\theta}^{2}}{g^{2} x^{3}+e^{2} x^{2}+c_{r}^{2}}},
$$

and the dimensionless parameters are

$$
\begin{aligned}
& e=\frac{3 G}{\sigma_{0}} \varepsilon, \quad g=\frac{G b \sqrt{3}}{\sigma_{0}} \vartheta, \\
& c_{r}=\frac{2 \sqrt{3} G}{\sigma_{0} b^{2}} C_{r}, \quad c_{\theta}=\frac{\sqrt{3}}{\beta^{2} \sigma_{0} b^{2}} C_{\theta}, \quad c_{z}=\frac{\sqrt{3}}{\beta \sigma_{0} b} C_{z} .
\end{aligned}
$$


Substituting (37.19) into the first integral (37.21) and employing the Dirichlet transformation, [8.575], we may express the normal force $N$ by means of a single integral. The result will be written in a dimensionless form,

$$
\hat{n}=n-\left(-\frac{q_{a r}+q_{b r}}{2}\right)=\frac{1+\beta^{2}}{2\left(1-\beta^{2}\right)} \Delta q+\frac{e}{1-\beta^{2}} \int_{\beta^{2}}^{1} p(x) d x,
$$

where

$$
n=\frac{N}{\sigma_{0} A}=\frac{1}{\pi b^{2}\left(1-\beta^{2}\right) \sigma_{0}} N
$$

is a dimensionless normal force, and the quantity $\hat{n}$ may be called the force reduced with respect to the action of pressure; the possibility of introducing such a reduced force results from the fact that-according to the yield condition adopted-an additional loading of the system by three-axial uniform tension or compression does not affect the yielding of the body. Similarly, the dimensionless torque may be determined by the formula

$$
m_{t}=\frac{M_{t} \sqrt{3}}{W_{0} \sigma_{0}}=\frac{2 g}{1-\beta^{4}} \int_{\beta^{2}}^{1} x p(x) d x,
$$

where $W_{0}$ denotes the elastic section modulus in torsion, and the dimensionless tangential pressures are

$$
\begin{array}{ll}
t_{a \theta}=\frac{\sqrt{3}}{\sigma_{0}} p_{a \theta}=c_{\theta}, & t_{b \theta}=\frac{\sqrt{3}}{\sigma_{0}} p_{b \theta}=\beta^{2} c_{\theta}, \\
t_{a z}=\frac{\sqrt{3}}{\sigma_{0}} p_{a z}=c_{z}, & t_{b z}=\frac{\sqrt{3}}{\sigma_{0}} p_{b z}=\beta c_{z} .
\end{array}
$$

Equations (37.23), (37.26), (37.28) and (37.29) determine, five types of loadings in terms of four parameters, namely $c_{\theta}, c_{z}$ and the ratios of the remaining parameters, e.g. $g / c_{r}$ and $e / c_{r}$, and hence they constitute parametrical equations of the plastic interaction surface. The admissible ranges of variability of the parameters were discussed in [8.575].

\subsection{Particular cases of combined surface loadings}

The tangential pressures $t_{\theta}$ and $t_{z}$ occur very seldom in engineering applications; a certain problem allowing for these pressures was discussed by V. L. Dobrovolsky [8.116]. More interesting is the case of simultaneous tension, torsion and internal and external radial pressures; this combined case is simpler than the general one, since hyperelliptic integrals reduce here to elliptic integrals. We present here this case following a paper by J. Skrzypek and M. Życzkowski [8.489].

Let us introduce a new variable of integration $y=1 / x$, facilitating the reduction 
of the cubic polynomials to the canonical form. Substituting in (37.23), (37.26) and (37.27) $c_{\theta}=c_{z}=0$, we rewrite these equations as follows:

$$
\begin{aligned}
& \Delta q=\frac{\varkappa_{r}}{\sqrt{3}} \int_{1}^{\beta^{-2}} \frac{y d y}{\sqrt{y\left(y^{3}+\frac{e^{2}}{c_{r}^{2}} y+\frac{g^{2}}{c_{r}^{2}}\right)}}, \\
& n=\frac{\beta^{2} q_{a}-q_{b}}{1-\beta^{2}}+\frac{e \varkappa_{r}}{\left(1-\beta^{2}\right) c_{r}} \int_{1}^{\beta^{-2}} \frac{d y}{y \sqrt{y\left(y^{3}+\frac{e^{2}}{c_{r}^{2}} y+\frac{g^{2}}{c_{r}^{2}}\right)}}, \\
& m_{t}=\frac{2 g \varkappa_{r}}{\left(1-\beta^{4}\right) c_{r}} \int_{1}^{\beta^{-2}} \frac{d y}{y^{2} \sqrt{y\left(y^{3}+\frac{e^{2}}{c_{r}^{2}} y+\frac{g^{2}}{c_{r}^{2}}\right)}},
\end{aligned}
$$

where $x_{r}=\operatorname{sign} c_{r}= \pm 1$, and the additional subscript $r$ of $q_{a}$ and $q_{b}$ has been dropped. Equations (37.30) determine the plastic interaction surface in terms of the parameters $e / c_{r}$ and $g / c_{r}$; we now reduce the elliptic integrals appearing in (37.30) to their normal form.

The trinomial under the radical has one real root and two conjugate complex roots, since its discriminant is positive:

$$
\Delta=\frac{g^{4}}{4 c_{r}^{4}}+\frac{e^{6}}{27 c_{r}^{6}}>0 .
$$

These roots are

$$
y_{1}=\omega_{1}-\omega_{2}, \quad y_{2,3}=-\frac{\omega_{1}-\omega_{2}}{2} \pm \frac{i}{2} \sqrt{3}\left(\omega_{1}+\omega_{2}\right),
$$

where

$$
\omega_{1}=\sqrt[3]{\sqrt{\bar{\Delta}-\frac{g^{2}}{2 c_{r}^{2}}}}, \quad \omega_{2}=\sqrt[3]{\sqrt{\Delta}+\frac{g^{2}}{2 c_{r}^{2}}} .
$$

In this case we reduce elliptic integrals to their normal form by introducing the new variable $\zeta$ as follows:

$$
\zeta=2 \arctan \sqrt{\frac{\alpha_{2} y}{\alpha_{1}\left[y-\left(\omega_{1}-\omega_{2}\right)\right]}},
$$

where the auxiliary positive parameters $\alpha_{1}$ and $\alpha_{2}$ are determined by the formulae

$$
\alpha_{1}^{2}=\omega_{1}^{2}+\omega_{2}^{2}+\omega_{1} \omega_{2}, \quad \alpha_{2}^{2}=3\left(\omega_{1}^{2}+\omega_{2}^{2}-\omega_{1} \omega_{2}\right) .
$$

Having introduced (37.34) into the first integral (37.30), we first obtain

$$
\begin{aligned}
\Delta q= & \frac{\varkappa_{r}}{\sqrt{3}} \frac{2\left(\omega_{2}-\omega_{1}\right) \sqrt{\alpha_{1} \alpha_{2}}}{\left(\alpha_{1}+\alpha_{2}\right)^{2}} \int_{\zeta_{1}}^{\zeta_{2}} \frac{d \zeta}{(b+\cos \zeta) \sqrt{1-k^{2} \sin ^{2} \zeta}}- \\
& -\frac{\varkappa_{r}}{\sqrt{3}} \frac{\omega_{2}-\omega_{1}}{\alpha_{1}+\alpha_{2}} \sqrt{\frac{\alpha_{1}}{\alpha_{2}}} \int_{\zeta_{1}}^{\zeta_{2}} \frac{d \zeta}{\sqrt{1-k^{2} \sin ^{2} \zeta}},
\end{aligned}
$$


where the following notation is used:

$$
\begin{aligned}
& k^{2}=\frac{3 \alpha_{1}^{2}+4 \alpha_{1} \alpha_{2}+\alpha_{2}^{2}}{8 \alpha_{1} \alpha_{2}}, \quad b=\frac{\alpha_{2}-\alpha_{1}}{\alpha_{2}+\alpha_{1}}, \\
& \zeta_{1}=2 \arctan \sqrt{\frac{\alpha_{2}}{\alpha_{1}\left[1-\left(\omega_{1}-\omega_{2}\right)\right]}}, \\
& \zeta_{2}=2 \arctan \sqrt{\frac{\alpha_{2}}{\alpha_{1}\left[1-\beta^{2}\left(\omega_{1}-\omega_{2}\right)\right]}} .
\end{aligned}
$$

Hence the integral under consideration consists of an elliptic integral of the first kind and of an elliptic integral of the third kind. The latter shows an apparent singularity, which should be removed before numerical computations. Rather cumbersome details are given in [8.489]; finally, one arrives at the following formula, no longer showing any singularities:

$$
\begin{aligned}
\Delta q= & \frac{\varkappa_{r}}{\sqrt{3}}\left[\operatorname{Arth} \frac{2 \sqrt{y\left(\omega_{2}^{3}-\omega_{1}^{3}\right)+3 y^{2} \omega_{1} \omega_{2}+y^{4}}}{\alpha_{1}\left(\alpha_{1}+\alpha_{2}\right)+2 y^{2}}+\right. \\
& \left.+\frac{\left(\alpha_{2}-\alpha_{1}\right)\left(\omega_{2}-\omega_{1}\right)}{2\left(\alpha_{2}+\alpha_{1}\right) \sqrt{\alpha_{1} \alpha_{2}}} \Pi\left(\zeta, \frac{k^{2}}{v}, k\right)-\frac{\omega_{2}-\omega_{1}}{2 \sqrt{\alpha_{1} \alpha_{2}}} F(\zeta, k)\right]_{y_{1}=1}^{y_{2}=\beta^{-2}} .
\end{aligned}
$$

In this formula the symbol Ar th denotes the inverse hyperbolic tangent, the symbols $F(\zeta, k)$ and $\Pi\left(\zeta, k^{2} / v, k\right)$-incomplete elliptic integrals of the first and of the third kind, respectively; further

$$
v=-\frac{\left(\alpha_{1}+\alpha_{2}\right)^{2}}{4 \alpha_{1} \alpha_{2}},
$$

and the notations (37.34), (37.35) and (37.37) hold.

A similar reduction of the remaining elliptic integrals (37.30) to their normal form results in the following formulae:

$$
\begin{aligned}
\hat{n}= & \frac{1+\beta^{2}}{2\left(1-\beta^{2}\right)} \Delta q+ \\
& +\frac{2 e \varkappa_{r}}{\left(1-\beta^{2}\right) c_{r} \alpha_{1}}\left\{\frac{\omega_{2}-\omega_{1}}{\sqrt{\alpha_{1} \alpha_{2}\left(\alpha_{1}+\alpha_{2}\right)} F(\zeta, k)-\frac{1}{\omega_{2}-\omega_{1}}} \sqrt{\frac{\alpha_{2}}{\alpha_{1}}} E(\zeta, k)-\right. \\
& -\frac{\left.\sqrt{\left(\omega_{2}-\omega_{1}+y\right)\left[y^{2}+\left(\omega_{2}-\omega_{1}\right) y+\alpha_{1}^{2}\right]}\right\}_{y_{1}=1}^{y_{2}=\beta^{-2}},}{\left[\alpha_{1}\left(\omega_{2}-\omega_{1}+y\right)+\alpha_{2} y\right] \sqrt{y}} \\
m_{t}= & \frac{4 g \varkappa_{r}}{3 c_{r}\left(1-\beta^{4}\right)\left(\omega_{2}-\omega_{1}\right) \alpha_{1}^{3}} \times \\
& \times\left\{\frac{3 \omega_{1} \omega_{2}}{\left(\omega_{2}-\omega_{1}\right) \sqrt{\alpha_{1} \alpha_{2}}}\left[\left(\alpha_{1}-\alpha_{2}\right) F(\zeta, k)+2 \alpha_{2} E(\zeta, k)\right]+\right. \\
+ & \left.\frac{\left[\left(6 \omega_{1} \omega_{2}-\alpha_{1}^{2}-\alpha_{1} \alpha_{2}\right) y-\left(\omega_{2}^{3}-\omega_{1}^{3}\right)\right] \sqrt{y^{4}+3 y^{2} \omega_{1} \omega_{2}+y\left(\omega_{2}^{3}-\omega_{1}^{2}\right)}}{y^{2}\left[\alpha_{1}\left(y-\omega_{1}+\omega_{2}\right)+\alpha_{2} y\right]}\right\}_{y_{1}=1}^{y_{2}=\beta^{-2}} .
\end{aligned}
$$


These formulae determine the plastic interaction surface as a function of the parameters $e / c_{r}$ and $g / c_{r}$ (the quantities $\omega$ and $\alpha$ depend on these ratios only).

The case under consideration was also discussed by E. J. Panarelli and P. G. Hodge [8.384], who did not reduce the elliptic integrals to their normal form, but gave some numerical results. P. Vernay [8.542] based his limit analysis of the tube on the Tresca and Coulomb yield conditions. Linear plastic hardening was considered by A. S. Kravtchuk [3.389], who found a rule of increase of loadings which ensures a simple loading process at any point of the tube (this rule is slightly different from the rule of proportional increase). Linear plastic hardening was also assumed by O. M. Sidebottom and S. C. Chu [8.483], who employed incremental constitutive equations and carried out experimental verification. The Ilyushin geometrical theory of plasticity was used by A. Úbaydillayev [8.536], whereas A. F. Revuzhenko [8.425] discussed initial pressure with tension followed by additional torsion. M. A. Zadoyan [8.565] considered the limit state of an orthotropic tube obeying the Hill yield condition (12.3).

Let us now consider more specified particular cases, starting with torsion combined with pressure difference. The assumption $n=0$ in formulae (37.30) complicates rather than simplifies the problem: we have to express one of the parameters $e / c$ and $g / c_{r}$ in terms of the other, and this requirement leads to transcendental equations containing elliptic integrals. A certain approximate solution, making use of power series, is given in [8.489]. On the other hand, essential simplification is obtained if $e=0$ (torsion combined with pressure difference under conditions of no elongation), and this case will be presented here.

Let us introduce the notation

$$
\left(\frac{g}{c_{r}}\right)^{2 / 3}=\mu,
$$

and then the substitution $e=0$ into (37.33) and (37.35) yields

$$
\omega_{1}=0, \quad \omega_{2}=\mu, \quad \alpha_{1}=\mu, \quad \alpha_{2}=\mu \sqrt{3} .
$$

In this case it is convenient to introduce a new variable of integration

$$
t=\frac{y}{\mu},
$$

since then the parameter $\mu$ appears only in the integration limits and not in the integrands. Namely, we obtain

$$
\begin{aligned}
\Delta q=\frac{x_{r}}{\sqrt{3}} & {\left[\operatorname{Arth} \frac{2 \sqrt{t+t^{4}}}{1+\sqrt{3}+2 t^{2}}+\frac{1}{\sqrt[4]{3}(1+\sqrt{3})^{2}} \Pi\left(\zeta, \frac{k^{2}}{v}, k\right)-\right.} \\
& \left.-\frac{1}{2 \sqrt[4]{3}} F(\zeta, k)\right]_{t_{1}=\mu^{-1}}^{t_{2}=\beta^{-2} \mu^{-1}}
\end{aligned}
$$

where the arguments of the integrals are

$$
k^{2}=\frac{2+\sqrt{3}}{4}, \quad \frac{k^{2}}{y}=-\frac{\sqrt{3}}{2}, \quad \zeta=2 \arctan \sqrt[4]{3} \sqrt{\frac{t}{t+1}} .
$$


The formula determining the torque is simplified much more and is expressed by means of elementary functions only:

$$
m_{t}=-\left.\frac{4}{3} \frac{\varkappa_{r}}{\left(1-\beta^{4}\right) \mu^{3 / 2}} \frac{\sqrt{t^{4}+t}}{t^{2}}\right|_{t_{1}=\mu^{-1}} ^{t_{2}=\beta^{-2} \mu^{-t}} .
$$

The reduced normal force $\hat{n}$ is proportional here to the pressure difference and is equal to

$$
\hat{n}=\frac{1+\beta^{2}}{2\left(1-\beta^{2}\right)} \Delta q .
$$

A quadrant of the plastic interaction curve $m_{t}=f(\Delta q)$ is shown in Fig. 189 for the ratio of the radii $\beta=a / b=1 / 2$.

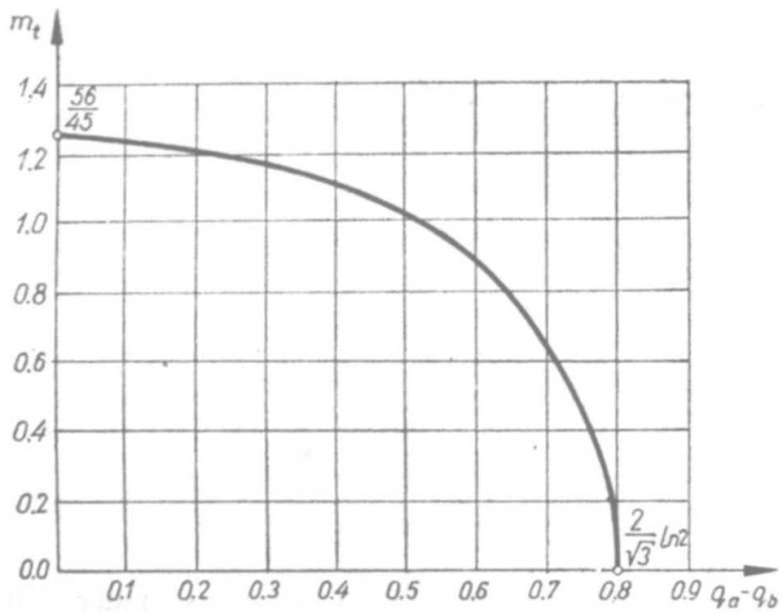

Fig. 189. Plastic interaction curve for torsion with pressure difference

Theoretical and experimental investigations of plastic thick-walled cylinders under internal pressure and torsion were carried out by B. Crossland and R. Hill [8.95]; a similar problem for concrete cylinders was analysed by A. M. Skudra [8.493], who applied the Malmeyster theory of locality of deformations.

Shakedown problems for a tube under variable pressure and torsion were considered by Zh. I. Tairova [8.519], who took plastic hardening into account. P. A. Pavlov [8.391] proposed a method of limit analysis of flanged pipe joints subject to pressure and torsion.

Another particular case of combined surface loadings, namely pressure difference with normal force, is simplest to analyse and very important for engineering applications. Substituting $g=0$ (no angle of twist) into formulae (37.33) and (37.35), we obtain here

$$
\begin{aligned}
& \omega_{1}=\omega_{2}=\frac{1}{\sqrt{3}} \frac{e}{c_{r}}=\frac{1}{\sqrt{3}} \eta, \text { where } \eta=\frac{e}{c_{r}}, \\
& \alpha_{1}=\alpha_{2}=\frac{e}{c_{r}}=\eta,
\end{aligned}
$$


and the elliptic integrals turn into elementary ones. Finally, one obtains

$$
\begin{aligned}
& \Delta q=\left.\frac{\varkappa_{r}}{\sqrt{3}} \operatorname{Arth} \frac{y}{\sqrt{y^{2}+\eta^{2}}}\right|_{y_{1}=1} ^{y_{2}=\beta^{-2}}, \\
& \hat{n}=\frac{1+\beta^{2}}{2\left(1-\beta^{2}\right)} \Delta q-\left.x_{r} \frac{\sqrt{y^{2}+\eta^{2}}}{y \eta\left(1-\beta^{2}\right)}\right|_{y_{1}=1} ^{y_{2}=\beta^{-2}} .
\end{aligned}
$$

The diagram of the function $n=f\left(q_{a}\right)$ for $\beta=a / b=1 / 2$ and for $q_{b}=0$ is shown in Fig. 190.

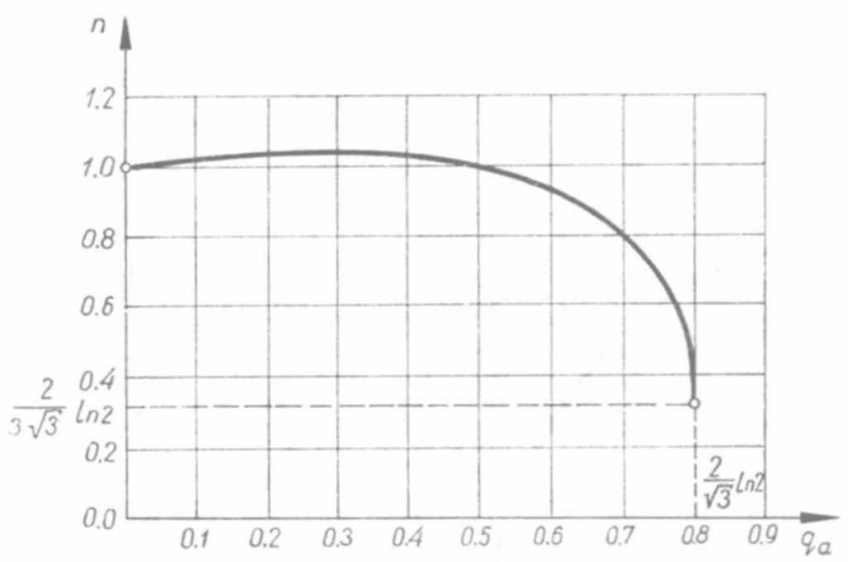

Fig. 190. Plastic interaction curve for tension with internal pressure

The bibliography concerning plastic thick-walled cylinders under normal pressures and axial force is fairly extensive. Even the earliest paper on plastic cylinders, published by B. de Saint-Venant [8.438], 1872, may be included here inasmuch as his solution, based on the Tresca yield condition, is valid without any changes in a certain interval of axial forces. The classical papers on elastic-plastic thick-walled cylinders under pressure and axial force are those of N. M. Belayev and A. K. Sinitsky [8.36], D. N. de G. Allen and D. G. Sopwith [8.6]; this problem is also treated in detail in the monographs by A. A. Ilyushin [1.8], V. V. Sokolovsky [2.163], W. Prager and P. G. Hodge [2.133], and A. Nadai [3.566]. W. T. Koiter [8.250] gave a solution based on the Tresca-Guest yield condition and the associated flow rule. D. Besdo [8.40] compared several numerical methods of solution, whereas R. Hill [8.197] gave a comparison of analytical solutions based on various yield conditions for isosensitive materials (which he called "centro-symmetric yield conditions"). A review of solutions was also given by R. Épain [8.127].

A general solution for thick-walled tubes under pressure and normal force, valid for arbitrary plastic hardening, was derived by A. A. Ilyushin and P. M. Ogibalov [8.212]; they employed the deformation theory of plasticity and took compressibility of material into account. As regards other solutions describing hardening in the combined case under consideration, let us mention the papers by $\mathrm{H}$. Jung 
[8.220] (closed-form solutions based on the Tresca-Guest hypothesis), B. R. Seth [8.454] (elastic-plastic transition in cylinders), J. Ryś [8.430] (effective calculation methods in the case of linear plastic hardening, based on the deformation theory), V. M. Zhigalkin [8.569] (Tresca-Guest, deformation theory), Zh. I. Tairova [8.518] (Tresca-Guest, kinematic hardening), Zh. Kultursynov [8.277, 8.278] (solutions employing the Ilyushin geometrical theory of plasticity), S. C. Chu and J. D. Vasilakis [8.84, 8.85] (incremental theory, various loading paths), V. Kortenska, D. Dimitrov and B. Ivanov [8.256] (parabolic and exponential hardening).

Numerous papers are devoted to finite plastic strains in thick-walled tubes under normal pressures and axial force. The earliest paper, published by D. Manning [8.325], 1945, assumed plane-strain conditions, and hence the axial force depended on pressures. A more general approach is due to C. W. MacGregor, L. F. Coffin and J. C. Fischer [8.314]; further papers were published by A. Nadai [8.357], V. A. Lomakin [8.310], P. M. Ogibalov [8.373], N. Perrone [8.394], S. A. Bocharova [8.45, 8.46, 8.47] (linear plastic hardening) and [8.48, 8.49] (plastic anisotropy), V. Kortenska, D. Dimitrov and B. Ivanov [8.257] (parabolic and exponential hardening) and by L. M. Levitan [8.305] (a numerical procedure appropriate for plastic hardening analysis). Stability of large plastic deformations in thick-walled cylinders was investigated by P. B. Mellor [8.335].

Plastic shakedown of thick-walled cylinders under variable pressures was discussed by P. G. Hodge [4.219], H. Faupel [8.138], N. C. Lind and Z. Mróz [8.307] and B. Fischer [8.144] (finite strains); many more papers deal with shakedown problems under variable thermal loading-they are reviewed in Sec. 37.4. Related problems of residual stresses in tubes were considered by V. V. Moskvitin [8.349], and R. I. Barnett [8.28] (optimal prestressing of tubular columns). Plastic deformations and maximal carrying capacity of multilayer pressure vessels under pressure and tension were discussed by T. W. Ting [8.531], M. Życzkowski [4.634], M. Galos [4.152], S. S. Hecker, C. H. Hamilton and L. J. Ebert [8.190], B. F. Trakhtenberg and R. I. Ulitskaya [8.533], W. Krzyś, M. Stodulski and A. Trojnacki [8.273] (experimental verification). Continuous non-homogeneity of a thick-walled cylinder was investigated by W. Olszak and W. Urbanowski [8.376]. G. E. Smith and A. J. M. Spencer [3.745] considered plastic deformations of thick-walled cylinders reinforced by two families of helical inextensible fibres. N. Bogdanova [8.52] discussed the elastic-plastic range in short cylinders, where there is a certain dependence of stresses on the variable $z$.

Let us also mention here some papers on thin-walled cylinders under pressure and axial tension. Maximal carrying capacity of a thin-walled cylinder under pressure and tension was evaluated by A. Troost [8.535] for arbitrary plastic hardening, geometry changes being taken into account. M. D. Coon and S. S. Gill [8.91] also allowed for geometrical effects in rigid-plastic thin-walled cylinders. A probabilistic approach to the limit analysis of a perfectly plastic tube was applied by $\mathbf{Z}$. Sobotka and J. Murzewski [8.497]. R. Dobrev [8.115] investigated limit states of a thinwalled tube under non-proportional pressure and tension. R. M. Haythornthwaite 
[8.189] analysed the influence of the wall thickness (deviations from ideal thinness of the wall) on the limit carrying capacity.

An important problem of instability of finite strains in thin-walled cylinders under pressure was discussed in Sec. 17.5; we mention here only some further papers on instability under pressure with tension, namely by R. P. Felgar [8.140], A. Krisch and R. Schweitzer [8.270], M. I. Sverdlov and I. Ya. Khavkin [8.511], F. I. Ruzanov [8.428] (anisotropy), A. Kumar and S. T. Ariaratnam [8.279, 8.280] (combined also with torsion).

\subsection{Body forces and thermal loadings}

Generalizations of the above solution to the case of body forces due to uniform or non-uniform rotation of cylinders were given by M. Życzkowski [4.630] and J. Skrzypek [4.540]. Skrzypek showed that in the case of a uniformly rotating thickwalled tube under simultaneous tension, torsion and pressure difference, formulae (37.38) and (37.40) are subject to minor changes only: the formula for the torque $m_{t}$ remains unchanged, the limit pressure difference $\Delta q$ should be changed by the term

$$
\Delta(\Delta q)=-\frac{\left(1-\beta^{2}\right) w}{2},
$$

(hence it decreases), whereas the limit normal force $n$ increases by the term

$$
\Delta n=\frac{1+\beta^{2}}{4} w
$$

with respect to the values given by (37.38) and by the first equation of (37.40) respectively. The symbol $w$ denotes here a dimensionless angular velocity squared,

$$
w=\frac{\gamma b^{2}}{g \sigma_{0}} \omega^{2},
$$

where $\gamma$ denotes the specific weight of the material of the tube. The increase of the limit normal force $n$ results from a safer stress state, closer to uniform triaxial tension.

In the particular case of no torsion and no pressure difference $\Delta q$ we may describe the plastic interaction curve by means of elementary functions:

$$
\begin{aligned}
& w=\frac{2}{\sqrt{3}\left(1-\beta^{2}\right)}\left[\operatorname{Arth} \frac{1}{\sqrt{1+\beta^{4} \frac{e^{2}}{c_{r}^{2}}}}-\operatorname{Arth} \frac{1}{\sqrt{1+\frac{e^{2}}{c_{r}^{2}}}}\right], \\
& n=\frac{1+\beta^{2}}{4} w+\frac{x_{r} c_{r}}{\left(1-\beta^{2}\right) e}\left[\sqrt{1+\frac{e^{2}}{c_{r}^{2}}}-\sqrt{1+\beta^{4} \frac{e^{2}}{c_{r}^{2}}}\right] .
\end{aligned}
$$

This curve is shown in Fig. 191 for $\beta=a / b=1 / 2$. 


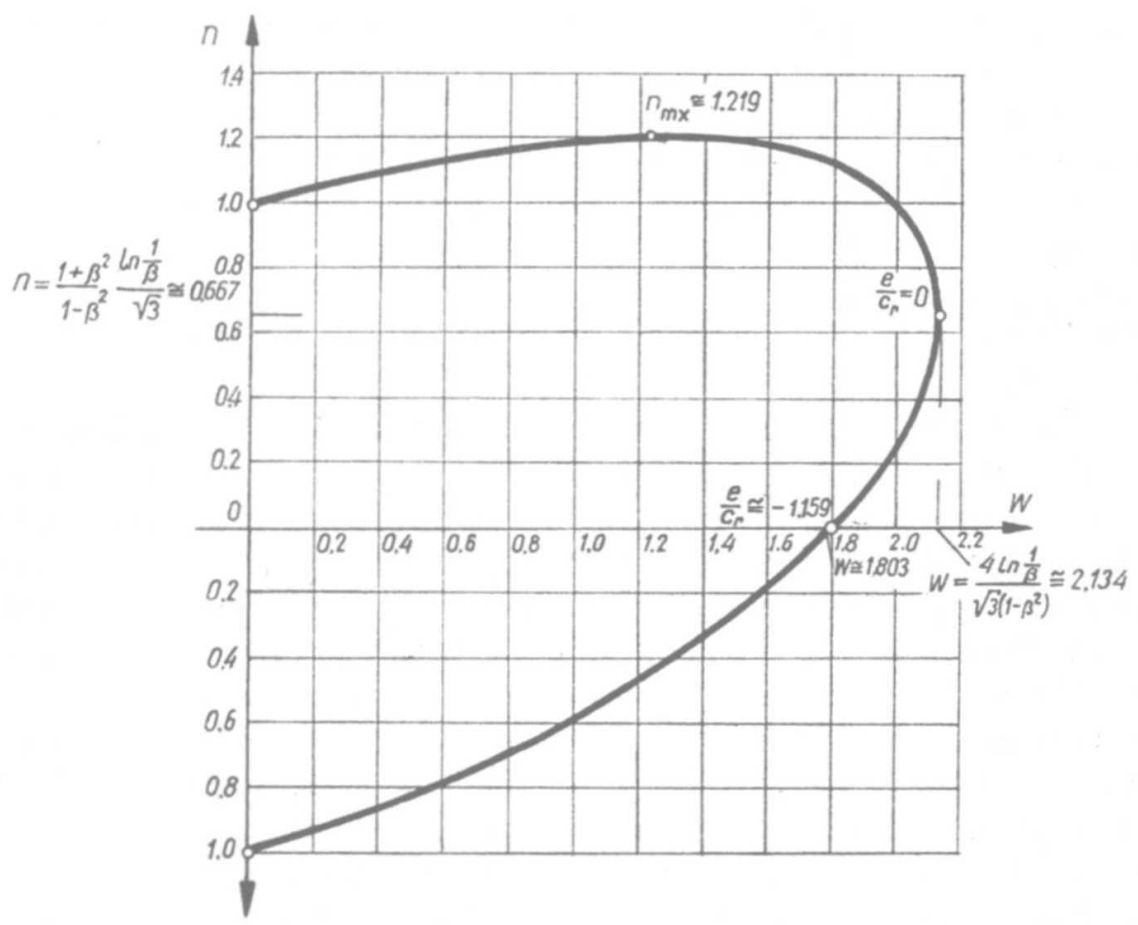

Fig. 191. Plastic interaction curve for tension with steady rotation

J. Skrzypek [4.540] considered also torsion with uniform rotation under the conditions of no elongation, torsion with uniform rotation with zero normal force, the limit state of a non-uniformly rotating cylinder and the problem of an optimal starting program. A. Ajovalasit and S. Noto La Diega [8.3] considered the limit state of a rotating solid cylinder under simultaneous normal force; they employed the Burzyński-Stassi d'Alia yield condition. The solutions quoted generalize those for rotating cylinders without axial force or without axial deformation; solid cylinders were considered by A. Nadai [3.566], A. G. Kostyuk [8.260], P. G. Hodge and M. Balaban [8.201] and W. Szczepiński [4.565], and hollow cylinders, by A. Nadai [3.566], I. N. Danilova [8.106], R. I. Mazing [8.333], Yu. V. Nemirovsky [8.364], V. I. Rozenblyum [2.145, 8.427], J. Lenard and J. B. Haddow [4.323, 4.324].

Propagation of elastic-plastic waves resulting from a collision of two rotating cylinders was investigated by $\mathrm{Kh}$. A. Rakhmatulin [8.420].

Cylinders are very often subject simultaneously to pressure and to the temperature gradient. If these two factors are independent, then we have a combined case according to the definition adopted. But even if they depend on each other, it is often convenient to consider the loading as a particular case of combined loadings. Of course, if we allow for the dependence of the physical properties of the ma- 
terial on temperature, then both the internal and the external temperature should be regarded as exertion factors, and not only their difference.

The earliest paper on elastic-plastic deformation of a thick-walled tube under pressure difference and thermal loadings was that of L. M. Kachanov [8.224], 1940. Many subsequent papers were based on the TG yield condition allowing for the dependence of yield-point stress $\sigma_{0}$ on temperature $T$; let us mention here the papers by T. Mura [8.354], E. Whalley [8.557], E. M. Morozov [8.348], N. Perrone [8.395] and V. I. Danilovskaya [8.108]. The HMH yield condition was employed by Yu. A. Samoylovitch [8.440].

Plastic hardening in a thick-walled tube under combined thermal loading and normal pressures was considered by D. R. Bland [8.43]; his analysis was based on the TG hypothesis, but the dependence of yield-point stress on temperature was not taken into account. B. F. Shorr [8.480] applied the method of successive elastic solutions. The Ramberg-Osgood hardening law (8.9) was employed by K. A. V. Pandalai and S. A. Patel [8.385], whereas a numerical procedure suitable for arbitrary plastic hardening combined with incremental constitutive equations was suggested by C. H. Austin [8.21]. The Seth theory of elastic-plastic transition was applied by B. N. Borah [8.53]. V. G. Tatarinov [8.522] analysed the influence of geometrical imperfections on the elastic-plastic behaviour of the tube; he applied the method of double Fourier series. Finite strains were considered by D. Annaratone [8.16], material anisotropy-by F. C. Weiler [8.554]. Multilayer pressure vessels in the plastic range were analysed by $\mathrm{Yu}$. N. Shevtchenko and V. G. Savtchenko [8.472] (various loading paths), Yu. V. Nemirovsky and M. L. Kheynloo [8.365] (non-linear hardening), M. S. Kenis and B. F. Trakhtenberg [8.234].

The interaction curves of the elastic carrying capacity for a thick-walled tube under a temperature gradient and internal pressure were investigated by $\mathrm{M}$. Życz-

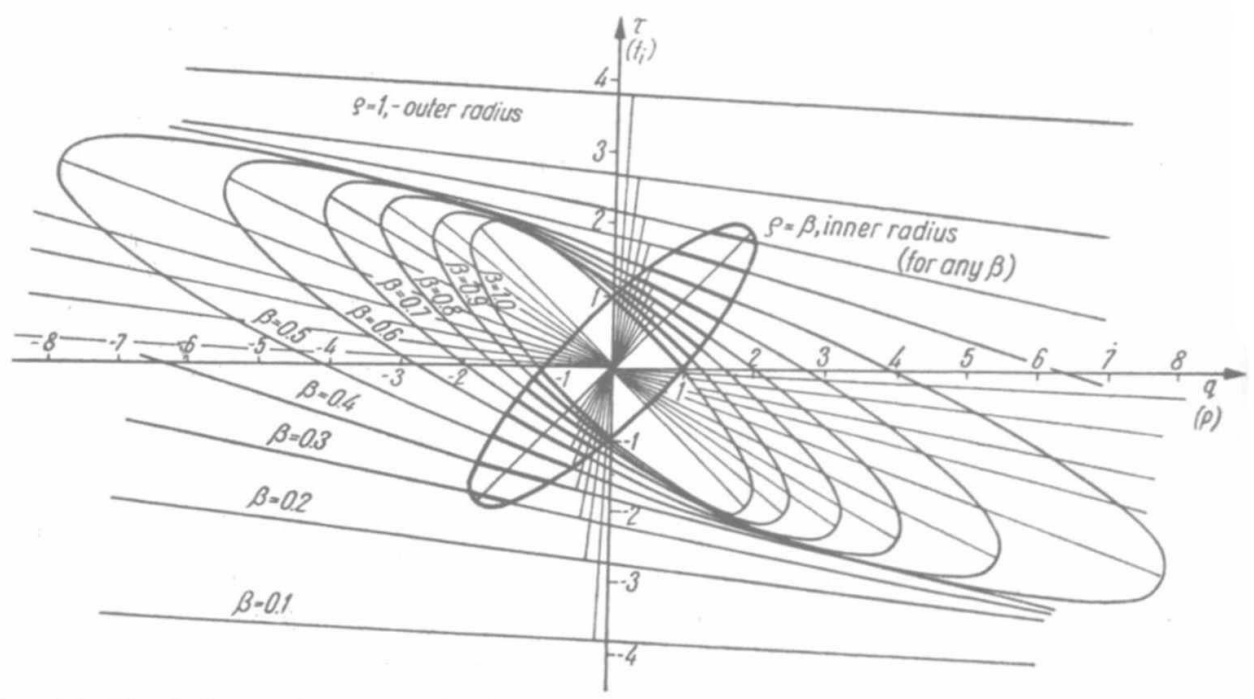

Fig. 192. Elastic interaction curves for internal pressure with temperature gradient 
kowski [1.24], W. Krzyś and A. P. Zieliński [8.272]. It was shown that the stress intensity may reach its upper bound only at the inner radius $\varrho=r / b=\beta$ or at the outer radius $\varrho=r / b=1$, (and not inside the interval $\beta<\varrho<1$ ) and for $\beta<0.275$ - only at the inner radius $\varrho=\beta$. The resulting elastic interaction curves in dimensionless coordinates $q=p / \bar{p}, \tau=T / \bar{T}$, are shown in Fig. 192; they consist of one ellipse $\sigma_{\left.e\right|_{Q=\beta}}=\sigma_{0}$, common for all values of $\beta$, and of a family of ellipses $\sigma_{\left.e\right|_{\varrho=1}}=\sigma_{0}$, depending on $\beta=a / b$.

It can be seen that a suitable temperature distribution may raise the elastic carrying capacity of a tube under internal pressure; this effect, called thermofrettage, was studied by P. M. Ogibalov [8.372].

Shakedown problems of thick-walled cylinders under variable temperature and/or pressure are of particular interest for engineering applications. For example, cyclic changes of temperature combined with constant internal pressure very often result in a certain form of non-shakedown, namely in ratcheting; this phenomenon was first investigated by D. R. Miller [8.345], 1959. Considerable attention was paid to such problems by D. A. Gokhfeld and his collaborator P. I. Ermakov [8.170, $8.171,8.130,8.174,4.173,4.174]$. Further papers are due to V. L. Fomin [8.148] (temperature fields with or without circular symmetry), R. I. Veytsman [8.543] (experimental), A. Sh. Dadashev [8.101, 8.104] (external pressure), R. W. Kauppila [8.233] (with internal heat generation), P. I. Ermakov, A. S. Nesmeyanov, A. F. Malygin and V. I. Morozov [8.131, 8.132] (with experimental verification), P. I. Ermakov, A. F. Malygin and B. E. Dorofeyev [8.133] (both pressure and temperature variable), T.Y. Chang and S. C. Chu [8.67] (kinematic hardening) and T. Uga [8.539]. Related problems of elastic-plastic cylinders under thermal impacts were studied by A. A. Ilyushin and P. M. Ogibalov [8.210] and J. Bree [8.59]. Stresses due to a phase change at a certain temperature were investigated by J. H. Weiner and J. V. Huddleston [8.555], H. G. Landau and E. E. Zwicky [8.294].

Some papers consider more general combined loadings of cylinders including thermal loadings. General equations allowing for circularly-symmetric body forces and thermal loadings were derived on the basis of the deformation theory of plasticity by M. Życzkowski [4.630] and D. D. Ivlev [8.215]. V. M. Sobolevsky considered in [8.494] a tube in an elastic medium, subject to pressure, normal force and temperature gradients, and in [8.496] a rotating tube under thermal loadings in the range of plastic hardening. T. B. Kammash, S. A. Murch and P. M. Naghdi [8.228] investigated plastic hardening of a tube under pressure, tension and circularly symmetric heat sources. Combined pressures, temperature gradient and body forces due to uniform rotation were discussed by V. K. Stryuk and M. E. Babeshko $[8.509,8.510,8.23,8.24]$; they employed both deformation and incremental theories of plasticity and allowed for boundary effects in short cylinders. Pressures combined with neutron irradiation were discussed by A. A. Ilyushin and P. M. Ogibalov [8.211]; pressures with a magnetic field-by V. P. Demutsky and R. V. Polovin [3.131] and by Yu. N. Shevtchenko [8.473]; pressures with torsion of a cylinder 
subject to ultrasounds--by I. G. Dovgyallo, V. I. Bord and S. S. Makarevitch [8.118].

Finally, let us mention a number of papers on shakedown problems of cylinders under more general combined loadings. K. R. Merckx [8.336] considered a tube under pressure and cyclic neutron irradiation. Variable thermal loadings of rotating cylinders were analysed by A. Sh. Dadashev [8.102] and by Yu. N. Shevtchenko [4.528]. Cyclic torsion and heating was discussed by D. G. Owen [8.380], and cyclic heating superposed on steady tension with torsion of cylinders was studied in detail by V. Sagar and D. J. Payne [8.434, 8.435].

\subsection{Combined loadings of thick-walled spheres}

The problems of combined loadings in thick-walled spheres, if conforming to the conditions of polar symmetry, are closely related to those arising in thick-walled cylinders, and hence some authors even consider these two cases jointly. However, the number of independent loadings is smaller here (e.g. the axial force in cylinders has no counterpart in thick-walled spheres), and thus the most important case of combined loadings in spheres is that of pressure difference with a temperature gradient.

M. G. Derrington and W. Johnson [8.114] investigated the onset of yielding and determined the optimal temperature distribution. V. M. Sobolevsky [8.495] considered an elastic-plastic sphere in an elastic medium subject to internal pressure and thermal loading. M. Rogoziński [8.426] assumed a linear dependence of the yield-point stress on temperature. Further papers are due to E. Whalley [8.557], W. Johnson and P. B. Mellor [8.219] (a detailed analysis of the formation of plastic zones), K. A. V. Pandalai and S. A. Patel [8.385] (plastic hardening of the Ramberg-Osgood type), S. A. Vulman [8.550] (eccentric hollow sphere), Yu. V. Nemirovsky and M. L. Kheynloo [8.365] (plastic hardening of multilayer spherical vessels). A thick-walled sphere under heat sources was considered by S. Glasstone [8.167], and under pressure combined with neutron irradiation-by A. A. Ilyushin and P. M. Ogibalov [8.211].

Shakedown problems of thick-walled spheres were discussed by D. A. Gokhfeld and P. I. Ermakov [8.173, 8.174], and by A. Sh. Dadashev [8.103]. H. Ishikawa [8.214] employed the incremental theory of plasticity for the analysis of transient thermal stresses in a hollow sphere.

\subsection{Experimental investigations of thin-walled tubes under combined loadings}

As it was mentioned in Sec. 37.1, the state of stress in thin-walled tubes under tension, torsion and internal pressure is both transversally and longitudinally homogeneous (or, strictly speaking, its non-homogeneity is in most cases negligibly small), there exists a direct transition between the levels $\mathscr{P}$ and $\mathscr{B}$, and hence numerous experimental investigations of tubes serve as a basis for the formulation or verification of physical laws at the level $\mathscr{P}$. Normal force results here in axial stresses 
$\sigma_{z}$ and internal pressure-in circumferential stresses $\sigma_{\theta}$, and within the range of small strains and small deflections their action may be regarded as similar; however, if finite strains are investigated, then certain differences appear (circumferential stresses increase not only because of the decreasing cross-sectional area but also because of the increasing diameter of the tube).

Extensive survey papers on experimental investigations of thin-walled tubes were published by M. J. Michno and W. N. Findley [8.339], and by K. Ikegami [8.208]. In what follows, we only mention some classical and more recent investigations, classifying them according to the type of loading employed.

In the simplest combined case-tension with internal pressure-we have biaxial stress states with fixed principal directions. J. Marin and J. A. Sauer [3.505], L. W. Hu and J. Marin [3.272] investigated in this way physical laws for aluminium alloys; A. M. Zhukov [3.866]-for the steel EI 415; N. Alberti [3.3]-yield condition for cast iron; J. Miastkowski and W. Szczepiński [3.521]-influence of prestraining on the yield condition for brass; G. B. Talypov and A. I. Chistyakov [8.520]-a similar effect for steel; J. F. Bratt and Ö. Kanan [8.58]-yield condition for an aluminium alloy in the range of biaxial compression; V. N. Bastun and N. I. Chernyak [3.34]-yield condition for an anisotropic steel; S. S. Hecker [3.238]-existence of corners in neutral surfaces for various metals; V. N. Bastun, V. M. Perepelin and L. M. Shkaraputa [8.31]-physical laws for titanium alloys; V. N. Bastun [3.38]-physical laws for steel at elevated temperatures.

The case of simultaneous tension and torsion is the most frequent one. The principal directions are then subject to change, but this change is coupled with the change of the ratio of principal stresses. This type of combined loadings was used in the investigations of the initial neutral surface (initial yield condition) by A. Ono [8.377] (copper, aluminium alloys), and of subsequent neutral surfaces by P. M. Naghdi, F. Essenburg and W. Koff [3.568] (aluminium alloy 245-T-4, various loading paths), Yu. I. Yagn and O. A. Shishmarev [8.561] (prestraining by torsion), J. Rogan and A. Shelton [3.677, 3.678] (steel En 25), S. Noto La Diega and A. Passannanti [8.367] (verification of the Stassi d'Alia hypothesis), J. F. Williams and N. L. Svenson [3.845] (aluminium, prestraining by tension), A. Phillips and J. L. Tang [3.637] (effect of loading path, aluminium), T. Inoue, K. Tanaka and T. Ishizaki [3.293] (aluminium alloys and steel at elevated temperatures), Y. Ohashi, K. Kawashima and S. Nagahiko [8.375] (steel), E. Shiratori, K. Ikegami, F. Yoshida, K. Kaneko and S. Koike [8.475] [aluminium alloy, deviations from the HMH hypothesis), A. Phillips and M. Ricciuti [8.398] (aluminium). Formation of plastic corners was investigated by A. Phillips and G. A. Gray [3.632], P. K. Bertsch and W. N. Findley [3.57], O. A. Shishmarev [8.476, 8.477] (nickel), M. J. Michno and W. N. Findley [3.526]. The effect of cyclic loading on subsequent neutral surfaces was investigated by F. P. Rybalko, A. V. Kostenko and L. P. Zelenin [8.429], R. L. Sierakowski and A. Phillips [3.740].

Simultaneous tension with torsion of thin-walled tubes was also frequently used for the formulation or verification of constitutive (physical) laws of plasticity. 
Let us mention here the papers by B. Budiansky, N. F. Dow, R. W. Peters and R. P. Shepherd [8.61] (aluminium alloy 14 S-T 4), A. Phillips [8.396] (aluminium 2S-0), A. M. Zhukov and Yu. N. Rabotnov [8.572, 3.865], (steel 20 and steel 25), E. A. Davis [8.112] (steel), J. Marin and H. A. B. Wiseman [8.329] (aluminium alloy 14S-TG), P. M. Naghdi, J. C. Rowley and C. W. Beadle' [3.567] (aluminium alloy), M. Feigen [8.139] (aluminium alloy), V. A. Sveshnikova [8.512] (copper, brass, aluminium), B. M. Malyshev [8.323, 8.324] (copper, brass, aluminium, steel), T. Ota, A. Shinda and H. Fukuoka [3.607] (steel, problem of anisotropy), B. Paul, W. Chen and Lee Li-Chih [3.619] (aluminium alloy, stepped loading), J. L. Schlafer and O. M. Sidebottom [8.449, 8.450, 8.481] (verification of the deformation and incremental theories), S. Smith and B. O. Almroth [3.746] (aluminium, deviations from the classical incremental theories), N. N. Kudryashov and B. A. Rytchkov [3.401], O. A. Shishmarev [8.478] (brass, steel, problem of anisotropy), O. M. Sidebottom and R. K. Bhattacharyya [8.482] (physical laws for a Tresca material), S. Komatsu and T. Sakimoto [8.254] (hypothesis of a uniform curve of plastic hardening in torsion with compression), Y. Ohashi, M. Tokuda and H. Yamashita [3.591, 3.593] (steel, trajectories with corners and trajectories of constant curvature). Various aspects of the Ilyushin geometrical theory of plasticity were verified by V. S. Lensky [3.430], L. S. Andreyev [3.7, 3.8], R. A. Vasin and A. B. Ibragimov [3.835], A. B. Ibragimov and I. D. Mashkov [3.283]. Finite plastic strains were investigated by V. I. Maksak and G. A. Doshtchinsky [8.320, 8.321].

In the case of simultaneous torsion and internal pressure the change of principal directions is also related to the ratio of principal stresses. This type of loading was used by S. S. Gill [8.166] (investigations of neutral loading), Yang Nan-Sheng [8.562] (physical laws for brass), Sh. N. Kats and L. M. Kachanov [8.232] (physical laws for steel), J. Parker and M. B. Basset [3.617] (investigations of plastic corners).

In the most general homogeneous case of tension with torsion and internal pressure, $\mathscr{P}_{3} \rightarrow \mathscr{B}_{3}$, we may control independently the principal directions and the ratio of principal stresses. This type of loading was employed by A. Phillips and L. Kaechele [8.397] (physical laws for aluminium 2S-0), K. Saitō and H. Igaki [8.439] (anisotropic hardening of copper), N. M. Mitrokhin [3.533] (hypothesis of a uniform curve of hardening for nickel), S. S. Gill and J. Parker [3.199] (physical laws for brass), N. Ya. Mikhaylov and Yu. I. Yagn [8.343] (loss of stability of plastic deformations), J. Parker and J. Kettlewell [8.388] (physical laws for copper), and by L.S. Andreyev [3.6] (verification of the Ilyushin postulate of isotropy).

\section{Combined loadings of cylinders without circular symmetry}

\subsection{General case of combined loadings of a thin-walled tube}

The problem of the limit carrying capacity of a thin-walled tube was analysed by A. A. Gvozdev [4.196] for a relatively general combined loading, consisting of an 
axial force $N$, two shear forces $V_{x}$ and $V_{y}$, two bending moments $M_{b x}$ and $M_{b y}$, and a twisting moment $M_{z}$. He employed a variational approach to statically partially admissible stress fields (satisfying the yield condition and the boundary conditions, and, in general, not satisfying the equilibrium equations), and hence he obtained an upper bound to the actual limit carrying capacity (cf. Sec. 20.4). General as it is, the solution obtained in [4.196] does not take into account the pressure $p$, which is one of the most essential loadings, e.g. for a pipe-line. Following the papers by J. Skrzypek and M. Życzkowski [8.491, 8.492], we now present a more general solution, allowing for internal pressure.

Consider a perfectly plastic thin-walled tube in a seven-fold combined case of loading (Fig. 193). Under the approximate assumption of no circumferential
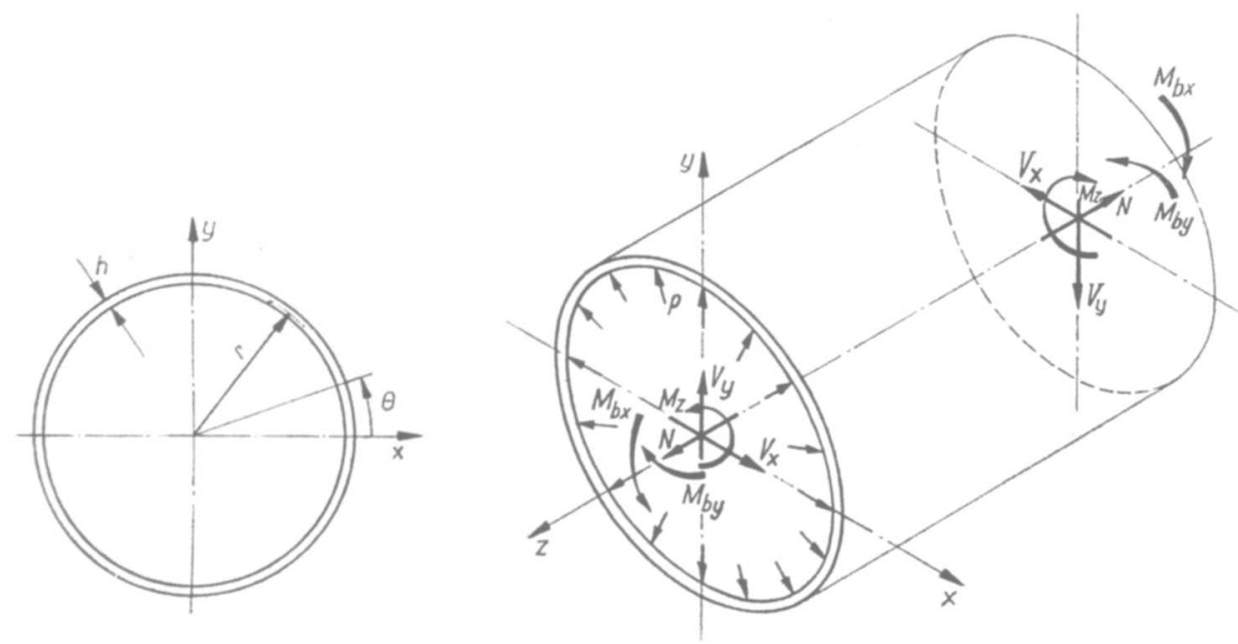

Fig. 193. Thin-walled tube in a seven-fold combined case of loading

bending in the wall of the tube, the state of stress is determined by the three components $\sigma_{z}, \sigma_{\theta}$ and $\tau_{z \theta}$ constant across the wall thickness, and hence the problem may be classified as $\mathscr{P}_{3} / \mathscr{B}_{7}$. Assume that these stress components satisfy the HMH yield condition in the form

$$
\sigma_{z}^{2}-\sigma_{z} \sigma_{\theta}+\sigma_{\theta}^{2}+3 \tau_{z \theta}^{2}=\sigma_{0}^{2}
$$

at any point of the tube (or, more accurately, at any point of the dangerous crosssection of the tube). Moreover, we assume the stresses $\sigma_{\theta}$ to be constant along the perimeter, and determined by the elementary pressure-vessel formula

$$
\sigma_{0}=\frac{r}{h} p=\text { const, }
$$


where $r$ denotes the mean radius and $h$ the wall thickness. On the other hand, the equilibrium equation [the third equation of (6.11)]

$$
\frac{\partial \tau_{z \theta}}{\partial \theta}=0
$$

will, in general, not be satisfied; form (38.3) should hold at least if the shearing forces $V_{x}$ and $V_{y}$ vanish (since then $\partial \sigma_{z} / \partial z=0$ ).

Formula (38.2) expresses directly the loading $p$ in terms of the stresses $\sigma_{\theta}$, whereas the remaining loadings are determined by the integral formulae

$$
\begin{aligned}
& N=\int_{0}^{2 \pi} \sigma_{z} h r d \theta, \quad M_{b x}=\int_{0}^{2 \pi} \sigma_{z} h r^{2} \sin \theta d \theta, \\
& M_{b y}=\int_{0}^{2 \pi} \sigma_{z} h r^{2} \cos \theta d \theta, \quad M_{t}=M_{z}=\int_{0}^{2 \pi} \tau_{z \theta} h r^{2} d \theta, \\
& V_{x}=\int_{0}^{2 \pi} \tau_{z \theta} h r \sin \theta d \theta, \quad V_{y}=\int_{0}^{2 \pi} \tau_{z \theta} h r \cos \theta d \theta .
\end{aligned}
$$

In what follows it will be more convenient to use dimensionless loadings, namely

$$
\begin{array}{lll}
n=\frac{N}{2 \pi r h \sigma_{0}}, & m_{x}=\frac{M_{b x}}{4 r^{2} h \sigma_{0}}, & m_{y}=\frac{M_{b y}}{4 r^{2} h \sigma_{0}}, \\
m_{z}=\frac{\sqrt{3} M_{z}}{2 \pi r^{2} h \sigma_{0}}, & v_{x}=\frac{\sqrt{3} V_{x}}{4 r h \sigma_{0}}, & v_{y}=\frac{\sqrt{3} V_{y}}{4 r h \sigma_{0}}
\end{array}
$$

and

$$
q=\frac{r}{h \sigma_{0}} p
$$

We now introduce an appropriate parametrization of the yield condition (38.1), generalizing the Gvozdev parametrization

$$
3 \tau_{z \theta}^{2}=\sigma_{0}^{2} \sin ^{2} \psi, \quad \sigma_{z}^{2}-\sigma_{z} \sigma_{\theta}+\sigma_{0}^{2}=\sigma_{0}^{2} \cos ^{2} \psi,
$$

which may be obtained by setting $\sigma_{\theta}=0$. The stress components may be expressed in terms of the new dependent variable $\psi=\psi(\theta)$ as follows:

$$
\begin{aligned}
& \tau_{z 0}=\frac{\sigma_{0}}{\sqrt{3}} \sin \psi, \quad \sigma_{0}=\frac{r}{h} p=\text { const }, \\
& \sigma_{z}=\frac{1}{2}\left|\sigma_{\theta} \mp \sqrt{4 \sigma_{0}^{2} \cos ^{2} \psi-3 \sigma_{\theta}^{2}}\right|,
\end{aligned}
$$

where the upper sign holds in a certain interval $\theta_{1}^{*}<\theta<\theta_{2}^{*}$ as yet unknown, and the lower sign, in the remaining interval $\theta_{2}^{*}<\theta<\theta_{1}^{*}+2 \pi$.

Substituting (38.8) into (38.4) and taking account of (38.5) we may now express the dimensionless loadings in terms of $\psi(\theta)$ and of the constant stress $\sigma_{\theta}$ as follows: 


$$
\begin{aligned}
& n=\frac{1}{2 \pi}\left\{\int_{\theta_{1}^{*}}^{\theta_{2}^{*}}\left[\frac{\sigma_{\theta}}{2 \sigma_{0}}-\sqrt{\cos ^{2} \psi-\frac{3}{4} \frac{\sigma_{\theta}^{2}}{\sigma_{0}^{2}}}\right] d \theta+\right. \\
& \left.+\int_{\theta_{2}^{*}}^{\theta_{1}^{*}+2 \pi}\left[\frac{\sigma_{\theta}}{2 \sigma_{0}}+\sqrt{\cos ^{2} \psi-\frac{3}{4} \frac{\sigma_{\theta}^{2}}{\sigma_{0}^{2}}}\right] d \theta\right\}, \\
& m_{x}=\frac{1}{4}\left\{\int_{\theta_{1}^{*}}^{\theta_{2}^{*}}\left[\frac{\sigma_{0}}{2 \sigma_{0}}-\sqrt{\cos ^{2} \psi-\frac{3}{4} \frac{\sigma_{\theta}^{2}}{\sigma_{0}^{2}}}\right] \sin \theta d \theta+\right. \\
& \left.+\int_{\theta_{2}^{*}}^{\theta_{1}^{*}+2 \pi}\left[\frac{\sigma_{\theta}}{2 \sigma_{0}}+\sqrt{\cos ^{2} \psi-\frac{3}{4} \frac{\sigma_{\theta}^{2}}{\sigma_{0}^{2}}}\right] \sin \theta d \theta\right\}, \\
& m_{y}=\frac{1}{4}\left\{\int_{\theta_{1}^{*}}^{\theta_{2}^{*}}\left[\frac{\sigma_{\theta}}{2 \sigma_{0}}-\sqrt{\cos ^{2} \psi-\frac{3}{4} \frac{\sigma_{\theta}^{2}}{\sigma_{0}^{2}}}\right] \cos \theta d \theta+\right. \\
& \left.+\int_{\theta_{2}^{*}}^{\theta_{1}^{*}+2 \pi}\left[\frac{\sigma_{\theta}}{2 \sigma_{0}}+\sqrt{\cos ^{2} \psi-\frac{3}{4} \frac{\sigma_{\theta}^{2}}{\sigma_{0}^{2}}}\right] \cos \theta d \theta\right\}, \\
& m_{z}=\frac{1}{2 \pi} \int_{0}^{2 \pi} \sin \psi d \theta \text {, } \\
& v_{x}=\frac{1}{4} \int_{0}^{2 \pi} \sin \psi \sin \theta d \theta, \quad v_{y}=\frac{1}{4} \int_{0}^{2 \pi} \sin \psi \cos \theta d \theta .
\end{aligned}
$$

Let us now apply a variational approach. For the assumed constant value of $\sigma_{\theta}$ (i.e. of the pressure $q$ ), and for fixed values of five other loadings we look for the maximal value of the remaining (seventh) one; the only unknown function is $\psi=\psi(\theta)$, but the limits of integration $\theta_{1}^{*}$ and $\theta_{2}^{*}$ are also subject to variation (isoperimetric problem with variable end-points). Introducing suitable multipliers $\lambda_{i}$, we write the Lagrangian functional as follows:

$$
\tilde{F}[\psi(\theta)]=\lambda_{1} 2 \pi n+\lambda_{2} 2 \pi m_{z}+\lambda_{3} 4 m_{y}+\lambda_{4} 4 v_{x}+\lambda_{5} 4 m_{x}+\lambda_{6} 4 v_{y} .
$$

Substituting the integral formulae (38.9) for the loadings and rearranging (38.10), we arrive at

$$
\tilde{F}[\psi(\theta)]=\int_{\theta_{1}^{*}}^{\theta_{2}^{*}} g_{1}[\theta, \psi(\theta)] d \theta+\int_{\theta_{2}^{*}}^{\theta_{1}^{*}+2 \pi} g_{2}[\theta, \psi(\theta)] d \theta,
$$


where the following notation is introduced:

$$
\begin{aligned}
g_{1,2}= & \left(\lambda_{1}+\lambda_{3} \cos \theta+\lambda_{5} \sin \theta\right)\left(\frac{\sigma_{\theta}}{2 \sigma_{0}} \mp \sqrt{\cos ^{2} \psi-\frac{3}{4} \frac{\sigma_{\theta}^{2}}{\sigma_{0}^{2}}}\right)+ \\
& +\left(\lambda_{2}+\lambda_{4} \sin \theta+\lambda_{6} \cos \theta\right) \sin \psi .
\end{aligned}
$$

The Lagrangian multipliers are ascribed here to all the six loadings $n, v_{x}, v_{y}, m_{x}$, $m_{y}, m_{z}$ for the sake of symmetry of notation; in fact, one of these multipliers is unnecessary (being equal to unity), and in the final formulae only five ratios of these multipliers appear.

We look for such functions $\psi=\psi_{1}(\theta)$ in $\theta_{1}^{*}<\theta<\theta_{2}^{*}$ and $\psi=\psi_{2}(\theta)$ in $\theta_{2}^{*}<\theta<\theta_{1}^{*}+2 \pi$, and for such values $\theta_{1}^{*}$ and $\theta_{2}^{*}$ which maximize the functional (38.11). The Euler-Lagrange equations for the functional (38.11)-in view of the absence of the derivative $\psi^{\prime}(\theta)$ in the integrands - are

$$
\frac{\partial g_{1}}{\partial \psi_{1}}=0 \quad \text { and } \quad \frac{\partial g_{2}}{\partial \psi_{2}}=0
$$

to be satisfied in the two intervals, respectively. The boundary angles will be found from the continuity conditions

$$
g_{1}=g_{2} \text { for } \theta=\theta_{1}^{*} \text { and } \theta=\theta_{2}^{*},
$$

which, after all, result from the general transversality conditions for the case under consideration.

The above four conditions with substituted (38.12) lead to the equations for $\psi_{1}$ and $\psi_{2}$

$$
\begin{aligned}
& \left(\lambda_{1}+\lambda_{3} \cos \theta+\lambda_{5} \sin \theta\right) \sin \psi_{1}+ \\
& \quad+\left(\lambda_{2}+\lambda_{4} \sin \theta+\lambda_{6} \cos \theta\right) \sqrt{\cos ^{2} \psi_{1}-\frac{3}{4} \frac{\sigma_{\theta}^{2}}{\sigma_{0}^{2}}}=0, \\
& \left(\lambda_{1}+\lambda_{3} \cos \theta+\lambda_{5} \sin \theta\right) \sin \psi_{2}- \\
& \quad-\left(\lambda_{2}+\lambda_{4} \sin \theta+\lambda_{6} \cos \theta\right) \sqrt{\cos ^{2} \psi_{2}-\frac{3}{4} \frac{\sigma_{\theta}^{2}}{\sigma_{0}^{2}}}=0,
\end{aligned}
$$

and for both boundary angles $\theta_{1}^{*}$ and $\theta_{2}^{*}$

$$
\lambda_{1}+\lambda_{3} \cos \theta^{*}+\lambda_{5} \sin \theta^{*}=0 .
$$

Solving (38.15) with respect to $\psi_{1}$ and $\psi_{2}$, we obtain

$$
\begin{aligned}
& \sin \psi_{1}=-\frac{x\left(\lambda_{2}+\lambda_{4} \sin \theta+\lambda_{6} \cos \theta\right) \sqrt{1-\frac{3}{4} \frac{\sigma_{\theta}^{2}}{\sigma_{0}^{2}}}}{\sqrt{\left(\lambda_{1}+\lambda_{3} \cos \theta+\lambda_{5} \sin \theta\right)^{2}+\left(\lambda_{2}+\lambda_{4} \sin \theta+\lambda_{6} \cos \theta\right)^{2}}}, \\
& \sin \psi_{2}=+\frac{x\left(\lambda_{2}+\lambda_{4} \sin \theta+\lambda_{6} \cos \theta\right) \sqrt{1-\frac{3}{4} \frac{\sigma_{\theta}^{2}}{\sigma_{0}^{2}}}}{\sqrt{\left(\lambda_{1}+\lambda_{3} \cos \theta+\lambda_{5} \sin \theta\right)^{2}+\left(\lambda_{2}+\lambda_{4} \sin \theta+\lambda_{6} \cos \theta\right)^{2}}},
\end{aligned}
$$


for the intervals $\left(\theta_{1}^{*}, \theta_{2}^{*}\right)$ and $\left(\theta_{2}^{*}, \theta_{1}^{*}+2 \pi\right)$, respectively; for the sake of brevity the following symbol has been introduced here:

$$
x=\operatorname{sign}\left(\lambda_{1}+\lambda_{3} \cos \theta+\lambda_{5} \sin \theta\right) \text {. }
$$

However, bearing in mind the definitions of the intervals " 1 " and " 2 " and in view of (38.16), we state that in the interval " 1 ", $\theta_{1}^{*}<\theta<\theta_{2}^{*}$ one obtains

$$
\varkappa=\varkappa_{1}=\operatorname{sign}\left(\lambda_{1}+\lambda_{3} \cos \theta+\lambda_{5} \sin \theta\right)=-1,
$$

whereas in the interval " 2 ", $\theta_{2}^{*}<\theta<\theta_{1}^{*}+2 \pi$ we have

$$
x=x_{2}=\operatorname{sign}\left(\lambda_{1}+\lambda_{3} \cos \theta+\lambda_{5} \sin \theta\right)=+1 ;
$$

hence, finally

$$
\sin \psi_{1}=\sin \psi_{2}=+\frac{\left(\lambda_{2}+\lambda_{4} \sin \theta+\lambda_{6} \cos \theta\right) \sqrt{1-\frac{3}{4} \frac{\sigma_{\theta}^{2}}{\sigma_{0}^{2}}}}{\sqrt{\left(\lambda_{1}+\lambda_{3} \cos \theta+\lambda_{5} \sin \theta\right)^{2}+\left(\lambda_{2}+\lambda_{4} \sin \theta+\lambda_{6} \cos \theta\right)^{2}}} .
$$

The boundary angles $\theta_{1}^{*}$ and $\theta_{2}^{*}$ are determined by Eq. (38.16), which, in general, has two roots in the interval $0<\theta<2 \pi$. However, it turns out that the values of these angles do not appear in the final equations of the plastic interaction surface. To prove this statement we calculate the root occurring in the functions $g_{1}$ and $g_{2},(38.12)$; in view of (38.21) we obtain

$$
\sqrt{\cos ^{2} \psi-\frac{3}{4} \frac{\sigma_{\theta}^{2}}{\sigma_{0}^{2}}}=\frac{\varkappa\left(\lambda_{1}+\lambda_{3} \cos \theta+\lambda_{5} \sin \theta\right) \sqrt{1-\frac{3}{4} \frac{\sigma_{\theta}^{2}}{\sigma_{0}^{2}}}}{\sqrt{\left(\lambda_{1}+\lambda_{3} \cos \theta+\lambda_{5} \sin \theta\right)^{2}+\left(\lambda_{2}+\lambda_{4} \sin \theta+\lambda_{6} \cos \theta\right)^{2}}},
$$

where $x$ is defined by (38.18), and hence $x=-1$ in the first interval and $x=+1$ in the second one. Thus these roots differ in sign only; on the other hand, the functions $g_{1}$ and $g_{2}$ differ also only in sign before that root, and hence the integrand is identical in both intervals. Finally, making use of the periodicity of that integrand, we obtain the following formulae for the loadings in the limit state:

$$
\begin{aligned}
& \boldsymbol{n}=\frac{\sigma_{\theta}}{2 \sigma_{0}}+
\end{aligned}
$$

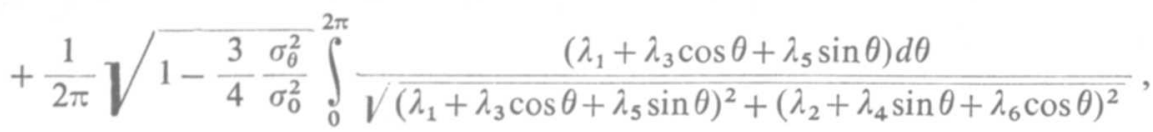

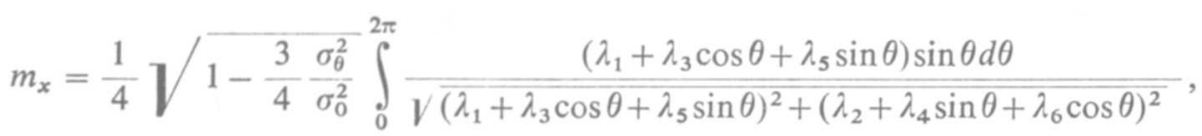

$$
\begin{aligned}
& m_{y}=\frac{1}{4} \sqrt{1-\frac{3}{4} \frac{\sigma_{\theta}^{2}}{\sigma_{0}^{2}}} \int_{0}^{2 \pi} \frac{\left(\lambda_{1}+\lambda_{3} \cos \theta+\lambda_{5} \sin \theta\right) \cos \theta d \theta}{\sqrt{\left(\lambda_{1}+\lambda_{3} \cos \theta+\lambda_{5} \sin \theta\right)^{2}+\left(\lambda_{2}+\lambda_{4} \sin \theta+\lambda_{6} \cos \theta\right)^{2}}},
\end{aligned}
$$




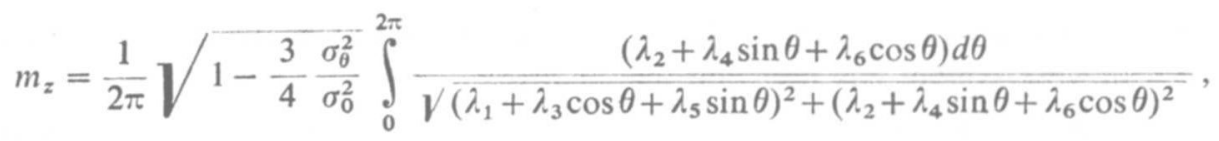

$$
\begin{aligned}
& v_{x}=\frac{1}{4} \sqrt{1-\frac{3}{4} \frac{\sigma_{\theta}^{2}}{\sigma_{0}^{2}}} \int_{0}^{2 \pi} \frac{\left(\lambda_{2}+\lambda_{4} \sin \theta+\lambda_{6} \cos \theta\right) \sin \theta d \theta}{\sqrt{\left(\lambda_{1}+\lambda_{3} \cos \theta+\lambda_{5} \sin \theta\right)^{2}+\left(\lambda_{2}+\lambda_{4} \sin \theta+\lambda_{6} \cos \theta\right)^{2}}},
\end{aligned}
$$

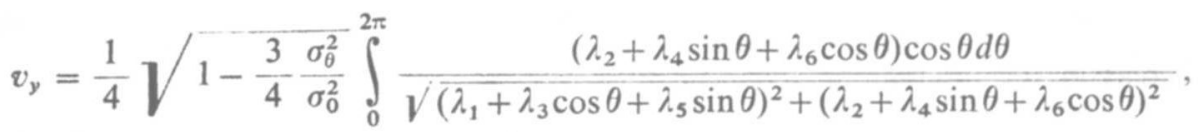

and additionally

$$
q=\frac{\sigma_{\theta}}{\sigma_{0}} .
$$

The integrals (38.23) are elliptic integrals. Their reduction to normal form presents some difficulties in the general case, but in particular cases the procedure is much simpler. These integrals determine the plastic interaction surface (hypersurface) in the function of five parameters, namely the ratios of the Lagrangian multipliers $\lambda_{i}$. In fact, they furnish an upper bound to the plastic interaction surface; J. Skrzypek [8.492] constructed kinematically admissible velocity fields, leading to the same results, and this fact also verifies the above statement.

\subsection{Particular cases for a thin-walled tube}

Suppose that in Eqs. (38.23) $\lambda_{2}=\lambda_{5}=\lambda_{6}=0$; then the denominators of the respective integrands, equal to $\left[\left(\lambda_{1}+\lambda_{3} \cos \theta\right)^{2}+\lambda_{4}^{2} \sin ^{2} \theta\right]^{1 / 2}$, become symmetric with respect to $\theta=0$ and $\theta=\pi$. Hence all the integrals whose numerators are antysymmetric with respect to $\theta=0$ or $\theta=\pi$ vanish-we obtain $m_{x}=m_{z}=v_{y}=0$. Thus we arrive at the four-fold combined case of bending and shear in the plane $x z$, tension and internal pressure. Let us introduce the following symbols for the ratios of $\lambda_{i}$ :

$$
\Lambda_{1}=\frac{\lambda_{1}}{\lambda_{3}}, \quad \Lambda_{4}=\frac{\lambda_{4}}{\lambda_{3}},
$$

and eliminate $\sigma_{\theta}$ by means of (38.24); then the remaining loadings may be represented as follows:

$$
\begin{aligned}
& n=\frac{q}{2}+\frac{1}{\pi} \sqrt{1-\frac{3}{4} q^{2}} \int_{0}^{\pi} \frac{\left(\Lambda_{1}+\cos \theta\right) d \theta}{\sqrt{\left(\Lambda_{1}+\cos \theta\right)^{2}+\Lambda_{4}^{2} \sin ^{2} \theta}}, \\
& m_{y}=\frac{1}{2} \sqrt{1-\frac{3}{4} q^{2}} \int_{0}^{\pi} \frac{\left(\Lambda_{1}+\cos \theta\right) \cos \theta d \theta}{\sqrt{\left(\Lambda_{1}+\cos \theta\right)^{2}+\Lambda_{4}^{2} \sin ^{2} \theta}}, \\
& v_{x}=\frac{1}{2} \sqrt{1-\frac{3}{4} q^{2}} \int_{0}^{\pi} \frac{\Lambda_{4} \sin ^{2} \theta d \theta}{\sqrt{\left(\Lambda_{1}+\cos \theta\right)^{2}+\Lambda_{4}^{2} \sin ^{2} \theta}} .
\end{aligned}
$$


Elliptic integrals (38.26) may be reduced to their normal form by means of the substitution

$$
\zeta=2 \operatorname{arccot} \sqrt{\frac{\alpha_{1}(1-\cos \theta)}{\alpha_{2}(1+\cos \theta)}},
$$

where

$$
\alpha_{1,2}^{2}=\left(\mp 1-\frac{\Lambda_{1}}{1-\Lambda_{4}^{2}}\right)^{2}+\frac{\Lambda_{1}^{2}+\Lambda_{4}^{2}}{1-\Lambda_{4}^{2}}-\frac{\Lambda_{1}^{2}}{\left(1-\Lambda_{4}^{2}\right)^{2}} .
$$

The final formulae contain complete elliptic integrals of all the three kinds, namely

$$
\begin{aligned}
& n= \frac{q}{2}-\frac{2}{\pi} \sqrt{\frac{1-\frac{3}{4} q^{2}}{1-\Lambda_{1}^{2}}}\left[\left(b-\Lambda_{1}\right) \mathbf{K}(k)-b \mathbf{\Pi}\left(\frac{1}{b^{2}-1}, k\right)\right], \\
& m_{y}= \sqrt{\frac{1-\frac{3}{4} q^{2}}{1-\Lambda_{1}^{2}}}\left\{\left(2 b^{2}-b \Lambda_{1}-1\right) \mathbf{K}(k)+\frac{b^{2}-1}{k^{\prime 2}+k^{2} b^{2}} \mathbf{E}(k)-\right. \\
&\left.-b\left[2 b-\Lambda_{1}-\frac{\left(1-2 k^{2}+2 b^{2} k^{2}\right) b}{k^{\prime 2}+k^{2} b^{2}} \mathbf{\Pi}\left(\frac{1}{b^{2}-1}, k\right)\right]\right\}, \\
& v_{x}=\Lambda_{4} \sqrt{\frac{1-\frac{3}{4} q^{2}}{1-\Lambda_{1}^{2}}}\left\{2\left(b^{2}-1\right) \mathbf{K}(k)-\frac{b^{2}-1}{k^{\prime 2}+k^{2} b^{2}} \mathbf{E}(k)+\right. \\
&\left.+b^{2}\left[2-\frac{1-2 k^{2}+b^{2} k^{2}}{k^{\prime 2}+k^{2} b^{2}}\right] \mathbf{\Pi}\left(\frac{1}{b^{2}-1}, k\right)\right\},
\end{aligned}
$$

where

$$
b=-\frac{\alpha_{1}+\alpha_{2}}{\alpha_{1}-\alpha_{2}}, \quad k^{2}=\frac{1-\Lambda_{1}^{2}-\Lambda_{4}^{2}}{1-\Lambda_{1}^{2}}, \quad k^{\prime 2}=1-k^{2} .
$$

The four-fold combined case under consideration was also analysed by $\mathrm{M}$. I. Erkhov [8.129].

Further particular cases were discussed in detail by J. Skrzypek and M. Życzkowski [8.491, 8.492]. We quote here the solution for the important case of bending with torsion, obtained earlier by A. A. Gvozdev [4.196]. Assuming in (38.23) $\lambda_{1}=\lambda_{4}=\lambda_{5}=\lambda_{6}=0$ and additionally $\sigma_{\theta}=0$, we obtain $n=m_{x}=v_{x}=v_{y}$ $=q=0$, and the elliptic integrals in the formulae for the remaining loadings may be reduced to their normal form as follows:

$$
\begin{aligned}
& m_{\nu}=m_{b}=\sqrt{1+\Lambda_{2}^{2}}\left[\mathbf{E}(k)-\frac{\Lambda_{2}^{2}}{1+\Lambda_{2}^{2}} \mathbf{K}(k)\right], \\
& m_{z}=m_{t}=\frac{2 \Lambda_{2}}{\pi \sqrt{1+\Lambda_{2}^{2}}} \mathbf{K}(k),
\end{aligned}
$$

where

$$
\Lambda_{2}=\frac{\lambda_{2}}{\lambda_{3}}, \quad k^{2}=\frac{1}{1+\Lambda_{2}^{2}} .
$$


Hence we have obtained Eqs. (28.44) in a slightly different notation. They furnish an upper bound also for a solid circular section; the corresponding plastic interaction curve is shown in Fig. 148 (upper bound).

Simultaneous bending with torsion of thin-walled tubes was also analysed by R. Hill and M. P.L. Siebel [6.94, 8.484], and by G. Guerra [8.185]. The limit carrying capacity in the four-fold combined case of bending, torsion, tension and pressure was discussed in detail by J. Skrzypek [8.492], L. D. Larson, W. F. Stokey and J. E. Panarelli [8.296].

\subsection{Effect of circumferential bending of the wall}

The solutions given in preceding section were based on the assumption of a purely membrane state in the wall of the tube, regarded as a circular cylindrical shell. This assumption was also adopted by M.I. Erkhov [8.128], A. I. Vinogradov and S. P. Fesik [8.545], who considered a more particular but very important case of simultaneous internal pressure with bending. However, in the plastic range a circumferential bending (moment $m_{\theta}$ ) may appear, and this effect may result in a certain decrease of the limit carrying capacity. In fact, the first plastification at the point where the maximal negative $\sigma_{z}$ meet the positive $\sigma_{\theta}$ results in an apparent weakening of the wall, and consequently in circumferential bending. This problem was investigated by M. Mrowiec and M. Życzkowski [7.206, 4.397].

By the use of a rather complicated statically admissible stress field, [7.206], and by employing the Hermite boundary approximation, the following equation of the plastic interaction surface was derived in [4.397]

$$
m=\sum_{j=0}^{k} \alpha_{j k} q^{j},
$$

where

$$
m=\frac{M_{b}}{\pi h r^{2} \sigma_{0}}, \quad q=\frac{r}{h \sigma_{0}} p .
$$

Equation (38.33) furnishes a lower bound for the three-fold combined case-pressure with overall bending and tension, allowing for circumferential bending effects.

Namely, the coefficients $\alpha_{j k}$ depend on the dimensionless normal force $\psi$,

$$
\psi=\frac{N}{2 \pi p r^{2}},
$$

defined differently in comparison to $n$, (38.5). In the case of a pipe with closed ends we have $\psi=1 / 2$; plane strain in the elastic range is characterized by $\psi=v$. Under the assumption of $k=4$ (approximation by a polynomial of the fourth degree) the following formulae for the coefficients $\alpha_{j k}=\alpha_{j k}(\psi)$ were derived:

$$
\alpha_{04}=\frac{4}{\pi}, \quad \alpha_{14}=-(1-2 \psi)\left(1-\frac{\pi}{16} t\right),
$$




$$
\begin{aligned}
\alpha_{24}= & -\frac{3}{2 \pi}+\frac{3}{8}(1-2 \psi)\left(1-\frac{\pi}{16} t\right) t, \\
\alpha_{34}= & \sqrt{1-\psi+\psi^{2}}\left[\frac{3}{\pi}-\frac{3}{4}(1-2 \psi)\left(1-\frac{\pi}{16} t\right) t\right]+ \\
& +3\left(1-\psi+\psi^{2}\right)(1-2 \psi)\left(1-\frac{\pi}{16} t\right)-\frac{16}{\pi}\left(1-\psi+\psi^{2}\right)^{3 / 2}+ \\
& +\frac{\left(1-\psi+\psi^{2}\right)^{3 / 2}}{2(1-2 \psi)}\left[4 \sqrt{1-\psi+\psi^{2}}+(2-\psi) t\right], \\
\alpha_{44}= & -\left(1-\psi+\psi^{2}\right)\left[\frac{3}{2 \pi}-\frac{3}{8}(1-2 \psi)\left(1-\frac{\pi}{16} t\right) t\right]- \\
& -2\left(1-\psi+\psi^{2}\right)^{3 / 2}(1-2 \psi)\left(1-\frac{\pi}{16} t\right)+\frac{12}{\pi}\left(1-\psi+\psi^{2}\right)^{2}- \\
& -\frac{\left(1-\psi+\psi^{2}\right)^{2}}{2(1-2 \psi)}\left[4 \sqrt{1-\psi+\psi^{2}}+(2-\psi) t\right],
\end{aligned}
$$

where $t=h / r$ denotes a dimensionless parameter of the wall thickness.

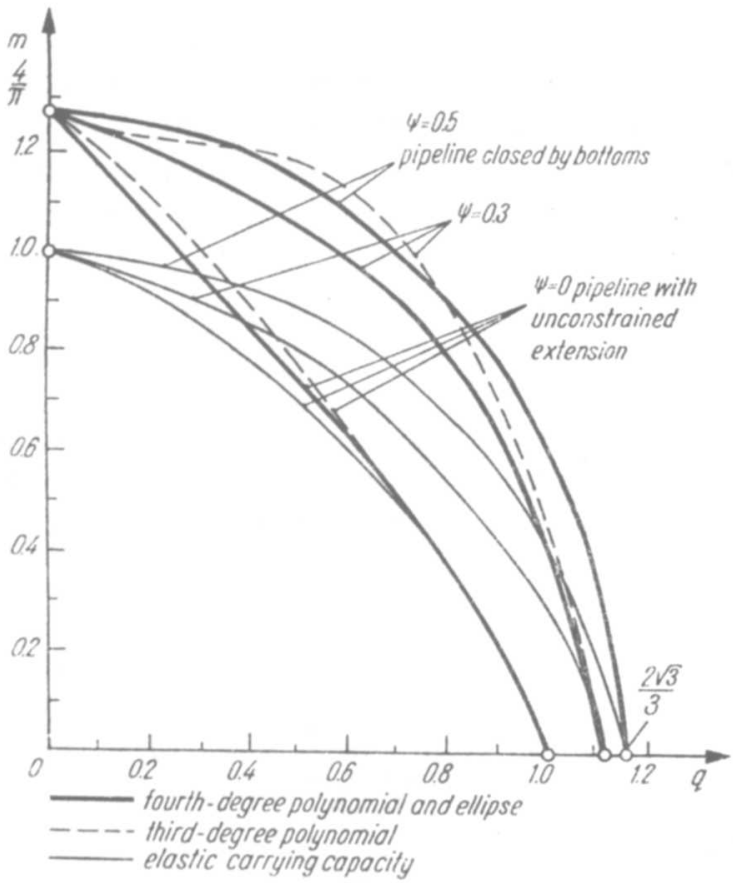

Fig. 194. Plastic interaction curves for a thin-walled pipe-line allowing for circumferential bending of the wall 
In the case of $\psi=1 / 2$ the above formulae become divergent; it turns out, however, that then the effect of circumferential bending vanishes and the following equation of an ellipse is sufficiently accurate:

$$
m=\frac{4}{\pi} \sqrt{1-\frac{3}{4} q^{2}} .
$$

Equation (38.37) follows from (38.23) (with slightly changed notation) and from the more general Erkhov's equations, [8.128]. It can be seen that in this case the derivative $d m / d q$ for $q=2 / \sqrt{3}$ increases infinitely, and this fact results in the divergence of (38.36) mentioned above. For $\psi=0$ the influence of the circumferential bending is fairly remarkable: the formula (38.33) with substituted (38.36) takes (for $t=h / r=0.1$ ) the form

$$
m=1.273-0.979 q-0.440 q^{2}+0.825 q^{3}-0.679 q^{4} \text {. }
$$

This parabola lies clearly below the ellipse (38.37), Fig. 194. However, it should be remembered that (38.38) jgives a lower bound to the actual limit carrying capacity of the tube, and hence the negative effect of circumferential bending resulting from this formula may be overestimated.

\subsection{General longitudinally homogeneous case of combined loadings of a thick-walled tube}

The general circularly symmetric case of combined loadings of a thick-walled tube, considered in Sec. 37, did not include a very important type of loading, namely bending. In fact, bending does not conform to circular symmetry. On the other hand, bending without shear may be considered within the class of logitudinally homogeneous cases: the stresses and strains then depend on $r$ and $\theta$ and do not depend on $z$ in cylindrical coordinates. Following the papers by M. Życzkowski and J. Skrzypek [4.638, 4.541, 4.543] we now consider the limit state of a thickwalled tube under simultaneous internal pressure, tension, torsion, and bending (Fig. 195).

Most of our assumptions coincide with those adopted in Sec. 37: we assume the HMH yield condition, law of similarity of deviators (Hencky-Ilyushin or Levy-Mises), and incompressibility of the material. Moreover, we assume full
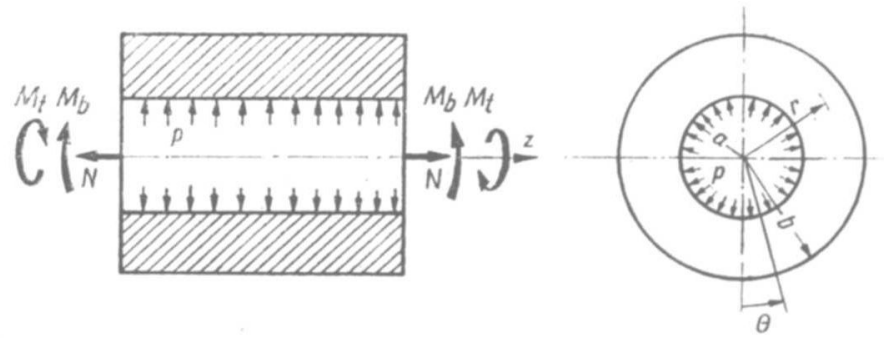

Fig. 195. A thick-walled tube under pressure, tension, torsion and bending 
plastification throughout the tube in the limit state. This assumption, almost obvious in circularly symmetric cases and satisfied also in the case of pure bending, is no longer obvious in the four-fold combined case under consideration. It turns out, in general, that this assumption may be regarded here only as an approximation. However, the approximation errors are in most cases insignificant, and admitting elastic (or rigid) zones in the limit state we would encounter essential complications.

Equations of internal equilibrium in cylindrical coordinates (6.11) where body forces and derivatives with respect to $z$ are omitted take the form

$$
\begin{aligned}
& \frac{\partial \sigma_{r}}{\partial r}+\frac{1}{r} \frac{\partial \tau_{r \theta}}{\partial \theta}+\frac{\sigma_{r}-\sigma_{\theta}}{r}=0, \\
& \frac{\partial \tau_{r \theta}}{\partial r}+\frac{1}{r} \frac{\partial \sigma_{\theta}}{\partial \theta}+2 \frac{\tau_{r \theta}}{r}=0, \\
& \frac{\partial \tau_{r z}}{\partial r}+\frac{1}{r} \frac{\partial \tau_{\theta z}}{\partial \theta}+\frac{\tau_{r z}}{r}=0 .
\end{aligned}
$$

Moreover, we use the compatibility equations (7.52), which are simplified here to the form

$$
\begin{aligned}
& \frac{\partial^{2} \varepsilon_{z}}{\partial r^{2}}=0, \quad \frac{\partial^{2} \varepsilon_{z}}{\partial \theta^{2}}+r \frac{\partial \varepsilon_{z}}{\partial r}=0, \quad \frac{\partial^{2}}{\partial r \partial \theta}\left(\frac{\varepsilon_{z}}{r}\right)=0, \\
& \frac{1}{r} \frac{\partial^{2} \varepsilon_{r}}{\partial \theta^{2}}+\frac{1}{r} \frac{\partial}{\partial r}\left(r^{2} \frac{\partial \varepsilon_{\theta}}{\partial r}\right)-\frac{1}{r} \frac{\partial^{2}\left(r \gamma_{r \theta}\right)}{\partial r \partial \theta}-\frac{\partial \varepsilon_{r}}{\partial r}=0, \\
& \frac{1}{r} \frac{\partial^{2}\left(r \gamma_{\theta z}\right)}{\partial r^{2}}-\frac{\partial^{2}}{\partial r \partial \theta}\left(\frac{\gamma_{z r}}{r}\right)-\frac{1}{r^{2}} \frac{\partial\left(r \gamma_{\theta z}\right)}{\partial r}=0, \\
& \frac{\partial^{2} \gamma_{z r}}{\partial \theta^{2}}-\frac{\partial^{2}\left(r \gamma_{\theta z}\right)}{\partial r \partial \theta}=0 .
\end{aligned}
$$

These nine equations together with the yield condition (37.3), the incompressibility equation (37.4) and the law of similarity of deviators (9.28) constitute a system of 16 equations with the following unknown functions: stresses, strains, and the function $\varphi$; moreover, three other "hidden" unknowns are the displacements (or velocities), and hence the total number of unknowns is also 16.

To reduce the number of equations we first introduce two stress functions of the type (19.37). Namely, we employ the following dimensionless stress functions $\Phi$ and $\Psi$

$$
\begin{array}{lll}
\sigma_{r}=\frac{2 \sigma_{0}}{\sqrt{3}}\left(\frac{1}{\varrho} \Phi^{\prime}+\frac{1}{\varrho^{2}} \Phi^{*}\right), & \tau_{r z}=\frac{2 \sigma_{0}}{\sqrt{3}} \frac{1}{\varrho} \Psi^{\prime}, \\
\sigma_{\theta}=\frac{2 \sigma_{0}}{\sqrt{3}} \Phi^{\prime \prime}, & \tau_{\theta z}=-\frac{2 \sigma_{0}}{\sqrt{3}} \Psi^{\prime}, \\
\tau_{r \theta}=-\frac{2 \sigma_{0}}{\sqrt{3}}\left(\frac{1}{\varrho} \Phi^{\prime \prime}-\frac{1}{\varrho^{2}} \Phi^{\cdot}\right), &
\end{array}
$$


where the dashes denote differentiation with respect to the dimensionless radius $\varrho=r / b$, and the dots-with respect to the angle $\theta$. Hence Eqs. (38.39) are satisfied identically.

The first three compatibility equations (38.40) determine the strain $\varepsilon_{z}$. The general integral, satisfying all these equations, has the form

$$
\varepsilon_{z}=C_{1}+C_{2} r \cos \left(\theta-\theta_{0}\right) \text {, }
$$

and introducing the dimensionless radius $\varrho$, we may write

$$
\varepsilon_{z}=\lambda+\varkappa \varrho \cos \theta,
$$

since for the system of coordinates shown in Fig. 195 we have $\theta_{0}=0 ; \lambda$ and $x$ denote the parameters proportional to axial extension and curvature (or their rates), respectively. By using Eqs. (37.4) and (9.28) we may eliminate the stress $\sigma_{z}$, which does not appear in the equilibrium equations

$$
\sigma_{z}=\frac{\sigma_{0} \sqrt{3}}{H} \varepsilon_{z}+\frac{1}{2}\left(\sigma_{r}+\sigma_{\theta}\right),
$$

where the dimensionless "plastic modulus" $H$ is introduced by the formula

$$
H=\frac{2 \sigma_{0}}{\sqrt{3}} \varphi .
$$

The stress functions $\Phi$ and $\Psi$ and the function $H$ will be regarded as the basic unknowns in our problem. They are governed by the yield condition (37.3) and the remaining compatibility conditions (38.40). Substituting (38.41) and (38.44) into (37.3), we obtain after some rearrangements

$$
\begin{aligned}
& \left\{\left(\boldsymbol{\Phi}^{\prime \prime}-\frac{1}{\varrho} \boldsymbol{\Phi}^{\prime}-\frac{1}{\varrho^{2}} \boldsymbol{\Phi}^{\cdot{ }^{\prime}}\right)^{2}+4\left[\left(\frac{1}{\varrho} \boldsymbol{\Phi}^{\prime \prime}-\frac{1}{\varrho^{2}} \Phi^{\prime}\right)^{2}+\left(\Psi^{\prime}\right)^{2}+\right.\right. \\
& \left.\left.+\left(\frac{1}{\varrho} \Psi^{\prime}\right)^{2}\right]-1\right\} H^{2}+3\left(\varkappa^{2} \varrho^{2} \cos ^{2} \theta+2 \varkappa \lambda \varrho \cos \theta+\lambda^{2}\right)=0 .
\end{aligned}
$$

The fourth compatibility equation (38.40) with substituted strains expressed in terms of stresses by using (9.28) takes the form

$$
\begin{gathered}
\left(\varrho^{2} \frac{\partial^{2}}{\partial \varrho^{2}}+3 \varrho \frac{\partial}{\partial \varrho}-\frac{\partial^{2}}{\partial \theta^{2}}\right)\left[\left(\Phi^{\prime \prime}-\frac{1}{\varrho} \Phi^{\prime}-\frac{1}{\varrho^{2}} \Phi^{*}\right) H\right]+ \\
+4\left(\varrho \frac{\partial^{2}}{\partial \varrho \partial \theta}+\frac{\partial}{\partial \theta}\right)\left[\left(\frac{1}{\varrho} \Phi^{\prime \prime}-\frac{1}{\varrho^{2}} \Phi^{\prime}\right) H\right]=0 .
\end{gathered}
$$

Finally, the last two compatibility equations, determining the function $\Psi$, are not independent; they may be integrated once and jointly result in the following equation, [4.541],

$$
\left(1+\varrho \frac{\partial}{\partial \varrho}\right)\left(H \Psi^{\prime}\right)+\frac{\partial}{\partial \theta}\left(\frac{H}{\varrho} \Psi^{\cdot}\right)+\vartheta \varrho=0,
$$

where $\vartheta$ is an integration constant proportional to the unit angle of twist (or its rate). 
The system of three non-linear partial differential equations (38.46), (38.47) and (38.48) is completed with six boundary conditions (or rather three boundary conditions in the language of partial differential equations): $\sigma_{r}=\tau_{r \theta}=\tau_{r z}=0$ for $\varrho=1$ and $\sigma_{r}=-p, \tau_{r \theta}=\tau_{r z}=0$ for $\varrho=a / b=\beta$. In view of (38.41) we write these conditions in the form

$$
\begin{array}{ll}
\Phi^{\prime}(1, \theta)+\Phi^{*}(1, \theta)=0, & \beta \Phi^{\prime}(\beta, \theta)+\Phi^{*}(\beta, \theta)=-\frac{\sqrt{3}}{2} \beta^{2} \frac{p}{\sigma_{0}}, \\
\Phi^{\prime \prime}(1, \theta)-\Phi^{*}(1, \theta)=0, & \beta \Phi^{\prime \prime}(\beta, \theta)-\Phi^{*}(\beta, \theta)=0, \\
\Psi^{*}(1, \theta)=0, & \Psi^{*}(\beta, \theta)=0 .
\end{array}
$$

These conditions do not allow for tangential pressures, which, however, appear rather seldom in engineering applications and are not shown in Fig. 195.

\subsection{A perturbation solution for prevailing pressure}

An exact solution of the system of three non-linear partial differential equations (38.46), (38.47) and (38.48) presents serious difficulties. Hence we apply two variants of the perturbation method, and then employ the Hermite boundary approximation, making also use of some other solutions for particular cases, obtained earlier. The perturbation method results not only in linearization, but also in uncoupling of the equations.

Under the assumption $x=\lambda=\vartheta=0$, corresponding to the action of internal pressure in plane strain conditions, the above equations are considerably simplified and we easily find their solution

$$
\begin{aligned}
& \Phi=\Phi_{000}=\frac{1}{2} \varrho^{2} \ln \varrho-\frac{1}{4} \varrho^{2}, \\
& H=H_{000}=\frac{D}{\varrho^{2}}, \quad \Psi=\Psi_{000}=0,
\end{aligned}
$$

where $D$ is a constant, which in the fully plastic state remains indeterminable. Now we assume the parameters $x / D, \lambda / D$ and $\vartheta / D$ to be small and look for the solution of the system under consideration in the form of a triple power series

$$
\begin{aligned}
& \Phi=\sum_{i=0}^{\infty} \sum_{j=0}^{\infty} \sum_{k=0}^{\infty} \Phi_{i j k}(\varrho, \theta) x^{i} \lambda^{j} \vartheta^{k}, \\
& H=\sum_{i=0}^{\infty} \sum_{j=0}^{\infty} \sum_{k=0}^{\infty} H_{i j k}(\varrho, \theta) x^{i} \lambda^{j} \vartheta^{k}, \\
& \Psi=\sum_{i=0}^{\infty} \sum_{j=0}^{\infty} \sum_{k=0}^{\infty} \Psi_{i j k}(\varrho, \theta) x^{i} \lambda^{j} \vartheta^{k} .
\end{aligned}
$$

We begin the process of solution with Eq. (38.48). Substitution of (38.51) makes it possible to determine the subsequent functions $\Psi_{i j k}$ by linear equations of the type

$$
\Psi_{i j k}^{\prime \prime}-\frac{1}{\varrho} \Psi_{i j k}^{\prime}+\frac{1}{\varrho^{2}} \Psi_{i j k}=f_{i j k}^{(\Psi)}(\varrho, \theta),
$$


where $f_{i j k}^{(y)}$ depend on functions previously evaluated. The general solution of (38.52) is assumed in the form of the Fourier series

$$
\Psi_{i j k}=\sum_{n=0}^{\infty} f_{n}(\varrho) \cos n \theta+\bar{\Psi}_{i j k}(\varrho, \theta),
$$

$\bar{\Psi}_{i j k}$ being a particular solution of the non-homogeneous equation (38.52). Performing the differentiations required, we obtain the following Euler equation for functions $f_{n}(\varrho)$ :

$$
f_{n}^{\prime \prime}-\frac{1}{\varrho} f_{n}^{\prime}-\frac{n^{2}}{\varrho^{2}} f_{n}=0 ;
$$

hence

$$
f_{n}(\varrho)=C \varrho^{m}, \quad m=1 \mp \sqrt{1+n^{2}} .
$$

Finally, the successive corrections $\Psi_{i j k}$ are determined by the general formula

$$
\Psi_{i j k}=\sum_{n=0}^{\infty}\left(C_{n 1} \varrho^{1-\sqrt{1+n^{2}}}+C_{n 2} \varrho^{1+\sqrt{1+n^{2}}}\right) \cos n \theta+\bar{\Psi}_{i j k}(\varrho, \theta) .
$$

Similarly, Eq. (38.46) gives the following system of equations with respect to $\Phi_{i j k}$ :

$$
\Phi_{i j k}^{\prime \prime}-\frac{1}{\varrho} \Phi_{i j k}^{\prime}-\frac{1}{\varrho^{2}} \Phi_{i j k}^{\ddot{\prime}}=f_{i j k}^{(\Phi)}(\varrho, \theta)
$$

with the general integral of the form

$$
\begin{aligned}
\boldsymbol{\Phi}_{i j k} & =C_{01}+C_{02} \varrho^{2}+\left(C_{11} \varrho+C_{12} \varrho \ln \varrho\right) \cos \theta+ \\
& +\sum_{n=2}^{\infty}\left[C_{n 1} \varrho \cos \left(\sqrt{n^{2}-1} \ln \varrho\right)+C_{n 2} \varrho \sin \left(\sqrt{n^{2}-1} \ln \varrho\right)\right] \cos n \theta+\bar{\Phi}_{i j k}(\varrho, \theta) .
\end{aligned}
$$

Finally, Eq. (38.47) gives a system of equations for unknown $H_{i j k}$

$$
H_{i j k}^{\prime \prime}+\frac{3}{\varrho} H_{i j k}^{\prime}-\frac{1}{\varrho^{2}} H_{i j k}^{*}=f_{i j k}^{(H)}(\varrho, \theta)
$$

and its general integral may be written as

$$
\begin{aligned}
& H_{i j k}=C_{01}+\frac{C_{02}}{\varrho^{2}}+\left(\frac{C_{11}}{\varrho}+\frac{C_{12}}{\varrho} \ln \varrho\right) \cos \theta+ \\
& +\sum_{n=2}^{\infty}\left[\frac{C_{n 1}}{\varrho} \cos \left(\sqrt{n^{2}-1} \ln \varrho\right)+\frac{C_{n 2}}{\varrho} \sin \left(\sqrt{n^{2}-1} \ln \varrho\right)\right] \cos n \theta+\bar{H}_{i j k}(\varrho, \theta) .
\end{aligned}
$$

The constants $C_{n 1}$ and $C_{n 2}$ are of course different for every function calculated, and for particular $i, j, k$. These constants are evaluated from boundary conditions and from the conditions of consistency of displacements in a doubly-connected 
body, [4.541]. However, it turns out that the boundary conditions may be satisfied exactly along one boundary line only (the inner or the outer radius), since the assumption of full plastification of the tube in the limit state is merely an approximate assumption. A detailed discussion of the problem of full plastification in the limit state is due to $\mathrm{Z}$. Kordas [8.255]. In the case under consideration the boundary conditions were satisfied exactly at the outer radius $\varrho=1$ and approximately at the inner boundary: instead of the "local" conditions the following "integral" formulae were applied:

$$
\frac{1}{2 \pi} \int_{0}^{2 \pi} \sigma_{r} d \theta=-p, \quad \int_{0}^{2 \pi} \tau_{r \theta} d \theta=0, \quad \int_{0}^{2 \pi} \tau_{r z} d \theta=0 .
$$

Finally, the stress distribution with an accuracy up to the second-order corrections is determined by the formulae

$$
\begin{aligned}
& \sigma_{r}=\frac{2 \sigma_{0}}{\sqrt{3}}\left[\ln \varrho+\left\{\frac{1}{8}\left(1-\varrho^{6}\right)+\frac{3}{52}\left[\frac{1}{\varrho} \cos (\sqrt{3} \ln \varrho)-\right.\right.\right. \\
& \left.\left.-\frac{2 \sqrt{3}}{\varrho} \sin (\sqrt{3} \ln \varrho)-\varrho^{6}\right] \cos 2 \theta\right\} \frac{x^{2}}{D^{2}}+\frac{3}{8}\left(1-\varrho^{4}\right) \frac{\lambda^{2}}{D^{2}}+ \\
& \left.+\frac{1}{12}\left(1-\varrho^{6}\right) \frac{\vartheta^{2}}{D^{2}}+\frac{1}{2}\left(\frac{1}{\varrho}-\varrho^{5}\right) \cos \theta \frac{x \lambda}{D^{2}}+\ldots\right\rceil \text {, } \\
& \sigma_{\theta}=\frac{2 \sigma_{0}}{\sqrt{3}}\left[(\ln \varrho+1)+\left\{\frac{1}{8}\left(1-7 \varrho^{6}\right)+\frac{3}{52}\left[\frac{1}{\varrho} \cos (\sqrt{3} \ln \varrho)-\right.\right.\right. \\
& \left.\left.-\frac{2 \sqrt{3}}{\varrho} \sin (\sqrt{3} \ln \varrho)-14 \varrho^{6}\right] \cos 2 \theta\right\} \frac{x^{2}}{D^{2}}+\frac{3}{8}\left(1-5 \varrho^{4}\right) \frac{\lambda^{2}}{D^{2}}+ \\
& \left.+\frac{1}{12}\left(1-7 \varrho^{6}\right) \frac{\vartheta^{2}}{D^{2}}+\frac{1}{2}\left(\frac{1}{\varrho}-7 \varrho^{5}\right) \cos \theta \frac{x \lambda}{D^{2}}+\ldots\right] \text {, } \\
& \sigma_{z}=\frac{2 \sigma_{0}}{\sqrt{3}}\left[\left(\ln \varrho+\frac{1}{2}\right)+\frac{3}{2} \varrho^{3} \cos \theta \frac{x}{D}+\frac{3}{2} \varrho^{2} \frac{\lambda}{D}+\left\{\frac{1}{8}\left(1-4 \varrho^{6}\right)+\right.\right. \\
& \left.+\frac{3}{52}\left[\frac{1}{\varrho} \cos (\sqrt{3} \ln \varrho)-\frac{2 \sqrt{3}}{\varrho} \sin (\sqrt{3} \ln \varrho)-\frac{15}{2} \varrho^{6}\right] \cos 2 \theta\right\} \frac{x^{2}}{D^{2}}+ \\
& +\frac{3}{8}\left(1-3 \varrho^{4}\right) \frac{\lambda^{2}}{D^{2}}+\frac{1}{12}\left(1-4 \varrho^{6}\right) \frac{\vartheta^{2}}{D^{2}}+\frac{1}{2}\left(\frac{1}{\varrho}-4 \varrho^{5}\right) \cos \theta \frac{x \lambda}{D^{2}}+\ldots \rrbracket \text {, } \\
& \tau_{r \theta}=\frac{2 \sigma_{0}}{\sqrt{3}}\left\{\frac{3}{104}\left[\frac{7}{\varrho} \cos (\sqrt{3} \ln \varrho)-\frac{\sqrt{3}}{\varrho} \sin (\sqrt{3} \ln \varrho)-7 \varrho^{6}\right] \sin 2 \theta \frac{x^{2}}{D^{2}}+\right. \\
& \left.+\frac{1}{2}\left(\frac{1}{\varrho}-\varrho^{5}\right) \sin \theta \frac{x \lambda}{D^{2}}+\ldots\right\} \text {, } \\
& \tau_{\theta z}=\frac{2 \sigma_{0}}{\sqrt{3}}\left[\frac{1}{2} \varrho^{3} \frac{\vartheta}{D}+\ldots\right\}, \\
& \tau_{z r}=0+\ldots
\end{aligned}
$$


The individual loadings may be determined by simple integrations; introducing dimensionless quantities, we obtain

$$
\begin{aligned}
n= & \frac{\sqrt{3}}{2 \pi b^{2} \sigma_{0}} N=\beta^{2} \ln \frac{1}{\beta}+\frac{3}{4}\left(1-\beta^{2}\right) \frac{\lambda}{D}-\frac{1}{8} \beta^{2}\left(1-\beta^{6}\right) \frac{\varkappa^{2}}{D^{2}}- \\
& -\frac{3}{8} \beta^{2}\left(1-\beta^{4}\right) \frac{\lambda^{2}}{D^{2}}-\frac{1}{12} \beta^{2}\left(1-\beta^{6}\right) \frac{\vartheta^{2}}{D^{2}}+\ldots, \\
m_{t}= & \frac{\sqrt{3}}{W_{0} \sigma_{0}} M_{t}=\frac{2}{3} \frac{1-\beta^{6}}{1-\beta^{4}} \frac{\vartheta}{D}+\ldots, \\
m_{b}= & \frac{2 \sqrt{3}}{\pi b^{3} \sigma_{0}} M_{b}=\left(1-\beta^{6}\right) \frac{\varkappa}{D}-\beta^{2}\left(1-\beta^{6}\right) \frac{\varkappa \lambda}{D^{2}}+\ldots
\end{aligned}
$$

and, by virtue of (38.61),

$$
\begin{aligned}
q_{m}= & \frac{\sqrt{3}}{2 \sigma_{0}} p_{m}=\ln \frac{1}{\beta}-\frac{1}{8}\left(1-\beta^{6}\right) \frac{\varkappa^{2}}{D^{2}}-\frac{3}{8}\left(1-\beta^{4}\right) \frac{\lambda^{2}}{D^{2}}- \\
& -\frac{1}{12}\left(1-\beta^{6}\right) \frac{\vartheta^{2}}{D^{2}}+\ldots
\end{aligned}
$$

The symbol $p_{m}$ denotes here the mean value of the internal pressure, resulting from formula (38.61); moreover, J. Skrzypek [4.541] investigated also other possibilities of approximate fulfilment of the boundary conditions, e.g. based on the Trefftz method.

Equations (38.63) and (38.64) may be regarded as a parametrical presentation of a fragment of the plastic interaction surface, determining it in a certain vicinity of the internal pressure in plane strain conditions. Inverting the series (38.63) with respect to $\varkappa / D, \lambda / D$ and $\vartheta / D$ and substituting these inverted series into (38.64), we obtain the following explicit formula:

$$
\begin{aligned}
q_{m}= & \ln \frac{1}{\beta}-\frac{1}{8\left(1-\beta^{6}\right)} m_{b}^{2}-\frac{2}{3\left(1-\beta^{4}\right)}\left(n-\beta^{2} \ln \frac{1}{\beta}\right)^{2}-\frac{3\left(1-\beta^{4}\right)^{2}}{16\left(1-\beta^{6}\right)} m_{t}^{2}- \\
& -\frac{8 \beta^{2}}{9\left(1-\beta^{4}\right)^{2}}\left(n-\beta^{2} \ln \frac{1}{\beta}\right)^{3}-\frac{\beta^{2}}{2\left(1-\beta^{4}\right)\left(1-\beta^{6}\right)}\left(n-\beta^{2} \ln \frac{1}{\beta}\right) m_{b}^{2}- \\
& -\frac{\beta^{2}\left(1-\beta^{4}\right)}{4\left(1-\beta^{6}\right)}\left(n-\beta^{2} \ln \frac{1}{\beta}\right) m_{t}^{2}-\ldots
\end{aligned}
$$

J. Skrzypek investigated in [4.541] the deviations of $\sigma_{r}$ at the inner radius $\varrho=\beta$ from its mean value $\left(-p_{m}\right)$; they are of order of several per cent.

\subsection{A perturbation solution for prevailing torsion}

Another variant of the perturbation method as applied to the system of equations $(38.46) \div(38.48)$ consists in assuming the solution in the form of a triple power series 


$$
\begin{aligned}
& \Phi=\sum_{i=0}^{\infty} \sum_{j=0}^{\infty} \sum_{k=0}^{\infty} \Phi_{i j k}(\varrho, \theta) x^{i} \lambda^{j} \xi^{k}, \\
& H=\sum_{i=0}^{\infty} \sum_{j=0}^{\infty} \sum_{k=0}^{\infty} H_{i j k}(\varrho, \theta) x^{i} \lambda^{j} \xi^{k}, \\
& \Psi=\sum_{i=0}^{\infty} \sum_{j=0}^{\infty} \sum_{k=0}^{\infty} \Psi_{i j k}(\varrho, \theta) x^{i} \lambda^{j} \xi^{k},
\end{aligned}
$$

where $\xi$ denotes a parameter proportional to internal pressure. The zeroth approximation corresponds here to the limit state under pure torsion; the exact solution in this case is given by

$$
\Phi_{000}=0, \quad H_{000}=\vartheta \varrho, \quad \Psi_{000}=-\frac{1}{2} \varrho .
$$

In the present variant of solution the most convenient way is to start with Eq. (38.47), which determines the successive functions $\Phi_{i j k}$ :

$$
\Phi_{i j k}^{\prime \prime \prime \prime}+\frac{4}{\varrho} \Phi_{i j k}^{\prime \prime \prime}+\frac{2}{\varrho^{2}} \Phi_{i j k}^{\prime \prime}-\frac{1}{\varrho^{4}} \Phi_{i j k}^{\ddot{1}}+\frac{1}{\varrho^{4}} \Phi_{i j k}^{\cdots}=f_{i j k}^{(\Phi)}(\varrho, \theta) \text {. }
$$

Looking for the general solution of this equation in the form of a Fourier series in $\theta$, we finally obtain, [4.543],

$$
\Phi_{i j k}=\sum_{n=0}^{\infty}\left(C_{n 1} \varrho^{m_{n 1}}+C_{n 2} \varrho^{m_{n 2}}+C_{n 3} \varrho^{m_{n 3}}+C_{n 4} \varrho^{m_{n 4}}\right) \cos n \theta+\bar{\Phi}_{l j k}(\varrho, \theta),
$$

where the exponents $m_{n 1}, m_{n 2}, m_{n 3}, m_{n 4}$ are defined as the roots of the quartic equations

$$
m^{4}-2 m^{3}-m^{2}\left(1+2 n^{2}\right)+2 m\left(1+n^{2}\right)-n^{2}\left(1-n^{2}\right)=0
$$

for $n=0,1,2, \ldots$ The symbol $\bar{\Phi}_{i j k}$ denotes, as before, a particular integral of the non-homogeneous equation (38.68).

The evaluation of the functions $\Psi_{i j k}$ and $H_{i j k}$ is much simpler. The substitution of (38.66) and (38.67) into (38.46) results in a system of very simple linear equations

$$
\Psi_{i j k}^{\prime}=f_{i j k}^{(\Psi)}(\varrho, \theta)
$$

with the solutions

$$
\Psi_{i j \mathrm{k}}=C(\theta)+\bar{\Psi}_{i j k}(\varrho, \theta),
$$

whereas (38.47) yields the system of equations

$$
H_{i j k}^{\prime}+\frac{1}{\varrho} H_{i j k}=f_{i j k}^{(H)}(\varrho, \theta),
$$

whose solutions may be written as

$$
H_{i j k}=\frac{C(\theta)}{\varrho}+\bar{H}_{i j k}(\varrho, \theta) \text {. }
$$


Having determined the integration constants from the boundary conditions and the conditions of consistency of displacements in a doubly-connected body, [4.543], we finally obtain

$$
\begin{aligned}
\sigma_{r}= & \frac{2 \sigma_{0}}{\sqrt{3}}\left\{2\left(1-\frac{1}{\varrho^{3}}\right) \frac{\xi}{\vartheta}+12\left[\frac{1-\beta^{9}}{\beta^{6}\left(1-\beta^{3}\right)}\left(\frac{1-\beta^{6}}{1-\beta^{9}}-\frac{1}{\varrho^{3}}\right)+\frac{1}{\varrho^{9}} \mid \frac{\xi^{3}}{\vartheta^{3}}+\right.\right. \\
& +\left[\frac{18 \ln \beta}{\beta^{\frac{5+\sqrt{17}}{2}}-\beta^{\frac{5-\sqrt{17}}{2}}}\left(\varrho^{\frac{-3+\sqrt{17}}{2}}-\varrho^{\frac{-3-\sqrt{17}}{2}}\right)-\frac{18 \ln \varrho}{\varrho^{4}}\right] \cos \theta \frac{x \lambda \xi}{\vartheta^{3}}+ \\
& +\left[\left(m_{21}-4\right) C_{21} \varrho^{m_{21}-2}+\ldots+\left(m_{24}-4\right) C_{24} \varrho^{m_{24}-2}+\right. \\
& \left.\left.+\frac{9}{2}\left(\frac{5}{\varrho^{3}}-\frac{14}{\varrho^{4}}\right)\right] \cos 2 \theta \frac{x^{2} \xi}{\vartheta^{3}}+\ldots\right\}
\end{aligned}
$$

and similar formulae for the remaining stress components. These formulae, up to third-order corrections, are given in [4.543]. The symbols $m_{21}, m_{22}, m_{23}$ and $m_{24}$ denote here real roots of Eq. (38.70) for $n=2$, i.e. of the equation

$$
m^{4}-2 m^{3}-9 m^{2}+10 m+12=0 .
$$

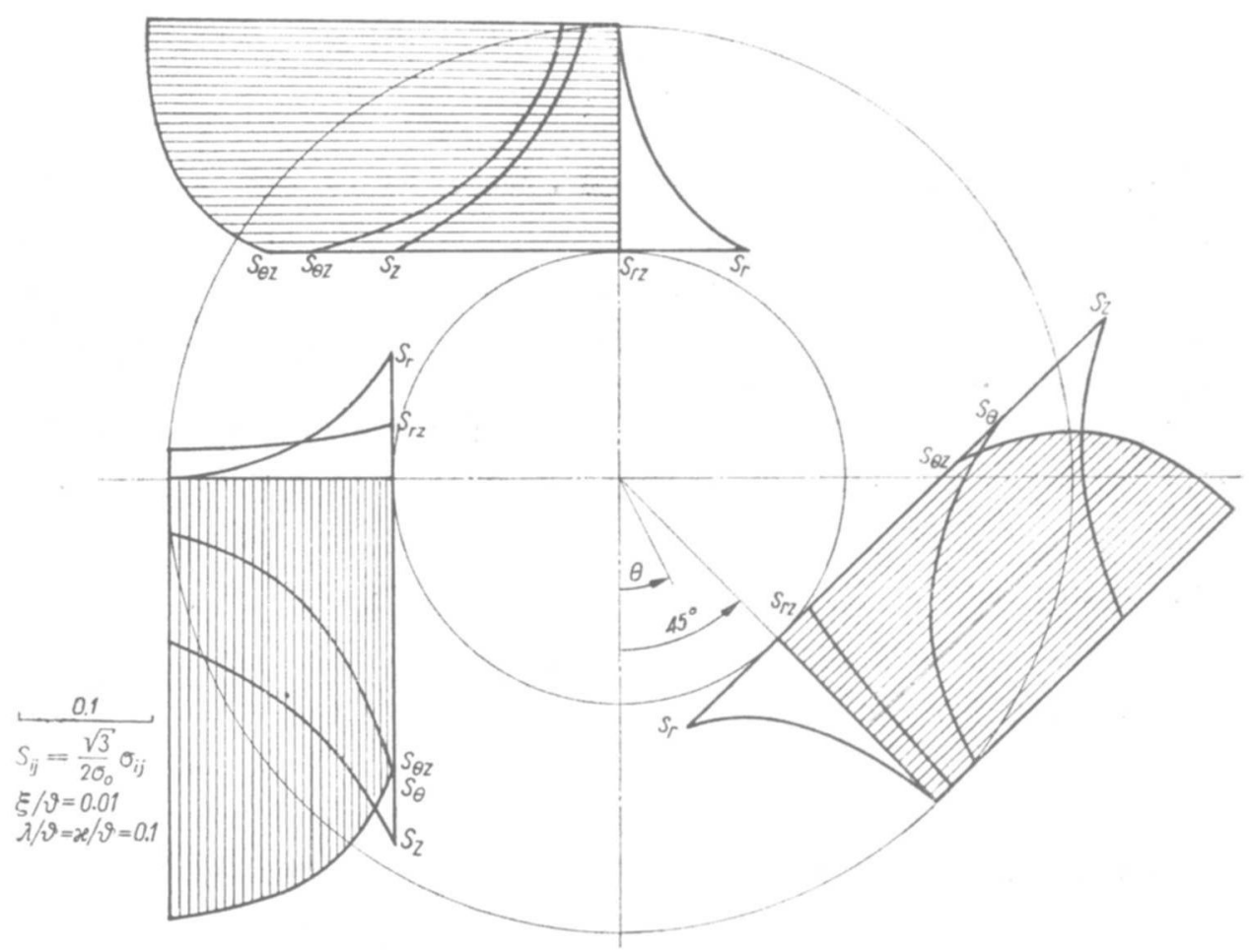

Fig. 196. Stress distribution in a fully plastic tube 
These roots may be evaluated exactly, namely

$$
m=\frac{1}{2} \pm \sqrt{\frac{21}{4} \pm \sqrt{13}} .
$$

Figure 196 shows the stress distribution for a tube with the ratio of radii $\beta=a / b$ $=1 / 2$, and for three values of the angle $\theta: \theta=45^{\circ}, \theta=180^{\circ}$ and $\theta=270^{\circ}$. The diagrams were carried out by using (38.75) up to the second-order corrections inclusive. The following values of the parameters were assumed: $\xi / \vartheta=0.01, x / \vartheta$ $=\lambda / \vartheta=0.1$.

Performing appropriate integrations, we determine the dimensionless loadings of the tube [introduced as in Eqs. (38.63)]

$$
\begin{aligned}
n= & 3(1-\beta) \frac{\lambda}{\vartheta}+\frac{2\left(1-\beta^{3}\right)}{\beta} \frac{\xi}{\vartheta}-\frac{54\left(1-\beta^{5}\right)}{5 \beta^{5}} \frac{\lambda \xi^{2}}{\vartheta^{3}}- \\
& -\frac{9}{2}\left[\beta \ln \frac{1}{\beta}-\frac{5}{2}(1-\beta)\right] \frac{x^{2} \lambda}{\vartheta^{3}}+\ldots . \\
m_{b}= & 2\left(1-\beta^{3}\right) \frac{x}{\vartheta}-\frac{9}{2}\left[\frac{1-\beta^{3}}{6}+1-\beta^{2}\right] \frac{\varkappa^{3}}{\vartheta^{3}}- \\
& -18\left[3(1-\beta)-\beta \ln \frac{1}{\beta}\right] \frac{x \lambda^{2}}{\vartheta^{3}}-\frac{36\left(1-\beta^{3}\right)}{\beta^{3}} \frac{x \xi^{2}}{\vartheta^{3}}+\ldots, \\
m_{t}= & \frac{4}{1-\beta^{4}}\left[\frac{1-\beta^{3}}{3}-\frac{1-\beta^{3}}{4} \frac{x^{2}}{\vartheta^{2}}-\frac{3(1-\beta)}{2} \frac{\lambda^{2}}{\vartheta^{2}}-\frac{6\left(1-\beta^{3}\right)}{\beta^{3}} \frac{\xi^{2}}{\vartheta^{2}}+\ldots\right],
\end{aligned}
$$

whereas the internal pressure is determined by the boundary condition for $\varrho=\beta$

$$
q=\frac{2\left(1-\beta^{3}\right)}{\beta^{3}} \frac{\xi}{\vartheta} .
$$

The first two series (38.78) may be inverted with respect to $\lambda / \vartheta$ and $x / \vartheta$; adding here the formula for $\xi / \vartheta$ in terms of $q$, (38.79), and substituting these expressions into the third equation of (38.78), we obtain the following explicit equation of a fragment of the plastic interaction surface in the vicinity of pure torsion:

$$
\begin{gathered}
m_{t}=\frac{4}{1-\beta^{3}}[ \\
{\left[\frac{1-\beta^{3}}{3}-\frac{9 \beta^{3}+\beta^{4}\left(1+\beta+\beta^{2}\right)}{8\left(1-\beta^{3}\right)} q^{2}-\frac{1}{6(1-\beta)} n^{2}+\right.} \\
\left.+\frac{\beta^{2}}{2 \sqrt{3}(1-\beta)} q n-\frac{1}{16\left(1-\beta^{3}\right)} m_{b}^{2}+\ldots\right] .
\end{gathered}
$$

\subsection{The Hermitian boundary approximation for the plastic interaction surface}

Application of the perturbation method in the remaining two cases, when either the parameters $\lambda, \vartheta$ and $\xi$ are small, or the parameters $x, \vartheta$ and $\xi$ are small, encounters essential difficulties. In fact, in the first case, where the zeroth approximation corresponds to pure bending, a discontinuity line (discontinuity of stresses $\sigma_{z}$ ) appears in it, and this results in the divergence of the power series in a certain neigh- 
bourhood of that line. The second case corresponds to pure tension in the zeroth approximation; then no discontinuity line appears, but nevertheless the series determining the function $m_{b}=m_{b}(n)$ is not an ordinary power series, but a generalized power series (23.36). Thus looking then for a solution of $(38.46) \div(38.48)$ in the form of an ordinary triple power series in $\varkappa, \vartheta$ and $\xi$ is evidently excluded. However, leaving bending out of consideration and regarding only the parameters $\vartheta$ and $\xi$ as small, we may determine a fragment of the plastic interaction surface in the vicinity of pure tension by the series, [4.543],

$$
n=\frac{\sqrt{3}\left(1-\beta^{2}\right)}{2}+\beta^{2} q-\frac{\sqrt{3} \beta^{2}}{1-\beta^{2}} q^{2}-\frac{\sqrt{3}\left(1-\beta^{4}\right)}{8} m_{t}^{2}+\ldots
$$

Making use of the series (38.65), (38.80), (38.81) and of (23.36), (28.20), (28.34) as well as of a lower bound derived by J. Skrzypek [8.490] for the limit carrying capacity of an inextensible pipe-line, J. Skrzypek and M. Życzkowski proposed in [4.543] two approximate equations of the plastic interaction surface $f\left(q, n, m_{b}\right.$, $\left.m_{t}\right)=0$. The multidimensional Hermitian boundary approximation was employed and the conditions of coincidence were imposed on the function itself and on its derivatives at the points corresponding to pure individual loadings. The simpler, seven-parameter approximation makes it possible to use the conditions of coincidence up to all first-order partial derivatives inclusive; it has the form (after the correction of some errors in [4.543]):

$$
\begin{aligned}
& \frac{2}{\ln ^{2}(1 / \beta)}\left[1-\frac{3+6 \beta^{2}-5 \beta^{4}}{6\left(1-\beta^{4}\right)} \ln \frac{1}{\beta}-\frac{\beta^{4}\left(9+18 \beta^{2}+13 \beta^{4}\right)}{9\left(1-\beta^{4}\right)^{2}} \ln ^{2} \frac{1}{\beta}+\ldots\right] q^{2}+ \\
& +\frac{4}{3\left(1-\beta^{2}\right)^{2}} n^{2}-\frac{8 \beta^{2}}{3\left(1-\beta^{2}\right)^{2}} q n+\frac{9\left(1-\beta^{4}\right)^{2}}{16\left(1-\beta^{3}\right)^{2}} m_{t}^{2}+\frac{3 \pi^{2}}{64\left(1-\beta^{3}\right)^{2}} m_{b}^{2}+ \\
& +\frac{4 \sqrt{3}}{9\left(1-\beta^{2}\right)^{3}}\left|m_{b}\right| n^{2}-\frac{1}{\ln ^{4}(1 / \beta)}\left[1-\frac{3+6 \beta^{2}-5 \beta^{4}}{3\left(1-\beta^{4}\right)} \ln \frac{1}{\beta}+\right. \\
& \left.+\frac{-2 \beta^{4}\left(15+30 \beta^{2}-11 \beta^{4}\right)}{9\left(1-\beta^{4}\right)^{2}} \ln ^{2} \frac{1}{\beta}+\ldots\right] q^{4}=1 .
\end{aligned}
$$

The presence of series in the approximate equation of the plastic interaction surface (38.82) results from the fact that the value $\overline{\bar{q}}$ for $n=0$ is determined by the series (38.65). For example, for $\beta=a / b=1 / 2$ we obtain

$$
\begin{aligned}
& 1.909 q^{2}+2.370 n^{2}-1.185 q n+0.6457 m_{t}^{2}+0.6043 m_{b}^{2}+ \\
& \quad+1.825\left|m_{b}\right| n^{2}-0,256 q^{4}=1 .
\end{aligned}
$$

Paper [4.543] proposed also a more accurate, eighteen-parameter Hermitian boundary approximation, ensuring the coincidence not only of the first partial derivatives, but also of almost all second derivatives. However, the final equation is rather lengthy, and the improvement of accuracy not too high.

A certain lower bound for the limit carrying capacity in the four-fold combined case under consideration was given by W. F. Stokey, D. B. Peterson and R. A. 
Wunder [8.507]; they based their analysis on the Tresca-Guest yield condition. The elastic carrying capacity of a thick-walled tube under internal pressure, tension and bending, was investigated by M. Mrowiec and M. Życzkowski [8.352]. Experimental investigations of the limit carrying capacity of thick-walled tubes under pressure with bending were carried out by B. P. Sergiyev [8.453].

\subsection{Elastic-plastic range. Other cases of non-symmetric combined loadings}

Thin-walled tubes under simultaneous pressure, tension, torsion and bending were considered in the elastic-plastic range by I. V. Stasenko [8.504], L. D. Larson, W. F. Stokey and W. E. Franzen [8.297], and in the range of plastic hardeningby I. V. Stasenko [8.505] (linear hardening). V. V. Kolokolchikov [8.253] analysed elastic-plastic bending of a rotating thin-walled tube. K. Wellinger, H. Gassmann and $\mathrm{H}$. Zanner [8.556] investigated, theoretically and experimentally, oval-shaped thin-walled tubes under constant bending and variable pressure.

Elastic-plastic deformations of a thick-walled tube under pressure not conforming to circular symmetry were considered by O. A. Grigan, V. A. Dintsyn, V. L. Livshits and A. L. Livshits [8.179]. J. B. Tiedemann, I. Vigness and T. E. Pardue [8.527] analysed, theoretically and experimentally, deflections of rotating thick-walled cylinders; they allowed for plastic hardening and Bauschinger effect.

Several papers discuss thermal loadings combined with other loading types where the conditions of circular symmetry are not preserved. V. L. Fomin considered non-symmetric temperature distributions combined with constant internal pressure: in [8.146] he employed the perturbation method to allow for the dependence of temperature on the angle $\theta$; in [8.149] he solved a similar problem in the range of plastic hardening, and in [8.147] he considered the dependence of temperature on the variable $z$. Thin-walled tubes under pressure, bending and thermal loadings were analysed by V. V. Danilovskaya [8.109] (comparison of elastic and limit carrying capacities), J. M. Chern and D. H. Pai [8.73] (including viscous effects). Thick-walled tubes under a similar combination of loadings were considered by K. R. Merckx [8.337] (power plastic hardening law), H. G. Edmunds and F. J. Beer [8.124] (incremental collapse as a result of variable bending), A. Pellisier-Tanon [8.392] (constant and variable loadings of cylinders of nuclear reactors), E. I. Makeranets and V. I. Odinokov [8.317] (oval-shaped cylinders under pressure and temperature). A. E. Kulago [8.274] investigated, theoretically and experimentally, a thick-walled elastic-plastic tube under a variable magnetic field.

\section{Disks and sheets under combined in-plane loadings}

\subsection{Annular disks under surface loadings}

Considering thick-walled tubes subject to internal and to external radial pressures, we found that in many cases the pressure difference is the only factor that affects 
the process; this conclusion is based on the assumption of the HMH yield condition (or any other yield condition described by a cylindrical yield surface), incompressibility of the material and plane strain conditions. Conversely, in jthe related case of annular disks in plane stress conditions, both pressures affect the process independently, and hence it is interesting to construct the relevant plastic interaction curve in the plane $p_{a}-p_{b}$.

We now determine this curve for an annular disk of constant thickness, with internal radius $a$ and external radius $b$, loaded by normal pressures $p_{a}$ and $p_{b}$. Under the assumption of a plane stress state the problem is statically internally determinate, since the equilibrium equation in polar coordinates and the yield condition contain only two unknowns, $\sigma_{r}$ and $\sigma_{\theta}$. Assume the HMH yield condition and introduce the Nadai-Sokolovsky parametrization (18.38); integrating the equilibrium equation (18.39), we arrive at the general solution in the inverse form (18.40). Denoting by $\zeta_{a}$ and $\zeta_{b}$ the values of $\zeta$ corresponding to $r=a$ and $r=b$ respectively, using (18.40) twice and eliminating the constant $C_{1}$, we obtain

$$
\beta^{2}=\frac{\left|\sin \left(\zeta_{b}-\pi / 3\right)\right|}{\left|\sin \left(\zeta_{a}-\pi / 3\right)\right|} \exp \left[\sqrt{3}\left(\zeta_{a}-\zeta_{b}\right)\right],
$$

where $\beta=a / b$. The values $\zeta_{a}$ and $\zeta_{b}$ will now be determined from the boundary conditions. They have the form $\sigma_{r}(a)=-p_{a}, \sigma_{r}(b)=-p_{b}$, but the parameters $\zeta_{a}$ and $\zeta_{b}$, determined from (18.38), are not unique, since we have to select an appropriate branch of the function arcus sinus. For prevailing internal pressure (compressive pressure), the circumferential stress is positive (with some exceptions), and we have simply

$$
\zeta_{a}=-\arcsin \left(\frac{\sqrt{3}}{2} q_{a}\right), \quad \zeta_{b}=-\arcsin \left(\frac{\sqrt{3}}{2} q_{b}\right),
$$

where the dimensionless loadings are $q_{a}=p_{a} / \sigma_{0}, q_{b}=p_{b} / \sigma_{0}$. For prevailing external pressure the stress $\sigma_{\theta}$ is negative, the parameter $\zeta$ exceeds the interval $-\pi / 2$ $\leqslant \zeta \leqslant \pi / 2$, and we have to choose another branch, namely

$$
\zeta_{a}=\pi+\arcsin \left(\frac{\sqrt{3}}{2} q_{a}\right), \quad \zeta_{b}=\pi+\arcsin \left(\frac{\sqrt{3}}{2} q_{b}\right) .
$$

Substituting (39.2) and (39.3) into (39.1) we may write jointly for both cases

$$
\frac{a^{2}}{b^{2}}=\beta^{2}=\frac{\sqrt{4-3 q_{b}^{2}} \pm q_{b}}{\sqrt{4-3 q_{a}^{2}} \pm q_{a}} \exp \left\{\mp \sqrt{3}\left[\arcsin \left(\frac{\sqrt{3}}{2} q_{a}\right)-\arcsin \left(\frac{\sqrt{3}}{2} q_{b}\right)\right\}\right. \text {. }
$$

Moreover, the absolute values of the arguments of arcus sinus must not be greater than one, and hence we have the limitations $\left|q_{a}\right| \leqslant 2 / \sqrt{3},\left|q_{b}\right| \leqslant 2 / \sqrt{3}$; it turns out that only the first of these limitations is essential, whereas the second is satisfied automatically.

Finally, the plastic interaction curve in the plane $q_{a}-q_{b}$ consists of the segments determined by Eq. (39.4) and of two straight segments $q_{a}= \pm 2 / \sqrt{3}$. This curve 


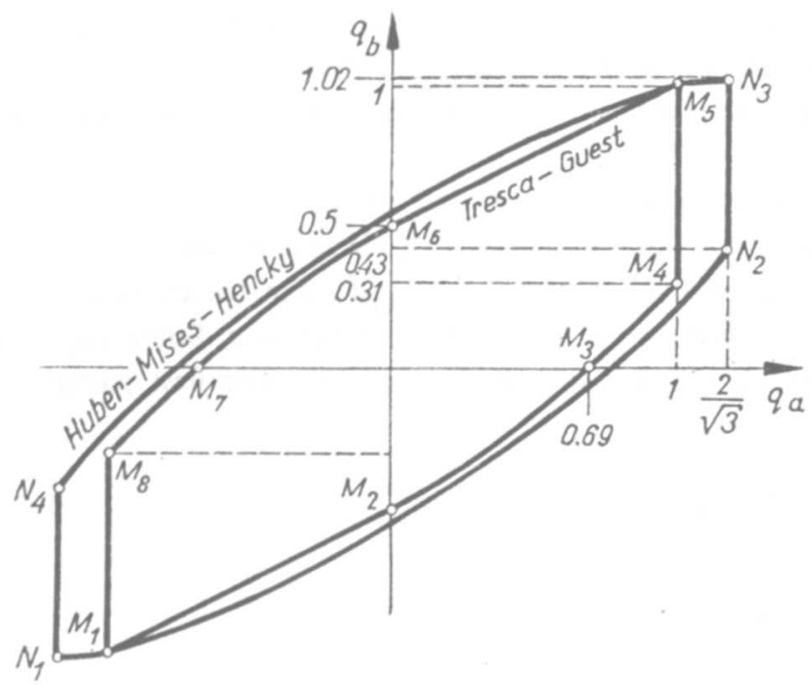

Fig. 197. Plastic interaction curves for an annular disk under internal and external pressure

is shown in Fig. 197 for $\beta=a / b=0.5$ together with the related curve based on the Tresca-Guest yield condition and consisting of six curved segments and two straight ones (W. Krzyś, M. Życzkowski [2.91]).

Linear plastic hardening of a disk under internal and external pressure was first discussed by G.'S. Shapiro [8.458], who obtained a solution by employing approximate expressions for stress and strain intensities. J. Ryś and M. Życzkowski [8.431] proposed a solution based on the perturbation method. Power plastic hardening was considered by A. G. Kostyuk [8.259], T. M. Makhonina [8.319] (who used the Nadai-Sokolovsky parametrization), J. Ryś and M. Życzkowski [8.431]. O. A. Zveryev and V. A. Palmov [8.574] proposed a method of solution for arbitrary isotropic hardening.

More general combined surface loadings of circularly-symmetric disks were considered by R. P. Nordgren and P. M. Naghdi [7.230, 7.231] (normal and tangential pressures), J. M. Chern and S. Nemat-Nasser [7.28], Zh. I. Tairova [8.517] (secondary plastic deformations under normal and tangential pressures) and $\mathrm{O}$. A. Yushtchenko [8.563] (simultaneous radial and axial pressure).

\subsection{Rectangular sheets. Sheets with holes}

Rectangular sheets are often subject to uniformly distributed in-plane loadings along the edges; then, under the assumption of material homogeneity and of constant thickness, we have homogeneous stress states in the sheet, $\mathscr{P}_{n} \rightarrow \mathscr{S}_{n} \rightarrow \mathscr{B}_{n}$, and the transition from the point to the body as a whole is immediate. Such cases may serve for the verification of constitutive equations of plasticity (e.g. V. I. Trofimov [8.534]). 
A non-homogeneous state of stress may be due to non-uniform loading along the edges. N. F. Ershov [8.135] considered linearly varying normal loading (in-plane bending); he found six possible types of stress distribution in the elastic-plastic range.

A non-homogeneous state of stress may also be introduced by the holes in the sheet. Most papers consider infinite sheets with circular holes; a typical combined loading consists here of two mutually perpendicular tractions at infinity, $p_{1 \infty}$ and $p_{2 \infty}$, and of normal pressure $p_{a}$ in the hole of radius $a$. Some papers take also the tangential pressure $t_{a}$ into consideration, Fig. 198. The first paper of this

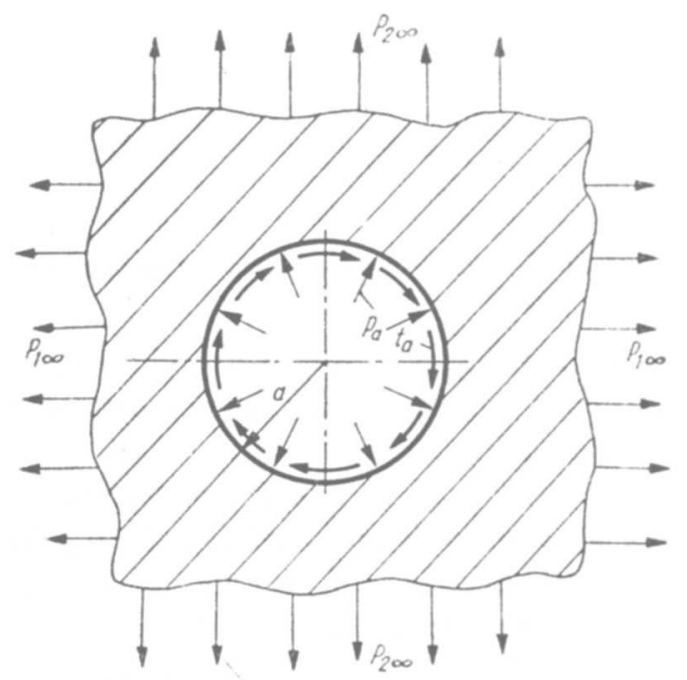

Fig. 198. An infinite sheet with a circular hole under combined loadings

type was published by L. A. Galin [4.150], who considered also in-plane bending of the sheet, but assumed plane-strain conditions (thick sheet) and material incompressibility; these assumptions facilitated the application of the complex-variable technique. Further elastic-plastic plane-strain solutions are due to O. S. Parasyuk [8.387] (who allowed for tangential pressure $t_{a}$ and applied Mikhlin's general integrals to this case, [8.344]), G. N. Savin and O. S. Parasyuk [4.510, 4.511]) (nonhomogeneous stress states at infinity), A. I. Kuznetzov [8.284] (homogeneous loadings, but a non-homogeneous material), I. Yu. Khoma [8.237] (in-plane bending with normal and tangential loadings in the hole), G. P. Cherepanov [8.71] (similar combined loadings for non-circular holes), A. S. Kosmodamiansky [8.258] (infinite sequence of equal circular holes in a line) and V. V. Kuznetzov [8.289, 8.290] (elliptic hole, loadings $p, p_{1 \infty}, p_{2 \infty}$ ),

Similar problems in plane-stress conditions are, as a rule, more difficult. The classical problem of an infinite sheet with an unloaded circular hole, uniformly stretched at infinity, was generalized by A. P. Sokolov [8.502] to two independent 
loadings, $p_{1 \infty}$ and $p_{2 \infty}$; he applied the Tresca-Guest yield condition and used the perturbation method with the difference $p_{1}-p_{2}$ as a small parameter. F. A. Gaydon and A. W. McCrum [8.162, 8.163] gave upper and lower bounds to the limit carrying capacity for a similar problem of a square sheet (with finite dimensions, HMH and TG yield conditions, respectively). Further papers are due to P. I. Perlin [8.393] (approximate method based on complex-variable approach), G. P. Cherepanov [8.72] (loadings $p_{a}, p_{1 \infty}, p_{2 \infty}$, TG yield condition) and A. P. Kharchenko [8.236] (elliptic hole free of load, biaxial tension at infinity, $p_{1 \infty}$ and $p_{2 \infty}$ ). Several perturbation solutions of related problems may be found in the book by D. D. Ivlev and L. V. Ershov [8.216]. Shakedown of a square sheet with a circular hole, subject to biaxial variable tension, was analysed by T. Belytschko [4.29] (finite element method), and by L. Corradi and A. Zavelani [4.94] (finite elements combined with linear programming).

Plastic hardening was considered by I. A. Tsurpal (cf. G. N. Savin [4.512]), R. Yu. Kerimov [8.235] (power plastic hardening law), and by L. M. Savelyev [8.444] (arbitrary hardening, circular unloaded hole, biaxial tension at infinity). Most of the above solutions are quoted in detail in the monograph by G. N. Savin [4.512], who also paid considerable attention to elastic-plastic deformations in the vicinity of cracks in sheets under combined loadings.

\subsection{Disks and sheets under body forces and thermal loadings}

In many engineering applications, particularly in the design of steam and gas turbines, the disks are subject to simultaneous action of surface loadings, body forces (rotation), and thermal loadings. Elastic analysis of such disks was initiated in the 19th Century; the earliest papers on elastic-plastic deformation of rotating disks were those by H. Hencky [2.53], 1924 (approximation of the HMH ellipse by an inclined straight line), F. László [8.298], A. Nadai and L. H. Donnell [8.356] (TG yield condition).

Combined loadings resulting only from body forces occur if a disk is subject to non-steady rotation; then the angular velocity $\omega$ and the angular acceleration $\dot{\omega}$ may be regarded as independent exertion factors. The onset of yielding (elastic interaction curve) in such cases was considered by S. R. Reid [8.423] (TG yield condition), T. Y. Reddy and H. Srinath [8.422] (uniform and non-uniform thickness, HMH yield condition). Basic equations for the plastic range, allowing also for thermal loadings, were derived by M. Życzkowski [4.630]; plastic interaction curve for a full disk under external radial pressure and centrifugal forces was given in $[8.576,1.26]$ (TG yield condition). The limit state of a rotating disk under additional tangential pressure was considered by F. M. Mazzolani [8.334]. Optimal plastic design of rotating circular and annular disks under simultaneous radial pressures was discussed by D. C. Drucker and R. T. Shield [8.120] and by J. Heyman [8.194, 8.195] (TG yield condition); T. Liszka and M. Życzkowski [4.337] employed the 
Burzyński-Stassi d'Alia yield condition and gave a comparison of elastic and plastic optimal design.

The earliest considerations on plastic deformations of non-uniformly heated rotating disks of arbitrary profile are due to A. S. Thompson [8.526], 1946. A more general method for this problem was given by S. S. Manson [8.327]. M. Sh. Mikeladze [8.340] and A. D. Kovalenko [8.261] applied direct numerical integration of governing non-linear differential equations. A. Mendelson [3.519] developed a method of successive approximations, whereas R. M. Shneyderovitch [8.479] reduced the problem to certain integral equations. A more detailed analysis of power plastic hardening in the case under consideration was given by Yu. N. Shevtchenko [8.464, 8.465, 8.466]. I. Palley [8.382] investigated the effect of loading path (in the plane angular velocity-temperature) on plastic deformations; this problem was also considered by Yu. N. Shevtchenko, who applied the variable stiffness method [8.468] and the method of successive elastic solutions, [8.471]. I. A. Birger [8.41] introduced here the concept of "simple loading", comparing the deformation and the incremental theories of plasticity; related problems were also discussed by Yu. N. Shevtchenko and R. G. Terekhov [8.467, 8.470]. Transient thermal stresses and residual stresses in disks subject to thermal and surface loadings were analysed by $\mathrm{H}$. Odenö [8.371]; secondary plastic deformations in rotating disks under surface and thermal loadings were considered by $\mathrm{Yu}$. N. Shevtchenko, I. A. Motovilovets, V. G. Savtchenko and V. N. Vasilenko [8.469]. I. A. Birger, M. F. Yakovlev and V. F. Guk $[8.42,8.186]$ proposed a numerical method of calculation of disks under combined loadings, allowing for temperaturedependent material constants and for the incremental theory of plasticity. Finite strains in non-uniformly heated rotating circular disks were considered by V. N. Alpert [8.7] (Tresca-Guest and Schmidt-Ishlinsky-Hill yield conditions with associated flow rules, isotropic hardening). R. G. Terekhov [8.523] carried out experimental investigations on plastic disks under body forces and thermal loadings. Various approaches to the calculation of non-uniformly heated rotating circular disks were compared critically by I. A. Kozlov [8.267].

Body forces and thermal loadings of disks are often varying in time and hence many papers discuss the relevant shakedown problems. The earliest paper was written by V. I. Rozenblyum [4.488], 1958, but it is D. A. Gokhfeld who paid most attention to this problem: in his own papers [4.166, 8.172, 8.175], with K. M. Kononov [8.169] (yield-point stress depending linearly on temperature, logarithmic temperature distribution), and with O.F. Chernyavsky [4.169] (mathematical programming methods). Further papers were published by M. G. Kabelevsky [8.221, 8.222, 8.223] (deformation and incremental theories and experimental verification), L. I. Fridman [8.156] (non-uniform heating, rotation and radial external pressure, disks of variable thickness), S. Amada and A. Machida [8.10, 8.11, $8.12,8.13$ ] (perfect plasticity, deformation and flow theories), H. A. Koenig, W. Vogel and L. Cernoch [8.249], I. V. Demyanushko and Yu. M. Temis [8.113] (who also took creep strains into account). 
A quite different shakedown [problem of disks was considered by K. L. Johnson [8.218], V. K. Garg, S. C. Anand and P. G. Hodge [8.160, 8.15]: they analysed elastic-plastic deformations of a wheel rolling on a rigid track.

Combined surface and thermal loadings of sheets with holes were discussed by A. M. El-Karamani [8.125], and by V. M. Mirsalimov [8.347] (surface loading in the hole and at infinity, non-uniform heating). Shakedown problems of sheets under tension and variable temperature were analysed by E. W. Parkes [8.389], and those of sheets with holes-by Yu. G. Korotkikh and A. K. Lyubimov [4.300] (finite difference method combined with iterations).

Propagation of elastic-plastic waves in sheets loaded by dynamic normal and tangential pressures as a result of oblique impact was analysed by $\mathrm{Kh}$. A. Rakhmatulin [8.420] and N. Cristescu [8.93]. Elastic-plastic half-plane under moving forces was considered by A. M. Skobeyev and L. M. Flitman [8.488].

\subsection{Decohesive carrying capacity of an infinite sheet under combined surface} and thermal loadings

In Sec. 18.4 we presented an example of a perfectly elastic-plastic infinite sheet with rigid circular inclusion under uniform tractions $p$ at infinity (Fig. 99); the process terminated with decohesive carrying capacity and not with limit carrying capacity. Now we generalize this problem to additional thermal loadings (uniform temperature increase $T$ ) and construct the corresponding interaction curves in the plane $p-T$ (K. Szuwalski and M. Życzkowski [8.516]).

Let us assume the temperature-independent $\mathrm{HMH}$ yield condition and first consider the elastic interaction curve (onset of yielding). General integrals (18.34) and (18.35) now take the form

$$
\begin{aligned}
\sigma_{r} & =A+\frac{B}{r^{2}}-\frac{\alpha}{2} E T, \\
\sigma_{\theta} & =A-\frac{B}{r^{2}}-\frac{\alpha}{2} E T, \\
u & =\frac{1}{E}\left[(1-v) A r-(1+v) \frac{B}{r}\right]+\frac{1+v}{2} \alpha T .
\end{aligned}
$$

The boundary conditions (18.35) yield

$$
A=p+\frac{\alpha}{2} E T, \quad B=\frac{a^{2}}{1+v}[(1-v) p+\alpha E T],
$$

$a$ being the radius of rigid inclusion, and $\alpha$ the coefficient of linear thermal expansion. The maximal stress intensity occurs at $r=a$, and substituting (39.6) into (39.5) and then into the $\mathrm{HMH}$ yield condition, we obtain the following equation of the elastic interaction curve:

$$
3 \tau^{2}+(1-v) q \tau+4\left(1-v+v^{2}\right) q^{2}-(1+v)^{2}=0,
$$


where

$$
q=\frac{p}{\sigma_{0}}, \quad \tau=\frac{\alpha E}{\sigma_{0}} T .
$$

Equation (39.7) describes a family of ellipses with Poisson's ratio $v$ as a parameter; they are shown in Fig. 199.

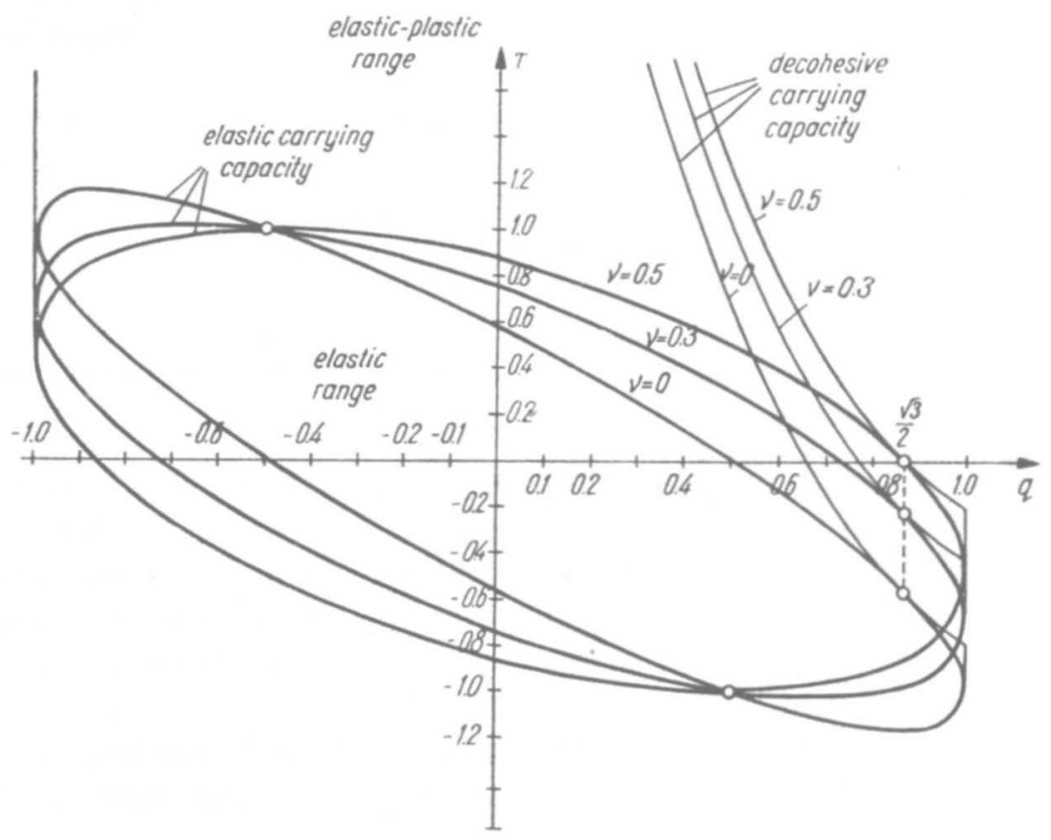

Fig. 199. Interaction curves for an infinite sheet with a rigid circular inclusion under thermal loadings

Exceeding these ellipses we enter the elastic-plastic range: the plastic zone $a<r<r^{*}$ is surrounded by the elastic zone $r>r^{*}$. The distribution of stresses in the plastic zone is statically determinate and hence given by (18.38) and (18.40) irrespective of any particular theory of plasticity and irrespective of thermal effects. However, to determine the constant $C_{1}$ we have to analyse displacements, and here we confine our considerations to the Hencky-Ilyushin deformation theory of plasticity, generalized so as to allow for thermal stresses. We assume the material to be incompressible except being subject to thermal expansion and replace the law of volume change $(9.23)$ by the following

$$
\varepsilon_{r}+\varepsilon_{\theta}+\varepsilon_{z}=3 \alpha T .
$$

Then (18.46) and (18.49) are generalized to

$$
\varepsilon_{\theta}=\frac{\sigma_{0}}{3 K \sqrt{3}} \sin \zeta+\alpha T+C_{2} \exp (-\sqrt{3} \zeta),
$$




$$
\varepsilon_{r}=\frac{\sigma_{0}}{3 K \sqrt{3}} \sin \left(\zeta+\frac{\pi}{3}\right)+\alpha T+\frac{C_{2}}{\cos \zeta} \sin \left(\zeta-\frac{\pi}{6}\right) \exp (-\sqrt{3} \zeta)
$$

Boundary conditions and continuity conditions (18.50) remain without change. However, being now interested only in decohesive carrying capacity, we substitute at once $\zeta_{a}=\pi / 2$, then $\varepsilon_{r}^{(p)}(a) \rightarrow \infty$, and the process terminates. Then six of the conditions (18.50) determine the constants $A, B, C_{1}, C_{2}, r^{*}$ and $\zeta^{*}$, and the seventh one furnishes the following equation of the interaction curve of decohesive carrying capacity:

$$
\tau=\frac{1}{2}\left[\sqrt{3\left(1-q^{2}\right)}-q\right] \exp \left[\sqrt{3}\left(\arccos q-\frac{1}{6} \pi\right)\right]-\frac{1-2 v}{\sqrt{3}},
$$

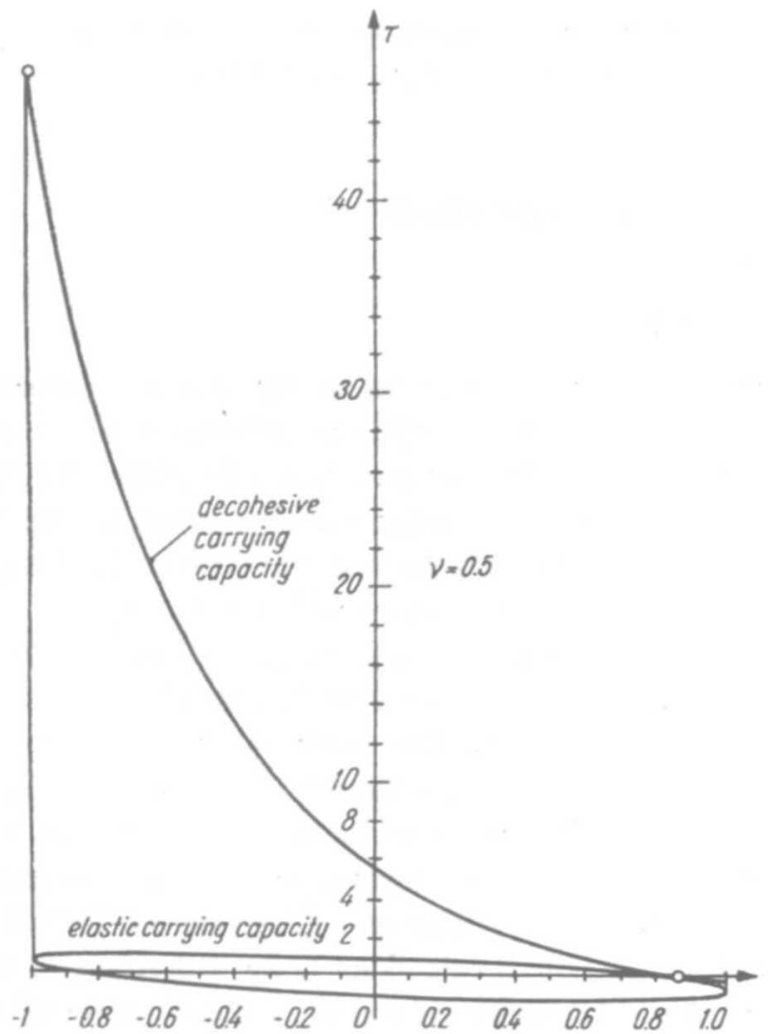

Fig. 200. A full interaction curve corresponding to decohesive carrying capsicity

for $-1 \leqslant q \leqslant 1$. These curves are shown partly in Fig. 199 for various $v$, and wholly-in Fig. 200 for $v=0.5$. Equation (39.11) may also be presented in a simpler form. Introducing a new parameter of uniform tension at infinity, $\chi$, by the formulae

$$
\chi=\arccos q-\frac{1}{6} \pi . \quad q=\cos \left(\chi+\frac{1}{6} \pi\right)
$$


where $-\frac{1}{6} \pi \leqslant \chi \leqslant \frac{5}{6} \pi$, we may write instead of (39.12)

$$
\tau=\sin \chi \exp (\chi \sqrt{3})-\frac{1-2 v}{\sqrt{3}} \text {. }
$$

The "inverse" criterion of decohesion, $\varepsilon_{r}^{(p)}(a) \rightarrow-\infty$, leads to antisymmetric curves

$$
\tau=-\frac{1}{2}\left[\sqrt{3\left(1-q^{2}\right)}+q\right] \exp \left[\sqrt{3}\left(\frac{5}{6} \pi-\arccos q\right)\right]+\frac{1-2 v}{\sqrt{3}} .
$$

However, employing a finite-strain-theory, we obtain no major changes with respect to (39.12), but essential changes with respect to (39.15) (cf. Sec. 18.5), and hence only (39.12) may be regarded as a termination of the deformation process in a perfectly elastic-plastic sheet with rigid inclusion. It should be stressed that the curve (39.12) is clearly concave, and hence the admissible domain of solutions does not exhibit the property of convexity in the case under consideration.

\section{Combined loadings of plates and shells}

\subsection{Plates under surface loadings}

Determination of limit carrying capacity for plates under several independent transverse loadings, in principle similar to that for beams, presents as a rule much greater difficulties. The paper by D. C. Drucker and H. G. Hopkins [8.119] on the limit carrying capacity of a circular plate under a concentrated central force and a uniformly distributed loading is probably the first one on combined loading of the plate as a whole. A simplified approach to this problem may be found in the book by J. B. Martin [8.330]. The elastic-plastic range of such a circular plate was considered by N. A. Forsman [8.150], whereas R. T. Shield [8.474] discussed the problem of optimal limit design of that plate. The latter paper was extended to several circularly-symmetric loadings by D. E. Charrett and G. I. N. Rozvany [8.68], and by R. Reiss [8.424]. The limit carrying capacity of circular plates under two circularly-symmetric distributed loadings of opposite sense, acting on unequal parts of those plates, was considered by W. Flügge and J. C. Gerdeen [8.145] (TG yield condition), whereas N. Jones [7.117] calculated finite deflections of rigidplastic circular plates under the above system of loadings. Equal sense of both independent distributed loadings was analysed by P. G. Hodge [4.223]. A. Sawczuk and Th. Jaeger [4.513] considered the following cases of combined loadings: concentrated force and edge couples, distributed loading and edge couples, concentrated force and ring of forces. V. M. Klyagin [8.248] derived the plastic interaction curve for a ribbed circular plate under simultaneous uniformly distributed loading and edge couples. B. Paul [8.390] considered annular plates (flanges) under a uniform twisting moment and radial pressure. 
Numerous papers are devoted to circular and annular plates under simultaneous action of normal forces and in-plane loading. A. S. Grigorev [8.180] investigated the limit carrying capacity of a circular plate under uniformly distributed normal loading, in-plane tension and centrifugal forces. Further papers on the limit state of circularly-symmetric plates submitted to combined loadings under consideration are due to P. G. Hodge and R. Sankaranarayanan [8.199] (TG yield condition with associated flow rule, detailed analysis of interaction curves for annular plates), J. B. Haddow [7.81] (simply supported circular plates, TG yield condition), Yu. R. Lepik [8.303] (annular plates, anisosensitive material), H. M. Haydl and A. N. Sherbourne [8.188] (simply supported and clamped circular plates, Ivanov's approximation for the $\mathrm{HMH}$ yield condition, numerical integration). Deflections of circularly-symmetric plates under simultaneous normal loading and in-plane tension were considered by Y. Ohashi and S. Murakami [7.235] (elastic-plastic plates, small deflections) and [8.374] (large deflections), Yu. R. Lepik [4.327] (large deflections of rigid-plastic plates), K. W. Neale and N. C. Lind [8.362] (large deflections of rigid-plastic plates, TG yield condition with FlüggeNakamura's approximation).

The limit carrying capacity of rectangular plates under simultaneous action of concentrated and distributed loadings was first discussed by A. Sawczuk [8.445], (yield-line approach) and then extensively investigated by Z. Sobotka: in [8.498] he analysed orthotropic clamped plates, and in [8.499]-isotropic plates simply supported along two opposite edges, in [8.500] he proposed a general lower bound approach, and in [8.501] he gave a detailed analysis for simply supported plates. Rectangular plates under simultaneous normal loadings and in-plane tension were considered by A. Sawczuk [8.444], J. B. Alblas [8.5] (two edges clamped and two edges free) and E. Elter [8.126] (influence of residual stresses, kinematic hardening), whereas Z. Marciniak [8.328] considered a related problem from the point of view of metal-forming processes. Elastic-plastic deflections of arbitrarily shaped plates under normal loadings and in-plane tension were considered by H. Armen, H. S. Levine and A. Pifko [8.18] (kinematic hardening of Ziegler's type, finite element method).

The limit carrying capacity of reinforced plates under several independent loadings was considered by F. N. Pannell [8.386]: he proved a theorem stating that the sum of reinforcements sufficient for individual loadings is sufficient for all the loadings acting simultaneously.

Shakedown analysis of plates under variable surface loadings was initiated by D. A. Gokhfeld [7.68] and J. A. König [4.303, 7.140]. Further papers are due to D. A. Gokhfeld and O. F. Chernyavsky [4.175] (generalized stresses), A. K. Lyubimov [7.176] (hardening and softening materials), M. A. Muspratt [8.355] (linear programming methods), Yu. Yu. Atkochiunas, R. P. Karkauskas and A. A. Cyras [8.19] (circular plates, finite element method), R. M. Kirakosyan [8.242] (cone-like distributed variable loadings), Y. Ohashi and K. Kawashima [7.244] (large deflections, plastic hardening described by a fifth-degree parabola, experimental verifi- 
cation). Optimal design of plates in shakedown conditions was considered by L. G. Lantukh and A. V. Perelmuter [8.295] (convex programming methods), J. A. König [8.268] (one-parameter variable loading), Yu. M. Pochtmân and Z. I. Pyatigorsky [8.408, 8.409] (design without calculations of the elastic response).

\subsection{Plates under body forces and thermal loadings}

Body forces due to the rotation of a plate under simultaneous normal pressure were first considered in the already mentioned paper by A. S. Grigorev [8.180]. Further papers on the limit state of rotating circular plates under pressure were published by M. Sh. Mikeladze [8.341] (simplified HMH yield condition), V. N. Alpert [8.8] (symmetric and antisymmetric pressure distributions, lower bound to the plastic interaction curve), A. N. Sherbourne and S. D. N. Murthy [8.461] (anisotropic plates, generalized TG and $\mathrm{HMH}$ yield conditions). Additional thermal effects were studied by Yu. N. Shevtchenko [8.463], I. A. Motovilovets and Yu. N. Shevtchenko [8.350]; they assumed plastic hardening and employed the method of successive elastic solutions.

The limit state of a circular plate under bending and thermal loadings was considered by N. Perrone [8.395] under the assumption of temperature-dependent yield-point stress; this problem was also studied by L. I. Polyakov and M. A. Rudis [8.414]. Further papers are due to A. V. Kolgadin [7.134] (elastic-plastic bending of rectangular plates in a temperature field), N. M. Adyasova, S. A. Kapustin and E. I. Sankov [8.1] (arbitrarily shaped plates with holes, various boundary conditions, finite difference method combined with iterations), T. P. Vinogradova and A. P. Shinkarenko [8.546] (rectangular plates, plastic hardening, finite differences), T. D. Semykina and A. A. Firtytch [8.452] (sandwich plate, TG yield condition, non-steady temperature field), V. M. Dolinsky and B. S. Kovalsky [8.117] (elastic-plastic bending of a circular plate resting on elastic-plastic foundation), E. Friedman and B. A. Boley [7.61] (effect of melting).

Yu. R. Lepik [8.302] investigated the limit carrying capacity of a circular plate under bending and simultaneous neutron irradiation. H. M. Haydl and A. N. Sherbourne [7.89] proved an analogy between irradiated and variable-thickness plates.

Shakedown of plates under variable thermal loadings was first analysed by H. Yüksel [7.392], who investigated the formation of individual plastic zones. Further papers are due to V. I. Rozenblyum [4.489] (arbitrarily shaped plate with clamped edges), D. A. Gokhfeld [7.68] (simply supported circular plate under pressure and a parabolic temperature distribution, both varying in time), [4.170] (clamped circular plate), and [4.174] (arbitrarily shaped plates), O. F. Chernyavsky [4.73] (Pontryagin's principle, without the replacement of the actual plate by a finite-degrees-of-freedom model), J. A. König [8.269] (generalization of Rozenblyum's solution for temperature-dependent elastic moduli), A. R. Belyakov and O.F. Chernyavsky [8.39] (circular plate under pressure, temperature and edge 
loadings), Z. Waszczyszyn and E. Pabisek [8.552, 8.553] (numerical analysis of modified elastic curves for a circular plate).

Let us also mention here the papers on optimal design by C. Polizzotto [8.412] (a general approach allowing for body forces), U. Lepik and Z. Mróz [8.304] (optimal design of a plastic plate under impulsive loading).

\subsection{Shells under surface loadings}

From among various cases of combined loadings of shells the case of the circular cylindrical shell under simultaneous radial pressure and axial loading is the one most often analysed in the plastic range. If the shell is sufficiently long and the pressure is distributed uniformly, then the state of stress may be regarded as homogeneous and very simple for analysis (homogeneous cases were discussed in Sec. 37), whereas possible complications result from edge effects, or from beamcolumn effects if the axial loading is compressive.

The earliest paper was that of P. G. Hodge [7.93], who employed the TrescaGuest yield condition and considered a clamped sandwich shell of finite length under simultaneous radial pressure and axial force. Plastic interaction curves for cylindrical shells clamped at one end were derived by E. T. Onat [7.252] and R. Sankaranarayanan [8.441]. P. G. Hodge and J. Panarelli considered in [4.200] uniform shells clamped at both ends and compared plastic interaction curves based on TG and HMH yield conditions and in [7.103]-shells under pressure, axial load and torque. Further papers were published by R. Dobrev [8.115] (effect of the loading path on the limit state), U. Lepik [7.158] (finite deflections of a rigid-plastic shell under tension and external pressure, TG yield condition and associated flow rule), M. Capurso and A. Gandolfi [8.65] (reinforced shells), M. Sayir [7.315] (axial tension with a ring of forces and with internal pressure), J. Klepaczko and J. A. König [8.247] (internal pressure with axial compression, geometry changes taken into account, experimental verification), L. M. Kull (8.276) (finite deflections of rigid-plastic sandwich shells), W. Wojewódzki and H. Stolarski [8.558] (internal pressure varying linearly along the axis), V. S. Gudramovitch and I. A. Diskovsky [8.183] (finite deflections under pressure with axial compression of a sandwich shell) and A. Ubaydillayev [8.537, 8.538] (axial tension and a ring of radial compressive forces).

Simultaneous action of internal pressure and edge moments was considered by V. D. Bordyug [8.54] (boundary layer method), H. S. Ho and D. P. Updike [8.198] (TG yield condition with associated flow rule, detailed analysis of various mechanisms of plastic collapse), H. M. Haydl and A. N. Sherbourne [8.187] (a simple approximate method and a comparison with more accurate analysis), and A. M. Khomyakov [8.238] (internal pressure combined with a local meridional moment). Other papers on combined loadings of cylindrical shells refer mainly to independent internal and external pressures: P. G. Hodge [4.223] considered uniform external pressure with two rings of internal forces at the ends, P. K. Agrawal and P. G. 
Hodge [8.2] - a ring of external forces and uniform internal pressure, W. Wojewódzki and H. Stolarski [8.559] — linearly varying pressure and a ring of forces, Yu. K. Kulikov and A. M. Khomyakov [8.275] -distributed loading and concentrated normal forces.

Rotationally symmetric shallow shells (panels) under combined loadings were considered first by Sh. Mikeladze [8.341] (a paraboloidal shell under pressure and compression in supporting plane). Further papers are due to O. N. Shabliy [8.455] (spherical shell, TG yield condition), O. N. Shabliy and P. F. Kupriychuk [8.456] (conical shell), I. S. Akhmedyanov and A. V. Kireyev [4.5] (spherical shell, elasticplastic range, arbitrary loading, Fourier series combined with iterations), H. M. Haydl and A. N. Sherbourne [7.86] (limit state of a spherical shell under pressure and compression in supporting plane, HMH yield condition including shear effects) and [8.462] (moderately large deflections of the above shell), and by M. S. Mikhalishin [8.342] (spherical shell made of anisosensitive plastic material). Toroidal shells under combined loadings were discussed by A. N. Semenenko [8.451] (limit carrying capacity under pressure and edge loadings) and J. Skrzypek [7.342] (rigidplastic sandwich shells under pressure and overall bending, finite deflections and finite strains). S. Palusamy and N. C. Lind [8.383] investigated experimentally shell-nozzle junctures under pressure, normal and shearing forces. Many solutions are reviewed in a more detailed form in a survey paper by P. G. Hodge [8.203].

Optimal plastic design of shells under combined loadings was initiated by E. T. Onat and W. Prager [7.253]: they considered optimal thickness variation of a cylindrical shell under uniformly distributed internal pressure and axial load. Their solution was extended by W. Freiberger [7.60] to arbitrary rotationally symmetric pressure distribution. Further papers are due to M. Save and R. T. Shield [8.443] (fixed and moving loads), M. R. Ibragimov [8.206] (several fixed loadings), P. Stehlin and L. Holsteinson [8.506] (orthotropic sandwich panels under compression and shear), F. G. Shamiyev and S. M. Gabibzade [8.457] (uniformly distributed internal pressure and ring of external forces). A related problem of the most inconvenient distribution of loading acting on a rotationally symmetric shell was discussed by M. A. Danelashvili [8.105].

Problems of plastic shakedown of shells were analysed mainly in connection with the design of pressure vessels. Hence shakedown analysis under thermal loadings was developed side by side with or even ahead of a similar analysis under purely surface loadings (cf. Sec. 37.4 and 40.4). Here we quote only the survey papers by D. G. Eyre and T. V. Galambos [8.136], A. Sawczuk [4.517], G. Maier and J. A. König [8.316], and the design guides by G. E. Findlay and J. Spence [8.143], F. A. Leckie and R. K. Penny [8.301] and S. Vejvoda [8.541], and give a brief review of papers on shakedown under purely surface loadings.

F. A. Leckie [8.299] considered shakedown of intersections of cylindrical and spherical shells and determined the optimal ratio of (constant) thicknesses of these shells; F. A. Leckie and R. K. Penny [8.300], E. Proctor and R. F. Flinders [8.418] discussed a similar problem of cylindrical nozzles in spherical vessels. A. Sawczuk 
[7.308, 8.446] developed a general method of evaluation of upper bounds to shakedown loadings for shells. Cylindrical! shells junder cyclic internal pressure were also discussed by U. S. Saidkarimov and V. A. Tolok [8.436, 8.437]. Further papers are due to N. P. Theophilos [8.524] (spherical shells, TG yield condition), J. D. Fox, H. Kraus and R. K. Penny [8.153] (elipsoidal heads of pressure vessels, twoparameter| system of self-stresses), W. A. Macfarlane and G. E. Findlay [8.313] (shell junctions), G. Guerlement [8.184] (torispherical heads), I. S. Chernyshenko and G. K. Sharshukov [8.83a] (spherical shell with a hole).

The theory of elastic-plastic stability of shells under combined loadings was developed mainly by E. I. Grigolyuk; we do not discuss the relevant papers because of the specific character of stability problems.

\subsection{Shells under body forces and thermal loadings}

The literature on plastic deformations of shells under body forces combined with other loadings is rather scarce. We shall only mention here the paper by $\mathrm{O}$. N. Shabliy and P. F. Kupriychuk [8.456] (shallow conical shell under pressure, ring of forces and centrifugal forces); some related papers on rotating cylinders were discussed in Sec. 37.4.

Conversely, relatively many papers deal with plastic shells under combined thermal loadings. Studies of thermoplasticity of shells were initiated by V. S. Chernina [8.74] (cooling of an infinitely long cylindrical shell consisting of two materials, perfect plasticity and linear hardening), E. T. Onat and S. Yamantürk [7.256] (multilayer shells, temperature-dependent yield-point stress, detailed analysis for a cylindrical shell). I. Z. Palley [8.381] investigated the effect of thermal loading history in a sandwich shell. An engineering method of calculation of the maximal carrying capacity of jet engine shells was developed by V. I. Feodosyev [8.142]. Further papers on the thermoplasticity of cylindrical shells are due to V. S. Zarubin [8.566] (sandwich shell, isotropic hardening, effect of loading history), L. I. Polyakov and M. A. Rudis [8.415] (temperature as a function of ?axial jvariable, temperature-dependent yield-point stress), V. V. Piskun [7.263, 8.399, 8.400] (non-steady temperature field, normal pressure and axial loading, incremental theory of plastic hardening, iterative procedure), S. A. Kapustin [7.125] (kinematic hardening of the Kadashevitch-Novozhilov type), S. A. Kapustin and N. M. Adyasova [8.229] (reinforced shells), and by I. B. Tarasov [8.521] (local thermal loading combined with internal pressure).

Rotationally symmetric shells under combined thermal loadings were considered by A. I. Borisyuk [7.11] (isotropic hardening, method of variable stiffnesses, detailed calculations for a conical shell), V. I. Makhnenko and E. A. Velikoivanenko [7.177] (non-steady temperature field' combined with pressure, method of variable stiffnesses, spherical shell), I. V. Prokhorenko [7.269] (temperature, pressure and edge loadings, method of variable stiffnesses), V. I. Makhnenko, L. A. Izbenko and Yu. A. Skosnyagin [8.318] (kinematic hardening), and by Yu. N. 
Shevtchenko [7.336] (open rotationally symmetric shells, isotropic hardening, method of elastic solutions). A. I. Borisyuk [8.56] considered thermal loadings combined with body forces (rotation of a conical shell), and A. I. Borisyuk with I. V. Prokhorenko [8.57]-thermal loadings combined with neutron irradiation.

Dynamic problems of thermoplasticity of rotationally symmetric shells were considered by V. G. Bazhenov and G. S. Mikhaylov [8.32, 8.33] (thermal shock, finite deflections, kinematic hardening), by the same authors with A. G. Ugodchikov [8.34] (temperature-dependent yield-point stress and a numerical example), and by A. P. Sinitsyn [8.486] (thermoplastic waves of combined stresses).

Problems of plastic shakedown of shells under thermal loadings were developed mainly by D. A. Gokhfeld and his collaborators. Let us mention here the papers by D. A. Gokhfeld [7.68] (cylindrical shell under a constant ring of forces and variable temperature), D. A. Gokhfeld, P. I. Ermakov and I. M. Plagov [7.67] (cylindrical shell under a moving heat source), I. M. Plagov [8.401, 8.402] (incremental collapse of cylindrical shells, application to ingot moulds), O. F. Chernyavsky [8.82] (conical shell of arbitrarily variable thickness under constant pressure and variable temperature distribution, perfect plasticity), D. A. Gokhfeld, A. G. Laptevsky and O. F. Chernyavsky [7.69, 7.70, 8.177, 7.71] (incremental collapse, TG yield condition, kinematic approach), I. M. Plagov and O. F. Chernyavsky [8.403] (incremental collapse of elements of metallurgical equipment), O. F. Chernyavsky and E. F. Chernyayev [8.83] (semi-infinite cylindrical shell under independently variable pressure, edge forces, edge moments and temperature), D. A. Gokhfeld, O. F. Chernyavsky and E. F. Chernyayev [7.72] (cyclic changes of level of heating medium). Other papers are due to A. I. Borisyuk [8.55] (rotationally symmetric shells where both surface loadings and temperature are variable, temperaturedependent elastic and plastic moduli, numerical calculations for a conical shell), Yu. G. Korotkikh and S. A. Kapustin [7.137] (thermal loading and ring of forces, mixed hardening, effects of loading history), F. J. Beer [8.35] (constant pressure and variable temperature, a comparison of linear and parabolic temperature distribution in the wall), R.S. Barsoum [8.30] (kinematic hardening, numerical method), R. M. Kirakosyan [8.243] (cylindrical shell of elliptic profile, cyclic changes of pressure and temperature), I. V. Prokhorenko and V. V. Piskun [8.419] (rotationally symmetric shells, incremental theory of plasticity, plastic hardening), V. S. Zarubin and A. A. Polyakov [8.567] (sandwich conical shells in membrane state).

Related shakedown problems of cylinders were discussed in Sec. 37.4.

\subsection{Interaction curves for a cylindrical shell under a ring of forces and axial loading}

As an example of the determination of interaction curves for shells under combined loadings we consider an infinitely long sandwich circular cylindrical shell under a ring of forces $2 Q$ at $x=0$ and axial loading $p_{x}^{*}$ at infinity (Fig. 201, [7.371, 7.372]). The material of the shell is assumed to be isotropic and incompressible, 


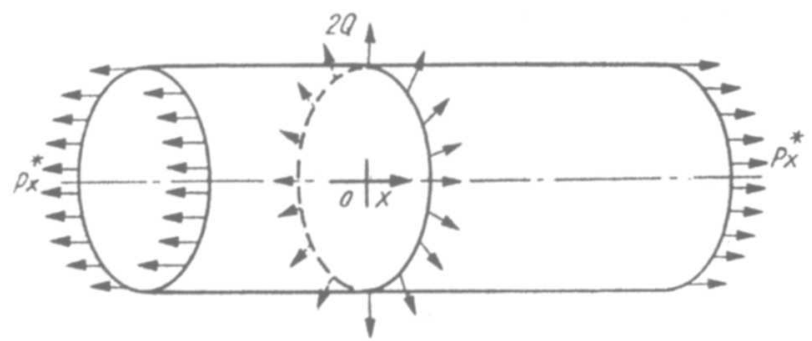

Fig. 201. A cylindrical shell under a ring of forces and axial loading

perfectly elastic-plastic, and subject to Huber-Mises-Hencky yield condition. The small-deflection theory is employed.

We first derive the equations of the elastic interaction curve. Let us introduce the dimensionless variables (34.19), and make use of the basic equations given in Sec. 34.4 with substituted $q_{r}(x) \equiv 0$. In the elastic range Hooke's law leads to (34.29) and to similar expressions for $\sigma_{x}^{+}$and $\sigma_{\theta}^{+}$; substituting these formulae into the equilibrium equations (34.23), we obtain

$$
\begin{aligned}
& 2 u^{\prime}-w=\frac{3}{2} p, \\
& 2 w^{\mathrm{IV}}+2 w-u^{\prime}=0,
\end{aligned}
$$

and after eliminating the axial displacement $u$,

$$
w^{\mathrm{IV}}+\frac{3}{4} w=\frac{3}{8} p \text {. }
$$

The corresponding homogeneous equation may be regarded as a particular case $(v=1 / 2)$ of the well-known governing equation for elastic cylindrical shells under end loads, which, however, is usually quoted without axial loading. The general solution of (40.2) is as follows:

$$
w=e^{-\beta x}\left(A_{1} \cos \beta x+A_{2} \sin \beta x\right)+e^{\beta x}\left(A_{3} \cos \beta x+A_{4} \sin \beta x\right)+\frac{p}{2},
$$

where $\beta^{4}=3 / 16$. In view of the symmetry of the shell we consider only its righthand side, $x \geqslant 0$. The condition at infinity, $w^{\prime}(\infty)=0$, yields $A_{3}=A_{4}=0$. Two further boundary conditions are $w^{\prime}(0)=0$ and

$$
\left.D \frac{d^{3} w^{*}}{d x^{* 3}}\right|_{x=0}=-Q,
$$

where

$$
D=\frac{2 E h H^{2}}{1-v^{2}}=\frac{8}{3} E h H^{2}
$$

denotes the elastic bending rigidity of the shell, and $w^{*}$ and $x^{*}$ are the physical (dimensional) deflection and the axial coordinate, respectively. Introducing into (40.4) dimensionless variables (34.19) and the dimensionless ring force

$$
q=\frac{1}{2 \sigma_{0} h} \sqrt{\frac{R}{H}} Q,
$$


we rewrite that boundary condition in the form

$$
w^{\prime \prime \prime}(0)=-\frac{3}{4} q .
$$

Making use of the above boundary conditions we obtain $A_{1}=A_{2}=-3 q / 16 \beta^{3}$, and hence

$$
w=-\frac{3 q}{16 \beta^{3}} e^{-\beta x}(\cos \beta x+\sin \beta x)+\frac{p}{2} .
$$

The corresponding elastic stress distribution is given by

$$
\begin{aligned}
\sigma_{x} & = \pm \frac{q \sigma_{0}}{2 \beta} e^{-\beta x}(\cos \beta x-\sin \beta x)+p \sigma_{0}, \\
\sigma_{\theta} & =\frac{q \sigma_{0}}{4 \beta} e^{-\beta x}\left[\frac{3}{4 \beta^{2}}(\cos \beta x+\sin \beta x) \pm(\cos \beta x-\sin \beta x)\right],
\end{aligned}
$$

where the upper and the lower signs refer to the outer and the inner layers, respectively. The stress intensity reaches its maximum at $x=0$, and equating this maximum to the yield-point stress $\sigma_{0}$, we determine the elastic interaction curve as follows:

$$
\left(p \pm \frac{q}{2 \beta}\right)^{2}+(\sqrt{3} \pm 1)^{2} \frac{q^{2}}{16 \beta^{2}}-(\sqrt{3} \pm 1)\left(p \pm \frac{q}{2 \beta}\right) \frac{q}{4 \beta}=1 \text {. }
$$

These two ellipses intersect at $q=0, p= \pm 1$, and at $p=0, q= \pm \sqrt{2 / \sqrt{3}}=$ \pm 1.0746 , and describe a curvilinear convex tetragon.

In what follows, we confine ourselves to the quadrant $q \geqslant 0, p \geqslant 0$. Then the maximal stress intensity in the elastic range is reached in the outer layer; exceeding the elastic carrying capacity, we arrive at a certain zone $0<x<x_{1}$, in which the outer layer is plastic (except for $p=\mathrm{J}$, when the elastic range is followed immediately by plastification of both layers, and for $q=0$, when the shell becomes plastic as a whole). Under the assumption of the Hencky-Ilyushin theory of plasticity, the zone under consideration is governed by Eq. (34.33) with substituted $q_{r}(x) \equiv 0$. In the elastic zone $x_{1}<x<\infty$ the general solution (40.3) with $A_{3}=A_{4}=0$ remains without change. This system of equations requires a total of 7 boundary conditions, which determine four integration constanis for Eq. (34.33), $A_{1}, A_{2}$, and the boundary coordinate $x_{1}$. They are as follows:

$$
\begin{aligned}
& w_{p}^{\prime}(0)=0, \quad m_{x p}^{\prime}(0)=q, \\
& w_{p}\left(x_{1}\right)=w_{e}\left(x_{1}\right), \quad w_{p}^{\prime}\left(x_{1}\right)=w_{e}^{\prime}\left(x_{1}\right), \\
& \left(\sigma_{e}^{+}\right)_{e}\left(x_{1}\right)=\sigma_{0} \text { or, equivalently, } \varphi_{p}^{+}\left(x_{1}\right)=\frac{1}{2 G}=\frac{3}{2 E}, \\
& m_{x p}\left(x_{1}\right)=m_{x e}\left(x_{1}\right), \quad m_{x p}^{\prime}\left(x_{1}\right)=m_{x e}^{\prime}\left(x_{1}\right),
\end{aligned}
$$


where the subscripts " $e$ " refer to the elastic zone, and the subscripts " $p$ " to the elastic-plastic zone. The last three conditions make it possible to determine the constants $A_{1}$ and $A_{2}$ :

$$
\begin{aligned}
A_{1}= & -\frac{3}{8 \beta^{2}} e^{\beta x_{1}}\left[\left(p-\frac{2}{\sqrt{3}} \sin \omega_{1}\right)\left(\sin \beta x_{1}+\cos \beta x_{1}\right)-\right. \\
& \left.-\frac{2}{\beta \sqrt{3}} \omega_{1}^{\prime} \cos \omega_{1} \cos \beta x_{1}\right], \\
A_{2}= & -\frac{3}{8 \beta^{2}} e^{\beta x_{1}}\left[\left(p-\frac{2}{\sqrt{3}} \sin \omega_{1}\right)\left(\sin \beta x_{1}-\cos \beta x_{1}\right)-\right. \\
& \left.-\frac{2}{\beta \sqrt{3}} \omega_{1}^{\prime} \cos \omega_{1} \sin \beta x_{1}\right],
\end{aligned}
$$

and yield the equation

$$
\left(2 \sqrt{3}-4 \omega_{1}^{\prime \prime}\right) \cos \omega_{1}+4 \omega_{1}^{\prime} \sin \omega_{1}+p \sqrt{3}=0
$$

where the subscripts "1" denote the values of the respective functions at $x=x_{1}$, and the subscript "+" of $\omega_{+}$has been dropped. Numerical integration of the governing equation (34.33) requires four initial conditions at the starting point $x=0$, and since (40.11) furnishes only two of them, we have to assume the remaining two and to solve a system of three equations at the point $x=x_{1}$, the coordinate of which is also unknown: two continuity conditions (40.11) and the condition (40.13).

Without going into the details of numerical solutions in the elastic-plastic range, we shall now discuss the decohesive carrying capacity. It is determined by the condition $\varepsilon_{x}^{+} \rightarrow \infty$ at $x=0$. Making use of (34.20), (34.25) and (34.27), we may write

$$
\frac{\varepsilon_{x}^{+}}{\varepsilon_{\theta}^{+}}=-\frac{u^{\prime}+w^{\prime \prime}}{w}=\frac{\sin (\omega-\pi / 6)}{\cos \omega},
$$

and hence the condition $\varepsilon_{x}^{+} \rightarrow \infty$ is equivalent to $\omega(0)=\pi / 2$ at $w(0) \neq 0$ (in order to eliminate $\varepsilon_{\theta}^{+}(0)=0$ ). This additional condition joins $p$ and $q$ and describes the interaction curve of the decohesive carrying capacity of the shell. Now Eq. (34.33) becomes singular at the starting point $x=0$ and a numerical solution must be completed by an appropriate generalized power series valid in the vicinity of that point. It turns out that the following generalized power series holds (for $x \geqslant 0$ )

$$
\omega(x)=\sum_{j=0}^{\infty} C_{j} x^{j / 2}=C_{0}+C_{1} x^{1 / 2}+C_{2} x+\ldots,
$$

where $C_{0}=\pi / 2$. The initial conditions $w^{\prime}(0)=0$ and $m_{x}^{\prime}(0)=q$ yield

$$
q=\frac{C_{1}^{2}}{\sqrt{3}}, \quad C_{2}=0,
$$


whereas substituting (40.15) into (34.33) and equating the coefficients of $x^{j / 2}$ on both sides make it possible to determine the subsequent coefficients of (40.15) in terms of $C_{1}$ and $C_{3}$ :

$$
\begin{aligned}
& C_{4}=\frac{\sqrt{3}}{15}, \\
& C_{5}=\frac{1}{6} C_{1}^{2} C_{3}-\frac{1}{2} \frac{C_{3}^{2}}{C_{1}}-\frac{1}{720} C_{1}^{5}, \\
& C_{6}=\frac{4 \sqrt{3}}{105}\left(\frac{C_{3}}{C_{1}}+\frac{p \sqrt{3}}{4 C_{1}^{2}}+\frac{C_{1}^{2}}{12}\right),
\end{aligned}
$$

The determination of the interaction curve of decohesive carrying capacity was carried out ${ }_{\text {; }}$ as follows. For an assumed value of axial loading $p$ and for arbitrarily chosen $C_{1}$ and $C_{3}$, the subsequent coefficients $C_{j}$ were found from (40.17); the series (40.15) was used in the vicinity of $x=0$ and then numerical integration of (34.33) (the Runge-Kutta procedure) was performed. Finally, the continuity conditions at $x=x_{1}$ and the condition (40.13) were treated as a system of equations with three unknowns $C_{1}, C_{3}$ and $x_{1}$, and solved by means of the falsi rule. Then the second coordinate of the interaction curve, $q$, was found from (40.16).

The procedure described above works for $0.2695 \leqslant p \leqslant 0.9720$, the corresponding values of $q$ being $q=1.2879$ and $q=0.3120$, respectively. For $p>0.9720$ there appears another zone of one-side plastification, namely plastification of the inner layer within a certain interval $x_{2}<x<x_{3}$; this zone is governed by Eq. (34.36). For $p$ very close to unity, even further zones of one-side plastification are possible. On the other hand, for $p<0.2695$ there appears a zone of two-side plastification $0<x<x_{1}$, governed by (34.40) and (34.41), and followed by a zone of one-side plastification $x_{1}<x<x_{2}$; in the latter zone either the outer layer is plastic (for $p>0.1$ ), or the inner layer is plastic (for $p<0.1$ ). In these cases the numerical procedures are more complicated; some details are given in [7.372]. For $p=0$ one obtains $q=1.9045$ and this point is common with the plastic interaction curve (curve of limit carrying capacity).

The whole quadrant of the interaction curve of decohesive carrying capacity is shown in Fig. 202 together with the elastic interaction curve and the plastic interaction curve; the latter curve determines the onset of yielding for a rigid-plastichardening shell, but, in general, cannot be reached by a perfectly elastic-plastic shell. The curve of decohesive carrying capacity is tangent to the elastic interaction curve at the point

$$
\begin{aligned}
& \bar{q}=\frac{\sqrt{3}-1}{\sqrt[4]{3}}=0.5562, \\
& \bar{p}=\sqrt{3}-1=0.7321 .
\end{aligned}
$$




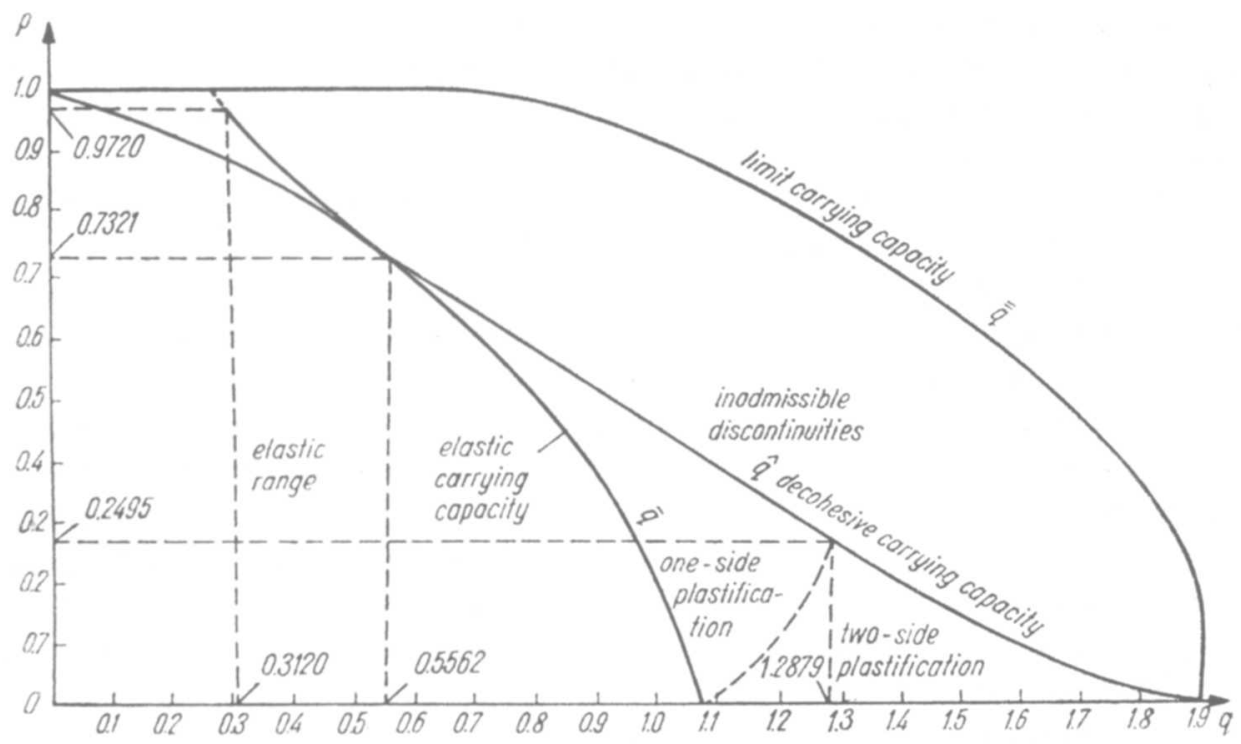

Fig. 202. Interaction curves for a cylindrical shell under a ring of forces and axial loading

Indeed, at this particular point of the elastic curve we have $\omega=\pi / 2$, but simultaneously $w=0, \varepsilon_{\theta}^{+}=0$ and $\varepsilon_{x}^{+}$in (40.14) may be arbitrary, not necessarily infinitely great.

It should be noted that the interaction curve of decohesive carrying capacity is concave. More details may be found in [7.371, 7.372, 7.373].

The decohesive carrying capacity of toroidal shells was investigated by J. Skrzypek [7.342], who compared the results of the deformation theory of plasticity and of the theory of plastic flow.

\subsection{Concluding remarks}

Problems of combined loadings of unbounded regions (half-plane, half-space, etc. should be considered separately. We do not devote a separate section to themsince very few of them have been published so far-but mention them briefly here)

Most of the papers are devoted to the propagation of elastic-plastic waves under combined dynamic loadings. The earliest investigations are due here to A. M. Skobeyev [8.487] (oblique impact), H. H. Bleich and I. Nelson [8.44] (independently acting normal and tangential pressures). Because of the large velocities of wave propagation, the viscous effects may be important; they were studied by S. Kaliski, W. K. Nowacki and E. Włodarczyk [8.226, 8.227]. Further papers are due to W. K. Nowacki [8.368] (bilinear theory of plasticity) and [8.369] (plastically passive waves under two-parameter loading); his solutions are summed up in the monograph [8.370]; T. C. T. Ting and N. Nan [8.528] (normal and tangential pressures), T. C. T. Ting [8.529] (interaction of shock waves due to two shear 
loadings), and [8.530] (plane and cylindrical waves, matrix approach), N. Cristescu [8.94] (coupling of waves), and A. P. Sinitsyn [8.485] (influence of the yield condition on wave propagation). Plastic waves due to combined loadings are discussed in detail in a survey by R. J. Clifton [8.88].

A static problem of combined pressure and punch loading on a half-space was considered by R. Negre and P. Stutz [8.363] (Coulomb yield condition).

In spite of a relatively extensive bibliography on combined loadings in the theory of plasticity, many problems are still open to theoretical or experimental investigations. Let us mention here some of them. From the point of view of engineering applications, the most important investigations at the level $\mathscr{P}$ of analysis are those concerning the physical criteria of termination of the process of plastic deformations (various forms of decohesion and material instability); it should be noted, however, that some approaches to this problem require also the subsequent levels of analysis (e.g. crack propagation). A more accurate description of plastic behaviour under cyclic combined loadings also requires further investigations. From the theoretical point of view the deviations from Drucker's postulate and the theory of plastic softening should be studied.

At the level $\mathscr{S}$ methods of analysis allowing for plastically passive processes should be developed. Such processes occur very often in engineering applications, but cause serious complications in analysis. A study of the effects of geometry changes at this level may also be interesting (thin-walled deformable sections of bars, sandwich sections of surface structures with a deformable core).

Particular attention should be paid to the development of the theory of combined loadings at the level $\mathscr{B}$. The effects of geometry changes are particularly important at this level and not yet sufficiently explored: they introduce new elements to the theory (concave interaction surfaces, effect of loading paths), and may be essential in applications. Various types of interaction surfaces (corresponding to decohesion, loss of stability, limits of shakedown) should be studied. The theory of elastic-plastic stability under combined loadings also requires further research. Plastic behaviour under dynamic combined loadings has not been studied thoroughly yet. Finally, let us mention here the problems of optimal plastic design of elements under combined loadings, constant or variable in time. 


\section{References*}

\section{Chapter I}

[1.1] E. Chwalla, Ưber die Behandlung der Stabilitätsfragen in den deutschen und österreichischen Stahlbaunormen, Stb 22 (1953), 4, 73.

[1.2] O. De Donato, G. Maier, On local unloading in piecewise-linear plasticity, TR ISTC 38 (1974), $20 \mathrm{pp}$.

[1.3] D. C. Drucker, Plasticity of metals: mathematical theory and structural applications, Proc. ASCE (1950).

[1.4] L. Finzi, Unloading processes in elastic-plastic structures, 9th ICAM 1956 (1957), 8, 137.

[1.5] P. G. Hodge, Jr., The linearization of the plasticity problems by means of non-homogeneous materials, IUTAM, NH, 1958 (1959), 147-156.

[1.6] - , Plastic analysis of structures, McGraw-Hill, New York 1959.

[1.7] A. A. Ilyushin, On the theory of small elastic-plastic deformations (in Russian), PMM 10 (1946), 3, 347-356.

[1.8] —, Plasticity (in Russian), Gos. Izd. Tekhn.-Teor. Lit., Moskva-Leningrad 1948.

[1.9] - , Normal and shearing stresses in pure bending of beams beyond the elastic limit and the analogy with bending of plates (in Russian), IS 19 (1954), 3-12.

[1.10] - , On the relation between stresses and small strains in the mechanics of continua (in Russian), PMM 18 (1954), 6, 641-666.

[1.11] -, Plasticity (in Russian), Izd. AN SSSR, Moskva 1963.

[1.12] V. D. Klyushnikov, Analytical theory of plasticity (in Russian), Izv. AN SSSR, Mekh. 1965/2, 82-87.

[1.13] B. Lücke, Die Berücksichtigung von Entlastungszonen und Zonen erneuter Belastung bei der elastisch-plastischen Blechbiegung mit endlichen Formänderungen, IA 43 (1973), 1, 34-43.

[1.14] J. Mandel, Note sur l'application du critère de Tresca au problème de la flexion circulaire d'une cylindre élastoplastique, AMS 24 (1972), 5-6, 863-871.

[1.15] W. Prager, Convexity in plasticity, AMS 24 (1972), 5-6, 827-836.

[1.16] J. Rychlewski, Note on the beginning of plastic deformation in a body under uniform pressure, AMS 17 (1965), 3, 405-412.

[1.17] R. M. Shneyderovitch, Strength at statical and repeated statical loading (in Russian), Mashinostroyenye, Moskva 1968.

[1.18] K. Szuwalski, M. Życzkowski, On the phenomenon of decohesion in perfect plasticity, IJSS 9 (1973), 1, 85-98.

[1.19] W. Świsterski, Wpływ ściśliwości materiału na odkształcenia sprężysto-plastyczne rozciąganego pręta o niejednorodności poprzecznej, CT 77 (1973), 4, 11-15.

[1.20] I. S. Voronyuk, On the position of the rotation axis of the cross-sections in the elements subject to bending (in Russian), SK, RMNTS 21 (1973), 18-28.

* Abbreviations are explained on pages 666-679. 
[1.21] J. Walczak, Nowoczesna miara wytężenia materiału, AMS, 3 (1951), 1, 5-26.

[1.22] V. Ya. Yablonko, On certain peculiarities of the system "testing machine-specimen" at low-cycle loading (in Russian), SDR PMTCN (1967), 162-169.

[1.23] M. Życzkowski, Wytężenie materiału w stanach podkrytycznych, RI 8 (1960), 4, 725-761 (Polish full text); BAP SST 8 (1960), 7, 333-341 (German summary).

[1.24] -, Powierzchnie graniczne w teorii wytężenia, RI 9 (1961), 4, 609-637 (Polish full text); BAP SST 9 (1961), 12, 667-676 (German summary).

[1.25] -, Computation of the "Exertion" of material in subcritical states, BAP SST 10 (1962), 6, 211-220 (English summary); RI 10 (1962), 2, 279-305 (Polish full text).

[1.26] —, Zum Begriff der Konstruktionsanstrengung, OIA 17 (1963), 3, 169-186.

[1.27] —, Combined loadings in the theory of plasticity, IJNLM 2 (1967), 2, 173-205.

[1.28] —, Obciążenia złożone w teorii plastyczności, IPPT PAN-PWN, Warszawa 1973.

\section{Chapter II}

[2.1] A. Abel, H. Muir, The Bauschinger effect and discontinuous yielding, PhM 26 (1972), 2, 489-504.

[2.2] - , - A new look at the Bauschinger effect, MA 4 (1972), 8, 267-271.

[2.3] -, -, The effect of cyclic loading on subsequent yielding, AMet 21 (1973), 2, 93-97.

[2.4] -, - , The Bauschinger effect and stacking fault energy, PhM 27 (1973), 3, 585-594.

[2.5] J. Bauschinger, UUber die Veränderung der Elastizitätsgrenze und des Elastizitätsmoduls verschiedener Metalle, CI, 1881, 289-348.

[2.6] -, Über die Veränderung der Elastizitätsgrenze und der Festigkeit des Eisens und Stahls ..., MMTL XV, München 1886, 1-116.

[2.7] D. Besdo, Einige Betrachtungen über Stoffgesetze der Plastomechanik Cosseratscher Kontinua, ZAMM 54 (1974), T70-T71.

[2.8] N. I. Bezukhov, Theory of elasticity and plasticity (in Russian), Gostekhizdat, Moskva 1953.

[2.9] H. F. Bohnenblust, P. Duwez, Some properties of a mechanical model of plasticity, JAM 15 (1948), 222-225.

[2.10] P. W. Bridgman, The compressibility of thirty metals, PAAAS 58 (1923), 163-242.

[2.11] $\mathrm{O}$. Bruhns, Vergleich einiger elastisch-plastischer Stoffgesetze bei Formänderungsprozessen mit Be- und Entlastung, ZAMM 52 (1972), 4, 40-42.

[2.12] - , On the description of cyclic deformation processes using a more general elastoplastic constitutive law, AMS 25 (1973), 3, 535-546.

[2.13] W. Burzyński, Studium nad hipotezami wytężenia, Akad. Nauk. Techn., Lwów 1928.

[2.14] G. I. Bykovtsev, V. V. Dudukalenko, D. D. Ivlev, On loading functions of an anisotropically hardening plastic material (in Russian), PMM 28 (1964), 4, 794-797.

[2.15] G. I. Bykovtsev, Yu. M. Myasnyankin, On the slip surfaces in the three-dimensional rigidplastic bodies (in Russian), DAN SSSR 167 (1966), 6, 1260-1262.

[2.16] G. I. Bykovtsev, D. D. Ivlev, Yu. M. Myasnyankin, On the relations on stress discontinuity surfaces in three-dimensional rigid-plastic, bodies (in Russian), PMM 32 (1968), 3, 472 477.

[2.17] P. C. T. Chen, A comparison of flow and deformation theories in a radially stressed annular plate, Trans ASME E40 (1973), 1, 283-287.

[2.18] N. I. Chernyak, D. A. Gavrilov, Deformation of metals at static repeated loading (in Russian), Nauk. Dumka, Kiev 1971.

[2.19] R. J. Clifton, On the equivalence of $F^{e} F^{p}$ and $\vec{F}^{p} \bar{F}^{e}$, Trans ASME E39 (1972), 2, 287-289.

[2.20] M. Como, Sull'influenza della comprimibilitá del materiale nei problemi di elasto-plasticitá, CoM 19 (1967), 2, 1-7. 
[2.21] N. Coutris, J. Ségura, Etude dans le domaine plastique des milieux avec couples de contrainte, CRAS 272 (1971), 17, A1126-A1129.

[2.22] I. S. Degtyarev, On the stress and strain rate discontinuities in three-dimensional problems of a compressible rigid-plastic body (in Russian), PMM 35 (1971), 5, 926-929.

[2.23] - , On the relations on the surfaces of velocity discontinuities in a compressible rigid-plastic body (in Russian), Izv. AN SSSR, MTT 1972/4, 170-172.

[2.24] U. Dehlinger, Die Fliessbedingung bei mehrachsigem Spannungszustand vielkristalliner Metalle, ZMet 35 (1943), 9, 182-184.

[2.25] W. Derski, S. Ziemba, Analiza modeli reologicznych, IPPT PAN-PWN, Warszawa 1968.

[2.26] A. G. Dorfman, S. D. Leytes, Elastic-plastic deformations and limit carrying capacity of a cylindrical bar subject to simultaneous torsion and tension (in Russian), SMRS 1964/2, 27-31.

[2.27] J. E. Dorn, A. J. Latter, Stress-strain relations for finite elasto-plastic deformations, $J A M$ 15 (1948), 234-236.

[2.28] D. C. Drucker, Some implications of work hardening and ideal plasticity, $Q A M 7$ (1950), 411-418.

[2.29] —, Coulomb friction, plasticity and limit loads, JAM 21 (1954), 71-74.

[2.30] F. Edelman, On the coincidence of plasticity solutions obtained with incremental and deformation theories, 1st USNCAM (1952), 493-498.

[2.31] A. C. Eringen, Nonlinear theory of continuous media, McGraw-Hill, New York 1962.

[2.32] H. Ford, Advanced mechanics of materials, Longmans, London 1963.

[2.33] A. M. Freudenthal, H. Geiringer, Mathematical theories of the inelastic continuum, HP6 (1958), 229-433.

[2.34] A. M. Freudenthal, Introduction to the mechanics of solids, Wiley, New York 1966.

[2.35] A. Gajewski, M. Życzkowski, An optimal forming of a bar compressed with subtangential force in elastic-plastic range, AMS 23 (1971), 2, 147-165.

[2.36] G. Gerard, S. Wildhorn, A study of Poisson's ratio in the yield region, NACA TN 2561, 1952.

[2.37] A. Gleyzal, A mathematical formulation of the general continuous deformation problem, QAM 6 (1949), 429.

[2.38] I. I. Goldenblat, Some problems of mechanics of deformable media (in Russian), Gostekhizdat, Moskva 1955.

[2.39] A. E. Green, W. Zerna, Theoretical elasticity, Clarendon Press, Oxford 1954.

[2.40] A. E. Green, P. M. Naghdi, Plasticity theory and multipolar continuum mechanics, Mat 12 (1965), 1, 21-26.

[2.41] - , - A general theory of an elastic-plastic continuum, ARMA 18 (1965), 4, 251-281.

[2.42] A. E. Green, B. C. McInnis, P. M. Naghdi, Elastic-plastic continua with simple force dipole, IJES 6 (1968), 7, 373-394.

[2.43] H. J. Greenberg, W. S. Dorn, E. H. Wetherell, A comparison of flow and deformation theories in plastic torsion of a square cylinder, "Plasticity", 2nd SNSM (1960), 279-296.

[2.44] W. L. Greenstreet, A. Phillips, A theory of an elastic-plastic continuum with special emphasis to artificial graphite, AMech 16 (1973), 1-2, 143-156.

[2.45] S. I. Gubkin, Plastic deformation of metals, vol. I-III (in Russian), Metallurgizdat, Moskva 1960.

[2.46] J. J. Guest, On the strength of ductile materials under combined stress, PhM 50 (1900), 69-132.

[2.47] Zh. H. Guo, Time derivatives of tensor fields in nonlinear continuum mechanics, AMS 15 (1963), 1, 131-163.

[2.48] G. B. Gurevitch, Fundamentals of the theory of algebraic invariants (in Russian), GTTI, Moskva-Leningrad 1948. 
[2.49] A. P. Gusenkov, Properties of the cyclic deformation diagrams at normal temperatures, (in Russian), SDR PMCTN (1967), 34-63.

[2.50] B. T. Haigh, The strain energy function and the elastic limit, Eng 109 (1920), 158-160.

[2.51] G. H. Handelman, W. H. Warner, Loading path and the incremental strain law, JMP 33 (1954), 2, 157-164.

[2.52] R. M. Haythornthwaite, A more rational approach to strain-hardening data, EP (1968), 201-218.

[2.53] H. Hencky, Zur Theorie plastischer Deformationen und der hierdurch im Material hervorgerufenen Nachspannungen, ZAMM 4 (1924), 4, 323-334.

[2.54] —, Úber die Form des Elastizitätsgesetzes bei ideal elastischen Stoffen, ZTP 9 (1928), 214-223, 457.

[2.55] A. V. Hershey, A review of the definitions of finite strain, 1st USNCAM (1951), 473-478.

[2.56] R. Hill, The mathematical theory of plasticity, Clarendon Press, Oxford 1950.

[2.57] -, On discontinuous plastic states, JMPS 1 (1952), 1, 19-30.

[2.58] -, Discontinuity relations in mechanics of solids, PSM 2 (1961), 247-276.

[2.59] M. Hlavaček, Pruzno-plastické látky se zpevněnim při uvažováni momentových napěti, StavC 21 (1973), 6-8, 441-451.

[2.60] P. G. Hodge, Jr., G. N. White, A quantitative comparison of flow and deformation theories of plasticity, JAM 17 (1950), 180-184.

[2.61] P. G. Hodge, Jr., On real and ideal materials, ExpM 11 (1971), 1, 12-18.

[2.62] -, Continuum mechanics, McGraw-Hill, New York 1970.

[2.63] O. Hoffman, G. Sachs, Introduction to the theory of plasticity for engineers, McGraw-Hill, New York 1953.

[2.64] T. C. Hsu, S. R. Davies, R. Royles, A study of the stress-strain relationship in the workhardening range, Trans ASME D89 (1967), 3, 453-458.

[2.65] T. C. Hsu, On the meaning of the stress invariants with particular reference to plasticity, BMEE 19 (1971), 1, 1-8.

[2.66] M. T. Huber, Właściwa praca odkształcenia jako miara wytężenia materiału, CT 22 (1904), 34-40, 49-50, 61-62, 80-81; Pisma, Vol. II, PWN, Warszawa 1956, 3-20.

[2.67] A. A. Ilyushin, Some problems of plastic deformations (in Russian), PMM 7 (1943), 4, 245-272.

[2.68] -, Relation between the Saint-Venant-Levy-Mises theory and the theory of small elasticplastic deformations (in Russian), PMM 9 (1945), 3, 207-218.

[2.69] - , Theory of plasticity at simple loading of the bodies exhibiting plastic hardening (in Russian), PMM 11 (1947), 2, 291-296.

[2.70] D. D. Ivlev, On the theory of simple deformation of plastic bodies (in Russian), PMM 19 (1955), 6, 734-735.

[2.71] - , On discontinuous solutions of three-dimensional problems of perfect plasticity (in Russian), PMM 22 (1958), 4, 480-486.

[2.72] —, Theory of perfect plasticity (in Russian), Nauka, Moskva 1966.

[2.73] D. D. Ivlev, On deformation theories of plasticity (in Russian), PGMSS (1969), 233-239.

[2.74] W. Jaunzemis, Continuum mechanics, Macmillan, New York 1967.

[2.75] C. F. Jenkin, A mechanical model illustrating the behaviour of metals under static and alternating loads, Eng 114 (1922), 603.

[2.76] W. Johnson, P. B. Mellor, Plasticity for mechanical engineers, Van Nostrand, LondonPrinceton 1962.

[2.77] W. Johnson, R. Sowerby, J. B. Haddow, Plane-strain slip-line fields: theory and bibliography, Arnold, London 1970.

[2.78] L. M. Kachanov, On a problem of combined loading (in Russian), PMM 19 (1955), 3, 371-375.

[2.79] —, Fundamentals of the theory of plasticity (in Russian), Gostekhizdat, Moskva 1956. 
[2.80] Yu. I. Kadashevitch, V. V. Novozhilov, Theory of plasticity taking residual microstresses into account (in Russian), PMM 22 (1958), 1, 78-89.

[2.81] A. E. Kalatinets, Combined loading of materials exhibiting full Bauschinger effect (in Russian), PTT (1974), 92-109.

[2.82] T. Kishi, T. Tanabe, The Bauschinger effect and its role in mechanical anisotropy, JMPS 21 (1973), 5, 303-315.

[2.83] Y. Kishino, Yield criterion derived through the consideration of plastic deformations with couple-stresses, TRTU 37 (1972), 1, 61-85.

[2.84] M. Kleiber, On the kinematics of elastic-plastic finite strains, BAP SST 21 (1973), 1, 25-31.

[2.85] V. D. Klyushnikov, On the conditions of proportional change of deviators in the theory of small elastic-plastic deformations (in Russian), Izv AN SSSR, OTN 1957/6, 138-139.

[2.86] O. M. Kochin, On unloading of elastic-plastic bodies (in Russian), UN 2 (1971), 129-137.

[2.87] V. V. Kolokoltchikov, Couple-stress theory of small elastic-plastic deformations (in Russian), VMU MM 1970/1, 76-84.

[2.88] S. Komljenović, Plastic flow with the non-symmetric stress tensor, BSCA RSFY A10 (1965), 6, 185.

[2.89] K. Kowalczyk, Liniowa schematyzacja wykresu naprężenie-odkształcenie przy obciążeniu cyklicznym i dowolnym, ustalonym udziale efektu Bauschingera, CT 77 (1973), 8, 25-34.

[2.90] K. Kowalczyk, M. Życzkowski, Badanie wpływu efektu Bauschingera na plastyczne przystosowanie się konstrukcji (na przykładzie układu prętowego), RI 22 (1974), 2, 145-167.

[2.91] W. Krzyś, M. Życzkowski, Sprężystość i plastyczność, PWN, Warszawa 1962.

[2.92] W. Krzyś, Optymalne kształtowanie z uwagi na stateczność ściskanych słupów cienkościennych o profilu zamkniętym, ZNPK M24, Kraków 1967.

[2.93] —, Optimale Formen gedrückter dünnwandiger Stützen im elastisch-plastischen Bereich, WZTUD 17 (1968), 2, 407-410 (German full text); BAP SST 21 (1973), 9, 409-419 (English summary).

[2.94] E. H. Lee, Elastic-plastic deformation at finite strains, Trans. ASME E36 (1969), 1, 1-6.

[2.95] S. Lees, PhM ser. 6, 44 (1922), 511.

[2.96] Th. Lehmann, Some thermodynamic considerations of phenomenological theory of nonisothermal elastic-plastic deformations, AMS 24 (1972), 5-6, 975-989.

[2.97] Yu. R. Lepik, L. Ya. Luht, On the influence of compressibility on bending of elastic-plastic plates (in Russian), TOP 5 (1964), 634-640.

[2.98] M. Levy, Mémoire sur les équations générales ... CRAS 70 (1870), 1323-1325.

[2.99] S. D. Leytes, On the elastic-plastic bending of a beam of rectangular cross-section (in Russian), Izv AN SSSR MM 1961/6, 133-139.

[2.100] —, Elastic-plastic deformations of a cylinder subject to simultaneous torque and tensile force (in Russian), IZh 5 (1965), 2, 382-387.

[2.101] T. H. Lin, M. Ito, Latent elastic strain energy due to the residual stresses in a plastically deformed polycrystal, Trans ASME E34 (1967), 606-611.

[2.102] H. Lippmann, [Eine Cosserat-Theorie des plastischen Fließens, AMech 8 (1969), 3-4, 255-284.

[2.103] J. Litoński, J. Klepaczko, Wpływ wstępnego plastycznego rozciągania na moduł Younga mosiądzu i niskowęglowej stali, RI 12 (1964), 2, 251-266.

[2.104] W. Lode, Versuche über den Einfluss der mittleren Hauptspannung auf die Fliessgrenze, ZAMM 5 (1925).

[2.105] W. Lode, Versuche über den Einfluss der mittleren Hauptspannung auf des Fliessen der Metalle-Eisen, Kupfer und Nickel, $Z P h 36$ (1926), 11-12.

[2.106] - , Der Einfluss der mittleren Hauptspannung auf das Fliessen der Metalle, FGI 303 (1928). 
[2.107] P. Ludwik, Elemente der technologischen Mechanik, Springer, Berlin 1909, Recent Russian translation: RP 15 (1971), 130-166.

[2.108] L. E. Malvern, Introduction to the mechanics of a continuous medium, Prentice-Hall, Englewood Cliffs 1969.

[2.109] J. Marin, L. W. Hu, On the validity of assumptions made in theories of plastic flow for metals, Trans ASME 75 (1953), 6, 1181-1190.

[2.110] G. Masing, Zur Heyn'schen Theorie der Verfestigung der Metalle durch Verborgen elastischer Spannungen, WVSK 3 (1923), 1.

[2.111] R. A. Mezhlumyan, On the function of transversal deformation (in Russian), PMM 16 (1952), 4, 491-494.

[2.112] R. Mises, Mechanik der festen Körper im plastisch deformablen Zustand, GNMPK 1 (1913), 4, 582-592.

[2.113] V. V. Moskvitin, Plasticity at variable loadings (in Russian), Izd. Mosk. Universiteta, Moskva 1965.

[2.114] Z. Mróz, On forms of constitutive laws for elastic-plastic solids, AMS 18 (1966), 1, 3-35.

[2.115] F. D. Murnaghan, The compressibility of solids under extreme pressures, KAV (1961), 121-136.

[2.116] J. Murzewski, Une théorie statistique du corps fragile quasihomogène, 9th ICAM 1956, (1957), vol. 5, 313-320.

[2.117] A. Nádai, Der Beginn des Fliessvorganges in einem tordierten Stab, ZAMM 3 (1923), 6, 442-444.

[2.118] —, Plastic behaviour of metals in the strain-hardening range, part I, JAP 8 (1937), 205-217.

[2.119] P. M. Naghdi, Stress-strain relations in plasticity and thermoplasticity, "Plasticity", 2nd SNSM (1960), 121-167.

[2.120] G. C. Nayak, O. C. Zienkiewicz, Convenient form of stress invariants for plasticity, Proc ASCE JSD 98 (1972), 4, 949-954.

[2.121] V. V. Novozhilov, On the physical meaning of stress invariants used in the theory of plasticity (in Russian), PMM 16 (1952), 5, 617-619.

[2.122] - On the class of combined loadings characterized by principal directions kept constant (in Russian), PMM 18 (1954), 4, 415-424.

[2.123] —, Theory of elasticity (in Russian), Sudpromgiz, Leningrad 1958.

[2.124] F. K. G. Odqvist, Kompatibilitätsgleichungen bei Zylinderkoordinaten, ZAMM 14 (1934), 2, 123-124.

[2.125] W. Olszak, P. Perzyna, A. Sawczuk (editors), Z. Marciniak, Z. Mróz, J. Rychlewski, W. Szczepiński, W. Urbanowski, M. Życzkowski, Teoria plastyczności, IPPT PANPWN, Warszawa 1965.

[2.126] W. R. Osgood, JAS 13 (1946), 45.

[2.127] T. Pełczyński, Wpływ stanu naprężeń na przejście materiału w stan plastyczny, PrzM 10 (1951), 175-179 and 204-208.

[2.128] P. Perzyna, Teoria lepkoplastyczności, IPPT PAN-PWN, Warszawa 1966.

[2.129] A. C. Pipkin, R. S. Rivlin, Mechanics of rate-independent materials, ZAMP 16 (1965), 3, 313-327.

[2.130] S. D. Ponomarev et al., Strength calculations in machine design (in Russian), Vol. I (1956), Vol. II (1958), Vol. III (1959), Mashgiz, Moskva.

[2.131] W. Prager, On isotropic materials with continuous transition from elastic to plastic state, Sth ICAM (1938), 234-237.

[2.132] —, Theory of plastic flow versus theory of plastic deformation, JAP 19 (1948), 6, 540-543.

[2.133] W. Prager, P. G. Hodge, Jr., Theory of perfectly plastic solids, Wiley, New York 1951.

[2.134] W. Prager, Discontinuous fields of plastic stress and flow, 2nd USNCAM (1955), 21-32.

[2.135] —, An elementary discussion of definitions of stress rate, QAM 18 (1961), 403-407. 
[2.136] W. Prager, Einführung in die Kontinuumsmechanik, Birkhäuser, Basel-Stuttgart 1961. English translation: Introduction to mechanics of continua, Ginn \& Co., New York 1961.

[2.137] L. Prandtl, Spannungsverteilung in plastischen Körpern, Ist ICM (1924), 43-54.

[2.138] -, Ein Gedenkenmodell zur kinetischen Theorie der festen Körper, ZAMM 8 (1928), 85-106.

[2.139] Yu. N. Rabotnov, Creep of structural elements (in Russian), Nauka, Moskva 1966.

[2.140] W. Ramberg, W. Osgood, Description of stress-strain curves by three parameters, NACA TN 902, July 1943.

[2.141] K. A. Reckling, Plastizitätstheorie und ihre Anwendung auf Festigkeitsprobleme, Springer Berlin-Heidelberg-New York 1967.

[2.142] A. Reuss, Berücksichtigung der elastichen Formänderungen in der Plastizitätstheorie, ZAMM 10 (1930), 3, 266-274.

[2.143] H. Richter, Verzerrungstensor, Verzerrungsdeviator und Spannungstensor bei endlichen Formänderungen, ZAMM 29 (1949), 65-75.

[2.144] M. Roš, A. Eichinger, Versuche zur Klärung der Frage der Bruchgefahr, EMPA Ber 14 (1926); 2nd ICAM (1926).

[2.145] V. I. Rozenblyum, On the influence of compressibility on plastic flow of elastic-plastic bodies (in Russian), Izv AN SSSR Mekh 1965/3, 95-101.

[2.146] G. Sachs, H. Shoji, Zug-Druckversuche on Messingkristallen (Bauschingereffect), $Z P h$ 45 (1927), 776-796.

[2.147] G. Sachs, Zur Ableitung einer Fliessbedingung, VDI Z 72 (1928), 734.

[2.148] M. S. Sarkisyan, On the relation between dissimilar deviators (in Russian), Izv AN SSSR MTT 1972/4, 64-69.

[2.149] A. Sawczuk, On yielding of Cosserat continua, AMS 19 (1967), 3, 471-480.

[2.150] L. I. Sedov, On the notions of simple loading and on admissible strain paths (in Russian). PMM 23 (1959), 2, 400-402.

[2.151] - , Introduction to the mechanics of a continuous medium (in Russian), Fizmatgiz, Moskva 1962; English translation: Addison-Wesley, Reading, Mass. 1965.

[2.152] -, Mechanics of a continuous medium (in Russian), Vol. 1, Izd. Nauka, Moskva 1970.

[2.153] S. V. Serensen, R. M. Shneyderovitch, Investigation of deformation processes and of criteria of metal fracture at cyclic loading in the elastic-plastic range (in Russian), SDR PMCTN (1967), 5-15.

[2.154] B. R. Seth, Generalized strain measure with applications to physical problems, IUTAM SOE (1962), Haifa.

[2.155] - Generalized strain and transition concepts for elastic-plastic deformation, creep and relaxation, 11th ICAM 1964 (1966), 383-389.

[2.156] -, Measure-concept in mechanics, IJNLM 1 (1966), 1, 35-40.

[2.157] M. R. Shammamy, Comparison of incremental and total theories with test data for circular torsion-tension members subjected to proportionate and non-proportionate loading, Doct. Diss. Univ. Ill., Urbana 1965, 98 pp.

[2.158] M. R. Shammamy, O. M. Sidebottom, Incremental versus total-strain theories for proportionate and non-proportionate loading of torsion-tension members, $\operatorname{Exp} M 7$ (1967), 12, 497-505.

[2.159] O. A. Shishmarev, E. Ya. Kuzmin, On the dependence of elastic constants of the metal on plastic deformations (in Russian), Izv AN SSSR OTN 1961/4, 167-169.

[2.160] H. Shoji, Ưber den Bauschingereffect, $Z P h 51$ (1928), 728-730.

[2.161] A. K. Sinitsky, Approximate solutions of elastic-plastic problems based on assumption of constant Poisson's coefficient (in Russian), Tr LPI (1950), 2.

[2.162] L. H. Sjodahl, J. B. Conway, Voce equation shown to be identical to the generalized strain concept, TMS AIME 245 (1969), 7, 1659-1660. 
[2.163] V. V. Sokolovsky, Theory of plasticity (in Russian), Gostekhizdat, Moskva 1946 and 1950; Wysshaya Shkola, Moskva 1969 (3rd edition).

[2.164] A. J. M. Spencer, Theory of invariants, part III of the volume "Continuum Physics", Academic Press, New York-London 1971.

[2.165] W. Szczepiński, On the effect of plastic deformation on yield condition, AMS 15 (1963), 2, 275-296.

[2.166] —, Wstęp do analizy procesów obróbki plastycznej, IPPT PAN-PWN, Warszawa 1967.

[2.167] G. B. Talypov, Influence of microstresses, causing the Bauschinger effect, on yield limit and stability in pure shear (in Russian), IUP 3 (1964), 237-345.

[2.168] - Investigations on the Bauschinger effect (in Russian), Izv AN SSSR MM 1964/6, 131-137.

[2.169] —, Analysis of experimental data on the Bauschinger effect and their theoretical treatment (in Russian), IZh MTT 1966/2, 108-115.

[2.170] - On the theory of plasticity taking the Bauschinger effect into account (in Russian), IZh MTT 1966/6, 81-88.

[2.171] —, Plasticity and strength of steel under combined loading (in Russian), Leningr. Univ., Leningrad 1968.

[2.172] J. J. Telega, O niektórych uogólnieniach twierdzeń nośności granicznej dla ośrodka Cosseratów, MTS 10 (1972), 3, 411-427.

[2.173] T. Y. Thomas, Extended compatibility conditions for the study of surfaces of discontinuity in continuum mechanics, JMM 6 (1957), 311-322, 907-908.

[2.174] - , Plastic flow and fracture in solids, Academic Press, New York-London 1961.

[2.175] H. Tresca, Mémoire sur l'écoulement des corps solides, MPDS 18 (1968), 733; 20 (1972), $75-135$.

[2.176] C. Truesdell, The mathematical foundations of elasticity and fluid dynamics, JRMA 1 (1952), 125-300.

[2.177] - Corrections and additions to "The mathematical foundations of elasticity and fluid dynamics", JRMA 2 (1953), 593-616.

[2.178] —, The simplest rate theory of pure elasticity, CPAM 8 (1955), 123-132.

[2.179] C. Truesdell, R. Toupin, The classical field theories, MP 3/1 (1962), 226-793.

[2.180] W. Truszkowski, Stress-strain relationship for tensile tested materials, AMS 8 (1956), 3, 581-595.

[2.181] K. Turski, Stress-strain equations for a reverse loading of a prestrained material, BAP SST 21 (1973), 10, 477-483.

[2.182] E. P. Unksov, An engineering theory of plasticity (in Russian), Mashgiz, Moskva 1959; English translation: Butterworths, London 1961.

[2.183] F. K. Van Iterson, Plasticity in engineering, Blackie and Son, London 1947.

[2.184] I. Vigness, T. E. Pardue, Poisson-ratio determinations for elastic and plastic strains of tensile specimens, NBS Circ. 528 (1954), 1-9.

[2.185] V. Z. Vlasov, The compatibility equations in curvilinear coordinates (in Russian), PMM 8 (1944), 4, 301-306.

[2.186] - , General theory of shells and its applications in engineering (in Russian), Gostekhizdat, Moskva 1959; German translation: Akademie-Verlag, Berlin 1955.

[2.187] E. Voce, A practical strain-hardening function, Met 51 (1955), 307, 219-226.

[2.188] J. Walczak, Wytrzymalość materialów oraz podstawy teorii sprężystości $i$ plastyczności, Vol. II, PWN, Kraków 1973.

[2.189] J. D. Weber, Hypothese des variables internes et visco-élasticité non-linéaire, IUTAM MVEMB 1974 (1975), 147-155.

[2.190] H. M. Westergaard, On the resistance of ductile materials to combined stresses, JFI 189 (1920), 627. 
[2.191] R. L. Woolley, The Bauschinger effect in some face-centred and body-centred cubic metals, PhM 44 (1953), 353, 597-618.

[2.192] A. Ylinen, A method of determining the buckling stress and required cross-sectional area for centrally loaded straight columns in elastic and inelastic range, Mem AIPC 16 (1956), 529-550.

[2.193] Y. Yoshimura, On the natural shearing strain, 2nd JCMAM (1952), 1-4.

[2.194] S. Zaremba, Sur une forme perfectionnée de la théorie de la relaxation, Bull. Int. Acad. Sci. Cracovie 8 (1903), 594-614.

[2.195] A. M. Zhukov, Elastic properties of a plastically deformed metal and combined loading (in Russian), IS 30 (1960), 3-16.

[2.196] —, Behaviour of metals in unloading and reverse loading (in Russian), IZh 1 (1961), $1,124-133$.

[2.197] - Some peculiarities of behaviour of a metal subject to elastic-plastic deformation (in Russian), VTP (1961), 30-57.

[2.198] H. Ziegler, D. McVean, On the notion of an elastic solid, RPAM (1967), 561-572.

[2.199] M. Życzkowski, Influence of compressibility of material on the stress distribution in elasticplastic plates, BAP SST 6 (1958), 1, 13-24 (English summary); ABM 5 (1958), 1, 53-87 (Polish full text).

\section{Chapter III}

[3.1] N. N. Afanasyev, Theory of fatigue strength under combined state of stress (in Russian), ZhTF 16 (1946), 4, 443-454.

[3.2] A. F. Akhmerov, Yu. B. Tyurin, A definition of rotating and of sliding modulus of hardening under combined stress (in Russian), $\operatorname{Tr}$ KAI 163 (1974), 92-98.

[3.3] N. Alberti, Conferme sperimentali di una condizione di plasticità, $R S 30$ (1960), 12, 19531959.

[3.4] L. S. Andreyev, On plastic deformations of metals under biaxial tension (in Russian), IZh 2 (1962), 3, 150-157.

[3.5] -, On the verification of laws of plasticity in the stress space (in Russian), IZh MTT 1966/2, 97-101.

[3.6] - , On the verification of the postulate of isotropy (in Russian), PM 5 (1969), 7, 122-125.

[3.7] - , On plastic deformation under loading trajectories with corners (in Russian), TM 9 (1970), 175-180.

[3.8] - Experimental investigation of plastic deformation under loading trajectories with corners (in Russian), Izv AN SSSR MTT (1971), 4, 143-149.

[3.9] B. D. Annin, B. P. Rusov, Experimental verification of the postulate of isotropy in the stress space (in Russian), DSS 3 (1969), 122-125; PP (1971), 119-120.

[3.10] J. H. Argyris, D. W. Scharpf, Methods of elasto-plastic analysis, ZAMP 23 (1972), 4, $517-$ 552.

[3.11] H. Armen, G. Isakson, A. Pifko, Discrete element methods for the plastic analysis of structures subjected to cyclic loading, AIAA/ASME 8th Conf. (1967), 148-161.

[3.12] R. A. Arutyunyan, On cyclic loading of an elastic-plastic medium (in Russian), Izv AN SSSR MM (1964), 4, 89-91.

[3.13] R. A. Arutyunyan, A. A. Vakulenko, On multiple loading of an elastic-plastic medium (in Russian), Izv AN SSSR MM (1965), 4, 53-61.

[3.14] R. A. Arutyunyan, On allowing for the Bauschinger effect and the plastic volume change in the theory of plasticity (in Russian), IUP 7 (1968), 87-94.

[3.15] Yu. M. Aryshensky, I. I. Kaluzhsky, V. V. Uvarov, Problems of the theory of plasticity of anisotropic media (in Russian), Tr FPI 69 (1973), 59-66. 
[3.16] E. K. Ashkenazi, Probiems of anisotropy of strength (in Russian), MPol (1965), 2.

[3.17] E. K. Ashkenazi, E. V. Ganov, Anisotropy of structural materials (in Russian), Mashinostroyenye, Leningrad 1972.

[3.18] G. Augusti, J. B. Martin, W. Prager, On the decomposition of stress and strain tensors into spherical and deviatoric parts, PNAS 63 (1969), 2, 239-241.

[3.19] V. D. Azzi, S. W. Tsai, Anisotropic strength of composites, ExpM 5 (1965), 9, 283-288.

[3.20] K. Sh. Babamuradov, Zh. Kultursynov, Numerical comparison of strain trajectories of a thin-walled tube, based on various theories of plasticity (in Russian), VVPM 10 (1972), 79-88.

[3.21] H. W. Babel, D. A. Eitman, R. W. McIver, The biaxial strengthening of tectured titanium, Trans ASME D89 (1967), 1, 13-18.

[3.22] G. Backhaus, Zur Fliessgrenze bei allgemeiner Verfestigung, ZAMM 48 (1968), 2, 99-108: BAP SST 16 (1968), 3, 223-232.

[3.23] - , Zur analytischen Darstellung des Materialverhaltens im plastischen Bereich, ZAMM 51 (1971), 6, 471-477.

[3.24] -, Zur analytischen Erfassung des allgemeinen Bauschingereffektes, AMech 14 (1972), $1,31-42$.

[3.25] -, Zum Stoffgesestz des elastisch-plastischen Körpers, ZAMM 52 (1972), 10, T293-T305.

[3.26] M. E. Backman, Form of the relation between stress and finite elastic and plastic strains under impulsive loading, JAP 35 (1964), 2524-2533.

[3.27] J. Bahuaud, M. Boivin, Nouvel aspect des formules du coefficient du Poisson sécant et tangent, JM 8 (1969), 4, 553-564.

[3.28] P. P. Balandin, On the problem of failure hypotheses (in Russian), VIT (1937), 1.

[3.29] W. M. Baldwin, Metals Technol. (1946).

[3.30] A. Baltov, A. Sawczuk, A rule of anisotropic hardening, AMech 1 (1965), 2, 81-92.

[3.31] G. I. Barenblatt, V. A. Gorodtsov, On the structure of the field of microstresses of uncontained plastic flow (in Russian), PMM 28 (1964), 2, 326-334.

[3.32] Yu. M. Bash, R. A. Vasin, K. E. Vega, On allowing for deformational anisotropy in the theory of plastic flow (in Russian), VTP (1961), 83-91.

[3.33] E. V. Bashkirov, Determination of deformational anisotropy of the material at variable loading (in Russian), RSK (1973), 105-108.

[3.34] V. N. Bastun, N. I. Chernyak, On application of some yield conditions for anisotropic steel (in Russian), PM 2 (1966), 1, 92-98.

[3.35] V. N. Bastun, On a certain case of dependence of the reduced elastic modulus on the state of stress (in Russian), PM 5 (1969), 2, 135-138.

[3.36] V. N. Bastun, N. I. Chernyak, On the applicability of hardening hypotheses within the range of small plastic strains (in Russian), PM 9 (1973), 12, 53-57.

[3.37] V. N. Bastun, On the yield condition of an anisotropically hardening material (in Russian), PPro (1974), 2, 88-96.

[3.38] -, Verification of hypotheses of the theory of small elastic-plastic deformations at elevated temperature (in Russian), TNEK 14 (1974), 24-27.

[3.39] V. N. Bastun, V. Ya. Bash, On the condition of existence of a uniform hardening curve in the case of deformational anisotropy (in Russian), PM 11 (1975), 3, 72-77.

[3.40] S. Batdorf, B. Budiansky, A mathematical theory of plasticity based on the concept of slip, NACA TN 1871 (1949),

[3.41] - , - Polyaxial stress-strain relations of a strain-hardening metal, JAM 21 (1954), 4, 323-326.

[3.42] A. B. Bayterekov, M. Ya. Leonov, Simple deformation of a plastic body (in Russian) Izv AN SSSR MTT (1974), 2, 88-96.

[3.43] V. L. Bazhanov, I. I. Goldenblat, N. A. Nikolayenko, A. M. Sinyukov, Analysis of Structures subject to thermal loadings (in Russian), Mashinostroyenye, Moskva 1969. 
[3.44] A. J. Becker, The strength and stiffness of steel under biaxial loading, Univ. of Illinois Bull. 13 (1916).

[3.45] S. M. Belevitch, Plane problem of thermoplasticity under combined loading (in Russian), UZGU 122 (1970), 33-44.

[3.46] J. F. Bell, The experimental foundations of solid mechanics, HP 6a/1, Springer, Berlin-Heidelberg-New York 1973.

[3.47] F. P. Belyankin, V. F. Yatsenko, G. G. Margolin, Limit deformability of anisotropic plastics under biaxial compression (in Russian), PM 3 (1967), 9, 90-94.

[3.48] I. A. Berezhnoi, V. V. Ignatyev, On some pecularities of transformation of singular loading functions for a two-dimensional plastic model (in Russian), Izv AN SSSR MTT (1969), 4, 144-146.

[3.49] I. A. Berezhnoi, D. D. Ivlev, E. V. Makarov, On deformational models of the theory of plasticity and of complex media (in Russian), PMM 34 (1970), 3, 553-557.

[3.50] I. A. Berezhnoi, V. V. Dudukalenko, D. D. Ivlev, On acquired anisotropy of plastic bodies (in Russian), MSS (1972), 41-46.

[3.51] I. A. Berezhnoi, V. I. Tseyler, On construction of loading surfaces for complex elasticplastic models (in Russian), Tr NIIMVU 10 (1973), 3-15.

[3.52] - , - On construction of loading surfaces for a quasi-plastic model of a group of wheels and the results of some experiments (in Russian), Izv AN SSSR MTT (1974), 4, 175-180.

[3.53] C. A. Berg, Construction of the equivalent plastic strain increment, SAM 51 (1972), 3, 311-316.

[3.54] A. Berio, L. Bortolotti, P. Manca, A. Paglietti, On the plastic behaviour of time dependent materials, theoretical and experimental investigation, AMS 24 (1972), 3, 351-361.

[3.55] I. Berman, P. G. Hodge Jr., A general theory of piecewise linear plasticity for initially anisotropic materials, AMS 11 (1959), 5, 513-540.

[3.56] S. A. Bernshteyn, A generalization of the octahedral theory of plasticity (in Russian), Izv AIA 109 (1958).

[3.57] P. K. Bertsch, W. F. Findley, An experimental study of subsequent yield surfaces - corners, normality, Bauschinger and allied effects, 4-th USNCAM (1962).

[3.58] I. F. Besseling, A theory of plastic flow for anisotropic hardening in plastic deformation of an initially isotropic material, Nat. Luchtvaartlaboratorium Amsterdam, Rep. S-410 (1953).

[3.59] J. Betten, Eine Bemerkung zum Potentialbegriff in der Plastomechanik, $A E 43$ (1972), $6,471-473$.

[3.60] I. A. Birger, Theory of plastic flow at non-isothermal loading (in Russian), Izv AN SSSR MM (1964), 1, 193-196.

[3.61] -, Theory of plastic flow and the calculation of disks (in Russian), $R P 12$ (1966), 183-199.

[3.62] I. A. Birger, I. V. Demyanushko, Theory of plasticity at non-isothermal loading (in Russian), IZh MTT (1968), 6, 70-77.

[3.63] J. F. W. Bishop, R. Hill, A theory of the plastic distortion of a polycrystalline aggregate under combined stress, PhM 42 (1951), 414-427.

[3.64] - , - A theoretical derivation of the plastic properties of a polycrystalline face-centered metal, PhM 42 (1951), 1298.

[3.65] D. R. Bland, The two measures of work-hardening, 9th ICAM 1956, 8 (1957), 45-50.

[3.66] —, The associated flow rule of plasticity, JMPS 6 (1957), 71-78.

[3.67] H. H. Bleich, On uniqueness in ideally elastoplastic problems in case of nonassociated flow rules, Trans ASME E39 (1972), 4, 983-987.

[3.68] E. I. Blinov, K. N. Rusinko, Analytical investigation of deviations from the postulate of isotropy and from the principle of fading memory (in Russian), Izv AN SSSR MTT (1973), $6,117-122$. 
[3.69] E. I. Blinov, On the deviations from the postulate of isotropy and from the principle of fading memory (in Russian), Tr FPI 65 (1974), 68-73.

[3.70] - Analytical investigation of the modulus of supplementary loading at an orthogonal corner of the loading trajectory (in Russian), $\operatorname{Tr}$ FPI 65 (1974), 73-79.

[3.71] B. A. Boley, J. H. Weiner, Theory of thermal stresses, Wiley, New York-London 1960.

[3.72] J. R. Booker, E. H. Davis, A general treatment of plastic anisotropy under conditions of plane strain, JMPS 20 (1972), 4, 239-250.

[3.73] C. I. Borş, Sur la théorie de la plasticité des corps orthotropes, BAP SST 14 (1966), 9, 887-890.

[3.74] A. I. Botkin, Izv VNIIG 26 (1940).

[3.75] A. N. Bramley, P. B. Mellor, Plastic flow in stabilized sheet steel, IJMS 8 (1966), 2, 101-114.

[3.76] B. Bresler, K. S. Pister, Failure of plain concrete under combined stresses, Proc ASCE 81 (1955), 674.

[3.77] P. W. Bridgman, The thermodynamics of plastic deformation and generalized entropy, Rev. Mod. Phys. 22 (1950), 56-63.

[3.78] G. Brinkmann, Stoffgesetze 2. Ordnung in der Plastizitätstheorie, ZAMM 50 (1970), Sonderh. 1-4, 103-104.

[3.79] C. Brutti, Netztafeln zur Ermittlung der Anstrengung nach v. Mises-Huber bei mehrachsigen Spannungszuständen, Kon 16 (1964), 5, 187-188.

[3.80] B. Budiansky, A reassessment of deformation theories of plasticity, Trans ASME E26 (1959), 2, 259-264.

[3.81] B. Budiansky, Z. Hashin, J. L. Sanders, The stress field of a slipped crystal and the early plastic behavior of polycrystalline materials, 2nd SNSM (1960), 239-258.

[3.82] B. Budiansky, T. T. Wu, Theoretical prediction of plastic strains of polycrystals, 4th USNCAM (1962), 1175-1185.

[3.83] H. D. Bui, Ecrouissage des métaux, CRAS 259 (1964), 4509-4512.

[3.84] -, Ecrouissage des métaux, CRAS AB262 (1966), A401-A404.

[3.85] H. D. Bui, Etude de l'evolution de la frontière du domaine élastique avec l'ecrouissage et relations de comportement élasto-plastique des métaux cubiques, BDER A (1969), 3, 5-108.

[3.86] H. D. Bui, J. Zarka, Fondements expérimentaux et modèles pour l'etude du comportement global des métaux, STA 47 (1973), 1, 45-64.

[3.87] W. Burzyński, Teoretyczne podstawy hipotez wytężenia, CT 47 (1929).

[3.88] —, Ưber die Anstrengungshypothesen, SB 94 (1929), 21, 259.

[3.89] H. J. Buschmann, H. G. Schöpf, P. Stilijanov, Dissipationsfunktionen und Fliessgesetze, WZTUD 20 (1971), 2, 405-408.

[3.90] G. I. Bykovtsev, On the implications of Drucker's postulate for plastically anisotropic media (in Russian), PMM 28 (1964), 2, 356-359.

[3.91] L. R. Calcote, The analysis of laminated composite structures, Van Nonstrand Reinhold, New York 1969.

[3.92] M. Capurso, Yield conditions for incompressible isotropic and orthotropic materials with different yield stress in tension and compression, Mec 2 (1967), 2, 118-125.

[3.93] J. Chakrabarty, A hypothesis of strain-hardening in anisotropic plasticity, IJMS 12 (1970), 2, 169-176.

[3.94] T. Y. Chang, Nonisothermal kinematic hardening law in plasticity, Proc ASCE, JEMD 99 (1973), 12, 423-428.

[3.95] K. F. Chernykh, On the forms of relations between symmetric tensors in continuum mechanics (in Russian), IZh MTT (1967), 3, 42-51.

[3.96] A. I. Chistyakov, Influence of preliminary plastic deformation on the yield-point stress of the steel St45 (in Russian), IUP 4 (1965), 189-192.

[3.97] - Influence of cyclic deformation on the Bauschinger effect and on the yield-point stress of the steel 20Ch (in Russian), IUP 6 (1967), 145-149. 
[3.98] P. C. Chou, B. M. McNamee, D. K. Chou, The yield criterion of laminated media, JCM 7 (1973), 1, 22-35.

[3.99] J. Christoffersen, The elastic-plastic behaviour of monocrystals, a finite-deformation theory, DCAMM 14 (1971), 38 pp.

[3.100] - Elastic-plastic materials with a circular yield cone, DCAMM 32 (1972), 20 pp.

[3.101] - , Potential functions in the deformation theories of plasticity, DCAMM 33 (1972), $9 \mathrm{pp}$.

[3.102] - A simple flow theory of elastic-plastic deformation applicable to materials with a singular yield surface, DCAMM 46 (1973), $22 \mathrm{pp}$.

[3.103] V. N. Churakov, On conditions and criteria of similarity of the theories of plasticity (in Russian), PM 5 (1969), 4, 94-110.

[3.104] P. Cicala, Sulle deformazione plastiche, AANL 8 (1950), 583.

[3.105] —, Sobre la teoriá de Batdorf y Budiansky de la deformación plastica, Rev. Univ. Córdoba (Argent.) 12 (1950), 2.

[3.106] C. Clavuot, H. Ziegler, Ưber einige Verfestigungsregeln, IA 28 (1959), 13-26.

[3.107] B. D. Coleman, M. E. Gurtin, Thermodynamics with internal state variables, JCP 47 (1967), 597-613.

[3.108] M. Como, S. D'Agostino, Strain hardening plasticity with Bauschinger effect, Mec 4 (1969), 2, 146-158.

[3.109] M. Como, A. Grimaldi, Analytical formulation of the theoretical subsequent yield surfaces of metals with strain-hardening and Bauschinger effect in an ideal tension-torsion test, Mec 4 (1969), 4, 286-297.

[3.110] - , - Il modello piano nell'analisi della plasticità dei metalli con incrudimento ed effetto Bauschinger, RS (1969), 1-2.

[3.111] - , -, Strain by shearing stresses in continuum media, Mec 5 (1970), 2, 117-125.

[3.112] - - , Holonomic stress-strain relations in plasticity, $R S$ (1970).

[3.113] - , - Plastic strains by continuous slip bands in metals under proportional loading, RS (1970).

[3.114] M. Como, S. D'Agostino, A. Grimaldi, Influence of the offset on the experimental yield surfaces of metals: a theoretical evaluation, AMS 25 (1973), 4, 685-693.

[3.115] F. Correia de Araújo, Elasticidade e plasticidade, Imprensa Portuguesa, Porto 1961.

[3.116] N. Cristescu, Sur l'effet de Bauschinger, CRAS 249 (1959), 5, 616-618.

[3.117] B. Crossland, The effect of fluid pressure on the shear properties of metals, Proc IME 169 (1954), 935-944.

[3.118] D. M. Cunningham, E. G. Thomsen, J. E. Dorn, Plastic flow of magnesium alloy under bi-axial stresses, Proc ASTM 47 (1947), 546-553.

[3.119] S. Cuomo, Sulla resistenza al fuoco dei continui elastoplastici, RIL ASL A102 (1968), 2, 343-400.

[3.120] J. Cerný, Mechanické vlastnosti vícefázové hmoty dle teorie lokálního charakteru deformaci, StavC 22 (1974), 5, 275-286.

[3.121] A. A. Cyras, A. E. Borkauskas, R. P. Karkauskas, Theory and methods of optimization of elastic-plastic systems (in Russian), Stroyizdat, Leningrad 1974.

[3.122] J. L. Dais, On stress-strain relations for isotropic rigid perfectly plastic solids, QAM 27 (1969), 2, 263-266.

[3.123] V. L. Danilov, On the formulation of deformational hardening rule (in Russian), Izv $A N$ SSSR MTT (1971), 6, 146-150.

[3.124] - , On changes of the yield surface in the deformation process (in Russian), Izv VUZ $M$ (1972), 4, 10-16.

[3.125] Z. B. Dao, Experimental verification of simplified variants of the theory of plasticity (in Russian), VMU MM (1966), 1, 107-118.

[3.126] N. K. Das Talukder, On kinematic hardening rule of Ziegler, JIEI MED 51 (1971), 11 part 6, 257-258. 
[3.127] N. N. Davidenkov, In favour and against a uniform theory of strength (in Russian), VIT (1947), 4, 121-129.

[3.128] E. A. Davis, Increase of stress with permanent strain and stress-strain relations in the plastic state for copper under combined stresses, JAM 10 (1943), A187-A196.

[3.129] -, Yielding and fracture of medium-carbon steel under combined stress, JAM 12 (1945), 1, A13-A24.

[3.130] - The Bailey flow rule and associated yield surface, Trans ASME E28 (1961), 2, 310.

[3.131] V. P. Demutsky, R. V. Polovin, On magnetoplastic flow (in Russian), PMM 33 (1969), 6, 1069-1075.

[3.132] I. V. Demyanushko, Yu. M. Temis, On the formulation of the plastic flow theory with anisotropic hardening for materials with physical fields taken into account (in Russian), Izv AN SSSR MTT (1975), 5, 111-119.

[3.133] V. Diaconiţa, Sul la théorie de la plasticité des corps orthotropes consolidables, CRAS 269 (1969), 17, A802-A804.

[3.134] G. J. Dienes, Effect of nuclear radiations on the mechanical properties of solids, JAP 24 (1953), 6.

[3.135] O. W. Dillon, J. Kratochvil, A strain gradient theory of plasticity, IJSS 6 (1970), 12, 1513-1533.

[3.136] J. E. Dorn, JAP 20 (1949), 15.

[3.137] G. A. Doshtchinsky, Theory of limit elastic state (in Russian), Izv TPI 85 (1957), 343-354.

[3.138] - A generalization of the condition of mean strain to brittle materials (in Russian), Izv TPI 96 (1959), 1, 37-44.

[3.139] G. A. Doshtchinsky, V. I. Maksak, Plastic deformation of steel under combined loading with constant stress intensity (in Russian), IZh MTT (1966), 3, 153-155.

[3.140] - , - Experimental investigation of plastic deformations under combined loading (in Russian), IZh MTT (1966), 5, 118-122.

[3.141] G. A. Doshtchinsky, A. M. Koreneva, Investigation of loading and reverse loading with constant stress intensity in the plastic range (in Russian), IZh MTT (1968), 4, 163-165.

[3.142] - , - , Plastic flow at constant stress intensity (in Russian), Izv AN SSSR, MTT (1970), 5, 163-167.

[3.143] G. A. Doshtchinsky, On experimental verification of the law of similarity of deviators in the theory of plasticity (in Russian), Izv TPI 188 (1974), 20-24.

[3.144] A. Drescher, Badanie mechanizmów plastycznego płynięcia materiałów ziarnistych, Prace IPPT PAN, Warszawa 1975.

[3.145] D. C. Drucker, Relation of experiments to mathematical theories of plasticity, Trans ASME 71 (1949), 349-357.

[3.146] -, A more fundamental approach to plastic stress-strain relations, 1st USNCAM (1951), $487-491$.

[3.147] D. C. Drucker, W. Prager, Soil mechanics and plastic analysis or limit design, $Q A M 10$ (1952), 157-165.

[3.148] D. C. Drucker, Limit analysis of two- and three-dimensional soil mechanics problems, JMPS 1 (1953), 217-226.

[3.149] - Stress-strain relations in the plastic range of metals-experiments and basic concepts, $R h 1$ (1956), 97-119.

[3.150] -, On uniqueness in the theory of plasticity, QAM 14 (1956), 1, 35-42.

[3.151] -, A definition of stable inelastic material, JAM 26 (1959), 1, 101-106.

[3.152] - Extension of the stability postulate with emphasis on temperature changes, 1nd SNSM (1960), 170-184.

[3.153] -, Basic concepts in plasticity, HEM (1962), Chapter 46.

[3.154] -, On the postulate of stability of material in the mechanics of continua, JM 3 (1964), 2, 235-249. 
[3.155] D. C. Drucker, The continuum theory of plasticity on the macroscale and the microscale, JMat 1 (1966), 4, 873-910.

[3.156] -, On time-independent plasticity of metals under combined stress at elevated temperature, RPAM (1967), 209-222.

[3.157] R. N. Dubey, M. J. Hillier, Yield criteria and the Bauschinger effect for a plastic solid, Trans ASME D94 (1972), 1, 228-230.

[3.158] R. N. Dubey, Z. Mróz, On the relations between stress and strain rates for elastic-plastic solids, AMS 25 (1973), 2, 383-388.

[3.159] T. D. Dudderar, J. Duffy, Neutron irradiation and the yield surface of copper, Trans ASME E34 (1967), 1, 200-206.

[3.160] V. V. Dudukalenko, On the condition of uniqueness for an anisotropically hardening plastic material (in Russian), PM 4 (1968), 9, 117-120.

[3.161] V. V. Dudukalenko, V. A. Minayev, On the deformation of a statistically non-homogenous plastic medium (in Russian), Izv AN SSSR MTT (1970), 3, 83-86.

[3.162] G. J. Dvorak, M. S. M. Rao, J. Q. Tarn, Yielding in unidirectional composites under external loads and temperature changes, JCM 7 (1973), 2, 194.

[3.163] - - - , Generalized initial yield surfaces for unidirectional composites, Trans ASME E41 (1974), 1, 249-253.

[3.164] F. Edelman, D. C. Drucker, Some extensions of elementary plasticity theory, JFI 251 (1951), 6, 581-605.

[3.165] M. A. Eisenberg, A. Phillips, On nonlinear kinematic hardening, AMech 5 (1968), 1, 1-13. [3.166] - - A theory of plasticity with non-coincident yield and loading surfaces, AMech 11 (1971) 3-4, 247-260.

[3.167] S. A. Elsufyev, On plastic deformation under combined loading (in Russian), $\operatorname{Tr}$ LPI 278 (1967), 29-31.

[3.168] S. A. Elsufyev, I. N. Izotov, On the relation between stresses and plastic strains for some combined loading trajectories (in Russian), IZh MTT (1968) 1, 140-142.

[3.169] S. A. Elsufyev, On the derivation of relations between stresses and plastic strains (in Russian), IUP 7 (1968), 136-140.

[3.170] J. L. Ericksen, Deformations possible in every isotropic, incompressible, perfectly elastic body, ZAMP 5 (1954), 466-486.

[3.171] -, Deformations possible in every compressible, isotropic, perfectly elastic material, JMP 34 (1955), 126-128.

[3.172] M. I. Estrin, A certain variant of the theory of combined loading (in Russian), DAN SSSR 206 (1972), 1, 43-46.

[3.173] F. Fardshisheh, E. T. Onat, Representation of elasto-plastic behavior by means of state variables, PPlast 1972 (1973), 89-114.

[3.174] F. J: Fava, On the plasticity theory for anisotropic materials, CIRP 15 (1967), 4, 411-415.

[3.175] M. M. Filonenko-Boroditch, On strength conditions for materials exhibiting different properties in tension and in compression (in Russian), IS 19 (1954), 13-36.

[3.176] -, Mechanical theories of strength (in Russian), Izdat. Moskovsk. Univ., Moskva 1961.

[3.177] L. Finzi, Principle of minimum differential elastic energy, AANL 8 (1955), 18, 274-280.

[3.178] L. Fischer, How to predict structural behavior of R. P. laminates, Modern Plastics (1960), 6.

[3.179] W. Flügge, Tensor analysis and continuum mechanics, Springer, Berlin-Heidelberg-New York 1972.

[3.180] N. Fox, On the derivation of the constitutive equations of ideal plasticity, AMech 7 (1969), 4, 248-251.

[3.181] —, Some problems of finite plastic deformation, AMS 24 (1972), 3, 373-381.

[3.182] R. M. W. Fréderking, O. M. Sidebottom, An experimental evaluation of plasticity theories for anisotropic metals, Trans ASME E38 (1971), 1, 15-21. 
[3.183] A. M. Freudenthal, M. Ronay, Second order effects in dissipative media, Proc RS A292 (1966), 1428, 14-50.

[3.184] —, Second-order strain accumulation in work hardening media, RhA 6 (1967), 2.

[3.185] A. M. Freudenthal, P. F. Gou, Second order effects in the theory of plasticity, AMech 8 (1969), 1-2, 34-52.

[3.186] L. B. Freund, Constitutive equations for elastic-plastic materials at finite strain, IJSS 6 (1970), 8, 1193-1209.

[3.187] Ya. B. Fridman, Mechanical properties of materials (in Russian), Oborongiz, Moskva 1946.

[3.188] H. Fromm, Discussion after the paper by R. Mises during the Third Congress on Mechanics in Stockholm (1930); R. Mises, Selecta, Providence 1963, 323-324.

[3.189] —, Grenzen des elastischen Verhaltens beanspruchter Stoffe, HPTM IV/1 (1931), 359.

[3.190] Y. C. Fung, Foundations of solid mechanics, Prentice-Hall, Englewood Cliffs 1965.

[3.191] P. K. Fung, D. J. Burns, N. C. Lind, Yield under high hydrostatic pressure, SFP 1972, (1974), 287-299.

[3.192] Z. Gabryszewski, Wybrane zagadnienia teorii plastyczności ciał anizotropowych, $Z N P W r$ 203 (1968), 3-54.

[3.193] G. A. Gemmerling, On the postulate of plasticity (in Russian), ZhPMTF (1964), 1, 80-84.

[3.194] G. A. Genev, On the problem of yield conditions (in Russian), IVSMTP (1956), 180-187.

[3.195] - , On the problem of the strength condition of concrete (in Russian), IVTPP (1958), 134-144.

[3.196] G. A. Genev, V. N. Kissyuk, On the problem of generalization of the theory of strength of concrete (in Russian), BZhB (1965), 2, 16-19.

[3.197] V. O. Geogdzhayev, Some problems of the theory of elastic-plastic deformation of anisotropic materials (in Russian), Tr MFTI 1 (1958), 69-96.

[3.198] -, On the strength criterion for anisotropic materials (in Russian), $\operatorname{Tr}$ MFTI 5 (1960), 23-29.

[3.199] S. S. Gill, J. Parker, Plastic stress-strain relationship. Some experiments on the effect of loading path and loading history, Trans ASME E26 (1959), 1, 77-87.

[3.200] D. A. Gokhfeld, I. A. Ivanow, O. S. Sadakov, Description of combined loading effects on basis of a structural model of medium (in Russian), UMDS (1975), 171-183.

[3.201] I. I. Goldenblat, Theory of small elastic-plastic deformations of anisotropic media (in Russian), Izv AN SSSR OTN (1955), 2.

[3.202] I. I. Goldenblat, V. A. Kopnov, Strength criteria of anisotropic reinforced plastics (in Russian), SMRS (1965), 5, 5-9.

[3.203] -, -, Strength criterion of anisotropic materials (in Russian), Izv AN SSSR Mekh (1965), $6,77-83$.

[3.204] - , - A generalized theory of plastic flow of anisotropic media (in Russian), SM (1966), 307-319.

[3.205] - , - Yield and strength criteria for structural materials (in Russian), Mashinostroyenye, Moskva 1968.

[3.206] M. L. Gorb, D. M. Karpinos, A. A. Ostrovsky, Investigation of the influence of plastic prestraining on the form of yield curves (in Russian), PPro (1970), 6, 64-68.

[3.207] J. Gouzou, A. Magnée, Critère de plasticité et loi de déformation de l'acier doux, CRAS C271 (1970), 9, 537-540.

[3.208] J. Gouzou, R. D'Haeyer, Treatment of plastic phenomena under combined stresses for body-centred cubic metals, MRBen 39 (1974), 11-19.

[3.209] L. V. Grebnev, Plasticity and strength of metals under combined stress (in Russian), PM 6 (1970), 2, 120-124.

[3.210] -, Investigation of plastic deformations of a metal under combined loading in the case of biaxial tension (in Russian), PM 7 (1971), 10, 111-114. 
[3.211] A. E. Green, Hypo-elasticity and plasticity, JRMA 5 (1956), 5, 725-734; Proc RS 234A (1956), 46-59.

[3.212] A. E. Green, P. M. Naghdi, A comment on Drucker's postulate in the theory of plasticity, AMech 1 (1965), 4, 334-338.

[3.213] - , - A thermodynamic development of elastic-plastic continua, IUTAM IACM 1966 (1968), 117-131.

[3.214] - , - Some remarks on elastic-plastic deformation at finite strain, IJES 9 (1971), 12, 1219-1229.

[3.215] —, -, Rate-type constitutive equations and elastic-plastic materials, IJES 11 (1973), 7, 725-734.

[3.216] R. J. Green, A plasticity theory for porous solids, IJMS 14 (1972), 4, 215-224.

[3.217] D. Grzesik, Berechnung von Fliessortkurven, MAn (1974), 231-248.

[3.218] G. Gudehus, Elastic-plastic constitutive equations for dry sand, AMS 24 (1972), 3, 395-402.

[3.219] J. B. Haddow, T. M. Hrudey, A finite strain theory for elastic-plastic deformation, $I J N L M$ 6 (1971), 4, 435-450.

[3.220] J. B. Haddow, T. M. Hrudey, The yield condition and flow rule for a metal subjected to finite elastic volume change, Trans ASME D93 (1971), 4, 708-712.

[3.221] H. T. Hahn, W. Jaunzemis, A dislocation theory of plasticity, IJES 11 (1973), 10, 10651078.

[3.222] H. T. Hahn, A finite-deformation theory of plasticity, IJSS 10 (1974), 1, 111-121.

[3.223] G. H. Handelman, C. C. Lin, W. Prager, On the mechanical behavior of metals in the strain-hardening range, QAM 4 (1947), 397-407.

[3.224] Y. Hara, The condition of slip in yielding, 15th JCAM (1966), 38-42.

[3.225] - On basic principles of the slip theory of plasticity, 20th JCAM 1970 (1971), 205-209.

[3.226] C. Hartung, T. Lehmann, Vergleich einiger Formänderungsgesetze für plastische Formănderungen, ZAMM 48 (1968), 8, 138-141.

[3.227] M. Hartzmann, Stress-strain relations for materials with different tension, compression yield strengths, AIAA 11 (1973), 3, 378-379.

[3.228] K. Hashiguchi, Theories of the yielding for frictional materials, Trans JSCE 4 (1973), 144-145.

[3.229] K. S. Havner, A path criterion for deformation plasticity theory, Proc ASCE JEMD 95 (1969), 3, 747-761.

[3.230] - A discrete model for the prediction of subsequent yield surfaces in polycrystalline plasticity, IJSS 7 (1971), 7, 719-730.

[3.231] K. S. Havner, On convergence of a discrete aggregate model in polycrystalline plasticity IJSS 7 (1971), 9, 1269-1275.

[3.232] - Aspects of theoretical plasticity at finite deformation and large pressure, ZAMP 25 (1974), 6, 765-781.

[3.233] -, An analytical model of large deformation effects in crystalline aggregates, SFP 1972 (1974), 93-106.

[3.234] I. Hayashi, K. Kawaguchi, H. Fukuda, An experimental study on the subsequent yield of mild steel, JJSTP 11 (1970), 108.

[3.235] R. M. Haythornthwaite, Stress and strain in soils, 2nd SNSM (1960), 185-193.

[3.236] —, Range of yield condition in ideal plasticity, Proc ASCE JEMD 87 (1961), 6, 117-133.

[3.237] - , The range of the yield condition in a stable ideally plastic material sensitive to hydrostatic pressure, 10th ICAM 1960 (1962), 296-297.

[3.238] S. S. Hecker, Experimental investigation of corners in the yield surface, AMech 13 (1972), $1-2,69-86$.

[3.239] J. D. Helfinstine, R. Lance, Yielding of fiber reinforced Tresca material, Proc ASCE JEMD 98 (1972), 4, 849-866. 
[3.240] K. Hellan, A note on equivalence of the postulates of isotropic hardening, Trans ASME H96 (1974), 1, 79-80.

[3.241] H. Hencky, Welche Umstände bedingen die Verfestigung bei der bildsamen Verformung von festen isotropen Körpern, $Z P h 55$ (1929), 145-155.

[3.242] —, Stb 16 (1943), 95.

[3.243] A. V. Hershey, The plasticity of an isotropic aggregate of anisotropic face-centred cubic crystals, JAM 21 (1954), 3, 241-249.

[3.244] R. Hill, Theory of yielding and plastic flow of anisotropic metals, Proc RS A193 (1948), 281-297.

[3.245] -, PhM 41 (1950), 733-744.

[3.246] -, The mechanics of quasi-static plastic deformation in metals, SurvM 7 (1956).

[3.247] —, New horizons in the mechanics of solids, JMPS 5 (1956), 66-74.

[3.248] - , On the problem of uniqueness in the theory of a rigid-plastic solid, JMPS 4 (1956), 4, 247-255; 5 (1957), 1, 1-8; 5 (1957), 3, 153-161; 5 (1957), 4, 312-317.

[3.249] -, A general theory of uniqueness and stability in elastic-plastic solids, JMPS 6 (1958), 236-249.

[3.250] - Some basic principles in the mechanics of solids without a natural time, JMPS 7 (1959), 3, 209-225.

[3.251] -, Continuum micromechanics of elastoplastic polycrystals, JMPS 13 (1965), 2, 89-101.

[3.252] - , Generalized constitutive relations for incremental deformations of metal crystals by multislip, JMPS 14 (1966), 2.

[3.253] -, Eigenmodal deformations in elastic-plastic continua, JMPS 15 (1967), 6, 371-386.

[3.254] - , On the classical constitutive relations for elastic/plastic solids, RPAM (1967), 241249.

[3.255] -, On constitutive inequalities for simple materials, JMPS 16 (1968), 4, 229-242; 16 (1968), 5, 315-322.

[3.256] - , Macroscopic measures of deformation and of plastic work for a micrononhomogeneous medium (in Russian), PMM 35 (1971), 1, 31-39.

[3.257] R. Hill, J. R. Rice, Elastic potentials and the structure of inelastic constitutive laws, SIAM JAMath 25 (1973), 3, 448-461.

[3.258] P. G. Hodge, Jr., W. Prager, A variational principle for plastic materials with strain-hardening, JMP 27 (1948), 1-10.

[3.259] P. G. Hodge, Jr., The theory of piecewise linear isotropic plasticity, IUTAM DF 1955 (1956).

[3.260] - A general theory of piecewise linear plasticity based on maximum shear, JMPS $\mathbf{5}$ (1957), 242-260.

[3.261] -, Piecewise linear plasticity, 9th ICAM 1956, 8, (1957), 65-72.

[3.262] K. Hohenemser, Fliessversuche an Rohren aus Stahl bei kombinierter Zug- und Torsionsbeanspruchung, ZAMM 11 (1931), 15-19.

[3.263] K. Hohenemser, W. Prager, Beitrag zur Mechanik des bildsamen Verhaltens von Flußstahl, ZAMM 12 (1932), 1, 1-14.

[3.264] G. S. Holister, C. Thomas, I. King, Yield behaviour of a split fibre in a two-dimensional elasto-plastic matrix: an experimental analysis, FST 1 (1969), 3, 227-241.

[3.265] K. A. Holsapple, Elastic-plastic materials as simple materials, ZAMM 53 (1973), 5, 261-270.

[3.266] - A finite elastic-plastic theory and invariance requirements, AMech 17 (1973), 3-4, 277-290.

[3.267] W. F. Hosford, Jr., W. A. Backofen, Strength and plasticity of textured metals, FDP (1964), 259-298.

[3.268] W. F. Hosford, Jr., A generalized isotropic yield criterion, Trans ASME E39 (1972), 2, 607-609. 
[3.269] T. C. Hsu, A theory of the yield locus and flow rule of anisotropic materials, JSA 1 (1966), 3, 204-215.

[3.270] - , The characteristics of coaxial and non-coaxial strain paths, JSA 1 (1966), 3, 216-222.

[3.271] - , The effect of the rotation of the stress axes on the yield criterion of prestrained materials, Trans ASME D88 (1966), 1, 61-70.

[3.272] L. W. Hu, J. Marin, Anisotropic loading functions for combined stresses in the plastic range, JAM 22 (1955), 1, 77-85.

[3.273] L. W. Hu, An experimental study of the fracture of metals under hydrostatic pressure, JMPS 4 (1956), 2, 96-103.

[3.274] - Modified Tresca's yield condition and associated flow rules for anisotropic materials and its applications, JFI 265 (1958), 187-204.

[3.275] L. W. Hu, J. F. Bratt, Effect of tensile plastic deformation on yield condition, JAM 25 (1958), 3, 411.

[3.276] L. W. Hu, Development of a triaxial stress testing machine and triaxial stress experiments, Proc SESA 16 (1959), 27-37.

[3.277] —, Plastic stress-strain relations and hydrostatic stress, 2nd SNSM (1960), 195-201.

[3.278] L. W. Hu, K. D. Pae, Inclusion of the hydrostatic stress component in formulation of the yield condition, JFI 275 (1963), 6, 491-502.

[3.279] M. T. Huber, Kryteria wytrzymalościowe w stereomechanice technicznej, Inst. Wydawn. SIMP, Warszawa 1948.

[3.280] J. W. Hutchinson, Plastic stress-strain relations of f.c.c. polycrystalline metals hardening according to Taylor's rule, JMPS 12 (1964), 1, 25.

[3.281] - Elastic-plastic behaviour of polycrystalline metals and composites, Proc RS A319 (1970), 247-272.

[3.282] V. A. Ibragimov, V. D. Klyushnikov, Some problems for media with falling stress-strain diagram (in Russian), Izv AN SSSR MTT (1971), 4, 116-121.

[3.283] A. B. Ibragimov, I. D. Mashkov, Problems of deformational anisotropy (in Russian), VMU MM (1971), 5, 92-96.

[3.284] V. A. Ibragimov, Some problems of the theory of softening media (in Russian), Izv AN SSSR MTT (1972), 4, 55-63.

[3.285] K. Ikegami, E. Shiratori, Application of Svensson's yield condition to some cases of fundamental preloading paths, Trans JSME 37 (1970), 1312-1329.

[3.286] A. A. Ilyushin, V.S. Lensky, On the laws of deformation of materials under combined loading (in Russian), AMSin 3 (1959), 3.

[3.287] A. A. Ilyushin, Problems of the general theory of plasticity (in Russian), PMM 24 (1960), 3, 399-411.

[3.288] - , On the increment of plastic deformation and the yield surface (in Russian), PMM 24 (1960), 4, 663-666.

[3.289] - , On the foundations of the general mathematical theory of plasticity (in Russian), VTP (1961), 3-29.

[3.290] -, On the postulate of plasticity (in Russian), PMM 25 (1961), 503-507.

[3.291] -, Once more on the postulate of isotropy, Izv AN SSSR MM (1962), 1, 201-204.

[3.292] -, Mechanics of continua (in Russian), Izdat. Moskovsk. Univers., Moskva 1971.

[3.293] T. Inoue, K. Tanaka, T. Izshizaki, Yield surfaces of metals at elevated temperatures, 16th JCMR (1973), 126-131.

[3.294] T. Inoue, K. Tanaka, Subsequent yield conditions of metal under cyclic loading at elevated temperatures, IA 44 (1975), 2, 53-62.

[3.295] - , - An elastic-plastic stress analysis of quenching when considering a transformation, IJMS 17 (1975), 5. 361-368 
[3.296] S. Irmay, Failure criteria of plastic solids in the space of stress invariants, IJT 6 (1968), 3, 165-173.

[3.297] A. Yu. Ishlinsky, Hypothesis of strength of shape change (in Russian), UZMU Mekhanika 46 (1940).

[3.298] -, A general theory of plasticity with linear hardening (in Russian), UMZ 6 (1954), 3, 314-324.

[3.299] I. A. Ivanov, Investigation of some properties of combined loading based on a model representing the non-homogeneity of the medium (in Russian), SNT CPI 92 (1971), 23-32.

[3.300] H. J. Ivey, Plastic stress-strain relations and yield surfaces for aluminium alloys, JMES 3 (1961), 1, 15-31.

[3.301] D. D. Ivlev, On the investigation of a linear tensorial relation in plasticity (in Russian), PMM 20 (1956), 2, 289-292.

[3.302] - , On construction of the theory of ideal plasticity (in Russian), PMM 22 (1958), 6, $850-855$.

[3.303] - On the relations describing plastic flow for the Tresca yield condition and for its generalizations (in Russian), DAN SSSR 124 (1959), 3, 546-549.

[3.304] -, On isotropic hardening of plastic bodies (in Russian), DAN SSSR 127 (1959), 4, 777-779.

[3.305] -, On the theory of ideal plastic anisotropy (in Russian), PMM 23 (1959), 6, 1107-1114.

[3.306] - , On the postulate of isotropy in the theory of plasticity (in Russian), Izv AN SSSR MM (1960), 2, 125-127.

[3.307] -, On V.S. Lensky's paper "Some new data on plasticity of metals under combined loading" (in Russian), Izv. AN SSSR MM (1960), 6, 179-181.

[3.308] D. D. Ivlev, T. N. Martynova, On allowing for compressibility of ideally plastic media (in Russian), PMM 25 (1961), 6, 1126-1128.

[3.309] D. D. Ivlev, On the theory of limit equilibrium of rotationally symmetric shells at piece-wise linear yield conditions (in Russian), Izv AN SSSR MM (1962), 6, 95-102.

[3.310] D. D. Ivlev, T. N. Martynova, On the theory of compressible, ideally plastic media (in Russian), PMM 27 (1963), 3, 589-592.

[3.311] D. D. Ivlev, On deformation theories of plasticity at singular loading surfaces (in Russian), PMM 31 (1967), 5, 887-889.

[3.312] D. D. Ivlev, G. I. Bykovtsev, Theory of a plastic hardening body (in Russian), Nauka, Moskva 1971.

[3.313] W. D. Iwan, On class of models for the yielding behaviour of continuous and composite systems, Trans ASME E34 (1967), 3, 612-617.

[3.314] L. R. Jackson, K. F. Smith, W. T. Lankford, Plastic flow in anisotropic sheet metal, MTTP 2440 (1948).

[3.315] A. W. Jenike, R. T. Shield, On the plastic flow of Coulomb solids beyond original failure, JAM 27 (1959), 599-602.

[3.316] K. R. Johnson, O. M. Sidebottom, Strain-history effect on isotropic and anisotropic plastic behavior, ExpM 12 (1972), 6, 264-271.

[3.317] J. J. Jonas, Flow stress under conditions of high temperature deformation, JSIR 32 (1973), $10,528-532$.

[3.318] G. de Josselin de Jong, Lower bound collapse theorem and lack of normality of strain rate to yield surface for soils, IUTAM RSM 1964 (1966).

[3.319] J. W. Justusson, A. Phillips, Stability and convexity in plasticity, AMech 2 (1966), 3, 251267.

[3.320] L. M. Kachanov, On experimental determination of loading surfaces and of the Bauschinger effect (in Russian), IUP 8 (1971), 108-112.

[3.321] - , On the problem of experimental determination of the yield surfaces (in Russian), Izv AN SSSR MTT (1971), 4, 177-179. 
[3.322] Yu. I. Kadashevitch, V. V. Novozhilov, Theory of plasticity taking the Bauschinger effect into account (in Russian), DAN SSSR 117 (1957), 4, 586.

[3.323] Yu. I. Kadashevitch, On the interrelation of two basic problems in the flow theory taking residual microstresses into account (in Russian), PMM 27 (1963), 378-379.

[3.324] - , On various variants of the tensorially linear relations in the theory of plasticity (in Russian), IUP 6 (1967), 39-45.

[3.325] - , A generalized theory of plastic flow (in Russian), IUP 6 (1967), 25-38.

[3.326] Yu. I. Kadashevitch, V. V. Novozhilov, On allowing for microstresses in the theory of plasticity (in Russian), IZh MTT (1968), 3, 82-91.

[3.327] - , - On the influence of the initial microstresses on macroscopic deformation of polycrystals (in Russian), PMM 32 (1968), 5, 908-922.

[3.328] Yu. I. Kadashevitch, N. V. Kirsanova, On a certain dynamic model of plastic deformation of metals (in Rusian), Tr LTITBP 22 (1969), 214-216.

[3.329] Yu. I. Kadashevitch, On the forms of relations between stresses and strains under combined loading (in Russian), $\operatorname{Tr}$ LTITBP 22 (1969), 217-218.

[3.330] - , On a quasistatistic variant of the theory of plastic flow (in Russian), Izv AN SSSR MTT (1973), 4, 167-171.

[3.331] V. Kafka, The general theory of isothermal elastic-plastic deformation of polycrystals based on the analysis of the microscopic state of stress, ZAMM 48 (1968), 4, 265-282.

[3.332] - Microscopic stress components as latent variables governing changes of plastic deformation properties, ZAMM 48 (1968), 8, 146-148.

[3.333] V. Kafka, R. Novotný, Changes in deformation properties of steel caused by previous plastic strain under tensile load applied in different directions, AT CSAV 14 (1969), 5 561-587.

[3.334] V. Kafka, Determination of material constants for quasihomogeneous strainhardening materials, AT CSAV 17 (1972), 2, 173-208.

[3.335] —, Theory of slow elastic-plastic deformation of polycrystalline metals with micro-stresses as latent variables descriptive of the state of the material, AMS 24 (1972), 3, 403-418.

[3.336] -, Structural theory of elastic-plastic deformation of undirectional fiber-reinforced materials, AT ĆSAV 18 (1973), 5, 418-436; 19 (1974), 1, 87-95.

[3.337] A. Kammerer, La surface d'écoulement (surface de charge) et la deformation plastique commençante, CRAS A278 (1974), 3, 169-172.

[3.338] K. R. Kenk, On stress-strain relation in torsion (in Russian), IZh MTT (1968), 5, 134-139.

[3.339] J. Kestin, On the application of the principles of thermodynamics to strained solid materials, IUTAM IACM 1966 (1968), 177-212.

[3.340] J. Kestin, J. R. Rice, Paradoxes in the application of thermodynamics to strained solids, a critical review of thermodynamics, Mono Book Corp., Baltimore 1970, 275-298.

[3.341] S. A. Khristianovitch, E. I. Shemyakin, On the theory of ideal plasticity (in Russian), IZh MTT (1967), 4, 86-97.

[3.342] - , , On plane plastic deformation under combined loading (in Russian), Izv AN SSSR MTT (1969), 5, 138-149.

[3.343] S. A. Khristianovitch, Deformation of a plastic hardening material (in Russian), Izv $A N$ SSSR MTT (1974), 2, 148-174.

[3.344] K. C. Khuan, On plastic hardening of a rigid-plastic material (in Russian), PMM 22 (1958), 4, 544-546.

[3.345] W. M. Kirkpatrick, 4th Int. Conf. Soil Mechanics 1 (1957), 712-178.

[3.346] H. Kitagawa, Y. Tomita, Note on incremental stress-strain relations of elasto-plastic materials referred to a convected coordinate system, ZAMM 52 (1972), 3, 183-186.

[3.347] M. Kleiber, Objective formulation of rate-type constitutive equation for elastic-ideally plastic solids, BAP SST 22 (1974), 1, 49-54.

[3.348] J. Klepaczko, O pewnym przypadku anizotropii, MTS 7 (1969), 2, 155-163. 
[3.349] Z. Klębowski, Obecny stan wytrzymałościowego obliczenia materiałów o własnościach uogólnionych, $\operatorname{PrzT}$ (1934), 11.

[3.350] - Energetyczne hipotezy wytężenia a możność opracowania ogólnej teorii wytężenia, KJMTH (1950), 165-180.

[3.351] -, Warunek wytrzymalościowy na tle hipotez wytężenia, Państw. Zakłady Wyd. Szkolnych, Warszawa 1950.

[3.352] -, Polski wkład w rozwiązywanie zagadnienia wytężenia, ZNPW 45 (1960), Mechanika 6, 17-28.

[3.353] L. G. Klinger, G. Sachs, Dependence of the stress-strain curves of cold worked metals upon the testing direction, JAS 15 (1948), 599.

[3.354] K. Klöppel, M. Yamada, Anisotrope Fliessbedingung, Stb 29 (1960), 6, 173-180.

[3.355] S. D. Klyatchko, On the assessment of the flow theory (in Russian), Izv AN SSSR MM (1962), 5, 159-164.

[3.356] V. D. Klyushnikov, On the laws of plasticity for a particular class of loading paths (in Russian), PMM 21 (1957), 4, 533-543.

[3.357] - , On the laws of plasticity for a plastic hardening material (in Russian), PMM 22 (1958), $1,97-118$.

[3.358] -, On a certain possible way of construction of the laws of plasticity (in Russian), PMM 23 (1959), 2, 282-291.

[3.359] - New representations in plasticity and the deformation theory (in Russian), PMM 23 (1959), 4, 722-731.

[3.360] - The loading surface and tolerances in its experimental determination (in Russian), DAN SSSR 221 (1975), 2, 299-300.

[3.361] I. V. Knets, Development of the concepts of a function of locality of deformations and of a slip function in the statistical theories of plasticity (in Russian), MPol (1969), 3, 422-430.

[3.362] -, Recent fundamental directions in the mathematical theory of plasticity (in Russian), Zinatne, Riga 1971.

[3.363] W. T. Koiter, Stress-strain relations, uniqueness and variational theorems for elasticplastic material with a singular yield surface, QAM 11 (1953), 3, 29-53.

[3.364] -, On the stress-strain relations and the general theorems in plasticity, Labor. voor Toegepaste Mechanica, Techn. Hogeschool Delft, 1953.

[3.365] -, General theorems for elastic-plastic solids, PSM 1 (1960), chapter IV, 165-221.

[3.366] D. Kolarov, A. Baltov, N. Bontcheva, Mechanics of plastic media (in Bulgarian), Izd. Blgarskata Akademia na Naukite, Sofia 1975.

[3.367] K. Kondo, On the fundamental equations of the macroscopic mechanical behaviour of microscopically non-uniform materials, MUS BPES 1 (1955), 470-483.

[3.368] —, Energy at plastic deformation and criterion for yielding, MUS BPES 1 (1955), 484-494.

[3.369] -, Mathematical analysis of yield points of an isotropic material, MUS BPES 1 (1955), 495-521.

[3.370] - Non-holonomic foundations of the theory of plasticity and yielding, MUS BPES 1 (1955), 522-562.

[3.371] E. A. Konyayev, I. Z. Palley, Approximate equations for non-isothermal deformation (in Russian), VDP 13 (1967), 137-140.

[3.372] A. M. Koreneva, G. A. Doshtchinsky, Experimental investigation of the neutral loading curve (in Russian), Izv TPI 183 (1968), 42-44.

[3.373] Yu. G. Korotkikh, S. M. Belevitch, Basic equation of thermoplasticity in the case of combined loading (in Russian), UZGU 89 (1969), 131-141.

[3.374] - - - Equations of state for static and dynamic problems of thermoplasticity in the case of combined loading (in Russian), UZGU 108 (1970), 80-90.

[3.375] Yu. G. Korotkikh, A mathematical model of thermoplasticity in the case of large elasticplastic deformations (in Russian), MRZUP 7 (1973), 17-27. 
[3.376] I. M. Korovin, Some problems of plasticity in the case of loading trajectory with a corner (in Russian), Izv AN SSSR MTT (1969), 3, 152-158.

[3.377] P. F. Koshelev, S. E. Belayev, Strength and plasticity of structural materials at low temperatures (in Russian), Mashinostroyenye, Moskva 1967.

[3.378] W. Kosiński, W. Wojno, Remarks on internal variable and history descriptions of material, AMS 25 (1973), 4, 709-713.

[3.379] A. G. Kostyuk, A statistical theory of plasticity of a polycrystalline material (in Russian), IZh MTT (1968), 6, 60-69.

[3.380] K. F. Kovalov, On the criterion of estimation of the Bauschinger effect (in Russian), $\operatorname{Tr} L P I$ 278 (1967), 38-41.

[3.381] I. A. Kozlov, A. A. Lebedev, V. L. Akhremenko, Stress-strain relation in the initial stage of plastic deformation under biaxial tension (in Russian), PM 3 (1967), 12, 118-121.

[3.382] J. A. König, Przybliżone określanie odkształceń płyt i powłok plastycznych w oparciu o niestowarzyszone prawo płynięcia, RI 13 (1965), 1, 55-65.

[3.383] J. A. König, W. Olszak, The yield criterion in the general case of nonhomogeneous stress and deformation fields, TACM (1974), 58-70.

[3.384] Fr. Körber, A. Eichinger, Die Grundlagen der bildsamen Verformung, MKWIE 22 (1940), 57-80.

[3.385] J. Kratochvil, O. W. Dillon, Thermodynamics of elastic-plastic material as a theory with internal state variables, JAP 40 (1969), 3207-3218.

[3.386] J. Kratochvil, On a finite strain theory of elastic-inelastic materials, AMech 16 (1973), 127-142.

[3.387] - , Finite-strain theory of inelastic behavior of crystalline solids, SFP 1972 (1974), 401-415.

[3.388] J. Kratochvil, N. Zárubová, A method of determination of inelastic constitutive equations, AMS 27 (1975), 5-6, 749-757.

[3.389] A. S. Kravtchuk, On the accuracy of the deformation theory in the case of a simple loading (in Russian), Izh MTT (1968), 6, 111-114.

[3.390] - , On the theory of plasticity for strain trajectories of medium curvature (in Russian), UN 2 (1971), 91-100.

[3.391] A. F. Kregers, Plastic deformations under combined tension and torsion (in Russian), MPol (1966), 1, 20-26.

[3.392] - Influence of the third stress invariant on characteristic curves of the material (in Russian), MPol (1968), 4, 613-617.

[3.393] - , Physical relations of an orthotropic material in the theory of locality of deformations (in Russian), MPol (1968), 6, 970-975.

[3.394] - Variants of the theory of locality of deformations (in Russian), MPol (1970), 2, 138146.

[3.395] - Investigation of the yield surfaces in the theory of locality of deformations (in Russian), MPol (1971), 4, 594-598.

[3.396] - Investigation of the loading surfaces in the theory of locality of deformations (in Russian), MPol (1971), 5, 796-800.

[3.397] E. Kröner, Zur plastischen Verformung des Vielkristalls, $A M 9$ (1961), 2, 155-161.

[3.398] -, Dislocation: a new concept in the continuum theory of plasticity, JMP 42 (1963), 1, 27-37.

[3.399] —, Initial studies of plasticity theory based upon statistical mechanics, IBS (1970), 137148.

[3.400] E. Kröner, C. Teodosiu, Lattice defect approach to plasticity and viscoplasticity, PPlast 1972 (1973), 45-88.

[3.401] N. N. Kudryashov, B. A. Rytchkov, Investigation of deformations of an aluminum alloy under combined loading (in Russian), DNT (1970), 68-80. 
[3.402] G. S. Kular, M. J. Hillier, Re-interpretation of some simple tension and bulge test data for anisotropic metals, IJMS 14 (1972), 10, 631-634.

[3.403] V. I. Kuneyev, K. N. Rusinko, Orthogonal supplementary loading of materials with different properties in tension and in compression (in Russian), Izv AN SSSR MTT (1971), $5,90-98$.

[3.404] V. I. Kuneyev, Combined deformation of materials with different properties in tension and in compression (in Russian), RKSTP (1974), 97-114.

[3.405] T. Kurtyka, Analiza wzmocnienia plastycznego typu Edelmana-Druckera, CT 85 (1980) (in print).

[3.406] V. A. Kuz'menko, On the problem of a uniform (generalized) strain-hardening curve (in Russian), PPro (1970), 10, 64-68.

[3.407] - , On the influence of the type of stress state on strain hardening of the material (in Russian), PPro (1972), 12, 44-49.

[3.408] -, New schemes of deformation of solids (in Russian), Naukova Dumka, Kiev 1973.

[3.409] R. H. Lance, D. N. Robinson, A maximum shear stress theory of plastic failure of fiberreinforced materials, JMPS 19 (1971), 2, 49-60.

[3.410] J. H. Laning, Proof of least work principle equivalent to the von Mises relations for an isotropic plastic material, Watertown Arsenal Labo. Memo. Rep. 893 (1943), 13.

[3.411] A. A. Lebedev, G. S. Pisarenko, Investigation of the strength of steel 1X18H9T under combined stress in the temperature range to $820^{\circ} \mathrm{C}$ (in Russian), 2nd SVVP (1963).

[3.412] A. A. Lebedev, On a possible combination of the yield condition and the brittle fracture condition (in Russian), PM 4 (1968), 8, 85-93.

[3.413] - , On the paper by Stassi d'Alia "Flow and fracture of metals under combined stress" (in Russian), PPro (1973), 5, 41-44.

[3.414] H. Le Boiteux, Sur un critère énergétique de limite élastique, CRAS 265 (1967), 8, A238A239.

[3.415] E. H. Lee, D. T. Liu, Finite-strain elastic-plastic theory with application to plane-wave analysis, JAP 38 (1967), 1, 19-27.

[3.416] - , - Finite-strain elastic-plastic theory, IUTAM IACM 1966 (1968), 213-222.

[3.417] E. H. Lee, P. Germain, Elastic-plastic theory at 'finite strain, PPlast 1972 (1973), 117-133.

[3.418] L. H. N. Lee, J. T. Horng, A work-hardening rule for finite elastic-plastic deformation of metals at elevated temperatures, 3rd SMiRT (1975), paper L3/3.

[3.419] T. Lehmann, Ein neuer Ansatz für plastische Formünderungen mit Kaltverfestigung, ZAMM 38 (1958), 7-8, 295-297.

[3.420] -, Zur Beschreibung grosser plastischer Formänderung unter Berücksichtigung der. Werkstoffverfestigung, RhA 2 (1962), 247-254.

[3.421] -, Anisotrope plastische Formänderungen, RhA 3 (1964), 281-285.

[3.422] T. Lehmann, K. Thermann, Grundlagen der Plastizitätstheorie und ihrer Anwendungen, VDI Z 113 (1971), 15, 1169-1183.

[3.423] T. Lehmann, Einige Bemerkungen zu einer allgemeinen Klasse von Stoffgesetzen für grosse elasto-plastische Formänderungen, IA 41 (1972), 4, 297-310.

[3.424] - $\mathrm{Zu}$ einigen nichtlinearen Stoffgesetzen für plastische Formänderungen, RhA 11 (1972), 4-12.

[3.425] -, Anisotrope plastische Formänderungen, RRST SMA 17 (1972), 5, 1077-1086.

[3.426] -, On large elastic-plastic deformations, SFP 1972 (1974), 571-585.

[3.427] T. Lehmann, G. Zander, Non-isothermic large elastic-plastic deformations, AMS 27 (1975), 5-6, 759-772.

[3.428] H. Leipholz, Ưber den Zusammenhang zwischen Fliessgesetz und Fliessbedingung, IA 34 (1965), 3, 194-197.

[3.429] V. S. Lensky, Experimental verification of the laws of isotropy and of fading memory in the case of combined loading (in Russian), Izv AN SSSR OTN (1958). 11. 15-24. 
[3.430] - , Some new data on plasticity of metals under combined loading (in Russian), Izv $A N$ SSSR MM (1960), 5, 93-100.

[3.431] -, Analysis of plastic behaviour of metals under complex loading, 2nd SNSM (1960), 259-278.

[3.432] - , Influence of radioactive irradiation on mechanical properties of solids (in Russian), IS 28 (1960), 97-133.

[3.433] - Certain peculiarities of the behaviour of metals subject to elastic-plastic deformation (in Russian), VTP (1961), 58-82.

[3.434] -, On erroneous remarks by D. D. Ivlev (in Russian), Izv AN SSSR MM (1961), 3, 172-175.

[3.435] - A hypothesis of local determinacy in the theory of plasticity (in Russian), Izv $A N$ SSSR MM (1962), 5, 154-158.

[3.436] - Simplified variants of the theory of plasticity (in Russian), PM 5 (1969), 3, 18-22.

[3.437] V.S. Lensky, V. A. Lomakin, A deformation theory of thermoplasticity (in Russian), TNEK 10 (1970), 37-50.

[3.438] V. S. Lensky, I. D. Mashkov, A verification of the laws of plasticity in the three-dimensional space of strain deviator (in Russian), UN 2 (1971), 158-166.

[3.439] A. Leon, IA 4 (1933), 421.

[3.440] M. Ya. Leonov, N. Yu. Shvayko, Combined plane strain (in Russian), DAN DSSR 159 (1964), 5, 1007-1010.

[3.441] - , - On stress-strain relations in the vicinity of a corner at the loading trajectory (in Russian), DAN SSSR 171 (1966), 2, 306-309.

[3.442] M. Ya. Leonov, Elements of the mathematical theory of plasticity (in Russian), Izv $A N$ KirgSSR (1970), 3, 3-10.

[3.443] -, Fundamental postulates of the theory of plasticity (in Russian), DAN SSSR 199 (1971), $1,51-54$.

[3.444] —, Elements of the theory of plasticity (in Russian), DAN SSSR 199 (1971), 2, 293-295.

[3.445] -, Elements of the analytical theory of plasticity (in Russian), DAN SSSR 205 (1972), 2, 303-306.

[3.446] M. Ya. Leonov, E. I. Blinov, An analytical investigation of the Bauschinger effect (in Russian), $\operatorname{Tr}$ FPI 56 (1972), 22-30.

[3.447] M. Ya. Leonov, V. Ya. Molotnikov, B. A. Rytchkov, On the development of the concept of slip in the theory of plasticity (in Russian), Izv AN KirgSSR (1973), 2, 4-11.

[3.448] M. Ya. Leonov, Basic equations of the theory of plasticity (in Russian), RKSTP (1974), 3-16.

[3.449] M. Ya. Leonov, V. Ya. Molotnikov, B. A. Rytchkov, Certain generalizations of the concept of slip in the theory of plasticity (in Russian), PTT (1974), 12-35.

[3.450] J. M. Lessells, C. W. MacGregor, Combined stress experiments on a nickel-chromemolybdenum steel, JFI 230 (1940), 163.

[3.451] T. H. Lin, A proposed theory of plasticity based on slips, 2nd USNCAM (1954), 461-468.

[3.452] - Analysis of elastic and plastic strain in a face-centered cubic crystal, JMPS 5 (1957), 143-149.

[3.453] -, On stress-strain relations based on slips, 3rd USNCAM (1958), 581-587.

[3.454] T. H. Lin, M. Ito, Theoretical plastic distortion of a polycrystalline aggregate under combined and reversed stresses, JMPS 13 (1965), 103-115.

[3.455] - , Theoretical plastic stress-strain relationship of a polycrystal and the comparisons with the von Mises and the Tresca plasticity theories, IJES 4 (1966), 5, 543-561.

[3.456] T. H. Lin, Theory of inelastic structures, Wiley, New York 1968.

[3.457] -, Physical theory of plasticity, AAM 11 (1971), 255-311.

[3.458] T. H. Lin, D. Salinas, Y. M. Ito, Initial yield surface of a unidirectionally reinforced composite, Trans ASME E39 (1972), 2, 321-326. 
[3.459] T. H. Lin, Y. M. Ito, C. L. Yu, A new slip theory of plasticity, Trans ASME E41 (1974), 3, 587-592.

[3.460] H. Lippmann, Matrixungleichungen und die Konvexität der Fliessfläche, ZAMM 50 (1970), Sonderh. 1-4, 134-137.

[3.461] -, Ansätze und Lösungsbeispiele zur Theorie des anisotropen plastischen Fliessens, MAn (1974), 257-278.

[3.462] S. I. Liu, G. Sachs, Am. Inst. Mining Met. Engrs. TR 2392 (1948).

[3.463] V. A. Lomakin, On the problem of large elastic-plastic deformations (in Russian), $V M U$ $M M$ (1954), 5, 41-45.

[3.464] - The problem of determination of stresses and strains in heat treatment processes (in Russian), Izv AN SSSR MM (1959), 1, 103-110.

[3.465] - , On the theory of nonlinear elasticity and plasticity of anisotropic media (in Russian), Izv AN SSSR MM (1960), 4, 60-64.

[3.466] - , On the theory of plasticity of anisotropic media (in Russian), $V M U M M(1964), 4$, 49-53.

[3.467] J. D. Lubahn, R. P. Felgar, Plasticity and creep of metals, Wiley, New York 1961.

[3.468] J. Lubliner, A simple theory of plasticity, IJSS 10 (1974), 3, 313-319.

[3.469] —, On loading, yield and quasi-yield hypersurfaces in plasticity theory, IJSS 11 (1975) 9, 1011-1016.

[3.470] P. Ludwik, L. Scheu, Vergleichende Zug- Druck- Dreh- und Walzversuche, SE 45 (1925) 11, 373-381.

[3.471] G. Maier, D. C. Drucker, Elastic-plastic continua containing unstable elements obeying normality and convexity relations, SB 84 (1966), 23, 447-450.

[3.472] G. Maier, Sui legami associati tra sforzi e deformazioni incrementali in elastoplasticità RIL ASL A100 (1966), 4, 809-838.

[3.473] -, Stabilità, unicità di soluzione e leggi di scorrimento per strutture elastoplastiche con legami incrementali sforzi-deformazioni associati, RIL ASL A100 (1966), 1, 3-27.

[3.474] —, On elastic-plastic structures with associated stress-strain relations allowing for work softening, Mec 2 (1967), 1, 55-64.

[3.475] —, Linear flow-laws of elastoplasticity: a unified general approach, $A A N L 47$ (1970), 5 266-276.

[3.476] W. M. Mair, H. L. D. Pugh, Effect of pre-strain on yield surfaces in copper, JMES 6 (1964), 2, 150-163.

[3.477] W. M. Mair, An investigation into the existence of corners on the yield surface, JSA (1967), 3, 188-195.

[3.478] M. Maitra, K. Majumdar, A. Das, Unified plastic yield criterion for ductile solids, AIAA 11 (1973), 10, 1428-1429.

[3.479] J. Majer, Beitrag zu den dreiachsigen Spannungs-Dehnungs-Beziehungen fester Stoffe, ÖIA 4 (1950), 2, 140-153.

[3.480] E. V. Makhover, Some problems of the theory of plasticity of anisotropic media (in Russian), DAN SSSR 58 (1947), 2, 209-212.

[3.481] V. I. Maksak, Plastic deformation of steel under combined loading (in Russian), Izv TPI 139 (1965), 250-253.

[3.482] -, On combined loading with constant directions of stress tensor axes (in Russian), Izv TPI 157 (1970), 38-43.

[3.483] N. N. Malinin, Applied theory of plasticity and creep (in Russian), Mashinostroyenye, Moskva 1974.

[3.484] A. K. Malmeyster, Deformation of a medium subject to twinning (in Russian), VDP 3 (1955), 97.

[3.485] —, Plasticity of a quasi-isotropic solid (in Russian), VDP 4 (1956), 37-48. 
[3.486] A. K. Malmeyster, On the fundamentals of the theory of local deformations (in Russian), Izv AN LatvSSR (1961), 8, 53-61.

[3.487] —, Fundamentals of the theory of locality of deformations, I, (in Russian), MPol (1965), 4, 12-27.

[3.488] —, Geometry of the theories of failure (in Russian), MPol (1966), 4, 519-534.

[3.489] -, Fundamentals of the theory of locality of deformations, II (in Russian), MPol (1969), 1, 22-29.

[3.490] V. I. Malyi, On the postulates of plasticity (in Russian), IZh MTT (1966), 4, 65-69.

[3.491] - , On the evenness of the components of the stress vector-functional in the theory of plasticity (in Russian), $V M U M M$ (1966), 6, 80-84.

[3.492] - Expansion of the stress functional with respect to a small parameter (in Russian), VMU MM (1967), 2, 73-80.

[3.493] J. Mandel, Généralisation de la théorie de plasticité de W. Koiter, IJSS 1 (1965), 273-295.

[3.494] - - Cours de mécanique des milieux continus, Vol. I-II, Gauthier-Villars, Paris 1966.

[3.495] - Equations constitutives et directeurs dans les milieux plastiques et viscoplastiques, IJSS 9 (1973), 725-740.

[3.496] - , Relation de comportement des milieux élastiques-plastiques et élastiques-viscoplastiques, notion de repère directeur, SFP 1972 (1974), 387-400.

[3.497] E. H. Mansfield, Biaxial yield criteria, JRAS 75 (1971), 732, 849-850.

[3.498] R. M. Mansurov, On plastic loading of initially isotropic media with deformational anisotropy (in Russian), UN 2 (1971), 137-145.

[3.499] - , On accounting for deformational anisotropy in the theory of plasticity (in Russian), Izv AN SSSR MTT (1974), 1, 140-141.

[3.500] Z. Marciniak, Graphical representation of states of stress and strain, AMS 9 (1957), 3, 261-274.

[3.501] -, Mechanika procesów tloczenia blach, Wyd. Nauk.-Techn., Warszawa 1961.

[3.502] - Influence of the sign change of the load on the strain hardening curve of a copper test piece subject to torsion, AMS 13 (1961), 6, 744-752.

[3.503] J. Marin, Failure theories of materials subject to combined stresses, Proc ASCE 61 (1935), 851-867.

[3.504] J. Marin, L. W. Hu, Plastic stress-strain relations for biaxial tension and variable stress ratios, Proc ASTM 52 (1952), 1095-1125.

[3.505] J. Marin, J. A. Sauer, Plastic stress-strain relations under radial and non-radial combined stress loading, JFI 256 (1953), 2, 119-128.

[3.506] J. Marin, L. W. Hu, Bi-axial plastic stress-strain relations of a mild steel for variable stress ratios, Trans ASME 18 (1956), 3, 499-509.

[3.507] J. Marin, Mechanical properties of materials for combined stresses based upon true stress and strain, JFI 263 (1957), 1, 35-46.

[3.508] —, Theories of strength for combined stresses and nonisotropic materials, JAS 24 (1957), 4, 265-266, 274.

[3.509] R. Marjanović, W. Szczepiński, Yield surfaces of the M-63 brass prestrained by cyclic biaxial loading, AMS 26 (1974), 2, 311-320.

[3.510] T. N. Martynova, Properties of equations of the theory of plasticity with material compressibility taken into account (in Russian), $\operatorname{Tr}$ VISI 13 (1967), 1, 93-100.

[3.511] M. Matsuura, Extension of the Drucker-Prager yield condition, 9th JCAM 1959 (1960), 113-116.

[3.512] K. H. Matucha, P. Wincierz, Experimentelle Bestimmung von Fliessortkurven, MAn (1974), 201-229.

[3.513] P. Mazilu, On a differential constitutive law, RRMPA 19 (1974), 2, 205-208.

[3.514] -, A note on the constitutive law of plastic flow, AMS 26 (1974), 2, 321-326. 
[3.515] P. V. McLaughlin, Plastic limit behavior and failure of filament reinforced materials, IJSS 8 (1972), 11, 1299-1318.

[3.516] E. Melan, Zur Plastizität des räumlichen Kontinuums, IA 9 (1938), 116-126.

[3.517] A. Meldahl, Brown Boveri Mittleilungen, Jul.-Aug. 1943, 204.

[3.518] —, Brown Boveri Rev., Zürich 1944, 260.

[3.519] A. Mendelson, Plasticity: Theory and applications, MacMillan, New York 1968.

[3.520] J. G. Merkle, An ellipsoidal yield function for materials that can both dilate and compact inelastically, NED 12 (1970), 3, 425-451.

[3.521] J. Miastkowski, W. Szczepiński, An experimental study of yield surfaces of prestrained brass, IJSS 1 (1965), 189-194 (English summary); MTS 3 (1965), 2, 55-66 (Polish full text).

[3.522] J. Miastkowski, Wpływ historii obciążenia na powierzchnię plastyczności, I, MTS 4 (1966), 2, 5-16.

[3.523] —, Wpływ historii obciążenia na powierzchnię plastyczności, II, MTS 6 (1968), 1, 3-31.

[3.524] -, Analysis of the memory effect of plastically prestrained material, AMS 20 (1968), 3, 261-277.

[3.525] M. J. Michno, W. N. Findley, Experiments to determine small offset yield surfaces for 304L stainless steel under combined tension and torsion, AMech 18 (1973), 3-4, 163-179.

[3.526],-- , Subsequent yield surfaces for annealed mild steel under dead-weight loading: aging, normality, convexity, corners, Bauschinger and cross effects, Trans ASME H96 (1974), 1, 56-64.

[3.527] - , - , Subsequent yield surfaces for annealed mild steel under servo-controlled strain and load histories: aging, normality, convexity, corners, Bauschinger and cross effects, Trans ASME H97 (1975), 1, 25-32.

[3.528] M. Sh. Mikeladze, Statics of anisotropic plastic shells (in Russian), Izd. AN Gruz. SSR, Tbilisi 1963.

[3.529] I. E. Mileykovsky, On a possible yield condition foc an anisotropic body (in Russian), IVSMTP (1956), 169-179.

[3.530] I. N. Mirolyubov, On the problem of generalization of the octahedral shearing stresses failure theory to brittle materials (in Russian), $\operatorname{Tr}$ LPI 25 (1953).

[3.531] R. Mises, Mechanik der plastischen Formănderung von Kristallen, ZAMM 8 (1928), 3, 161-185.

[3.532] —, Three remarks on the theory of the ideal plastic body, RAV (1949), 415-429.

[3.533] N. M. Mitrokhin, Influence of the type of the stress deviator on plastic deformation of nickel subject to simple loading (in Russian), NTI LPI (1959), 11, 81-89.

[3.534] N. M. Mitrokhin, Yu. I. Yagn, On the systematic character of deviations from the laws of plasticity, (in Russian), DAN SSSR 135 (1960), 4, 796-799.

[3.535] R. K. Mittal, Biaxial loading of aluminum and a generalization of the parabolic law, JMat 6 (1971), 1, 67-81.

[3.536] O. Mohr, Welche Umstände bedingen die Elastizitätsgrenze und den Bruch eines Materials? VDI Z 44 (1900), 1524-1530, 1572-1577; 45 (1901), 740.

[3.537] -, Abhandlungen aus dem Gebiete der technischen Mechanik, 2. Auflage, Ernst und Sohn, Berlin 1914.

[3.538 J. L. M. Morrison, The yield of mild steel with particular reference to the effect of size of specimen, Proc IME 142 (1940), 3, 193-223.

[3.539] -, The criterion on yield of gun steels, Proc IME 159 (1948), 81-94.

[3.540] J. L. M. Morrison, W. M. Shepherd, An experimental investigation of plastic stress-strain relations, Proc IME 163 (1950), 1-19.

[3.541] V. V. Moskvitin, On secondary plastic deformations (in Russian), PMM 16 (1952), 3, 323-330. 
[3.542] V. V. Moskvitin, Elastic-plastic deformations of bodies subject to repeated loadings (in Russian), PMM 19 (1955), 6, 714-724.

[3.543] -, On the conditions of a simple repeated loading (in Russian), VMU MM (1962), 2, $72-75$.

[3.544] V. V. Moskvitin, E. Palne, On the theorem on variable loadings (in Russian), IZh MTT (1966), 3, 144-147.

[3.545] V. V. Moskvitin, Zh. I. Tairova, On a simple possibility of analysis of combined cyclic loadings (in Russian), VMU MM (1968), 3, 119-122.

[3.546] V. V. Moskvitin, Some problems of plasticity in the case of variable loadings (in Russian), PPMCTN (1969), 25-36.

[3.547] -, Cyclic plasticity and cumulative deterioration (in Russian), DAN SSSR 184 (1969), 4, 796-799.

[3.548] -, On a certain possibility of accounting for cumulative deterioration in cyclic plasticity (in Russian), VMU MM (1969), 5, 117-121.

[3.549] -, On simple variable loadings in thermoplasticity (in Russian), VMU $M M$ (1969), 5, 122-124.

[3.550] N. S. Mozharovsky, E. A. Antipov, Plasticity and fracture of heat-resistant materials under programmed variations of temperature and stresses (in Russian), TNEK 13 (1973), 61-67.

[3.551] Z. Mróz, Non-associated flow laws in plasticity, JM 2 (1963), 1, 21-42.

[3.552] -, Admissible loading paths in the deformation theories of plasticity, AMS 16 (1964), 5, 1091-1102.

[3.553] -, On non-linear flow laws in the theory of plasticity, BAP SST 12 (1964), 11, 789-797,

[3.554] -, On the description of anisotropic workhardening, JMPS 15 (1967), 3, 163-175.

[3.555] -, An attempt to describe the behaviour of metals under cyclic loads using a more general workhardening model, AMech 7 (1969), 199-212.

[3.556] - , On the theory of steady plastic cycles in structures, 1st SMiRT (1971), L5-6, 1-13.

[3.557] Z. Mróz, C. Goss, O złożonych modelach wzmocnienia plastycznego, MTS 10 (1972), 2, 259-279.

[3.558] Z. Mróz, Mathematical models of inelastic concrete behaviour, INSC (1973), 47-72.

[3.559] -, A description of workhardening of metals with application to variable loading, SFP 1972 (1974), 551-570.

[3.560] Z. Mróz, N. C. Lind, Simplified theories of cyclic plasticity, AMech 22 (1975), 1-2, 131-152,

[3.561] Z. Mróz, Description of plastic and viscous hardening of materials for steady and cyclic loading, 3rd SMiRT (1975), L5/6.

[3.562] J. F. Mulhern, T. G. Rogers, A. J. M. Spencer, A continuum model for fibre-reinforced plastic materials, Proc RS A301 (1967), 473-492.

[3.563] T. Mura, Residual stresses due to thermal treatments, RRFE $M U$ (1957), 10, 14.

[3.564] J. Murzewski, A probability theory of plastic and brittle behaviour of quasi-homogeneous materials, AMS 12 (1960), 2, 203-227.

[3.565] J. Murzewski, Z. Mendera, Yield surface of steel determined by semiempirical method, BAP SST 11 (1963), 7, 35-42.

[3.566] A. Nádai, Theory of flow and fracture of solids, vol. 1, McGraw-Hill, New York-TorontoLondon 1950.

[3.567] P. M. Naghdi, J. C. Rowley, C. W. Beadle, Experiments concerning the yield surface and the assumptions of linearity in the plastic stress-strain relations, JAM 22 (1955), 3, 416-420.

[3.568] P. M. Naghdi, F. Essenburg, W. Koff, An experimental study of initial and subsequent yield surfaces in plasticity, JAM 25 (1958), 2, 101-209.

[3.569] P. M. Naghdi, J. A. Trapp, On finite elastic-plastic deformation of metals, Trans ASME E41 (1974), 1, 254-260. 
[3.570] P. M. Naghdi, J. A. Trapp, Restrictions on constitutive equations of finitely deformed elastic-plastic materials, QJMAM 28 (1975), 1, 25-46.

[3.571],,-- On the nature of normality of plastic strain rate and convexity of yield surfaces in plasticity, Trans ASME EA2 (1975), 1, 61-66.

[3.572] B. Nayroles, Structure algébraique des théories classiques de plasticité, STA (1973), 1, 193-217.

[3.573] —, J. J. Moreau's approach to a fundamental problem: the quasi-static evolution of a perfectly elasto-plastic body, AMS 28 (1976), 1, 115-132.

[3.574] Yu. V. Nemirovsky, On elastic-plastic behaviour of a reinforced layer (in Russian), ZhPMTF (1969), 6, 81-89.

[3.575] H. Neuber, Anisotropic nonlinear stress-strain laws and yield conditions, IJSS 5 (1969), 12, 1299-1310.

[3.576] Q. S. Nguyen, Évolution de la contrainte et de l'écrouissage pour certains matériaux a écrouissage positif, STA 47 (1973), 1, 247-250.

[3.577] Q. S. Nguyen, H. D. Bui, Sur les matériaux élastoplastiques à écrouissage positif ou négatif, $J M 13$ (1974), 2, 321-342.

[3.578] V. N. Nikolayevsky, Mechanical properties of soils and the theory of plasticity (in Russian), INT MTDT 6 (1972).

[3.579] V. N. Nikolayevsky, N. M. Syrnikov, G. M. Shefter, Dynamics of elastic-plastic media subject to dilatation (in Russian), UMDS (1975), 397-413.

[3.580] V. V. Novozhilov, On stress-strain relations in a nonlinearly elastic medium (in Russian), PMM 15 (1951), 2, 183-194.

[3.581] - , On a certain direction in the theory of plasticity (in Russian), Izv AN SSSR MM (1961), $3,176-181$.

[3.582] -, Once more on the postulate of isotropy (in Russian), Izv AN SSSR MM (1962), 1, 205-208.

[3.583] - , On the forms of stress-strain relations in initially isotropic inelastic bodies (in Russian), PMM 27 (1963), 5, 794-812.

[3.584] —, On combined loading and perspectives of phenomenological approach to the analysis of microstresses (in Russian), PMM 28 (1964), 3, 393-400.

[3.585] W. J. O'Donnell, J. Porowski, Yield surfaces for perforated materials, Trans ASME E40 (1973), 1, 263-270.

[3.586] F. K. G. Odqvist, Die Verfestigung von flusseisenähnlichen Körpern, ZAMM 13 (1933), $5,360-363$.

[3.587] P. M. Ogibalov, Yield conditions for combined stresses in a body subject to unsteady thermal regime (in Russian), VMU (1949).

[3.588] - Theory of plastic deformations at elevated temperatures of the body (in Russian), $V M U$ (1950), 12.

[3.589] P. M. Ogibalov, I. A. Kiyko, High-parameter mechanics (in Russian), Mosk. Univers., Moskva 1966.

[3.590] P. M. Ogibalov, The Ilyushin laws and methods in the mechanics of deformable solids and media (in Russian), MPol (1971), 1, 36-40.

[3.591] Y. Ohashi, M. Tokuda, Precise measurement of plastic behaviour of mild steel tubular specimens subjected to combined torsion and axial force, JMPS 21 (1973), 4, 241-261.

[3.592] Y. Ohashi, M. Tokuda, S. Mizuno, A precise stress-strain relation of mild steel in the proportional deformation under combined loading, BJSME 17 (1974), 111, 1135-1142.

[3.593] Y. Ohashi, M. Tokuda, H. Yamashita, Plastic deformation of mild steel under combined load of axial force and torsion with strain trajectories of constant curvature, BJSME 18 (1975), 120, 579-586.

[3.594] J. G. Oldroyd, On the formulation of rheological equations of state, Proc RS 200A (1950), 523-541. 
[3.595] W. Olszak, W. Urbanowski, Ortotropia i niejednorodność w teorii plastyczności, AMS 8 (1956), 1, 85-110.

[3.596] - , - The plastic potential and the generalized distortion energy in the theory of nonhomogeneous anisotropic elastic-plastic bodies, AMS 8 (1956), 4, 671-694.

[3.597] - , - Plastic non-homogeneity: a survey of theoretical and experimental research, IUTAM NH 1958 (1959), 259-298.

[3.598] W. Olszak, On some basic aspects of the theory of non-homogeneous loose and cohesive media, AMS 11 (1959), 6, 651-766.

[3.599] W. Olszak, J. Rychlewski, W. Urbanowski, Plasticity under non-homogeneous conditions, AAM 7 (1962), 131-214.

[3.600] W. Olszak, Z. Mróz, P. Perzyna, Recent trends in the development of the theory of plasticity, Pergamon Press-PWN, Oxford-Warsżawa 1963.

[3.601] W. Olszak, P. Perzyna, The constitutive equations of the flow theory for a non-stationary yield condition, 11th ICAM 1964 (1966), 545-553.

[3.602] W. Olszak, Koncepcja naroża plastycznego w świetle teorii i doświadczenia, Ksiega jubil. A. Krupkowskiego, Warszawa 1965, 349-370.

[3.603] W. Olszak, W. Urbanowski, Anisotropie im elastisch-plastischen Bereich, MAn (1974), 279-301.

[3.604] E. Ondraček, Př́ispěvek k teorii plastického potenciálu, AMat 8 (1963), 3, 163-179.

[3.605] W. R. Osgood, Combined-stress tests on 24S-T aluminium-alloy tubes, JAM 14 (1947), 247-253.

[3.606] J. R. Osias, J. L. Swedlow, Finite elasto-plastic deformation, IJSS 10 (1974), 3, 321-339.

[3.607] T. Ota, A. Shindo, H. Fukuoka, Anisotropy of mild steel by combined tension-torsion tests, 8th JCAM 1958 (1959), 221-224.

[3.608] - , - , - A consideration on anisotropic yield criterion, 9th JCAM 1959 (1960), 117.

[3.609] D. R. Owen, Thermodynamics of materials with elastic range, ARMA 31 (1968), 2, 91-112,

[3.610] D. R. J. Owen, J. Holbeche, O. C. Zienkiewicz, Elastic-plastic analysis of fibrereinforced materials, FST 1 (1969), 3, 185-207.

[3.611] D. R. Owen, A mechanical theory of materials with elastic range, ARMA 37 (1970), 2, 85-110.

[3.612] A. Paglietti, Universal deformations for thermo-elastic-plastic materials, AMS 27 (1975), 5-6, 773-789.

[3.613] I. Z. Palley, Application of the theory of residual microstresses to non-isothermal deformation (in Russian), Izv AN SSSR Mekh (1965), 2, 110-113.

[3.614] - On accounting for effects of the Bauschinger type in the analysis of inelastic deformation in variable temperatures (in Russian), PDAD 5 (1969), 163-169.

[3.615] A. C. Palmer, G. Maier, D. C. Drucker, Normality relations and convexity of yield surfaces for unstable materials or structural elements, Trans ASME E34 (1967), 2, 464-470.

[3.616] V. M. Panferov, R. Kh. Ismagilov, L. M. Ilyin, E. Z. Korol, V. F. Tyurin, On the deformation of a solid in the case of combined loading with fixed principal directions of stresses and strains (in Russian), NTIM MU (1971), 8, 5-21.

[3.617] J. Parker, M. B. Bassett, Plastic stress-strain relationships-some experiments to derive a subsequent yield surface, Trans ASME E31 (1964), 4, 676-682.

[3.618] B. Paul, A modification of the Coulomb-Mohr theory of fracture, Trans ASME E28 (1961), 2, 259-268.

[3.619] B. Paul, W. Chen, Lee Li-Chih, An experimental study of plastic flow under stepwise increments of tension and torsion, 4th USNCAM 2 (1062), 1031-1038.

[3.620] B. Paul, Macroscopic criteria for plastic flow and brittle fracture, Fracture (collection of papers), vol. 2, 313-496, Acad. Press, New York 1968.

[3.621] H. Payne, The slip theory of plasticity for aggregates, JMPS 7 (1959), 2, 126-134.

[3.622] T. Pełczyński, Zagadnienia wytężenia materiałów, rys historyczny, OP 3 (1962), 1, 9-49. 
[3.623] N. Perrone, P. G. Hodge, Strain hardening solutions with generalized kinematic models, 3rd USNCAM (1958), 641-648.

[3.624] P. Perzyna, Simple material and plastic material, AMS 18 (1966), 3, 241-258.

[3.625] P. Perzyna, W. Wojno, Thermodynamics of a rate sensitive plastic material, AMS 20 (1968), 5, 499-511.

[3.626] P. Perzyna, Plasticity of irradiated materials, BAP SST 21 (1973), 10, 499-513.

[3.627] P. Perzyna, A. Sawczuk, Problems in thermoplasticity, NED 24 (1973), 1, 1-55.

[3.628] P. Perzyna, Internal variable description of plasticity, PPlast (1973), 145-176.

[3.629] P. P. Petrishtchev, Application of the theory of small elastic-plastic deformations to an anisotropic body (in Russian), Izd. Mosk. Gosud. Univ., Moskva 1951.

[3.630] A. Phillips, An experimental investigation of plastic stress-strain relations, 9th ICAM 1956, 9 (1957), 23-33.

[3.631] —, Pointed vertices in plasticity, 2nd SNSM (1960), 202-214.

[3.632] A. Phillips, G. A. Gray, Experimental investigation of corners in the yield surface, Trans ASME D83 (1961), 2, 275-288.

[3.633] A. Phillips, R. L. Sierakowski, On the concept of the yield surface, AMech 1 (1965), 1, 29-35.

[3.634] A. Phillips, M. Eisenberg, Observations on certain inequality conditions in plasticity, IJNLM 1 (1966), 2, 247-256.

[3.635] A. Phillips, Yield surfaces of pure aluminium at elevated temperatures, IUTAM ThI 1968 (1970), 241-258.

[3.636] A. Phillips, C. S. Liu, J. W. Justusson, An experimental investigation of yield surfaces at elevated temperatures, AMech 14 (1972), 2-3, 119-146.

[3.637] A. Phillips, J. L. Tang, The effect of loading path on the yield surface at elevated temperatures, IJSS 8 (1972), 4, 463-474.

[3.638] A. Phillips, R. Kasper, On the foundations of thermoplasticity, an experimental investigation, Trans ASME E40 (1973), 4, 891-896.

[3.639] A. Phillips, J. L. Tang, M. Ricciuti, Some new observations of yield-surfaces, AMech 20 (1974), 1-2, 23-39.

[3.640] A. Phillips, The foundations of thermoplasticity, experiments and theory, TACM (1974), $1-21$.

[3.641] -, Experimental plasticity, some thoughts on its present status and possible future trends, PPlast 1972 (1973), 193-231.

[3.642] A. Phillips, G. Weng, A new hardening law in thermoplasticity (in Russian), UMDS (1975), 550-558.

[3.643] G. S. Pisarenko, A. A. Lebedev, Deformation and fracture of materials under combined stress (in Russian), Izd. Naukowa Dumka, Kiev 1969.

[3.644] G. S. Pisarenko, A. A. Lebedev, V. P. Lamashevsky, An experimental investigation of the laws of deformation of carbon steel under combined stresses at low temperatures (in Russian), PPro (1969), 5, 42-47.

[3.645] B. E. Pobedrya, The notation of a simple process in the case of finite deformations, $P P$ (1971), 166-170.

[3.646] A. A. Polyakov, L. T. Shirogorodsky, Variable non-isothermal deformation of structural materials (in Russian), TNEK 11 (1971), 136-139.

[3.647] J. Porowski, W. J. O'Donnell, Effective plastic constants for perforated materials, Trans ASME J96 (1974), 3, 234-241.

[3.648] W. R. Powell, A note on yield curves in cyclic work softening, Trans ASME E35 (1968), 4, 822-824.

[3.649] W. Prager, Einfluss der Deformation auf die Fliessbedingung von zähplastischen Körpern, ZAMM 15 (1935), 1/2, 76-80.

[3.650] -, Mécanique des solides isotropes au delà du domaine élastique, Mem SM 87 (1937), $66 \mathrm{pp}$. 
[3.651] W. Prager, Strain-hardening under combined stresses, JAP 16 (1945), 12, 837-840.

[3.652] - , The stress-strain laws of the mathematical theory of plasticity, JAM 15 (1948), 3, 226-233.

[3.653] -, Recent developments in the mathematical theory of plasticity, JAP 20 (1949), 235-241.

[3.654] - On the use of singular yield conditions and associated flow rules, JAM 20 (1953), 317-320.

[3.655] —, The theory of plasticity: a survey of recent achievements, Proc IME 169 (1955), 41-57.

[3.656] -, A new method of analyzing stresses and strains in work-hardening plastic solids, JAM 23 (1956), 4, 493-496.

[3.657] —, Non-isothermal plastic deformation, Proc KNAW 61 (1958), 176-182.

[3.658] -, On plastic analysis of layered structures (in Russian), PMSS (1961), 302-309.

[3.659] -, Plastic failure of fiber-reinforced materials, Trans ASME E36 (1969), 3, 542-544.

[3.660] H. L. D. Pugh, D. Green, The effect of hydrostatic pressure on the plastic flow and fracture of metals, Proc IME 170 (1956)).

[3.661] Yu. N. Rabotnov, A model illustrating some properties of a plastic hardening body (in Russian), PMM 23 (1959), 1, 164-169.

[3.662] -, Elastic-plastic state of a composite structure (in Russian), PGMSS (1969), 411-415.

[3.663] D. Radenković, Théorèmes limites pour un matériau de Coulomb à dilatation non standartisée, CRAS 252 (1961), 26, 4103-4104.

[3.664] —, Théorie des charges limites, extension à la mécanique des sols, $S P 1961$ (1962), 116, 129-142.

[3.665] B. Raniecki, A. Sawczuk, Thermal effects in plasticity, ZAMM 55 (1975), 6, 333-342; 7-8, 363-374.

[3.666] Y. R. Rashid, On nonlinear kinematic hardening plasticity theory, NED 29 (1974), 1, 135-140.

[3.667] S. I. Ratner, Change of mechanical properties of metals under hydrostatic pressure (in Russian), ZhTF 19 (1949), 3, 408.

[3.668] K. A. Reckling, Experimente zur Feststellung der Werkstoffanisotropie und zur Überprüfung der Hillschen Verfestigungshypothese, Stb 38 (1969), 2, 43-51.

[3.669] M. Reiner, A mathematical theory of dilatancy, AJM 67 (1945), 350-362.

[3.670] L. Rendulic, Eine Betrachtung zur Frage der plastischen Grenzzustände, BI 19 (1938), 159-164.

[3.671] A. Reuss, Flieszpotential oder Gleitebenen? ZAMM 12 (1932), 15.

[3.672] -, Vereifachte Berechnung der plastischen Formänderungsgeschwindigkeiten bei Voraussetzung der Schubspannungsflieszbedingung, ZAMM 13 (1933), 5, 356-260.

[3.673] —, 4th ICAM 1934 (1935), 241.

[3.674] J. R. Rice, Inelastic constitutive relations for solids: an internal-variable theory and its application to metal plasticity, JMPS 19 (1971), 6, 433-455.

[3.675] R. S. Rivlin, Large elastic deformations of isotropic materials, IV, PhTRS A241 (1948), 379-397.

[3.676] R. S. Rivlin, J. L. Ericksen, Stress-deformation relations for isotropic materials, JRMA 4 (1955), 323-425.

[3.677] J. Rogan, A. Shelton, Yield and subsequent flow behaviour of some annealed steels under combined stress, JSA 4 (1969), 2, 127-137.

[3.678] -, - Effect of pre-stress on the yield and flow of En25 steel, JSA 4 (1969), 2, 138-161.

[3.679] I. D. Rogozin, On the theory of slip (in Russian), DSS 4 (1970), 148-155.

[3.680] I. D. Rogozin, The Lode-Nadai diagram for the theory of slip (in Russian), DSS 7 (1971), 215-217.

[3.681] V. I. Rozenblyum, On the condition of full plasticity for a perfectly plastic medium (in Russian), Izv AN SSSR MTT (1969), 4, 116-122.

[3.682] -, On the theorem on unloading (in Russian), IUP 9 (1973), 121-128. 
[3.683] Ya. I. Rudayev, On the conditions of applicability of the theory of small elastic-plastic deformations to non-proportional loading (tension with torsion) of circular bars (in Russian), DNT (1970), 55-67.

[3.684] —, On monotonic deformation of thin-walled tubes (in Russian), $\operatorname{Tr}$ FPI 65 (1974), 103-108.

[3.685] K. N. Rusinko, On plastic deformations at simple and variable repeated loadings (in Russian), Izv AN KirgSSR (1970), 5, 20-28.

[3.686] -, A generalization of Cicala's formula (in Russian), Izv AN SSSR MTT (1971), 6, 37-44.

[3.687] K. N. Rusinko, E. I. Blinov, Analytical investigation of deformations for stepwise loading trajectories (in Russian), PM 7 (1971), 11, 52-58.

[3.688] K. N. Rusinko, V. I. Kuneyev, Investigation of a corner (in Russian), $\operatorname{Tr}$ FPI 56 (1972), 55-63.

[3.689] K. N. Rusinko, A. E. Kalatinets, S. S. Dreval, Problems of the concept of slip in the theory of plasticity (in Russian), PM 10 (1974), 1, 3-19.

[3.690] B. A. Rytchkov, N. Yu. Shvayko, Limits of applicability of the deformation theory for broken loading trajectories of a linear anisotropically hardening medium (in Russian), Izv AN KirgSSR (1967), 2, 25-30.

[3.691] K. Saito, H. Igaki, M. Sugimoto, A study of the equivalent stress and the equivalent plastic strain rate, BJSME 15 (1972), 79, 33-39.

[3.692] G. D. Sandel, Über die Festigkeitsbedingungen, Dissertation, TH Stuttgart 1919; Leipzig 1925.

[3.693] —, SB 95 (1930), 335.

[3.694] J. L. Sanders, Plastic stress-strain relations based on linear loading functions, 2nd USNCAM (1954), 455-460.

[3.695] V. Ya. Sausin', The trace of fading memory at tension followed by a transverse compression (in Russian), MPol (1969), 4, 597-602.

[3.696] - , The relation between auxiliary stress and strain vectors at successive radial loadings of a plastic material (in Russian), MPol (1970), 2, 241-245.

[3.697] A. Sawczuk, Linear theory of plasticity of anisotropic bodies and its applications to problems of limit analysis, AMS 11 (1959), 5, 541-556.

[3.698] A. Sawczuk, P. Stutz, Sur le critère d'écoulement et de la loi de déformation plastique, CRAS 266 (1968), 2, A87-A89.

[3.699] A. Sawczuk, W. J. O'Donnell, J. Porowski, Plastic analysis of perforated plates for orthotropic yield criteria, IJMS 17 (1975), 6, 411-417.

[3.700] M. Sayir, Zur Fließbedingungen der Plastizitätstheorie, IA 39 (1970), 6, 414-432.

[3.701] F. Schleicher, Der Spannungszustand an der Fliessgrenze, ZAMM 6 (1926), 199-216.

[3.702] E. Schmid, Yield point of crystals, critical shear stress law, Ist ICAM (1924), 342.

[3.703] E. Schmid, W. Boas, Kristallplastizität mit besonderer Berücksichtigung der Metalle, Springer, Berlin 1935; English translation: Plasticity of crystals, Chapman \& Hall, London 1950 and 1968.

[3.704] R. Schmidt, Über den Zusammenhang von Spannungen und Formaenderungen im Verfestigungsgebiet, IA 3 (1932), 215-235.

[3.705] L. I. Sedov, Notions of various rates of tensors (in Russian), PMM 24 (1960), 3, 393-398.

[3.706] L. I. Sedov, M. E. Eglit, A construction of non-holonomic models of continua accounting for finite strains and some physico-chemical effects (in Russian), DAN SSSR 142 (1962), 1, 54-57.

[3.707] S. V. Serensen, On strength criteria for variable loadings in the case of plane and spatial stress states (in Russian), IS I/1 (1941), 3-12.

[3.708] B. R. Seth, Anisotropic plasticity (in Russian), PMTDT (1970), 419-425.

[3.709] —, Yield conditions in plasticity, AMS 24 (1972), 5-6, 769-776.

[3.710] M. J. Sewell, A plastic flow rule at a yield vertex, JMPS 22 (1974), 6, 469-490. 
[3.711] Yu. N. Shevtchenko, Unloading theorems in the theory of small elastic-plastic deformations in the case of non-uniform heating (in Russian), PM 2 (1966), 7, 11-21.

[3.712] -, On the theories of thermoplasticity of a hardening material (in Russian), TNEK 6 (1966), 5-22.

[3.713] - , Theorems on simple variable loading and unloading in the theory of small elasticplastic deformations in the case of non-uniform heating (in Russian), TNEK 9 (1970). $148-165$.

[3.714] - A deformation theory of thermoplasticity with kinematic hardening (in Russian), TNEK 10 (1970), 57-67.

[3.715] - Equations of thermoplasticity of irradiated materials (in Russian), TNEK 12 (1972), 39-52.

[3.716] Yu. N. Shevtchenko, R. G. Terekhov, Verification of a certain consequence of the postulate of isotropy (in Russian), PPro (1972), 2, 42-45.

[3.717] Yu. N. Shevtchenko, V. G. Savtchenko, R. G. Terekhov, A. I. Borisyuk, On the problem of applicability of the theory of small elastic-plastic deformations to the calculations of finite strains (in Russian), PPro (1972), 6, 3-6.

[3.718] Yu. N. Shevtchenko, R. G. Terekhov, Experimental verification of the hypothesis of existence of a thermomechanical surface in the theory of thermoplasticity (in Russian), TNEK 13 (1973), 43-47.

[3.719] - , - On the postulate of thermoplasticity (in Ukrainian), DAN URSR (1974), A7, 636-640.

[3.720] R. T. Shield, On Coulomb's law of failure in soils, JMPS 4 (1955), 1, 10-16.

[3.721] R. Shield, H. Ziegler, On Prager's hardening rule, ZAMP 9a (1958), 260-276.

[3.722] E. Shiratori, K. Ikegami, H. Okano, The influence of prestrain on subsequent yield surfaces, I, BJSME 11 (1968), 45, 413-418.

[3.723] E. Shiratori, K. Ikegami, Experimental study of the subsequent yield surface by using cross-shaped specimens, JMPS 16 (1968), 6, 373-394.

[3.724] - , -, Studies of the anisotropic yield condition, JMPS 17 (1969), 6, 473-491.

[3.725] E. Shiratori, K. Ikegami, K. Kaneko, The influence of the Bauschinger effect on the subsequent yield condition, BJSME 16 (1973), 100, 1482-1490.

[3.726],,--- , The stress vector and the subsequent yield surface in loading along the strain path with a corner, BJSME 17 (1974), 113, 1405-1412.

[3.727] O. A. Shishmarev, Influence of the type of stress deviator on plastic deformation of steel (in Russian), IZh MTT (1966), 5, 112-118.

[3.728] - Experimental investigation of yield points of steel under simple and combined loading (in Russian), IZh MTT (1968), 2, 187-190.

[3.729] - Comparison of the results of the theory of plastic flow with experimental data for combined loading (in Russian), Izv VUZ M (1971), 1, 32-37.

[3.730] K. K. Shkerbelis, Influence of combined loading on the deformation of concrete (in Russian), IBZB 3 (1958), 57-60.

[3.731] H. P. Shrivastava, Z. Mróz, R. N. Dubey, Yield criterion and second-order effects in plane-stress, AMech 17 (1973), 1-2, 137-143.

[3.732],,,--- Yield criterion and the hardening rule for a plastic solid, ZAMM 53 (1973), $10,625-633$.

[3.733] H. P. Shrivastava, R. N. Dubey, Yield condition and hardening rule for density varying materials, ZAMM 54 (1974), 9, 594-596.

[3.734] N. Yu. Shvayko, Yu. V. Klyshevitch, Approximation and generalization of stress ratestrain rate relations in the neighbourhood of a corner at the loading trajectory (in Russian), Izv AN KirgSSR (1968), 6, 21-29.

[3.735] N. Yu. Shvayko, Ya. I. Rudayev, On the limits of applicability of the deformation theory of plasticity in tension with torsion of circular bars (in Russian), Tr FPI 54 (1971), 145-152. 
[3.736] N. Yu. Shvayko, On a possible general form of the hardening function for a linear planeplastic medium (in Russian), GTU 14 (1972), 109-120.

[3.737] O. M. Sidebottom, Note on the effective plastic strain for a Tresca material, Trans ASME E38 (1971), 4, 1049-1050.

[3.738] F. Sidoroff, The geometrical concept of intermediate configuration and elastic-plastic finite strain, AMS 25 (1973), 2, 299-309.

[3.739] -, On the formulation of plasticity and viscoplasticity with internal variables, AMS 27 (1975), 5-6, 807-819.

[3.740] R. L. Sierakowski, A. Phillips, The effect of repeated loading on the yield surface, AMech 6 (1968), 2-3, 217-231.

[3.741] Yu. I. Sirotin, On the theory of perfect elastoplasticity of crystals (in Russian), Izv $A N$ SSSR MTT (1970), 1, 39-47.

[3.742] G. A. Smirnov-Alyayev, Fundamentals of the mathematical theory of plasticity of a considerably deforming body (in Russian), STMM LGU 3 (1939).

[3.743] G. A. Smirnov-Alyayev, V. M. Rozenberg, Theory of plastic deformations of metals (in Russian), Mashgiz, Moskva-Leningrad 1956.

[3.744] G. F. Smith, On the yield condition for anisotropic materials, QAM 20 (1962), 3, 241-247.

[3.745] G. F. Smith, A. J. M. Spencer, A continuum theory of a plastic-rigid solid reinforced by two families of inextensible fibres, QJMAM 23 (1970), 4, 489-504.

[3.746] S. Smith, B. O. Almroth, An experimental investigation of plastic flow under biaxial stress, ExpM 10 (1970), 6, 217-224.

[3.747] Z. Sobotka, Základni vztahy přirůstkové theorie plasticity a nové pojeti plastického potenciálu pro anisotropické hmoty, StavC 14 (1966), 6.

[3.748] —, Anisotropie, deviace a deviaćni uhel, StavČ 15 (1967), 6, 359-364.

[3.749] - Energetic yield condition for the plane deformation of the anisotropic materials, StavC 15 (1967), 8, 435-452.

[3.750] —, Dvojparametricka kvadratická podminka plasticity, Stav ${ }^{\searrow} 15$ (1967), 8, 494-500.

[3.751] —, The cubic yield condition for incompressible bodies, AT CSAV 12 (1967), 6, 830-832.

[3.752] - Energy yield conditions for anisotropic materials and for anisotropic Cosserat continua (in Russian), BKMSS 1966 (1968), 33-45.

[3.753] —, Theorie des plastischen Fliessens von anisotropen Körpern, ZAMM 49 (1969), 1-2, 25-32.

[3.754] -, Yield condition of the constant limiting distortion energy for anisotropic bodies, $A T C$ CSAV 14 (1969), 3, 352-365.

[3.755] - , The relations of the theory of plasticity for materials with different mechanical properties in tension and in compression, AT CSAV 16 (1971), 2, 320-324.

[3.756] - The plastic flow of orthotropic materials with different mechanical properties in tension and in compression, AT CSAV 16 (1971), 6, 772-776.

[3.757] -, Tensorial expansion for the plastic flow of partially compressible media, AT CSAV 18 (1973), 1, 1-16.

[3.758] —, Plastické přetvařeni hmot $\mathrm{s}$ ruznými mechanickými vlastnostmi $\mathrm{v}$ tahu a $\mathrm{v}$ tlaku, StavC 21 (1973), 6-8, 403-417.

[3.759] - Plasticity of anisotropic materials with positive and negative shear effects, Stav $\varnothing 21$ (1973), 9, 684-687.

[3.760] -, Second-order plastic flow of isotropic partially compressible media, AT CSAV 20 (1975), 3, 301-321.

[3.761] V. V. Sokolovsky, Some remarks on linearization of the equations of plasticity (in Russian), PMM 25 (1961), 5, 931-932.

[3.762] A. J. M. Spencer, Deformations of fibre-reinforced materials, Clarendon Press, Oxford 1972. 
[3.763] A. J. M. Spencer, J. E. Ferrier, Some solutions for a class of plastic-elastic solids, SFP 1972 (1974), 9-24.

[3.764] F. Stassi D'Alia, Un paraboloide di rivoluzione quale condizione di plasticità, Ing (1951).

[3.765] -, La condizione di plasticità secondo recenti ricerche, Rendiconti del corso Costruzioni Milano (1960), 60-71.

[3.766] - , Flow and fracture of materials according to a new limiting condition of yielding, $\mathrm{Mec} 2$ (1967), 3, 178-195.

[3.767] -, Limiting conditions of yielding for anisotropic materials, Mec 4 (1969), 4, 349-363.

[3.768] F. Stassi, Flow and fracture of metals under combined stress (in Russian), PPro (1973), $5,32-40$.

[3.769] F. D. Stockton, D. C. Drucker, On fitting mathematical theories of plasticity to experimental results, JCS 5 (1950), 239-247.

[3.770] F. D. Stockton, Experimental evidence of nonlinearity in plastic stress-strain relations, Techn. Rep. 88, Brown Univ. 1953.

[3.771] M. Stodulski, Dwuparametrowa rodzina obszarów plastycznego przystosowania się pręta rozciąganego i skręcanego, (in print).

[3.772] P. Stutz, Remarque sur l'écoulement plastique dans le cas d'une relation tensorielle linéaire entre contrainte et vitesse de deformation, CRAS 265 (1967), 16, A481-A484.

[3.773] - Remarque sur l'écoulement plastique dans le cas d'une relation tensorielle non linéaire entre contraintes et vitesse de deformation, CRAS 266 (1968), 23, A1149-A1152.

[3.774] M. Sugimoto, H. Igaki, K. Saito, Equivalent stress (rate) and equivalent strain rate for work-hardening materials (theory of anisotropic plasticity based on maximum shear stress hypothesis), BJSME 16 (1973), 102, 1857-1866.

[3.775] N. G. Suturina, On the form of subsequent yield surface of steel (in Russian), IUP 6 (1967), 156-160.

[3.776] N. L. Svensson, Anisotropy and the Bauschinger effect in cold rolled aluminium, JMES 8 (1966), 2, 162-172.

[3.777] N. G. Sverdlova, On the changes of yield surface in the case of arbitrary direction of loading (in Russian), IUP 7 (1968), 144-147.

[3.778] J. L. Swedlow, Character of the equations of elasto-plastic flow in three independent variables, IJNLM 3 (1968), 3, 325-336.

[3.779] W. Szczepiński, Experimental investigations of behaviour of the yield surface of metals under combined loading (in Russian), BKMSS 1966 (1968), 169-179.

[3.780] W. Szczepiński, J. Miastkowski, An experimental study of the effect of the prestraining history on the yield surfaces of an aluminium alloy, JMPS 16 (1968), 3, 153-162.

[3.781] W. Szczepiński, K. Turski, On the lines of influence of plastic deformation, AMS 21 (1969), 1, 49-57.

[3.782] W. Szczepiński, Stany graniczne i kinematyka ośrodków sypkich, IPPT PAN - PWN, Warszawa 1974.

[3.783] G. B. Talypov, V. N. Kamentsev, Investigation of yield-point stresses and of some other effects in the case of combined loading (in Russian), UZMU 280 (1960).

[3.784] G. B. Talypov, Yield-point stresses and fracture of low-carbon steel in the case of simple and of combined loading: the influence of aging (in Russian), Izv AN SSSR MM (1961), $6,125-130$.

[3.785] - , On the theory of plasticity of an initially isotropic hardening material (in Russian), IUP 5 (1966), 198-203.

[3.786] G. B. Talypov, Variation of the yield surface of an initially isotropic metal during the process of plastic deformation (in Russian), PMTDT (1970), 445-451.

[3.787] M. Tanaka, Y. Miyagawa, On generalized kinematic hardening theory of plasticity, IA 44 (1975), 4, 255-268. 
[3.788] M. Tanaka, Elastoplastic constitutive laws based on combined isotropic and kinematic hardening, TROU 25 (1975), 1230-1253.

[3.789] I. I. Tarasenko, Yield conditions and strength conditions of metals (in Russian), SNT LISI 23 (1956), 102-113.

[3.790] G. I. Taylor, C. F. Elam, Distortion of an aluminum crystal during a tensile test, Proc RS A102 (1923), 643.

[3.791] G. I. Taylor, H. Quinney, The plastic distortion of metals, PhTRS A230 (1931), 323-362.

[3.792] G. I. Taylor, Plastic strain in metals, JIM 62 (1938), 306-324.

[3.793] - A connection between the criterion of yield and the strain-ratio relationship in plastic solids, Proc RS A191 (1947), 441-446.

[3.794] C. Teodosiu, A dynamic theory of dislocations and its applications to the theory of the elastic-plastic continuum, FADT (1970), 837-876.

[3.795] R. G. Terekhov, Verification of the postulate of isotropy in the case of combined loading with changing principal directions of the stress tensor (in Russian), PM 6 (1970), 10, 89-92.

[3.796] - Investigations of a thermomechanical surface in the theory of thermoplasticity in the case of unloading (in Ukrainian), DAN URSR A (1973), 5, 471-474.

[3.797] G. A. Teters, A variant of the theory of locality (in Russian), MPol (1967), 5, 800-802.

[3.798] G. A. Teters, Combined loading and stability of shells made of polymeric materials (in Russian), Zinatne, Riga 1969.

[3.799] P. S. Theocaris, C. R. Hazell, Experimental investigation of subsequent yield surfaces using the moiré method, JMPS 13 (1965), 5, 281-294.

[3.800] T. Y. Thomas, Interdependence of the yield condition and the stress-strain relation for plastic flow, Proc NAS 40 (1954), 7, 593-597.

[3.801] —, Combined elastic and Mises' stress-strain relations, Proc NAS 41 (1955), 908-910.

[3.802] —, Combined elastic and Prandtl-Reuss' stress-strain relations, Proc NAS 41 (1955), 720 726.

[3.803] —, Velocity of dislocations in crystals, JMM 18 (1968), 6, 571-584.

[3.804] T. Tokuoka, Generalized Prandtl-Reuss materials, I-III, MFEKU 33 (1971), 3, 186-219.

[3.805] -, Yield conditions and flow rules derived from hypo-elasticity, ARMA 42 (1971), 4, 239-252.

[3.806] —, Constitutive equations of thermo-plastic materials, Trans JSASS 16 (1973), 31, 51-59.

[3.807] —, Fundamental relations of plasticity derived from hypo-elasticity, SFP 1972 (1974), 1-8.

[3.808] L. A. Tolokonnikov, V. V. Shevelev, S. P. Yakovlev, Experimental verification of the equations of plastic flow for an anisotropic body (in Russian), PM 4 (1968), 2, 16-19.

[3.809] L. A. Tolokonnikov, S. P. Yakovlev, On the formulation of the yield condition and associated flow rule for an anisotropic body (in Russian), Izv VUZ M 7 (1969), 17-20.

[3.810] L. A. Tolokonnikov, S. P. Yakovlev, V. M. Lyalin, Plastic flow of orthotropic bodies (in Russian), $P M 7$ (1971), 6, 11-16.

[3.811] L. A. Tolokonnikov, N, M. Matchenko, On visualization of equations of limit states for initially anisotropic bodies (in Russian), PPro (1974), 3, 54-56.

[3.812] C. Torre, Einfluss der mittleren Hauptnormalspannung auf die Fliess- und Bruchgrenze, ÖIA 1 (1947), 4-5, 316-342.

[3.813] —, Die Grenzzustände statisch beanspruchter Stoffe, SAAWT 15 (1949), 116-145.

[3.814] —, Die Mechanik der Grenzbeanspruchungen, ÖIA 4 (1950), 1, 93-108.

[3.815] —, Grenzbedingungen für spröden Bruch und plastisches Verhalten bildsamer Metalle, ÖIA 4 (1950), 2, 174-189.

[3.816] D. Trifan, Stress theory of plastic flow, JMP 35 (1956), 1, 44-51.

[3.817] A. Troost, J. Betten, Plastische Querzahlen anisotroper Verkstoffe, $A E 43$ (1972), 11, $811-812$. 
[3.818] A. Troost, J. Betten, Zur Frage der Konvexität von Fliessbedingungen bei plastischer Inkompressibilität und Kompressibilität, MRC 1 (1974), 2, 73-78.

[3.819] C. Truesdell, Hypo-elasticity, JRMA 5 (1955), 1, 83-133.

[3.820] S. W. Tsai, E. M. Wu, A general theory of strength for anisotropic materials, JCM 5 (1971), 1, 58-80.

[3.821] K. Turski, Badanie wpływu odkształcenia plastycznego na zachowanie się metalu przy różnych drogach wtórnego obciążenia, MTS 9 (1971), 1, 155-199.

[3.822] —, Yield criterion for a prestrained material, BAP SST 20 (1972), 10, 795-800.

[3.823] A. G. Ugodchikov, Yu. G. Korotkikh, Constitutive equations of nonisothermal elasticplastic deformation and methods of their practical application, SFP 1972 (1974), 535-549.

[3.824] A. A. Vakulenko, On the relations between stresses and strains in inelastic media, (in Russian), DAN SSSR 118 (1958), 665-668.

[3.825] —, Thermodynamic investigation of stress-strain relations in isotropic elastic-plastic media (in Russian), DAN SSSR 126 (1959), 736-739.

[3.826] - On stress-strain relations in isotropic and initially isotropic inelastic media (in Russian), IUP 2 (1963), 3-46.

[3.827] A. A. Vakulenko, I. Z. Palley, On the theory of plasticity for a medium subject to deformation at variable temperatures (in Russian), IUP 5 (1966), 188-197.

[3.828] A. A. Vakulenko, M. L. Kachanov, "Microstresses" in the theory of plasticity, PMTDT (1970), 99-103.

[3.829] A. A. Vakulenko, L. M. Kachanov, Theory of plasticity (in Russian), a survey in the volume Mechanics in USSR during 50 years (1972), vol. 3, 79-118.

[3.830] K. C. Valanis, On the thermodynamic foundation of classical plasticity, AMech 9 (1970), 3-4, 278-291.

[3.831] —, A theory of viscoplasticity without a yield surface, AMS 23 (1971), 4, 517-551.

[3.832] —, Observed plastic behavior of metals vis-a-vis the endochronic theory of plasticity, SFP 1972 (1974), 235-255.

[3.833] R. A. Vasin, On the form of hardening function in the flow theory (in Russian), $P M 3$ (1967), 7, 60-64.

[3.834] - Some problems of stress-strain relations in the case of combined loading (in Russian), UN 1 (1971), 59-126.

[3.835] R. A. Vasin, A. B. Ibragimov, On the investigation of deformational anisotropy in the case of combined loading (in Russian), PP (1971), 126-129.

[3.836] S. D. Volkov, A statistical failure theory (in Russian), Mashgiz, Moskva-Sverdlovsk 1960.

[3.837] R. G. Vos, W. H. Armstrong, Improved hardening theory for cyclic plasticity, AIAA 11 (1973), 3, 400-401.

[3.838] Y.S. Wang, A simplified theory of the constitutive equations of metal plasticity at finite deformation, Trans ASME E40 (1973), 4, 941-947.

[3.839] W. Warkenthin, Beitrag zur Formulierung der Sicherheitsnachweise gegen Fließen und Dauerbruch bei mehrachsigen Spannungszuständen, Sch 20 (1970), 3, 119-122.

[3.840] W. A. Warner, G. H. Handelman, Modified incremental strain law for work hardening materials, QJMAM 9 (1956), 3, 279-293.

[3.841] Z. Waszczyszyn, M. Życzkowski, Some applications in mechanics of approximate formulae for real roots of a cubic equation, BAP SST 14 (1966), 3, 149-158.

[3.842] F. C. Weiler, Anisotroriz, elastic-plastic-thermal stress analyses of solid structures, 1st SMiRT (1971), paper D3-3.

[3.843] C. H. Wells, P. R. Paslay, A small-strain plasticity theory for planar slip materials, Trans ASME E36 (1969), 1, 15-21.

[3.844] G. N. White, D. C. Drucker, Effective stress and effective strain in relation to stress theories of plasticity, JAP 21 (1950), 10, 1013-1021. 
[3.845] J. F. Williams, N. L. Svensson, Effect of tensile prestrain on yield locus of 1100-F aluminium, JSA 5 (1970), 128.

[3.846] - , - A rationally based yield criterion for work hardening materials, Mec 6 (1971), 2, 104-114.

[3.847] J. C. Wilson, R. G. Berggren, Effect of neutron irradiation on steel, Proc ASTM 55 (1955).

[3.848] J. Woodthorpe, R. Pearce, The anomalous behaviour of aluminium sheet under balanced biaxial tension, IJMS 12 (1970), 4, 341-347.

[3.849] Yu. I. Yagn, New methods of the strength calculations (in Russian), VIT (1931), 6.

[3.850] Yu. I. Yagn, O. A. Shishmarev, Some experimental data concerning elastic limits of plastically prestrained nickel specimens (in Russian), DAN SSSR 119 (1958), 1, 46-48.

[3.851] Yu. I. Yagn, L. V. Grebnev, Influence of the rotation of principal stress axes on flow and fracture of metals (in Russian), Tr LPI 278 (1967), 66-69.

[3.852] S. P. Yakovlev, V. F. Kuzin, Plane plastic flow of an anisotropic body (in Russian), PM 5 (1969), 11, 127-130.

[3.853] R. N. Yong, E. McKyes, V. Silvestri, Yield and failure of clays, AMS 24 (1972), 3, 511528.

[3.854] Y. Yoshimura, Comment on the slip theory of Batdorf and Budiansky, BJSME 1 (1958), 2, 109-113.

[3.855] Y. Yoshimura, Y. Takenaka, Strain history effects in plastic deformation of metals, IUTAM SOE (1962), 729.

[3.856] L. B. Zagovaylov, N. Yu. Shvayko, On the problem of stress-strain relations in the neighbourhood of a corner at the loading trajectory (in Russian), GTU 16 (1973), 134-142.

[3.857] V. V. Zakharov, A strength criterion for layered masses (in Russian), PlastM (1961), 8.

[3.858] V. S. Zarubin, A. A. Polyakov, A model of polycrystalline material under non-isothermal loading (in Russian), TNEK 8 (1969), 249-256.

[3.859] - , - A model of non-isothermal plastic deformation of a polycrystalline material (in Russian), TNEK 9 (1970), 253-266.

[3.860] - , - Non-isothermal plasticity of a polycrystalline hardening material (in Russian), TNEK 10 (1970), 189-194.

[3.861] V. S. Zarubin, A. A. Polyakov, Application of a computational model of plastic deforg̀ mation of a polycrystalline material to the analysis of transformation of the loading surface (in Russian), TNEK 12 (1972), 68-71.

[3.862] J. Zawadzki, Ciśnienie zredukowane jako jeden z parametrów wytężenia, RI 5 (1957), 3, 357-398.

[3.863] A. M. Zhukov, Strength and plasticity of the alloy D16T in the case of combined stresses (in Russian), Izv AN SSSR OTN (1954), 6, 61-70.

[3.864] -, Plastic properties and fracture of steel subject to biaxial stress state (in Russian), IS 20 (1954), 37-48.

[3.865] - , Plastic deformation of steel in the case of combined loading (in Russian), Izv AN SSSR OTN (1954), 11, 53-61.

[3.866] - Combined loading and the theories of plasticity of isotropic materials (in Russian), Izv AN SSSR OTN (1955), 8, 89-92.

[3.867] - Certain peculiarities of the neutral loading curve (in Russian), Izv AN SSSR OTN (1958), 8, 32-40.

[3.868] - , Plastic deformations of the alloy AKG in the case of simple and combined loadings (in Russsian), $R P 12$ (1966), 260-289.

[3.869] A. G. Zhuravlev, On the criterion of simple loading in a non-homogeneous medium (in Russian), VMU $M M$ (1962), 6, 39-42.

[3.870] H. Ziegler, A modification of Prager's hardening rule, QAM 17 (1959), 1, 55-65.

[3.871] -, Bemerkung zu einem Hauptachsenproblem in der Plastizitătsheorie, ZAMP 11 (1960), 2, 
[3.872] H. Ziegler, Plastizität ohne Thermodynamik, ZAMP 21 (1970), 5, 798-805.

[3.873] H. Ziegler, J. Nänni, Ch. Wehrli, Zur Konvexität der Fliessfläche, ZAMP 24 (1973), 1, 140-144.

[3.874] -, -, -, Zur Konvexităt der Dissipationsflächen, ZAMP 25 (1974), 1, 76-82.

[3.875] O. C. Zienkiewicz, The finite element method in engineering science, McGraw-Hill, London (1971).

[3.876] A. F. Zilauts, On mathematical foundations of the theory of local deformations (in Russian), Izv AN LatvSSR (1965), 3, 84-89.

[3.877] M. Życzkowski, On the accuracy of approximations of the Huber yield condition, AMS 26 (1974), 1, 65-79.

[3.878] -, Eine dreifache Analogie für kinematische Verfestigung von plastischen Körpern, Festschrift Alf Pflïger, Hannover 1977, 289-294.

\section{Chapter IV}

[4.1] H. Abé, Finite deformation theory for the stretching of a thin elastic-plastic plate of finite thickness, TRTU 38 (1973), 2, 539-564; IJSS 11 (1975), 9, 999-1010.

[4.2] L. G. Afendik, Elastic-plastic bending of thin-walled tubes with large curvature (in Russian), PM 4 (1968), 6, 45-51.

[4.3] L. G. Afendik, M. F. Kolesnitchenko, Experimental investigation of elastic-plastic bending of thin-walled tubes (in Russian), VLPI (1968), 25, 135-141.

[4.4] A. C. Aitken, Studies in practical mathematics II, The evaluation of the latent roots and latent vectors of a matrix, Proc RSE 57 (1937), 269-304.

[4.5] I. S. Akhmedyanov, A. V. Kireyev, Integration of differential equations of elastic-plastic bending of a spherical shell under arbitrary loading (in Russian), $\operatorname{Tr} K A I 63$ (1972), 33-39.

[4.6] D. N. de G. Allen, R. V. Southwell, Relaxation methods applied to engineering problems, XIV, Plastic straining of two-dimensional stress-systems, PhTRS A202 (1950), 379-414.

[4.7] S. C. Anand, S. L. Lee, E. C. Rossow, Finite element analysis of elastic-plastic plane stress problems based upon Tresca yield criterion, IA 39 (1970), 2, 73-86.

[4.8] I. N. Ananev, Yu. I. Nyashin, A comparison of certain methods of stress analysis in the plastic range using the finite element technique (in Russian), SNT PPI 138 (1973), 3-10.

[4.9] J. H. Argyris, Elastoplastic matrix displacement analysis of three-dimensional continua, JRAS 69 (1965), 633-636.

[4.10] -, Elasto-plastic analysis of three-dimensional media, AT ASH 54 (1966), 1-2, 219-237.

[4.11] J. H. Argyris, D. W. Scharpf, J. B. Spooner, The elasto-plastic calculation of general structures and continua, 3rd CDSC (1968), 345-384.

[4.12] - , - - , Die elastoplastische Berechnung von allgemeinen Tragwerken und Kontinua, IA 37 (1969), 5, 326-352.

[4.13] J. H. Argyris, A. S. L. Chan, Applications of finite elements in space and time, $I A 41$ (1972), 4, 235-257; Static and dynamic elasto-plastic analysis by the method of finite elements in space and time, SFP 1972 (1973), 147-175.

[4.14] H. Armen, G. Isakson, A. Pifko, Discrete element methods for the plastic analysis of structures subjected to cyclic loading, IJNME 2 (1970), 189-206.

[4.15] J.-L. Auriault, J. Kravtchenko, R. Negre, R. Sibille, Etude des singularites du champ statique en plasticité coulombienne et leurs applications, PRMS 1974 (1977), 17-67.

[4.16] V. Ya. Bachinsky, I. N. Tkachenko, On the limit state of a bar made of a Prandtl material with limited deformability (in Russian), SK RMNTS 20 (1972), 73-75.

[4.17] J. F. Baker, M. R. Horne, J. Heyman, The steel skeleton, vol. II, Cambridge Univ. Press 1956.

[4.18] Yu. F. Balandin, Thermal fatigue of metals (in Russian), Sudostroyenye, Leningrad 1967.

[4.19] H. Balmer, J. S. Doltsinis, M. König, Elastoplastic and creep analysis with the ASKA program system, CMAME 3 (1974), 87-104. 
[4.20] N. V. Banichuk, Calculation of loading of an elastic-plastic body (in Russian), Izv AN SSSR MTT (1969), 1, 128-135.

[4.21] G. Bartelds, The application of the finite element displacement method to problems of elastoplastic deformation, CTAS (1972), 99-115.

[4.22] V. N. Bastun, On the influence of the geometrical form of a structure on its carrying capacity (in Russian), PM 9 (1973), 8, 57-63.

[4.23] H. Baumgärtel, Endlich-dimensionale analytische Störungstheorie, Akademie-Verlag, Berlin 1972.

[4.24] J. Bäcklund, H. Wennerström, Finite element analysis of elasto-plastic shells, IJNME 8 (1974), 2, 415-424.

[4.25] L. S. Beedle, Plastic design of steel frames, Wiley, New York 1958.

[4.26] L. M. Belenky, On the estimation of carrying capacity of frames with geometric non-linearity taken into account (in Russian), Izv VUZM 7 (1969), 9-12.

[4.27] A. R. Belyakov, O. F. Chernyavsky, On a certain mechanism of cumulation of plastic deformation in the conditions of combined cyclic loading (in Russian), SNT CPI 151 (1974), 56-66.

[4.28] T. Belytschko, M. Velebit, Finite element method for elastic plastic plates, Proc ASCE JEMD 98 (1972), 1, 227-242.

[4.29] T. Belytschko, Plane stress shakedown analysis by finite elements, IJMS 14 (1972), 9, 619-625.

[4.30] J. F. Besseling, The complete analogy between the matrix equations and the continuous field equations of structural analysis, Proc. Int. Symp. Analogue and Digital Techn. Appl. to Aeron., Liège 1963.

[4.31] J. Betten, Zum Traglastverfahren bei nichtlinearem Stoffgesetz, IA 44 (1975), 3, 199-207.

[4.32] P. P. Bijlaard, Theory of local plastic deformations, Mem AIPC 6 (1940/41), 27-44.

[4.33] I. A. Birger, Certain general methods of solution of problems of plasticity theory (in Russian), PMM 15 (1951), 6, 765-770.

[4.34] - Method of supplementary strains in the problems of plasticity theory (in Russian), Izv AN SSSR MM (1963), 1, 48-56.

[4.35] -, Methods of elastic solutions in the theory of plastic flow (in Russian), Izv AN SSSR MM (1964), 2, 116-118.

[4.36] —, Structural analysis with plasticity and creep taken into account (in Russian), Izv AN SSSR Mekh (1965), 2, 113-119.

[4.37] - , General computational algorithms for problems of the theory of elasticity, plasticity and creep (in Russian), UMDS (1975), 51-73.

[4.38] A. Biron, P. G. Hodge, Jr., Nonlinear programming method for limit analysis of rotationally symmetric shells, IJNME 3 (1968), 2, 201-213.

[4.39] A. Biron, U. S. Chawla, Numerical method for limit analysis of rotationally symmetric shells, BAP SST 18 (1970), 3, 185-192.

[4.40] J. F. W. Bishop, On the complete solution to problems of deformation of a plastic-rigid material, JMPS 2 (1953), 43-53.

[4.41] H. Bleich, Úber die Bemessung statisch unbestimmter Stahltragwerke unter Berücksichtigung der elastisch-plastischen Verhaltens des Baustoffes, BI 13 (1932), 19/20, 261.

[4.42] V. I. Blokh, Stress functions in the theory of elasticity (in Russian), PMM 14 (1950), 4, 415-422.

[4.43] -, Theory of elasticity, (in Russian), Izd. Kharkovsk. Univ., Kharkov 1964.

[4.44] V. V. Bokov, E. A. Sidorov, On the convergence of the method of variable parameters of elasticity (in Russian), UZGU 113 (1972), 143-147.

[4.45] A. Borkauskas, A. Cyras, On the duality in limit analysis and design of plates, BAP SST 16 (1968), 6, 241-248.

[4.46] A. E. Borkauskas, R. P. Karkauskas, Computational aspects of application of mathemat- 
ical programming methods to limit analysis of plates and shells (in Russian), IMS (1971), 2, 47-54.

[4.47] P. B. Bowden, A criterion for inhomogeneous plastic deformation, PhM 22 (1970), 177, $455-462$.

[4.48] L. L. Boyko, On the method of elastic solutions in the theory of plasticity (in Russian), $V M U$ MM (1970), 5, 108-116.

[4.49] -, On two methods of solution of an elastic-plastic problem (in Russian), VMU $M M$ (1971), 3, 102-109.

[4.50] E. F. Boyle, A. Jennings, Accelerating the convergence of elastic-plastic stress analysis, IJNME 7 (1973), 2, 232-235.

[4.51] P. W. Bridgman, The stress distribution at the neck of a tension specimen, Trans ASM 32 (1944), 553.

[4.52] G. L. Brovko, V. S. Lensky, On convergence of the method of homogeneous linear approximations in the theory of plasticity of non-homogeneous bodies (in Russian), PMM 36 (1972), 3, 519-527.

[4.53] E. P. Brumfitt, J. Schroeder, A variational principle in stress-rate dependent thermoplasticity, 3rd CANCAM (1971), 177-178.

[4.54] R. Brzeziński, J. A. König, Evaluation of shakedown deflections of framed structures by linear programming, SPAS (1972), 101-116.

[4.55] -, - , Deflections of elastic-plastic framed structures at shakedown, JSM 2 (1973), 211228.

[4.56] D. L. Bykov, V. A. Shatchnev, On a certain generalization of the method of elastic solutions (in Russian), PMM 33 (1969), 2, 290-298.

[4.57] D. L. Bykov, On certain methods of solution of plasticity problems (in Russian), UN 4 (1975), 119-139.

[4.58] C. R. Calladine, Limit analysis of curved tubes, JMES 16 (1974), 2, 85-87.

[4.59] M. Capurso, On the incremental solution of elasto-plastic continua in the range of large displacements, $\mathrm{Mec} 5$ (1970), 2, 98-106.

[4.60] M. Capurso, G. Maier, Incremental elastoplastic analysis and quadratic optimization, Mec 5 (1970), 2, 107-116.

[4.61] M. Capurso, Limit analysis of continuous media with piecewise linear yield condition, Mec 6 (1971), 1, 53-58.

[4.62] -, A displacement bounding principle in shakedown of structures subjected to cyclic loads, IJSS 10 (1974), 1, 77-92.

[4.63] G. Ceradini, C. Gavarini, Calcolo a rottura e programmazione lineare, GGC 103 (1965), $1-2,48-64 ; 106$ (1968), 2-3, 125-137; 106 (1968), 4, 163-180.

[4.64] G. Ceradini, A maximum principle for the analysis of elastic plastic systems, $\mathrm{Mec} \mathbf{1}$ (1966), $3-4,77-82$.

[4.65] —, Sull'adattamento dei corpi elasto-plastici soggetti ad azioni dinamiche, GGC 107 (1969), 4-5, 239-250.

[4.66] A. Charnes, H. Greenberg, Plastic collapse and linear programming, BAMS 57 (1951), 6.

[4.67] A. Charnes, C. E. Lemke, O. C. Zienkiewicz, Virtual work, linear programming and plastic limit analysis, Proc RS 251A (1959), 110-116.

[4.68] W. H. Chen, Necking of a bar, IJSS 7 (1971), 7, 685-717.

[4.69] G. P. Cherepanov, Elastic-plastic problems (in Russian), IZh MTT (1969), 2, 82-91.

[4.70] -, Mechanics of brittle fracture (in Russian), Nauka, Moskva 1974.

[4.71] L. F. Chernous'ko, N. V. Banichuk, Variational methods in mechanics and automatic control (in Russian), Nauka, Moskva 1973.

[4.72] V. I. Chernov, O. N. Ivanchenko, V. P. Bardzilovsky, On the method of successive approximations in elastic-plastic analysis (in Russian), PM 11 (1975), 3, 120-123.

[4.73] O. F. Chernyavsky, On the solution of problems of limit analysis and of shakedown by 
means of the Pontryagin maximum principle (in Russian), Izv AN SSSR MTT (1970), 4, 99-105.

[4.74] E. Chianese, Collasso incrementale localizzato per materiali non omogenei, RASFM 36 (1969/70), 145-166.

[4.75] A. N. Chizhevsky, On acceleration of convergence of the method of elastic solutions in physically nonlinear problems (in Russian), PP (1971), 188-193.

[4.76] J. Christoffersen, A note on necking of cylindrical bars, DCAMM 58 (1973), $10 \mathrm{pp}$.

[4.77] E. Chwalla, Die Stabilität zentrisch und exzentrisch gedrückter Stäbe aus Baustahl, SBAWW (1928).

[4.78] —, Theorie des aussermittig gedrückten Stabes aus Baustahl, Stb 7 (1934), 161.

[4.79] R. W. Clough, The finite element method in plane stress analysis, Proc ASCE JSD (1960), 345-378.

[4.80] M. G. Cockroft, A. Latham, A simple criterion of fracture for ductile metals, Rep NELG (1966).

[4.81] L. F. Coffin, Study of the effects of cyclic thermal stresses on a ductile metal, Trans ASME 76 (1954), 931-950.

[4.82] -, The stability of metals under cyclic plastic strain, Paper ASME A100 (1959), 12 pp.

[4.83] —, Failure of structural metals subjected to strain-cycling conditions, Trans ASME D81 (1959), 208.

[4.84] M. Z. Cohn, S. K. Ghosh, S. R. Parimi, Unified approach to theory of plastic structures, Proc ASCE JEMD 98 (1972), 5, 1133-1158.

[4.85] I. F. Collins, The upper bound theorem for rigid/plastic solids generalized to include Coulomb friction, JMPS 17 (1969), 5, 323-338.

[4.86] G. Colonnetti, Sul problema delle coazioni elastiche, RAL 27 (1918), Ser. 5a.

[4.87] —, De l'équilibre des systemes élastiques dans lesquels se produisent des déformations plastiques, JMPA Ser. IX, 17 (1938), 233-255.

[4.88] —, Elastic equilibrium in the presence of permanent set, $Q A M 7$ (1950), 353.

[4.89] —, L'équilibre des corps déformables, Dunod, Paris 1955.

[4.90] L. Comtet, Advanced combinatorics, D. Reidel, Dordrecht-Boston 1974.

[4.91] R. M. Cooper, G. A. Shifrin, An experiment on circular plates in the plastic range, 2nd US NCAM (1954), 527-534.

[4.92] L. Corradi, G. Maier, Inadaptation theorems in the dynamics of elastic-work-hardening structure, IA 43 (1973), 1, 44-57.

[4.93] -, -, Dynamic non-shakedown theorem for elastic perfectly-plastic continua, JMPS 22 (1974), 5, 401-413.

[4.94] L. Corradi, A. Zavelani, A linear programming approach to shakedown analysis of structures, CMAME 3 (1974), 1, 37-53.

[4.95] L. Corradi, G. Maier, Extremum theorems for large displacement analysis of discrete elastoplastic structures with piecewise linear yield surfaces, JOTA 15 (1975), 1, 51-67.

[4.96] G. R. Cowper, E. T. Onat, The initiation of necking and buckling in plane plastic flow, 4th US NCAM (1962), vol. 2, 1023-1029.

[4.97] H. Craemer, Fallacies and paradoxa in the plasticity theory, AT ASH 10 (1955), 1-2, 73-82.

[4.98] J. G. Crose, A. H. S. Ang, Nonlinear analysis method for circular plates, Proc ASCE JEMD 95 (1969), 4, 979-999.

[4.99] A. A. Cyras, Linear programming methods in the analysis of elastic-plastic systems (in Russian), Stroyizdat, Moskva 1969.

[4.100] —, Theory of optimization in the limit analysis of a deformable solid (in Russian), Mintis, Vilnius 1971.

[4.101] J. Datsko, C. T. Yang, Correlation of bendability of materials with their tensile properties, Trans ASME B82 (1960), 4; 309-313.

[4.102] J. Datsko, Material properties and manufacturing process, New York 1966. 
[4.103] N. N. Davidenkov, N. I. Spiridonova, Analysis of stresses in the neck of a tension specimen, $Z L 11$ (1945), 6, 583-593 (Russian original); Proc ASTM 46 (1946), 1147-1159 (English translation).

[4.104] N. N. Davidenkov, V. A. Likhatchev, Irreversible deformation of metals under cyclic thermal loadings (in Russian), Mashgiz, Moskva 1962.

[4.105] J. M. Davies, Variable repeated loading and the plastic design of structures, SEng 48 (1970), 5, 181-194.

[4.106] P. J. Davis, Interpolation and approximation, Blaisdell, New York-Toronto-London 1964.

[4.107] O. De Donato, Second shakedown theorem allowing for cycles of both loads and temperature, RIL ASL A104 (1960), 1, 265-277.

[4.108] —, Some extremum theorems for the elasto-plastic boundary value problem in the presence of large displacements, $\mathrm{Mec} 5$ (1970), 3, 210-218.

[4.109] —, On piecewise linear constitutive laws in plasticity, TR ISTC 662 (1974), 36 pp.

[4.110] G. Del Piero, On a mathematical theory of elastic-plastic materials, AMS 27 (1975), 2, 253-271.

[4.111] C. S. Desai, J. F. Abel, Introduction to the finite element method, Van Nostrand Reinhold, New York 1972.

[4.112] P. Dewhurst, I. F. Collins, A matrix technique for constructing slip-line field solutions to a class of plane strain plasticity problems, IJNME 7 (1973), 3, 357-378.

[4.113] L. Dietrich, J. Miastkowski, W. Szczepiński, Nośność graniczna elementów konstrukcji, PWN, Warszawa 1971.

[4.114] G. Dincă, Operatori monotoni in teoria plasticitătii, Acad. RSR, Bucuresti 1972.

[4.115] K. S. Dinno, S. S. Gill, A method for calculating the lower bound limit pressure for thick shells of revolution with specific reference to cylindrical vessels with torispherical ends, IJMS 16 (1974), 6, 415-427.

[4.116] —, - , A lower-bound limit pressure analysis for the oblique intersection of a flush cylindrical nozzle and the torus of a cylindrical vessel with a torispherical end, JSA 9 (1974), $4,247-262$.

[4.117] S. Dorosz, An upper bound to maximum residual deflections of elastic-plastic structures at shakedown, BAP SST 24 (1976), 3, 167-174.

[4.118] D. C. Drucker, H. J. Greenberg, W. Prager, The safety factor for an elastic-plastic body in plane strain, JAM 18 (1951), 371-378.

[4.119] D. C. Drucker, H. J. Greenberg, E. H. Lee, W. Prager, On plastic rigid solutions and limit design theorems for elastic plastic bodies, 1st US NCAM (1951), 533-538.

[4.120] D. C. Drucker, W. Prager, H. J. Greenberg, Extended limit design theorems for continuous media, QAM 9 (1952), 4, 381-389.

[4.121] D. C. Drucker, Limit analysis and design, $A M R 7$ (1954), 10, 421-423.

[4.122] -, On uniqueness in the theory of plasticity, QAM 14 (1956), 1, 35-42.

[4.123] -, Variational principles in the mathematical theory of plasticity, Proc SAM 8 (1958), 7-22.

[4.124] D. C. Drucker, J. R. Rice, Plastic deformation in brittle and ductile fracture, EFM 1 (1970), 4, 577-602.

[4.125] R. N. Dubey, S. T. Ariaratnam, Bifurcation in elastic-plastic solids in plane stress, $Q A M$ 27 (1969), 3, 381-390.

[4.126] - , -, Necking instabilities in elastic-plastic plates, IJES 10 (1972), 2, 145-154.

[4.127] A. Dudek, M. Życzkowski, Niektóre przypadki nośności granicznej rozciąganych prętów zwitych, RI 24 (1976), 1, 119-133.

[4.128] R. A. Dulnev, Thermal fatigue of metals (in Russian), Mashinostroyenye, Moskva 1971.

[4.129] M. Duszek, Effect of geometry changes on the carrying capacity of cylindrical shells, BAP SST 13 (1965), 4, 325-333. 
[4.130] M. Duszek, A. Sawczuk,'Load-deflection relations for rigid-plastic cylindrical shells beyond the incipient collapse, IJMS 12 (1970), 10, 839-848.

[4.131] M. K. Duszek, On minimum principles in finite plasticity, BAP SST 21 (1973). 2, 131-138.

[4.132] —, Geometrycznie nieliniowa teoria konstrukcji sztywno-plastycznych, IPPT PAN, Warszawa 1975.

[4.133] J. Dutheil, L'exploitation des phénomènes d'adaptation dans les ossatures en acier doux, AIT BTP (1948), 2.

[4.134] C. Dyrbye, P. Lange Hansen, Studies on the load carrying capacities of steel structures, Res. Lab. of Build. Techn., Techn. Univ. of Denmark, Bull. No. 3, 1954.

[4.135] P. I. Ermakov, A. S. Nesmeyanov, B. E. Dorofeyev, V. I. Morozov, Influence of various factors on the cumulation of strains in simple elements under variable temperature (in Russian), SNT CPI 151 (1974), 125-131.

[4.136] L. V. Ershov, D. D. Ivlev, On an approximate solution of axially-symmetric elastic-plastic problems by means of the small parameter method (in Russian), $V M U M M(1958), 2$.

[4.137] G. Espey, Instability of thin-walled tubes subjected to internal pressure, Trans ASME B68 (1946), 281.

[4.138] D. G. Eyre, T. V. Galambos, Deflection analysis for shakedown, Proc ASCE JSD 96 (1970), 7, 1359-1376.

[4.139] S. M. Feynberg, A principle of limit state (in Russian), PMM 12 (1948), 1, 63-68.

[4.140] M. M. Filonenko-Boroditch, Theory of elasticity (in Russian), 4th ed., Fizmatgiz, Moskva 1949.

[4.141] B. Finzi, Integrazione delle equazioni indefinite della meccanica dei sistemi continui, RAL 4 (1934), 19.

[4.142] J. Foulkes, Linear programming and structural design, 2nd SLP (1955), 1, 177-184.

[4.143] J. Foulkes, E. T. Onat, Tests of the behavior of circular plates under transverse load, BUTR OOR-3172/3 (1955).

[4.144] E. N. Fox, The existence of exact solutions in limit analysis for homogeneous isotropic plates of rigid perfectly-plastic material, EP (1968), 147-181.

[4.145] V. Franciosi, Il procedimento del "limit design" per carico non proporzionale, RS 5 (1954), 3, 23-28.

[4.146] A. M. Freudenthal, Inelastic behavior of engineering materials and structures, Wiley, New York 1950.

[4.147] J. Fritzsche, Die Tragfähigkeit von Balken aus Baustahl bei beliebig oft wiederholter Belastung, BI 12 (1931), 827.

[4.148] H. Gajewski, Ein Verfahren zur Konstruktion statisch zulässiger Spannungsfelder, ZAMM 47 (1967), 1, 19-30.

[4.149] —, Zur Lösung einer Klasse konvexer Minimumprobleme der Plastizitätstheorie, ZAMM 49 (1969), 1-2, 84-89.

[4.150] L. A. Galin, Plane elastic-plastic problem (in Russian), PMM 10 (1946), 3, 367-386.

[4.151] R. H. Gallagher, J. Padlog, P. P. Bijlaard, Stress analysis of heated complex shapes, JARS 32 (1962), 700-707.

[4.152] M. Galos, On the load-carrying capacity of multilayer pressure vessels, BAP SST 16 (1968), 11-12, 511-520 (English summary); ABM 15 (1968), 1, 113-129 (Polish full text).

[4.153] C. Gavarini, Plastic analysis of structures and duality in linear programming, $\mathrm{Mec} 1$ (1966), 3-4, 95-97.

[4.154] —, Sul rientro in fase elastica delle vibrazioni forzate elasto-plastiche, GGC 107 (1969) $4-5$. 
[4.155] C. Gavarini, G. Beolchini, Sull'adattamento delle strutture elasto-plastiche in presenza di effetti del $2^{\circ}$ ordine instabilizzanti, GGC 108 (1970), 3, 237-251.

[4.156] C. Gavarini, L'analyse limite des structures au moyen de la programmation mathématique, STA 47 (1973), 2, 439-442.

[4.157] S. V. Gavrilenkov, S. Kerimbayev, On the influence of the non-homogeneity of the stress field on the load carrying capacity (in Russian), PPro (1973), 9, 14-17.

[4.158] H. Geiringer, Beitrag zum vollständigen ebenen Plastizitätsproblem, 3rd ICAM, Stockholm (1930).

[4.159] H. Geiringer, W. Prager, Mechanik isotroper Körper im plastischen Zustand, EEN 13 (1933), 311.

[4.160] H. Geiringer, Some recent results in the theory of an ideal plastic body, AAM 3 (1953), 197-294.

[4.161] - On the characteristics of v. Mises equations of a perfectly plastic body, MSPSC (1956), $125-130$.

[4.162] V. O. Geogdzhayev, On the elastic-plastic equilibrium of a half-plane (in Russian), $P M 10$ (1974), 1, 127-130.

[4.163] J. Gerij, Thermo-élastoplasticité cyclique par la méthode des éléments finis, STA 47 (1973), 2, 311-329.

[4.164] S. S. Gill, A simple illustration of incremental collapse, BMEE 6 (1967), 1, 3-8.

[4.165] A. Gjelsvik, Extension of incremental collapse theorems, Proc ASCE JEMD 97 (1971), 5, 1603-1607.

[4.166] D. A. Gokhfeld, On shakedown problems in the conditions of repeated thermal loadings (in Russian), TNET 1 (1961), 138-149.

[4.167] - , On the possibility of cumulation of plastic strains as a result of cyclic thermal loadings (in Russian), $R P 7$ (1961), 64-75.

[4.168] - , On strength ealculations in the case of repeated thermal field and loading (in Russian), RP 11 (1965), 209-228.

[4.169] D. A. Gokhfeld, O. F. Chernyavsky, Application of the simplex method to shakedown problems (in Russian), TNEK 6 (1966), 99-108.

[4.170] D. A. Gokhfeld, On the application of Koiter's theorem to shakedown problems of nonuniformly heated elastic-plastic bodies (in Russian), PM 3 (1967), 8, 41-49.

[4.171] —, Theorems and methods of the shakedown theory of elastic-plastic bodies (in Russian), TNEK 7 (1967), 93-102.

[4.172] D. A. Gokhfeld, O. F. Chernyavsky, Application of linear programming methods to some two-dimensional problems of limit analysis and shakedown in the static approach (in Russian), TNEK 7 (1967), 103-107.

[4.173] D. A. Gokhfeld, Theory of shakedown under repeated loadings (in Russian), PPMCTN (1969), 109-117.

[4.174] -, Load carrying capacity of structures in the conditions of variable temperature (in Russian), Mashinostroyenye, Moskva 1970.

[4.175] D. A. Gokhfeld, O. F. Chernyavsky, Generalized variables in the problems of shakedown of plates and shells (in Russian), 7th VKTOP (1970), 194-200.

[4.176] - , - Application of linear programming methods to shakedown problems in kinematic approach (in Russian), TNEK 9 (1970), 273-283.

[4.177] - , - The shakedown theory, its present state, applicational value and development trends (in Russian), SNT CPI 151 (1974), 3-32.

[4.178] S. S. Golushkevitch, Statics of limit states of soils (in Russian), Gos. Izd. Tekhn. Lit., Moskva 1957.

[4.179] M. L. Gorb, A. A. Ostrovsky, N. I. Chernyak, On the problem of determination of the 
limit curve corresponding to the appearance of local deformations (yield layers) (in Russian), PPro (1971), 10, 27-31.

[4.180] A. P. Goryachev, E. I. Sankov, Numerical realization of the finite element method for plane physically non-linear problems (in Russian), UZGU 134 (1971), 20-28.

[4.181] G. Gaudel, M. Boivin, J. Bahuaud, Problèmes de fatigue et de rupture, $M M E$ (1973), 278, 35-39.

[4.182] J. Gouzou, Theoretical basis of forming limit diagram, MRBen 35 (1973), 41-50.

[4.183] H. J. Greenberg, Complementary minimum principles for an elastic-plastic material, QAM 7 (1949), 1, 85-95.|

[4.184] - , On the variational principles of plasticity, BUTR A11-54 (1949), March.

[4.185] H. J. Greenberg, W. Prager, On limit design of beams and frames, BUTR A18-1 (1949), and Trans ASCE 117 (1952), 447-484.

[4.186] A. S. Grigorev, The stress state in momentless cylindrical shells at large strains (in Russian). PMM 21 (1957), 6, 827-832.

[4.187] -, Equilibrium of a momentless shell of revolution at large strains (in Russian), PMM 25 (1961), 6, 1083-1090.

[4.188] —, The stress state and the carrying capacity of flexible plates and shells at large deformations, IASS NSP (1964).

[4.189] M. Grüning, Die Tragfähigkeit statisch unbestimmten Tragwerke aus Stahl bei beliebig häufig wiederholter Belastung, Springer, Berlin 1926.

[4.190] S. I. Gubkin, S. I. Dobrovolsky, B. B. Boyko, Photoplasticity (in Russian), Akad. Nauk Beloruss. SSR, Minsk 1957.

[4.191] J. S. Gunasekera, J. M. Alexander, Matrix analysis of the large deformation of an elasticplastic axially symmetric continuum, SFP 1972 (1973), 125-146.

[4.192] M. E. Gurtin, A generalization of the Beltrami stress functions in continuum mechanics, ARMA 13 (1963), 321-329.

[4.193] A. A. Gvozdev, Determination of the failure load for statically indeterminate systems subject to plastic deformations (in Russian), PSt 8 (1934).

[4.194] —, Determination of the value of failure load for statically indeterminate systems subject to plastic deformations, KPD 1936 (1938) (Russian original); IJMS 1 (1960), 4, 322-335 (English translation).

[4.195] -, On limit analysis (in Russian), IS 5/1 (1948), 32-57.

[4.196] -, Calculation of the load carrying capacity of structures by means of limit analysis (in Russian), Stroyizdat, Moskva 1949.

[4.197] A. Haar, Th. Kármán, Zur Theorie der Spannungszustände in plastischen und sandartigen Medien, GNMPK (1909), 2, 204-218.

[4.198] H. Haase, Bruchlinientheorie von Platten, Werner-Verlag, Düsseldorf 1962.

[4.199] G. H. Handelman, A variational principle for a state of combined plastic stress, QAM 1 (1944), 351-353.

[4.200] E. W. Hart, Theory of the tensile test, $A M 15$ (1967), 351-355.

[4.201] R. M. Haythornthwaite, Beams with full end fixity, Eng 183 (1957), 110-112.

[4.202] S. S. Hecker, C. H. Hamilton, L. J. Ebert, Elastic-plastic analysis: a simplified approach, SerM 3 (1969), 11, 793-798.

[4.203] H. Hencky, Ưber einige statisch bestimmte Fälle 'des Gleichgewichtes in plastischen Körpern, ZAMM 3 (1923), 4, 241-251.

[4.204] -, Die Bewegungsgleichungen beim nichtstationären Fliessen plastischer Massen, ZAMM 5 (1925), 144.

[4.205] J. Heyman, Plastic design of portal frames, Cambridge Univ. Press 1957.

[4.206] —, The significance of shake down loading, 9th C AIPC (1972), 3-10.

[4.207] —, Plastic design and limit state design, SE 51 (1973), 4, 127-131. 
[4.208] H. D. Hibbitt, P. V. Marcal, J. R. Rice, A finite element formulation for problems of large strain and large displacement, IJSS 6 (1970), 8, 1069-1086.

[4.209] R. Hill, A variational principle of maximum plastic work in classical plasticity, QJMAM 1 (1948), 18-28.

[4.210] —, A comparative study of some variational principles in the theory of plasticity, JAM 17 (1950), 1, 64-66.

[4.211] —, On the state of stress in a plastic-rigid body at the yield point, PhM 42 (1951), 868-875.

[4.212] - A note on estimating yield point loads in a plastic-rigid body, $P h M 43$ (1952), 353-355.

[4.213] —, Comments on Dr Lee's paper, PhM 43 (1952), Ser. 7, 560.

[4.214] —, Stability of rigid-plastic solids, JMPS 6 (1957), 1, 1-8.

[4.215] M. J. Hillier, Tensile plastic stability under complex stress, IJMS 5 (1963), 1, 57-67.

[4.216] —, Tensile plastic instability of thin tubes, IJMS 7 (1965), 8, 531-538.

[4.217] H. S. Ho, Shakedown in elastic-plastic systems under dynamic loadings, Pap ASME APMW-27 (1971), $6 \mathrm{pp}$.

[4.218] - , On the general method of construction of yield surfaces, 4th CANCAM (1973), 187188.

[4.219] P. G. Hodge, Jr., Shake-down of elastic-plastic structures, Residual Stresses, W. R. Osgood, ed., Reinhold, New York 1954.

[4.220] -, Minimum principles of piecewise linear isotropic plasticity, JRMA 5 (1956), 917938.

[4.221] - , The mathematical theory of plasticity, in Elasticity and Plasticity, Wiley, New York 1958.

[4.222] —, The practical significance of limit analysis, JAS 25 (1958), 11, 724-726.

[4.223] —, Plastic analysis of rotationally symmetric plates and shells, Prentice-Hall, Englewood Cliffs 1963.

[4.224] —, Yield-point load determination by non-linear programming, 11th ICAM 1964 (1966), 554-561.

[4.225] P. G. Hodge, Jr., C. K. Sun, General properties of yield-point surfaces, Trans ASME E35 (1968), 1, 107-110.

[4.226] P. G. Hodge, Jr., Numerical applications of minimum principles in plasticity, EP (1968), 237-256.

[4.227] —, Limit analysis with multiple load parameters, IJSS 6 (1970), 661-675.

[4.228] - , A consistent finite element model for the two-dimensional continuum, IA 39 (1970), 6, 375-382.

[4.229] - , A consistent finite-difference model for the two-dimensional continuum, MSS (1972), 605-614.

[4.230] - , Computer solutions of plasticity problems, PPlast 1972 (1974), 261-282.

[4.231] A. Hoffmann, M. Livolant, R. Roche, Plastic analysis of shells by finite element method, 2nd SMiRT (1973), paper 16/2.

[4.232] L. D. Hofmeister, G. A. Greenbaum, D. A. Evensen, Large strain, elasto-plastic finite element analysis, AIAA 9 (1971), 7, 1248-1254.

[4.233] R. Hooke, An experimental evaluation of the rigid-plastic theory of bending of plates, CET IEA 11 (1969), 2, 141-149.

[4.234] H. G. Hopkins, The method of characteristics and its application to the theory of stress waves in solids, EP (1968), 277-315.

[4.235] -, Mathematical methods in plasticity theory, PPlast 1972 (1974), 235-259.

[4.236] M. R. Horne, Effect of variable repeated loads in the plastic theory of structures, ES (1949), 141-151.

[4.237] —, The stability of elastic-plastic structures, PSM 2 (1961), 277-322. 
[4.238] T. R. Hsu, A. W. M. Bertels, Improved approximation of constitutive elasto-plastic stressstrain relationship for finite element analysis, AIAA 12 (1974), 10, 1450-1452.

[4.239] J. W. Hutchinson, J. P. Miles, Bifurcation analysis of the onset of necking in an elastic/plastic cylinder under uniaxial tension, JMPS 22 (1974), 1, 61-71.

[4.240] V. A. Ikrin, V. V. Filippov, Bounds on deformations cumulated at shakedown of plane frames (in Russian), SMRS (1974), 3, 22-25.

[4.241] A. A. Ilyushin, The method SN-EVM in the theory of plasticity (in Russian), PPMM (1971), 166-178.

[4.242] - On a certain model explaining the approximate method SN-EVM in the theory of plasticity (in Russian), UN 1 (1971), 52-58.

[4.243] —, On the relations and methods of the modern theory of plasticity (in Russian), UMDS (1975), 240-255.

[4.244] A. Ingerslev, The strength of rectangular slabs, JISE 1 (1923), 1.

[4.245] T. Inoue, K. Tanaka, M. Aoki, A theoretical investigation on the thermal ratcheting, JSMSJ 22 (1973), 224-228.

[4.246] T. Inoue, K. Tanaka, Temperature dependent analysis of elastoplastic thermal stresses by finite element method, MFEKU 35 (1973), 1, 1-14.

[4.247] B. M. Irons, R. C. Tuck, A version of the Aitken accelerator for computer iteration, IJNME 1 (1969), 275-278.

[4.248] G. R. Irwin, Fracture, HP VI (1958), 551-590.

[4.249] A. Yu. Ishlinsky, On the stability of viscoplastic flow of flat and circular bars (in Russian), PMM 7 (1943), 2, 109-130.

[4.250] D. D. Ivlev, Approximate solution of elastic-plastic problems of the theory of perfect plasticity by means of the small parameter method (in Russian), DAN SSSR 113 (1957), 2.

[4.251] - Approximate solution of plane elastic-plastic problems of the theory of perfect plasticity by means of the small parameter method (in Russian), VMU MM (1957), 5.

[4.252] D. D. Ivlev, A. D. Chernyshov, On the equations of perfect plasticity expressed in terms of velocity components (in Russian), PMM 35 (1971), 1, 183-185.

[4.253] D. D. Ivlev, On evaluation of diplacements in elastic-plastic problems of the theory of perfect plasticity (in Russian), UMDS (1975), 236-240.

[4.254] R. J. Izbicki, Application of a two-parameter pertubation method to problems of loadcarrying capacity, AMS 22 (1970), 4, 421-438.

[4.255] I. N. Izotov, A. G. Mityukov, Experimental investigations of the conditions of localized plastic deformations under plane and spatial stress states (in Russian), PPro (1973), 10, $37-42$.

[4.256] J A. Jacobs, Relaxation methods applied to problems of plastic flow, PhM 41 (1950), 349-361, 458-467.

[4.257] M. Janas, Nośność graniczna luków i sklepień, Arkady, Warszawa, 1967.

[4.258] -, Kinematical compatibility problems in yield-line theory, MCR 19 (1967), 58, 33-44.

[4.259] J. Javornicky, Photoplasticity, Elsevier, Amsterdam 1974.

[4.260] P. S. Jensen, Finite difference technique for variable grids, CS 1 (1971), 17-27.

[4.261] K. Ježek, Die Tragfähigkeit des exzentrisch beanspruchten und des querbelasteten Druckstabes aus einem ideal plastischen Material, SBAWW IIa, 143 (1934).

[4.262] -, Die Festigkeit von Druckstäben aus Stahl, Springer, Wien 1937.

[4.263] K. W. Johansen, Brudlinienteorier, Gjellerup, Copenhagen 1943; English ed.: Yield-line theory, Cement and Concr. Ass., London 1962.

[4.264] L. M. Kachanov, Variational pronciples for elastic-plastic media (in Russian), PMM 6 (1942), 2-3, 187-196.

[4.265] -, Mechanics of plastic media (in Russian), Gostekhizdat, Moskva-Leningrad 1948.

[4.266] -, Fundamentals of fracture mechanics (in Russian), Nauka, Moskva 1974. 
[4.267] S. A. Kalanta, Yu. A. Nagyavitchius, A. A. Cyras, Application of nonlinear programming to the solution of problems of shallow cylindrical shells (in Russian), LMS (1972), 1, 35-47.

[4.268] S. Kaliski, Pewne problemy brzegowe dynamicznej teorii sprężystości i cial niesprężystych, wyd. WAT, Warszawa 1957.

[4.269] R. Kao, A comparison of Newton-Raphson methods and incremental procedures for geometrically nonlinear analysis, CS 4 (1974), 1091-1097.

[4.270] M. A. Kaplan, The stress and deformation in mild steel during axisymmetric necking, Trans ASME E40 (1973), 1, 271-276.

[4.271] S. A. Kapustin, L. K. Kiselev, V. A. Trúbitsyn, Basic relations of a mixed finite element method for the analysis of elastic-plastic plates (in Russian), MRZUP 8 (1974), 117-126.

[4.272] T. Kármán, Untersuchungen über Knickfestigkeit, MFIW 81 (1910).

[4.273] T. Kato, Perturbation theory for linear operators, Springer, Berlin-Heidelberg-New York 1966.

[4.274] M. Kawahara, K. Horii, Large strain, elastic-plastic numerical analysis by means of finite element method, Trans JSCE 3 (1972), 2, 154-155.

[4.275] G. Kazinczy, Kisérletek befalozott tartókkal, Betonszemle 2 (1914), 4, 68; 2 (1914), 5, 83; 2 (1914), 6, 101.

[4.276] —, Die Weiterentwicklung der Elastizitätstheorie, Technika, Budapest 1931.

[4.277] Z. Kączkowski, R. Tribiłło, Pewne uogólnienia metody różnic skończonych, AIL 21 (1975), 2, 287-293.

[4.278] R. M. Kirakosyan, A theorem on shakedown under variable external loadings in the case of arbitrary plastic hardening (in Russian), DAN ArmSSR 52 (1971), 4, 209-215.

[4.279] - Minimal principles and certain theorems on elastic-plastic equilibrium of bodies under non-stationary forces and thermal loadings (in Russian), Izv AN ArmSSR M 26 (1973), 2, 51-61.

[4.280] V.A. Kiselev, Limit loadings in bending of elastic-plastic beams (in Russian), SMRS (1973), 5, 30-34.

[4.281] N. C. Kist, Leidt een sterkteberekening..., Inaugural lecture, Delft Technical University, Oct. 2, 1917.

[4.282] -, Die Zaehigkeit des Materials als Grundlage für die Berechnung von Bruecken, Hochbauten und aehnlichen Konstruktionen aus Flusseisen, EB 11 (1920), 425.

[4.283] H. Kitagawa, Y. Tomita, An incremental finite element anylysis of two-dimensional large strain and large displacement problems for elasto-plastic material, 21st JCAM (1973), 243-255.

[4.284] H. J. Kleemola, M. A. Nieminen, On the strain-hardening parameters of metals, $M T 5$ (1974), 8, 1863-1866.

[4.285] M. Kleiber, Yield condition for the constrained bodies, BAP SST 21 (1973), 5, 341-346.

[4.286] —, Plasticity of discretized bodies, BAP SST 21 (1973), 7-8, 369-378.

[4.287] -, Podstawy teorii plastycznego płynięcia ciał dyskretyzowanych, RI 21 (1973), 2, 279-292.

[4.288] - , Lagrangian and Eulerian finite element formulation for large strain elasto-plasticity, BAP SST 23 (1975), 3, 117-126.

[4.289] J. Klepaczko, Generalized conditions for stability in tension tests, IJMS 10 (1968), 4, 297-313.

[4.290] -, Uogólnione warunki stateczności w próbie rozciągania, MTS 8 (1970), 1, 75-91.

[4.291] V. D. Klyushnikov, The method of elastic solutions in the theory of plastic flow, ZhPMTF (1965), 1, 133-135.

[4.292] A. S. Kobayashi, P. R. Trumpler, Elastic stresses in a rotating disk of general profile, IJMS 2 (1960), 1-2, 13-29.

[4.293] W. T. Koiter, Some remarks on plastic shakedown theorems, 8th ICAM 1952, 1 (1955), 220. 
[4.294] W. T. Koiter, A new general theorem on shake-down of elastic-plastic structures, Proc KNAW B59 (1956), 24-34.

[4.295] L. Konieczny, Shake-down theorems for beam structures, BAP SST 22 (1974), 5, 353-362 (English summary); MTS 8 (1970), 3, 257-276 (Polish full text).

[4.296] D. C. A. Koopman, R. H. Lance, On linear programming and plastic limit analysis, JMPS 13 (1965), 2, 77-87.

[4.297] Z. Kordas, M. Życzkowski, |Investigations of the shape of thick-walled non-circular cylinders showing full plasticization at the collapse, BAP SST 18 (1970), 10, 839-847 (English summary); RI 18 (1970), 3, 371-390 (Polish full text).

[4.298] Z. Kordas, Kształtowanie rurociągu o zmiennej średnicy, wykazującego pełne uplastycznienie w stadium zniszczenia, MTS 11 (1973), 4, 455-465.

[4.299] Z. Kordas, W. Skraba, Kształtowanie cylindrów grubościennych pod działaniem ciśnienia wewnętrznego i zginania $\mathrm{z}$ warunku całkowitego uplastycznienia $\mathrm{w}$ stadium zniszczenia, RI 25 (1977), 1, 37-52.

[4.300] Yu. G. Korotkikh, A. K. Lyubimov, On a certain method of solution of elastic-plastic problems of cyclic loadings (in Russian), UZGU 89 (1969), 96-103.

[4.301] Yu. G. Korotkikh, A. A. Kravtchenko, Solution of contact problems of thermoplasticity in the case of combined loadings in curvilinear coordinates (in Russian), UZGU 89 (1969), 142-149.

[4.302] Yu. G. Korotkikh, S. M. Belevitch, On the solution of plane problems of combined loadings in thermoplasticity (in Russian), TNEK 9 (1970), 231-240.

[4.303] J. A. König, Theory of shake-down of elastic-plastic structures, AMS 18 (1966), 2, 227-238.

[4.304] -, A shakedown theorem for temperature dependent elastic moduli, BAP SST 17 (1969), 3, 247-255.

[4.305] -, Podstawowe twierdzenia z zakresu teorii dostosowywania się konstrukcji sprężystoplastycznych do obciążeń zmiennych w czasie, MTS 8 (1970), 2, 149-158.

[4.306] —, On shakedown criteria, PPlast 1972 (1974), 408-411.

[4.307] J. Kravtchenko, J. Lanier, Application de la méthode de Riemann à la solution de certains problèmes de la plasticité plane pour materiaux à angle de frottement interne nul, CRAS 265 (1967), 25, A866-A868.

[4.308] A. S. Kravtchuk, On selecting a basis for realization of the method of ?successive solutions in combined loadings processes (in Russian), UN 2 (1971), 100-106.

[4.309] R. Krohn, Knickfestigkeit, Berlin 1923.

[4.310] Yu. A. Krutkov, Tensor of the stress functions and general solutions in static elasticity (in Russian), Izd. AN SSSR, Moskva-Leningrad 1949.

[4.311] V. I. Krylov, V. V. Bobkov, P. I. Monastyrnyi, Numerical methods of higher mathematics (in Russian), Vol. 2, Vysheyshaya Shkola, Minsk 1975.

[4.312] V. P. Kryzhanovsky, L. G. Lantukh, A. V. Perelmuter, On shakedown analysis of elasticplastic structures (in Russian), SMTS 15 (1971), 137-144.

[4.313] B. N. Kuznetsov, On the advisability of shakedown analysis of bar structures (in Russian), $\operatorname{Tr}$ CNIISK 36 (1974), 60-66.

[4.314] A. A. Labutin, On the methods of construction of the diagrams of limit plasticity (in Russian), ST LMI 54 (1966), 148-155.

[4.315] —, Diagrams of limit plasticity and their practical application (in Russian), $\operatorname{Tr} K P I 1$ (1971), 2, 192-205.

[4.316] W. T. Lankford, E. Saibel, Some problems in unstable plastic flow under biaxial tension, MTTP 2238 (1947).

[4.317] W. Lansing, W. R. Jensen, W. Falby, Matrix analysis methods for inelastic structures, Ist CMMSM (1966).

[4.318] P. K. Larsen, E. P. Popov, A note on incremental equilibrium equations and approximate constitutive relations in large inelastic deformations, AMech 19 (1974), 1-2, 1-14. 
[4.319] J. Ledziński, Z. Waszczyszyn, Analiza zjawiska ,przeskoku” w zakresie sprężysto-plastycznym na modelu układu kratowego Misesa, MTS 4 (1966), 2, 71-82.

[4.320] C. H. Lee, S. Kobayashi, New solutions to rigid-plastic deformation problems using a matrix method, Trans ASME B95 (1973), 3, 865-873.

[4.321] E. H. Lee, On the significance of the limit theorems for a plastic-rigid body, PhM 43 (1952), Ser. 7, 549-560.

[4.322] -, Plastic flow of a $V$-notched bar pulled in tension, JAM 19 (1952), 331-336.

[4.323] J. G. Lenard, Plastic flow and fracture of a rotating cylinder, SIAM JAMath 21 (1971), $4,565-572$.

[4.324] J. Lenard, J. B. Haddow, Plastic collapse speeds for rotating cylinders, IJMS 14 (1972), 5, 285-292.

[4.325] V. S. Lensky, G. L. Brovko, The method of homogeneous linear approximations in uncoupled problems of thermo-radiational elasticity and plasticity (in Russian), TNEK 11 (1971), 100-113.

[4.326] Yu. R. Lepik, Plastic flow of flexible circular plates of a rigid-plastic material (in Russian), Izv AN SSSR MM (1960), 2, 78-87.

[4.327] -, On axisymmetric bending of flexible circular rigid-plastic plates (in Russian), IZh MTT (1966), 4, 104-110.

[4.328] M. Levy, CRAS 73 (1871), 16, 1098-1103.

[4.329] S. D. Leytes, On the problem of mechanism of the loss of stability (in Russian), IS 7 (1950), 123-130.

[4.330] -, Stability of compressed steel bars (in Russian), Gos. Izdat. Lit. po Stroit. i Arkh., Moskva 1954.

[4.331] M. J. Lighthill, A technique for rendering approximate solutions to physical problems uniformly valid, PhM 40 (1949), 1179-1201.

[4.332] - A technique for rendering approximate solutions to physical problems uniformly valid, $Z F l 9$ (1961), 267-275.

[4.333] C. C. Lin, On a perturbation theory based on the method of characteristics, JMP 33 (1954), $117-134$.

[4.334] T. H. Lin, Analogy between the inelastic strain gradient and body forces in cubic crystals and isotropic media, Proc NAS, March 1966.

[4.335] H. Lippmann, On the incremental extremum theorems for elastic-plastic media, MRC 1 (1974), 1, 33-36.

[4.336] - Extremum and variational principles in plasticity, RI 23 (1975), 3, 393-422.

[4.337] T. Liszka, M. Życzkowski, Optymalne kształtowanie nierównomiernie nagrzanych tarcz wirujących z uwagi na nośność sprężystą i graniczną, MTS 14 (1976), 2, 283-302.

[4.338] T. Liszka, J. Orkisz, Zmodyfikowana metoda różnic skończonych przy nieregularnej siatce węzłów w problemach mechaniki (in print).

[4.339] B. I. Lyubarov, On the analysis of elastic-plastic systems under variable repeated loading (in Russian), SMRS (1974), 2, 28-32.

[4.340] G. Maier, Extremum theorems for the analysis of elastic-plastic structures containing unstable elements, Mec 2 (1967), 4, 235-242.

[4.341] - A quadratic programming approach for certain classes of nonlinear structural problems, Mec 3 (1968), 2, 121-130.

[4.342] -, Quadratic programming and theory of \{elastic-plastic structures, $\operatorname{Mec} 3$ (1968), 4, । 265-273.

[4.343] -, Some theorems for plastic strain rates and plastic strains, $J M 8$ (1968), 1, 5-19.

[4.344] —, Sul comportamento flessionale instabile nelle travi inflesse elastoplastiche, RIL ASL A102 (1968), 4, 648-677.

[4.345] - Complementary plastic work theorems in piecewise-linear elastoplasticity, IJSS 5 (1969), 3, 261-270. 
[4.346] - , Teoremi di minimo in termini finiti per continui elastoplastici con leggi constitutive linearizzate a tratti, RIL ASL A103 (1969), 5, 1066-1080.

[4.347] —, Shakedown theory in perfect elastoplasticity with associated and non-associated flowlaws: a finite element linear programming approach, Mec 4 (1969), 3, 250-260.

[4.348] -, A matrix structural theory of piecewise linear elastoplasticity with interacting yield planes, Mec 5 (1970), 1, 54-66.

[4.349] - A minimum principle for incremental elastoplasticity with non-associated flow laws, JMPS 18 (1970), 5, 319-330.

[4.350] - Incremental plastic analysis in the presence of large displacements and physical instabilizing effects, IJSS 7 (1971), 4, 345-372.

[4.351] —, Shakedown of plastic structures with unstable parts, Proc ASCE JEMD 98 (1972), $5,1322-1327$.

[4.352] —, Mathematical programming methods in structural analysis, SVME 1972 (1973), Vol. 2, session $8,32 \mathrm{pp}$.

[4.353] G. Maier, A. Zavelani, J. C. Dotreppe, Equilibrium branching due to flexural softening, Proc ASCE JEMD 99 (1973), 4, 897-901.

[4.354] G. Maier, D. C. Drucker, Effects of geometry change on essential features of inelastic behavior, Proc ASCE JEMD 99 (1973), 4, 819-834.

[4.355] G. Maier, A shakedown matrix theory allowing for work-hardening and second-order geometric effects, SFP 1972 (1973), 417-433.

[4.356] G. Maier, E. Vitiello, Bounds on plastic strains and displacements in dynamic shakedown of work-hardening structures, Trans ASME E41 (1974), 2, 434-440.

[4.357] H. Maier-Leibnitz, Versuche zur weiteren Klärung der Frage der tatsächlichen Tragfähigkeit durchlaufender Träger aus Baustahl, Stb 9 (1936), 153.

[4.358] V. I. Maksak, V. I. Chernomortchenko, Stability in tension and experimental investigations of strength of thin-walled tubes (in Russian), PPro (1970), 5, 26-27.

[4.359] N. N. Malinin, Zh. L. Petrosyan, Stresses in the smallest section of a neck in a circular tension specimen (in Russian), Izv VUZ M (1967), 6, 34-39.

[4.360] N. N. Malinin, Stability of biaxial plastic tension of anisotropic sheets and cylindrical shells (in Russian), Izv AN SSSR MTT (1971), 2, 115-118.

[4.361] S. S. Manson, Fatigue: a complex subject-some simple approximations, ExpM 5 (1965), 7, 193-226.

[4.362] —, Thermal stress and low cycle fatigue, McGraw-Hill, New York 1966.

[4.363] P. V. Marcal, I. P. King, Elastic-plastic analysis of two-dimensional stress systems by the finite element method, IJMS 9 (1967), 3, 143-155.

[4.364] P. V. Marcal, A comparative study of numerical methods of elastic-plastic analysis, AIAA 6 (1968), 1, 157-158.

[4.365] - Finite-element analysis of combined problems of non-linear material and geometric behavior, CAAM (1969), 133-149.

[4.366] Z. Marciniak, Analiza stateczności cienkościennej powłoki walcowej poddanej rozciąganiu w stanie plastycznym, $R I 6$ (1958), 4, 527-535.

[4.367] Z. Marciniak, K. Kuczyński, Limit strains in the processes of stretchforming sheet metal, IJMS 9 (1967), 9, 609-620.

[4.368] Z. Marciniak, Analysis of necking preceding fracture of sheet metal under tension, MI 60 (1968), 8, 701-709.

[4.369] —, Odksztalcenia graniczne przy tloczeniu blach, WNT, Warszawa 1971.

[4.370] Z. Marciniak, K. Kuczyński, T. Pokora, Influence of the plastic properties of a material on the forming limit diagram for sheet metal in tension, IJMS 15 (1973), 10, 789-806.

[4.371] A. A. Markov, Variational principles in the theory of plasticity (in Russian), PMM 9 (1947), 339-350. 
[4.372] J. B. Martin, A. R. S. Ponter, On dual energy theorems for a class of elastic-plastic problems due to G. Maier, JMPS 20 (1972), 5, 301-306.

[4.373] J. B. Martin, On the kinematic minimum principle for the rate problem in classical plasticity, JMPS 23 (1975), 2, 123-128.

[4.374] J. Massau, Mémoire sur l'intégration des équations aux dérivées partielles, Ghent 1899; reprinted Edition du Centenaire, Bruxelles-Mons 1952.

[4.375] F. A. McClintock, A. S. Argon, Mechanical behavior of materials, Addison-Wesley, Reading 1966.

[4.376] P. V. McLaughlin, S. C. Batterman, On extending the range of applicability of the limit theorems, Trans ASME E37 (1970), 2, 518-521.

[4.377] P. V. McLaughlin, Properties of work-hardening materials with a limit surface, Trans ASME E40 (1973), 3, 803-807.

[4.378] R. M. McMeeking, J. R. Rice, Finite-element formulations for problems of large elasticplastic deformation, IJSS 11 (1975), 5, 601-616.

[4.379] E. Melan, Theorie statisch unbestimmter Systeme aus idealplastischem Baustoff, SBAWW IIa 145 (1936), 195-218.

[4.380] - , Der Spannungszustand eines Mises-Henckyschen Kontinuums bei veränderlicher Belastung, SBAWW IIa 147 (1938), 73-87.

[4.381] R. M. Mello, D. S. Griffin, Plastic collapse loads for piping elbows using inelastic analysis, Trans ASME J96 (1974), 3, 177-183.

[4.382] P. B. Mellor, The ultimate strength of thin-walled shells and circular diaphragms subjected to hydrostatic pressure, IJMS 1 (1960), 216.

[4.383] —, Tensile instability in thin-walled tubes, JMES 4 (1962), 3, 251-256.

[4.384] R. J. Melosh, R. Levy, Nonproportional loading limit for structures, CS 4 (1974), 2, 453-465.

[4.385] A. Mendelson, S. S. Manson, Practical solution of plastic deformation problems in the elastic-plastic range, NASA TR R-28 (1959).

[4.386] H. Mierzejewski, Podstawy mechaniki cial plastycznych, Akademia Nauk Technicznych, Warszawa 1927.

[4.387] J. P. Miles, Bifurcation in plastic flow under uniaxial tension, JMPS 19 (1971), 2, 89-102.

[4.388] -, Fluid-pressure eigenstates and bifurcation in tension specimens under lateral pressure, JMPS 21 (1973), 3, 145-162.

[4.389] R. Miner, Cumulative damage in fatigue, JAM 12 (1945), 159-164.

[4.390] H. van Minh, R. Sowerby, J. L. Duncan, Variability of forming limit curves, IJMS 16 (1974), 1, 31-44.

[4.391] - , - , - Probabilistic model of limit strains in sheet metal, IJMS 17 (1975), 5, 339-350.

[4.392] B. Yu. Mirzabekyan, M. I. Reytman, Evaluation of the load-carrying capacity of shells by means of linear programming (in Russian), IZh MTT (1968), 1, 122-124.

[4.393] R. Mises, Ubber die Stabilitätsprobleme der Elastizitätstheorie, ZAMM 3 (1923), 406-422.

[4.394] J. J. Moreau, On unilateral constraints, friction and plasticity, New Variational Techniques in Mathematical Physics, CIME (1973), Edizioni Cremonese.

[4.395] N. S. Mozharovsky, E. A. Antipov, G. I. Gontcharov, On the cumulation of plastic strains in a metal under cyclic thermoplasticity and their influence on fracture criteria (in Russian), VKPI $M$ (1969), 6, 24-28.

[4.396] E. Mönch, Der heutige Stand der Photoplastizität, SAAWT 25 (1959), 5, 174-180.

[4.397] M. Mrowiec, M. Życzkowski, Limit interaction curves for thin-walled pipe-line under internal pressure and bending, BAP SST 16 (1968), 10, 451-460 (English summary); RI 15 (1967), 4, 617-627 (Polish full text).

[4.398] Z. Mróz, B. Raniecki, Variational principles in uncoupled thermoplasticity, IJES 11 (1973), 11, 1133-1141. 
[4.399] T. M. Mulcahy, A plasticity model for cyclic strain accumulation, Trans ASME E38 (1971), 4, 869-874.

[4.400] T. Mura, S. Lee, Application of variational principles to limit analysis, QAM 21 (1963), 3, 243-248.

[4.401] T. Mura, W. H. Rimawi, S. L. Lee, Extended theorems of limit analysis, QAM 23 (1965), 2.

[4.402] T. Mura, S. L. Lee, R. H. Bryant, W. H. Rimawi, Limit analysis by direct method of variation, Proc ASCE JEMD 93 (1967), 67-78.

[4.403] A. Nádai, Plasticity, McGraw-Hill, New York 1931.

[4.404] —, The principle of minimum work applied to states of finite, homogeneous, plane plastic strain, 1st USNCAM 1951 (1952), 479-485.

[4.405] J. C. Nagtegaal, D. M. Parks, J. R. Rice, On numerically accurate finite element solutions in the fully plastic range, CMAME 4 (1974), 2, 153-177.

[4.406] Yu. A. Nagyavitchius, A. A. Cyras, Evaluation of the limit load for shallow cylindrical shells by means of linear programming, (in Russian) LMS (1968), 2, 68-78.

[4.407] L. P. Natanson, A constructive theory of functions (in Russian), Gostekhizdat, MoskvaLeningrad 1949.

[4.408] G. C. Nayak, O. C. Zienkiewicz, Elasto-plastic stress analysis, a generalization for various constitutive relations including strain softening, IJNME 5 (1972), 1, 113-135.

[4.409] A. Nayfeh, Perturbation methods, Wiley, New York-London 1973.

[4.410] B. Nayroles, Possibilités théoriques de simulation analogique discrête du mouvement des structures élastoplastiques, CGFR 1 (1965), 1, 21-24.

[4.411] —, Essai de théorie fonctionnelle des structures rigides plastiques parfaites, $J M 9$ (1970), 3, 491-506.

[4.412] B. G. Neal, Plastic collapse and shake-down theorems for structures of strain-hardening material, JAS 17 (1950), 297-306.

[4.413] -, The plastic methods of structural analysis, Wiley, New York 1956.

[4.414] K. W. Neale, A general variational theorem for the rate problem in elasto-plasticity, IJSS 8 (1972), 7, 865-876.

[4.415] A. Needleman, A numerical study of necking in circular cylindrical bars, JMPS 20 (1972), $2,111-127$.

[4.416] S. Nemat-Nasser, On elastic, work-hardening solids, IJSS 4 (1968), 8, 787-797.

[4.417] H. Neuber, Kerbspannungslehre, Springer, Berlin-Göttingen-Heidelberg 1958.

[4.418] Q. S. Nguyen, J. Zarka, Quelques méthodes de resolution numerique en plasticité classique et en viscoplasticité, STA 47 (1973), 2, 407-437.

[4.419] D. H. Nguyen, Analyse limite rigide plastique par une méthode quasi-directe, STA 47 (1973), 2, 453-458.

[4.420] D. Niepostyn, Nośność graniczna plyt prostokqtnych, NOT-Arkady, Warszawa 1962.

[4.421] -, Nośność graniczna plyt kolowo-symetrycznych, NOT-Arkady, Warszawa 1963.

[4.422] V. V. Novozhilov, On plastic weakening (in Russian), PMM 29 (1965), 4, 681-689.

[4.423] V. V. Novozhilov, O. G. Rybakina, Perspectives of formulation of a strength criterion in the case of combined loading (in Russian), IZh MTT (1966), 5, 103-111.

[4.424],-- , On the perspectives of formulation of a strength criterion in the case of combined loading (in Russian), PPMCTN (1969), 71-80.

[4.425] J. T. Oden, Finite elements of nonlinear continua, McGraw-Hill, New York 1972.

[4.426] -, Mathematical aspects of finite-element approximations in continuum mechanics, MechT 2 (1975), 159-250.

[4.427] I. A. Oding, Yu. V. Kostotchkin, Deformation and fracture due to thermal fatigue (in Russian), MTOM (1960), 4, 26-29.

[4.428] K. Ohji, M. Saito, Slip fracture surface of a circular pipe under internal pressure, 21st JCAM (1973), 347-354. 
[4.429] W. Olszak, P. Perzyna, Extremum theorems in the theory of plasticity of non-homogeneous and anisotropic bodies, AMS 9 (1957), 6, 695-712.

[4.430] - , - Remarks on the validity of variational theorems in the mechanics of inelastic bodies, IUTAM NH 1958 (1959), 157-165.

[4.431] E. T. Onat, On certain second-order effects in the limit design of frames, JAS 22 (1955), 681-684.

[4.432] E. T. Onat, R. M. Haythornthwaite, Load carrying capacity of circular plates at large deflection, JAM 23 (1956), 1, 49-55.

[4.433] E. T. Onat, The effects of non-homogeneity caused by strain-hardening on the small deformations of a rigid-plastic solid, IUTAM NH 1958 (1959), 171-180.

[4.434] - The influence of geometry changes on the load-deformation behaviour of plastic solids, 2nd SNSM (1960), 225-238.

[4.435] J. Orkisz, Problem odciążenia obrotowo-symetrycznych powłok w stanie błonowym przy dużych odkształceniach niesprężystych, MTS 3 (1965), 1, 63-83.

[4.436] -, Skończone odkształcenia niesprężystych wiotkich osiowo-symetrycznych powłok ortotropowych w stanie błonowym, RI 15 (1967), 4, 679-693.

[4.437] -, Skończone odkształcenia wiotkich osiowo-symetrycznych powłok w stanie błonowym w świetle teorii płynięcia plastycznego, MTS 5 (1967), 4, 463-473.

[4.438] A. Ostrowski, Solution of equations and systems of equations, Academic Press, New YorkLondon 1960.

[4.439] D. R. J. Owen, E. M. Salonen, Three-dimensional elastoplastic finite element analysis, IJNME 9 (1975), 1, 209-218.

[4.440] A. C. Palmer, A limit theorem for materials with non-associated flow laws, JM 5 (1966), 2, 217-222.

[4.441] -, Limit analysis of cylindrical shells by dynamic programming, IJSS 5 (1969), 4, 289-303.

[4.442] V. Panc, General theory of limiting states of stress in isotropic solids, (preliminary information), AT CSAV 14 (1969), 6, 832-840.

[4.443] V. M. Panferov, On convergence of the method of elastic solutions in the theory of elasticplastic shells (in Russian), PMM 13 (1949), 1, 79-94.

[4.444] - On applicability of variational methods to the problems of the theory of small elasticplastic deformations (in Russian), PMM 16 (1952), 3, 319-322.

[4.445] E. W. Parkes, Wings under repeated thermal stress, AirE 26 (1954), 402.

[4.446] E. O. Paton, B. N. Gorbunov, Load carrying capacity of welded beams subject to plastic deformations, (in Russian), Vid. Vseukrainskoy Akad. Nauk, Kiev 1935.

[4.447] B. Paul, P. G. Hodge, Jr., Carrying capacity of elastic-plastic shells under hydrostatic pressure, 3rd USNCAM (1958), 631-640.

[4.448] J. H. Percy, Quadrilateral finite elements in elastic-plastic plane stress analysis, AIAA 5 (1967), 367.

[4.449] N. Perrone, R. Kao, A general finite difference method for arbitrary meshes, CS 5 (1975), 45-58.

[4.450] V. A. Petushkov, Solution of boundary-value problems of cyclic plasticity by the finite element method (in Russian), Mash (1974), 1, 72-77.

[4.451] A. H. Philippidis, The general proof of the principle of maximum plastic resistance, JAM 15 (1948), 241-242.

[4.452] A. Phillips, Variational principles in the theory of finite plastic deformations, QAM 7 (1949), 110-114.

[4.453] S. Piechnik, The influence of bending on the limit state of a circular bar subject to torsion, AMS 13 (1961), 1, 77-106.

[4.454] S. Piechnik, M. Życzkowski, On the plastic interaction curve for bending and torsion of a circular bar, AMS 13 (1961), 5, 669-692.

[4.455] S. Piechnik, Steady-state creep of solid bar under combined load, Trans RITS 190 (1962). 
[4.456] S. Piechnik, Stationary creep of a solid circular bar and tube under torsion and tension, Trans RITS 192 (1962).

[4.457] —, Combined tension-bending creep for a solid bar, Trans RITS 194 (1962).

[4.458] A. A. Pikovsky, Statics of bar systems with compressed elements (in Russian), Gos. Izd. Fiz.-Mat. Lit., Moskva 1961.

[4.459] E. Plenard, Intéret pratique d'une nouvelle caractéristique mécanique: la limite d'accomodation, RMet 65 (1968), 12, 845-862.

[4.460] A. R. S. Ponter, Convexity conditions and energy theorems for time independent materials, JMPS 16 (1968), 4, 283-288.

[4.461] -, An upper bound on the small displacements of elastic, perfectly plastic structures, Trans ASME E39 (1972), 4, 959-963.

[4.462] A. R. S. Ponter, J. B. Martin, Some extremal properties and energy theorems for inelastic materials and their relationship to the deformation theory of plasticity, JMPS 20 (1972), $5,281-300$.

[4.463] G. G. Pope, The application of the matrix displacement method in plane elasto-plastic problems, Ist CMMSM (1966).

[4.464] E. P. Popov, P. Sharifi, A refined curved element for thin shells of revolution, IJNME 3 (1971), 4, 495-508.

[4.465] W. Prager, P. S. Symonds, Stress analysis in elastic-plastic structures, 3rd SAMath 1949 (1950), 187-197.

[4.466] W. Prager, The general theory of limit design, 8th ICAM 1952 (1955), 2, 65-72.

[4.467] —, Théorie générale des états limites d'équilibre, JMPA 34 (1955), 395-406.

[4.468] —, Probleme der Plastizitätstheorie, Birkhäuser, Basel-Stuttgart 1955; English translation: An introduction to plasticity, Addison-Wesley 1959.

[4.469] —, Shakedown in elastic-plastic media subjected to cycles of load and temperature, MSPSC (1956), 239-244.

[4.470] —, Plastic design and thermal stresses, BWJ 3 (1956), 8, 355-359.

[4.471] -, Programmation linéaire en théorie des constructions, Mem CERES 3 (1962), 33.

[4.472] —, Limit analysis: the development of a concept, PPlast 1972 (1974), 3-24.

[4.473] —, Bauschinger adaptation of rigid, workhardening trusses, MRC 1 (1974), 5-6, 253-256.

[4.474] L. Prandtl, Anwendungsbeispiele zu einem Henckyschen Satz über das plastische Gleichgewicht, ZAMM 3 (1923), 6, 401-406.

[4.475] A. M. Protsenko, Limit equilibrium of systems with a finite number of degrees of freedom at large deformations, (in Russian), IZh MTT (1968), 5, 70-75.

[4.476] —, Convergence of the problem of limit equilibrium (in Russian), PMM 34 (1970), 2, 370-372.

[4.477] -, Extremal boundary-value problems in the theory of plasticity (in Russian), Izv $A N$ SSSR MTT (1972), 5, 28-32.

[4.478] —, Extremal boundary-value problems for an elastic-plastic body (in Russian), Izv AN SSSR MTT (1974), 1, 80-87.

[4.479] D. Radaj, Matrizenverschiebungsmethode für temperaturabhängig elastisch-plastische Tragwerke und Kontinua, AMech 14 (1972), 1, 71-78.

[4.480] D. Radenković, Q. S. Nguyen, La dualité des théorèmes limites pour une structure en matériau rigide-plastique standard, AMS 24 (1972), 5-6, 991-998.

[4.481] B. Raniecki, On extremum principles in uncoupled thermoplasticity, BAP SST 20 (1972), 10, 801-806.

[4.482] —, Jednoznaczność i zasady wariacyjne w termoplastyczności, Term (1975), 175-222.

[4.483] H. Reissner, Eigenspannungen und Eigenspannungsquellen, ZAMM 11 (1931), 1.

[4.484] R. M. Richards, J. R. Blacklock, Finite element analysis of inelastic structures, AIAA 7 (1969), 3, 432-438. 
[4.485] O. Richmond, H. L. Morrison, Application of a perturbation technique based on the method of characteristics to axisymmetric plasticity, Pap ASME (1968).

[4.486] M. Roš, J. Brunner, Die Knicksicherheit von an beiden Enden gelenkig gelagerten Stäben aus Konstruktionsstahl, 2nd ICAM (1926), 378-386.

[4.487] V. I. Rozenblyum, On shakedown of non-uniformly heated elastic-plastic bodies (in Russian), Izv AN SSSR OTN (1957), 7, 136-138.

[4.488] —, On the theory of shakedown of elastic-plastic bodies (in Russian), Izv AN SSSR OTN (1958), 6, 47-53.

[4.489] - On shakedown analysis of non-uniformly heated elastic-plastic bodies (in Russian), ZhPMTF (1965), 5, 98-101.

[4.490] C. Ruiz, The inclusion of the incremental collapse problem in undergraduate course, BMEE 6 (1967), 1, 9-21.

[4.491] J. Rychlewski, O podstawowych twierdzeniach teorii przystosowania ciał sprężystoplastycznych, 11th PKMCS (1967).

[4.492] A. R. Rzhanitsyn, Structural analysis with plastic properties of materials taken into account (in Russian), Stroyizdat, Moskva 1949 and 1954.

[4.493] -, Limit analysis of shells by means of linear programming (in Russian), 6th VKTOP (1966), 656-665.

[4.494] G. Sacchi, G. Buzzi Ferraris, Sul criterio cinematico di calcolo a rottura di piastre inflesse mediante programmazione lineare, RIL ASL 101 (1967).

[4.495] G. Sacchi, M. Save, A note on the limit loads of non-standard materials, Mec 3 (1968), $1,43-45$.

[4.496] - , - On the evaluation of the limit load for rigid-perfectly plastic continua, $\mathrm{Mec} 3$ (1968) $3,1-8$.

[4.497] G. Sacchi, Limit analysis and design of structures having elements with random distribution of yield stresses, with associated and non-associated flow laws, Mec 6 (1971), 1, 65-68.

[4.498] G. Sacchi, G. Maier, M. Save, Limit design of frames for movable loads by linear programming, IUTAM OSD 1973 (1975), 415-432.

[4.499] G. Sachs, J. D. Lubahn, Failure of ductile metals in tension, Trans ASME 68 (1946), 271-276.

[4.500] M. A. Sadowsky, A principle of maximum plastic resistance, JAM 10 (1943), A65-A68.

[4.501] J. Salençon, Écoulement plastique libre et analyse limite pour les matériaux standards et non standards, AIT BTP 25 (1972), 89-100, 295-296.

[4.502] —, Théorie des charges limites, STA 47 (1973), 2, 287-309.

[4.503] —, Théorie de la plasticité pour les applications a la mécanique des sols, Eyrolles, Paris 1974.

[4.504] - , Un example de non validité de la théorie classique des charges limites pour un système non standard, PPlast 1972 (1974), 432-439.

[4.505] H. Sanecki, M. Życzkowski, Basic equations of perfect plasticity parametrized by general stress functions, AMS 29 (1977), 2, 359-362. Erratum: AMS 29, 6

[4.506] S. O. Sarkisyan, On the method of elastic solutions in the theory of cylindrical shells (in Russian), Izv AN ArmSSR M 25 (1972), 5, 14-22.

[4.507] - , On certain methods in physically non-linear theory of sandwich plates (in Russian), Izv AN ArmSSR M 26 (1973), 3, 37-46.

[4.508] M. A. Save, C. E. Massonnet, Plastic analysis and design of plates, shells and disks, NorthHolland, Amsterdam-London 1972.

[4.509] M. Save, Théorie de la stabilisation plastique, RMet 20 (1974), 1, 37-42.

[4.510] G. N. Savin, O. S. Parasyuk, Influence of a non-homogeneous stress field on the plastic zone near a hole (in Ukrainian), DAN URSR (1948), 3.

[4.511] - , - Plastic zones near a hole in a non-homogeneous plane stress field (in Russian), UZLU FM 12 (1949), 3.

[4.512] G. N. Savin, Stress distribution around holes (in Russian), Naukova Dumka, Kiev 1968. 
[4.513] A. Sawczuk, T. Jaeger, Grenztragfähigkeitstheorie der Platten, Springer, Berlin-GöttingenHeidelberg 1963.

[4.514] A. Sawczuk, Nośność graniczna ram plaskich, NOT-Arkady, Warszawa 1964,

[4.515] —, On initiation of the m:mbrane action in rigid-plastic plates, $J M 3$ (1964), 1, 15-23.

[4.516] - Estimation of the post-yield load-deflection relationship of perfectly plastic plates, TPS 1963 (1966), 435-440.

[4.517] —, Shakedown analysis of elastic-plastic structures, NED 28 (1974), 1, 121-136.

[4.518] M. Sayir, H. Ziegler, Der Verträglichkeitssatz der Plastizitätstheorie und seine Anwendung auf räumlich unstetige Felder, ZAMP 20 (1969), 78-93.

[4.519] J. Schroeder, A. N. Sherbourne, A general variational theorem in classical plasticity, JMP 46 (1967), 2, 175-187.

[4.520] S. V. Serensen, P. I. Kotov, On the methods of experimental investigations of thermal fatigue (in Russian), $Z L 24$ (1958), 9, 1097-1106.

[4.521] S. V. Serensen, Problems of load-carrying capacity at low-cycle fatigue (in Russian), PPMCTN (1969), 6-25.

[4.522] S. V. Serensen, R. M. Shneyderovitch, A fracture criterion for cyclic elastic-plastic deformation (in Russian), PPMCTN (1969), 80-93.

[4.523] M. J. Sewell, The governing equations and extremum principles of elasticity and plasticity generated from a single functional, TR 1227, Math. Res. Cent. Madison, Wisc. 1972.

[4.524] - Plastic buckling, Chapter 5 of "Stability", ed. by H. H. E. Leipholz, Waterloo 1972, Study No. 6, 85-197.

[4.525] D. I. Shakhnovsky, On locally-adiabatic plastic flow of metals (in Russian), NTVZMI (1973), 2, 93-95.

[4.526] D. Shanks, Non-linear transformations of divergent and slowly convergent sequences, JMP 34 (1955), 1, 1-42.

[4.527] F. R. Shanley, A proposed mechanism of fatigue failure, IUTAM CF 1955 (1956), 251-259.

[4.528] Yu. N. Shevtchenko, Thermoplasticity under variable loading conditions (in Russian), Naukova Dumka, Kiev 1970.

[4.529] —, Approximate solution methods of the problems of repeated loading in thermoplasticity (in Russian), PP (1971), 383-391.

[4.530] - Method of elastic solutions in the theory of plastic flow in the case of non-isothermal loading processes (in Russian), TNEK 15 (1975), 45-49.

[4.531] R. T. Shield, On the plastic flow of metals under conditions of axial symmetry, Proc RS 233A (1955), 267-287.

[4.532] R. M. Shneyderovitch, O. A. Levin, N. A. Makhutov, M. D. Novopashin, Measurement methods of fields of cyclic elastic-plastic deformations (in Russian), $Z L 38$ (1972), 10, 1246-1253.

[4.533] E. M. Shoemaker, Some paradoxes associated with elastic-plastic limit load analysis, AMS 20 (1968), 4, 473-483.

[4.534] - , A re-examination of the critical speed of a rotating disk, BIPI 14 (1968), 433-440.

[4.535] E. M. Shoemaker, W. P. Chen, On uniqueness of the limit load for unbounded regions, AMS 21 (1969), 5, 679-689.

[4.536] E. M. Shoemaker, On velocity discontinuities in elastic-plastic boundary value problems, AMS 26 (1974), 2, 327-331.

[4.537] —, On the collapse load for non-standard plastic materials, AMech 20 (1974), 3-4, 263-271.

[4.538] E. Siebel, Zur Mechanik des Zugversuchs, WF 2 (1944), 5.

[4.539] G. Sines, Yielding and plastic instability under biaxial stress in design of metal pressure vessels, JMat 4 (1969), 2, 377-392.

[4.540] J. Skrzypek, Limit state of thick-walled rotating cylinders under combined loa ting, $B A P$ SST 15 (1967), 4, 189-204 (English summary); RI 14 (1966), 4, 629-652 (Polish full text). 
[4.541] J. Skrzypek, On the limit carrying capacity of a pipe-line cross-section, IJSS 4 (1968), 12, 1203-1219.

[4.542] J. Skrzypek, M. Życzkowski, Non-symmetrical plastic collapse modes of a symmetrically loaded circular cylinder, AMech 7 (1969), 2-3, 213-223.

[4.543] - , - Approximate determination of the limit surface of the cross-section of a thickwalled tube under combined loads, BAP SST 18 (1970), 7, 267-274 (English summary); MTS 8 (1970), 2, 107-126 (Polish full text).

[4.544] Z. Sobotka, Theorie plasticity, t. I-II, CSAV, Praha 1954 and 1955.

[4.545] —, Teorie plasticity desek, Academia, Praha 1973.

[4.546] V. V. Sokolovsky, On a certain form of representation of the stress components in the theory of plasticity (in Russian), DAN SSSR 61 (1948), 2.

[4.547] A. J. M. Spencer, Perturbation methods in plasticity, JMPS 9 (1961), 3, 279-288; 10 (1962), 1, 17-26; 10 (1962), 2, 165-177.

[4.548] -, The approximate solution of certain problems of axially-symmetric plastic flow, JMPS 12 (1964), 231-243.

[4.549] J. F. Steffensen, Remarks on iteration, SAT 16 (1933), 64-72.

[4.550] B. Storåkers, Plastic and visco-plastic instability of a thin tube under internal pressure, torsion and axial tension, IJMS 10 (1968), 6, 519-529.

[4.551] - Bifurcation and instability modes in thick-walled rigid-plastic cylinders under pressure, JMPS 19 (1971), 6, 339-353.

[4.552] - , Uniqueness and stability at finite deformation of inelastic solids, Inst. Hallfasthetslära Stockholm 186 (1973), 58 pp.

[4.553] -, On uniqueness and stability of elastic-plastic deformation, AMS 27 (1975), 5-6, 821-839.

[4.554] J. A. Stricklin, W. Haisler, W. A. Riesemann, Self-correcting initial-value formulations in nonlinear structural mechanics, AIAA 9 (1971), 10, 2066-2067.

[4.555] - , - , - Evaluation of solution procedures for material and/or geometrically nonlinear structural analysis, AIAA 11 (1973), 3, 292-299.

[4.556] H. Strifors, B. Storåkers, Uniqueness and stability of rigid-plastic spherical shells subjected to finite deformations under hydrostatic pressure, JMPS 21 (1973), 3, 125-134.

4.557] F. Stüssi, C. F. Kollbrunner, Beitrag zum Traglastverfahren, BT 13 (1935), 264.

[4.558] J. L. Swedlow, T. A. Cruse, Formulation of boundary integral equations for three-dimensional elasto-plastic flow, IJSS 7 (1971), 12, 1673-1683.

[4.559] J. L. Swedlow, A procedure for solving problems of elasto-plastic flow, CS 3 (1973), 4, 879-898.

[4.560] H. W. Swift, Plastic instability under plane stress, JMPS 1 (1952), 1.

[4.561] P. S. Symonds, W. Prager, Elastic-plastic analysis of structures subjected to loads varying arbitrarily between prescribed limits, JAM 17 (1950), 315-323.

[4.562] P. S. Symonds, Shakedown in continuous media, JAM 18 (1951), 85-89.

[4.563] P. S. Symonds, B. G. Neal, The interpretation of failure loads in the plastic theory of continuous beams and frames, JAS 19 (1952), 1, 15-22.

[4.564] P. S. Symonds, Limit analysis, HEM (1962), Chapter 49, 28 pp.

[4.565] W. Szczepiński, Dynamic expansion of a rotating solid cylinder of mild steel, AMS 19 (1967), 1, 75-87.

[4.566] -, Projektowanie elementów maszyn metoda nośności granicznej, IPPT PAN-PWN, Warszawa 1968.

[4.567] -, Limit analysis and plastic design of structural elements of complex shape, PAS 12 (1972), $1-47$.

[4.568] K. Szuwalski, Tran-le Binh, Nośność rozdzielcza idealnie sprężysto-plastycznych belek statycznie niewyznaczalnych, CT 81 (1977), 4, 10-15.

[4.569] -, Nośność rozdzielcza statycznie niewyznaczalnych układów prętowych z materiału asymptotycznie idealnie plastycznego, RI (in print). 
[4.570] K. Szuwalski, Nośność rozdzielcza pierścieniowej tarczy kołowo-symetrycznej ze sztywną inkluzją, MTS 17 (1979), 4, 589-602.

[4.571] -, Wpływ charakterystyk materiałowych na nośność rozdzielczą tarczy nieograniczonej z kołową sztywną inkluzją, PKMS (in print).

[4.572] —, Idealnie sprężysto-plastyczna tarcza o profilu hiperbolicznym, MTS (in print).

[4.573] —, Nośność graniczna i rozdzielcza skokowo niejednorodnych tarcz wirujących, $A B M$ (in print).

[4.574] A. K. Tadros, P. B. Mellor, Some comments on the limit strains in sheet metal stretching, IJMS 17 (1975), 3, 203-210.

[4.575] $\mathrm{Zh}$. I. Tairova, A theorem on variable loadings at non-uniform heating in the theory of plastic flow (in Russian), Izv AN AzbSSR FTM (1968), 5, 34-38.

[4.576] S. Tang, Nonlinear analysis of instability in tension, Proc ASCE JEMD 97 (1971), 5, 1487-1494.

[4.577] J. F. Tavernelli, L. F. Coffin, A complilation and interpretation of cyclic strain fatigue tests on metals, Proc ASMet 51 (1959), 438-453.

[4.578] J. J. Telega, Zastosowanie programowania liniowego do wyznaczania nośności granicznej konstrukcji, MTS 9 (1971), 1, 7-52.

[4.579] -, Ośrodki idealnie-plastyczne pod działaniem obciążeń wieloparametrowych, RI 20 (1972), 2, 241-251.

[4.580] -, On the limit analysis and certain variational principle for rigid plastic solids, BAP SST 22 (1974), 5, 421-426.

[4.581] I. G. Teregulov, On the methods of reduction of continual nonlinear problems of solid mechanics to discrete problems, Izv AN SSSR MTT (1972), 5, 21-27.

[4.582] G. Thierauf, Elastisch-plastische Formänderungen biegesteifer Tragwerke nach der Spannungstheorie II. Ordnung, IA 42 (1973), 5, 285-295.

[4.583] T. Y. Thomas, Characteristic surfaces in the Prandtl-Reuss plasticity theory, JRMA 5 (1956), 2, 251-262.

[4.584] P. F. Thomason, An analysis of necking in axi-symmetric tension specimens, IJMS 11 (1969), 5, 481-490.

[4.585] E. G. Thomsen, C. T. Yang, S. Kobayashi, Mechanics of plastic deformation in metal processing, Macmillan, New York 1965.

[4.586] Tran-le Binh, M. Życzkowski, The Stüssi-Kollbrunner paradox in the light of the concept of decohesive carrying capacity, AMS 28 (1976), 4, 607-614.

[4.587] W. Truszkowski, Deformation of metal at the neck of a strained test-piece, BAPSL A1 (1952), 373.

[4.588] H. S. Tsien, The Poincaré-Lighthill-Kuo method, $A A M 4$ (1956), 281-349.

[4.589] M. J. Turner, R. W. Clough, H. C. Martin, L. C. Topp, Stiffness and deflection analysis of complex structures, JAS 23 (1956), 9, 805-823, 854.

[4.590] V. O. Vakorin, A. S. Fedorov, On the formation of a "neck" in pure bending of a strip (in Russian), $\operatorname{Tr}$ LKI 62 (1968), 19-22.

[4.591] S. Valliappan, Elasto-plastic analysis of anisotropic work-hardening materials, AMS 24 (1972), 3, 465-481.

[4.592] J. A. Van den Broek, Theory of limit design, Wiley, New York 1948.

[4.593] M. Van Dyke, Perturbation methods in fluid mechanics, Academic Press, New YorkLondon 1964.

[4.594] R. G. Venkateswara, A. V. Krishna Murty, An alternate form of the Ramberg-Osgood formula for matrix displacement analysis, NED 17 (1971), 3, 297-308.

[4.595] A. M. Vinogradova, On the formation of a neck in tension of hollow cylindrical specimens (in Russian), Izv AN SSSR MTT (1971), 6, 150-157.

[4.596] -, On the stability of equilibrium in tension (in Russian), Izv AN SSSR MTT (1973), S, 66-72. 
[4.597] E. Vitiello, Upper bounds to plastic strains in shake-down of structures subjected to cyclic loads, Mec 7 (1972), 3, 205-213.

[4.598] V. K. Vorontsov, P. I. Polukhin, Photoplasticity (in Russian), Izd. Metallurgya, Moskva 1969.

[4.599] P. P. Vorovitch, Yu. P. Krasovsky, On the method of elastic solutions (in Russian), DAN SSSR 126 (1959), 4, 740-743.

[4.600] A. J. Wang, W. Prager, Thermal and creep effects in work-hardening elastic-plastic solids, JAS 21 (1954) 5, 343-344, 360.

[4.601] K. Washizu, Variational methods in elasticity and plasticity, Pergamon Press, Oxford 1968 and 1975.

[4.602] W. Wasow, On the convergence of an approximation method of M. J. Lighthill, JRMA 4 (1955), 751-767.

[4.603] A. W. Wegmuller, Elastic-plastic finite element analysis of plates, $I A 44$ (1975), 2, 63-77.

[4.604] J. R. Whiteman, A bibliography for finite elements, Academic Press, London 1975.

[4.605] M. Wnuk, Limit state of a bar with arbitrary cross-section under tension and torsion, BAP SST 10 (1962), 6, 221-230.

[4.606] - , Yield curves for bars of various cross-sections under combined torsion and tension, BAP SST 11 (1963), 11, 619-626.

[4.607] - Effect of torsion on the tension of a prismatic bar with cross-section of arbitrary shape in the elastic plastic range, BAP SST 11 (1963), 11, 627-635.

[4.608] - Porównanie nośności sprężystej oraz plastycznej w przypadku obciążenia złożonego, ABM 11 (1964), 1, 97-108.

[4.609] R. H. Wood, Plastic and elastic design of slabs and plates, Thames and Hudson, London 1961.

[4.610] - A partial failure of limit analysis for slabs, and the consequences for future research, MCR 21 (1969), 67, 79-90; discussion 22 (1970), 71, 109-114.

[4.611] C. Woźniak, Constrained continuous media, BAP SST 21 (1973), 3, 109-116; 4, 167-182.

[4.612] E. H. Wu, E. A. Witmer, Finite element analysis of large elastic plastic transient deformations of simple structures, AIAA 9 (1971), 1719-1724.

[4.613] M. H. Lee Wu, A simple method of determining plastic stresses and strains in rotating disks with non-uniform metal properties, JAM 19 (1952), 489-495.

[4.614] Y. Yamida, N. Yoshimura, T. Sakurai, Plastic stress-strain matrix and its application for the solution of elastic-plastic problems by the finite element method, IJMS 10 (1968), 343-354.

[4.615] Y. Yamamoto, Variational principles of equilibrium of an elastic-plastic body, $Q A M 10$ (1952), 3, 215-224.

[4.616] - , Rate of convergence for the iterative approach in elastic-plastic analysis of continua, IJNME 7 (1973), 4, 497-508.

[4.617] T. Yokobori, An interdisciplinary approach to fracture and strength of solids, WoltersNoordhoff, Groningen 1968.

[4.618] A. Zavelani-Rossi, A new linear programming approach to limit analysis, $S V M E 1972$ (1973), 2, 8.

[4.619] A. Zavelani, A compact linear programming procedure for optimal design in plane stress, JSM 2 (1973), 4, 301-324.

[4.620] A. Zavelani-Rossi, Finite element techniques in plane limit problems, $\operatorname{Mec} 9$ (1974).

[4.621] A. M. Zhukov, On the problem of formation of a neck in a tension specimen (in Russian), IS 5/1 (1949), 34-51.

[4.622] H. Ziegler, Some extremum principles in irreversible thermodynamics with application to continuum mechanics, PSM 4 (1963), 92-193.

[4.623] O. C. Zienkiewicz, S. Valliappan, I. P. King, Elastic-plastic solutions of engineering problems, initial stress, finite element approach, IJNME 1 (1969), 75-100. 
[4.624] O. C. Zienkiewicz, The finite element method: from intuition to generality, $A M R 23$ (1970), $3,249-256$.

[4.625] O. C. Zienkiewicz, I. C. Cormeau, Visco-plasticity and plasticity-an alternative for finite element solution of material nonlinearities, LNCS 10 (1974), 259-287.

[4.626] - , -, Visco-plasticity, plasticity and creep in elastic solids-a unified numerical solution approach, IJNME 8 (1974), 4, 821-845.

[4.627] Z. Zudans, M. M. Reddi, H. C. Tsai, DYPLAS, a finite element dynamic elastic-plastic large deformation analysis program, NED 27 (1974), 3, 398-412.

[4.628] M. Życzkowski, Simultaneous tension and torsion of a circular bar in the elasto-plastic range, BAP SST 3 (1955), 2, 51-55 (English summary); RI 3 (1955), 2, 285-322 (Polish full text).

[4.629] -, Computation of the critical forces of non-prismatic elastic bars by the method of partial interpolation, BAP SST 4 (1956), 4, 221-233 (English summary); RI 4 (1956), 3, 367-412 (Polish full text).

[4.630] -, Certain general equations for plane circularly symmetrical plastic states, AMS 10 (1958), 4, 463-478.

[4.631] -, Potenzieren von verallgemeinerten Potenzreihen mit beliebigem Exponent, ZAMP 12 (1961), 6, 572-576.

[4.632] —, Tablice wspólczynników przy potęgowaniu szeregów potęgowych, ZM 6 (1963), 4, 395-406.

[4.633] - , The "onefold optimum approximation" and some of its applications in mechanics, BAP SST 11 (1963), 6, 199-208 (English summary); RI 11 (1963), 3, 463-490 (Polish full text).

[4.634] - , Plastic deformation and strength of an initially expanded multi-layer pressure vessel, BAP SST 12 (1964), 2, 89-98 (English summary); ABM 11 (1964), 1, 83-96 (Polish full text).

[4.635] -, Operations on generalized power series, ZAMM 45 (1965), 4, 235-244.

[4.636] - , Plastic interaction curves for combined bending and tension of beams with arbitrary cross-section, AMS 17 (1965), 2, 307-330.

[4.637] - Równania przemieszczeniowe teorii idealnej plastyczności we współrzędnych krzywoliniowych, 11th PKMCS (1967).

[4.638] —, Limit state of a thick-walled tube under internal pressure and bending, AMS 20 (1968), 3, 313-325.

[4.639] M. Życzkowski, J. Skrzypek, Influence of bending, torsion and tension on the stationary creep of a pipe line, AMS 23 (1971), 4, 465-480.

[4.640] -, Stationary creep and creep rupture of a thick-walled tube under combined loadings, 2nd IUTAM CiS 1970 (1972), 315-329.

[4.641] M. Życzkowski, M. Galos, Plastic torsion and tension of naturally uniformly twisted bars, AMS 24 (1972), 5-6, 1039-1055.

[4.642] M. Życzkowski, K. Szuwalski, Decohesive carrying capacity in thermal stress problems, 3rd SMiRT (1975), paper L2/4.

[4.643] - , - On the termination of the process of finite plastic deformations, J. Mec. Appl. (in print).

\section{Chapter V}

[5.1] A. F. Akhmerov, Investigation of the processes of bending with tension of plane-curved details (in Russian), $\operatorname{Tr} K A I 36$ (1957), 49-66.

[5.2] - Elastic-plastic analysis under combined loadings tension-bending (in Russian), Izv VUZ AT (1969), 3, 127-138.

[5.3] Yu. N. Aleshinsky, Analysis of structural elements beyond the elastic limit (in Russian), NDVS S (1958), 1, 52-63. 
[5.4] Yu. N. Aleshinsky, Solution and improvement of analysis of elements of steel structures beyond the elastic limit (in Russian), $\operatorname{Tr} M I I Z D T 108$ (1959), 5-81.

[5.5] - Elastic-plastic analysis of sections with a generalized profile under longitudinal force and bending moment (in Russian), $\operatorname{Tr} I D N P L 30$ (1968), 3-30.

[5.6] K. I. Amariei, B. T. Kolundžija, Analiza u sukcesivnim etapama elasto-plastičnog ponašanja konstrukcija majuči u obzir uticaj aksjalnih sila, Iz 28 (1974), 2, 1-10.

[5.7] B. Barbarito, La rottura delle sezioni pressoinflesse nel cemento armato, GGC (1960), 9.

[5.8] B. Barbarito, S. Aiello, La rottura delle sezioni pressoinflesse in conglomerato precompresso, Cem 59 (1962), 6, 13-19.

[5.9] F. Baron, M.S. Venkatesan, Inelastic response for arbitrary histories of loads, Proc ASCE JEMD 95 (1969), 3, 763-786.

[5.10] A. J. Barrett, Unsymmetrical bending and bending combined with axial loading of a beam of rectangular cross section into the plastic range, JRAS 57 (1953), 503-509.

[5.11] - , Beam strength and curvature under combined tension and bending in the plastic range, JAS 22 (1955), 1, 71-72.

[5.12] S. C. Batterman, L. P. Felton, Optimal plastic design of doubly symmetric closed structures, IJSS 8 (1972), 6, 733-750.

[5.13] Z. Bažant, Mézni stav prư̌ezu namáhaných šikmým mimostřednim tlakem nebo tahem, SVUTB (1965), 3, 207-220.

[5.14] J. Bäcklund, Large deflection analysis of elasto-plastic beams and frames, IJMS 18 (1976), 6, 269-278.

[5.15] G. E. Belsky, On qualitative investigations of stability of beam-columns (in Russian), SMRS (1967), 2, 23-27.

[5.16] N. I. Bezukhov, O. V. Luzhin, Application of the methods of elasticity and plasticity to the solution of engineering problems (in Russian), Vysshaya Shkola, Moskva 1974.

[5.17] H. U. Bilek, Die Berücksichtigung des nichtlinearen Krümmungsausdruck bei der Berechnung von Traglast und Gleichgewichtslagen idealelastisch-idealplastischer Stäbe mit Rechteckquerschnitt, Diss. Dokt.-Ing. Univ. Stuttgart, 1973.

[5.18] B. S. Bilobran, On the plastic bending with tension of thin-walled tubes (in Russian), VLPI 7 (1965), 207-212.

[5.19] C. Birnstiel, J. Michalos, Ultimate load of $H$-columns under biaxial bending, Proc ASCE JSD 89 (1963), 2, 161-197.

[5.20] B. A. Boley, Temperature and deformation in rods and plates melting under internal heat generation, Ist SMiRT (1971), 5, L2-3/1-13.

[5.21] D. S. Brooks, The elasto-plastic behaviour of rectangular sections carrying biaxial bending, Trans IEA CE 6 (1964), 1, 41-47.

[5.22] E. H. Brown, Plastic asymmetrical bending of beams, IJMS 9 (1967), 2, 77-82.

[5.23] J. Bródka, M. Kwaśniewski, Stan graniczny dwuteowników obciążonych mimośrodowo, AIL 15 (1969), 3, 473-490.

[5.24] T. I. Campbell, T. M. Charlton, Finite deformation of a fully fixed beam comprised of a non-linear material, IJMS 15 (1973), 5, 415-428.

[5.25] F. Campus, Plastification de l'acier doux en flexion plane composée, BARB CS 49 (1963), $4,315-319$.

[5.26] E. Cegielski, Modyfikowane krzywe nośności sprężystej przy zginaniu z rozciąganiem belek o przekroju prostokątnym, CT 80 (1976), 4-M, 24-30.

[5.27] W. F. Chen, T. Atsuta, Interaction equations for biaxially loaded sections, Proc ASCE JSD 98 (1972), 5, 1035-1052.

[5.28] -, -, Ultimate strength of biaxially loaded steel H-columns, Proc ASCE JSD 99 (1973), 3, 469-489; 100 (1974), 10, 2149.

[5.29] W.F. Chen, Plastic $H$-columns under repeated biaxial loading, $R C T$ AIPC 13 (1973), 1-7. 
[5.30] W. F. Chen, T. Atsuta, Theory of beam-columns, Vol. 1, In-plane behavior and design, McGraw-Hill, New York 1977.

[5.31] E. R. Chorna, N. L. Chernov, Analysis of deformation of thin-walled bars under biaxial bending beyond the elastic limit (in Ukrainian), PM 10 (1964), 5, 493-502.

[5.32] N. L. Chernov, S. D. Kudryavtsev, Elastic-plastic deformations of thin-walled bars under moving loads (in Russian), PM 4 (1968), 11, 73-79.

[5.33] C. Cichoń, Z. Waszczyszyn, Numeryczne obliczanie sprężystoplastycznego łuku kolowego o przekroju typu sandwich, AIL 17 (1971), 3, 529-543.

[5.34] C. Cichoń, Numeryczna metoda analizy procesów wielokrotnych dla materiałów z liniowym wzmocnieniem, ABM 22 (1975), 4, 398-406.

[5.35] M. Z. Cohn, T. Rafay, Collapse load analysis of frames considering axial forces, Proc ASCE JEMD 100 (1974), 4, 773-794.

[5.36] M. Como, La sezione a doppio $\mathrm{T}$ nella fase di completa plasticizzacione in presenza di sforzo normale e momento flettente, RASFM, Serie IV, 4 (1959).

[5.37] D. A. DaDeppo, R. Schmidt, Finite extensional deformation of a rigid plastic arch, IJSS 10 (1974), 9, 1017-1034.

[5.38] H. Dietmann, Berechnung der Fließkurven von Bauelementen bei kleinen Verformungen, TU Stuttgart 1968.

[5.39] I. L. Dikovitch, Statics of elastic-plastic beams of naval structures (in Russian), Sudostroyenye, Leningrad 1967.

[5.40] H. Djalaly, Comportement élasto-plastique d'une barre sollicitée en compression et flexion déviée, CoMet 9 (1972), 4, 25-33.

[5.41] A. G. Dorfman, S. D. Leytes, Elastic-plastic stability of eccentrically compressed bars with unsymmetric I- and T-section (in Russian), ITS 14 (1965), 5-22.

[5.42] J. Dutheil, La stabilité des colonnes en acier soumisses au flambement-déversement, AIT BTP 10 (1957), 679-711.

[5.43] T. J. Dwyer, T. V. Galambos, Plastic behavior of tubular beam columns, Proc ASCE JSD 91 (1965), 4, 153-168.

[5.44] N. F. Ershov, Elastic-plastic deformations of strips under combined loading (in Russian), $\operatorname{Tr}$ GPI 22 (1966), 3, 31-41.

[5.45] L. Finzi, Sulle condizioni al contorno di aste elastoplastiche presso o tensoinflesse studiate coi metodi della meccanica non lineare, RIL ASL Sci. Mat.-Fiz. A95 (1961), 1, 127-130.

[5.46] J. M. Frankland, R. E. Roach, Strength under combined tension and bending in the plastic range, JAS 21 (1954), 7, 449-453; 22 (1955), 11, 795-797.

[5.47] W. Freiberger, W. Prager, Plastic twisting of thick-walled circular ring sectors, JAM 23 (1956), 461-463.

[5.48] W. Freiberger, Elastic-plastic torsion of circular ring sectors, QAM 14 (1956), 259-265.

[5.49] J. Fritzsche, Die Tragfähigkeit von Balken aus Stahl mit Berücksichtigung des plastischen Verformungsvermögens, BI 11 (1930), 851.

[5.50] L. A. Galin, Elastic-plastic torsion of prismatic bars (in Russian), PMM 13 (1949), 3, 285-296.

[5.51] M. Galos, M. Życzkowski, Analytical calculation of limit carrying capacity of isotropic bars subject to torsion, BAP SST 12 (1964), 2, 69-87 (English summary); RI 12 (1964), 2, 269-298 (Polish full text).

[5.52] M. Galos, Obliczanie nośności granicznej skręcanych prętów o dowolnej niejednorodności poprzecznej, RI 16 (1968), 2, 233-260.

[5.53] M. Galos, M. Życzkowski, Plastyczne skręcanie prętów kołowo zakrzywionych o dowolnej niejednorodności poprzecznej, RI 18 (1970), 4, 683-701.

[5.54] D. H. Garbaccio, Bending with axial force of bars in plasticity, Diss. Abstr. 15 (1955), 6, 1041-1042. 
[5.55] A. V. Gemmerling, E. N. Kuznetsov, Shakedown of beam-columns (in Russian), SMRS (1967), 6, 21-25.

[5.56] —, Analysis of bar systems (in Russian), Stroyizdat, Moskva 1974.

[5.57] A. R. Gent, H. R. Milner, The ultimate load capacity of elastically restrained H-columns under biaxial bending, Proc ICE 41 (1968), 685-704.

[5.58] E. Giangreco, Przegląd ostatnich prac włoskich w dziedzinie nośności granicznej, RI 14 (1966), 1, 3-33.

[5.59] G. Giannelli, Sul calcolo a rottura delle sezioni in cemento armato pressoinflesse, GGC 96 (1958), 12, 828-836.

[5.60] S. S. Gill, Effect of deflexion on the plastic collapse of beams with distributed load, IJMS 15 (1973), 6, 465-471.

[5.61] K. Girkmann, Bemessung von Rahmentragwerken unter Zugrundelegung eines idealplastischen Stahles, $S B A W W$ (Abt. IIa) 140 (1931), 679.

[5.62] D. Goldner, Plastic bending in tubular beams, MD 45 (1973), 24, 152-155.

[5.63] B. N. Gorbunov, V. G. Chudnovsky, Analysis of beams under biaxial plastic bending, (in Russian), ST KISI (1935), 11.

[5.64] B. N. Gorbunov, S. A. Palchevsky, Analysis of beams under biaxial bending with plastic deformations taken into account (in Russian), StP (1939), 9.

[5.65] P. Grundy, Shakedown of bars in bending and tension, Proc ASCE JEMD 95 (1969), 3, 519-529.

[5.66] G. Gurfinkel, A. Robinson, Determination of strain distribution and curvature in a reinforced concrete section subjected to bending moment and longitudinal load, JACI 64 (1967), 7, 398-403.

[5.67] A. Gürkök, H. G. Hopkins, The effect of geometry changes on the load carrying capacity of beams under transverse load, SIAM JAMath 25 (1973), 3, 500-521.

[5.68] H. B. Harrison, The plastic behaviour of mild steel beams of rectangular section bent about both principal axes, SEng 41 (1963), 7, 231-237.

[5.69] G. A. Harstead, C. Birnstiel, K. C. Leu, Inelastic $H$-column under biaxial bending, Proc ASCE JSD 94 (1968), 10, 2371-2398.

[5.70] F. Hartmann, Der einseitige Druck bei Stäben aus Baustahl, ZÖIAV (1933), 21-23.

[5.71] —, Knickung, Kippung, Beulung, F. Deuticke, Leipzig-Wien 1937.

[5.72] R. M. Haythornthwaite, Plastic behavior of beams with elastic end constraints, 9th ICAM 1956, 8 (1957), 59.

[5.73] T. R. Higgins, Plastic design of steel-a progress report, JBSCE 45 (1958), 3, 207-226.

[5.74] P. G. Hodge, Jr., Post-yield behavior of a beam with partial end fixity, IJMS 16 (1974), $6,385-388$.

[5.75] V. Iannelli, Sul problema della pressoflessione elastoplastica, RIL ASL A106 (1972), 2, 239-296.

[5.76] -, Ritorni elastici per carichi crescenti nella flessione composta in campo elastoplastico, RIL ASL A107 (1973), 3, 555-579.

[5.77] K. Ingerle, Die Biegesteifigkeit eines rechteckigen Stabelementes aus einem ideal-elastischplastischen Werkstoff, ÖIZ 12 (1969), 10, 361-364.

[5.78] D. D. Ivlev, On torsion of helical bars made of perfectly plastic material (in Russian), Izv AN SSSR OTN MM (1961), 5, 124-126.

[5.79] A. Jakubowicz, Techniczna teoria zginania prętów dla pewnego przypadku materiału fizykalnie nieliniowego, RI 10 (1962), 3, 443-458.

[5.80] E. Jőgi, On the analysis of elastic-plastic shallow arches (in Russian), UZTGU 129 (1962). $460-468$.

[5.81] -, Symmetric deformation of an elastic-plastic shallow circular arch (in Russian), UZTGU 150 (1964), 231-238. 
[5.82] N. Jones, Review of the plastic behavior of beams and plates, AIAA Paper 343 (1972); ISP 19 (1972), 218, 313-327.

[5.83] -, Influence of in-plane displacements at the boundaries of rigid-plastic beams and plates, IJMS 15 (1973), 7, 547-561.

[5.84] M. P. Kamat, G. W. Swift, D. E. Killian, Beam-column element for inelastic analysis, IJNME 10 (1976), 5, 1177-1178.

[5.85] V. N. Kissyuk, Minimal weight design of one-dimensional systems under deformation constraints allowing for the effect of normal force on the limit bending moment (in Russian), NMRSK (1971), 163-167.

[5.86] I. A. Kiyko, Torsion of a bar of non-homogeneous perfectly plastic material (in Russian), IZh MTT (1967), 2, 155-157.

[5.87] A. M. Kochetkov, On bending of beams with large displacements (in Russian), IZh MTT 5 (1965), 6, 1081-1086.

[5.88] L. O. Kolesnikov, A method of analysis of large displacements of a bar in the elasticplastic range (in Ukrainian), PM 4 (1958), 3, 317-325.

[5.89] V. P. Kolomiets, A method of analysis of stresses and strains in a beam section under combined loading allowing for the actual stress-strain diagram (in Russian), Izv VUZ AT (1966), 1, 63-72.

[5.90] I. Konishi, N. Shiraishi, T. Taniguchi, H. Furuta, Structural analysis and design of rigid frames based on plastic theorem, MFEKU 37 (1975), 4, 219-236.

[5.91] T. Kozłowski, Zagadnienie stanów granicznych stalowych łuków, CT 65 (1960), 4, 1-7.

[5.92] J. A. König, A method of shakedown analysis of frames and arches, IJSS 7 (1971), 4, 327-344.

[5.93] J. Krużelecki, W. Krzyś, Nowa metoda doboru zastępczego przekroju wielopunktowego w analizie sprężysto-plastycznego zginania, RI 26 (1978), 2, 291-306.

[5.94] I. N. Kudryavtsev, Biaxial bending in the range of plastic deformations (in Russian), Izd. V.I.A. RKKA im. Kuybysheva, 1940.

[5.95] S. D. Kudryavtsev, Plastic biaxial bending of thin-walled bars with unsymmetric I-section (in Russian), PM 2 (1966), 10, 54-62.

[5.96] - Elastic-plastic deflections under biaxial bending of thin-walled beams with unsymmetric I-section (in Russian), Izv VUZ SA (1968), 3, 31-35.

[5.97] —, Theoretical and experimental investigations of elastic-plastic deformations of thinwalled bars under moving loads (in Russian), Izv VUZ M (1969), 3, 45-50.

[5.98] B. N. Kuznetsov, Shakedown of statically determinate bars under various loading programs (in Russian), SMRS (1971), 1, 21-23.

[5.99] —, Shakedown of bars made of materials with different yield point stresses in tension and in compression (in Russian), SMRS (1971), 5, 44-46.

[5.100] D. Lamblin, G. Guerlement, Interaction curves for bending and axial forces of perfectly plastic curved I-beams, JSM 1 (1972), 2, 187-212.

[5.101] M. G. Lay, Yielding of uniformly loaded steel members, Proc ASCE JSD 91 (1965), 6, 49-66.

[5.102] N. A. Lebedinets, Evaluation of elastic-plastic deformations of hingeless arches (in Russian), SMTS 1 (1965), 145-157.

[5.103] L. N. Lebedinets, N. A. Lebedinets, Design of cross-sections of hingeless steel arches based on limit loads (in Russian), $\operatorname{Tr}$ DIIZDT 110 (1970), 102-106.

[5.104] - , - Evaluation of elastic-plastic displacements of shallow arches with geometry changes taken into account (in Russian), $\operatorname{Tr}$ DIIZDT (1971), 160-164.

[5.105] Yu. R. Lepik, Bending of an elastic-plastic bar in the case of prestressing (in Russian), UZTGU 73 (1959), 157-167.

[5.106] S. D. Leytes, Computer-aided analysis of stability of eccentrically compressed elasticplastic bars (in Russian), SM (1966), 68-76. 
[5.107] - Investigation of finite deformations of eccentrically compressed elastic-plastic bars (in Russian), PM 2 (1966), 4, 72-77.

[5.108] A. M. Lokoshtchenko, S. A. Shesterikov, Elastic-perfectly-plastic ring under external pressure (in Russian), Izv AN SSSR MTT (1970), 3, 125-126.

[5.109] A. Z. Lokshin, V. A. Tulin, Spreading of plastic zones and deformation of semi-finished strips under free bending with tension (in Russian), $\operatorname{Tr}$ LKI 75 (1971), 103-112.

[5.110] Y. Luscouarch, Programme de calcul en élasto-plasticité des structures planes, CoMét 13 (1976), 1, 40-50.

[5.111] O. V. Luzhin, Biaxial bending of prismatic rectangular beams with plastic hardening taken into account (in Russian), Izv VUZ SA (1959), 11-12.

[5.112] B. I. Lyubarov, Variable repeated loading of eccentrically compressed elastic-plastic bars (in Russian), IMEFM (1965), 94-104.

[5.113] —, Some problems of shakedown of elastic-plastic bodies (in Russian), SMRS (1967), $1,31-34$.

[5.114] - , On shakedown of cross-sections of bars under variable repeated loading (in Russian), NMRSK (1968), 177-183.

[5.115] A. A. Makarevitch, Stress analysis in a rectangular bar under bending with tension allowing for an arbitrary stress-strain diagram (in Russian), Izv VUZ SA (1967), 12, 163-172.

[5.116] —, Limit state of a section of a reinforced elastic-plastic beam (in Russian), $\operatorname{Tr}$ NISI (1970), 2, 80-94.

[5.117] B. P. Makarov, On the behaviour of beam-columns in the elastic-plastic range (in Russian), SMRS (1965), 5, 35-37.

[5.118] P. J. Marshall, J. S. Ellis, Ultimate biaxial capacity of box steel columns, Proc ASCE JSD 96 (1970), 9, 1873-1887.

[5.119] Ch. Massonnet, M. Save, Calcul plastique des constructions, Centre Belgo-Luxembourgeois d'Information de l'Acier, Vol. I, Bruxelles 1961; Vol. II, Bruxelles 1963.

[5.120] - , - Résistance limite d'une poutre courbe á parois minces soumise á flexion, Mem AIPC 23 (1963), 245-261.

[5.121] - , - Résistance limite d'une poutre courbe en caisson soumisse á flexion pure, $V H C$ (1964), 229-239.

[5.122] J. F. McDonough, The capacity of the biaxially loaded beam column at ultimate load, Doct. Diss. Univ. Cincinnati (1968), 145 pp.; Diss. Abstr. B29 (1969), 10, 3735.

[5.123] J. Mielniczuk, Nośność graniczna pręta z karbami obciążonego siłą podhużną i momentem zginającym, RI 19 (1971), 3, 407-421.

[5.124] A. Mioduchowski, Sprężysto-plastyczne skręcanie wycinka torusa, $R I 19$ (1971), 4, 629-636.

[5.125] F. E. Monasa, Deflections and stability behavior of elasto-plastic flexible bars, Trans ASME EA1 (1974), 2, 537-538.

[5.126] G. A. Morris, S. J. Fenves, Approximate yield surface equations, Proc ASCE JEMD 95 (1969), 4, 937-954.

[5.127] N. L. Moshensky, Application of an electronic model to the solution of the problem of elastic-plastic combined bending of a bar (in Russian), $\operatorname{Tr}$ LKI 38 (1962), 115-125.

[5.128] M. Mrowiec, Nośność graniczna niejednorodnego łuku kolistego o zmiennym przekroju, obciążonego ciśnieniem wewnętrznym, CT 68 (1963), 4, 4-8.

[5.129] K. K. Mukhanov, Yu. V. Sobolev, Eccentric compression of a stepped column under various ratios of loadings (in Russian), $M K$ (1966), 307-324.

[5.130] A. Nádai, Der Beginn des Fliessvorganges in einem tordierten Stab, ZAMM 3 (1923), 6, 442-444.

[5.131] T. Nonaka, Some interaction effects in a problem of plastic beam dynamics, Trans ASME E34 (1967), 3, 623-630, 631-637, 638-643.

[5.132] T. Ohta, T. Yamasaki, Elasto-plastic analysis of steel structures, Trans JSCE 3 (1972), 2, 152-153. 
[5.133] E. T. Onat, W. Prager, The influence of axial forces in the collapse loads of frames, Proc lst MCSM (1953), 40-42.

[5.134] -, -, Limit analysis of arches, JMPS 1 (1953), 1, 77-89.

[5.135] J. Orkisz, Principles of choosing a multipointed equivalent cross-section for elastic-plastic beams, BAP SST 10 (1962), 10, 405-414.

[5.136] - , Interaction curves for multi-point equivalent cross-sections of elastic-plastic beams, BAP SST 10 (1962), 11, 451-460.

[5.137] J. Orkisz, M. Życzkowski, Differential equations of elastic-plastic bending of beams with multi-point cross-sections, BAP SST 12 (1964), 4, 227-236.

[5.138] J. Orkisz, Sprężysto-plastyczne ugięcia prętów słabo zakrzywionych o dowolnym przekroju, RI 14 (1966), 4, 673-680.

[5.139] J. Orkisz, M. Życzkowski, Application of the method of equivalent multipoint cross-sections to the calculation of finite elastic-plastic deflections of beams, BAP SST 14 (1966), 11-12, 651-660 (English summary); RI 14 (1966), 4, 681-698 (Polish full text).

[5.140] A. Ormerod, The plastic bending of beams loaded in non-principal planes, CEPWR 54 (1954), 1179-1191.

[5.141] M. V. Pankova, On the limit carrying capacity of a curved beam (in Russian), IZh MTT (1969), 2, 170-172.

[5.142] D. Pantchovski, A graphical and a graphoanalytical method of calculation of beams with asymmetric cross-section, loaded by bending and normal force in the elastic-plastic range (in Bulgarian), GVTUZ PM 3, 1967 (1968), 2, 147-159.

[5.143] A. Phillips, Bending with axial force of curved bars in plasticity, $J A M 19$ (1952), 327-330.

[5.144] V. V. Pinadzhyan, On the limit state of short eccentrically compressed $H$-columns in the case of biaxial eccentricity of applied loadings (in Russian), DAN ArmSSR 21 (1955), 2.

[5.145] —, Some problems of the limit state of compressed elements of steel structures (in Russian), Izd. Akad. Nauk Arm. SSR, Erevan 1956.

[5.146] —, Strength and deformations of compressed bars of metal structures (in Russian), Nauka, Erevan 1971.

[5.147] F. A. Pivnev, A method of deflection analysis of elastic-plastic two-hinged steel arches (in Russian), $\operatorname{Tr} K I I Z D T 28$ (1958), 153-172.

[5.148] G. Prathap, T. K. Varadan, The inelastic large deformation of beams, Trans ASME E43 (1976), 4, 689-690.

[5.149] C. V. Rabinovitch, A graphoanalytical method of limit analysis in the case of bending with tension (in Russian), VM (1958), 2, 19-22.

[5.150] M. Radwańska, Z. Waszczyszyn, Calculation of finite elastic-plastic deflections of weakly curved bars, BAP SST 21 (1973), 7-8, 333-340 (English summary); RI 20 (1972), 4, 479-496 (Polish full text).

[5.151] A. Raithel, B. Barbarito, La rottura delle sezioni pressoinflesse nel cemento armato, GGC 6 (1959).

[5.152] M. I. Razumikhin, Yu. B. Drobot, Analysis of the technological process of bending with tension of rolled elements (in Russian), KSP (1965), 9, 18-22.

[5.153] G. Ritter, Zweiachsige Biegung mit Längskraft für Rechteckquerschnitte nach dem Traglastverfahren, WZHABW 12 (1965), 1, 81-89.

[5.154] J. M. Roberts, T. Sheppard, On the mechanics of the tension-levelling process, JIM 99 (1971), Oct., 239-301.

[5.155] G. I. Rozenblat, Some problems of the analysis of arches beyond the elastic limit (in Russian), ST MISI 27 (1957), 133-161,

[5.156] A. R. Rzhanitsyn, On some relations for a rectangular section of a beam beyond the elastic limit (in Russian), Izv AN SSSR MM (1963), 6, 17-19.

[5.157] —, On the problem of a momentary stiffness of a cross-section (in Russian), SMRS (1966), 2, 7-11. 
[5.158] B. de Saint-Venant, Mémoire sur l'etablissement des équations differentielles..., JMPA II, 16 (1871), 373-382.

[5.159] S. Santathadaporn, W. F. Chen, Tangent stiffness method for biaxial bending, Proc ASCE JSD 98 (1972), 1, 153-163.

[5.160] M. Save, On yield conditions in generalized stresses, $Q A M 19$ (1961), 3, 259-267.

[5.161] A. Sawczuk, M. Janas, Load-carrying capacities of arches with non-homogeneous properties of the cross-section, BAPSST9 (1961), 5, 283-290.

[5.162] V. G. Sebeshev, Limit equilibrium of a circular two-hinged arch (in Russian), Izv VUZ SA, (1972), 1, 35-41.

[5.163] F. B. Seely, J. O. Smith, Advanced mechanics of materials, 2nd ed., Wiley, New York 1952.

[5.164] S. V. Serensen, V. P. Kogayev, R. M. Shneyderovitch, Load-carrying capacity and strength calculations of machine elements (in Russian), 2nd ed., Mashgiz, Moskva 1963.

[5.165] H. Shakir-Khalil, G. S. Tadros, Plastic resistance of mild steel rectangular sections, SEng $\mathbf{5 1}$ (1973), 7, 239-249.

[5.166] M. K. Shen, G. Thierauf, Berechnung der Verformungen eines elastisch-plastischen Stabelementes unter gleichzeitiger Wirkung von Biegemoment und Normalkraft, BI 46 (1971), 9, 342-344.

[5.167] R. M. Shneyderovitch, Elastic-plastic bending of bars and bar systems (in Russian), Izv AN SSSR OTN (1958), 3, 130-134.

[5.168] P. I. Sikalo, Deformation of flexible steel beam-columns in the elastic-plastic range (in Russian), SMTS 10 (1970), 147-155.

[5.169] Ya. Z. Skomorovsky, On the analysis of elastic-plastic deformations in the manufacturing process of thin-walled tubes (in Russian), Tr VNII SMT 25 (1971), 337-368.

[5.170] R. A. Skripnikova, Spatial deformation of an inelastic thin-walled bar under compression with biaxial eccentricity (in Russian), SMRS (1974), 3, 32-35.

[5.171] Z. Sobotka, Approximate solution for yield states of torsion with shear and normal force, Stav C 23 (1975), 11, 889-892.

[5.172] B. L. Srivastava, S. C. Goyal, Effect of normal force on fully plastic moment of I-beam sections, JIEI MED 52 (1972), 5/3, 173-174; 11, 356-363.

[5.173] L. K. Stevens, Carrying capacity of mild-steel arches, Proc ICE 7 (1957), 493-514.

[5.174] —, Moment-curvature relationship for axially loaded members, CEPWR 53 (1958), 626, 904-906.

[5.175] I. A. Strelbitskaya, Biaxial bending of metal beams beyond the elastic limit (in Russian), ST ISM AN USSR 18 (1953).

[5.176] O. I. Strelbitska, Plastic moments for I and channel sections in biaxial bending (in Ukrainian), PM 4 (1958), 4, 453-461.

[5.177] O. I. Strelbitska, Yu. L. Biryukovitch, E. O. Pryadko, Experimental investigations on biaxial bending of rolled beams beyond the elastic limit (in Ukrainian), DAN URSR (1960), 1, 22-26.

[5.178] H. Sudzuki, E. Takano, H. Negisi, Passive unloading in plastic bending with tension (in Japanese), RUEC 17 (1964), 27-35; 18 (1965), 55-60.

[5.179] N. Kh. Suyunshkaliyev, Loading surfaces for a thin-walled tube under simultaneous bending and tension (in Russian), $\operatorname{Tr}$ TPI 156 (1975), 17-19.

[5.180] W. Swida, Die Berechnung von Stählernen Bogen unter Berücksichtigung der Tragfähigkeitsreserve im elastisch-plastischen Zustand, Stb 19 (1950), 3, 17-20; 19 (1950), 4, 29-34; 20 (1951), 2, 25-28.

[5.181] H. Tanaka, Traglastberechnung steifer Rahmen unter Berücksichtigung von Längskräften, BB 12 (1958), 11, 478-483.

[5.182] B. V. Terentev, Strength of an eccentrically compressed or stretched bar with a monosymmetric section in the limit state (in Russian), MPK 2 (1972), 87-90. 
[5.183] G. Thierauf, Traglastberechnung und Bemessung von Stockwerkrahmen mit Hilfe der linearen Programmierung, Stb 44 (1975), 1, 19-26.

[5.184] A. R. Toakley, Axial load effects in optimum rigid-plastic design, BS 5 (1970), 2, 111-115.

[5.185] A. D. Tomlenov, Theory of plastic deformation of metals, (in Russian), Metallurgia, Moskva 1972.

[5.186] O. N. Totsky, Biaxial bending of a steel beam in the plastic range (in Russian), SMRS (1964), 6-9.

[5.187] Y. Tozawa, Effect of additional tension on the elastic response in elastic-plastic bending (in Japanese), RRFT CU 7 (1956), 11, 1-9.

[5.188] L. L. Valter, A graphical method of limit analysis for a steel bar under simultaneous bending moment and normal force (in Russian), $\operatorname{Tr} L K I 16$ (1955), 56-59.

[5.189] P. I. Valukonis, A. P. Cižas, Conditions of plastic flow in an asymmetric I-section under simultaneous normal force and bending moment (in Russian), LMS (1972), 2, 61-66.

[5.190] - , - A design problem of elastic-plastic bar systems under simultaneous normal force and bending moment (in Russian), LMS (1973), 2, 75-81.

[5.191] R. V. Vebra, A. A. Cyras, Optimal plastic design of arches (in Russian), LMS (1970), 1, 85-95.

[5.192] S. Vinnakota, Y. Aoshima, Inelastic behaviour of rotationally restrained columns under biaxial bending, SEng 52 (1974), 7, 245-255.

[5.193] A. M. Wahl, Mechanical springs, McGraw-Hill, New York-Toronto-London 1963.

[5.194] A. J. Wang, W. Prager, Plastic twisting of a circular ring sector, JMPS 3 (1955), 169-175.

[5.195] Z. Waszczyszyn, M. Życzkowski, Finite elastic deflections of a stretchable beam on immovable supports, AMS 14 (1962), 1, 61-82.

[5.196] —, - Finite deflections of elastic-plastic beams, the stretchability of their axes being taken into account, BAP SST 11 (1963), 10, 547-558.

[5.197] Z. Waszczyszyn, Zastosowanie metody kollokacji do obliczania sprężysto-plastycznych ugięć belek o skrępowanej przesuwności podpór, RI 13 (1965), 2, 247-268.

[5.198] —, Uproszczone obliczanie sprężysto-plastycznych ugięć belek o skrępowanej przesuwności podpór, AIL 11 (1965), 3, 417-442.

[5.199] - Experimental verification of finite elastic-plastic deflections of beams on immovable supports, BAP SST 14 (1966), 3, 199-207 (English summary); MTS 3 (1965), 2, 35-54 (Polish full text).

[5.200] —, Application of multi-point equivalent cross-sections to the calculation of finite defiections of elastic-plastic beams with stretchable axis, AMech 3 (1967), 2, 219-235.

[5.201] - Application of the theory of finite deflections to calculations of elastic-plastic arches, AIL 16 (1970), 1, 55-74.

[5.202] J. C. Wilhoit, J. E. Merwin, The effect of axial tension on moment carrying capacity of pipe line stressed beyond the elastic limit, 3rd Annual Offshore Technol. Conf., Houston 1971, 293-296.

[5.203] J. Wölfel, Zustandsdiagramme des elastisch-plastisch verformten Balkens, MBT 11 (1962), $8,417-422$.

[5.204] M. Yamadá, K. Shirakawa, Elasto-plastische Biegeformänderungen von Stahlstützen mit I-Querschnitt, II, Stb 40 (1971), 3, 65-74.

[5.205] Y. Yokoo, T. Nakamura, M. Keii, The minimum weight design of multi-story building frames based upon the axial force-bending moment interaction yield condition, IUTAM OSD 1973 (1975), 497-517.

[5.206] K. Yoshimura, Inelastic behavior of steel $H$-shape columns subjected to repeated bending, MFE KyuU 34 (1975), 3, 157-193.

[5.207] M. A. Zadoyan, Plastic torsion of an incomplete torus (in Russian), DAN SSSR 223 (1975), 2, 318-321. 
[5.208] H. Ziegler, Ưber den Zusammenhang zwischen der Fliessbedingung eines starrplastischen Körpers und seinem Fliessgesetz, ZAMP 11 (1960), 5, 413-426.

[5.209] -, Über den Zusammenhang zwischen der Fliessbedingung eines elastisch-plastischen Körpers und seinem Fliessgesetz, ZAMP 12 (1961), 1, 53-68.

[5.210] —, On the theory of plastic potential, QAM 19 (1961), 39-44.

[5.211] M. Życzkowski, Theory of finite deflections of elastic-plastic beams, 2nd CMI 1956 (1957), 24-32 (English text); CT 62 (1957), 3, 1-4 (Polish text).

\section{Chapter VI}

[6.1] G. Albrecht, Beitrag zur mittragenden Breite von Plattenbalken im elasto-plastischen Bereich, TWM IKIB (1976), 7, $117 \mathrm{pp.}$

[6.2] Yu. N. Aleshinsky, Effect of shearing stresses on the load-carrying capacity of sections of a bar under bending with normal force (in Russian), $\operatorname{Tr} M I I Z D T 108$ (1959), 82-116.

[6.3] S. A. Ambartsumyan, M. A. Zadoyan, On the problem of elastic-plastic bending of beams (in Russian), Izv AN SSSR OTN (1958), 10, 130-132.

[6.4] E. Amstutz, Berechnung von Wabenträgern nach der Plastizitätstheorie, $S B 88$ (1970), 9, 167-173.

[6.5] R. Askari, Calcul de la charge ultime des grillages compte tenu de la rigidité à la torsion des poutres, CoMet 11 (1974), 1, 39-57.

[6.6] G. Augusti, Calcolo a rottura delle travi di parete sottile, IC 6 (1966), 20, 637-660.

[6.7] - , Full plastic torque of I-beams, IJMS 8 (1966), 641-649.

[6.8] G. Augusti, R. Scuderi, Sul collasso plastico di travi a mensola con sezione a doppio $T, \mathrm{G}$. Genovese, Napoli 1967.

[6.9] G. Augusti, On the limit analysis of I-beams with warping restraints, EP (1968), 21-40.

[6.10] B. Åkesson, J. Bäcklund, Plastische Traglast gemischt tordierter Stäbe mit offenem Profil, Stb 42 (1973), 3.

[6.11] J. F. Baker, J. W. Roderick, Further tests on beams and portals, Trans IW 3 (1940), 83.

[6.12] V. I. Batsikadze, Bending with torsion of beams beyond the elastic limit (in Russian), $\operatorname{Tr} \operatorname{GrPI}(1957), 9,35-42$.

[6.13] Z. Bažant, Mezni stav obdélnikového průřezu pri současném pủsobeni ohybovych momentů $M_{x}, M_{y}$, posouvajicich sil $T_{x}, T_{y}$ a osove sily $N$, Stav $C$ 15 (1967), 7, 401-412.

[6.14] -, Mezní stav obdélnikového prủřezu a tenkostěnného průřezu tvaru i př́i složeném namáháni, StavC 17 (1969), 1, 51-61.

[6.15] Z. Bažant, Mezní plastický stav masívnich průřezu a tenkostěnných prủřezu otevřených i uzavřených profilů při složeném namáháni, SVUTB (1971), 1-4, 177-198.

[6.16] Z. Bažant, S. Smiřák, Plastic limit analysis of thin walled rectangular section under bending, shear and torsion, SVUTB (1971), 1-4, 199-205.

[6.17] Z. Bažant, Plastic analysis of cross sections under combined stress, SVUTB (1972), 1-4, 89-95.

[6.18] L. M. Belenky, On the effect of concentration of loading in the problems of elastic-plastic bending (in Russian), SMRS (1963), 5, 28-32.

[6.19] C. Berardi, Il momento flettente limite in presenza della caratteristica tagliante nei profilati metallici a doppio $T, A C M 8$ (1956), 2, 79-83.

[6.20] J. Betten, Bemerkungen zum Versuch von Hohenemser, ZAMM 55 (1975), 3, 149-157.

[6.21] E. A. Beylin, On the limit state of bars under bending and torsion (in Russian), Sb. 17 Nauchn. Konf. Leningr. Inzh.-Str. Instituta, Sekcya Matem.-Sopr. Mat. (1959), 11-16.

[6.22] - , On the limit state of bars under bending and bending with compression (in Russian), SMRS (1961), 1, 18-23.

[6.23] - , On the limit state of a bar under torsion with additional normal force (in Russian), ST LIIZDT 190 (1962), 229-235. 
[6.24] N. I. Bezukhov, On the theory of plastic bending (in Russian), VIT (1936), 10.

[6.25] - Effect of loading path on the load carrying capacity of a cross-section under combined stress (in Russian), Izv AIA 8 (1953).

[6.26] B. F. Bochkov, Elastic-plastic bending of frames (in Russian), $\operatorname{Tr} \operatorname{GPI} 14$ (1960), 10, 35-62.

[6.27] B. Boni, M. Kleiber, Limit analysis of plane bending-and-torque supporting grids, AIL 21 (1975), 1, 69-87.

[6.28] N. S. Boulton, B. Boonsukha, Plastic collapse loads for circular-arc bow girders, Proc ICE 13 (1959), June, 161-178.

[6.29] N. S. Boulton, Plastic twisting and bending of an I-beam in which the warp is restricted, IJMS 4 (1962), 6, 491-502.

[6.30] J. E. Bower, Design of beams with web openings, Proc ASCE JSD 94 (1968), 3, 783-807.

[6.31] J. E. Bower, Ultimate strength of beams with rectangular holes, Proc ASCE JSD 94 (1968), 6, 1315-1337.

[6.32] D. S. Brooks, The elasto-plastic behaviour of a circular bar loaded by axial force and torque in the strain hardening range, IJMS 11 (1969), 1, 75-85.

[6.33] B. M. Broude, Limit analysis of beams allowing for shearing stresses (in Russian), ITS 5 (1951).

[6.34] —, Limit states of steel beams (in Russian), Gos. Izd. Stroit. i Arkh., Moskwa-Leningrad 1953.

[6.35] J. Bródka, Zginanie i skręcanie dwuteownika cienkościennego w fazie odkształceń sprężystoplastycznych, IBud 18 (1961), 10, 395-401.

[6.36] P. G. Bytchkov, Load-carrying capacity of prismatic bars under bending with additional torsion (in Russian), Tr MTI (1955), 3, 181-207.

[6.37] -, Load-carrying capacity of non-circular sections under pure and restrained torsion and bending (in Russian), $\operatorname{Tr} M T I$ (1957), 5, 257-274.

[6.38] C. R. Calladine, Engineering plasticity, Pergamon Press, Oxford 1969.

[6.39] —, A plastic theory for collapse of plate girders under combined shearing force and bending moment, SEng 51 (1973), 4, 147-154.

[6.40] M. Capurso, Stati limiti nelle lastre travi, GGC 105 (1967), 6-7, 427-443.

[6.41] W. P. Chen, E. M. Shoemaker, Collapse loads of cantilever beams under end shear, AMech 13 (1972), 191-203.

[6.42] E. R. Chernaya, Analysis of deformation of thin-walled bars under oblique bending with torsion beyond the elastic limit (in Russian), PM 1 (1965), 11, 71-80.

[6.43] -, Experimental investigation of I-beams under oblique bending with torsion beyond the elastic limit (in Russian), SK RMNTS 11 (1969), 63-70.

[6.44] N. L. Chernov, E. R. Chernaya, Experimental investigation of thin-walled beams under biaxial bending with torsion due to moving load beyond the elastic limit (in Russian), PM 2 (1966), 2, 63-69.

[6.45] N. L. Chernov, On elastic-plastic deflections of I-beams under moving load (in Russian), PM 3 (1967), 8, 50-59.

[6.46] N. L. Chernov, E. R. Chernaya, Strength and deformability of thin-walled bars under biaxial bending with torsion due to moving load beyond the elastic limit (in Russian), SMTS 6 (1968), 141-149.

[6.47] N. L. Chernov, S. D. Kudryavtsev, Deformability of thin-walled bars taking account of shear under moving load beyond the elastic limit (in Russian), SMTS 11 (1970), 140-149.

[6.48] C. Clavuot, Welle und Rohr aus starrplastischem Material unter Zug und Torsion, Diss. Doct. Math. ETH Zürich, Leemann A. G., 1959.

[6.49] R. J. Clifton, An analysis of combined longitudinal and torsional waves in a thin-walled tube, Report No. 5, Brown University, May 1960.

[6.50] - An analysis of combined longitudinal and torsional plastic waves in a thin-walled tube, USNCAM 5 (1966), 465-480. 
[6.51] R. J. Clifton, Elastic-plastic boundaries in combined longitudinal and torsional plastic wave propagation, Trans ASME E35 (1968), 4, 782-786.

[6.52] M. Como, Il problema dello sforzo di taglio nel calcolo a rottura nelle sezioni in cemento armato, AITCN 38.

[6.53] I. V. Davidov, P. I. Zaytsev, An investigation of the effect of the shearing force on the load-carrying capacity of beams made of various kinds of steel (in Russian), DPM 3 (1966), 70-74.

[6.54] I. V. Davidov, Limit load of a thin-walled I-beam under oblique bending with torsion (in Russian), STVF 24 (1971), 37-43.

[6.55] V. I. Davydov, Shearing stresses in plastic bending (in Russian), $\operatorname{Tr}$ GPIII (1956), 3, 49-60.

[6.56] K. S. Dinno, S. S. Gill, The plastic torsion of I-sections with warping restraints, IJMS, 6 (1964), 27-43.

[6.57] K. S. Dinno, W. Merchant, A procedure for calculating the plastic collapse of I-sections under bending and torsion, SEng 43 (1965), 7, 219-221.

[6.58] D. C. Drucker, The effect of shear on the plastic bending of beams, JAM 23 (1956), 4, 509-514.

[6.59] V. I. Dunayevsky, V. S. Sviderskaya, I. Z. Entin, Effect of the shearing force on the strain distribution in elastic-plastic bending of beams (in Russian), Izv VUZ M 12 (1970), 16-18.

[6.60] G. Yu. Dzhanelidze, A. I. Lurie, The St.-Venant Problem for naturally twisted rods (in Russian), DAN SSSR 24 (1939), 1-2.

[6.61] G. Yu. Dzhanelidze, Kirchhoff conditions for naturally twisted rods (in Russian), $\operatorname{Tr} L P I$ (1946), 1.

[6.62] F. Ellyin, R. Deloin, The effect of shear on yielding of structural members, IJSS 8 (1972), 3, 297-314.

[6.63] M. I. Erkhov, Limit equilibrium of perfectly plastic bars with arbitrary section under combined stress state (in Russian), $\operatorname{Tr} C N I I S K 4$ (1961), 151-167.

[6.64] D. G. Eyre, Inelastic shear deflection of steel beams, Proc ASCE JSD 99 (1973), 10, 19851997.

[6.65] L. E. Farmer, P. M. Ferguson, T-beams under combined bending, shear and torsion, JACI 64 (1967), 11, 757-766.

[6.66] B. Fontaine, Comportement des tubes métalliques munis de fenêtres et soumis à flexion et torsion, $A c B 31$ (1966), 7-8, 351-354.

[6.67] V. Franciosi, E. Giangreco, Il calcolo a rottura in presenza di sforzo normale, momento flettende e sforzo di taglio, GGC 92 (1954), 5, 354-361.

[6.68] H. Fukuoka, Infinitesimal plane waves in elastic-plastic tubes under combined tensiontorsion loads, 16th JCAM 1966 (1967), 109-113.

[6.69] -, Combined tension-torsion elastic-plastic waves as propagating singular surfaces, 20th JCAM 1970 (1971), 231-235.

[6.70] H. Fukuoka, T. Masui, Incremental impact loading of plastically prestressed aluminum by combined tension-torsion load, BJSME 18 (1975), 116, 104-113.

[6.71] A. Galli, V. Franciosi, Introduzione della caratteristica tagliante nel calcolo a rottura, Ing 27 (1953), 8, 893-897.

[6.72] P. V. Galptchian, Plastic state of prismatic bars with sections in the form of circular sectors and circular ring sectors under simultaneous torsion and bending (in Russian), Izv $A N$ ArmSSR M 29 (1976), 3, 31-41.

[6.73] F. A. Gaydon, On the combined torsion and tension of a partly plastic circular cylinder, QJMAM 5 (1952), 1, 29-41.

[6.74] F. A. Gaydon, H. Nuttall, On the combined bending and twisting of various sections, JMPS 6 (1957), 1, 17-26.

[6.75] A. V. Gemmerling, Load-carrying capacity of I-beams (in Russian), Sbornik Statey CNIPS "Eksperimentalnye Issledovanya Stalnykh Konstrukciy", Moskva 1950. 
[6.76] S. S. Gill, J. K. G. Loucher, An experimental investigation of plastic collapse of structural members under combined bending and torsion, SEng 42 (1964), 12, 423-428.

[6.77] R. P. Goel, L. E. Malvern, Biaxial plastic simple waves with combined kinematic and isotropic hardening, Trans ASME E37 (1970), 4, 1100-1106.

[6.78] Yu. V. Goldshteyn, M. A. Solomeshtch, On the optimal design of elastic-plastic bar systems (in Russian), Izv VUZ SA 12 (1970), 43-48.

[6.79] C. D. Goode, M. A. Helmy, Design of rectangular beams subjected to combined bending and torsion, Con 1 (1967), 7, 241-244.

[6.80] V. N. Gorelov, A. S. Neymark, On the stress state in an orthotropic plastic hollow cylinder under tension with torsion (in Russian), $M e K u 9$ (1976), 20-25.

[6.81] B. Goschy, I szelvényü tartó határállapota egyidejü hajlitás és nyirás esetén, $M S z$ 13 (1963), $10,470-473$.

[6.82] S. C. Goyal, The effect of shear on the plastic bending of I-beam, 7th CMI (1961), 77-106.

[6.83] A. P. Green, A theory of the plastic yielding due to bending of cantilevers and fixed-ended beams, JMPS 2 (1954), 1, 1-15; 3 (1955), 2, 143-155.

[6.84] A. P. Green, B. B. Hundy, Plastic yielding of I-beams, Eng 184 (1957), 74-76, 112-115.

[6.85] O. I. Greste, The fully plastic resistance of prismatic beams subjected to combined bending and torsion, Univ. N.S.W., Kensington-Sydney 1968.

[6.86] A. P. Gusenkov, M. P. Gavrilov, Strength under certain regimes of combined low-cycle fatigue loading (in Russian), Mash (1975), 1, 62-69.

[6.87] A. H. Hall, N. M. Newmark, Shear deflection of wide-flange steel beams in the plastic range, Trans ASCE 122 (1957), 666-687.

[6.88] C. Hartung, Vergleich zweier Formänderungsgesetze für plastische Werkstoffe mit isotroper Werkstoffverfestigung unter Zug-, Druck- und Schubbeanspruchung bei endlichen Formänderungen, RhA 8 (1969), 1, 60-70.

[6.89] A. W. Hendry, The stress distribution in a simply supported beam of I-section carrying a central concentrated load, Proc SESA 7 (1949), 91.

[6.90] J. Heyman, The limit design of space frames, JAM 18 (1951), 157-162.

[6.91] —, The limit design of a transversely loaded square grid, JAM 19 (1952), 153-158.

[6.93] J. Heyman, V. L. Dutton, Plastic design of plate girders with unstiffened webs, WMF 22 (1954), 265.

[6.93] J. Heyman, The full plastic moment of an I-beam in the presence of shear force, JMPS 18 (1970), 5, 359-365.

[6.94] R. Hill, M. P. L. Siebel, On combined bending and twisting of thin tubes in the plastic range, PhM 42 (1951), 722-733.

[6.95] - , - On the plastic distortion of solid bars by combined bending and twisting, JMPS 1 (1953), 3, 207-214.

[6.96] P. G. Hodge, Jr., Interaction curves for shear and bending of plastic beams, JAM 24 (1957), 3, 453-456.

[6.97] P. G. Hodge, R. Sankaranarayanan, The determination of safe loads of beams subjected to combined twisting and bi-axial bending moments, Trans ASME E26 (1959), 3, 442-447.

[6.98] M. R. Horne, The plastic theory of bending of mild steel beams with particular reference to the effect of shear forces, Proc RS A207 (1951).

[6.99] -, The full plastic moments of sections subjected to shear force and axial load, $B W J$ 5 (1958), 4, 170-178.

[6.100] B. C. Hoskin, Torsional rigidity of a prismatic bar of circular section under combined tension and torsion, AJAS 12 (1961), 2, 255-256.

[6.101] O. I. Ibrayev, An estimation of the effect of loading path on the load-carrying capacity of bars (in Russian), DAN UzbSSR (1964), 3, 9-13.

[6.102] -, Effect of loading path on the load-carrying capacity of bars in the case of linear plastic hardening (in Russian), Izv VUZ SA (1965), 2, 40-45. 
[6.103] E. O. Imegwu, Plastic flexure and torsion, JMPS 8 (1960), 2, 141-146.

[6.104] —, Combined plastic bending and torsion, JMPS 10 (1962), 3, 277-282.

[6.105] H. Ishikawa, Elastic-plastic bars under simultaneous bending and torsion (in Japanese), Trans JSME 38 (1972), 308, 700-714.

[6.106] -, A theoretical method for solving an elasto-plastic bending problem, BJSME 15 (1972), $86,928-939$.

[6.107] -, On the elastoplastic problem of cantilever subject to combined bending and twisting, BJSME 15 (1972), 90, 1481-1493.

[6.108] —, Elasto-plastic stress analysis of prismatic bar under combined bending and torsion, ZAMM 53 (1973), 1, 17-30.

[6.109] S. I. Ivanov, A solution of the problem of fully plastic torsion with tension of prismatic bars using the method of elastic solutions combined with finite differences (in Russian), $\operatorname{Tr}$ KuyAI (1958), 4, 59-67.

[6.110] W. Johnson, N. R. Chitkara, A. S. Ranshi, Plane-stress yielding of cantilevers in bending due to combined shear and axial load, JSA 9 (1974), 2, 67-77.

[6.111] P. Juhás, Plastická únosnost ocel'ovych nosnikov namáhaných ohybom a smykom, Stav $\check{C}$ 23 (1975), 10, 761-781.

[6.112] L. M. Kachanov, Stability of elastic-plastic equilibrium of a shaft under compression with torsion (in Russian), DAN SSSR 88 (1953), 4, 627-630.

[6.113] B. P. Kishkin, Solution of stress concentration problems in the range of elastic-plastic deformations (in Russian), UN 1 (1971), 136-146.

[6.114] F. E. Klimenko, Effect of the shearing force on the load-carrying capacity of a section of beams in bending (in Russian), DLPI M 3 (1958), 1-2, 47-54.

[6.115] K. Klöppel, M. Yamada, Fliesspolyeder des Rechteck- und I-Querschnittes unter der Wirkung von Biegemoment, Normalkraft und Querkraft, Stb 27 (1958), 11, 284-290; 28 (1959), 4, 112.

[6.116] V. O. Kolgadin, Elastic-plastic deformations of a thin-walled bar under biaxial bending with torsion (in Ukrainian), PM 10 (1964), 1, 55-63.

[6.117] - , Torsion with tension of a narrow rectangular bar beyond the elastic limit (in Russian), PM 1 (1965), 7, 132-135.

[6.118] V. A. Kolgadin, Deformation analysis in an I-beam under bending with torsion beyond the elastic limit (in Russian), PM 1 (1965), 10, 72-77.

[6.119] C. F. Kollbrunner, N. Hajdin, Dünnwandige Stäbe, Band 2, Stäbe mit deformierbaren Querschnitten, Nicht-elastisches Verhalten dünnwandiger Stäbe, Springer, Berlin-HeidelbergNew York 1975.

[6.120] S. Komatsu, T. Sakimoto, Elasto-plastic behavior of steel members under combined torsion and compression, TROU 23 (1973), 681-696.

[6.121] I. M. Korovin, Combined deformation of prismatic bars (in Russian), Izv AN SSSR MTT (1970), 2, 138-146.

[6.122] V. V. Kozlyakov, On the problem of elastic-plastic bending of I-beams made of linearly hardening material accounting of shear and axial forces (in Russian), Tr LKI 46 (1964), 25-37.

[6.123] N. A. Kravtsov, Investigation of the effect of the shearing force on the limit moment in continuous beams under moving load (in Russian) Izv VUZ M 8 (1963), 93-100.

[6.124] V. Krrupka, Mezní stav nosnikú tvaru i při krutu za ohybu, ISt 2 (1954), 1, 31-36.

[6.125] V. N. Kukudzhanov, Elastic-plastic bending of thin-walled bars allowing for shearing stresses (in Russian), $\operatorname{Tr} M F T I 1$ (1958), 97-114.

[6.126] L. P. Kunitsky, Load-carrying capacity of steel beams with shear forces taken into account (in Russian), ST KISI 12 (1959), 188-217.

[6.127] - , Load-carrying capacity of steel beams under moving loads with shear forces taken into account (in Russian), SMTS 2 (1965), 148-159. 
[6.128] M. Kurata, K. Sonoda, Minimum weight design of grillage girder, Trans JSCE 3 (1972), 2, 136-137.

[6.129] T. Kusuda, Effect of shearing and normal forces on the load-carrying capacity of a beam section (in Japanese), JSNA 105 (1959), 127-135.

[6.130] A. I. Kuzin, On plastic beam analysis under combined stresses (in Russian), $\operatorname{Tr}$ GISI 45 (1964), 79-90.

[6.131] - , On the problem of experimental evaluation of limit load of steel beams (in Russian), $\operatorname{Tr}$ GISI 50 (1967), 95-101.

[6.132] - Peculiarities of the development of plastic deformations in metal beams under combined state of stress (in Russian), Tr GISI 57 (1970), 67-75.

[6.133] A. I. Kuznetsov, On inelastic cyclic deformation (in Russian), IUP 9 (1973), 51-62.

[6.134] P. Lampert, Bruchwiderstand von Stahlbetonbalken unter Torsion und Biegung, Dis, Dokt. ETH Zürich, R. Langer, Zürich 1970.

[6.135] B. B. Lampsi, Strength of I-beams (in Russian), Izv VUZ SA (1959), 1, 66-80.

[6.136] - , On the problem of formation of a yield hinge in the web of a metallic I-beam (in Russian), $\operatorname{Tr}$ GISI 67 (1974), 10-15.

[6.137] R. H. Lance, D. N. Robinson, Limit analysis of ductile fiber-reinforced structures, Proc ASCE JEMD 98 (1972), 1, 195-209.

[6.138] T. van Langendonck, Einfluss der Querkraft auf die Grösse des Biegemoments, das die plastischen Gelenke erzeugt, BT 33 (1965), 3, 84-87.

[6.139] C. F. A. Leth, The effect of shear stresses on the carrying capacity of I-beams, DAM Rept, A11-107, Brown Univ., Providence 1954.

[6.140] S. D. Leytes, An approximate analysis of elastic-plastic deformations on the basis of the hypothesis of stress stabilization (in Russian), 2nd VSTPM (1964), 244.

[6.141] U.S. Lindholm, Some experiments in dynamic plasticity under combined stress, $M B M D L$ (1968), 77-95.

[6.142] J. Lindner, Näherungsweise Ermittlung der Traglasten von auf Biegung und Torsion beanspruchten I-Trägern, $B T 48$ (1971), 5, 160-170.

[6.143] J. Lipkin, R. J. Clifton, An experimental study of combined longitudinal and torsional plastic waves in a thin-walled tube, 12th ICAM 1968 (1969), 292-304.

[6.144] -, -, Plastic waves of combined stresses due to longitudinal impact of a pretorqued tube, Trans ASME E37 (1970), 4, 1107-1120.

[6.145] E. Longbottom, J. Heyman, Experimental verification of the strengths of plate girders designed in accordance with the revised British Standard 153, Proc ICE 5 (1956), 2, 462-521.

[6.146] Le-Wu Lu, Linearized interaction curves for plastic beams under combined bending and twisting, AIAA 1 (1963), 3, 706-707.

[6.147] K. Lundgren, Vlasov torsion of elastic-ideally-plastic beams of thin-walled open cross, section, IJMS 18 (1976), 3, 105-109.

[6.148] S. M. Makky, Fracture surfaces in round bars under combined tension and torsion, ZAMM 45 (1965), 6, 433-440.

[6.149] G. F. Malikov, Calculation of highly curved beams based on the limit equilibrium method (in Russian), $\operatorname{Tr}$ MEI 29 (1957), 5-18.

[6.150] J. B. Martin, On the elastic-plastic behaviour of certain bents under transverse loading, IJMS 3 (1961), 1-2, 1-12.

[6.151] P. C. Massey, The torsional rigidity of steel I beams already yielding under uniform bending moment, CEPWR 58 (1963), 680, 367-371; 681, 488-492.

[6.152] G. Mets, Investigation of a plastically deformed metal under combined state of stress (in Russian), Tr TalPI A289 (1970), 39-50.

[6.153] R. A. Mezhlumyan, Bending and torsion of thin-walled cylindrical shells beyond the elastic limit (in Russian), PMM 14 (1950), 254-264. 
[6.154] R. A. Mezhlumyan, Applied theory of elastic-plastic shells and its application to structural analysis (in Russian), IS 10 (1951), 35-70.

[6.155] - , An inverse problem of applied theory of plasticity and load-carrying capacity of structures in the case of plastic hardening (in Russian), Izv AN SSSR OTN (1955), 12, 80-95.

[6.156] H. Mii, Plastic deformation of light-metal bars strained with combined tension and torsion, JJSAM 3 (1950), 195-198.

[6.157] -, Plastic deformation of light-metal bars strained with combined bending and torsion, JJSAM 5 (1952), 11-14.

[6.158] M. Mikkola, An analysis of physically nonlinear structures, Julk. Valtion tekn. tutkimnslaitos 112 (1966), Helsinki, 98 pp.

[6.159] P. M. Miller, Numerical analysis of combined bending and torsion of a prismatic bar using rigid-plastic and workhardening plasticity theories, Doct. Diss. Michigan State Univ. (1966).

[6.160] P. M. Miller, L. E. Malvern, Numerical solution of Piechnik's equation for the combined bending and torsion of rigid-plastic bars, Proc 10th MCSM (1967), Colorado State Univ., 571-580.

[6.161] - , - Numerical analysis of combined bending and torsion of a work-hardening plastic square bar, Trans ASME E34 (1967), 4, 1005-1010.

[6.162] I. N. Mirolyubov, An approximate strength analysis of non-uniformly stressed bars with plastic strains taken into account (in Russian), $\operatorname{Tr} L V M I$ (1957), 6, 28-69.

[6.163] A. N. Mitinsky, Shearing stresses in elastic-plastic bending (in Russian), $\operatorname{Tr}$ VNTOS III/3 (1939).

[6.164] V. A. Monakhov, V. I. Sebekina, On the load-carrying capacity of shallow arches (in Russian), SMRS (1976), 5, 30-35.

[6.165] G. A. Morris, S. J. Fenves, Elastic-plastic analysis of frameworks, Proc ASCE JSD 96 (1970), 5, 931-946.

[6.166] J. Mutermilch, E. Olszewski, M. Lubiński, Wymiarowanie konstrukcji stalowych według stanów granicznych z uwzględnieniem naprężeń stycznych i odkształceń, AIL 2 (1956), $1-2,15-44$.

[6.167] C. D. Myers, M. A. Eisenberg, The effect of radial inertia on combined plastic stress wave propagation in thin-walled tubes, Trans ASME E41 (1974), 3, 619-624.

[6.168] B. G. Neal, Effect of shear force on the fully plastic moment of an I-beam, JMES 3 (1961), 3, 258-266.

[6.169] -, Effect of shear and normal forces on the fully plastic moment of an I-beam, JMES 3 (1961), 3, 279-285.

[6.170] - The effect of shear and normal forces on the fully plastic moment of a beam of rectangular cross-section, Trans ASME E28 (1961), 2, 269-274.

[6.171] —, Limit load of a cantilever in plane stress, EP (1968), 473-488.

[6.172] Yu. V. Nemirovsky, Effect of shear and of rotational inertia on the dynamic bending of plastic beams (in Russian), DSS 19-20 (1974), 83-100.

[6.173] G. M. Nosov, Evaluation of optimal depth of plastic beams with shear force taken into account (in Russian), Tr DPI 54 (1960), 101-113.

[6.174] M. Ohnami, K. Shiozawa, Influence of strain history on plastic deformation of polycrystalline metals subjected to cyclic stressing, 15th JCMR (1972), 106-111.

[6.175] T. Ohta, T. Nakazawa, K. Saito, Elastic-plastic analysis of steel tubes subject to bending and torsion (in Japanese), TRKU 46 (1974), 4, 502-509; 47 (1974), 2, 66-71.

[6.176] E. T. Onat, R. T. Shield, Remarks on combined bending and twisting of thin tubes in the plastic range, JAM 20 (1953), 345-348.

[6.177] -, - , The influence of shearing forces on the plastic bending of wide beams, 2nd USNCAM 1954 (1955), 535-537. 
[6.178] J. Oudin, Y. Ravalard, Contribution à l'étude du comportement limite des plaques rectangulaires en déformation plane, JM 13 (1974), 2, 225-244.

[6.179] S. A. Palchevsky, Evaluation of the load-carrying capacity of steel bars in some cases of combined stress state (in Russian), ST KISI 8 (1948).

[6.180] B. V. Panov, Static load-carrying capacity of cold-worked bars under tension with torsion (in Russian), Mash (1971), 1, 65-71.

[6.181] P. F. Papkovitch, Fundamentals of the theory of elastic-plastic statically determinate beams (in Russian), Tr VNTOS 111 (1939), 3.

[6.182] H. Payne, S. J. Czyzak, On the torsion of a thin-wall cylinder following a plastic extension, JMPS 8 (1960), 1, 39-44.

[6.183] A. Phillips, Introduction to plasticity, Ronald Press, New York 1956.

[6.184] G. M. Pinsker, Relations between generalized stresses in the limit state of open thin-walled profiles (in Russian), $\operatorname{Tr}$ VNIPIE 7 (1975), 118-126.

[6.185] V. M. Radhakrishnan, Stress ratchetting under tension and cyclic torsion, NED 34 (1975), 3, 379-387.

[6.186] -, On stress ratchetting, Trans ASME E42 (1975), 4, 884-886.

[6.187] Z. M. Rakhimbekova, Elastic-plastic bending of beams in the case of linear plastic hardening allowing for shearing deformations (in Russian), ST MISI 84 (1970), 306-311.

[6.188] Z. M. Rakhimbekova, L. V. Bozhkova, Effect of the shearing force on the magnitude of deflection in bending of steel beams (in Russian), $\operatorname{Tr}$ VNIUI 43 (1972), 221-226.

[6.189] Z. M. Rakhimbekova, On the problem of shearing stress distribution along the height of an I-beam beyond the elastic limit (in Russian), Tr VNIUI 44 (1972), 170-175.

[6.190] Yu. A. Rakovshtchik, Simultaneous bending and torsion of a circular bar beyond the elastic limit (in Russian), Izv AN SSSR OTN MM (1959), 3, 158-160.

[6.191] A. S. Ranshi, N. R. Chitkara, W. Johnson, Plane stress-plastic collapse loads for tapered cantilevers and haunched beam;, IJMS 16 (1974), 12, 867-885.

[6.192] - , - - , Plastic yielding of I-beams under shear, and shear and axial loading, IJMS 18 (1976), 7/8, 375-386.

[6.193] E. A. Rayevskaya, Limit analysis of a cantilever I-beam under restrained torsion beyond the elastic limit, (in Russian), IS 20 (1954), 93-100.

[6.194] —, Load-carrying capacity of I-beams under simultaneous bending and torsion (in Russian), Avtoref. Diss. Kand. Tekhn. Nauk MISI, Moskva 1955.

[6.195] —, A theoretical estimation of the load-carrying capacity of I-beams under simultaneous bending and torsion (in Russian), ITS 7 (1957), 269-298.

[6.196] - An experimental investigation of load-carrying capacity of I-beams under simultaneous bending and torsion (in Russian), SNR MINK 15 (1959), 2, 100-120.

[6.197] K. A. Reckling, Der ebene Spannungszustand bei der plastischen Balkenbiegung, Festschrift I. Szabo, W. Ernst Verlag, Berlin-München-Düsseldorf 1971, 39-46.

[6.198] —, Secondary effects upon the plastic bending of beams, SPAS (1972), 74-87.

[6.199] —, Beitrag zum Traglastverfahren, speziell für die Balkenbiegung mit Querkräften, Stb 44 (1975), 12, 358-361.

[6.200] I. P. Renne, On the limit state under transverse bending of rectangular beams (in Russian), Tr TulMI 7 (1955), 163-174.

[6.201] I. P. Renne, V. A. Ogorodnikov, V. G. Nakhaytchuk, A procedure for construction of plasticity diagrams based on experiments with cylindrical specimens under simultaneous torsion and tension (in Russian), PPro (1976), 6, 111-114.

[6.202] G. I. N. Rozvany, Optimal plastic design for bending and shear, Proc ASCE JEMD 99 (1973), 5, 1107-1109.

[6.203] -, Optimal design of flexural systems, Pergamon Press, Oxford-New York 1976.

[6.204] Ya. I. Rudayev, On a certain problem of essentially non-monotonic loading (tension with torsion) of a circular cylinder (in Russian), $\operatorname{Tr}$ FPI 56 (1972), 70-76. 
[6.205] J. Rutecki, Skręcanie rury cienkościennej o przekroju prostokątnym poza granicą sprężystości, AMS 8 (1956), 1, 29-40.

[6.206] A. R. Rzhanitsyn, Combined loading of thin-walled profiles with an undeformable contour in the elastic and inelastic range (in Russian), Trudy Lab. Stroit. Mekh. CNIPS, Stroyizdat, Moskva 1941.

[6.207] - , Approximate solutions to problems of the theory of plasticity (in Russian), IVSMTP (1956), 6-65.

[6.208] R. Sankaranarayanan, P. G. Hodge, Jr., On the use of linearized yield conditions for combined stresses in beams, JMPS 7 (1958), 1, 22-36.

[6.209] G. Schröder, R. Zeller, Mechanische Eigenschaften von plastisch vortordierten Rundstäben, Ind. Anz. 95 (1973), 74, 1709-1710.

[6.210] V. G. Sebeshev, I. A. Chaplinsky, Minimal weight design of arches based on the limit equilibrium method (in Russian), Izv VUZ SA (1974), 5, 45-63.

[6.211] - , - Theoretical and experimental investigation of load-carrying capacity of two-hinged arches (in Russian), Izv VUZ SA (1975), 9, 43-49.

[6.212] P. Seetharam, Behaviour and collapse loads of rigid-plastic prismatic bents, IE 15 (1971), $10,15-32$.

[6.213] H. Shakir-Khalil, Plastic resistance of sections under biaxial bending and torsion, SEng 54 (1975), 3, 99-103.

[6.214] G. S. Shapiro, On the limit state and elastic-plastic state of structures (in Russian), Izv AN SSSR MM (1963), 4, 138-144.

[6.215] A. N. Sherbourne, J. van Oostrom, Plastic analysis of castellated beams, CS 2 (1972), 1-2, 79-140.

[6.216] A. Shindo, Y. Seguchi, O. Shinmura, H. Kojima, Elastic-plastic problems of compressible straight bar under combined loading condition, BJSME 21 (1978), 151, 30-36.

[6.217] R. M. Shneyderovitch, M. B. Groman, Static load-carrying capacity of shafts (in Russian), Izv AN SSSR OTN (1958), 3, 130-134.

[6.218] R. M. Shneyderovitch, Elastic-plastic deformation of a circular bar under bending with torsion (in Russian), VPMK (1959), 296-314.

[6.219] R. M. Shneyderovitch, Static load-carrying capacity of members under elastic-plastic deformation (in Russian), VM (1959), 1, 14-24.

[6.220] N. Yu. Shvayko, Combined deformation of an elastic-plastic body (in Russian), VPP (1964), 43-64.

[6.221] J. O. Smith, O. M. Sidebottom, Inelastic behavior of load-carrying members, Wiley, New York 1965.

[6.222] N. A. Smolyago, Elastic-plastic deformations of a bar under simultaneous bending, torsion and tension, (in Russian), ST BTISM 3 (1974), 48-52.

[6.223] N. K. Snitko, Stability of bar systems (in Russian), Stroyizdat, Moskva 1952.

[6.224] Z. Sobotka, Plasticity of steel beams under combined bending, shear and normal load, Stav $C^{23}$ (1975), 9, 678-690.

[6.225] V. V. Sokolovsky, Theory of plasticity (in Russian), AN SSSR, Moskva 1946.

[6.226] M. C. Steele, The plastic bending and twisting of square section members, JMPS 3 (1955), 2, 156-166.

[6.227] A. I. Strelbitskaya, Some relations between generalized stresses in the limit state of a thinwalled profile (in Russian), ST ISM AN USSR 10 (1949); 12 (1950); 14 (1950); 15 (1950).

[6.228] -, Limit loads of thin-walled beams under simultaneous bending and torsion (in Russian), ST ISM AN USSR 19 (1954).

[6.229] O. I. Strelbytska, Formulae for the bimoment in the elastic-plastic state of an I-beam (in Ukrainian), DAN URSR (1954), 6, 435-438.

[6.230] —, Limit state of thin-walled profiles made of steel without a perfectly plastic platform under bending with torsion (in Ukrainian), PM 1 (1955), 1, 98-117. 
[6.231] A. I. Strelbitskaya, Investigation of the behaviour of a thin-walled channel section beyond the elastic limit (in Russian), ST ISM AN USSR 21 (1956), 14-26.

[6.232] O. I. Strelbytska, Load-carrying capacity of thin-walled bars under combined stresses (in Ukrainian), PM 2 (1956), 3, 306-317.

[6.233] - , Determination of limit loads of beams under bending with torsion (in Ukrainian), DAN URSR (1957), 6, 543-547.

[6.234] O. I. Strelbytska, Effect of torsion on the value of load-carrying capacity of hingeless frames (in Ukrainian), DAN URSR (1958), 9, 937-942.

[6.235] A. I. Strelbitskaya, Strength analysis of thin-walled bars beyond the elastic limit (in Russian), Izd. AN USSR, Kiev 1958.

[6.236] - , Determination of load-carrying capacity of beams under bending with shearing stresses taken into account (in Russian), Izv VUZ SA (1959), 3, 119-127.

[6.237] O. I. Strelbytska, E. O. Pryadko, G. I. Evseyenko, Experiments on steel beams under plastic bending with torsion (in Ukrainian), DAN URSR (1960), 9, 1174-1179.

[6.238] - , - - Experimental investigations of a channel beam under plastic bending with torsion (in Ukrainian), DAN URSR (1961), 9, 1127-1131.

[6.239] O. I. Strelbytska, Limit analysis of frames under bending with torsion (in Ukrainian), PM 7 (1961), 6, 639-648.

[6.240] -, Elastic-plastic stress state in thin-walled bars under bending with torsion (in Ukrainian) PM 8 (1962), 3, 258-270.

[6.241] O. I. Strelbytska, G. I. Evseyenko, Biaxial bending with torsion of thin-walled bars beyond the elastic limit (in Ukrainian), DAN URSR (1962), 10, 1308-1312.

[6.242] O. I. Strelbytska, Load-carrying capacity of thin-walled bars under oblique bending with torsion (in Ukrainian), PM 9 (1963), 3, 275-288.

[6.243] A. I. Strelbitskaya, Limit state of thin-walled profiles under biaxial bending with torsion (in Russian), 4th TOP 1962 (1964), 921-928.

[6.244] -, Limit state of frames made of thin-walled bars under bending with torsion (in Russian), Naukova Dumka, Kiev 1964.

[6.245] A. I. Strelbitskaya, G. I. Evseyenko, Experimental investigation of plastic biaxial bending with torsion in thin-walled bars (in Russian), PM 1 (1965), 9, 65-80.

[6.246] A. I. Strelbitskaya, Plastic deformations and load-carrying capacity of metal structures (in Russian), PM 3 (1967), 10, 100-108.

[6.247] A. I. Strelbitskaya, G. I. Evseyenko, Experimental investigation of elastic-plastic behaviour of thin-walled structures (in Russian), Naukova Dumka, Kiev 1968.

[6.248] F. Stüssi, Ưber den Verlauf der Schubspannungen in auf Biegung beanspruchten Balken aus Stahl, SB 98 (1931), 2.

[6.249] G. Svéd, D. S. Brooks, Elasto-plastic behaviour of a round bar subjected to axial force and torque, $A T$ ASH 50 (1965), 1-4, 337-351.

[6.250] M. Škaloud, Ultimate load theories for shear webs in the light of experimental evidence, StavČ 23 (1975), 9, 691-702.

[6.251] S. Šmiřak, $\mathrm{K}$ otázce mezni plastické únosnosti průřezu v ohybu při soucasném pŭsobeni smyku a tahu ci tlaku, SVUTB (1967), 3-4, 463-475.

[6.252] T. C. T. Ting, On the initial slope of elastic-plastic boundaries in combined longitudinal and torsional wave propagation, Trans ASME E36 (1969), 2, 203-211.

[6.253] - , Plastic wave propagation in linearly work-hardening materials, Trans ASME E40 (1973), 4, 1045-1049.

[6.254] M. Tochacek, Stress analysis of thin-walled bar with open section in elastic-plastic state by method of elastic solution, AP CVUT 1 (1966), 2, 129.

[6.255] G. S. Trofimov, On the strength of bent profiles under variable repeated loadings (in Russian), Tr BSKI 12 (1966), 6, 74-80. 
[6.256] T. Usami, Inelastic behavior of thin-walled open sections under combined axial force, bending and twisting, Trans JSCE 5 (1974), 50-51.

[6.257] D. Valalas, Intervention de l'effort tranchant dans la rupture d'un matériau plastique, ATPB (1964), 6, 465-472.

[6.258] L. P. Vinokurov, A. G. Yurev, Approximate limit analysis of beams and plates (in Russian), Belgorodsky Tekhnol. Inst. Stroit. Mat., Belgorod 1972, 75 pp.

[6.259] R. Vukotić, Granično stanje kombinovano napregnutog štapa, MatK 14 (1969), 4, 15-21.

[6.260] P. F. Walsh, Limit analysis of grids including torsion, CET IEA 12 (1970), 1, 90-93.

[6.261] R. Windels, Traglasten von Balkenquerschnitten bei Angriff von Biegemoment, Längskraft und Querkraft, Stb 39 (1970), 1, 10-16.

[6.262] -, Der Querkrafteinfluß bei plastischer Balkenbiegung, Stb 43 (1974), 3, 82-85.

[6.263] M. Wnuk, Upper and lower bounds to the plastic interaction curve for the combined tension and torsion, BAP SST 11 (1963), 2, 33-40 and 41-45; (English summary); |RI 11 (1963), 1, 179-200 (Polish full text).

[6.264] -, Wymiarowanie prętów jednocześnie skręcanych i rozciąganych w oparciu o teorię nośności granicznej, $A B M 10$ (1963), 2, 189-196.

[6.265] W. J. Worley, Inelastic behaviour of aluminium alloy I-beams with web cutouts, Univ. Illinois Engng. Exper. Stat. Bull. 448 (1958), 36 pp.

[6.266] H. C. Wu, H. C. Lin, Combined plastic waves in a thin-walled tube, IJSS 10 (1974), 8, $903-$ 917.

[6.267] Xu Bing-Hang, Elastic-plastic bending of bars with shear and normal forces taken into account (in Russian), $\operatorname{Tr}$ LKI 35 (1962), 21-27.

[6.268] Y. Yamada, K. Takatsuka, Elastic-plastic analysis of beams with uniform cross-section under combined loadings, Mon. J. Inst. Ind. Sci., Univ. Tokyo 23 (1971); 12, 531-535; 25 (1973), 6, 238-242.

[6.269] T. Yamasaki, O. Tosyaki, Elastic-plastic analysis of rectangular bars under constant torque and increasing bending moment (in Japanese), TRKU 40 (1967), 5, 717-724.

[6.270] A. Ylinen, The effect of shearing force on the buckling load beyond the limit of proportionality, $A T$ ASH 50 (1965), 1-4, 403-414.

[6.271] S. M. Yufin, Shakedown and incremental collapse of beam-columns in the presence of shearing force (in Russian), $\operatorname{Tr}$ GPI 31 (1975), 14, 86-90.

[6.272] M. A. Zadoyan, On two problems of the theory of perfect plasticity (in Russian), Izv AN Arm SSR FM 17 (1964), 6, 73-81.

[6.273] —, Plastic state of a thick-walled cylindrical tube under simultaneous torsion and bending (in Russian), DAN ArmSSR 56 (1973), 4, 214-220.

[6.274] - The problem of plastic state of cylindrical bars under bending with torsion (in Russian), MDTK (1975), 196-203.

[6.275] M. A. Zadoyan, L. A. Levonyan, Plastic state of a prismatic rectangular bar under simultaneous torsion and bending (in Russian), Izv AN ArmSSR T 28 (1975), 6, 10-16.

[6.276] A. Z. Zarifyan, Load-carrying capacity of I-beams under eccentric transverse loading (in Russian), PM 5 (1969), 7, 71-75.

\section{Chapter VII}

[7.1] E. Aarend, Yu. Lepik, L. Luht, Large deflections of a flexible circular simply supported elastic-plastic plate (in Russian), UZTGU 102 (1961), 377-384.

[7.2] H. Abe, A systematic formulation of nonlinear thin elastic-plastic shell theories, IJES 13 (1975), 11, 1003-1014.

[7.3] N. V. Akhvledyani, V. N. Shayshmelashvili, On the calculation of the load-carrying capacity of shells (in Russian), SAN GSSR 13 (1952), 10.

[7.4] N. V. Akhvledyani, On limit analysis of rotationally symmetric reinforced concrete shells (in Russian) SAN GSSR 18 (1957), 205-210. 
[7.5] V. N. Alpert, Load-carrying capacity of circular plates under antisymmetric loading (in Russian), IZh MTT (1966), 1, 90-93.

[7.6] -, On the yield condition for rigid-plastic shells (in Russian), Izv AN SSSR MTT (1972) 6, 131-139.

[7.7] Y. Ando, K. Iida, T. Kawai, G. Yagawa, F. Kikuchi, Finite element large deflection analysis of elastic-plastic shells of revolution subjected to axisymmetric loading, BJSME 15 (1972) 85, 796-802.

[7.8] A. L. L. Baker, A plastic design theory for reinforced and prestressed concrete shell roofs MCR 4 (1950), 27-34.

[7.9] A. Biron, A. Sawczuk, Plastic analysis of rib-reinforced cylindrical shells, Trans ASME E34 (1967), 37-42.

[7.10] A. Biron, Limit analysis of cylindrical shells with longitudinal rib reinforcement, IJSS 6 (1970), 7, 893-908.

[7.11] A. I. Borisyuk, Axially symmetric elastic-plastic stress state in shells of revolution (in Russian), PM 2 (1966), 11, 47-54.

[7.12] - , Analysis of shells based on the theory of small elastic-plastic deformations allowing for the compressibility of material (in Russian), TNEK 12 (1972), 52-58.

[7.13] M. W. Braestrup, Yield lines in discs, plates and shells, Rapp. Afd. Baerende Konstr., Danm. Tekn. Hejsk. 14 (1970).

[7.14] J. F. Brotchie, Elastic-plastic analysis of transversely loaded plates, Proc ASCE JEMD 86 (1960), 5, 57-90.

[7.15] O Bruhns, Zur Theorie elastoplastischer Schalentragwerke, IA 42 (1973), 4, 234-244.

[7.16] T. Buriyev, On basic relations of the general theory of shells under repeated and variable elastic-plastic loadings (in Russian), VVPM 10 (1972), 89-98.

[7.17] G. I. Bykovtsev, On the limit equilibrium of anisotropic plates and shells of revolution (in Russian), Izv AN SSSR MM (1964), 6, 69-77.

[7.18] G. I. Bykovtsev, Yu. P. Listrova, G. A. Murlina, On improved accuracy of the limit analysis of rotationally symmetric shells (in Russian), PM 7 (1971), 4, 28-34.

[7.19] C. R. Calladine, Simple ideas in the large-deflection plastic theory of plates and slabs, EP (1968), 93-127.

[7.20] —, On the derivation of yield conditions for shells, Trans ASME E39 (1972), 3, 852-853.

[7.21] M. Capurso, Sul calcolo dei carichi di collasso di piastre semplicemente inflesse di materiale con diversa resistenza a trazione e compressione, TI 31 (1966), 10, 545-555.

[7.22] - , Sull'analisi limite delle superfici di rivoluzione con nervature eccentriche, CoM 19 (1967), 3, 170-179; 19 (1967), 4, 219-233.

[7.23] M. Capurso, A. Gandolfi, Sul collasso rigido-plastico dei gusci nervati di rivoluzione CoM 19 (1967), 6, 418-424.

[7.24] M. Capurso, Comportamento elasto-plastico delle piastre sottili metalliche nel campo dei grandi spostamenti, CoM 20 (1968), 1, 42-51.

[7.25] M. Capurso, A. Gandolf, Sull'analisi limite delle piastre nervate, $\operatorname{CoM} 20$ (1968), 4, 254-262.

[7.26] M. Capurso, R. Ramasco, Sul calcolo elasto-plastico delle piastre circolari e delle volte di rivoluzione ribassate in regime di grandi spostamenti, CoM 21 (1969), 5, 386-403.

[7.27] J. Chakrabarty, Influence of transverse anisotropy on the plastic collapse of plates and shells, Trans ASME B97 (1975), 1, 125-130.

[7.28] J. M. Chern, S. Nemat-Nasser, Twisting and expansion of a hole in a non-uniform disk, IJSS 6 (1970), 6, 829-857.

[7.29] I. S. Chernyshenko, On elastic-plastic equilibrium of rotationally symmetric shells with finite deflections (in Russian), PM 2 (1966), 9, 26-36.

[7.30] -, Elastic-plastic bending of a cylindrical shell with finite deflections (in Russian), PM4 (1968), 2, 36-40. 
[7.31] I. S. Chernyshenko, State of stress in flexible shells with zero Gaussian curvature beyond the elastic limit (in Russian), PM 6 (1970), 8, 36-41.

[7.32] A. A. Cyras, A. E. Borkauskas, R. P. Karkauskas, Theory and methods of optimization of elastic-plastic systems (in Russian), Stroyizdat, Leningrad 1974.

[7.33] M. Dacko, Zginanie sprężysto-plastycznej kołowo-symetrycznej płyty o dużych ugięciach, BWAT 19 (1970), 9, 13-29.

[7.34] N. K. Das Talukder, R. K. Chakrabarti, On limit analysis of orthotropic shells of revolution, AMS 23 (1971), 3, 353-360.

[7.35] R. Dobrev, Elastic-plastic bending of a square plate, 1st BCTAM 1969 (1971), vol. 1, 351-362.

[7.36] D. C. Drucker, Limit analysis of cylindrical shells under axially-symmetric loading, Proc 1st MCSM (1953), 158-163.

[7.37] D. C. Drucker, R. T. Shield, Limit analysis of symmetrically loaded thin shells of revolution, Trans ASME E26 (1959), 1, 61-68.

[7.38] M. Duszek, Plastic analysis of cylindrical shells subjected to large defiections, ASM 18 (1966), 5, 599-614.

[7.39] -, Analiza plastyczna dwuwarstwowych powłok walcowych uwzględniająca wpływ zmian ksztaltu, RI 15 (1967), 4, 653-663.

[7.40] - , Plastic behaviour of shallow spherical shells with large deflections (in Russian), BAP SST 15 (1967), 9, 767-777.

[7.41] —, Plastic analysis of shallow spherical shells at moderately large deflections, IUTAM TTS 1967 (1969), 374-388.

[7.42] M. Duszek, A. Sawczuk, O związkach podstawowych teorii powlok plastycznych, $R I 18$ (1970), 4, 715-731.

[7.43] M. Duszek, Równania teorii dużych ugięć powłok plastycznych, RI 20 (1972), 3, 389-407.

[7.44] —, Geometrycznie nieliniowa teoria konstrukcji sztywno-plastycznych, IPPT PAN, Warszawa $1975,158 \mathrm{pp}$.

[7.45] -, A systematic study of kinematics of shells at large strains and displacements, BAP SST 26 (1978), 1, 39-47.

[7.46] G. Eason, The load carrying capacities of cylindrical shells subjected to a ring of force, JMPS 7 (1959), 169-181.

[7.47] —, The elastic-plastic bending of a simply supported plate, JAM 28 (1961), 3, 395-401.

[7.48] F. Ellyin, The effect of yield surfaces on the limit pressure of intersecting shells, IJSS 5 (1969), 7, 713-725.

[7.49] -, Elastic-plastic behavior of intersecting shells, Proc ASCE JEMD 95 (1969), 1, 69-94.

[7.50] M. I. Erkhov, Finite relation between forces and moments in plastic deformation of shells (in Russian), SMRS (1959), 3, 38-41.

[7.51] - , Plastic state of shells, plates and bars made of a perfectly plastic material (in Russian), Izv AN SSSR OTN MM (1960), 6, 151-154.

[7.52] - , Symmetric deformation of a cylindrical shell beyond the elastic limit (in Russian), $\operatorname{Tr}$ CNIISK 4 (1961), 176-198.

[7.53] - , Load-carrying capacity of a momentless unsymmetrically loaded cylindrical shell (in Russian), $\operatorname{Tr}$ CNIISK 10 (1962), 84-90.

[7.54] —, Limit equilibrium of shallow shells of revolution (in Russian), SMRS (1966), 4, 18-22.

[7.55] —, Theory of perfectly plastic bodies and structures (in Russian), Nauka, Moskva 1978.

[7.56] M. F. Ershov, Equilibrium of flexible elastic-plastic shells and plates (in Ukrainian), $P M$ 8 (1962), 5, 489-499.

[7.57] E. Faccioli, Incrudimento cinematico della piastra circolare sottile con la condizione di plasticità iniziale di von Mises, RS 37 (1967), 11, 1028-1045.

[7.58] S. M. Feynberg, Plastic flow of an axially symmetric shallow shell (in Russian) PMM 21 (1957), 4, 544-549. 
[7.59] W. Flügge, T. Nakamura, Plastic analysis of shells of revolution under axisymmetric loads, IA 34 (1965), 4, 238-247.

[7.60] W. Freiberger, Minimum weight design of cylindrical shells, JAM 23 (1956), 4, 576-580.

[7.61] E. Friedman, B. A. Boley, Stresses and deformations in melting plates, JSR 7 (1970), 3 324-333.

[7.62] A. N. Frolov, Nonlinear deformation of shells of revolution (in Russian), Izv AN SSSR MTT (1973), 1, 157-162.

[7.63] R. F. Gabbasov, On lower bounds to the load-carrying capacity of shells of revolution (in Russian), ST MISI 53 (1968), 47-56.

[7.64] J. C. Gerdeen, F. A. Simonen, D. T. Hunter, Large deflection analysis of elastic-plastic shells of revolution, 11th SSDM (1970), 239-249.

[7.65] J. C. Gerdeen, Large deflection analysis of elastic-plastic shells using numerical integration, AIAA 9 (1971), 6, 1012-1018.

[7.66] H. Gesund, Y. P. Kaushik, Yield line analysis of punching failures in slabs, Mem AIPC 30 (1970), 1, 41-60.

[7.67] D. A. Gokhfeld, P. I. Ermakov, I. M. Plagov, Elastic-plastic deformation of a cylindrical shell under repeated effects of a moving axially symmetric heat source (in Russian), TNEK 6 (1966), 89-98.

[7.68] D. A. Gokhfeld, Some shakedown problems of plates and shells (in Russian), 6th TOP (1966), 284-291.

[7.69] D. A. Gokhfeld, A. G. Laptevsky, O. F. Chernyavsky, Incremental collapse of cylindrical shells under variable temperature (in Russian), TNEK 11 (1971), 164-168.

[7.70] - , , , Incremental collapse of shells under variable temperature (in Russian), 8th TOP 1971 (1973), 114-119.

[7.71] D. A. Gokhfeld, O. F. Chernyavsky, Incremental collapse of shells (in Russian), PKZS 2 (1975), 15-19.

[7.72] D. A. Gokhfeld, O. F. Chernyavsky, E. F. Chernyayev, Incremental collapse of cylindrical shells under cyclic changes of the level of heat carrier (in Russian), TNEK 16 (1976), 94-98.

[7.73] B. A. Gorlatch, Physically nonlinear relations of the theory of axially symmetric shells allowing for compressibility of the material (in Russian), $\operatorname{Tr}$ KuyAI 48 (1971), 86-92.

[7.74] - Elastic-plastic deformations of an axisymmetrically loaded spherical shell at finite deflections (in Russian), $\operatorname{Tr}$ KuyAI 60 (1973), 74-83.

[7.75] A. S. Grigorev, On the bending of a circular plate beyond the elastic limit (in Russian), PMM 16 (1952), 1, 111-115.

[7.76] —, Bending of a circular clamped plate beyond the elastic limit (in Russian), Izv AN SSSR OTN MM (1962), 6, 83-87.

[7.77] Gu Guo-ji, Gu Qiu-lin, On the large deflection in elastic-plastic bending of circular plates (in Chinese), AMSin 2 (1958), 3.

[7.78] W. Gutkowski, W. Szczepiński, Statycznie dopuszczalne pola momentów plastycznych dla płyt, RI 19 (1971), 1, 3-13.

[7.79] A. N. Guz, I. S. Chernyshenko, K. I. Shnerenko, Spherical vessel heads weakened by holes (in Russian), Naukova Dumka, Kiev 1970.

[7.80] A. A. Gvozdev, A. M. Protsenko, Perspectives of application of limit analysis of shells (in Russian), 7th TOP 1969 (1970), 736-748.

[7.81] J. B. Haddow, Yield-point loading curves for circular plates, IJMS 11 (1969), 5, 455-459.

[7.82] M. Hamada, M. Tanaka, A numerical method for solving elastic-plastic problems of rotationally symmetric shells, BJSME 14 (1971), 74, 724-734; 16 (1973), 100, 1522-1531.

[7.82a] M. Hamada, M. Tanaka, A numerical method considering the Bauschinger effect for large deflection analysis of elastic-plastic circular plates, BJSME 15 (1972), 87, 1029-1038. 
[7.83] H. M. Haydl, A. N. Sherbourne, Effect of transverse shear on limit load of cylindrical shells, NED 22 (1972), 2, 290-295.

[7.84] - , Y Yield surfaces for thin shells accounting for transverse shear, AMS 25 (1973), 4, 663-674.

[7.85] -, -, Limit loads of variable-thickness circular plates accounting for transverse shear, JSA 8 (1973), 2, 108-112.

[7.86] —, —, Rigid-plastic collapse of compression bent shallow shells, IJMS 15 (1973), 9, 727-739.

[7.87] -, -, Collapse of clamped variable thickness circular plates, Proc ASCE JEMD 99 (1973), 6, 1309-1314.

[7.88] - , - , Plastic analysis of shallow spherical shells, Trans ASME E41 (1974), 3, 593-598.

[7.89],-- , Similarity in plastic analysis of irradiated and variable thickness plates and shells, ZAMM 54 (1974), 7, 445-446.

[7.90] - , - , Shear-bending collapse of cylindrical shells, RRST SMA 19 (1974), 5, 871-877.

[7.91] R. M. Haythornthwaite, The deflection of plates in the elastic-plastic range, 2nd USNCAM 1954 (1955), 521-526.

[7.92] R. M. Haythornthwaite, E. T. Onat, The load carrying capacity of initially flat circular steel plates under reversed loading, JAS 22 (1955), 12, 867-869.

[7.93] P. G. Hodge, Jr., The rigid-plastic analysis of symmetrically loaded cylindrical shells, JAM 21 (1954), 4, 336-342.

[7.94] -, Displacements in an elastic-plastic cylindrical shell, JAM 23 (1956), 74-79.

[7.95] P. G. Hodge, Jr., F. Romano, Deformations of an elastic-plastic cylindrical shell with linear strain-hardening, JMPS 4 (1956), 145-161.

[7.96] P. G. Hodge, Piecewise linear isotropic plasticity applied to a circular cylindrical shell with symmetric radial loading, JFI 263 (1957), 1, 13-33.

[7.97] P. G. Hodge, S. V. Nardo, Carrying capacity of an elastic-plastic cylindrical shell with linear strain-hardening, JAM 25 (1958), 1, 79-85.

[7.98] P. G. Hodge, Jr., Plastic analysis of circular conical shells, JAM 27 (1960), 4, 696-700.

[7.99] - , Yield conditions for rotationally symmetric shells under axisymmetric loading, JAM 27 (1960), 2, 323-331.

[7.100] -, The Mises yield condition for rotationally symmetric shells, QAM 18 (1961), 4, 305-311.

[7.101] - A comparison of yield conditions in the theory of plastic shells, MSS (1961), 165-177 (English edition); 458-470 (Russian edition).

[7.102] —, Piecewise-linear bounds on the yield-point load of shells, JMPS 11 (1963), 1, 1-12.

[7.103] P. G. Hodge, Jr., J. Panarelli, Plastic analysis of cylindrical shells under pressure, axial loads and torque, DM 2 (1965), 2, 262-277.

[7.104] H. G. Hopkins, A. J. Wang, Load carrying capacities for circular plates of perfectlyplastic material with arbitrary yield condition, JMPS 3 (1954), 117-129.

[7.105] A. A. Ilyushin, An approximate theory of elastic-plastic deformations of an axially symmetric shell (in Russian), PMM 8 (1944), 6, 15-24.

[7.106] - , Finite relation between forces and moments and the corresponding deformations in the theory of shells (in Russian), PMM 9 (1945), 1, 101-110.

[7.107] V. N. Ionov, Stresses and strains in shells with zero Gaussian curvature (in Russian), IS 29 (1960), 63-76.

[7.108] G. V. Ivanov, Approximation of the finite relation between forces and moments in shells subject to the Mises yield condition (in Russian), IZh MTT (1967), 6, 74-75.

[7.108a] -, Elastic-plastic flow of shells subject to the Mises yield condition (in Russian), Izv AN SSSR MTT (1969), 3, 85-90.

[7.109] D. D. Ivlev, Yu. P. Listrova, Yu. V. Nemirovsky, On the theory of the limit state of layered plates and shells of revolution (in Russian), Izv AN SSSR MM (1964), 4, 77-86.

[7.110] M. Janas, Limit analysis of non-symmetric shells by a generalized yield line method, IASS NSP 1963 (1964), 997-1010. 
[7.111] M. Janas, Yield loci for nonsymmetric plastic shells, BAP SST 13 (1965), 1, 9-17.

[7.112] —, Large plastic deformations of reinforced concrete slabs, IJSS 4 (1968), 61-74.

[7.113] M. Janas, A. König, Nośność graniczna powlok, przekrycia i zbiorniki, Arkady, Warszawa, 1968.

[7.114] M. Janas, A. Sawczuk, Problems in plastic analysis of shells, Prace IPPT PAN (1970), 4, 34 pp. (paper for presentation at the IASS Colloquium Madrid 1969).

[7.115] M. Janas, Arching action in elastic-plastic plates, JSM 1 (1973), 3, 277-293.

[7.116] K. W. Johansen, Critical notes on calculation and design of cylindrical shells, 3rd C AIPC (1948), 601-606.

[7.117] N. Jones, Combined distributed loads on rigid-plastic circular plates with large deflections, IJSS 5 (1969), 1, 51-64.

[7.118] -, The influence of large deflections on the behavior of rigid-plastic cylindrical shells loaded impulsively, Trans ASME E37 (1970), 2, 416-425.

[7.119] - , Consistent equations for the large deflections of structures, BMEE 9 (1970), 3, 235-248.

[7.120] N. Jones, R. M. Walters, Large deflections of rectangular plates, JShR 15 (1971), 2, 164-171.

[7.121] Zh. V. Kachalov, Yu. P. Listrova, V. N. Potapov, On the effect of geometry changes on the load-carrying capacity of circular plates (in Russian), Izv AN SSSR MTT (1972), 3, 131-133.

[7.122] L. M. Kachanov, Elastic-plastic problems of the theory of shells and plates (in Russian). 6th TOP (1966), 954-959.

[7.123] S. Kaliszky, Optimum limit design for reinforced concrete structures, Rep. Univ. Southampton, Dept. Civil Engng. (1964).

[7.124] S. A. Kapustin, On the calculation of elastic-plastic shells in displacements (in Russian), UZGU 89 (1969), 67-76.

[7.125] —, Thermoplastic deformations of shells under combined and non-isothermal loadings (in Russian), UZGU 108 (1970), 28-37.

[7.126] G. Kazinczy, The limit design of shells, 3rd C AIPC (1948).

[7.127] K. O. Kemp, The yield criterion for orthotropically reinforced concrete slabs, IJMS 7 (1965), 11, 737-746.

[7.128] R. Yu. Kerimov, Investigation of the elastic-plastic state of stress in a cylindrical shell with a circular hole (in Russian), PM 9 (1973), 10, 18-22.

[7.129] F. Kikuchi, Y. Ando, Application of simplified hybrid displacement method to large deflection analysis of elastic-plastic plates and shells, JFEUT B32 (1973), 1, 117-135.

[7.130] R. M. Kirakosyan, On the bending of prestressed circular plates beyond the elastic limit (in Russian), 6th TOP (1966), 472-475.

[7.131] P. Klement, Theorie der elastisch-plastischen Zylinderschale, ÖIA 16 (1962), 3, 199-211.

[7.132] K. A. Koba, O. N. Shabliy, Large deflections of rigid-plastic shallow shells of revolution, clamped along the contour (in Russian), DPM 15 (1972), 3-11.

[7.133] V. A. Kolgadin, A. I. Strelbitskaya, Elastic-plastic state of rectangular plates under transverse loading (in Russian), 6th TOP (1966), 484-490.

[7.134] V. A. Kolgadin, Elastic-plastic bending of rectangular plates in a temperature field (in Russian), TNEK 8 (1969), 175-182.

[7.135] V. I. Korolev, Small elastic-plastic deformations of plates and shells (in Russian), PM 4 (1968), 4, 16-24.

[7.136] -, Elastic-plastic deformations of shells (in Russian), Mashinostroyenye, Moskva 1971.

[7.137] Yu. G. Korotkikh, S. A. Kapustin, Investigation of thermoplastic deformations of a cylindrical shell under various loading histories (in Russian), Mash (1971), 6, 61-65.

[7.138] J. A. König, Deformations of cylindrical elastic-plastic shells, BAP SST 12 (1964), 1, 13-20.

[7.139] J. A. König, J. Rychlewski, Limit analysis of circular plates with jump non-homogeneity, IJSS 2 (1966), 3, 493-513.

[7.140] J. A. König, Shakedown theory of plates, AMS 21 (1969), 5, 623-637. 
[7.141] M. W. Kwieciński, Yield condition for orthotropically reinforced slab, AMS 18 (1966), $5,615-626$.

[7.142] P. Ladevèze, R. Valid, Calcul et essais d'une plaque circulaire encastrée sous pression unilatérale en grande deformation, RAe 137 (1970), 197-204.

[7.143] R. H. Lance, D. N. Robinson, Plastic analysis of filled, reinforced, circular cylindrical shells, IJMS 15 (1973), 1, 65-79.

[7.144] H. G. Landau, J. H. Weiner, Transient and residual stresses in heat-treated plates, JAM 25 (1958), 4, 459-465.

[7.145] G. Landgraf, Aufstellung von Fliessbedingungen für Schnittgrössen von Flächentragwerken, ZAMM 48 (1968), 5, 317-323.

[7.146] A. Langenbach, Elastisch-plastische Deformationen von Platten, ZAMM 41 (1961), 3, 126-134.

[7.147] F. Laudiero, A. Tralli, Finite element incremental analysis of elastoplastic plate bending, Mec 8 (1973), 3, 190-202.

[7.148] S. L. Lee, B. J. Thorn, Outer and inner bounds on Tresca limit loads of cylindrical shells, JFI 277 (1964), 6, 517-533.

[7.149] Ya. A. Lellep, On the limit equilibrium of annular plates under tension with bending (in Russian), PM 8 (1972), 6, 72-76.

[7.150] Ya. A. Lellep, Yu. R. Lepik, On evaluation of load-carrying capacity of axially symmetric shells in the case of piecewise linear yield conditions (in Russian), Izv AN SSSR MTT (1973), 2, 144-150.

[7.151] Yu. R. Lepik, Equilibrium of flexible elastic-plastic plates with large deflections (in Russian), IS 24 (1956), 37-51.

[7.152] - , On the equilibrium of flexible plates beyond the elastic limit (in Russian), PMM 21 (1957), 6, 833-842.

[7.153] —, Evaluation of the residual deflection and of residual forces after unloading of flexible elastic-plastic plates (in Russian), Izv AN SSSR OTN MM (1959), 3, 154-157.

[7.154] —, Large deflections of circular rigid-plastic plates, clamped along the contour (in Russian), lst TOP (1961).

[7.155] - , On the load-carrying capacity of non-homogeneous plates and shells (in Russian), Izv AN SSSR MM (1963), 4, 167-171.

[7.156] - Equilibrium of elastic-plastic and rigid-plastic plates and shells (in Russian), IZh 4 (1964), 3, 601-616.

[7.157] —, Thermal stresses in flexible non-homogeneous plates beyond the elastic limit (in Russian), UZTGU 177 (1965), 168-179.

[7.158] U. Lepik, Large deflections of rigid-plastic cylindrical shells under axial tension and external pressure, NED 4 (1966), 1, 29-38.

[7.159] Yu. R. Lepik, Large deflections of a rigid-plastic shell under internal and external pressure (in Russian), 6th TOP (1966), 534-541.

[7.160] -, On the limit equilibrium of plates whose material has different yield-point stresses in tension and in comrression (in Russian), 7th TOP 1969 (1970), 360-364.

[7.161] —, On the optimal design of rigid-plastic shells of revolution allowing for transverse forces (in Russian), MDTK (1975), 243-250.

[7.162] T. H. Lin, S. R. Lin, B. Mazelsky, Elastoplastic bending of rectangular plates with large deflection, Trans ASME E39 (1972), 4, 978-982.

[7.163] Yu. P. Listrova, On the load-carrying capacity of a cylindrical shell subject to the yield condition of maximal reduced stress (in Russian), Izv AN SSSR MM (1963), 2, 173-176.

[7.164] Yu. P. Listrova, M. A. Rudis, On the limit equilibrium of non-homogeneous plates and shells of revolution subject to piecewise linear yield conditions (in Russian), Izv AN SSSR $M M$ (1965), 2, 88-94. 
[7.165] Yu. P. Listrova, M. A. Rudis, Some problems of limit equilibrium of shells of revolution accounting for thermal effects (in Russian), TNEK 5 (1965), 270-278.

[7.166] Yu. P. Listrova, N. I. Mokashova, On the limit equilibrium of structures whose material has different yield-point stresses in tension and in compression (in Russian), IZh MTT (1967), 6, 81-85.

[7.167] Yu. P. Listrova, V. N. Potapov, M. A. Rudis, Limit equilibrium of non-homogeneous shells of revolution whose material has different yield-point stresses (in Russian), PM 5 (1969), 11, 34-39.

[7.168] Yu. P. Listrova, V. N. Potapov, Load-carrying capacity of isotropic structures whose material has different yield-point stresses accounting for thermal effects (in Russian), Izv VUZ M (1970), 2, 5-8.

[7.169] Yu. P. Listrova, G. A. Murlina, On the limit equilibrium of plates and shells of revolution made of a compressible material (in Russian), PM 6 (1970), 7, 79-84.

[7.170] Yu. P. Listrova, G. A. Murlina, T. D. Semykina, On the deformation of shells of revolution made of a rigid-plastic-hardening material accounting for thermal effects (in Russian), Izv AN SSSR MTT (1971), 2, 59-63.

[7.171] A. Z. Lokshin, V. I. Mamay, Large elastic-plastic deflections of a circular plate (in Russian), $\operatorname{Tr} L K I 53$ (1966), 49-55.

[7.172] O. Lomacky, B. Hyman, Stress analysis of thin elasto-plastic shelis, Pap ASME WA/PVP-3 (1970).

[7.173] P. A. Lukash, Calculation of shallow shells and plates accounting for physical and geometrical nonlinearity (in Russian), $\operatorname{Tr}$ CNIISK 7 (1961), 268-320.

[7.174] V. A. Lukin, I. V. Shirko, Elastic-plastic bending of flexible circular plates (in Russian), 7th TOP 1969 (1970), 370-374.

[7.175] M. E. Lunchick, Yield failure of stiffened cylinders under hydrostatic pressure, 3rd USNCAM (1958), 589-594.

[7.176] A. K. Lyubimov, On the state of stress in plates under variable elastic-plastic deformation, (in Russian), UZGU 108 (1970), 58-64.

[7.177] V. I. Makhnenko, E. A. Velikoivanenko, A thermoplastic problem for a shallow shell of revolution (in Russian), TNEK 7 (1967), 57-64.

[7.178] N. N. Malinin, Zh. L. Petrosyan, Large plastic deformations of a flat membrane under uniformly distributed pressure (in Russian), Izv VUZ M (1968), 10, 30-37.

[7.179] P. V. Marcal, C. E. Turner, Numerical analysis of the elastic-plastic behaviour of axisymmetrically loaded shells of revolution, JMES 5 (1963), 5, 232-237.

[7.180] P. V. Marcal, A stiffness method for elastic-plastic problems, IJMS 7 (1965), 4, 229-238.

[7.181] P. V. Marcal, W. R. Pilgrim, A stiffness method for elastic-plastic shells of revolution, JSA 1 (1966), 4, 339-350.

[7.182] P. V. Marcal, Large deflection analysis of elastic-plastic shells of revolution, AIAA 8 (1970), 9, 1627-1633.

[7.183] Ch. Massonnet, General theory of elasto-plastic membrane-plates, EP (1968), 443-471.

[7.184] C. Mazzarella, I domini di plasticizzazione per le lastre formate da materiale con limite plastici a trazione ed a compressione diversi in condizioni di simmetria radiale, GGC 105 (1967), 6-7, 472-483; 105 (1967), 8, 511-523.

[7.185] P. V. McLaughlin, S. C. Batterman, Limit surfaces for fibrous composite plates, AIAA 8 (1970), 12, 2136-2140.

[7.186] P. V. McLaughlin, Plastic behaviour of two-layer sandwich structures, Trans ASME E40 (1973), 1, 257-262.

[7.187] R. E. Melchers, G. I. N. Rozvany, Optimum design of reinforced concrete tanks, Proc ASCE JEMD 96 (1970), 6, 1093-1106.

[7.188] G. Menditto, La piastra circolare allo stato limite, RIL ASL 98 (1964), 1, 335-356. 
[7.189] G. Menditto, Studio dello stato limite di una piastra circolare di materiale rigido-plastico con il criterio di plasticizzazione dello Stassi d'Alia, GGC 103 (1965), 6-7, 38-336.

[7.190] - , Lo stato limite della piastra circolare in differenti condizioni di vincolo e di carico, RIL ASL 99 (1965), 1, 70-93.

[7.191] M. Sh. Mikeladze, On the load-carrying capacity of initially anisotropic shells (in Russian), DAN SSSR 98 (1954), 1, 921-923.

[7.192] - , On the plastic equilibrium of a multilayer anisotropic shell (in Russian), DAN SSSR 102 (1955), 2, 229-232.

[7.193] -, On the plastic flow of anisotropic shells (in Russian), Izv AN SSSR OTN (1955), 8, 67-80.

[7.194] —, Rigid-plastic analysis of anisotropic plates and shells, 9th ICAM 1956 (1957), 8.

[7.195] -, A general theory of anisotropic rigid-plastic shells (in Russian), Izv AN SSSR OTN (1957), 1, 85-94.

[7.196] -, Analysis of weight and strength of rigid-plastic orthotropic shells (in Russian); AMS 11: (1959), 1, 17-31.

[7.197] -, An engineering theory of thin plastic shells (in Russian), SAN GSSR 40 (1965), 3, 551-558.

[7.198] -, The purposes and problems of the technical theory of thin plastic shells, IUTAM.TTS 1967 (1969), 85-92.

[7.199] —, Introduction to the engineering theory of thin elastic-plastic shells (in Russian), Metsnereba, Tbilisi 1969.

[7.200] M. S. Mikhalishin, O.N. Shabliy, Rigid-plastic behaviour of shells and plates made of heteromodulic hardening material (in Russian), PPro (1970), 11, 78-85.

[7.201] M. S. Mikhalishin, Yu. V. Nemirovsky, O. N. Shabliy, On the theory of limit equilibrium: of bimetallic shells of revolution and of circular plates (in Russian), ZhPMTF (1974), 2, 139-151,

[7.202] M. M. Mohaghegh, M. D. Coon, Plastic analysis of thick circular plates, IJMS 15 (1973), $11,935-942$.

[7.203] P. Montague, M. R. Horne, Elastic-plastic axisymmetric analysis of a thin-walled cylindrical shell subjected to a radial pressure difference, Trans ASME E35 (1968), 4, 787-795.

[7.204] C. T. Morley, On the yield criterion of an orthogonally reinforced concrete slab element, JMPS 14 (1966), 1, 33.

[7.205] A. J. Morris, Elastic-plastic analysis of loaded plates, Enr 222 (1966), 5770, 304-305.

[7.206] M. Mrowiec, Stan graniczny rurociągu cienkościennego w przypadku ciśnienia wewnętrznego i zginania, RI 14 (1966), 3, 513-527.

[7.207] Z. Mróz, A. Sawczuk, Load-carrying capacity of annular plates (in Russian), Izv. AN SSSR (1960), 3, 72-78.

[7.208] Z. Mróz, The load carrying capacity of orthotropic shells, AMS 12 (1960), 1, 85-107.

[7.209] Z. Mróz, Xu Bing-ye, The load carrying capacities of symmetrically loaded spherical shells, AMS 15 (1963), 2, 245-266.

[7.210] Z. Mróz, Optimum design of reinforced concrete shells of revolution, IASS NSP 1963 (1964), 732-748.

[7.211] H. F. Muensterer, F. P. J. Rimrott, Elastic-plastic response of a sandwich cylinder subjected to internal pressure, JSA 6 (1971), 4, 273-278.

[7.212] T. Mura, J. S. Kao, S. L. Lee, Limit analysis of circular orthotropic plastes, Proc ASCE JEMD 90 (1964), 5, 375-395.

[7.213] J. Myszkowski, Biegung dünner Kreisplatten im plastischen Materialbereich, ZAMM 50 (1970), Sonderheft 1-4, 137-138.

[7.214] - Endliche Durchbiegungen beliebig eingespannter dünner Kreis- und Kreisringplatten im plastischen Materialbereich, IA 40 (1971), 1, 1-13. 
[7.215] P. M. Naghdi, Bending of elastoplastic circular plates with large deflections, JAM 19 (1952), 3, 293-300.

[7.216] Yu. A. Nagyavitchius, A. A. Cyras, On the problem of linearization of the yield conditions for shallow cylindrical shells (in Russian), LMS (1969), 1, 43-50.

[7.217] T. Nakam ıra, Plastic analysis of shells of revolution under axisymmetric loads, Doct. Diss. Stanford Univ. (1961).

[7.218] —, Limit analysis of nonsymmetric sandwich shells, IASS NSP 1963 (1964), 768-784.

[7.219] Yu. V. Nemirovsky, Yu. N. Rabotnov, Limit equilibrium of reinforced cylindrical shells (in Russian), Izv AN SSSR OTN MM (1963), 3, 83-94.

[7.220] —, —, Limit analysis of ribbed plates and shells, IASS NSP 1963 (1964), 786-807.

[7.221] Yu. V. Nemirovsky, On the limit state of layered and structurally orthotropic cylindrical shells (in Russian), IZh MTT (1966), 5, 130-138.

[7.222] -, Limit equilibrium of wafer-like cylindrical shells (in Russian), IZh MTT (1967), 3, 52-59.

[7.223] —, On elastic-plastic behaviour of a reinforced layer (in Russian), ZhPMTF (1969), 6, 81-89.

[7.224] —, Limit equilibrium of multilayer reinforced axisymmetric shells (in Russian), Izv $A N$ SSSR MTT (1969), 6, 80-89.

[7.225] —, Load-carrying capacity of cylindrical bimetallic shells (in Russian), Izv VUZ M (1969), $11,54-61$.

[7.226] Yu. V. Nemirovsky, O. N. Shabliy, M. S. Mikhalishin, Limit state of structures made of miterials with different yield-point stresses in tension and in compression (in Russian), PM 9 (1973), 10, 3-11.

[7.227] Yu. V. Nemirovsky, Momentless plastic axisymmetric shells (in Russian), MDTK (1975), 327-333.

[7.228] M. P. Nielsen, Yield conditions for reinforced concrete shells in the membrane state, IASS NSP 1963 (1964), 1030-1040.

[7.229] D. Niepostyn, The limit analysis of an orthotropic circular cylinder, AMS 8 (1956), 3, 565-580.

[7.230] R. P. Nordgren, P. M. Naghdi, Finite twisting and expansion of a hole in a rigid/plastic plate, Trans ASME E30 (1963), 4, 605-612.

[7.231] - , - Loading and unloading solutions for an elastic-plastic annular plate in the state of plane stress under com'sined pressure and couple, IJES 1 (1963), 33-70.

[7.232] V. V. Novozhilov, Theory of thin shells (in Russian), Sudpromgiz, Leningrad 1951 (1st ed.) and 1962 (2nd ed.). English translation: Noordhoff, Groningen 1959.

[7.233] Y. Ohashi, S. Murakami, Elastic-plastic bending of circular plates accounting for longitudinal forces (in Japanese), Trans JSME 29 (1963), 206, 1581-1591; 31 (1965), 224, 501-513; 31 (1965), 224, 514-520.

[7.234] —, -, The elasto-plastic bending of a clamped thin circular plate, IIth ICAM 1964 (1966), 212-223.

[7.235] —, -, One-side elastic-plastic bending of a circular plate combined with radial tension (in Russian), IZh 5 (1965), 1, 94-102.

[7.236] -, 一, Large deflection in elasto-plastic bending of a simply supported circular plate under a uniform load, Trans ASME E33 (1965), 4, 866-870.

[7.237] Y. Ohashi, S. Murakami, A. Endo, Elasto-plastic bending of an annular plate at large deflection, IA 35 (1967), 5, 340-350.

[7.238] Y. Ohashi, S. Murakami, K. Kawashima, Large elasto-plastic bending of a simply supported thin circular plate with local unloading process, JSME 1967 Semi-International Symposium, Tokyo, 2 (1968), 157-166.

[7.239] Y. Ohashi, S. Murakami, Study on the axisymmetric elasto-plastic deformation of thin plates, MFENU 20 (1968), 2, 355-433. 
[7.240] Y. Ohashi, N. Kamiya, Large deflection of a supported circular plate having a non-linear stress-strain diagram, ZAMM 48 (1968), 3, 159-171.

[7.241] Y. Ohashi, K. Kawashima, On the residual deformation of elasto-plastically bent thin circular plate after perfect unloading, ZAMM 49 (1969), 5, 275-286.

[7.242] - , - On stress-strain relation of mild steel in unloading and inverse loading for bending analysis of thin circular plate, BJSME 13 (1970), 56, 215-221.

[7.243] -, - , Analysis of unloading on a thin circular plate of mild steel using actual unloading stress-strain relation, ZAMM 50 (1970), 8, 467-478.

[7.244] -, - , Large elasto-plastic deflection of a circular plate of mild steel under cyclic loading, ZAMM 52 (1972), 11, 505-514.

[7.245] Y. Ohashi, T. Okouchi, The elasto-plastic deformation of a supported short cylindrical shell of mild steel under internal pressure, IJMS 17 (1975), 4, 267-282.

[7.246] H. Okamura, K. Yoshida, Elasto-plastic analysis of rectangular steel plates with consideration of their large deflections and rib-stiffened construction, Trans JSCE 3 (1972), 2, 170-171.

[7.247] W. Olszak, J. Murzewski, Elastic-plastic bending of non-homogeneous orthotropic circular plates, AMS 9 (1957), 4, 467-485; 5, 605-629.

[7.248] W. Olszak, A. Sawczuk, Problems of the limit analysis and design of non-homogeneous axially symmetric shells, 2nd CSRC (1957), 249-256.

[7.249] —, -, Die Grenztragfähigkeit von zylindrischen Schalen bei verschiedenen Formen der Plastizitätsbedingung, AT ASH 26 (1959), 1/2, 55-77.

[7.250] -, -, Inelastic behaviour in shells, Noordhoff, Groningen 1967.

[7.251] E. T. Onat, W. Prager, Limit analysis of shells of revolution, I-II, Proc KNAW B57 (1954), 5, 534-548.

[7.252] E. T. Onat, The plastic collapse of cylindrical shells under axially symmetric loading, QAM 13 (1955), 63-72.

[7.253] E. T. Onat, W. Prager, Limits of economy of material in shells, deIng 67 (1955), 10, 46-49.

[7.254] E. T. Onat, Analysis of shells of revolution composed of workhardening material, JMPS 7 (1958), 1, 45-59.

[7.255] -, Plastic analysis of shallow conical shells, Proc ASCE JEMD 86 (1960), 6; 10th ICAM 1960 (1962).

[7.256] E. T. Onat, S. Yamantürk, On thermally stressed elastic-plastic shells, JAM 29 (1962), 1, 108-114.

[7.257] E. T. Onat, Plastic shells, IASS NSP 1963 (1964), 649-659.

[7.258] A. Oschatz, Bestimmung der Traglast von Kreis- und Kreisringplatten mit Berücksichtigung der Querkraftschubspannungen, ZAMM 48 (1968), 5, 325-332.

[7.259] O. M. Paliy, V. S. Chuvikovsky, Elastic-plastic axisymmetric bending of a circular cylindrical shell (in Russian), PM 2 (1966), 6, 49-54.

[7.260] B. Paul, Carrying capacity of elastic-plastic shells with various end conditions under hydrostatic compression, JAM 26 (1959), 4, 553-560.

[7.261] T. H. H. Pian, Yield conditions of plates and shells by Mises-Hencky criterion, AFOSR TN 60-608 (1960).

[7.262] M. Pignataro, Carico di collasso per piastre circolari di materiale ad ortotropia polare, CoM 22 (1970), 1, 33-41; 2, 84-96.

[7.263] V. V. Piskun, Elastic-plastic axisymmetric stress state of a circular cylindrical shell of constant thickness under unsteady heating (in Russian), TNEK 6 (1966), 79-88.

[7.264] L. I. Polyakov, Yu. T. Polyakov, M. A. Rudis, On the load-carrying capacity of a sandwich circular shell (in Russian), Izv AN SSSR MM (1962), 6, 163-165.

[7.265] E. P. Popov, M. Khojasteh-Bakht, S. Yaghmai, Analysis of elastic-plastic circular plates, Proc ASCE JEMD 93 (1967), 6, 49-65. 
[7.266] E. P. Popov, M. Khojasteh-Bakht, P. Sharifi, Elastic-plastic analysis of some pressure vessel heads, Pap ASME WA/PVP-7 (1969).

[7.267] V. S. Popov, On the construction of the limit yield surface for shells of revolution (in Russian), Izv VUZ M (1974), 6, 23-27.

[7.268] V. S. Popov, On the construction of the limit yield surface for sandwich structures (in Russian), Izv VUZ M (1974), 7, 9-12.

[7.269] I. V. Prokhorenko, Thermoplastic state of stress in shells of revolution (in Russian), TNEK 11 (1971), 127-131.

[7.270] S. Qu, Approximate yield conditions for axisymmetrically loaded shell of revolution (in Chinese), ASN MM 1 (1965), 2, 206-208.

[7.271] —, Approximate yield conditions for perfectly plastic shells (in Chinese), AMSin 8 (1965), 3, 173-185.

[7.272] Yu. N. Rabotnov, An approximate engineering theory of elastic-plastic shells (in Russian), PMM 15 (1951), 2, 167-174.

[7.273] M. I. Reitman, Analysis of the equations of the theory of perfectly plastic shells, AMS 19 (1967), 4, 595-601.

[7.274] M. I. Reitman, L. I. Yarin, Parametrical optimization of reinforced concrete structures with the aid of computers (in Russian), Stroyizdat, Moskva 1974.

[7.275] M. Robinson, A comparison of yield surfaces for thin shells, IJMS 13 (1971), 4, 345-354.

[7.276] —, The effect of transverse shear stresses on the yield surface for thin shells, IJSS 9 (1973), $7,819-828$.

[7.277] V. I. Rozenblyum, An approximate theory of equilibrium of plastic shells (in Russian), PMM 18 (1954), 3, 289-302.

[7.278] -, On the yield condition for thin shells (in Russian), PMM 24 (1960), 2, 364-366.

[7.279] - , On the calculation of load-carrying capacity of perfectly plastic axisymmetric shells (in Russian), IUP 4 (1965), 207-218.

[7.280] - , On the complete system of equations of plastic equilibrium of thin shells (in Russian), IZh MTT (1966), 3, 127-132.

[7.281] V. V. Rozhdestvensky, On the problem of limit states of cross-sections of thin shells (in Russian), IVSMTP (1956), 223-232.

[7.282] J. Rychlewski, Limit analysis of helicoidal shells, IASS NSP 1963 (1964), 819-841.

[7.283] - , On the general theory of perfectly plastic shells (in Russian), 6th TOP (1966), 873-880.

[7.284] J. Rychlewski, G. S. Shapiro, Perfectly plastic plates and shells (in Russian), 6th TOP (1966), 987-995.

[7.285] A. R. Rzhanitsyn, Analysis of reinforced concrete shells by the limit equilibrium method (in Russian), Teor. Rasch. Zhelezobet. Konstr. CNIISK, Moskva 1958, 155-175.

[7.286] - Analysis of shells by the limit equilibrium method (in Russian), IVTPP (1958), 7-35.

[7.287] -, Analysis of shallow shells by the limit equilibrium method (in Russian), SMRS (1959), $1,5-11$.

[7.288] E. Saareste, K. Soonets, Large deflections of a square plate in the range of elastic-plastic deformations (in Russian), UZTGU 281 (1971), 241-255.

[7.289] R. Sankaranarayanan, A generalized square yield condition for shells of revolution, Proc IAS A59 (1964), 3, 127-140.

[7.290] R. Sankaranarayanan, W. Olszak, The load carrying capacities of plates and shells, IASS NSP 1963 (1964), 842-866.

[7.291] - , - A note on the load carrying capacity of anisotropic plates and shells, BAP SST 14 (1966), 4, 329-336.

[7.292] R. Sankaranarayanan, Limit analysis of non-homogeneous shells of revolution, $B A P$ SST 14 (1966), 7, 633-636 (English summary); RI 14 (1966), 2, 231-240 (Polish full text). 
[7.293] R. Sankaranarayanan, Yield surfaces for non-homogeneous anisotropic shells of revolution, Proc IAS A65 (1967), 1, 49-61.

[7.294] M. Save, A consistent limit-analysis theory for reinforced concrete slabs, MCR 19 (1967), $58,3-12$.

[7.295] -, Théorie des charges limites des plaques et coques, STA 47 (1973), 2, 331-372.

[7.296] A. Sawczuk, Some problems of load carrying capacities of orthotropic and non-homogeneous plates, AMS 8 (1956), 4, 549-563.

[7.297] -, Yield condition for anisotropic shells, BAP SST 8 (1960), 6, 273-277.

[7.298] A. Sawczuk, J. Rychlewski, On yield surfaces for plastic shells, AMS 12 (1960), 1, 29-53.

[7.299] A. Sawczuk, P. G. Hodge, Jr., Comparison of yield conditions for circular cylindrica shells, JFI 269 (1960), 5, 362-374.

[7.300] A. Sawczuk, On the theory of anisotropic plastic plates and shells, AMS 13 (1961), 3, 355365 .

[7.301] A. Sawczuk, W. Olszak, A method of limit analysis of reinforced concrete tanks, SSCM (1962), 416-437.

[7.302] -, -, Zagadnienia powlok niesprężystych, MTS 1 (1963), 1, 37-73.

[7.303] A. Sawczuk, M. Duszek, A note on the interaction of shear and bending in plastic plates, AMS 15 (1963), 3, 411-426.

[7.304] A. Sawczuk, W. Olszak, Problems of inelastic shells, TPS 1963 (1966), 89-106.

[7.305] A. Sawczuk, On formulation of the equations of limit analysis of structures, ZAMM 46 (1966), Sonderheft, 28-32.

[7.306] - , On yield criteria and collapse modes for plates, IJNLM 2 (1967), 3, 233-243.

[7.307] A. Sawczuk, P. G. Hodge, Jr., Limit analysis and yield-line theory, Trans ASME E35 (1968), 2, 357-362.

[7.308] A. Sawczuk, On incremental collapse of shells under cyclic loading, IUTAM TTS 1967 (1969), 328.

[7.309] -, Yield surfaces, Univ. of Waterloo, Sol. Mech. Div. TN 1 (1971).

[7.310] -, Lagrangian formulation of large deflection theories for plastic shells, BAP SST 19 (1971), 6, 437-442.

[7.311] -, A note on anisotropic plate theory, AMS 23 (1971), 6, 961-967.

[7.312] A. Sawczuk, M. Janas, J. A. König, Analiza plastyczna konstrukcji, Zakład im. Ossolińskich PAN, Warszawa 1972.

[7.313] A. Sawczuk, Zagadnienia plastycznosci i analizy konstrukcji plastycznych, ZNPWr, Monografie (1979).

[7.314] M. Sayir, Kollapsbelastung von rotationssymetrischen Zylinderschalen, ZAMP 17 (1966), 2, 353-360.

[7.315] - , Die rotationssymmetrische dünne Zylinderschale aus idealplastischem Material, ZAMP 19 (1968), 2, 185-219.

[7.316] J. Schroeder, A. N. Sherbourne, A general theorem for thin shells in classical plasticity, JMP 47 (1968), 1, 95-108.

[7.317] —, -, Bounds for unsymmetrical yield point loads of thin shells, JMP 47 (1968), 2, 249-261.

[7.318] J. Schroeder, P. Roy, Yield points for partially loaded spherical domes, Proc ASCE JEMD 97 (1971), 1, 63-76.

[7.319] - , -, Upper bounds to limit pressure of branch-pipe lateral connections, Pap ASME $P V P-43$ and $P V P-44,12$ pp. and 8 pp., (1971).

[7.320] V. I. Sebekina, On the limit equilibrium of anisotropic shells under axisymmetric loadings (in Russian), SMRS (1966), 4, 13-18.

[7.321] O. N. Shabliy, Inelastic deformation of shallow shells of revolution made of hardening materials (in Russian), PM 6 (1970), 8, 24-29. 
[7.322] O. N. Shabliy, Rigid-plastic behaviour of sheıls and plates made of a linearly hardening material (in Russian), PM 7 (1971), 4, 35-43.

[7.323] -, Inelastic deformation of shells of revolution made of an anisotropic hardening material (in Russian), PM 8 (1972), 4, 30-38.

[7.324] O. N. Shabliy, K. A. Koba, Large deflections of circular and annular plates made of rigidplastic-hardening material (in Russian), PPro (1972), 3, 78-85.

[7.325] O. N. Shabliy, M. S. Mikhalishin, Limit equilibrium of cylindrical shells made of a perfectly rigid-plastic material with different yield-point stresses in tension and in compression (in Russian), PPro (1973), 8, 23-29.

[7.326] O. N. Shabliy, K. A. Koba, Finite deflections of annular shallow shells of revolution made of a rigid-plastic-hardening material (in Russian), PM 10 (1974), 3, 9-16.

[7.327] O. N. Shabliy, N. P. Zhuk, Limit equilibrium of a cylindrical shell accounting for shearing stresses (in Russian), PM 10 (1974), 7, 69-76.

[7.328] O. N. Shabliy, K. A. Koba, On a more accurate theory of finite deflections of rigid-plastic shells of revolution and of plates (in Russian), PM 12 (1976), 8, 3-10.

[7.329] - , - Analysis of rigid-plastic shells of revolution and of plates accounting for finite deflections (in Russian), PM 12 (1976), 9, 58-66.

[7.330] B. W. Shaffer, M. Senator, An elasticplastic analysis of a cylindrical shell with partially plastic cross sections, 4th USNCAM 2 (1962), 1113-1121.

[7.331] G. S. Shapiro, Elastic-plastic bending of a circular plate and the existence of solution of the rigid-plastic problem (in Russian), Izv AN SSSR OTN MM (1961), 2, 142-146.

[7.332] - , On yield surface for ideally plastic shells, MSS (1961), 414-418 (English version); 504-507 (Russian version).

[7.333] -, On the behaviour of plates and shells beyond the elastic limit (in Russian), 2nd VSTPM 1964 (1966), 378-386.

[7.334] A. N. Sherbourne, N. K. Srivastava, Elastic-plastic bending of restrained pin-ended circular plates, IJMS 13 (1971), 3, 231-241.

[7.335] Yu. M. Shevtchenko, O. I. Borisyuk, Effect of compressibility of the material in the problems of elastic-plastic deformations of thin shells (in Ukrainian), DAN URSR (1971), A11, 1035-1039.

[7.336] Yu. N. Shevtchenko, Elastic-plastic state of stress in unclosed shells of revolution under non-isothermal loading processes (in Russian), TNEK 16 (1976), 67-75.

[7.337] R. T. Shield, D. C. Drucker, Limit strength of thin-walled pressure vessels with an ASME standard torispherical head, 3rd USNCAM 1958 (1959), 665-672.

[7.338] A. Shindo, Y. Seguchi, T. Shirai, K. Denpo, A numerical approach to finite elastic-plastic deflections of circular plates, BJSME 15 (1972), 80, 158-170.

[7.339] I. V. Shirko, Elastic-plastic bending of a clamped plate (in Russian), $\operatorname{Tr}$ MFTI 3 (1959), 180-193.

[7.340] I. V. Shirko, V. L. Yakushev, Elastic-plastic bending of a cylindrical shell by using the Prandtl-Reuss theory (in Russian), Izv VUZ M (1973), 2, 21-26.

[7.341] N. A. Shoeb, W. C. Schnobrich, Elastic-plastic analysis of cylindrical shells, Proc ASCE JEMD 98 (1972), 1, 47-59.

[7.342] J. Skrzypek, Odksztalcenia plastyczne, analiza form utraty nośności geometrycznie nieliniowych powlok toroidalnych, ZNPK PNT (1979), $194 \mathrm{pp}$.

[7.343] V. V. Sokolovsky, Elastic-plastic bending of circular and annular plates (in Russian), PMM 8 (1944), 2, 141-166.

[7.344] - , Plastic bending of a circular plate (in Russian), IZh 3 (1963), 3, 563-568.

[7.345] K. Soonets, On the behaviour of rotationally symmetric circular cylindrical shells accounting for elastic-plastic deformations (in Russian), UZTGU 342 (1974), 311-322.

[7.346] N. K. Srivastava, A. N. Sherbourne, Elastic plastic bending of circular plates, Proc ASCE JEMD 97 (1971), 1, 13-31. 
[7.347] V. N. Stelmashuk, An approximate engineering theory of perfectly rigid-plastic shells of revolution (in Russian), TNEK 7 (1967), 65-70.

[7.348] A. I. Strelbitskaya, V. A. Kolgadin, Analysis of bending of rectangular plates beyond the elastic limit (in Russian), PM 2 (1966), 10, 44-53.

[7.349] A. I. Strelbitskaya, A survey on the bending of plates beyond the elastic limit (in Russian), PM 2 (1966), 12, 1-17.

[7.350] A. I. Strelbitskaya, V. A. Kolgadin, S. I. Matoshko, Application of the methods of the theory of plasticity to the problems of inelastic bending of rectangular plates (in Russian), PM 3 (1967), 8, 31-40.

[7.351] A. I. Strelbitskaya, V. A. Kolgadin, R. I. Rybakova, Effect of mixed boundary conditions on the elastic-plastic state of plates in bending (in Russian), PM 3 (1967), 11, 29-36.

[7.352] A. I. Strelbitskaya, V. A. Kolgadin, Stress and strain state of plates in bending accounting for plastic hardening of the material (in Russian), PM 5 (1969), 2, 85-95.

[7.353] A. I. Strelbitskaya, V. A. Kolgadin, S. I. Matoshko, On the investigation of elastic-plastic bending of plates, non-homogeneous in the elastic range (in Russian), PM 5 (1969), 10, $106-111$.

[7.354] - , - , , Bending of rectangular plates beyond the elastic limit (in Russian), Naukova Dumka, Kiev 1971.

[7.355] A. I. Strelbitskaya, S. A. Goloborodko, R. I. Rybakova, Elastic-plastic bending of rectangular flexible plates with various side ratios (in Russian), $P M 7$ (1971), 8, 59-68.

[7.356] A. I. Strelbitskaya, On the determination of plastic coefficients for plates accounting for plastic hardening of the material (in Russian), PM 7 (1971), 10, 106-111.

[7.357] - , Behaviour of flexible plates beyond the elastic limit in the case of homogeneous boundary conditions (in Russian), PM 7 (1971), 12, 71-81.

[7.358] - Elastic-plastic deformations and load-carrying capacity of shallow shells (survey) (in Russian), PM 9 (1973), 8, 3-21.

[7.359] - Elastic-plastic behaviour of shallow shells under uniformly distributed loading (in Russian), PM 11 (1975), 10, 25-35.

[7.360] - , On the solution of elastic-plastic problems of shallow shells under transverse loading (in Russian), PM 12 (1976), 4, 47-59.

[7.361] J. A. Stricklin, Pao-Tan Hsu, T. H. H. Pian, Large elastic, plastic and creep deflections of curved beams and axisymmetric shells, AIAA 2 (1964), 9, 1913-1920.

[7.362] W. Szczepiński, Lower bounds on bearing capacity of shells and plates loaded at the edges by distributed moments, AMS 26 (1974), 1, 95-104.

[7.363] M. Tanaka, Large deflection analysis of elastic-plastic circular plates with combined isotropic and kinematic hardening, IA 41 (1972), 5, 342-356.

[7.364] S. C. Tang, Elasto-plastic and large deformation analysis of thin shells by the deformation theory of plasticity, CS 6 (1976), 4-5, 297-303.

[7.365] B. Tekinalp, Elastic-plastic bending of a built-in circular plate under a uniformly distributed load, JMPS 5 (1957), 2, 135-142.

[7.366] V. R. Terrovere, Uniform strength reinforcement of the edge zone of shells of revolution (in Russian), PM 6 (1970), 10, 42-48.

[7.367] —, Load-carrying capacity and optimal design of an ellipsoidal shell (in Russian), PM 8 (1972), 2, 68-72.

[7.368] - Effect of finite geometry changes on the load-carrying capacity of shells of revolution (in Russian), PM 12 (1976), 10, 15-20.

[7.369] B. J. Thorn, J. S. Kao, S. L. Lee, Nonregular progression in strain hardening shells, Proc ASCE JEMD 92 (1966), 1, 59-80.

[7.370] S. Timoshenko, S. Woinowsky-Krieger, Theory of plates and shells, McGraw-Hill, New York 1959. 
[7.371] Tran-le Binh, M. Życzkowski, Problem of the decohesive carrying capacity of a cylindrical shell under ring of forces and tension (in print).

[7.372] -, -, Interaction curves corresponding to decohesive carrying capacity of a cylindrical shell under combined loading (in print).

[7.373] Tran-le Binh, Combined loadings of perfectly elastic-plastic cylindrical shells resulting in decohesive carrying capacity (in print).

[7.374] I. S. Tsurkov, Elastic-plastic equilibrium of shells of revolution in the range of small axisymmetric deformations (in Russian), Izv AN SSSR OTN (1956), 11, 106-110.

[7.375] - Elastic plastic equilibrium of shallow shells in the range of small deformations (in Russian), Izv AN SSSR OTN (1957), 6, 139-142.

[7.376] - , On the problem of elastic-plastic bending of shallow metal shells with finite deflections (in Russian), IZh 1 (1961), 1, 145-153.

[7.377] R. Valid, La théorie linéaire des coques et son application aux calculs inélastiques, Publ. ONERA 147 (1973), 147 pp.

[7.378] V. V. Vasilev, Axisymmetric elastic-plastic state of a shell of revolution (in Ukrainian), PM 7 (1961), 3, 272-278.

[7.379] - Elastic-plastic deformations of metal pressure vessels, reinforced by a unidirectional system of glass fibres (in Russian), MPol (1969), 6, 1069-1074.

[7.380] - , A convenient form of equations of elastic-plastic state of a shell of revolution under combined loading (in Russian), SMTS 15 (1971), 132-136.

[7.381] T. P. Vinogradova, Elastic-plastic bending of flexible rectangular plates (in Russian), PM 6 (1970), 5, 73-81.

[7.382] Yu. M. Voltchkov, G. V. Ivanov, V. N. Solodovnikov, Relations between curvature rates and moment rates in the problem of elastic-plastic bending of plates (in Russian), Izv AN SSSR MTT (1970), 2, 171-175.

[7.383] A. N. Vozyanov, On the effect of compressibility of the material on elastic-plastic bending of shells and plates (in Russian), Tr LKI 62 (1968), 23-28.

[7.384] Z. Waszczyszyn, Finite elastic-plastic deflections of circular symmetric plates, BAP SST 15 (1967), 4, 293-302.

[7.385] —, Ścisłe równania i obliczanie skończonych sprężysto-plastycznych ugięć ortotropowych powłok obrotowo-symetrycznych, RI 16 (1968), 3, 363-383.

[7.386] -, Obliczanie skończonych ugięć sprężysto-plastycznych plyt i powlok obrotowo-symetrycznych, ZNPK PNT 5 (1970), 203 pp.

[7.387] -, Calculation of sandwich shells of revolution at large elastic-plastic deflections, AMS 24 (1972), 3, 483-498.

[7.388] —, Postcritical behaviour of an idealized circular plate beyond the elastic limit (in Russian), Izv AN SSSR MTT (1973), 5, 125-130.

[7.389] J. H. Weiner, An elastoplastic thermal-stress analysis of a free plate, JAM 23 (1956), 3, $397-401$.

[7.390] R. H. Wood, The importance of shear in the yield criterion for the bending of slabs, JSA 6 (1971), 1, 13-19.

[7.391] S. Yaghmai, E. P. Popov, Incremental analysis of large deflections of shells of revolution IJSS 7 (1971), 10, 1375-1393.

[7.392] $\mathrm{H}$. Yüksel, Elastic, plastic stresses in free plate with periodically varying surface temperature, JAM 25 (1958), 4, 603-606.

[7.393] A. Zavelani Rossi, Sul criterio statico di calcolo a rottura nei sistemi bidimensionali piani, RIL ASL A102 (1968), 1, 124-165.

[7.394] —, Sul criterio cinematico di calcolo a rottura nei sistemi bidimensionali piani, RIL ASL A102 (1968), 1, 108-123. 
[7.395] N. P. Zhuk, O. N. Shabliy, On the limit equilibrium of shells of revolution and of circular plates accounting for shearing stresses (in Russian), PM 8 (1972), 7, 35-41.

[7.396] - , - Limit equilibrium of a circular plate accounting for shearing stresses (in Russian), PM 9 (1973), 6, 47-53.

\section{Chapter VIII}

[8.1] N. M. Adyasova, S. A. Kapustin, E. I. Sankov, Calculation of elastic-plastic thin plates accounting for in-plane forces (in Russian), UZGU 122 (1970), 13-21.

[8.2] P. K. Agrawal, P. G. Hodge, Jr., Yield-point ring load of filled circular cylindrical shells, IJMS 12 (1970), 11, 935-947.

[8.3] A. Ajovalasit, S. Noto La Diega, Influenza della tensione assiale sul comportamento plastico dei cilindri rotanti, TI 33 (1968), 10, 655-661.

[8.4] N. V. Akhvledyani, G. I. Lezhava, Investigations of the load-carrying capacity of trusses under combined loadings (in Russian), ISM (1970) 72-77.

[8.5] J. B. Alblas, On the limit analysis of a rectangular plate under combined stresses, $A S R$ 12 (1964), 4, 435-453.

[8.6] de G. Allen D. N., D. G. Sopwith, The stresses and strains in a partly plastic thick tube under internal pressure and end load, 7th ICAM (1948); Proc RS A205 (1951), 69-83.

[8.7] V. N. Alpert, Finite elastic-plastic deformations of a non-uniformly heated rotating disk (in Russian), PM 1 (1965), 9, 58-64.

[8.8] - , Load-carrying capacity of a rotating disk under a transverse loading (in Russian), $\operatorname{Tr}$ KuyAI 45 (1970), 255-260.

[8.9] R. J. Alvarez, C. Birnstiel, Inelastic analysis of multistory multibay frames, Proc ASCE JSD 95 (1969), 11, 2477-2503.

[8.10] S. Amada, Elasto-plastic stress analysis of discs, Pap SRI 36 (1971), 1-15.

[8.11] -, Cyclic thermal and centrifugal loadings in discs, ICMBM 1971, 3 (1972), 21-30.

[8.12] S. Amada, A. Machida, Non-steady elastic-plastic thermal stresses in rotating discs (in Japanese), Trans JSME 38 (1972), 316, 3038-3045.

[8.13] - , - Elastic-plastic stress analysis of rotating discs: discs subjected to transient thermal and constant centrifugal loading, Pap SRI 46 (1973), 40 pp.

[8.14] C. Amariei, L'analyse élasto-plastique des structures soumises aux charges variables, Conf. Privind. Calc. Seismic Struct. Iaşi 1 (1970). 27-37.

[8.15] S. C. Anand, V. K. Garg, Shakedown of rolling wheel under hub loading, Proc ASCE JEMD 100 (1974), 6, 1237-1251.

[8.16] D. Annaratone, Criteri di dimensionamento dei cilindri ad altissima pressione, Ina (1970), 9, 581-596.

[8.17] J. M. Aribert, P. Jouve, Simulation du comportement élasto-plastique d'une ossature en flexion plane, CoMet 9 (1972), 3, 5-16.

[8.18] H. Armen, H. S. Levine, A. Pifko, Elastic-plastic behavior of plates under combined bending and stretching, 1lth SSDM (1970), 224-238.

[8.19] Yu. Yu. Atkochiunas, R. P. Karkauskas, A. A. Cyras, Non-linear analysis of circular elastic-plastic plates under variable repeated loading by using finite element discretization (in Russian), LMS (1972), 2, 75-91.

[8.20] G. Augusti, A. Baratta, Theory of probability and limit analysis of structures under multiparameter loading, SFP 1972 (1973), 347-364.

[8.21] C. H. Austin, A numerical solution for the expansion of thick-walled cylindrical pressure vessels, Doct. Diss. Univ. Pittsburgh (1967); Diss. Abstracts B29 (1968), 1, 196.

[8.22] K. B. Ayers, Behaviour patterns in I beams carrying a pure bending moment and subjected to a repeated thermal cycle, JMES 6 (1964), 4, 343-352.

[8.23] M. E. Babeshko, State of stress in a short solid cylinder under non-isothermal loading (in Russian), TNEK 11 (1971), 121-127. 
[8.24] M. E. Babeshko, V. K. Stryuk, On the calculation of stresses in a solid cylinder based on the theory of plastic flow with isotropic hardening (in Russian), PM 10 (1974), 6, 27-33.

[8.25] G. Backhaus, Grundlagen der Dimensionierung im plastischen Bereich, MIL 8 (1969), 3, 116-120.

[8.26] J. F. Baker, Comportement et conception des ossatures métalliques, RSou 3 (1950), 95-114.

[8.27] J. F. Baker, M. R. Horne, L. G. Johnson, The use of plastic theory in the design of a fabricating shop, Proc ICE, Dec. 1961.

[8.28] R. L. Barnett, Optimum prestressed tubular columns, Proc ASCE JSD 96 (1970), 2, 291-307.

[8.29] R. P. Baronas, Problems of optimization of a part of parameters of elastic-plastic frames (in Russian), LMS (1973), 1 (12), 89-103.

[8.30] R. S. Barsoum, A convergent method for cyclic plasticity analysis with application to nuclear components, IJNME 6 (1973), 2, 227-236.

[8.31] V. N. Bastun, V. M. Perepelin, L. M. Shkaraputa, Peculiarities of the deformation of titanium alloys in plane stress conditions (in Russian), PM 8 (1972), 4, 93-99.

[8.32] V. G. Bazhenov, G S. Mikhaylov, On a certain solution method of problems of statics and dynamics of axisymmetric elastic-plastic shells allowing for large deflections and combined non-isothermal loading (in Russian), UZGU 108 (1970), 91-97.

[8.33] - , - A numerical analysis of dynamic behaviour of elastic-plastic shells of revolution under intensive heating (in Russian), TNEK 11 (1971), 143-148.

[8.34] V. G. Bazhenov, G. S. Mikhaylov, A. G. Ugodchikov, Dynamic problems of thermoplasticity of shells of revolution (in Russian), 8th TOP 1971 (1973), 93-99.

[8.35] F. J. Beer, Plastic growth of pressurized shell through interaction of steady pressure with cyclic thermal stresses, TSTF (1971), 340-367.

[8.36] N. M. Belayev, A. K. Sinitsky, Stresses and strains in thick-walled cylinders in the elasticplastic range (in Russian), Izv AN SSSR OTN (1938), 2, 3-54.

[8.37] L. M. Belenky, Combined bending of rigid-plastic beams under transverse concentrated forces (in Russian), Tr KTIRP 33 (1970), 30-34.

[8.38] - Peculiarities of plastic deformation of beams under combined bending (in Russian), Tr KTIRP 33 (1970), 35-43.

[8.39] A. R. Belyakov, O.F. Chernyavsky, Analysis of shakedown conditions of a circular plate under a multiparameter loading (in Russian), TNEK 14 (1974), 48-52.

[8.40] D. Besdo, Principal- and slip-line methods of numerical analysis in plane and axiallysymmetric deformations of rigid plastic media, JMPS 19 (1971), 6, 313-328.

[8.41] I. A. Birger, Theory of plastic flow and the calculation of disks (in Russian), RP 12 (1966), 183-199.

[8.42] I. A. Birger, M. F. Yakovlev, V. F. Guk, A numerical method of strength calculation of a disk allowing for plastic deformations (in Russian), TPM 6 (1975), 3, 31-37.

[8.43] D. R. Bland, Elastoplastic thick-walled tubes of work-hardening material subject to internal and external pressures and to temperature gradients, JMPS 4 (1956), 4, 209-229.

[8.44] H. H. Bleich, I. Nelson, Plane waves in an elastic-plastic half-space due to combined surface pressure and shear, Trans ASME E33 (1966), 1, 149-158.

[8.45] S. A. Bocharova, State of stress in a tube under a uniform internal pressure and a longitudinal force in the range of large plastic deformations (in Russian), RP 9 (1963), 196-218.

[8.46] —, State of stress in a thick-walled tube under internal pressure and longitudinal force (in Russian), Izv VUZ $M$ (1967), 2, 16-23.

[8.47] - , On the limits of applicability of the theory of small plastic deformations to the calcur lation of thick-walled tubes under internal pressure and longitudinal force (in Russian), Izv VUZ M (1967), 3, 15-18.

[8.48] —, State of stress in an anisotropic cylinder under internal pressure and longitudinal force in the range of large plastic deformations (in Russian), Izv VUZ $M$ (1971), 7, 5-10. 
[8.49] S. A. Bocharova, Determination of internal pressure and of longitudinal force acting on an anisotropic cylinder in the range of large plastic deformations (in Russian), Izv VUZ $M$ (1971), 8, 10-15.

[8.50] S. R. Bodner, Buckling of an elasto-plastic column subjected to axial load and a transverse temperature gradient, IJMS 4 (1962), 425-437.

[8.51] A. N. Bogdanov, Plastic flow of a curved bar under certain combinations of normal and tangential loads acting on the free end (in Russian), $\operatorname{Tr}$ LIVT 88 (1967), 9-15.

[8.52] N. Bogdanova, Combined stress and strain states of elastic-plastic hollow cylindrical bodies (in Bulgarian), TMysl 2 (1965), 2, 31-38.

[8.53] B. N. Borah, Thermo-elastic-plastic transition of tubes under uniform pressure and steady state temperature, IJPAM 3 (1972), 1, 82-91.

[8.54] V. D. Bordyug, The boundary layer method in the theory of thin inelastic shells (in Russian), SK 6 (1970), 155-198.

[8.55] A. I. Borisyuk, Elastic-plastic state of stress in shells of revolution under axisymmetric repeated loadings (in Russian), TNEK 8 (1969), 157-165.

[8.56] - Investigations on an axisymmetric thermoelastic-plastic state of stress in a conical shell with various boundary conditions (in Russian), TNEK 13 (1973), 32-36.

[8.57] A. I. Borisyuk, I. V. Prokhorenko, Axisymmetric elastic-plastic state of a shell of revolution under non-uniform heating and irradiation (in Russian), TNEK 15 (1975), 57-64.

[8.58] J. F. Bratt, Ö. Kanan, Determination of the yield condition in the third quadrant of the stress plane, Trans ASME E33 (1966), 1, 228.

[8.59] J. Bree, Elastic-plastic behaviour of thin tubes subjected to internal pressure and intermittent high-heat fluxes with application to fast-nuclear-reactor fuel elements, JSA 2 (1967), 3, 226-238.

[8.60] A. R. Brunsvold, H. V. Ahmed, C. C. Stone, Thermal ratcheting with kinematic hardening in a two-bar assembly with unequal areas and properties, Trans ASME J98 (1976), 3, 256-263.

[8.61] B. Budiansky, N. F. Dow, R. W. Peters, R. P. Shepherd, Experimental studies of polyaxial stress-strain laws of plasticity, 1st USNCAM 1951 (1952), 503-512.

[8.62] D. Burgreen, Structural growth induced by thermal cycling, Trans ASME D90 (1968), $4,469-475$.

[8.63] Gh. Buzdugan, Efectul variaţiei uniforme a temperaturii asupra capacităţii portante a grinzilor solicitate la incovoiere elasto-plastica, MCM 10 (1958), 1, 21-24.

[8.64] C. R. Calladine, The effect of cross-section on the creep buckling behaviour of columns, IJMS 4 (1962), 387-407.

[8.65] M. Capurso, A. Gandolfi, Sul collasso plastico dei tubi circolari nervati soggetti a pressione e sforzo assiale, CoM 20 (1968), 2, 102-118.

[8.66] G. Ceradini, Sul calcolo delle strutture elastoplastiche, CoM 17 (1965), 3, 177; 17 (1965), 5, 327-332.

[8.67] T. Y. Chang, S. C. Chu, Elastic-plastic deformation of cylindrical pressure vessels under cyclic loading, NED 27 (1974), 2, 228-237.

[8.68] D. E. Charrett, G. I. N. Rozvany, Extensions of the Prager-Shield theory of optimal plastic design, IJNLM 7 (1972), 1, 51-64.

[8.69] W. F. Chen, S. Santathadaporn, Curvature and the solution for eccentrically loaded columns, Proc ASCE JEMD 95 (1969), 1, 21-40.

[8.70] W. F. Chen, Further studies of inelastic beam-column problem, Proc ASCE JSD 97 (1971), 2, 529-544.

[8.71] G. P. Cherepanov, Inverse elastic-plastic problem under plane strain conditions (in Russian), Izv AN SSSR OTN MM (1963), 1, 57-60.

[8.72] - , On a certain method of solution of elastic-plastic problems (in Russian), PMM 27 (1963), 3, 428-435. 
[8.73] J. M. Chern, D. H. Pai, Inelastic analysis of a straight tube under combined bending, pressure and thermal loads, Trans ASME J97 (1975), 3, 155-162.

[8.74] V.S. Chernina, Elastic-plastic deformation of a welded non-homogeneous cylindrical shell (in Russian), Izv AN SSSR OTN MM (1960), 1, 133-140.

[8.75] M. L. Chernov, Load-carrying capacity of steel beams under a moving system of forccs (in Ukrainian), PM 2 (1956), 4, 409-419.

[8.76] -, Deformation of steel beams beyond the elastic limit under a moving system of forces (in Ukrainian), PM 3 (1957), 4, 420-431.

[8.77] —, The effect of loading motion on the deformation of steel beams beyond the elastic limit (in Ukrainian), PM 10 (1964), 1, 46-54.

[8.78] N. L. Chernov, V. L. Tarasenko, Elastic-plastic deflections of two-span steel beams on flexible supports under moving loads (in Russian), NTGK 12 (1969), 105-117.

[8.79] N. L. Chernov, Shakedown analysis of continuous beams under moving loads (in Russian), Izv VUZ SA (1970), 6, 23-29.

[8.80] —, Elastic-plastic bending of I-beams allowing for shearing stresses and contact compressive stresses under moving loads (in Russian), Izv VUZ SA (1972), 8, 3-9.

[8.81] —, On the gradual development of plastic deformations in steel beams under moving loads (in Russian), Izv VUZ SA (1973), 1, 3-7.

[8.82] O.F. Chernyavsky, Load-carrying capacity of a perfectly plastic conical shell under temperature variations (in Russian), TNEK 10 (1970), 166-172.

[8.83] O. F. Chernyavsky, E. F. Chernyayev, Shakedown of cylindrical shells under repeated thermal and mechanical loadings (in Russian), TNEK 14 (1974), 52-55.

[8.83a] I. S. Chernyshenko, G. K. Sharshukov, On elastic-plastic analysis of shells of revolution under cyclic loadings (in Russian), PPro (1976), 4, 99-102.

[8.84] S. C. Chu, A more rational approach to the problem of an elastoplastic thick-walled cylinder, JFI 294 (1972), 1, 57-65.

[8.85] S. C. Chu, J. D. Vasilakis, Inelastic behavior of thick-walled cylinders subject to nonproportionate loading, ExpM 13 (1973), 3, 113-119.

[8.86] E. Chwalla, Das Tragvermögen gedrückter Baustahlstäbe mit krummer Achse und zusătzlicher Querbelastung, Stb 8 (1935), 34.

[8.87] C. Cichoń, The shakedown of circular arches for movable loads, RI 23 (1975), 4, 641-656.

[8.88] R. J. Clifton, Plastic waves: theory and experiment, MechT 1 (1972), 102-167.

[8.89] M. Z. Cohn, D. E. Grierson, An automatic approach to the analysis of plastic frames under fixed and variable loading, SEng 49 (1971), 7, 291-297.

[8.90] M. Z. Cohn, S. R. Parimi, Optimal design of plastic structures for fixed and shakedown loadings, Trans ASME E40 (1973), 2, 595-599.

[8.91] M. D. Coon, S. S. Gill, The effect of change of geometry on the rigid plastic limit load of cylinders, IJMS 10 (1968), 5, 355-368.

[8.92] A. D. Cox, A. J. M. Spencer, A note on the approximate calculation of yield-point loads for ideal plastic-rigid solids taking account of body forces, ZAMP 14 (1963), 2, 172-175.

[8.93] N. Cristescu, On the propagation of elastic-plastic waves under combined loadings (in Russian), PMM 23 (1959), 6, 1124-1129.

[8.94] —, On the coupling of plastic waves as related to the yield condition, RRST SMA 16 (1971), 4, 797-809.

[8.95] B. Crossland, R. Hill, On the plastic behaviour of thick tubes under combined torsion and internal pressure, JMPS 2 (1953), 1, 27-38.

[8.96] S. Cuomo, Sulla resistenza di travi inflesse di acciaio ad elevate temperature, GGC 98 (1960), 11, 861-883.

[8.97] A. A. Cyras, Minimal weight design of frames under variable repeated loading (in Russian), SMRS (1965), 6. 
[8.98] A. A. Cyras, A proof of fundamental theorems of optimization of a perfectly elastic-plastic body (in Russian), LMS (1971), 1, 27-34.

[8.99] -, Theory of optimization of an elastic-plastic body under variable repeated loadings (in Russian), SMRS (1972), 1, 1-6.

[8.100] A. Cyras, K. Vislavicius, Optimization of elastic-plastic structures under movable loading, MRC 2 (1975), 4, 181-185.

[8.101] A. Sh. Dadashev, Elastic-plastic equilibrium and shakedown of a thick-walled tube under internal pressure and temperature gradient (in Russian), Izv AN AzbSSR FTM (1965) $4,123-132$.

[8.102] -, On shakedown of non-uniformly heated rotating cylinders (in Russian), Izv AN AzbSSR FTM (1965), 5, 50-54.

[8.103] - , On shakedown of a hollow sphere under a temperature field and internal pressure (in Russian), UZAU FM (1966), 2, 19-22.

[8.104] -, Elastic-plastic equilibrium and shakedown of a tube with internal coating subject to external pressure and temperature gradient (in Russian), SDZ TUP (1968), 70-81.

[8.105] M. A. Danelashvili, On inconvenient loading of reinforced shells of revolution (in Russian), ISM (1970), 67-71.

[8.106] I. N. Danilova, On the effect of the magnitude of a bore-hole on the load-carrying capacity of a rotor (in Russian), Izv AN SSSR OTN (1956), 5, 122-123.

[8.107] —, Effect of non-uniform heating and of compressibility of the material on the load-carrying capacity of a solid rotor (in Russian), Izv AN SSSR OTN (1958), 6, 104-106.

[8.108] V. I. Danilovskaya, Elastic-plastic symmetric deformation of a thick-walled tube taking account of non-uniform temperature distribution along the radius (in Russian), $P M 1$ (1965), 6, 8-13.

[8.109] V. V. Danilovskaya, A comparative analysis of elastic carrying capacity and of limit carrying capacity of a pipe-line (in Russian), $\operatorname{Tr}$ LKI 46 (1964), 103-111.

[8.110] P. K. Das, D. C. Chandler, B. K. Foster, The plastic bending of beams and their failure by low cycle fatigue, Trans ASME H95 (1973), 3, 161-169.

[8.111] J. M. Davies, Collapse and shakedown loads of plane frames, Proc ASCE JSD 93 (1967), $3,35-50$.

[8.112] E. A. Davis, Combined tension-torsion tests with fixed principal directions, JAM 22 (1955), $3,411-415$.

[8.113] I. V. Demyanushko, Yu. M. Temis, Kinetics of the stress-strain state of disks under cyclic non-isothermal loading (in Russian), Izv AN SSSR MTT (1975), 3, 90-98.

[8.114] M. G. Derrington, W. Johnson, The onset of yield in a thick spherical shell subject to internal pressure and uniform heat flow, $A S R$ A7 (1959), 6, 408-420.

[8.115] R. Dobrev, Limit state of uniform and sandwich cylindrical shells under non-proportional axial and transverse loadings (in Bulgarian), Izv ITM BAN 3 (1966), 23-42.

[8.116] V. L. Dobrovolsky, Solution of two problems for a perfectly plastic material (in Russian), $\operatorname{Tr}$ MIEM 53 (1975), 147-152.

[8.117] V. M. Dolinsky, B. S. Kovalsky, Elastic-plastic bending of a circular plate resting on an elastic-plastic foundation (in Russian), SMTS 12 (1970), 48-50.

[8.118] I. G. Dovgyallo, V. I. Bord, S. S. Makarevitch, I. P. Nekhayev, On the stress analysis in some cases of combined loading (in Russian), Prochnost i Plast. Mat. v Ultrazvuk. Pole I, Minsk 1973, 43-47.

[8.119] D. C. Drucker, H. G. Hopkins, Combined concentrated and distributed load on ideallyplastic circular plates 2nd USNCAM 1954 (1955), 517-520.

[8.120] D. C. Drucker, R. T. Shield, Bounds on minimum weight design, QAM 15 (1957), 3, 269-281.

[8.121] M. Duszek, A. Sawczuk, Unstable limit analysis solution for multistorey frames, Stav $\check{C} \mathbf{2 3}$ (1975), 9, 638-649. 
[8.122] M. Duszek, A. Sawczuk, Stable and unstable states of rigid plastic frames at the yield-point load, JSM 4 (1976), 1, 33-47.

[8.123] C. Dyrbye, Continuous girders with distributed live load, Mem AIPC 30 (1970), 1, 1-13.

[8.124] H. G. Edmunds, F. J. Beer, Notes on incremental collapse of pressure vessels, JMES 3 (1961), 3, 187-199.

[8.125] A. M. El-Karamani, Stress concentration around a circular hole in an elastic-plastic medium under a temperature field and all-round tension (in Russian), $V M U M M(1972), 4,91-97$.

[8.126] E. Elter, Einfluß der Rest- oder Eigenspannungen auf die Deformation der Platten, WZTUD 21 (1972), 1, 163-168.

[8.127] R. Épain, Résistance des cylindres épais élastoplastiques, SP (1962), 37-66.

[8.128] M. I. Erkhov, Load-carrying capacity of an overground thin-walled pipe-line (in Russian), SMRS (1960), 6, 19-32.

[8.129] —, Problems of strength of perfectly plastic shells (in Russian), SK 4 (1969), 74-164.

[8.130] P. I. Ermakov, Analysis of irreversible deformation of a cylinder under temperature variations (in Russian), TNEK 4 (1964), 214-222.

[8.131] P. I. Ermakov, A. S. Nesmeyanov, A. F. Malygin, V. I. Morozov, Analysis of irreversible deformation of thin-walled cylinders under cyclic operations of pressure and temperature field (in Russian), SNT CPI 92 (1971), 49-55.

[8.132] P. I. Ermakov, A. S. Nesmeyanov, V. I. Morozov, Elastic-plastic deformation of a thickwalled cylinder under repeated operations of pressure and temperature field (in Russian), SNT CPI 92 (1971), 56-60.

[8.133] P. I. Ermakov, A. F. Malygin, B. E. Dorofeyev, On the cumulation of irreversible deformations of thick-walled tubes under temperature variations (in Russian), PPro (1974), 2, 24-27.

[8.134] P. I. Ermakov, A. S. Nesmeyanov, V. I. Morozov, Irreversible deformation of a hexagonal tube under cyclic operations of temperature field and pressure (in Russian), TNEK 14 (1974), 45-48.

[8.135] N. F. Ershov, Elastic-plastic deformations of strips under combined loading (in Russian), $\operatorname{Tr}$ GPI 22 (1966), 3, 31-41.

[8.136] D. G. Eyre, T. V. Galambos, Variable repeated loading-a literature survey, Civ. and Environmental Eng. Dept., Washington Univ. (St. Louis), Struct. Div. Rep. 3 (1967).

[8.137] —, -, Shakedown of bars under extreme loads, Proc ASCE JSD 101 (1975), 9, 2005-2009,

[8.138] H. Faupel, Residual stresses in heavy-wall cylinders, JFI 259 (1955), 5, 405.

[8.139] M. Feigen, Inelastic behavior under combined tension and torsion, 2nd USNCAM 1954 (1955), 469-476.

[8.140] R. P. Felgar, Plastic analysis of the instability of pressure vessels subjected to internal pressure and axial load, Trans ASME D84 (1962), 2, 278-286.

[8.141] L. P. Felton, R. B. Nelson, Optimized components in frame synthesis, AIAA 9 (1971), $6,1027-1031$.

[8.142] V. I. Feodosyev, Strength of details of jet engines under thermal loadings (in Russian) Oborongiz, Moskva 1963.

[8.143] G. E. Findlay, J. Spence, Applying the shakedown concept to pressure vessel design, Enr 226 (1968), 63-65.

[8.144] B. Fischer, Zur zyklischen, elastoplastischen Beanspruchung eines dickwandigen Zylinders bei endlichen Verzerrungen, MIMB 9 (1977).

[8.145] W. Flügge, J. C. Gerdeen, Collapse of a simply supported circular plate under two uniform loads, IJSS 3 (1967), 4, 677-689.

[8.146] V. L. Fomin, Elastic-plastic equilibrium of a non-uniformly heated tube under internal pressure (in Russian), Izv AN SSSR OTN MM (1960), 3, 55-59.

[8.147] - Elastic-plastic equilibrium of a hollow cylinder under an axisymmetric temperature. field (in Russian), Izv AN SSSR OTN MM (1961), 5, 127-128. 
[8.148] V. L. Fomin, On shakedown of elastic-plastic tubes under the operation of a thermal field and a uniform external pressure (in Russian), Izv AN SSSR OTN MM (1962), 1, 149-152.

[8.149] —, Plane deformation of hardening hollow cylinders under internal pressure and stationary thermal field (in Russian), IUP 3 (1964), 161-171.

[8.150] N. A. Forsman, On elastic-plastic bending of plates (in Russian), ZhPMTF (1960), 4, $79-80$.

[8.151] J. Foulkes, Minimum weight design and the theory of plastic collapse, QAM 10 (1953) 347-358.

[8.152] -, The minimum weight design of structural frames, Proc RS A223 (1954), 482.

[8.153] J. D. Fox, H. Kraus, R. K. Penny, Shakedown of pressure vessels with ellipsoidal heads, Pap ASME PVP-34 (1971), 11 pp.

[8.154] V. Franciosi, G. Augusti, R. Sparacio, Collapse of arches under repeated loading, Proc ASCE JSD 90 (1964), 1, 165-201.

[8.155] L. I. Fridman, Changes of plastic deformations due to multiple non-uniform heatings (in Russian), TNEK 3 (1963), 120-131.

[8.156] -, Shakedown of non-uniformly heated rotating disk (in Russian), TNEK 7 (1967), 108-113.

[8.157] -, Shakedown of turbine blades (in Russian), PDAD 6 (1971), 170-182.

[8.158] Y. Fukumoto, H. Yoshida, Deflection stability of beams under repeated loads, Proc ASCE JSD 95 (1969), 7, 1443.

[8.159] - , - Failure of arches under variable repeated loading, Mem AIPC 30 (1970), 1, 15-40.

[8.160] V. K. Garg, S. C. Anand, P. G. Hodge, Jr., Elastic-plastic analysis of a wheel rolling on a rigid track, IJSS 10 (1974), 9, 945-956.

[8.161] B. E. Gatewood, R. W. Gehring, Allowable axial loads and bending moments for inelastic structures under nonuniform temperature distribution, JAS 29 (1962), 5, 513-520.

[8.162] F. A. Gaydon, A. W. McCrum, A theoretical investigation of the yield point loading of a square plate with a central circular hole, JMPS 2 (1954), 3, 156-169.

[8.163] F. A. Gaydon, On the yield-point loading of a square plate with concentric circular hole, JMPS 2 (1954), 3, 170-176.

[8.164] M. M. Ghamian, S. Krishnasamy, A. N. Sherbourne, Inelastic deformations of mildsteel beams under symmetrical and unsymmetrical cyclic bending, ExpM 14 (1974), 2, 49-56.

[8.165] A. F. M. Ghani, A. Roushan, Shakedown analysis of nonprismatic beams, Proc ASCE JSD 93 (1967), 6, 25-54.

[8.166] S. S. Gill, Three "neutral" loading tests, JAM 23 (1956), 4, 497-502.

[8.167] S. Glasstone, Nuclear reactor engineering, Macmillan, London 1956.

[8.168] D. A. Gokhfeld, Certain results of experimental investigation of shakedown under thermal loadings (in Russian), TNET 2 (1962), 133-140.

[8.169] D. A. Gokhfeld, K. M. Kononov, Shakedown of non-uniformly heated rotating disks (in Russian), Izv VUZ M (1962), 4, 85-91.

[8.170] D. A. Gokhfeld, P. I. Ermakov, Shakedown of thick-walled tubes subject to non-uniform heating (in Russian), ZhPMTF (1963), 3, 107-110.

[8.171] - , - Calculation of thick-walled cylinders subject to repeated operations of pressure and temperature (in Russian), TNEK 3 (1963), 111-119.

[8.172] D. A. Gokhfeld, Shakedown of multiparameter systems under non-uniform heating (in Russian), TNEK 4 (1964), 191-203.

[8.173] D. A. Gokhfeld, P. I. Ermakov, Shakedown of thick-walled spherical vessels subject to repeated operations of a thermal field (in Russian), RP 10 (1964), 137-147.

[8.174] D. A. Gokhfeld, On incremental collapse due to temperature variations (in Russian), PM 1 (1965), 6, 26-32.

[8.175] - , Load-carrying capacity of turbine disks in non-stationary regimes (in Russian), Mash (1965), 6, 61-68. 
[8.176] D. A. Gokhfeld, A. E. Kharitonchik, On incremental buckling due to temperature variations (in Russian), TNEK 6 (1966), 219-226.

[8.177] D. A. Gokhfeld, A. G. Laptevsky, Analysis of conditions of cyclic plastic deformations in a slag car shell under repeated operations of a thermal field (in Russian), SNT CPI 151 (1974), 46-55.

[8.178] I. G. Grechykho, A beam-column in the elastic-plastic range (in Russian), SMRS (1960), 6, 23-27.

[8.179] O. A. Grigan, V. A. Dintsyn, V. L. Livshits, A. L. Livshits, Elastic-plastic problem for a thick-walled cylinder under an arbitrary loading (in Russian), IZh MTT (1968), 1, $142-146$.

[8.180] A. S. Grigorev, Load-carrying capacity of circular plates under bending with tension (in Russian), IS 24 (1956), 52-61.

[8.181] O. Gross, W. Prager, Minimum-weight design for moving loads, 4th USNCAM 2 (1962), 1047-1052.

[8.182] P. Grundy, F. Tin Loi, Deflection analysis of strain hardening structures under repeated loading, IJMS 20 (1978), 1, 21-36.

[8.183] V. S. Gudramovitch, I. A. Diskovsky, Rigid-plastic analysis of cylindrical shells under axial force combined with lateral pressure allowing for large deflections (in Russian), SMTS 16 (1972), 49-51.

[8.184] G. Guerlement, Application de la théorie de la stabilisation plastique au dimensionnement des réservoirs, $R M e c 20$ (1974), 1, 43-48.

[8.185] G. Guerra, Contributo allo studio dell'equilibrio dei prismi di de Saint Venant, Aer 41 * (1961), 6, 347-351.

[8.186] V. F. Guk, An algorithm of numerical solution of the problem of thermal stresses in a disk employing the theory of plastic flow (in Russian), $C A$ (1975), 37-45.

[8.187] H. M. Haydl, A. N. Sherbourne, Rigid-plastic collapse of cylindrical shells, Trans ASME B95 (1973), 1, 215-218.

[8.188] - , - Limit loads of circular plates under combined loadings, Trans ASME E40 (1973), 3, 799-802.

[8.189] R. M. Haythornthwaite, Analysis of complex stress states in classical plasticity, DM 3 (1967), 41-52.

[8.190] S. S. Hecker, C. H. Hamilton, L. J. Ebert, Elasto-plastic analysis of residual stresses and axial loading in composite cylinders, JMat 5 (1970), 4, 868-900.

[8.191] J. Heyman, Plastic design of beams and plane frames for minimum material consumption, QAM 8 (1951), 373.

[8.192] -, Plastic design of plane frames for minimum weight, SEng 31 (1953), 125.

[8.193] -, Minimum weight of frames under shakedown loading, Proc ASCE JEMD 84 (1958), 4, $1790 / 1-1790 / 25$.

[8.194] -, Rotating disks-insensitivity of design, 3rd USNCAM (1958), 551-556.

[8.195] -, Plastic design of rotating disc, Proc IME 172 (1958), 531.

[8.196] A. B. Higginbotham, R. D. Hanson, Axial hysteretic behavior of steel members, Proc ASCE JSD 102 (1976), 7, 1365-1381.

[8.197] R. Hill, Plastic analysis of pressurized cylinders under axial load, IJMS 18 (1976), 3, 145-148.

[8.198] H. S. Ho, D. P. Updike, Limit analysis for combined edge and pressure loading on a cylindrical shell, Pap ASME PVP-22 (1971), 9 pp.

[8.199] P. G. Hodge, Jr., R. Sankaranarayanan, Plastic interaction curves for annular plates in tension and bending, JMPS 8 (1960), 3, 153-163.

[8.200] P. G. Hodge, Jr., J. Panarelli, Interaction curves for circular cylindrical shells according to the Mises or Tresca yield criterion, Trans ASME E29 (1962), 2, 375-380.

[8.201] P. G. Hodge, Jr., M. Balaban, Elastic-plastic analysis of a rotating cylinder, IJMS 4 (1962), 6, 465 . 
[8.202] P. G. Hodge, Jr., A. J. Kalinowski, Shakedown interaction curve for a circular arch, DM 8 (1970), 529-540.

[8.203] P. G. Hodge, Jr., Plastic analysis and pressure-vessel safety, AMR 24 (1971), 7, 741-747.

[8.204] M. R. Horne, Determination of the shape of fixed-ended beams for maximum economy according to the plastic theory, 4th C AIPC (1952), 119.

[8.205] - , The effect of variable repeated loads in building structures designed by plastic theory, Mem AIPC 14 (1954), 53.

[8.206] M. R. Ibragimov, Minimal weight design of a shell under several independent loadings (in Russian), Mat. Nauchn. Konf. Mol. Uch. AN Azerb. SSR, Baku 1966, 10-19.

[8.207] S. Igarashi, C. Matsui, K. Yoshimura, Experimental study on beam-columns under repeated bending, RCT AIPC 13 (1973), 171-177.

[8.208] K. Ikegami, A historical perspective of the experimental study of subsequent yield surfaces for metal (in Japanese), JSMSJ 24 (1975), 261, 491-505; 24 (1975), 263, 709-719; English translation by Brit. Ind. and Sci. Int. Transl. Service, London 1976.

[8.209] V. A. Ikrin, On the construction of optimal non-proportional loading trajectories for beams and plane frames (in Russian), SNT CPI 113 (1973), 25-37.

[8.210] A. A. Ilyushin, P. M. Ogibalov, On plastic deformations of a thick-walled tube under thermal impulses and pressure (in Russian), Izv AN SSSR OTN (1958), 12, 85-89.

[8.211] —, - , On the strength of a thick-walled tube and of a hollow sphere subject to irradiation (in Russian), IS 28 (1960), 134-144.

[8.212] —, -, Elastic-plastic deformations of hollow cylinders (in Russian), Izdat. Moskovskogo Universiteta, Moskva 1960.

[8.213] K. Inoue, K. Ogawa, Nonlinear analysis of strain-hardening frames subjected to variable loading, TROU 24 (1974), 763-781.

[8.214] H. Ishikawa, Transient thermoelastoplastic stress analysis for a hollow sphere using the incremental theory of plasticity, IJSS 13 (1977), 7, 645-656.

[8.215] D. D. Ivlev, On a certain particular solution of general equations of the theory of perfect plasticity in cylindrical coordinates (in Russian), DAN SSSR 123 (1958), 6, 988-990.

[8.216] D.D. Ivlev, L.V. Ershov, The perturbation method in the theory of an elastic-plastic body (in Russian), Nauka, Moskva 1978.

[8.217] K. Ježek, Die Tragfähigkeit axial gedrückter und auf Biegung beanspruchter Stahlstäbe, Stb 9 (1936), 12.

[8.218] K. L. Johnson, A shakedown limit in rolling contact, 4th USNCAM (1962), 2, 971-975.

[8.219] W. Johnson, P. B. Mellor, Elasticplastic behaviour of thick-walled spheres of non-workhardening material subject to a steady-state radial temperature gradient, IJMS 4 (1962), 147-158.

[8.220] H. Jung, Ưber die Spannungen in dickwandigen Röhren bei elastisch-plastischer Beanspruchung, WZHSM 2 (1958), 251-256.

[8.221] M. G. Kabelevsky, On cyclic, non-isothermal deformation of disks (in Russian), TNEK 6 (1966), 29-40.

[8.222] -, On the applicability of the deformation theory to the analysis of turbine disks under combined loadings (in Russian), TNEK 7 (1967), 49-56.

[8.223] -, Stress and strain analysis in disks under non-stationary and cyclic loading (in Russian), TNEK 9 (1970), 219-231.

[8.224] L. M. Kachanov, Elastic-plastic equilibrium of non-uniformly heated thick-walled cylinders subject to internal pressure (in Russian), ZhTF 10 (1940), 14, 1167-1172.

[8.225] L. F. Kahn, R. D. Hanson, Inelastic cycles of axially loaded steel members, Proc ASCE JSD 102 (1976), 5, 947-959.

[8.226] S. Kaliski, W. K. Nowacki, E. Włodarczyk, Plane biwaves in an elastic-viscoplastic semispace, PVP 8 (1967), 2, 175-190. 
[8.227] S. Kaliski, W. K. Nowacki, E. Włodarczyk, Propagation of plane loading and unloading biwaves in an elastic-viscoplastic semi-infinite body, PVP 8 (1967), 3, 293-331.

[8.228] T. B. Kammash, S. A. Murch, P. M. Naghdi, The elastic-plastic cylinder subjected to radially distributed heat source, lateral pressure and axial force with application to nuclear reactor fuel elements, JMPS 8 (1960), 1, 1-25.

[8.229] S. A. Kapustin, N. M. Adyasova, On the calculation of elastic-plastic reinforced shells under surface and thermal loadings (in Russian), MRZUP 6 (1972), 56-64.

[8.230] J. A. Karczewski, Analiza sprężysto-plastycznej pracy obciążonych zmiennie przestrzennych konstrukcji prętowych, ZNPW Budown. 46 (1976), 3-114.

[8.231] B. Kato, H. Akiyama, Inelastic bar subjected to thrust and cyclic bending, Proc ASCE JSD 95 (1969), 1, 33-56.

[8.232] Sh. N. Kats, L. M. Kachanov, On the plastic deformation under combined loading (in Russian), Izv AN SSSR OTN (1957), 11, 172-173.

[8.233] R. W. Kauppila, A boiler tube problem: elastic-plastic behavior of a thick-walled cylinder caused by sinusoidal inside surface temperature, internal heat generation and external heat flux, Doct. Diss. Univ. Michigan (1968), 145 pp.; Diss. Abstrs. B29 (1969), 8, 2902-2903.

[8.234] M. S. Kenis, B. F. Trakhtenberg, Elastic-plastic strains and stresses in multilayer expanded cylinders (in Russian), Izv VUZ M (1972), 11, 15-20.

[8.235] R. Yu. Kerimov, Analysis of elastic-plastic stress state and of plastic zones in a plate with circular hole in tension (in Russian), Izv AN AzbSSR FTM (1972), 4, 130-134.

[8.236] A. P. Kharchenko, Deformations around an elliptic hole in an elastic-plastic body (in Russian), PM 10 (1974), 4, 48-55.

[8.237] I. Yu. Khoma, Plastic zones around a circular hole in non-uniform plane stress field taking into account normal and tangential loadings (in Ukrainian), $P M 9$ (1963), 2, 167-173.

[8.238] A. M. Khomyakov, Limit analysis of a cylindrical shell under internal pressure and a local meridional couple (in Russian), Izv VUZ AT (1974), 4, 103-109.

[8.239] R. M. Kirakosyan, On elastic-plastic bending of a beam under moving loading (in Russian), Izv AN ArmSSR M 21 (1968), 5-6, 103-114.

[8.240] -, On elastic-plastic bending of a beam under a moving force (in Russian), Izv AN ArmSSR M 22 (1969), 1, 68-76.

[8.241] -, Elastic-plastic pure bending of a rotating circular shaft (in Russian), Izv AN ArmSSR M 23 (1970), 3, 23-35.

[8.242] —, Elastic-plastic axisymmetric bending of a clamped circular plate under a cone-like distributed variable loading (in Russian), Izv AN ArmSSR M 25 (1972), 1, 42-50.

[8.243] —, On a certain elastic-plastic problem for a shell with elliptic cross-section (in Russian), Izv AN ArmSSR M 26 (1973), 4, 49-59.

[8.244] V. N. Kissyuk, Elastic-plastic one-dimensional systems of minimal weight under moving loads (in Russian), SK 1 (1969), 67-70.

[8.245] - Elastic-plastic trusses of minimal weight under variable repeated loading (in Russian), SK 8 (1970), 176-180.

[8.246] -, Rigid-plastic bar systems (frames) in shakedown conditions (in Russian), $S K 8$ (1970), 181-185.

[8.247] J. Klepaczko, J. A. König, Ściskanie osiowe powłoki cylindrycznej z równoczesnym ciśnieniem wewnętrznym, RI 14 (1966), 2, 263-275.

[8.248] V. M. Klyagin, A ribbed circular plate under combined loadings (in Russian), Izv VUZ SA (1973), 2, 42-45.

[8.249] H. A. Koenig, W. Vogel, L. Cernoch, Elasto-plastic cyclic analysis of structural members, Pap ASME GT-1 (1972), 5 pp.

[8.250] W. T. Koiter, On partially plastic thick-walled tubes, Biezeno Anniv. Volume, H. Stain, Haarlem-Antwerpen-Djakarta (1953), 233. 
[8.251] A. Kolář, Mezní stavy únosnosti spojitých nosnikủ při pohyblivem zatiženi, StavC 16 (1968), 8, 491-504.

[8.252] —, Přispěvek $\mathrm{k}$ řešeni mezní únosnosti spojitých nosnikũ při pohyblivem zatiženi, Stav C 17 (1969), 4, 301-315.

[8.253] V. V. Kolokolchikov, Rotation of an elastic-plastic thin-walled tube subject to bending (in Russian), $V M U$ MM (1968), 2, 79-83.

[8.254] S. Komatsu, T. Sakimoto, Elasto-plastic behavior of steel members under combined torsion and compression, TROU 23 (1973), 681-696.

[8.255] Z. Kordas, Problematyka określania kształtów ciał wykazujących całkowite uplastycznienie w stadium zniszczenia, ZNPK PNT (1977), 7.

[8.256] V. Kortenska, D. Dimitrov, B. Ivanov, Analysis of the stress distribution in a thick-walled tube of strain-hardening material (in Bulgarian), MashB 22 (1973), 3, 103-107.

[8.257],,,--- Stress distribution in thick-walled tubes in the plastic range (in Bulgarian), TPM 4 (1973), 3, 41-47.

[8.258] A. S. Kosmodamiansky, Elastic-plastic problem for an isotropic medium weakened by an infinite number of equal excavations (in Russian), Izv AN SSSR OTN MM (1961), 4, 187-188.

[8.259] A. G. Kostyuk, On the equilibrium of an annular plate subject to a power hardening law (in Russian), PMM 14 (1950), 3.

[8.260] —, Stresses in a solid rotating cylinder beyond the elastic limit (in Russian), PMM 18 (1954), 4, 453-456.

[8.261] A. D. Kovalenko, Plates and shells as applied to turbine rotors (in Russian), Akad. Nauk Ukr. SSR, Kiev 1955.

[8.262] K. Kowalczyk, M. Życzkowski, Badanie wpływu efektu Bauschingera na plastyczne przystosowanie się konstrukcji (na przykładzie układu prętowego), RI 22 (1974), 2, 145-167.

[8.263] K. Kowalczyk, Powierzchnie graniczne dla modelu sprężysto-plastycznego pręta przy uwzględnieniu zmian geometrii, MTS 17 (1979), 2, 203-215.

[8.264] K. Kowalczyk, M. Życzkowski, Modyfikowane krzywe neutralne dla geometrycznie nieliniowego modelu pręta (in print).

[8.265] K. Kowalczyk, Wpływ wzmocnienia plastycznego i wstępnej krzywizny na powierzchnie graniczne dla modelu pręta geometrycznie nieliniowego, PKMS 11 (1981).

[8.266] —, Obszary plastycznego przystosowania dla modelu resoru piórowego (in print).

[8.267] I.A. Kozlov, Investigations of the load-carrying capacity of elements of turbine rotors (in Russian), Naukova Dumka, Kiev 1969.

[8.268] J. A. König, Optymalne projektowanie płyt na obciążenie cykliczne, $A B M 18$ (1971), 4, 575-589.

[8.269] —, O projektowaniu konstrukcji z uwzględnieniem przystosowania w przypadku gdy stałe sprężyste zależą od temperatury, RI 20 (1972), 3, 423-434.

[8.270] A. Krisch, R. Schweitzer, Die Spannungen im ausbauchenden Rohr unter Innendruck und einer zusătzlichen Längskraft, $A E 37$ (1966), 7, 599-605.

[8.271] S. G. Krishnasamy, A. N. Sherbourne, Model I-shaped beams under alternating deflections, Proc ASCE JSD 97 (1971), 3, 1015-1019.

[8.272] W. Krzyś, A. P. Zieliński, Nośność sprężysta rury obciążonej ciśnieniem wewnętrznym i różnicą temperatur, CT 76 (1972), 4, 32-35.

[8.273] W. Krzyś, M. Stodulski, A. Trojnacki, Analiza procesu formowania doświadczalnego wielowarstwowego zbiornika roztlaczanego, $A B M 23$ (1976), 4, 515-528.

[8.274] A. E. Kulago, Plastic deformation of a tube under a quasi-stationary magnetic field (in Russian), VMU MM (1969), 4, 118-121.

[8.275] Yu. K. Kulikov, A. M. Khomyakov, Load-carrying capacity of thin shells under a local normal force (in Russian), Izv VUZ AT (1976), 2, 131-134.

[8.276] L. M. Kull, Calculation of flexible rigid-plastic cylindrical shells under combined loadings (in Russian), PM 5 (1969), 11, 28-33. 
[8.277] Zh. Kultursynov, Combined loading of a thick-walled tube (in Russian), VVPM 3 (1970), 182-191.

[8.278] - On the construction of strain trajectories for a thick-walled tube under combined loading (in Russian), VVPM 11 (1972), 45-49.

[8.279] A. Kumar, S. T. Ariaratnam, Uniqueness of deformation of thin-walled rigid-plastic cylinders under internal pressure, tension and torque, IJSS 11 (1975), 11, 1211-1217.

[8.280] - , - Uniqueness and stability of rigid-plastic cylinders under internal pressure and axial tension, IJSS 12 (1976), 7, 525-535.

[8.281] V. V. Kupriyanov, Elastic-plastic analysis of frames under variable repeated loading allowing for normal and shearing forces (in Russian), $\operatorname{Tr}$ CNIISK 10 (1962), 96-107.

[8.282] N. S. Kurdin, Elastic-plastic state of a bar subject to centrifugal forces and torque (in Russian), Izv AN SSSR OTN MM (1959), 3, 152-154.

[8.283] A. I. Kuzin, On the problem of evaluation of the load-carrying capacity of steel beams (in Russian), $\operatorname{Tr}$ GISI 52 (1970), 44-53.

[8.284] A. I. Kuznetzov, The problem of torsion and plane strain of non-homogeneous plastic bodies, AMS 10 (1958), 4, 447-462.

[8.285] B. N. Kuznetsov, Evaluation of deflections of an elastic-plastic beam under a concentrated moving load (in Russian), $\operatorname{Tr}$ CNIISK 35 (1974), 132-137.

[8.286] -, Shakedown of beam-columns and frames (in Russian), SMRS (1975), 2, 19-21.

[8.287] - , On shakedown analysis of frames (in Russian), $\operatorname{Tr}$ CNIISK 41 (1976), 67-74.

[8.288] - Evaluation of displacements of a two-span beam under variable repeated loading (in Russian), SMRS (1976), 5, 38-41.

[8.289] V. V. Kuznetsov, Stress concentration around an elliptic hole in an elastic-plastic body (in Russian), $P M 8$ (1972), 5, 85-91.

[8.290] - , On evaluation of the strain state in an elastic-plastic thick plate with an elliptic hole (in Russian), $P M 9$ (1973), 9, 133-137.

[8.291] D. Lamblin, M. Save, Minimum-volume plastic design of beams for movable loads, Mec 6 (1971), 3, 157-163.

[8.292] D. Lamblin, Minimum-weight plastic design of continuous beams subjected to one single moveable load, JSM 1 (1972), 1, 133-157.

[8.293] B. B. Lampsi, Load-carrying capacity of steel beams (in Russian), BIPI (1958), 1-2, 381-394.

[8.294] H. G. Landau, E. E. Zwicky, Transient and residual thermal stresses in an elastic-plastic cylinder, JAM 27 (1960), 481-488.

[8.295] L. G. Lantukh, A. V. Perelmuter, On the problem of shakedown of plates (in Russian), PPro (1970), 6, 40-43.

[8.296] L. D. Larson, W. F. Stokey, J. E. Panarelli, Limit analysis of a thin-walled tube under internal pressure, bending moment, axial force and torsion, Trans ASME EA1 (1974), 3, 831-832.

[8.297] L. D. Larson, W. F. Stokey, W. E. Franzen, An approximate model for an elastic-plastic pipe element under combined loading, Trans ASME J97 (1975), 1, 22-28.

[8.298] F. László, Geschleuderte Umdrehungskoerper im Gebiet bleibender Deformation, ZAMM 9 (1929), 281.

[8.299] F. A. Leckie, Shakedown pressures for flush cylinder-sphere shell intersections, JMES 7 (1965), 4, 367-371.

[8.300] F. A. Leckie, R. K. Penny, Shakedown loads for radial nozzles in spherical pressure vessels, IJSS 3 (1967), 743-751.

[8.301] —, -, Shakedown as a guide to the design of pressure vessels, Trans ASME B91 (1969), 799.

[8.302] Yu. R. Lepik, On the load-carrying capacity of circular plates subject to irradiation (in Russian), UZTGU 129 (1962), 482-486. 
[8.303] Yu. R. Lepik, On the load-carrying capacity of circular plates under bending with tension and under bending with compression (in Russian), Izv AN SSSR MTT (1972), 3, 125-130.

[8.304] U. Lepik, Z. Mróz, Optimal design of plastic structures under impulsive and dynamic pressure loading, IJSS 13 (1977), 657-674.

[8.305] L. M. Levitan, Numerical analysis of stresses and strains in a thick-walled tube at finite deformations (in Russian), $\operatorname{Tr}$ CNIITM $117^{\prime}$ (1974), 21-24.

[8.306] S. D. Leytes, On the spatial stability of beam-columns in the elastic-plastic range (in Russian), SMRS (1961), 4, 24-29.

[8.307] N. C. Lind, Z. Mróz, A study of cyclic plasticity, RCT AIPC 13 (1973), 9-22.

[8.308] H. Lippmann, Zur Theorie des plastischen Fließens, ZAMM 52 (1972), 4, 3-17.

[8.309] R. K. Livesley, Optimum design of structural frames for alternative system of loading, CEPWR 54 (1959), 636, 739-740.

[8.310] V. A. Lomakin, Large deformations of a tube and of a hollow sphere (in Russian), IS 21 (1955), 61-73.

[8.311] B. V. Lopatin, Limit analysis of beams subject to a temperature gradient, (in Russian), SNT IEI (1955), 6, 9-15.

[8.312] Le-Wu Lu, H. Kamalvand, Ultimate strength of laterally loaded columns, Proc ASCE JSD 94 (1968), 6, 1505-1524.

[8.313] W. A. Macfarlane, G. E. Findlay, A simple technique for calculating shakedown loads in pressure vessels, Proc IME 186 (1972), 4, 45-52.

[8.314] C. W. MacGregor, L. F. Coffin, J. C. Fischer, The plastic flow of thick-walled tubes with large strains, JAP 9 (1947), 3, 291-297.

[8.315] H. Maeda, H. Namikawa, On the rapid bending of the column subjected to axial and lateral loads, TRKanU (1961), 3, 1-11.

[8.316] G. Maier, J. A. König, Shakedown analysis of elastoplastic structures: a review of recent developments, 3rd SMiRT (1975), paper L5/1.

[8.317] E. I. Makeranets, V. I. Odinokov, Calculation of plastic flow of infinitely long hollow oval cylinders (in Russian), Izv AN SSSR MTT (1976), 2, 103-110.

[8.318] V. I. Makhnenko, L. A. Izbenko, Yu. A. Skosnyagin, Numerical solution of an axisymmetric problem of thermoplasticity for a shell of revolution (in Russian), TNEK 12 (1972), 62-67.

[8.319] T. M. Makhonina, Elastic-plastic state of a disk in the case of power plastic hardening (in Russian), RP 5 (1960), 212-225.

[8.320] V. I. Maksak, G. A. Doshtchinsky, An experimental method and analysis of large plastic deformations in the case of simple loading (in Russian), Izv TPI 173 (1970), 3-9.

[8.321] - , - Investigations of large plastic deformations in the case of combined loading (in Russian), Izv TPI 173 (1970), 10-12.

[8.322] L. I. Malament, An elastic-plastic process in a continuous beam under moving load (in Russian), IMK (1940).

[8.323] V. M. Malyshev, Plastic flow under simultaneous continuous tension and torsion in the range of small torques (in Russian), VMU $M M$ (1958), 55-68.

[8.324] -, Step-wise torsion of tubes under continuous tension (in Russian), VMU MM (1958), 2, 33-39.

[8.325] D. Manning, The overstrain of tube by internal pressure, Eng (1945), 101.

[8.326] E. H. Mansfield, Loading and heating of a simple structure with linear work-hardening, JRAS 67 (1963), 626, 92-102.

[8.327] S. S. Manson, Analysis of rotating disks of arbitrary contour and radial temperature distribution in the region of plastic deformation, 1st USNCAM 1951 (1952), 569-577.

[8.328] Z. Marciniak, Wykreślne wyznaczanie rozkładu naprężeń przy plastycznym zginaniu blach z uwzględnieniem siły osiowej, RI 6 (1958), 4, 539-545. 
[8.329] J. Marin, H. A. B. Wiseman, Plastic stress-strain relations for aluminium alloy 14S-T6 subjected to combined tension and torsion, TMS AIME 5 (1953), sec. 2, 9, 1181-1190.

[8.330] J. B. Martin, Plasticity: fundamentals and general results, MIT Press, Cambridge (Mass.) 1975.

[8.331] C. E. Massonnet, Forty years of research on beam-columns in steel, SMA 1 (1976), 1, 27-157.

[8.332] R. Mayeda, W. Prager, Minimum-weight design of beams for multiple loading, IJSS 3 (1967), 6, 1001-1011.

[8.333] R. I. Mazing, Elastic-plastic deformation of a fast rotating cylinder (in Russian), Izv $A N$ SSSR OTN MM (1959), 3, 143-147.

[8.334] F. M. Mazzolani, L'influenza di azioni tangenziali di perimetro sulla velocità limite degli anelli rotanti, RASFM 36 (1969/70), 309-324.

[8.335] P. B. Mellor, Tensile instability conditions in ideal pressure containers, Eng 215 (1963), No. 5583, 174-179.

[8.336] K. R. Merckx, Cyclic operation of pressure piping with $\gamma$ heating, Pap ASME (1959), Met. $-4,55 \mathrm{pp}$.

[8.337] -, Plastic bending of rods or tubes with radial temperature distributions, NSE 5 (1959), 3, 202-203.

[8.338] J. D. Meyer, K. H. Gerstle, Shakedown of strain-hardening structures, Proc ASCE JSD 98 (1972), 1, 95-110.

[8.339] M. J. Michno, Jr., W. N. Findley, An historical perspective of yield surface investigations for metals, IJNLM 11 (1976), 1, 59-82.

[8.340] M. Sh. Mikeladze, Elastic-plastic deformations in fast rotating disks with variable thickness (in Russian), IS 15 (1953), 21-34.

[8.341] -, Estimation of the load-carrying capacity of shallow shells of revolution and of circular plates under bending with tension (in Russian), PM 1 (1965), 2, 40-47.

[8.342] M. S. Mikhalishin, Limit equilibrium of a shallow spherical shell under bending with compression or tension (in Russian), PM 12 (1976), 8, 11-15.

[8.343] N. Ya. Mikhaylov, Yu. I. Yagn, Experimental investigation of the limit carrying capacity of thin-walled nickel tubes under tension, torsion and internal pressure changing along various loading paths (in Russian), DAN SSSR 135 (1960), 3, 545-548.

[8.344] S. G. Mikhlin, Theory of plasticity (in Russian), Izd. AN SSSR, Moskva 1934.

[8.345] D. R. Miller, Thermal-stress ratchet mechanism in pressure vessels, Trans ASME D81 (1959), 2, 190-196.

[8.346] A. Mioduchowski, K. Thermann, Zur zyklischen elasto-plastischen Torsion des Kreiszylinders bei endlichen Verzerrungen, IA 42 (1973), 5, 309-318.

[8.347] V. M. Mirsalimov, Solution of elasto-plastic problems for a plane with a circular hole under a non-uniform temperature field (in Russian), DAN AzbSSR 29 (1973), 10, 7-11.

[8.348] E. M. Morozov, Approximate calculation of thermoplastic stresses in a tube (in Russian), Izv VUZ M (1961), 9, 11-18.

[8.349] V. V. Moskvitin, Secondary plastic deformations in hollow thick-walled cylinders (in Russian), VMU MM (1951), 8.

[8.350] I. A. Motovilovets, Yu. N. Shevtchenko, Elastic-plastic state of stress in plates subject to a three-dimensional temperature field (in Russian), 4th TOP 1962 (1964), 715-723.

[8.351] A. Mrázik, Experimentalne vyšetrovanie pružnoplastických deformácii ocelóvých nosnikov pri pohyblivom opakovanom zaraženi, Stavč 17 (1969), 3, 227-244.

[8.352] M. Mrowiec, M. Życzkowski, Nośność sprężysta rurociągu grubościennego, RI 10 (1962), 3, 519-530.

[8.353] T. M. Mulcahy, An assessment of kinematic hardening thermal ratcheting, Trans ASME H96 (1974), 3, 214-221. 
[8.354] T. Mura, Unstable plastic yield of a hollow cylinder under internal pressure and thermal stress, RRFE MU (1957), 9, 20-29.

[8.355] M. A. Muspratt, Shakedown of steel plates, Trans ASME E38 (1971), 4, 1088-1090.

[8.356] A. Nadai, L. H. Donnell, Stress distribution in rotating disks of ductile material after the yield point has been reached, Trans ASME 51 (1929), 173-181.

[8.357] A. Nadai, Theory of the plastic distortion of thick-walled cylinders, $R A V$ (1949), 430-448.

[8.358] J. C. Nagtegaal, W. Prager, Optimal layout of a truss for alternative loads, IJMS 15 (1973), 7, 583-592.

[8.359] J. C. Nagtegaal, A superposition principle in optimal plastic design for alternative loads, IJSS 9 (1973), 12, 1465-1471.

[8.360] B. G. Neal, P. S. Symonds, A method for calculating the failure load for a framed structure subjected to fluctuating loads, JICE 35 (1950), 186.

[8.361] B. G. Neal, The behaviouriof framed structures under repeated loading, QJMAM4 (1951), 78.

[8.362] K. W. Neale, N. C. Lind, Limit analysis of plates under combined loads, Proc ASCE JEMD 96 (1970), 5, 711-728.

[8.363] R. Negre, P. Stutz, Schémas résolutifs pour le problème du poinçon de révolution dans le cas de la plasticité parfaite, CRAS 270 (1970), 3, A198-A201.

[8.364] Yu. V. Nemirovsky, On the elastic-plastic equilibrium and the load-carrying capacity of a hollow rotating tube (in Russian), Izv AN SSSR OTN MM (1959), 3, 148-151.

[8.365] Yu. V. Nemirovsky, M. L. Kheynloo, The Lamé problem for multilayer spherical vessels and cylindrical tubes in the nonlinear theory of elasticity (in Russian), PM 8 (1972), 4, $39-41 . \frac{2}{3}$

[8.366] T. Nonaka, An elastic-plastic analysis of a bar under repeated axial loading, IJSS 9 (1973), $5,569-580$.

[8.367] S. Noto La Diega, A. Passannanti, Variazioni dei carichi limiti di scorrimento a trazione ed a torsione semplice per effetto di deformazioni permanenti respettivamente di torsione e di trazione, TI 36 (1971), 11-12, 377-383.

[8.368] W. K. Nowacki, On certain closed form solution for the plane shear-pressure wave in elastic-plastic half-space, AMS 20 (1968), 5, 575-593.

[8.369] - , The unloading wave in an elastic-plastic semi-space with a rigid unloading in the case of two-parameter loads, AMS 21 (1969), 4, 515-535.

[8.370] —, Zagadnienia falowe $w$ teorii plastyczności, IPPT PAN-PWN, Warszawa 1974.

[8.371] H. Odenö, Transient thermal stresses in elasto-plastic discs, JMES 11 (1969), 4, 384-391.

[8.372] P. M. Ogibalov, Deformation of a tube subject to internal pressure and variable temperature (in Russian), IS 20 (1954), 55-58.

[8.373] - , Large elastic-plastic symmetric deformations of a thick-walled tube (in Russian), NDVS FM (1959), 1, 110-116.

[8.374] Y. Ohashi, S. Murakami, Axisymmetric elasto-plastic deformation of circular plates under combined action of lateral load and membrane force, MFENU 21 (1969), 1, 79-121.

[8.375] Y. Ohashi, K. Kawashima, S. Nagahiko, Precise experimental results and an analytical formulation of the subsequent plastic deformation of mild steel subjected to pre-stressing, BJSME 18 (1975), 125, 1218-1225.

[8.376] W. Olszak, W. Urbanowski, Sprężysto-plastyczny grubościenny walec niejednorodny pod działaniem parcia wewnętrznego i siły podłużnej, AMS 7 (1955), 3, 315-336.

[8.377] A. Ono, Stress and strain in metals undergoing plastic flow-tubewall subjected to axial pull and torque, Proc JA 29 (1953), 8, 446-451.

[8.378] Zh. G. Ovanesova, V. V. Abramov, Elastic-plastic analysis of a multi-span beam made of a composite material under simultaneous mechanical and thermal loadings (in Russian), PPro (1973), 10, 62-65.

[8.379] - , - Elastic-plastic analysis of beams on elastic foundation under simultaneous mechanical and thermal loadings (in Russian), SMTS 23 (1974), 91-97. 
[8.380] D. G. Owen, Inelastic deformations in a solid circular cylinder subjected to cyclic torsion and heating, IJMS 16 (1974), 5, 311-327.

[8.381] I. Z. Palley, Effect of loading history on the stress state in a sandwich shell (in Russian), VDP 9 (1962), 115-126.

[8.382] -; Effect of combined loading in a non-uniformly heated rotating disk (in Russian), Izv AN LatvSSR (1963), 2, 41-45.

[8.383] S. Palusamy, N. C. Lind, Experiments on the plastic limit behavior of shell-nozzle junctures subjected to nonsymmetric loading, Pap ASME PVP-45 (1971), 12 pp.

[8.384] J. E. Panarelli, Ph. G. Hodge, Jr., Interaction of pressure, end load, and twisting moment for a rigid-plastic circular tube, Trans ASME E30 (1964), 3, 396-400.

[8.385] K. A. V. Pandalai, S. A. Patel, Stress analysis in the inelastic range in the presence of temperature, JASI 17 (1965), 3, 149-153.

[8.386] F. N. Pannell, The general principle of superposition in the design of rigid-plastic plates, CCE 61 (1966), 9, 323-326.

[8.387] O.S. Parasyuk, Elastic-plastic problems with non-biharmonic plastic state (in Russian), DAN SSSR 63 (1948), 4.

[8.388] J. Parker, J. Kettlewell, Plastic stress-strain relationships-further experiments on the effect of loading history, Trans ASME E28 (1961), 3, 439-446.

[8.389] E. W. Parkes, The inelastic behaviour of aluminium alloy tension members when subjected to heating on one face, EP (1968), 489-527.

[8.390] B. Paul, Carrying capacity of elastic-plastic shells with various end conditions under hydrostatic compression, JAM 26 (1959), 4, 553-560.

[8.391] P. A. Pavlov, Evaluation of the load-carrying capacity of tubular shafts with flanges under simultaneous tension and torsion (in Russian), NTI LPI (1960), 4, 102-104.

[8.392] A. Pellissier-Tanon, Fondements mécaniques des méthodes de calcul associées à la limitation de la déformation plastique dans les appareils à pression, RFM 48 (1973), 19-33.

[8.393] P. I. Perlin, An approximate method of solution of elastic-plastic problems (in Russian), IS 28 (1960), 145-150.

[8.394] N. Perrone, Strain-hardening solutions to axisymmetric disks and tubes, Pap ASME A29 (1959), 9 pp.

[8.395] -, Collapse load of structures with temperature-dependent yield stresses, 4th USNCAM (1962), 705-713.

[8.396] A. Phillips, Combined tension and torsion tests for aluminium alloy 2S-0, JAM 19 (1952), $4,496-500$.

[8.397] A. Phillips, L. Kaechele, Combined stress tests in plasticity, JAM 23 (1956), 1, 43-48.

[8.398] A. Phillips, M. Ricciuti, Fundamental experiments in plasticity and creep of aluminiumextension of previous results, IJSS 12 (1976), 3, 159-171.

[8.399] V. V. Piskun, State of stress in a plastic hardening cylindrical shell under non-isothermal loading (in Russian), TNEK 9 (1970), 210-219.

[8.400] - , Analysis of the deformation process of a heated elastic-plastic cylindrical shell (in Russian), TNEK 12 (1972), 58-62.

[8.401] I. M. Plagov, On the problem of deformation of metal moulds subject to temperature variations (in Russian), SNT CPI 45 (1968), 61-67.

[8.402] —, Incremental collapse of a cylindrical shell under repeated operations of a non-stationary axisymmetric temperature field (in Russian), TNEK 8 (1969), 165-170.

[8.403] I. M. Plagov, O. F. Chernyavsky, Incremental collapse of elements of metallurgical equipment subject to temperature variations (in Russian), SNT CPI 151 (1974), 33-45.

[8.404] Yu. M. Pochtman, Z. I. Pyatigorsky, On the optimal design of certain structures with respect to shakedown (in Russian), SMRS (1973), 2, 23-25.

[8.405] -, --, Optimal design of structures with respect to shakedown under quasistatic loadings (in Russian), DAN SSSR 210 (1973), 5, 1030-1032. 
[8.406] Yu. M. Pochtman, Z. I. Pyatigorsky, On the safety factor of certain optimal structures made of a perfectly elastic-plastic material (in Russian), PPro (1973), 11, 47-49.

[8.407] - , - Optimal design of structures with respect to shakedown as a method of determination of their limit state (in Russian), DAN SSSR 216 (1974), 1237-1239.

[8.408] - , -, Optimal shakedown design of plates in the case of unknown loading history (in Russian), SMRS (1976), 3, 7-10.

[8.409] -, - , On the effect of loading history on the limit state of certain structures optimal with respect to shakedown (in Russian), Izv AN ArmSSR M 29 (1976), 4, 72-79.

[8.410] - , - Calculations and optimal design of structures with respect to shakedown (in Russian), Nauka, Moskva 1978.

[8.411] C. Polizzotto, Optimum plastic design for multiple sets of loads, $\operatorname{Mec} 9$ (1974), 3, 206-213.

[8.412] -, Optimum plastic design of structures under combined stresses, IJSS 11 (1975), 5, 539-553.

[8.413] C. Polizzotto, C. Mazzarella, T. Panzeca, Optimum design for work-hardening adaptation, CMAME 12 (1977), 2, 129-144.

[8.414] L. I. Polyakov, M. A. Rudis, Some problems of limit equilibrium of circular plates allowing for temperature effects (in Russian), TNEK 4 (1964), 233-240.

[8.415] - , - On the limit equilibrium of a sandwich cylindrical shell allowing for temperature effects (in Russian), TNEK 6 (1966), 70-78.

[8.416] W. Prager, Optimum plastic design of a portal frame for alternative loads, Trans ASME E34 (1967), 3, 772-773.

[8.417] —, Foulkes mechanism in optimal plastic design for alternative loads, IJMS 13 (1971), 11, 971-973.

[8.418] E. Proctor, R. F. Flinders, Shakedown investigations on partial penetration welded nozzles in a spherical shell, NED 8 (1968), 171-185.

[8.419] I. V. Prokhorenko, V. V. Piskun, Thermoelasto-plastic state of shells of revolution under repeated loadings (in Russian), TNEK 13 (1973), 36-38.

[8.420] Kh. A. Rakhmatulin, On the propagation of elastic-plastic waves under combined loading (in Russian), PMM 22 (1958), 6, 133-137.

[8.421] Yu. A. Rakovshtchik, Evaluation of residual displacements of beam-columns beyond the elastic limit (in Russian), SMRS (1971), 4, 65-66.

[8.422] T. Y. Reddy, H. Srinath, Effect of acceleration stresses on the yielding of rotating disks, IJMS 16 (1974), 8, 593-596.

[8.423] S. R. Reid, On the influence of acceleration stresses on the yielding of disks of uniform thickness, IJMS 14 (1972), 11, 755-763.

[8.424] R. Reiss, The method of stress variation applied to minimal design for multiple loading, IJSS 12 (1976), 2, 135-146.

[8.425] A. F. Revuzhenko, Combined loading of a thick-walled tube made of a perfectly plastic material (in Russian), DSS 6 (1970), 149-157.

[8.426] M. Rogoziński, Some problems of thermoplasticity of a spherical shell, IUTAM NH 1958 (1959), 215-226.

[8.427] V. I. Rozenblyum, On plastic analysis of thick-walled cylinders (in Russian), IZh MTT (1966), 4, 133-137.

[8.428] F. I. Ruzanov, Stability of the plastic state of anisotropic cylindrical shells under tension (in Russian), Mash (1974), 3, 100-105.

[8.429] F. P. Rybalko, A. V. Kostenko, L. P. Zelenin, Effect of tension on plastic deformation due to alternating torsion (in Russian), Izv VUZ F (1961), 1, 24-30.

[8.430] J. Ryś, Efektywne obliczanie rur grubościennych w przypadku liniowego wzmocnienia plastycznego, RI 17 (1969), 1, 109-134.

[8.431] J. Ryś, M. Życzkowski, Obliczanie tarcz z otworem kołowym w przypadku plastycznego wzmocnienia materiału, ABM 18 (1971), 3, 479-492. 
[8.432] G. Sacchi, A. Zavelani-Rossi, Sulla progettazione ottimale, mediante programmazione lineare, di strutture soggette a carichi fissi e viaggianti, RIL ASL A104 (1970), 3, 485-497.

[8.433] G. Sacchi, G. Maier, M. Save, Limit design of frames for movable loads by linear programming, IUTAM OSD 1973 (1975), 415-432.

[8.434] V. Sagar, D. J. Payne, Elastic-plastic deformations of thickwalled circular cylinders under transient heating and combined axial tension and torsion loads, JMPS 22 (1974), 1, 47-59.

[8.435] - , - Incremental collapse of thick-walled circular cylinders under steady axial tension and torsion loads and cyclic transient heating, JMPS 23 (1975), 1, 39-53.

[8.436] U. S. Saidkarimov, Computer-aided calculations of thin cylindrical shells under cyclic loading (in Russian), VVPM 3 (1970).

[8.437] U. S. Saidkarimov, V. A. Tolok, Elastic-plastic analysis of thin cylindrical shells under cyclic loading (in Russian), PM 8 (1972), 7, 15-18.

[8.438] B. de Saint-Venant, Sur l'intensité des forces capables de déformer, avec continuité, des blocs ductiles cylindriques pleins ou évidés, et placés dans diverses circonstances, CRAS 74 (1872), 1009-1015.

[8.439] K. Saitō, H. Igaki, Anisotropy induced by plastic deformation (in Japanese), Trans JSME 23 (1957), 135, 893-904.

[8.440] Yu. A. Samoylovitch, On a certain simplified solution of the problem of elastic-plastic equilibrium of a cylinder in a non-uniform temperature field (in Russian), IFZ 9 (1965), $5,597-602$.

[8.441] R. Sankaranarayanan, Plastic interaction curves for circular cylindrical shells under combined lateral and axial pressure, JFI 270 (1960), 5, 359-366.

[8.442] M. Save, W. Prager, Minimum weight design of beams subjected to fixed and moving loads, JMPS 11 (1963), 4, 255-267.

[8.443] M. A. Save, R. T. Shield, Minimum-weight design of sandwich shells subjected to fixed and moving loads, 11th ICAM 1964 (1966), 341-349.

[8.444] L. M. Savelyev, Biaxial tension of a plate with a circular hole beyond the elastic limit (in Russian), $\operatorname{Tr}$ KuyAI 60 (1973), 61-73.

[8.445] D. D. Savvin, On the problem of bending of rotating bars beyond the elastic limit (in Russian), Avtoreferat Kand. Diss., Novocherkassk 1956.

[8.446] A. Sawczuk, Evaluation of upper bounds to shakedown loads for shells, JMPS 17 (1969), 4, 291-301.

[8.446a] - Zagadnienia nośności granicznej elementów rozciąganych i zginanych w zastosowaniu do teorii zbiorników prostokątnych, RI3 (1955), 2, 255-284.

[8.446b]-, $\mathrm{Z}$ zagadnień jednoczesnego działania sił skupionych i obciążeń ciągłych w teorii nośności granicznej płyt, ZNPW B 6 (1956), 41-59.

[8.447] U. Säverin, Bestimmung der Tragfähigkeitsgrenzen des druck- und biegebelasteten Durchlaufträgers unter variabler, wiederholter Belastung, Sbf 6 (1967), 1-2, 82-99.

[8.448] C. G. Schilling, F. W. Schutz, L. S. Beedle, Behavior of welded single-span frames under combined loading, WJ 35 (1956), 5, 234-252.

[8.449] J. L. Schlafer, An experimental study of the incremental and total strain theories for nonproportionately loaded thin-walled cylinders, Doct. Diss. Univ. of Mlinois; Diss. Abstr. B29 (1969), 8, 2927.

[8.450] J. L. Schlafer, O. M. Sidebottom, Experimental evaluation of incremental theories for nonproportionate loading of thin-walled cylinders, ExpM 9 (1969), 11, 500-506.

[8.451] A. N. Semenenko, Load-carrying capacity of a toroidal shell (in Russian), Izv VUZ M (1970), 12, 18-21.

[8.452] T. L. Semykina, A. A. Firtytch, On the calculation of an elastic-plastic sandwich plate loaded by pressure in a non-stationary temperature field (in Russian), Izv AN SSSR MTT (1970), 3, 131-135. 
[8.453] B. P. Sergiyev, Limit analysis of thick-walled pipe-lines under simultaneous bending moment and pressure (in Russian), KNM (1976), 12, 12-14.

[8.454] B. R. Seth, Elastic-plastic transition in shells and tubes under pressure, ZAMM 43 (1963), $7-8,345-351$.

[8.455] O. N. Shabliy, Load-carrying capacity of a shallow spherical shell under compression with bending (in Russian), PM 1 (1965), 4, 19-24.

[8.456] O. N. Shabliy, P. F. Kupriychuk, Evaluation of load-carrying capacity of shallow shells of revolution (in Russian), PM 2 (1966), 7, 50-57.

[8.457] F. G. Shamiyev, S. M. Gabibzade, Optimal design of shells under oppositely oriented systems of loadings (in Russian), 11th NS AN AzbSSR (1974), 54-56.

[8.458] G. S. Shapiro, On integration in quadratures of plane, one-dimensional problems of the theory of plastic hardening (in Russian), PMM 13 (1949), 6.

[8.459] L. A. Shapovalov, Effect of non-uniform heating on stability of a compressed bar (in Russian), PMM 22 (1958), 1, 119-123.

[8.460] A. N. Sherbourne, S. Krishnasamy, Indeterminate systems under cyclic alternating loading MCon 1 (1968), 4, 327-349.

[8.461] A. N. Sherbourne, S. D. N. Murthy, Plastic analysis of anisotropic disks and plates, Proc ASCE JEMD 98 (1972), 1, 11-26.

[8.462] A. N. Sherbourne, H. M. Haydl, Plastic analysis of shallow spherical shells under combined loading at moderately large deflections, ZAMM 54 (1974), 2, 73-82.

[8.463] Yu. N. Shevtchenko, Bending of a disk under non-uniform heating in the case of power plastic hardening (in Russian), TNET 1 (1961), 103-109.

[8.464] -, State of stress in fast rotating, non-uniformly heated disks in the case of power law of plastic hardening (in Russian), TNET 2 (1962), 75-80.

[8.465] —, Elastic-plastic state of stress in a disk in an axisymmetric temperature field (in Russian), TNEK 3 (1963), 99-110.

[8.466] —, Elastic-plastic state of stress in a disk accounting for loading history (in Russian), TNEK 5 (1965), 241-249.

[8.467] —, Approximate solution methods of thermoplasticity problems accounting for loading history (in Russian), TNEK 7 (1967), 37-48.

[8.468] - , Numerical determination of the elastic-plastic state of stress in a disk accounting for loading history (in Russian), DPM 5 (1967), 18-25.

[8.469] Yu. N. Shevtchenko, I. A. Motovilovets, V. G. Savtchenko, V. N. Vasilenko, Elasticplastic state of stress in a disk of asymmetric profile under repeated heating (in Russian), PM 4 (1968), 2, 28-35.

[8.470] Yu. N. Shevtchenko, R. G. Terekhov, Application of the theory of plastic flow to thermal stress analysis in disks (in Russian), TNEK 8 (1969), 196-207.

[8.471] Yu. N. Shevtchenko, Elastic-plastic state of stress in a disk under repeated loading (in Russian), TNEK 8 (1969), 143-156.

[8.472] Yu. N. Shevtchenko, V. G. Savtchenko, Elastic-plastic state of stress in a multilayer cylinder accounting for loading history (in Russian), TNEK 11 (1971), 113-119.

[8.473] A. Yu. Shevtchenko, Elastic-plastic state of stress in a long thick-walled cylinder exposed to a magnetic field (in Russian), PM 9 (1973), 8, 50-56.

[8.474] R. T. Shield, Optimum design methods for multiple loading, ZAMP 14 (1963), 1, 38-45.

[8.475] E. Shiratori, K. Ikegami, F. Yoshida, K. Kaneko, S. Koike, The subsequent yield surfaces after preloading under combined axial load and torsion, BJSME 19 (1976), 134, 877-883.

[8.476] O. A. Shishmarev, Investigation of the fragment of the yield surface opposite to the loading point (in Russian), Izv AN SSSR OTN MM (1962), 4, 159-164.

[8.477] -, Investigation of the yield surfaces of plastically deformed nickel specimens (in Russian), IZh 3 (1963), 4, 740-747. 
[8.478] O. A. Shishmarev, Experimental study on one type of plastic anisotropy not considered in simplified flow theories, SFP 1972 (1973), 491-505.

[8.479] R. M. Shneyderovitch, Plastic analysis of disks (in Russian), VM (1954), 3, 14-20.

[8.480] B. F. Shorr, On the calculation of non-uniformly heated cylinders in the elastic-plastic range (in Russian), Izv AN SSSR OTN MM (1960), 6, 57-62.

[8.481] O. M. Sidebottom, Evaluation of finite plasticity theories for nonproportionate loading of torsion-tension members, ExpM 12 (1972), 1, 18-24.

[8.482] O. M. Sidebottom, R. K. Bhattacharyya, Evaluation of finite-plasticity theories for torsiontension members made of Tresca materials, ExpM 13 (1973), 238-245.

[8.483] O. M. Sidebottom, S. C. Chu, Bursting pressure of thick-walled cylinders subjected to internal and external pressures, axial load and torsion, ExpM 15 (1975), 6, 209-218.

[8.484] M. P. L. Siebel, The combined bending and twisting of thin cylinder in the plastic range, JMPS 1 (1953), 189-206.

[8.485] A. P. Sinitsyn, Propagation of elastic-plastic waves under combined state of stress (in Russian), RUUPV (1973), 306-313.

[8.486] —, Thermoplastic waves in a cylindrical shell under combined state of stress (in Russian), SMTS 21 (1973), 31-37.

[8.487] A. M. Skobeyev, On a plane elastic-plastic wave (in Russian), PMM 29 (1965), 3, 509-515.

[8.488] A. M. Skobeyev, L. M. Flitman, Moving load on an inelastic half-plane (in Russian), PMM 34 (1970), 1, 189-192.

[8.489] J. Skrzypek, M. Życzkowski, Limit state of a thick-walled tube under simultaneous torsion, tension and pressure gradient, BAP SST 13 (1965), 5, 285-294 (English summary); RI 13 (1965), 2, 281-296 (Polish full text).

[8.490] J. Skrzypek, Lower bound of the limit carrying capacity of the thick-walled inextensible pipe-line, BAP SST 17 (1969), 11-12, 525-532 (English summary); ABM 16 (1969), 1, 81-91 (Polish full text).

[8.491] J. Skrzypek, M. Życzkowski, Generalization of Gvozdev's problem of limit load carrying capacity of a thin-walled tube to the case of pressure, BAP SST 19 (1971), 12, 545-554 (English summary); RI 18 (1970), 3, 471-483 (Polish full text).

[8.492] J. Skrzypek, An approximate solution of the problem of limit carrying capacity of a thinwalled tube under combined loadings, BAP SST 19 (1971), 12, 555-562 (English summary); PKMS 6 (1972), 7-25 (Polish full text).

[8.493] A. M. Skudra, Plastic deformations of a quasiisotropic body under tension with subsequent torsion (in Russian), IBZB 5 (1960), 189-197.

[8.494] V. M. Sobolevsky, Elastic- and elastic-plastic state of stress of a circular cylindrical tube in an elastic medium under internal pressure, axial force and radial heat flux (in Russian), UZBINK (1957), 207-252.

[8.495] - Elastic and elastic-plastic state of stress of a hollow sphere in an elastic medium under internal pressure and radial heat flux (in Russian), Izv VUZ E (1958), 3, 103-110.

[8.496] - Elastic and elastic-plastic state of stress of a non-uniformly heated rotating circular cylindrical tube (in Russian), IFZ 2 (1959), 3, 52-61.

[8.497] Z. Sobotka, J. Murzewski, A general method for computing the distribution of a function of random variables with application to the yield condition, AMS 14 (1962), 1, 19-34.

[8.498] Z. Sobotka, Únosnost vetknutých ortotropických obdélnikových desek s rovnoměrným zatižením a s osamělými břemeny, Stav $\breve{C} 11$ (1963), 10, 589-607.

[8.499] —, Mezní osamělé břemeno a rovnoměrné zatiženi na isotropických obdélníkových deskách podepřených na dvou protějších stranách, StavC 18 (1970), 4, 277-310.

[8.500] —, Dolní mez plastické únosnosti obdélníkových desek s rovnoměrným zatižením a s osamělým břemenem, StavČ 22 (1974), 5, 237-260.

[8.501] —, Únosnost obdélnikových desek $s$ osamělým břemenem a s rovnoměrným zatižením, Stav ¿ 23 (1975), 5, 282-318. 
[8.502] A. P. Sokolov, On elastic-plastic state of a plate (in Russian), DAN SSSR 10 (1948), 1.

[8.503] R. Sparacio, Collasso da carichi ripetuti in sollecitazione composta, Ing 36 (1962), 10, 899. 903; 11, 1033-1043.

[8.504] I. V. Stasenko, Elastic-plastic bending of a thin-walled tube (in Russian), Izv VUZ M (1968), 4, 41-46.

[8.505] - , Bending of a thin-walled tube in the case of linear plastic hardening (in Russian), Izv VUZ M (1970), 1, 8-12.

[8.506] P. Stehlin, L. Holsteinson, Minimum weight design of orthotropic sandwich panels loaded in compression and shear, MFF 122 (1971), 75 pp.

[8.507] W. F. Stokey, D. B. Peterson, R. A. Wunder, Limit loads for tubes under internal pressure, bending moment, axial force and torsion, NED 4 (1966), 2, 193-201.

[8.508] N. S. Streletsky, On the problem of collapse of trusses under cyclic loading (in Russian), Izv AN SSSR OTN (1946), 12.

[8.509] V. K. Stryuk, Elastic-plastic state of stress in a short cylinder (in Russian), TNEK 9 (1970), 183-191.

[8.510] - Thermoelastoplastic state of stress in a thick-walled cylinder of finite length (in Russian), TNEK 10 (1970), 57-67.

[8.511] M. I. Sverdlov, I. Ya. Khavkin, On two types of the loss of stability and strength of cylindrical shells (in Russian), Izv VUZ M (1972), 10, 13-19.

[8.512] V. A. Sveshnikova, On plastic deformation of hardening materials (in Russian), Izv AN SSSR OTN (1956), 1, 155-161.

[8.513] P. S. Symonds, B. G. Neal, The calculation of failure loads on plane frames under arbitrary loading programmes, JICE 35 (1950), 41.

[8.514] - , - Recent progress in the plastic methods of structural analysis, JFI 252 (1951), 383, 469.

[8.515] P. S. Symonds, Cyclic loading tests on small frames, 4th C AIPC (1953), 109.

[8.516] K. Szuwalski, M. Życzkowski, A concave interaction curve corresponding to decohesive carrying capacity under thermal loadings, JTS (in print).

[8.517] Zh. I. Tairova, Secondary plastic deformations in an annular plate under pressure and couple (in Russian), UZAUFM (1967), 6, 41-49,

[8.518] -, A thick-walled tube under axial force, internal and external lateral pressures (in Russian), MDTT (1970), 96-106.

[8.519] - , A thick-walled tube made of an incompressible plastic hardening material under pulsating pressure and variable torque (in Russian), Izv AN AzbSSR FTM (1970), 4, 3-7.

[8.520] G. B. Talypov, A. I. Chistyakov, Effect of large plastic prestraining on yield surfaces of low-carbon steel (in Russian), IUP 3 (1964), 249-251.

[8.521] I. B. Tarasov, Elastic-plastic state of stress in a cylindrical shell under local operation of a stationary temperature field combined with internal pressure (in Russian), ST MISI 100 (1972), 57-61 and 142-145.

[8.522] V. G. Tatarinov, Elastic-plastic state of a high-pressure vessel with technological imperfections accounting for thermal stresses (in Russian), VPSVD 1 (1969), 97-109.

[8.523] R. G. Terekhov, Experimental investigation of elastic-plastic deformation of rotating, non-uniformly heated disks (in Russian), PM 2 (1966), 10, 100-107.

[8.524] N. P. Theophilos, Inelastic characteristics of spherical shells with axisymmetric boundaries, Doct. diss. Buffalo State Univ. 1968; Diss. Abstr. B29 (1968), 6, 2033.

[8.525] G. Thierauf, EinfluB wiederholter Belastung auf das elastisch-plastische Verhalten eines I-Trägers aus Baustahl, BT 49 (1972), 6, 210-213.

[8.526] A. S. Thompson, Stresses in rotating disks at high temperatures, JAM 13 (1946), A45-A52.

[8.527] J. B. Tiedemann, I. Vigness, T. E. Pardue, Rotating beam deflection studies, NRLR No. 4139 (1953), $17 \mathrm{pp}$. 
[8.528] T. C. T. Ting, N. Nan, Plane waves due to combined compressive and shear stresses in a half space, Trans ASME E36 (1969), 2, 189-197.

[8.529] T. C. T. Ting, Interaction of shock waves due to combined two shear loadings, IJSS 5 (1969), 5, 415-435.

[8.530] --, Elastic-plastic boundaries in the propagation of plane and cylindrical waves of combined stress, QAM 27 (1970), 4, 441-449.

[8.531] T. W. Ting, Fracture of closed circular pipes under internal pressure and axial tension, JMM 9 (1960), 6, 821-867.

[8.532] T. G. Toridis, R. K. Wen, Inelastic response of beams to moving loads, Proc ASCE JEMD 92 (1966), 43.

[8.533] B. F. Trakhtenberg, R. I. Ulitskaya, Calculation of expanded multilayer structures of cylindrical type (in Russian), MPMDM (1974), 47-53.

[8.534] V. I. Trofimov, Elastic-plastic behaviour of mild structural steel under simple and combined loading (in Russian), IPPPSM (1955), 69-84.

[8.535] A. Troost, Belastbarkeit dünnwandiger Behälter durch Innendruck und Lăngskräfte, Mll 9 (1955), 23-24, 1054-1061.

[8.536] A. Ubaydillayev, Elastic-plastic deformations of a thick-walled tube under combined loading (in Russian), VVPM 31 (1975), 108-120.

[8.537] - Elastic-plastic deformations of a cylindrical shell (in Russian), VVPM 39 (1976), 147-157.

[8.538] —, Combined loading of a finite cylindrical shell (in Russian), VVPM 40 (1976), 119-128.

[8.539] T. Uga, Thermocyclic deformation of high-pressure vessels (in Japanese), PPMach 15 (1975), 3, 16-25.

[8.540] J. A. Van den Broek, Columns subject to uniformly distributed transverse loads, EJ 24 (1941), 115.

[8.541] S. Vejvoda, Praktické využiti teorie přizpúsobení při navrchováni tlakových nádob, Stroj 23 (1973), 7, 395-400.

[8.542] P. Vernay, Analyse limite d'un tube creux soumis à des efforts de torsion, de charge axiale et de pressions radiales pour les matériaux de Tresca et de Coulomb, Thèse doct. méc. sol., Univ. Grenoble 1971.

[8.543] R. I. Veytsman, On the strength of tubes subject to internal pressure and variable thermal loadings in the plastic range (in Russian), VMUst (1964), 234-238.

[8.544] V.P. Vikulenkov, Calculation of statically indeterminate, elastic-plastic systems under combined loading (in Russian), Izv VUZ M (1973), 4, 16-21.

[8.545] A. I. Vinogradov, S. P. Fesik, Certain problems of elastic-plastic bending (in Russian) $\operatorname{Tr}$ KIIZDT 40 (1960), 89-115.

[8.546] T. P. Vinogradova, A. P. Shinkarenko, On the problem of bending of physically nonlinear rectangular plates by using the method of variable directions (in Russian), UZGU 122 (1970), 22-27.

[8.547] V. E. Voronkov, V. V. Moskvitin, Rotation of an initially bent elastic-plastic bar (in Russian), IZh MTT (1966), 6, 147-149.

[8.548] V. E. Voronkov, Behaviour of an elastic-plastic bar in bending under subsequent rotation (in Russian), PPMCTN (1969), 136-143.

[8.549] -, Rotation of elastic-plastic bars in bending (in Russian), VMU $M M(1970), 6,73-79$.

[8.550] S. A. Vulman, An approximate solution of the elastic-plastic problem for hollow bodies with a surface close to spherical (in Russian), Izv AN SSSR MTT (1971), 1, 119-122.

[8.551] M. Wakabayashi, T. Nonaka, S. Morino, The experimental study on the inelastic behavior of steel frames with a rectangular cross-section subjected to vertical and horizontal loading, BDPRI 18 (1969), 3, 65-82.

[8.552] Z. Waszczyszyn, Numerical shakedown analysis of circular plates subjected to cycles of pressure and temperature, 3rd SMiRT (1975), paper L5/11. 
[8.553] Z. Waszczyszyn, E. Pabisek, Numerical analysis of circular elastic-plastic plates under cycles of pressure and temperature, NED 45 (1978), 1, 173-183.

[8.554] F. C. Weiler, Anisotropic elastic-plastic stress analysis of axisymmetric solids, CAGD 1970 (1972), 375-405.

[8.555] J. H. Weiner, J. V. Huddleston, Transient and residual stresses in heat-treated cylinders, JAM 26 (1959), 1, 31-39.

[8.556] K. Wellinger, H. Gassmann, H. Zenner, Festigkeitsverhalten unrunder Rohre unter zügiger und schwellender Innendruckbeanspruchung, $R R R 7$ (1968), 6, 223-230.

[8.557] E. Whalley, The design of pressure vessels subjected to thermal stress, IJMS 1 (1960), $2-3,301-308$, and 4, 379-395.

[8.558] W. Wojewódzki, H. Stolarski, Nośność graniczna powłoki walcowej obciążonej liniowozmiennym ciśnieniem i siłą osiową, RI 17 (1969), 4, 669-692.

[8.559] —, -, Krzywe nośności granicznej powłoki walcowej obciążonej liniowo-zmiennym ciśnieniem i pierścieniową siłą poprzeczną, $P N P W(1971), 6,23-49$.

[8.560] E. W. Wright, Unbraced multistory steel frames with wind load, Proc ASCE JSD 98 (1972), 1, 185-202.

[8.561] Yu. I. Yagn, O. A. Shishmarev, Investigations of plastic deformation of thin-walled tubular specimens under simultaneous tension and torsion (in Russian), ZL 24 (1958), 10, 1243-1245.

[8.562] Yang Nan-Sheng, Plastic stress-strain relations for brass (in Chinese), AMSin 2 (1958), $1,43-65$.

[8.563] O. A. Yushtchenko, On a certain case of axisymmetric deformation in perfect plasticity (in Ukrainian), DAN URSR (1957), 1, 18-22.

[8.564] M. A. Zadoyan, On a certain particular solution of equations of perfect plasticity (in Russian), DAN ArmSSR 39 (1964), 5, 265-269.

[8.565] - , On certain solutions of plastic flow equations for an anisotropic medium (in Russian), IZh MTT (1966), 2, 91-96.

[8.566] V.S. Zarubin, Investigation of the loading history of a non-uniformly heated cylindrical shell (in Russian), TNEK 4 (1964), 223-232.

[8.567] V.S. Zarubin, A. A. Polyakov, Non-isothermal alternating deformation in the case of biaxial state of stress (in Russian), TNEK 14 (1974), 19-21.

[8.568] A. Zavelani-Rossi, Optimal shake-down design of reinforced concrete beams, RIL ASL A105 (1971), 147-164.

[8.569] V. M. Zhigalkin, Stresses and strains in an elastic-plastic-hardening tube (in Russian), FTPRPI (1970), 3, 13-22.

[8.570] N. D. Zhudin, Deformation of continuous steel beams in the elastic-plastic range under moving load (in Russian), ST ISM AN USSR 17 (1952).

[8.571] —, Behaviour of continuous steel beams in the elastic-plastic range under moving load (in Russian), ST ISM AN USSR 18 (1953).

[8.572] A. M. Zhukov, Yu. N. Rabotnov, Investigation of plastic deformations of steel under combined loading (in Russian), IS 18 (1954), 105-112.

[8.573] A. Zingali, F. Braga, Calcolo dei sistemi elasto-plastici nel campo dei grandi spostamenti per carichi communque variabili, GGC 108 (1970), 6, 435-450.

[8.574] O. A. Zveryev, V. A. Palmov, Axisymmetric deformation of an elastic-plastic-hardening plate (in Russian), Izv AN SSSR MTT (1970), 2, 178-181.

[8.575] M. Życzkowski, The limit load of a thick-walled tube in a general circularly symmetrical case, AMS 8 (1956), 2, 155-179; 9th ICAM 1956, 8 (1957), 129-139.

[8.576] —, Limit state of non-homogeneous rotating circular disks, 9th ICAM 1956, 6 (1957), 351-360 (English summary); RI 5 (1957), 1, 49-96 (Polish full text).

[8.577] -, Discussion to the paper by G. Maier [4.355], SFP 1972 (1973), 492-495.

[8.578] M. Życzkowski, A. Zaborski, Creep rupture phenomena in creep buckling, IUTAM MVEMB 1974 (1975), 283-290. 


\section{Index of abbreviations used in bibliography}

Journals (J), Irregular series (I), Collections of papers (C), Proceedings of congresses and
symposia* (P) $^{*}$

$A A M$

$A A N L$

$A B M$

$A c B$

$A C M$

$A E$

Aer

AFOSR TN

AIAA

AIL

AirE

AIT BTP

AITCN

$A J A S$

$A J M$

$A M$

AMat

AMech

$A M R$

$A M S$

AMSin

AP CVUT

$A R M A$

$A S N M M$

$A S R$

$A T A S H$

$A T \check{C} S A V$

$A T P B$

$B A M S$

BAPSL

$B A P$ SST

$B A R B C S$
Advances in Applied Mechanics, Academic Press, New York (I)

Atti della Accademia Nazionale dei Lincei, Rendiconti Classe di Scienze

Fisiche, Matematiche e Naturali, Roma (J)

Archiwum Budowy Maszyn - the Archive of Mechanical Engineering,

Polska Akademia Nauk, Warszawa (J)

Acier (Belgique) (J)

Acciaio e Costruzioni Metallichi (J)

Archiv für das Eisenhüttenwesen, Düsseldorf (J)

Aerotecnica, Roma (J)

Aero. Str. Laboratory Technical Report, Massachusetts Institute of Technology, Cambridge, Mass. (I)

American Institute of Aeronautics and Astronautics Journal, New York (J)

Archiwum Inżynierii Lądowej, Polska Akademia Nauk, Warszawa (J) Aircraft Engineering, London (J)

Annales de l'Institut Technique du Bâtiment et des Travaux Publics, Paris (I)

Atti Istituto Tecnica delle Construzioni, Napoli (I)

Australian Journal of Applied Science (J)

American Journal of Mathematics, Baltimore (J)

Acta Metallurgica, Naw York (J)

Aplikace Matematiky, Praha (J)

Acta Mechanica, Springer, Wien-New York (J)

Applied Mechanics Reviews, ASME, New York (J)

Archiwum Mechaniki Stosowanej-Archives of Mechanics, - Polska

Akademia Nauk, Warszawa (J)

Acta Mechanica Sinca, Peking (J)

Acta Polytechnica, Práce ČVUT v Praze, Praha (I)

Archive for Rational Mechanics and Analysis, Springer, Berlin (J)

Acta Sci. Natur. Scholar. Sup. Sinens, Mat.-Mech., Peking (I)

Applied Science Research, den Haag (J)

Acta Technica Academiae Scientiarum Hungaricae, Budapest (J)

Acta Technica Ceskoslovenské Akademie Ved, Praha (J)

Annales des Travaux Publics de Belgique, Bruxelles (I)

Bulletin of the American Mathematical Society (J)

Bulletin de l'Académie Polonaise des Sciences et des Lettres (until 1952), Warszawa (J)

Bulletin de l'Académie Polonaise des Sciences, Série des Sciences Techniques (Cl. IV), Warszawa (since 1953)

Bulletin de l'Académie Royale Belg., Classe des Sciences, Bruxelles (J)

* In the case of certain congresses and symposia two dates are given: the first date refers to the congress itself, whereas the second one (bracketed) gives the year of publication of the proceedings. 
$B B$

BCTAM

$B D E R$

$B D P R I$

$B I$

$B I P I$

BJSME

BKMSS

$B M E E$

$B S$

$B S C A R S F Y$

$B T$

$B U T R$

$B W A T$

$B W J$

$B Z h B$

$C A$

CAAM

$C A G D$

$C A I P C$

CANCAM

CCE

CDSC

Cem

CEPWR

CET IEA

CGFR

CI

CIRP

CMAME

CMI

CMMSM

CoM

CoMet

Con

$C P A M$

CRAS

CS

CSRC

$C T$

CTAS

DAN ArmSSR

DAN AzbSSR

DAN SSSR
Bauplanung-Bautechnik, Berlin (J)

Bulgarian Congress of Theoretical and Applied Mechanics (P)

Bulletin des Diverses Etudes et Recherches, Paris (J)

Bulletin Disaster Prevent. Research Institute Japan (J)

Bauingenieur, Berlin (J)

Buletinul Institului Politechnic din Iaşi, Iaşi (J)

Bulletin of the Japan Society of Mechanical Engineers, Tokyo (J)

Blgarska Konf. Mekh. Spl. Sred., Varna 1966 (1968) (P)

Bulletin of Mechanical Engineering Education (J)

Building Science ( $\mathrm{J}$ )

Bulletin Scientifique, Conseil des Académies des Sciences et des Arts de la RSF de Yougoslavie, Zagreb (J)

Bautechnik, Berlin (J)

Brown University Technical Report, Providence, R. I. (I)

Biuletyn Wojskowej Akademii Technicznej, Warszawa (I)

British Welding Journal, London (J)

Beton i Zhelezobeton, Moskva (J)

Chislennyi Analiz, Kiev 1975 (C)

Computational Approaches in Applied Mechanics, ASME, New York 1969 (C)

Continuum Aspects Graphite Design 1970 (1972), Oak Ridge (P)

Congrés de l'Association Internationale des Ponts et Charpents (P)

Canadian Congress of Applied Mechanics; 3rd, Calgary 1971; 4th, Montreal $1973(\mathrm{P})$

Concrete and Constructional Engineering, London (J)

Conference on Dimensioning and Strength Calculations, Budapest: 2nd (1965); 3rd (1968) (P)

il Cemento (J)

Civil Engineering and Public Works Review, London (J)

Civil Engineering Transactions, Institution of Engineers, Australia, Sydney (J)

Cahiers du Groupe Français de Rhéologie (I)

Civilingenieur (J)

Annals of the CIRP (College International pour Recherche Scientifique des Techniques de Production Mécanique), Paris (I)

Computer Methods in Applied Mechanics and Engineering (J)

Congress of Mechanics in India (P)

Conference on Mathematical Methods in Structural Mechanics (P)

Costruzioni Metallichi (J)

Construction Métallique, Paris (J)

Concrete, London (J)

Communications on Pure and Applied Mathematics, New York (J)

Comptes Rendus hebdomadaires des séances de l'Académie des Sciences, Paris (J)

Computers and Structures, Pergamon Press, Oxford (J)

Symposium Concrete Shell Roof Construction; 2nd, Oslo 1957 (P)

Czasopismo Techniczne, Lwów (until 1939); Kraków (since 1945) (J);

Contributions to the Theory of Aircraft Structures, Delft 1972 (C)

Doklady Akademii Nauk Armyanskoy SSR, Erevan (J)

Doklady Akademii Nauk Azerbaydzhanskoy SSR, Baku (J)

Doklady Akademii Nauk SSSR, Moskva (J) 


DAN URSR
DAN UzbSSR
DCAMM
deIng
DLPI M
DM
DNT
DPM
DSS
EB
EEN
EFM
EJ
EMPA Ber
Eng
Enr
EP
ES
ExpM
FADT
FDP
FGI
FST
FTPRPI
GGC
GNMPK
GTU
GVTUZ PM

HEM

$H P$

HPTM

$I A$

IASS NSP

IBS

IBud

$I B Z B$

IC

$I C M, I C A M$
Dopovidy Akademii Nauk Ukrainskoy RSR, Kiev (J)

Doklady Akademii Nauk Uzbetskoy SSR, Tashkent (J)

The Danish Center for Applied Mathematics and Mechanics Reports, København-Lyngby (I)

de Ingenieur, den Haag (J)

Doklady Lvovskogo Politekhnicheskogo Instituta, Mekhanika, Lvov (I)

Developments in Mechanics, Wiley, New York (I)

Deformatsya Neuprugogo Tela, Ilim, Frunze 1970 (C)

Dinamika i Protchnost Mashin (I)

Dinamika Sploshnoy Sredy, Novosibirsk (I)

Eisenbau (J)

Ergebnisse der Exacten Naturwissenschaften, Springer (I)

Engineering Fracture Mechanics (J)

Engineering Journal, Montreal (J)

Eidgenössische Materialprüfungsanstalt, Berichte, Zürich (I)

Engineering, London ( $\mathrm{J}$ )

Engineer, New York (J)

Engineering Plasticity, Cambridge University Press, Cambridge 1968 (C)

Engineering Structures, Academic Press, New York 1949 (C)

Experimental Mechanics, Westport, Conn. (J)

Fundamental Aspects of Dislocation Theory, 1970 (C)

Fundamentals of Deformation Processing, Syracuse University Press, Syracuse, N. Y., 1964 (C)

Forschungsarbeiten auf dem Gebiete des Ingenieurwesens, VDI (I)

Fibre Science and Technology, London (J)

Fiziko-Tekhnicheskie Problemy Razrabotki Poleznykh Iskopayemykh

Giornale del Genio Civile, Roma (J)

Göttinger Nachrichten, Mathematisch-Physikalische Klasse, Göttingen (I)

Gidroaeromekhanika i Teorya Uprugosti, Mezhvuz. Nauchn. Sbornik (I)

Godishnik na Vysshite Tekhnicheski Uchebni Zavedenya, Prilozhna Mekhanika, Sofia (I)

Handbook of Engineering Mechanics, Edited by W. Flügge, McGrawHill, New York 1962 (C)

Handbuch der Physik-Encyclopedia of Physics, Edited by S. Flügge, Springer (C)

Handbuch der Physik und Technischen Mechanik, A. Barth 1931 (C) Ingenieur-Archiv, Springer, Berlin (J)

International Association of Shell Structures, Symposium on Nonclassical Shell Problems, Warszawa 1963, North-Holland-PWN (1964) (P) Inelastic Behavior of Solids, Edited by M. F. Kanninen et al., Columbus 1969, McGraw-Hill, New York (1970) (P)

Inżynieria i Budownictwo, Warszawa (J)

Issledovaniya po Betonu i Zhelezobetonu, Moskva-Riga (I)

Ingegneria Civ. (J)

International Congress of (Applied) Mechanics; 1st, Delft 1924 (1925); 2nd, Zürich 1926; 3rd, Stockholm 1930; 4th, Cambridge, England 1934; 5th, Cambridge, Mass. 1938; 6th, Paris 1946; 7th, London 1948; 8th, Istanbul 1952 (1955); 9th, Bruxelles 1956 (1957); 10th, Stresa 1960 (1962); 11th, München 1964 (1966); 12th, Stanford 1968 (1969); 13th, Moskva 1972 (1974); 14th, Delft 1976 (P) 
[3.542] V. V. Moskvitin, Elastic-plastic deformations of bodies subject to repeated loadings (in Russian), PMM 19 (1955), 6, 714-724.

[3.543] - , On the conditions of a simple repeated loading (in Russian), VMU $M M$ (1962), 2, 72-75.

[3.544] V. V. Moskvitin, E. Palne, On the theorem on variable loadings (in Russian), IZh MTT (1966), 3, 144-147.

[3.545] V. V. Moskvitin, Zh. I. Tairova, On a simple possibility of analysis of combined cyclic loadings (in Russian), VMU MM (1968), 3, 119-122.

[3.546] V. V. Moskvitin, Some problems of plasticity in the case of variable loadings (in Russian), PPMCTN (1969), 25-36.

[3.547] -, Cyclic plasticity and cumulative deterioration (in Russian), DAN SSSR 184 (1969), 4, 796-799.

[3.548] -, On a certain possibility of accounting for cumulative deterioration in cyclic plasticity (in Russian), VMU MM (1969), 5, 117-121.

[3.549] -, On simple variable loadings in thermoplasticity (in Russian), VMU $M M$ (1969), 5, 122-124.

[3.550] N. S. Mozharovsky, E. A. Antipov, Plasticity and fracture of heat-resistant materials under programmed variations of temperature and stresses (in Russian), TNEK 13 (1973), 61-67.

[3.551] Z. Mróz, Non-associated flow laws in plasticity, JM 2 (1963), 1, 21-42.

[3.552] - Admissible loading paths in the deformation theories of plasticity, AMS 16 (1964), 5, 1091-1102.

[3.553] —, On non-linear flow laws in the theory of plasticity, BAP SST 12 (1964), 11, 789-797,

[3.554] -, On the description of anisotropic workhardening, JMPS 15 (1967), 3, 163-175.

[3.555] - An attempt to describe the behaviour of metals under cyclic loads using a more general workhardening model, AMech 7 (1969), 199-212.

[3.556] - , On the theory of steady plastic cycles in structures, Ist SMiRT (1971), L5-6, 1-13.

[3.557] Z. Mróz, C. Goss, O złożonych modelach wzmocnienia plastycznego, MTS 10 (1972), 2, 259-279.

[3.558] Z. Mróz, Mathematical models of inelastic concrete behaviour, INSC (1973), 47-72.

[3.559] - , A description of workhardening of metals with application to variable loading, SFP 1972 (1974), 551-570.

[3.560] Z. Mróz, N. C. Lind, Simplified theories of cyclic plasticity, AMech 22 (1975), 1-2, 131-152,

[3.561] Z. Mróz, Description of plastic and viscous hardening of materials for steady and cyclic loading, 3rd SMiRT (1975), L5/6.

[3.562] J. F. Mulhern, T. G. Rogers, A. J. M. Spencer, A continuum model for fibre-reinforced plastic materials, Proc RS A301 (1967), 473-492.

[3.563] T. Mura, Residual stresses due to thermal treatments, RRFE MU (1957), 10, 14.

[3.564] J. Murzewski, A probability theory of plastic and brittle behaviour of quasi-homogeneous materials, AMS 12 (1960), 2, 203-227.

[3.565] J. Murzewski, Z. Mendera, Yield surface of steel determined by semiempirical method, BAP SST 11 (1963), 7, 35-42.

[3.566] A. Nádai, Theory of flow and fracture of solids, vol. 1, McGraw-Hill, New York-TorontoLondon 1950.

[3.567] P. M. Naghdi, J. C. Rowley, C. W. Beadle, Experiments concerning the yield surface and the assumptions of linearity in the plastic stress-strain relations, JAM 22 (1955), 3, 416-420.

[3.568] P. M. Naghdi, F. Essenburg, W. Koff, An experimental study of initial and subsequent yield surfaces in plasticity, JAM 25 (1958), 2, 101-209.

[3.569] P. M. Naghdi, J. A. Trapp, On finite elastic-plastic deformation of metals, Trans ASME E41 (1974), 1, 254-260. 
[3.570] P. M. Naghdi, J. A. Trapp, Restrictions on constitutive equations of finitely deformed elastic-plastic materials, QJMAM 28 (1975), 1, 25-46.

[3.571] —, - , On the nature of normality of plastic strain rate and convexity of yield surfaces in plasticity, Trans ASME EA2 (1975), 1, 61-66.

[3.572] B. Nayroles, Structure algébraique des théories classiques de plasticité, STA (1973), 1, 193-217.

[3.573] —, J. J. Moreau's approach to a fundamental problem: the quasi-static evolution of a perfectly elasto-plastic body, AMS 28 (1976), 1, 115-132.

[3.574] Yu. V. Nemirovsky, On elastic-plastic behaviour of a reinforced layer (in Russian), ZhPMTF (1969), 6, 81-89.

[3.575] H. Neuber, Anisotropic nonlinear stress-strain laws and yield conditions, IJSS 5 (1969), $12,1299-1310$.

[3.576] Q.S. Nguyen, Évolution de la contrainte et de l'écrouissage pour certains matériaux a écrouissage positif, STA 47 (1973), 1, 247-250.

[3.577] Q. S. Nguyen, H. D. Bui, Sur les matériaux élastoplastiques à écrouissage positif ou négatif, $J M 13$ (1974), 2, 321-342.

[3.578] V. N. Nikolayevsky, Mechanical properties of soils and the theory of plasticity (in Russian), INT MTDT 6 (1972).

[3.579] V. N. Nikolayevsky, N. M. Syrnikov, G. M. Shefter, Dynamics of elastic-plastic media subject to dilatation (in Russian), UMDS (1975), 397-413.

[3.580] V. V. Novozhilov, On stress-strain relations in a nonlinearly elastic medium (in Russian), PMM 15 (1951), 2, 183-194.

[3.581] - , On a certain direction in the theory of plasticity (in Russian), Izv AN SSSR MM (1961), 3, 176-181.

[3.582] - , Once more on the postulate of isotropy (in Russian), Izv AN SSSR MM (1962), 1, 205-208.

[3.583] —, On the forms of stress-strain relations in initially isotropic inelastic bodies (in Russian), PMM 27 (1963), 5, 794-812.

[3.584] - , On combined loading and perspectives of phenomenological approach to the analysis of microstresses (in Russian), PMM 28 (1964), 3, 393-400.

[3.585] W. J. O'Donnell, J. Porowski, Yield surfaces for perforated materials, Trans ASME E40 (1973), 1, 263-270.

[3.586] F. K. G. Odqvist, Die Verfestigung von flusseisenähnlichen Körpern, ZAMM 13 (1933), 5, 360-363.

[3.587] P. M. Ogibalov, Yield conditions for combined stresses in a body subject to unsteady thermal regime (in Russian), VMU (1949).

[3.588] - , Theory of plastic deformations at elevated temperatures of the body (in Russian), VMU (1950), 12.

[3.589] P. M. Ogibalov, I. A. Kiyko, High-parameter mechanics (in Russian), Mosk. Univers., Moskva 1966.

[3.590] P. M. Ogibalov, The Ilyushin laws and methods in the mechanics of deformable solids and media (in Russian), MPol (1971), 1, 36-40.

[3.591] Y. Ohashi, M. Tokuda, Precise measurement of plastic behaviour of mild steel tubular specimens subjected to combined torsion and axial force, JMPS 21 (1973), 4, 241-261.

[3.592] Y. Ohashi, M. Tokuda, S. Mizuno, A precise stress-strain relation of mild steel in the proportional deformation under combined loading, BJSME 17 (1974), 111, 1135-1142.

[3.593] Y. Ohashi, M. Tokuda, H. Yamashita, Plastic deformation of mild steel under combined load of axial force and torsion with strain trajectories of constant curvature, BJSME 18 (1975), 120, 579-586.

[3.594] J. G. Oldroyd, On the formulation of rheological equations of state, Proc RS 200A (1950), 523-541. 
[3.595] W. Olszak, W. Urbanowski, Ortotropia i niejednorodność w teorii plastyczności, AMS 8 (1956), 1, 85-110.

[3.596] - , - The plastic potential and the generalized distortion energy in the theory of nonhomogeneous anisotropic elastic-plastic bodies, AMS 8 (1956), 4, 671-694.

[3.597] - , - Plastic non-homogeneity: a survey of theoretical and experimental research, IUTAM NH 1958 (1959), 259-298.

[3.598] W. Olszak, On some basic aspects of the theory of non-homogeneous loose and cohesive media, AMS 11 (1959), 6, 651-766.

[3.599] W. Olszak, J. Rychlewski, W. Urbanowski, Plasticity under non-homogeneous conditions, AAM 7 (1962), 131-214.

[3.600] W. Olszak, Z. Mróz, P. Perzyna, Recent trends in the development of the theory of plasticity, Pergamon Press-PWN, Oxford-Warszawa 1963.

[3.601] W. Olszak, P. Perzyna, The constitutive equations of the flow theory for a non-stationary yield condition, 11th ICAM 1964 (1966), 545-553.

[3.602] W. Olszak, Koncepcja naroża plastycznego w świetle teorii i doświadczenia, Księga jubil. A. Krupkowskiego, Warszawa 1965, 349-370.

[3.603] W. Olszak, W. Urbanowski, Anisotropie im elastisch-plastischen Bereich, MAn (1974), 279-301.

[3.604] E. Ondraček, Přispěvek k teorii plastického potenciálu, AMat 8 (1963), 3, 163-179.

[3.605] W. R. Osgood, Combined-stress tests on 24S-T aluminium-alloy tubes, JAM 14 (1947), 247-253.

[3.606] J. R. Osias, J. L. Swedlow, Finite elasto-plastic deformation, IJSS 10 (1974), 3, 321-339.

[3.607] T. Ota, A. Shindo, H. Fukuoka, Anisotropy of mild steel by combined tension-torsion tests, 8th JCAM 1958 (1959), 221-224.

[3.608] - , - , - A consideration on anisotropic yield criterion, 9th JCAM 1959 (1960), 117.

[3.609] D. R. Owen, Thermodynamics of materials with elastic range, ARMA 31 (1968), 2, 91-112,

[3.610] D. R. J. Owen, J. Holbeche, O. C. Zienkiewicz, Elastic-plastic analysis of fibrereinforced materials, FST 1 (1969), 3, 185-207.

[3.611] D. R. Owen, A mechanical theory of materials with elastic range, ARMA 37 (1970), 2, 85-110.

[3.612] A. Paglietti, Universal deformations for thermo-elastic-plastic materials, AMS 27 (1975), 5-6, 773-789.

[3.613] I. Z. Palley, Application of the theory of residual microstresses to non-isothermal deformation (in Russian), Izv AN SSSR Mekh (1965), 2, 110-113.

[3.614] - On accounting for effects of the Bauschinger type in the analysis of inelastic deformation in variable temperatures (in Russian), PDAD 5 (1969), 163-169.

[3.615] A. C. Palmer, G. Maier, D. C. Drucker, Normality relations and convexity of yield surfaces for unstable materials or structural elements, Trans ASME E34 (1967), 2, 464-470.

[3.616] V. M. Panferov, R. Kh. Ismagilov, L. M. Ilyin, E. Z. Korol, V. F. Tyurin, On the deformation of a solid in the case of combined loading with fixed principal directions of stresses and strains (in Russian), NTIM MU (1971), 8, 5-21.

[3.617] J. Parker, M. B. Bassett, Plastic stress-strain relationships-some experiments to derive a subsequent yield surface, Trans ASME E31 (1964), 4, 676-682.

[3.618] B. Paul, A modification of the Coulomb-Mohr theory of fracture, Trans ASME E28 (1961), 2, 259-268.

[3.619] B. Paul, W. Chen, Lee Li-Chih, An experimental study of plastic flow under stepwise increments of tension and torsion, 4th USNCAM 2 (1062), 1031-1038.

[3.620] B. Paul, Macroscopic criteria for plastic flow and brittle fracture, Fracture (collection of papers), vol. 2, 313-496, Acad. Press, New York 1968.

[3.621] H. Payne, The slip theory of plasticity for aggregates, JMPS 7 (1959), 2, 126-134.

[3.622] T. Pełczyński, Zagadnienia wytężenia materiałów, rys historyczny, OP 3 (1962), 1, 9-49. 
[3.570] P. M. Naghdi, J. A. Trapp, Restrictions on constitutive equations of finitely deformed elastic-plastic materials, QJMAM 28 (1975), 1, 25-46.

[3.571] - , - On the nature of normality of plastic strain rate and convexity of yield surfaces in plasticity, Trans ASME EA2 (1975), 1, 61-66.

[3.572] B. Nayroles, Structure algébraique des théories classiques de plasticité, STA (1973), 1, 193-217.

[3.573] —, J. J. Moreau's approach to a fundamental problem: the quasi-static evolution of a perfectly elasto-plastic body, AMS 28 (1976), 1, 115-132.

[3.574] Yu. V. Nemirovsky, On elastic-plastic behaviour of a reinforced layer (in Russian), ZhPMTF (1969), 6, 81-89.

[3.575] H. Neuber, Anisotropic nonlinear stress-strain laws and yield conditions, IJSS 5 (1969), $12,1299-1310$.

[3.576] Q.S. Nguyen, Évolution de la contrainte et de l'écrouissage pour certains matériaux a écrouissage positif, STA 47 (1973), 1, 247-250.

[3.577] Q. S. Nguyen, H. D. Bui, Sur les matériaux élastoplastiques à écrouissage positif ou négatif, JM 13 (1974), 2, 321-342.

[3.578] V. N. Nikolayevsky, Mechanical properties of soils and the theory of plasticity (in Russian), INT MTDT 6 (1972).

[3.579] V. N. Nikolayevsky, N. M. Syrnikov, G. M. Shefter, Dynamics of elastic-plastic media subject to dilatation (in Russian), UMDS (1975), 397-413.

[3.580] V. V. Novozhilov, On stress-strain relations in a nonlinearly elastic medium (in Russian), PMM 15 (1951), 2, 183-194.

[3.581] - , On a certain direction in the theory of plasticity (in Russian), Izv AN SSSR MM (1961), 3, 176-181.

[3.582] -, Once more on the postulate of isotropy (in Russian), Izv AN SSSR MM (1962), 1, 205-208.

[3.583] —, On the forms of stress-strain relations in initially isotropic inelastic bodies (in Russian), PMM 27 (1963), 5, 794-812.

[3.584] -, On combined loading and perspectives of phenomenological approach to the analysis of microstresses (in Russian), PMM 28 (1964), 3, 393-400.

[3.585] W. J. O'Donnell, J. Porowski, Yield surfaces for perforated materials, Trans ASME E40 (1973), 1, 263-270.

[3.586] F. K. G. Odqvist, Die Verfestigung von flusseisenähnlichen Körpern, ZAMM 13 (1933), 5, 360-363.

[3.587] P. M. Ogibalov, Yield conditions for combined stresses in a body subject to unsteady thermal regime (in Russian), VMU (1949).

[3.588] - , Theory of plastic deformations at elevated temperatures of the body (in Russian), VMU (1950), 12.

[3.589] P. M. Ogibalov, I. A. Kiyko, High-parameter mechanics (in Russian), Mosk. Univers., Moskva 1966.

[3.590] P. M. Ogibalov, The Ilyushin laws and methods in the mechanics of deformable solids and media (in Russian), MPol (1971), 1, 36-40.

[3.591] Y. Ohashi, M. Tokuda, Precise measurement of plastic behaviour of mild steel tubular specimens subjected to combined torsion and axial force, JMPS 21 (1973), 4, 241-261.

[3.592] Y. Ohashi, M. Tokuda, S. Mizuno, A precise stress-strain relation of mild steel in the proportional deformation under combined loading, BJSME 17 (1974), 111, 1135-1142.

[3.593] Y. Ohashi, M. Tokuda, H. Yamashita, Plastic deformation of mild steel under combined load of axial force and torsion with strain trajectories of constant curvature, BJSME 18 (1975), 120, 579-586.

[3.594] J. G. Oldroyd, On the formulation of rheological equations of state, Proc RS 200A (1950), 523-541. 
[3.595] W. Olszak, W. Urbanowski, Ortotropia i niejednorodność w teorii plastyczności, AMS 8 (1956), 1, 85-110.

[3.596] - , - The plastic potential and the generalized distortion energy in the theory of nonhomogeneous anisotropic elastic-plastic bodies, AMS 8 (1956), 4, 671-694.

[3.597] - , - Plastic non-homogeneity: a survey of theoretical and experimental research, IUTAM NH 1958 (1959), 259-298.

[3.598] W. Olszak, On some basic aspects of the theory of non-homogeneous loose and cohesive media, AMS 11 (1959), 6, 651-766.

[3.599] W. Olszak, J. Rychlewski, W. Urbanowski, Plasticity under non-homogeneous conditions, AAM 7 (1962), 131-214.

[3.600] W. Olszak, Z. Mróz, P. Perzyna, Recent trends in the development of the theory of plasticity, Pergamon Press-PWN, Oxford-Warszawa 1963.

[3.601] W. Olszak, P. Perzyna, The constitutive equations of the flow theory for a non-stationary yield condition, 11th ICAM 1964 (1966), 545-553.

[3.602] W. Olszak, Koncepcja naroża plastycznego w świetle teorii i doświadczenia, Księga jubil. A. Krupkowskiego, Warszawa 1965, 349-370.

[3.603] W. Olszak, W. Urbanowski, Anisotropie im elastisch-plastischen Bereich, MAn (1974), 279-301.

[3.604] E. Ondrað̌ek, Přispěvek k teorii plastického potenciálu, AMat 8 (1963), 3, 163-179.

[3.605] W. R. Osgood, Combined-stress tests on 24S-T aluminium-alloy tubes, JAM 14 (1947), 247-253.

[3.606] J. R. Osias, J. L. Swedlow, Finite elasto-plastic deformation, IJSS 10 (1974), 3, 321-339.

[3.607] T. Ota, A. Shindo, H. Fukuoka, Anisotropy of mild steel by combined tension-torsion tests, 8th JCAM 1958 (1959), 221-224.

[3.608] - , - , - A consideration on anisotropic yield criterion, 9th JCAM 1959 (1960), 117.

[3.609] D. R. Owen, Thermodynamics of materials with elastic range, ARMA 31 (1968), 2, 91-112,

[3.610] D. R. J. Owen, J. Holbeche, O. C. Zienkiewicz, Elastic-plastic analysis of fibrereinforced materials, FST 1 (1969), 3, 185-207.

[3.611] D. R. Owen, A mechanical theory of materials with elastic range, ARMA 37 (1970), 2, 85-110.

[3.612] A. Paglietti, Universal deformations for thermo-elastic-plastic materials, AMS 27 (1975), 5-6, 773-789.

[3.613] I. Z. Palley, Application of the theory of residual microstresses to non-isothermal deformation (in Russian), Izv AN SSSR Mekh (1965), 2, 110-113.

[3.614] —, On accounting for effects of the Bauschinger type in the analysis of inelastic deformation in variable temperatures (in Russian), PDAD 5 (1969), 163-169.

[3.615] A. C. Palmer, G. Maier, D. C. Drucker, Normality relations and convexity of yield surfaces for unstable materials or structural elements, Trans ASME E34 (1967), 2, 464-470.

[3.616] V. M. Panferov, R. Kh. Ismagilov, L. M. Ilyin, E. Z. Korol, V. F. Tyurin, On the deformation of a solid in the case of combined loading with fixed principal directions of stresses and strains (in Russian), NTIM MU (1971), 8, 5-21.

[3.617] J. Parker, M. B. Bassett, Plastic stress-strain relationships-some experiments to derive a subsequent yield surface, Trans ASME E31 (1964), 4, 676-682.

[3.618] B. Paul, A modification of the Coulomb-Mohr theory of fracture, Trans ASME E28 (1961), 2, 259-268.

[3.619] B. Paul, W. Chen, Lee Li-Chih, An experimental study of plastic flow under stepwise increments of tension and torsion, 4th USNCAM 2 (1062), 1031-1038.

[3.620] B. Paul, Macroscopic criteria for plastic flow and brittle fracture, Fracture (collection of papers), vol. 2, 313-496, Acad. Press, New York 1968.

[3.621] H. Payne, The slip theory of plasticity for aggregates, JMPS 7 (1959), 2, 126-134.

[3.622] T. Pełczyński, Zagadnienia wytężenia materiałów, rys historyczny, OP 3 (1962), 1, 9-49. 
[3.623] N. Perrone, P. G. Hodge, Strain hardening solutions with generalized kinematic models, 3rd USNCAM (1958), 641-648.

[3.624] P. Perzyna, Simple material and plastic material, AMS 18 (1966), 3, 241-258.

[3.625] P. Perzyna, W. Wojno, Thermodynamics of a rate sensitive plastic material, AMS 20 (1968), 5, 499-511.

[3.626] P. Perzyna, Plasticity of irradiated materials, BAP SST 21 (1973), 10, 499-513.

[3.627] P. Perzyna, A. Sawczuk, Problems in thermoplasticity, NED 24 (1973), 1, 1-55.

[3.628] P. Perzyna, Internal variable description of plasticity, PPlast (1973), 145-176.

[3.629] P. P. Petrishtchev, Application of the theory of small elastic-plastic deformations to an anisotropic body (in Russian), Izd. Mosk. Gosud. Univ., Moskva 1951.

[3.630] A. Phillips, An experimental investigation of plastic stress-strain relations, 9th ICAM 1956, 9 (1957), 23-33.

[3.631] —, Pointed vertices in plasticity, 2nd SNSM (1960), 202-214.

[3.632] A. Phillips, G. A. Gray, Experimental investigation of corners in the yield surface, Trans ASME D83 (1961), 2, 275-288.

[3.633] A. Phillips, R. L. Sierakowski, On the concept of the yield surface, AMech 1 (1965), 1, 29-35.

[3.634] A. Phillips, M. Eisenberg, Observations on certain inequality conditions in plasticity, IJNLM 1 (1966), 2, 247-256.

[3.635] A. Phillips, Yield surfaces of pure aluminium at elevated temperatures, IUTAM ThI 1968 (1970), 241-258.

[3.636] A. Phillips, C. S. Liu, J. W. Justusson, An experimental investigation of yield surfaces at elevated temperatures, AMech 14 (1972), 2-3, 119-146.

[3.637] A. Phillips, J. L. Tang, The effect of loading path on the yield surface at elevated temperatures, IJSS 8 (1972), 4, 463-474.

[3.638] A. Phillips, R. Kasper, On the foundations of thermoplasticity, an experimental investigation, Trans ASME E40 (1973), 4, 891-896.

[3.639] A. Phillips, J. L. Tang, M. Ricciuti, Some new observations of yield-surfaces, AMech 20 (1974), 1-2, 23-39.

[3.640] A. Phillips, The foundations of thermoplasticity, experiments and theory, TACM (1974), $1-21$.

[3.641] —, Experimental plasticity, some thoughts on its present status and possible future trends, PPlast 1972 (1973), 193-231.

[3.642] A. Phillips, G. Weng, A new hardening law in thermoplasticity (in Russian), UMDS (1975), $550-558$.

[3.643] G. S. Pisarenko, A. A. Lebedev, Deformation and fracture of materials under combined stress (in Russian), Izd. Naukowa Dumka, Kiev 1969.

[3.644] G. S. Pisarenko, A. A. Lebedev, V. P. Lamashevsky, An experimental investigation of the laws of deformation of carbon steel under combined stresses at low temperatures (in Russian), PPro (1969), 5, 42-47.

[3.645] B. E. Pobedrya, The notation of a simple process in the case of finite deformations, $P P$ (1971), 166-170.

[3.646] A. A. Polyakov, L. T. Shirogorodsky, Variable non-isothermal deformation of structural materials (in Russian), TNEK 11 (1971), 136-139.

[3.647] J. Porowski, W. J. O'Donnell, Effective plastic constants for perforated materials, Trans ASME J96 (1974), 3, 234-241.

[3.648] W. R. Powell, A note on yield curves in cyclic work softening, Trans ASME E35 (1968), 4, 822-824.

[3.649] W. Prager, Einfluss der Deformation auf die Fliessbedingung von zähplastischen Körpern, ZAMM 15 (1935), 1/2, 76-80.

[3.650] - Mécanique des solides isotropes au delà du domaine élastique, Mem SM 87 (1937), $66 \mathrm{pp}$. 
[3.651] W. Prager, Strain-hardening under combined stresses, JAP 16 (1945), 12, 837-840.

[3.652] -, The stress-strain laws of the mathematical theory of plasticity, JAM 15 (1948), 3, 226-233.

[3.653] -, Recent developments in the mathematical theory of plasticity, JAP 20 (1949), 235-241.

[3.654] -, On the use of singular yield conditions and associated flow rules, JAM 20 (1953), $317-320$.

[3.655] -, The theory of plasticity: a survey of recent achievements, Proc IME 169 (1955), 41-57.

[3.656] - , A new method of analyzing stresses and strains in work-hardening plastic solids̄, JAM 23 (1956), 4, 493-496.

[3.657] -, Non-isothermal plastic deformation, Proc KNAW 61 (1958), 176-182.

[3.658] -, On plastic analysis of layered structures (in Russian), PMSS (1961), 302-309.

[3.659] - Plastic failure of fiber-reinforced materials, Trans ASME E36 (1969), 3, 542-544.

[3.660] H. L. D. Pugh, D. Green, The effect of hydrostatic pressure on the plastic flow and fracture of metals, Proc IME 170 (1956)).

[3.661] Yu. N. Rabotnov, A model illustrating some properties of a plastic hardening body (in Russian), PMM 23 (1959), 1, 164-169.

[3.662] -, Elastic-plastic state of a composite structure (in Russian), PGMSS (1969), 411-415.

[3.663] D. Radenković, Théorèmes limites pour un matériau de Coulomb à dilatation non standartisée, CRAS 252 (1961), 26, 4103-4104.

[3.664] - Théorie des charges limites, extension à la mécanique des sols, $S P 1961$ (1962), 116, 129-142.

[3.665] B. Raniecki, A. Sawczuk, Thermal effects in plasticity, ZAMM 55 (1975), 6, 333-342; 7-8, 363-374.

[3.666] Y. R. Rashid, On nonlinear kinematic hardening plasticity theory, NED 29 (1974), 1, 135-140.

[3.667] S. I. Ratner, Change of mechanical properties of metals under hydrostatic pressure (in Russian), ZhTF 19 (1949), 3, 408.

[3.668] K. A. Reckling, Experimente zur Feststellung der Werkstoffanisotropie und zur Überprüfung der Hillschen Verfestigungshypothese, Stb 38 (1969), 2, 43-51.

[3.669] M. Reiner, A mathematical theory of dilatancy, AJM 67 (1945), 350-362.

[3.670] L. Rendulic, Eine Betrachtung zur Frage der plastischen Grenzzustände, BI 19 (1938), 159-164.

[3.671] A. Reuss, Flieszpotential oder Gleitebenen? ZAMM 12 (1932), 15.

[3.672] —, Vereifachte Berechnung der plastischen Formänderungsgeschwindigkeiten bei Voraussetzung der Schubspannungsflieszbedingung, ZAMM 13 (1933), 5, 356-260.

[3.673] —, 4th ICAM 1934 (1935), 241.

[3.674] J. R. Rice, Inelastic constitutive relations for solids: an internal-variable theory and its application to metal plasticity, JMPS 19 (1971), 6, 433-455.

[3.675] R. S. Rivlin, Large elastic deformations of isotropic materials, IV, PhTRS A241 (1948), 379-397.

[3.676] R. S. Rivlin, J. L. Ericksen, Stress-deformation relations for isotropic materials, JRMA 4 (1955), 323-425.

[3.677] J. Rogan, A. Shelton, Yield and subsequent flow behaviour of some annealed steels under combined stress, JSA 4 (1969), 2, 127-137.

[3.678] -, -, Effect of pre-stress on the yield and flow of En25 steel, JSA 4 (1969), 2, 138-161.

[3.679] I. D. Rogozin, On the theory of slip (in Russian), DSS 4 (1970), 148-155.

[3.680] I. D. Rogozin, The Lode-Nadai diagram for the theory of slip (in Russian), DSS 7 (1971), 215-217.

[3.681] V. I. Rozenblyum, On the condition of full plasticity for a perfectly plastic medium (in Russian), Izv AN SSSR MTT (1969), 4, 116-122.

[3.682] -, On the theorem on unloading (in Russian), IUP 9 (1973), 121-128. 
[3.683] Ya. I. Rudayev, On the conditions of applicability of the theory of small elastic-plastic deformations to non-proportional loading (tension with torsion) of circular bars (in Russian), DNT (1970), 55-67.

[3.684] -, On monotonic deformation of thin-walled tubes (in Russian), $\operatorname{Tr}$ FPI 65 (1974), 103-108.

[3.685] K. N. Rusinko, On plastic deformations at simple and variable repeated loadings (in Russian), Izv AN KirgSSR (1970), 5, 20-28.

[3.686] -, A generalization of Cicala's formula (in Russian), Izv AN SSSR MTT (1971), 6, 37-44.

[3.687] K. N. Rusinko, E. I. Blinov, Analytical investigation of deformations for stepwise loading trajectories (in Russian), PM 7 (1971), 11, 52-58.

[3.688] K. N. Rusinko, V. I. Kuneyev, Investigation of a corner (in Russian), Tr FPI 56 (1972), $55-63$.

[3.689] K. N. Rusinko, A. E. Kalatinets, S. S. Dreval, Problems of the concept of slip in the theory of plasticity (in Russian), PM 10 (1974), 1, 3-19.

[3.690] B. A. Rytchkov, N. Yu. Shvayko, Limits of applicability of the deformation theory for broken loading trajectories of a linear anisotropically hardening medium (in Russian), Izv AN KirgSSR (1967), 2, 25-30.

[3.691] K. Saito, H. Igaki, M. Sugimoto, A study of the equivalent stress and the equivalent plastic strain rate, BJSME 15 (1972), 79, 33-39.

[3.692] G. D. Sandel, Uber die Festigkeitsbedingungen, Dissertation, TH Stuttgart 1919; Leipzig 1925.

[3.693] - $S B 95$ (1930), 335.

[3.694] J. L. Sanders, Plastic stress-strain relations based on linear loading functions, 2nd USNCAM (1954), 455-460.

[3.695] V. Ya. Sausin', The trace of fading memory at tension followed by a transverse compression (in Russian), MPol (1969), 4, 597-602.

[3.696] - The relation between auxiliary stress and strain vectors at successive radial loadings of a plastic material (in Russian), MPol (1970), 2, 241-245.

[3.697] A. Sawczuk, Linear theory of plasticity of anisotropic bodies and its applications to problems of limit analysis, AMS 11 (1959), 5, 541-556.

[3.698] A. Sawczuk, P. Stutz, Sur le critère d'écoulement et de la loi de déformation plastique, CRAS 266 (1968), 2, A87-A89.

[3.699] A. Sawczuk, W. J. O'Donnell, J. Porowski, Plastic analysis of perforated plates for orthotropic yield criteria, IJMS 17 (1975), 6, 411-417.

[3.700] M. Sayir, Zur Fließbedingungen der Plastizitätstheorie, IA 39 (1970), 6, 414-432.

[3.701] F. Schleicher, Der Spannungszustand an der Fliessgrenze, ZAMM 6 (1926), 199-216.

[3.702] E. Schmid, Yield point of crystals, critical shear stress law, 1st ICAM (1924), 342.

[3.703] E. Schmid, W. Boas, Kristallplastizität mit besonderer Berücksichtigung der Metalle, Springer, Berlin 1935; English translation: Plasticity of crystals, Chapman \& Hall, London 1950 and 1968 ,

[3.704] R. Schmidt, Uber den Zusammenhang von Spannungen und Formaenderungen im Verfestigungsgebiet, IA 3 (1932), 215-235.

[3.705] L. I. Sedov, Notions of various rates of tensors (in Russian), PMM 24 (1960), 3, 393-398.

[3.706] L. I. Sedov, M. E. Eglit, A construction of non-holonomic models of continua accounting for finite strains and some physico-chemical effects (in Russian), DAN SSSR 142 (1962), 1, 54-57.

[3.707] S. V. Serensen, On strength criteria for variable loadings in the case of plane and spatial stress states (in Russian), IS I/1 (1941), 3-12.

[3.708] B. R. Seth, Anisotropic plasticity (in Russian), PMTDT (1970), 419-425.

[3.709] -, Yield conditions in plasticity, AMS 24 (1972), 5-6, 769-776.

[3.710] M. J. Sewell, A plastic flow rule at a yield vertex, JMPS 22 (1974), 6, 469-490. 
[3.711] Yu. N. Shevtchenko, Unloading theorems in the theory of small elastic-plastic deformations in the case of non-uniform heating (in Russian), PM 2 (1966), 7, 11-21.

[3.712] -, On the theories of thermoplasticity of a hardening material (in Russian), TNEK 6 (1966), 5-22.

[3.713] - Theorems on simple variable loading and unloading in the theory of small elasticplastic deformations in the case of non-uniform heating (in Russian), TNEK 9 (1970), $148-165$.

[3.714] -, A deformation theory of thermoplasticity with kinematic hardening (in Russian), TNEK 10 (1970), 57-67.

[3.715] - Equations of thermoplasticity of irradiated materials (in Russian), TNEK 12 (1972), 39-52.

[3.716] Yu. N. Shevtchenko, R. G. Terekhov, Verification of a certain consequence of the postulate of isotropy (in Russian), PPro (1972), 2, 42-45.

[3.717] Yu. N. Shevtchenko, V. G. Savtchenko, R. G. Terekhov, A. I. Borisyuk, On the problem of applicability of the theory of small elastic-plastic deformations to the calculations of finite strains (in Russian), PPro (1972), 6, 3-6.

[3.718] Yu. N. Shevtchenko, R. G. Terekhov, Experimental verification of the hypothesis of existence of a thermomechanical surface in the theory of thermoplasticity (in Russian), TNEK 13 (1973), 43-47.

[3.719] - , - On the postulate of thermoplasticity (in Ukrainian), DAN URSR (1974), A7, 636-640.

[3.720] R. T. Shield, On Coulomb's law of failure in soils, JMPS 4 (1955), 1, 10-16.

[3.721] R. Shield, H. Ziegler, On Prager's hardening rule, ZAMP 9a (1958), 260-276.

[3.722] E. Shiratori, K. Ikegami, H. Okano, The influence of prestrain on subsequent yield surfaces, I, BJSME 11 (1968), 45, 413-418.

[3.723] E. Shiratori, K. Ikegami, Experimental study of the subsequent yield surface by using cross-shaped specimens, JMPS 16 (1968), 6, 373-394.

[3.724] —, -, Studies of the anisotropic yield condition, JMPS 17 (1969), 6, 473-491.

[3.725] E. Shiratori, K. Ikegami, K. Kaneko, The influence of the Bauschinger effect on the subsequent yield condition, BJSME 16 (1973), 100, 1482-1490.

[3.726],,,--- , The stress vector and the subsequent yield surface in loading along the strain path with a corner, BJSME 17 (1974), 113, 1405-1412.

[3.727] O. A. Shishmarev, Influence of the type of stress deviator on plastic deformation of steel (in Russian), IZh MTT (1966), 5, 112-118.

[3.728] - Experimental investigation of yield points of steel under simple and combined loading (in Russian), IZh MTT (1968), 2, 187-190.

[3.729] - Comparison of the results of the theory of plastic flow with experimental data for combined loading (in Russian), Izv VUZ M (1971), 1, 32-37.

[3.730] K. K. Shkerbelis, Influence of combined loading on the deformation of concrete (in Russian), IBZB 3 (1958), 57-60.

[3.731] H. P. Shrivastava, Z. Mróz, R. N. Dubey, Yield criterion and second-order effects in plane-stress, AMech 17 (1973), 1-2, 137-143.

[3.732] - , - - , Yield criterion and the hardening rule for a plastic solid, ZAMM 53 (1973), $10,625-633$.

[3.733] H. P. Shrivastava, R. N. Dubey, Yield condition and hardening rule for density varying materials, ZAMM 54 (1974), 9, 594-596.

[3.734] N. Yu. Shvayko, Yu. V. Klyshevitch, Approximation and generalization of stress ratestrain rate relations in the neighbourhood of a corner at the loading trajectory (in Russian), Izv AN KirgSSR (1968), 6, 21-29.

[3.735] N. Yu. Shvayko, Ya. I. Rudayev, On the limits of applicability of the deformation theory of plasticity in tension with torsion of circular bars (in Russian), Tr FPI 54 (1971), 145-152. 
[3.736] N. Yu. Shvayko, On a possible general form of the hardening function for a linear planeplastic medium (in Russian), GTU 14 (1972), 109-120.

[3.737] O. M. Sidebottom, Note on the effective plastic strain for a Tresca material, Trans ASME E38 (1971), 4, 1049-1050.

[3.738] F. Sidoroff, The geometrical concept of intermediate configuration and elastic-plastic finite strain, AMS 25 (1973), 2, 299-309.

[3.739] -, On the formulation of plasticity and viscoplasticity with internal variables, AMS 27 (1975), 5-6, 807-819.

[3.740] R. L. Sierakowski, A. Phillips, The effect of repeated loading on the yield surface, AMech 6 (1968), 2-3, 217-231.

[3.741] Yu. I. Sirotin, On the theory of perfect elastoplasticity of crystals (in Russian), Izv AN SSSR MTT (1970), 1, 39-47.

[3.742] G. A. Smirnov-Alyayev, Fundamentals of the mathematical theory of plasticity of a considerably deforming body (in Russian), STMM LGU 3 (1939).

[3.743] G. A. Smirnov-Alyayev, V. M. Rozenberg, Theory of plastic deformations of metals (in Russian), Mashgiz, Moskva-Leningrad 1956.

[3.744] G. F. Smith, On the yield condition for anisotropic materials, QAM 20 (1962), 3, 241-247.

[3.745] G. F. Smith, A. J. M. Spencer, A continuum theory of a plastic-rigid solid reinforced by two families of inextensible fibres, QJMAM 23 (1970), 4, 489-504.

[3.746] S. Smith, B. O. Almroth, An experimental investigation of plastic flow under biaxial stress, ExpM 10 (1970), 6, 217-224.

[3.747] Z. Sobotka, Základni vztahy přirůstkové theorie plasticity a nové pojeti plastického potenciálu pro anisotropické hmoty, Stav C 14 (1966), 6.

[3.748] —, Anisotropie, deviace a deviaćni uhel, Stav $C^{15}$ (1967), 6, 359-364.

[3.749] - Energetic yield condition for the plane deformation of the anisotropic materials, Stav C 15 (1967), 8, 435-452.

[3.750] -, Dvojparametricka kvadratickả podminka plasticity, Stav $\check{C} 15$ (1967), 8, 494-500.

[3.751] - , The cubic yield condition for incompressible bodies, AT ČSAV 12 (1967), 6, 830-832.

[3.752] - Energy yield conditions for anisotropic materials and for anisotropic Cosserat continua (in Russian), BKMSS 1966 (1968), 33-45.

[3.753] —, Theorie des plastischen Fliessens von anisotropen Körpern, ZAMM 49 (1969), 1-2, 25-32.

[3.754] - , Yield condition of the constant limiting distortion energy for anisotropic bodies, AT CSAV 14 (1969), 3, 352-365.

[3.755] - , The relations of the theory of plasticity for materials with different mechanical properties in tension and in compression, AT CSAV 16 (1971), 2, 320-324.

[3.756] -, The plastic flow of orthotropic materials with different mechanical properties in tension and in compression, $A T C \check{C}$ AV 16 (1971), 6, 772-776.

[3.757] -, Tensorial expansion for the plastic flow of partially compressible media, $A T$ CSAV 18 (1973), 1, 1-16.

[3.758] -, Plastické přetvařeni hmot s ruznými mechanickými vlastnostmi v tahu a $\mathrm{v}$ tlaku, StavC 21 (1973), 6-8, 403-417.

[3.759] -, Plasticity of anisotropic materials with positive and negative shear effects, StavC 21 (1973), 9, 684-687.

[3.760] -, Second-order plastic flow of isotropic partially compressible media, AT CSAV 20 (1975), 3, 301-321.

[3.761] V. V. Sokolovsky, Some remarks on linearization of the equations of plasticity (in Russian), PMM 25 (1961), 5, 931-932.

[3.762] A. J. M. Spencer, Deformations of fibre-reinforced materials, Clarendon Press, Oxford 1972. 
[3.763] A. J. M. Spencer, J. E. Ferrier, Some solutions for a class of plastic-elastic solids, SFP 1972 (1974), 9-24.

[3.764] F. Stassi D'Alia, Un paraboloide di rivoluzione quale condizione di plasticità, Ing (1951).

[3.765] - , La condizione di plasticità secondo recenti ricerche, Rendiconti del corso Costruzioni Milano (1960), 60-71.

[3.766] - Flow and fracture of materials according to a new limiting condition of yielding, $\mathrm{Mec} 2$ (1967), 3, 178-195.

[3.767] - , Limiting conditions of yielding for anisotropic materials, Mec 4 (1969), 4, 349-363.

[3.768] F. Stassi, Flow and fracture of metals under combined stress (in Russian), PPro (1973), 5, 32-40.

[3.769] F. D. Stockton, D. C. Drucker, On fitting mathematical theories of plasticity to experithental results, JCS 5 (1950), 239-247.

[3.770] F. D. Stockton, Experimental evidence of nonlinearity in plastic stress-strain relations, Techn. Rep. 88, Brown Univ. 1953.

[3.771] M. Stodulski, Dwuparametrowa rodzina obszarów plastycznego przystosowania się pręta rozciąganego i skręcanego, (in print).

[3.772] P. Stutz, Remarque sur l'écoulement plastique dans le cas d'une relation tensorielle linéaire entre contrainte et vitesse de deformation, CRAS 265 (1967), 16, A481-A484.

[3.773] -, Remarque sur l'écoulement plastique dans le cas d'une relation tensorielle non linéaire entre contraintes et vitesse de deformation, CRAS 266 (1968), 23, A1149-A1152.

[3.774] M. Sugimoto, H. Igaki, K. Saito, Equivalent stress (rate) and equivalent strain rate for work-hardening materials (theory of anisotropic plasticity based on maximum shear stress hypothesis), BJSME 16 (1973), 102, 1857-1866.

[3.775] N. G. Suturina, On the form of subsequent yield surface of steel (in Russian), IUP 6 (1967), 156-160.

[3.776] N. L. Svensson, Anisotropy and the Bauschinger effect in cold rolled aluminium, JMES 8 (1966), 2, 162-172.

[3.777] N. G. Sverdlova, On the changes of yield surface in the case of arbitrary direction of loading (in Russian), IUP 7 (1968), 144-147.

[3.778] J. L. Swedlow, Character of the equations of elasto-plastic flow in three independent variables, IJNLM 3 (1968), 3, 325-336.

[3.779] W. Szczepiński, Experimental investigations of behaviour of the yield surface of metals under combined loading (in Russian), BKMSS 1966 (1968), 169-179.

[3.780] W. Szczepiński, J. Miastkowski, An experimental study of the effect of the prestraining history on the yield surfaces of an aluminium alloy, JMPS 16 (1968), 3, 153-162.

[3.781] W. Szczepiński, K. Turski, On the lines of influence of plastic deformation, AMS 21 (1969), 1, 49-57.

[3.782] W. Szczepiński, Stany graniczne i kinematyka ośrodków sypkich, IPPT PAN - PWN, Warszawa 1974.

[3.783] G. B. Talypov, V. N. Kamentsev, Investigation of yield-point stresses and of some other effects in the case of combined loading (in Russian), UZMU 280 (1960).

[3.784] G. B. Talypov, Yield-point stresses and fracture of low-carbon steel in the case of simple and of combined loading: the influence of aging (in Russian), Izv AN SSSR MM (1961), $6,125-130$.

[3.785] -, On the theory of plasticity of an initially isotropic hardening material (in Russian), IUP 5 (1966), 198-203.

[3.786] G. B. Talypov, Variation of the yield surface of an initially isotropic metal during the process of plastic deformation (in Russian), PMTDT (1970), 445-451.

[3.787] M. Tanaka, Y. Miyagawa, On generalized kinematic hardening theory of plasticity, IA 44 (1975), 4, 255-268. 
[3.788] M. Tanaka, Elastoplastic constitutive laws based on combined isotropic and kinematic hardening, TROU 25 (1975), 1230-1253.

[3.789] I. I. Tarasenko, Yield conditions and strength conditions of metals (in Russian), SNT LISI 23 (1956), 102-113.

[3.790] G. I. Taylor, C. F. Elam, Distortion of an aluminum crystal during a tensile test, Proc RS A102 (1923), 643.

[3.791] G. I. Taylor, H. Quinney, The plastic distortion of metals, PhTRS A230 (1931), 323-362.

[3.792] G. I. Taylor, Plastic strain in metals, JIM 62 (1938), 306-324.

[3.793] - A connection between the criterion of yield and the strain-ratio relationship in plastic solids, Proc RS A191 (1947), 441-446.

[3.794] C. Teodosiu, A dynamic theory of dislocations and its applications to the theory of the elastic-plastic continuum, FADT (1970), 837-876.

[3.795] R. G. Terekhov, Verification of the postulate of isotropy in the case of combined loading with changing principal directions of the stress tensor (in Russian), PM 6 (1970), 10, 89-92.

[3.796] - , Investigations of a thermomechanical surface in the theory of thermoplasticity in the case of unloading (in Ukrainian), DAN URSR A (1973), 5, 471-474.

[3.797] G. A. Teters, A variant of the theory of locality (in Russian), MPol (1967), 5, 800-802.

[3.798] G. A. Teters, Combined loading and stability of shells made of polymeric materials (in Russian), Zinatne, Riga 1969.

[3.799] P. S. Theocaris, C. R. Hazell, Experimental investigation of subsequent yield surfaces using the moiré method, JMPS 13 (1965), 5, 281-294.

[3.800] T. Y. Thomas, Interdependence of the yield condition and the stress-strain relation for plastic flow, Proc NAS 40 (1954), 7, 593-597.

[3.801] —, Combined elastic and Mises' stress-strain relations, Proc NAS 41 (1955), 908-910.

[3.802] —, Combined elastic and Prandtl-Reuss' stress-strain relations, Proc NAS 41 (1955), 720726.

[3.803] -, Velocity of dislocations in crystals, JMM 18 (1968), 6, 571-584.

[3.804] T. Tokuoka, Generalized Prandtl-Reuss materials, I-III, MFEKU 33 (1971), 3, 186-219.

[3.805] -, Yield conditions and flow rules derived from hypo-elasticity, ARMA 42 (1971), 4, 239-252.

[3.806] —, Constitutive equations of thermo-plastic materials, Trans JSASS 16 (1973), 31, 51-59.

[3.807] - Fundamental relations of plasticity derived from hypo-elasticity, SFP 1972 (1974), 1-8.

[3.808] L. A. Tolokonnikov, V. V. Shevelev, S. P. Yakovlev, Experimental verification of the equations of plastic flow for an anisotropic body (in Russian), PM 4 (1968), 2, 16-19.

[3.809] L. A. Tolokonnikov, S. P. Yakovlev, On the formulation of the yield condition and associated flow rule for an anisotropic body (in Russian), Izv VUZ M 7 (1969), 17-20.

[3.810] L. A. Tolokonnikov, S. P. Yakovlev, V. M. Lyalin, Plastic flow of orthotropic bodies (in Russian), $P M 7$ (1971), 6, 11-16.

[3.811] L. A. Tolokonnikov, N, M. Matchenko, On visualization of equations of limit states for initially anisotropic bodies (in Russian), PPro (1974), 3, 54-56.

[3.812] C. Torre, Einfluss der mittleren Hauptnormalspannung auf die Fliess- und Bruchgrenze, ÖIA 1 (1947), 4-5, 316-342.

[3.813] —, Die Grenzzustände stațisch beanspruchter Stoffe, SAAWT 15 (1949), 116-145.

[3.814] —, Die Mechanik der Grenzbeanspruchungen, ÖIA 4 (1950), 1, 93-108.

[3.815] - , Grenzbedingungen für spröden Bruch und plastisches Verhalten bildsamer Metalle, ÖIA 4 (1950), 2, 174-189.

[3.816] D. Trifan, Stress theory of plastic flow, JMP 35 (1956), 1, 44-51.

[3.817] A. Troost, J. Betten, Plastische Querzahlen anisotroper Verkstoffe, AE 43 (1972), 11, 811-812. 
[3.818] A. Troost, J. Betten, Zur Frage der Konvexität von Fliessbedingungen bei plastischer Inkompressibilität und Kompressibilităt, MRC 1 (1974), 2, 73-78.

[3.819] C. Truesdell, Hypo-elasticity, JRMA 5 (1955), 1, 83-133.

[3.820] S. W. Tsai, E. M. Wu, A general theory of strength for anisotropic materials, JCM 5 (1971), 1, 58-80.

[3.821] K. Turski, Badanie wpływu odkształcenia plastycznego na zachowanie się metalu przy różnych drogach wtórnego obciążenia, MTS 9 (1971), 1, 155-199.

[3.822] —, Yield criterion for a prestrained material, BAP SST 20 (1972), 10, 795-800.

[3.823] A. G. Ugodchikov, Yu. G. Korotkikh, Constitutive equations of nonisothermal elasticplastic deformation and methods of their practical application, SFP 1972 (1974), 535-549.

[3.824] A. A. Vakulenko, On the relations between stresses and strains in inelastic media, (in Russian), DAN SSSR 118 (1958), 665-668.

[3.825] —, Thermodynamic investigation of stress-strain relations in isotropic elastic-plastic media (in Russian), DAN SSSR 126 (1959), 736-739.

[3.826] - , On stress-strain relations in isotropic and initially isotropic inelastic media (in Russian), IUP 2 (1963), 3-46.

[3.827] A. A. Vakulenko, I. Z. Palley, On the theory of plasticity for a medium subject to deformation at variable temperatures (in Russian), IUP 5 (1966), 188-197.

[3.828] A. A. Vakulenko, M. L. Kachanov, "Microstresses" in the theory of plasticity, PMTDT (1970), 99-103.

[3.829] A. A. Vakulenko, L. M. Kachanov, Theory of plasticity (in Russian), a survey in the volume Mechanics in USSR during 50 years (1972), vol. 3, 79-118.

[3.830] K. C. Valanis, On the thermodynamic foundation of classical plasticity, AMech 9 (1970), 3-4, 278-291.

[3.831] - , A theory of viscoplasticity without a yield surface, AMS 23 (1971), 4, 517-551.

[3.832] —, Observed plastic behavior of metals vis-a-vis the endochronic theory of plasticity, SFP 1972 (1974), 235-255.

[3.833] R. A. Vasin, On the form of hardening function in the flow theory (in Russian), $P M 3$ (1967), 7, 60-64.

[3.834] -, Some problems of stress-strain relations in the case of combined loading (in Russian), UN 1 (1971), 59-126.

[3.835] R. A. Vasin, A. B. Ibragimov, On the investigation of deformational anisotropy in the case of combined loading (in Russian), PP (1971), 126-129.

[3.836] S. D. Volkov, A statistical failure theory (in Russian), Mashgiz, Moskva-Sverdlovsk 1960.

[3.837] R. G. Vos, W. H. Armstrong, Improved hardening theory for cyclic plasticity, AIAA 11 (1973), 3, 400-401.

[3.838] Y.S. Wang, A simplified theory of the constitutive equations of metal plasticity at finite deformation, Trans ASME E40 (1973), 4, 941-947.

[3.839] W. Warkenthin, Beitrag zur Formulierung der Sicherheitsnachweise gegen Fließen und Dauerbruch bei mehrachsigen Spannungszuständen, Sch 20 (1970), 3, 119-122.

[3.840] W. A. Warner, G. H. Handelman, Modified incremental strain law for work hardening materials, QJMAM 9 (1956), 3, 279-293.

[3.841] Z. Waszczyszyn, M. Życzkowski, Some applications in mechanics of approximate formulae for real roots of a cubic equation, BAP SST 14 (1966), 3, 149-158.

[3.842] F. C. Weiler, Anisotror ì, elastic-plastic-thermal stress analyses of solid structures, 1st SMiRT (1971), paper D3-3.

[3.843] C. H. Wells, P. R. Paslay, A small-strain plasticity theory for planar slip materials, Trans ASME E36 (1969), 1, 15-21.

[3.844] G. N. White, D. C. Drucker, Effective stress and effective strain in relation to stress theories of plasticity, JAP 21 (1950), 10, 1013-1021. 
[3.845] J. F. Williams, N. L. Svensson, Effect of tensile prestrain on yield locus of 1100-F aluminium, JSA 5 (1970), 128.

[3.846] - , - A rationally based yield criterion for work hardening materials, Mec 6 (1971), 2, 104-114.

[3.847] J. C. Wilson, R. G. Berggren, Effect of neutron irradiation on steel, Proc ASTM 55 (1955).

[3.848] J. Woodthorpe, R. Pearce, The anomalous behaviour of aluminium sheet under balanced biaxial tension, IJMS 12 (1970), 4, 341-347.

[3.849] Yu. I. Yagn, New methods of the strength calculations (in Russian), VIT (1931), 6.

[3.850] Yu. I. Yagn, O. A. Shishmarev, Some experimental data concerning elastic limits of plastically prestrained nickel specimens (in Russian), DAN SSSR 119 (1958), 1, 46-48.

[3.851] Yu. I. Yagn, L. V. Grebnev, Influence of the rotation of principal stress axes on flow and fracture of metals (in Russian), $\operatorname{Tr}$ LPI 278 (1967), 66-69.

[3.852] S. P. Yakovlev, V. F. Kuzin, Plane plastic flow of an anisotropic body (in Russian), PM 5 (1969), 11, 127-130.

[3.853] R. N. Yong, E. McKyes, V. Silvestri, Yield and failure of clays, AMS 24 (1972), 3, 511528.

[3.854] Y. Yoshimura, Comment on the slip theory of Batdorf and Budiansky, BJSME 1 (1958), 2, 109-113.

[3.855] Y. Yoshimura, Y. Takenaka, Strain history effects in plastic deformation of metals, IUTAM SOE (1962), 729.

[3.856] L. B. Zagovaylov, N. Yu. Shvayko, On the problem of stress-strain relations in the neighbourhood of a corner at the loading trajectory (in Russian), GTU 16 (1973), 134-142.

[3.857] V. V. Zakharov, A strength criterion for layered masses (in Russian), PlastM (1961), 8.

[3.858] V. S. Zarubin, A. A. Polyakov, A model of polycrystalline material under non-isothermal loading (in Russian), TNEK 8 (1969), 249-256.

[3.859] - , - A model of non-isothermal plastic deformation of a polycrystalline material (in Russian), TNEK 9 (1970), 253-266.

[3.860] - , - Non-isothermal plasticity of a polycrystalline hardening material (in Russian), TNEK 10 (1970), 189-194.

[3.861] V.S. Zarubin, A. A. Polyakov, Application of a computational model of plastic defors mation of a polycrystalline material to the analysis of transformation of the loading surface (in Russian), TNEK 12 (1972), 68-71.

[3.862] J. Zawadzki, Ciśnienie zredukowane jako jeden z parametrów wytężenia, RI 5 (1957), 3, 357-398.

[3.863] A. M. Zhukov, Strength and plasticity of the alloy D16T in the case of combined stresses (in Russian), Izv AN SSSR OTN (1954), 6, 61-70.

[3.864] - Plastic properties and fracture of steel subject to biaxial stress state (in Russian), IS 20 (1954), 37-48.

[3.865] -, Plastic deformation of steel in the case of combined loading (in Russian), Izv $A N$ SSSR OTN (1954), 11, 53-61.

[3.866] - Combined loading and the theories of plasticity of isotropic materiais (in Russian), Izv AN SSSR OTN (1955), 8, 89-92.

[3.867] -, Certain peculiarities of the neutral loading curve (in Russian), Izv AN SSSR OTN (1958), 8, 32-40.

[3.868] -, Plastic deformations of the alloy AKG in the case of simple and combined loadings (in Russsian), $R P 12$ (1966), 260-289.

[3.869] A. G. Zhuravlev, On the criterion of simple loading in a non-homogeneous medium (in Russian), VMU MM (1962), 6, 39-42.

[3.870] H. Ziegler, A modification of Prager's hardening rule, QAM 17 (1959), 1, 55-65.

[3.871] —, Bemerkung zu einem Hauptachsenproblem in der Plastizitätsheorie, ZAMP 11 (1960), 2, 
[3.872] H. Ziegler, Plastizität ohne Thermodynamik, ZAMP 21 (1970), 5, 798-805.

[3.873] H. Ziegler, J. Nänni, Ch. Wehrli, Zur Konvexität der Fliessfläche, ZAMP 24 (1973), 1, $140-144$.

[3.874] - , - -, Zur Konvexität der Dissipationsflächen, ZAMP 25 (1974), 1, 76-82.

[3.875] O. C. Zienkiewicz, The finite element method in engineering science, McGraw-Hill, London (1971).

[3.876] A. F. Zilauts, On mathematical foundations of the theory of local deformations (in Russian), Izv AN LatvSSR (1965), 3, 84-89.

[3.877] M. Życzkowski, On the accuracy of approximations of the Huber yield condition, AMS 26 (1974), 1, 65-79.

[3.878] - Eine dreifache Analogie für kinematische Verfestigung von plastischen Körpern, Festschrift Alf Pflüger, Hannover 1977, 289-294.

\section{Chapter IV}

[4.1] H. Abé, Finite deformation theory for the stretching of a thin elastic-plastic plate of finite thickness, TRTU 38 (1973), 2, 539-564; IJSS 11 (1975), 9, 999-1010.

[4.2] L. G. Afendik, Elastic-plastic bending of thin-walled tubes with large curvature (in Russian), PM 4 (1968), 6, 45-51.

[4.3] L. G. Afendik, M. F. Kolesnitchenko, Experimental investigation of elastic-plastic bending of thin-walled tubes (in Russian), VLPI (1968), 25, 135-141.

[4.4] A. C. Aitken, Studies in practical mathematics II, The evaluation of the latent roots and latent vectors of a matrix, Proc RSE 57 (1937), 269-304.

[4.5] I. S. Akhmedyanov, A. V. Kireyev, Integration of differential equations of elastic-plastic bending of a spherical shell under arbitrary loading (in Russian), $\operatorname{Tr} K A I 63$ (1972), 33-39.

[4.6] D. N. de G. Allen, R. V. Southwell, Relaxation methods applied to engineering problems, XIV, Plastic straining of two-dimensional stress-systems, PhTRS A202 (1950), 379-414.

[4.7] S. C. Anand, S. L. Lee, E. C. Rossow, Finite element analysis of elastic-plastic plane stress problems based upon Tresca yield criterion, IA 39 (1970), 2, 73-86.

[4.8] I. N. Ananev, Yu. I. Nyashin, A comparison of certain methods of stress analysis in the plastic range using the finite element technique (in Russian), SNT PPI 138 (1973), 3-10.

[4.9] J. H. Argyris, Elastoplastic matrix displacement analysis of three-dimensional continua, JRAS 69 (1965), 633-636.

[4.10] -, Elasto-plastic analysis of three-dimensional media, AT ASH 54 (1966), 1-2, 219-237.

[4.11] J. H. Argyris, D. W. Scharpf, J. B. Spooner, The elasto-plastic calculation of general structures and continua, 3rd CDSC (1968), 345-384.

[4.12] - , - , - Die elastoplastische Berechnung von allgemeinen Tragwerken und Kontinua, IA 37 (1969), 5, 326-352.

[4.13] J. H. Argyris, A. S. L. Chan, Applications of finite elements in space and time, IA 41 (1972), 4, 235-257; Static and dynamic elasto-plastic analysis by the method of finite elements in space and time, SFP 1972 (1973), 147-175.

[4.14] H. Armen, G. Isakson, A. Pifko, Discrete element methods for the plastic analysis of structures subjected to cyclic loading, IJNME 2 (1970), 189-206.

[4.15] J.-L. Auriault, J. Kravtchenko, R. Negre, R. Sibille, Etude des singularites du champ statique en plasticité coulombienne et leurs applications, PRMS 1974 (1977), 17-67.

[4.16] V. Ya. Bachinsky, I. N. Tkachenko, On the limit state of a bar made of a Prandtl material with limited deformability (in Russian), SK RMNTS 20 (1972), 73-75.

[4.17] J. F. Baker, M. R. Horne, J. Heyman, The steel skeleton, vol. II, Cambridge Univ. Press 1956.

[4.18] Yu. F. Balandin, Thermal fatigue of metals (in Russian), Sudostroyenye, Leningrad 1967.

[4.19] H. Balmer, J. S. Doltsinis, M. König, Elastoplastic and creep analysis with the ASKA program system, CMAME 3 (1974), 87-104. 
[4.20] N. V. Banichuk, Calculation of loading of an elastic-plastic body (in Russian), Izv AN SSSR MTT (1969), 1, 128-135.

[4.21] G. Bartelds, The application of the finite element displacement method to problems of elastoplastic deformation, CTAS (1972), 99-115.

[4.22] V. N. Bastun, On the influence of the geometrical form of a structure on its carrying capacity (in Russian), PM 9 (1973), 8, 57-63.

[4.23] H. Baumgärtel, Endlich-dimensionale analytische Störungstheorie, Akademie-Verlag, Berlin 1972.

[4.24] J. Bäcklund, H. Wennerström, Finite element analysis of elasto-plastic shells, IJNME 8 (1974), 2, 415-424.

[4.25] L. S. Beedle, Plastic design of steel frames, Wiley, New York 1958.

[4.26] L. M. Belenky, On the estimation of carrying capacity of frames with geometric non-linearity taken into account (in Russian), Izv VUZM 7 (1969), 9-12.

[4.27] A. R. Belyakov, O. F. Chernyavsky, On a certain mechanism of cumulation of plastic deformation in the conditions of combined cyclic loading (in Russian), SNT CPI 151 (1974), 56-66.

[4.28] T. Belytschko, M. Velebit, Finite element method for elastic plastic plates, Proc ASCE JEMD 98 (1972), 1, 227-242.

[4.29] T. Belytschko, Plane stress shakedown analysis by finite elements, IJMS 14 (1972), 9. 619-625.

[4.30] J. F. Besseling, The complete analogy between the matrix equations and the continuous field equations of structural analysis, Proc. Int. Symp. Analogue and Digital Techn. Appl. to Aeron., Liège 1963.

[4.31] J. Betten, Zum Traglastverfahren bei nichtlinearem Stoffgesetz, IA 44 (1975), 3, 199-207.

[4.32] P. P. Bijlaard, Theory of local plastic deformations, Mem AIPC 6 (1940/41), 27-44.

[4.33] I. A. Birger, Certain general methods of solution of problems of plasticity theory (in Russian), PMM 15 (1951), 6, 765-770.

[4.34] -, Method of supplementary strains in the problems of plasticity theory (in Russian), Izv AN SSSR MM (1963), 1, 48-56.

[4.35] -, Methods of elastic solutions in the theory of plastic flow (in Russian), Izv AN SSSR MM (1964), 2, 116-118.

[4.36] -, Structural analysis with plasticity and creep taken into account (in Russian), Izv AN SSSR Mekh (1965), 2, 113-119.

[4.37] - , General computational algorithms for problems of the theory of elasticity, plasticity and creep (in Russian), UMDS (1975), 51-73.

[4.38] A. Biron, P. G. Hodge, Jr., Nonlinear programming method for limit analysis of rotationally symmetric shells, IJNME 3 (1968), 2, 201-213.

[4.39] A. Biron, U. S. Chawla, Numerical method for limit analysis of rotationally symmetric shells, BAP SST 18 (1970), 3, 185-192.

[4.40] J. F. W. Bishop, On the complete solution to problems of deformation of a plastic-rigid material, JMPS 2 (1953), 43-53.

[4.41] H. Bleich, UUber die Bemessung statisch unbestimmter Stahltragwerke unter Berücksichtigung der elastisch-plastischen Verhaltens des Baustoffes, BI 13 (1932), 19/20, 261.

[4.42] V. I. Blokh, Stress functions in the theory of elasticity (in Russian), PMM 14 (1950), 4, 415-422.

[4.43] —, Theory of elasticity, (in Russian), Izd. Kharkovsk. Univ., Kharkov 1964.

[4.44] V. V. Bokov, E. A. Sidorov, On the convergence of the method of variable parameters of elasticity (in Russian), UZGU 113 (1972), 143-147.

[4.45] A. Borkauskas, A. Cyras, On the duality in limit analysis and design of plates, BAP SST 16 (1968), 6, 241-248.

[4.46] A. E. Borkauskas, R. P. Karkauskas, Computational aspects of application of mathemat- 
ical programming methods to limit analysis of plates and shells (in Russian), IMS (1971), 2, 47-54.

[4.47] P. B. Bowden, A criterion for inhomogeneous plastic deformation, PhM 22 (1970), 177, 455-462.

[4.48] L. L. Boyko, On the method of elastic solutions in the theory of plasticity (in Russian), VMU MM (1970), 5, 108-116.

[4.49] -, On two methods of solution of an elastic-plastic problem (in Russian), VMU $M M$ (1971), 3, 102-109.

[4.50] E. F. Boyle, A. Jennings, Accelerating the convergence of elastic-plastic stress analysis, IJNME 7 (1973), 2, 232-235.

[4.51] P. W. Bridgman, The stress distribution at the neck of a tension specimen, Trans ASM 32 (1944), 553.

[4.52] G. L. Brovko, V. S. Lensky, On convergence of the method of homogeneous linear approximations in the theory of plasticity of non-homogeneous bodies (in Russian), PMM 36 (1972), 3, 519-527.

[4.53] E. P. Brumfitt, J. Schroeder, A variational principle in stress-rate dependent thermoplasticity, 3rd CANCAM (1971), 177-178.

[4.54] R. Brzeziński, J. A. König, Evaluation of shakedown deflections of framed structures by linear programming, SPAS (1972), 101-116.

[4.55] - , -, Deflections of elastic-plastic framed structures at shakedown, JSM 2 (1973), 211228.

[4.56] D. L. Bykov, V. A. Shatchnev, On a certain generalization of the method of elastic solutions (in Russian), PMM 33 (1969), 2, 290-298.

[4.57] D. L. Bykov, On certain methods of solution of plasticity problems (in Russian), UN 4 (1975), 119-139.

[4.58] C. R. Calladine, Limit analysis of curved tubes, JMES 16 (1974), 2, 85-87.

[4.59] M. Capurso, On the incremental solution of elasto-plastic continua in the range of large displacements, Mec 5 (1970), 2, 98-106.

[4.60] M. Capurso, G. Maier, Incremental elastoplastic analysis and quadratic optimization, Mec 5 (1970), 2, 107-116.

[4.61] M. Capurso, Limit analysis of continuous media with piecewise linear yield condition, Mec 6 (1971), 1, 53-58.

[4.62] - A displacement bounding principle in shakedown of structures subjected to cyclic loads, IJSS 10 (1974), 1, 77-92.

[4.63] G. Ceradini, C. Gavarini, Calcolo a rottura e programmazione lineare, GGC 103 (1965), 1-2, 48-64; 106 (1968), 2-3, 125-137; 106 (1968), 4, 163-180.

[4.64] G. Ceradini, A maximum principle for the analysis of elastic plastic systems, $\operatorname{Mec} 1$ (1966), 3-4, 77-82.

[4.65] —, Sull'adattamento dei corpi elasto-plastici soggetti ad azioni dinamiche, GGC 107 (1969), 4-5, 239-250.

[4.66] A. Charnes, H. Greenberg, Plastic collapse and linear programming, BAMS 57 (1951), 6.

[4.67] A. Charnes, C. E. Lemke, O. C. Zienkiewicz, Virtual work, linear programming and plastic limit analysis, Proc RS 251A (1959), 110-116.

[4.68] W. H. Chen, Necking of a bar, IJSS 7 (1971), 7, 685-717.

[4.69] G. P. Cherepanov, Elastic-plastic problems (in Russian), IZh MTT (1969), 2, 82-91.

[4.70] —, Mechanics of brittle fracture (in Russian), Nauka, Moskva 1974.

[4.71] L. F. Chernous'ko, N. V. Banichuk, Variational methods in mechanics and automatic control (in Russian), Nauka, Moskva 1973.

[4.72] V. I. Chernov, O. N. Ivanchenko, V. P. Bardzilovsky, On the method of successive approximations in elastic-plastic analysis (in Russian), PM 11 (1975), 3, 120-123.

[4.73] O. F. Chernyavsky, On the solution of problems of limit analysis and of shakedown by 
means of the Pontryagin maximum principle (in Russian), Izv AN SSSR MTT (1970), 4, 99-105.

[4.74] E. Chianese, Collasso incrementale localizzato per materiali non omogenei, RASFM 36 (1969/70), 145-166.

[4.75] A. N. Chizhevsky, On acceleration of convergence of the method of elastic solutions in physically nonlinear problems (in Russian), PP (1971), 188-193.

[4.76] J. Christoffersen, A note on necking of cylindrical bars, DCAMM 58 (1973), $10 \mathrm{pp.}$

[4.77] E. Chwalla, Die Stabilität zentrisch und exzentrisch gedrückter Stäbe aus Baustahl, SBAWW (1928).

[4.78] - , Theorie des aussermittig gedrückten Stabes aus Baustahl, Stb 7 (1934), 161.

[4.79] R. W. Clough, The finite element method in plane stress analysis, Proc ASCE JSD (1960), 345-378.

[4.80] M. G. Cockroft, A. Latham, A simple criterion of fracture for ductile metals, Rep NELG (1966).

[4.81] L. F. Coffin, Study of the effects of cyclic thermal stresses on a ductile metal, Trans ASME 76 (1954), 931-950.

[4.82] —, The stability of metals under cyclic plastic strain, Paper ASME A100 (1959), 12 pp.

[4.83] - Failure of structural metals subjected to strain-cycling conditions, Trans ASME D81 (1959), 208.

[4.84] M. Z. Cohn, S. K. Ghosh, S. R. Parimi, Unified approach to theory of plastic structures, Proc ASCE JEMD 98 (1972), 5, 1133-1158.

[4.85] I. F. Collins, The upper bound theorem for rigid/plastic solids generalized to include Coulomb friction, JMPS 17 (1969), 5, 323-338.

[4.86] G. Colonnetti, Sul problema delle coazioni elastiche, RAL 27 (1918), Ser. 5a.

[4.87] -, De l'équilibre des systemes élastiques dans lesquels se produisent des déformations plastiques, JMPA Ser. IX, 17 (1938), 233-255.

[4.88] —, Elastic equilibrium in the presence of permanent set, QAM 7 (1950), 353.

[4.89] —, L'équilibre des corps déformables, Dunod, Paris 1955.

[4.90] L. Comtet, Advanced combinatorics, D. Reidel, Dordrecht-Boston 1974.

[4.91] R. M. Cooper, G. A. Shifrin, An experiment on circular plates in the plastic range, 2nd US NCAM (1954), 527-534.

[4.92] L. Corradi, G. Maier, Inadaptation theorems in the dynamics of elastic-work-hardening structure, IA 43 (1973), 1, 44-57.

[4.93] - , - Dynamic non-shakedown theorem for elastic perfectly-plastic continua, JMPS 22 (1974), 5, 401-413.

[4.94] L. Corradi, A. Zavelani, A linear programming approach to shakedown analysis of structures, CMAME 3 (1974), 1, 37-53.

[4.95] L. Corradi, G. Maier, Extremum theorems for large displacement analysis of discrete elastoplastic structures with piecewise linear yield surfaces, JOTA 15 (1975), 1, 51-67.

[4.96] G. R. Cowper, E. T. Onat, The initiation of necking and buckling in plane plastic flow, 4th US NCAM (1962), vol. 2, 1023-1029.

[4.97] H. Craemer, Fallacies and paradoxa in the plasticity theory, AT ASH 10 (1955), 1-2, 73-82.

[4.98] J. G. Crose, A. H. S. Ang, Nonlinear analysis method for circular plates, Proc ASCE JEMD 95 (1969), 4, 979-999.

[4.99] A. A. Cyras, Linear programming methods in the analysis of elastic-plastic systems (in Russian), Stroyizdat, Moskva 1969.

[4.100] - , Theory of optimization in the limit analysis of a deformable solid (in Russian), Mintis, Vilnius 1971.

[4.101] J. Datsko, C. T. Yang, Correlation of bendability of materials with their tensile properties, Trans ASME B82 (1960), 4; 309-313.

[4.102] J. Datsko, Material properties and manufacturing process, New York 1966. 
Fourier, J. B. J., 289, 292, 377, 378, 498, 516, 536

Fox, E. N., 136, 198, 233

Fox, J. D., 537

Franciosi, V., 268, 399, 400, 403, 480

Frankland, J. M., 306

Franzen, W. E., 523

Frederking, R. M. W., 93, 202

Freiberger, W., 346, 434, 536

Freudenthal, A. M., 28, 35, 39, 46, 51, 100, 103, $136,171,181,230$

Freund, L. B., 198

Fridman, L. I., 482, 528

Fridman, Ya. B., 107

Friedman, E., 427, 441, 534

Fritzsche, J., 249, 295

Frolov, A. N., 440

Fromm, H., 88, 95

Fukuda, H., 168, 188

Fukumoto, Y., 480, 481

Fokuoka, H., 113, 202, 357, 502

Fung, P. K., 95

Fung, Y. C., 145

Furuta, H., 335

Gabbasov, R. F., 434

Gabibzade, S. M., 536

Gabryszewski, Z., 205

Gajewski, A., 58

Gajewski, H., 265, 289

Galambos, T. V., 251, 316, 479, 481, 536

Galileo, G., 103, 107, 244, 434, 435, 460

Galin, L. A., 292, 295, 526

Gallagher, R. H., 284, 288

Galli, A., 399, 400

Galos, M., 215, 273, 295, 342, 343, 366, 495

Galptchian, P. V., 383

Gandolfi, A., 423, 437, 535

Ganov, E. V , 112

Garbaccio, D. H., 337

Garg, V. K., 529

Gassmann, H., 523

Gatewood, B. E., 482

Gaudel, G., 245

Gauss, K., 278, 417, 457

Gavarini, C., 250, 290, 291, 479

Gavrilenkov, S. V., 215

Gavrilov, D. A., 59

Gavrilov, M. P., 357

Gaydon, F. A., 356, 383, 527

Gehring, R. W., 482
Geiringer, H., 28, 29, 39, 51, 100, 103, 136, 176, 253,281

Gemmerling, A. V., 311, 322, 385

Gemmerling, G. A., 124

Genev, G. A., 102, 103

Gent, A. R., 338

Geogdzhayev, V. O., 113, 201, 203, 204, 222

Gerard, G., 73

Gerdeen, J. C., 438, 440, 532

Gerij, J., 285

Germain, P., 198

Gerstle, K. H., 479

Gesund, H., 459

Ghamian, M. M., 479

Ghani, A. F. M., 479

Ghosh, S. K., 291

Giangreco, E., 335, 403

Giannelli, G., 335

Gill, S. S., 139, 250, 291, 322, 385, 408, 495, 502

Girkmann, K., 304, 388, 397, 435

Gjelsvik, A., 269

Glasstone, S., 500

Gleyzal, A., 49

Goel, R. P., 357

Gokhfeld, D. A., 147, 250, 291, 311, 434, 441, $482,499,500,528,533,534,538$

Goldenblat, I. I., 27, 34, 44, 46, 50, 95, 97, 107 , 113, 114, 200, 203, 204

Goldner, D., 333

Goldshteyn, Yu. B., 403

Goloborodko, S. A., 457

Golushkevitch, S. S., 222

Gontcharov, G. I., 250

Goode, C. D., 384

Gorb, M. L., 169, 216

Gorbunov, B N., 249, 341, 342

Gorelov, V. N., 354

Gorlatch, V. A., 440, 455

Gorodtsov, V. A., 147

Goryachev, A. P., 285

Goschy, B., 400

Goss, C., 165

Gou, P. F., 100, 181

Gouzou, J., 97, 183, 245

Goyal, S. C., 334, 400

Gray, G. A., 170, 501

Grebnev, L. V., 138, 139, 143

Grechukho, I. G., 478

Green, A. E., 27, 46, 50, 65, 88, 95, 124, 126, 197, 198, 205

Green, A. P., 399-403 
Green, G., 41

Green, R. J., 184

Greenbaum, G. A., 285

Greenberg, H. J., 86, 208, 264, 265, 271, 272, 290,479

Greenstreet, W. L., 73, 195

Greste, O. I., 384

Grierson, D. E., 480

Griffin, D. S., 215

Griffith, A. A., 246

Grigan, O. A., 523

Grigolyuk, E. I., 537

Grigorev, A. S., 215, 420, 533, 534

Grimaldi, A., 156, 157, 168, 173, 192

Groman, M. B., 382

Gross, O., 481

Grundy, P., 311, 479

Grüning, M., 249, 479

Grzesik, D., 113

Gu, Guo-ji, 439

Gu, Qiu-lin, 439

Gubkin, S. I., 49, 292

Gudehus, G., 125

Gudramovitch, V. S., 535

Guerlement, G., 334, 537

Guerra, G., 510

Guest, J. J.:

Chapter II, 71

Chapter III, 97-107, 112, 113, 118, 123, 125, 129-136, 144, 147, 149, 154, 161, 162, 201, 204

Chapter IV, 255, 262, 265, 271, 280

Chapter VI, 348,390

Chapter VII, 413, 415, 423-440, 444, 445, 450-460

Chapter VIII, 464, 483, 494, 495, 498, 523-528, 532-538

Guk, V. F., 528

Gunasekera, J. S., 285

Guo, Zh. H., 39, 47

Gurevitch, G. B., 34

Gurfinkel, G., 322

Gurtin, M. E., 199, 256

Gusenkov, A. P., 59, 357

Gutkowski, W., 423

Guz, A. N., 440

Gürkök, A., 322

Gvozdev, A. A., 206, 208, 209, 265, 269, 331, $335,339,352,413,502,504,509$

Haar, A , 271

Haase, H., 209, 413
Hadamard, J., 20

Haddow, J. B., 51, 104, 198, 239, 281, 459, 497, 533

Hahn, H. T., 198

Haigh, B. T., 18, 29, 69, 90, 105, 248

Haisler, W. E., 287, 288

Hajdin, N., 408

Hall, A. H., 401

Hamada, M., 439

Hamilton, C. H., 288, 495

Hamilton, W. R., 136, 173

Handelman, G. H., 84, 181, 272, 361, 372, 373, 385-387

Hanson, R. D., 479

Hara, Y., 103, 192

Harrison, H. B., 342, 478

Harstead, G. A., 338

Hart, E. W., 216

Hartmann, F., 322

Hartung, C., 181, 356

Hartzman, M., 102

Hashiguchi, K., 104

Hashin, Z., 191

Havner, K. S., 175, 192, 198

Hayashi, I., 168

Haydl, H. M., 456-459, 533-536

Haythornthwaite, R. M., 53, 100, 103, 123, 125, $126,214,322,420,434,438,495$

Hazell, C. R., 170, 423

Heaviside, O., 222

Hecker, S. S., 171, 288, 495, 501

Helfinstine, J. D., 114

Hellan, K., 143

Helmy, M. A., 384

Hencky, H.:

Chapter I, 15

Chapter II, 48, 49, 69-86

Chapter III, 89, 96-111, 117, 118, 124, 125, $129-138,142-147,154,155,160,167,172$, $173,180,187,188,192,197,200,203$

Chapter IV, 218, 235, 240, 255-257, 260-263, 271, 280-284, 290

Chapter VI, 348, 352-357, 362, 370-374, 386, $387,390,394,399$

Chapter VII, 413-418, 421-430, 436-451, 455, $458-460$

Chapter VIII, 484, 498, 501, 503, 512, 524-530, $533-536,539,540$

Hendry, A. W., 401

Hermite, Ch., 276, 277, 365, 380, 510, 515, 521-523 
Hershey, A. V., 48, 99

Hertz, H., 29

Heyman, J., 209, 214, 250, 384, 400, 401, 478, $479,480,527$

Hibbitt, H. D., 285

Higginbotham, A. B., 479

Higgins, T. R., 334, 335

Hill, R.:

Chapter II, 29, 39

Chapter III, 99, 100, 110-114, 122, 123, 130, $142,143,160,175-179,182,191,192$, 198-205

Chapter IV, 206-208, 211, 214-217, 222, 233, 255, 265, 268, 271, 272

Chapter V, 294

Chapter VI, 354, 361, 372, 373, 382-389

Chapter VII, 436, 439, 445, 460, 461

Chapter VIII, 464, 492-494, 510, 528

Hillier, M. J., 112, 203, 216

Hlavaček, M., 27

Ho, H. S., 250, 263, 535

Hodge, P. G, Jr.:

Chapter I, 6

Chapter II, 28, 29, 55, 86

Chapter III, 112, 144, 147, 154, 161, 162, 176 $181,182,204$

Chapter IV, 207-209, 213, 214, 247, 250, 262, 264, 265, 268, 271, 272, 278, 282, 283, 291

Chapter V, 322

Chapter VI, 384, 393-395, 401

Chapter VII, 413, 416, 423, 426, 427, 431, 434, $435,438-445,450,455$

Chapter VIII, 462, 466, 480, 492-497, 528, $532-536$

Hoffman, A. 285

Hoffman, O., 49

Hofmeister, L. O., 285

Hohenemser, K., 87, 88, 104, 135, 180, 181, 483

Holbeche, J., 114

Holister, G. S., 115

Holsapple, K. A., 198, 199

Holsteinson, L., 536

Hooke, R., 56, 57, 70-73, 107, 117, 127, 138, $153,176,196,214,218,219,354,448,449$, 476, 539

Hopkins, H. G., 262, 278-281, 322, 423, 532 Horii, K., 285

Horne, M. R., 209, 213, 247, 249, 392, 393, 398, $400,404,440,480,481$

Horng, J. T., 198

Hosford, W. F., 99, 111
Hoskin, B. C., 356

Hrudey, T. M., 104, 198

Hsu, P. T., 438

Hsu, T. C., 37, 48, 56, 93, 113, 126, 200

Hsu, T. R., 288

Hu, L. W., 84, 90, 95, 96, 99, 102, 111, 112, 169 , 170, 191, 201, 436, 461, 501

Huber, M. T.:

Chapter I, 15

Chapter II, 69, 71, 74, 76, 81, 82

Chapter III, 89, 95-101, 104-111, 117, 118, $124,125,129-131,133,137,138,144-147$, $154,155,160,167,173,178,180,187,188$, 192,200

Chapter IV, 218, 235, 240, 253, 256, 257, 260, $262,271,280,290$,

Chapter VI, 348, 353, 362, 370, 374, 386, 387, 390, 394, 399

Chapter VII, 413, 415, 418, 422-424, 427-429, $436,442-447,450,451,485-460$

Chapter VIII, 484, 498, 501, 503, 512, 524-529, 533-536, 539

Huddleston, J. V., 499

Hundy, B. B., 401

Hunter, D. T., 438, 440

Hutchinson, J., 192, 217

Hyman, B., 440

Iannelli, V., 321

Ibragimov, A. B., 126, 170, 502

Ibragimov, M. R., 195, 196, 536

Ibragimov, V. A., 215

Ibrayev, O. I., 388

Igaki, H., 162, 204, 502

Igaraschi, S., 479

Ignatyev, V. V., 130

Iida, K., 440

Ikegami, K., 123, 169, 170, 171, 203, 501

Ikrin, V. A., 251, 480

Ilyin, L. M., 181

Ilyushin, A. A.:

Chapter I, 3, 15, 16, 24

Chapter II, 29, 33, 36, 45, 53, 55, 73-79, 83-86

Chapter III, 89, 91, 97, 120, 122, 126, 130, 135, $140,143,172-175,179,181,183,185-187$, $192,197,203$

Chapter IV, 235, 252-258, 271, 284, 286, 292

Chapter V, 295

Chapter VI, 348, 352, 355-357, 362, 370, 373, 386 
Ilyushin, A. A. (cont.):

Chapter VII, 413, 417, 420, 421, 424, 425, 437, $438,440,446-456,459$

Chapter VIII, 466, 472, 484, 492-495, 499-502, $512,530,540$

Imegwu, E. O., 383

Ingerle, K., 306

Ingerslev, A., 206

Inoue, $\mathrm{K}, 480$

Inoue, T., 163, 171, 195, 249, 285, 501

Ionov, V. N. 457

Irmay, S., 90

Irons, B. M., 289

Irwin, G. R., 246

Isakson, G., 149, 285

Ishikawa, H., 383, 402, 500

Ishizaki, T., 171, 501

Ishlinsky, A. Yu., 100, 123, 132, 146-150, 161, $163,203,216,255,439,464,528$

Ismagilov, R., Kh., 181

Ito, Y. M., 66, 99, 115, 191

Ivanchenko, O. N., 288

Ivanov, B., 495

Ivanov, G. V., 424, 454, 533

Ivanov, I. A., 147

Ivanov, S. I., 366

Ivey, H. J., 160, 170, 178

Ivlev, D. D., 39, 51, 76, 88, 93, 98-100, 112, $123,125,136,140,144-147,158,160,167,173$, $175,181,187,255,273,281,346,435-437,499$, 527

Iwan, W. D., 147

Izbenko, L. A., 537

Izbicki, R. J., 281

Izotov, I. N., 143, 217

Jackson, L. R., 110, 201

Jacobi, C. G. J., 345, 347, 381

Jacobs, J. A., 281

Jaeger, Th., 209, 413, 532

Jakubowicz, A., 337

Janas, M., 209, 268, 335, 403, 413, 435, 437, 460

Jaunzemis, W., 27, 29, 34, 51, 198, 256

Javornicky, J., 292

Jenike, A. W., 125

Jenkin, C. F., 62

Jennings, A., 289

Jensen, P. S., 282

Jensen, W. R., 288

Ježek, K., 213, 295, 316, 478

Johansen, K. W., 209, 435
Johnson, K. L., 529

Johnson, K. R., 169

Johnson, L. G., 480

Johnson, W., 35, 51, 216, 281, 399, 401-404, 500

Jonas, J. J., 107

Jones, N., 322, 413, 434, 440, 457, 532

Josselin de Jong, G. De., 125

Jõgi, E., 323

Jouve, P., 478

Juhás, P., 401

Jung, H., 494

Justusson, J. W., 106, 165, 171

Kabelevsky, M. G., 528

Kachalov, Zh. V., 439

Kachanov, L. M., 45, 75, 84, 86, 168, 185, 246, $271,388,413,498,502$

Kachanov, M. L., 147

Kadashevitch, Yu, I., 59, 64, 146, 147, 154, 180, 181, 194, 537

Kaechele, L., 502

Kafka, V., 115, 147

Kahn, L. F., 479

Kalanta, S. A., 291

Kalatinets, A. E., 59, 192

Kalinowski, A. J., 480

Kaliski, S., 259, 543

Kaliszky, S., 437

Kaluzhsky, I. I., 113, 200

Kamalvand, H., 478

Kamat, M. P., 323

Kamentsev, V. N., 97, 170

Kamerer, A., 165

Kamiya, N., 439

Kammash, T. B., 499

Kanan, Ö., 501

Kaneko, K., 169, 171, 501

Kantorovitch, L. V., 217

Kao, J. S., 422, 440

Kao, R., 282, 288

Kaplan, M. A., 216

Kapustin, S. A., 285, 441, 456, 457, 534, 537, 538

Karczewski, J. A., 480

Karkauskas, R. P., 88, 290, 291, 445, 533

Kármán, T., 36, 215, 271

Karpinos, D. M., 169

Kasper, R., 163, 194

Kato, B., 479

Kato, T., 275

Kats, Sh. N., 502 
Kauppila, R. W., 499

Kaushik, Y. P., 459

Kawaguchi, K., 168

Kawahara, M., 285

Kawai, T., 440

Kawashima, K., 439, 441, 501, 533

Kazinczy, G., 206, 249, 265, 435

Kączkowski, Z., 282

Keii, M. 335

Kemp, K O., 422

Kenis, M. S., 498

Kenk, K. R., 171

Kerimbayev, S., 215

Kerimov, R. Yu., 527

Kestin, J., 158

Kettlewell, J., 502

Kharchenko, A. P., 527

Kharitontchik, A. E., 482

Khavkin, I. Ya., 496

Kheynloo, M. L., 498, 500

Khojasteh-Bakht, M., 438-440

Khoma, I. Yu., 526

Khomyakov, A. M., 535, 536

Khristyanovitch, S. A., 118, 183

Khuan Ke-Chzhi, 154

Kikuchi, F., 440, 457

Killian, D. E., 323

King, I. P., 115, 284-288

Kirakosyan, R. M., 250, 269, 439, 480, 481, 533, 538

Kirchhoff, G. R., 35, 68, 263, 410, 411, 456

Kireyev, A. V., 292, 535

Kirkpatrick, W. M., 103

Kirsanova, N. V., 146

Kiselev, L. K., 285

Kiselev, V. A., 246

Kishi, T., 62, 203

Kishino, Y., 27

Kishkin, B. P., 366

Kissyuk, V. N., 103, 335, 480, 481

Kist, W. C., 206

Kitagawa, H., 198, 285

Kiyko, I. A., 106, 343

Kleiber, M., 65, 198, 263, 285, 385

Klepaczko, J., 65, 111, 216, 535

Klębowski, Z., 95, 102

Kleemola, H. J., 216

Kleitz, M., 31

Klement, P., 440, 450

Klimenko, F. E., 398

Klinger, L. J., 201
Klöppel, K., 111, 403, 404

Klyagin, V. M., 532

Klyushnikov, V. D., 10, 84, 156, 163, 168, 171, 173, 192, 196, 215, 286

Klyatchko, S. D., 178, 180, 183, 184

Klyshevitch, Yu. V., 192

Knets, I. V., 191, 192

Koba, K. A., 434, 439, 440, 459

Kobayashi, A. S., 243, 281, 285

Kochetkov, A. M., 315

Kochin, D. M., 65, 126

Koenig, H. A., 528

Koff, W., 170, 501

Kogayev, V. P., 321, 356, 382

Koike, S., 501

Koiter, W. T., 118, 136, 181, 182, 191, 249, 250, 265, 268-272, 494

Kojima, H , 383

Kolář, A., 480

Kolarov, D., 164

Kolesnikov, L. O., 315, 322

Kolesnitchenko, M. F., 215

Kolgadin, V. A., 366, 389, 413, 419, 420, 424, 441, 456, 534

Kollbrunner, C. F., 222, 229, 230, 233, 408

Kolokoltchikov, V. V., 27, 523

Kolomiets, V. P., 322

Kolosov, G. V., 292

Kolundžija, B. T., 323

Komatsu, S., 366, 502

Komljenović, S., 27

Kondo, K., 189

Konieczny, L., 250, 479

Konishi, I., 335

Kononov, K. M., 528

Konyayev, E. A., 194

Koopman, D. C. A., 290

Kopnov, V. A., 95, 97, 107, 113, 114, 200, 203, 204,

Kordas, Z., 217, 517

Koreneva, A. M., 168

Korol, E. Z., 181

Korolev, V. I., 413, 440

Korotkith, Yu., G., 194, 198, 255, 282, 441, 529, 538

Korovin, I. M., 183, 356, 366

Kortenska, V., 495

Koshelev, P. F., 107

Kosiński, W., 199

Kosmodamiansky, A. S., 526

Kostenko, A. V., 501 
Kostotchkin, Yu., V., 249

Kostyuk, A. G., 192, 197

Kotov, P. I., 249

Kovalenko, A. D., 528

Kovalov, K. F., 155

Kovalsky, B. S., 534

Kowalczyk, K., 61, 251, 468, 474, 475, 479, 525

Kozlov, I. A., 137, 528

Kozlyakov, V. V., 404

Kozłowski, T., 335

König, J. A., 130, 163, 249-251, 294, 309, 311, $413,422,437-441,450,480,533-536$

König, M., 285

Körber, F., 138, 197

Krasovsky, Yu. P., 286

Kratochvil, J., 158, 199

Kraus, H., 537

Kravtchenko, A. A., 255

Kravtchenko, J., 281

Kravtchuk, A. S., 143, 174, 288, 492

Kravtsov, N. A., 401

Kregers, A. F., 138, 156, 192, 204, 356

Krisch, A., 496

Krishna Murty, A. U., 284

Krishnasamy, S. G., 479

Krohn, R., 215

Kronecker, L., 26

Kröner, E., 158, 192, 199

Krutkov, Yu. A., 256

Krużelecki, J., 318

Krylov, V. I., 289

Kryzhanovsky, V. P., 266

Krzyś, W., 29, 58, 318, 369, 495, 499, 525

Křupka, V., 408

Kuczyński, K., 245

Kudryashov, N. N., 192, 502

Kudryavtsev, I. N., 337, 339

Kudryavtsev, S. D., 337, 339, 401, 480, 481

Kukudzhanov, V. N., 398, 400

Kulago, A. E., 523

Kular, G. S., 112

Kulikov, Yu. K., 536

Kull, L. M., 535

Kultursynov, Zh., 185, 495

Kumar, A., 496

Kuneyev, V. I., 192

Kunitsky, L. P., 398, 401, 480

Kuo, Y. H., 275, 281

Kupriyanov, V. V., 480

Kupriychuk, P. F., 536, 537

Kurata, M., 384
Kurdin, N. S., 481

Kurtyka, T., 159

Kusuda, T., 403

Kutta, W., 278, 542

Kuzin, A. I., 399, 478

Kuzin, V. F., 111

Kuz'menko, V. A., 109, 138

Kuzmin, E. Ya., 65

Kuznetsov, A. I., 356, 526

Kuznetsov, B. N., 250, 311, 479, 480, 481

Kuznetsov, V. V., 526

Kwaśniewski, M., 334

Kwieciński, M. W., 422

Labutin, A. A., 245

Ladevèze, P., 439

Lagrange, J., 40-43, 47, 49, 51, 196, 197, 225 . $240,271,296,313,343,351,394,457,505$, 506, 508

Lamasnevsky, P., 138

Lamblin, O., 334, 481

Lamé, G., 253, 286

Lampert, P., 383, 389

Lampsi, B. B., 400, 401, 478

Lance, R. H., 114, 290, 403, 437, 459

Landau, H. G., 441, 499

Landgraf, G., 458

Lange Hansen, P., 213

Langenbach, A., 457

Langendonck, T. Van, 392

Lanier, J., 281

Laning, J. H., 178-180, 183, 184, 187-189, 438

Lankford, W. T., 110, 201, 216

Lansing, W., 288

Lantukh, L. G., 266, 534

Laplace, C. E. P., 365

Laptevsky, A. G., 441, 538

Larsen, P. K., 285

Larson, L. O., 510, 523

Lászlo, F., 527

Latham, A. A., 245

Latter, A. J., 49, 197

Laudiero, F., 424

Lay, M. G., 322

Lebedev, A. A., 95, 106, 107, 109, 137, 138

Lebedinets, L. N., 335

Lebedinets, N. A., 323, 335

Le Boiteux, H., 102

Leckie, F. A., 536

Ledziński, J., 215

Lee, C. H., 285 
Lee, E. H., 65, 198, 208, 222

Lee, L. C., 170, 502

Lee, L. H. N., 198

Lee, S. L., 268, 285, 422, 434, 440

Lees, S., 62

Lehmann, T., 65, 158, 181, 198, 203, 204

Leipholz, H., 123

Lellep, Ya. A., 435, 445

Lemke, C. E., 290

Lenard, J., 239, 245, 497

Lensky, V. S., 90, 107, 187, 189, 194, 195, 286, 502

Leon, A., 103

Leonov, M. Ya., 192, 356

Lepik, Yu. R. (U..), 73, 214, 321, 413, 420, 433$435,438-445,457,459,533-535$

Lessels, J. M., 96

Leth, C. F. A., 400

Leu, K. C., 338

Levin, O. A., 250

Levine, H. S., 533

Levitan, L. M., 495

Levonyan, L. A., 383

Levy, M.:

Chapter II, 78, 79, 83-86

Chapter III, 97, 116, 124, 125, 130, 135, 136, 149, 178, 198

Chapter IV, 252, 253, 256, 258, 262, 263,

Chapter VI, 352, 355, 361, 362, 373, 386

Chapter VII, 424, 438, 442, 451

Chapter VIII, 484

Leytes, S. D., 84, 150, 154, 213, 315, 316, 356, 398, 478, 512 .

Lezhava, G. I., 479

Lie, M. S., 199

Lighthill, M. J., 275, 281

Likhatchev, V. A., 249

Lin, C. C., 181, 281

Lin, H. C., 357

Lin, S. R., 457

Lin, T. H., 66, 99, 115, 156, 191, 264, 288, 413, 457

Lind, N. C., 95, 165, 495, 533, 536

Lindholm, V. S., 357

Lindner, J., 388

Lipkin, J., 357

Lippmann, H., 27, 113, 123, 271, 272, 479

Listrova, Yu. P., 433, 435, 437, 439, 441

Liszka, T., 261, 282, 527

Litoński, J., 65

Liu, C. S., 106, 171
Liu, D. T., 198

Liu, S. I., 159

Livesley, R. K., 479

Livolant, M., 285

Livshits, A. L., 523

Livshits, V. L., 523

Lode, W., 32, 35, 46, 75, 78, 80, 92, 96, 101, 118 , $131,155,173,178,191,483$

Lokoshtchenko, A. M., 323

Lokshin, A. Z., 316, 439

Lomacky, O., 440

Lomakin, V. A., 194, 195, 197, 203, 204, 495

Longbottom, E., 401

Lopatin, B. V., 481

Love, A. E. H., 263, 410, 411, 456

Lu, Le-Wu, 384, 478

Lubahn, J. D., 138, 164, 216

Łubiński, M., 401

Lubliner, J., 199

Lücke, B., 16

Ludwik, P., 48, 56, 138, 216

Luht, L. Ya., 73, 420, 439

Lukash, P. A., 457

Lukin, V. A., 439

Lunchick, M. E., 436

Lundgren, K., 408

Lurie, A. I., 367, 456

Luscouarch, Y., 323

Luzhin, O. V., 339

Lyalin, V. M., 112, 201

Lyapunov, A. M., 134

Lyubarov, V. I., 266, 311, 339, 384, 401, 479

Lyubimov, A. K., 282, 441, 529, 533

Macfarlane, W. A., 537

MacGregor, C. W., 96, 495

Machida, A., 528

Maeda, H., 478

Magnée, A., 97

Maier, G., 195, 215, 246, 250, 269, 272, 284. 285, 291, 467, 468, 481, 536

Maier-Leibnitz, H., 229

Mair, W. M., 169, 171

Maitra, M., 104

Majer, J., 104

Majumdar, K., 104

Makarevitch, A. A., 322, 335

Makarevitch, S. S., 500

Makarov, B. P., 316

Makarov, E. V., 147

Makeranets, E. I., 523 
Makhnenko, V. I., 441, 537

Makhonina, T. M., 525

Makhover, E. V., 200

Makhutov, N. A., 250

Makky, S. N., 356

Maksak, V. I., 168, 183, 216, 502

Malament, L. I., 480

Malikov, G. F., 399

Malinin, N. N., 204, 216, 416

Malmeyster, A. K., 93, 113, 191, 204, 356, 493

Malvern, L. E., 31, 47, 49, 121, 264, 357, 383

Malygin, A. F., 499

Malyi, V. I., 122, 143, 189

Malyshev, B. M., 502

Mamay, V. I., 439

Manca, P., 147

Mandel, J., 24, 118, 135, 162, 180, 182, 199, 216, 373,382

Manning, D., 495

Mansfield, E. H., 107, 482

Manson, S. S., 248, 249, 288, 528

Mansurov, R., 160

Marcal, P. V., 285, 288, 438, 440

Marciniak, Z., 93, 97, 139, 216, 245, 246, 533

Margolin, G. G., 111

Marin, J., 84, 95, 111-113, 138, 169, 191, 461, 501,502

Marjanović, R., 171

Markov, A. A., 272

Marshall, P. J., 338

Martin, J. B., 204, 272, 385, 532

Martin, H. C., 284

Martynova, T. N., 125

Mashkov, I. D., 90, 170, 502

Masing, G., 59, 62, 174

Massau, J., 280

Massey, P. C., 385

Massonnet, Ch. E., 209, 295, 334, 413, 453, 454, 457, 478

Masui, T., 357

Matchenko, N. M., 90

Matoshko, S. I., 413, 420, 456

Matsui, C., 479

Matsuura, M., 102

Matucha, K. H., 113

Maxwell, J. C., 69, 256

Mayeda, R., 478

Mazelsky, B., 457

Mazilu, P., 184

Mazing, R. I., 497

Mazzarella, C., 443, 480
Mazzolani, F. M., 527

McClintock, F. A., 246

McCrum, A. W., 527

McDonough, J. F., 338

McInnis, B. C., 27

McIver, R. W., 202

McKyes, E., 125

McLaughlin, P. V., 115, 215, 268, 456, 460

McMeeking, R. M., 285

McNamee, B. M., 114

McVean, D., 48

Melan, E., 132, 146, 148-150, 154, 161, 163, 176, $178,203,249,250,265,266,307,464,476,479$

Melchers, R. F., 437

Meldahl, A., 90, 92, 94, 95, 97, 123, 161

Mello, R. M., 215

Mellor, P. B., 35, 111, 202, 213, 216, 245, 495, 500

Melosh, R. J., 266

Mendelson, A., 178, 288, 528

Mendera, Z., 103

Menditto, G., 422

Merchant, W., 384, 408

Merckx, K. R., 500, 523

Merkle, J. G., 102

Merwin, J. E., 333

Mesnager, A., 48

Mets, G., 357

Meyer, J. D., 479

Mezhlumyan, R. A., 73, 408

Miastkowski, J., 97, 168-170, 209, 501

Michalos, J., 338

Michno, M. J., 170, 171, 501

Mielniczuk, J., 335

Mierzejewski, H., 263

Mii, H., 356, 385

Mikeladze, M. Sh., 113, 413, 436, 460, 461, 528, 534,536

Mikhalishin, M. S., 435, 439, 445, 536

Mikhaylov, G. S., 538

Mikhaylov, N. Ya., 502

Mikhlin, S. G., 526

Mikkola, M., 402

Miles, J. P., 217

Mileykovsky, I. E., 111

Miller, D. R., 499

Miller, P. M., 383

Milner, H. R., 338

Minayev, V. A., 147

Miner, R., 248

Mioduchowski, A., 347, 479 
Mirolyubov, I. N., 102, 126, 373, 382

Mirsalimov, V. M., 528

Mirzabekyan, B. Yu., 291

Mises, R.:

Chapter I, 15

Chapter II, 54, 70, 71, 74, 76, 78-86

Chapter III, 89, 96-118, 124, 125, 129-138, $144-147,149,154,155,160,167,173,178$, $180,187,188,192,198-200,204$

Chapter IV, 211, 215, 218, 235, 240, 252, 253, 256-262, 271, 280, 290

Chapter VI, 348, 352-355, 361, 362, 370, 373, $374,386,387,390,394,399$

Chapter VII, 413, 415, 418, 422-424, 427-429, $435-438,442-447,450,451,455,458-461$

Chapter VIII, 484, 498, 501, 503, 512, 524, $525,527,529,533-536,539$

Mitinsky, A. N., 392

Mitrokhin, N. M., 138, 502

Mittal, R. K., 197

Mityukov, A. G., 217

Miyagawa, Y., 161

Mizuno, S., 173

Mohaghegh, M. M., 459

Mohr, O., 103, 262

Mokashova, N. I., 435

Molotnikov, V. Ya., 192

Monakhov, V. A., 403

Monasa, F. E., 323

Monastyrnyi, P. I., 289

Montague, P., 440

Moreau, J. J., 289

Morera, G., 256

Morino, S., 478

Morley, C. T., 422

Morozov, E. M., 498

Morozov, V. I , 250, 499

Morris, A. J., 459

Morris, G. A., 340, 389

Morrison, H. L., 281

Morrison, J. L. M., 96, 99, 180

Moshensky, N. L., 322, 478

Moskvitin, V. V., 59, 62, 149, 174, 184, 194, 249, 250, 481, 495

Motovilovets, I. A., 528, 534

Mozharovsky, N. S., 106, 250

Mönch, E., 292

Mrázik, A., 481

Mrowiec, M., 277, 335, 416, 510, 523

Mróz, Z., 67, 87, 124. 136. 145, 161, 165, 173,
$179-183,194,198,218,250,271,272,356,423$,

431, 434, 437, 455, 495, 535

Muensterer, H. F., 450

Muir, H., 60

Mukhanov, K. K., 322, 478

Mulcahy, T. M., 250, 482

Mulhern, J. F., 114

Mura, T., 195, 268, 422, 498

Murakami, S., 420, 438, 439, 533

Murch, S. A., 499

Murlina, G. A., 433, 435, 441

Murnaghan, F. D., 49

Murthy, S. D. N., 534

Murzewski, J., 103, 109, 419, 495

Muskhelishvili, N. I., 292

Muspratt, M. A., 533

Mutermilch, J., 401

Myasnyankin, Yu. M., 51

Myers, C. D., 357

Myszkowski, J., 439

Nádai, A., 48, 73, 172, 196, 216, 235, 240, 261, $272,295,352,494,495,497,524,525,527$

Nagahiko, S., 501

Naghdi, P. M., 27, 60, 65, 88, 124, 126, 162, $170,194,198,205,416,426,438,499,501$, 502,525

Nagtegaal, J. C., 285, 479

Nagyavitchius, Yu. A., 291, 427, 445

Nakamura, T., 335, 431, 432, 434, 445, 455, 533

Nakazawa, T., 382

Nakhaytchuk, V. G., 357

Namikawa, H., 478

Nan, N., 543

Nardo, S. V., 440

Natanson, I. P., 276

Nayak, G. C., 36, 285

Nayfeh, A., 275

Nayroles, B., 175, 285, 289

Nänni, J., 123

Neal, B. G., 209, 230, 249, 334, 390, 398-400, $403,404,480$

Neale, K. W., 272, 533

Needleman, A., 217

Negisi, H., 311

Negre, R., 281, 544

Nelson, I., 543

Nelson, R. B., 479

Nemat-Nasser, S., 288, 416, 525

Nemirovsky, Yu, V., 115, 399, 416, 435-438, $445,497,498,500$ 
Nesmeyanov, A. S., 250, 499

Neuber, H., 113, 204, 210

Newmark, N. M., 401

Newton, I., 278

Neymark, A. S., 354

Nguyen, D. H., 285

Nguyen, Q. S., 183, 196, 262, 268

Nielsen, P. M., 416

Nieminen, M. A., 216

Niepostyn, D., 209, 413, 436

Nikolayenko, N. A., 107

Nikolayevsky, V. N., 102, 118, 125

Nonaka, T., 335, 478, 479

Nordgren, R. P., 416, 525

Nosov, G. M., 399

Noto La Diega, S., 497, 501

Novopashin, M. D., 250

Novotny, R., 147

Novozhilov, V. V., 40, 59, 64, 71, 75, 87-89, $136,146,147,154,173,180,183,187,194$, $248,410,456,537$

Nowacki, W. K., 543

Nuttall, H., 383

Nyashin, Yu. I., 288

Oden, J. T., 284

Odenö, H., 528

Oding, I. A., 249

Odinokov, V. I., 523

O'Donnell, W. J., 115, 416, 423

Odqvist, F. K. G., 50, 141-144, 146, 161, 177, $183,185,188,193,197$

Ogawa, K., 480

Ogibalov, P. M., 106, 189, 194, 494, 495, 499, 500

Ogorodnikov, V. A., 356

Ohashi, Y., 104, 173, 188, 420, 438-441, 501, 502,533

Ohji, K., 245

Ohnami, M., 357

Ohta, T., 321, 382

Okamura, H., 457

Okano, H., 169

Okouchi, T., 440

Oldroyd, J. G., 197

Olszak, W., 103, 104, 107, 111, 136, 156, 163, $184,198,200,271,272,413,419,433,435,436$, $437,445,495$

Olszewski, E., 401
Onat, E. T., 199, 214, 215, 217, 335, 383, 399, $428,430,431,434,437-440,444,445,454$, $535,536,537$

Ondraček, E., 100

Ono, A., 501

Oostrom, van, J., 408

Orkisz, J., 215, 282, 317-321

Ormerod, A., 337

Oschatz, A., 459

Osgood, W. R., 55, 57, 138, 243, 284, 316, 356, 383, 498, 500,

Osias, J. R., 198

Ostrovsky, A. A., 169, 216

Ostrowski, A., 278

Ota, T., 113, 188, 202, 502

Oudin, J., 399

Ovanesova, Zh. G., 482

Owen, D. G., 500

Owen, D. R. J., 114, 199, 285

Pabisek, E., 535

Padlog, J., 284, 288

Pae, K. D., 102

Paglietti, A., 147, 199

Pai, D. H., 523

Palchevsky, S. A., 342, 396, 397, 400

Paliy, D. M., 438

Palley, I. Z., 163, 194, 528, 537

Palmer, A C., 195, 268, 291, 434

Palmov, V. A., 525

Palne, E., 174

Palusamy, S., 536

Panarelli, J. E., 416, 492, 510, 535

Panc, V., 245

Pandalai, K. A. V., 498, 500

Panferov, V. M., 271, 286

Pankova, M. V., 306

Pannell, F. N., 533

Panov, B. V., 354

Pantchovski, D., 338

Panzeca, T., 480

Papkovitch, P. F., 393

Parasyuk, O. S., 292, 394, 526

Pardue, T. E., 73, 523

Parimi, S. R., 291, 480

Parker, J., 139, 168, 502

Parkes, E. W., 250, 529

Parks, D. M., 285

Paslay, P. R., 146

Passannanti, A., 501

Patel, S. A., 498, 500 
Paton, E. O., 249

Paul, B., 95, 97, 99, 103, 108, 109, 170, 213, 246, $440,502,532$

Pavlov, P. A., 493

Payne, D. J., 500

Payne, H., 156, 191, 356

Pearce, R., 111

Pellisier, A., 523

Pełczyński, T., 34, 95, 107, 108, 112

Penny, R. K., 536, 537

Percy, J. H., 285

Perelmuter, A. V., 266, 534

Perepelin, V. M., 501

Perlin, P. I., 527

Perrone, N., 147, 282, 495, 498, 534

Perzyna, P., 29, 136, 158, 184, 195, 198, 199, 271, 272, 413

Peters, R. W., 502

Peterson, D. B., 522

Petrishtchev, P. P., 203

Petrosyan, Zh. L., 216, 416

Petushkov, V. A., 285

Philippidis, A. H., 272

Phillips, A., 73, 106, 126, 146, 156, 163-165, $168-171,175,178,180,194,195,272,323$, 402, 501, 502

Pian, T. H. H., 427, 438

Piechnik, S., 273, 274, 276, 372-375, 387

Pifko, A., 149, 285, 533

Pignataro, M., 440

Pilgrim, W. R., 440

Pikovsky, A. A., 213

Pinadzhyan, V. V., 321, 337

Pinsker, G. M., 408

Piola, G., 35

Pipkin, A. C., 65, 175, 199

Pisarenko, G. S., 95, 106, 107, 138

Piskun, V. V., 441, 537, 538

Pister, K. S., 102

Pivnev, F. A., 323

Plagov, I. M., 441, 538

Plenard, E., 250

Pobedrya, B. E., 197

Pochtman, Yu, M., 480, 534

Poincaré, H., 273, 275, 281

Poisson, S. D., 45, 68, 107, 152, 237, 239, 258, $357,365,419,530$

Pokora, T., 245

Polizzotto, C., 479, 480, 535

Polovin, R. V., 195, 499

Polukhin, P. I., 292
Polyakov, A. A., 156, 195, 538

Polyakov, L. I., 445, 534, 537

Polyakov, Yu. T., 445

Poncelet, J. V., 103, 107, 244

Ponomarev, S. D., 45, 286

Ponter, A. R. S., 251, 271, 272

Pontryagin, L. S., 291, 534

Pope, G. G., 284, 288

Popov, E. P., 285, 438, 440

Popov, V. S., 435, 439, 445

Porowski, J., 115, 416, 423

Potapov, V. N., 435, 439

Powell, W. R., 195

Poynting, J. H., 181

Prager, W.:

Chapter I, 19

Chapter II, 29, 35, 39, 57, 86

Chapter III, 87, 89, 96, 99, 100, 102, 104, 112, $114,118,125,132,135,136,145-151,154$, $160,161,163,173,175-184,187,193,198$, 203, 204

Chapter IV, 206-208, 224, 249, 250, 264, 265, $271,272,281,284,290$

Chapter V, 294, 310, 335, 346

Chapter VI, 383, 393, 401

Chapter VII, 428, 430, 433, 434, 438, 445

Chapter VIII, 478-482, 494, 536

Prandtl, L.:

Chapter II, 54, 75, 79-86

Chapter III, 97, 116, 124, 130, 131, 135, 136, $140,149,153,178,198$

Chapter IV, 218, 220, 235, 252-259, 281

Chapter VI, 356, 361, 365, 374, 385-387

Chapter VII, 420, 438, 440, 450, 454, 457

Prathap, G., 316

Proctor, E., 536

Prokhorenko, I. V., 441, 537, 538

Protsenko, A. M., 214, 271, 289, 291, 413

Pryadko, E. O., 337, 385

Pugh, H. L. D., 95, 169

Pyatigorsky, Z. I., 480, 534

Qu, S., 455

Quinney, H., 96, 100, 143, 483

Rabinovitch, C. V., 334

Rabotnov, Yu. N., 84, 115, 155-157, 336, 436, 437, 443, 450, 459, 502

Radaj, D., 285

Radenković, D., 125, 268

Radhakrishnan, V. M., 357 
Radwańska, M., 323

Rafay, T., 335

Raithel, A., 335

Rakhimbekova, Z. M., 400, 401

Rakhmatulin, Kh., A., 497, 529

Rakovshtchik, Yu. A., 382, 385, 478

Ramasco, R., 439

Ramberg, W., 57, 243, 284, 316, 356, 383, 498, 500

Raniecki, B., 194, 272

Rankine, W. J. M., 434

Ranshi, A. S., 399, 401, 402, 404

Rao, M. S. M., 115

Raphson, 278

Rashid, Y. R., 147

Ratner, S. I., 95

Ravalard, Y., 399

Rayevskaya, E. A., 384, 408

Razumikhin, M. I., 340

Reckling, K. A., 35, 64, 202, 230, 365, 384, 398, 400

Reddi, M. M., 285

Reddy, T. Y., 527

Reid, S. R., 527

Reiner, M., 136

Reiss, R., 532

Reissner, H. 271, 287

Reitman, M. I., 291, 437, 457

Rendulic, L., 102

Renne, I. P., 356, 402

Reuss, A.:

Chapter II, 79-86

Chapter III, 97, 98, 116, 118, 124, 130, 131, $135,136,140,145,149,153,178,198$

Chapter IV, 218, 220, 235, 252-258

Chapter VI, 356, 361

Chapter VII, 420, 438, 440, 450, 454, 457

Revuzhenko, A. F., 492

Rhzanitsyn, A. R., 209, 230, 270, 291, 322, 373, $382,383,394,395,397,405,435,437,450,479$

Riccati, J. F., 315

Ricciuti, M., 171, 501

Rice, J. R., 158, 198, 199, 246, 285

Richards, R. M., 285

Richmond, O., 281

Richter, H., 49

Riemann, B., 50, 189, 281

Riesemann, W. A., 287, 288

Rimawi, W. H., 268

Rimrott, F. P. J., 450

Ritter, G., 340
Ritz, W., 289

Rivlin, R. S., 65, 136, 175, 199

Roach, R. E., 306

Roberts, J. M., 335

Robinson, A., 322

Robinson, D. N., 114, 403, 437, 459

Robinson, M., 454, 458

Roche, R., 285

Roderick, J. W., 401

Rogan, J., 168, 501

Rogers, T. G., 114

Rogozin, I. O., 191

Rogoziński, M., 500

Romano, F., 440

Ronay, M., 181

Rossow, E. C., 285

Roš, M., 45, 71, 96, 137, 215, 483

Roushan, A., 479

Rowley, J. C., 170, 502

Roy, P., 457

Royles, R., 48, 56

Rozenberg, V. M., 197

Rozenblat, G. I., 323

Rozenblyum, V. I., 73, 126, 136, 194, 250, 356, 443, 455, 456, 497, 528, 534

Rozhdestvensky, V. V., 425, 427

Rozvany, G. I. N., 401, 437, 532

Rudayev, Ya. J., 173, 192, 356

Rudis, M. A., 433, 435, 445, 534, 537

Ruiz, C., 250

Runge, C., 278, 542

Rusinko, K. N., 192

Rusov, B. P., 188

Rutecki, J., 408

Ruzanov, F. I., 496

Rybakina, O. G., 248

Rybakova, R. I., 420, 457

Rybalko, F. P., 501

Rychlewski, J., 24, 104, 107, 250, 281, 413, 422,

423, 425, 441, 451, 456, 459

Ryder, E. I., 475

Ryś, J., 495, 525

Rytchkov, B. A., 173, 192, 502

Saareste, E., 457

Sacchi, G., 209, 268, 290, 291, 481

Sachs, G., 49, 59, 71, 159, 201, 216

Sadakov, O. S., 147

Sadowsky, M. A., 272

Sagar, V., 500

Saibel, E., 216 
Saidkarimov, U. S., 537

Saint-Venant, B. de, 78, 103, 107, 112, 244, 245, 256, 294, 361, 406, 407, 494

Saitō, K., 162, 204, 382, 502

Saito, M., 245

Sakimoto, T., 366, 502

Sakurai, T., 284

Salençon, J., 268, 281

Salinas, D., 115

Salonen, E. M., 285

Samoylovitch, Yu. A., 498

Sandel, G. D., 102, 103, 156

Sanders, J. L., 162, 182, 191

Sanecki, H., 256

Sankaranarayanan, R., 384, 389, 433, 435, 436, 533, 535

Sankov, E. I., 285, 535

Santathadaporn, S., 339, 478

Sarkisyan, M. S., 37, 135, 173

Sarkisyan, S. O., 286

Sauer, J. A., 501

Sausin, V. Ya., 192

Save, M., 209, 268, 291, 294, 295, 422, 423, 481, 536

Savelyev, L. M., 527

Savin, G. N., 292, 526, 527

Savtchenko, V. G., 197, 498, 528

Savvin $_{\text {, D. D., } 481}$

Sawczuk, A.:

Chapter II, 27

Chapter III, 112, 115, 136, 160, 161, 163, 169, 194, 195, 201, 203

Chapter IV, 209, 214, 250, 268

Chapter V, 335

Chapter VI, 403

Chapter VII, 413, 422, 423, 425, 433-435, 437, $441,443,445,451,456-459,461$

Chapter VIII, 478, 532, 533, 536

Sayir, M., 99, 100, 111-113, 123, 268, 535

Säverin, U., 479

Scharpf, D. W., 88, 285, 288

Scheu, L., 138

Schilling, C. G., 478

Schlafer, J. L., 502

Schleicher, F., 101, 102

Schmid, E., 113, 189

Schmidt, R., 99, 100, 123, 137, 143, 255, 335, $439,464,528$

Schnobrich, W. C., 450

Schöpf, H. G., 123

Schroeder, J., 272, 457, 458
Schröder, G., 357

Schutz, F. W., 478

Schwedler, J. W., 390, 395

Schweitzer, R., 496

Scuderi, R., 408

Sebekina, V. I., 403, 436

Sebeshev, V. G., 335, 403, 404

Sedov, L. I., 47, 65, 84, 197-199

Seely, F. B., 316

Seetharam, P., 385

Seguchi, Y., 383, 439

Segura, J., 27

Semenenko, A. N., 536

Semykina, T. D., 441, 534

Senator, M., 445

Serensen, S. V., 59, 102, 248, 249, 321, 357, 382

Sergiyev, B. P., 523

Seth, B. R., 48, 112, 495, 498

Sewell, M. J., 182, 217, 268

Shabliy, O. N., 434, 435, 439, 440, 445, 458, 459, 536,537

Shaffer, B. W., 445

Shakhnovsky, D. I., 215

Shakir-Khalil, H., 340, 389

Shamiyev, F. G., 536

Shammamy, M. R., 86, 356

Shanks, D., 289

Shanley, F. R., 248, 475

Shapiro, G. S., 399, 411, 413, 420, 423, 451, 457, 458,525

Shapovalov, L. A., 482

Sharifi, P., 285, 438, 440

Sharshukov, G. K., 537

Shatchnev, V. A., 286

Shayshmelashvili, V. N., 437

Shefter, G. M., 118

Shelton, A., 168, 501

Shemyakin, E. I., 118, 183

Shen, M. K., 322

Shepherd, R. P., 502

Shepherd, W. M., 180

Sheppard, T., 335

Sherbourne, A. N., 272, 404, 434, 439, 456, 458, $459,479,533-536$

Shesterikov, S. A., 322

Shevtchenko, Yu. N., 126, 188, 194, 197, 250, $286,441,455,498,499,500,528,534,538$

Shevelev, V. V., 200

Shield, R. T., 87, 88, 103, 125, 147, 149, 281, $383,399,432,434,455,527,533$

Shifrin, G. A., 214, 438 
Shindo, A., 113, 188, 202, 383, 439, 502

Shinkarenko, A. P., 534

Shinmura, O., 383

Shiozawa, K., 357

Shirai, T., 439

Shiraishi, N., 335

Shirakawa, K., 311, 479

Shiratori, E., 123, 169, 170, 171, 203, 501

Shirko, I. V., 420, 439, 440

Shishmarev, O. A., 65, 99, 168, 170, 180, 188, 501,502

Shkaraputa, L. M., 501

Shkerbelis, K. K., 103

Shnerenko, K. I., 440

Shneyderovitch, R. M., 3, 59, 174, 249, 250, 321, $322,356,382,528$

Shoeb, N. A., 450

Shoemaker, E. M., 208, 222, 398

Shoji, H., 59

Shorr, B. F., 498

Shrivastava, H. P., 159, 161, 356

Shvayko, N. Yu., 173, 192, 356

Sibille, R., 281

Sidebottom, O. M., 86, 93, 143, 169, 202, 356, $361,492,502$

Sidoroff, F., 189, 199

Sidorov, E. A., 288

Siebel, M. P. L., 373, 382, 383, 510

Sierakowski, R. L., 163-165, 168, 170, 171, 175, 501

Sikalo, P. I., 316

Silvestri, V., 125

Simonen, F. A., 438, 440

Simpson, T., 438

Sines, G., 216

Sinitsky, A. K., 73, 494

Sinitsyn, A. P., 538, 544

Sinyukov, A. M., 107

Sirotin, Yu. J., 113

Sjodahl, L. H., 56

Skobeyev, A. M., 529, 543

Skomorovsky, Ya. Z., 322

Skosnyagin, Yu. A., 537

Skraba, W., 217

Skripnikova, R. A., 338

Skrzypek, J., 217, 273, 274, 277, 418, 450, 489, $496,497,503,508-510,512,522,536,543$

Skudra, A. M., 493

Smirnov-Alayev, G. A., 197

Smith, G. E., 115, 495

Smith, G. F., 110
Smith, J. O., 316, 361

Smith, K. F., 110, 201

Smith, S., 183, 502

Smolyago, N. A., 388

Snitko, N. K., 396

Sobolev, Yu. V., 323, 478

Sobolevsky, V. M., 499, 500

Sobotka, Z., 100, 102, 104, 111, 113, 136, 200, $209,346,365,396,397,403,404,413,459$, 495,533

Sokolov, A. P., 526

Sokolovsky, V. V., 36, 45, 130, 235, 260, 281, $361,413,420,426,439,494,524,525$

Solodovnikov, V. N., 424

Solomeshtch, M. A., 403

Sonoda, K., 384

Soonets, K., 440, 457

Sopwith, D. G., 494

Southwell, R. V., 281, 383

Sowerby, R., 51, 245, 281

Sparacio, R., 480-482

Spence, J., 536

Spencer, A. J. M., 34, 114, 115, 198, 201, 273, 281, 481, 495

Spiridonova, N. I., 216

Spooner, J. B., 285

Srinath, H., 527

Srivastava, B. L., 334

Srivastava, N. K., 434, 439

Stasenko, I. V., 523

Stassi d'Alia, F., 102, 106, 113, 261, 422, 443, 497, 501, 528

Steele, M. C., 383

Steffensen, J. F., 288, 289

Stehlin, P., 536

Stelmashuk, V. N., 445

Stevens, L. K., 306, 316, 323

Stilijanov, P., 123

Stockton, F. D., 100, 138, 191

Stodulski, M., 157, 495

Stokey, W. F., 510, 522, 523

Stolarski, H., 535, 536

Stone, C. C., 482

Storåkers, B., 214, 216, 217

Strelbitskaya, A. I., (Strelbytska, O. I.), 337, $339,342,384,385,388,389,400,403-408$, $413,419,420,424,456,457$

Streletsky, N. S., 479

Stricklin, J. A., 287, 288, 438

Strifors, H., 217

Stryuk, V. K., 499 
Stutz, P., 136, 268, 544

Stüssi, F., 222, 229, 230, 233, 392

Sudzuki, H., 311

Sugimoto, M., 162, 204

Sun, C. K., 208, 462, 466

Suturina, N. G., 170

Suyunshkaliyev, N. Kh., 322

Svéd, G., 361

Svensson, N. L., 147, 161, 203, 501

Sverdlov, M. I., 496

Sverdlova, N. G., 169

Sveshnikova, V. A., 502

Sviderskaya, V. S., 402

Swedlow, J. L., 100, 198, 292

Swida, W., 323

Swift, H. W., 323

Symonds, P. S., 208, 230, 249, 271, 356, 479, 480

Synge, J. L., 284

Syrnikov, N. M., 118

Szczepiński, W., 45, 97, 118, 168-171, 209, 214, 265, 281, 423, 497, 501

Szuwalski, K., 18, 57, 221, 222, 229, 233, 234, 239, 240, 529

Škaloud, M., 401

Šmiłak, S., 403, 408

Swisterski, W., 25

Tadros, A. K., 245

Tadros, G. S., 340, 389

Tairova, Z. I., 149, 266, 493, 495, 525

Takano, E., 311

Takatsuka, K., 389

Takenaka, Y., 161

Talypov, G. B., 59, 60, 96, 154, 160, 168, 170, 180, 501

Tanabe, T., 62, 203

Tanaka, H., 335

Tanaka, K., 165, 171, 195, 249, 285, 501

Tanaka, M., 161, 180, 439

Tang, J. L., 163, 169, 171, 501

Tang, S. C., 457

Taniguchi, T., 335

Tarasenko, I. I., 103

Tarasenko, V. L., 480

Tarasov, I. B., 537

Tarn, J. Q., 115

Tatarinov, V. G., 498

Tavernelli, J. F., 248

Taylor, G. I., 96, 100, 123, 143, 189, 276, 483

Tekinalp, B., 420

Telega, J. J., 27, 268, 291
Temis, Yu. M., 163, 528

Teodosiu, C., 199

Teregulov, I. G., 285

Terekhov, R. G. 126, 188, 194, 197, 528

Terentev, B. V., 323

Terrovere, V. R., 416, 434

Teters, G. A., 192

Theocaris, P. S., 170, 423

Theophilos, N. P., 537

Thermann, K., 185, 479

Thierauf, G., 285, 323, 479

Thomas, C., 115

Thomas, T. Y., 39, 51, 123, 136, 195, 198, 245, 253

Thomason, P. F., 216

Thompson, A. S., 528

Thomsen, E. G., 138, 168, 281

Thorn, B. J., 434, 440

Tiedemann, J. B., 523

Timoshenko, S. P., 35, 446

Ting, T. C. T., 357, 543

Ting, T. W., 495

Tin Loi, F., 475

Tkachenko, I. N., 246

Toakley, A. R., 334

Tochacek, M., 408

Tokuda, M., 104. 173, 188, 502

Tokuoka, T., 194, 198, 205

Tolok, V. A., 537

Tolokonnikov, L. A., 90, 112, 200, 201

Tomita, Y., 198, 285

Tomlenov, A. D., 335

Topp, L. P., 284

Toridis, T. G., 481

Torre, C., 102, 125

Tosyaki, O., 383

Totsky, O. N., 342

Toupin, R., 50, 51

Tozawa, Y., 311

Trakhtenberg, B. F., 495, 498

Tralli, A., 424

Tran-Le Binh, 230, 233, 446, 538-543

Trapp, J. A., 198

Trefftz, E., 518

Tresca, H.:

Chapter I, 10

Chapter II, 71, 78

Chapter III, 97-99, 103-107, 112, 113, 118, $123,125,129-136,144,147,149,154,161$, $162,201,204$

Chapter IV, 255, 262, 265, 271, 280 
Tresca, H. (cont.):

Chapter VI, 348, 390

Chapter VII, 413, 415, 422-424, 427-437, 439, $440,444,445,450,451,455-460$

Chapter VIII, 464, 492, 494, 495, 498, 502, 523, 525, 527

Tribillo, K., 262

Trifan, D., 165, 180

Trofimov, G. S., 384

Trofimov, V. I., 525

Trojnacki, A., 495

Troost, A., 111, 123, 495

Truesdell, C., 39, 40, 49-51, 180, 197

Trumpler, P. R., 243

Truszkowski, W., 55, 216

Tsai, H. C., 285

Tsai, S. W., 114

Tseyler, V. I., 156

Tsien, H. S., 275

Tsurkov, I. S., 440, 457

Tsurpal, I. A., 527

Tuck, R. C., 289

Tulin, V. A., 316

Turbitsyn, V. A., 285

Turner, C. E., 440

Turner, M. J., 284

Turski, K., 62, 168, 169, 171

Tyurin, V. F., 181

Tyurin, Yu. B., 162

Ubaydillayev, A., 492, 535

Uga, T., 499

Ugodchikov, A. G., 194, 538

Ulitskaya, R. I., 495

Unksov, E. P., 45

Updike, D. P., 535

Urbanowski, W., 33, 45, 104, 107, 111, 200, 495

Usami, T., 408

Uvarov, V. V., 113, 200

Vakorin, V. O., 216

Vakulenko, A. A., 147, 149, 155, 158, 185, 194, 357

Valalas, D., 402

Valanis, K. C., 175, 199

Valid, R., 413, 439

Valliappan, S. I, 284, 285, 287

Valter, L. L., 334

Valukonis, P. I., 334, 335

Van den Broek, J. A., 209, 265, 478

Van Dyke, M., 275
Van Iterson, F. K., 69

Van Minh, H., 245

Varadan, T. K., 316

Vasilakis, J. D., 495

Vasilenko, V. N., 528

Vasilev, V. V., 437, 440

Vasin, R. A., 126, 183, 189, 502

Vebra, R. V., 335

Vega, K. E., 126

Vejvoda, S., 536

Velebit, M., 285

Velikoivanenko, E. A., 441, 537

Venkatesan, M. S., 322

Venkateswara, R. G., 284

Vernay, P., 492

Veytsman, R. I., 499

Vigness, I., 73, 523

Vikulenkov, V. P., 481

Vinnakota, S., 338

Vinogradov, A. I., 510

Vinogradova, A. M., 216

Vinogradova, T. P., 456, 534

Vinokurov, L. P., 384

Vitiello, E., 250, 251

Vislavicius, K., 481

Vlasov, V. Z., 50

Voce, E., 56

Vogel, W., 528

Volkov, S. D., 103, 109

Voltchkov, Yu. M., 424

Voronkov, V. E., 481

Vorontsov, V. K., 292

Voronyuk, I. S., 15, 316

Vorovitch, P. P., 286

Vos, R. G., 155

Vozyanov, A. N., 455

Vukotić, R., 403

Vulman, S. A., 500

Wahl, A. M., 346

Wakabayashi, M., 478

Walczak, J., 19, 28

Walsh, P. F., 384

Walters, R. M., 457

Wang, A. J., 272, 346, 423

Wang, Y. S., 199

Warkenthin, W., 112

Warner, W. H., 84, 182

Washizu, K., 271

Wasow, W., 275 
Waszczyszyn, Z., 98, 215, 311, 314, 321-323, $438-440,450,535$

Weber, J. D., 49

Wegmuller, A. W., 285

Wehrli, Ch., 123

Weiler, F. C., 205, 498

Weiner, J. H., 194, 441, 499

Wellinger, K., 523

Wells, C. H., 146

Wen, R. K., 481

Weng, G., 163, 170

Wennerström, H., 285

Westergaard, H. M., 29, 90, 105, 107

Wetherell, E. H., 86

Whalley, E., 498, 500

White, G. N., 86, 139, 144

Whiteman, J. R., 284

Wildhorn, S., 73

Wilhoit, J. C., 333

Williams, J. F., 147, 161, 501

Wilson, J. C., 107

Wincierz, P., 113

Windels, R., 398, 403

Wiseman, H. A. B., 502

Witmer, E. A., 285

Włodarczyk, E., 543

Wnuk, M., 273, 277, 361, 365

Wojewódzki, W., 535, 536

Wojno, W., 158, 199

Wood, R. H., 209, 222, 413, 459

Woodthorpe, J., 111

Woolley, R. L., 62

Worley, W. J., 401

Woźniak, C., 263

Wöhler, A., 248

Wölfel, J., 323, 403

Wright, E. W., 478

Wu, E. H., 285

Wu, E. M., 114

Wu, H. C., 357

Wu, M. H. Lee, 214

Wu, T. T., 192

Wunder, R. A., 523

Xu Bing-Hang, 403

Xu, Bing-Ye, 431, 434, 455

Yablonko, V. Ya., 3

Yagawa, G., 440

Yaghmai, S., 438, 439

Yakushev, V. L., 440
Yamadá, M., 111, 311, 403, 404, 479

Yamada, Y., 389

Yamamoto, Y., 272, 284, 288

Yamantürk, S., 445, 537

Yamasaki, T., 321, 383

Yamashita, H., 188, 502

Yang, C. T., 244, 281

Yang, N. S., 502

Yarin, L. I., 437

Yatsenko, V. F., 111

Yagn, Yu. I., 102, 138, 143, 168, 170, 501, 502

Yakovlev, M. F., 528

Yakovlev, S. P., 111, 112, 200, 201

Ylinen, A., 57, 224, 227, 403

Yokobori, T., 246

Yokoo, Y., 335

Yong, R. N., 125

Yoshida, F., 501

Yoshida, H., 480, 481

Yoshida, K., 457

Yoshimura, K., 311, 479

Yoshimura, Y., 49, 156, 161, 191, 284

Young, T., 65, 286

Yu, C. L., 191

Yufin, S. M., 403

Yurev, A. G., 384

Yushtchenko, O. A., 525

Yüksel; H., 441, 534

Zaborski, A., 468

Zadoyan, M. A., 346, 382, 383, 388, 389, 401, 487, 492

Zagovaylov, L. B., 192

Zakharov, K. V., 114

Zander, G., 198

Zanner, H., 523

Zaremba, S., 39

Zarifyan, A. Z., 385

Zarka, J., 161, 262

Zarubin, V. S., 156, 195, 537, 538

Zarubova, N., 199

Zaytsev, P. I., 400

Zavelani Rossi, A., 215, 290, 291, 423, 468, 480-482, 527

Zawadzki, J., 102

Zelenin, L. P., 501

Zeller, R., 357

Zerna, W., 46, 50

Zhigalkin, V. M., 495

Zhudin, N. D., 480

Zhuk, N. P., 458, 459 
Zhukov, A. M., 65, 96, 104, 111, 112, 138, 139, $170,216,501,504$

Zhuravlev, A. G., 194

Zhuravsky, D. I., 390, 395

Ziegler, H., 48, 87, 88, 123, 132, 147-150, 152, $177,184,268,272,294,366,423,439,533$, Zilauts, A. F., 192

Zieliński, A. P., 499

Ziemba, S., 19, 64

Zienkiewicz, O. C., 36, 88, 114, 284-290

Zingali, A., 479

Zudans, Z., 285

Zveryev, O. A., 525
Zwicky, E. E., 499

Zyczkowski, M.,

Chapter I, 18, 19-21

Chapter II, 29, 57, 58, 61, 73

Chapter III, 98, 130, 149

Chapter IV, 215, 217, 221, 222, 230, 240, 251, 256, 261, 273-277

Chapter V, 295, 311, 314, 321, 342

Chapter VI, 348, 366, 369, 372, 375

Chapter VII, 416, 446

Chapter VIII, 468, 474, 475, 479, 483-496, 498, 499, 503-509, 510-512, 513-523, 525, 527, $529-532,538-543$ 


\section{Subject index}

Acceleration of iterations, 288-289

Angular velocity, 8, 481, 527

Airy's stress function, 256

Alternating plasticity, 247, 466

Alternators, 50, 255

Aluminium, 202, 501-502

Amplitude, 18, 247

Analogy

for kinematic hardening, 149-154

sand-hill, 295

with three-phase current, 34

Analytic functions, 292

Anisosensitivity, 94, 100, 138, 143, 200, 422

acquired, 94, 145

Anisotropic material (body), 70, 94, 109-115, $160,199-205,423,460-461$

Anisotropy

acquired (induced), 94, 157

structural, 113-115, 201

Applicability

of the deformation theory, 84,124

of the yield conditions, 107, 112

Approximation

Chebyshev best, 277

Hermitian, 276-277, 365, 380, 510-512, 515, 521-523

onefold optimal, 277, 333-334

Arches, 323, 335, 384, 404, 481

Axial force, 5; see Normal force

Axiator, 31, 35, 127, 204

Axiatoric subspace, 89

Bar, 2, 293, 348, 462-482

in tension, 223-226

naturally curved, $4,209,306,323,334,342-$ $347,399,403$

naturally twisted, $4,273,366-372$

non-prismatic, 4, 209

thin-walled, 208, 349, 352, 384, 404-408

under thermal loadings, $481-482$
Bar system, 226-229, 478-482

Bauschinger effect, 58-62, 94, 144, 154, 160, $166,177,247,479$

full, 59

ideal, 59,174

in Naghdi-Eringen sense, 60

overideal, 168

Beam, 23, 295-323, 389-402, 478-482

of rectangular cross-section, 231-233, 300-302, 307-316

on immovable supports, 322

Bending

biaxial (oblique), 335-342

biaxial with normal force, 335-338, 339-340, 349,478

circumferential of the wall, 510-512

pseudo-oblique, 156, 336

spherical, 418

with decohesion, 246

with restricted torsion, 408

with shear, 270, 389-402

with tension (normal force), 274, 277, 293-335

with tension and shear, 402-404

with tension and torsion, 388

with torsion, 269, 372-385

Bending effects, 416

Bending moments

in a bar or beam, 5, 293, 295-342, 374, 385, $394,406,469,503$

in a surface structure, 7, 8, 23, 409, 419, 430, 447,

Bimoment, 5, 349, 404-408

Birectangular stress distribution, 323, 421

Bitrapeziform stress distribution, 295, 417-419, 439

Body as a whole, 2, 462-544

Body

couples, 27

forces, 37, 273, 462, 481-482, 496-497, 527-528, 534 
Boundary conditions, 278

in displacements, 252, 484

in stresses, 36, 252, 265, 269, 290, 349, 352, $362,369,393-396,488,515$

Boundary layer, 535

Bounds for displacements at shakedown, 251, 479

Bounds for limit carrying capacity

lower, 264, 349-350, 365, 381, 388-390, 396, $407,423,453$

upper, 267, 269, 350-352, 365, 373, 381, 389, $391,393,434,453,458,503-510,537$

Brass, 171, 188, 501-502

Bulging, 216-217

Burzyński plane, 91

Cartesian coordinates, 26, 38, 39, 255, 350, 361, 373

Cast iron, 501

Centrifugal force, 214

Centroidal curve, 342

Characteristics, 278-281, 343, 457

Circularly symmetric states, 51, 482-502, 524-525

Classification of combined loadings, 21-25

Coefficient of transversal deformation, 72-73, 184,419

Collapse load, 213

Column, 24, 213, 215, 295, 316, 321, 337, 403, $466,468-478$

Compatibility matrix, 283

Comparative states, 71

Complementary specific energy, 224

Complex variable, 526, 527

Composites, 114, 268

Compressibility, 24, 126, 235, 254-255, 273, 348, $357-361, \quad 417-421,438$

plastic, 155

Concavities, 171, 466, 473-477, 532, 543, 544

Concrete, 145, 335, 399, 408, 416, 422, 437, 480, 482,493

Conditions of perfect plasticity, 70-72, 252

for anisotropic bodies, 109-115

for isotropic bodies, 94-109

Cone

circular, 101, 103

elliptic, 106, 422

Consistency condition, 148, 150, 175, 177

Consistent systems, 9, 412, 467, 468-470

Constant strain approach, 288

Constant stress approach, 287
Constitutive equations, $1,10,15,22,58,117$, $141,170,185,189,284,286,413,437,454$, $468,472,502$

of perfect plasticity, $135-136$

of plastic anisotropy, 199-205

of plastic hardening, 172-199

for a cross-section, 294-302, 318-320, 335$337,472-473$

Continuity condition, 366, 506 for material description, 175 for structures, $232,314,450$ relaxed, 222, 233, 239

Continuous body (medium), 18 constrained, 263

Contraction of a tensor, 27

Control

function, 3

point, 156, 170, 171, 177, 307

variable, 13,16

Convergence, 275, 286, 288, 364

in the opposite direction, 289

Convexity, 122, 124, 268, 462, 468, 532

Copper, 187, 188, 202, 501-502

Corner on a trajectory, $79,187,502$

Cosserat continuum, 27

Couple stresses, 27

Crack, 246, 527, 544

Cross effects, 170

Criterion of decohesion, 215, 221, 226, 240, 24I physical, 244-246, 265, 474

Criterion of exertion, 69-71, 95

Criterion of shakedown, 249

Cross-section of bar structures, 2, 4-6 annular, 330, 335, 346, 352, 354

arbitrary, 361-366

bisymmetric, 301, 304, 320, 328, 333, 338

circular, 329-330, 335, 346, 347, 352, 374-382, 481

I, 334, 337-340, 383, 388, 390, 399-401

monosymmetric, 15, 295-300, 317-318, 324-336

multipoint substitutive, 316-321

narrow rectangular, 363-364, 366, 382-383, 388

rectangular, 300-302, 307-316, 329, 339, $340-342,384,388,396-399,403,470$

Cross-section of surface structure, 6-8, 409-461

Curvature, 5, 373, 385, 514

of the strain-neutral layer, 410

of the trajectory, 185, 502

Curvature increment, 410, 411

Curvilinear coordinates, 262-263 
Curvilinear tetragons, $473-477$

Cycle, 119

thermal, 269, 482

Cyclic

collapse, 247

loading, 60-62, 149, 246-251, 439, 465, 544

permutation, 28

plasticity $60-62$

work-softening, 195

Cylinder; see Tube

Cylindrical coordinates, 29-30, 38, 43, 50, 258$259,261,348,352,374,425,469,483,513$

Decohesion, 18, 221, 251, 544

partial, 222

Decohesive carrying capacity, 18, 221-246, 253, 531-532, 541-543

Decomposition of strains 65, 196

additive, 65

multiplicative, 65

Deformable cross-sections, 295, 467, 544

Deformation process, 3 ; see also Loading process and Process

Deparametrization, 263, 326

Deviator, 31, 35, 127, 140, 204, 280, 413, 424, $428,487,512$

Deviatoric

law, 181

plane, 92

space (subspace), 89, 185-189

stresses, 257

strains, 253

Dilatation, 44

Dirac function, 20, 222

Directional cosines, 26, 260

Discontinuities, 275, 277, 405, 423

admissible, 38, 51, 264, 268, 424

inadmissible, 18, 51, 221, 233, 238, 241, 268

Discontinuity surface (line), 38-39, 344, 349, 521

Discretization, 278

Disk

annular, 523-525

decohesive carrying capacity, 233, 239, 531-532

rotating, 214, 239, 527-528

under thermal loadings, 527-532

Displacement equations, 253-255, 361-366, 387-388, 457

Displacement vector, 39-40, 252

Dissipation, 1, 10
Dissipated energy, 66, 143, 167, 246, 266, 294, 465

Distance function, 19

Distribution of plastic zones, 301

Distributions (generalized functions), 222, 264

Divergent series, 289

Double neutral surfaces, 163-165

Drive,

hydraulic, 8

mechanical, 8

Drucker's postulate 120-135, 178, 195, 208, 267, $462,466,544$

Dynamic loadings, 2, 283; see also Impact

Dynamic programming, 291

Eccentric compression, 25, 466, 478

Eccentricity, 9, 24, 466

Effective

strain, 45

stress, 36,70

value of a tensor, 32

Eigenvalues, 28

Elastic carrying capacity, 17, 206, 209, 231, 235, $253,355,365,523$

Elastic limit, 13, 52, 69, 266

conventional, 53

Elastic range, 67-70, 231, 297, 472, 476

Elastic section modulus, 325, 467, 489

Elastica, 299, 321

Elastic-plastic

carrying capacity, 213

interface, $75,292,365$

range, 209, 299, 364-366, 384, 408, 416-420, $437-439, \quad 446-450,526,530,532$

Ellipsoid, 101, 422

Elliptic integrals, 321, 381-382, 487, 490-492, 508-509

Endurance limit, 248

Equations

elliptic, 275, 377, 386

hyperbolic, 275, 281

of compatibility, 24, 49-51, 236, 240, 252, 287, $295,352,369,468,485,513$

of internal equilibrium, 37, 235, 240, 251, 264, 269, 286, 287, 290, 343, 349, 350, 362, 368, $373,386,392,446,468,471,484,513,539$ parabolic, 275, 377

Equipressure cross-sections, 92

Eulerian coordinates, 40, 65, 196

Euler's angles, 33, 160

Even materials, 93 
Evolution laws, 158

Excessive work, 127

Exertion of the material, 18-21

Exertion factor, 3, 5-9, 12-16, 19-22, 58, 82,

$119,195,304,462,481,527$

accessory, 9, 11, 105, 450, 466

basic, $9,16,87,412,462$

Experimental verification, 167-171, 202, 205, $314,337,338,357,385,389,401,402,408$, $461,482,499,500-502,525,528,533,535$

Extensions in a surface structure, 410, 417

Extra-stresses, 114

Falsi rule, 278

Fatigue, 18

for multiaxial stress states, 248

limit, 247

low-cycle, 18, 208, 247

thermal, 249

Fibre-reinforced, 114, 436

Finite differences, 281-282

Finite elements, 282-285, 467-468

Finite (large) deflections, 311-316, 320-321, 416, $434,437,438,440,445,457,459,532,535,536$

Finite (large) strains, 41-43, 48, 50, 138, 196-199, 205, 225-226, 240-243, 272, 416, 467, 495, 532, 536

Flow rule, 78, 129

associated, 117, 124, 148, 201, 208, 294, 423, $428,434,442,494,535$

non-associated, 125, 130, 201, 269

for a cross-section, 294, 423

Fourier series, 289, 292, 377, 378, 498, 516, 519

Frames, 335, 384, 389, 480

Fracture mechanics, 249

Friction, dry, 62, 146

Functional, 16, 264, 267, 268, 289, 350-352, 391, 505

analysis, 289

\section{Gaussian coordinates, 409}

\section{Generalized}

displacements, 9, 209, 210, 223, 464, 467

external forces, 9, 207, 223, 467, 478, 483

plastic strain rates, 207, 442

strains, 5, 9, 285, 304, 354, 360, 411, 452, 458, 467

stresses, 5, 9, 207, 265, 285, 301, 304, 349, 350, $354,360,387,388,390,404,409,411,444$, $452,458,467,478$
Generic stress point, 156

Geometric

hardening, 210, 214

softening, 210,214

stiffness matrix, 468

Geometrically determinate, 12, 287

Geometry changes, 15, 25, 84, 123, 196, 207-215, $217,244,272,462,466-477,495,535,544$

Gradients

of displacement, 42

of plastic potential, 118

geometric, 208, 294

static, 208, 294, 390

Gravity, specific, 8

Haigh diagram, 247-248

Half-plane, 543

Half-space, 543

Hardening; see Plastic hardening

Hardening modulus, 55

Harmonic function, 365

Heat sources, 8, 499

Hencky-Prandtl nets, 281

Hermite's boundary approximation, 276, 365, $380,510-512,515,521-523$

Hidden parameters, 158

Hole, 292, 416, 526, 529, 534

Hooke's law, 64, 67-69, 117, 127, 153, 245, 354, 448,476

Humidity, 4, 6, 8, 11, 69, 93, 105, 184

Hyperboloid, 101

Hyperplanes, 444

Hypersphere, 187

Hypersurface, 277, 407, 431, 442, 444

Hypotheses of failure, 69, 71, 147, 206, 244, 334

Huber-Haigh-Hencky, 70, 96

Sharpened pencil, 107

Tresca-Guest, 97-99, 161

Hypothesis

of stabilization, $84,154,356,398$

of local determinacy, 189

of plane cross-sections (Bernoulli), 263, 296, $352,373,381,469$

of straight normals (Love-Kirchhoff), 263, $410,411,456$

Hysteresis loop, 248

Ilyushin space, $89, \quad 185-189$

Impact, 529, 543

Inadaptation (non-shakedown), 247, 268-269 
Incompressibility, 3, 15, 72, 79, 110, 153, 166, 204, 225, 253-254, 262, 266, 354-357, 373, $399,417,424,437,446,484,512,526$

Incremental collapse, 247, 482, 523, 538

Incremental laws, 175

associated, 175, 204

non-associated, 181, 204

Independence of exertion factors, 25, 462, 467

Inextensional deformation, 417

Infinitesimal element, 2, 5, 37, 38

Initial strain process, 288, 479

Initial stress process, 287

Instability, 18, 216-217, 496

in Drucker's sense, 125, 134

in Lyapunov's sense, 134

Intensity

of a tensor, 32

of plastic strains, 139

Interaction (limit) surfaces (curves), 16, 21, 293, 533, 544; see also Surface

decohesive, 18, 467, 531, 542-543

elastic. 17, 22, 266, 415, 463, 498, 527, 529, 539-540

for a cross-section, 302-306, 323-336

for simple models, 468-477

plastic, 17, 22, 207, 304, 323-336, 339, 341, $349,364,372,374,383,394,398,403,415$, $421,425,435,442,453,463,490,508,510$, $518,524,527,542$

Invariance, 5, 8, 11, 27

Invariant

basic, 27-28, 34, 36, 44, 115, 135

common of several tensors, $12,34,70,146$, 158,182

cylindrical, 29-31, 36, 44, 92, 260

geometrical, 185

Lode's parameter, $32,46,75,78,80,92,118$, 131,173

octahedral, 29, 36, 45, 71

of finite strains, 46

of the axiator, $31,44,90$

of the deviator, $31,44,90,105$

polynomial, 34

principal, 28-29, 36, 44

symmetric, 28,30

Inversion of a series, 518

Irradiation. 4, 11, 107, 456, 462, 499, 500, 534, 538

Irreversibility, 1, 2, 9, 73, 163

condition, 175

Isoperimetric problem, 351, 394, 505
Isosensitivity, 93-94, 100, 109, 173, 417, 494

Isotropic tensor functions, 136

Isotropy, 93-94, 94-109, 135-199

Iterations, 282, 286-289, 529

Kinematic approach, 267, 458

Kinematically admissible, 266-269, 270, 395, $398,400,443,508$

Kinematically determinate, 268

Kinematics of a rigid frame, 149-154

Kronecker's symbol, 26

\section{Lagrangian}

coordinates, 40, 65, 196, 225, 240, 296, 313

function, 351, 394, 505

multipliers, 351, 506

Law

of critical shear stress, 189

of shape change, $68,72,79,252,253$

Hencky-llyushin, 73-78, 115, 203, 252, 352, $373,417,424,447,512,530$

Levy-Mises, 78-79, 116, 252, 352, 373, 424, 512

Nadai-Davis, 196-197, 240-243

Prager-Laning, 178-179, 183, 187

Prandtl-Reuss, 79-82, 116, 130, 131, 252, 440

of similarity of deviators, $75,78,118,129,132$, $149,153,197,200,280,413,424,428$, 487,512

of volume change, $68,72-73,75,118,203,252$, 417,530

Layer, 446, 459, 495, 500

Length of the trajectory, 39, 46, 141, 185

Levels of analysis of plastic deformations, 2-9

Limit analysis, 210, 401, 423, 437, 445, 479, 481, 495

Limit state, 18, 222, 421, 423, 445, 527, 534, 536

Limit carrying capacity 206-221, 228, 231, 253, $265,532,542$

of the cross-section of a bar, 294, 299, 323-325, $339-342,343-347,349-352,365,400,510$ of the cross-section of a surface structure, $421-437,442-445,451-461$

of the second kind, 212

Limit surfaces (curves), 17, 90, 276, 293, 413; see also Interaction surfaces

cylindrical, 95-100, 105, 151, 261

rotationally symmetric, 100-103, 104, 261

with cutoffs, 108

Limited interaction, 434, 439, 440, 455 
Limiting procedure, 208, 228

Linear programming, 290-291, 533

Linearization, 290, 459, 478, 515

Lines of maximal slope, 343-347

Load carrying capacity, 206, 213; see also Limit carrying capacity

Loading

combined, 21, 76, 273, 462

hard, 3, 8, 13, 247

in broader sense, 11

in narrower sense, 10-16

proportional, 14, 15, 19

reverse, 11, 13, 16, 58, 63, 139

secondary, 11,13

simple, 14, 83, 169, 201, 528

soft, $3,8,13$

thermal, 222, 266, 286, 467, 481, 523, 529-532, 537-538

variable, $18,246-251,533$

Loading multipliers, 8, 253, 270

Loading path (trajectory), 16, 169, 272, 304, 388, $399,456,495,498,501,528,535,544$

Loading process, 3,16

Loading program, 246, 464

Loading surface, 18, 164

Localized deformation, 245

Lode's parameter, $32,46,75,78,80,92,118$, 131,173

Magneto-plasticity, 4, 499, 523

Material (body, medium)

anisosensitive, 94, 138, 143, 200, 435, 436, 439, $443,445,460,533$

anisotropic, 70, 94, 109-115, 138, 199-205, $423,460-461$

asymptotically perfectly plastic, 57-58, 210, 223-224, 227-229

indifferently (neutrally) stable, 121, 208

isosensitive, 94, 100, 109, 173, 417, 494

perfectly elastic-plastic, $54,72,73-86,126-136$, 181, 226, 243, 296-316, 319-320, 335-339, 354-366, 416-420, 438, 446-450, 463-466, 529-532, 538-543

perfectly rigid-plastic, $54,136,210,435,442$, 462

rigid-plastic, 15, 56, 78, 221, 265, 294

stable, 121,128

stable in certain directions, 122,128

temporarily stable, 122

Material coordinates, 40, 65

Material derivatives, 47
Material stability, 120-135, 544

Material tensor, 104

Mathematical programming, 289-291, 528

Matrix, 179-180, 278, 279, 283-284, 437-438, $468-474,480$

Maximal carrying capacity, 213, 214, 291, 295, $440,474,495$

Mean stress, 18, 109, 247

Mean value of shearing stresses, 71

Mechanism of plastic collapse, 17, 18, 207, 214, $218,221,226,294,459$

Mechanisms of plastic flow, 162

dependent, 182

independent, 162

Melting, 441, 534

Membrane forces, 8, 409, 417, 430, 441, 461

Membrane state, 410, 413-416, 460, 510

Methods

analytical, 262-277

numerical, 277-292

of characteristics, 278-281

of collocation, 314

of direct integration, 278

of finite differences, 281-282, 439, 440, 457, 529,534

of finite elements, 282-285, 389, 424, 450, 457, $478,480,533$

of integral equations, 292

of iterations, 286-289, 457, 529

of mathematical programming, 289-291, 528

of reduction of plasticity equations, 253-262

of strained coordinates (PLK), 275

of successive elastic solutions, 286-288, 366, $404,408,440,457,478,498,534$

SN-EVM (Ilyushin), 292

variational, 270-273, 350-352, 394, 397, 427, 439,505

Microcracks, 184

Micrononhomogeneity, 160

Microstresses, 66, 143, 146, 150, 180

Model of Ryder-Shanley, 475

Models of plastic bodies, 62-64

Mohr's circle, 262

Moment states, 416-424

Moments of inertia, 297, 336, 392

Monocrystal, 109

Movable (moving) loads, 9, 291, 389, 401, 480, 529

Multilayer pressure vessels, 495, 500, 537

Multipoint (multilayer) cross-section, 6, 316-321, $438,441,445,461$ 
Necking, 18, 52, 216-217, 222, 225, 245, 295

Neutral

axis, 296, 324, 337, 340

layer, 304

surface (curve); see Surface

surface, physical 427,436

Nickel, 168, 501

Non-existence of mechanism of plastic collapse, 222

Non-homogeneity, 4, 6, 15, 24, 25, 59, 147, 200, $209,223,273,335,343,346-347,419,422$, $433,435,456,484-487,526$

substitutive, 343

Non-linear programming, 291, 443

Normal forces

in a bar, $5,293-335,353-372,387,395.407$, $469,488,503$

in a surface structure, 7, 8, 23; see also Membrane forces

reduced with respect to pressure, 489

Normality rule, 123, 124, 208, 462, 468

Notation

engineering, 26, 35, 43, 68, 75, 110

indicial (mathematical), 35, 43, 50, 110, $451-453$

incremental, 81

vectorial, 179,185

Notch, 222, 251, 335, 366, 372

Octahedra! strains, 45

Octahedral stresses, 36, 71

Odqvist parameter, 142-145, 158, 161, 177, 183, $185,193,197$

Operations on power series, 274-275, 326, 518

Optimal design, 58, 290, 335, 399, 401, 434, 445, $459,461,478-481,527,532,534-536,544$

Optimal temperature distribution, 499, 500

Orthotropy, 110, 200, 201, 419, 422, 436-437, $438,440,445,450,460-461,492$

Overlapping, 230

Own weight, 223-229

Parabolic cylinder, 431, 435

Paraboloid, 101, 103, 124, 431

Paradox

$\mathrm{Hu}, 90$

Stüssi-Kollbrunner, 222, 229-233

Parametrization, 253

of equilibrium equations, 255

of the yield condition, 218, 235, 240, 260-262, $350,447,504,524$
Perfectly elastic-plastic material, see Material

Perfectly plastic flow, 52, 70, 158, 175 uncontained, 207

Permutation symbols, 50, 255

Perturbation method, 273-275, 281, 358, 364-365, $370-372,374-382,387,418,439,481,515-$ $521,523,525,527$

Photoplasticity, 292

Plane body plastic model, 192

Plane-plastic medium, 192

Plane strain, 97, 280-281, 399, 526

Plane stress, 97, 290, 399, 524-532

Plastic hardening, 52, 55-58, 137-199, 201-205, $244,247,321-323,366,389$, 401-402, 408, $437-441,472,494,498,523$

anisosensitive, 144

anisotropic, general, 157-163, 198

apparent, 59, 145

generalized isotropic, 144-145

isosensitive, 144

isotropic, 61, 141-144, 166, 168, 191, 198, 202, $356,424,528,537$

kinematic, $61,132,145-154,165,168,178$, $203,424,533,537,538$

kinematic complete, 147

kinematic direct, 147

linear, 55, 144, 146, 159, 166, 382, 401, 408, $438,472,537$

mixed, 154-155, 162, 166, 169, 439

non-proportional, 203, 204

overkinematic, 168

piece-wise linear (multiple linear), 56, 161-162, 204, 215, 272, 318-319

polynomial, 84

power, 56, 84, 174, 337, 402, 408, 525, 527

proportional, 202

true, 59

Plastic

corners, 136, 155-157, 170-171, 181-182, 184, 192, 501

hinge, 230, 481

moduli, 110, 200

platform, 214

potential, 115-118, 135, 176, 199, 204, 271

reaction, 294

softening, 124, 165, 195-196, 215, 272, 285, 533,544

strain, 64-66

waves, $357,497,538,543-544$

work, 66-67, 141, 143, 144, 158, 175, 177, 193, 202, 249 
Plastification (yielding)

full, 217-221, 323-334, 343, 359, 389, 390, $424-437,513,517$

one-side, 297-298, 300-302, 312-313, 315, 337, $446,472,540$

two-side, 298-299, 302, 310, 314, 315, 337, $444,446,473,542$

Plates, 290, 416-424, 532-535

circular, 23, 423, 434, 438, 532

perforated, 115,423

reinforced, 437, 459, 533

rectangular, 533, 534

under body forces, 534

under normal forces and in-plane loading, 533

under thermal loadings, 534

Point of the body, 2-4, 87-205

Polycrystalline aggregate, 109, 189-192

Polycrystalline material, 113

Polyhedrons, 290, 434, 444

Pontryagin's maximum principle, 291, 534

Post-yield behaviour, 214

Postulate

of continuous description, 84, 139

of isotropy, 186-189, 192, 502

of $I_{2}$-isotropy, 187

of plasticity, 122

of thermoplasticity, 126

Power of plastic strains, 80

Power series, 229, 273-275, 286, 364, 492, 515, 518

generalized, 229, 274-275, 323-333, 522, 541

Prager spaces, 87

Prandtl stress function, 256, 257, 361, 372, 385-387

Pressure

external, 488, 535

hydrostatic, 24, 72, 95

internal, 23, 482, 487, 498, 501, 503-523, 535

normal, 488, 524, 526, 543

tangential, 488, 515, 525, 526, 543

variable, 493, 495, 499, 523

Pressure vessel, 215, 216, 495, 500, 536-537

Prestressing, optimal, 20, 21

Prevailing

bending, 454, 455

membrane forces, 454

pressure, $515-518$

torsion, 518-521

Principal

axes, 5, 260

diagonal, 26
Principal

directions, 6, 28, 29, 33, 45, 112, 130, 136, 139, $196,413,416,417,434,501$

initial curvatures, 409, 413, 416

plastic strains, 160

strains, 45, 92, 138, 166, 424

stresses, 35, 90, 91, 98, 107, 160, 427, 501

values, 28, 33-34

Principle

of maximal power, 271

of maximal plastic resistance, 272

of maximal plastic work, 272

of rigidification, 25, 35, 52, 84, 197, 207, 210-214

of superposition, 2, 385

of virtual work, 5, 264, 267, 282, 284, 467

Probability, 20

Process (deformation process)

cyclic, 119

fully active, 10

mixed, 14

neutral, 72, 77, 82, 140, 141, 167

non-isothermal (anisothermal), 193, 205

partly active, 10

plastically active, $10,14,17,58,72,77,81$, $139,155,172,176,193,225,304,438$

plastically passive, $10,17,58,72,74,81,139$, $167,172,176,225,304,438,544$

proportional in strains, 83

proportional in stresses, 83

rectilinear, 86

simple, $83,86,139,172,268,357$

spontaneous, 11-14

Proportional limit, 58, 69

Pseudo-state variables, 158

Pseudo-statically-determinate, 280

Purely plastic range, 210

Quadratic forms, 451-456, 458, 459, 461

Quadratic programming, 291

Quasi-cycle, 119-120

Quasi-cyclic (quasi-repeated) processes, 165, 174,246

Quasi-cyclic tests, 171

Quasi-total differential, 176

Ratcheting, 247

thermal, 249, 482, 499

Rate of dissipated work, 207

Reduced strain, 137, 201 
Reduced stress, 70-72, 137, 201, 206

Relations along characteristics, 280

Relaxation method, 281, 383

Reloading, 163

Reversibility, 119

Rotational symmetry, 258, 424, 446-450, 536, 538-543

in a narrower sense, 259

Rupture, 107-109, 244-246

Safety factor, 19

Sandwich structure, 6, 410, 435

cross-section, 426, 441-450, 459-460, 544

Schematizations of the stress-strain diagram, $53-58,207$

special, 57

Secant modulus, 53, 202, 358

Second-order constitutive laws, 184

Second-order effects, 181

Secondary plastic strains, 310

Seismic analysis, 479

Self-stresses, 249, 266, 310, 464, 537

Semi-empirical laws, 183

Shakedown domains, 250, 465, 474, 476, 477, 479

rectangular, 250-251, 465

Shakedown problems, 2, 18, 246-251, 265-266, 268-269, 291

of cross-sections, $294,307,311,356,384,401$, $403,434,441$

of bars and beams, 463-466, 474-479

of surface structures, 528-529, 533-535, 536-538

of tubes and cylinders, 495, 500, 523

under thermal loadings, 250, 482, 500, 534, 538

Shear (shearing) forces

in a bar or beam, 5, 293, 298, 344, 390-402, 406,503

in a surface structure, 7, 410, 457-459, 536

Shear tests, 51

Shearing strain, 5, 43, 76-79, 81, 352 extremal, 45, 183

Shearing stress (tangential stress), 35, 76-79, 81, $342-347,392,405,458$

extremal, 37

maximal, 37, 71, 137, 183

Sheet

with holes, 526-527, 529

with rigid circular inclusion, 234-243, 529-532
Shells, 535-543

anisotropic, 461

circular cylindrical, 425, 427, 431, 443-450, 535-536, 538-543

conical, 434, 536, 537

multilayer, 495, 500, 537

of moderate thickness, 456,460

reinforced, 437, 535, 537

rotationally symmetric, $440,446,459,536$, 537-538

sandwich, 441-450, 535-543

shallow, 536

spherical, 434, 459, 536, 537

toroidal, 450, 536, 543

under pressure and axial forces, 535

under thermal loadings, 537-538

Simple deformation process, 83, 169, 172, 201

Simpler combined case in surface structures, $425,460-461$

Singular point, 281

Singularities, 329, 364

Slip lines, 51, 403

Slips, 189-192

Small parameter, $273,358,364,370,375,418$, 515

Solution

in the broader sense, 16

in the narrower sense, 16

Space

nine-dimensional, 87-88, 117, 127, 147

six-dimensional, 88, 189

five-dimensional, $89,185-189$

three-dimensional, 90-91, 95-105

two-dimensional (plane), 91-93

of dependent variables, 16

of independent (control) variables, 16

of the exertion factors, 16-18, 21, 87, 193, 208, 277

of plastic strains, 142,167

of principal strains, 166

of principal stresses, 90-91, 95-105

of principal values of a tensor, 29, 90, 204

Spatial coordinates, 40, 65, 196

Spheres, thick-walled, 500

Spherical coordinates, 20, 38, 44, 190

Spin tensor, 47

Springs, 346, 475

Stability 468,544

in Drucker's sense, 126-135

Stabilization of stresses, 83, 154, 356, 398

Stages of deformation processes, 243-244 
Starting axis, 324-333

State variables, 157

internal, 158, 171, 183, 194, 199, 284

Statically admissible, 263-266, 270, 349, 384, 392,510

Statically determinate, $12,265,287,292,342-347$, 399, 441, 476

Statically partially admissible, 269-270, 346, $350,365,393,396,458,503-510$

Statically indeterminate bars, 462-466, 479, 481

Statically indeterminate beams, 229-233, 265, 398,404

Steel, 188, 202, 245, 501-502

Stiffness matrix, 284, 438

Strain, 39-51

conventional (Cauchy), 52

elastic, 64

logarithmic (natural, Hencky), 47-49, 51, 138, $142,196,197,216,240$

plastic, 64-66

reduced, 137, 201

residual, 476

Strain

axiator, 44

components, 3, 43, 252

deviator, 44, 82

intensity, 45, 76, 137, 201, 286, 437, 439

measure generalized, 48

quasi-cycle, 120

rate, $46,78,272$

tensors, $40-43$

Strain-hardening, 64, 142, 203

Stress, 35-39

nominal, 52

reduced, 70-72, 137, 201, 206

residual, 249, 271, 310, 338, 476, 533

true, 52, 138, 197, 212, 216, 240

Stress

axiator, 35,68

components, 3, 11, 35, 252

deviator, 35, 74, 78, 83, 181, 197

functions, 255-259, 361, 372, 383, 513

intensity, 36, 70, 137, 201

quasi-cycle, $120,126,155,171$

rates, 39, 80, 175, 272

space, $16,87,90-91,95-105,188$

tensor, 12, 35, 116

Stress-strain diagram, 51-67

true, 52

Strength, 18, 103, 244
Stretchability of the axis, 295, 311-314, 320 321

Stretching tensor, 47

Subdifferential set, 118

Successive elastic solutions, 286-289, 366, 404, $408,440,457,478,498,534$

Summation convention, 9, 26, 35, 278

plane, 411,451

Supplementary loadings, 286-287

Supplementary strains, 287-288

Surface (curve)

concave, $122,171,466,473-477,532,543,544$

convex, 19, 122, 208, 462

initial neutral, 69, 473, 501

inner neutral, 164

loading, 18, 164

modified elastic, 18, 251, 266, 307-311, 464, $468,474,477,535$

neutral, $18,69,75-76,79,126,156,163$

neutral double, 163-165

neutral in the strain space, 165-167

of maximal carrying capacity, 215

outer neutral, 164

subsequent neutral, $69,137-171,215,307-311$

thermomechanical, 194

yield, 19, 163, 164, 207, 431

Surface structure, 2, 409-461, 532-543

multilayer, 438

reinforced, 436-437

Symmetry of the tensor, 37

System of coordinates, 5, 262

System of differential equations, 278-281

Systems of structural analysis, 285

Tangent modulus, 53, 57

Tangential forces, 7

Temperature, 5, 8, 11, 69, 93, 102, 105-107, 115, $119,162,171,184,193-195,249,272,287$, $433,441,455,462,481,482,497-500,501$, $523,529-532,534,537-538$

Tension

with internal pressure, 188

with torsion, $22,168,187,192,352-372$

with torsion and biaxial bending, 385-389

Tensor,

curvilinear, 43

fourth-order, 67, 109, 201

of curvatures, 6,12

of directions and similarity, $33,74,78,80,82$

of elastic moduli, 67

of inelastic deformation, 199 
Tensor

of membrane forces, 414

of plastic moduli, 109-113, 201

of residual microstresses, 146

of stress functions, 255

of transformed stresses, 200

second-order, 26-34, 116

skew-symmetric, 27

symmetric, 424

Tensor spaces, 88,89

Tensorial linearity, 170

Tensorial non-linearity, 181, 182, 187

Testing machine, 8

Theorem

Ilyushin's on simple loading, 83

Koiter's on non-shakedown, 268-269

Melan's on shakedown, 265-266, 307, 464, 476,479

Schwedler-Zhuravsky, 390, 395

Theory (of plasticity)

based on slip, 189-192, 356

functional, 198

geometrical (Ilyushin), 185-189, 356, 492, 495

incremental (flow, anholonomic), 175-185, 204-205, 286, 288, 438, 440, 502, 528

of elastic-plastic flow (Prandtl-Reuss), 80

of finite plastic strains, 196-199, 240-243

of locality of deformations, 191, 204, 493

of plastic anisotropy, 199-205

of plastic flow (Levy-Mises), 78-86, 373, 438

of plastic softening, 195-196

of small elastic-plastic deformations (Hencky-

Ilyushin), 73-78, 82-86, 115, 203, 252, 352.

$373,417,424,447,512,530$

rate-type, 198

total (deformation, holonomic), 172-174, 203-204, 271, 284, 502

Thermofrettage, 499

Thermomechanical surface, 194

Thermoplasticity, 4, 163, 193-195, 205, 255 . $272,282,285,286,445,456,467,528-532$

Thin-walled section, 5, 383, 389, 390, 399-401, 403-408

deformable, $295,467,544$

Time factor, 1, 78, 80

Torsion,

pure, 295

restricted, 408

with rotation, 481
Torsion with shear, 342-347, 349

Trajectory of the process, 16,18

Transformation 16, 82, 246

of tensors, 26

Dirichlet's, 489

Transition from one level to another, 22, 206-292

Transposition of a matrix, 284

Transversality condition, 506

Triaxial (hydrostatic) compression, 24, 95

Trigonometric representation, 438

Truss, 212, 467, 479

Tube, thick-walled, 23, 24, 174, 210, 217, 258, $268,330,482-500,512-523$

rotating, 214, 496-497

under tension, torsion and pressures, 489-496

under tension, torsion, pressure and bending, 512-523

under thermal loadings, 497-500

Tube, thin-walled, 4, 12, 168, 185, 331, 500-510 curved, 215

under general combined loadings, 502-510 under tension with torsion, 218-221

under tension, torsion and internal pressure, $483,495,500-502$

Twisting moment

in a bar or beam, 5, 293, 344, 353-389, 395, 488,503

in a surface structure, $7,409,416,457$

Ultimate load (strength), 213

Ultrasounds, 500

Uncoupling of the equations, 259, 515

Uniaxial tension-compression test, 12, 25, $51-67,137,145,173$

Uniform curve of plastic hardening, 137-141, 154, 172, 201, 202, 204, 502

Uniqueness, 123, 126, 161, 208, 210, 214, 217, $271,391,398,468$

condition, 175

Unit angle of natural twist, (initial angle of twist), 4, 366

Unit angle of twist, 5, 273, 352, 385, 485, 514

Unit angle increment, 411, 469

Unit distortion energy, 69

Unit elongation, 5, 385, 485, 514

Unit vector, 127

Unit sphere, 20

Unloading, 10-16, 58, 63, 164

full (total), 310, 439

proportional, 14 
Unloading modulus, 65,126

Unstretchability of the axis, 299-300, 314-316, 321

Vacancy, 230

Variable repeated loading, 188, 246-251, 265266, 268-269, 356

Variable stiffness method, 286-289, 537

Variational approach, 350-352, 394, 397, 427, 439,505

Variational principles, 271-273

Vector spaces, 87-93

Virtual work, 9, 264, 267, 282, 284, 304, 411. 462, 464, 467, 469

Warping, 361, 365, 373, 387

Warping group, 5; see Bimoment

Warping moment, 5, 349, 404-408

Work-hardening, 64, 143, 203

Yield condition, 70-72, 78, 94-115, 348, 413 approximate, 129

averaged, 455

Burzyński, 101-103, 113, 422, 443, 497, 528

elliptic "ad hoc", 104-105, 262, 348, 458

for the cross-section, 299, 426
Yield condition,

Huber-Mises-Hencky (HMH), 15, 71, 81, 89, $96,101,109,129-135,348,353,413,418$, $424-427,442-443,484,503,524,529$, 539-543

Mises-Hill, 109-112, 199-200, 460-461

of maximal normal stress (Galileo), 244, $434-435,460$

Schmidt-Ishlinsky-Hill, 100, 435, 464

Tresca-Guest (TG), 71, 97-99, 112-113, 129-135, 204, 348, 427-434, 440, 444-445, $460,464,525,533$

Yield lines, 435, 533

Yield point (yield stress)

conventional, 53

in shear, 76, 343

lower, 13, 52

second, 215

upper, 13, 52

Yield-point load, 206

Yield surface, 19, 163, 164, 207; see also Interaction surface, plastic

Zone

elastic, 252, 297, 354, 392

plastic, 252, 297, 354, 392, 419, 480, 482, 500, 534 